\title{
Laboratory Guide to \\ Early Life History Stages of Northeast Pacific Fishes
}

Ann C. Matarese, Arthur W. Kendall, Jr., Deborah M. Blood, and Beverly M. Vinter

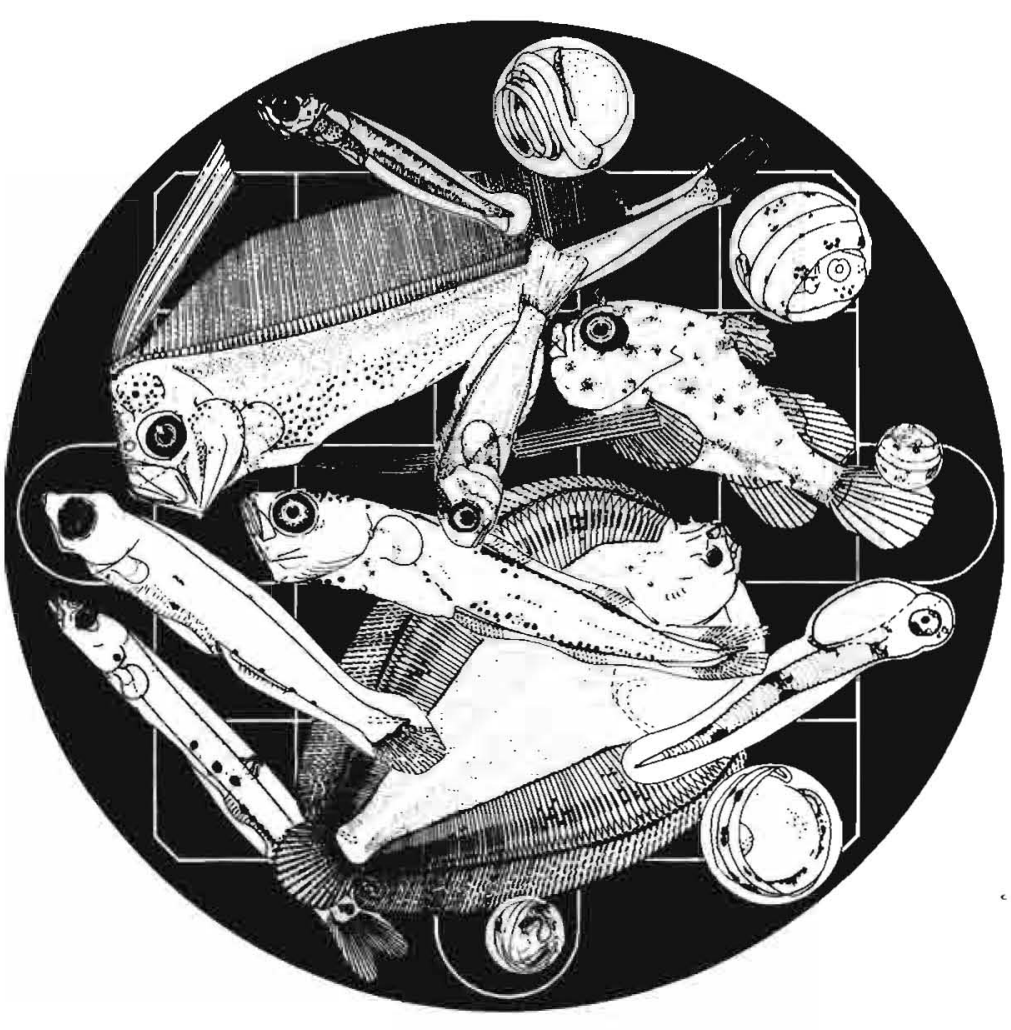

U.S. Department of Commerce 


\section{NOAA TECHNICAL REPORT NMFS}

The major responsibilities of the National Marine Fisheries Service (NMFS) are to monitor and assess the abundance and geographic distribution of fishery resources, to understand and predict fluctuations in the quantity and distribution of these resources, and to establish levels for their optimum use. NMFS is also charged with the development and implementation of policies for managing national fishing grounds, development and enforcement of domestic fisheries regulations, surveillance of foreign fishing off United States coastal waters, and the development and enforcement of international fishery agreements and policies. NMFS also assists the fishing industry through marketing service and economic analysis programs, and mortgage insurance and vessel construction subsidies. It collects, analyzes, and publishes statistics on various phases of the industry.

The NOAA Technical Repor NMFS series was established in 1983 to replace two subcategories of the Technical Reports series: "Special Scientific Report-Fisheries" and "Circular." The series contains the following types of reports: Scientific investigations that document long-term continuing programs of NMFS; intensive scientific reports on studies of restricted scope; papers on applied fishery problems; technical reports of general interest intended to aid conservation and management; reports that review in considerable detail and at a high technical level certain broad areas of research; and technical papers originating in economics studies and from management investigations. Since this is a formal series, all submitted papers receive peer review and those accepted receive professional editing before publication.

Copies of NOAA Technical Reports NMFS are available free in limited numbers to governmental agencies, both Federal and State. They are also available in exchange for other scientific and technical publications in the marine sciences. Individual copies may be obtained from; U.S. Department of Commerce, National Technical Information Service, 5285 Port Royal Road, Springfield, VA 22161. Although the contents have not been copyrighted and may be reprinted entirely, reference to source is appreciated.

48. Widow rockfish: Proceedings of a workshop, Tiburon, California, December 11-12, 1980, by William H. Lenarz and Donald R. Gunderson (editors). January 1987, $57 \mathrm{p}$.

49. Reproduction, movements, and population dynamics of the southern kingfish, Menticirrhus americanus, in the northwestern Gulf of Mexico, by Stephen M. Harding and Mark E. Chittenden, Jr. March 1987, 21 p.

50. Preparation of acetate peels of valves from the ocean quahog, Arctica islandica, for age determinations, by John W. Ropes. March 1987, 5 p.

51. Status, biology, and ecology of fur seals: Proceedings of an international workshop, Cambridge, England, 23-27 April 1984, by John P. Croxall and Roger L. Gentry (editors). June 1987, 212 p.

52. Limited access alternatives for the Pacific groundfish fishery, by Daniel D. Huppert (editor). May 1987, 45 p.

53. Ecology of east Florida sea turles: Proceedings of the Cape Canaveral, Florida, sea turtle workshop, Miami, Florida, February 26-27, 1985, by Wayne N. Witzell (convener and editor). May 1987, $80 \mathrm{p}$.

54. Proximate and fatty acid composition of 40 southeastern U.S. finfish species, by Janet A. Gooch, Malcolm B. Hale, Thomas Brown, Jr., James C. Bonnet, Cheryl G. Brand, and Lloyd W. Reiger. June 1987, 23 p.

55. Proximate composition, energy, fatty acid, sodium, and cholesterol content of finfish, shellfish, and their products, by Judith Krzynowek and Jenny Murphy. July 1987, $53 \mathrm{p}$.

56. Some aspects of the ecology of the leatherback turtle Dermochelys coriacea at Laguna Jolova, Costa Rica, by Harold F. Hirth and Larry H. Ogren. July 1987, 14 p.

57. Food habits and dietary variability of pelagic nekton off Oregon and Washington, 1979-1984, by Richard D. Brodeur, Harriet V. Lorz, and William G. Pearcy. July 1987, 32 p.

58. Stock assessment of the Gulf menhaden, Brevoortia patronus, fishery, by Douglas S. Vaughan. September 1987, 18 p.

59. Atlantic menhaden, Brevoortia tyrannus, purse seine fishery, 1972-84, with a brief discussion of age and size composition of the landings, by Joseph W. Smith, William R. Nicholson, Douglas S. Vaughan, Donnie L. Dudley, and Ethel A. Hall. September 1987,23 p.

60. Gulf menhaden, Brevoortia patronus, purse seine fishery, 1974-85, with a brief discussion of age and size composition of the landings, by Joseph W. Smith, Eldon J. Levi, Douglas S. Vaughan, and Ethen A. Hall. December 1987, 8 p.

61. Manual for starch gel electrophoresis: A method for the detection of genetic variation, by Paul B. Aebersold, Gary A. Winans, David J. Teel, George B. Milner, and Fred M. Utter. December 1987. 19 p.

62. Fishery publication index, 1980-85; Technical memoradum index, 1972-85, by Cynthia S. Martin, Shelley E. Arenas, Jacki A. Guffey, and Joni M. Packard. December 1987, $149 \mathrm{p}$.

63. Stock assessment of the Atlantic menhaden, Brevoortia tyrannus, fishery, by Douglas S. Vaughan and Joseph W. Smith. January 1988, 18 p.
64. Illustrated key to penaeoid shrimps of commerce in the Americas, by Isabel Pérez Farfante. April 1988, 32 p.

65. History of whaling in and near North Carolina, by Randall R. Reeves and Edward Mischell. March 1988, 28 p.

66. Atlas and zoogeography of common fishes in the Bering Sea and northeastern Pacific, by M. James Allen and Gary B. Smith. April 1988, 151 p.

67. Index numbers and productivity measurement in multispecies fisheries: An application to the Pacific coast trawl fleet, by Dale Squires. July 1988, 34 p.

68. Annotated bibliography II of the hard clam Mercenaria mercenaria, by J.L. McHugh and Marjorie W. Sumner. September 1988, $59 p$.

69. Environmental quality and aquaculture systems: Proceedings of the thirteenth U.S.-Japan meeting on aquaculture, Mie, Japan, October $24-25$, 1984, edited by Carl J. Sindermann. October $1988,50 \mathrm{p}$.

70. New and innovative advances in biology/engineering with potential for use in aquaculture: Proceedings of the fourteenth U.S.-Japan meeting on aquaculture, Woods Hole, Massachusetts, October 16-17, 1985, edited by Albert K. Sparks. November 1988,69 p.

71. Greenland turbot Reinhardtius hippoglossoides of the eastern Bering Sea and Aleutian Islands region, by Miles S. Alton, Richard G. Bakkala, Gary E. Walters, and Peter T. Munro. December 1988, $31 \mathrm{p}$.

72. Age determination methods for northwest Allantic species, edited by Judy Penttila and Louise M. Dery. December 1988, 135 p.

73. Marine flora and fauna of the Eastern United States. Mollusca: Cephalopoda, by Michael Vecchione, Clyde F.E. Roper, and Michael J. Sweeney. February 1989, $23 \mathrm{p}$.

74. Proximate composition and fatty acid and cholesterol content of 22 species of northwest Atlantic finfish, by Judith Krzynowek, Jenny Murphy, Richard S. Maney, and Laurie J. Panunzio. May 1989, 35 p.

75. Codend selection of winter founder Pseudopleuronectes americanus, by David G. Simpson. March 1989, 10 p.

76. Analysis of fish diversion efficiency and survivorship in the fish return system at San Onofre Nuclear Generating Station, by Milton S. Love, Meenu Sandhu, Jeffrey Stein, Kevin T. Herbinson, Robert H. Moore, Michael Mullin, and John S. Stephens, Jr. April 1989, 16 p.

77. Illustrated key to the genera of free-living marine nematodes of the order Enoplida, by Edwin J. Keppner and Armen C. Tarjan. July 1989, 26 p.

78. Survey of fishes and water properties of south San Francisco Bay, California, 1973-82, by Donald E. Pearson. August 1989, 21 p.

79. Species composition, distribution, and relative abundance of fishes in the coastal habitat off the southeastern United States, by Charles A. Wenner and George R. Sedberry. July 1989,49 p.

80. Laboratory guide to early life history stages of northeast Pacific fishes, by Ann C. Matarese, Arthur W. Kendall, Jr., Deborah M. Blood, and Beverly M. Vinter. October $1989,651 \mathrm{p}$. 
NOAA Technical Report NMFS 80

\section{Laboratory Guide to Early Life History Stages of Northeast Pacific Fishes}

Ann C. Matarese

Arthur W. Kendall, Jr.

Deborah M. Blood

Beverly M. Vinter

October 1989

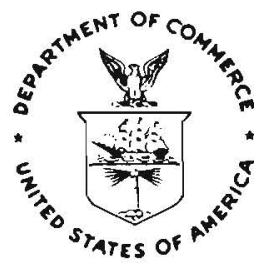

U.S. DEPARTMENT OF COMMERCE

Robert Mosbacher, Secretary

National Oceanic and Atmospheric Administration

John A. Knauss, Under Secretary for Oceans and Atmosphere

National Marine Fisheries Service

James Brennan, Assistant Administrator for Fisheries 
The National Marine Fisheries Service (NMFS) does not approve, recommend or endorse any proprietary product or proprietary material mentioned in this publication. No reference shall be made to NMFS, or to this publication furnished by NMFS, in any advertising or sales promotion which would indicate or imply that NMFS approves, recommends or endorses any proprietary product or proprietary material mentioned herein, or which has as its purpose an intent to cause directly or indirectly the advertised product to be used or purchased because of this NMFS publication. 


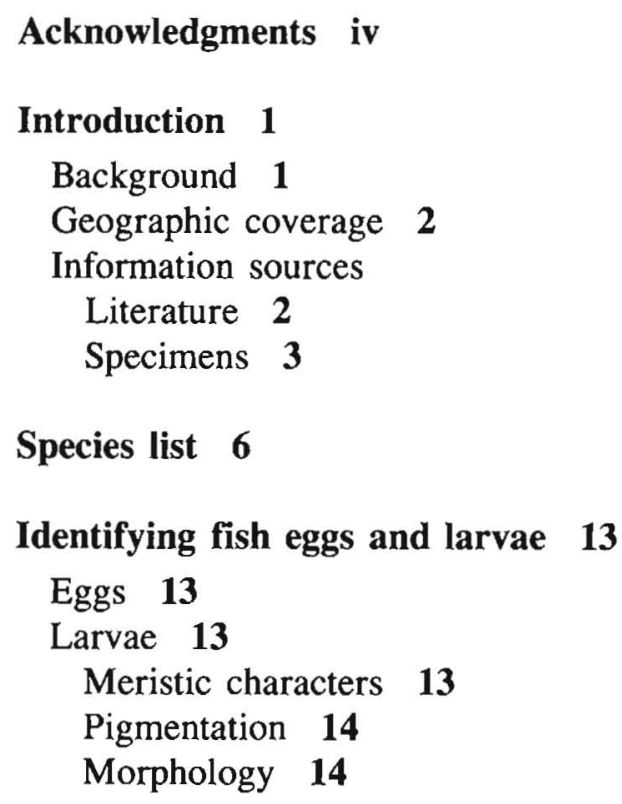

Using this laboratory guide 28

Format 28

Meristics 28

General life history $\mathbf{2 8}$

Early life history 29

\section{Taxon accounts}

Elopomorpha $\mathbf{3 1}$

Clupeiformes $\mathbf{4 3}$

Salmoniformes $\mathbf{5 1}$

Stomiiformes $\mathbf{8 3}$

Scopelomorpha 125

Gadiformes 181

Ophidiiformes 211

Lophiiformes 221

Gobiesociformes 229

Atherinomorpha 235

Lampriformes $\mathbf{2 4 3}$

Beryciformes 249

Zeiformes 257

Gasterosteiformes 261

Scorpaeniformes $\mathbf{2 6 7}$

Perciformes 483

Pleuronectiformes $\mathbf{5 6 5}$

Tetraodontiformes $\mathbf{6 2 7}$

\section{Citations 630}

Index to scientific names 642

Index to common names $\mathbf{6 5 0}$ 


\section{Acknowledgments}

A project of this scope and magnitude always involves many people whose talents and skills were essential throughout its development and completion. This guide began as an idea in 1979 and has gradually evolved over the last 10 years with the assistance of numerous people. During the early stages, two major projects contracted with the University of Washington (UW) served as starting points. Kevin Howe, formerly of the UW, was asked to compile a checklist of fishes from Point Conception, California, north into the Arctic Ocean, and to set up a meristic database system. His assembled data form the basis of meristic sections on our taxonomic text pages and meristic tables. Kathryn Garrison, formerly of the UW, and Bruce Miller (UW) synthesized the available literature on the reproduction and early life history of Puget Sound fishes, and this enabled us to write many of our sections on life history.

During the several years we spent collecting data and examining material, a number of scientists donated specimens and/or unpublished data. We would like to thank the following people: Jeffrey Marliave (Vancouver Public Aquarium), David Misitano (NWAFC Mukilteo Laboratory), Albert Giorgi (NWAFC Coastal Zone and Estuarine Studies), Kevin Bailey (NWAFC Resource Assessment and Conservation Engineering), William Watson (Marine Ecological Consultants), Bruce Mundy (formerly Oregon State University), A. J. Paul (University of Alaska), Conrad Mahnken (NWAFC Manchester Field Station), Sally L. Richardson (deceased), and H. Geoffrey Moser (NMFS Southwest Fisheries Center). The complete files of the late E. H. Ahlstrom were kindly made available by $\mathrm{H}$. Geoffrey Moser.

Throughout the planning, organizing, writing, and layout, many people contributed their special talents and we thank the following for their time: Theodore Pietsch, Kevin Howe, and Steven Leipertz, UW (meristic database); Kevin Howe and Steven Leipertz, UW, and Richard Bates, NWAFC, (literature database); Ralph Mintel, Richard Bates, and Michael McPhail, NWAFC (programming);
William Rugen, NWAFC (proofreading and verification); James Peacock, NWAFC (layout, typesetting); Jack McCormick and Nancy Peacock, NMFS Scientific Publications (format and editorial assistance); William Richards, formerly NMFS Scientific Editor (editorial assistance); and Michael Fahay, NMFS Northeast Fisheries Center, who has encouraged all of us along the way and who spent several days reviewing our very preliminary first draft.

We are grateful to the following, who in reviewing sections from an earlier draft made many suggestions and corrections and generously provided us with unpublished data from their personal data files: James Allen (ecology and life history), G. David Johnson (Perciformes), Wayne Laroche (Scorpaenidae, Agonidae, Carangidae), Douglas Markle (Gadiformes, Ophidiiformes), H. Geoffrey Moser (Myctophidae, Stomiiformes, Pleuronectiformes, Scorpaenidae), Bruce Mundy (meristic data), Barbara Sumida MacCall (Carangidae, Pleuronectidae, Stromateoidei), Betsy Washington (Scorpaeniformes). James Allen, Robert Lea, and Alex Peden reviewed the adult distributions and nomenclature.

The following shared unpublished figures with us: Kathryn Garrison and Jeffrey Marliave (stichaeids); Betsy Washington (cottids); William Watson (Atherinops and Atherinopsis); and Lucy Wold and Guillermo Moreno (Sebastes mystinus).

The following reviewed the entire draft and made many valuable suggestions, and we thank them sincerely for their time and efforts: Michael Fahay, Jeffrey Leis, Joanne Lyczkowski-Shultz, Douglas Markle, Jeffrey Marliave, Gerald McGowen, H. Geoffrey Moser, Bruce Mundy, Muneo Okiyama, William Richards, Betsy Washington, and William Watson.

Finally, we all express a special thanks to our friend and colleague Jean Dunn for his many contributions throughout the various stages of this guide. His years of experience with the early-lifehistory stages of North Pacific fishes and extensive knowledge of the literature were a valuable addition to this work. 


\section{Laboratory Guide to Early Life History Stages of Northeast Pacific Fishes}

\author{
ANN C. MATARESE \\ ARTHUR W. KENDALL, JR. \\ DEBORAH M. BLOOD \\ BEVERLY M. VINTER \\ Northwest and Alaska Fisheries Center \\ National Marine Fisheries Service, NOAA \\ 7600 Sand Point Way N.E. \\ Seattle, Washington 98115
}

\begin{abstract}
This laboratory guide presents taxonomic information on eggs and larvae of fishes of the Northeast Pacific Ocean (north of California) and the eastern Bering Sea. Included are early-lifehistory series, illustrations, and comparative descriptions of $\mathbf{2 3 2}$ species expected to spawn here, out of a total 627 species known to occur in marine waters of this area. Meristic and general life-history data are included, as well as diagnostic characters to help identify eggs and larvae. Most of this information has been gleaned from literature, with the addition of 200 previously unpublished illustrations.
\end{abstract}

\section{Introduction}

\section{Background}

The importance of early-life-history studies to fisheries investigations and phylogenetic research has increased dramatically during the last decade. Early-life-history stages are now routinely used in fisheries studies to investigate the interannual variation in recruitment (e.g., Wooster 1983), and in studies of the phylogeny of fishes (e.g., Moser et al. 1984b). The fact that early-life-history stages of many species remain unknown in the northeastern Pacific Ocean limits their use in these disciplines, as well as in research on ecology, behavior, and the biological effects of pollution on fishes.

At the Northwest and Alaska Fisheries Center (NWAFC), progress in the identification and understanding of early-life-history stages of marine fishes has steadily increased. Our investigations beginning in 1965 were primarily aimed at determining spawning grounds and distribution of eggs and larvae for only one target species: the Pacific hake, Merluccius productus. Studies in the late sixties and early seventies concentrated on ascertaining abundance and horizontal distribution of the eggs and larvae of major taxa occurring off the northeast Pacific coast and in the Gulf of Alaska and Bering Sea. In addition to baseline studies on distribution and abundance, work has increasingly emphasized the use of egg and/or larval surveys (targeted on species such as walleye pollock, Theragra chalcogramma) to generate estimates of spawning stock biomass and to test hypotheses concerning the multitude of possible factors involved in the stock/recruitment relationship (e.g., feeding, predation, growth, and transport). These studies require the accurate identification of all early-life-history stages, from the egg stage to newly settled juveniles and adults. The ability to make such identifications is a direct result of taxonomic studies in our laboratory, especially for the family Gadidae. These studies have enabled identification of all gadid species occurring in the Northeast Pacific Ocean and have allowed additional research on development, osteology, and systematics (e.g., Matarese et al. 1981, Dunn and Vinter 1984, and Dunn and Matarese 1984).

The primary purpose of this laboratory guide is to allow expansion of the use of early-life-history studies in fisheries research by providing descriptive information necessary to insure accurate identification of eggs, larvae, and early juveniles of marine fishes in the northeastern Pacific Ocean and Bering Sea. The guide is designed to aid in species identification; thus, it includes little of the developmental information usually found in ontogenetic descriptions. We have placed emphasis on the accuracy and quality of illustrations, whether from the literature or originals drawn for this guide. In some cases, however, when specimens were not available, we reproduced substandard figures from the literature. Diagnostic characters are included, which enable identification of early-life-history stages of closely related and/or similar-looking taxa, as are comparative tables and figures which outline similarities and differences among taxa. Detailed information is included for species where considerable early-life-history data are available. For poorly known taxa, available information is given, sometimes limited to characters of adults of the species in the study area and a description of early-life-history stages of related species from other areas. 


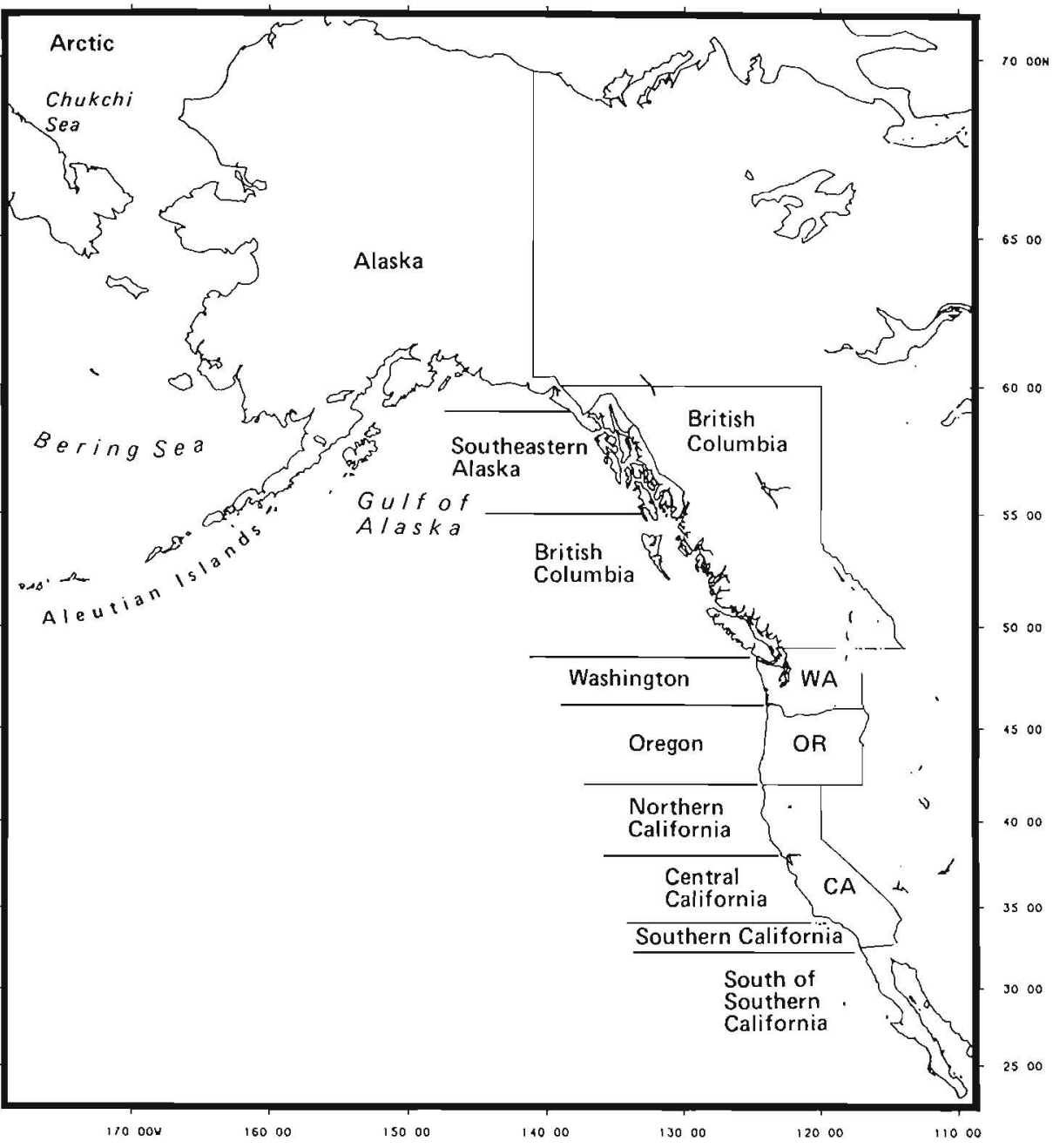

Figure 1
Study area showing geographic ranges used in species accounts.

\section{Geographic coverage}

The geographic area covered is the Pacific Ocean between approximately $38^{\circ} \mathrm{N}$ and $66^{\circ} \mathrm{N}$ and west to $180^{\circ}$; however, we have included only those taxa with northern limits of distribution between Oregon and the northern Bering Sea (Fig. 1). Taxa restricted to California waters or the Arctic are excluded. Because most ichthyoplankton surveys concentrate on coastal areas, usually within 200 miles $(370 \mathrm{~km})$ of shore, this guide emphasizes coastal, nearshore species rather than strictly oceanic species. Taxa that usually do not produce planktonic early-life-history stages are generally excluded (e.g., Embiotocidae) as are strictly freshwater and estuarine species or spawners. Sources useful in identifying early-life-history stages of freshwater and estuarine species found adjacent to our study area include Wang $(1981,1986)$ and Auer (1982). In general, we have based coverage on species' likelihood of spawning in the Northeast Pacific Ocean rather than on the occurrence of adults. The geographic distribution of spawning is generally more restricted than the overall range of a species. Some low-latitude oceanic species (e.g., most members of the family Scombridae) which occur off Oregon and Washington are not included because they usually spawn much further south and their eggs and larvae do not occur in our area.

\section{Information sources}

Literature In producing this guide, we first compiled meristic and life history information from the literature. This was accomplished largely through a computer-based meristic information file generated by Kevin Howe at the University of Washington, and a compendium of life history information produced under the direction of Bruce Miller, University of Washington (Garrison and Miller 1982). Information from these sources was augmented and updated as it was incorporated in this guide. The meristic database was compiled by examining specimens and original radiographs, and from the literature (original descriptions, revisions, or general sources). When possible, counts are from the left side of the body. Since Garrison and Miller (1982) is not generally available, original sources cited by them are referred to in this guide. Published earlylife-history information and illustrations were used when available, supplemented when possible by data from our field collections. Among the most useful published sources were Fitch and Lavenberg (1968, 1971, 1975), Miller and Lea (1972), Hart (1973), Eschmeyer et al. (1983), Moser et al. (1984b), Ozawa (1986a), and the many papers authored or co-authored by Ahlstrom, Moser, and Richardson (see Citations). Okiyama (1988) was received too late to be fully included in this guide. 
Specimens In addition to data gleaned from the literature, we have included some unpublished data and original illustrations obtained from specimens in our collections (Table 1). Specimens were from two major sources: (1) Investigations in the Kodiak Island region in the Gulf of Alaska conducted 1977-79, and (2) studies undertaken in cooperation with the U.S.S.R. beginning in 1980 to investigate the distribution and abundance of ichthyoplankton off northern California, Oregon, and Washington and in the Gulf of Alaska.
Some specimens were also derived from work in the Bering Sea during 1971-72, 1976-78, and 1979. Dunn (1986) provides a list of ichthyoplankton surveys conducted by the NWAFC from which were obtained many of the specimens examined or illustrated for this guide. Occasionally specimens were obtained from other research collections or from rearing experiments conducted primarily by Jeffrey Marliave (Vancouver Public Aquarium) and David Misitano (NWAFC).

Table 1

Collection data for original illustrations by Northwest and Alaska Fisheries Center (NWAFC). ${ }^{\mathrm{a}}$

\begin{tabular}{|c|c|c|c|c|c|c|}
\hline Taxon & $\begin{array}{c}\mathrm{SL} \\
(\mathrm{mm})\end{array}$ & Cruise & $\begin{array}{l}\text { Station } \\
\text { number }\end{array}$ & Gear $^{b}$ & Date & $\begin{array}{l}\text { Location or } \\
\mathrm{N}^{\circ} \quad \mathrm{W}^{\circ}\end{array}$ \\
\hline Thalassenchelys coheni & 190.0 & & & & 150284 & Puget Sound, $\mathrm{WA}^{\mathrm{c}}$ \\
\hline Clupea pallasi & $\begin{array}{l}10.4 \\
15.0 \\
19.0 \\
23.8\end{array}$ & $\begin{array}{l}4 \text { MF81 } \\
\text { SF7703 } \\
\text { SF7603 }\end{array}$ & $\begin{array}{l}\text { G005A } \\
\text { P-4 } \\
13\end{array}$ & $\begin{array}{l}6 \mathrm{~B} 5 \\
\mathrm{~N} \\
\mathrm{~B} \\
\text { seine }\end{array}$ & $\begin{array}{l}200581 \\
050477 \\
180576 \\
140672\end{array}$ & $\begin{array}{l}58^{\circ} 19.6^{\prime}, 153^{\circ} 54.5^{\prime} \\
\text { Str. Juan de Fuca, WA } \\
\text { Str. Juan de Fuca, WA }\end{array}$ \\
\hline Nansenia candida & 5.1 & 1PO84 & $\mathrm{G} 053 \mathrm{~A}$ & $6 \mathrm{~B} 5$ & 220384 & $44^{\circ} 40.1^{\prime}, 127^{\circ} 40.0^{\prime}$ \\
\hline Bathylagus milleri & 15.0 & $2 \mathrm{MF78}$ & G074A & TT & 020778 & $57^{\circ} 07.0^{\prime}, 151^{\circ} 01.0^{\prime}$ \\
\hline Bathylagus ochorensis & 7.9 & $1 E Q 83$ & G056A & B & 030583 & $44^{\circ} 00.0^{\prime}, 127^{\circ} 39.0^{\prime}$ \\
\hline Bathylagus pacificus & $\begin{array}{l}11.6 \\
17.6\end{array}$ & 3MF79 & S25A & $\begin{array}{l}6 \mathrm{~B} 5 \\
6 \mathrm{~B} 5\end{array}$ & 170679 & $\begin{array}{l}54^{\circ} 05.2^{\prime}, 170^{\circ} 59.0^{\prime} \\
\text { Gulf of Alaska }\end{array}$ \\
\hline Macropinna microstoma & 11.1 & 1PO84 & G066A & $6 \mathrm{~B} 5$ & 240384 & $43^{\circ} 40.0^{\prime}, 124^{\circ} 37.0^{\prime}$ \\
\hline Danaphos oculatus & 22.4 & & M161 & & 230784 & $6 \mathrm{~km} \mathrm{~W}$ of Newport, OR \\
\hline Tactosioma macropus & 14.3 & 1PO80 & G011A & $6 \mathrm{~B} 5$ & 030880 & $47^{\circ} 18.7^{\prime}, 125^{\circ} 13.3^{\prime}$ \\
\hline Lampanyctus regalis & 5.2 & $1 \mathrm{PO} 80$ & G061A & $6 \mathrm{~B} 5$ & 120880 & $38^{\circ} 00.0^{\prime}, 125^{\circ} 42.5^{\prime}$ \\
\hline Diaphus theta & $\begin{array}{r}4.6 \\
6.3 \\
16.0\end{array}$ & $\begin{array}{l}\text { K6703 } \\
1 \text { PO80 } \\
\text { IPO80 }\end{array}$ & $\begin{array}{l}50 \\
\text { G085A } \\
\text { G064A }\end{array}$ & $\begin{array}{l}1 \mathrm{MN} \\
6 \mathrm{~B} 5 \\
6 \mathrm{~B} 5\end{array}$ & $\begin{array}{l}080567 \\
200880 \\
130880\end{array}$ & $\begin{array}{l}42^{\circ} 00.0^{\prime}, 127^{\circ} 35.0^{\prime} \\
40^{\circ} 20.0^{\prime}, 125^{\circ} 20.0^{\prime} \\
42^{\circ} 22.5^{\prime}, 126^{\circ} 17.5^{\prime}\end{array}$ \\
\hline Stenobrachius leucopsarus & $\begin{array}{r}4.9 \\
6.3 \\
18.0\end{array}$ & $\begin{array}{l}2 \mathrm{KE} 72 \\
1 \mathrm{PO} 84 \\
4 \mathrm{DI} 78\end{array}$ & $\begin{array}{l}\text { G50A } \\
\text { G112A } \\
\text { G051A }\end{array}$ & $\begin{array}{l}6 \mathrm{~B} 5 \\
6 \mathrm{~B} 5 \\
\mathrm{TT} 1\end{array}$ & $\begin{array}{lll}08 & 05 & 72 \\
02 & 04 & 84 \\
13 & 04 & 78\end{array}$ & $\begin{array}{l}57^{\circ} 46.0^{\prime}, 149^{\circ} 21.0^{\prime} \\
40^{\circ} 40.1^{\prime}, 126^{\circ} 47.0^{\prime} \\
55^{\circ} 26.8^{\prime}, 153^{\circ} 53.7^{\prime}\end{array}$ \\
\hline Melanonus zugmayeri & 17.0 & Ocean ACRE-9 & $9-11 \mathrm{~N}$ & & 190370 & $32^{\circ} 03.0^{\prime}, 64^{\circ} 05.0^{\prime}$ \\
\hline Boreogadus saida & 16.3 & Glacier (OCSEAP) & 11 & $6 \mathrm{~B} 5$ & 110876 & $70^{\circ} 47.0^{\prime}, 162^{\circ} 14.0^{\prime}$ \\
\hline Gadus macrocephalus & 4.1 & FOX8611I (sample 111) & 166 & MOC & 180586 & $57^{\circ} 40.6^{\prime}, 155^{\circ} 09.8^{\prime}$ \\
\hline Theragra chalcogramma & $\begin{array}{l}\text { egg } \\
3.5\end{array}$ & composite & & & -0482 & $\begin{array}{l}\text { Gulf of Alaska } \\
\text { Gulf of Alaska }^{d}\end{array}$ \\
\hline Ophidiidae & $\begin{array}{l}15.6 \\
29.8\end{array}$ & $\begin{array}{l}\text { IEQ83 } \\
\text { IEQ83 }\end{array}$ & $\begin{array}{l}\text { G044A } \\
\text { G073A }\end{array}$ & $\begin{array}{l}6 \mathrm{~B} 5 \\
6 \mathrm{~B} 5\end{array}$ & $\begin{array}{l}300483 \\
060583\end{array}$ & $\begin{array}{l}45^{\circ} 20.0^{\prime}, 124^{\circ} 48.0^{\prime} \\
43^{\circ} 20.2^{\prime}, 127^{\circ} 57.0^{\prime}\end{array}$ \\
\hline Brosmophycis marginata & 10.6 & & & & & Puget Sound ${ }^{e}$ \\
\hline Gobiesox maeandricus & 7.0 & & & & 120276 & Brit. Columbia ${ }^{f}$ \\
\hline Cololabis saira & $\begin{array}{l}6.7 \\
7.4\end{array}$ & $\begin{array}{l}\text { ITK80 } \\
\text { IDA81 }\end{array}$ & $\begin{array}{l}\text { G091 } \\
\text { G039A }\end{array}$ & $\begin{array}{l}\mathrm{N} \\
\mathrm{N}\end{array}$ & $\begin{array}{lll}08 & 05 & 80 \\
02 & 11 & 81\end{array}$ & $\begin{array}{l}42^{\circ} 00.0^{\prime}, 125^{\circ} 55.0^{\prime} \\
45^{\circ} 37.0^{\prime}, 124^{\circ} 52.0^{\prime}\end{array}$ \\
\hline Trachipterus altivelis & $\begin{array}{r}9.4 \\
24.0\end{array}$ & $\begin{array}{l}\text { K6805 } \\
\text { IPO82 }\end{array}$ & $\begin{array}{l}30 \\
\text { G46A }\end{array}$ & $\begin{array}{l}1 \mathrm{MN} \\
6 \mathrm{~B} 5\end{array}$ & $\begin{array}{l}23 \quad 1068 \\
290582\end{array}$ & $\begin{array}{l}45^{\circ} 44.0^{\prime}, 124^{\circ} 38.0^{\prime} \\
40^{\circ} 40.5^{\prime}, 126^{\circ} 48.4^{\prime}\end{array}$ \\
\hline Melamphaes sp. & 3.7 & IEQ83 & G034A & $6 \mathrm{~B} 5$ & 280483 & $46^{\circ} 00.0^{\prime}, 128^{\circ} 31.0^{\prime}$ \\
\hline Sebastes brevispinis & 4.6 & SEI77-9 & 21 & trawl & 120777 & $58^{\circ} 28.3^{\prime}, 139^{\circ} 30.0^{\prime}$ \\
\hline Sebastes caurinus & 5.1 & & & & 050777 & Reared by C. Mahnken ${ }^{8}$ \\
\hline Sebastes jordani & 4.6 & Marathon 85-1 & 229 & trawl & 250585 & $48^{\circ} 09.6^{\prime}, 125^{\circ} 05.3^{\prime}$ \\
\hline Sebastes melanops & 4.0 & & & & 070284 & Newport, $\mathrm{OR}^{\mathrm{h}}$ \\
\hline Sebastes polyspinis & 6.1 & Poseydon 85-1 & & trawl & -0785 & Gulf of Alaska \\
\hline Sebastes rufus & 36.0 & $\begin{array}{l}\text { (Groundfish Comm. } \\
\text { Invest.) }\end{array}$ & 8 & MWT & 040685 & Farrallon Is., $\mathrm{CA}^{\mathrm{i}}$ \\
\hline Sebastes variegatus & 4.6 & SEI77-9 & 21 & trawl & 120777 & $58^{\circ} 28.3^{\prime}, 139^{\circ} 30.0^{\prime}$ \\
\hline Sebastes zacentrus & 4.7 & SEI77-9 & 33 & trawl & 160777 & $59^{\circ} 40.1^{\prime}, 143^{\circ} 03.5^{\prime}$ \\
\hline Sebastolobus sp. & egg & IPO81 & G065A & $\mathrm{N}$ & 220581 & $43^{\circ} 40.0^{\prime}, 125^{\circ} 01.4^{\prime}$ \\
\hline
\end{tabular}


Table 1 (continued)

\begin{tabular}{|c|c|c|c|c|c|c|}
\hline Taxon & $\begin{array}{l}\text { SL. } \\
(\mathrm{mm})\end{array}$ & Cruise & $\begin{array}{l}\text { Station } \\
\text { number }\end{array}$ & Gear $^{b}$ & Date & $\begin{array}{l}\text { Location or } \\
\mathrm{N}^{\circ} \quad \mathrm{W}^{\circ}\end{array}$ \\
\hline Anoplopoma fimbria & $\begin{array}{r}5.6 \\
8.8 \\
12.0\end{array}$ & $\begin{array}{l}\text { 1PO84 } \\
\text { MF77B-6 } \\
\text { MF77B-6 }\end{array}$ & $\begin{array}{l}\text { G019B } \\
83(1) \\
2(4)\end{array}$ & $\begin{array}{l}6 \mathrm{~B} 5 \\
\mathrm{~N} \\
\mathrm{~N}\end{array}$ & $\begin{array}{l}150384 \\
150577\end{array}$ & $\begin{array}{l}46^{\circ} 40.5^{\prime}, 124^{\circ} 59.0^{\prime} \\
55^{\circ} 40.7^{\prime}, 155^{\circ} 23.0^{\prime} \\
54^{\circ} 22.6^{\prime}, 166^{\circ} 42.5^{\prime}\end{array}$ \\
\hline Blepsias bilobus & $\begin{array}{l}12.4 \\
16.7 \\
24.8\end{array}$ & $\begin{array}{l}\text { 3MF79 } \\
\text { 3MF79 } \\
\text { 3MF79 }\end{array}$ & $\begin{array}{l}\text { V02A } \\
\text { S12A } \\
\text { S } 40 \mathrm{~A}\end{array}$ & $\begin{array}{l}\mathrm{N} \\
\mathrm{N} \\
\mathrm{N}\end{array}$ & $\begin{array}{l}020679 \\
060679 \\
210679\end{array}$ & $\begin{array}{l}56^{\circ} 03.5^{\circ}, 166^{\circ} 33.9^{\prime} \\
56^{\circ} 35.7^{\prime}, 165^{\circ} 54.3^{\prime} \\
56^{\circ} 31.6^{\prime}, 166^{\circ} 42.0^{\prime}\end{array}$ \\
\hline Blepsias cirrhosus & $\begin{array}{l}11.5 \\
16.8\end{array}$ & & & & $\begin{array}{l}-0480 \\
-0480\end{array}$ & $\begin{array}{l}\text { Friday Harbor, WA } \\
\text { Friday Harbor, WA }\end{array}$ \\
\hline Chitonotus pugetensis & $\begin{array}{l}3.0 \\
4.9\end{array}$ & & & & & $\begin{array}{l}\text { Puget Sound, } \mathrm{WA}^{\mathrm{j}} \\
\text { Puget Sound, } \mathrm{WA}^{j}\end{array}$ \\
\hline Gymnocanthus A & $\begin{array}{r}9.6 \\
11.9\end{array}$ & $\begin{array}{l}\text { MF76A } \\
\text { MF76A }\end{array}$ & $\begin{array}{l}\text { B07 } \\
\text { B17 }\end{array}$ & $\begin{array}{l}\text { 6B5 } \\
6 \mathrm{~B} 5\end{array}$ & $\begin{array}{l}040576 \\
200576\end{array}$ & $\begin{array}{l}56^{\circ} 49.7^{\prime}, 169^{\circ} 39.0^{\prime} \\
54^{\circ} 42.3^{\prime}, 165^{\circ} 25.9^{\prime}\end{array}$ \\
\hline Hemilepidotus jordani & $\begin{array}{l}10.6 \\
18.4\end{array}$ & $\begin{array}{l}\text { 1SH81 } \\
\text { 3MF79 }\end{array}$ & $\begin{array}{l}066 \\
\text { V06A }\end{array}$ & $\begin{array}{l}\mathrm{N} \\
\mathrm{N}\end{array}$ & $\begin{array}{l}190381 \\
030679\end{array}$ & $\begin{array}{l}57^{\circ} 03.0^{\prime}, 155^{\circ} 53.0^{\prime} \\
56^{\circ} 02.8^{\prime}, 166^{\circ} 33.6^{\prime}\end{array}$ \\
\hline Myoxocephalus B & $\begin{array}{r}9.1 \\
12.2\end{array}$ & $\begin{array}{l}1 \mathrm{MD} 82 \\
1 \mathrm{MD} 82\end{array}$ & $\begin{array}{l}\text { G135A } \\
\text { G135A }\end{array}$ & $\begin{array}{l}6 \mathrm{~B} 5 \\
6 \mathrm{~B} 5\end{array}$ & $\begin{array}{l}290582 \\
290582\end{array}$ & $\begin{array}{l}54^{\circ} 54.1^{\prime}, 158^{\circ} 39.0^{\prime} \\
54^{\circ} 54.1^{\prime}, 158^{\circ} 39.0^{\prime}\end{array}$ \\
\hline Myoxocephalus G & 8.7 & 2KE72 & G39A & 6B5 & 060572 & $57^{\circ} 33.0^{\prime}, 152^{\circ} 06.0^{\prime}$ \\
\hline Nautichthys oculofasciatus & 8.3 & & & & -0480 & Friday Harbor, WA \\
\hline Psychrolutes paradoxus & 18.0 & & & & & Mukilteo, WA \\
\hline Synchirus gilli & $\begin{array}{l}10.5 \\
16.8\end{array}$ & & & $\mathrm{~N}$ & $\begin{array}{l}150579 \\
160579\end{array}$ & $\begin{array}{l}\text { Neah Bay, WA } \\
\text { Neah Bay, WA }\end{array}$ \\
\hline Agonidae A & $\begin{array}{r}4.7 \\
10.0\end{array}$ & IPO82 & $\mathrm{G} 026 \mathrm{~A}$ & 6B5 & 140582 & $\begin{array}{l}44^{\circ} 01.5^{\prime}, 124^{\circ} 33.8^{\prime} \\
\text { Gulf of Alaska }\end{array}$ \\
\hline Agonomalus mozinoi & 8.2 & MF77B-5 & $3(2)$ & $6 \mathrm{~B} 5$ & 260477 & $54^{\circ} 38.7^{\prime}, 167^{\circ} 14.0^{\prime}$ \\
\hline Cyclopteridae & 4.0 & 3 MF81 & G058A & $5 \mathrm{~B} 5$ & 010581 & $56^{\circ} 55.9^{\prime}, 154^{\circ} 55.8^{\prime}$ \\
\hline Nectoliparis pelagicus & $\begin{array}{r}7.9 \\
20.5\end{array}$ & $\begin{array}{l}\text { MF77B-6 } \\
\text { MF77B-6 }\end{array}$ & $\begin{array}{l}55(1) \\
46(1)\end{array}$ & $\begin{array}{l}\mathrm{TT} \\
6 \mathrm{~B} 5\end{array}$ & $\begin{array}{l}130577 \\
130577\end{array}$ & $\begin{array}{l}55^{\circ} 46.7^{\prime}, 169^{\circ} 25.1^{\prime} \\
55^{\circ} 44.4^{\prime}, 171^{\circ} 31.3^{\prime}\end{array}$ \\
\hline Paraliparis sp. & 28.5 & $4 \mathrm{DI} 78$ & 25 & $6 \mathrm{~B} 5$ & 010478 & $57^{\circ} 58.3^{\prime}, 150^{\circ} 02.2^{\prime}$ \\
\hline Bathymaster A & $\begin{array}{r}9.0 \\
29.6\end{array}$ & $\begin{array}{l}\text { 2MF78 } \\
\text { MF77B-5 }\end{array}$ & $\begin{array}{l}\text { G35A } \\
2(2)\end{array}$ & $\begin{array}{l}6 \mathrm{~B} 5 \\
\mathrm{~N}\end{array}$ & $\begin{array}{l}250678 \\
260477\end{array}$ & $\begin{array}{l}56^{\circ} 02.0^{\prime}, 154^{\circ} 08.4^{\prime} \\
54^{\circ} 23.3^{\prime}, 166^{\circ} 44.0^{\prime}\end{array}$ \\
\hline Ronquilus jordani & $\begin{array}{r}7.7 \\
10.4\end{array}$ & & $\begin{array}{l}803 \mathrm{E} \\
912 \mathrm{E}\end{array}$ & & $\begin{array}{l}-0572 \\
-0572\end{array}$ & Newport, OR \\
\hline Anoplarchus purpurescens & $\begin{array}{r}6.1 \\
9.0 \\
12.0 \\
12.0 \\
12.0\end{array}$ & & & & & $\begin{array}{l}\text { Puget Sound, } \mathrm{WA}^{\mathrm{j}} \\
\text { Puget Sound, } \mathrm{WA}^{\mathrm{j}} \\
\text { Puget Sound, WA } \\
\text { Puget Sound, } \mathrm{WA}^{\mathrm{j}} \\
\text { Puget Sound, } \mathrm{WA}^{j}\end{array}$ \\
\hline Bryozoichthys-Chirolophis & $\begin{array}{l}16.5 \\
29.0\end{array}$ & $\begin{array}{l}\text { MF76A } \\
\text { MF77B-6 }\end{array}$ & $\begin{array}{l}10 \\
42\end{array}$ & $\begin{array}{l}6 \mathrm{~B} 5 \\
\mathrm{~N}\end{array}$ & 120577 & $\begin{array}{l}56^{\circ} 29.8^{\prime}, 171^{\circ} 34.1^{\prime} \\
56^{\circ} 45.6^{\prime}, 171^{\circ} 30.8^{\prime}\end{array}$ \\
\hline Lumpenus sagitla & $\begin{array}{l}17.3 \\
35.1\end{array}$ & $\begin{array}{l}1 \mathrm{MD} 82 \\
4 \mathrm{MF} 81\end{array}$ & $\begin{array}{l}\text { G028A } \\
\text { G028A }\end{array}$ & $\begin{array}{l}6 \mathrm{~B} 5 \\
6 \mathrm{~B} 5\end{array}$ & $\begin{array}{l}080482 \\
210581\end{array}$ & $\begin{array}{l}56^{\circ} 40.0^{\prime}, 155^{\circ} 27.0^{\prime} \\
57^{\circ} 29.5^{\prime}, 155^{\circ} 43.0^{\prime}\end{array}$ \\
\hline Plectobranchus evides & $\begin{array}{r}9.2 \\
16.9 \\
31.3\end{array}$ & & $\begin{array}{l}1143 \mathrm{E} \\
1107 \mathrm{E} \\
933 \mathrm{E}\end{array}$ & & $\begin{array}{l}240473 \\
200473 \\
-0672\end{array}$ & $\begin{array}{l}\text { Newport, OR } \\
\text { Newport, OR } \\
\text { Newport, OR }\end{array}$ \\
\hline Xiphister atropurpureus & 8.0 & & & & 200378 & Brit. Columbia ${ }^{f}$ \\
\hline Delolepis gigantea & 17.5 & $1 \mathrm{MF} 80$ & $34 \mathrm{~A} ?$ & $\mathrm{~N}$ & 270380 & $57^{\circ} 52.4^{\prime}, 154^{\circ} 38.9^{\prime}$ \\
\hline Lyconectes aleutensis & 16.0 & MF77B-5 & $8(2)$ & $\mathrm{N}$ & 250477 & $54^{\circ} 37.0^{\prime}, 166^{\circ} 13.9^{\prime}$ \\
\hline Apodichthys flavidus & $\sim 15$ & & & & & Brit. Columbia' \\
\hline Pholis sp. & $\begin{array}{r}9.2 \\
23.0\end{array}$ & 2MF78 & $\mathrm{G} 034 \mathrm{~A}$ & $6 \mathrm{~B} 5$ & 250678 & $\begin{array}{l}\text { Brit. Columbia }{ }^{f} \\
56^{\circ} 33.3^{\prime}, 154^{\circ} 51.2^{\prime}\end{array}$ \\
\hline Anarhichas orientalis & 21.0 & MF77B-5 & $8(2)$ & $\mathrm{N}$ & 250477 & $54^{\circ} 37.0^{\prime}, 166^{\circ} 13.9^{\prime}$ \\
\hline Ammodytes hexapterus & $\begin{array}{r}9.8 \\
32.3\end{array}$ & $\begin{array}{l}1 \mathrm{SH} 81 \\
1 \mathrm{CH} 83\end{array}$ & $\begin{array}{l}177 \\
\text { S008A }\end{array}$ & $\begin{array}{l}\text { 6B5 } \\
\mathrm{N}\end{array}$ & $\begin{array}{lll}22 & 04 & 81 \\
18 & 05 & 83\end{array}$ & $\begin{array}{l}55^{\circ} 54.0^{\prime}, 156^{\circ} 36.0^{\prime} \\
59^{\circ} 03.2^{\prime}, 147^{\circ} 31.7^{\prime}\end{array}$ \\
\hline Clevelandia ios & $\begin{array}{r}3.4 \\
15.0\end{array}$ & & & TT & $\begin{array}{l}040582 \\
200166\end{array}$ & $\begin{array}{l}\text { Bohom Bay, OR } \\
\text { Yaquina Bay, OR }\end{array}$ \\
\hline Coryphopterus nicholsi & 4.4 & & & & 210585 & Dabob Bay, WA' \\
\hline Lepidogobius lepidus & $\begin{array}{r}3.5 \\
20.8\end{array}$ & & & $\begin{array}{l}\text { TT (midwater) } \\
6 \mathrm{ft} \text {. otter trawl }\end{array}$ & 190584 & $\begin{array}{l}\text { Yaquina Bay, OR } \\
1 \text { mi off Newport, OR }\end{array}$ \\
\hline
\end{tabular}




\begin{tabular}{|c|c|c|c|c|c|c|}
\hline \multicolumn{7}{|c|}{ Table 1 (continued) } \\
\hline Taxon & $\underset{(\mathrm{mm})}{\mathrm{SL}}$ & Cruise & $\begin{array}{l}\text { Station } \\
\text { number }\end{array}$ & Gear $^{b}$ & Date & $\begin{array}{l}\text { Location or } \\
\mathrm{N}^{\circ} \quad \mathrm{W}^{\circ}\end{array}$ \\
\hline Citharichthys sordidus & $\begin{array}{l}4.5 \\
7.0\end{array}$ & $\begin{array}{l}1 \mathrm{EQ} 83 \\
1 \mathrm{EQ} 83\end{array}$ & $\begin{array}{l}\text { G042A } \\
\text { G042A }\end{array}$ & & $\begin{array}{l}300483 \\
300483\end{array}$ & $\begin{array}{l}45^{\circ} 20.0^{\prime}, 124^{\circ} 06.0^{\prime} \\
45^{\circ} 20.0^{\prime}, 124^{\circ} 06.0^{\prime}\end{array}$ \\
\hline Atheresthes stomias & $\begin{array}{l}10.0 \\
13.4 \\
25.6\end{array}$ & $\begin{array}{l}\text { MF76A-W } \\
\text { DE-4 } \\
\text { DE-4 }\end{array}$ & $\begin{array}{l}36 \\
14 \mathrm{KH} 2 \\
16 \mathrm{~J}\end{array}$ & $\begin{array}{l}6 \mathrm{~B} 5 \\
6 \mathrm{~B} 5 \\
6 \mathrm{~B} 5\end{array}$ & $\begin{array}{l}250576 \\
250771 \\
020871\end{array}$ & $\begin{array}{l}55^{\circ} 29.0^{\prime}, 165^{\circ} 50.8^{\prime} \\
56^{\circ} 45.0^{\prime}, 168^{\circ} 05.0^{\prime} \\
56^{\circ} 15.0^{\prime}, 171^{\circ} 21.0^{\prime}\end{array}$ \\
\hline Glyptocephalus zachirus & 11.5 & 2MF78 & G68A & TT & 010778 & $56^{\circ} 17.3^{\prime}, 152^{\circ} 55.8^{\prime}$ \\
\hline Hippoglossoides elassodon & $\begin{array}{r}5.0 \\
7.9 \\
15.0 \\
18.0\end{array}$ & $\begin{array}{l}\text { 2MF78 } \\
\text { 2MF78 } \\
\text { 3MF79 } \\
\text { SEI77-9 }\end{array}$ & $\begin{array}{l}\text { G66A } \\
\text { G66A } \\
\text { S33A } \\
43\end{array}$ & $\begin{array}{l}\mathrm{TT} \\
\mathrm{TT} \\
6 \mathrm{~B} 5 \\
6 \mathrm{~B} 5\end{array}$ & $\begin{array}{l}300678 \\
300678 \\
190679 \\
220777\end{array}$ & $\begin{array}{l}55^{\circ} 59.7^{\prime}, 153^{\circ} 33.2^{\prime} \\
55^{\circ} 59.7^{\prime}, 153^{\circ} 33.2^{\prime} \\
56^{\circ} 16.2^{\prime}, 166^{\circ} 29.6^{\prime} \\
58^{\circ} 19.5^{\prime}, 150^{\circ} 53.0^{\prime}\end{array}$ \\
\hline Hippoglossus stenolepis & $\begin{array}{l}14.4 \\
18.0\end{array}$ & $\begin{array}{l}\text { 4DI78 } \\
3 \mathrm{MF} 81\end{array}$ & $\begin{array}{l}\text { D4SC } \\
\text { G012A }\end{array}$ & $\begin{array}{l}\text { TT } \\
6 \mathrm{~B} 5\end{array}$ & $\begin{array}{l}140478 \\
270481\end{array}$ & $\begin{array}{l}56^{\circ} 14.5^{\prime}, 153^{\circ} 22.2^{\prime} \\
57^{\circ} 57.0^{\prime}, 154^{\circ} 13.3^{\prime}\end{array}$ \\
\hline Lepidopsetta bilineata & $\begin{array}{r}4.3 \\
7.4 \\
10.8 \\
16.3\end{array}$ & $\begin{array}{l}4 \mathrm{DI78} \\
5 T 179 \\
\text { DE-4 } \\
\text { 2MF78 }\end{array}$ & $\begin{array}{l}\text { G009A } \\
\text { G024A } \\
\text { DEAA2 } \\
\text { G023A }\end{array}$ & $\begin{array}{l}6 \mathrm{~B} 5 \\
6 \mathrm{~B} 5 \\
6 \mathrm{~B} 5 \\
6 \mathrm{~B} 5\end{array}$ & $\begin{array}{l}300378 \\
190579 \\
260771 \\
240678\end{array}$ & $\begin{array}{l}58^{\circ} 22.0^{\prime}, 150^{\circ} 12.8^{\prime} \\
56^{\circ} 23.7^{\prime}, 155^{\circ} 45.0^{\prime} \\
57^{\circ} 30.0^{\prime}, 169^{\circ} 30.0^{\prime} \\
57^{\circ} 19.5^{\prime}, 152^{\circ} 23.9^{\prime}\end{array}$ \\
\hline Lepidopsetra 2 & $\begin{array}{r}6.3 \\
9.7 \\
16.4\end{array}$ & $\begin{array}{l}\text { 2MF78 } \\
\text { 2MF78 } \\
\text { SEI77-9 }\end{array}$ & $\begin{array}{l}\text { G044A } \\
\text { D01A } \\
43\end{array}$ & $\begin{array}{l}\text { TT } \\
\text { TT } \\
6 \mathrm{~B} 5\end{array}$ & $\begin{array}{l}260678 \\
280678 \\
220777\end{array}$ & $\begin{array}{l}57^{\circ} 61.1^{\prime}, 151^{\circ} 17.4^{\prime} \\
56^{\circ} 42.3^{\prime}, 153^{\circ} 33.7^{\prime} \\
58^{\circ} 19.5^{\prime}, 150^{\circ} 53.0^{\prime}\end{array}$ \\
\hline Microstomus pacificus & $\begin{array}{l}15.0 \\
26.0\end{array}$ & $\begin{array}{l}\text { MF84-6 } \\
1 \text { PO80 }\end{array}$ & $\begin{array}{l}\text { B1 } \\
\text { G095A }\end{array}$ & $\begin{array}{l}6 \mathrm{~B} 3 \\
6 \mathrm{~B} 5\end{array}$ & $\begin{array}{l}250884 \\
230880\end{array}$ & $\begin{array}{l}\text { Bering Sea }{ }^{\mathrm{m}} \\
39^{\circ} 20.0^{\prime}, 124^{\circ} 22.0^{\prime}\end{array}$ \\
\hline Parophrys vetulus & $\begin{array}{r}4.5 \\
10.0 \\
17.5\end{array}$ & $\begin{array}{l}\text { K6805 } \\
6502 \\
\text { SF7702 }\end{array}$ & $\begin{array}{l}32 \\
14 \mathrm{~F} \\
2\end{array}$ & $\begin{array}{l}1 \mathrm{MN} \\
6 \mathrm{~B} 3\end{array}$ & $\begin{array}{lll}23 & 10 & 68 \\
14 & 05 & 65 \\
23 & 02 & 77\end{array}$ & $\begin{array}{l}45^{\circ} 44.0^{\prime}, 124^{\circ} 07.0^{\prime} \\
46^{\circ} 10.0^{\circ}, 124^{\circ} 42.0^{\prime} \\
\text { Str. Juan de Fuca, WA }\end{array}$ \\
\hline Platichthys stellatus & $\begin{array}{l}4.8 \\
6.6 \\
8.2 \\
9.0\end{array}$ & 4 MF81 & G029A & $6 \mathrm{~B} 5$ & $\begin{array}{lll}21 & 05 & 81 \\
29 & 04 & 78 \\
29 & 0478 \\
05 & 0670\end{array}$ & $\begin{array}{l}57^{\circ} 24.8^{\prime}, 155^{\circ} 37.0^{\prime} \\
\text { Str. Juan de Fuca, WA } \\
\text { Str. Juan de Fuca, WA } \\
\text { Puget Sound, WA }\end{array}$ \\
\hline Pleuronectes quadrituberculatus & $\begin{array}{l}6.3 \\
7.8\end{array}$ & $\begin{array}{l}\text { 3MF79 } \\
\text { 3MF79 }\end{array}$ & $\begin{array}{l}\text { V014A } \\
\text { V015A }\end{array}$ & $\begin{array}{l}6 \mathrm{~B} 5 \\
6 \mathrm{~B} 5\end{array}$ & $\begin{array}{l}050679 \\
050679\end{array}$ & $\begin{array}{l}57^{\circ} 02.9^{\prime}, 165^{\circ} 02.8^{\prime} \\
57^{\circ} 03.8^{\prime}, 165^{\circ} 03.2^{\prime}\end{array}$ \\
\hline Psettichthys melanostictus & $\begin{array}{r}2.5 \\
6.9 \\
8.1 \\
13.9\end{array}$ & & $591 \mathrm{E}$ & & $\begin{array}{l}150284 \\
070384 \\
270384 \\
-06-\end{array}$ & $\begin{array}{l}\text { Puget Sound, } W^{j} \\
\text { Puget Sound, } \mathrm{WA}^{\mathrm{j}} \\
\text { Puget Sound, WA } \\
\text { Newport, OR }\end{array}$ \\
\hline 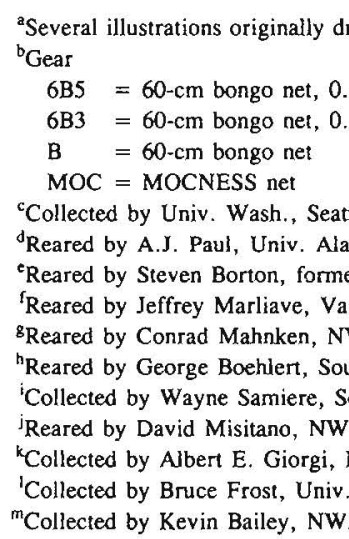 & $\begin{array}{l}\text { for this p } \\
\text {-mm mesh } \\
\text { mm mesh } \\
\text { WA } 98195 \\
\text { Inst. Mar } \\
\text { of Searte } \\
\text { ser Public } \\
\text { FC, Coasta } \\
\text { est Fish. } \\
\text { west Fish. } \\
\text { C, Environ. } \\
\text { AFC, Coas } \\
\text { ash., Schoo } \\
\text { C, Resource }\end{array}$ & 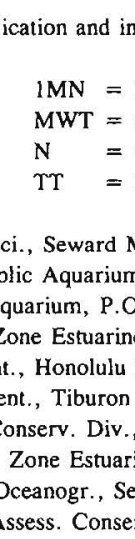 & $\begin{array}{l}\text { ard, AK } 95 \\
\text { le, WA } 98 \\
\text { ncouver, } \\
\text { ster Field } \\
\text { HI } 96822 \\
\text { CA } 94920 \\
\text { Stn., P.O } \\
\text { Montlake } \\
\text { 5. } \\
\text { Sand Point }\end{array}$ & $\begin{array}{l}\text { v6B } 3 x \\
\text { ox 38, } \\
\text { ikilteo, } \\
\text { thle, WA } \\
\text { Seattle, }\end{array}$ & VA 98353. & endall and Matarese 1987) \\
\hline
\end{tabular}


The following list of species found in the study area was compiled from the literature. The order of higher taxa generally follows J. Nelson (1984); genera and species are listed alphabetically within families. The list is annotated with page numbers indicating where taxa are described in the guide. Page numbers are out of sequence in instances (e.g., myctophids, cottids) where taxa are grouped according to larval similarities, rather than alphabetically. Letters in place of page numbers indicate that a species is not given individual treatment for the following reasons: $\mathrm{A}=$ anadromous, $\mathrm{D}=$ direct development, $\mathrm{F}=$ freshwater spawner, $S=$ spawns south of study area, $U=$ inadequate early-life-history information available, $\mathrm{V}=$ viviparous. In these cases, available meristic and ecological information is given in appropriate higher-category (e.g., order, family) accounts.

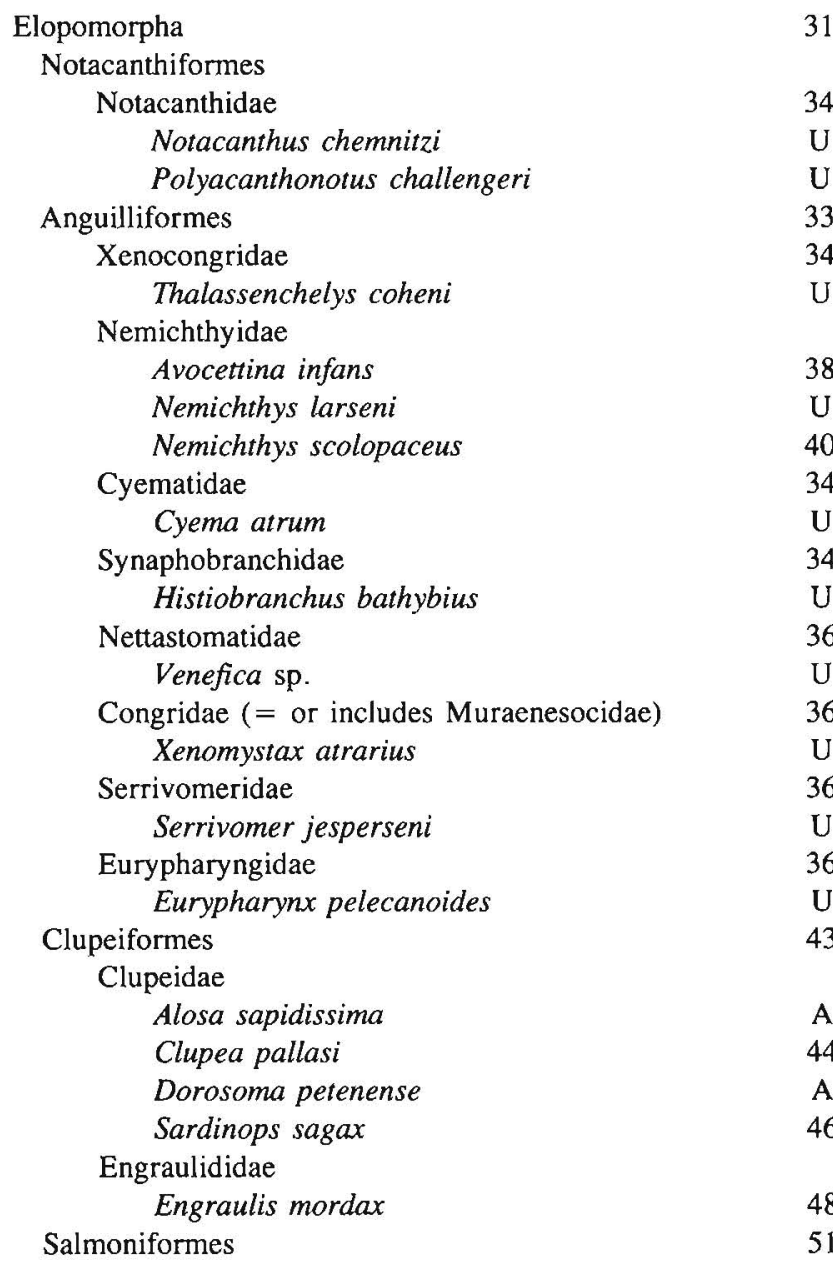

Argentinoidei

Argentinidae

Argentina sialis

Nansenia candida

Bathylagidae

Bathylagus bericoides

Bathylagus milleri

Bathylagus ochotensis

Bathylagus pacificus

Bathylagus wesethi

Leuroglossus schmidti

Leuroglossus stilbius
Therobrommus callorhinus

Opisthoproctidae

Bathylychnops exilis

Dolichopteryx sp.

Dolichopteryx longipes

Macropinna microstoma

Alepocephalidae

Alepocephalus tenebrosus

Bathylaco nigricans

Ericara salmoneum

Leptochilichthys agassizi

Narcetes stomias

Talismania bifurcata

Platytroctidae

Holtbymia innesi

Holtbyrnia latifrons

Maulisia argipalla

Pellisolus eubranchus

Sagamichthys abei

Salmonoidei

Osmeridae

Allosmerus elongatus

Hypomesus olidus

Hypomesus pretiosus

Mallotus villosus

Osmerus mordax

Spirinchus starksi

Spirinchus thaleichthys

Thaleichthys pacificus

Salmonidae

Coregoninae

Coregonus laurettae

Coregonus nasus

Coregonus pidschian

Coregonus sardinella

Stenodus leucichthys

Salmoninae

Oncorhynchus gorbuscha

Oncorhynchus keta

Oncorhynchus kisutch

Oncorhynchus nerka

Oncorhynchus tshawytscha

Parasalmo clarki

Parasalmo gairdneri

Parasalmo mykiss

Parasalmo penshinensis

Salvelinus alpinus

Salvelinus leucomaenis

Salvelinus malma

Stomiiformes

Gonostomatoidei

Gonostomatidae

Cyclothone acclinidens

Cyclothone atraria

Cyclothone pallida

Cyclothone pseudopallida

Cyclothone signata

Cyclothone sp.

Gonostoma atlanticum

Gonostoma gracile
70

72

72

74

76

D

D

D

D

D

D

77

U

$\mathrm{U}$

$\mathrm{U}$

$\mathrm{U}$

U

79

$\mathrm{U}$

U

$\mathrm{U}$

80

$\mathrm{A} / \mathrm{F}$

$\mathrm{U}$

A

A

F 
Sternoptychidae

Argyropelecus affinis

Argyropelecus hemigymnus

Argyropelecus lychnus

Argyropelecus sladeni

Sternoptyx diaphana

Sternoptyx pseudobscura

Danaphos oculatus

Stomioidei

Chauliodontidae

Chauliodus macouni

Melanostomiidae

Bathophilus flemingi

Eustomias sp.

Opostomias mitsuii

Tactostoma macropus

Malacosteidae

Aristostomias scintillans

Idiacanthidae

Idiacanthus antrostomus

Idiacanthus fasciola

Scopelomorpha

Aulopiformes

Scopelarchidae

Benthalbella dentata

Benthalbella linguidens

Notosudidae

Scopelosaurus harryi

Bathysauridae

Bathysaurus mollis

Synodontidae

Synodus lucioceps

Paralepididae

Lestidiops ringens

Notolepis rissoi

Paralepis atlantica

Anotopteridae

Anotopterus pharao

Alepisauridae

Alepisaurus ferox

Myctophiformes

Neoscopelidae

Neoscopelus macrolepidotus

Myctophidae

Ceratoscopelus townsendi

Diaphus theta

Dorsadena yaquinae

Electrona rissoi

Lampadena urophaos

Lampanyctus fernae

Lampanyctus jordani

Lampanyctus regalis

Lampanyctus ritteri

Loweina rara

Notoscopelus resplendens

Notoscopelus japonicus

Parvilux ingens

Protomyctophum crockeri

Protomyctophum thompsoni

Stenobrachius leucopsarus
Stenobrachius nannochir

Symbolophorus californiensis

Taaningichthys bathyphilus

Tarletonbeania crenularis

Tarletonbeania taylori

Gadiformes

Moridae

Antimora microlepis

Laemonema longipes

Melanonidae

Melanonus zugmayeri

Merlucciidae

Merluccius productus

Gadidae

Boreogadus saida

Eleginus gracilis

Gadus macrocephalus

Microgadus proximus

Theragra chalcogramma

Macrouridae

Albatrossia pectoralis

Coryphaenoides acrolepis

Coryphaenoides armatus

Coryphaenoides cinereus

Coryphaenoides filifer

Coryphaenoides leptolepis

Coryphaenoides liocephalus

Coryphaenoides longifilis

Coryphaenoides yaquinae

Nezumia stelgidolepis

Ophidiiformes

Ophidioidei

Ophidiidae

Bassozetus sp.

Chilara taylori

Dicrolene filamentosa

Holcomycteronus profundissimus

Spectrunculus grandis

Spectrunculus radcliffei

Bythitoidei

Aphyonidae

Barathronus pacifica

Sciadonus pedicellaris

Bythitidae

Brosmophycis marginata

Cataetyx rubrirostris

Batrachoidiformes

Batrachoididae

Porichthys notatus

Lophiiformes

Ceratioidei

Ceratiidae

Ceratias holboelli

Ceratias sp.

Oneirodidae

Bertella idiomorpha

Chaenophryne longiceps

Chaenophryne melanorhabdus

Oneirodes bulbosus

Oneirodes thompsoni
U

154

174

158

$\mathrm{U}$

181

182

U

$\mathrm{U}$

184

186

189

190

192

194

196

198

200

$\mathrm{U}$

202

U

U

204

206

U

U

U

208

212

U

214

U

U

U

U

217

U

U

218

U

D

221

222

222

U

U

222

U

224

U

226 
Gobiesociformes

Gobiesocidae

Gobiesox maeandricus

Rimicola muscarum

Atherinomorpha

Beloniformes

Scomberesocidae Cololabis saira

Atheriniformes

Atherinidae

Atherinops affinis

Atherinopsis californiensis

Lampriformes

Lampridae

$$
\text { Lampris guttatus }
$$

Trachipteridae

Trachipterus altivelis

Beryciformes

Anoplogastridae

Anoplogaster cornuta

Melamphaidae

Melamphaes lugubris

Melamphaes parvus

Poromitra crassiceps

Scopeloberyx robustus

Scopelogadus bispinosus

Cetomimoidei

Barbourisiidae Barbourisia rufa

Cetomimidae

Cetomimus sp.

Cetostoma regani

Gyrinomimus sp.

Rondeletiidae

Rondeletia loricata

Zeiformes

Oreosomatidae

Allocyttus sp.

Gasterosteiformes

Aulorhynchidae Aulorhynchus flavidus

Gasterosteidae

Gasterosteus aculeatus

Pungitius pungitius

Syngnathidae

Syngnathus leptorhynchus 264

Scorpaeniformes

Scorpaenoidei

Scorpaenidae

Sebastinae

Sebastes aleutianus

Sebastes alutus

Sebastes auriculatus

Sebastes aurora

Sebastes babcocki

Sebastes borealis

Sebastes brevispinis

Sebastes caurinus

Sebastes chlorostictus

Sebastes ciliatus

Sebastes crameri

Sebastes diploproa
Sebastes elongatus $\quad 302$

Sebastes emphaeus $\quad 304$

Sebastes entomelas $\quad 306$

Sebastes flavidus 308

Sebastes glaucus U

Sebastes goodei U

Sebastes helvomaculatus $\quad 310$

Sebastes jordani $\quad 312$

Sebastes maliger U

Sebastes melanops $\quad 314$

Sebastes melanostictus U

Sebastes melanostomus $\quad 316$

Sebastes miniatus $\quad 318$

Sebastes mystinus $\quad 320$

Sebastes nebulosus U

Sebastes nigrocinctus U

Sebastes paucispinis $\quad 322$

Sebastes pinniger $\quad 324$

Sebastes polyspinis U

Sebastes proriger $\quad 326$

Sebastes rastrelliger U

Sebastes reedi $\quad 328$

Sebastes ruberrimus U

Sebastes rufus $\quad 330$

Sebastes saxicola $\quad 332$

Sebastes variegatus

Sebastes wilsoni U

Sebastes zacentrus $\quad 334$

Sebastolobinae 336

Sebastolobus alascanus $\quad 336$

Sebastolobus altivelis $\quad 336$

Sebastolobus macrochir U

Anoplopomatoidei

Anoplopomatidae

Anoplopoma fimbria 338

Erilepis zonifer U

Hexagrammoidei

Hexagrammidae 341

Oxylebius pictus $\quad 342$

Zaniolepis frenata $\quad 344$

Zaniolepis latipinnis $\quad 344$

Ophiodon elongatus $\quad 346$

Pleurogrammus monopterygius $\quad 348$

Hexagrammos decagrammus $\quad 352$

Hexagrammos lagocephalus $\quad 354$

Hexagrammos octogrammus $\quad 356$

Hexagrammos stelleri 358

Cottoidei

Cottidae 360

Archaulis biseriatus U

Artediellichthys nigripinnis U

Artedielliscus sp. U

Artediellus camchaticus U

Artediellus gomojunovi U

Artediellus miacanthus $\quad \mathrm{U}$

Artediellus ochotensis U

Artediellus pacificus U

Artediellus scaber U

Artedius corallinus $\quad 420$

Artedius fenestralis $\quad 414$

Artedius harringtoni $\quad 416$

Artedius lateralis $\quad 418$ 


\begin{tabular}{|c|c|}
\hline Artedius meanyi & 384 \\
\hline Artedius notospilotus & 420 \\
\hline Ascelichthys rhodorus & 386 \\
\hline Asemichthys taylori & $\mathrm{U}$ \\
\hline Blepsias bilobus & 448 \\
\hline Blepsias cirrhosus & 450 \\
\hline Chitonotus pugetensis & 388 \\
\hline Clinocottus acuticeps & 422 \\
\hline Clinocottus embryum & 424 \\
\hline Clinocottus globiceps & 426 \\
\hline Clinocottus recalvus & 428 \\
\hline Cottoid A & 438 \\
\hline Cottus aleuticus & $\mathrm{U}$ \\
\hline Cottus asper & 444 \\
\hline Dasycottus setiger & 440 \\
\hline Enophrys bison & 390 \\
\hline Enophrys diceraus & $U$ \\
\hline Enophrys lucasi & $\mathrm{U}$ \\
\hline Eurymen gyrinus & $\mathrm{U}$ \\
\hline Gilbertidia sigalutes & 434 \\
\hline Gymnocanthus detriscus & $\mathrm{U}$ \\
\hline Gymnocanthus galeatus & $\mathrm{U}$ \\
\hline Gymnocanthus pistilliger & $\mathrm{U}$ \\
\hline Gymnocanthus tricuspis & 394 \\
\hline Hemilepidotus gilberti & 372 \\
\hline Hemilepidotus hemilepidotus & 374 \\
\hline Hemilepidotus jordani & 376 \\
\hline Hemilepidotus papilio & $\mathrm{U}$ \\
\hline Hemilepidotus spinosus & 378 \\
\hline Hemilepidotus zapus & 380 \\
\hline Hemitripterus bolini & $\mathrm{U}$ \\
\hline Hemitripterus villosus & 452 \\
\hline Icelinus borealis & $\mathrm{U}$ \\
\hline Icelinus burchami & $\mathrm{U}$ \\
\hline Icelinus filamentosus & $\mathrm{U}$ \\
\hline Icelinus oculatus & $\mathrm{U}$ \\
\hline Icelinus tenuis & $\mathrm{U}$ \\
\hline Icelus canaliculatus & $\mathrm{U}$ \\
\hline Icelus euryops & $\mathrm{U}$ \\
\hline Icelus scutiger & $\mathrm{U}$ \\
\hline Icelus spatula & U \\
\hline Icelus spiniger & $\mathrm{U}$ \\
\hline Icelus uncinalis & $U$ \\
\hline Jordania zonope & $\mathrm{U}$ \\
\hline Leptocottus armatus & 446 \\
\hline Malacocottus kincaidi & $\mathrm{U}$ \\
\hline Malacocottus sp. & $\mathrm{U}$ \\
\hline Malacocottus zonurus & 442 \\
\hline Megalocottus laticeps & $\mathrm{U}$ \\
\hline Megalocottus platycephalus & $U$ \\
\hline Microcottus sellaris & $\mathrm{U}$ \\
\hline Myoxocephalus axillaris & $U$ \\
\hline Myoxocephalus brandti & $\mathrm{U}$ \\
\hline Myoxocephalus jaok & $\mathrm{U}$ \\
\hline Myoxocephalus niger & $\mathrm{U}$ \\
\hline Myoxocephalus polyacanthocephalus & U \\
\hline Myoxocephalus quadricornis & $\mathrm{U}$ \\
\hline Myoxocephalus scorpioides & $\mathrm{U}$ \\
\hline Myoxocephalus scorpius & 402 \\
\hline Myoxocephalus sp. & $\mathrm{U}$ \\
\hline Myoxocephalus stelleri & $U$ \\
\hline Myoxocephalus verrucosus & $\mathrm{U}$ \\
\hline
\end{tabular}

Nautichthys oculofasciatus

Nautichthys pribilovius

Nautichthys robustus

Oligocottus maculosus

Oligocottus rimensis

Oligocottus snyderi

Paricelinus hopliticus

Phallocottus obtusus

Porocottus bradfordi

Porocottus quadrifilis

Psychrolutes paradoxus

Psychrolutes phrictus

Radulinus asprellus

Radulinus boleoides

Rhamphocottus richardsoni

Scorpaenichthys marmoratus

366

Sigmistes caulias

Sigmistes smithi

Stelgistrum beringianum

Stelgistrum concinnum

Sternias xenostethus

Stlegicottus xenogrammus

Synchirus gilli

Taurocottus brashnikovi

Thecopterus aleuticus

Thyriscus anoplus

Triglops forficata

Triglops jordani

Triglops macellus

Triglops metopias

Triglops pingeli

Triglops scepticus

Zesticelus profundorum

U

U

U

U

U

U

Agonomalus mozinoi

Agonopsis yulsa

Agonus acipenserinus

Agonus decagonus

Anoplagonus inermis

Aspidophoroides bartoni

Aspidophoroides olriki

Bathyagonus alascanus

Bathyagonus infraspinatus

Bathyagonus nigripinnis

Bathyagonus pentacanthus

Bothragonus swani

Hypsagonus quadricomis

Ocella dodecaedron

Ocella impi

Ocella verrucosa

Odontopyxis trispinosa

Pallasina barbata

Percis japonicus

Sarritor frenatus

Sarritor leptorhynchus

Stellerina xyosterna

Xeneretmus latifrons

Xeneretmus leiops

Xeneretmus triacanthus 
Cyclopteropsis phrynoides

Eumicrotremus andriashevi

Eumicrotremus barbatus

Eumicrotremus birulai

Eumicrotremus derjugini

Eumicrotremus gyrinops

Eumicrotremus orbis

Eumicrotremus soldatovi

Eumicrotremus taranetzi

Lethotremus muticus

Pelagocyclus vitiazi

Liparidinae

Acantholiparis caecus

Acantholiparis opercularis

Acantholiparis sp.

Careproctus abbreviatus

Careproctus attenuatus

Careproctus bowersianus

Careproctus cameliae

Careproctus canus

Careproctus colletti

Careproctus cypselurus

Careproctus ectenes

Careproctus filamentosus

Careproctus furcellus

Careproctus gilberti

Careproctus longifilis

Careproctus melanurus

Careproctus microstomus

Careproctus mollis

Careproctus opisthotremus

Careproctus oregonensis

Careproctus ostentum

Careproctus ovigerum

Careproctus pellucidus

Careproctus phasma

Careproctus pycnosoma

Careproctus rastrinus

Careproctus scottae

Careproctus simus

Careproctus sp.

Careproctus spectrum

Careproctus zachirus

Crystallichthys cyclospilus

Crystallichthys mirabilis

Elassodiscus caudatus

Elassodiscus tremebundus

Gyrinichthys minytremus

Liparis bristolensis

Liparis callyodon

Liparis catharus

Liparis cyclopus

Liparis dennyi

Liparis florae

Liparis fucensis

Liparis gibbus

Liparis grebnitzki

Liparis mednius

Liparis megacephalus

Liparis micraspidophorus

Liparis mucosus

Liparis ochotensis
Liparis pulchellus

Liparis rutteri

Liparis tunicatus

Lipariscus nanus

Nectoliparis pelagicus

Odontoliparis ferox

Osteodiscus cascadiae

Paraliparis cephalus

Paraliparis dactylosus

Paraliparis deani

Paraliparis holomelas

Paraliparis latifrons

Paraliparis megalopus

Paraliparis melanobranchus

Paraliparis mento

Paraliparis paucidens

Paraliparis pectoralis

Paraliparis rosaceus

Paraliparis ulochir

Polypera beringiana

Polypera greeni

Rhinoliparis attenuatus

Rhinoliparis barbulifer

Temnocora candida

Perciformes

Percoidei

Carangidae

Naucrates ductor

Seriola lalandi

Trachurus symmetricus

Bramidae

Brama japonicus

Taractes asper

U

U

U

U

476

U

U

$\mathrm{U}$

$\mathrm{U}$

478

U

$\mathrm{U}$

U

$\mathrm{U}$

U

U

$\mathrm{U}$

$\mathrm{U}$

U

U

U

U

480

U

483

Caristiidae

Caristius macropus

Sciaenidae

Atractoscion nobilis

Genyonemus lineatus

Seriphus politus

Pentacerotidae

Pseudopentaceros sp.

494

Embiotocidae

Amphistichus koelzi

Amphistichus rhodoterus

Brachyistius frenatus

Cymatogaster aggregata

Embiotoca lateralis

Hyperprosopon argenteum

Hyperprosopon ellipticum

Hypocritichthys analis

Phanerodon furcatus

Rhacochilus vacca

Zoarcoidei

Bathymasteridae

Bathymaster caeruleofasciatus

Bathymaster leurolepis

Bathymaster signatus

Ronquilus jordani

V

V

V

V

V

V

V

V

$\mathrm{V}$

V

496

U

$\mathrm{U}$

U

Zoarcidae

Bothrocara brunneum

Bothrocara hollandi

Bothrocara molle 
Bothrocara pusillum

Bothrocara remigerum

Derepodichthys alepidotus

Gymnelus bilabrus

Gymnelus hemifasciatus

Gymnelus popovi

Gymnelus viridus

Krusensterniella pavlovskii

Lycenchelys altus

Lycenchelys camchaticus

Lycenchelys crotalinus

Lycenchelys hippopotamus

Lycenchelys jordani

Lycenchelys longirostris

Lycenchelys microporus

Lycenchelys pliciferus

Lycenchelys rassi

Lycenchelys ratmanovi

Lycenchelys roseus

Lycenchelys volki

Lycodapus derjugini

Lycodapus dermatinus

Lycodapus endemoscotus

Lycodapus fierasfer

Lycodapus leptus

Lycodapus mandibularis

Lycodapus pachysoma

Lycodapus parviceps

Lycodapus poecilis

Lycodapus psarosomatus

Lycodes brevipes

Lycodes concolor

Lycodes cortezianus

Lycodes diapterus

Lycodes mucosus

Lycodes pacifica

Lycodes palearis

Lycodes raridens

Lycodes turneri

Lyconema barbatum

Melanostigma pammelas

Nalbantichthys elongatus

Opaeophacus acrogeneius

Pachycara bulbiceps

Puzanovia rubra

Taranetzella lycoderma

Stichaeidae

Stichaeinae

Stichaeini

Eumesogrammus praecisus

Stichaeus punctatus

Chirolophini

Bryozoichthys lysimus

Bryozoichthys marjorius

Chirolophis decoratus

Chirolophis nugator

Chirolophis snyderi

Chirolophis tarsodes

Gymnoclinus cristulatus

Lumpeninae

Lumpenini

Acantholumpenus mackayi
Anisarchus medius

Lumpenella longirostris

Lumpenus fabricii

Lumpenus maculatus

Lumpenus medius

Lumpenus sagitta

Poroclinus rothrocki

Opisthocentrini

Allolumpenus hypochromus

Opisthocentrus ocellatus

Plectobranchus evides

Xiphisterinae

Alectrini

Alectridium aurantiacum

Anoplarchus insignis

Anoplarchus purpurescens

Xiphisterini

Cebidichthys violaceus

Phytichthys chirus

Xiphister atropurpureus

Xiphister mucosus

Cryptacanthodidae

Delolepis gigantea

Lyconectes aleutensis

Pholididae

Apodichthys flavidus

Pholis clemensi

Pholis dolichogaster

Pholis fasciata

Pholis gilli

Pholis laeta

Pholis ornata

Pholis schultzi

Xererpes fucorum

Anarhichantidae

Anarhichas orientalis

Anarrhichthys ocellatus

Ptilichthyidae

Ptilichthys goodei

Zaproridae

Zaprora silenus

U

U

U

U

U

U

U

504

U

508

510

504

U

U

512

504

U

514

516

518

520

520

522

$\mathrm{U}$

U

U

U

U

$\mathrm{U}$

U

U

U

524

526

528

530

Scytalinidae

Scytalina cerdale

Trachinoidei

Trichodontidae

Arctoscopus japonicus $\quad \mathrm{U}$

Trichodon trichodon $\quad 532$

Blennioidei

Clinidae

Gibbonsia metzi

Gibbonsia montereyensis

Heterostichus rostratus

Icosteoidei

Icosteidae

Icosteus aenigmaticus

Ammodytoidei

Ammodytidae

Ammodytes hexapterus

Gobioidei

Gobiidae 
Aphanopus carbo

Benthodesmus elongatus

Benthodesmus tenuis

$\mathrm{U}$

Lepidopus fitchi

$\mathrm{U}$

Scombridae

Euthynnus pelamis

Sarda chiliensis

Scomber japonicus

Thunnus alalunga

Thunnus albacares

Thunnus obesus

Thunnus thynnus

Acanthuroidei

Luvaridae*

Luvarus imperialis

Stromateoidei

Centrolophidae

Icichthys lockingtoni

Tetragonuridae

Tetragonurus cuvieri

Stromateidae

Peprilus simillimus

Pleuronectiformes

Paralichthyidae

Citharichthys sordidus

Citharichthys stigmaeus

Paralichthys californicus

Pleuronectidae

Acanthopsetta nadeshnyi

Atheresthes evermanni

Atheresthes stomias

Clidoderma asperrimum

Eopsetta jordani

Glyptocephalus zachirus

588

Hippoglossoides elassodon $\quad 590$

$S$

$\mathrm{S}$

554

$\mathrm{S}$

$S$

$\mathrm{S}$

S

Hippoglossoides robustus

Hippoglossus stenolepis

Inopsetta ischrya

Isopsetta isolepis

Lepidopsetta bilineata

Lepidopsetta 2

Limanda aspera

Limanda proboscidea

Limanda sakhalinensis

Liopsetta glacialis

Lyopsetta exilis

Microstomus pacificus

Parophrys vetulus

Platichthys stellatus
Pleuronectes quadrituberculatus

Pleuronichthys coenosus

Pleuronichthys decurrens

Psettichthys melanostictus

Reinhardtius hippoglossoides

Cynoglossidae

Symphurus atricauda

Tetraodontiformes

Molidae

Mola mola

*Luvaridae is placed in the Scombroidei in J. Nelson (1984), but other studies (Leis and Richards 1984) have shown it belongs in the Acanthuroidei. 
This guide is designed primarily to assist in identifying eggs and larvae of fishes collected by sampling plankton. This process is usually divided into two main steps: 1) Sorting fish material out of the total plankton, and 2) identifying fish eggs and larvae of interest. Sorting procedures such as outlined in Kramer et al. (1972) are usually used. Matarese and Sandknop (1984) discuss identification of fish eggs, and Powles and Markle (1984) discuss identification of fish larvae. Eggs should be fixed and maintained in 3-5\% buffered formalin, whereas larvae are better preserved if transferred to $70 \%$ ethanol after fixation in $3-5 \%$ buffered formalin (see Lavenberg et al. 1984). Identification is usually accomplished using a dissecting microscope at magnifications of 7 to $70 \times$, with lighting from above and below a clear or frosted glass stage. A calibrated ocular micrometer is needed for various measurements of eggs and larvae. Specimens are frequently examined as whole mounts in their preservation medium in dishes or watch glasses ( $\sim 50 \mathrm{~mm}$ diameter). Fine probes and forceps can be used carefully to manipulate the specimens. A piece of white paper inserted between the specimen dish and the stage makes pigment more readily visible.

Since the larvae, and certainly the eggs, do not look like their parents, different characters are required to identify them. In practice, it is often convenient to separate the eggs and larvae in a sample into similar-appearing types, before recording specific observations or trying to identify them. Generally, it is best to identify all specimens in a sample to the lowest taxonomic level possible, even if the collection was intended for certain target species; without careful examination, other species could be misidentified as those of a target species.

In addition to characters of the eggs and larvae themselves, clues to their identity are found in information such as geographic area, depth, and date of collection.

\section{Eggs}

For pelagic fish eggs, the following identifying characters are recorded as known in this guide (see Matarese and Sandknop 1984 for more details): Shape (most are spherical, a few ellipsoidal); size (most are $\sim 1 \mathrm{~mm}$ in diameter, range $\sim 0.5-4.0 \mathrm{~mm}$ for pelagic eggs covered in this guide); oil globules (present in many species, vary in number and size); yolk (homogeneous or segmented); chorion (smooth in most, but sculptured in some); perivitelline space (narrow $[<0.1$ egg diameter] in many, but reaches one-half egg diameter in some); and embryonic characters (shape, pigment, gut length, early fin development, and myomere count of the embryo in late-stage eggs). Few taxonomic generalizations can be made for fish eggs; closely related species may have eggs that are as dissimilar as species in different orders (Table 2). However, the yolk is usually segmented in primitive teleosts (e.g., clupeiforms, anguilliforms, and salmoniforms) and homogeneous in more advanced forms. Also, oil globules are absent in pleuronectids covered in this guide.

\section{Larvae}

Fish larvae possess a larger suite of characters than the eggs, and may undergo dramatic changes with development. To begin to identify a larval fish, the following should be noted: Size (NL or SL) and stage of development (e.g., preflexion, flexion, postflexion, transforming [Kendall et al. 1984]) as well as its general appearance (e.g., long and slender, short and deep bodied, lightly or heavily pigmented). Figure 2 illustrates anatomical features and measurements used to describe larvae. With practice, it is often possible to group larvae according to order or family (Fig. 3).

Meristic characters Meristic characters are essential and should be determined, understanding that fin elements are gradually added during larval development. Myomeres are the first meristic character to stabilize, and the number usually reflects the number of adult vertebrae. The number of vertebrae varies among the fishes covered in this guide from $<20$ (molids) to $>200$ (e.g., most elopomorphs) (see Figure 4 where, for fishes occurring in the study area, vertebral counts are arranged by family in ascending order). Use of polarized light often facilitates counting myomeres. Myosepta are frequently more discernable than the myomeres, and if they are counted, one should be added to account for the myomeres anterior and posterior to the first and last myosepta.

The developing median fins contain several bits of taxonomic information. Dipping the larva in a potassium hydroxide solution and then in an Alizarin Red solution (see Hollister 1934 for solution recipes) for a few seconds, then rinsing and examining it in its preservative allows fin rays and head spines to be seen more clearly, since they stain red. However, this procedure may interfere with future attempts to clear and stain the larva. The principal caudal fin count is often an ordinal character (Table 2) and is very useful and relatively easy to determine in larvae, since it generally reaches its adult state shortly after flexion. The number, position, and order of development of the dorsal and anal fins, and their composition in terms of spines and soft rays, are important characters (Table 2). In several fishes (e.g., Sebastes), the final dorsal and anal spine develop first as soft rays, and change to spines during transformation. Thus, in late larvae, the median fins are different in the composition of spines and soft rays than in the adults. Fin rays that become elongate in larvae often develop precociously, otherwise second dorsal and anal soft rays generally develop concurrently when present. Spinous dorsal fins may develop before, concurrently, or after the second dorsal fin. When there is a gap between the spinous and soft-ray portions of the dorsal fin, spines generally develop after the soft rays.

In the sequence of paired fin development, the pectorals often develop early in the larval period, and the pelvics late. The pectoral fins form in the egg as larval pectoral fins, without fin rays. The fin rays generally develop much later in the larval stage. The length of the pectoral fin, as well as its number of rays, is a useful character. While the number of pectoral rays may vary within and among species in a genus, the pelvic fin position and formula are generally stable at a high level of classification (e.g., order; see Table 2). The pelvic fin is absent in anguilliforms and members of some other groups (e.g., zoarcids, ammodytids, and molids). In primitive fishes (e.g., clupeiforms, myctophiforms) it contains no spines, is abdominal, and generally contains more than five soft rays. In most perciforms and scorpaeniforms, the basic pelvic fin count is I,5, and it is thoracic in position. This count is reduced in some; notably in the northern Pacific, cottids often have fewer than five soft rays. The pelvic fin is modified into a sucking disc in gobiesociforms and in some cyclopterids.

Other meristic characters (e.g., gill rakers, secondary caudal fin rays, scales) develop too late to be generally useful in identifying larvae, but may be essential when working with pelagic juveniles. 
Pigmentation Pigmentation available as taxonomic characters on larvae is limited to melanophores, since other pigment cells (e.g., xanthophores) do not retain their color in currently-used fixatives and preservatives. Melanophore patterns are very useful for identifying larval fishes. The relative size, position, and sometimes the number of melanophores in series should be noted. In some cases, pigmentation consists of a group of melanophores in a specific area; in others the pigmentation consists of an individual melanophore. Pigmentation generally changes as larvae develop. Movement of individual melanophores is rather limited, but addition or loss of melanophores is common. Usually preflexion larvae are less pigmented than later larvae, and late in the larval period, as transformation occurs, the larval pigment pattern is overgrown by the largely superficial pattern of the juvenile. Between the preflexion and transformation stages in most fish there is a definite larval pigment pattern which is relatively stable, and unique to a species in many cases. Although the position of melanophores is a species characteristic, the degree of contradiction seems to be physiologically moderated. Thus, larvae of the same species could have a different overall pigmented appearance, lighter or darker. Several species in the Northeast Pacific have heavily pigmented larvae, which are readily recognized in samples but may be confused with one another (Table 3, Fig. 5).

Morphology Larval shape can vary from stout and robust to quite slender and elongate (Table 2). Several fishes in the study area have elongate larvae which may be confused with one another (Table 4, Fig. 6). The ratio of body depth at the pectoral fin to standard length is usually sufficient to characterize overall body shape. The size and shape of the head and eye may also be important. The length of the gut, measured as the ratio of preanal to standard length, is quite useful. As with other characters, larval shape characters vary with development, so the size and stage of development should be noted when comparing shape of an unknown larva to illustrations and descriptions of known specimens.

Head spines, when present, may be more numerous and accentuated in larvae than in adults. Among Northeast Pacific fishes, larval head spines are most prevalent in cottids and scorpaenids, although they also occur in some members of groups such as perciforms and pleuronectids (Table 2).
Once the above data on an unknown specimen or group of similar specimens are assembled, actual identification becomes largely a process of eliminating species whose characters do not match the unknown specimen. Keys are not presented in this guide and generally do not work well with fish larvae, because the larvae change so much with development, and the larvae of all species in a study area are rarely known. With larvae, particularly, the first attempt should be to identify the unknown to order or family, based on meristic values, shape, and general appearance. Table 2 and the figures of larval representatives of various orders (Fig. 4) should be of assistance in this. Once an idea of the appropriate order/ suborder is established, more detailed information can be obtained in the material at the beginning of each ordinal account, including general life-history characters, species represented in the study area, and meristic and early-life-history characters. Illustrations, meristics, and other information given in the individual taxon accounts in this guide should then be compared with the unknown specimens to find the most likely species. Differences between the unknown and described larvae should be noted. If an unknown specimen does not match any larval descriptions given here, check meristic tables of the most likely taxa to see if the unknown specimen fits a species whose larvae have not yet been described. 

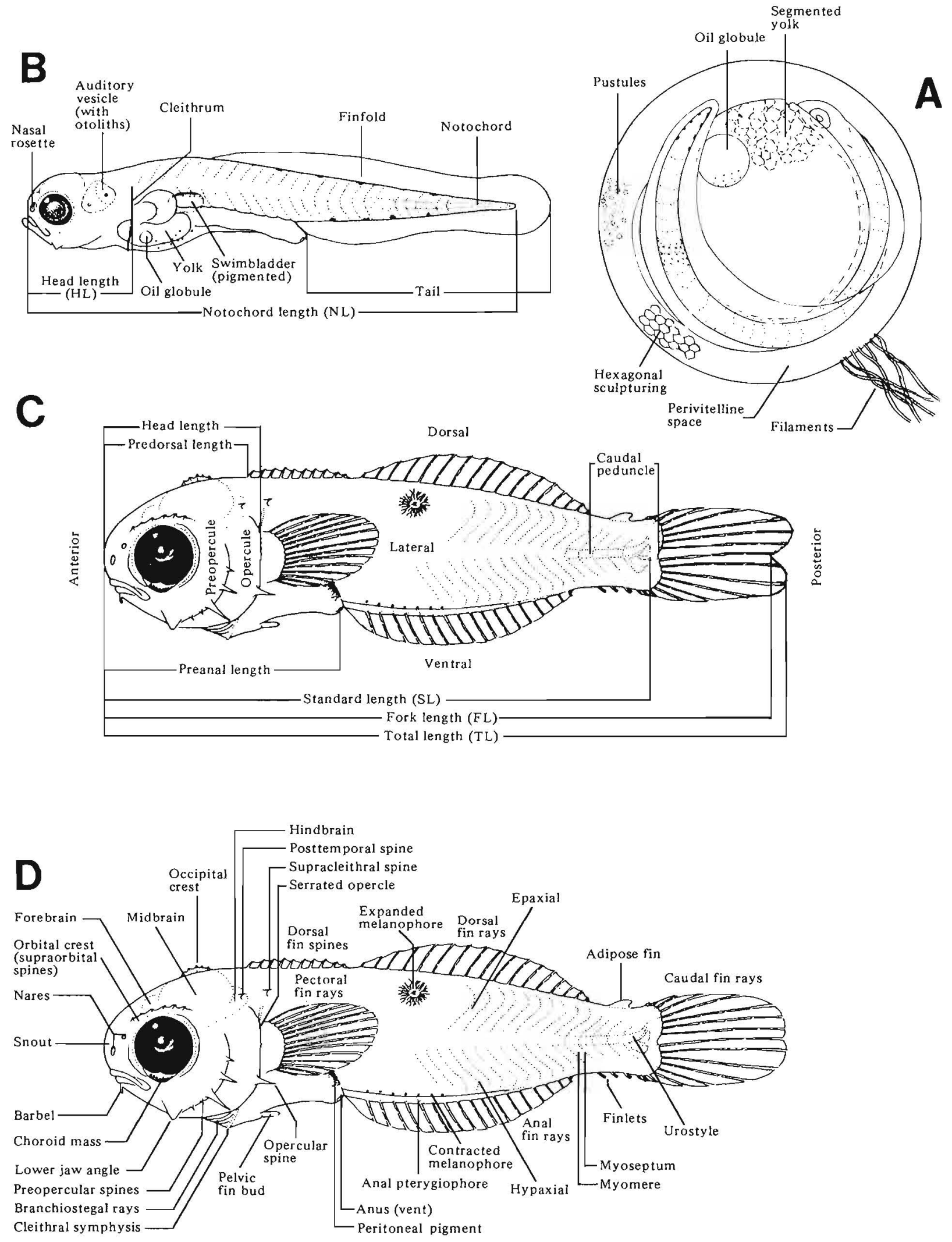

Figure 2

Examples of features used to describe early stages of fishes: A, egg; B, preflexion larva; C, late larva showing base points for measurements; D, late larva showing morphological features (B-D, after Fahay 1983). 

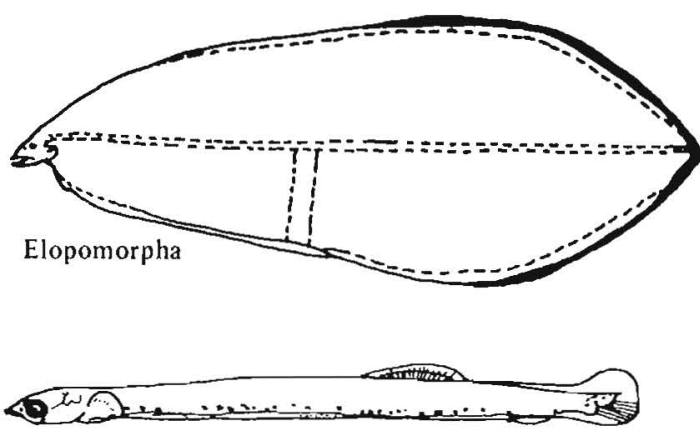

Clupeiformes

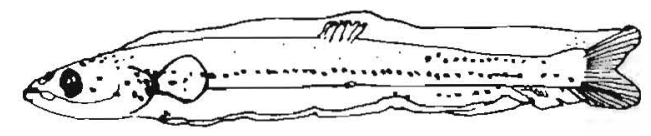

Argentinoidei
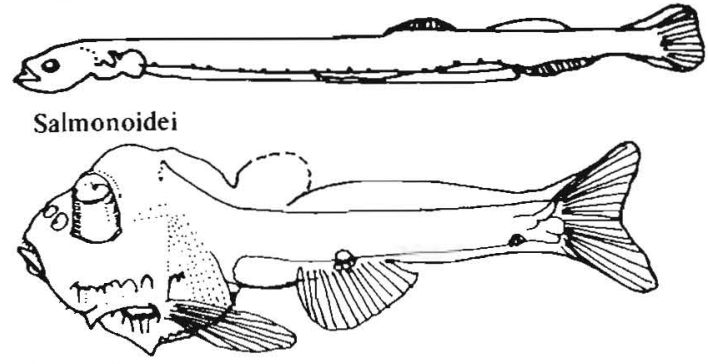

Stomiiformes
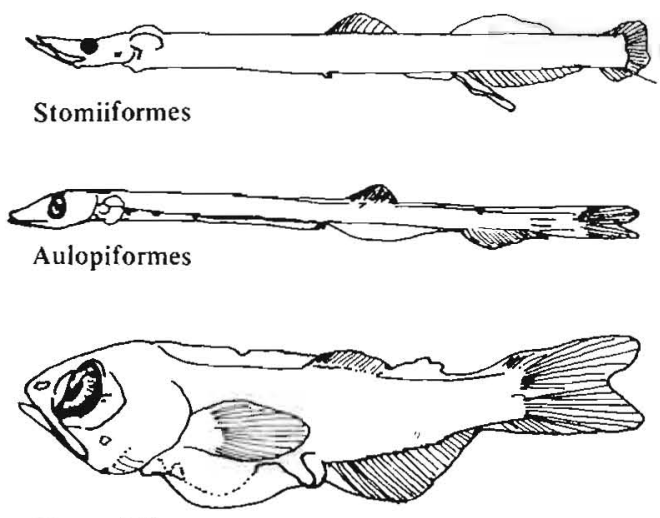

Myctophiformes

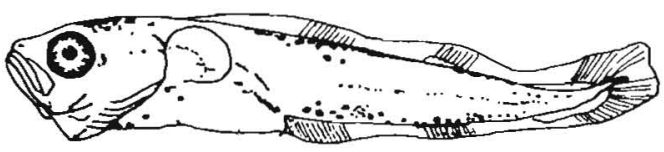

Gadiformes

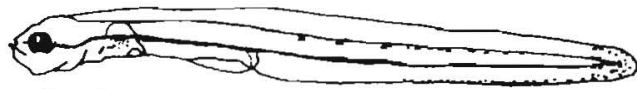

Ophidiiformes
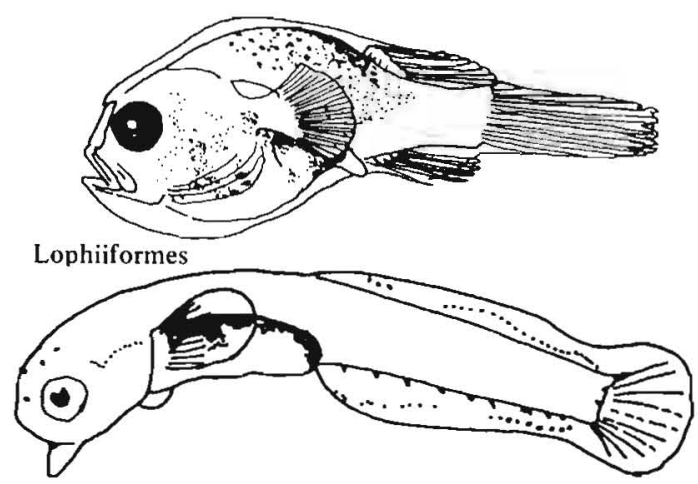

Gobiesociformes
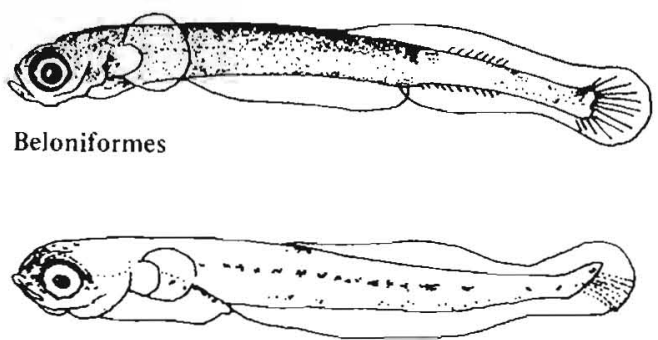

Atheriniformes

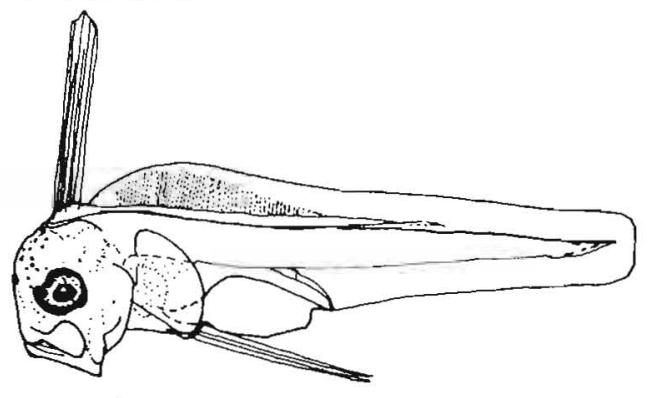

Lampriformes
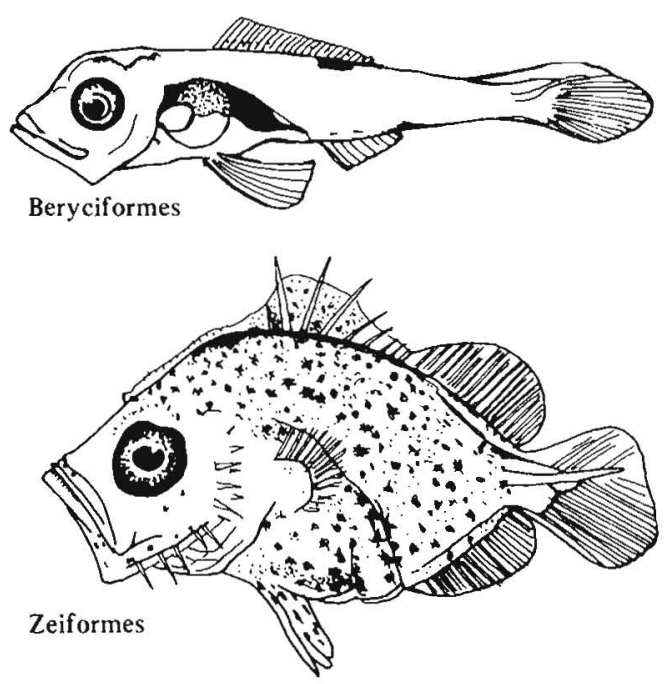

Figure 3

Representative postflexion larvae of higher categories of fishes of the Northeast Pacific Ocean. 


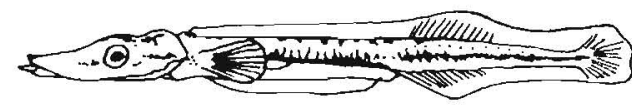

Gasterosteiformes

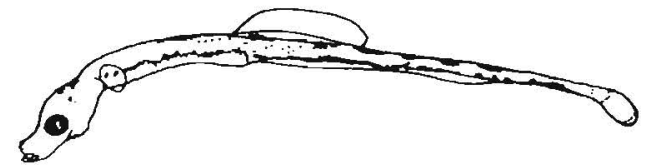

Gasterosteiformes

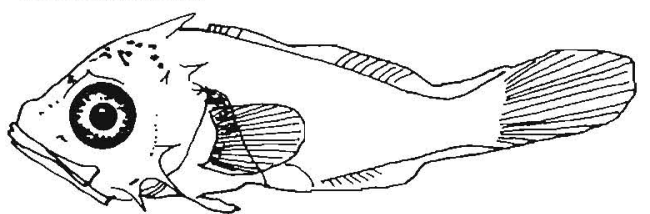

Scorpaenoidei

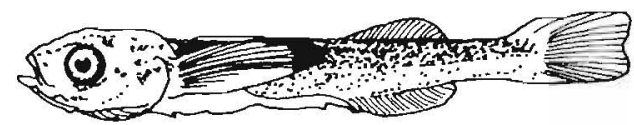
Anoplopomatoidei

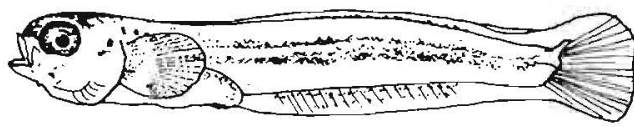

Hexagrammoidei

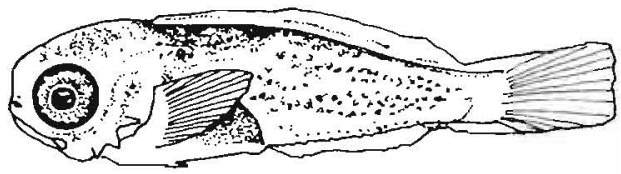

Cottoidei

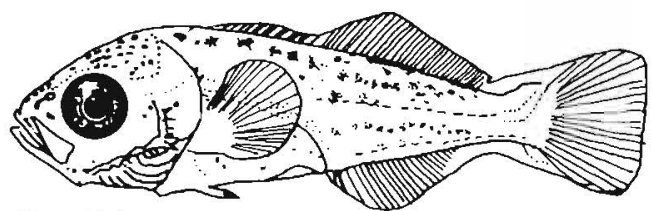

Percoidei

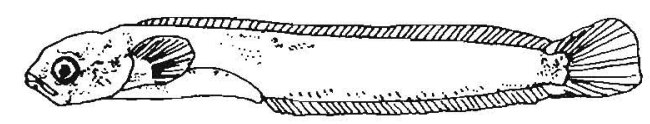

Zoarcoidei

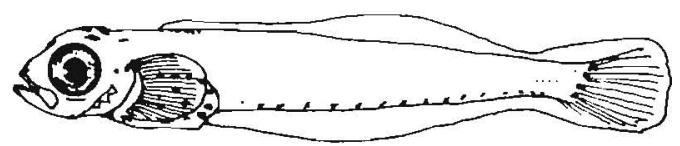

Trachinoidei

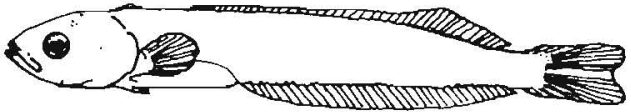

Blennioidei

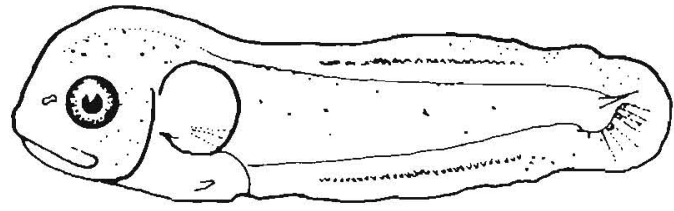

lcosteoidei
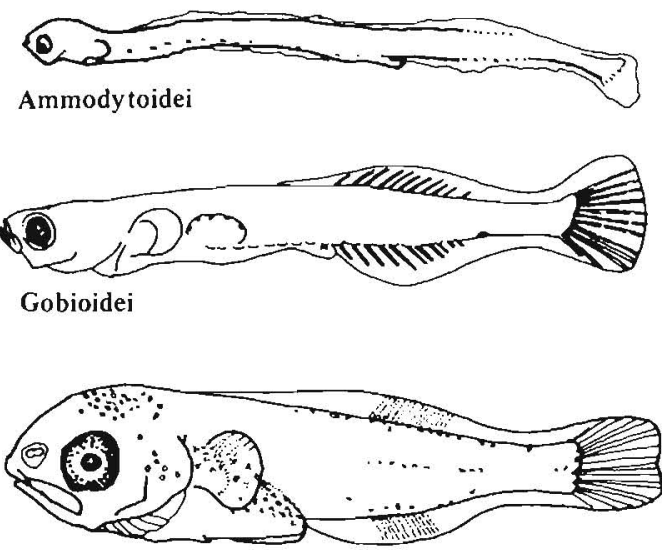

Scombroidei

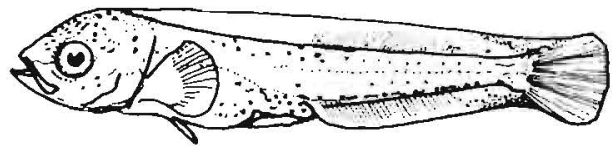

Stromateoidei
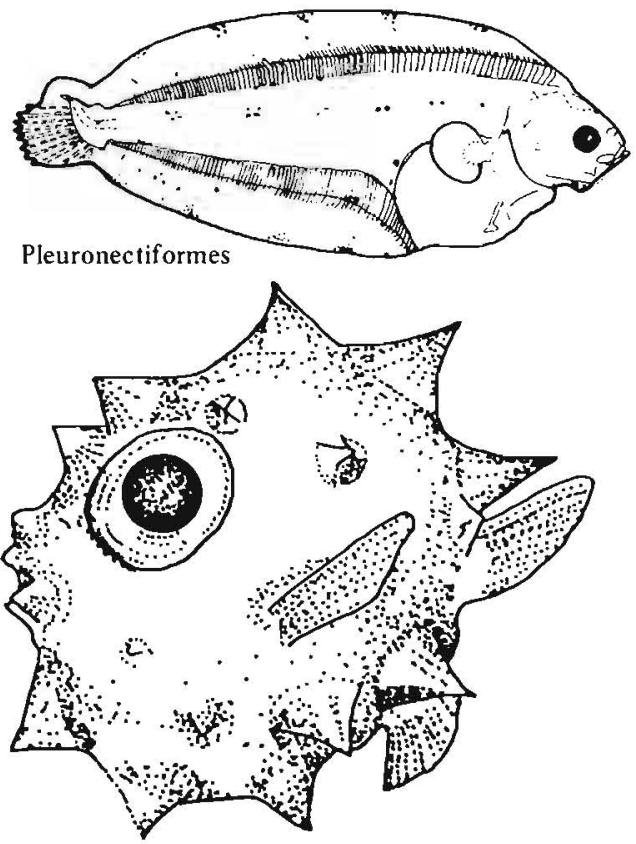

Tetraodontiformes

Figure 3 (continued) 
Table 2

Ordinal/subordinal egg, larval, and meristic characters of Northeast Pacific fishes (Ahlstrom and Moser 1976, Fahay 1983, Leis and Rennis 1983, in part).

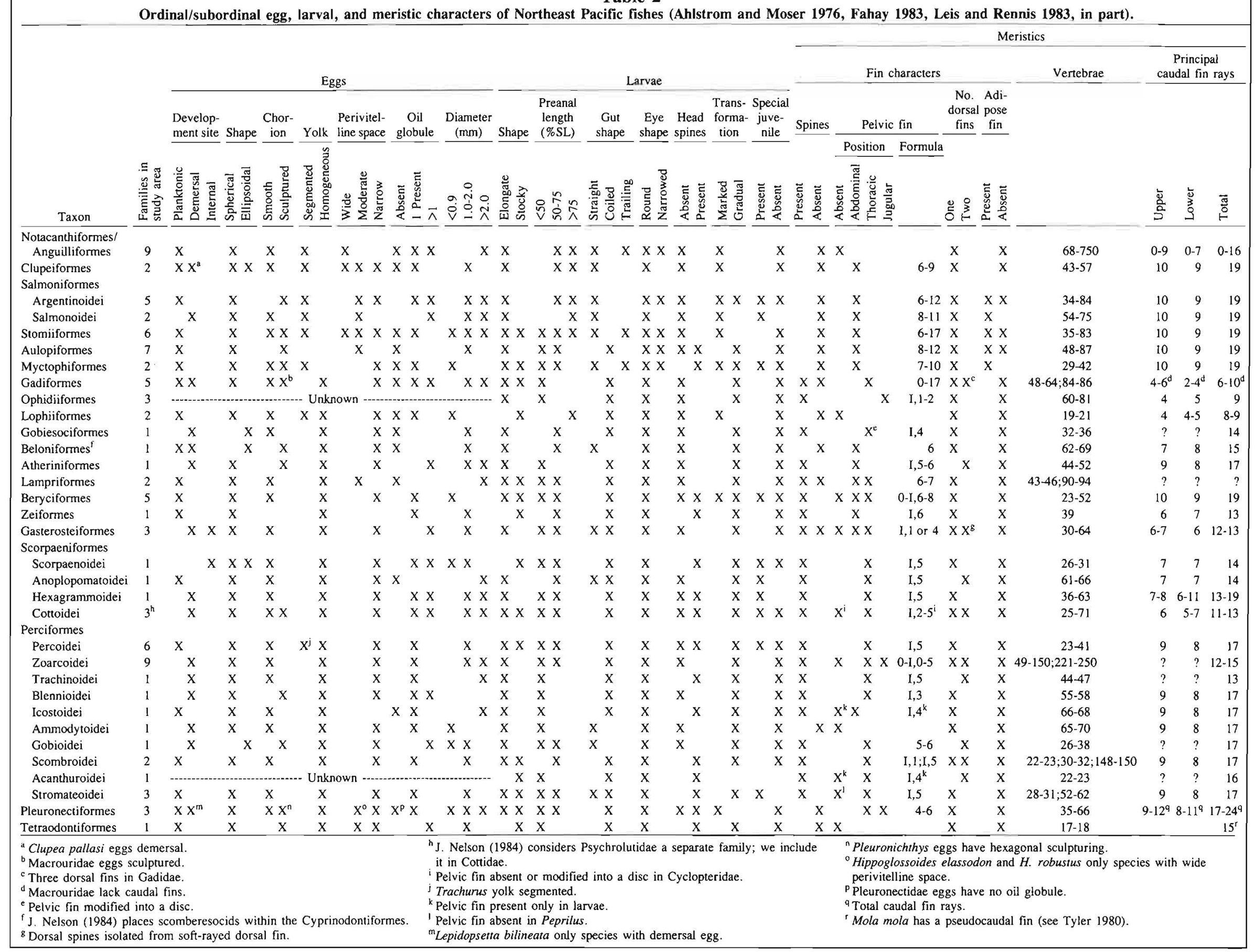




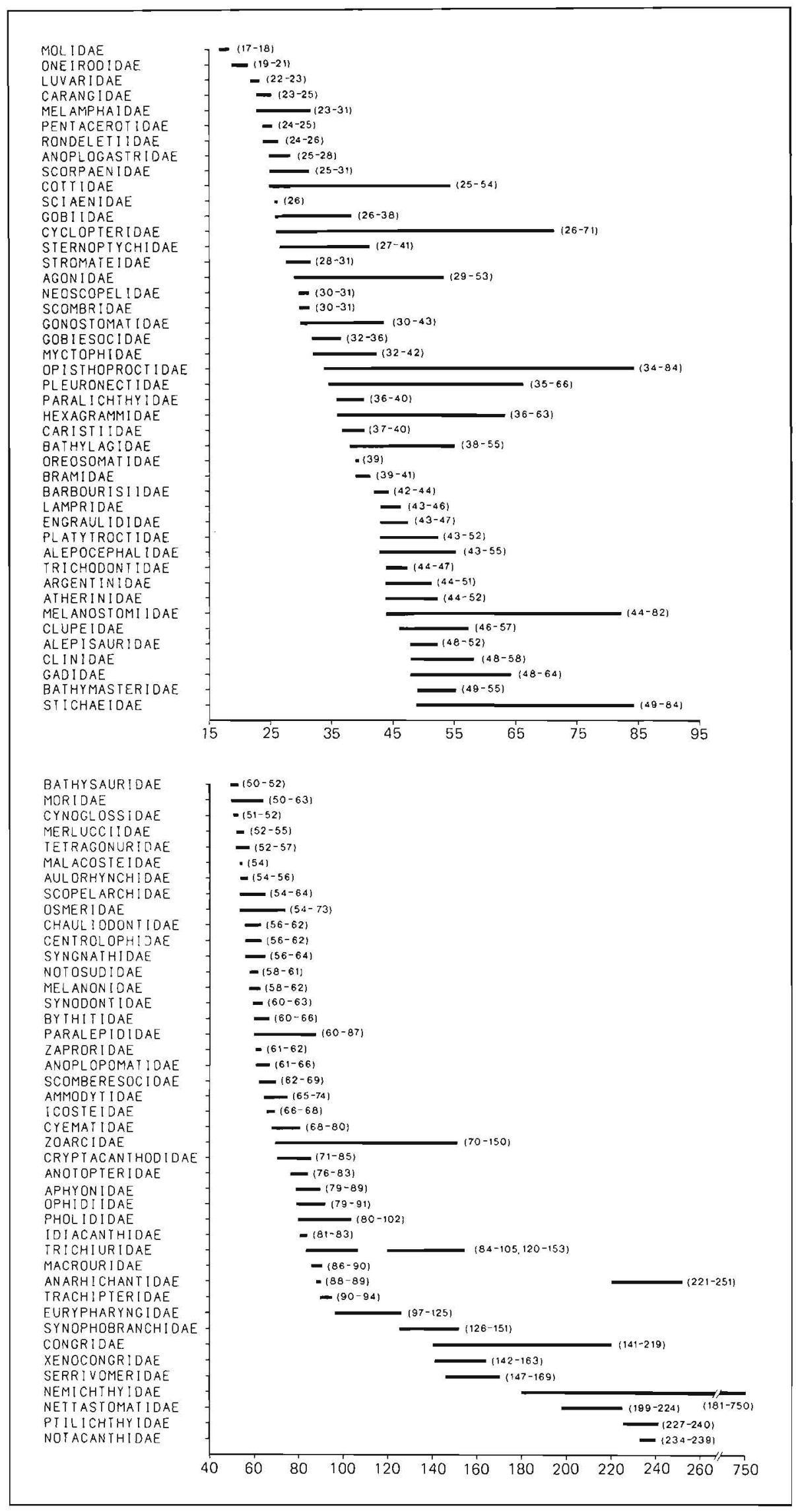

Figure 4

Ranges of total vertebral counts for families in the study area. 
Table 3

Distinguishing features of some commonly collected heavily pigmented larvae.*

\begin{tabular}{|c|c|c|c|c|c|}
\hline Taxon & Distribution & $\begin{array}{c}\text { Total } \\
\text { vertebrae }\end{array}$ & Description of pigment & $\begin{array}{l}\text { Other diagnostic } \\
\text { feanures }\end{array}$ & $\begin{array}{l}\text { Page } \\
\text { reference }\end{array}$ \\
\hline \multicolumn{6}{|l|}{ Myomeres 25-50 } \\
\hline Rhamphocottus richardsoni & SSC-Bering Sea & $26-28$ & $\begin{array}{l}\text { Small spots, densely distributed on } \\
\text { head, gut, and } 3 / 4 \text { body; absent on } \\
\text { peduncle and tail }\end{array}$ & $\begin{array}{l}\text { Subterminal mouth, deep body; after } \\
\text { flexion head spines and prickles by } \\
9-10 \mathrm{~mm}\end{array}$ & 366 \\
\hline Myoxocephalus G & Wash.-Bering Sea & $34-37$ & $\begin{array}{l}\text { Spots concentrated dorsally on head } \\
\text { and gut, and over anterior } 1 / 2 \text { body; } \\
\text { absent over gut }\end{array}$ & Head spines develop during flexion & 400 \\
\hline Hemilepidotus spp. & SSC-SE Alaska & $35-39$ & $\begin{array}{l}\text { Spots widely distributed on head, gut, } \\
\text { and body }\end{array}$ & Head spines develop during flexion & 372 \\
\hline Scorpaenichihys marmoralus & SSC-SE Alaska & $35-37$ & $\begin{array}{l}\text { Dense, uniformly distributed spots } \\
\text { over head, gut, and } 3 / 4 \text { body; absent } \\
\text { on tail }\end{array}$ & $\begin{array}{l}\text { Head bumps and preopercular spines } \\
\text { develop after flexion; preanal finfold }\end{array}$ & 382 \\
\hline Blepsias spp. & Cent. Calif.-Bering Sea & $37-39$ & $\begin{array}{l}\text { Dense, large spots evenly concentrated } \\
\text { over dorsal head, gut, and }>3 / 4 \text { body; } \\
\text { absent on tail tip }\end{array}$ & $\begin{array}{l}\text { Head spines and bumps develop dur- } \\
\text { ing flexion }\end{array}$ & 448 \\
\hline Pleuronichthys spp. & SSC-Bering Sea & $37-41$ & $\begin{array}{l}\text { Dense spots concentrated over head, } \\
\text { gut, and }>3 / 4 \text { body; dorsal and anal } \\
\text { finfold }\end{array}$ & Slender body with wide finfolds & 616 \\
\hline Radulinus spp. & SSC-Gulf of Alaska & $38-40$ & $\begin{array}{l}\text { Widely distributed over lateral gut sur- } \\
\text { face and almost } 3 / 4 \text { body }\end{array}$ & $\begin{array}{l}\text { Preopercular spines not prominent, gut } \\
\text { coiled }\end{array}$ & 406 \\
\hline Hemitripterus villosus & $\begin{array}{l}\text { Gulf of Alaska- } \\
\text { Bering Sea }\end{array}$ & $39-41$ & $\begin{array}{l}\text { Spots evenly distributed to } 3 / 4 \text { body } \\
\text { and into dorsal and anal finfold }\end{array}$ & Large size at development & 452 \\
\hline Nautichthys oculofasciatus & Cent. Calif.-Bering Sea & $40-41$ & $\begin{array}{l}\text { Large, widely distributed spots to } 3 / 4 \\
\text { body; dorsal and anal finfold pigment } \\
\text { at midbody }\end{array}$ & $\begin{array}{l}\text { Long, pigmented precocious pector- } \\
\text { als; head spines, bumps, ridges devel- } \\
\text { op after flexion }\end{array}$ & 454 \\
\hline 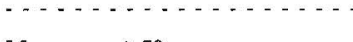 & 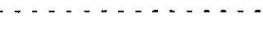 & $\cdots \cdots$ & $\ldots$ & $\ldots \ldots \ldots \ldots \ldots \ldots$ & $\cdots$ \\
\hline \multicolumn{6}{|l|}{ Myomeres >50 } \\
\hline $\begin{array}{l}\text { Hexagrammos spp./ } \\
\text { Pleurogrammus monopterygius }\end{array}$ & S. Calif-Bering Sea & $50-63$ & $\begin{array}{l}\text { Widely distributed on dorsal head and } \\
\text { gut, above and below notochord }\end{array}$ & Gut length $<50 \%$ SL & 348 \\
\hline Ophiodon elongatus & SSC-Gulf of Alaska & $56-59$ & $\begin{array}{l}\text { Widely distributed on dorsal head and } \\
\text { gut, more concentrated on dorso- and } \\
\text { ventrolateral surface; chin and isthmus }\end{array}$ & $\begin{array}{l}\text { Pointed snout and large terminal } \\
\text { mouth }\end{array}$ & 346 \\
\hline Zaprora silenus & Cent. Calif.-Bering Sea & $61-62$ & $\begin{array}{l}\text { Small spots densely concentrated over } \\
\text { entire body, except ventrally on gut, } \\
\text { dorsal finfold, and posterior edge of } \\
\text { gill cover; lightly on caudal peduncle } \\
\text { and anal finfold }\end{array}$ & Large size at development & 530 \\
\hline Anoplopoma fimbria & SSC-Bering Sea & $61-66$ & Widely distributed over $>3 / 4$ body & $\begin{array}{l}>15 \mathrm{~mm} \text {, pectoral fins long, pig- } \\
\text { mented; gut length }>50 \% \mathrm{SL} \text {; anus } \\
\text { curves ventrad }\end{array}$ & 338 \\
\hline Cololabis saira & SSC-Bering Sea & $62-69$ & $\begin{array}{l}\text { Small spots densely concentrated, ven- } \\
\text { tral tailtip without pigment }\end{array}$ & Long preanal finfold & 236 \\
\hline Lyconectes aleutensis & N. Calif.-Bering Sea & $73-75$ & $\begin{array}{l}\text { Heavily concentrated over }>3 / 4 \text { body, } \\
\text { isthmus; lighter over gut and absent on } \\
\text { peduncle }\end{array}$ & Large size at development & 520 \\
\hline Delolepis gigantea & N. Calif--Bering Sea & $81-83$ & $\begin{array}{l}\text { Widely distributed to } 3 / 4 \text { body, absent } \\
\text { over gut and isthmus }\end{array}$ & Large size at development & 520 \\
\hline $\begin{array}{l}\text { *Other heavily pigmented larvae } \\
\text { Anarhichas orientalis (p. } 52 \\
\text { Naucrates ductor (p. 484) } \\
\text { Peprilus simillimus (p. 562) } \\
\text { Oxylebius pictus (p. 342) } \\
\text { Zaniolepis (p. } 344 \text { ) }\end{array}$ & at may be less frequently & countered i & lude the following species: & & \\
\hline
\end{tabular}


A Rhamphocottus richardsoni

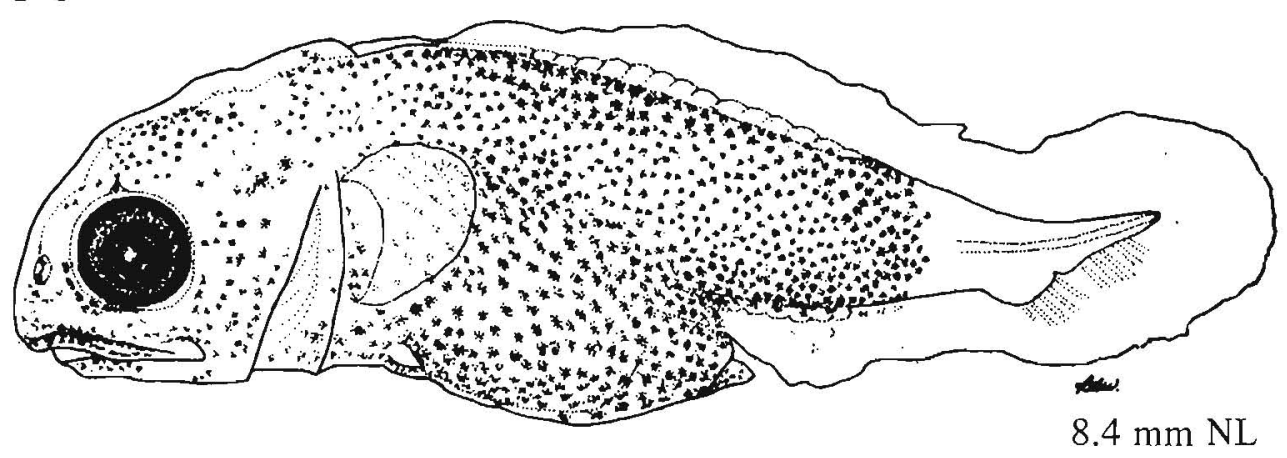

B Myoxocephalus G

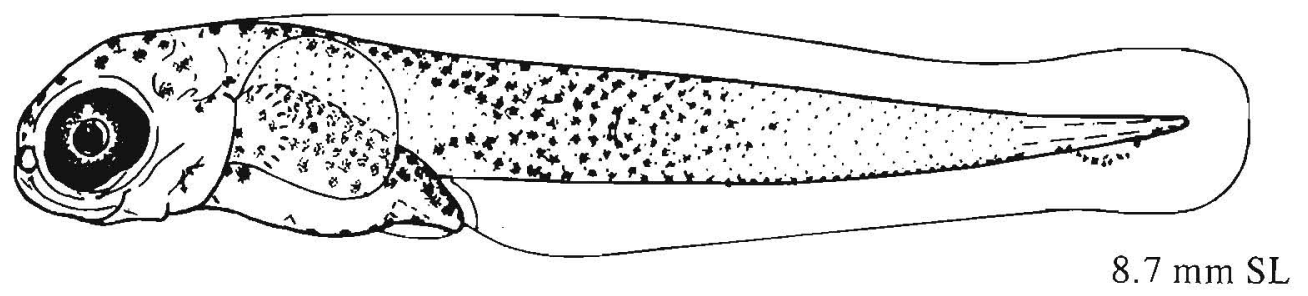

C Hemilepidotus spp.(Hemilepidotus spinosus)

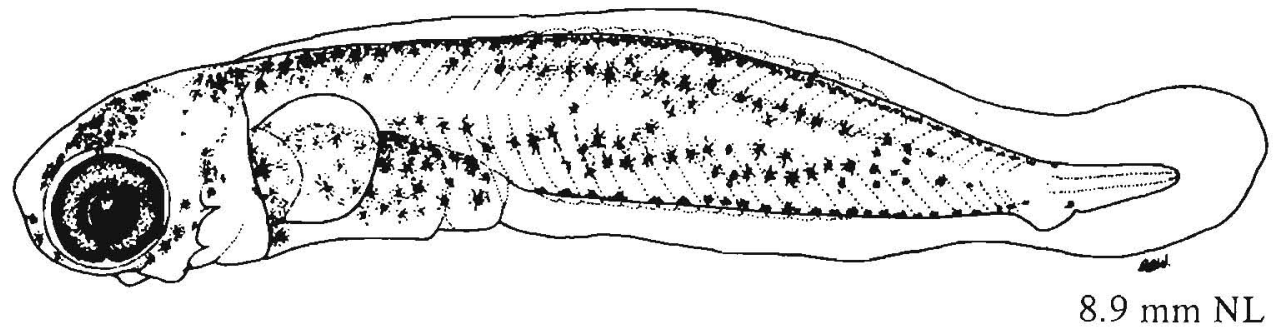

Figures A, C, Richardson and Washington 1980; B, NWAFC original (B. Vinter).

Figure 5

Commonly collected heavily pigmented larvae with $25-50$ myomeres (A-I), and $>50$ myomeres (J-P). 
D Scorpaenichthys marmoratus

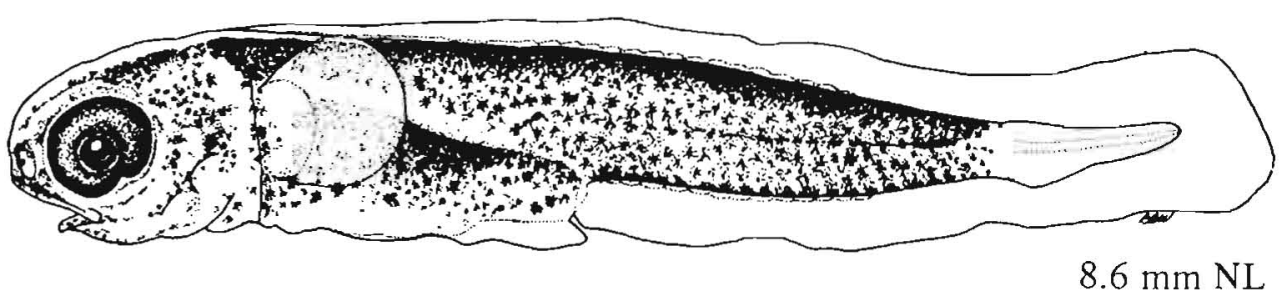

E Blepsias spp.(Blepsias cirrhosus)

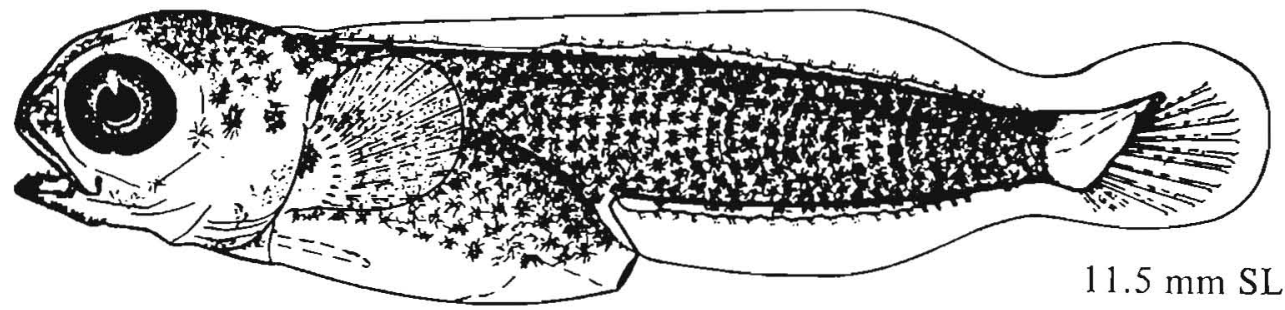

F Pleuronichthys spp.(Pleuronichthys decurrens)

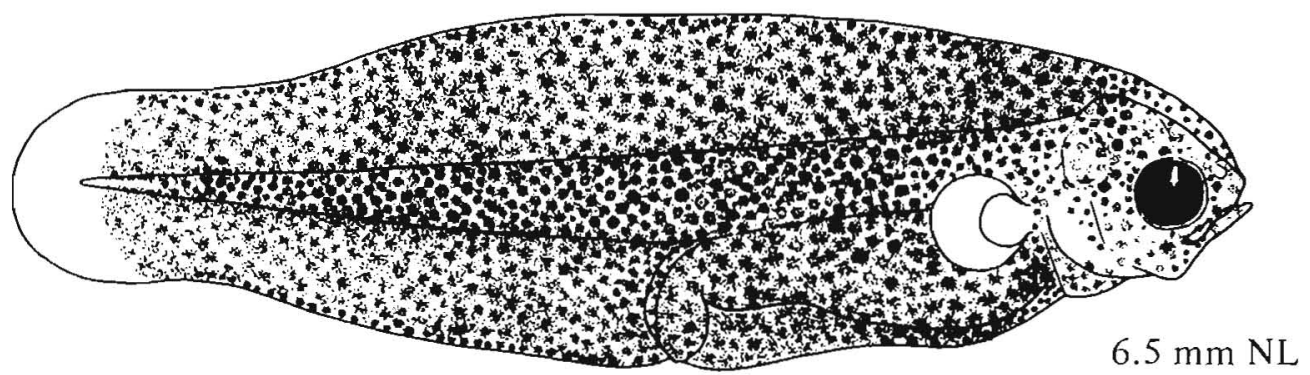

Figure D, Richardson and Washington 1980; E, NWAFC original (B. Vinter); F, Sumida et al. 1979.

Figure 5 (continued) 


\section{G Radulinus spp.(Radulinus asprellus)}

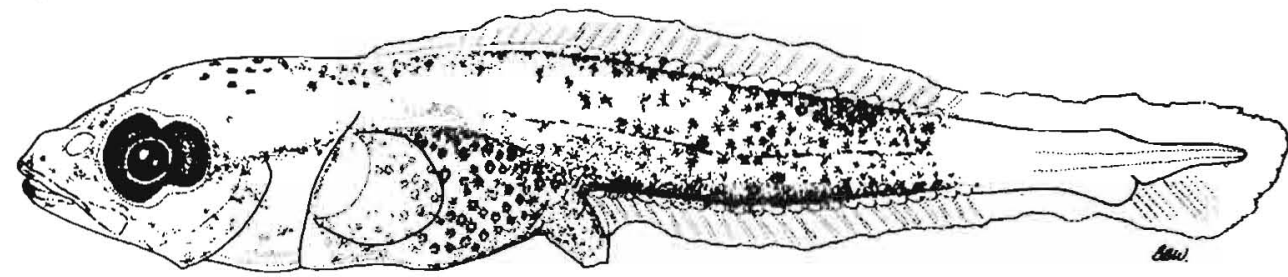

$9.6 \mathrm{~mm} \mathrm{NL}$

\section{Hemitripterus villosus}

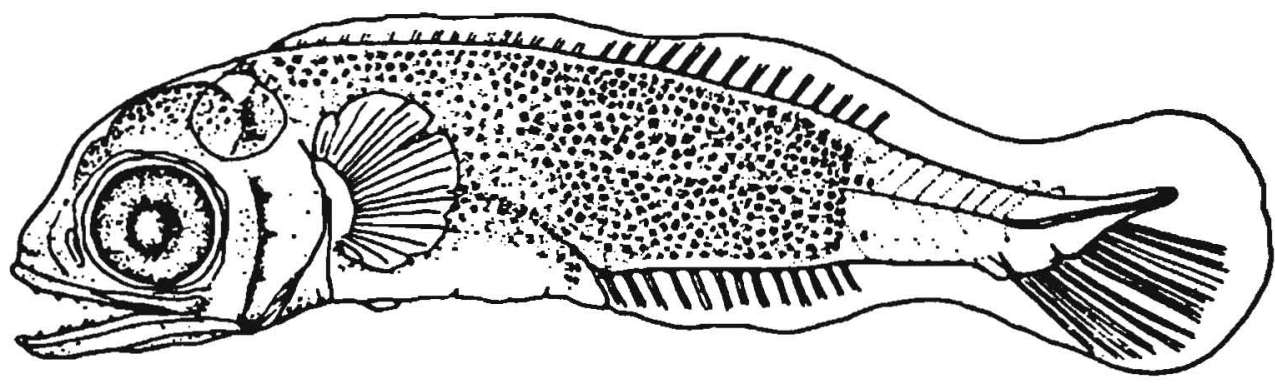

$14.4 \mathrm{~mm} \mathrm{SL}$

\section{| Nautichthys oculofasciatus}

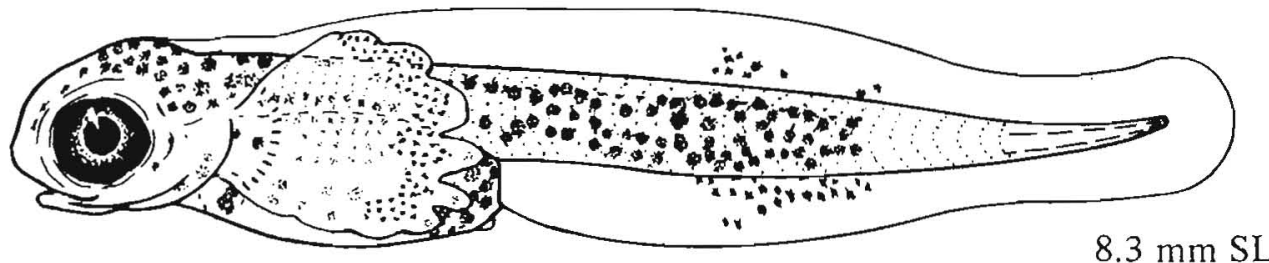




\section{Hexagrammos spp. (Hexagrammos octogrammus)}

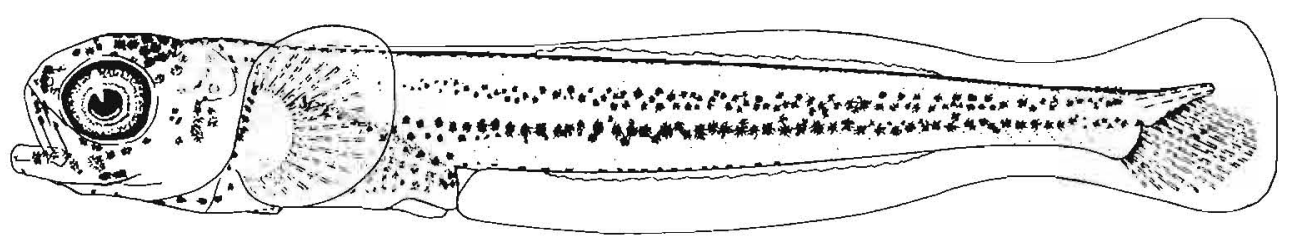

$15.0 \mathrm{~mm} \mathrm{SL}$

\section{Ophiodon elongatus}

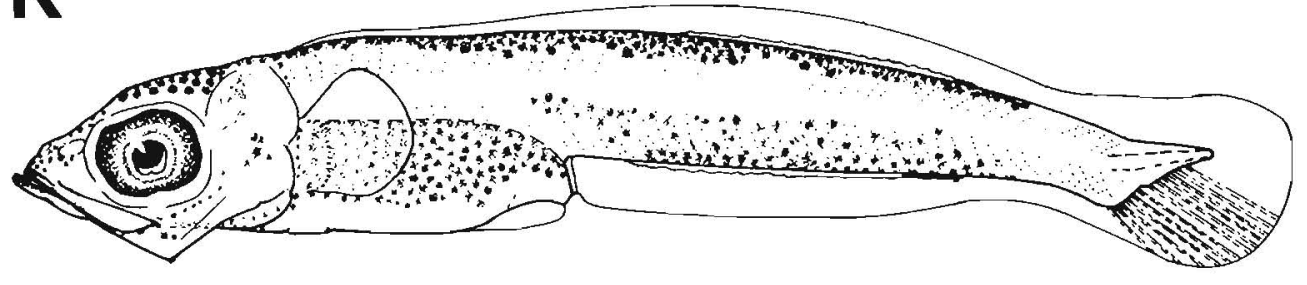

$12.2 \mathrm{~mm} \mathrm{SL}$

\section{Laprora silenus}

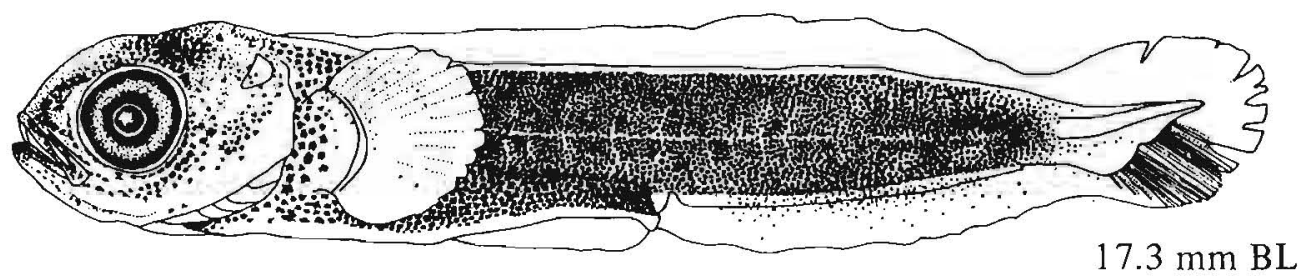

$17.3 \mathrm{~mm} \mathrm{BL}$

\section{Anoplopoma fimbria}

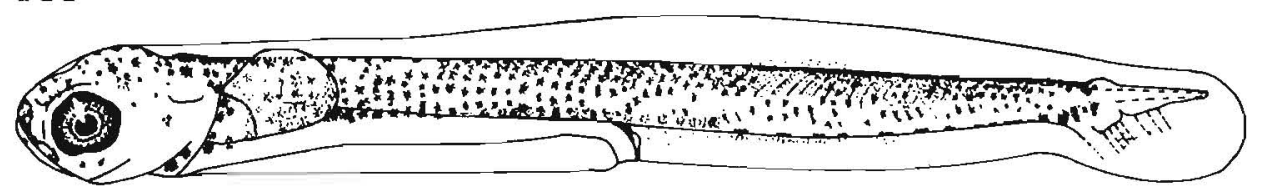

\section{$12.0 \mathrm{~mm} \mathrm{SL}$}

Figures $J-K$, Kendall and Vinter $1984 ; \mathrm{L}$, Haryu and Nishiyama $1981 ; \mathrm{M}$, NWAFC original (B. Vinter).

Figure 5 (continued) 


\section{$\mathbf{N}^{\text {Cololabisis arita }}$}

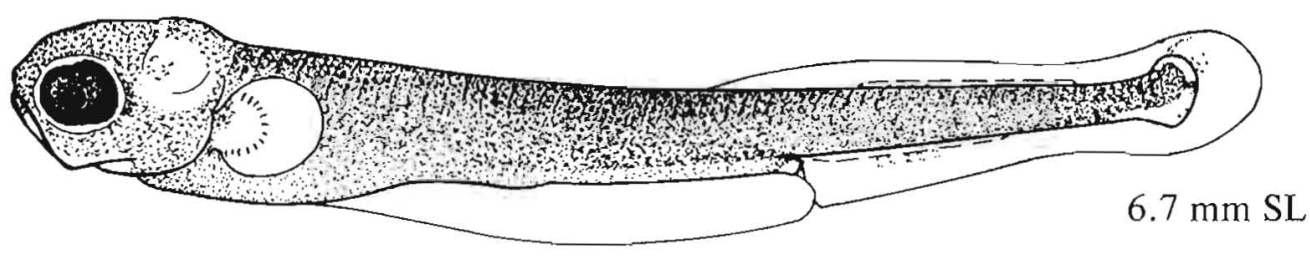

\section{Lyconectes aleutensis}

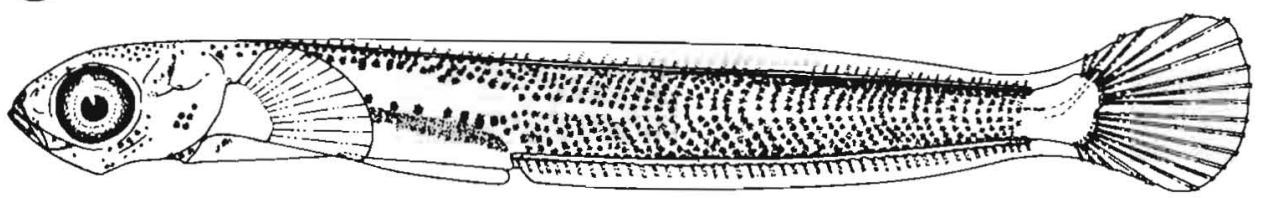

\section{$16.5 \mathrm{~mm} \mathrm{SL}$}

\section{Delolepis gigantea}

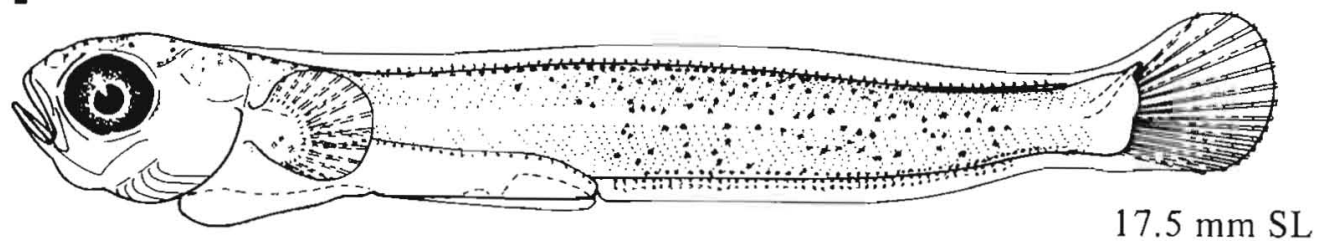


Table 4

Comparison of selected diagnostic characters of some commonly collected families with similar-looking elongate larvae.

\begin{tabular}{|c|c|c|c|c|c|c|c|c|}
\hline \multirow[b]{2}{*}{ Taxon } & \multicolumn{4}{|c|}{ Myomeres } & \multirow[b]{2}{*}{$\begin{array}{l}\text { Preanal } \\
\text { length }\end{array}$} & \multirow[b]{2}{*}{$\begin{array}{l}\text { Presence of } \\
\text { adipose } \\
\text { fin }\end{array}$} & \multirow[b]{2}{*}{$\begin{array}{c}\text { Diagnostic } \\
\text { pigment }\end{array}$} & \multirow[b]{2}{*}{$\begin{array}{c}\text { Page } \\
\text { reference }\end{array}$} \\
\hline & Preanal & Postanal & Total & $\begin{array}{l}\text { Number between } \\
\text { dorsal fin inser- } \\
\text { tion and anal } \\
\text { fin origin } \\
\text { (flexion stage) }\end{array}$ & & & & \\
\hline \multicolumn{9}{|c|}{ Short dorsal and anal fin bases } \\
\hline Clupeidae & $28-32$ & $19-23$ & $46-57$ & $6-8$ & $80 \%$ & None & Gut & 44 \\
\hline Engraulididae & $24-26$ & $19-21$ & $43-47$ & $0-2$ & $67-75 \%$ & None & Isthmus, gut & 48 \\
\hline Osmeridae ${ }^{\mathrm{b}}$ & - & - & $54-73$ & $9-11$ & $80 \%$ & Yes & Single row pvm; ${ }^{\mathrm{c}}$ ventral gut midline & 79 \\
\hline \multicolumn{9}{|c|}{ Long dorsal and anal fin bases } \\
\hline Bathymasteridae & $13-16$ & $34-39$ & $49-55$ & Overlaps & $<50 \%$ & None & $\begin{array}{l}\text { Urostyle or slash-like pigment along } \\
\text { posterior hypaxial and epaxial myo- } \\
\text { meres }\end{array}$ & 496 \\
\hline Stichaeidae & $14-31$ & $34-59$ & $50-83$ & Overlaps & $<50 \%$ & None & Gut, anus, $\mathrm{pvm}^{\mathrm{c}}$ & 500 \\
\hline Pholididae & - & - & $80-102$ & Overlaps & $>50 \%$ & None & Gut, $\mathrm{pvm}^{\mathrm{c}}$ & 522 \\
\hline Ammodytidae & $40-47$ & $23-25$ & $65-74$ & Overlaps & $60 \%$ & None & Double row pvm ${ }^{c}$ & 540 \\
\hline
\end{tabular}




\section{ELONGATE LARVAE}

Short dorsal and anal fin bases

A Clupeidae (Clupea pallasi)

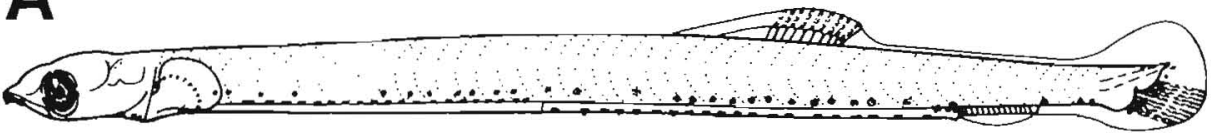

$19.0 \mathrm{~mm} \mathrm{SL}$

B Engraulididae (Engraulis mordax)

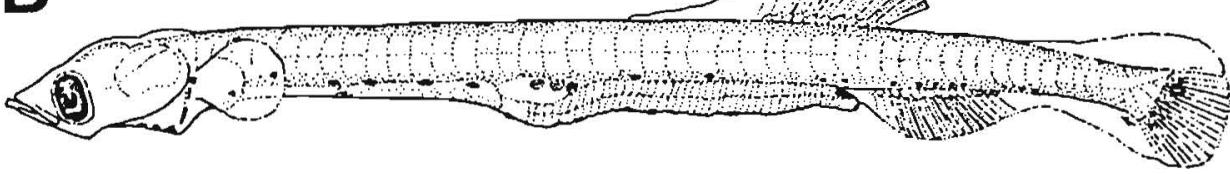

C Osmeridae (Mallotus villosus)

$11.5 \mathrm{~mm}$

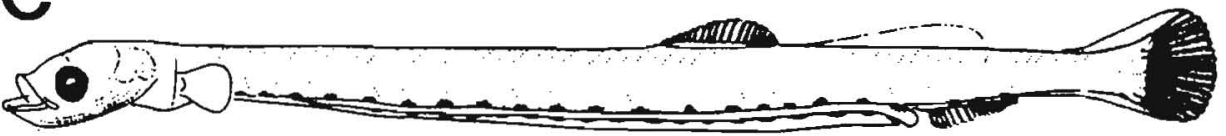

\section{$16.0 \mathrm{~mm}$}

Long dorsal and anal fin bases

D Bathymasteridae (Ronquilus jordani)

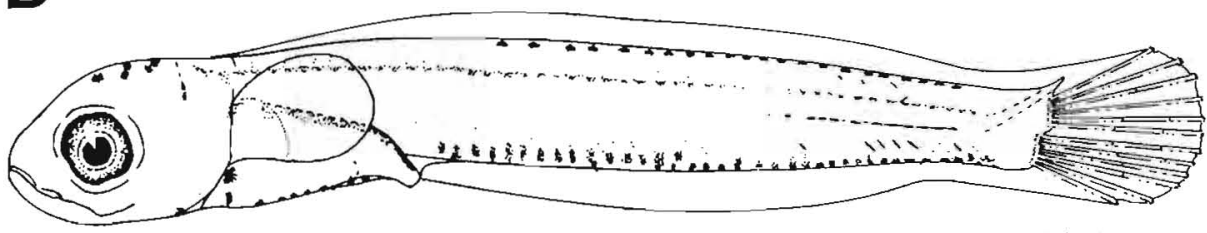

Stichaeidae (Anoplarchus purpurescens)

$10.4 \mathrm{~mm} \mathrm{SL}$

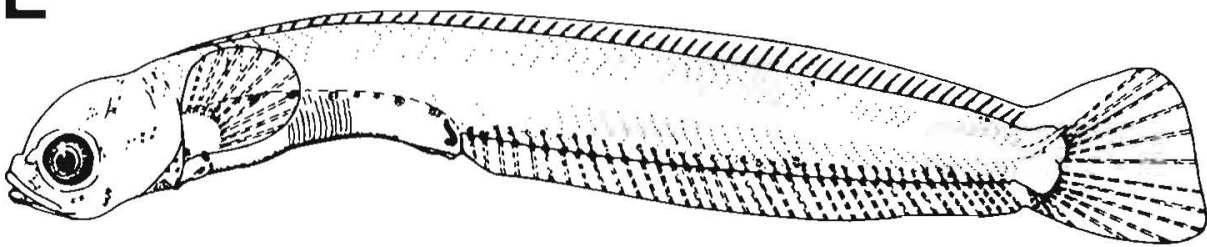

F Pholididae (Pholis sp.)

$12.0 \mathrm{~mm} \mathrm{SL}$

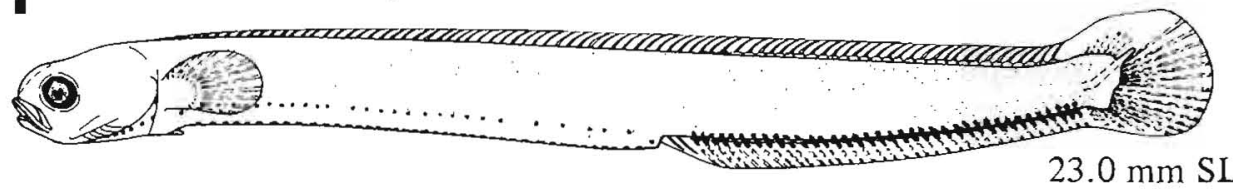

G

Ammodytidae (Ammodytes hexapterus)

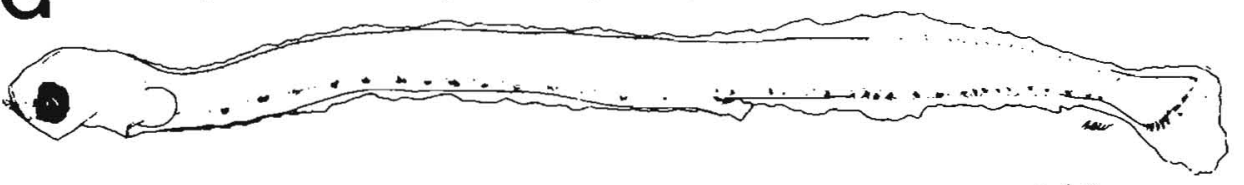

$16.0 \mathrm{~mm}$

Figures A, D-F, NWAFC originals (B. Vinter); B, Kramer and Ahlstrom 1968; C, Fahay 1983 (after Templeman 1948); G, Stevens et al. 1984

Figure 6

Commonly collected elongate larvae. 


\section{Using this laboratory guide}

\section{Format}

This laboratory guide has been designed to be practical and easy to use. Only information deemed necessary for accurate and timely identification has been included. A two-page format is provided for each taxon where sufficient early-life-history information exists. The left page includes pertinent information for identification and the right page includes illustrations, usually with notations indicating important diagnostic features. The left page is divided into two columns: Information on meristic characters and life history features is presented on the left, and developmental information on the right. Blanks within the format indicate that particular information was not available (e.g., egg size, fecundity), to point out gaps in knowledge, and allow researchers to insert new information as it becomes available. The family name appears at the top of each page for quick reference. The phylogenetic sequence generally follows J. Nelson (1984) unless otherwise indicated. Species names along with their authorities appear at the upper right corner of the left page, and common names (from Robins et al. 1980, Hubbs et al. 1979, Shiino 1976, and others) are included at the upper left corner of the right page. Nomenclature generally follows Robins et al. (1980) and Steyskal (1980) unless a more recent revision is available (usually from Moser et al. 1984b). Exceptions are noted in the text.

For taxa treated at the species level, available illustrations are arranged on the right-hand page as follows: Late-stage egg is in the upper right-hand corner, and order of larvae is shown (top to bottom) as yolksac, preflexion, flexion, postflexion, and either a transforming juvenile or special prejuvenile stage. Blank spaces indicate that stages were not available for illustration. When necessary for identification purposes, additional illustrations such as dorsal and ventral views are provided. Most illustrations were compiled from the literature; Moser et al. (1984b) provided over 100 illustrations. In a few cases, illustrations from the literature were redrawn, modified, or corrected; when this occurred, it is indicated. In addition to the published illustrations, original illustrations of 124 fish eggs and fish larvae by Beverly Vinter are included. Collection data are provided for original illustrations (Table 1).

Introductory sections are provided for each order and for taxa (usually genera or families) with difficult identification problems or for taxa that contain numerous similar species (e.g., Cottidae and Sebastes). Important diagnostic features are summarized, and, in some cases, tables are provided to aid in identification. In the Northeast Pacific Ocean, early-life-history stages of many species are either undescribed, incompletely described, or without adequate illustrations. For these taxa, identification material is provided at the lowest taxonomic level possible, usually family level (e.g., Osmeridae) or generic level (e.g., Cyclothone). Family level descriptions include a summary of available early and general lifehistory information, tables of meristic characters, and brief accounts of early-life-history characters from closely related taxa described from other geographic areas. For taxa where no early-life-history data are available (e.g., Cetomimoidei), life history summaries and meristic data are provided.

\section{Meristics}

Data summaries of meristic structures, except those for the caudal fin or from recently completed research, are from the NWAFC meristic database. The range (high and low value) and mode are presented for each entry. Ranges reported here generally represent the most extreme values ever recorded; in many cases, these values are not likely to be observed. These values may have been seen on specimens collected outside our study area. Thus, in using this guide more emphasis should be placed on the reported modal values. An " $X$ " appears when data are unavailable. For fin ray counts, $\mathrm{R}=$ rays (soft rays) and $\mathrm{S}=$ spines; for gill raker counts, $\mathrm{U}=$ upper and $\mathrm{L}=$ lower. In addition to pelvic fin-ray counts, fin position is given. Position is indicated by the following descriptors: Abdominal, thoracic, jugular, absent, or modified (e.g., pelvic disc in cyclopterids). The total vertebral count given may not equal the sum of the precaudal and caudal vertebral counts in some cases, since these counts may have originated from different sources.

Data for caudal fin-ray counts have been gleaned from published material, as well as from original observations. Caudal fin-ray counts are reported in the following sequence: Upper secondary, upper principal + lower principal, lower secondary, with ranges for each when available. Total caudal fin rays or total principal caudal fin rays are reported if no other data are available. Blanks in the caudal field indicate that no data are available.

For some taxa, it was necessary to forego the standard meristic format in order to present the data or provide additional information (e.g., members of the family Gadidae have three dorsal fins and two anal fins). Departures from the standard format are either explained on the page where they occur or in introductory sections preceding species descriptions for certain taxa.

\section{General life history}

Life history data are provided as ancillary information which may aid in identification of eggs and larvae. These data were extracted from Garrison and Miller (1982) and supplemented by the general literature and original, unpublished material.

Geographic ranges are from the NWAFC meristic database and the literature. Allen and Smith (1988) provided a significant amount of new information. Range information is restricted primarily to the study area. Thus, the limits of the southern range beyond the California-Mexico border, the northern range beyond the Arctic to the north and east, and the western range beyond the Bering Sea are not specified.

The following general locations are used to approximate geographic range within the study area (abbreviations in parentheses are used hereafter in the text when necessary):

South of southern California (SSC)

Southern California, $32-34^{\circ} \mathrm{N}$ (S. Calif. or S. California)

Central California, $34-38^{\circ} \mathrm{N}$ (Cent. Calif. or Cent. California)

Northern California, $38-42^{\circ} \mathrm{N}$ (N. Calif. or N. California)

Oregon, $42-46^{\circ} \mathrm{N}$

Washington, $46-48^{\circ} 30^{\prime} \mathrm{N}$ (Wash.)

British Columbia, $48^{\circ} 30^{\prime}-55^{\circ} \mathrm{N}$ (Brit. Col.)

Southeastern Alaska, $55-59^{\circ} \mathrm{N}$ (SE Alaska)

Gulf of Alaska, $54-60^{\circ} \mathrm{N}$

Aleutian Islands, $51-55^{\circ} \mathrm{N}$ (Aleutian Is.)

Bering Sea, $54-66^{\circ} \mathrm{N}$

Chukchi Sea, north of $66^{\circ} \mathrm{N}$

Arctic 
In addition to geographic range, general ecological descriptors are incorporated in the NWAFC meristic database. The following descriptors are used.

\section{Pelagic environment}

Nearshore shelf pelagic: Extends from the shore seaward to include waters overlying an ocean bottom $<200 \mathrm{~m}$. Equivalent to the neritic province of Hedgpeth (1957) and other authors.

Oceanic: Waters overlying an ocean bottom $>200 \mathrm{~m}$. The following subdivisions based on water depth are used: Epipelagic 0-200 m; Mesopelagic 200-1000 m; Bathypelagic $>1000 \mathrm{~m}$.

\section{Benthic environment}

Intertidal, nearshore: Extends from high tide to low tide. Equivalent to the littoral province of many authors.

Nearshore shelf demersal: All bottom from low tide to $200 \mathrm{~m}$ (= epibenthal in text). Equivalent to the sublittoral zone of Hedgpeth (1957) and other authors.

Mesobenthal: Deep sea beyond the continental shelf at depths of $200-500 \mathrm{~m}$. Lowest part of the shelf and upper part of continental slope (Fedorov 1973).

Bathybenthal: Deep sea along the continental slope at depths of 500-2500 m. Middle and lower sections of continental slope (Fedorov 1973).

Freshwater or anadromous: Generally the mouth and lower reaches of rivers and streams.

Other information under Life History includes reproductive mode (e.g., viviparous, ovoviviparous, oviparous) and indicates whether eggs and larvae are pelagic or demersal. Data on spawning are divided into four categories: Season, area, mode, and migration. Spawning often varies among geographic regions and populations, so care has been taken to provide as much specific geographic information as available. Fecundity values are given as counts of ripening eggs in individual females: Total ranges or (in a few cases) as a function of length in the form $F=a L^{b}$. Ages at first maturity and longevity values were extracted from the general literature. If available, age/length differences between sexes are noted.

\section{Early life history}

Egg and oil globule diameter measurements in millimeters are usually given as ranges (high and low values) with modal values as available in parentheses after the range. Precision varies among literature sources, but specimens used for original measurements were measured to the nearest $0.1 \mathrm{~mm}$. Egg diameters on the illustrations are as they were given in the source. Incubation time, when available, is given in number of days to hatching for a specific temperature $\left({ }^{\circ} \mathrm{C}\right)$. When describing embryonic pigment patterns, emphasis was placed on those characteristics which aid in identification. Diagnostic characters usually provide a summary of important features and comparisons with similar fish eggs.

Figure 2 provides examples of features used to describe and identify early stages of fishes. Original measurements of larvae are in millimeters and given in standard length (SL). Some measurements extracted from the literature were given as body length (BL), notochord length (NL), total length (TL), head length (HL), or percentages of these. Preanal length is usually expressed as a percentage of SL. If specific values are not available, preanal lengths are given as $<50 \% \mathrm{SL}, 50-75 \% \mathrm{SL}$, or $>75 \% \mathrm{SL}$. Definitions for developmental stages are from Ahlstrom et al. (1976) and Kendall et al. (1984). Transformation is defined as acquisition of the adult complement of fin rays, and in some taxa this is accompanied by squamation. Sequence of fin development is usually described as the order in which fin rays accept alizarin stain, inferring ossification. For some taxa the sequence is determined by completion of the ossification of a fin element rather than the initiation of ossification (e.g., G.D. Johnson 1984); this is noted in the text. When three or more fins develop simultaneously, semicolons are used to separate the sequence of formation of one or more fins (e.g., dorsal; anal; caudal, pectoral, and pelvic). Otherwise, commas are used to separate fins developing individually in sequence (e.g., dorsal, anal, caudal, pectoral, and pelvic). Precocious fin development is usually noted. When describing pigment, those melanophores or patterns of melanophores important in identifying the taxon are stressed. Generally only melanophores are mentioned, since other pigment is not visible in formalin-preserved specimens. The general appearance, shape, and relative size of melanophores or groups of melanophores are indicated by imprecise descriptors such as spot, patch, or blotch. Diagnostic pigment characteristics are also indicated directly on the illustration page. The description of pigment is often brief and in telegraphic style and is not meant to be a substitute for more detailed discussions available in complete early-lifehistory descriptions. References are provided when more complete early-life-history descriptions are available. Under Diagnostic Characters, a brief summary of key features is provided which may help to distinguish a larval specimen from other similar larvae in either closely related taxa or from morphologically similar groups. When possible, comparative information is included in tables and is cross-referenced.

Data from the files of the late E.H. Ahlstrom are footnoted as "E.H. Ahlstrom notes." These files contain original lecture notes for classes conducted on ichthyoplankton taxonomy between 1971 and 1977, early-life-history data for most orders of fishes, and notes on teleost caudal fins. The files were made available to Kendall \& Matarese by H. Geoffrey Moser (Southwest Fish. Cent., Natl. Mar. Fish. Serv., NOAA), as authors contributing to Ontogeny and Systematics of Fishes (Moser et al. 1984b). 



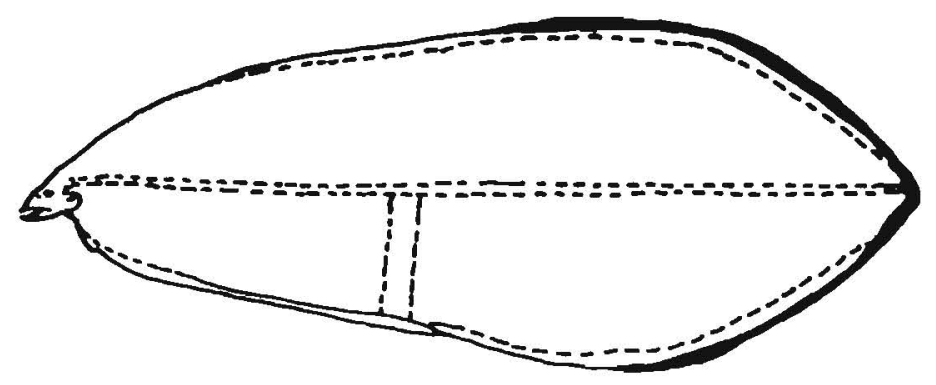

\section{Elopomorpha: Notacanthiformes} Anguilliformes

The notacanthiform fishes (spiny eels) and anguilliform fishes (true eels) generally occur worldwide. The spiny eels are primarily a deep-sea group and are distinguished from the true eels by a number of characters including the absence or reduction of the caudal fin, the presence of well developed pelvic fins and fin spines, a short dorsal fin, and the presence in larvae of a thin postcaudal filament. According to Castle (1984), the Notacanthiformes consist of 3 families, 6 genera, and about 22 species; the Anguilliformes, a much larger order, include 21 families, 153 genera, and about 720 species.

The early life history of eels has been studied for many years primarily due to the presence of a distinctive leptocephalus larval phase, but many species remain inadequately known. Eggs are generally large, pelagic, possess segmented yolks, and have one or more oil globules. Although few larvae have been collected, at least 12 taxa from at least 9 families are thought to occur in the study area. Since early life histories are incomplete, this section (except for the nemichthyids) will be described by family.

Families in study area: Notacanthidae

Xenocongridae

Nemichthyidae

Cyematidae

Synaphobranchidae

Nettastomatidae

Congridae

Serrivomeridae 


\section{ELOPOMORPHA}

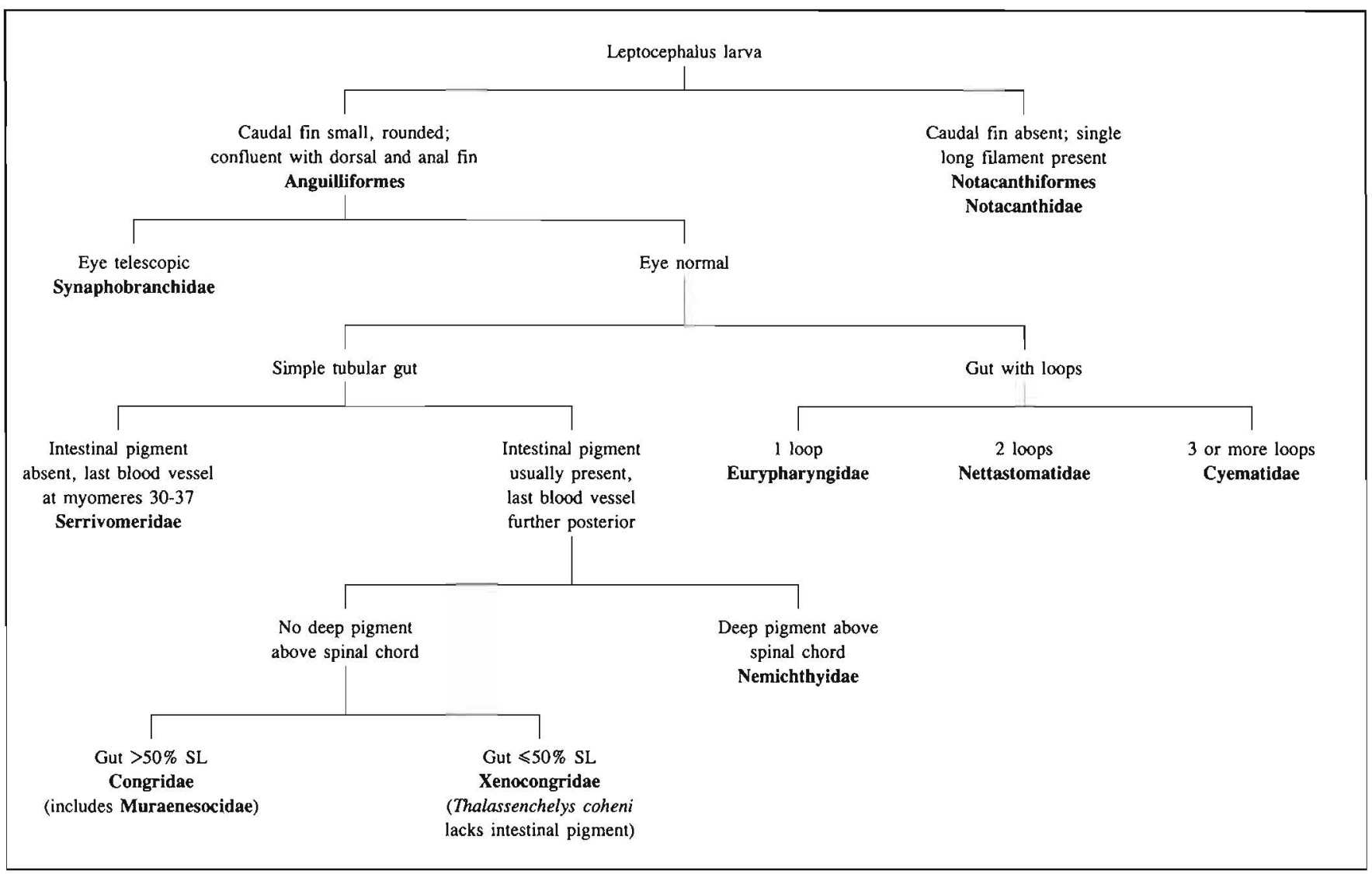

Key to elopomorph leptocephali in the Northeast Pacific (after Smith 1979 and Fahay 1983, in part). 
Table 5

Meristic characters of superorder Elopomorpha.

\begin{tabular}{|c|c|c|c|c|c|c|c|}
\hline \multirow[b]{2}{*}{ Taxon } & \multirow[b]{2}{*}{ Distribution } & Vertebrae & \multicolumn{5}{|c|}{ Fins } \\
\hline & & $\begin{array}{l}\text { Precaudal Caudal } \\
\text { (Total) }\end{array}$ & Dorsal & Anal & Pectoral & Pelvic & Caudal \\
\hline \multicolumn{8}{|l|}{ Notacanthiformes } \\
\hline Notacanthidae & & $(234-244)$ & & & & & \\
\hline Notacanthus chemnitzi & Cent. Calif. ${ }^{\text {a-Oregon }}$ & $\begin{array}{l}47-55 \\
\quad(234-239)\end{array}$ & $\begin{array}{l}\text { VII-X or } \\
\text { IX,2-3 }\end{array}$ & $\begin{array}{l}\text { XI-XXV, } \\
115-132\end{array}$ & $10-17$ & $\begin{array}{l}\text { I-IV, } \\
6-10\end{array}$ & Absent \\
\hline Polyacanthonotus challengeri & Oregon-Bering Sea & $(242-244)$ & XXXII-XXXV & $161-162$ & $12-13$ & 1,8 & Reduced \\
\hline \multicolumn{8}{|l|}{ Anguilliformes } \\
\hline Xenocongridae & & $(97-163)$ & & & & & \\
\hline Thalassenchelys coheni & & $\begin{array}{c}67-74 \quad 83-92 \\
(142-163)\end{array}$ & $280-350$ & $218-260$ & Absent & Absent & Absent \\
\hline Nemichthyidae & & $(170-400+)$ & & & & & \\
\hline Avocettina infans & SSC-Aleutian Is. & $(181-201)$ & $300-350$ & $265-270$ & & Absent & Reduced \\
\hline Nemichthys larseni & SSC-Oregon & $\begin{array}{l}79-84 \\
\quad(400-750)\end{array}$ & & & $10-12$ & Absent & \\
\hline Nemichthys scolopaceus & SSC-Aleutian Is. & $(293-750)$ & $307-450$ & $312-454$ & $10-14$ & Absent & Reduced \\
\hline Cyematidae & & $(74-108)$ & & & & & \\
\hline Cyema atrum & SSC-Oregon & $\begin{array}{l}38-43 \\
\quad(74-80)\end{array}$ & $79-83$ & $72-86$ & $12-15$ & Absent & 5 \\
\hline Synaphobranchidae & & $(126-172)$ & & & & & \\
\hline Histiobranchus bathybius & Bering Sea & $(126-151)$ & $265-302$ & $188-203$ & $15-17$ & Absent & 18 \\
\hline Nettastomatidae & & $(186-290)$ & & & & & \\
\hline Venefica sp. A & Cent. Calif.-Wash. & $(199-224)$ & $310+$ & 325 & & Absent & 12 \\
\hline Congridae & & $(120-261)$ & & & & & \\
\hline Xenomystax atrarius & SSC-Brit. Col. & $\begin{array}{c}50-57 \quad 107-123 \\
(141-219)\end{array}$ & $253-292$ & $189-214$ & $11-14$ & Absent & $7-8$ \\
\hline Serrivomeridae & & $(137-170)$ & & & & & \\
\hline Serrivomer jesperseni & Brit. Col. & $\begin{array}{l}89-125 \\
\quad(147-169)\end{array}$ & $141-161$ & $127-161$ & $6-7$ & Absent & $\begin{array}{l}\text { Reduced } \\
\text { (6 principal) }\end{array}$ \\
\hline Eurypharyngidae & & $(97-125)$ & & & & & \\
\hline Eurypharynx pelecanoides & N. Calif. ${ }^{b}$ & $(97-125)$ & $155-196$ & $118-147$ & 11 & Absent & Absent in adult \\
\hline
\end{tabular}

\section{Anguilliformes}

Primary characters used in identifying anguilliform leptocephali are the following (Ref: E.H. Ahlstrom notes, Castle 1984, Fahay 1983, Smith 1979):

Body shape May vary from slender to deep-bodied, and the tail may be tapered or rounded.

Head characteristics Size of the head relative to the body may vary; also important are head shape (blunt, sharp, or elongate), snout shape, nasal organ (size and position), eyes (round, narrow, or telescopic), and teeth (presence/absence, fanglike if present).

Number of myomeres Usually 100-250; exceptions include Cyema $(<100)$ and Nemichthys $(>750)$. Preanal and predorsal counts are also useful.

Gut characteristics Gut may be a simple straight tube or more complex with loops or swellings. Relative length of gut can range from $<50 \%$ SL to $>90 \% \mathrm{SL}$, although most eels have gut lengths between $50-70 \%$ SL.

Position of vertical blood vessels Variation in the position of the last blood vessel.

Pigmentation General body pigment may be located above/below the gut, along body midline, above/below notochord internally, or along the dorsal body margin. Pigment may be in the form of fine stippling or large stellate melanophores (blotches). Head pigment also varies.

Size at transformation Maximum size before transformation can vary from $<100 \mathrm{~mm} \mathrm{SL}$ (most families) to $\sim 400 \mathrm{~mm} \mathrm{SL}$ (nemichthyids).

Fins Dorsal and anal fins are confluent with the caudal fin, caudal fin is usually markedly reduced with 10 or fewer rays (about 5-11 with highest numbers in synaphobranchids); pectoral fin is moderate, reduced, or lacking, and fin rays usually form late, i.e., after the leptocephalus stage. Pelvic fin is absent. 
Notacanthidae (234-244 myomeres)* Notacanthus chemnitzi and Polyacanthonotus challengeri both occur in the study area but their early life histories are unknown, as is the case of most notacanthids. Leptocephalus giganteus (identity unknown) from outside the study area is presented for comparison only. Notacanthid larvae are easily separable from larvae of true eels. Among the most notable characters are the following: Greatly elongate shape, thin postcaudal filament, and pigment which occurs in a ventral series.

Xenocongridae (97-163 myomeres) Thalassenchelys coheni (142-163) may or may not belong with the xenocongrids. These larvae are quite unusual, with a short, deep body, rounded tail, and lack of pigment. They appear to be widely distributed in the northeastern Pacific from Washington to south of southern California.

Cyematidae (74-80 myomeres, most 74-78) Cyema atrum larvae are identified by their deep body shape, pointed head and tail, 3-4 gut loops on posterior half of gut, and low myomere count. Pigment is scattered over the lateral body surface, on the snout and lower jaw, and along the gut, especially on the loops. Other general features include gut length $\sim 67 \%$ SL and dorsal fin origin approximately above anus. Size at transformation is $60-70 \mathrm{~mm}$ SL.

Synaphobranchidae (subfamily Synaphobranchinae) (126-151 myomeres) Synaphobranchinae are represented in the Northeast Pacific by Histiobranchus bathybius but their larvae are unknown. Larvae in other members of the subfamily are identified by their telescopic eye, general lack of pigment, and the broad white stripe formed by an opaque central area of myomeres around the notochord. The body is moderately elongate and the head is short and pointed. The dorsal fin origin is anterior to the anus. The gut is usually relatively simple, unpigmented, and about $75 \%$ SL. Some taxa have loops in the gut. Ventral pigment is lacking, and lateral pigment is restricted to the postanal body. Some genera have a prominent pigment spot laterally, near the level of the anus. Size at transformation is $130-170 \mathrm{~mm} \mathrm{SL}$.

\footnotetext{
*Vertebral range is given for taxa in the study area. When no data are available, the range of counts for family is given (Table 5).
}

Ref: E.H. Ahlstrom notes, Castle 1984, Castle and Raju 1975, Fahay 1983. 


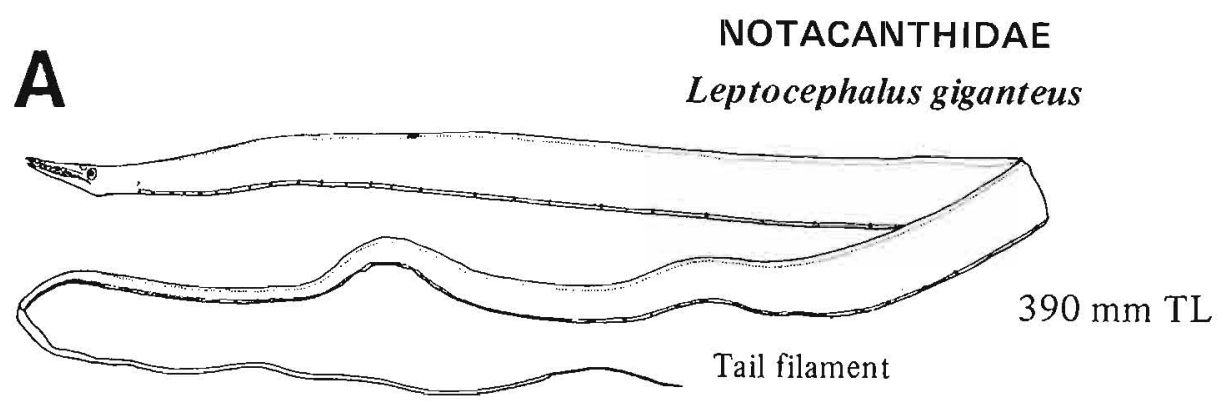

\section{XENOCONGRIDAE}

\section{Thalassenchelys coheni}
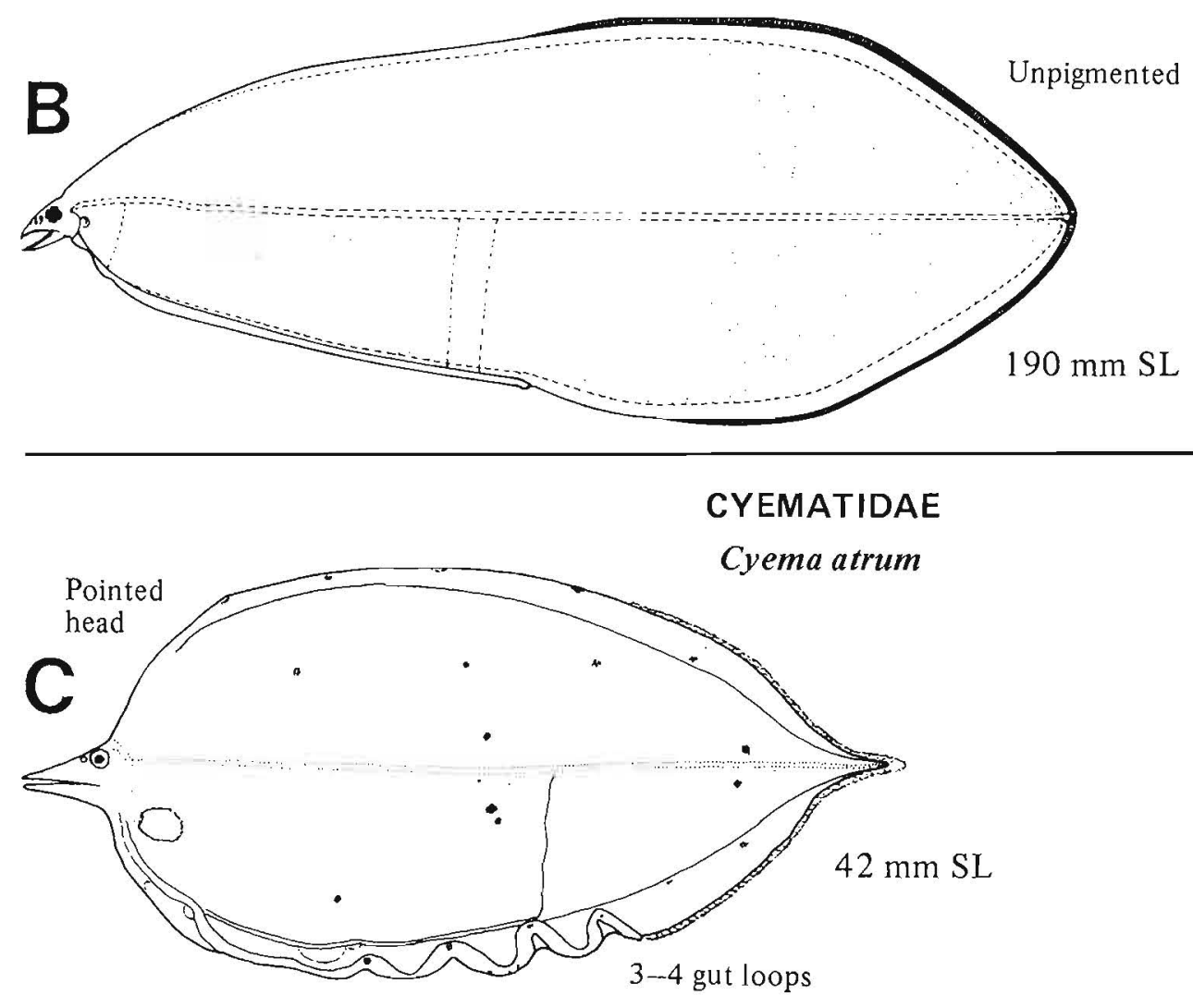

\section{SYNAPHOBRANCHIDAE}

D

Synaphobranchus sp.
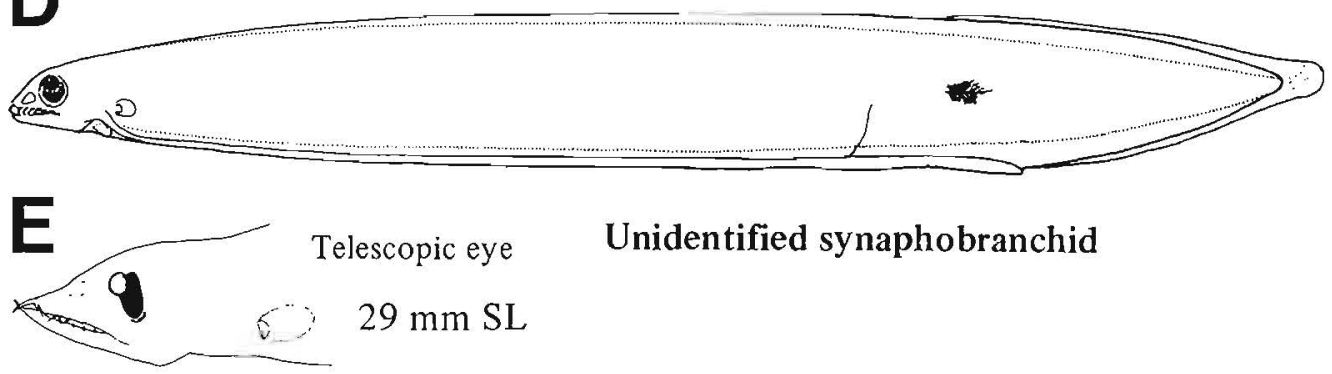

Unidentified synaphobranchid

Figures A, D, Castle 1984; B, NWAFC original (B. Vinter); C, E, Smith 1984. 


\section{ELOPOMORPHA}

Nettastomatidae (186-290 myomeres) Nettastomatids are represented in the Northeast Pacific by Venefica sp., but their larvae are unknown. Larvae in the family are identified by their gut characteristics (presence of two loops and gut length $<50 \%$ SL) and moderately long head. The body is deep to elongate and the tail is pointed. The dorsal fin origin is posterior to the head but well anterior, at myomeres 11-12. Ventral and lateral pigment is variable. Size at transformation is also variable but occurs between 120 and $200 \mathrm{~mm}$ SL.

Congridae (141-219 myomeres) Congrids are represented in the area by Xenomystax atrarius, a member of the subfamily Muraenesocinae (larvae unknown). Xenomystax is closely related to Paraxenomystax (Smith 1979). Members of this subfamily are sometimes considered part of a separate family. Their larvae have a moderately elongate body with the gut length about $75 \%$ SL. Pigment occurs on the head, widely spaced along the throat to anus, and below the lateral midline in the form of a row of large spots. Size at transformation is probably similar to the congrids with most at $100 \mathrm{~mm}$ SL but some up to 200-300 mm SL.

Serrivomeridae (147-169 myomeres) Serrivomer jesperseni larvae are identified by a head shape that is sharp and slightly concave, pointed tail, simple gut with a length of $\sim 75 \% \mathrm{SL}$, and a small nasal organ near the eye. The dorsal fin origin is located anterior to the anus. The last blood vessel occurs between myomeres $30-37$. Pigment may be located variously over the lateral body surface, but ventral pigment is lacking. Other pigment appears along the dorsal and anal fin bases and in a cluster on the orbit above the eye. Size at transformation is $60 \mathrm{~mm}$ SL.

Eurypharyngidae (97-125 myomeres) Eurypharynx larvae are identified by their short, deep head and body, gut about $50-67 \%$ SL with one loop posteriorly, and pointed tail. Ventral pigment is limited to the gut loop. Size at transformation is $30-40 \mathrm{~mm} \mathrm{SL}$. 

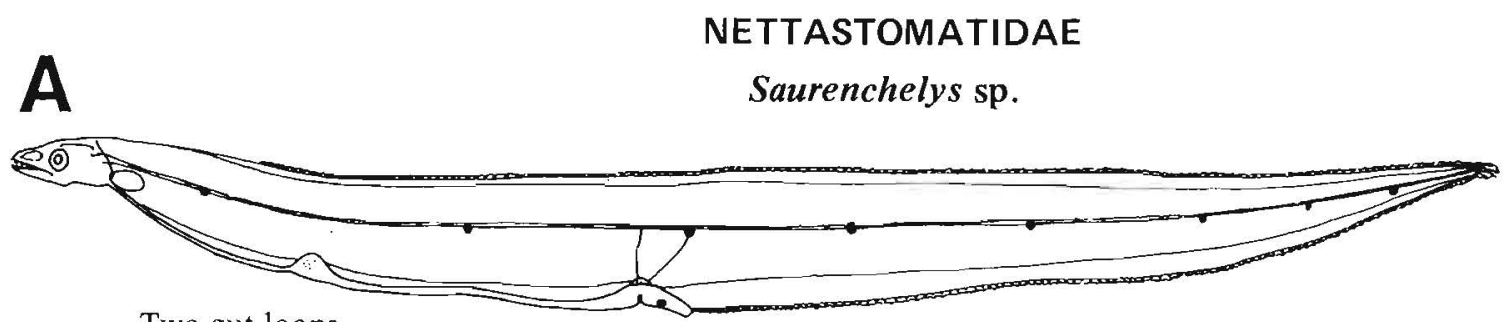

Two gut loops

\section{CONGRIDAE}

Paraxenomystax sp.

B

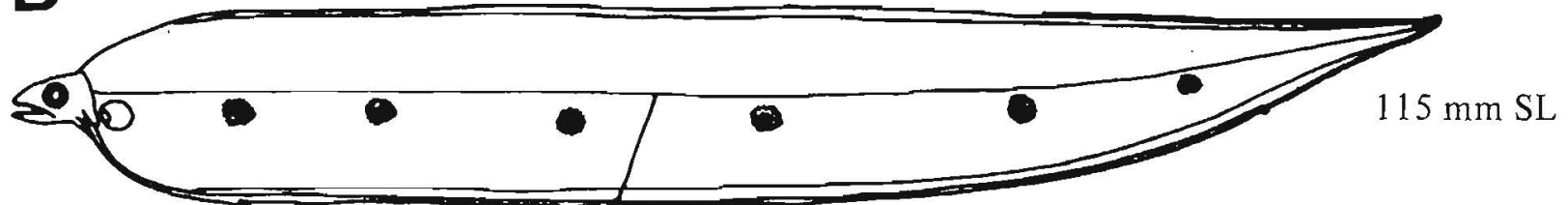

C

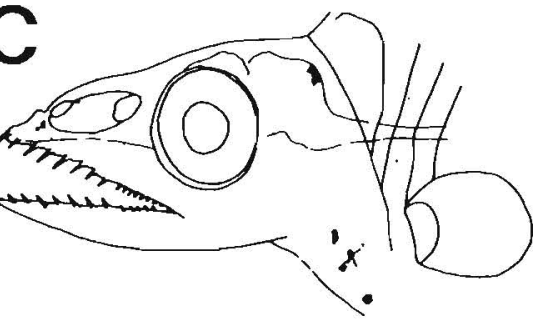

Large spots below lateral line

D

SERRIVOMERIDAE

Serrivomer jesperseni

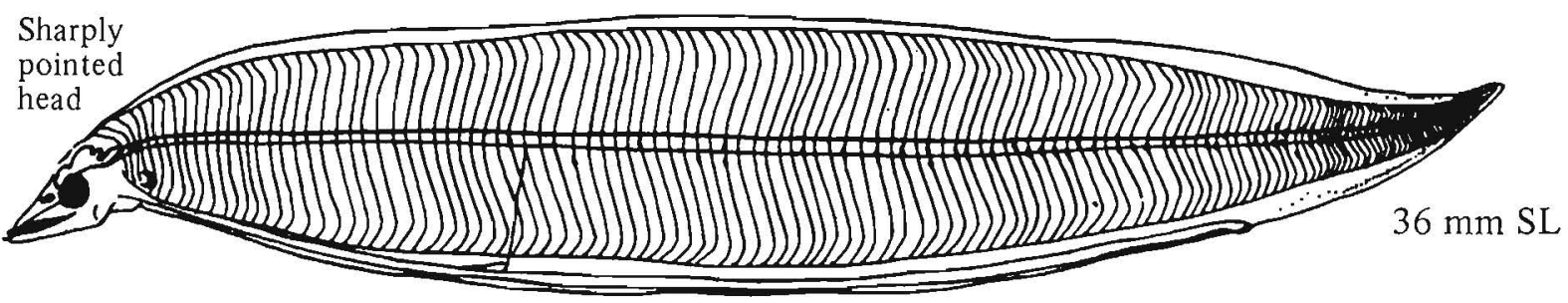

\section{EURYPHARYNGIDAE}

Eurypharynx pelecanoides

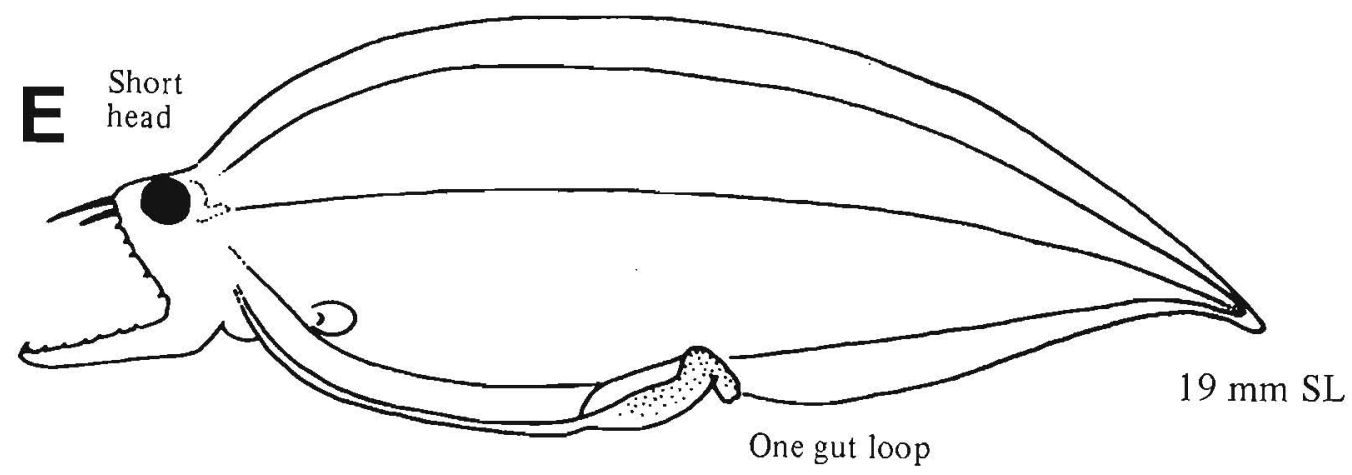

Figure A, Smith 1984; B-C, Smith 1979; D, Bauchot 1959; E, Fahay 1983 (after Smith 1979), 


\section{MERISTICS}

\begin{tabular}{|c|c|}
\hline \multirow[t]{3}{*}{ Vertebrae } & Total: $181-193-201$ \\
\hline & Precaudal: X-X-X \\
\hline & Caudal: X-X-X \\
\hline Branchiostegal rays & $X-X-X$ \\
\hline Caudal fin & Reduced \\
\hline Pelvic fin & Absent \\
\hline \multirow[t]{2}{*}{ Dorsal fin } & R: $279-339-432$ \\
\hline & D100: $130-164-210^{a}$ \\
\hline Pectoral fin & $\mathrm{R}: 14-16-18$ \\
\hline \multirow[t]{2}{*}{ Anal fin } & R: $240-299-372$ \\
\hline & A100: $103-138-176^{\mathrm{a}}$ \\
\hline Gill rakers & U: X-X-X $\quad$ L: X-X-X \\
\hline \multicolumn{2}{|l|}{ LIFE HISTORY } \\
\hline Range & $\begin{array}{l}\text { South of southern California to } \\
\text { Aleutian Is., } 51-55^{\circ} \mathrm{N}\end{array}$ \\
\hline Ecology & $\begin{array}{l}\text { Meso- and bathypelagic, } \\
510-4580 \mathrm{~m}\end{array}$ \\
\hline ELH pattern & $\begin{array}{l}\text { Oviparous, pelagic eggs, pelagic } \\
\text { larvae }\end{array}$ \\
\hline \multirow[t]{4}{*}{ Spawning } & Season: \\
\hline & Area: \\
\hline & Mode: \\
\hline & Migration: \\
\hline Fecundity & Range/function: \\
\hline
\end{tabular}

Age at first maturity Longevity

\section{EARLY LIFE HISTORY DESCRIPTION}

\section{EGGS}

Diameter

No. of oil globules

Oil globule diameter

Yolk

Envelope

Hatch size

Incubation time/temp.

Pigment

Diagnostic characters

\section{LARVAE}

Preanal length

Length at flexion

Length at transformation

Sequence of fin Dorsal and anal (late) development

\section{Pigment}

- Small spots on top of spinal cord, at least posteriorly

- Row of spots dorsally along gut length, ventral row anterior to stomach

- Several groups (usually three) of internal spots along body subaxially-about four spots in each group ${ }^{b}$

- Dorsal and anal fin bases

\section{Diagnostic characters}

Distinguished from Nemichthys spp. by

- Body less elongate

- Caudal structure (tail more round, not filiform)

- Position of liver and last blood vessel, 30th and 70-88th myomere, respectively

- Number of myomeres (181-201)

\footnotetext{
${ }^{2}$ The total dorsal and anal fin ray counts and the number of lateral line pores and vertebrae are often of no value; they are not comparable because caudal parts are often missing and regeneration may have occurred. Nielsen and Smith (1978) introduced artificial lengths/counts with a greater comparative value which we employ here (see their Materials and Methods section for a complete discussion), e.g., D200, A200 $=$ number of fin rays anterior to vertebrae no. 201.

${ }^{b}$ According to Smith (1979), there are three blotches of internal lateral pigment spots which become less prominent with growth.
}

Ref: Castle 1984, Nielsen and Smith 1978, Smith 1979 

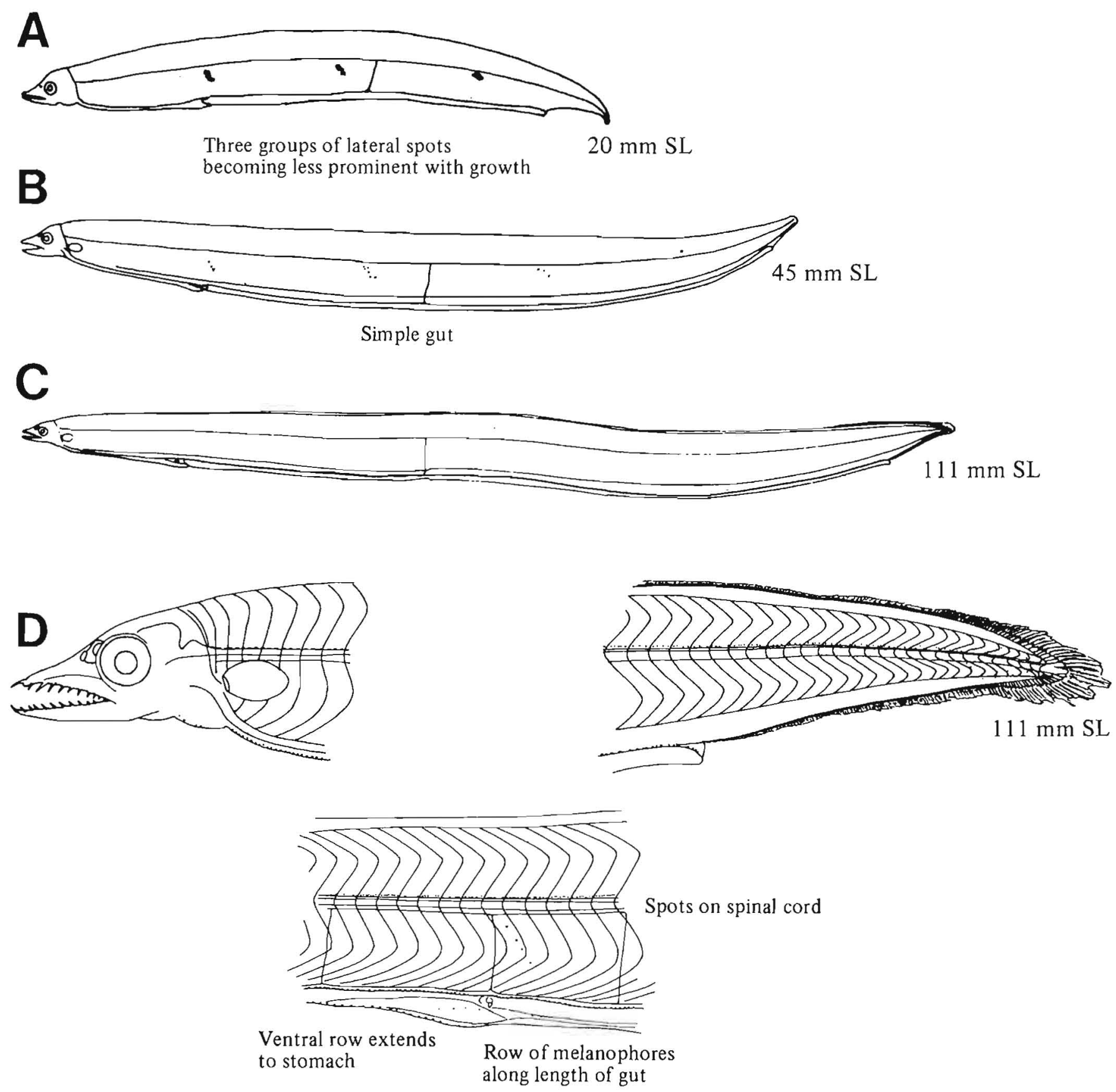

Figures A-D, Smith 1979. 


\section{MERISTICS}

\begin{tabular}{ll} 
Vertebrae & Total: $300-300-300$ \\
& Precaudal: $77-85-105$ \\
& Caudal: X-X-X \\
Branchiostegal rays & $7-X-15$ \\
Caudal fin & Reduced \\
Pelvic fin & Absent \\
Dorsal fin & R: $330-330-330$ \\
& D200: $170-207-253^{\text {a }}$ \\
Pectoral fin & R: $10-11-14$ \\
Anal fin & R: $320-320-320$ \\
& A200:186-218-273 a \\
Gill rakers & U: X-X-X L: X-X \\
& \\
LIFE HISTORY & \\
\hline
\end{tabular}

\begin{tabular}{|c|c|}
\hline Range & $\begin{array}{l}\text { South of southern California to } \\
\text { Brit. Col., } 48^{\circ} 30^{\prime}-55^{\circ} \mathrm{N}\end{array}$ \\
\hline Ecology & $\begin{array}{l}\text { Epi-, meso-, and bathypelagic, } \\
91-1829 \mathrm{~m}\end{array}$ \\
\hline ELH pattern & $\begin{array}{l}\text { Oviparous, pelagic eggs, pelagic } \\
\text { larvae }\end{array}$ \\
\hline Spawning & $\begin{array}{l}\text { Season: } \\
\text { Area: } \\
\text { Mode: } \\
\text { Migration: }\end{array}$ \\
\hline $\begin{array}{l}\text { Fecundity } \\
\text { Age at first maturity } \\
\text { Longevity }\end{array}$ & Range/function: \\
\hline
\end{tabular}

\section{EARLY LIFE HISTORY DESCRIPTION}

\section{EGGS}

Diameter

No. of oil globules

Oil globule diameter

Yolk

Envelope

Hatch size

Incubation time/temp.

Pigment

Diagnostic characters

\section{LARVAE}

Preanal length

Length at flexion

Length at transformation $\sim 200 \mathrm{~mm} \mathrm{SL}$

Sequence of fin Dorsal and anal (late) development

Pigment

- Three prominent spots laterally below midline in small larvae (lower on body than on Avocettina infans)

- Above gut along length

- Above spinal cord

Diagnostic characters

- Elongate with filiform tail

- Number of myomeres $(>300)$

Distinguished from $A$. infans by

- Position of liver (myomere 40) and last vertical blood vessel (80-100th myomere)

Nemichthys larseni larvae are not known but adults occur within the study area. See Table 5 for data on adults. Additional meristic characters from Nielsen and Smith (1978) are: D200, 173-205-222; A200, 164-200-208. See $A$. infans (p. 38, footnote a).

\footnotetext{
${ }^{a}$ See Avocettina infans.

Ref: Castle 1965, 1984; Nielsen and Smith 1978
} 


\section{A}

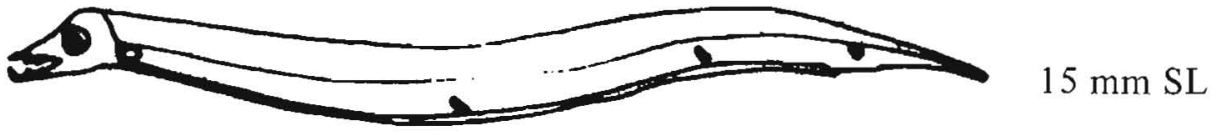

B

Three spots laterally below midline

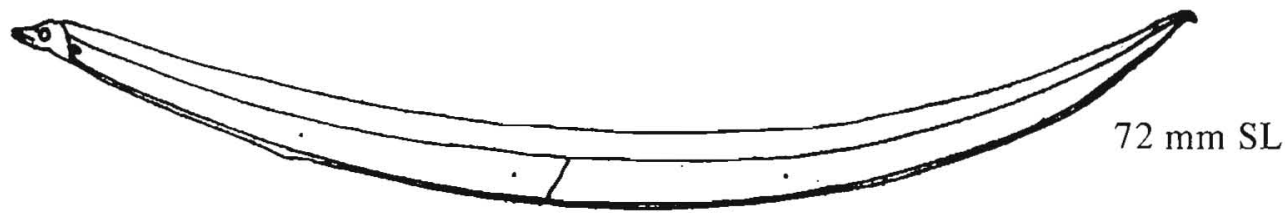

C

Lateral spots less intense

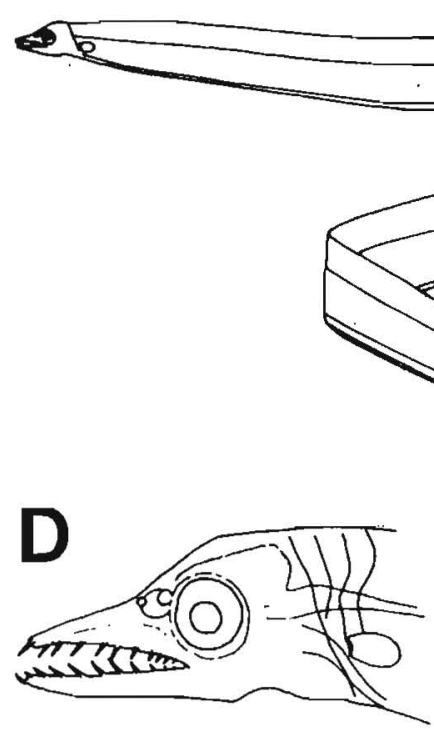

E
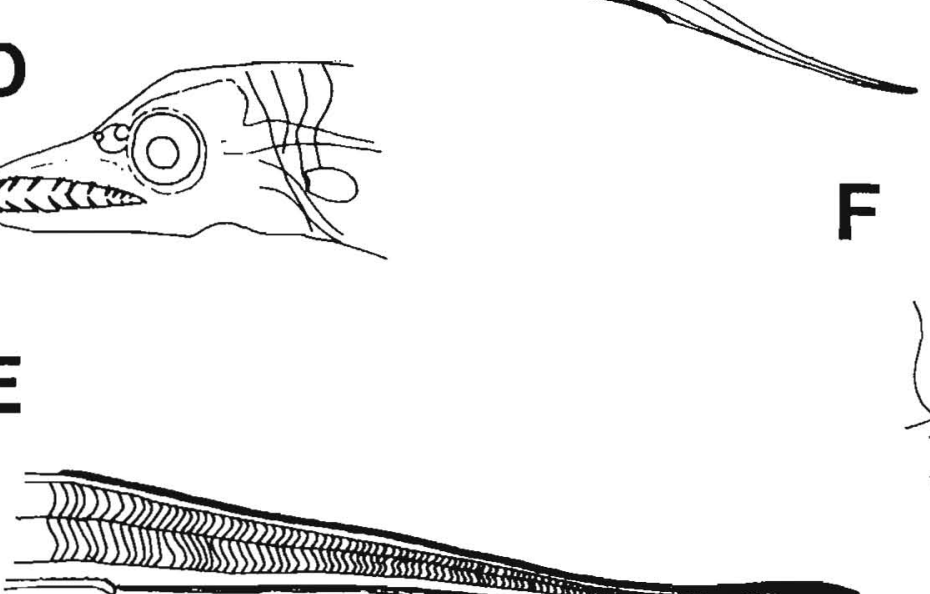

$182 \mathrm{~mm} \mathrm{SL}$

Melanophores above spinal cord

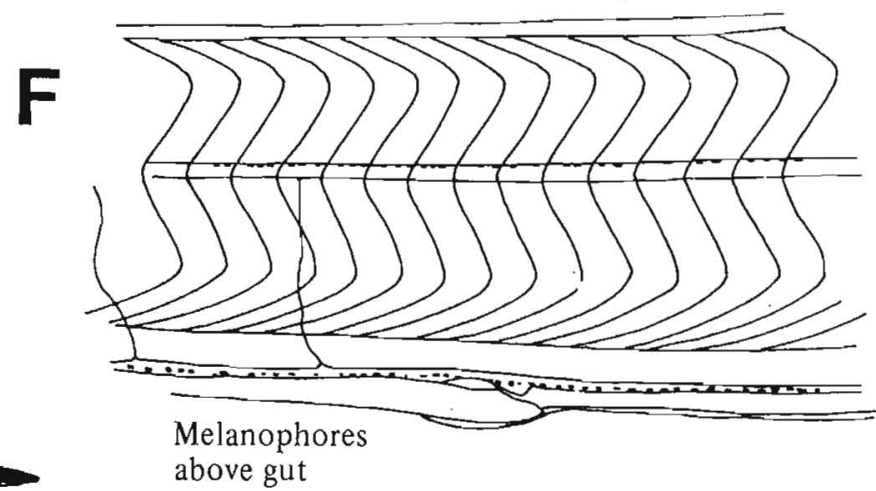

Filiform tail

Figures A-F, Smith 1979. 



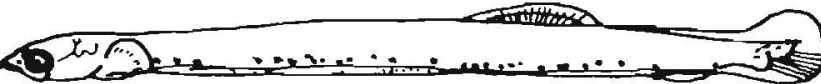

\section{Clupeiformes}

The Clupeiformes (herrings, anchovies) are generally small, coastal marine fishes that occur worldwide. Most species form schools and swim near the surface in nearshore waters, feeding on plankton. They have specialized gill rakers for straining large amounts of water. The order consists of 4 families, 78 genera, and about 317 species (McGowan and Berry 1984). Clupeids generally lay demersal eggs whereas engraulidids have pelagic eggs that are sometimes ellipsoidal in shape. Larvae are elongate and similar in appearance but may be distinguished by myomere counts and pigmentation characters.

Families in study area: Clupeidae Engraulididae 


\section{MERISTICS}

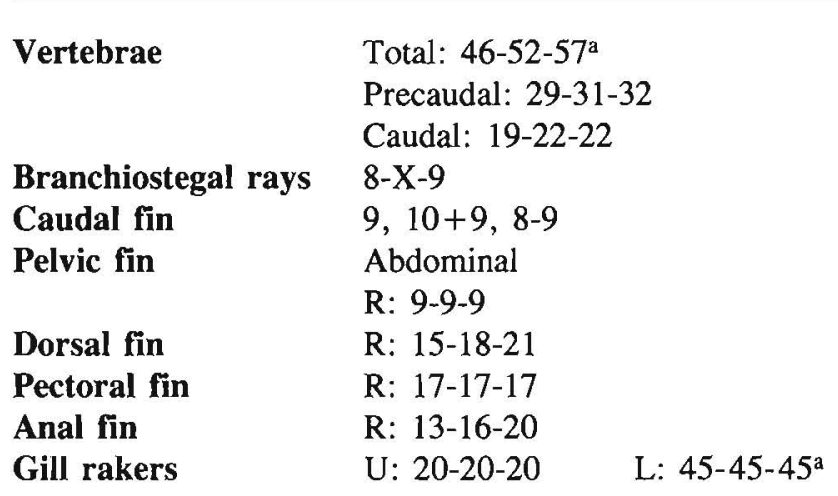

\section{LIFE HISTORY}

$\begin{array}{ll}\text { Range } & \text { South of southern California to } \\ & \text { Arctic, not specific } \\ \text { Ecology } & \text { Nearshore shelf pelagic, } \\ & 0-137 \mathrm{~m}^{\mathrm{b}} 475 \mathrm{~m}^{\mathrm{c}} \\ \text { ELH pattern } & \text { Oviparous; demersal, adhesive } \\ & \text { attached eggs; pelagic larvae } \\ \text { Spawning } & \text { Season: Jan-Apr (California), } \\ & \text { Mar-June (Alaska) } \\ & \text { Area: Demersal (usually on } \\ & \text { vegetation), nearshore } \\ & \text { Mode: Schools } \\ \text { Migration: Inshore } & \\ \text { Fecundity } & \text { Range/function: } 6300-41,000 / \\ & \mathrm{F}=0.000000436 \times \mathrm{L}^{4} \cdot 71 \\ \text { Age at first maturity } & \mathrm{L}=\text { SL mm } \\ & 2-4 \mathrm{yr}^{\mathrm{f}}(\text { Puget Sound })^{\mathrm{f}} \\ & 2-6 \mathrm{yr}^{\mathrm{f}}(\text { Bering Sea) } \\ \text { Longevity } & >19 \mathrm{yr}^{\mathrm{h}}\end{array}$

\footnotetext{
${ }^{a}$ McGowan and Berry (1984) report total vertebral counts of 53-60 and lower gill raker counts of 37-52.

${ }^{b}$ Alaska Department of Fish and Game 1985

'Allen and Smith 1988

${ }^{\mathrm{d}}$ Scattergood et al. 1959

e Schaefer 1937

${ }^{f}$ Katz 1942

${ }^{8}$ Rudomilov 1972

${ }^{\mathrm{h}}$ Fitch and Lavenberg 1975
}

Ref: Garrison and Miller 1982, Grant 1986, McGowan and Berry 1984

\section{EARLY LIFE HISTORY DESCRIPTION}

\section{EGGS}

Diameter

No. of oil globules

Oil globule diameter

Yolk

Envelope

Hatch size

Incubation time/temp.

Pigment

\section{$1.3-1.7 \mathrm{~mm}$}

None

Segmented

Smooth, clear, thick

5.6-7.5 $\mathrm{mm}$ SL $(7 \mathrm{~mm})$;

yolk absorbed 9-10 $\mathrm{mm} \mathrm{SL}$ $14-15 \mathrm{~d} / 8.5^{\circ} \mathrm{C}$

\section{Diagnostic characters \\ - Wide perivitelline space}

\section{LARVAE}

Preanal length

Length at flexion

$72 \% \mathrm{SL}$

Length at transformation

Sequence of fin

development

$18 \mathrm{~mm} \mathrm{SL}$

Pigment

- Isthmus, thoracic region

- Gut: Dorsal, midventral (midventral melanophores on intestine usually paired, sometimes slightly offset)

- Caudal, hypural

Diagnostic characters (see Table 4)

Distinguished from Engraulis mordax (p. 48) by

- More myomeres (usually $>50$ )

- Longer gut (consistently $>70 \%$ SL)

- Placement of dorsal fin relative to anus (6-7 myomeres between dorsal and anal fins)

- Less isthmus pigment

- Prominent swimbladder in E. mordax

- Dorsal spots at notochord tip usually not in $E$. mordax

- Generally more pigmented

Distinguished from Sardinops sagax by

- Presence of posteroventral pigment on gut

- Dorsal spots at notochord tip usually not in $S$. sagax

See also Mallotus villosus (p. 80) and Ammodytes hexapterus (p. 540) 
B

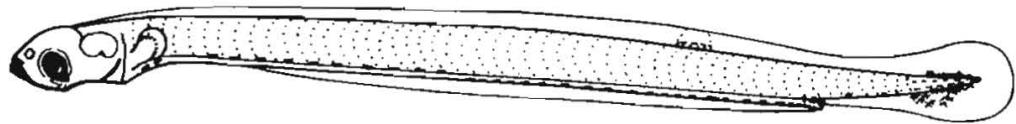

Number of myomeres, gut length

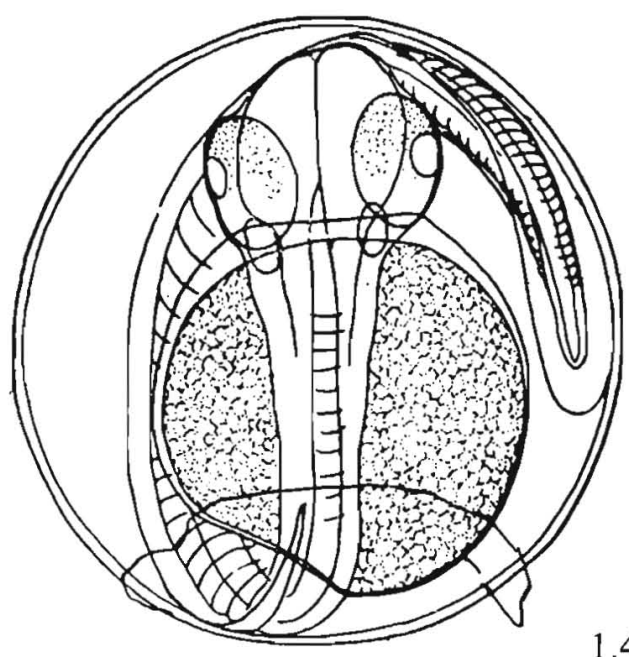

A

$1.4 \mathrm{~mm}$

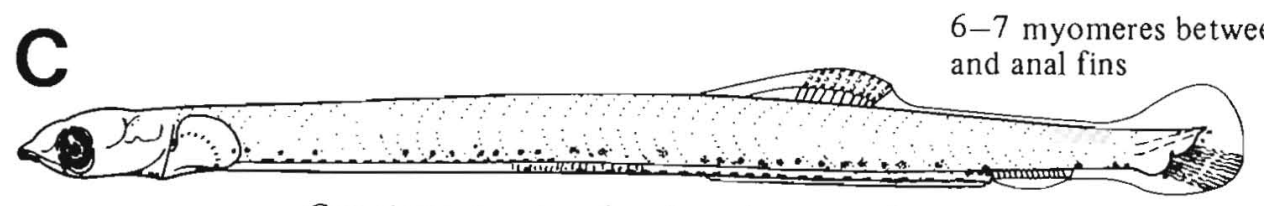

Gut pigment: anterodorsal, posteroventral

D

$19.0 \mathrm{~mm} \mathrm{SL}$

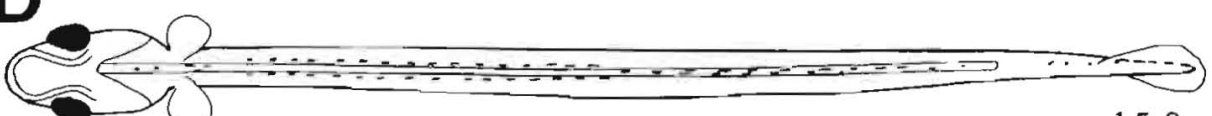

$15.0 \mathrm{~mm} \mathrm{SL}$

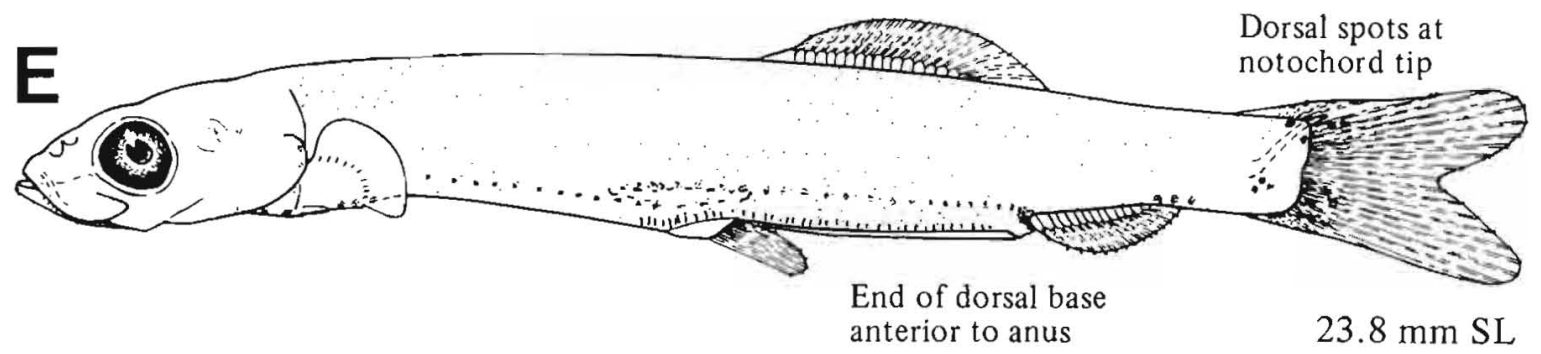

Figure A, Uchida et al. 1958; B-E (D, ventral view), NWAFC originals (B. Vinter). 
MERISTICS $^{a}$

\begin{tabular}{ll} 
Vertebrae & Total: $48-X-54$ \\
& Precaudal: $28-29-30$ \\
& Caudal: $22-22-23$ \\
Branchiostegal rays & $6-X-10$ \\
Caudal fin & $7-9,10+9,6-8$ \\
Pelvic fin & Abdominal \\
& R: $8-8-8$ \\
Dorsal fin & R: $17-X-20$ \\
Pectoral fin & R: $17-17-17$ \\
Anal fin & R: $17-X-20$ \\
Gill rakers & U: $21-X-23$ \\
& \\
& \\
LIFE HISTORY & \\
\hline
\end{tabular}

$\begin{array}{ll}\text { Range } & \begin{array}{l}\text { South of southern California to } \\ \text { SE Alaska, } 55-59^{\circ} \mathrm{N}\end{array} \\ \text { Ecology } & \text { Nearshore shelf pelagic, 0-80 m } \\ \text { ELH pattern } & \text { Oviparous, pelagic eggs, pelagic } \\ & \text { larvae } \\ \text { Spawning } & \begin{array}{l}\text { Season: Winter-summer } \\ \text { Area: Near surface in coastal } \\ \text { and offshore waters }\end{array} \\ & \begin{array}{l}\text { Mode: Pelagic, schools } \\ \text { Migration: }\end{array} \\ \text { Fecundity } & {\text { Range/function: } 30,000-65,000^{\mathrm{c}}}^{\mathrm{c}} \\ \text { Age at first maturity } & 1-2 \mathrm{yr}^{\mathrm{b}} \\ \text { Longevity } & 10 \mathrm{yr}^{\mathrm{b}}, \text { possibly } 25 \mathrm{yr}^{\mathrm{d}}\end{array}$

\section{EARLY LIFE HISTORY DESCRIPTION}

\section{EGGS}

Diameter

No. of oil globules

$1.34-2.05 \mathrm{~mm}$

Oil globule diameter

Yolk

Envelope

Hatch size

One

$0.16 \mathrm{~mm}$

Irregularly segmented

Smooth, thin

3.5-3.75 mm SL

Incubation time/temp.

Pigment

$$
13-16^{\circ} \mathrm{C}^{\mathrm{e}}
$$

\title{
Diagnostic characters \\ - Wide perivitelline space
}

\author{
LARVAE \\ Preanal length \\ $>75 \%$ SL \\ Length at flexion \\ 9.0-14.0 mm SL \\ Length at transformation $35 \mathrm{~mm} \mathrm{SL}$ \\ Sequence of fin \\ development \\ Caudal, dorsal, anal, \\ pelvics, pectorals \\ Pigment \\ - Row of melanophores along dorsal surface of gut \\ and along ventral midline \\ - Melanophores over anterior gut appear dash-like, \\ and those over posterior gut become larger and \\ more intense with development
}

Diagnostic characters (see Table 4)

- See Clupea pallasi (p. 44)

- Dash-like melanophores along anterior surface of gut

Distinguished from $C$. pallasi by

- Lack of posteroventral pigment on gut

Distinguished from Engraulis mordax (p. 48) by

- Dorsal and anal fin placement (6-8 myomeres between fins)

\footnotetext{
${ }^{a}$ Meristics include information from McGowan and Berry 1984.

bFrey 1971

cHart 1973

'Fitch and Lavenberg 1971

${ }^{\circ}$ Garrison and Miller 1982

Ref: McGowan and Berry 1984.
} 
B

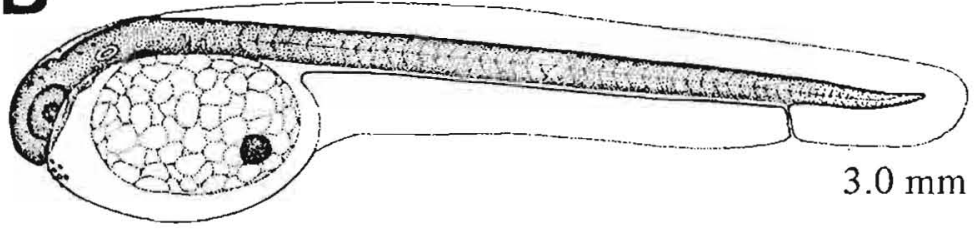

C
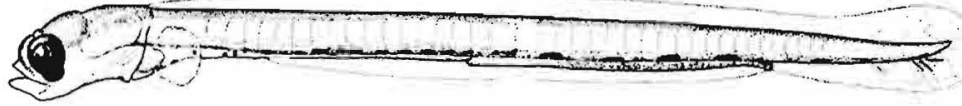

Gut pigment restricted to dorsal surface

$6.1 \mathrm{~mm}$

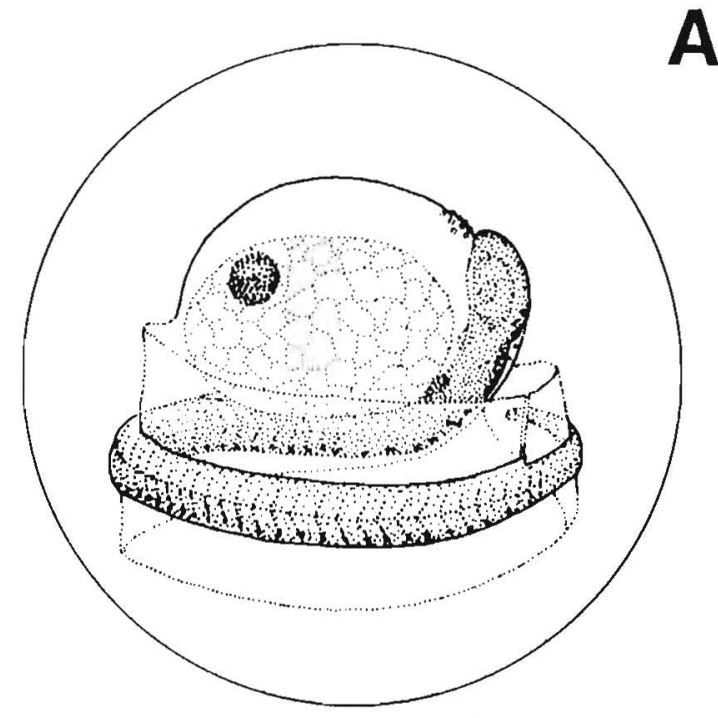

$1.34-2.05 \mathrm{~mm}$

D

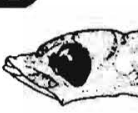

Melanophores appear dash-like

along the anterior half of gut

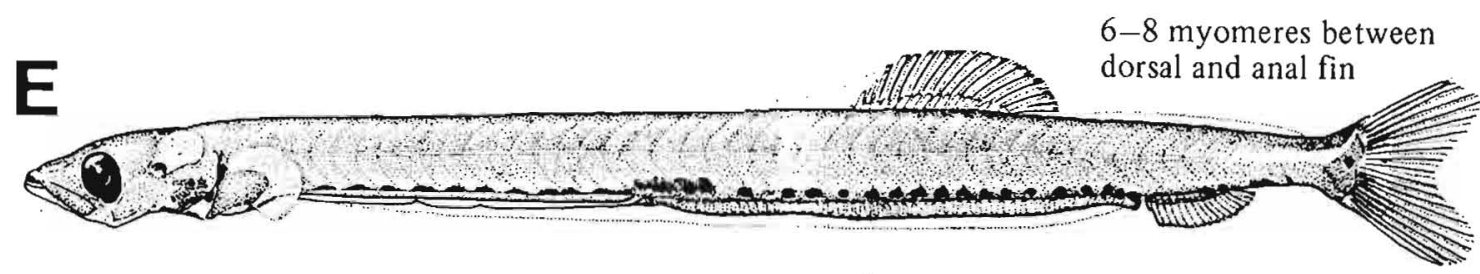

$19.7 \mathrm{~mm}$

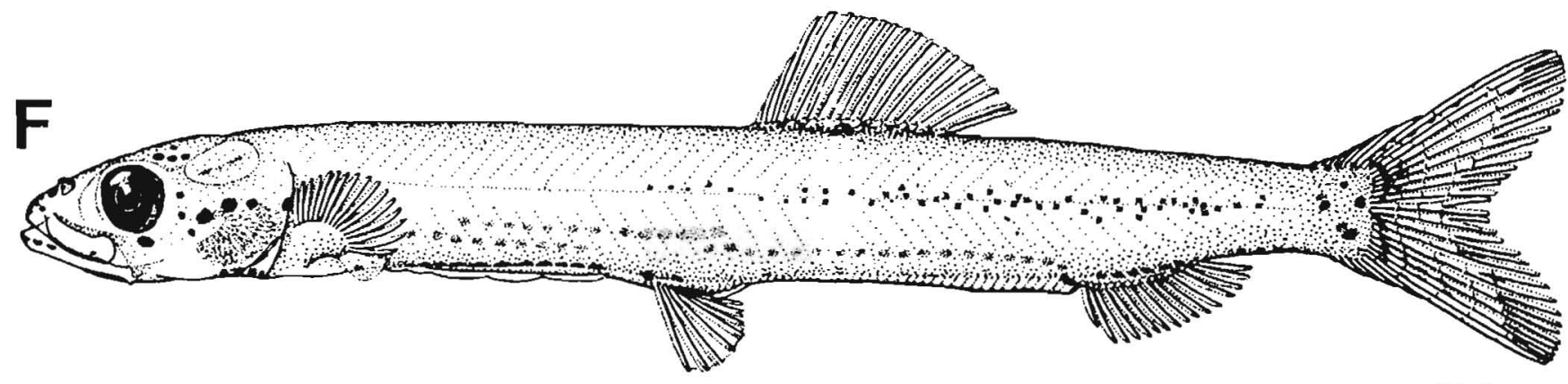

$31.3 \mathrm{~mm}$

Figure A, Matarese and Sandknop 1984; B-F, Kramer 1970. Stippling in these figures is not pigment. 


\section{MERISTICS}

\begin{tabular}{ll} 
Vertebrae & Total: $43-46-47$ \\
& Precaudal: $24-25-26$ \\
& Caudal: $19-20-21$ \\
Branchiostegal rays & $14-14-14$ \\
Caudal fin & $8-10,10+9,8-10$ \\
Pelvic fin & Abdominal \\
& R: $6-6-6$ \\
Dorsal fin & R: $14-X-19$ \\
Pectoral fin & R: $13-X-20$ \\
Anal fin & R: $19-X-26$ \\
Gill rakers & U: $28-X-41 \quad$ L: $37-X-45$ \\
& \\
LIFE HISTORY & \\
\hline
\end{tabular}

$\begin{array}{ll}\text { Range } & \begin{array}{c}\text { South of southern California to } \\ \text { Brit. Col., } 48^{\circ} 30^{\prime}-55^{\circ} \mathrm{N} \\ \text { Ecology }\end{array} \\ \begin{array}{l}\text { Epi- and mesopelagic, 0-300 } \mathrm{m}^{\mathrm{a}} \\ \text { ELH pattern }\end{array} & \begin{array}{l}\text { Oviparous, pelagic eggs, pelagic } \\ \text { larvae }\end{array} \\ \text { Spawning } & \text { Season: May }{ }^{\mathrm{b}} \text { to mid-Augc; } \\ & \text { year-round (California) } \\ & \text { Area: Pelagic } \\ & \text { Mode: } \\ & \text { Migration: } \\ \text { Fecundity } & \text { Range/function: } 4025^{\mathrm{e}}-30,000^{\mathrm{r}} \\ \text { Age at first maturity } & 1-2 \mathrm{yr}^{\mathrm{g}} \\ \text { Longevity } & 7 \mathrm{yr}^{\mathrm{h}}\end{array}$

\section{EARLY LIFE HISTORY DESCRIPTION}

\section{EGGS}

Diameter

No. of oil globules

Oil globule diameter

Yolk

Envelope

Hatch size

Incubation time/temp.

Pigment

\section{Diagnostic characters \\ - Ovoid \\ - Narrow perivitelline space}

\section{LARVAE}

Preanal length

\section{Length at flexion}

Length at transformation

Sequence of fin development

Pigment

- Isthmus

- Row of melanophores along dorsal surface of gut continuing along ventral body midline

- Ventral pigment on gut beginning about midway

Diagnostic characters (see Table 4)

- See Clupea pallasi (p. 44)

- Isthmus pigment present

- Dorsal and anal fin placement (0-2 myomeres between fins)

- Midventral melanophores on intestine usually staggered, not single as in osmerids, and usually not as evenly paired as in C. pallasi

\footnotetext{
${ }^{a}$ Pacific Fisheries Management Council 1978

${ }^{\circ}$ Kendall and Clark 1982

cBlackburn 1973

${ }^{d}$ Lasker and Smith 1977

'Frey 1971

Baxter 1967

${ }^{8}$ Clark and Phillips 1952

${ }^{h}$ Hart 1973

Ref: McGowan and Berry 1984, Wang 1981.
} 
B

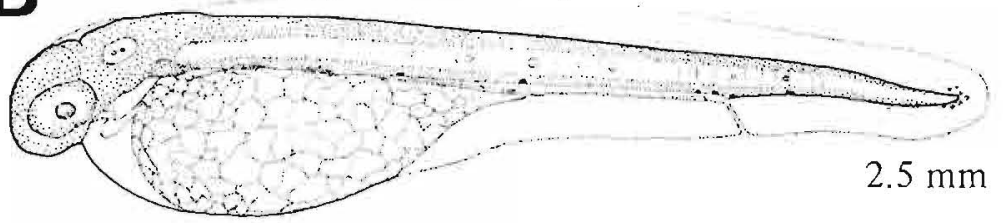

C
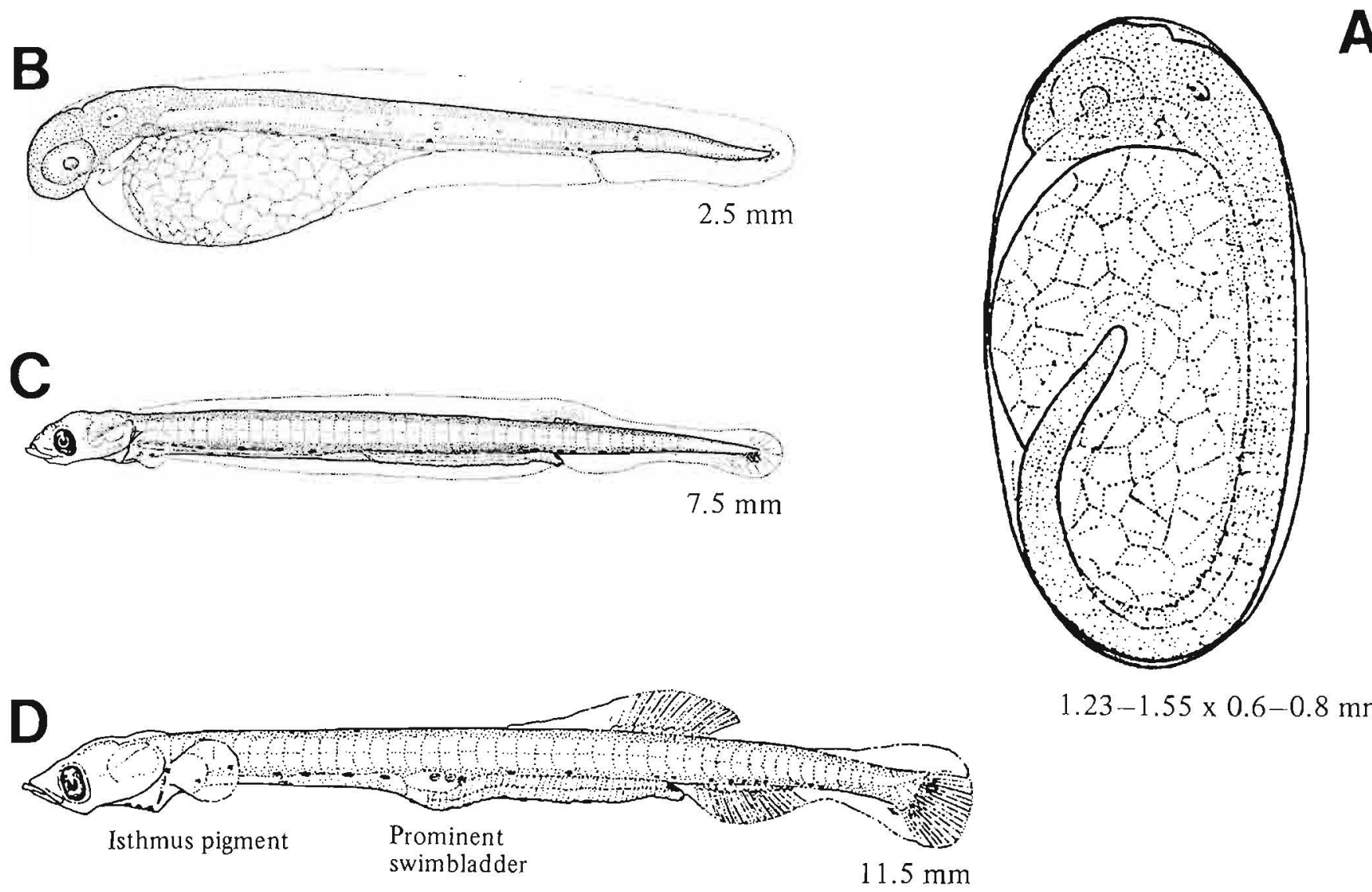

$1.23-1.55 \times 0.6-0.8 \mathrm{~mm}$

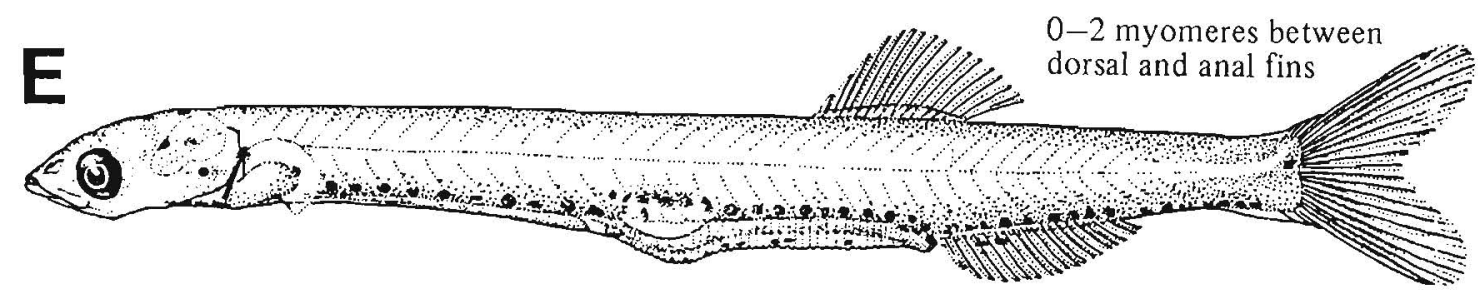

$18.4 \mathrm{~mm}$

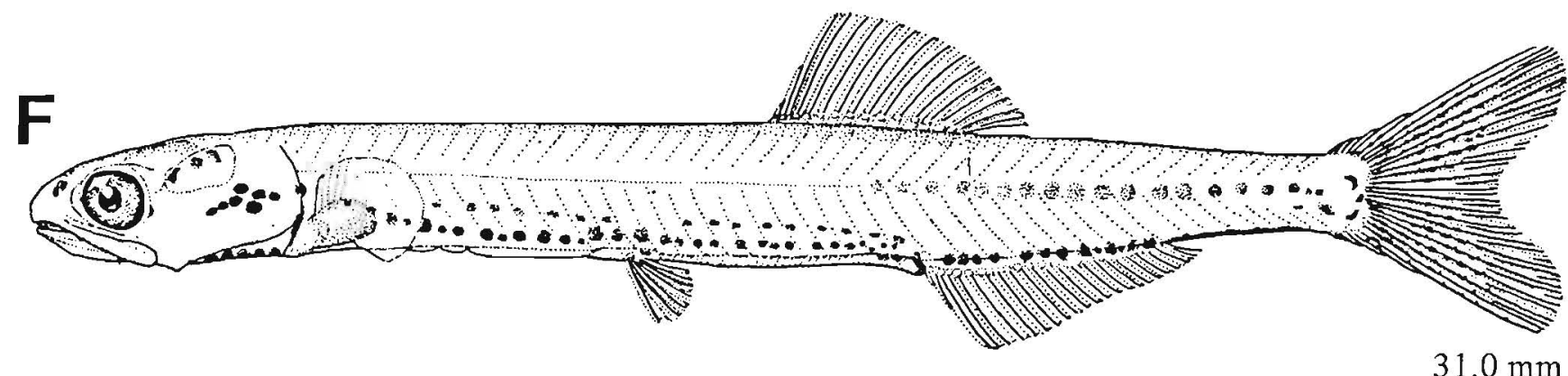

Figure A, Bolin 1936; B-F, Kramer and Ahlstrom 1968. Stippling in these figures is not pigment. 


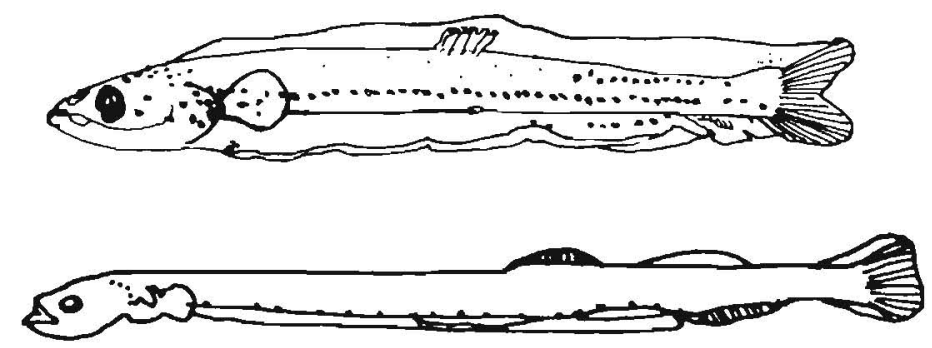

\section{Salmoniformes}

The salmoniform fishes (salmons, smelts, deep sea smelts, and others) are primarily freshwater spawners except for members of the Argentinoidei, the largest taxon of salmoniforms with marine eggs and larvae in our area. There is much disagreement as to the composition of the order (see Fink 1984). In our area, it consists of 4 suborders, 15 families, 90 genera, and about 320 species (J. Nelson 1984). The argentinoids are mostly deep-sea fishes. Eggs are pelagic and have distinctive pustules on the inner surface of the egg membranes; larvae have a variety of forms. Larval characters include presence/absence of eyestalks, unique development of median fins, and distinctive pigment patterns. Pigment patterns for bathylagids are discussed according to the categories described by Ahlstrom et al. (1984b) - species with large, isolated melanophores and those with a linear series of small melanophores.

A summary of meristic characters is included for Alepocephalidae and Platytroctidae. Although salmon parr are occasionally collected in plankton tows, they are not treated here. Information on their early life history is reviewed in Kendall and Behnke (1984).

Families in study area: Argentinidae

Bathylagidae

Opisthoproctidae

Alepocephalidae

Platytroctidae

Osmeridae

Salmonidae 


\section{MERISTICS}

\begin{tabular}{ll}
\hline Vertebrae & Total: $47-X-51$ \\
& Precaudal: 30-31-32 \\
& Caudal: $16-18-18$ \\
Branchiostegal rays & $5-5-5$ \\
Caudal fin & $12,10+9,11$ \\
Pelvic fin & Abdominal \\
& R: $10-11-12$ \\
Dorsal fin & R: $10-X-13$ \\
Pectoral fin & R: $11-X-18$ \\
Anal fin & R: $12-X-15$ \\
Gill rakers & U: $7-8-9$ \\
& \\
& \\
LIFE HISTORY & \\
\hline Range & South of southern California to \\
& Oregon, $42-46^{\circ} \mathrm{N}$ \\
Ecology & Epi- and mesopelagic, 11-274 m \\
ELH pattern & Oviparous, pelagic eggs, pelagic \\
& larvae \\
Spawning & Season: Jan-spring \\
& Area: \\
& Mode: \\
Fecundity & Migration: \\
Age at first maturity & Range/function: \\
Longevity & $>5$ yr ${ }^{a}$
\end{tabular}

${ }^{\text {a }}$ Fitch and Lavenberg 1968

${ }^{\mathrm{b}}$ Transformation: Morphological changes (deepening of body, lengthening of snout, enlargement of eye) and folding of anterior gut to form stomach, along with masking of larval pigment, occur at $25-30 \mathrm{~mm}$. Pelagic juveniles may occur at $50-100 \mathrm{~mm}$.

Ref: Ahlstrom et al. $1984 \mathrm{~b}$.

\section{EARLY LIFE HISTORY DESCRIPTION}

\section{EGGS}

Diameter

No. of oil globules

Oil globule diameter

Yolk

Envelope

Hatch size

$1.31-1.66 \mathrm{~mm}$

One, at vegetal pole

$0.27-0.46 \mathrm{~mm}$

Segmented

Pustules (pronounced raised bumps on inner surface)

Incubation time/temp.

Pigment

Diagnostic characters

- Pustules more pronounced than in bathylagid eggs

\section{LARVAE}

Preanal length

Length at flexion

$76-84 \%$ SL

Length at transformation $25-30 \mathrm{~mm} \mathrm{SL}$ (prolonged)

Sequence of fin

Caudal, dorsal and anal, development pectorals, pelvics

Pigment

- Internal head pigment

- Series of ventral trunk blotches extending from pectoral fin to end of gut

- 1-2 large blotches postanal with a large caudal blotch

- Blotches expand with development (see figure)

Diagnostic characters

- Transverse rugae lining gut

- Fin rays form in finfold, away from body margin

Distinguished from Nansenia candida by

- Presence of internal head pigment

- Caudal pigment on preflexion larvae

- Fewer spots over gut 

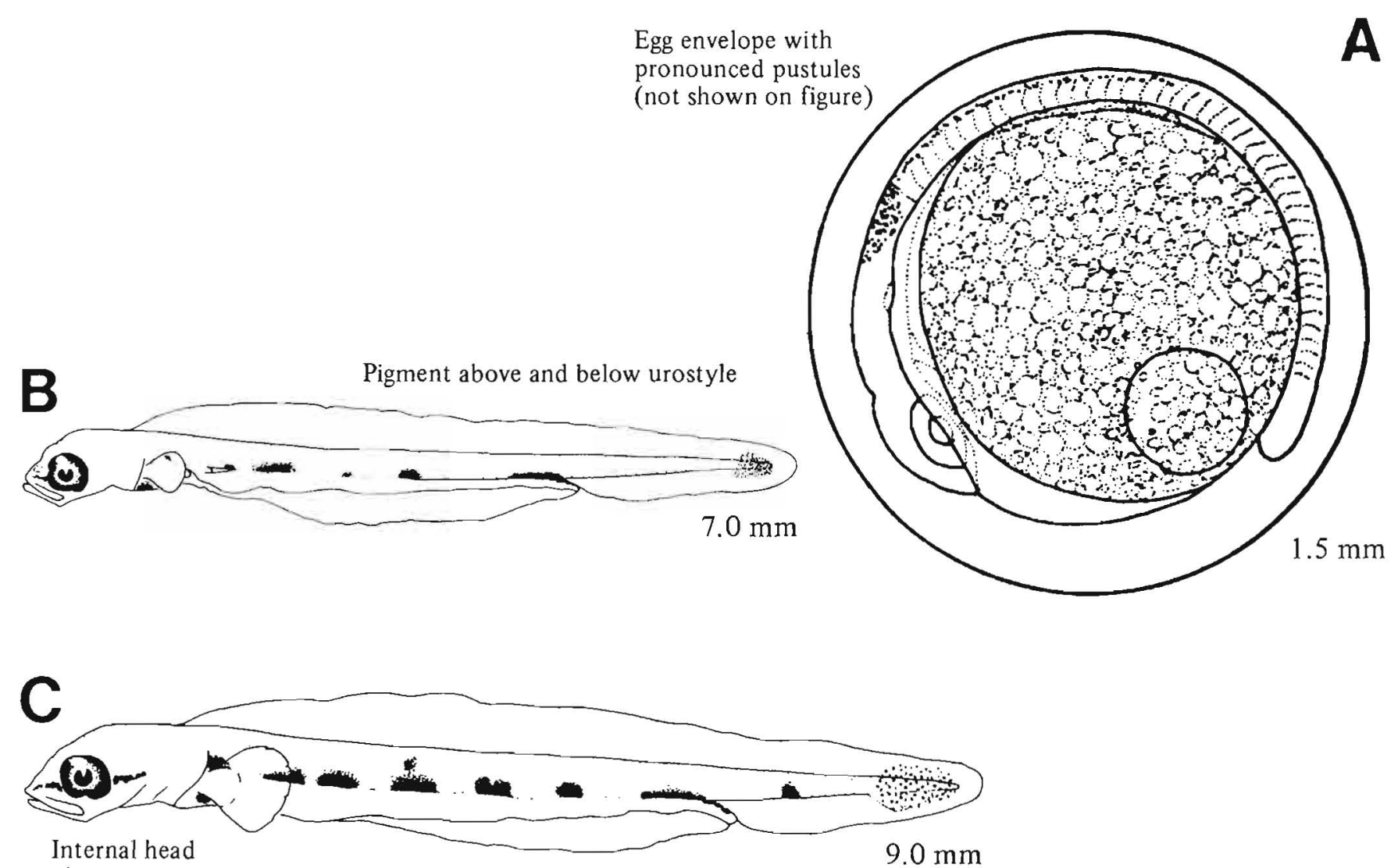

pigment

Fin rays form in finfold, away from body margin
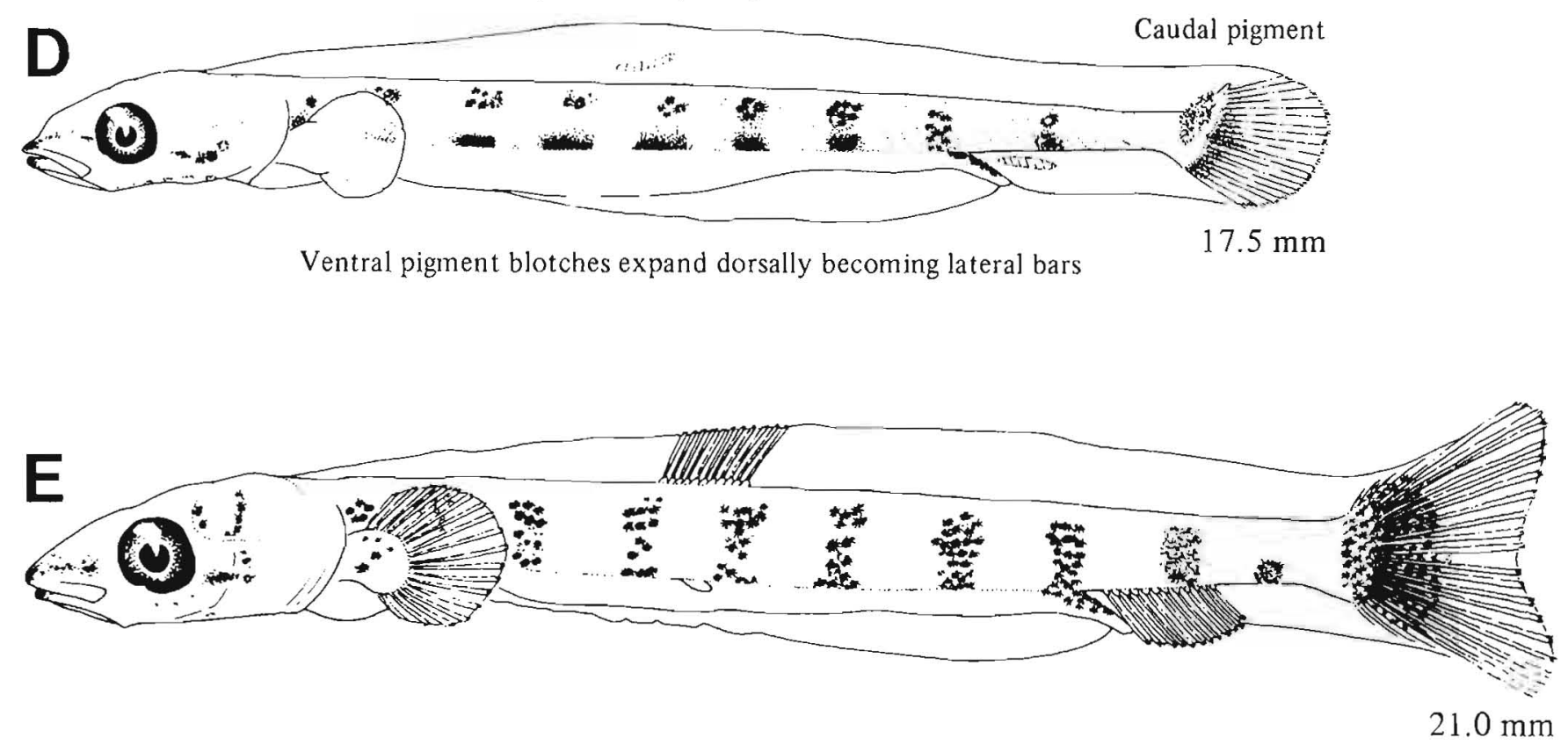

Figures A-E, Ahlstrom et al. $1984 \mathrm{~b}$. 


\section{MERISTICS}

\begin{tabular}{|c|c|}
\hline \multirow[t]{3}{*}{ Vertebrae } & Total: $44-X-47$ \\
\hline & Precaudal: $\mathrm{X}-\mathrm{X}-\mathrm{X}$ \\
\hline & \multirow{2}{*}{$\begin{array}{l}\text { Caudal: } X-X-X \\
3-3-3\end{array}$} \\
\hline Branchiostegal rays & \\
\hline Caudal fin & $11,10+9,14$ \\
\hline Pelvic fin & Abdominal \\
\hline & $\mathrm{R}: 9-10-10$ \\
\hline Dorsal fin & $\mathrm{R}: 9-\mathrm{X}-10^{\mathrm{b}}$ \\
\hline Pectoral fin & $\mathrm{R}: 9-\mathrm{X}-11^{\mathrm{b}}$ \\
\hline Anal fin & $\mathrm{R}: 8-\mathrm{X}-9$ \\
\hline Gill rakers & $\mathrm{U}: 12-12-12$ \\
\hline \multicolumn{2}{|l|}{ LIFE HISTORY } \\
\hline Range & $\begin{array}{c}\text { South of southern California to } \\
\text { Gulf of Alaska, } 54-60^{\circ} \mathrm{N}\end{array}$ \\
\hline Ecology & $\begin{array}{l}\text { Epi- and mesopelagic, } \\
200-1000 \mathrm{~m}^{\mathrm{c}}\end{array}$ \\
\hline ELH pattern & $\begin{array}{l}\text { Oviparous, pelagic eggs, pelagic } \\
\text { larvae }\end{array}$ \\
\hline Spawning & Season: \\
\hline & Area: \\
\hline & Mode: \\
\hline & Migration: \\
\hline Fecundity & \multirow[t]{2}{*}{ Range/function: } \\
\hline $\begin{array}{l}\text { Age at first maturity } \\
\text { Longevity }\end{array}$ & \\
\hline
\end{tabular}

\section{EARLY LIFE HISTORY DESCRIPTION}

\section{EGGS}

Diameter

No. of oil globules

Oil globule diameter

Yolk

Envelope

Hatch size

Incubation time/temp.

Pigment

Diagnostic characters

- Pustules

\section{LARVAE}

Preanal length

Length at flexion

$1.39-1.56 \mathrm{~mm}$

One, at vegetal pole

$0.41-0.49 \mathrm{~mm}$

Pustules

Length at trat

Sequence of fin development

Pigment

- Embedded pigment above gut

- Superficial head pigment (>8.4 mm SL)

- With development, embedded line of melanophores running length of body

- Conspicuous caudal pigment

- Ventral pigment from isthmus along anterior $2 / 3$ of gut

\section{Diagnostic characters}

- Transverse rugae lining gut only in posterior section, anteriorly an elongate s-shaped fold is present along with longitudinal rugae

- Fin rays form in finfold, away from body margin

\footnotetext{
${ }^{a}$ Referred to by Ahlstrom as northern Nansenia (E.H. Ahlstrom notes).

${ }^{b}$ Ahlstrom et al. 1984b

${ }^{c}$ Cohen 1958
}

Ref: Ahlstrom et al. 1984b. 

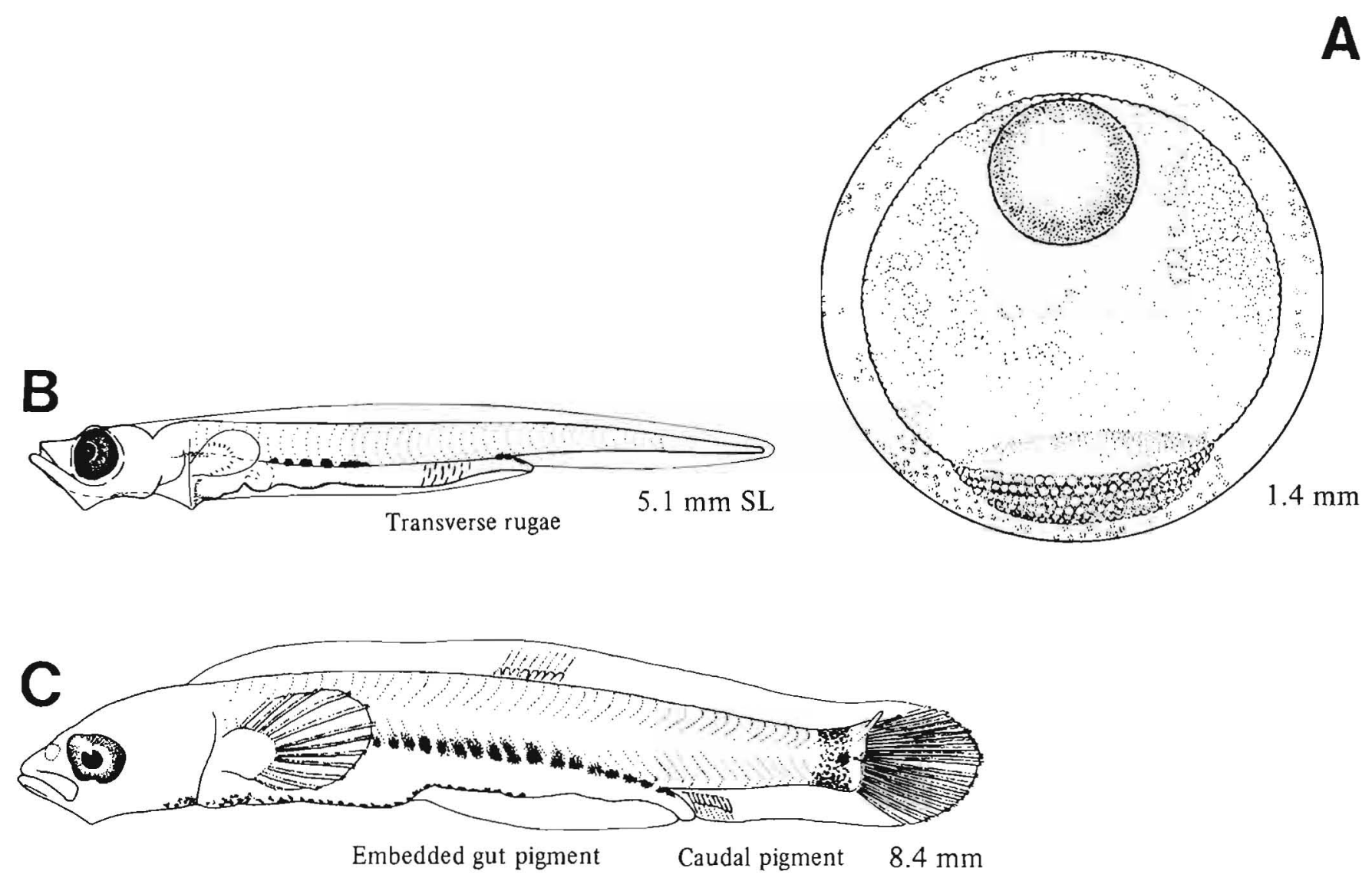

Figures A, C, Ahlstrom et al. 1984b; B, NWAFC original (B. Vinter). 


\section{MERISTICS}

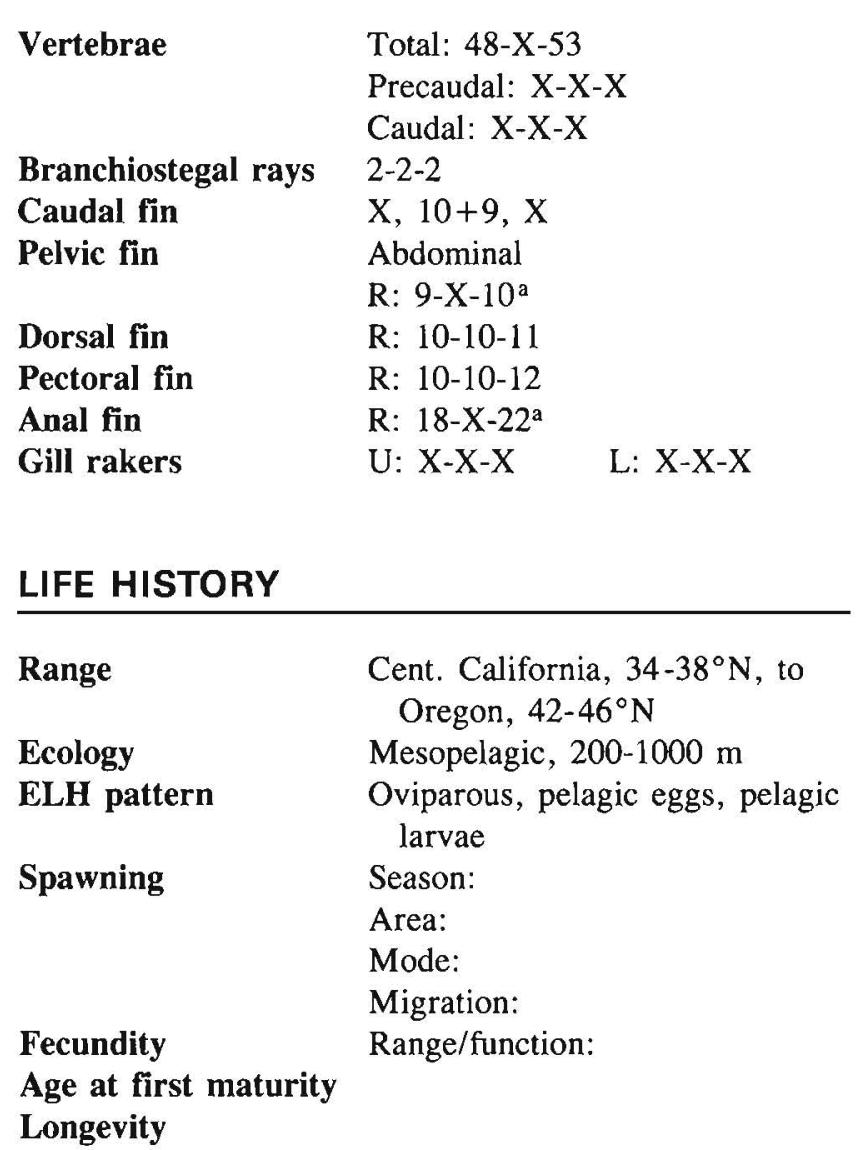

Ahlstrom et al. $1984 b$

${ }^{b}$ Transformation: Direct, marked in all species; slender body becoming deeper with development, large head and eyes, gut coils and becomes covered by black peritoneal sheath.

Ref: Ahlstrom et al. $1984 b$.

\section{EARLY LIFE HISTORY DESCRIPTION}

\section{EGGS}

Diameter

No. of oil globules

Oil globule diameter

Yolk

Envelope

Hatch size

Incubation time/temp.

Pigment

Diagnostic characters

\section{LARVAE}

Preanal length $\quad 84-89 \%$ SL

Length at flexion

Length at transformation ${ }^{b}$

Sequence of fin Caudal, dorsal, anal, development pectorals, pelvics

Pigment - Linear series of small melanophores

- Series of melanophores (up to 18) along lateral gut

- With development to late postflexion, additional pigment appears on lower jaw, isthmus, opercle, pectoral fin base, and lateral caudal peduncle

Diagnostic characters

- Anterior section of gut smaller in diameter compared with other species (see illustration)

- Eye stalks longer and persist into later larval stages (65\% HL)

- Fin rays form in finfold, away from body margin Distinguished from other bathylagids with eyestalks by

- Linear series of small melanophores

- B. pacificus has large, isolated spots, long eyestalks, and less intense pigment

- B. ochotensis has shorter stalks and more intense pigment 

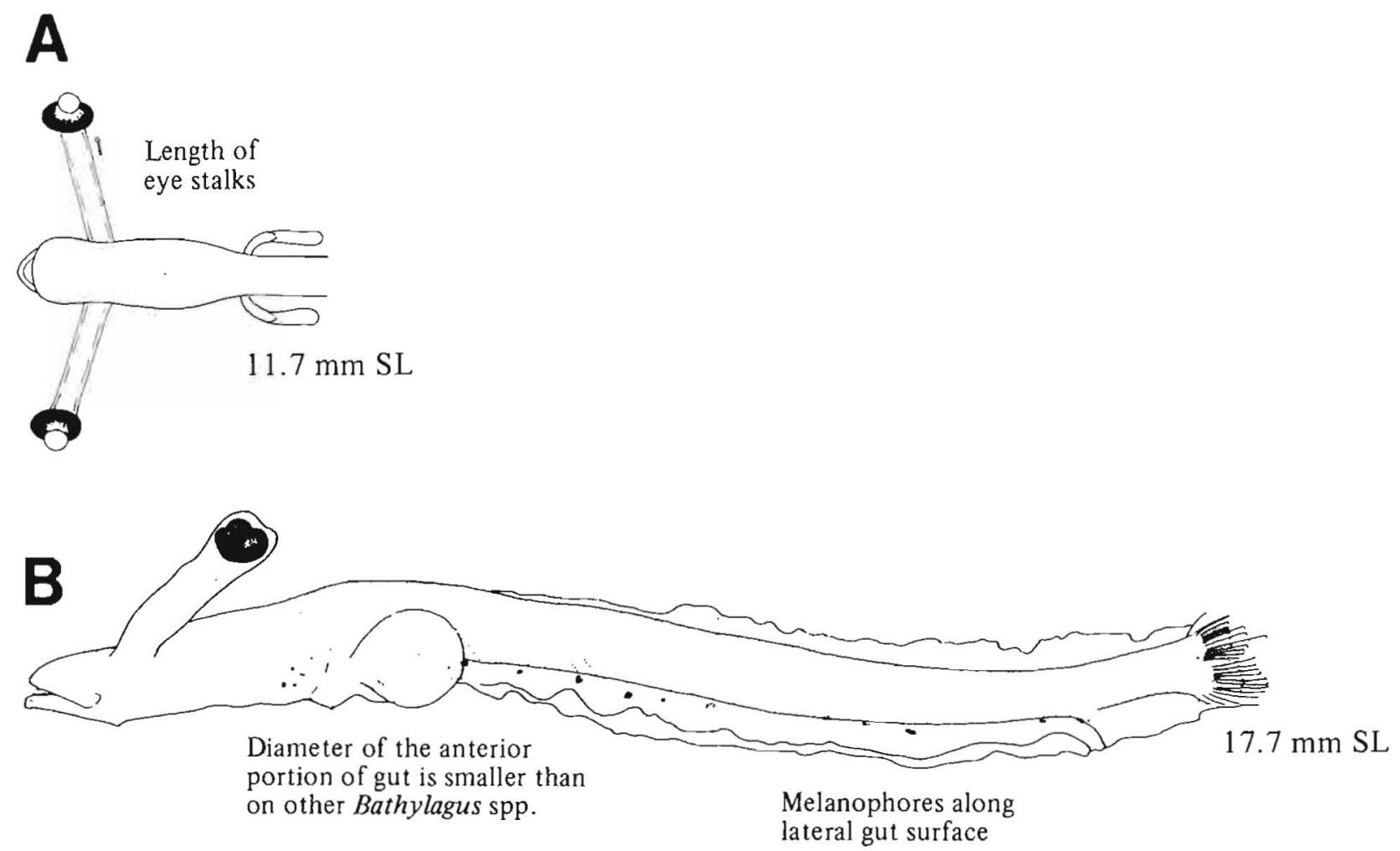

Figures A-B, Ahlstrom et al. 1984b (North Atlantic specimen). 


\section{MERISTICS}

\begin{tabular}{ll} 
Vertebrae & Total: $50-\mathrm{X}-55$ \\
& Precaudal: $18-\mathrm{X}-21$ \\
& Caudal: $30-\mathrm{X}-34$ \\
Branchiostegal rays & $2-2-2$ \\
Caudal fin & $16-18,10+9,15-17$ \\
Pelvic fin & Abdominal \\
& R: $6-\mathrm{X}-8$ \\
Dorsal fin & R: $6-\mathrm{X}-9$ \\
Pectoral fin & R: $11-\mathrm{X}-16$ \\
Anal fin & R: $20-\mathrm{X}-28$ \\
Gill rakers & U: $X-\mathrm{X}-\mathrm{X} \quad$ L: $25-\mathrm{X}-27^{\mathrm{a}}$ \\
& \\
& \\
LIFE HISTORY & \\
\hline
\end{tabular}

$\begin{array}{ll}\text { Range } & \begin{array}{c}\text { S. California, } 32-34^{\circ} \mathrm{N}, \text { to } \\ \text { Bering Sea, } 54-66^{\circ} \mathrm{N}\end{array} \\ \text { Ecology } & \text { Epi- and mesopelagic, } 60-1000 \mathrm{~m} \\ \text { ELH pattern } & \text { Oviparous, pelagic eggs, pelagic } \\ & \text { larvae } \\ \text { Spawning } & \text { Season: } \\ & \text { Area: } \\ & \text { Mode: } \\ & \text { Migration: } \\ \text { Fecundity } & \text { Range/function: } \\ \text { Age at first maturity } & \\ \text { Longevity } & \end{array}$

EARLY LIFE HISTORY DESCRIPTION

\section{EGGS}

Diameter

No. of oil globules

Oil globule diameter

$\begin{array}{ll}\text { Yolk } & \text { Segmented } \\ \text { Envelope } & \text { Pustules on inner surface }\end{array}$

Hatch size

Incubation time/temp.

Pigment

Diagnostic characters

\section{LARVAE}

Preanal Iength

Length at flexion

Length at transformation

$59-61 \% \mathrm{SL}$
Sequence of fin Caudal, dorsal, anal, development pectorals, pelvics

Pigment - Large isolated melanophores

- Preflexion larvae: Pigment on lower jaw, midgut, and tail

- Flexion larvae -Opposing dorsal and ventral midline melanophores - Large melanophores on head and pectoral fin base - Large lateral blotch at base of caudal fin

Diagnostic characters

- Gut shorter (usually only 50\% SL) than in other species

- Eye rounder and larger than in other bathylagids (not stalked)

- Fin rays develop in finfold, away from body margin

Distinguished from other bathylagids without eyestalks by

- Large isolated spots

- $B$. wesethi has a series of melanophores along hypaxial region, large eye, and spots posteriorly along dorsal midline

- Leuroglossus spp. larvae have smaller eyes and no dorsal spots

\footnotetext{
${ }^{a}$ E.H. Ahlstrom notes
}

Ref: Ahlstrom et al. 1984b. 


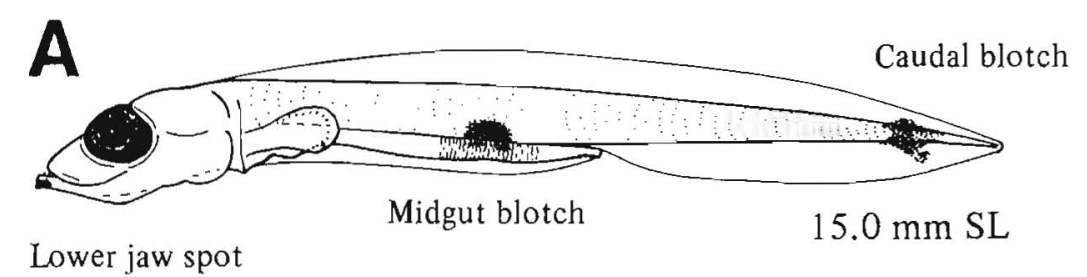

Lower jaw spot

B No eye stalks

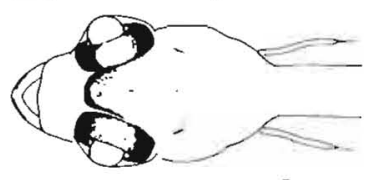

$9.5 \mathrm{~mm} \mathrm{SL}$

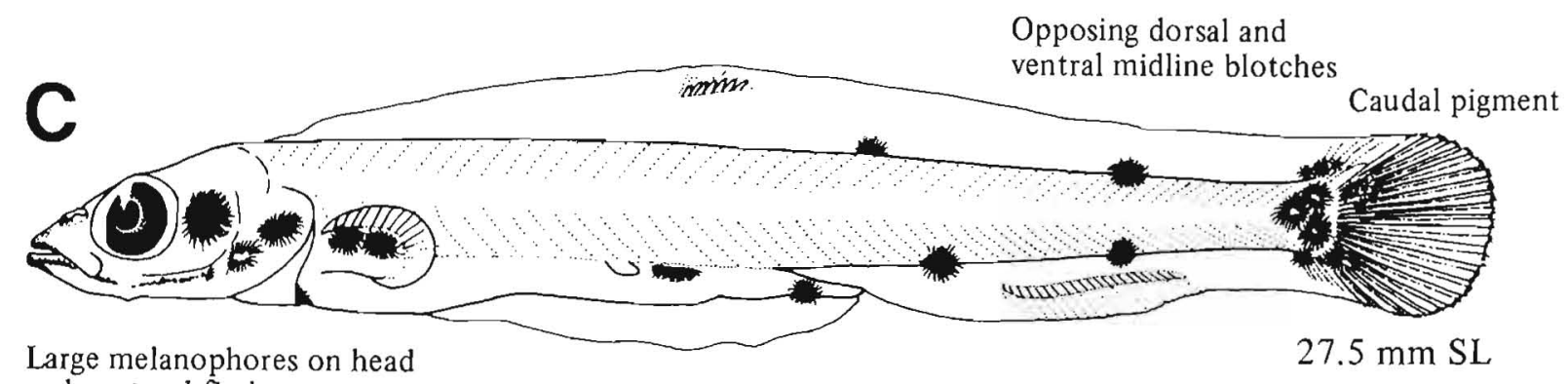

and pectoral fin base 


\section{MERISTICS}

$\begin{array}{ll}\text { Vertebrae } & \text { Total: } 47-\mathrm{X}-49 \\ & \text { Precaudal: } 25-26-27 \\ & \text { Caudal: } 21-22-23 \\ \text { Branchiostegal rays } & 2-2-2 \\ \text { Caudal fin } & 13-14,10+9,15-16 \\ \text { Pelvic fin } & \text { Abdominal } \\ & \text { R: } 9-10-10 \\ \text { Dorsal fin } & \text { R: } 9-11-12 \\ \text { Pectoral fin } & \text { R: } 9-10-11 \\ \text { Anal fin } & \text { R: } 12-X-15 \\ \text { Gill rakers } & \text { U: X-X-X } \quad \text { L: } 28-28-28^{a} \\ & \\ \text { LIFE HISTORY } & \end{array}$

$\begin{array}{ll}\text { Range } & \begin{array}{c}\text { South of southern California to } \\ \text { Bering Sea, } 54-66^{\circ} \mathrm{N}\end{array} \\ \begin{array}{l}\text { Ecology } \\ \text { ELH pattern }\end{array} & \begin{array}{l}\text { Oviparous, pelagic eggs, pelagic } \\ \text { larvae }\end{array} \\ \text { Spawning } & \text { Season: Jan-Feb } \\ & \text { Area: Off continental slope } \\ & \text { Mode: } \\ & \text { Migration: } \\ \text { Fecundity } & \text { Range/function: } \\ \text { Age at first maturity } & \\ \text { Longevity } & \end{array}$

E.H. Ahlstrom notes

${ }^{b}$ Wang 1981

${ }^{c}$ Ahlstrom 1965

${ }^{d}$ Numerous globules at vegetal pole which coalesce to one clump at each equatorial

pole.

Ref: Ahlstrom et al. 1984b.

\section{EARLY LIFE HISTORY DESCRIPTION}

\section{EGGS}

Diameter

$0.92-1.1 \mathrm{~mm}$

No. of oil globules

Oil globule diameter

Yolk

Envelope

$>10$, usually coalesce to 1 in late stage ${ }^{d}$

Hatch size

Incubation time/temp.

Pigment

- Unpigmented

Diagnostic characters

- Lack of pigmentation in late-stage eggs

\section{LARVAE}

Preanal length

$81-90 \%$ SL

Length at flexion

Length at transformation

Sequence of fin development
Segmented

Pustules on inner surface
- Series of melanophores develops on hypaxial myomeres

- Epaxial melanophores limited to posterior body

- Series along posterior gut

- May occur on urostyle at sizes $<7.9 \mathrm{~mm} \mathrm{SL}$

Diagnostic characters

- Eye stalks

Distinguished from other bathylagids with linear series of smaller melanophores by

- Posterior gut melanophores larger and fewer

- Anterior region of gut lacks pigment

- Epaxial myomere series limited to posterior region

- Fin rays form in finfold, away from body margin

Distinguished from $B$. bericoides by

- Small $B$. bericoides larvae are unavailable but presumably eye stalks are longer at comparable stages

Distinguished from $B$. pacificus by

- Series of melanophores as opposed to isolated spots 

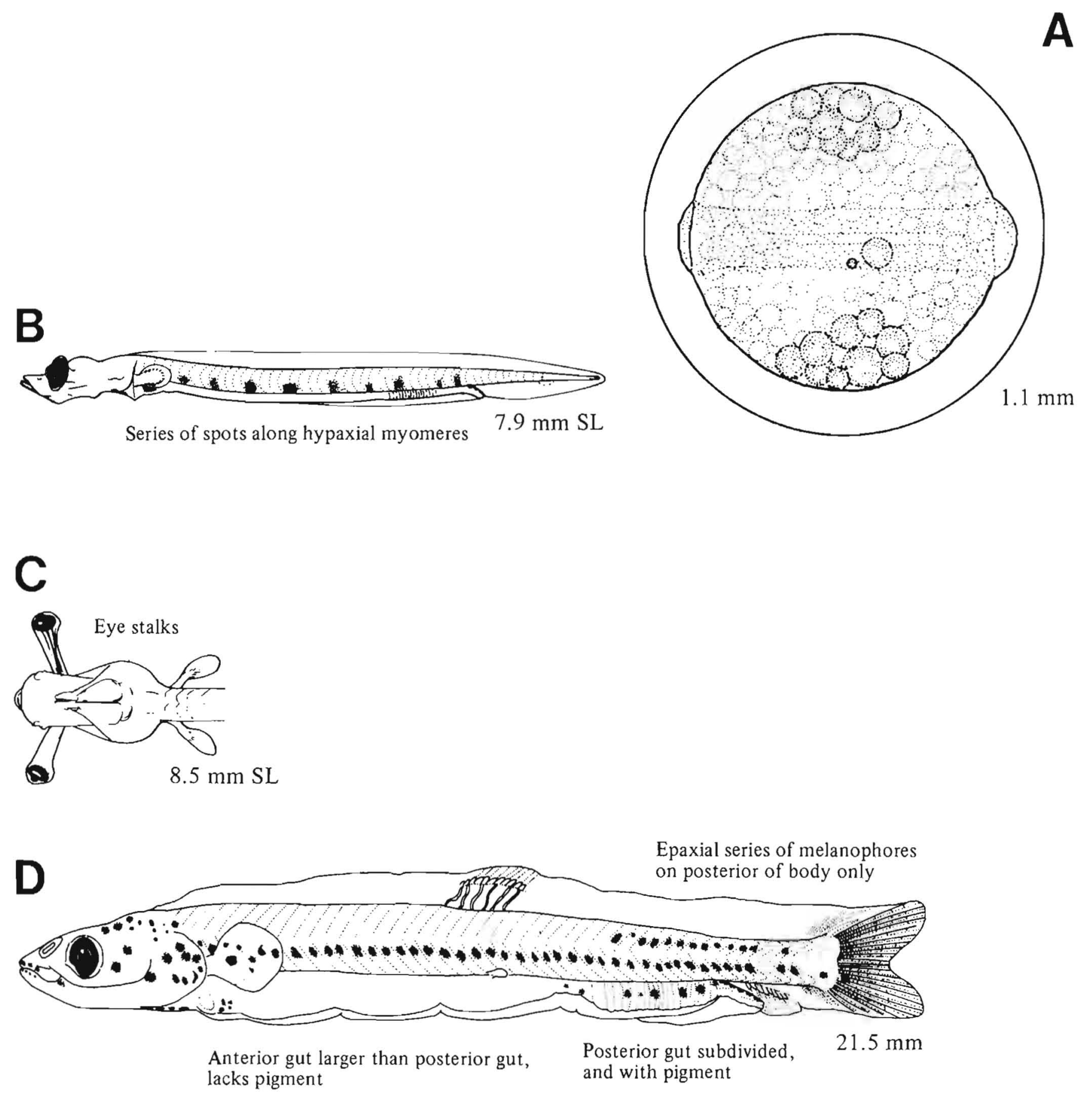

Figures A, C-D, Ahlstrom et al. 1984b; B, NWAFC original (B. Vinter). 
MERISTICS

\begin{tabular}{|c|c|}
\hline \multirow[t]{3}{*}{ Vertebrae } & Total: $44-X-49$ \\
\hline & Precaudal: $18-X-25$ \\
\hline & Caudal: $21-\mathrm{X}-28$ \\
\hline Branchiostegal rays & $2-2-2$ \\
\hline Caudal fin & $13,10+9,13-14$ \\
\hline Pelvic fin & Abdominal \\
\hline & $\mathrm{R}: 7-\mathrm{X}-10$ \\
\hline Dorsal fin & $\mathrm{R}: 8-\mathrm{X}-9$ \\
\hline Pectoral fin & $\mathrm{R}: 7-\mathrm{X}-11$ \\
\hline Anal fin & $\mathrm{R}: 15-\mathrm{X}-22$ \\
\hline Gill rakers & $\mathrm{L}: 28-\mathrm{X}-32^{\mathrm{a}}$ \\
\hline \multicolumn{2}{|l|}{ LIFE HISTORY } \\
\hline Range & $\begin{array}{l}\text { S. California, } 32-34^{\circ} \mathrm{N} \text {, to } \\
\text { Bering Sea, } 54-66^{\circ} \mathrm{N}\end{array}$ \\
\hline Ecology & \\
\hline ELH pattern & $\begin{array}{l}\text { Oviparous, pelagic eggs, pelagic } \\
\text { larvae }\end{array}$ \\
\hline Spawning & Season: Feb-Mar; ${ }^{b}$ spring $^{c}$ \\
\hline & Area: \\
\hline & Mode: \\
\hline & Migration: \\
\hline Fecundity & \multirow[t]{2}{*}{ Range/function: } \\
\hline $\begin{array}{l}\text { Age at first maturity } \\
\text { Longevity }\end{array}$ & \\
\hline
\end{tabular}

\section{EARLY LIFE HISTORY DESCRIPTION}

\section{EGGS}

Diameter

No. of oil globules

Oil globule diameter

Yolk

Envelope

Segmented

Hatch size

Incubation time/temp.

Pigment

Diagnostic characters

\section{LARVAE}

$\begin{array}{ll}\text { Preanal length } & 76-85 \% \text { SL } \\ \begin{array}{l}\text { Length at flexion } \\ \text { Length at transformation }\end{array} & \\ \begin{array}{l}\text { Sequence of fin } \\ \text { development }\end{array} & \begin{array}{c}\text { Caudal, dorsal, anal, } \\ \text { pectorals, pelvics }\end{array}\end{array}$

Pigment - Large isolated melanophores

- Early larvae have large lateral blotch at midbody and another posteriad, becoming located on trunk with development

- Third blotch forms midway between and below other two blotches

- Fourth lateral trunk blotch forms in some late larval specimens between pectoral fin and large midbody blotch

- Other melanophores form lateral to liver and terminal section of gut

- Also isthmus/thoracic, dorsal gut, caudal, and two bands on body (early)

Diagnostic characters

- Stalked eyes

- Fin rays form in finfold, away from body margin

- Only bathylagid with stalked eyes and pigment consisting of large isolated spots
${ }^{a}$ E.H. Ahlstrom notes

${ }^{\mathrm{b}}$ Wang 1981

${ }^{C}$ Fitch and Lavenberg 1968

Ref: Ahlstrom et al. 1984b. 

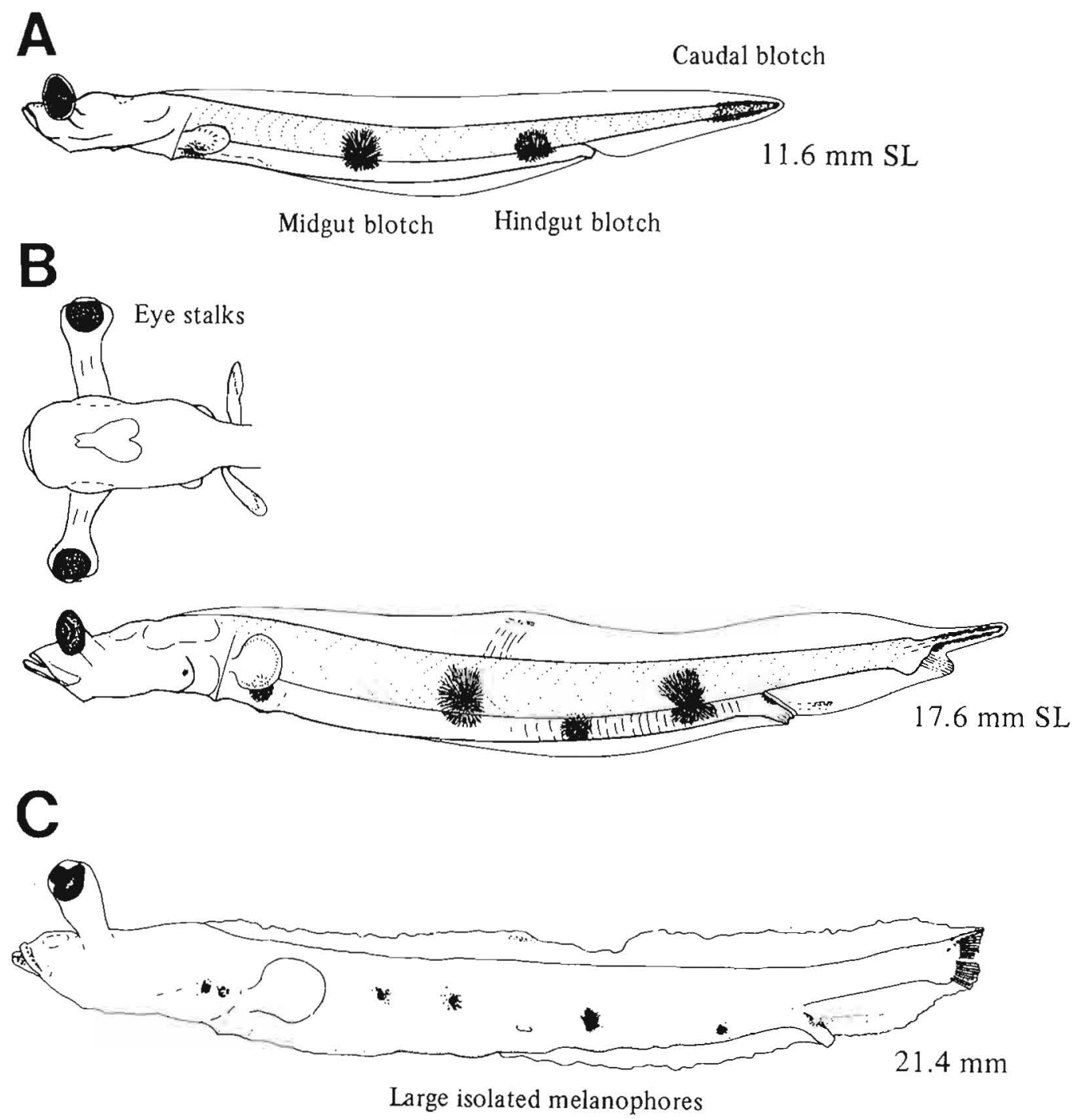


\section{MERISTICS}

$\begin{array}{ll}\text { Vertebrae } & \text { Total: } 43-\mathrm{X}-46 \\ & \begin{array}{l}\text { Precaudal: } 23-23-23 \\ \text { Caudal: } 22-22-22\end{array} \\ \text { Branchiostegal rays } & 2-2-2 \\ \text { Caudal fin } & 14-15,10+9,14-15 \\ \text { Pelvic fin } & \text { Abdominal } \\ & \text { R: } 9-\mathrm{X}-11 \\ \text { Dorsal fin } & \text { R: } 11-12-13 \\ \text { Pectoral fin } & \text { R: } 9-10-11 \\ \text { Anal fin } & \text { R: } 14-\mathrm{X}-16 \quad \\ \text { Gill rakers } & \text { U: } 8-8-8 \quad \text { L: } 16-\mathrm{X}-17\end{array}$

\section{LIFE HISTORY}

$\begin{array}{ll}\text { Range } & \begin{array}{c}\text { South of southern California to } \\ \text { Oregon, 42-46 }\end{array} \\ \text { Epology } & \begin{array}{l}\text { Epi- and mesopelagic, 40-1000 m } \\ \text { Oviparous, pelagic eggs, pelagic } \\ \text { larvae }\end{array} \\ \text { ELH pattern } & \text { Season: } \\ \text { Spawning } & \text { Area: } \\ & \text { Mode: } \\ & \text { Migration: } \\ \text { Fecundity } & \text { Range/function: } \\ \text { Age at first maturity } & \\ \text { Longevity } & \end{array}$

${ }^{2}$ Oil globules may only partially coalesce.

${ }^{b}$ Data on preflexion and postflexion larvae are from E.H. Ahlstrom notes. Hlustrations are unavailable.

Ref: Ahlstrom 1965, 1969; Ahistrom et al. 1984b

\section{EARLY LIFE HISTORY DESCRIPTION}

\section{EGGS}

Diameter

No. of oil globules

$0.90-1.10 \mathrm{~mm}$

Oil globule diameter

$12-20$, subequal $^{\mathrm{a}}$

Yolk

Envelope

Hatch size

Incubation time/temp.

Pigment

- No pigment over oil globules

Diagnostic characters

\section{LARVAE}

Preanal length

$79-94 \%$ SL

Length at flexion $>6 \mathrm{~mm}$, by $11 \mathrm{~mm} \mathrm{SL}$

Length at transformation $\sim 25 \mathrm{~mm} \mathrm{SL}$

Sequence of fin Caudal, dorsal, anal, development pectorals, pelvics

Pigment - Linear series of small melanophores

- Initially a series of paired spots dorsolaterally to gut extending from pectoral fin base to terminal section, becoming embedded with development (6-8 pairs developing to 7-8 pairs)

- Notochord tip (dorsal and ventral)

At flexion

- Series of melanophores develops along hypaxial region, and soon after a series develops along epaxial

- More lateral spots are added

- Median finfold

- Increased head pigment

Diagnostic characters

- Eyes not stalked

- Pigment pattern unique among Bathylagus spp. (including Leuroglossus spp.)

- Fin rays form in finfold, away from body margin

- Only bathylagid without stalked eyes and pigment consisting of a linear series of small melanophores 


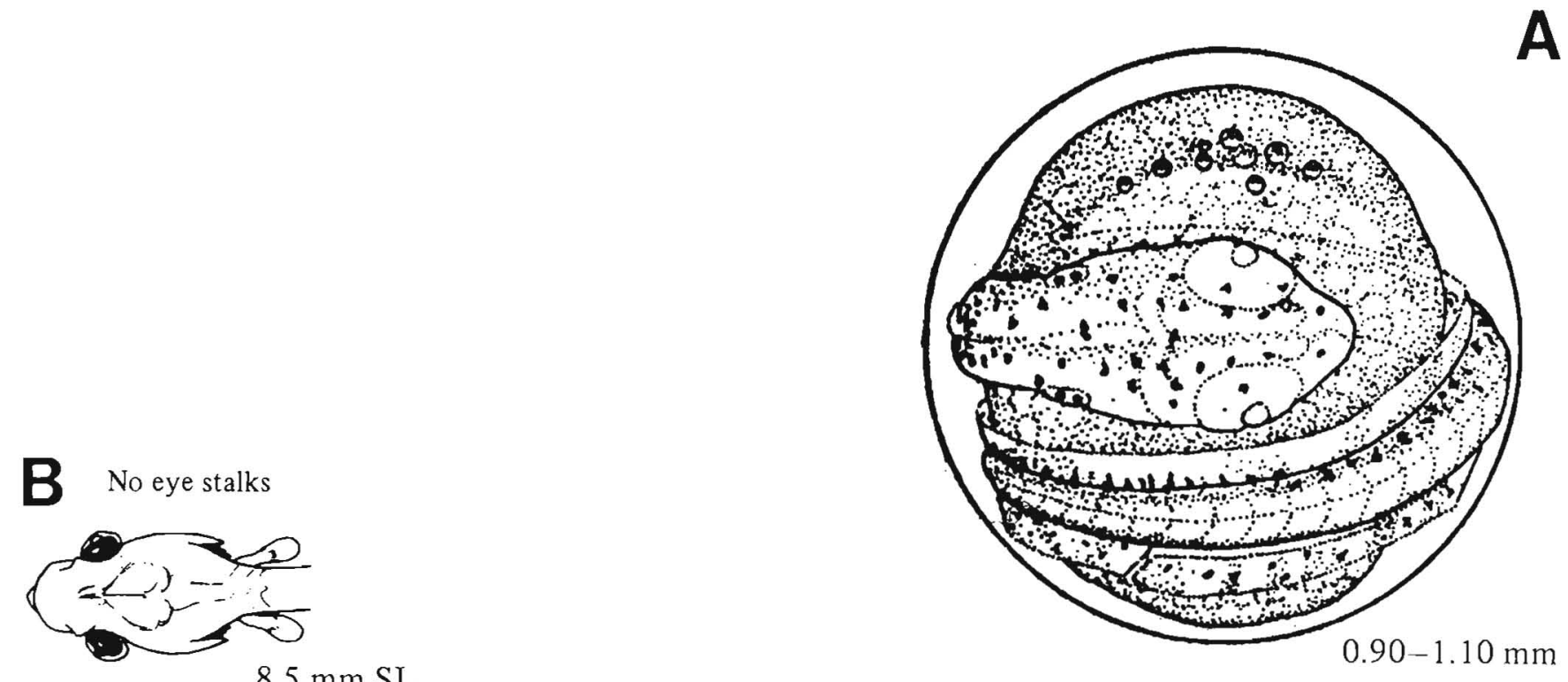

C

Median finfold pigment
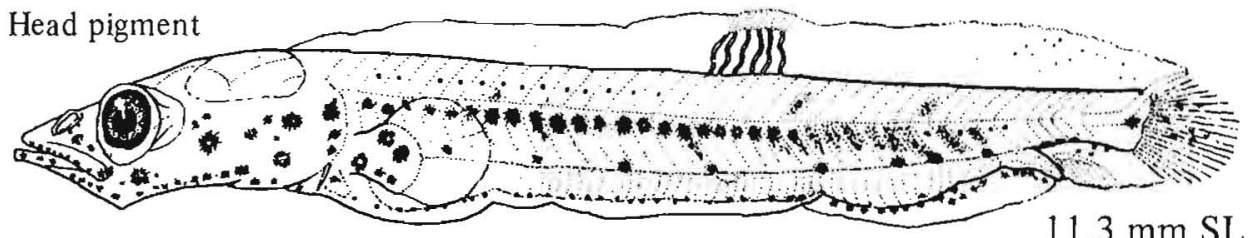

7-8 pairs dorsolateral

Transverse rugae gut melanophores along entire gut 


\section{MERISTICS}

\begin{tabular}{ll} 
Vertebrae & Total: $47-X-52$ \\
& Precaudal: $26-X-29$ \\
& Caudal: X-X-X \\
Branchiostegal rays & $2-2-2$ \\
Caudal fin & $15-17,10+9,15^{\text {b }}$ \\
Pelvic fin & Abdominal \\
& R: $8-X-9$ \\
Dorsal fin & R: $10-X-11$ \\
Pectoral fin & R: $8-X-9$ \\
Anal fin & R: $11-X-14$ \\
Gill rakers & U: $8-X-9$ \\
& \\
& \\
LIFE HISTORY $17-X-19$ \\
\hline
\end{tabular}

$\begin{array}{ll}\text { Range } & \text { Brit. Col., } 48^{\circ} 30^{\prime}-55^{\circ} \mathrm{N} \text {, to } \\ & \text { Bering Sea, } 54-66^{\circ} \mathrm{N} \\ \text { Ecology } & \text { Epi-, meso-, and bathypelagic, } \\ & 0-1800 \mathrm{~m}^{\mathrm{c}} \\ \text { Oviparous, pelagic eggs, pelagic } & \text { larvae } \\ \text { ELH pattern } & \text { Season: Fall-winter; }{ }^{\mathrm{b}} \text { summer }^{\mathrm{d}} \\ \text { Spawning } & \text { Area: Off continental slope } \\ & \text { Mode: } \\ & \text { Migration: } \\ \text { Fecundity } & \text { Range/function: } \\ \text { Age at first maturity } & \\ \text { Longevity } & \end{array}$

\section{EARLY LIFE HISTORY DESCRIPTION}

\section{EGGS}

Diameter

No. of oil globules

Oil globule diameter

Yolk

Envelope

Hatch size

Incubation time/temp.

Pigment

- Tip of notochord

\section{Diagnostic characters}

- Egg diameter

\section{LARVAE}

Preanal length

Length at flexion

Length at transformation

Sequence of fin development
$1.65-1.90 \mathrm{~mm}$

$<5$ up to 9 , usually coalescing to $1^{\mathrm{e}}$

$0.35-0.40 \mathrm{~mm}, 0.47$ after fusion ${ }^{\mathrm{e}}$

Segmented

Pustules
$72-78 \%$ SL

$13-18 \mathrm{~mm} \mathrm{SL}$

31-35 mm SL

Caudal, dorsal and anal, pectorals, pelvics gment - Large isolated melanophores

- Series of 5-6 spots on posterior gut

- Midtrunk patch

- Lower trunk blotches

- 1-2 postanal lateral blotches in larger larvae

Diagnostic characters

- Eye stalks short and only in early larvae

- Fin rays form in finfold, away from body margin

- More pigmented than L. stilbius

Distinguished from Bathylagus milleri by

- Eye stalks short

- Lack of dorsal spots

\footnotetext{
a Placed in Bathylagus by Ahistrom et al. 1984b.

${ }^{b}$ Dunn 1983

${ }^{\mathrm{c}}$ Fedorov 1973

dAhlstrom 1969

'Similar migrations, see L. stilbius.
}

Ref: Ahlstrom 1969, Ahlstrom et al. 1984b, Dunn 1983. 
B

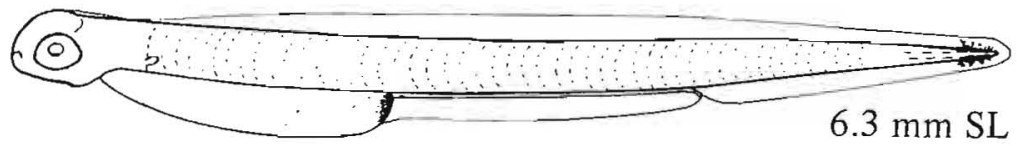

C
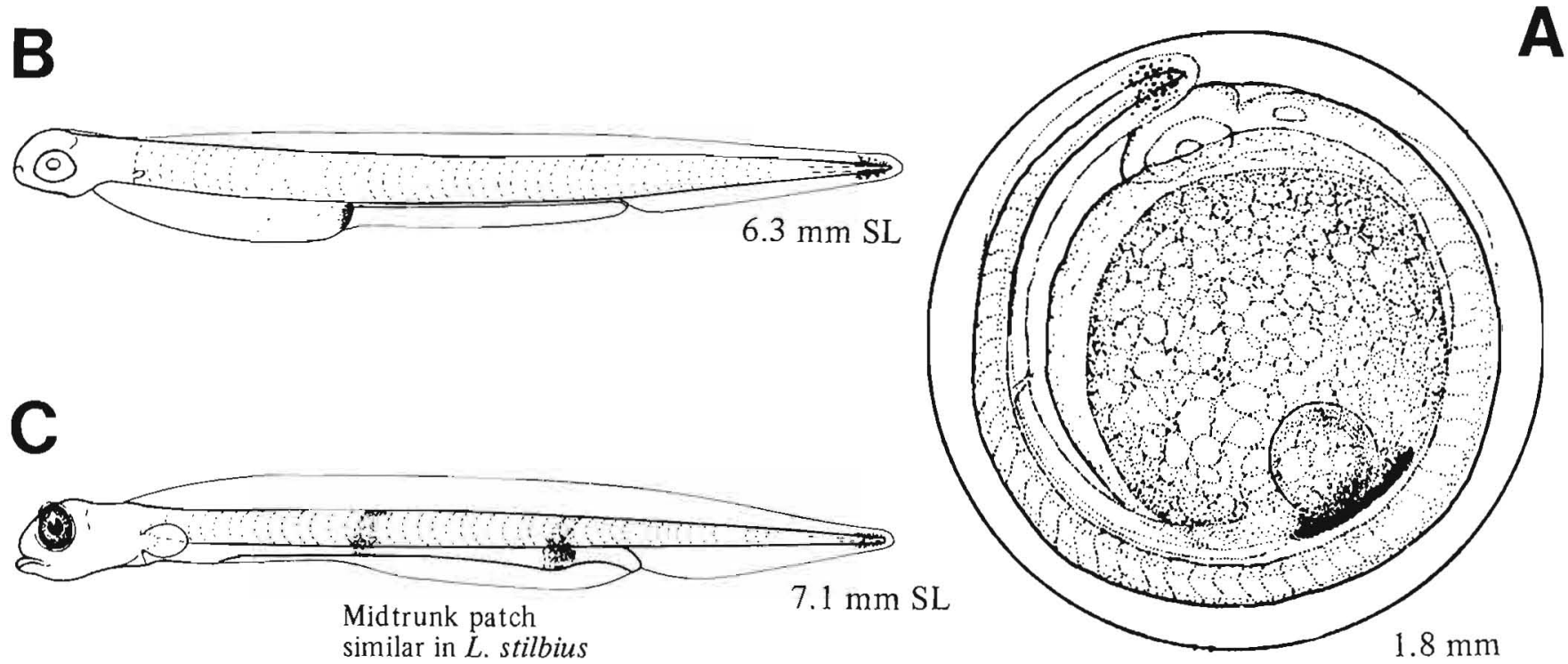

D

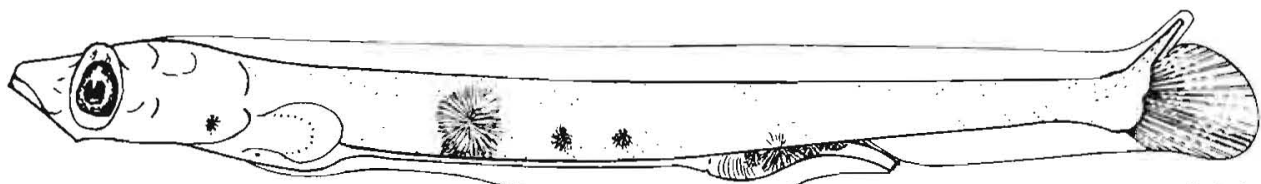

$16.1 \mathrm{~mm} \mathrm{SL}$
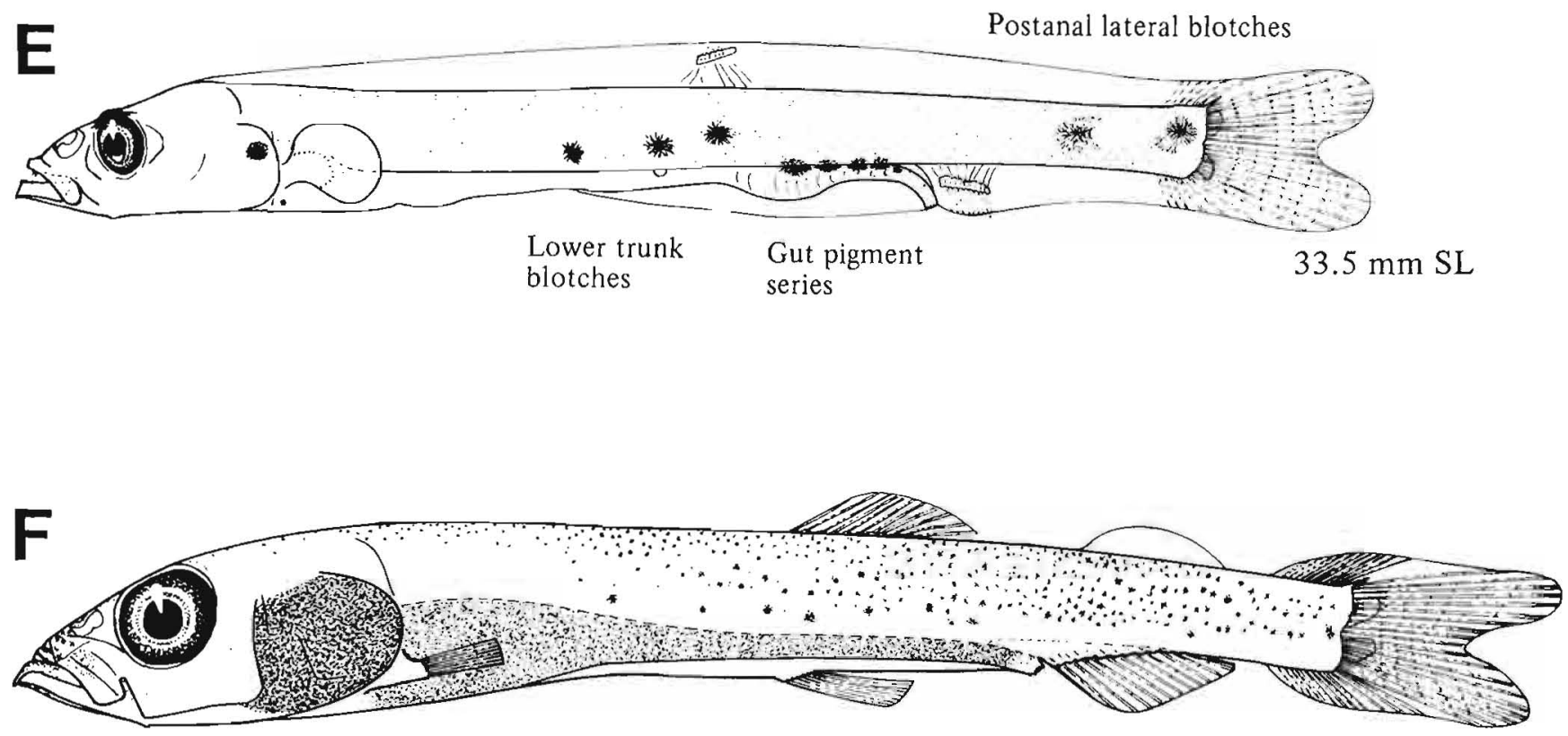

$33.1 \mathrm{~mm} \mathrm{SL}$

Figure A, Ahlstrom 1969; B-F, Dunn 1983. 


\section{MERISTICS}

\begin{tabular}{ll}
\hline Vertebrae & Total: $38-\mathrm{X}-42$ \\
& Precaudal: $20-21-22$ \\
& Caudal: $19-20-21$ \\
Branchiostegal rays & $2-2-2$ \\
Caudal fin & $12-16,10+9,13-15$ \\
Pelvic fin & Abdominal \\
& R: $8-X-10^{\text {b }}$ \\
Dorsal fin & R: $9-\mathrm{X}-11$ \\
Pectoral fin & R: $8-X-11$ \\
Anal fin & R: $11-X-14$ \\
Gill rakers & U: $7-X-9$ \\
& \\
& \\
LIFE HISTORY & \\
\hline
\end{tabular}

$\begin{array}{ll}\text { Range } & \begin{array}{c}\text { South of southern California to } \\ \text { Oregon, } 42-46^{\circ} \mathrm{N}\end{array} \\ \text { Ecology } & \begin{array}{l}\text { Evi- and mesopelagic, } 0-690 \mathrm{~m} \\ \text { Oviparous, pelagic eggs, pelagic } \\ \text { larvae } \\ \text { ELH pattern }\end{array} \\ \text { Spawning } & \begin{array}{l}\text { Season: Winter-spring } \\ \text { Area: } \\ \text { Mode: }\end{array} \\ & \text { Migration: } \\ \text { Fecundity } & \text { Range/function: } \\ \text { Age at first maturity } & \\ \text { Longevity } & \end{array}$

a Placed in Bathylagus by Ahlstrom et al. 1984b.

${ }^{b}$ Ahistrom et al. $1984 b$

'Ahistrom 1965

${ }^{d}$ After fertilization, 15-25 oil globules at vegetal pole; 2-5 oil globules during early stage which coalesce to 2 (sometimes 1) of equal size at opposite poles. These oil globules migrate toward and coalesce under the embryo prior to hatching.

${ }^{e}$ Data on preflexion and postflexion larvae are from E.H. Ahlstrom notes. Llustrations are unavailable.

Ref: Ahlstrom 1965, 1969; Ahlstrom et al. $1984 \mathrm{~b}$.

\section{EARLY LIFE HISTORY DESCRIPTION}

\section{EGGS}

Diameter

No. of oil globules ${ }^{d}$

Oil globule diameter

Yolk

Envelope

$1.10-1.21 \mathrm{~mm}$

Hatch size

Finely segmented

Pustules

Incubation time/temp.

$3 \mathrm{~mm} \mathrm{SL}$

Pigment

- Yolk membrane above oil globule

- Notochord tip

Diagnostic characters

- Egg diameter: Smaller than in L. schmidti

\section{LARVAE $^{\text {e }}$}

Preanal length

Length at flexion

Length at transformation $25-29 \mathrm{~mm} \mathrm{SL}$

Sequence of fin development

Caudal, dorsal and anal, pectorals, pelvics

Pigment - Large isolated melanophores

- Midtrunk patch between pectoral fin base and anus in smaller larvae is similar to $L$. schmidti

- Series of 5-6 melanophores on posterior gut

Diagnostic characters

- Eye stalks short and only in early larvae

- Fin rays form in finfold, away from body margin

Distinguished from $L$. schmidti by

- Lack of lower trunk and postanal blotches 


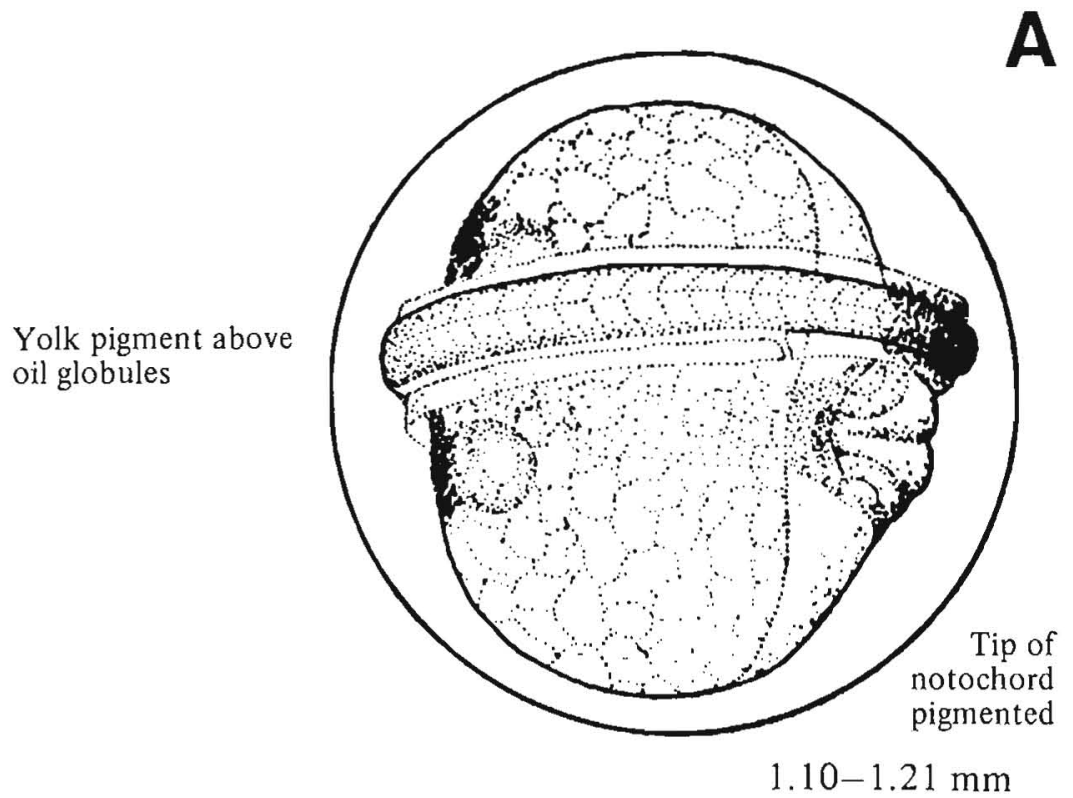

B Eye stalks short

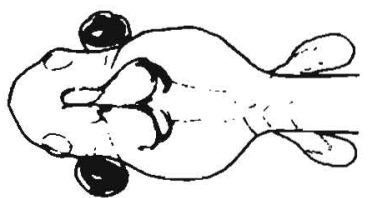

$8.5 \mathrm{~mm} \mathrm{SL}$

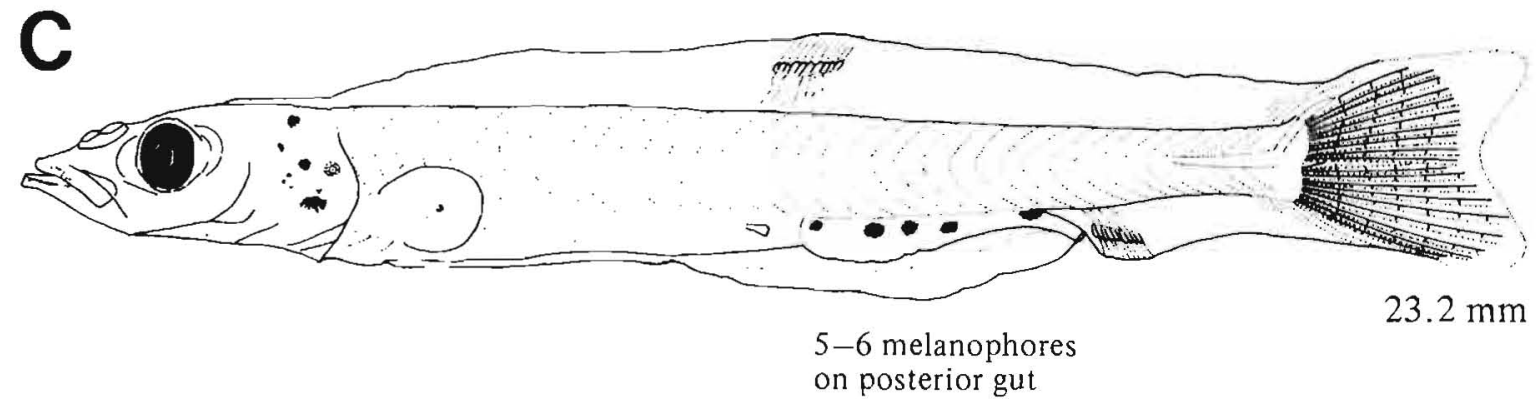

Figure A, Ahlstrom 1969; B-C, Ahlstrom et al. $1984 \mathrm{~b}$. 


\section{MERISTICS}

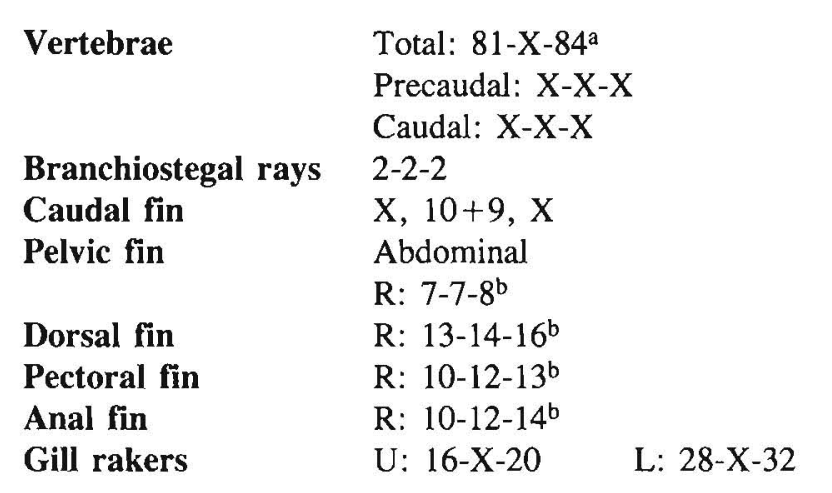

LIFE HISTORY

$\begin{array}{ll}\text { Range } & \text { N. California, } 38-42^{\circ} \mathrm{N} \text {, to } \\ & \text { Brit. Col., } 48^{\circ} 30^{\prime}-55^{\circ} \mathrm{N} \\ \text { Ecology } & \text { Epi- and mesopelagic } \\ \text { ELH pattern } & \begin{array}{c}\text { Oviparous, eggs probably pelagic, } \\ \text { pelagic larvae }\end{array} \\ \text { Spawning } & \begin{array}{l}\text { Season: } \\ \text { Area: }\end{array} \\ & \text { Mode: } \\ & \text { Migration: } \\ \text { Fecundity } & \text { Range/function: } \\ \text { Age at first maturity } & \begin{array}{l}\text { (Both sexes appear to mature at } \\ 400 \text { mm SL) }\end{array} \\ \text { Longevity } & 5 \mathrm{yr}^{\mathrm{c}}\end{array}$

${ }^{3}$ Ahlstrom et al. $1984 \mathrm{~b}$

biein and Bond 1985

${ }^{\mathrm{c}}$ Fitch and Lavenberg 1968

${ }^{d}$ Transformation (family): Marked by deepening of body and attainment of melanistic integument and large scales.

Ref: Ahjstrom et al. 1984b, Stein and Bond 1985.

\section{EARLY LIFE HISTORY DESCRIPTION}

\section{EGGS}

Diameter

No. of oil globules

2.2-2.6 (ripe ovarian eggs)

Oil globule diameter

Yolk

Envelope

Hatch size

Incubation time/temp.

Pigment

Diagnostic characters

\section{LARVAE}

Preanal length $\quad 80-82 \%$ SL

Length at flexion

Length at transformation $\mathrm{Up}$ to $124 \mathrm{~mm} \mathrm{SL}^{\mathrm{d}}$

Sequence of fin Caudal, pectorals and pelvics, development dorsal and anal

\section{Pigment}

- Dorsal blotches (six pairs), which extend into finfold, and ventral (eight pairs) lateral blotches; blotches alternate except for postanal ones which form a band

- Large caudal blotch

- Head heavily pigmented

- Lower gill arches heavily pigmented

- Pectoral and pelvic fin bases

\section{Diagnostic characters}

- Gut elongate

- Sac-like stomach (elongate and pointed at tip)

Distinguished from other opisthoproctids by

- Elongate snout with unique triangular flap at tip

- Round eyes (anterodorsal)

- Distinctive heavy pigment pattern

- Number of myomeres (81-84) 

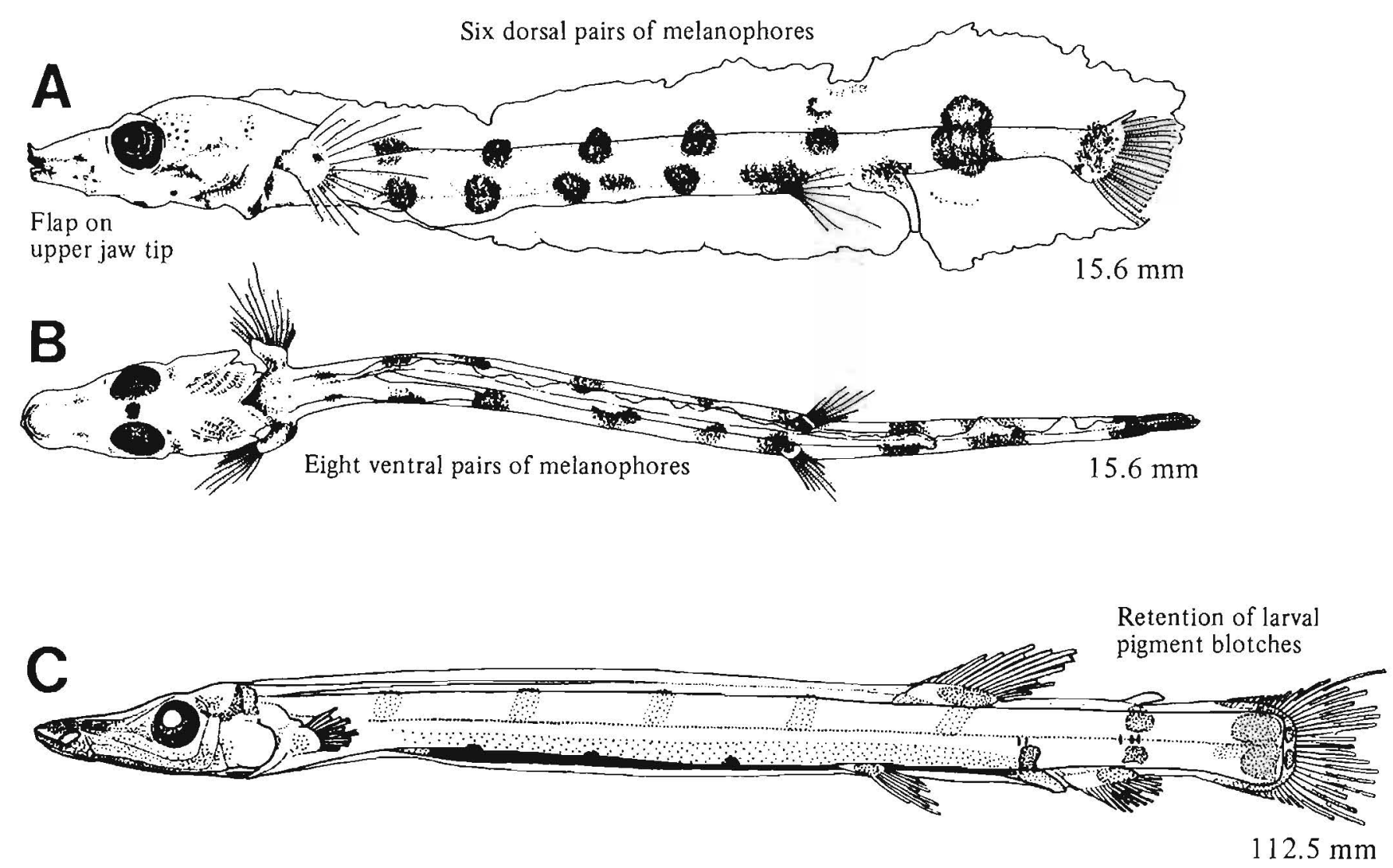

Figure A-B (B, ventral view), Ahlstrom et al. 1984b; C, Cohen 1960. 


\section{MERISTICS}

\begin{tabular}{ll}
\hline Vertebrae & Total: $40-X-44$ \\
& Precaudal: $X-X-X$ \\
& Caudal: X-X-X \\
Branchiostegal rays & $2-2-2$ \\
Caudal fin & X, $10+9, X$ \\
Pelvic fin & Abdominal \\
& R: $8-X-9$ \\
Dorsal fin & R: $10-X-11$ \\
Pectoral fin & R: $13-13-13$ \\
Anal fin & R: $8-X-9$ \\
Gill rakers & U: $X-X-X \quad$ L: $X-X-X$
\end{tabular}

\section{LIFE HISTORY}

\begin{tabular}{|c|c|}
\hline Range & $\begin{array}{l}\text { South of southern California to } \\
\text { Oregon, } 42-46^{\circ} \mathrm{N}\end{array}$ \\
\hline Ecology & Epi- and mesopelagic, $152-457 \mathrm{~m}$ \\
\hline ELH pattern & $\begin{array}{l}\text { Oviparous, eggs probably pelagic, } \\
\text { pelagic larvae }\end{array}$ \\
\hline Spawning & $\begin{array}{l}\text { Season: } \\
\text { Area: } \\
\text { Mode: } \\
\text { Migration: }\end{array}$ \\
\hline $\begin{array}{l}\text { Fecundity } \\
\text { Age at first maturity }\end{array}$ & Range/function: \\
\hline Longevity & $5 \mathrm{yr}^{\mathrm{b}}$ \\
\hline
\end{tabular}

According to A.E. Peden (Brit. Col. Prov. Mus., Victoria, B.C., Canada V8V 1 X4, pers. commun., 22 Jan. 1987), several species may be in the area with $D$. Longipes having the more southerly distribution. Other species may occur north of Oregon and at least one form occurs off British Columbia.

${ }^{\mathrm{b}}$ Fitch and Lavenberg 1968

Ref: Ahlstrom et al. 1984b.

\section{EARLY LIFE HISTORY DESCRIPTION}

\section{EGGS}

Diameter

No. of oil globules

Oil globule diameter

Yolk

Envelope

Hatch size

Incubation time/temp.

Pigment

Diagnostic characters

\section{LARVAE}

Preanal length

$74-75 \%$ SL

Length at flexion

Length at transformation See Bathylychnops exilis

Sequence of fin development

Pectorals and pelvics probably right after caudal, before dorsal and anal

Pigment - Genus

- Lateral series of melanophores above gut; some species develop serial melanophores on hypaxial myomeres

- Head pigment: Jaws, internal snout, gill arches

\section{Diagnostic characters}

- Gut elongate (sac-like stomach, elongate and pointed at tip)

- Tubular eyes

- Elongate pectoral and pelvic fin rays

- Number of myomeres (40-44), less than B. exilis (81-84) and more than Macropinna microstoma $(34-37)$ 


\section{A Dolichopteryx binocularis}

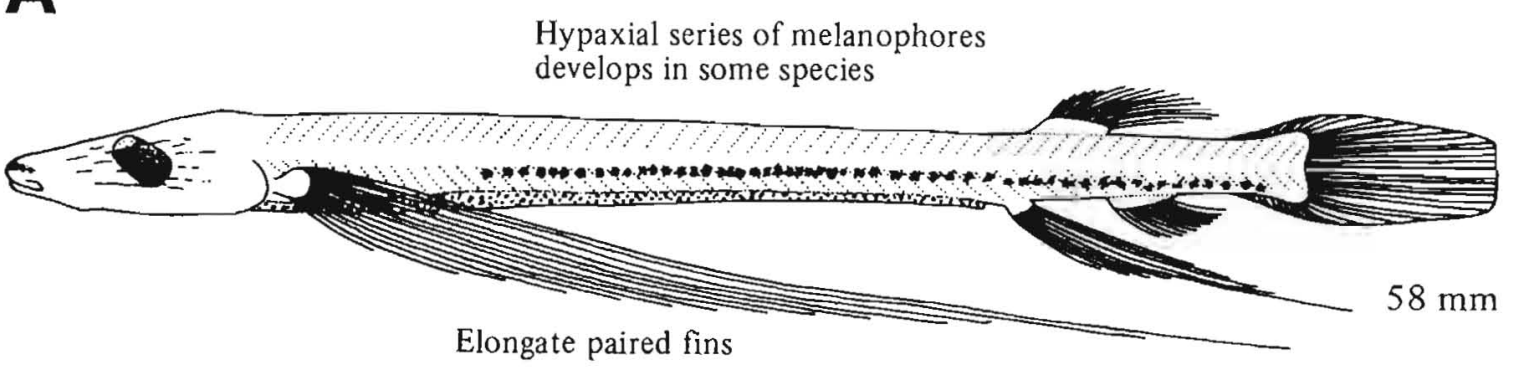

Figure A, Ahlstrom et al. 1984b (after Roule and Angel 1930, Mediterranean specimen). 


\section{MERISTICS}

\begin{tabular}{ll} 
Vertebrae & Total: $34-\mathrm{X}-37$ \\
& Precaudal: X-X-X \\
& Caudal: X-X-X \\
Branchiostegal rays & $3-3-3$ \\
Caudal fin & X, $10+9, \mathrm{X}$ \\
Pelvic fin & Abdominal \\
& R: $9-\mathrm{X}-10$ \\
Dorsal fin & R: $11-\mathrm{X}-12$ \\
Pectoral fin & R: $17-\mathrm{X}-19$ \\
Anal fin & R: $14-14-14 \quad$ L: X-X-X \\
Gill rakers & U: X-X-X \\
& \\
LIFE HISTORY & \\
\hline
\end{tabular}

$\begin{array}{ll}\text { Range } & \begin{array}{c}\text { South of southern California to } \\ \text { Bering Sea, } 54-66^{\circ} \mathrm{N}\end{array} \\ \text { Ecology } & \begin{array}{l}\text { Epi- and mesopelagic, } 99-891 \mathrm{~m} \\ \text { Oviparous, eggs probably pelagic, } \\ \text { pelagic larvae }\end{array} \\ \text { ELH pattern } & \text { Season: } \\ \text { Spawning } & \text { Area: } \\ & \text { Mode: } \\ & \text { Migration: } \\ \text { Fecundity } & \text { Range/function: } \\ \text { Age at first maturity } & \\ \text { Longevity } & \end{array}$

EARLY LIFE HISTORY DESCRIPTION

\section{EGGS}

Diameter

No. of oil globules

Oil globule diameter

Yolk

Envelope

Hatch size

Incubation time/temp.

Pigment

Diagnostic characters

\section{LARVAE}

Preanal length

$59-64 \%$ SL

Length at flexion

Length at transformation See Bathylychnops exilis

Sequence of fin development

Caudal, pectorals and pelvics, dorsal and anal

Pigment

- Series of melanophores on each hypaxial myomere

- Heavy embedded blotch at pelvic fin base; expands dorsad and ventrad

- Caudal blotch

- Above terminal section on gut ventral to liver

- Lower jaw

Diagnostic characters

- Deeper body and shorter gut than $B$. exilis and Dolichopteryx longipes

- Head with pronounced hump or bend at nape

- Tubular eyes directed dorsally

- Number of myomeres (34-37) 

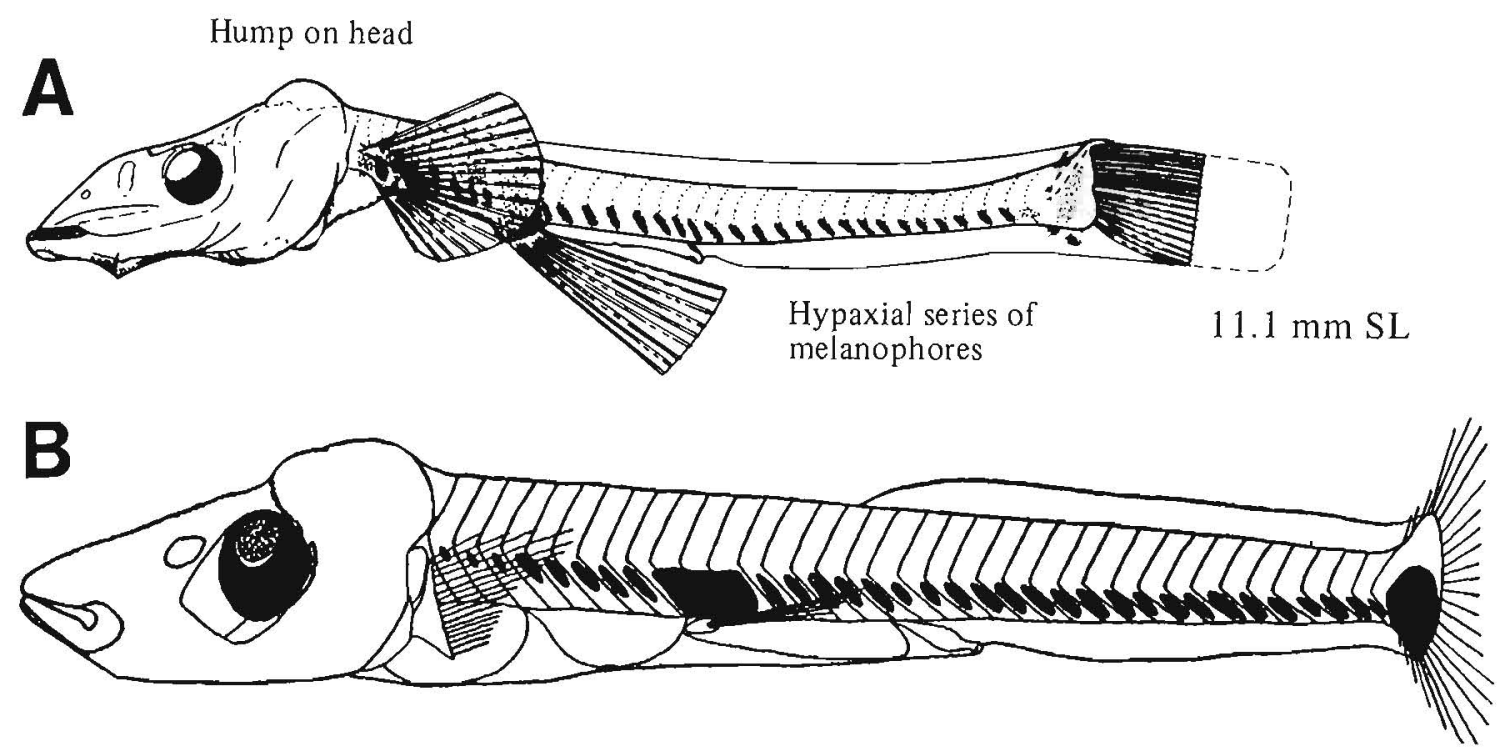

$12.0 \mathrm{~mm}$

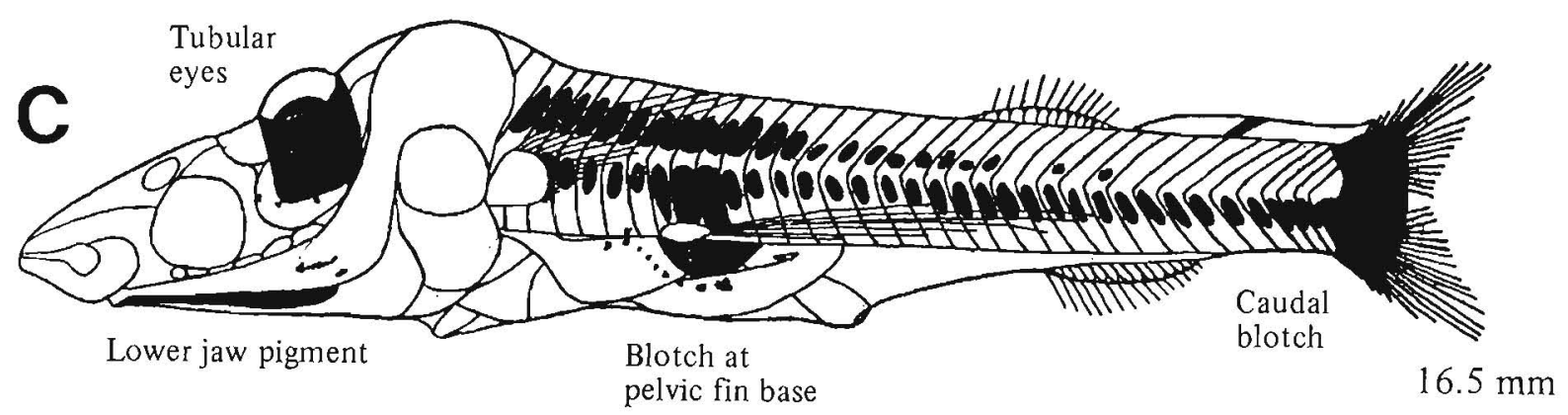

Figure A, NWAFC original (B. Vinter); B-C, Chapman 1939. 
Members of the slickhead family are found worldwide in the deep sea, with six species in six genera found within the limits of this study area. Adults occur at depths of 45 to $5500 \mathrm{~m}$ but are primarily taken in hauls near the ocean bottom below $600 \mathrm{~m}$. Juveniles occur in midwater (Fitch and Lavenberg 1968). Reproductive characteristics are unknown. Little information on alepocephalid early-life-history stages has appeared since Beebe (1933) in which they were found to hatch from large eggs (3-4 mm) and have direct development. There is no close relationship between alepocephalids and argentinoids because alepocephalids have large eggs, direct development, and share no specialized ontogenetic characters with argentinoids (Ahlstrom et al. 1984b).

\begin{tabular}{|c|c|c|c|c|c|c|c|c|c|}
\hline \multirow[b]{3}{*}{ Taxon } & \multicolumn{8}{|c|}{$\begin{array}{c}\text { Table } 6 \\
\text { Meristic characters of family Alepocephalidae. }\end{array}$} & \multirow[b]{3}{*}{ Branchiostegals } \\
\hline & \multirow[b]{2}{*}{ Distribution } & Vertebrae & \multicolumn{4}{|c|}{ Fins } & \multicolumn{2}{|c|}{ Gill rakers } & \\
\hline & & $\begin{array}{l}\text { Precaudal Caudal } \\
\text { (Total) }\end{array}$ & Dorsal & Anal & Pectoral & Pelvic & Upper & Lower & \\
\hline Alepocephalus tenebrosus & Cent. Calif.-Bering Sea & $(53-55)$ & 17 & $17-18$ & 10 & $6-7$ & $7-8$ & $17-18$ & $6-7$ \\
\hline Bathylaco nigricans & SSC-Oregon & $(45-46)$ & $17-22$ & $11-12$ & $6-11$ & $6-9$ & $3-5$ & $8-13$ & $9-10$ \\
\hline Ericara salmoneum & S. Calif.-Bering Sea & & $17-19$ & $4-28$ & 12 & 6 & & & 6 \\
\hline Leptochilichthys agassizi & SSC-Oregon & & 14 & 13 & 11 & 10 & 8 & 19 & 13 \\
\hline Narcetes stomias & SSC-Wash. & & $17-21$ & $14-16$ & $10-11$ & $8-9$ & $3-4$ & $12-14$ & 8 \\
\hline Talismania bifurcata* & SSC-Brit. Col. & $\begin{array}{l}16-17 \quad 27-28 \\
(43-46)\end{array}$ & 22 & $21-23$ & $10-12$ & $6-7$ & $7-8$ & $15-18$ & 7 \\
\hline
\end{tabular}


Tubeshoulders are found in all oceans, and five species in four genera are known in the Northeast Pacific Ocean and Bering Sea (Matsui and Rosenblatt 1987). Adults are commonly taken in midwater trawls but have been found at the surface (at night) to below $1000 \mathrm{~m}$ (Fitch and Lavenberg 1968). Little is known of their reproduction and early life history except that juveniles of Sagamichthys abei migrate upward at night to within $200 \mathrm{~m}$ of the surface to feed (Hart 1973).

Table 7

Meristic characters of family Platytroctidae.*

\begin{tabular}{|c|c|c|c|c|c|c|c|c|c|c|}
\hline \multirow[b]{2}{*}{ Taxon } & \multirow[b]{2}{*}{ Distribution } & \multicolumn{2}{|c|}{ Vertebrae } & \multicolumn{4}{|c|}{ Fins } & \multicolumn{2}{|c|}{ Gill rakers } & \multirow[b]{2}{*}{ Branchiostegal } \\
\hline & & \multicolumn{2}{|c|}{ (Total) } & Dorsal & Anal & Pectoral & Pelvic & Upper & Lower & \\
\hline Holtbymia innesi & Bering Sea & \multicolumn{2}{|c|}{$(46-48)$} & $18-19$ & 17 & 16 & 9 & 7 & $15-16$ & 8 \\
\hline Holibymia latifrons & SSC-Brit. Col. & \multicolumn{2}{|c|}{$(46-50)$} & $17-20$ & $14-16$ & $16-20$ & $8-9$ & $6-8$ & $17-19$ & $8-9$ \\
\hline Maulisia argipalla & SSC-Bering Sea & \multicolumn{2}{|c|}{$(46-47)$} & $17-20$ & $15-17$ & $18-19$ & $7-8$ & $7-8$ & $16-18$ & 8 \\
\hline Pellisolus eubranchus & Oregon & \multicolumn{2}{|c|}{$(42-44)$} & $17-19$ & $15-16$ & $18-21$ & $6-8$ & $5-6$ & $17-18$ & $8-9$ \\
\hline Sagamichthys abei & S. Calif.-Brit. Col. & \multicolumn{2}{|c|}{$(50-52)$} & $16-18$ & $14-16$ & $14-18$ & $9-10$ & $7-8$ & $16-18$ & $6-8$ \\
\hline
\end{tabular}

*Taxonomy and meristic data from Matsui and Rosenblat (1987). The northernmost record of Miroriculs taningi (reported also as Normichthys campbelli) is $35^{\circ} \mathrm{N}$ but it may occur further north. 

Smelts are confined to the Northern Hemisphere, and seven species in six genera are found in the northeastern Pacific. Some species spawn intertidally, others are anadromous. Spawning is protracted and en masse. Spent fish return to deeper water except for Thaleichthys pacificus, which experiences high, though not complete, mortality after spawning (Garrison and Miller 1982). Osmerid eggs are generally $0.80-1.1 \mathrm{~mm}$, strong to feebly adhesive, and have numerous oil globules. The adhesive membrane results from the rupturing of an outer "chorion" during spawning which turns out and onto the substrate (Hearne $1983,1984)$. In general, larval characteristics include an elongate body shape, gut $75 \%$ of standard length, subterminal mouth, conspicuous choroid fissure, stalked pectorals, no dorsal melanophores, a single row of melanophores along the ventral midline of the gut, and a single row of melanophores on the ventral midline of the tail (Hearne 1984). All osmerid larvae possess a single midventral row of melanophores below the gut. Spirinchus starksi larvae have a greater number of ventral melanophores than Spirinchus thaleichthys or Thaleichthys pacificus (Hearne 1983). Myomere counts may be of additional use: Osmerids are the most abundant larvae in the nearshore waters $(0-20 \mathrm{~km})$ off Oregon; usually $>50 \%$ of the larvae collected from January through June are osmerids (B. Mundy, NMFS Southwest Fish. Cent., Honolulu Lab., Honolulu, HI 96822-2396, pers. commun., 1 Oct. 1986.). Presently, it is possible to identify only one of these larvae to genus or species. A complete developmental series of Mallotus villosus is known. They are common in our ichthyoplankton collections off Oregon, Washington, and in the Gulf of Alaska.

\begin{tabular}{|c|c|c|c|c|c|c|c|c|c|c|}
\hline \multirow[b]{3}{*}{ Taxon } & \multicolumn{9}{|c|}{$\begin{array}{c}\text { Table } 8 \\
\text { Meristic characters of family Osmeridae. }\end{array}$} & \multirow[b]{3}{*}{ Branchiostegals } \\
\hline & \multirow[b]{2}{*}{ Distribution } & \multicolumn{2}{|c|}{ Vertebrae } & \multicolumn{4}{|c|}{ Fins } & \multicolumn{2}{|c|}{ Gill rakers } & \\
\hline & & $\begin{array}{l}\text { Precauda } \\
\qquad(\mathrm{T}\end{array}$ & Caudal & \multirow{2}{*}{$\frac{\text { Dorsal }}{9-11}$} & \multirow{2}{*}{$\frac{\text { Anal }}{14-17}$} & \multirow{2}{*}{$\begin{array}{c}\text { Pectoral } \\
12-14\end{array}$} & \multirow{2}{*}{$\frac{\text { Pelvic }}{8}$} & \multicolumn{2}{|c|}{$\begin{array}{l}\text { Upper Lower } \\
\text { (Total) }\end{array}$} & \\
\hline Allosmerus elongalus & S. Calif.-Brit. Col. & $40-44$ & $23-27$ & & & & & $10-13$ & $23-28$ & $6-7$ \\
\hline Hypomesus pretiosus & S. Calif.-Bering Sea & $\begin{array}{r}42-44 \\
(62\end{array}$ & $22-24$ & $8-11$ & $12-17$ & $14-17$ & 8 & $10-13$ & $21-25$ & $7-8$ \\
\hline Mallotus villosus & Wash.-Arctic & $(62$ & & $10-14$ & $16-23$ & $16-21$ & 9 & $8-13$ & $24-35$ & $7-8$ \\
\hline Osmerus mordax & -Brit. Col.-Arctic & $(58$ & & $8-11$ & $12-16$ & $11-14$ & 8 & $\begin{array}{r}8-11 \\
(26\end{array}$ & $\begin{array}{l}18-24 \\
37)\end{array}$ & $6-8$ \\
\hline Spirinchus starksi & S. Calif.-SE Alaska & $33-36$ & $25-29$ & $8-11$ & $|5-2|$ & $10-11$ & 8 & $8-13$ & $24-31$ & $7-8$ \\
\hline Spirinchus thaleichthys & Cent. Calif.-Bering Sea & $\begin{array}{r}29-31 \\
(54\end{array}$ & ${ }^{24-27}$ & $8-10$ & $15-22$ & $10-12$ & 8 & $10-13$ & $26-34$ & $7-8$ \\
\hline Thaleichthys pacificus & Cent. Calif.-Bering Sea & $(65$ & & $10-13$ & $18-23$ & $10-12$ & 8 & $4-6$ & $13-18$ & $6-8$ \\
\hline
\end{tabular}




\section{MERISTICS}

\begin{tabular}{|c|c|}
\hline \multirow[t]{3}{*}{ Vertebrae } & Total: $62-X-73$ \\
\hline & Precaudal: X-X-X \\
\hline & Caudal: X-X-X \\
\hline Branchiostegal rays & $7-X-8$ \\
\hline Caudal fin & $X, 10+9, X$ \\
\hline \multirow[t]{2}{*}{ Pelvic fin } & Abdominal \\
\hline & $\mathrm{R}: 9-\mathrm{X}-9$ \\
\hline Dorsal fin & $\mathrm{R}: 10-\mathrm{X}-14$ \\
\hline Pectoral fin & $\mathrm{R}: 16-\mathrm{X}-21$ \\
\hline Anal fin & $\mathrm{R}: 16-\mathrm{X}-23$ \\
\hline Gill rakers & $\mathrm{L}: 24-\mathrm{X}-35$ \\
\hline \multicolumn{2}{|l|}{ LIFE HISTORY } \\
\hline Range & $\begin{array}{l}\text { Washington, } 46-48^{\circ} 30^{\prime} \mathrm{N} \text {, to } \\
\text { Arctic, not specific }\end{array}$ \\
\hline Ecology & $\begin{array}{l}\text { Nearshore shelf pelagic, } \\
0-200 \mathrm{~m} ;{ }^{\text {a }} 750 \mathrm{~m}^{\mathrm{b}}\end{array}$ \\
\hline ELH pattern & $\begin{array}{l}\text { Oviparous, demersal attached } \\
\text { eggs, pelagic larvae }\end{array}$ \\
\hline \multirow[t]{4}{*}{ Spawning } & $\begin{array}{l}\text { Season: Fall (British Columbia); } \\
\text { spring (Bering Sea) }{ }^{\mathrm{d}}\end{array}$ \\
\hline & Area: Gravel beaches ${ }^{c}$ \\
\hline & Mode: Schools \\
\hline & Migration: \\
\hline Fecundity & $\begin{array}{l}\text { Range/function: } 3000-6600 \\
\text { (British Columbia) }^{c}\end{array}$ \\
\hline Age at first maturity & $2+\mathrm{yr}^{\mathrm{e}}$ \\
\hline Longevity & $>3 \mathrm{yr}^{\mathrm{f}}$ \\
\hline
\end{tabular}

\section{EARLY LIFE HISTORY DESCRIPTION}

\section{EGGS}

Diameter $\quad 1 \mathrm{~mm}$

No. of oil globules

Oil globule diameter

Yolk

Envelope Adhesive

Hatch size 3-6 mm

Incubation time/temp.

Pigment

Diagnostic characters

\section{LARVAE}

Preanal length $\quad \sim 75 \%$ SL

Length at flexion After yolk absorption, $\sim 7-8 \mathrm{~mm}$ SL

Length at transformation

Sequence of fin Probably caudal, dorsal, development anal, pelvics, pectorals

Pigment

- Single row of melanophores along ventral gut midline

- Single row of postanal ventral melanophores developing into double row (ventral view)

Diagnostic characters (see Table 4)

Distinguished from clupeiforms by

- Higher number of myomeres (62-73)

- Pigment: Single rather than double row of midventral melanophores below gut

- Greater gut length (75\% SL)

- Presence of adipose fin

Distinguished from other osmerids by

- Combination of greater number of pectoral fin rays (16-21) and anal fin rays (16-23)

\footnotetext{
${ }^{a}$ Andriashev 1954

${ }^{b}$ Allen and Smith 1988

${ }^{c}$ Hart and McHugh 1944

${ }^{d}$ Baxter 1975

'Trumble 1973

' Hart 1973

Ref: Templeman 1948
} 
A

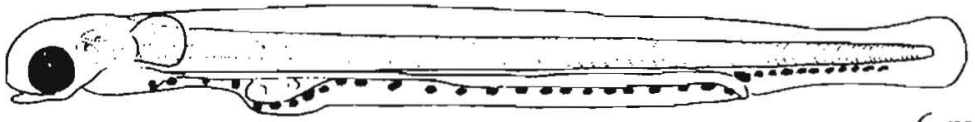

Single row of melanophores

$6 \mathrm{~mm}$

along ventral gut

B

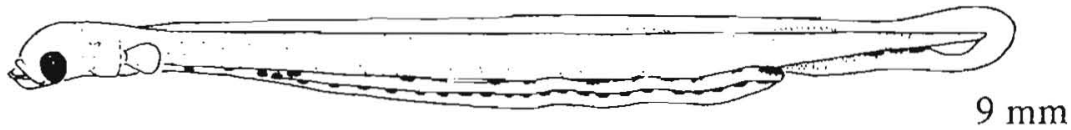

c

Adipose fin

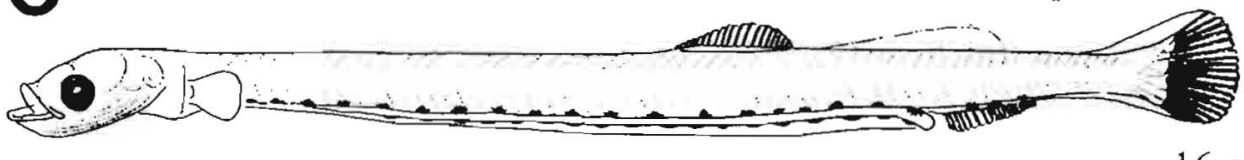

$16 \mathrm{~mm}$

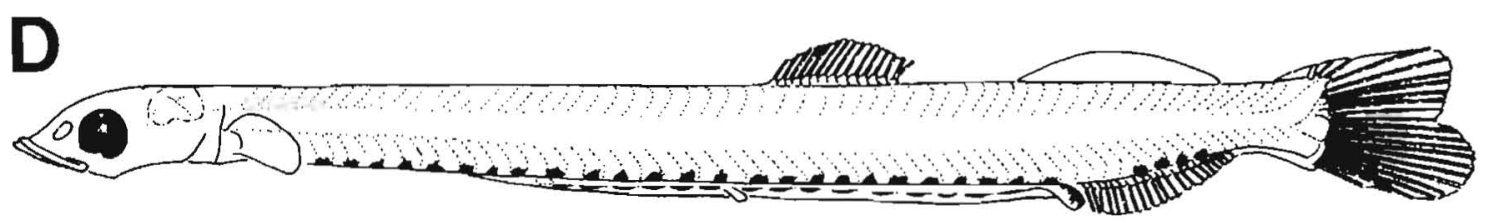

$29 \mathrm{~mm}$

E

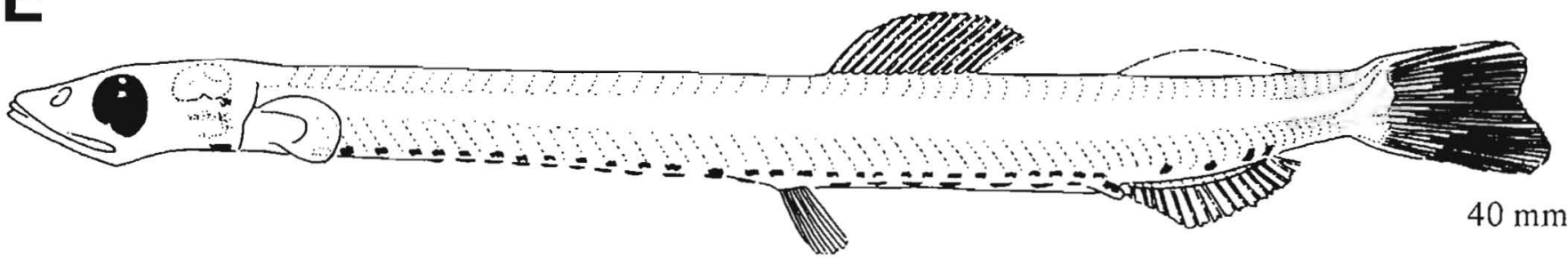

Figures A-E, Fahay 1983 (A, after Bigelow and Schroeder 1953; B-E, after Templeman 1948; Atlantic specimens) 


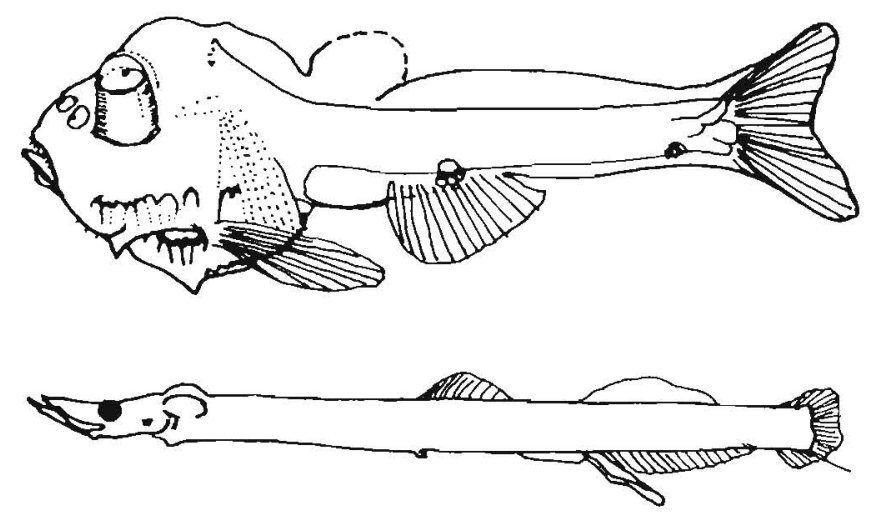

\section{Stomiiformes}

Fishes of the order Stomiiformes are mostly tropical to temperate, with many being deep-sea. This highly diverse order contains 9 families, 53 genera, and about 248 species (J. Nelson 1984). A total of 6 families, 12 genera, and 22 species are found within the study area. Body forms among stomiiforms range from eel-like (idiacanthids) to deep and extremely compressed (sternoptychids). Most species have luminescent organs, teeth on both premaxilla and maxilla, mouth extending past the eye, an adipose fin, and a dark-brown or black color (primarily silver in Gonostomatidae and Sternoptychidae). Presently, there is no consensus on the relationships within the order (Weitzman 1974, Fink and Weitzman 1982, Ahlstrom et al. 1984c, Fink 1984). Herein, we treat the gonostomatids and sternoptychids together, and the chauliodontids, melanostomiids, malacosteids, and idiacanthids together. For convenience, we will call the former families the gonostomatoids, and the latter the stomioids, without phylogenetic implications. The identification of gonostomatoid larvae requires a knowledge of developmental data from larvae, juveniles, and adults (including fin rays, teeth, and photophores). Of importance are pigment patterns, position of dorsal and anal fins (caution must be used, since positions change with growth), presence of an adipose fin, and photophores (number, pattern, and sequence of development) (Ahlstrom et al. 1984c). In addition to pigment patterns and meristic and photophore characters, gut structure (trailing or not) is an important feature to consider in the identification of stomioids.

Families in study area: Gonostomatidae

Sternoptychidae

Chauliodontidae

Melanostomiidae

Malacosteidae

Idiacanthidae 


\section{Table 9}

Definitions of alphabetical symbols used for designating photophores in deep-bodied sternoptychids and other stomiiform fishes (Ahlstrom et al. 1984c, in part).

\begin{tabular}{|c|c|}
\hline Code & Definitions \\
\hline \multicolumn{2}{|c|}{ Gonostomatoids } \\
\hline so & Symphyseal photophores (organs) located at tip of lower jaw. \\
\hline Orb & Photophores associated with the eye located anterior and posterior of orbit. \\
\hline Op & Photophores on opercle series generally three, coded as follows $1 /(1+1)$. \\
\hline $\mathrm{Br}(\mathrm{BRP})$ & Photophores located on the branchiostegal membranes. \\
\hline Is (I) & Photophores located on the isthmus. \\
\hline IP & Photophores of the ventral series found from the isthmus to the base of the pectoral fin. \\
\hline PV & Photophores of the ventral series found from the pectoral fin base to the pelvic (ventral) fin base. \\
\hline VAV & Photophores of the ventral series found from the pelvic (ventral) fin base to the anal fin base. \\
\hline $\mathrm{AC}$ & Photophores of the ventral series found from the anal fin base to caudal fin base. \\
\hline IC & Summary of photophores of the ventral series from the isthmus to caudal fin base (IP+PV+VAV+AC). \\
\hline IV & Summary of photophores of the ventral series from isthmus to pelvic (ventral) fin base (IP + PV). \\
\hline OV & Photophores of the lateral series from the opercle to pelvic (ventral) fin base. \\
\hline VA (VALA) & Photophores of the lateral series from the pelvic (ventral) fin base to the anal fin base. \\
\hline OAA & Summary of photophores of OV plus VA series. \\
\hline $\mathrm{OA}(\mathrm{OAB})$ & Summary of lateral photophores from the opercle to anal fin base $(O V+V A)$. \\
\hline $\mathrm{OAC}(\mathrm{OC})$ & $\begin{array}{l}\text { Entire lateral series on body sides just dorsal to ventral series and extending from opercular border, or just medial to it, over anal fin to caudal } \\
\text { fin base. }\end{array}$ \\
\hline ODM & Photophores (organs) found dorsal to the lateral midline (found only in Gonostoma gracile). \\
\hline \multicolumn{2}{|c|}{ Deep-bodied Sternoptychids } \\
\hline so & Subopercle photophore which is equivalent to posteriormost photophore in opercular series of gonostomatoids. \\
\hline PO & Photophore located anterior to orbit. \\
\hline PTO & Photophore located posterior to orbit and may be equivalent to upper photophore of opercular series of gonostomatoids. \\
\hline PRO & Preopercular photophore, used for PO photophore dorsal to ventral limb or preopercle. \\
\hline $\mathrm{Br}$ & Same as gonostomatoid definition. \\
\hline Is & Same as gonostomatoid definition. \\
\hline $\mathrm{AB}$ & $\begin{array}{l}\text { Photophores of ventral series located abdominally between pectoral fin base and pelvic fin base and equivalent to PV in gonostomatoids, plus a } \\
\text { few posterior photophores of the IP series. }\end{array}$ \\
\hline PAN & Photophores found anterior to anal fin and may be equivalent to VAV or VA in gonostomatoids. \\
\hline AN & Photophores found above anal fin. \\
\hline SC & Photophores found on lower (sub) caudal peduncle. Together with AN group may be equivalent to AC in gonostomatoids. \\
\hline SAB & Photophores located above (supra) to the abdominal series and may be equivalent to VA in gonostomatoids. \\
\hline SP & Photophores located above (supra) the pectoral fin and may be equivalent to OV in gonostomatoids. \\
\hline SAN & Photophores located above (supra) to anal photophores and equivalent to part of AC series. \\
\hline
\end{tabular}

Table 10

Photophore distribution in selected gonostomatoid genera (Ahlstrom et al. 1984c, in part). See Table 9 for photophore definitions.

\begin{tabular}{|c|c|c|c|c|c|c|c|c|c|c|}
\hline & $\begin{array}{l}\text { No. of } \\
\text { rows }\end{array}$ & SO & ORB & OP & $\mathrm{BR}$ & IS & IV & VAV & $\mathrm{AC}$ & $\begin{array}{l}\text { Photophores in } \\
\text { groups of glands? }\end{array}$ \\
\hline Argyropelecus & 2 & No & 2 & 2 & 6 & Yes & 18 & 4 & 10 & Yes \\
\hline Cyclothone & 2 & No & 1 & 2 & $8-11$ & No & $12-14$ & $4-5$ & $12-16$ & No \\
\hline Danaphos & 2 & No & I & $2-3$ & 6 & Yes & 18 & 5 & $22-26$ & Yes \\
\hline Gonostoma & 2 & Yes & 1 & $2-3$ & 9 & No & $11-16$ & $3-10$ & $15-23$ & No \\
\hline Sternoptyx & 2 & No & 2 & 2 & 3 & Yes & 15 & 3 & 7 & Yes \\
\hline
\end{tabular}




\section{MAJOR PHOTOPHORE GROUPS}

A Gonostomatoid
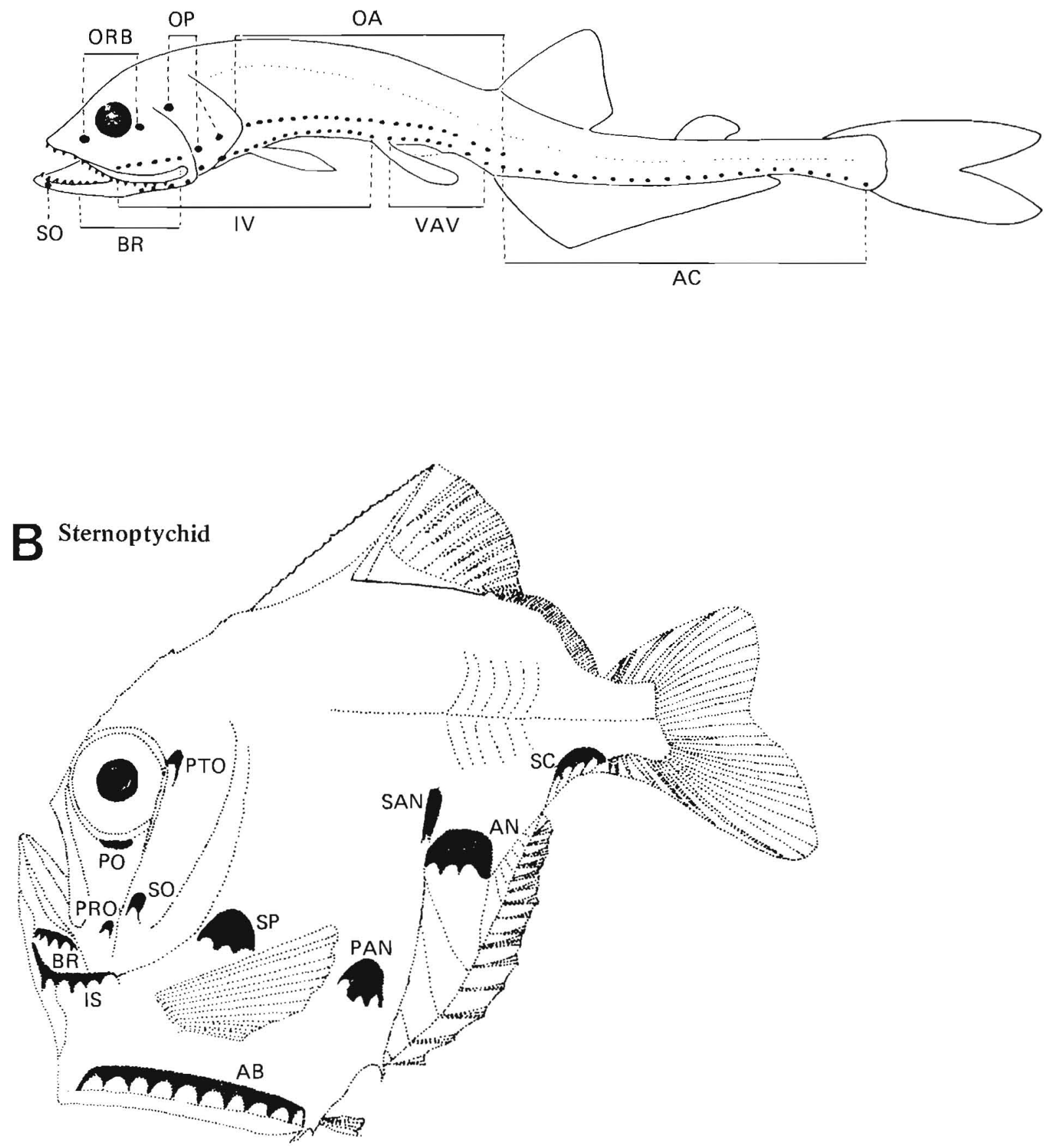

Figure A, Fahay 1983 (after Ozawa 1976); B, Badcock and Baird 1980 (redrawn). 
Table 11

Fin position and condition of adipose fin in selected gonostomatoid fishes (Ahlstrom et al. 1984c, in part).

\begin{tabular}{llll}
\hline \multicolumn{1}{c}{ Genera } & \multicolumn{1}{c}{ Adult } & \multicolumn{1}{c}{ Larvae } \\
\hline Argyropelecus & Anal origin opposite last dorsal fin ray & Anal origin behind dorsal fin \\
$\begin{array}{l}\text { Cyclothone } \\
\text { Danaphos }\end{array}$ & Anal origin opposite dorsal fin or slightly behind & Same as adult \\
Gonostoma & Anal origin behind dorsal fin & Same as adult \\
Stemoptyx & Anal origin opposite or 3-4 rays in advance of dorsal origin & Same as adult & Absent \\
\hline
\end{tabular}




\begin{tabular}{|c|c|c|c|c|c|c|c|c|c|c|c|}
\hline \multirow[b]{3}{*}{ Taxon } & \multicolumn{10}{|c|}{$\begin{array}{l}\text { Table } 12 \\
\text { Meristic characters of family Gonostomatidae. }\end{array}$} & \multirow[b]{3}{*}{ Branchiostegals } \\
\hline & \multirow[b]{2}{*}{ Distribution } & \multicolumn{2}{|c|}{ Vertebrae } & \multicolumn{5}{|c|}{ Fins } & \multicolumn{2}{|c|}{ Gill rakers } & \\
\hline & & \multicolumn{2}{|c|}{ (Total) } & \multirow{2}{*}{$\frac{\text { Dorsal }}{13-15}$} & \multirow{2}{*}{$\frac{\text { Anal }}{18-20}$} & \multirow{2}{*}{$\frac{\text { Pectoral }}{9-10}$} & \multirow{2}{*}{$\begin{array}{c}\text { Pelvic } \\
6\end{array}$} & \multirow{2}{*}{$\begin{array}{l}\text { Caudal } \\
6,10+9,6^{a}\end{array}$} & \multirow{2}{*}{$\frac{\text { Upper }}{6-9}$} & \multirow{2}{*}{$\begin{array}{l}\text { Lower } \\
14-17\end{array}$} & \\
\hline Cyclothone acclinidens & SSC-Oregon & $13-14$ & $17-19$ & & & & & & & & $13-15$ \\
\hline Cyclothone atraria & SSC-Bering Sea ${ }^{b}$ & $12-13$ & $18-20$ & $13-15$ & $18-21$ & $9-10$ & 6 & & $6-8$ & $13-16$ & $12-14$ \\
\hline Cyclothone pallida & SSC-Brit. Col. & $13-14$ & $17-21$ & $13-15$ & $17-19$ & $9-11$ & $6-7$ & & $7-10$ & $14-18$ & $13-15$ \\
\hline Cyclothone pseudopallida & SSC-Bering Sea & $12-13^{a}$ & $18-20^{a}$ & $13-15$ & $18-21$ & $9-10$ & 6 & & $4-6$ & $12-14$ & $13-15$ \\
\hline Cyclothone signata & SSC-Gulf of Alaska & 13 & $17-19$ & $13-14$ & $18-20$ & $9-10$ & 6 & $6-8,10+9,6-7^{a}$ & $3-5$ & $10-11$ & $12-14$ \\
\hline Gonostoma atlanticum & SSC-Oregon & $(38$ & & $16-18$ & $27-30$ & $9-10$ & $6-7$ & & $6-7$ & 11 & $11-12$ \\
\hline Gonostoma gracile & Gulf of Alaska-Bering Sea & $18-19$ & $22-24$ & $9-12$ & $22-30$ & $8-10$ & $6-8$ & & & & $11-12$ \\
\hline
\end{tabular}

${ }^{2}$ E.H. Ahlstrom notes

${ }^{b}$ Records from north of British Columbia are questionable (A.E. Peden, Brit. Col. Prov. Mus., Victoria, B.C., Canada V8V 1X4, pers. commun., 22 Jan. 1987). 
This worldwide genus of lightfishes may constitute the most abundant genus of fish in the sea. These small (to $80 \mathrm{~mm}$ ) bathypelagic fishes are found most commonly at depths of $300 \mathrm{~m}$ and greater and have been found as deep as $5300 \mathrm{~m}$. Very little information on life history is available. The eggs are unknown but larvae are common in offshore tropical and temperate areas. Cyclothone spp. larvae are discussed at the generic level since only partial series are available for the five species in the study area. Gorbunova (1982a) described the larvae of eight species from the Pacific including four from the study area (all species except $C$. atraria). The descriptions of $C$. acclinidens, $C$. pallida, and $C$. pseudopallida by Gorbunova (1982a) have been questioned by Ozawa and Oda (1986) who described the larvae (>7 mm SL) of five species of Cyclothone (all species from the study area except $C$. signata). Since preflexion larvae and transforming larvae linking larval series to adults are generally unavailable, Ozawa and Oda (1986) discussed the need for further investigation.

Preanal length for Cyclothone spp. larvae is about $50 \%$ SL. Lengths at flexion and transformation are 4-5 mm SL and about $14 \mathrm{~mm} \mathrm{SL}$, respectively. Caudal and dorsal fins form first, followed in sequence by the anal, pelvic, and pectoral fins. Photophores are described in Table 10.

Pigment characters for Cyclothone spp., based on described species, include

- 2-3 spots along gut with posteriormost spot at anus (sometimes more spots in larger specimens)

- One spot at cleithral symphysis

- Pigment on swimbladder

- Lateral series posterior to pectoral fins, ending prior to swimbladder

- Series over anal fin base (varies from 5-12 spots and may or may not be evenly spaced; number and spacing of series may be species-specific)

- Some species develop dorsal series similar to anal base series

- Spots at base of anal fin pterygiophores

- Spots usually appear above and below urostyle and in hypural area

Diagnostic characters for Cyclothone spp. larvae include

- Distinct dark streak or intense melanophore over and parallel to parhypural on caudal fin base

- Pigmentation over gut and along ventral margin of tail

- Anal fin origin opposite dorsal fin or slightly behind (no ontogenetic movement)

- Conspicuous swimbladder

- No adipose fin

- Larvae generally elongate

Diagnostic characters for species

- C. acclinidens (melanophore on dorsal part of tail end)

- C. pseudopallida (predorsal melanophores $2-4$, see figure)

- C. atraria (predorsal melanophores 6 or 7 , see figure)

- C. pallida (predorsal melanophores 9-12, see figure)

- C. signata (no melanophore on dorsal part of tail end, and no predorsal melanophores)

\section{Distinguish from Gonostoma}

- See Tables 10-12

- Fewer anal fin rays (16-21 vs. 21-31)

- Fewer vertebrae (29-33 vs. $37-40)$

- SO photophore absent

- Caudal pigment (present in Gonostoma atlanticum but not in G. gracile)

Larger postflexion larvae, which differ greatly from adults, undergo a metamorphic stage during which most of the photophores become pigmented simultaneously, the anterior portion of the body shortens, and the anus changes position from near the anal fin to nearer the ventral fin bases.

Ref: Ahlstrom et al. 1984c; Fahay 1983; Gorbunova 1982a; Grey 1964; Hart 1973; Jespersen and Tåning 1926; Mukacheva 1954, 1964 ; Ozawa and Oda 1986. 

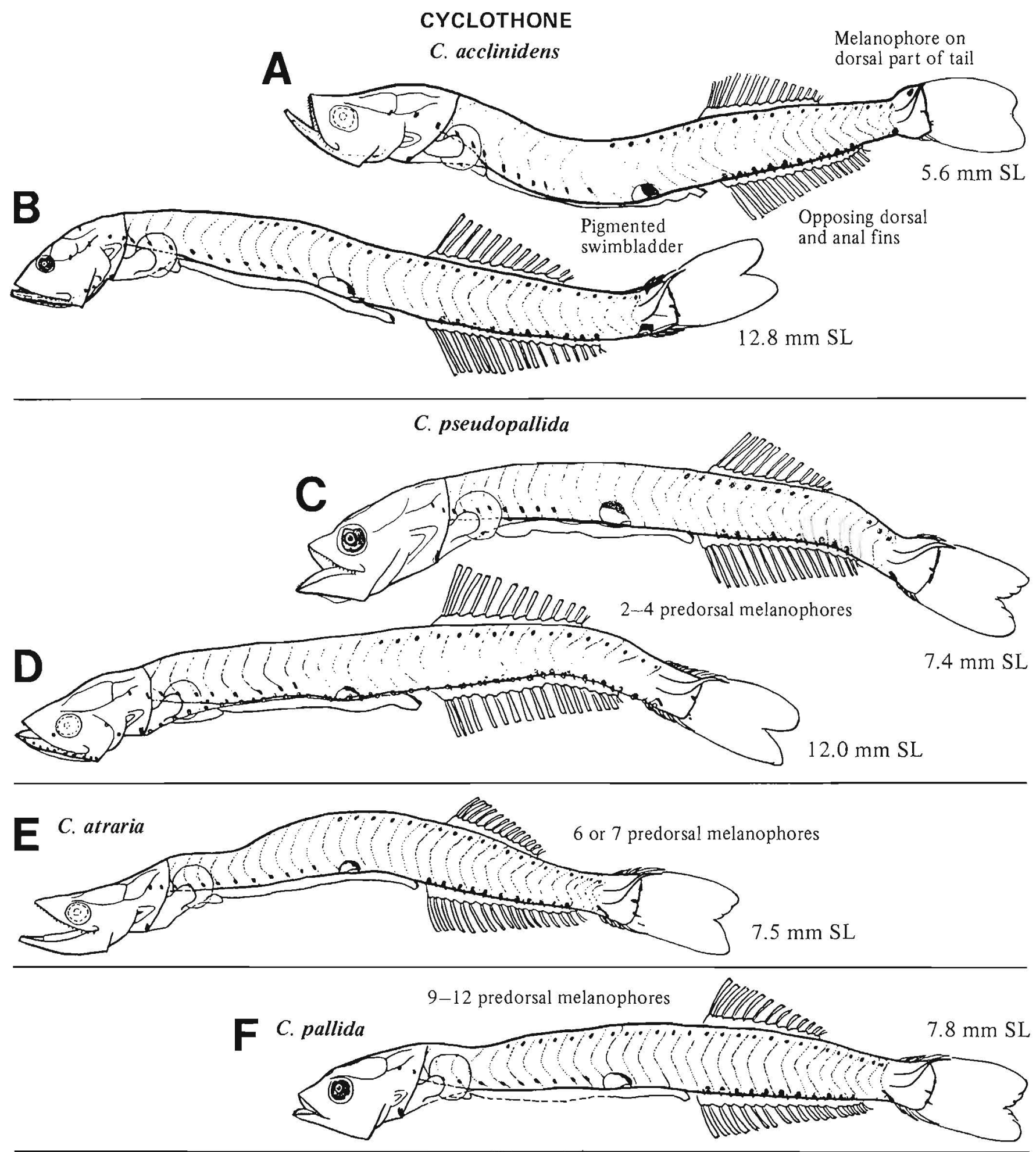

\section{G C. signata}

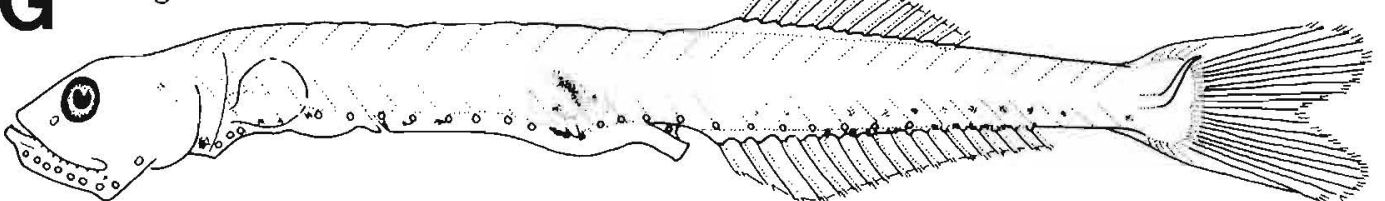

$9.0 \mathrm{~mm} \mathrm{SL}$

Figures A-F, Ozawa and Oda 1986; G, Ahlstrom et al. 1984c 
MERISTICS

G. atlanticum Norman 1930

Vertebrae

Total: $38-X-39$

Precaudal: $X-X-X$

Caudal: $X-X-X$

Branchiostegal rays

Pelvic fin

Dorsal fin

Pectoral fin

Anal fin

Gill rakers

11-X-12

Abdominal

R: 6-7-7

R: $16-18-18$

R: $9-\mathrm{X}-10$

$\mathrm{R}: 27-28-30$

U: $6-\mathrm{X}-7$

$\mathrm{L}: 11-\mathrm{X}-11$

\section{MERISTICS}

G. gracile Günther 1878

$\begin{array}{ll}\text { Vertebrae } & \text { Total: } 40-\mathrm{X}-42 \\ & \begin{array}{l}\text { Precaudal: } 18-\mathrm{X}-19 \\ \text { Caudal: } 22-\mathrm{X}-24\end{array} \\ \text { Branchiostegal rays } & \text { 11-X-12 } \\ \text { Pelvic fin } & \text { Abdominal } \\ & \text { R: } 6-\mathrm{X}-8 \\ \text { Dorsal fin } & \text { R: } 9-\mathrm{X}-14 \\ \text { Pectoral fin } & \text { R: } 8-\mathrm{X}-11 \\ \text { Anal fin } & \text { R: } 22-\mathrm{X}-30 \\ \text { Gill rakers } & \text { U: } 7-8-8 \quad \text { L: } 12-13-14\end{array}$

\section{LIFE HISTORY}

Range
(G. atlanticum)
(G. gracile)

Ecology

ELH pattern

Spawning

Age at first

maturity

Longevity
South of southern California to Oregon, $42-46^{\circ} \mathrm{N}$

Gulf of Alaska, $54-60^{\circ} \mathrm{N}$ to

Meso- and bathypelagic ${ }^{a}$ Oviparous, pelagic larvae

Season: Sept-Apr (Japan)

$1 \mathrm{yr}$ (males - then undergo sex reversal at $70-80 \mathrm{~mm} \mathrm{SL}$ )

$2 \mathrm{yr}$ (females)

$1 \mathrm{yr}$ (males) Bering Sea, $54-66^{\circ} \mathrm{N}$
EARLY LIFE HISTORY DESCRIPTION

\section{EGGS (G. denudatum)}

Diameter

No. of oil globules

$0.80-0.81 \mathrm{~mm}$

Oil globule diameter

One

Yolk

Envelope

Hatch size

Incubation time/temp.

Pigment

Diagnostic characters

\section{LARVAE (G. gracile) \\ Preanal length \\ Length at flexion \\ Length at transformation \\ Sequence of fin development \\ Caudal, dorsal and anal,
pectorals and pelvics}

Pigment

- Internal head pigment

- Swimbladder and peritoneum

Diagnostic characters

G. gracile distinguished from other Gonostoma spp. by

- Anal fin origin extremely in advance of dorsal fin origin

G. gracile distinguished from $G$. atlanticum by

- Lack of pigment on caudal peduncle and body surface

G. atlanticum distinguished from Cyclothone spp. by

- Caudal and ventral pigment

- Swimbladder position

Photophores (see Table 10)

In $G$. gracile and $G$. atlanticum, photophores develop as a group. This differs from other species of Gonostoma where photophores develop gradually.

${ }^{a} G$. allanticum mesopelagic only.

${ }^{\mathrm{b}} G$. gracile only. 


\section{GONOSTOMA}

\section{Gonostoma atlanticum}

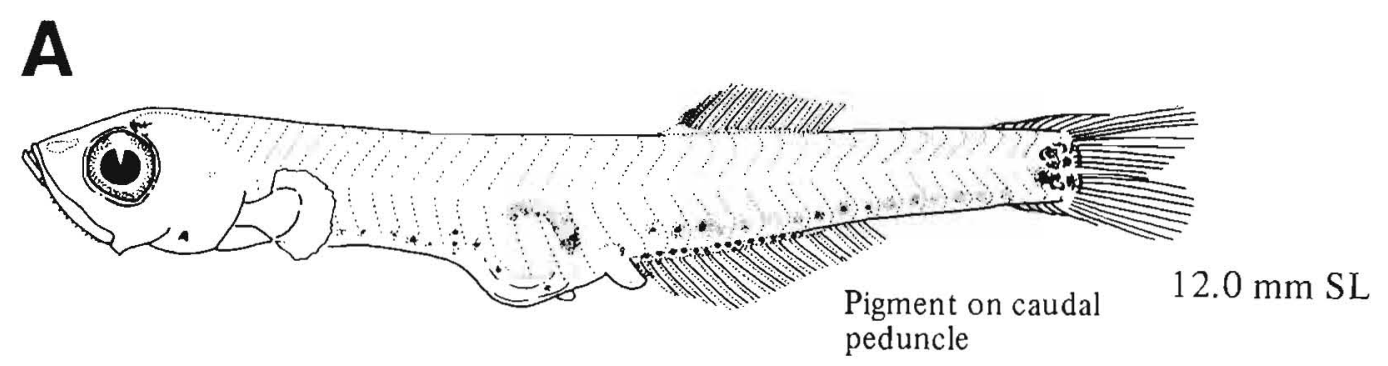

\section{Gonostoma gracile}

B

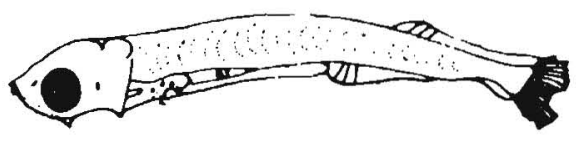

$6.0 \mathrm{~mm} \mathrm{SL}{ }^{\circ}$

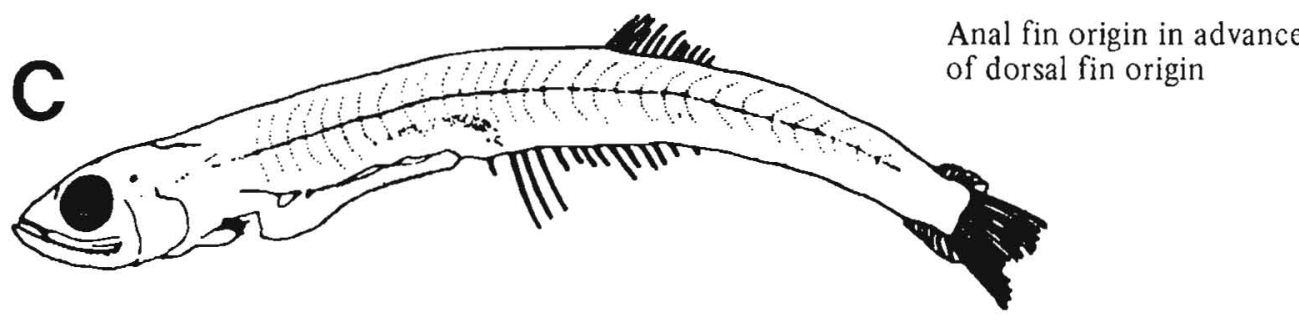

$11.5 \mathrm{~mm} \mathrm{SL}$ o
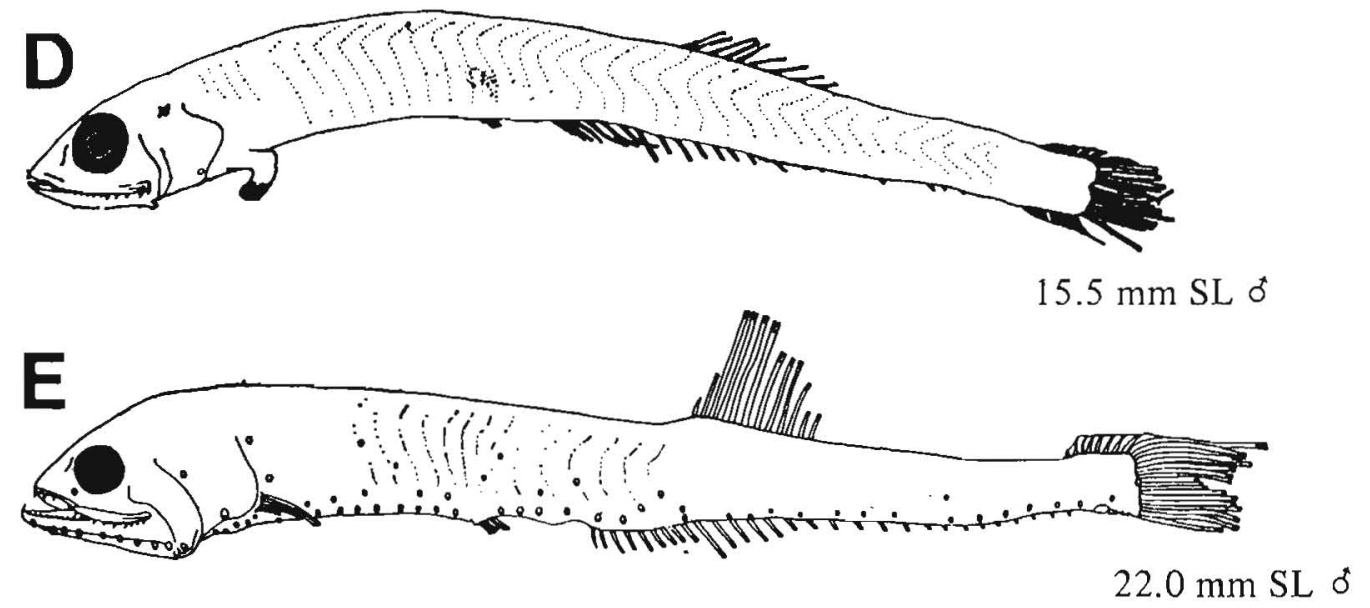

Figure A, Ahlstrom et al. 1984c (after Ahlstrom 1974); B-E, Kawaguchi and Marumo 1967 (western Pacific specimens). 


\section{STERNOPTYCHIDAE}

Found in all temperate and tropical seas, hatchetfishes are represented in the northeastern Pacific by seven species in three genera. Adults are found 100-5000 m below the surface (Schultz 1961). Sternoptychid eggs and larvae are planktonic, constituting the third most abundant family in the ichthyoplankton of open ocean waters (after myctophids and gonostomatids) (Ahlstrom 1972, Loeb 1979). Argyropelecus hemigymnus has eggs ranging from 0.92 to $1.04 \mathrm{~mm}$. The yolk is coarsely segmented and contains one oil globule (Ahlstrom 1974). Development of sternoptychids is distinctly divided into two periods (Sanzo 1935): Larval period, and the period of metamorphosis marked by a sharp reduction in body length, change in shape, and photophore and fin-ray development. Distribution and patterns of photophores, especially their sequence of development, are instrumental in larval identification. The following characters may aid in distinguishing between the three sternoptychid genera in the study area (see Tables 13-14).

\begin{tabular}{lccc} 
& Argyropelecus & Danaphos & Sternoptyx \\
\hline Anal fin rays & $11-13$ & $24-25$ & $14-16$ \\
Pectoral fin rays & $10-11$ & $13-14$ & $10-11$ \\
Vertebrae & $34-40$ & 38 & $28-31$ \\
Adipose fin & Present/absent & Absent & Absent \\
Photophores & & & \\
Br & 6 & 6 & 3 \\
Orb & 2 & 1 & 2 \\
IV & 18 & 18 & 15 \\
VAV & 4 & 5 & 3 \\
AC & 10 & $22-26$ & 7
\end{tabular}




\begin{tabular}{|c|c|c|c|c|c|c|c|c|c|}
\hline \multirow[b]{3}{*}{ Taxon } & \multirow[b]{3}{*}{ Distribution } & \multicolumn{6}{|c|}{$\begin{array}{l}\text { Table } 13 \\
\text { Meristic characters of family Sternoptychidae. }\end{array}$} & \multirow[b]{2}{*}{ Gill rakers } & \multirow[b]{3}{*}{ Branchiostegals } \\
\hline & & Vertebrae & \multicolumn{5}{|c|}{ Fins } & & \\
\hline & & $\begin{array}{l}\text { Precaudal Caudal } \\
\text { (Total) }\end{array}$ & Dorsal & Anal & Pectoral & Pelvic & Caudal & $\begin{array}{l}\text { Upper Lower } \\
\text { (Total) }\end{array}$ & \\
\hline Argyropelecus affinis & SSC-Oregon & $(39-41)$ & 9 & $12-13$ & $10-11$ & 6 & $10-12,10+9,4$ & $(18-22)$ & 10 \\
\hline Argyropelecus hemigymnus & SSC-Wash. & $(37-39)$ & 8 & 11 & $10-11$ & 6 & $10,10+9,5$ & $(18-24)$ & 10 \\
\hline Argyropelecus lychnus & SSC-Brit. Col. & $(35-37)$ & 9 & 12 & $10-11$ & 6 & $9-11,10+9,6$ & $8-9 \quad 12-14$ & 10 \\
\hline Argyropelecus sladeni & SSC-Oregon & $(36-38)$ & 9 & 12 & $10-11$ & 6 & $10,10+9,6$ & $(17-21)$ & 10 \\
\hline Danaphos oculatus & SSC-Brit. Col. & $(38)$ & 6 & $24-25$ & $10-11$ & & $8,10+9,3$ & $11-13$ & 10 \\
\hline Stemoptyx diaphana & SSC-Oregon & $(28-30)$ & $9-11$ & $13-16$ & $10-11$ & & & $6-9$ & 6 \\
\hline Sternopryx pseudobscura & SSC-Brit. Col. & $(27-30)$ & $9-11$ & $13-15$ & $10-11$ & & & $7-9$ & 6 \\
\hline
\end{tabular}

Table 14

Characters useful in separating larvae of Argyropelecus found in the Northeast Pacific (Belyanina 1984, in part).

\begin{tabular}{|c|c|c|c|c|}
\hline Character & A. affinis & A. hemigymnus & A. lychnus & A. sladeni \\
\hline $\begin{array}{l}\text { Photophores: SAB, } \\
\text { PAN, AN, SC in a } \\
\text { continuous row* }\end{array}$ & Yes & No & No & No \\
\hline Caudal pigment & $\begin{array}{l}\text { At or before transfor- } \\
\text { mation }(\sim 8 \mathrm{~mm} \mathrm{SL})\end{array}$ & $\begin{array}{l}\text { At or before transfor- } \\
\text { mation }(\sim 8 \mathrm{~mm} \mathrm{SL})\end{array}$ & No & $>13 \mathrm{~mm} \mathrm{SL}$ \\
\hline $\begin{array}{l}\text { Number of } \\
\text { postabdominal spines }\end{array}$ & 2 & $\begin{array}{l}1 \text { (posterior one } \\
\text { reduced) }\end{array}$ & 2 & 2 \\
\hline Number of dorsal fin rays & 9 & 8 & 9 & 9 \\
\hline Eye shape (telescopic) & Round & Cylindrical & Round & Round \\
\hline $\begin{array}{l}\text { Presence of well-developed } \\
\text { upper preopercular spine }\end{array}$ & No & No & Yes (pointed downward) & Yes (pointed upward) \\
\hline Presence of frontal crest & No & No & Yes & Yes \\
\hline
\end{tabular}


MERISTICS

$\begin{array}{ll}\text { Vertebrae } & \text { Total: } 39-\mathrm{X}-41 \\ & \begin{array}{l}\text { Precaudal: } \mathrm{X}-\mathrm{X}-\mathrm{X} \\ \text { Caudal: } \mathrm{X}-\mathrm{X}-\mathrm{X}\end{array} \\ & 10-10-10 \\ \text { Branchiostegal rays } & 10-12,10+9,4 \\ \text { Caudal fin } & \text { Abdominal } \\ \text { Pelvic fin } & \text { R: } 6-6-6 \\ & \text { R: } 9-9-9 \\ \text { Dorsal fin } & \text { R: } 10-11-11 \\ \text { Pectoral fin } & \text { R: } 12-X-13 \\ \text { Anal fin } & \text { U: } X-X-X \quad \text { L: } X-X-X \\ \text { Gill rakers } & \end{array}$

\section{LIFE HISTORY}

$\begin{array}{ll}\text { Range } & \begin{array}{c}\text { South of southern California to } \\ \text { Oregon, } 42-46^{\circ} \mathrm{N} \\ \text { Epi- and mesopelagic, 100-610 m } \\ \text { Ecology }\end{array} \\ \begin{array}{l}\text { Oviparous, pelagic eggs, pelagic } \\ \text { larvae }\end{array} \\ \text { Spawning } & \text { Season: } \\ & \text { Area: } \\ & \text { Mode: } \\ & \text { Migration: } \\ \text { Fecundity } & \text { Range/function: } \\ \text { Age at first maturity } & \end{array}$

\section{EARLY ILIFE HISTORY DESCRIPTION}

\section{EGGS}

Diameter

No. of oil globules

Oil globule diameter

Yolk

Envelope

Hatch size

Incubation time/temp.

Pigment

\section{Diagnostic characters}

\section{LARVAE}

Preanal length

$$
\begin{aligned}
& <50 \% \text { SL increasing with } \\
& \text { development to } \sim 50 \% \text { SL }
\end{aligned}
$$

Length at flexion

Length at transformation $<8.1 \mathrm{~mm} \mathrm{SL}$

Sequence of fin development

Caudal and pectorals, dorsal and anal, pelvics

Pigment

- Initially, lightly pigmented on anterior body, increases posteriorly with development

- Characteristic spot occurs on caudal peduncle above subcaudal photophores; in larvae $>11 \mathrm{~mm} \mathrm{SL}$, spot increases to a patch

Diagnostic characters

- Photophores: A. affinis distinguished by the location of supra-abdominal (SAB), preanal (PAN), anal (AN), and subcaudal (SC) photophores in a continuous straight line; $\mathrm{AN}$ and $\mathrm{SC}$ groups are separated by a gap

- Eyes telescopic

- Spines

-At $8 \mathrm{~mm}$ SL, a spine anterior to spinous dorsal plate

-At $11 \mathrm{~mm} \mathrm{SL}$, long posteriormost spine on spinous dorsal plate

-At $>11 \mathrm{~mm}$ SL, enlarged postabdominal spines 

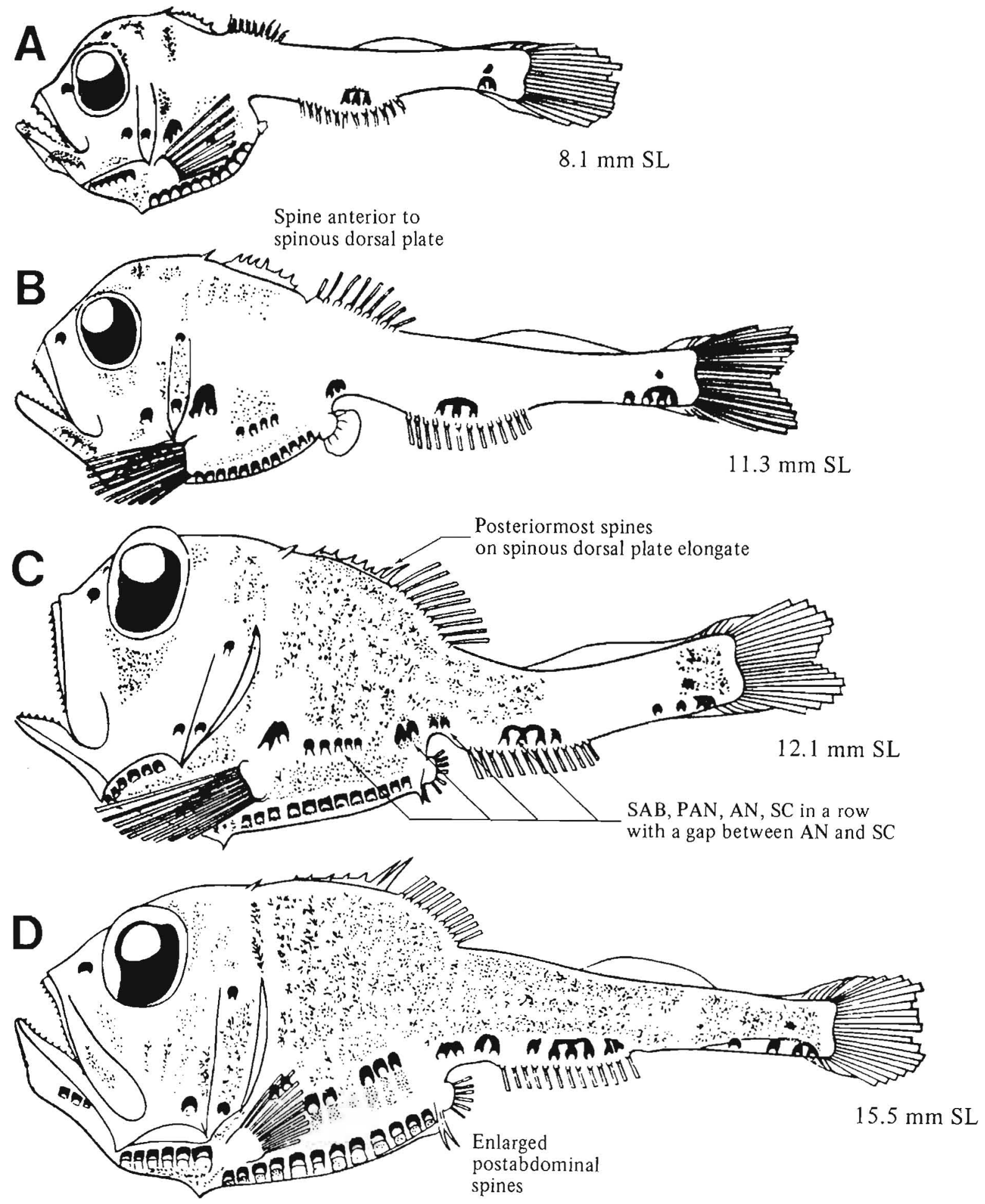

Figures $A-D$, Belyanina 1984 . 


\section{MERISTICS}

\begin{tabular}{ll} 
Vertebrae & Total: $37-\mathrm{X}-39$ \\
& Precaudal: X-X-X \\
& Caudal: X-X-X \\
Branchiostegal rays & $10-10-10$ \\
Caudal fin & $10,10+9,5$ \\
Pelvic fin & Abdominal \\
& R: $6-6-6$ \\
Dorsal fin & R: $8-8-8$ \\
Pectoral fin & R: $10-X-11$ \\
Anal fin & R: $11-11-11 \quad$ \\
Gill rakers & U: X-X-X $\quad$ L: X-X-X \\
& \\
LIFE HISTORY & \\
\hline
\end{tabular}

$\begin{array}{ll}\text { Range } & \begin{array}{c}\text { South of southern California to } \\ \text { Washington, } 46-48^{\circ} 30^{\prime} \mathrm{N}\end{array} \\ \text { Ecology } & \begin{array}{l}\text { Epi- and mesopelagic, 100-731 m } \\ \text { Oviparous, pelagic eggs, pelagic } \\ \text { larvae }\end{array} \\ \text { Spawning } & \text { Season: } \\ & \text { Area: } \\ & \text { Mode: } \\ & \text { Migration: } \\ \text { Fecundity } & \text { Range/function: } \\ \text { Age at first maturity } & \\ \text { Longevity } & \end{array}$

EARLY LIFE HISTORY DESCRIPTION

\section{EGGS}

Diameter

No. of oil globules

0.92-1.04 mm

Oil globule diameter

Yolk

Envelope

Hatch size

Incubation time/temp.

Pigment

- Embryo unpigmented

One

$0.26-0.28 \mathrm{~mm}$

Segmented

$2.5 \mathrm{~mm} \mathrm{SL}$; yolk absorbed

$$
4.7 \mathrm{~mm} \mathrm{SL}
$$

2-2.5 d (temperature unknown)

Diagnostic characters

\section{LARVAE}

Preanal length

$<50 \%$ SL increasing with development to $\sim 50 \% \mathrm{SL}$

Length at flexion

Length at transformation

$$
\begin{aligned}
& >6 \mathrm{~mm} \mathrm{SL} \text {; body shrinks } \\
& 2-3 \mathrm{~mm} \text {, gut shortens, } \\
& \text { head deepens, and eyes } \\
& \text { become telescopic } \\
& \text { Caudal and pectorals, dorsal } \\
& \text { and anal, pelvics }
\end{aligned}
$$

Sequence of fin development

\section{Pigment}

- Unpigmented at hatching (except eyes) and through transformation

- After transformation, pigment appears on head in patches and on caudal peduncle

\section{Diagnostic characters}

- Elongate compressed body

- Photophores and sequence of development ( 6-10 $\mathrm{mm}$ SL): Lower OP, BR, posterior IV, anterior IV, posterior $\mathrm{AC}$, anterior $\mathrm{AC}, \mathrm{OA}, \mathrm{ORB}$, and VAV

- SAB, PAN, AN, and SC photophores not in continuous row

- Postabdominal spines: In larvae $>10-12 \mathrm{~mm} \mathrm{SL}$, anterior spine becomes thinner and elongated and bends under the posterior spine; posterior spine gradually reduces and becomes spur-shaped

- Number of dorsal fin rays (eight); other sternoptychids usually have nine

- Eye shape more cylindrical than other Argyropelecus spp. 
B

Unpigmented
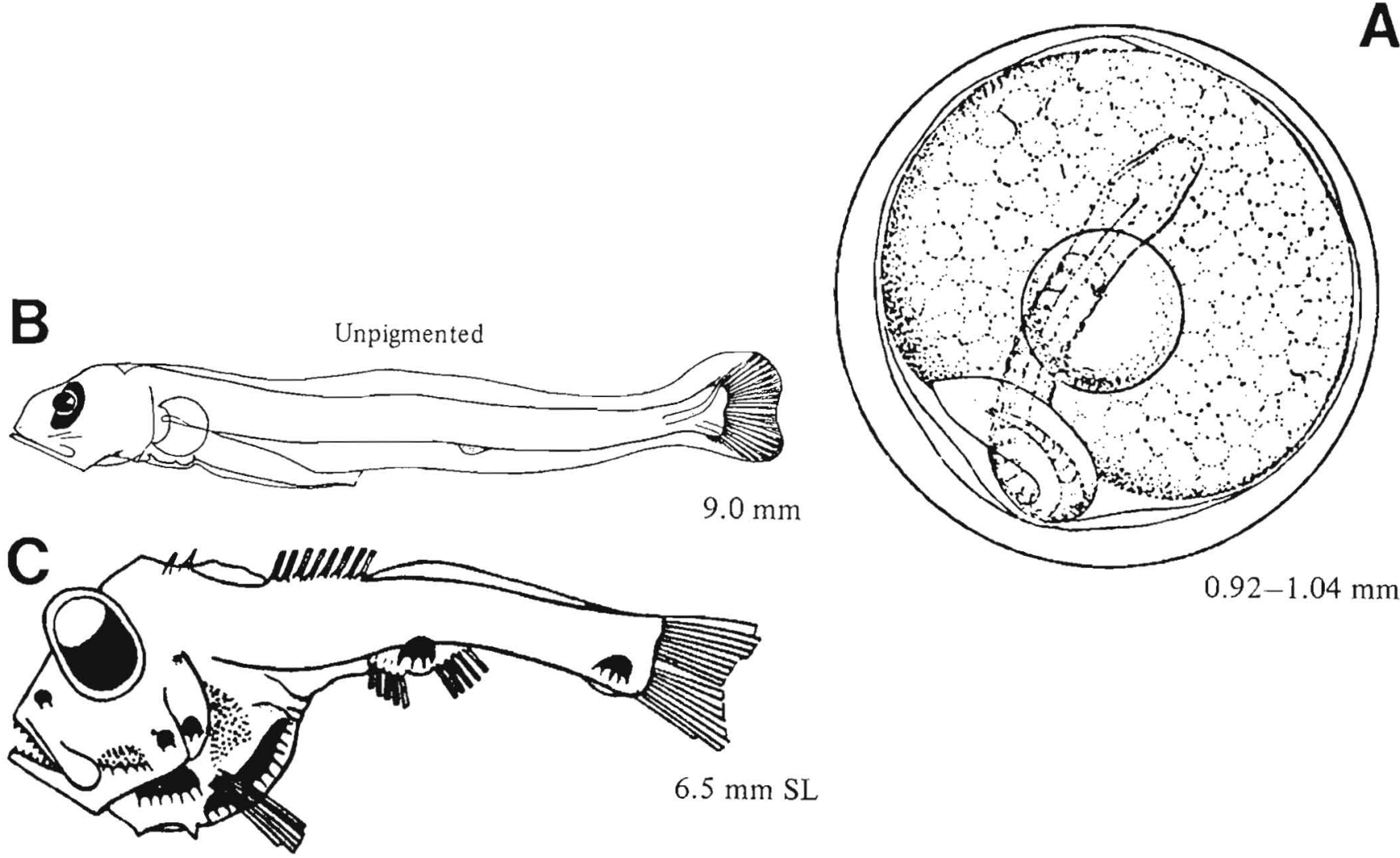

$0.92-1.04 \mathrm{~mm}$
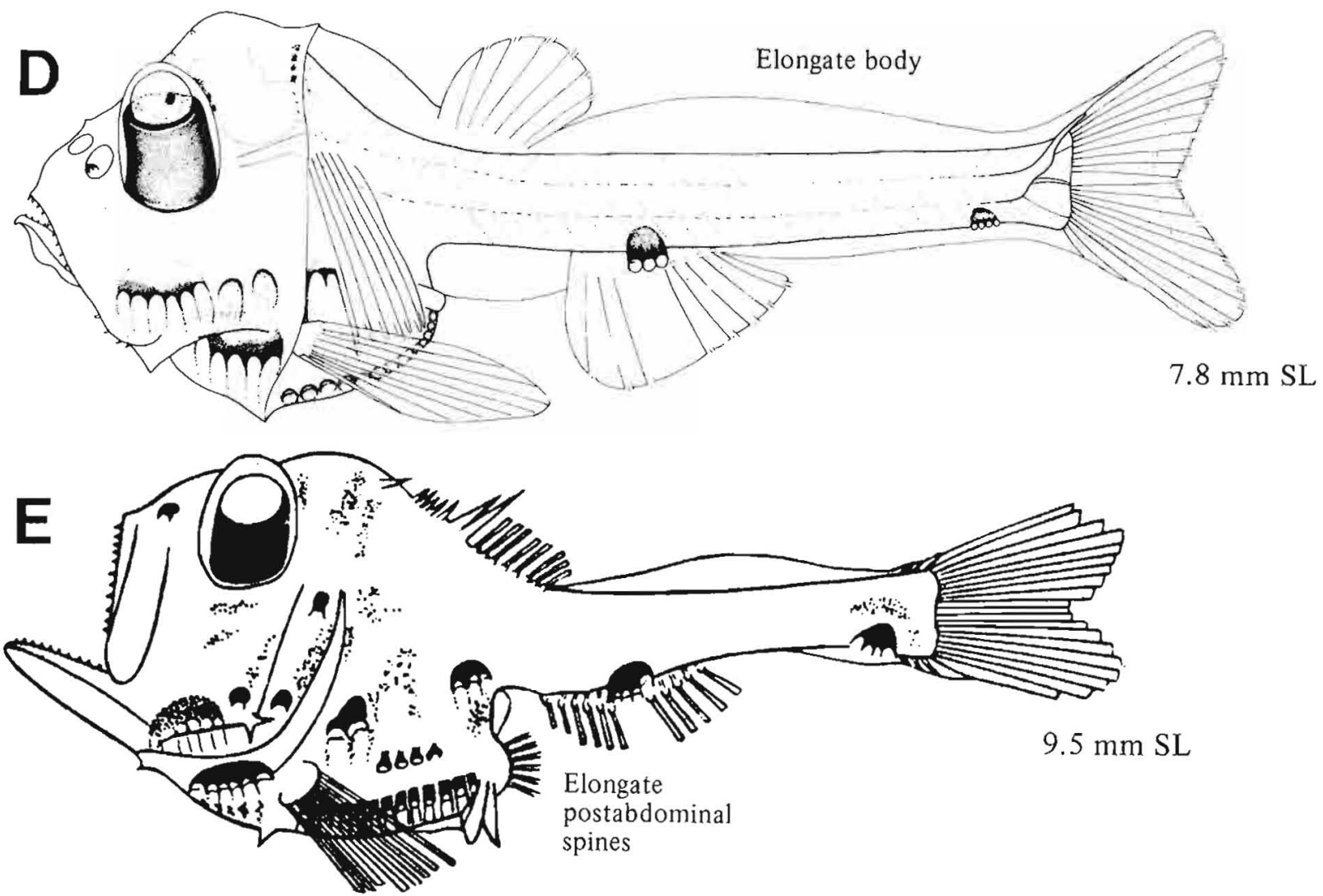

Figure A, Sanzo 1931a; B, Jespersen and Taning 1926; C, E, Belyanina 1984; D, Ahlstrom et al. 1984c (after Sanzo 1931a; A-B, D, Mediterranean specimens). 


\section{MERISTICS}

\begin{tabular}{ll} 
Vertebrae & Total: $35-\mathrm{X}-37$ \\
& Precaudal: X-X-X \\
& Caudal: X-X-X \\
Branchiostegal rays & $10-10-10$ \\
Caudal fin & $9-11,10+9,6$ \\
Pelvic fin & Abdominal \\
& R: $6-6-6$ \\
Dorsal fin & R: $9-9-9$ \\
Pectoral fin & R: $10-\mathrm{X}-11$ \\
Anal fin & R: $12-12-12$ \\
Gill rakers & U: $8-X-9$ \\
& \\
& \\
LIFE HISTORY $12-\mathrm{X}-14$ \\
\hline
\end{tabular}

$\begin{array}{ll}\text { Range } & \begin{array}{c}\text { South of southern California to } \\ \text { Brit. Col., } 48^{\circ} 30^{\prime}-55^{\circ} \mathrm{N} \\ \text { Epi-, meso-, and bathypelagic, } \\ 198-396 \mathrm{~m} ; 4066 \mathrm{~m}^{\mathrm{a}}\end{array} \\ \text { Ecology } & \begin{array}{l}\text { Oviparous, pelagic egg, pelagic } \\ \text { larvae }\end{array} \\ \text { ELH pattern } & \text { Season: } \\ \text { Spawning } & \begin{array}{l}\text { Area: } \\ \text { Mode: } \\ \text { Migration: }\end{array} \\ & \text { Range/function: } \\ \text { Fecundity } & \\ \text { Age at first maturity } & \\ \text { Longevity } & \end{array}$

\section{EARLY LIFE HISTORY DESCRIPTION}

\section{EGGS}

Diameter

No. of oil globules

Oil globule diameter

Yolk

Envelope

Hatch size

Incubation time/temp.

Pigment

Diagnostic characters

\section{LARVAE}

Preanal length

Length at flexion

Length at transformation

Sequence of fin development

Pigment

- Lightly pigmented, restricted to preanal body

Diagnostic characters

- Upper preopercular spine bent downward whereas same spine in $A$. sladeni is pointed slightly upward

- Pigmentation: Specimens described to $15 \mathrm{~mm} \mathrm{SL}$ appear to lack pigment on postanal body, specifically in the peduncle area

- Presence of frontal crest

\footnotetext{
${ }^{\mathrm{a}} \mathrm{Hart} 1973$
}

Ref: Ahistrom et al. 1984c, Belyanina 1984. 

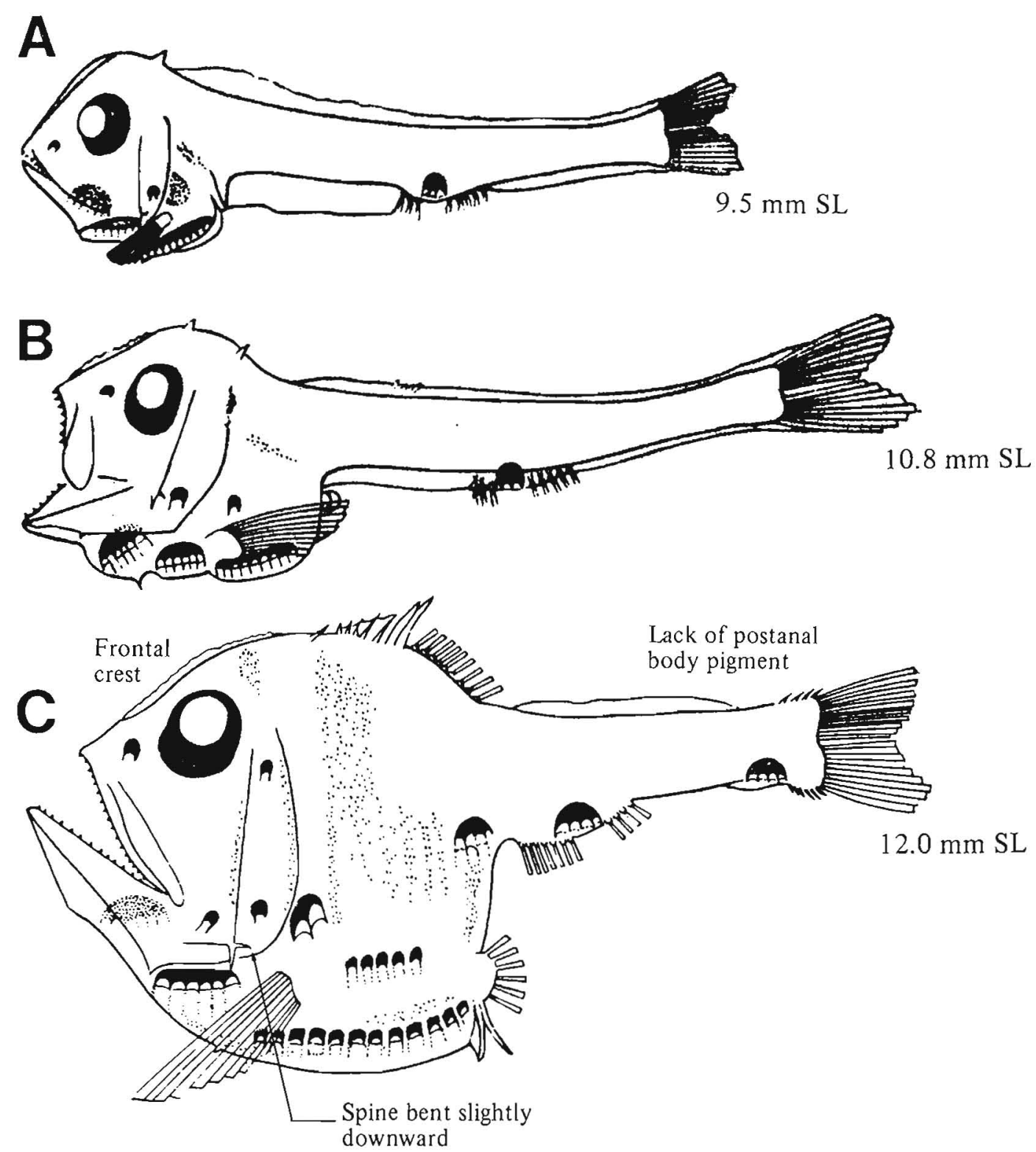

Figures A-C, Belyanina 1984 . 


\section{MERISTICS}

\begin{tabular}{|c|c|}
\hline \multirow[t]{3}{*}{ Vertebrae } & Total: $36-\mathrm{X}-38$ \\
\hline & Precaudal: $\mathrm{X}-\mathrm{X}-\mathrm{X}$ \\
\hline & Caudal: $X-X-X$ \\
\hline Branchiostegal rays & $10-10-10$ \\
\hline Caudal fin & $10,10+9,6$ \\
\hline \multirow[t]{2}{*}{ Pelvic fin } & Abdominal \\
\hline & $R: 6-6-6$ \\
\hline Dorsal fin & $\mathrm{R}: 9-9-9$ \\
\hline Pectoral fin & $\mathrm{R}: 10-\mathrm{X}-11$ \\
\hline Anal fin & $\mathrm{R}: 12-12-12$ \\
\hline Gill rakers & $\mathrm{U}: \mathrm{X}-\mathrm{X}-\mathrm{X}$ \\
\hline \multicolumn{2}{|l|}{ I_IFE HISTORY } \\
\hline Range & $\begin{array}{l}\text { South of southern California to } \\
\text { Oregon, } 42-46^{\circ} \mathrm{N}\end{array}$ \\
\hline Ecology & Epi- and mesopelagic, $101-610 \mathrm{~m}$ \\
\hline ELH pattern & $\begin{array}{l}\text { Oviparous, pelagic eggs, pelagic } \\
\text { larvae }\end{array}$ \\
\hline \multirow[t]{4}{*}{ Spawning } & Season: \\
\hline & Area: \\
\hline & Mode: \\
\hline & Migration: \\
\hline Fecundity & Range/function: \\
\hline Age at first maturity & \\
\hline Longevity & \\
\hline
\end{tabular}

\section{EARLY LIFE HISTORY DESCRIPTION}

\section{EGGS}

Diameter

No. of oil globules

Oil globule diameter

Yolk

Envelope

Hatch size

Incubation time/temp.

Pigment

Diagnostic characters

\section{LARVAE}

Preanal length $50-60 \%$ SL

Length at flexion

Length at transformation

Sequence of fin development

Caudal and pectorals, dorsal and anal, pelvics

\section{Pigment}

- Initially, appears anteriorly on body in patches and, with development, increases posteriorly

- Spot on peduncle does not appear as early as $A$. affinis, first appearing as a patch in larvae $>13 \mathrm{~mm} \mathrm{SL}$

Diagnostic characters

- Spines: Upper preopercular spine is developed (pointed slightly upward) and short frontal crests are present

Distinguished from $A$. affinis and $A$. hemigymnus by

- Combination of nine dorsal spines and SAB, PAN, $\mathrm{AN}$, and SC photophores not in continuous line 


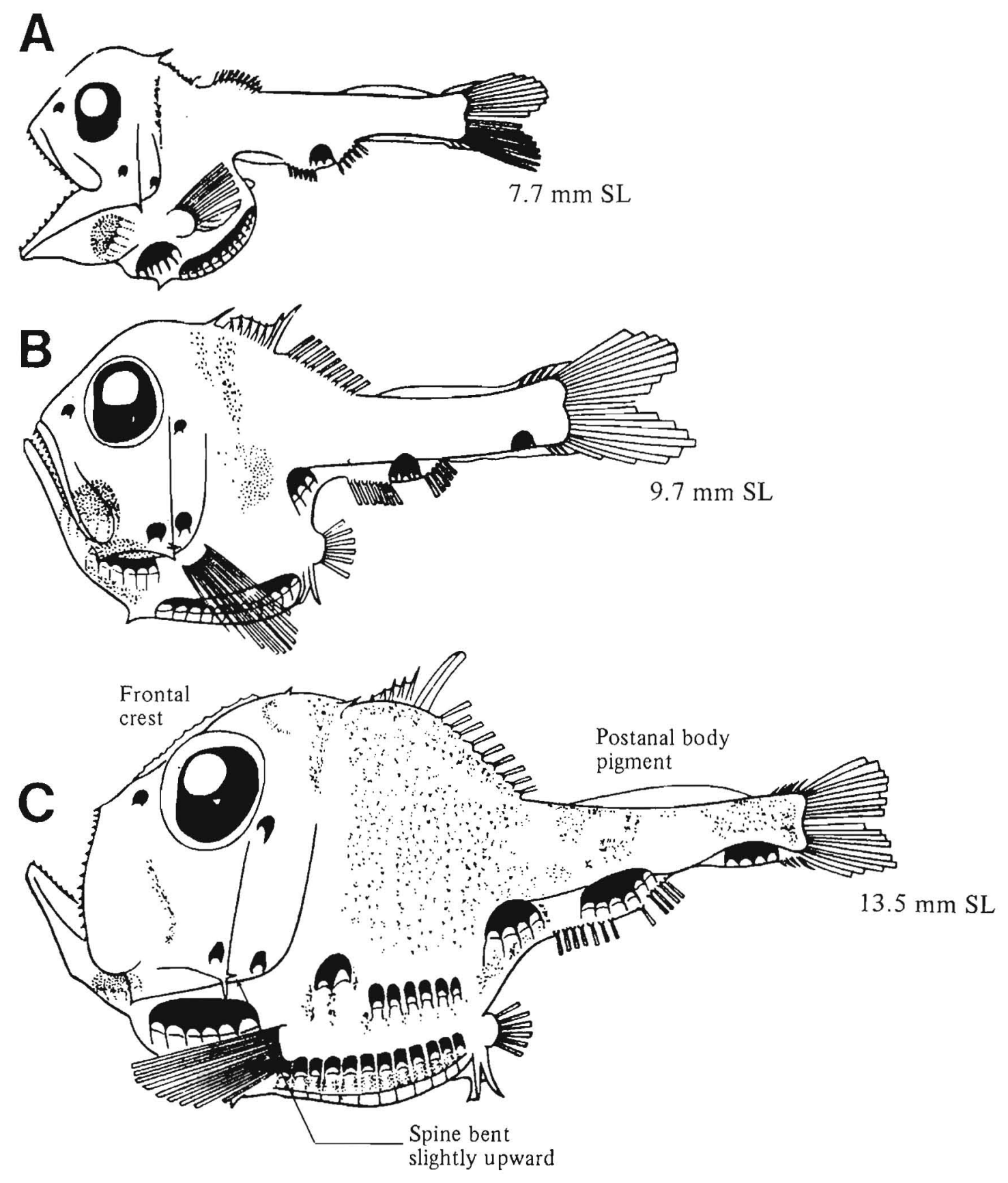

Figures A-C, Belyanina 1984 . 


\section{MERISTICS}

\section{S. diaphana Hermann 1781}

Vertebrae

Branchiostegal rays

Pelvic fin

Dorsal fin

Pectoral fin

Anal fin

Gill rakers
Total: $28-29-30$

Precaudal: X-X-X

Caudal: X-X-X

\section{6-6-6}

Abdominal

$\mathrm{R}$ : X-X-X

R: $9-10-11$

R: $10-\mathrm{X}-11$

$R: 13-14-16$

U: X-X-X

L: X-X-X

\section{MERISTICS}

Vertebrae

Branchiostegal rays

Pelvic fin

Dorsal fin

Pectoral fin

Anal fin

Gill rakers

\section{S. pseudobscura Baird 1971}

Total: $27-29-30$

Precaudal: X-X-X

Caudal: X-X-X

\section{6-6-6}

Abdominal

$\mathrm{R}: \mathrm{X}-\mathrm{X}-\mathrm{X}$

R: 9-10-11

R: $10-X-11$

R: $13-14-15$

$\mathrm{U}$ : X-X-X

\section{LIFE HISTORY}

$\begin{array}{ll}\text { Range } & \begin{array}{c}\text { South of southern California to } \\ \text { Brit. Col., } 48^{\circ} 30^{\prime}-55^{\circ} \mathrm{N}^{\mathrm{a}} \\ \text { Meso- and bathypelagic }\end{array} \\ \begin{array}{l}\text { Ecology } \\ \text { ELH pattern }\end{array} & \begin{array}{l}\text { Oviparous, eggs probably pelagic, } \\ \text { pelagic larvae }\end{array} \\ \text { Spawning } & \begin{array}{l}\text { Season: } \\ \text { Area: }\end{array} \\ & \text { Mode: } \\ & \text { Migration: } \\ \text { Fecundity } & \text { Range/function: } \\ \text { Age at first maturity } & \\ \text { Longevity } & \end{array}$

\footnotetext{
${ }^{a} S$. diaphana only to Oregon, $42-46^{\circ} \mathrm{N}$.

${ }^{b}$ Although not well documented, geographic variation among and within species is clearly present.

${ }^{c}$ A dramatic change in morphology occurs at transformation. Elongate larvae become deep-bodied juveniles. Body length cannot be associated with development.

${ }^{d}$ Badcock and Baird (1980) cite a personal communication from E.H. Ahlstrom stating that neither of the two forms of Sternoptyx spp. from the study area have caudal pigment. A figure of $S$. pseudodiaphana is provided as an example of an early larva with caudal pigment.
}

Ref: Badcock and Baird 1980, Belyanina 1983.

\section{EARLY LIFE HISTORY DESCRIPTION}

\section{EGGS}

Diameter

No. of oil globules

Oil globule diameter

Yolk

Envelope

Hatch size

Incubation time/temp.

Pigment

\section{Diagnostic characters}

LARVAE - Genus ${ }^{b}$

Preanal length

Length at flexion

Length at transformation $6-14 \mathrm{~mm} \mathrm{SL}^{\mathrm{c}}$

Sequence of fin Pectorals and caudal, anal, development dorsal and pelvics

Pigment

- Head and tip of lower jaw

- Peritoneum, becoming embedded with development

- Presence/absence of pigment on caudal peduncle; according to Belyanina (1983) some specimens of $S$. diaphana have pigment whereas specimens of $S$. pseudobscura have none ${ }^{d}$

Diagnostic characters

- Prior to metamorphosis, larvae of the two species are indistinguishable; both with spiny ridges on frontal and parietal bones, opercular and posttemporal spines

Distinguish S. pseudobscura juveniles from $S$. diaphana by

- Rounder body

- Posterior anal pterygiophores longer

- SAN higher in position

Photophores (see Table 10)

Order of development in Sternoptyx spp. larvae: SO, Br2, AB7, I4, SP3, PTO, AN1 followed by PAN, SC, PRO, and SAN in early juveniles; finally $\mathrm{SC}$. 


\section{STERNOPTYX}

A Sternoptyx sp.

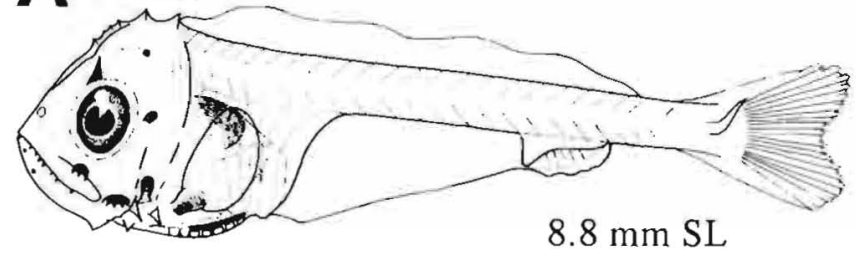

B S. pseudodiaphana

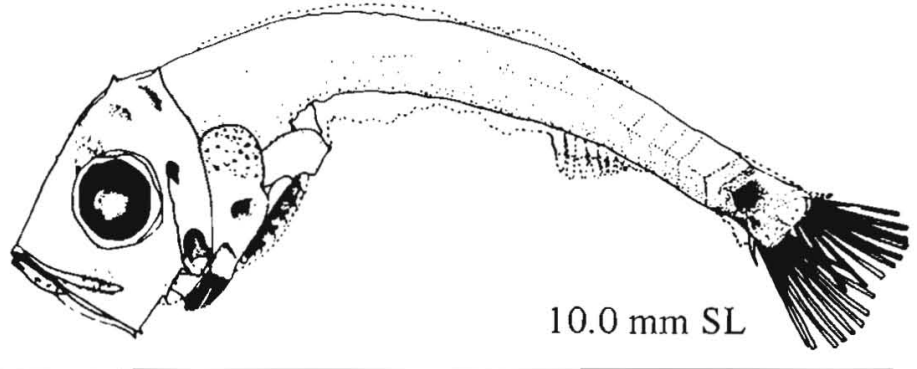

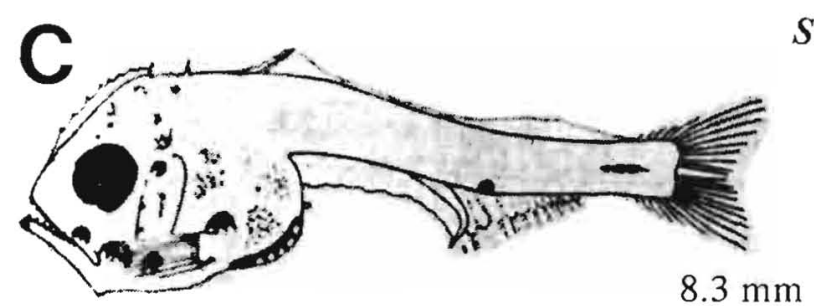

S. diaphana

D
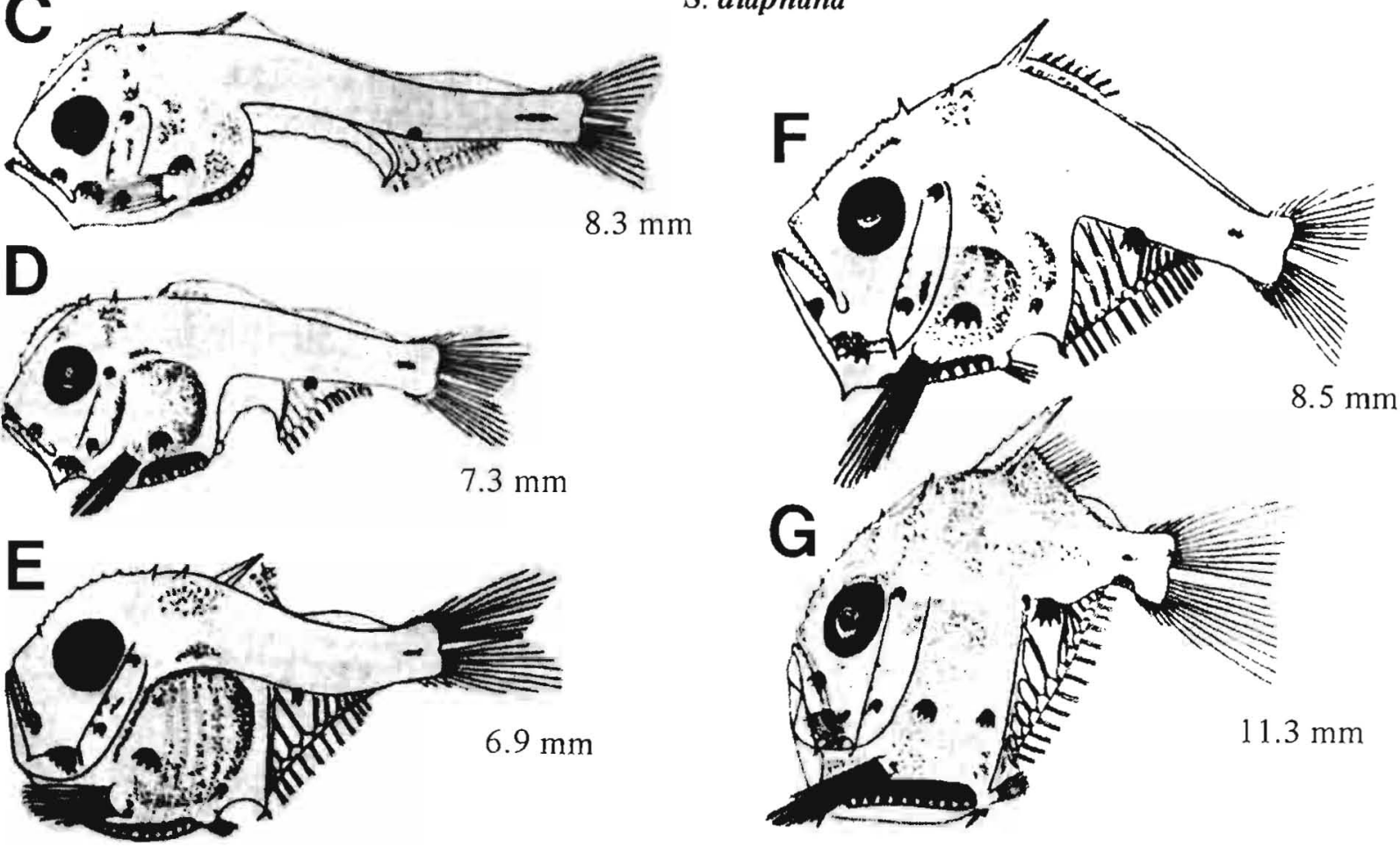

S. pseudobscura
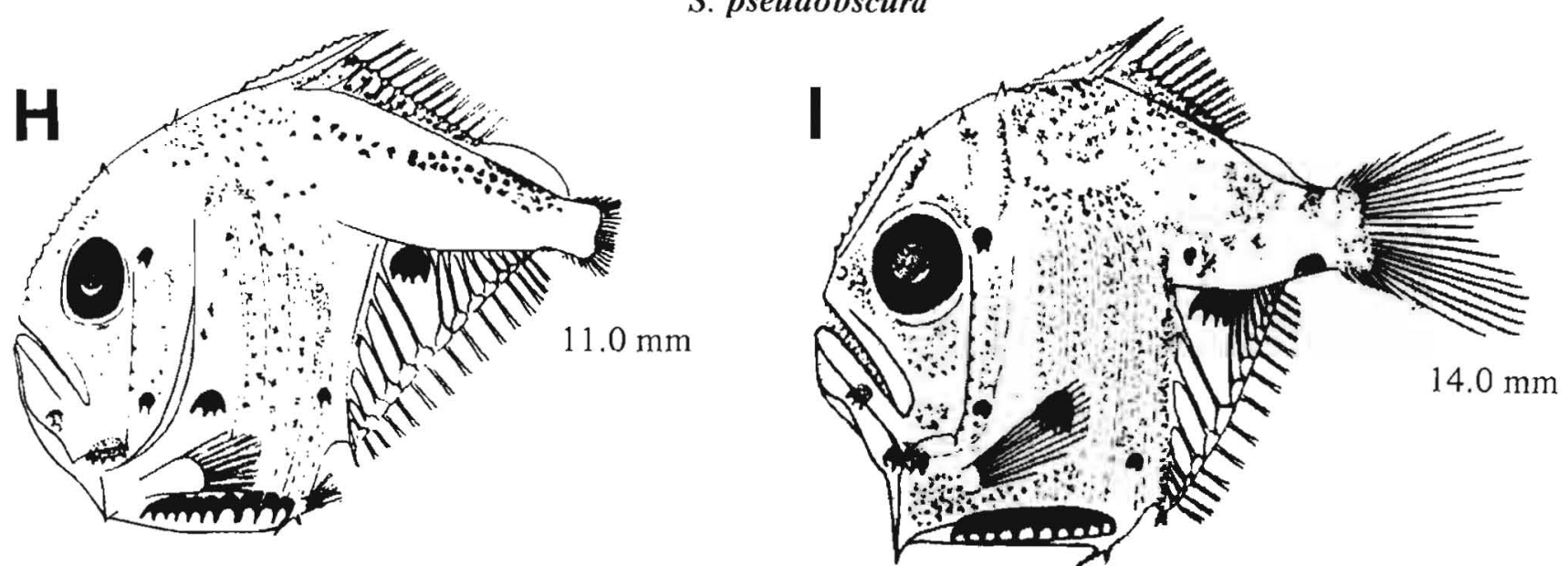

Figure A, Ahlstrom et al. 1984c; B, Badcock and Baird 1980 (eastern North Atlantic specimen); C-I, Belyanina 1984. 
MERISTICS

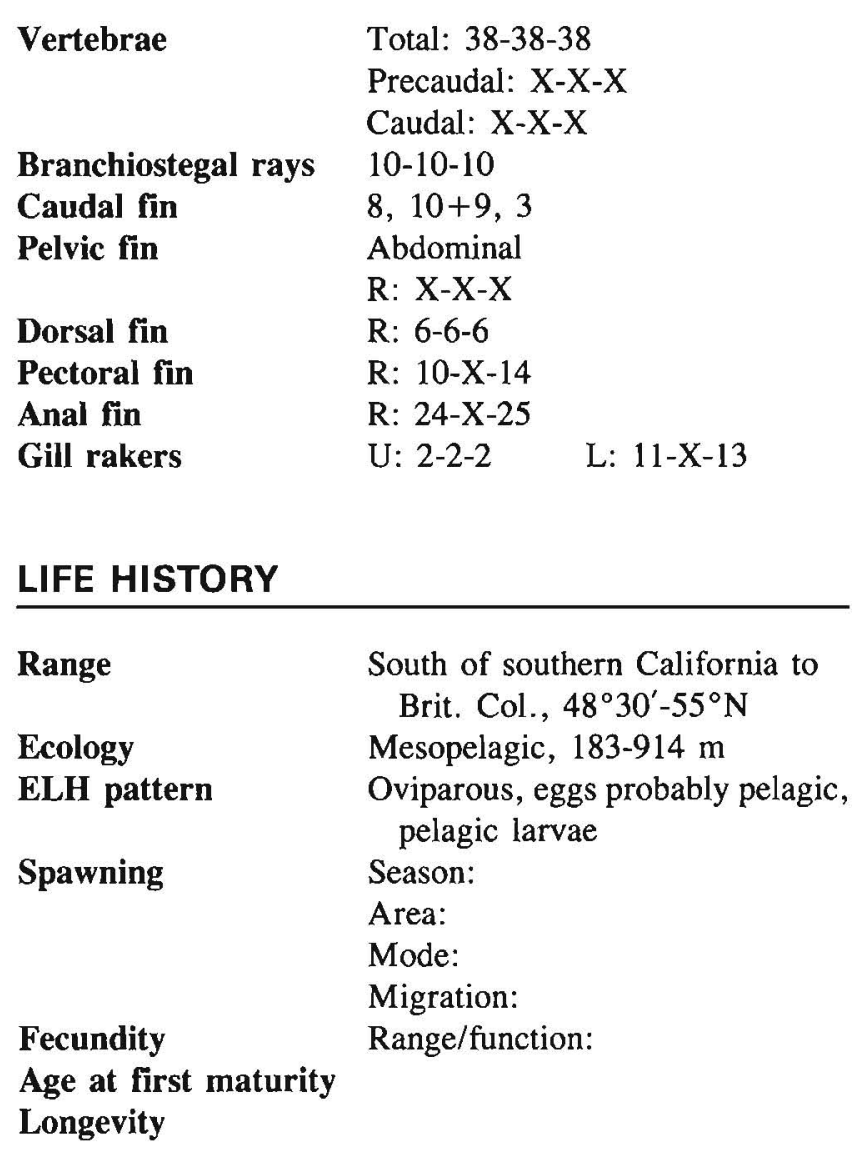

EARLY LIFE HISTORY DESCRIPTION

\section{EGGS}

Diameter

No. of oil globules

Oil globule diameter

Yolk

Envelope

Hatch size

Incubation time/temp.

Pigment

Diagnostic characters

\section{LARVAE}

Preanal length $\quad<50 \%$ SL (short gut)

Length at flexion

Length at transformation $\sim 45-50 \mathrm{~mm} \mathrm{SL}$

Sequence of fin development

Pigment

- Concentrated on preanal body in area below eye and over gut; smaller larvae generally unpigmented except over peritoneum

Diagnostic characters

- Larvae elongate

- Photophores begin formation $\sim 16.5 \mathrm{~mm}$ SL (see Table 15)

- Photophores in clusters with common bases

- Metamorphosis gradual with the initial formation of photophores in the BR and PV groups

Distinguished from Argyropelecus spp. and Sternoptyx spp. by

- Long anal fin (24-25 fin rays) and short dorsal fin (6 fin rays) 
Table 15

Development of photophores in Danaphos oculatus (Ahlstrom et al. 1984c).* See Table 9 for definitions of photophore groups.

\begin{tabular}{cccccccccc}
\hline \multirow{2}{*}{$\begin{array}{c}\text { Size } \\
\text { (mm SL) }\end{array}$} & ORB & OP & SO & BR & IP & PV & VAV & AC & OA \\
\cline { 2 - 8 } Adult & 1 & 3 & 0 & $(6)$ & $(3)+(4)$ & $(11)$ & $(5)$ & $(3)+16+(4)+1$ & 6 \\
16.5 & 0 & 0 & 0 & $(2)$ & 0 & 0 & 0 & 0 & 0 \\
16.5 & 0 & 0 & 0 & $(3)$ & 0 & $(3)$ & 0 & 0 & 0 \\
19.2 & 0 & 0 & 0 & $(4)$ & 0 & $(10)$ & 0 & 0 & 0 \\
21.0 & 1 & 1 & 0 & $(5)$ & $(2)+(4)$ & $(10 / 11)$ & 0 & $(2)+0+0+0$ & 0 \\
21.3 & 1 & 1 & 0 & $(4 / 5)$ & $(3)+(4)$ & $(10)$ & 0 & $(3)+0+(2)+0$ & 0 \\
21.8 & 1 & 2 & 0 & $(5)$ & $(3)+(4)$ & $(11)$ & $(2)$ & $(3)+8+(4)+0$ & 2 \\
24.2 & 1 & 2 & 0 & $(6)$ & $(3)+(4)$ & $(11)$ & $(2)$ & $(3)+9+(4)+0$ & 2 \\
\hline
\end{tabular}

*Parenthetical numbers indicate photophores found in common glands; nonparenthetical numbers indicate photophores are single.

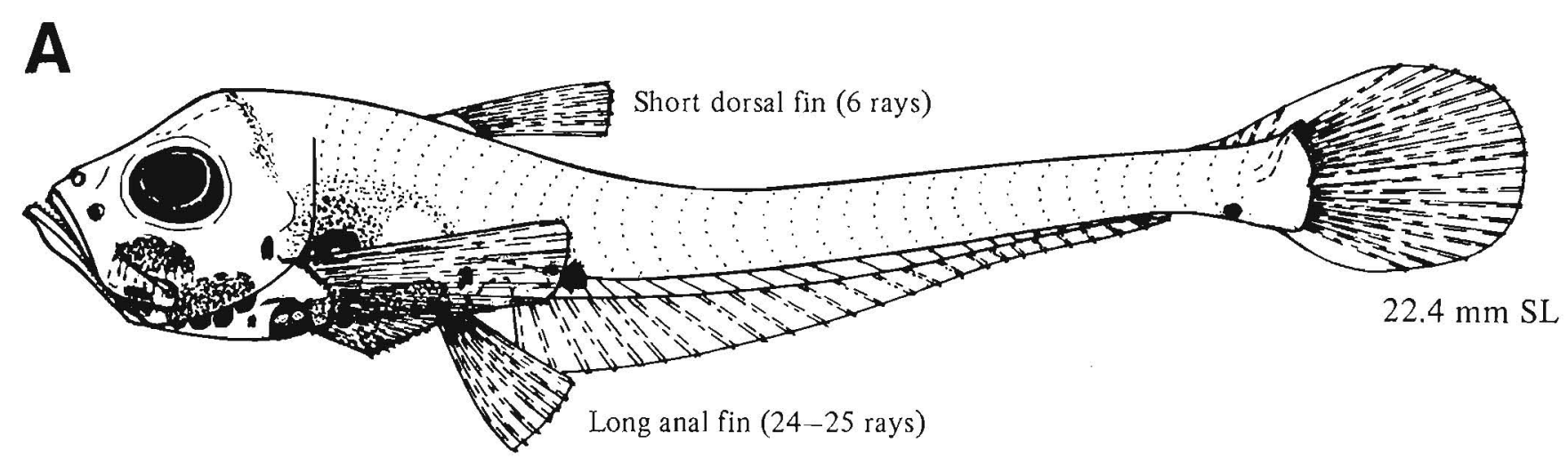

Figure A, NWAFC original (B. Vinter). 

Table 16

Meristic characters of stomioid genera. Most frequent count or range is followed by overall range or infrequent count in parentheses (Kawaguchi and Moser 1984, in part).

\begin{tabular}{|c|c|c|c|c|c|}
\hline \multirow[b]{2}{*}{ Taxon } & \multirow[b]{2}{*}{ Vertebrae } & \multicolumn{4}{|c|}{ Fin rays } \\
\hline & & Dorsal & Anal & Pectoral & Pelvic \\
\hline \multicolumn{6}{|l|}{ Chauliodontidae } \\
\hline Chauliodus & $51-62$ & $6,7(5-7)$ & $10-12(10-13)$ & $12,13(11-14)$ & $7(6-8)$ \\
\hline \multicolumn{6}{|c|}{ Melanostomiidae } \\
\hline Bathophilus & $38-45(33-50)$ & $13-16(9-18)$ & $15-16(9-18)$ & $1-37$ & $11-16(4-26)$ \\
\hline Eustomias & $56-69$ & $21-25(20-30)$ & $32-46$ & $0-13$ & $7(6-8)$ \\
\hline Opostomias & 60 & 21 & 24 & $1+4$ & 8 \\
\hline Tactostoma & $80-82$ & $14-16$ & $19-22$ & 0 & $8-10$ \\
\hline \multicolumn{6}{|l|}{ Malacosteidae } \\
\hline Aristostomias & $44-56$ & $18-26$ & $24-32$ & $6-10(3-17)$ & 6 \\
\hline \multicolumn{6}{|l|}{ Idiacanthidae } \\
\hline Idiacanthus & $79-85$ & $54-74$ & $34(33-39)$ & 0 & 6 \\
\hline
\end{tabular}

\section{Table 17}

Photophore counts of stomioid genera. Most frequent count or range is followed by overall range or infrequent count in parentheses (Kawaguchi and Moser 1984, in part). Photophore groups as defined by Ahlstrom et al. (1984c) (see Table 9).

\begin{tabular}{|c|c|c|c|c|c|c|}
\hline \multirow[b]{2}{*}{ Taxon } & \multicolumn{6}{|c|}{ Photophore groups } \\
\hline & IP & PV & VAV & $\mathrm{AC}$ & ov & VA \\
\hline \multicolumn{7}{|l|}{ Chauliodontidae } \\
\hline \multicolumn{7}{|l|}{ Melanostomiidae } \\
\hline Bathophilus & $5(4-6)$ & $12-18$ & $11-13(11-17)$ & $5-7 \quad(5-9)$ & $13-14(10-16)$ & $9-11(8-17)$ \\
\hline Eustomias & $7-8(9)$ & $27-33(24-36)$ & $13-17(11-21)$ & $17-23(15-25)$ & $26-33(24-37)$ & $13-18(12-22)$ \\
\hline Opostomias & $4+4$ & 27 & 17 & 16 & 27 & 17 \\
\hline Tactostoma & 8 & 46 & 19 & 12 & 43 & 18 \\
\hline \multicolumn{7}{|l|}{ Malacosteidae } \\
\hline Aristostomias & $5+3$ & $15-17(14-19)$ & $15-18$ & $9-11(12)$ & $16-19(14-20)$ & $15-17(14-18)$ \\
\hline \multicolumn{7}{|l|}{ Idiacanthidae } \\
\hline Idiacanthus & $\mathrm{IP}+\mathrm{PV}=31-36$ & & $16-18(15)$ & $13-18$ & $22-25$ & $31-35(30-36)$ \\
\hline
\end{tabular}

\section{Table 18}

Pigment characters and gut structure (NT $=$ not trailing, $\mathrm{T}=$ trailing freely) in larvae and transforming specimens of stomioids (Kawaguchi and Moser 1984, in part).

\begin{tabular}{|c|c|c|c|c|c|c|}
\hline Taxon & $\begin{array}{l}\text { Length } \\
\text { of larvae } \\
(\mathrm{mm})\end{array}$ & $\begin{array}{l}\text { Length of } \\
\text { transforming } \\
\text { specimens (mm) }\end{array}$ & $\begin{array}{c}\text { Dorsal myomere } \\
\text { melanophores } \\
\text { ( } n / \text { myomere) }\end{array}$ & $\begin{array}{l}\text { Epaxial myoseptum } \\
\text { melanophores }\end{array}$ & $\begin{array}{l}\text { Hypaxial myoseptum } \\
\text { melanophores }\end{array}$ & $\begin{array}{c}\text { Gut } \\
\text { structure }\end{array}$ \\
\hline \multicolumn{7}{|l|}{ Chauliodontidae } \\
\hline Chauliodus macouni & $38-49$ & $35-44$ & 0 & 0 & 0 & $\mathrm{NT}^{*}$ \\
\hline \multicolumn{7}{|l|}{ Melanostomiidae } \\
\hline Bathophilus flemingi & $2.9-23.8$ & - & 1 to several & 0 & 0 & NT \\
\hline Eustomias sp. & 33 & - & 7 total & 0 & 0 & $\mathrm{~T}$ \\
\hline Eustomias sp. & 13 & - & 7 total & 0 & 0 & $\mathbf{T}$ \\
\hline Eustomias spp. (4 types) & $6-45$ & - & $5-11$ total & 0 & 0 & $\mathrm{~T}$ \\
\hline Opostomias mitsuii & $15-21$ & - & 1 & $\begin{array}{c}0-1 \\
(2-3 \text { posteriorly) }\end{array}$ & $\begin{array}{c}1-2 \\
(3-5 \text { posteriorly) }\end{array}$ & NT \\
\hline Tactostoma macropus & $5-44$ & 49 & $0-1$ & 0 & $1-3$ & $\mathrm{NT}$ \\
\hline \multicolumn{7}{|l|}{ Malacosteidae } \\
\hline Aristostomias scintillans & $43-47$ & 45 & 14 total & 0 & 0 & $\mathrm{~T}$ \\
\hline \multicolumn{7}{|l|}{ Idiacanthidae } \\
\hline Idiacanthus antrostomus & $4.5-71$ & $67>$ & 0 & 0 & 1 & $\mathrm{~T}$ \\
\hline
\end{tabular}


MERISTICS

\begin{tabular}{|c|c|}
\hline Vertebrae & $\begin{array}{l}\text { Total: } 56-60-62 \\
\text { Precaudal: } 56-56-56 \\
\text { Caudal: } 4-4-4\end{array}$ \\
\hline Branchiostegal rays & $16-X-21$ \\
\hline Caudal fin & $X, 10+9, X$ \\
\hline Pelvic fin & $\begin{array}{l}\text { Abdominal } \\
\text { R: } 6-7-8\end{array}$ \\
\hline Dorsal fin & $R: 5-6-7$ \\
\hline Pectoral fin & $\mathrm{R}: 10-\mathrm{X}-13$ \\
\hline Anal fin & $\mathrm{R}: 10-\mathrm{X}-13$ \\
\hline Gill rakers & $\mathrm{U}: 3-3-3$ \\
\hline \multicolumn{2}{|l|}{ LIFE HISTORY } \\
\hline Range & $\begin{array}{l}\text { South of southern California to } \\
\text { Bering Sea, } 54-66^{\circ} \mathrm{N}\end{array}$ \\
\hline Ecology & $\begin{array}{l}\text { Epi-, meso-, and bathypelagic, } \\
76-4231 \mathrm{~m}\end{array}$ \\
\hline ELH pattern & $\begin{array}{l}\text { Oviparous, pelagic eggs, pelagic } \\
\text { larvae }\end{array}$ \\
\hline \multirow[t]{4}{*}{ Spawning } & Season: \\
\hline & Area: \\
\hline & Mode: \\
\hline & Migration: \\
\hline Fecundity & Range/function: \\
\hline Age at first maturity & \\
\hline Longevity & $>8 \mathrm{yr}^{\mathrm{a}}$ \\
\hline
\end{tabular}

\section{EARLY LIFE HISTORY DESCRIPTION}

\section{EGGS}

Diameter

No. of oil globules

Oil globule diameter

Yolk

Envelope

Hatch size

Incubation time/temp.

Pigment

- Pigment in caudal region of yolksac larvae, but soon disappears

Diagnostic characters

- Wide perivitelline space, $0.5 \mathrm{~mm}$

- Yolk segmented

- Large egg size

\section{LARVAE}

Preanal length

Length at flexion

Long, $>75 \%$ SL

Length at transformation

Sequence of fin development

$>15 \mathrm{~mm} \mathrm{SL}$

Adult $\sim 35-46 \mathrm{~mm} \mathrm{SL}^{\mathrm{b}}$

Dorsal, anal, and pelvics form late in postflexion larvae in adult position

Pigment

- According to Kawaguchi and Moser (1984), unpigmented except for fan-shaped caudal finfold in yolksac larvae. We have not collected any yolksac larvae and therefore have not observed this pigment. A figure of a yolksac C. sloani is included for comparison only.

Diagnostic characters

- Morphology: Small head, elliptical eyes, long gut, slender body

- Median finfold shape

- Lack of pigmentation

- Slightly trailing gut

"Fitch and Lavenberg 1968

${ }^{\mathrm{b}}$ Marked shrinkage at transformation.

Ref: Kawaguchi and Moser 1984. 

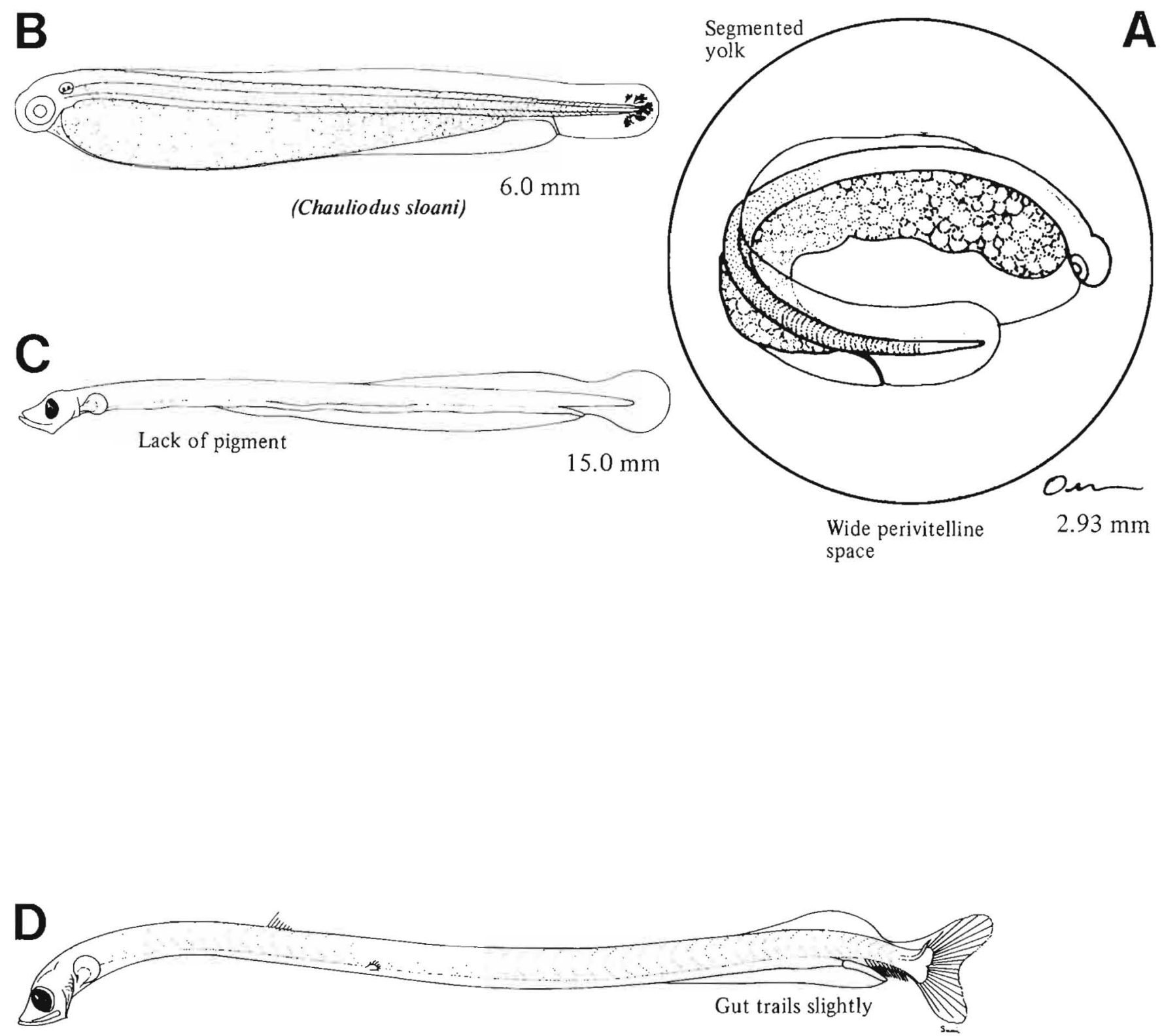

$45.2 \mathrm{~mm}$

Figure A, Matarese and Sandknop 1984; B, Mito 1961 (western Pacific specimen); C-D, Kawaguchi and Moser 1984. 


\section{MERISTICS}

\begin{tabular}{|c|c|}
\hline \multirow[t]{3}{*}{ Vertebrae } & Total: $44-46-48$ \\
\hline & Precaudal: $\mathrm{X}-\mathrm{X}-\mathrm{X}$ \\
\hline & Caudal: X-X-X \\
\hline Branchiostegal rays & $10-10-10$ \\
\hline Caudal fin & $X, 10+9, X$ \\
\hline \multirow[t]{2}{*}{ Pelvic fin } & Abdominal \\
\hline & $\mathrm{R}: 15-15-17$ \\
\hline Dorsal fin & $R: 15-15-16$ \\
\hline Pectoral fin & $\mathrm{R}:$ 4-5-7, jugular \\
\hline Anal fin & $\mathrm{R}: 16-17-17$ \\
\hline Gill rakers & $\mathrm{U}: \mathrm{X}-\mathrm{X}-\mathrm{X}$ \\
\hline \multicolumn{2}{|l|}{ LIFE HISTORY } \\
\hline Range & $\begin{array}{l}\text { South of southern California to } \\
\text { Brit. Col., } 48^{\circ} 30^{\prime}-55^{\circ} \mathrm{N}\end{array}$ \\
\hline Ecology & Meso - and bathypelagic \\
\hline ELH pattern & $\begin{array}{l}\text { Parity and eggs unknown, pelagic } \\
\text { larvae }\end{array}$ \\
\hline \multirow[t]{4}{*}{ Spawning } & Season: \\
\hline & Area: \\
\hline & Mode: \\
\hline & Migration: \\
\hline Fecundity & Range/function: \\
\hline $\begin{array}{l}\text { Age at first maturity } \\
\text { Longevity }\end{array}$ & \\
\hline
\end{tabular}

\section{EARLY LIFE HISTORY DESCRIPTION}

\section{EGGS}

Diameter

No. of oil globules

Oil globule diameter

Yolk

Envelope

Hatch size

Incubation time/temp.

Pigment

Diagnostic characters

\section{LARVAE \\ Preanal length $\quad>75 \%$ SL \\ Length at flexion \\ Length at transformation $\leqslant 25 \mathrm{~mm} \mathrm{SL}$ \\ Sequence of fin Dorsal, anal, and caudal development before pectorals and pelvics}

Pigment

- One to several melanophores per myomere along dorsum with opposing series along ventral surface

- Head, finfolds, median fins

- Anterodorsal surface of gut and on hindgut

- Pigment more concentrated in larger specimens

Diagnostic characters

- Deep bodied

- Large head, jaws

- Gut large (highly developed trailing s-shaped terminus)

- No midlateral pigment

- Large pigmented median finfolds

- Origin of dorsal and anal fin opposite each other

Ref: Kawaguchi and Moser 1984, Ozawa and Aono 1986. 


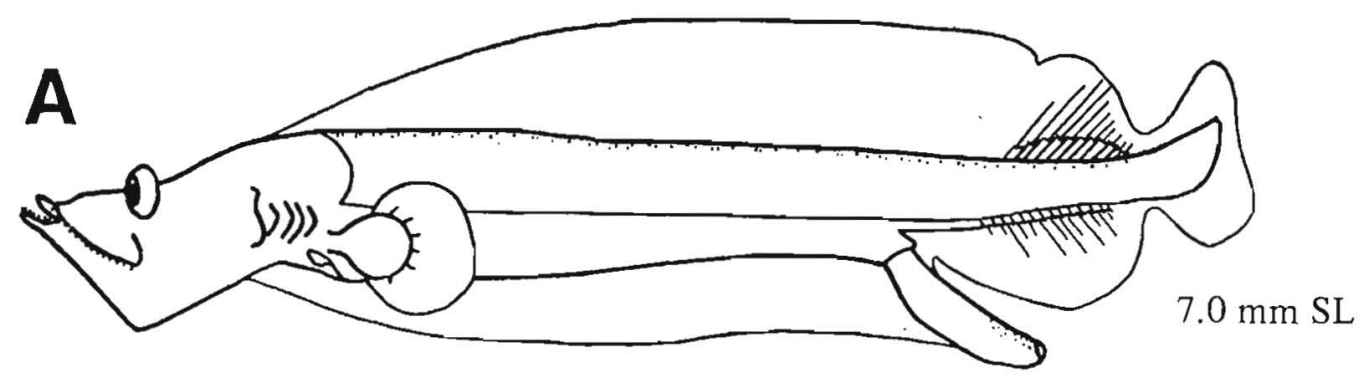

(Bathophilus sp.)

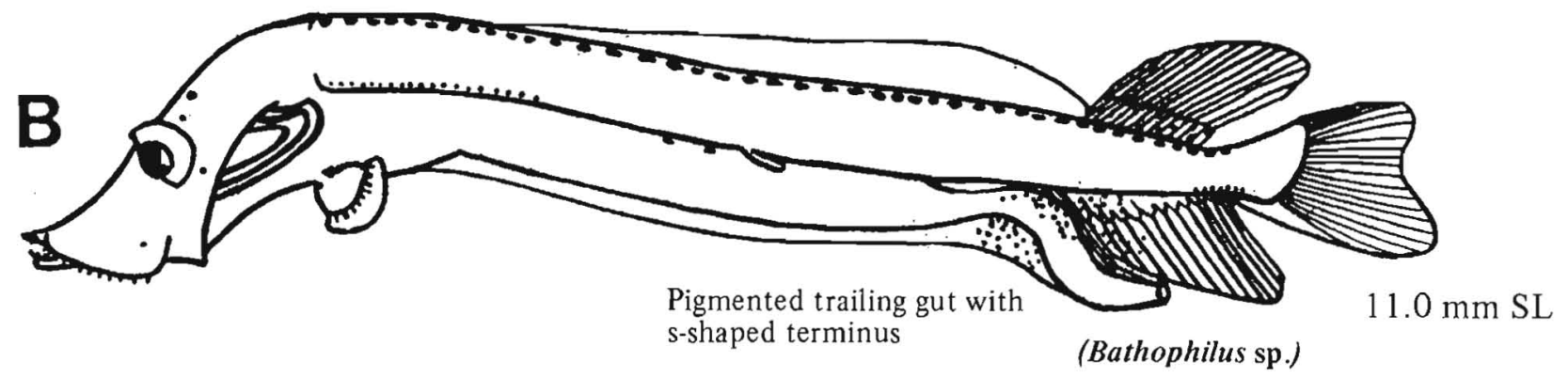

Pigment in finfolds

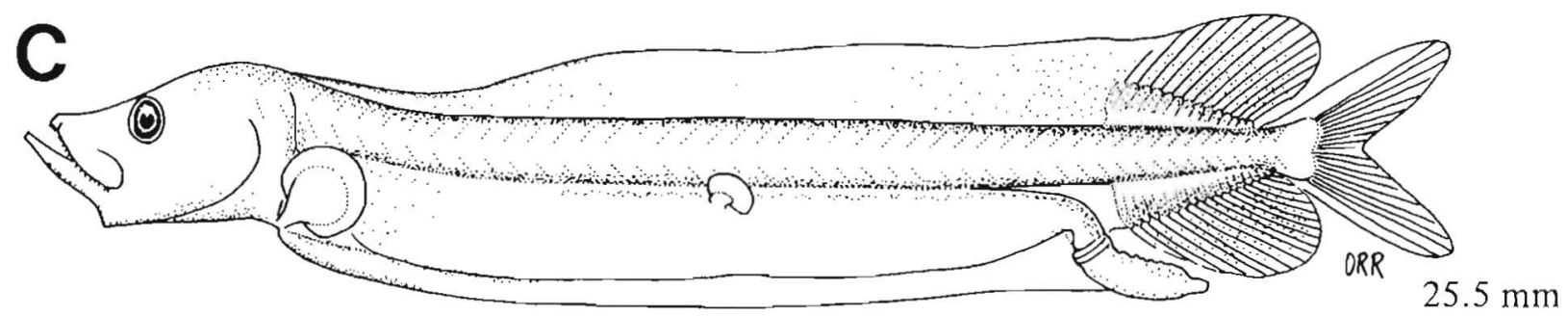

Figures A-B, Beebe and Crane 1939 (North Atlantic specimens); C, Kawaguchi and Moser 1984. 


\section{MERISTICS}

Vertebrae

Total: $72-\mathrm{X}-78$

Precaudal: $\mathrm{X}-\mathrm{X}-\mathrm{X}$

Caudal: X-X-X

Branchiostegal rays

Caudal fin

Pelvic fin

$\mathrm{X}-\mathrm{X}-\mathrm{X}$

$\mathrm{X}, 10+9, \mathrm{X}$

Abdominal?

$\mathrm{R}: 6-\mathrm{X}-8$

Dorsal fin

R: $20-X-30$

$\mathrm{R}: 0-\mathrm{X}-13$

Pectoral fin

Anal fin

Gill rakers

R: $32-X-46$

U: X-X-X L: X-X-X

\section{LIFE HISTORY}

$\begin{array}{ll}\text { Range } & \text { Oregon, } 42-46^{\circ} \mathrm{N} \\ \text { Ecology } & \\ \text { ELH pattern } & \begin{array}{l}\text { Parity and eggs unknown, pelagic } \\ \text { larvae }\end{array} \\ \text { Spawning } & \text { Season: } \\ & \text { Area: } \\ & \text { Mode: } \\ & \text { Migration: } \\ \text { Fecundity } & \text { Range/function: }\end{array}$

Age at first maturity

Longevity

\section{EARLY LIFE HISTORY DESCRIPTION}

\section{EGGS}

Diameter

No. of oil globules

Oil globule diameter

Yolk

Envelope

Hatch size

Incubation time/temp.

Pigment

\section{Diagnostic characters}

\author{
LARVAE - Genus \\ Preanal length \\ $>75 \%$ SL (trailing gut) \\ Length at flexion \\ Length at transformation $\sim 45 \mathrm{~mm} \mathrm{SL}$ \\ Sequence of fin \\ development \\ Pigment \\ - 5-11 large melanophores along dorsal midline \\ - Lower jaw symphysis
}

Diagnostic characters

- Morphology: Slender body, head elongate and flat

- Gut: Long, slender, deflected ventrad at anal fin origin, trailing from body

- Dorsal pigment patches

- Origin of dorsal fin behind that of anal fin

Distinguished from Aristostomias scintillans (p. 118) by

- Unpigmented gut

- Lack of ventral body pigment

- $<20$ dorsal spots (5-11)

${ }^{\text {a }}$ Possibly 1-4 species in the area, although only one larval form has been collected. Ref: Kawaguchi and Moser 1984, Ozawa and Aono 1986. 


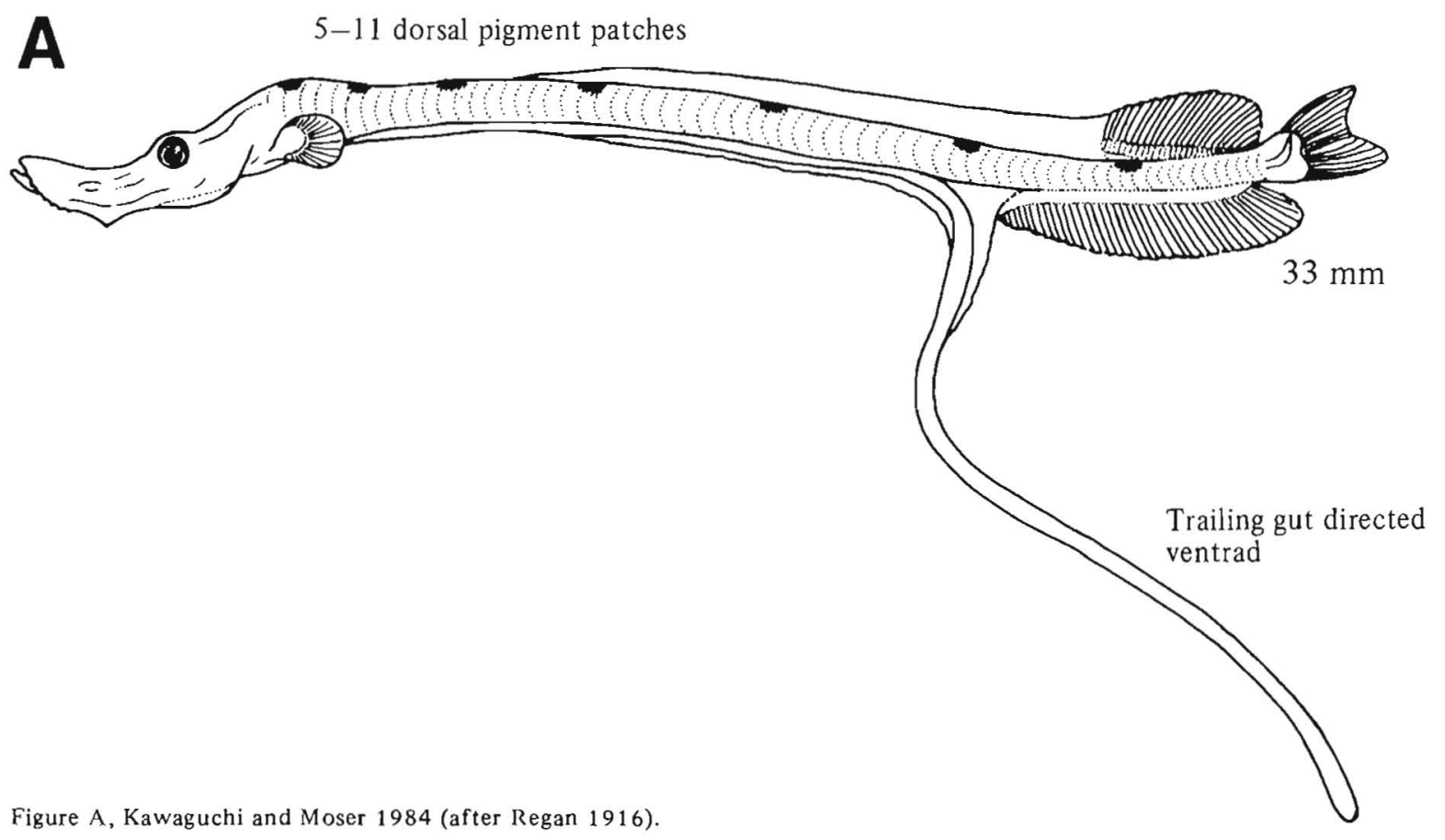




\section{MERISTICS}

\begin{tabular}{|c|c|}
\hline Vertebrae & $\begin{array}{l}\text { Total: } \mathrm{X}-\mathrm{X}-\mathrm{X} \\
\text { Precaudal: } \mathrm{X}-\mathrm{X}-\mathrm{X} \\
\text { Caudal: } \mathrm{X}-\mathrm{X}-\mathrm{X}\end{array}$ \\
\hline Branchiostegal rays & $10-X-15$ \\
\hline Caudal fin & $X, 10+9, X$ \\
\hline Pelvic fin & $\begin{array}{l}\text { Abdominal } \\
\text { R: } 7-X-8\end{array}$ \\
\hline Dorsal fin & $\mathrm{R}: 21-\mathrm{X}-23$ \\
\hline Pectoral fin & $\mathrm{R}: 5-5-5$ \\
\hline Anal fin & $\mathrm{R}: 21-\mathrm{X}-24$ \\
\hline Gill rakers & $\mathrm{U}: 2-2-2$ \\
\hline \multicolumn{2}{|l|}{ LIFE HISTORY } \\
\hline Range & $\begin{array}{l}\text { S. California, } 32-34^{\circ} \mathrm{N} \text {, to } \\
\text { Brit. Col., } 48^{\circ} 30^{\prime}-55^{\circ} \mathrm{N}\end{array}$ \\
\hline Ecology & Epi- and mesopelagic \\
\hline ELH pattern & $\begin{array}{l}\text { Parity and eggs unknown, pelagic } \\
\text { larvae }\end{array}$ \\
\hline Spawning & Season: \\
\hline & $\begin{array}{l}\text { Area: } \\
\text { Mode: } \\
\text { Migration: }\end{array}$ \\
\hline $\begin{array}{l}\text { Fecundity } \\
\text { Age at first maturity } \\
\text { Longevity }\end{array}$ & Range/function: \\
\hline
\end{tabular}

\section{EARLY LIFE HISTORY DESCRIPTION}

\section{EGGS}

Diameter

No. of oil globules

Oil globule diameter

Yolk

Envelope

Hatch size

Incubation time/temp.

Pigment

Diagnostic characters

\section{LARVAE}

Preanal length

Length at flexion

Length at transformation

Sequence of fin development
Caudal, dorsal, and anal before pectorals and pelvics
Pigment

- One expanded melanophore per myomere along dorsum

- 1-2 melanophores on each hypaxial myoseptum

- Area under dorsal fin appears banded

- Dorsally on head

- Gill arch

- Gut terminus

Diagnostic characters

- Morphology

-Moderately deep body

- Large mouth

- Large finfold

-Elongate sloping snout

-Eyes small

-No trailing gut

- Pigment pattern

- Origin of dorsal fin slightly before that of anal fin

-13-15 myomeres between pelvic and anal fins 


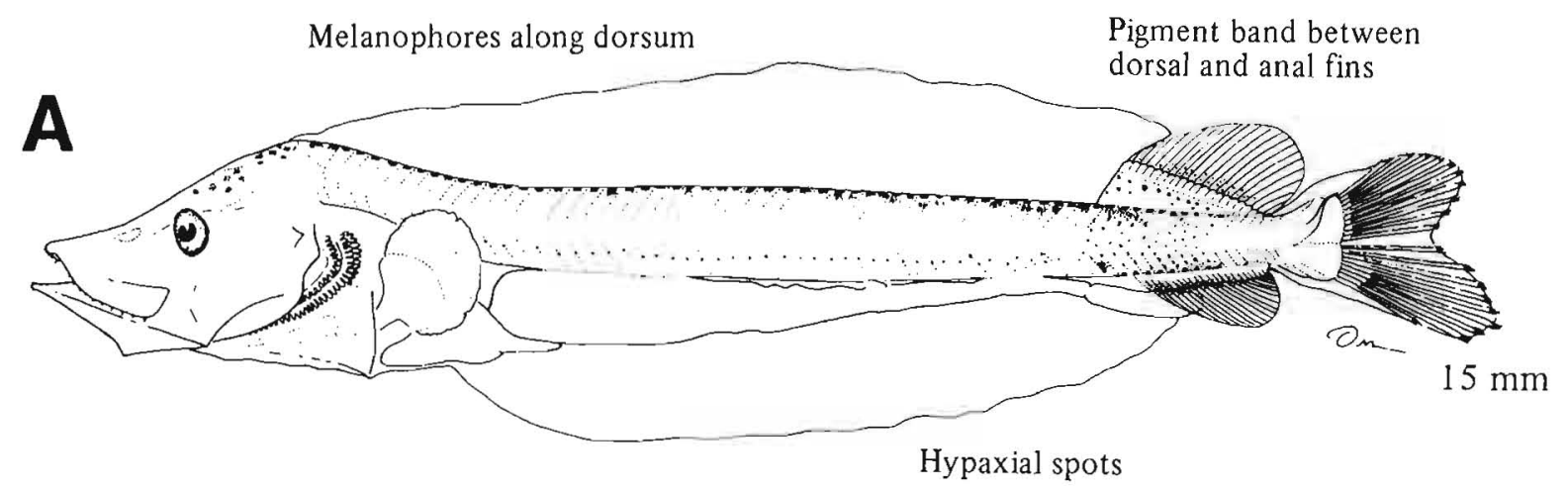

Figure A, Kawaguchi and Moser 1984 (western Pacific specimen). 


\section{MERISTICS}

\begin{tabular}{ll}
\hline Vertebrae & Total: $80-\mathrm{X}-82$ \\
& $\begin{array}{l}\text { Precaudal: X-X-X } \\
\text { Caudal: X-X-X }\end{array}$ \\
Branchiostegal rays & $13-13-13$ \\
Caudal fin & $\mathrm{X}, 10+9, \mathrm{X}$ \\
Pelvic fin & Abdominal \\
& $\mathrm{R}: 8-9-10$ \\
Dorsal fin & R: $14-\mathrm{X}-17$ \\
Pectoral fin & Absent in adults \\
Anal fin & R: $19-19-22$ \\
Gill rakers & $\mathrm{U}: \mathrm{X}-\mathrm{X}-\mathrm{X}$ \\
& \\
& \\
LIFE HISTORY & \\
\hline
\end{tabular}

\section{EARLY LIFE HISTORY DESCRIPTION}

\section{EGGS}

Diameter

No. of oil globules $1.39-1.54 \mathrm{~mm}$

Oil globule diameter

Yolk

Envelope

Hatch size

Incubation time/temp.

Pigment

\section{Diagnostic characters}

- Oil globule large and ventral

- Egg round

\section{LARVAE}

Preanal length $\quad>75 \%$ SL

Length at flexion

Length at transformation $44-49 \mathrm{~mm} \mathrm{SL}$

Sequence of fin development

Caudal, dorsal and anal, pectorals and pelvics

\section{Pigment}

- In early larvae, one melanophore per myomere along dorsum and 1-3 melanophores on hypaxial myosepta; postflexion larvae gradually lose the dorsal then ventral melanophores

- Lower jaw, isthmus, pectoral fin base, cleithrum, gut terminus, caudal peduncle

Diagnostic characters

- Morphology: Body elongate and flat, gut slender, finfold moderate

- Pectoral fin lost at transformation

- Decrease in pigment with development

- No trailing gut

- Finfolds unpigmented

Distinguished from Chauliodus macouni (p. 108) by

- Presence of pigment

- Posterior position of dorsal fin (origin of dorsal and anal fin opposite each other)

\footnotetext{
${ }^{2}$ Fisher and Pearcy 1983

Ref: Kawaguchi and Moser 1984, Ozawa and Aono 1986.
} 


\section{A}

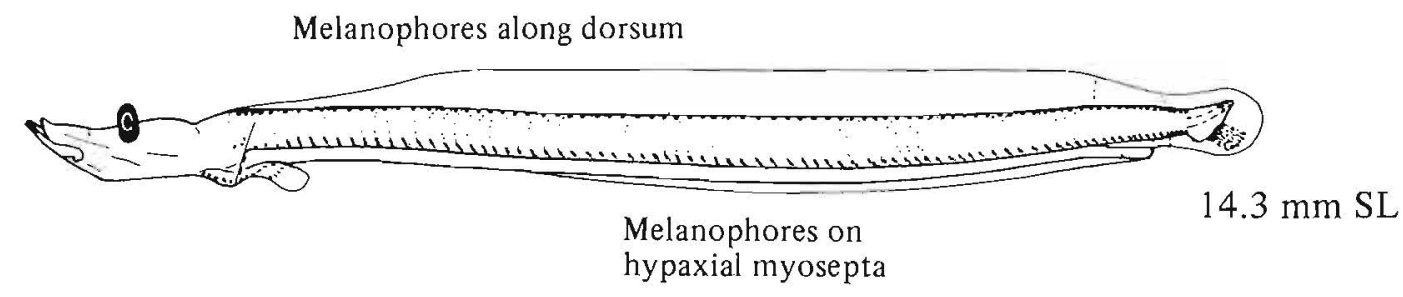

B

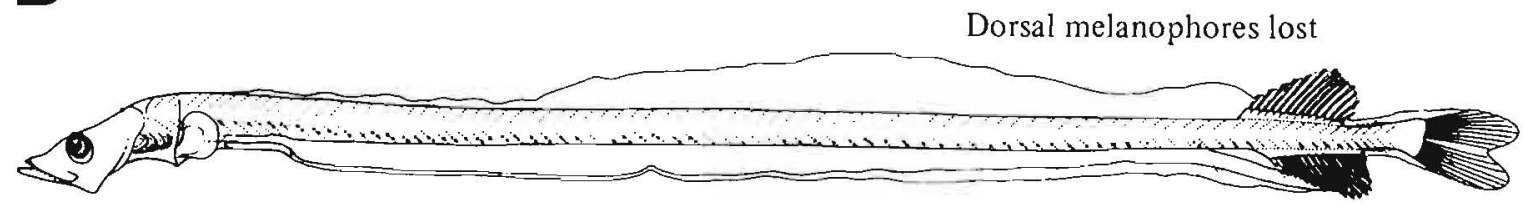

Figure A, NWAFC original (B. Vinter); B, Kawaguchi and Moser 1984. 


\section{MERISTICS}

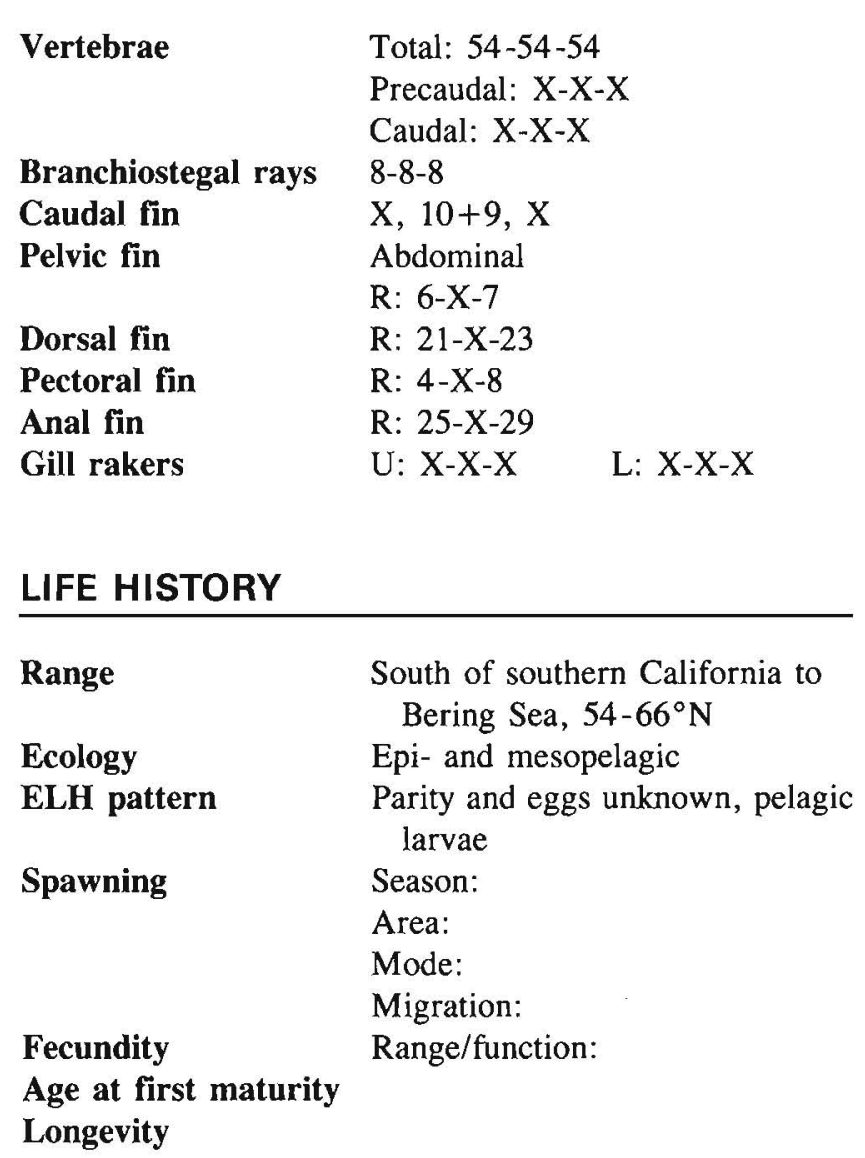

\section{EARLY ILIFE HISTORY DESCRIPTION}

\section{EGGS}

Diameter

No. of oil globules

Oil globule diameter

Yolk

Envelope

Hatch size

Incubation time/temp.

Pigment

Diagnostic characters

\section{LARVAE}

Preanal length

Length at flexion

Length at transformation $45 \mathrm{~mm} \mathrm{SL}$

Sequence of fin Dorsal, anal, and caudal; development pectorals, pelvics

Pigment

- Series of paired melanophores along dorsum; initially 14 pairs which increase in number with development

- Paired ventral series develops initially posteriorly and increases in number with development

- Brain, snout, lower jaw, otic region, gular-isthmus region, caudal fin, gut

\section{Diagnostic characters}

- Morphology: Body and gut slender, trailing gut, large flat head, finfold moderate

Distinguished from Eustomias spp. (p. 112) by

- Pigment on trailing gut

- Pigment along ventral midline 


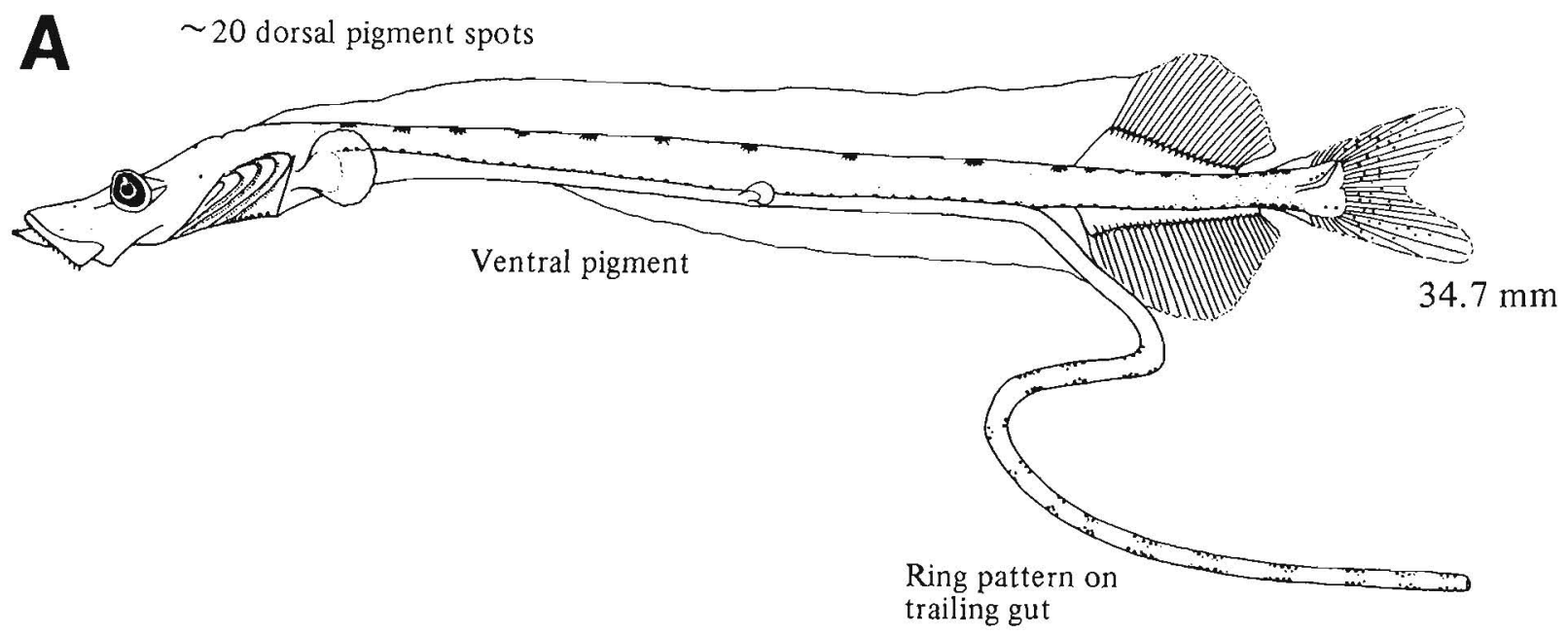

Figure A, Kawaguchi and Moser 1984. 


\section{MERISTICS}

\begin{tabular}{|c|c|}
\hline \multirow{3}{*}{ Vertebrae } & Total: $81-X-83$ \\
\hline & Precaudal: $\mathrm{X}-\mathrm{X}-\mathrm{X}$ \\
\hline & Caudal: X-X-X \\
\hline Branchiostegal rays & $12-X-18$ \\
\hline Caudal fin & $X, 10+9, X$ \\
\hline \multirow[t]{2}{*}{ Pelvic fin $^{\mathrm{a}}$} & Abdominal \\
\hline & $R: 6-6-6$ \\
\hline Dorsal fin & R: $54-X-66$ \\
\hline Pectoral fin & Absent in adults \\
\hline Anal fin & $\mathrm{R}: 28-\mathrm{X}-43$ \\
\hline Gill rakers & $\mathrm{U}: \mathrm{X}-\mathrm{X}-\mathrm{X}$ \\
\hline \multicolumn{2}{|l|}{ LIFE HISTORY } \\
\hline Range & $\begin{array}{l}\text { South of southern California to } \\
\text { N. California, } 38-42^{\circ} \mathrm{N}\end{array}$ \\
\hline Ecology & Meso- and bathypelagic \\
\hline ELH pattern & $\begin{array}{l}\text { Parity and eggs unknown, pelagic } \\
\text { larvae }\end{array}$ \\
\hline \multirow[t]{4}{*}{ Spawning } & Season: \\
\hline & Area: \\
\hline & Mode: \\
\hline & Migration: \\
\hline Fecundity & Range/function: $14,000^{a}$ \\
\hline \multicolumn{2}{|l|}{ Age at first maturity } \\
\hline Longevity & $>6 \mathrm{yr}$ (females) ${ }^{\mathrm{a}}$ \\
\hline & $<1$ yr $(\text { males })^{\mathrm{a}}$ \\
\hline
\end{tabular}

\section{EARLY LIFE HISTORY DESCRIPTION}

\section{EGGS}

Diameter

No. of oil globules

Oil globule diameter

Yolk

Envelope

Hatch size

Incubation time/temp.

Pigment

Diagnostic characters

\section{LARVAE}

Preanal length

Length at flexion

Length at transformation $35-70 \mathrm{~mm} \mathrm{SL}$

Sequence of fin Caudal, dorsal and anal, development $\quad$ pectorals, ${ }^{\mathrm{b}}$, pelvics $^{\mathrm{c}}$

Pigment - Genus

- Melanophore on posterior margin of each hypaxial myomere

- Isthmus

- Series along trailing gut

Diagnostic characters - Genus

- Morphology

-Extremely slender body

-Elongate flat head

-Elliptical eyes on stalks with cartilaginous support rods $(27 \% \mathrm{BL})$

-Trailing gut

-Small finfold

Distinguished from 1 . fasciola by

- Longer eye stalk and trailing gut

Since specimens are often damaged and pigment patterns are similar, the two species may be difficult to separate. In addition to the above characters, the two species differ in size at various stages of development. I. antrostomus larvae are 4.5-71.0 $\mathrm{mm} \mathrm{SL}$, transforming at sizes $>67 \mathrm{~mm} \mathrm{SL}$. I. fasciola larvae are $16-28 \mathrm{~mm} \mathrm{SL}$, transforming at sizes between 35 and $48 \mathrm{~mm} \mathrm{SL}$.

\footnotetext{
${ }^{a}$ Fitch and Lavenberg 1968

${ }^{b}$ Pectoral fins lost at transformation.

${ }^{c}$ Pelvic fins develop in transforming females.

Ref: Kawaguchi and Moser 1984.
} 


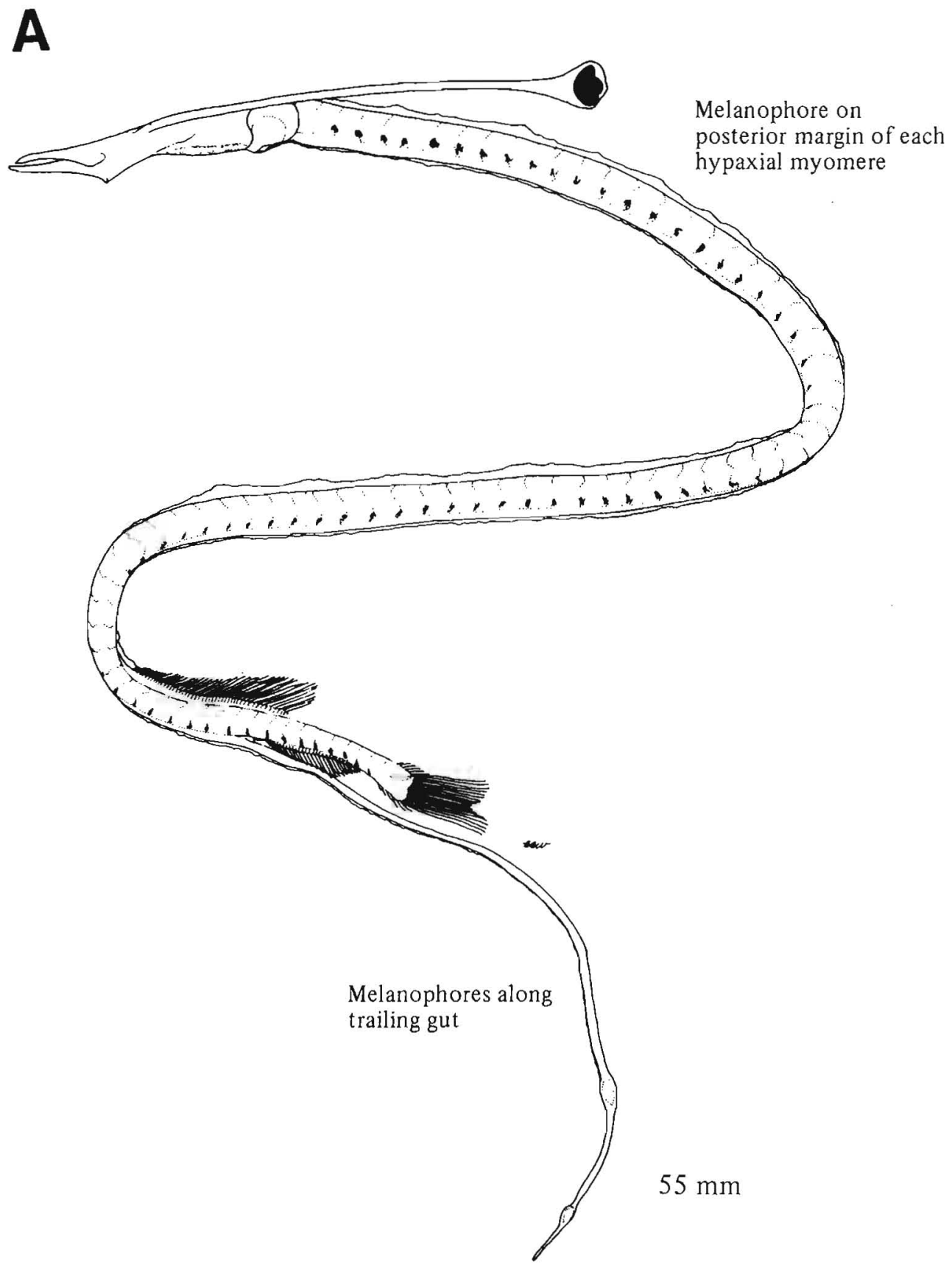




\section{MERISTICS}

\begin{tabular}{|c|c|}
\hline \multirow[t]{3}{*}{ Vertebrae } & Total: $\mathrm{X}-\mathrm{X}-\mathrm{X}$ \\
\hline & Precaudal: X-X-X \\
\hline & Caudal: X-X-X \\
\hline Branchiostegal rays & $12-X-18$ \\
\hline Caudal fin & $\mathrm{X}, 10+9, \mathrm{X}$ \\
\hline \multirow[t]{2}{*}{ Pelvic fin } & Abdominal \\
\hline & $R: 6-6-6$ \\
\hline Dorsal fin & $R: 56-X-77$ \\
\hline Pectoral fin & Absent in adults \\
\hline Anal fin & $\mathrm{R}: 38-\mathrm{X}-54$ \\
\hline Gill rakers & $\mathrm{U}: \mathrm{X}-\mathrm{X}-\mathrm{X} \quad \mathrm{L}: \mathrm{X}-\mathrm{X}-\mathrm{X}$ \\
\hline \multicolumn{2}{|l|}{ LIFE HISTORY } \\
\hline Range & $\begin{array}{l}\text { South of southern California to } \\
\text { Oregon, } 42-46^{\circ} \mathrm{N}\end{array}$ \\
\hline Ecology & Meso- and bathypelagic \\
\hline ELH pattern & $\begin{array}{l}\text { Parity and eggs unknown, pelagic } \\
\text { larvae }\end{array}$ \\
\hline \multirow[t]{4}{*}{ Spawning } & Season: \\
\hline & Area: \\
\hline & Mode: \\
\hline & Migration: \\
\hline Fecundity & Range/function: \\
\hline $\begin{array}{l}\text { Age at first maturity } \\
\text { Longevity }\end{array}$ & \\
\hline
\end{tabular}

\section{EARLY LIFE HISTORY DESCRIPTION}

\section{EGGS}

Diameter

No. of oil globules

Oil globule diameter

Yolk

Envelope

Hatch size

Incubation time/temp.

Pigment

Diagnostic characters

Ref: Kawaguchi and Moser 1984. 


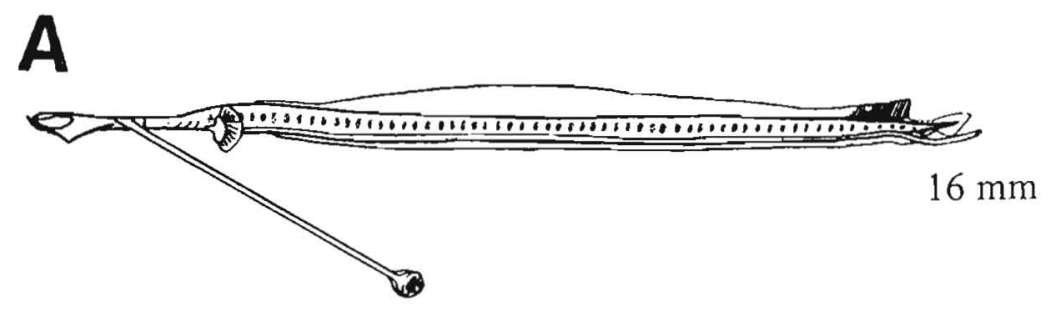

B

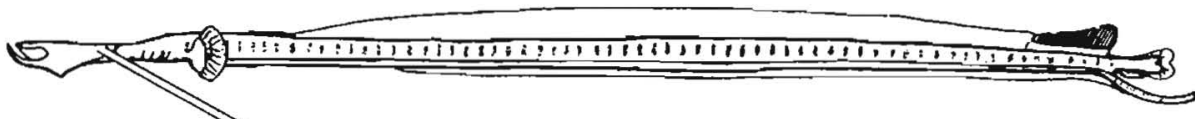

$25 \mathrm{~mm}$

C

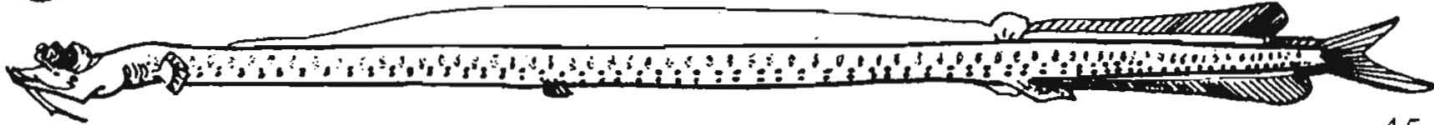

$45 \mathrm{~mm} \mathrm{SL}$ ?

D (50)

$40 \mathrm{~mm}$ SL ${ }^{\circ}$

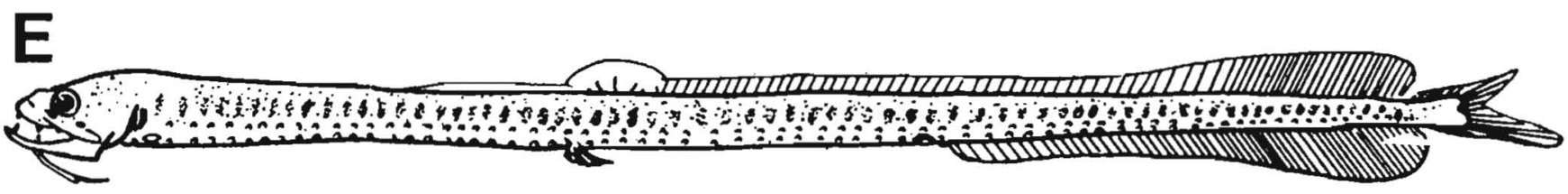

$48 \mathrm{~mm} \mathrm{SL}$ ?

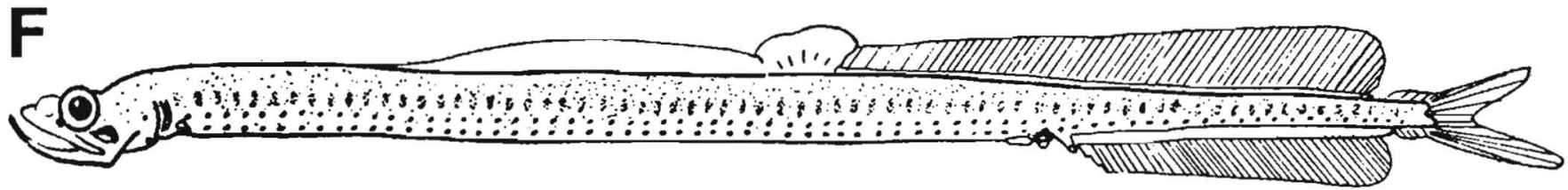

$35 \mathrm{~mm} \mathrm{SL} \mathrm{ơ}$

Figures A-F, Beebe 1934 (North Atlantic specimens). 


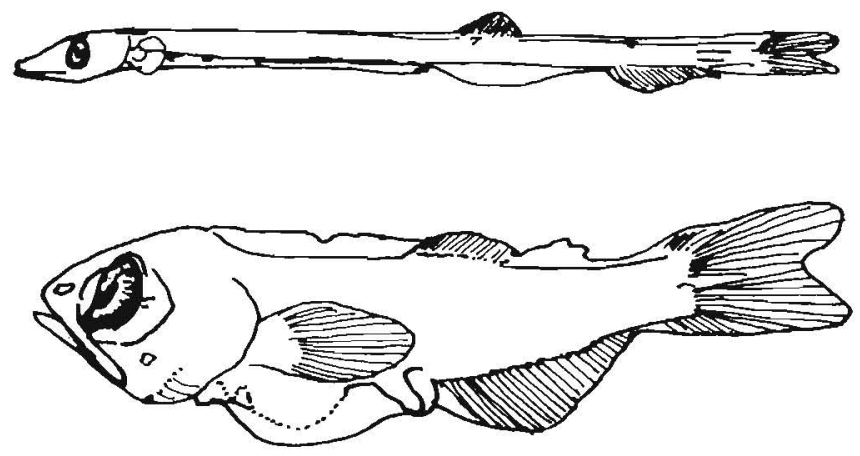

\section{Scopelomorpha: Aulopiformes Myctophiformes}

The myctophiforms (Myctophidae and Neoscopelidae) and aulopiforms (J. Nelson 1984) are treated together here. According to J. Nelson (1984), these orders consist of 14 families, and about 75 genera with 429 species. Although several families are benthic (e.g., synodontids), most families consist of deep-sea, pelagic, and benthopelagic forms. Many families (not myctophids) are synchronous hermaphrodites. The diverse early-life-history stages are generally well known and can be distinguished by morphological characters (head, gut, body) and pigment patterns. Additionally, photophore patterns and development are especially helpful in myctophid fishes, while gut pigment (i.e., number and development of peritoneal patches) is an important character distinguishing some of the other families.

Families in study area: Scopelarchidae

Notosudidae

Synodontidae

Bathysauridae

Paralepididae

Anotopteridae

Alepisauridae

Neoscopelidae

Myctophidae 


\section{MERISTICS}

B. dentata (Chapman 1939)

Vertebrae

Branchiostegal rays

Caudal fin

Pelvic fin

Dorsal fin

Pectoral fin

Anal fin

Total: 54-54-55

Precaudal: $\mathrm{X}-\mathrm{X}-\mathrm{X}$

Caudal: $\mathrm{X}-\mathrm{X}-\mathrm{X}$

8-8-8

$\mathrm{X}, 10+9, \mathrm{X}$

Abdominal

R: 9-9-9

R: $6-7-8$

R: $21-24-25$

R: $17-19-21$

MERISTICS

$B$. linguidens

(Mead and Böhlke 1953)

$\begin{array}{ll}\text { Vertebrae } & \text { Total: } 64-64-6 \\ & \text { Precaudal: } X-X \\ & \text { Caudal: } X-X-X \\ \text { Branchiostegal rays } & \text { X-X-X } \\ \text { Caudal fin } & \text { X, } 10+9, X \\ \text { Pelvic fin } & \text { Abdominal } \\ \text { Dorsal fin } & \text { R: } 8-X-9 \\ \text { Pectoral fin } & \text { R: } 24-X-25 \\ \text { Anal fin } & \text { R: } 28-X-30\end{array}$

\section{LIFE HISTORY}

Range
Ecology
ELH pattern

Spawning

Fecundity
Age at first maturity
Longevity

Longevity

\section{EARLY LIFE HISTORY DESCRIPTION}

\section{EGGS}

Diameter

No. of oil globules

Oil globule diameter

Yolk

Envelope

Hatch size

Incubation time/temp.

Pigment

Diagnostic characters

\section{LARVAE ${ }^{b}$}

Preanal length

Length at flexion

Length at transformation $50 \mathrm{~mm} \mathrm{SL}{ }^{\mathrm{d}}$

Sequence of fin development

\section{Anus moves posteriad during transformation}

Caudal; dorsal, anal, and dorsal part of pectoral; pelvics; ventral part of pectoral

Pigment

- No "accessory" pigment

- No "dermal" pigment; develops in transforming larvae and persists in adults

Diagnostic characters

- Gut expansion (in larvae, anus anterior to pelvic fin base but moves posteriad during transformation)

- Pelvic fin origin ahead of dorsal fin origin

- Adipose fin develops posterior to anal fin

- No peritoneal pigment in larvae

\footnotetext{
${ }^{a} B$. linguidens only to Oregon, $42-46^{\circ} \mathrm{N}$.

${ }^{b} B$. dentata only, $B$. linguidens larvae are incompletely known.

cJohnson 1974a

${ }^{\mathrm{d}}$ Larvae of Benthalbella spp. are unique among scopelarchids in achieving a large size (50-100 $\mathrm{mm} \mathrm{SL}$ ) while retaining a purely larval form and then exhibiting a very rapid transformation. The largest known larvae of $B$. linguidens $=85.5 \mathrm{~mm} \mathrm{SL}$, and may transform at larger sizes than $B$. dentata. Transformation is complete when peritoneal pigment first appears (uniformly in mesentary dorsal to gut from between pectoral fin bases to behind pelvic fins).
}

Ref: R.K. Johnson 1984. 


\section{B. linguidens}

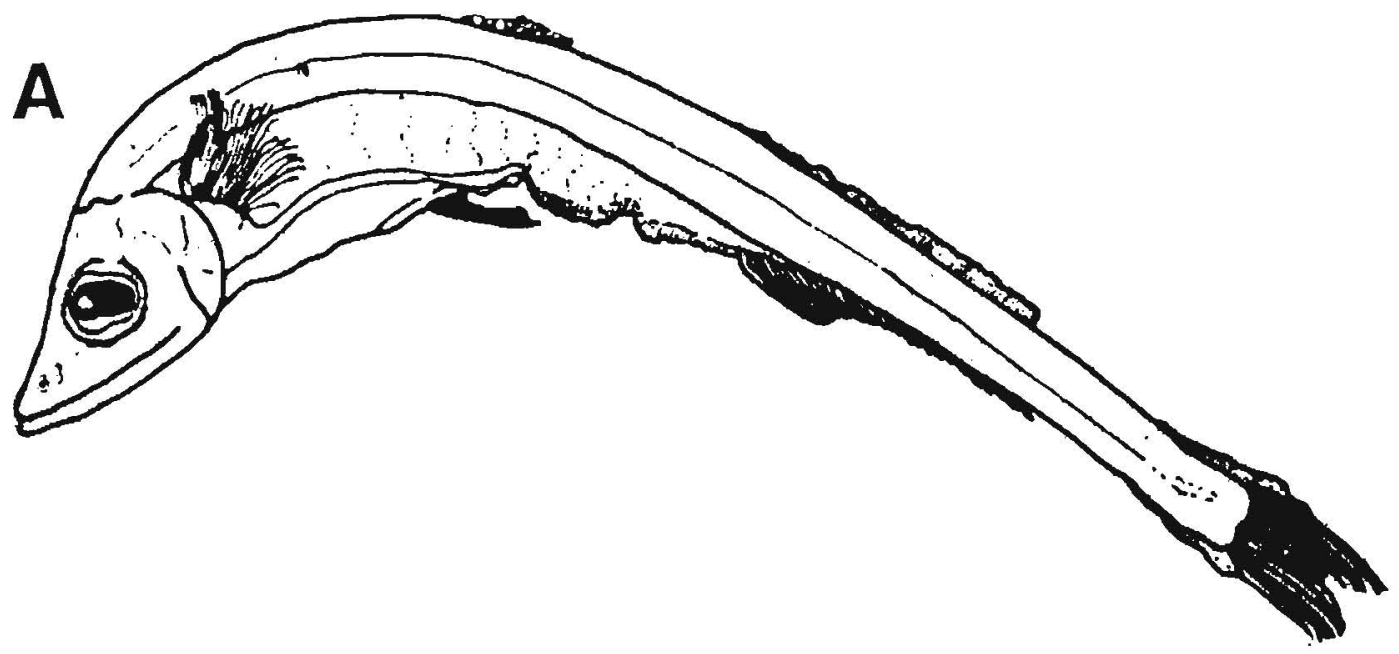

$49.0 \mathrm{~mm} \mathrm{SL}$

\section{B. dentata}

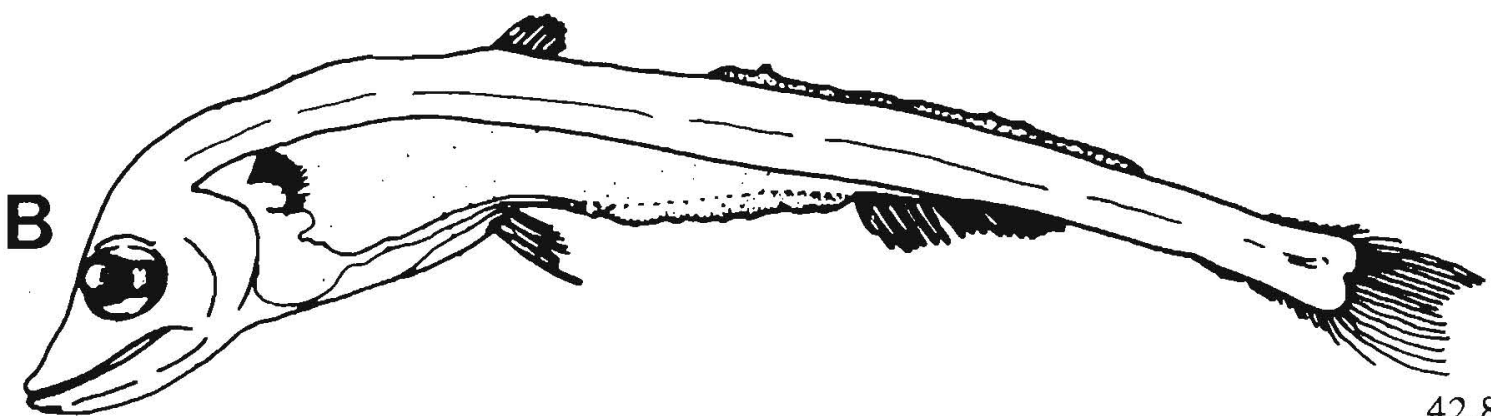

$42.8 \mathrm{~mm} \mathrm{SL}$

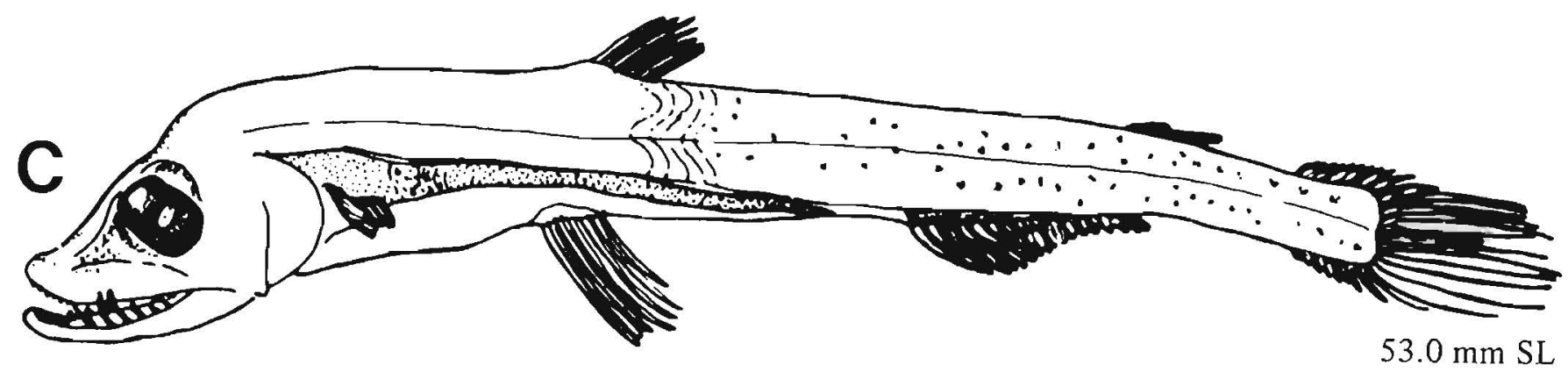

Figures $\mathrm{A}-\mathrm{C}$, Johnson 1974a. 


\section{MERISTICS}

\begin{tabular}{ll}
\hline Vertebrae & Total: $58-\mathrm{X}-61$ \\
& $\begin{array}{l}\text { Precaudal: X-X-X } \\
\text { Caudal: X-X-X }\end{array}$ \\
Branchiostegal rays & $10-10-10$ \\
Caudal fin & X, $10+9, \mathrm{X}$ \\
Pelvic fin & Abdominal \\
& R: $9-\mathrm{X}-10$ \\
Dorsal fin & R: $10-\mathrm{X}-12$ \\
Pectoral fin & R: $10-\mathrm{X}-14$ \\
Anal fin & R: $16-\mathrm{X}-19$ \\
Gill rakers & U: $2-2-2$ \\
& \\
& \\
LIFE HISTORY &
\end{tabular}

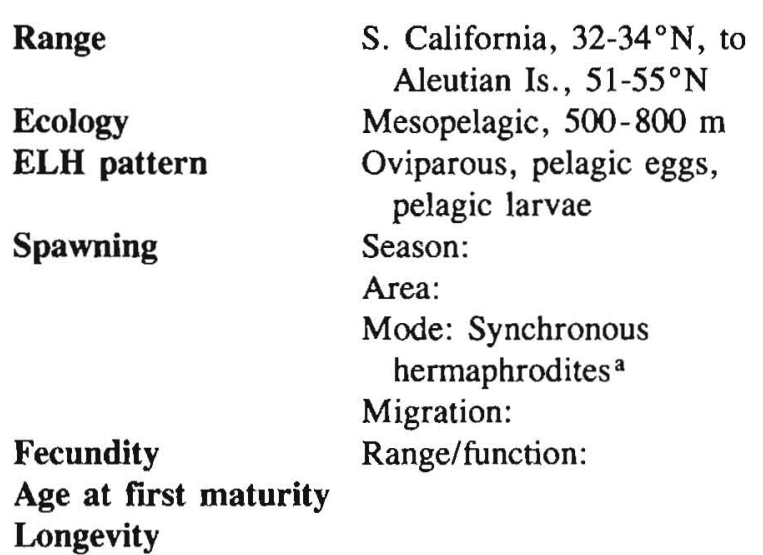

\section{EARLY LIFE HISTORY DESCRIPTION}

\section{EGGS}

Diameter

No. of oil globules

Oil globule diameter

Yolk

Envelope

Hatch size

Incubation time/temp.

Pigment

Diagnostic characters

\section{LARVAE}

Preanal length

Length at flexion

Length at transformation $25-45 \mathrm{~mm} \mathrm{SL}$

Sequence of fin development

Probably small

Pigment

- Restricted to tail along hypural region

Diagnostic characters-Family

- Hypural pigment: Exact distribution of pigment on the hypural region is diagnostic for species within the family

- Long body, becoming compressed toward tail

- Depressed head

- Posteriorly protruding lobes in brain (corpus cerebelli)

- Conical mass of choroid tissue around narrowed eye; long axis of the narrow eye is horizontal rather than vertical or oblique as it is in most other narrow-eyed larvae

- Anus at midbody; widely separated from anal fin

- Maxillary teeth

\footnotetext{
a Okiyama 1984
}

Ref: Okiyama 1984, Ozawa 1978 

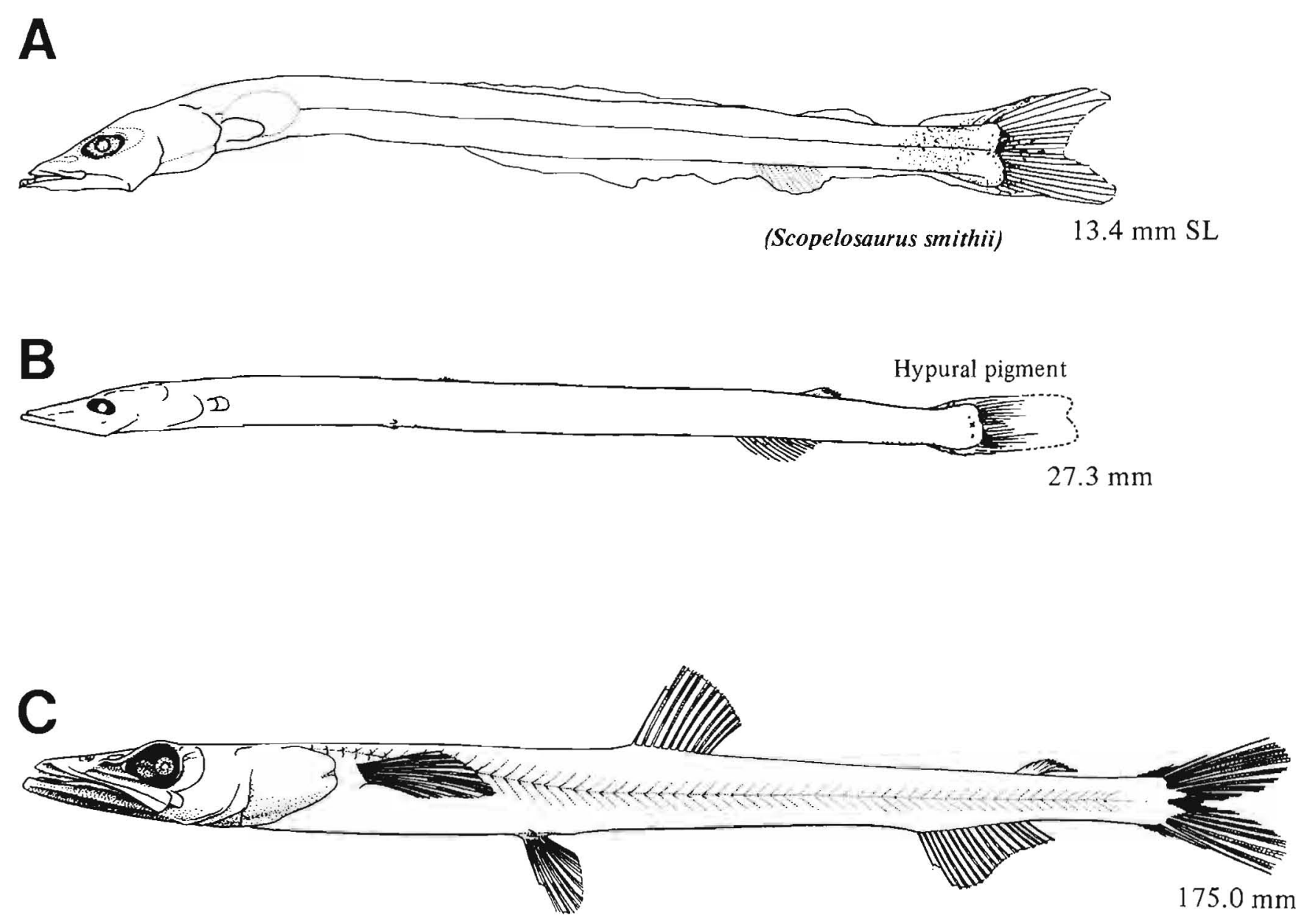

Figure A, Okiyama 1984 (southwestern Pacific specimen); B-C, Bertelsen et al. 1976 (North Atlantic specimens). 


\section{MERISTICS}

$\begin{array}{ll}\text { Vertebrae } & \begin{array}{l}\text { Total: } 50-X-52 \\ \text { Precaudal: X-X-X } \\ \text { Caudal: X-X-X }\end{array} \\ \text { Branchiostegal rays } & 8-8-8 \\ \text { Caudal fin } & 7,10+9,6 \\ \text { Pelvic fin } & \text { Abdominal } \\ & \text { R: } 8-8-8 \\ \text { Dorsal fin } & \text { R: } 15-X-17 \\ \text { Pectoral fin } & \text { R: } 16-X-17 \\ \text { Anal fin } & \text { R: } 11-13-13 \quad \\ \text { Gill rakers } & \text { U: } 5-X-6 \quad \text { L: } 14-X-16\end{array}$

\section{LIFE HISTORY}

\begin{tabular}{ll}
\hline Range & $\begin{array}{c}\text { South of southern California to } \\
\text { Oregon, } 42-46^{\circ} \mathrm{N} \\
\text { Bathybenthal } \\
\text { Ecology } \\
\text { ELH pattern }\end{array}$ \\
$\begin{array}{l}\text { Oviparous, pelagic eggs, } \\
\text { pelagic larvae }\end{array}$ \\
Season: \\
Area: \\
Mode: Synchronous \\
hermaphrodites
\end{tabular}

akiyama 1984

${ }^{b}$ Wenner 1978

'B. mollis larvae originally described as "Macristium" larvae (Johnson 1974b). An illustration of $B$. ferox is provided for comparison and to show fin rays intact.

${ }^{d}$ Changes at transformation include shortening of fins, expansion of gape, backward shift of dorsal fin origin, and darkening of body surface, oral cavity, and peritoneum. According to Sulak et al. (1985), the large sizes attained by these larvae suggest a long oceanic existence prior to transformation.

Ref: Johnson 1974b, Okiyama 1984, Sulak et al. 1985.

\section{EARLY LIFE HISTORY DESCRIPTION}

\section{EGGS}

Diameter

Number of oil globules

Ovarian eggs of $B$. ferox $1.2 \mathrm{~mm}^{\mathrm{b}}$

Oil globule diameter

Yolk

Envelope

Hatch size

Incubation time/temp.

Pigment

Diagnostic characters

\section{LARVAE}

Preanal length $70 \%$ SL

Length at flexion

Length at transformation $>83 \mathrm{~mm} \mathrm{SL}$ (gradual) $^{\mathrm{d}}$

Sequence of fin development

Pigment

- Peritoneal patches, about five; about six short bars laterally on gut

- About 17 vertical bars along body, 8 shorter bars in interspaces between vertical bars

- Several spots on head and fin rays

Diagnostic characters-Family

- Elongate fins

- Anterior placement of dorsal and pelvic fins

- Raised dorsal and anal fin bases

- Long gut terminating in front of anal origin

- Pigment: Peritoneal (5-6 sections) and lateral bars 

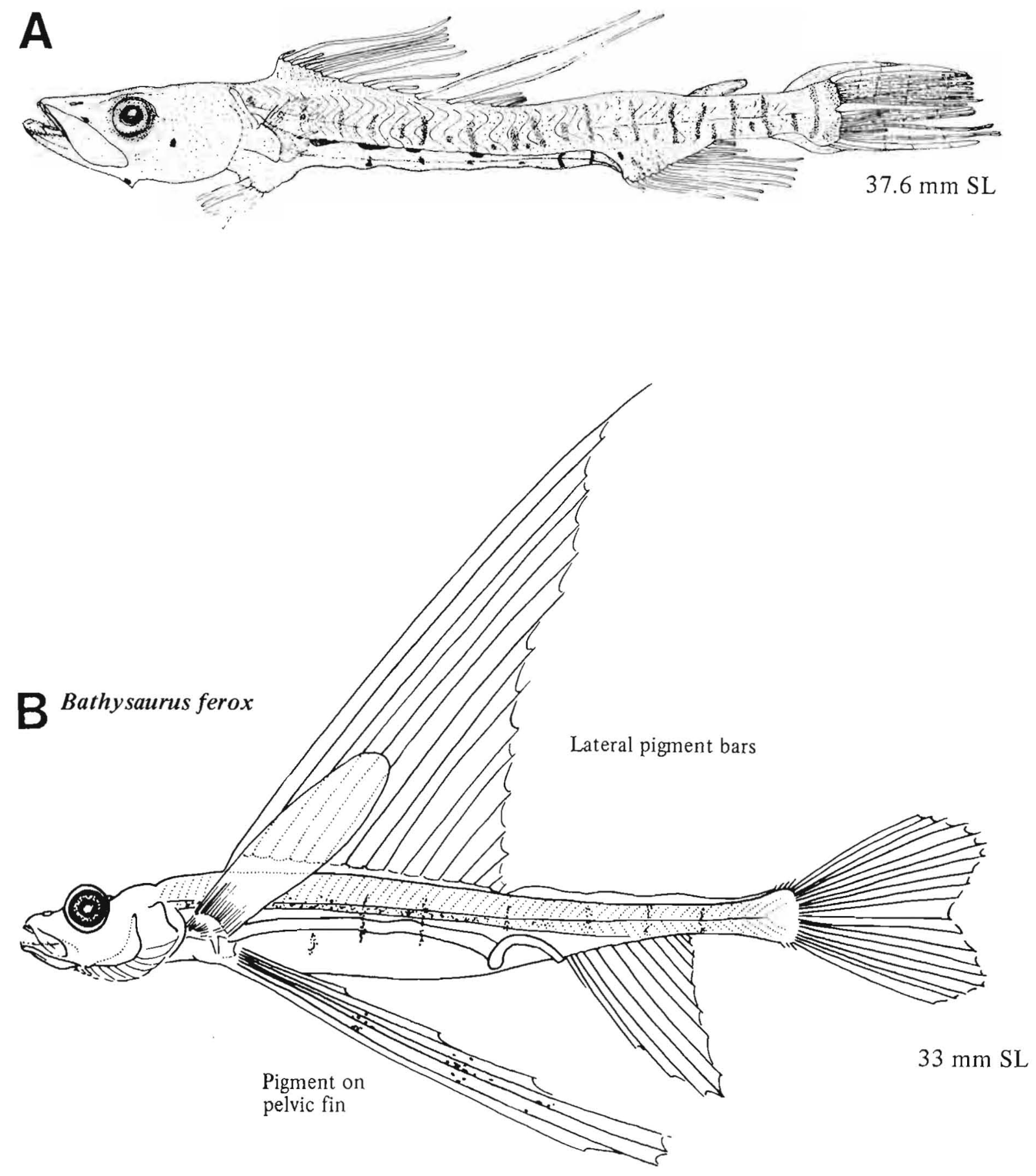

Figure A, Johnson 1974b (Gulf of Mexico specimen); B, Marshall 1960 (Atlantic specimen). 


\section{MERISTICS}

Vertebrae

Caudal fin

Pelvic fin

Dorsal fin

Pectoral fin

Anal fin

Gill rakers
Total: $60-62-63$

Precaudal: 49-52-54

Caudal: 8-10-11

18-18-18

$\mathrm{X}, 10+9, \mathrm{X}$

Abdominal

R: $8-8-8$

R: $11-\mathrm{X}-13$

$\mathrm{R}: 13-\mathrm{X}-14$

R: $12-\mathrm{X}-14$

$\mathrm{U}: \mathrm{X}-\mathrm{X}-\mathrm{X}$

L: X-X-X

\section{LIFE HISTORY}

\begin{tabular}{|c|c|}
\hline Range & $\begin{array}{l}\text { South of southern California to } \\
\text { N. California, } 38-42^{\circ} \mathrm{N}^{\mathrm{a}}\end{array}$ \\
\hline Ecology & Epi- and mesodemersal \\
\hline ELH pattern & $\begin{array}{l}\text { Oviparous, pelagic eggs, } \\
\text { pelagic larvae }\end{array}$ \\
\hline Spawning & $\begin{array}{l}\text { Season: } \\
\text { Area: } \\
\text { Mode: } \\
\text { Migration: }\end{array}$ \\
\hline $\begin{array}{l}\text { Fecundity } \\
\text { Age at first maturity } \\
\text { Longevity }\end{array}$ & Range/function: \\
\hline
\end{tabular}

a During "El Niño" years, adults occur as far north as Puget Sound, Washington. Postflexion larvae were collected off Oregon during the strong El Niño summer of 1982 (B. Mundy, NMFS Southwest Fish. Cent., Honolulu Lab., 2570 Dole St., Honolulu, HI 96822, pers. commun., 1 Oct. 1986).

${ }^{b}$ See Sumida et al. 1979.

${ }^{c}$ Data on preflexion and postflexion larvae are from E.H. Ahlstrom notes. nlustrations are unavailable.

Ref: Okiyama 1974, 1984; Ozawa 1986c; Sumida et al. 1979.

\section{EARLY LIFE HISTORY DESCRIPTION}

\section{EGGS}

\begin{tabular}{ll}
$\begin{array}{l}\text { Diameter } \\
\text { No. of oil globules } \\
\text { Oil globule diameter } \\
\text { Yolk }\end{array}$ & $1.33-1.44 \mathrm{~mm}$ \\
Envelope & None \\
Hatch size & Hexagonal surface \\
\hline
\end{tabular}

Incubation time/temp.

Pigment

- Unpigmented until late stage of development when pigment first appears along dorsal midline

- Few scattered yolksac spots

- Late-stage embryo resembles newly hatched larva with distinctive pigment along gut

Diagnostic characters

- Hexagonal surface similar to Pleuronichthys coenosus (p. 616) except hexagons are wider $(0.047 \mathrm{~mm}$ vs. $0.035 \mathrm{~mm})$ and irregularly arranged ${ }^{\mathrm{b}}$

\section{LARVAE}

Preanal length $\quad>50 \%$ SL

Length at flexion $\sim 10.5 \mathrm{~mm} \mathrm{SL}$

Length at transformation

Sequence of fin Caudal, anal, dorsal, development pectorals, pelvics

Pigment

- Seven evenly spaced pairs of "peritoneal patches" which form gradually

- Spot on postanal ventral midline midway between anus and tail

- Spot in area where hypurals are forming

Diagnostic characters

- Distinctive peritoneal patches (seven)

- Preanal finfold

- Deeper body than other Synodus spp. 


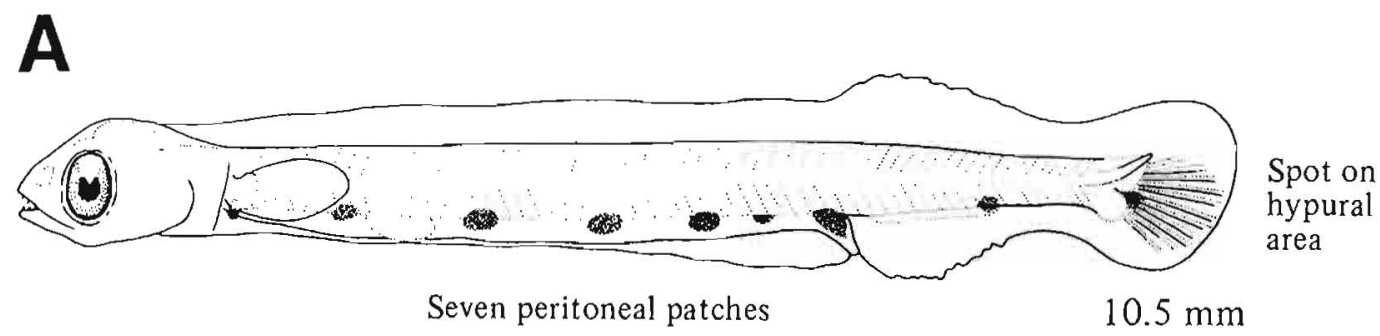




\section{MERISTICS}

\begin{tabular}{ll} 
Vertebrae & Total: $84-\mathrm{X}-87$ \\
& Precaudal: $41-41-41$ \\
& Caudal: X-X-X \\
Branchiostegal rays & $8-8-8$ \\
Caudal fin & $13,10+9,13$ \\
Pelvic fin & Abdominal \\
& R: $8-10-11$ \\
Dorsal fin & R: $9-12-12$ \\
Pectoral fin & R: $11-11-12$ \\
Anal fin & R: $26-26-33$ \\
Gill rakers & U: $3-\mathrm{X}-9$ \\
& \\
& \\
LIFE HISTORY & \\
\hline Range & S1-X-31 \\
Ecology & South of southern California to \\
ELH pattern & Brit. Col., $48^{\circ} 30^{\prime}-55^{\circ} \mathrm{N}$ \\
& $\begin{array}{l}\text { Epi-, meso-, and bathypelagic } \\
\text { Oviparous, eggs unknown, } \\
\text { pelagic larvae }\end{array}$ \\
& $\begin{array}{c}\text { Season: } \\
\text { Area: }\end{array}$ \\
& $\begin{array}{l}\text { Mode: Synchronous } \\
\text { hermaphrodites }\end{array}$ \\
& Migration \\
Fecundity & Range/function: \\
Age at first maturity & \\
Longevity &
\end{tabular}

a Okiyama 1984

${ }^{\mathrm{b}}$ Peritoneal pigment patches are sequentially formed with gradual lengthening of the gut. The number of patches (called "sections" by Okiyama 1984) may be speciesspecific. In general, these patches develop by $5-10 \mathrm{~mm} \mathrm{SL}$ and persist until $15-45$ $\mathrm{mm}$ SL. Other characteristic paralepidid pigment may include dorsum of body, caudal peduncle, and caudal and pectoral fins.

${ }^{c}$ General morphological characters in paralepidids are a long compressed body, shor gut increasing in length with development, head increasing in relative size with development, elongate snout, well-developed preanal finfold, and eyes initially ovoid, becoming round with development.

Ref: Okjyama 1984.

\section{EARLY LIFE HISTORY DESCRIPTION}

\author{
EGGS \\ Diameter \\ No. of oil globules \\ Oil globule diameter \\ Yolk \\ Envelope \\ Hatch size \\ Incubation time/temp. \\ Pigment
}

Diagnostic characters

\section{LARVAE}

Preanal length

Length at flexion

Length at transformation $\sim 45 \mathrm{~mm} \mathrm{SL}$

Sequence of fin

Caudal, anal, dorsal, development pectorals, pelvics (family)

Pigment

- Tip of jaw, two dorsal and one ventral midline spot

- Peritoneal patches increase during development to about seven

- With development, head, dorsal and anal fin bases, and caudal peduncle

Diagnostic characters - Family

- Pigment, especially peritoneal patches ${ }^{b}$

- Elongation of gut with development

- Morphology ${ }^{\mathrm{c}}$

Distinguished from Notolepis rissoi and Paralepis atlantica by

Lestidiops Notolepis Paralepis

No. peritoneal patches

$\begin{array}{lccc}\text { (flexion larvae) } & 3 & 1 & 2 \\ \text { (postflexion larvae) } & 7 & 12 & 3 \\ \text { o. myomeres } & 84-87 & 72-74 & 76-83\end{array}$


A

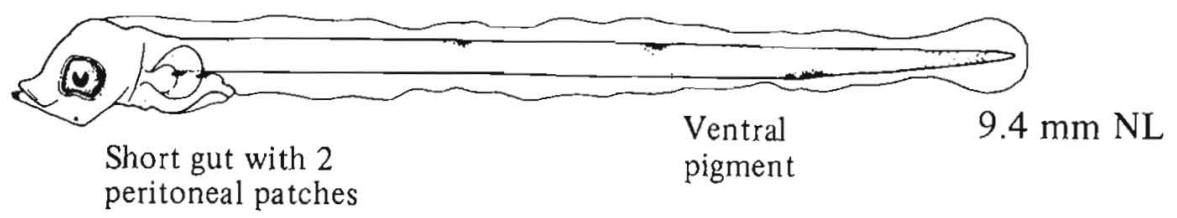

Short gut with 2

ventral

$9.4 \mathrm{~mm} \mathrm{NL}$

peritoneal patches

B

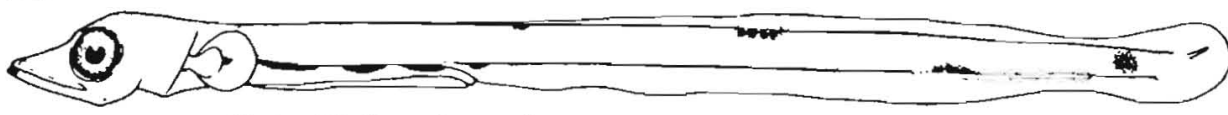

Gut with 3 peritoneal patches

$16.5 \mathrm{~mm} \mathrm{NL}$

Pigment above and below notochord along peduncle

C

Dorsal fin base pigment

Elongate gut with 7 peritoneal patches
Anal fin base pigment
$28.5 \mathrm{~mm} \mathrm{SL}$

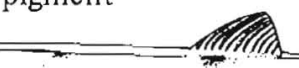

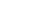




\section{MERISTICS}

Vertebrae

Caudal fin

Pelvic fin

Dorsal fin

Pectoral fin

Gill rakers
Anal fin
Total: $72-X-74$

Precaudal: 37-X-39

Caudal: $39-\mathrm{X}-41$

8-8-8

$\mathrm{X}, 10+9, \mathrm{X}$

Abdominal

R: $8-\mathrm{X}-12$

R: 9-X-13

R: $10-\mathrm{X}-13$

R: $29-X-34$

U: $3-X-9$

L: $18-\mathrm{X}-36$

\section{EARLY LIFE HISTORY DESCRIPTION}

\section{EGGS}

Diameter

No. of oil globules

Oil globule diameter

Yolk

Envelope

Hatch size

Incubation time/temp.

Pigment

Diagnostic characters

\section{LARVAE}

Preanal length

\section{Range}

South of southern California to Brit. Col., $48^{\circ} 30^{\prime}-55^{\circ} \mathrm{N}$

Ecology

ELH pattern

Epi-, meso-, and bathypelagic

Oviparous, eggs unknown, pelagic larvae

Spawning

Season:

Area:

Mode: Synchronous hermaphrodites $^{\text {a }}$

Migration:

Fecundity

Range/function:

Age at first maturity

Longevity

Length at flexion

Length at transformation

Sequence of fin development

Pigment

- Peritoneal patches, 1 increasing with development to 12 (see Lestidiops ringens)

- Above and below notochord on caudal peduncle

- Additional pigment along dorsum from dorsal fin origin to tail with development

Diagnostic characters

- See L. ringens (p. 134)

- Pigment, peritoneal patches

- Morphology

${ }^{\mathrm{a}}$ Okiyama 1984

Ref: Okiyama 1984 


\section{A}

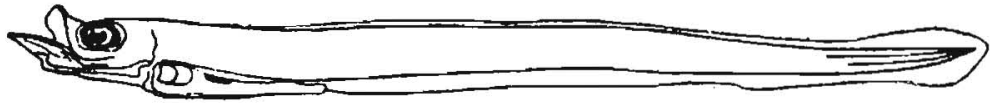

One peritoneal patch $\quad 9.5 \mathrm{~mm} \mathrm{SL}$

B

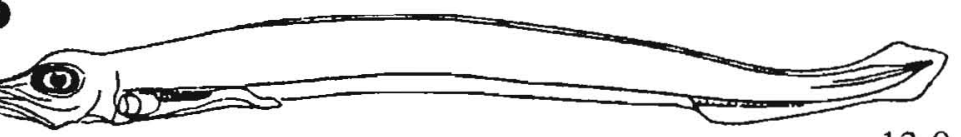

$13.0 \mathrm{~mm} \mathrm{SL}$
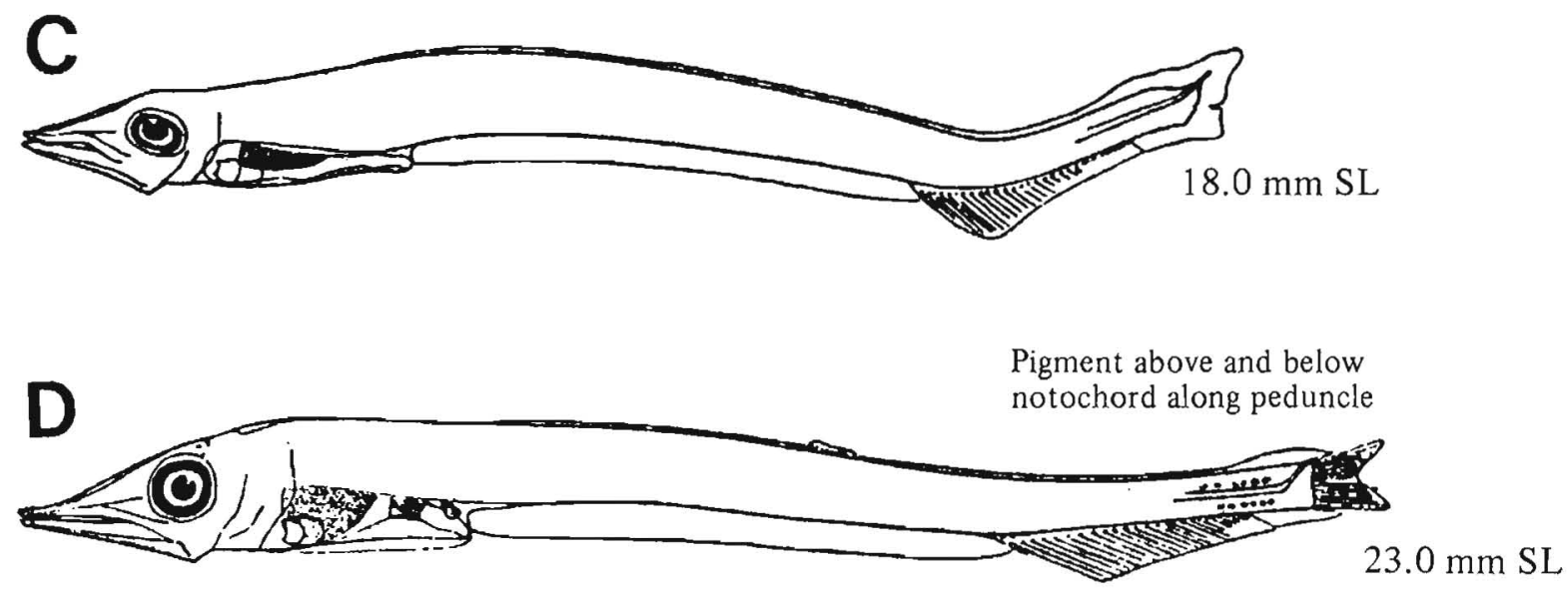

E

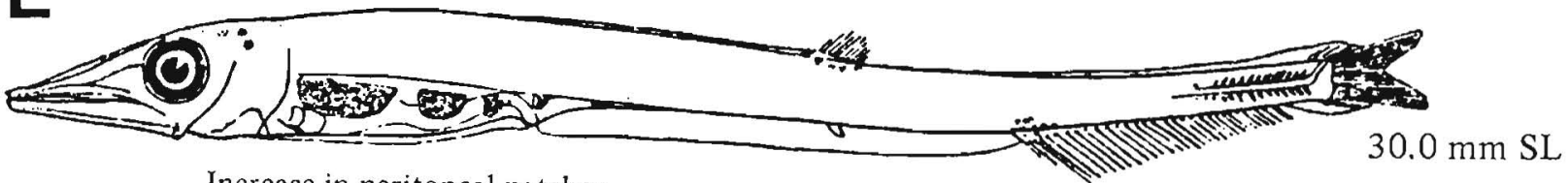

Increase in peritoneal patches

with gradual lengthening of gut

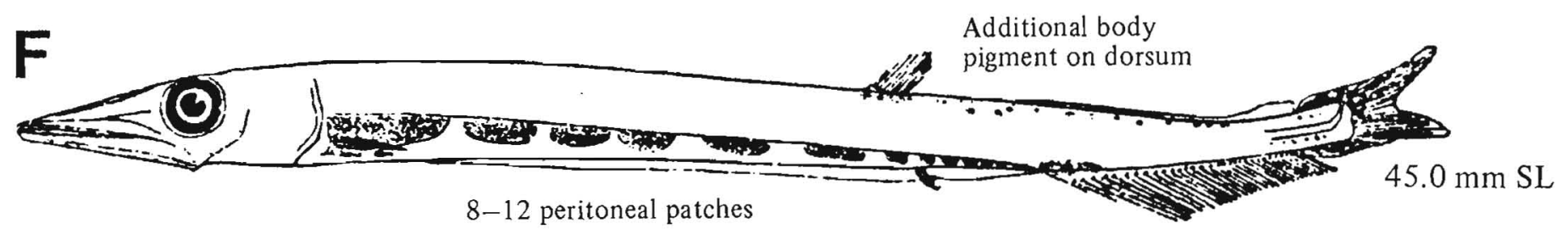

Figures A-F, Rofen 1966 a (North Atlantic specimens). 


\section{MERISTICS}

Vertebrae

Total: $60-67-69$

Precaudal: $38-40-41$

Caudal: X-X-X

Branchiostegal rays $\quad 8-8-8$

Caudal fin

Pelvic fin

$\mathrm{X}, 10+9, \mathrm{X}$

Abdominal

R: $9-\mathrm{X}-10$

Dorsal fin

Pectoral fin

Anal fin

Gill rakers

$\mathrm{R}: 15-\mathrm{X}-18$

R: $20-X-23$

U: $7-X-9$

L: $26-\mathrm{X}-32$

\section{LIFE HISTORY}

Range

South of southern California to SE Alaska, $55-59^{\circ} \mathrm{N}$

Ecology

ELH pattern

Spawning

Epi-, meso-, and bathypelagic

Oviparous, eggs unknown, pelagic larvae

Season:

Area:

Mode: Synchronous hermaphrodites $^{\mathrm{a}}$

Migration:

Age at first maturity

Longevity

\section{EARLY LIFE HISTORY DESCRIPTION}

\section{EGGS}

Diameter

No. of oil globules

Oil globule diameter

Yolk

Envelope

Hatch size

Incubation time/temp.

Pigment

Diagnostic characters

\section{LARVAE}

\section{Preanal length}

Length at flexion

Length at transformation

Sequence of fin development

Caudal, anal, dorsal, pectorals, pelvics (family)

- Peritoneal patches, one increasing with development to three (see Lestidiops ringens, p. 134)

- Few spots above and below notochord in caudal peduncle region which, with development, form a patch

- Patch at base of dorsal and anal fin, on cranium, on upper jaw (late in development)

Diagnostic characters

- See L. ringens

- Pigment, peritoneal patches

- Morphology

${ }^{a}$ Okiyama 1984

Ref: Okjyama 1984. 
A

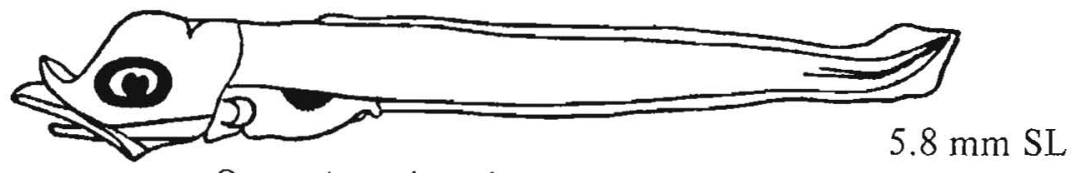

One peritoneal patch

B

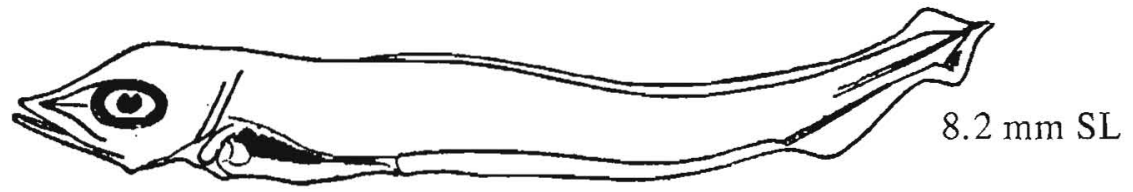

C

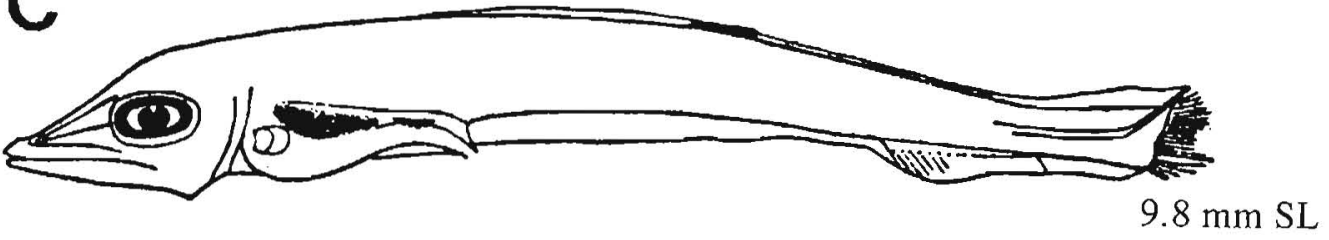

D

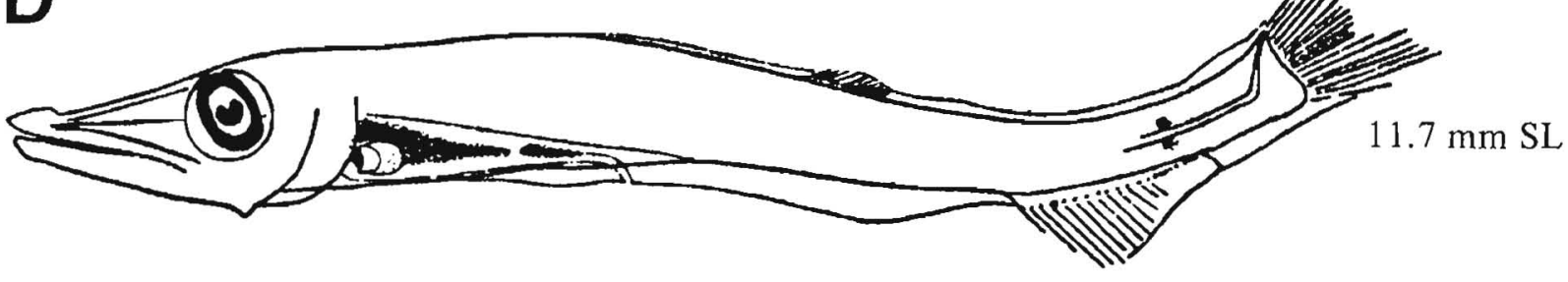

E

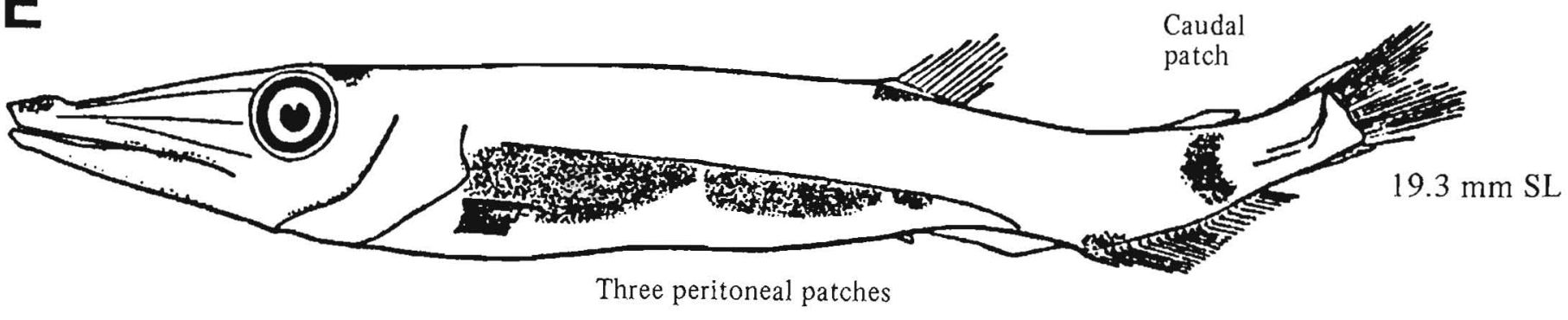

Figures A-E, Rofen 1966 a (North Atlantic specimens). 
MERISTICS

$\begin{array}{ll}\text { Vertebrae } & \text { Total: } 76-80-83 \\ & \begin{array}{l}\text { Precaudal: } 48-52-54 \\ \text { Caudal: } 27-29-31\end{array} \\ \text { Branchiostegal rays } & 7-7-7 \\ \text { Caudal fin } & 14-15,10+9,14 \\ \text { Pelvic fin } & \text { Abdominal } \\ & \text { R: } 9-\mathrm{X}-11 \\ \text { Dorsal fin } & \text { Absent } \\ \text { Pectoral fin } & \text { R: } 12-\mathrm{X}-16 \\ \text { Anal fin } & \text { R: } 14-\mathrm{X}-17 \\ \text { Gill rakers } & \text { U: X-X-X } \quad \text { L: X-X-X } \\ & \\ \text { LIFE HISTORY } & \end{array}$

\begin{tabular}{ll}
\hline Range & S. California, $32-34^{\circ} \mathrm{N}$, to \\
Bering Sea, $54-66^{\circ} \mathrm{N}$ \\
Ecology & $\begin{array}{l}\text { Epi- and mesopelagic } \\
\text { Oviparous, eggs unknown, } \\
\text { ELH pattern }\end{array}$ \\
$\begin{array}{l}\text { pelagic larvae } \\
\text { Spawning }\end{array}$ & $\begin{array}{l}\text { Area: } \\
\text { Mode: Synchronous } \\
\text { hermaphrodites }\end{array}$ \\
& $\begin{array}{l}\text { Migration: } \\
\text { Range/function: }\end{array}$ \\
$\begin{array}{l}\text { Fecundity } \\
\text { Age at first maturity } \\
\text { Longevity }\end{array}$ &
\end{tabular}

\section{EARLY LIFE HISTORY DESCRIPTION}

\section{EGGS}

Diameter

No. of oil globules

Oil globule diameter

Yolk

Envelope

Hatch size

Incubation time/temp.

Pigment

Diagnostic characters

\section{LARVAE $^{\text {b }}$}

Preanal length

Length at flexion

Length at transformation $<50 \mathrm{~mm} \mathrm{SL}$

Sequence of fin development

Only pectoral anlagen present at $14.2 \mathrm{~mm} \mathrm{SL}$

Pigment

- No peritoneal pigment patches, instead uniform peritoneal pigment

- Snout, jaw

- Dorsal midline

- Tail tip

\section{Diagnostic characters}

- Morphology: Long slender body, large head, pointed snout

- Fleshy prolongation at jaw tips

- Two large canine teeth

- Gut extending to midbody 


\section{A}

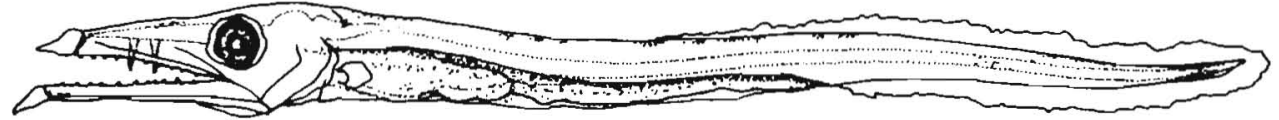

Fleshy prolongation

$14.2 \mathrm{~mm} \mathrm{SL}$ of jaws

B

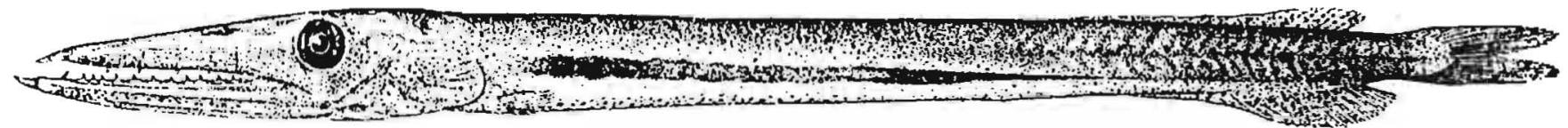

$50.0 \mathrm{~mm} \mathrm{SL}$

Figure A, Okiyama 1984; B, Rofen 1966 b (North Atlantic specimen). 


\section{MERISTICS}

\section{Vertebrae}

Total: $48-50-52$

Precaudal: 19-23-26

Caudal: 24-27-31

Branchiostegal rays

\section{8-8-8}

Caudal fin

Pelvic fin

Abdominal

R: $8-\mathrm{X}-10$

Dorsal fin

R: $30-X-45$

Pectoral fin

Anal fin

Gill rakers

R: $12-X-15$

$\mathrm{R}: 14-\mathrm{X}-17$

U: $2-\mathrm{X}-6$

L: $16-X-24$
$\mathrm{X}, 10+9, \mathrm{X}$

\section{EARLY LIFE HISTORY DESCRIPTION}

\section{EGGS}

Diameter

No. of oil globules

Oil globule diameter

Yolk

Envelope

Hatch size

Incubation time/temp.

Pigment

Diagnostic characters

\section{LARVAE}

Preanal length

Length at flexion

Length at transformation ${ }^{d}$

Sequence of fin

Pectorals, caudal, dorsal, Epi-, meso-, and bathypelagic, 0-1829 m

ELH pattern

Oviparous, eggs unknown, pelagic larvae

Spawning

Season: May (California) ${ }^{a}$

Area:

Mode: Synchronous hermaphrodites ${ }^{b}$

Migration:

Fecundity

Range/function:

Age at first maturity

Longevity development

\section{Pigment} anal, pelvics

- Genus: Preflexion larvae appear to be unpigmented except for pectoral fins

- Postflexion

- Heavy gut pigment

-Pigment patch at anal fin origin

-Pectoral fins

- Saddle in area of adipose fin

-Patch along midline over gut

Diagnostic characters

Distinguished from other species of Alepisaurus by

- Four preopercular spines

- Bony ridges on head

- Pigment patch at anal fin base

For family

- Large head and mouth

- Prominent canines on dentary

- Small fins

- Short gut

\footnotetext{
${ }^{2}$ Fitch and Lavenberg 1968

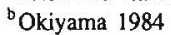

${ }^{\mathrm{c}}$ Dlustrations of preflexion and postflexion $A$. brevirostris are provided for comparison.

${ }^{\mathrm{d}}$ Transformation is gradual.

Ref: Okiyama 1984.
} 

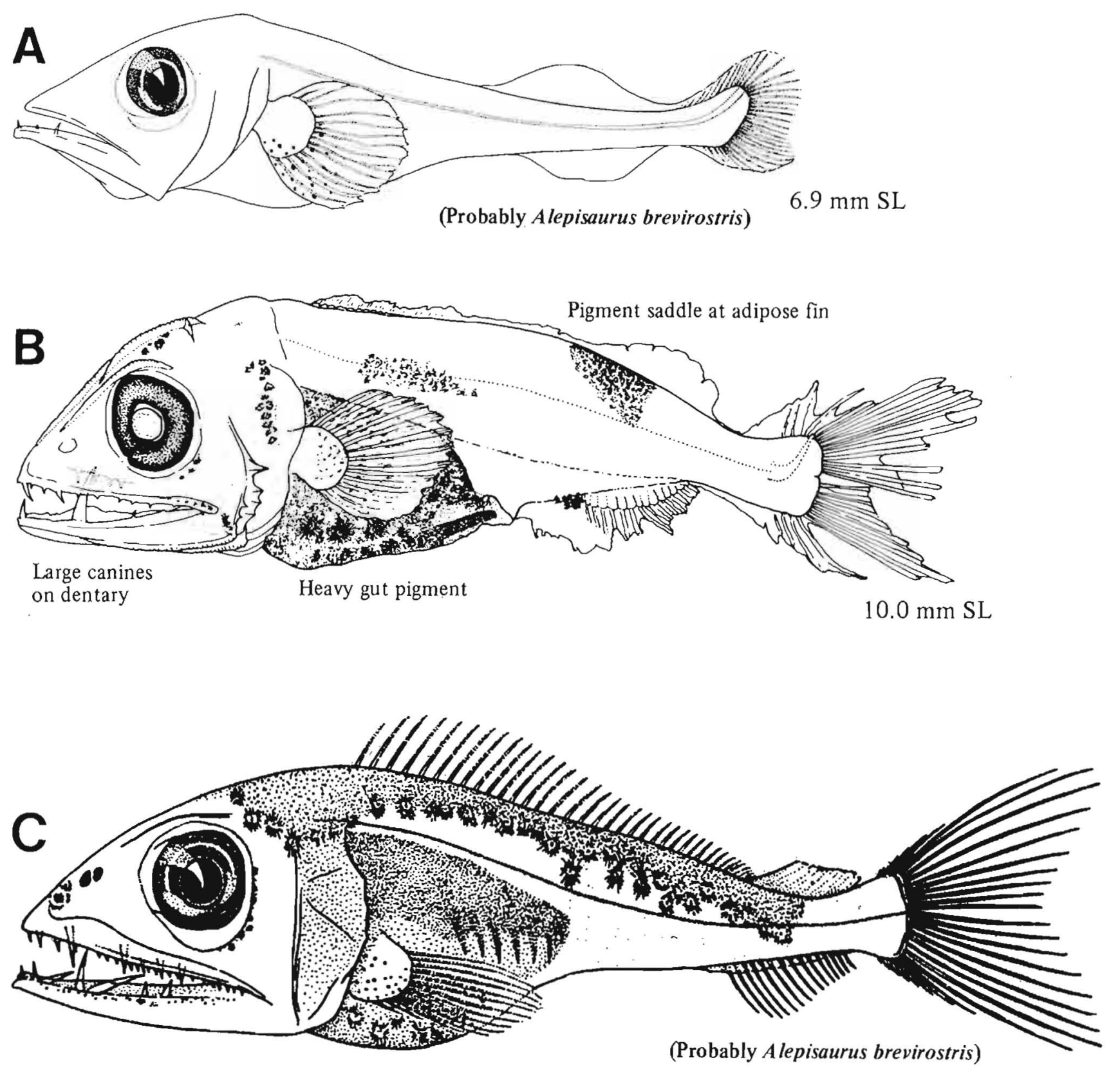

Figures A, C, Rofen 1966c (specimens collected off Bermuda); B, Okiyama 1984 (Hawaiian specimen). 


\section{MERISTICS}

Vertebrae $^{\mathrm{a}}$

Caudal fin

Pelvic fin

Dorsal fin

Pectoral fin

Anal fin

Gill rakers ${ }^{b}$
Total: $30-X-31$

Precaudal: $\mathrm{X}-\mathrm{X}-\mathrm{X}$

Caudal: X-X-X

8-8-8

$6-8,10+9,5-7$

Abdominal

R: 8-8-8

R: $11-X-13$

$\mathrm{R}: 18-\mathrm{X}-19$

R: $10-X-13$

U: $3-3-3$

(T: 10-X-12)

\section{LIFE HISTORY}

\section{Range $^{\mathrm{c}}$ \\ Ecology \\ ELH pattern}

Spawning

Fecundity

Age at first maturity

Longevity
Brit. Col., $48^{\circ} 30^{\prime}-55^{\circ} \mathrm{N}$

Mesopelagic, $300-800 \mathrm{~m}$

Oviparous, pelagic eggs, pelagic larvae

Season:

Area:

Mode:

Migration:

Range/function:

\section{EARLY LIFE HISTORY DESCRIPTION}

EGGS (Ripe ovarian)

$\begin{array}{ll}\text { Diameter } & 0.83-0.98 \mathrm{~mm} \\ \text { No. of oil globules } & \text { One } \\ \text { Oil globule diameter } & 0.39-0.61 \mathrm{~mm}\end{array}$

Yolk

Envelope

Hatch size

Incubation time/temp.

Pigment

Diagnostic characters

LARVAE (Postflexion only)

Preanal length $\sim 70 \%$ SL

Length at flexion

Length at transformation Fin rays formed by $10.0 \mathrm{~mm} \mathrm{SL}$

Sequence of fin development Pectorals, dorsal, anal, caudal, pelvics

Pigment

- Small dorsal patches on peritoneum and hindgut

Diagnostic characters

- Pigment patch over hindgut (not solid as in other myctophiforms)

- Small preopercular spines

Distinguished from most myctophiforms by

- Lack of peritoneal pigment patches

For family

- Morphology

-Deep body

- Large head

- Large pectoral fin

- Round eye without choroid tissue

- Lightly pigmented

${ }^{a}$ Genus vertebral range 29-35.

${ }^{b}$ Gill raker counts vary geographically.

' Peden and Hughes (1986); previously only off Japan and Hawaii.

Ref: Maruyama 1970; Nafpaktitis 1977; Okiyama 1974, 1984. 


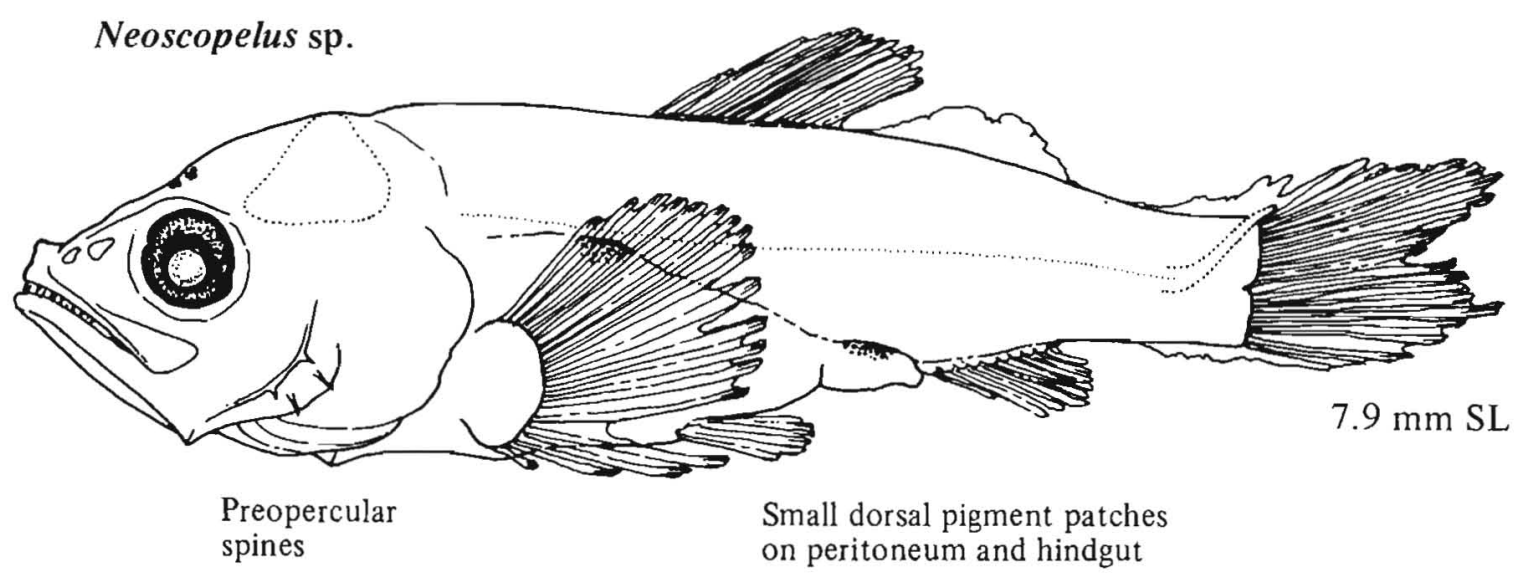

Figure A, Okiyama 1984 (southwestern Japan specimen). 
Lanternfishes are worldwide in distribution, having 32 genera with 235 species found from the Arctic to the Antarctic. Myctophids generally have large eyes, large terminal mouths, photophores, and a black body color. In the Northeast Pacific, 20 species occur within two subfamilies: Myctophinae and Lampanyctinae. Most of the species (13) are members of the Lampanyctinae.

Myctophids are oviparous and presumably all produce planktonic eggs (Moser et al. 1984a). Eggs of only two species have been described: Electrona rissoi by Sanzo (1939) and Lampanyctodes hectoris by Robertson (1977). Eggs of both species are small $(<1.0 \mathrm{~mm})$, possess a single large oil globule, segmented yolk, smooth chorion, and at least Lampanyctodes eggs possess a fragile chorion. We have identified several types of myctophid eggs with these characteristics, in particular a fragile chorion. At least two of these types are probably Diaphus and Stenobrachius (based on egg characters and the presence of yolksac larvae of those species in the same samples).

The larval photophore complements and the sequence of appearance of photophores are useful characters. Most myctophids develop the $\mathrm{Br} 2$ during the larval period (in the Northeast Pacific the only exception is Taaningichthys). The Br2 is located posteroventral to the orbit but during transformation assumes a position beneath the orbit on the branchiostegal membrane. Three myctophine genera and eleven lampanyctine genera develop additional photophores during the larval period, with the $\mathrm{Br} 2$ always first to appear (Moser et al. 1984a). Of these 14 genera, only 4 are represented in our study area: Ceratoscopelus, Lampadena, Diaphus, and Notoscopelus.

The taxonomic section on myctophids here is arranged according to the intrafamilial classification of the group (Table 19), since certain larval characters reflect this classification (e.g., larval eye shape).

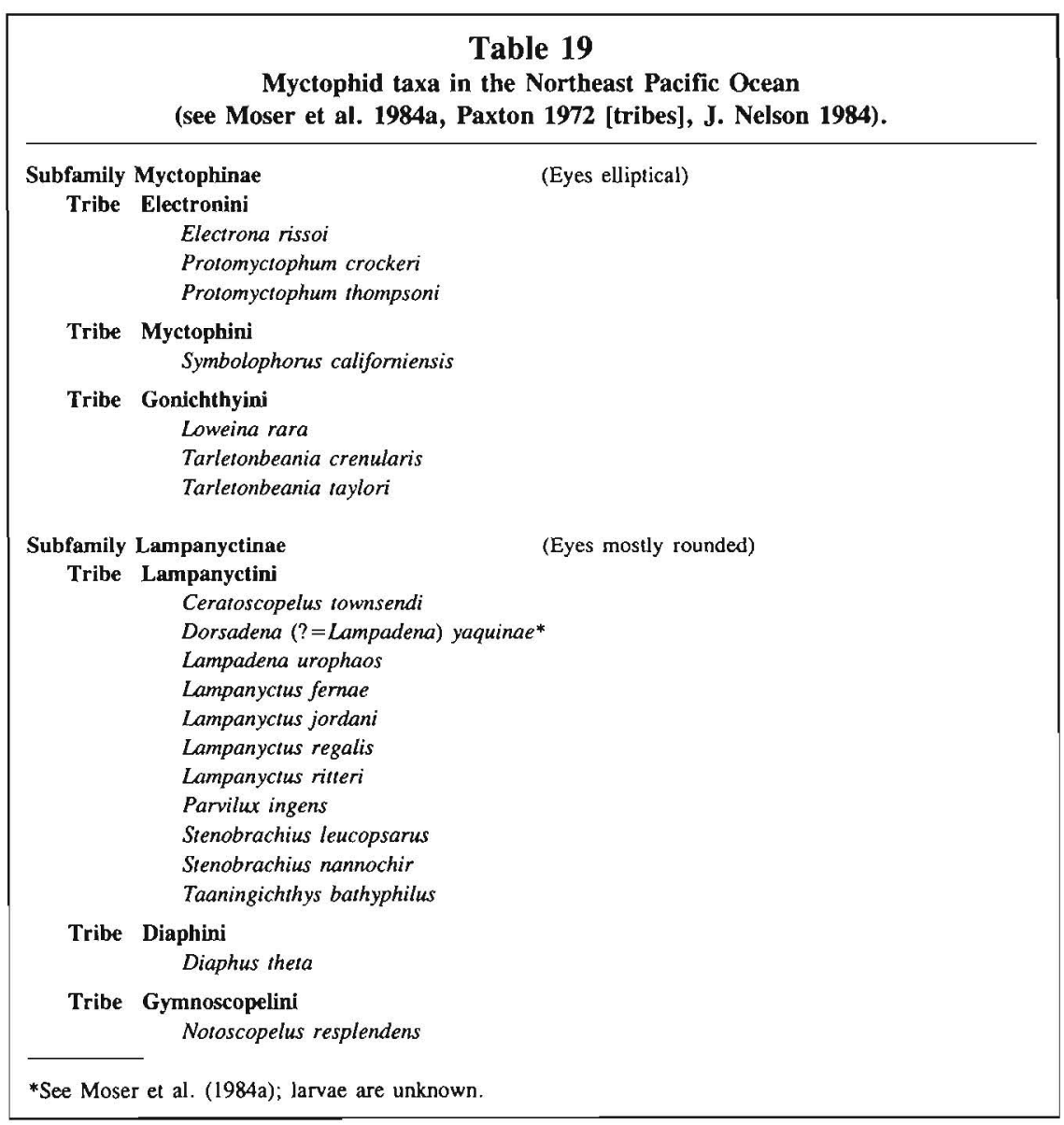




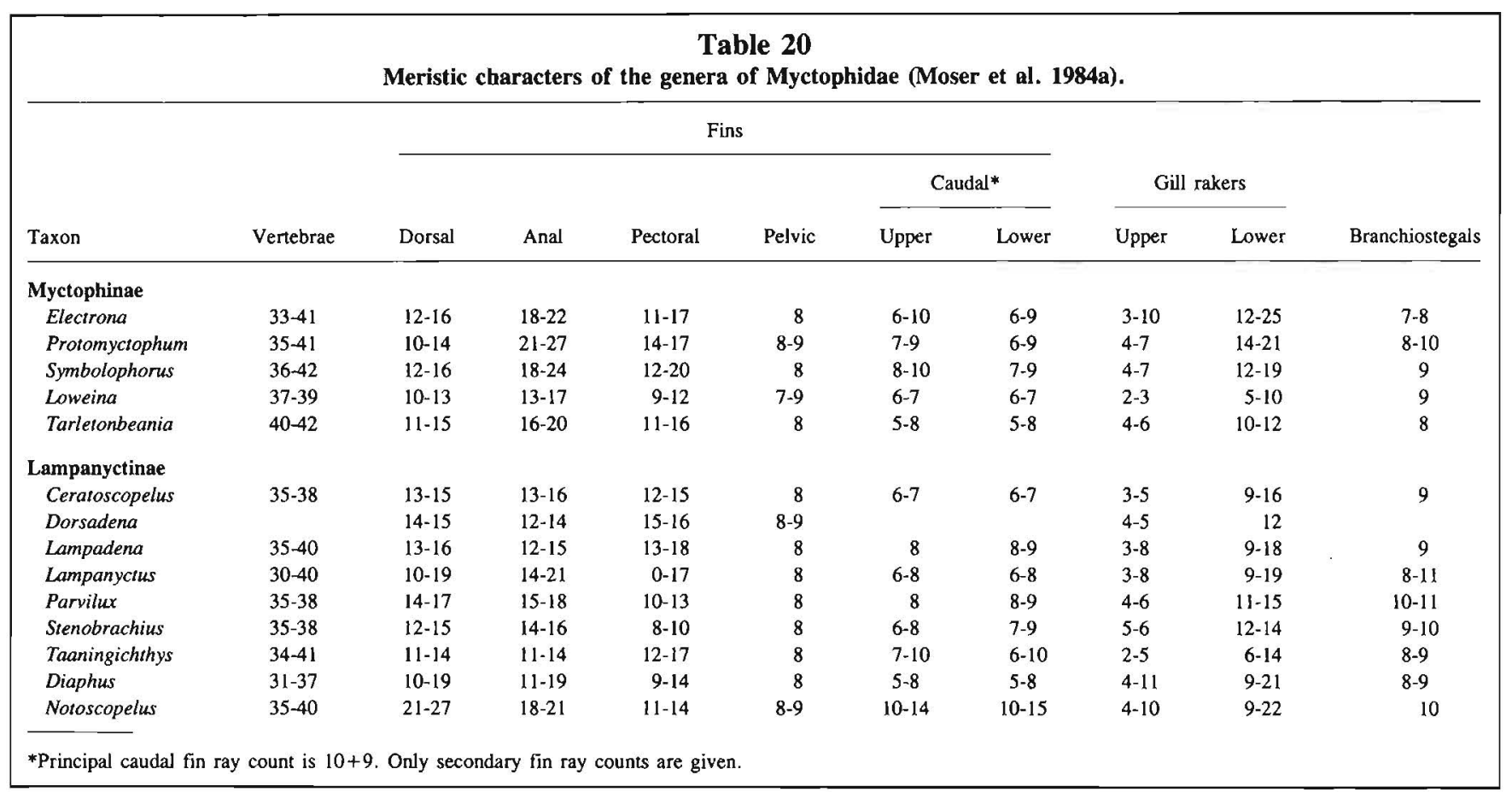

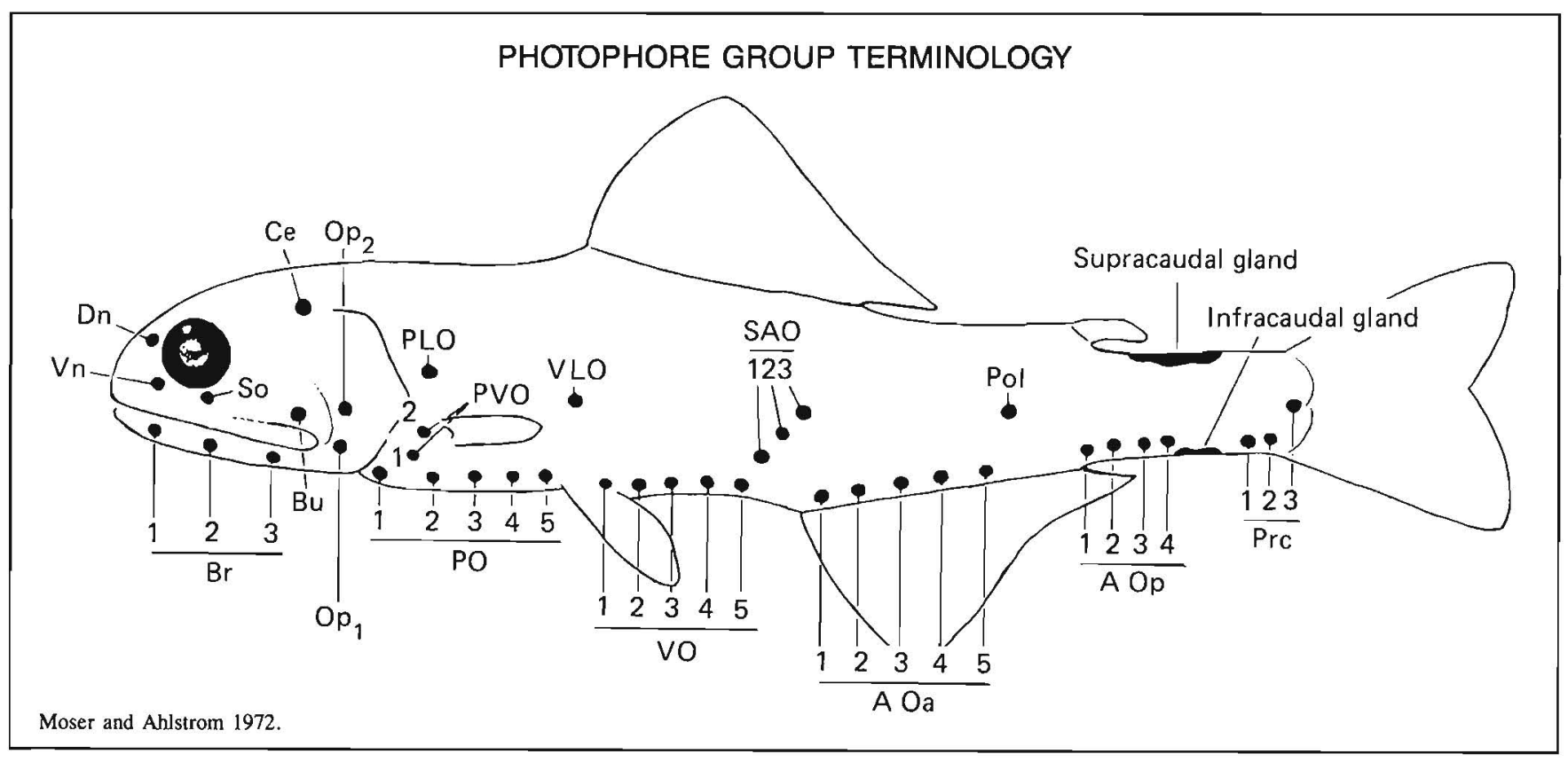




\section{MERISTICS}

\begin{tabular}{|c|c|}
\hline Vertebrae & $\begin{array}{l}\text { Total: } 32-\mathrm{X}-34 \\
\text { Precaudal: X-X-X } \\
\text { Caudal: X-X-X }\end{array}$ \\
\hline Branchiostegal rays & $8-8-8$ \\
\hline Caudal fin & $6-8,10+9,6-7$ \\
\hline Pelvic fin & $\begin{array}{l}\text { Abdominal } \\
\mathrm{R}: 8-8-8\end{array}$ \\
\hline Dorsal fin & $R: 13-X-15$ \\
\hline Pectoral fin & $R: 13-X-16$ \\
\hline Anal fin & $R: 18-19-20$ \\
\hline Gill rakers & $\mathrm{L}: 18-\mathrm{X}-21$ \\
\hline \multicolumn{2}{|l|}{ LIFE HISTORY } \\
\hline Range & $\begin{array}{l}\text { South of southern California to } \\
\text { Oregon, } 42-46^{\circ} \mathrm{N}\end{array}$ \\
\hline Ecology & Epi- and mesopelagic \\
\hline ELH pattern & $\begin{array}{l}\text { Oviparous, pelagic eggs, } \\
\text { pelagic larvae }\end{array}$ \\
\hline \multirow{4}{*}{ Spawning } & Season: \\
\hline & Area: \\
\hline & Mode: \\
\hline & Migration: \\
\hline $\begin{array}{l}\text { Fecundity } \\
\text { Age at first maturity } \\
\text { Longevity }\end{array}$ & Range/function: \\
\hline
\end{tabular}

\section{EARLY LIFE HISTORY DESCRIPTION}

\section{EGGS}

Diameter

No. of oil globules

$0.80-0.84 \mathrm{~mm}$

Oil globule diameter

Yolk

Envelope

Hatch size

Incubation time/temp.

Pigment

Diagnostic characters
One

$0.28 \mathrm{~mm}$

Segmented

Smooth, fragile

\section{LARVAE}

Preanal length

Length at flexion

Length at transformation

Sequence of fin development

Pigment

- Lower jaw symphysis (see $7.9 \mathrm{~mm}$ )

- Pectoral fin blade (see $6.3 \mathrm{~mm}$ )

Diagnostic characters

- Photophores: Early Br2, usually forms at transformation in other species

- Morphology: Body moderately slender, head large

- Gut saccular, s-shaped

- Space between anus and anal fin origin but not as large as in Protomyctophum spp.

- Eyes very narrow

- Transforms earlier than most myctophids (9-10 mm SL)

Distinguished from other myctophids with elliptical eyes by

- Pigment on lower jaw tip and pectoral fin

- Lack of pigment on postanal body 

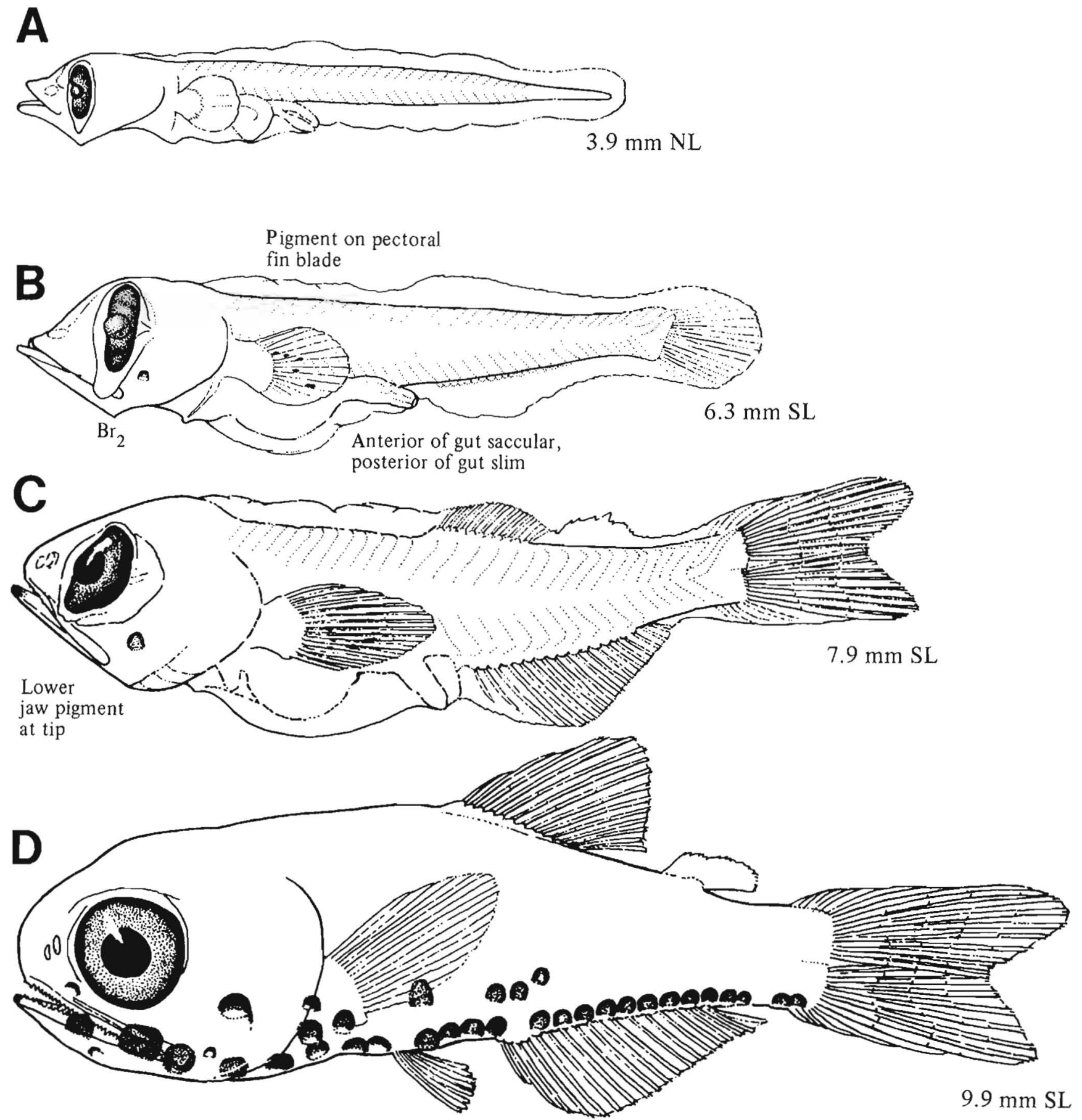

Figures A-D, Moser and Ahlstrom 1970. 


\section{MERISTICS}

\begin{tabular}{ll}
\hline Vertebrae & Total: $36-X-37$ \\
& Precaudal: X-X-X \\
& Caudal: X-X-X \\
Branchiostegal rays & $8-X-12$ \\
Caudal fin & $7-9,10+9,7-8$ \\
Pelvic fin & Abdominal \\
& R: $8-8-8$ \\
Dorsal fin & R: $11-12-13$ \\
Pectoral fin & R: $13-16-17$ \\
Anal fin & R: $19-22-24$ \\
Gill rakers & U: $4-5-6$ \\
& \\
LIFE HISTORY & \\
\hline & \\
Range & South of southern California to \\
& Washington, $46-48^{\circ} 30^{\prime} \mathrm{N}^{\mathrm{a}}$ \\
Ecology & Epi- and mesopelagic, $0-500$ m \\
ELH pattern & Oviparous, eggs probably \\
& pelagic, pelagic larvae \\
Spawning & Season: \\
& Area: \\
& Mode: \\
Fecundity & Migration: \\
Age at first maturity & Range/function: \\
Longevity & \\
&
\end{tabular}

\section{EARLY LIFE HISTORY DESCRIPTION}

\section{EGGS}

Diameter

No. of oil globules

Oil globule diameter

Yolk

Envelope

Hatch size

Incubation time/temp.

Pigment

Diagnostic characters

\section{LARVAE}

Preanal length $\quad<50 \%$ SL

Length at flexion $\quad 7.2-8.8 \mathrm{~mm} \mathrm{SL}$

Length at transformation $12-15 \mathrm{~mm} \mathrm{SL}$, as large as $18 \mathrm{~mm} \mathrm{SL}$

Sequence of fin Caudal, pectorals, anal, development dorsal, pelvics

Pigment

- Preflexion larvae unpigmented

- Large melanophore over gut

- Postanal ventral melanophores in juveniles

Diagnostic characters

- Single spot over gut and lack of pigment elsewhere (flexion and postflexion larvae)

For Genus

Distinguished from other myctophids with elliptical eyes by

- Head small, body slender

- Gut short

- Wide space between anus and anal fin

- Larvae relatively lightly pigmented

- Eyes moderately narrow

- No enlarged fins

Distinguished from $P$. thompsoni by

- Lack of postanal ventral melanophores in preflexion larvae and presence of only one spot over the gut in larger larvae

\footnotetext{
${ }^{\mathrm{s}}$ Wisner 1974

Ref: Moser and Ahlstrom 1970, Moser et al. 1984a.
} 


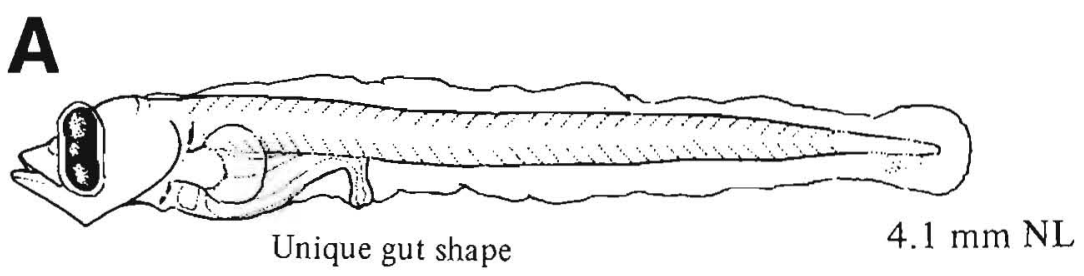

B
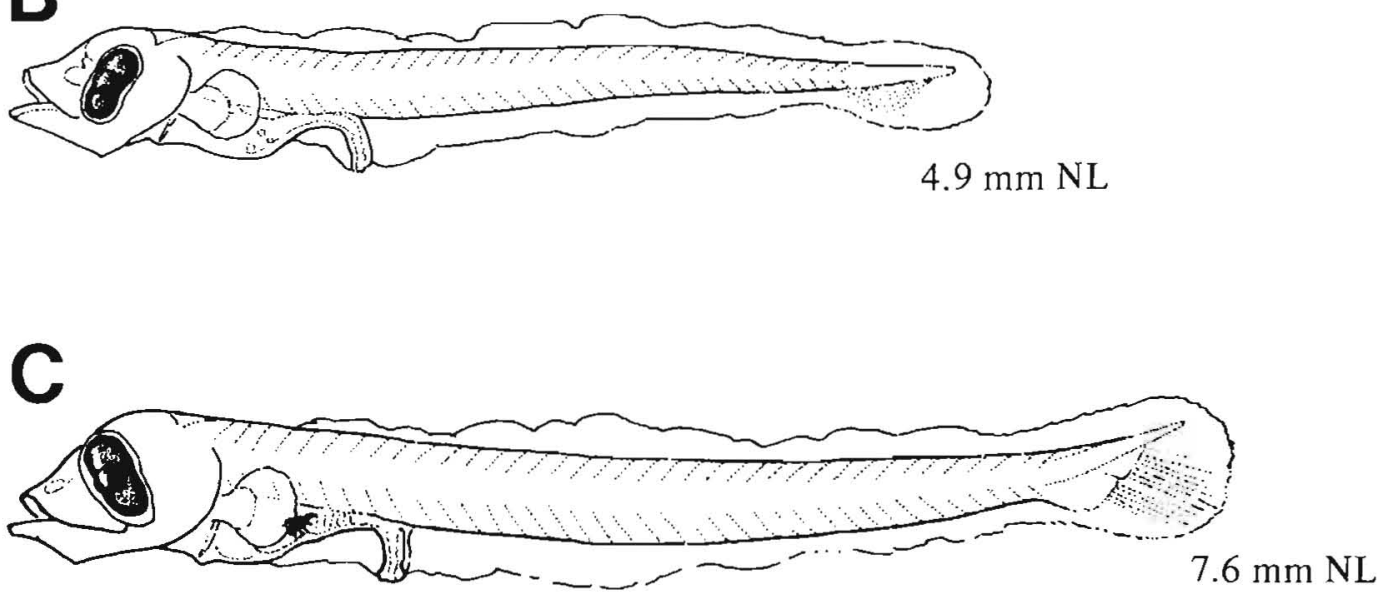

Large melanophore on anterior of gut

D

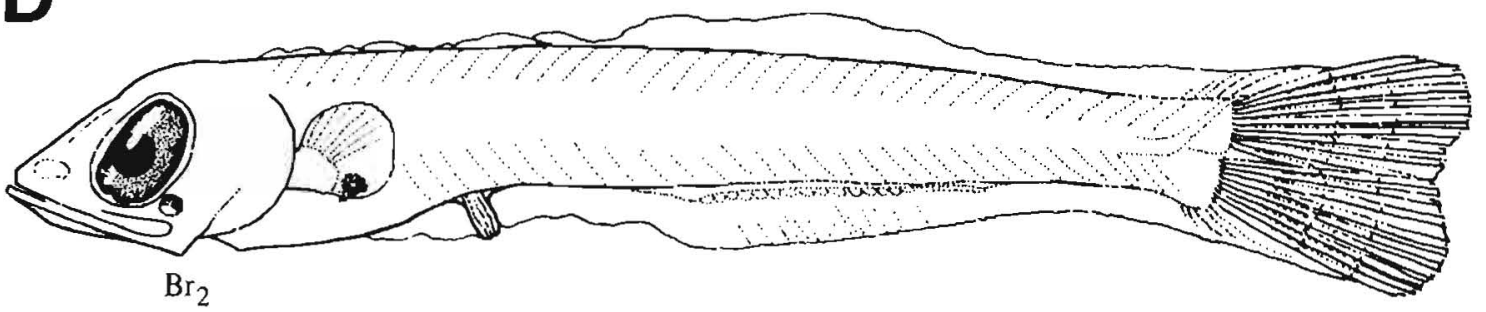

$12.4 \mathrm{~mm} \mathrm{SL}$

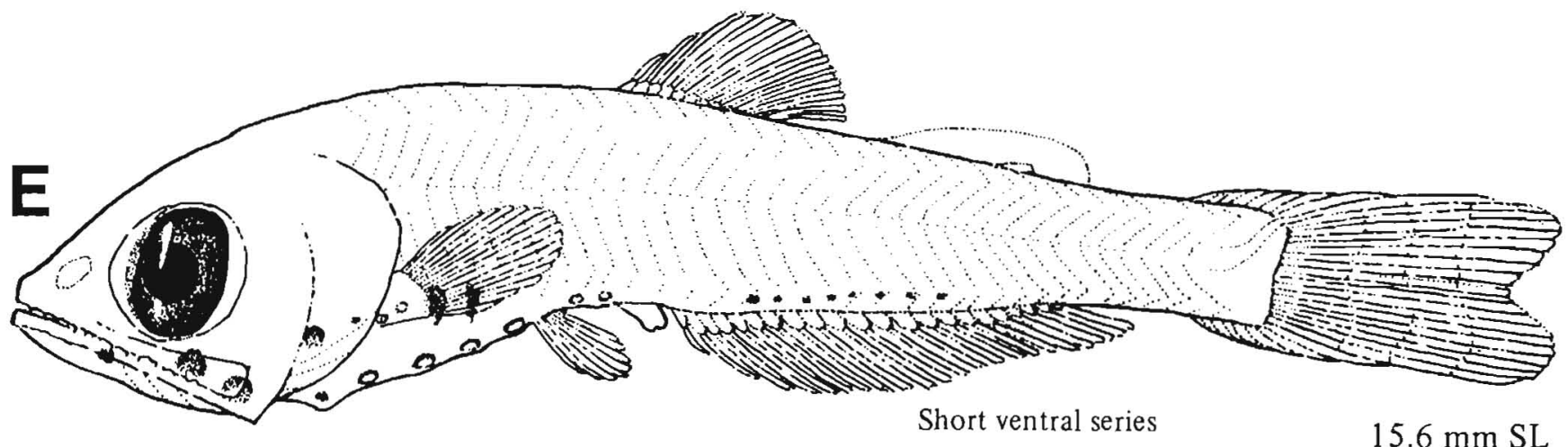

Figures A-E, Moser and AhIstrom 1970. 


\section{MERISTICS}

Vertebrae

Total: $37-38-39$

Precaudal: $\mathrm{X}-\mathrm{X}-\mathrm{X}$

Caudal: X-X-X

Branchiostegal rays

Caudal fin

Pelvic fin

$8-\mathrm{X}-12$

$7-9,10+9,6-8$

Abdominal

R: 8-8-8

Dorsal fin

R: $11-12-13$

Pectoral fin

Anal fin

R: $14-15-17$

Gill rakers

R: $21-23-25$

$\mathrm{U}: 3-4-4$

L: $13-14-16$

\section{LIFE HISTORY}

$\begin{array}{ll}\text { Range } & \begin{array}{c}\text { Cent. California, } 34-38^{\circ} \mathrm{N}^{\mathrm{a}} \text { to } \\ \text { Bering Sea, } 54-66^{\circ} \mathrm{N}\end{array} \\ \text { Ecology } & \text { Epi- and mesopelagic } \\ \text { ELH pattern } & \text { Oviparous, eggs probably } \\ & \text { pelagic, pelagic larvae } \\ \text { Spawning } & \text { Season: } \\ & \text { Area: } \\ & \text { Mode: } \\ & \text { Migration: } \\ \text { Fecundity } & \text { Range/function: } \\ \text { Age at first maturity } & \\ \text { Longevity } & \end{array}$

\section{EARLY LIFE HISTORY DESCRIPTION}

\section{EGGS}

Diameter

No. of oil globules

Oil globule diameter

Yolk

Envelope

Hatch size

Incubation time/temp.

Pigment

Diagnostic characters

\section{LARVAE}

Preanal length

$<50 \%$ SL

Length at flexion

7-10.0 mm SL

Length at transformation

Sequence of fin development

14-17 mm SL

Caudal, pectorals, anal, dorsal, pelvics

Pigment

- Postanal ventral melanophores $>10$ in preflexion larvae decreasing to $<10$ in flexion larvae; not present in postflexion larvae

- Dorsolateral surface of gut in postflexion larvae

Diagnostic characters

- See P. crockeri (p. 150)

- Narrow eye

- Postanal ventral melanophore pattern

${ }^{a}$ R.N. Lea, Calif. Dep. Fish Game, 2201 Garden Road, Monterey, CA 93940, pers. commun., 19 Feb. 1987.

Ref: Moser and Ahlstrom 1970 

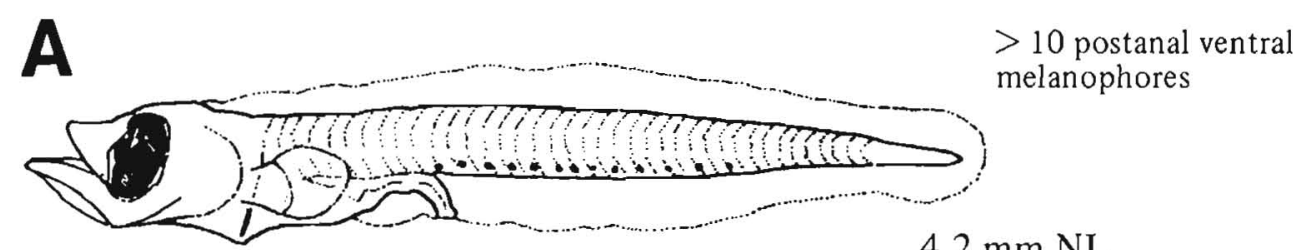

\section{B}

$4.2 \mathrm{~mm} \mathrm{NL}$

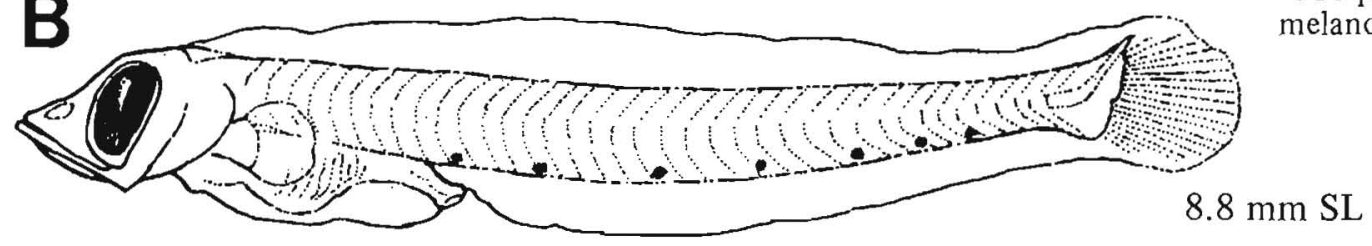

C

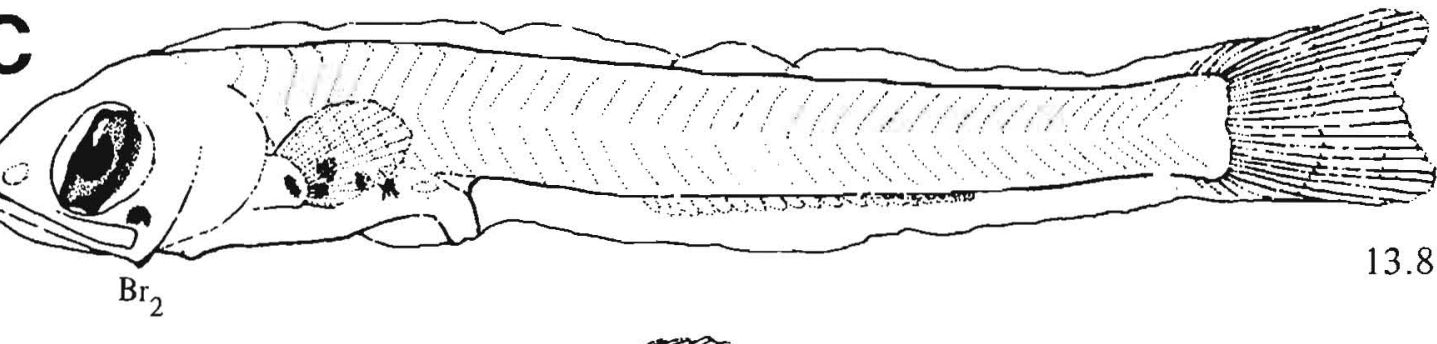
No postanal ventral melanophores

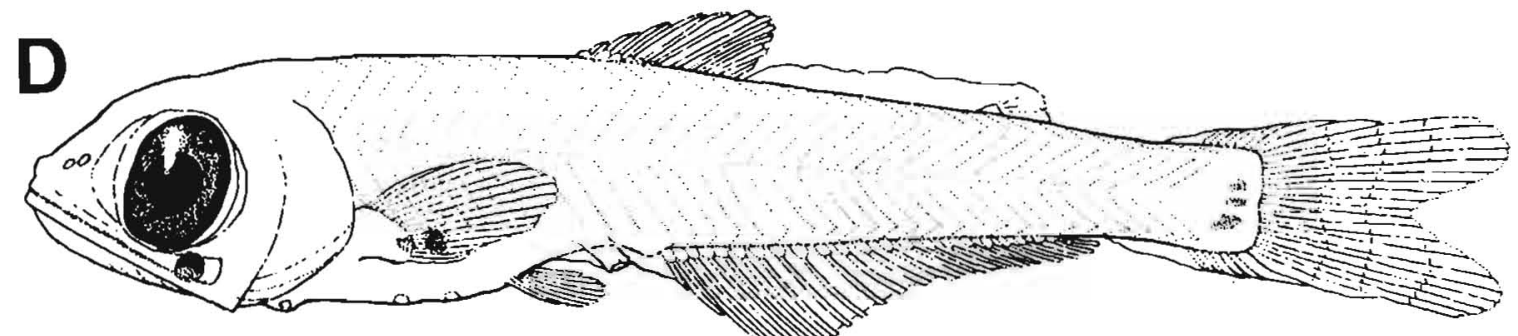

$18.6 \mathrm{~mm} \mathrm{SL}$

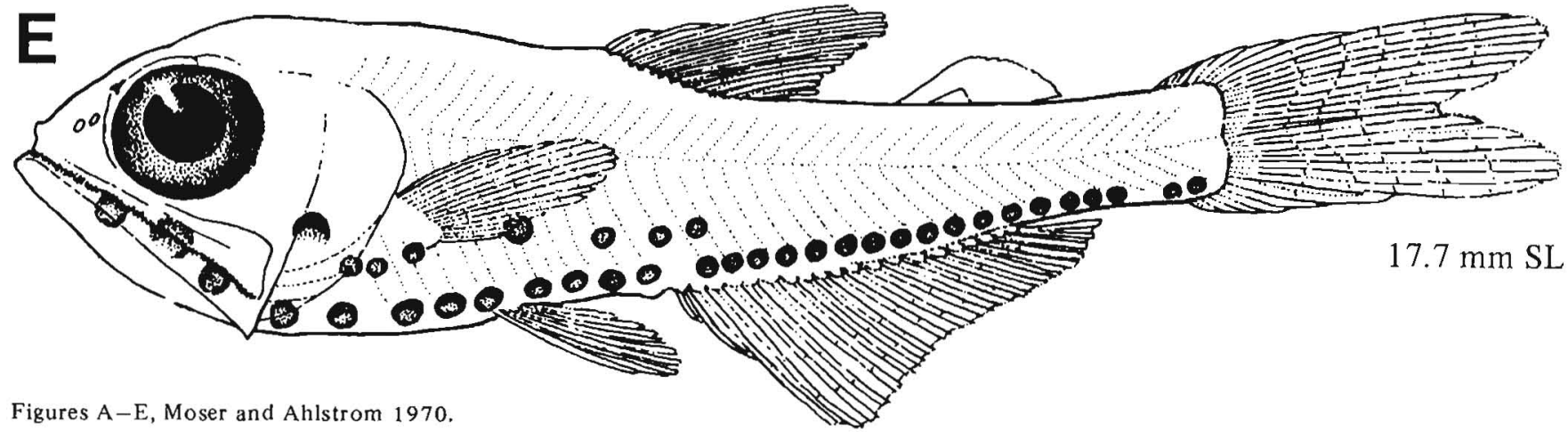




\section{MERISTICS}

Vertebrae

Total: $37-\mathrm{X}-40$

Precaudal: 16-16-17

Caudal: 22-23-23

Branchiostegal rays

Caudal fin

Pelvic fin

$8-\mathrm{X}-12$

$8-9,10+9,8-9$

Abdominal

R: 8-8-8

Dorsal fin

R: $13-14-15$

Pectoral fin

R: $15-17-19$

Anal fin

Gill rakers
R: $19-20-22$
L: $15-16-17$

\section{EARLY LIFE HISTORY DESCRIPTION}

\section{EGGS}

Diameter

No. of oil globules

Oil globule diameter

Yolk

Envelope

Hatch size

Incubation time/temp.

Pigment

Diagnostic characters

\section{LARVAE}

Preanal length

Length at flexion $\quad 8.0-10.0 \mathrm{~mm} \mathrm{SL}$

Length at transformation $23.0-24.0 \mathrm{~mm} \mathrm{SL}$

Sequence of fin development

Pectorals, pelvics, caudal, anal, dorsal: pectoral fin base large and wing-shaped with supernumerary rays developing first

Pigment

- Paired fins

- Lateral gut and anus

- Postanal ventral melanophores (preflexion)

Age at first maturity

Longevity

$7 \mathrm{yr}^{\mathrm{a}}$
- Also on snout, hindbrain, cleithral region, isthmus (lower jaw)

Diagnostic characters

Distinguished from other myctophids with elliptical eyes by

- Broad head

- Eyes slightly stalked with conical choroid mass (narrow)

- Early pectoral fin development, pectorals large with wing-shaped base

- Early pelvic fin development (unusual for myctophids)

- Dorsal fin rays develop in finfold

- Slightly enlarged median finfold

- Pigment on paired fins

${ }^{\mathrm{a}}$ Fitch and Lavenberg 1968

Ref: Moser and Ahlstrom 1970, 1974; Moser et al. 1984a. 
A

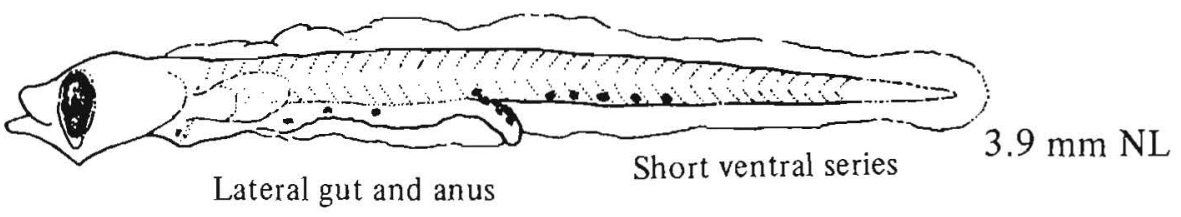

B

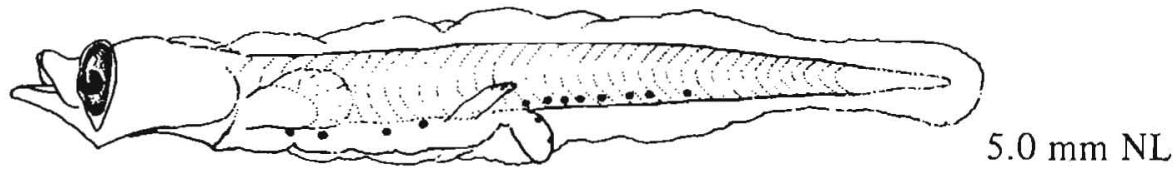

C

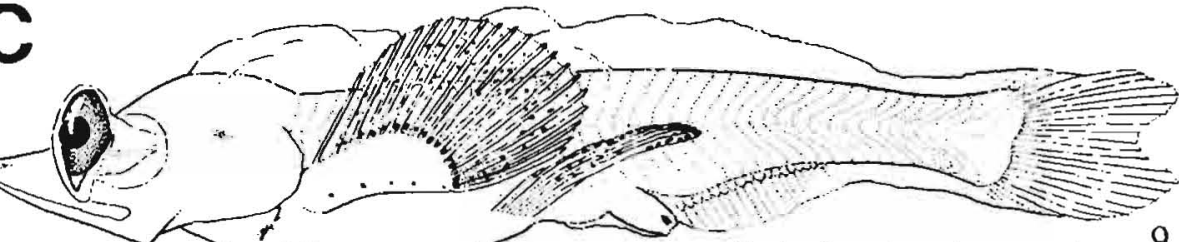

Early forming pigmented $9.6 \mathrm{~mm} \mathrm{SL}$ pectoral and pelvic fins
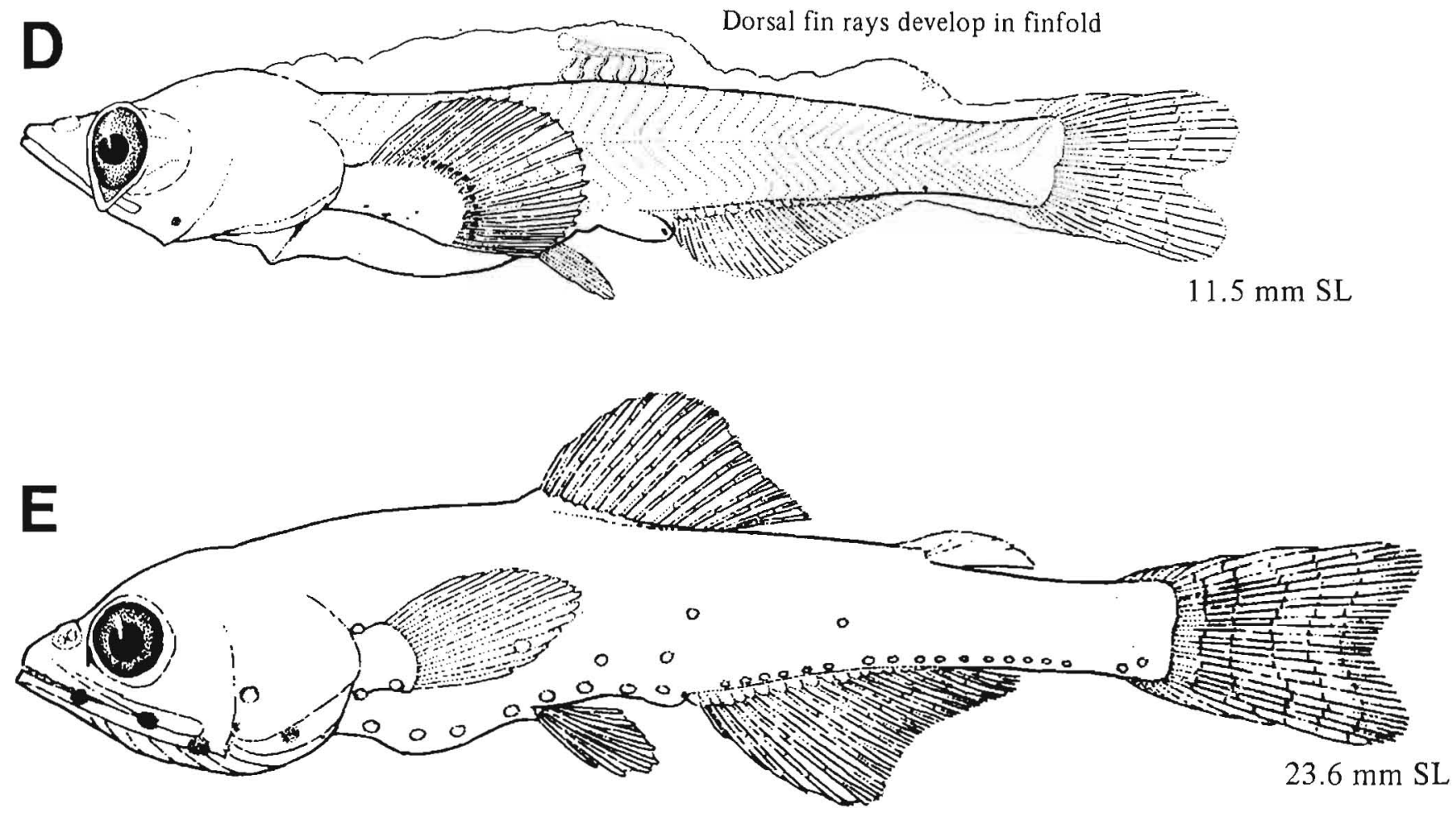

Figures A-E, Moser and Ahlstrom 1970. 
MERISTICS

\begin{tabular}{|c|c|}
\hline \multirow[t]{3}{*}{ Vertebrae } & \\
\hline & Precaudal: X-X-X \\
\hline & \\
\hline Branchiostegal rays & 9-9-9 \\
\hline Caudal fin & $6-7,10+9,6-7$ \\
\hline Pelvic fin & Abdominal \\
\hline Dorsal fin & $\mathrm{R}: \mathrm{X}-\mathrm{X}-\mathrm{X}$ \\
\hline Pectoral fin & $\begin{array}{l}\text { R: } 16-X-18 \text { (larvae) }^{a} \\
\text { R: } 9-X-12 \text { (adults) }^{-1}\end{array}$ \\
\hline Anal fin & $\mathrm{R}: \mathrm{X}-\mathrm{X}-\mathrm{X}$ \\
\hline Gill rakers & $\mathrm{U}: \mathrm{X}-\mathrm{X}-\mathrm{X}$ \\
\hline \multicolumn{2}{|l|}{ LIFE HISTORY } \\
\hline Range $^{b}$ & $\begin{array}{l}\text { South of southern California to } \\
\text { N. California, } 38-42^{\circ} \mathrm{N},{ }^{\mathrm{c}} \text { and } \\
\text { Oregon, } 42-46^{\circ} \mathrm{N}^{\mathrm{d}}\end{array}$ \\
\hline Ecology & Unknown \\
\hline ELH pattern & $\begin{array}{l}\text { Oviparous, eggs unknown, } \\
\text { pelagic larvae }\end{array}$ \\
\hline Spawning & Season: \\
\hline & Area: \\
\hline & Mode: \\
\hline & Migration: \\
\hline $\begin{array}{l}\text { Fecundity } \\
\text { Age at first maturity } \\
\text { Longevity }\end{array}$ & Range/function: \\
\hline
\end{tabular}

\section{EARLY LIFE HISTORY DESCRIPTION}

\section{EGGS}

Diameter

No. of oil globules

Oil globule diameter

Yolk

Envelope

Hatch size

Incubation time/temp.

Pigment

Diagnostic characters

\section{LARVAE}

Preanal length

Length at flexion

$55 \%$ SL increasing with development to $80 \%$ SL

Length at transformation $>20.0 \mathrm{~mm} \mathrm{SL}$

Sequence of fin Pectorals, caudal, anal and development dorsal, pelvics

Pigment

- Preflexion and flexion larvae

-Dark band of pigment between olfactory and optic lobes of the brain

- Blotch develops medially to each pectoral fin base

-Two blotches on dorsal surface of gut merging into one with development

- Three postanal body blotches

-Median finfold, lower pectoral ray

-Dorsal and ventral margins of caudal peduncle

\section{Diagnostic characters}

- Among largest myctophid larvae at transformation (>20.0 mm SL)

- Morphology: Early larvae slender but relative body depth doubles in later stages

- Only $\mathrm{Br} 2$ develops during larval period

Distinguished from all other myctophids except Tarletonbeania crenularis by

- Enlarged median finfolds

Distinguished from $T$. crenularis (p. 158) by

- Eye moderately narrow, no choroid tissue on ventral surface of eye

- Pigment: Band on head between eyes

\footnotetext{
${ }^{a}$ Larvae have six more pectoral fin rays than adults.

${ }^{\mathrm{b}}$ Range for larvae; adults widespread.

${ }^{c}$ Moser and Ahlstrom 1970

${ }^{\mathrm{d}}$ Richardson and Pearcy 1977

Ref: Moser and Ahlstrom 1970
} 


\section{A}
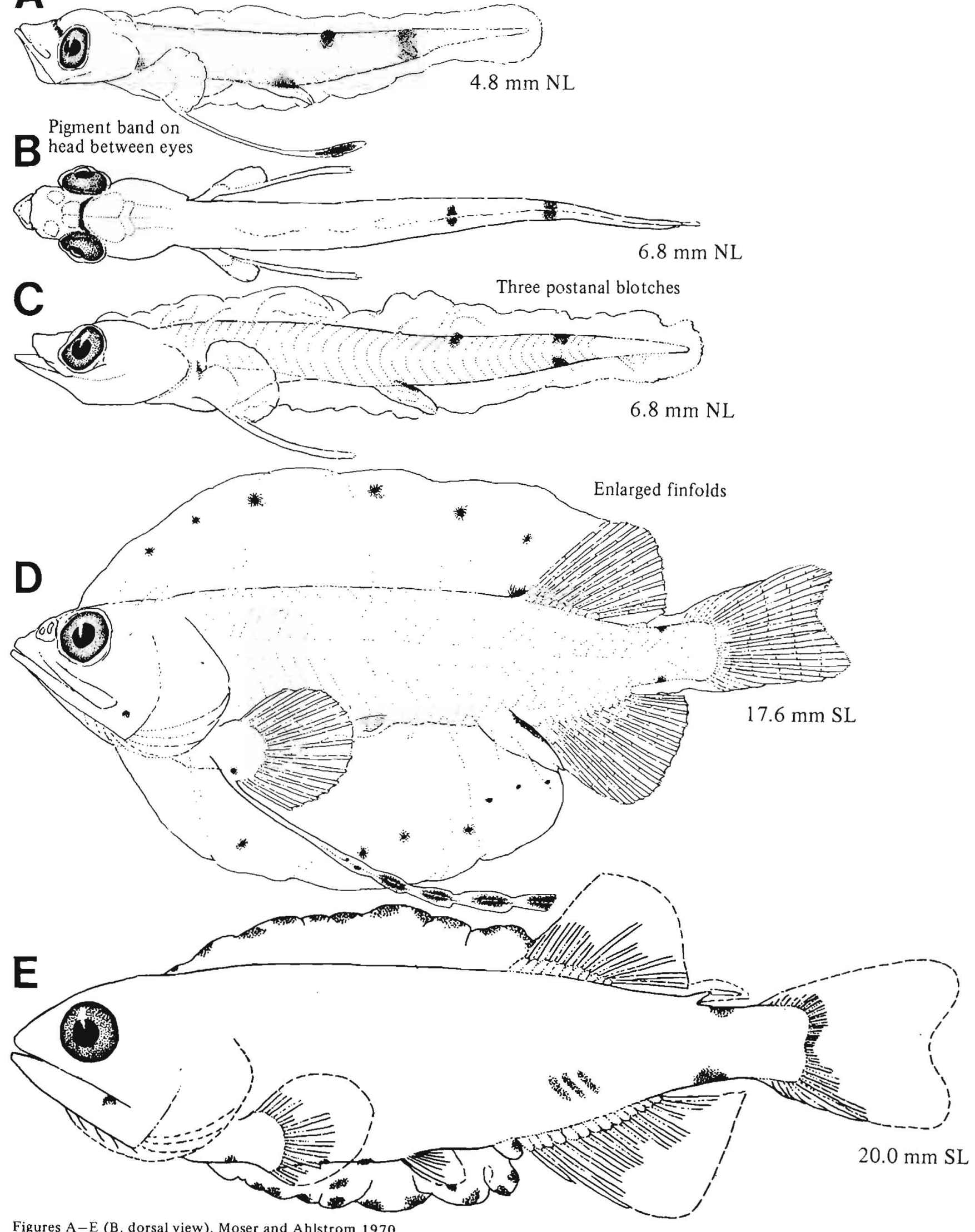

Figures A-E (B, dorsal view), Moser and Ahlstrom 1970. 


\section{MERISTICS}

\begin{tabular}{|c|c|}
\hline \multirow[t]{3}{*}{ Vertebrae } & Total: $39-41-42$ \\
\hline & Precaudal: $17-17-19$ \\
\hline & Caudal: $22-23-24$ \\
\hline Branchiostegal rays & $8-9-11$ \\
\hline Caudal fin & $5-7,10+9,5-7$ \\
\hline Pelvic fin & Abdominal \\
\hline & $\mathrm{R}: 7-8-9$ \\
\hline Dorsal fin & $\mathrm{R}: 11-12-14$ \\
\hline Pectoral fin & $\mathrm{R}: 11-13-15$ \\
\hline Anal fin & $\mathrm{R}: 17-18-20$ \\
\hline Gill rakers & $\mathrm{U}: 4-5-6 \quad \mathrm{~L}: 10-11-12$ \\
\hline \multicolumn{2}{|l|}{ LIFE HISTORY } \\
\hline Range & $\begin{array}{l}\text { South of southern California to } \\
\text { Bering Sea, } 54-66^{\circ} \mathrm{N}\end{array}$ \\
\hline Ecology & Epi- and mesopelagic, $0-832 \mathrm{~m}$ \\
\hline ELH pattern & $\begin{array}{c}\text { Oviparous, eggs probably } \\
\text { pelagic, pelagic larvae }\end{array}$ \\
\hline Spawning & $\begin{array}{l}\text { Season: Winter-spring; }{ }^{a} \\
\text { Nov-Feb }\end{array}$ \\
\hline & Area: \\
\hline & Mode: \\
\hline & Migration: \\
\hline Fecundity & Range/function: \\
\hline $\begin{array}{l}\text { Age at first maturity } \\
\text { Longevity }\end{array}$ & \\
\hline
\end{tabular}

\section{EARLY LIFE HISTORY DESCRIPTION}

\section{EGGS}

Diameter

No. of oil globules

Oil globule diameter

Yolk

Envelope

Hatch size

Incubation time/temp.

Pigment

Diagnostic characters

LARVAE

Preanal length

Length at flexion

Length at transformation

Sequence of fin development

Pigment

- Preflexion: Postanal band located at posteriormost 4-5 myomeres, on anus, and over gut

- Flexion: Head, finfold, 1-2 postanal melanophores

- Postflexion: Dorsal midline anterior to fin

\section{Diagnostic characters}

Distinguished from Loweina rara (p. 156) by

- See L. rara

Distinguished from all other myctophids except $L$. rara by

- Finfold: Dorsal and ventral finfolds greatly enlarged and conspicuously pigmented (more on dorsal)

- Eyes narrow with lunate choroid mass

- Only $\mathrm{Br} 2$ develops during larval period

- Enlarged ventral pectoral fin ray (lost during transformation)

- Postanal pigment band

Note: $T$. taylori adults occur in the area but their larvae are inadequately known. Pertseva-Ostroumova (1964) illustrated a $12-\mathrm{mm}$ SL larva as $T$. crenularis taylori, but it has no features that distinguish it from $T$. crenularis.

\footnotetext{
${ }^{a}$ Ahlstrom 1965

${ }^{\mathrm{b}}$ Wang 1981
}

Ref: Moser and Ahlstrom 1970, 1974; Moser et al. 1984a. 
A

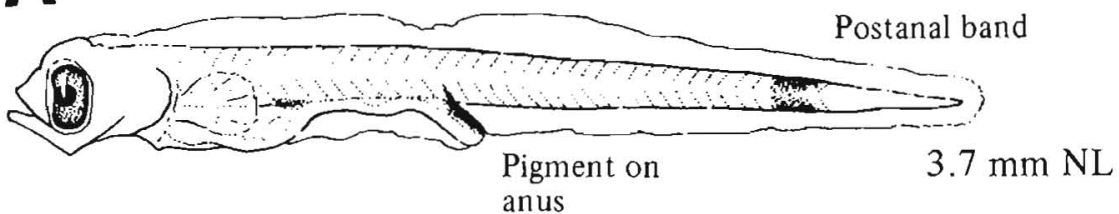

B

Pigment on
anus

$3.7 \mathrm{~mm} \mathrm{NL}$

0
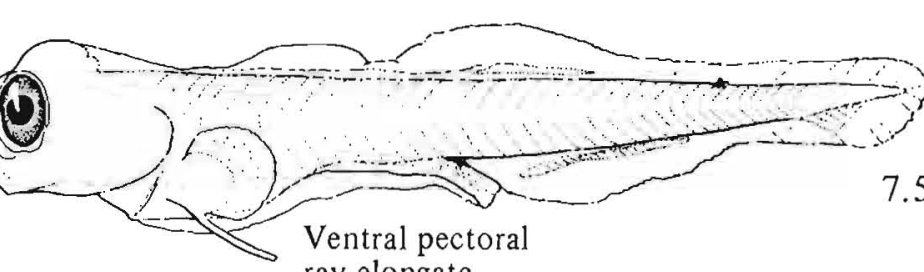

$7.5 \mathrm{~mm} \mathrm{NL}$

ray elongate Enlarged
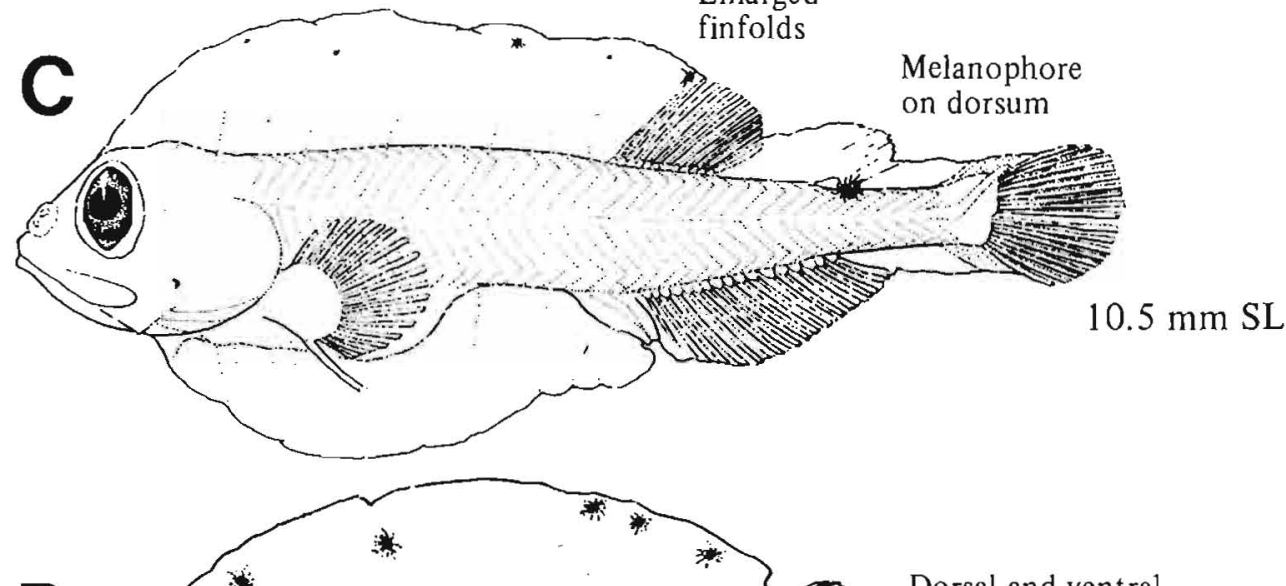

Dorsal and ventral

D melanophores

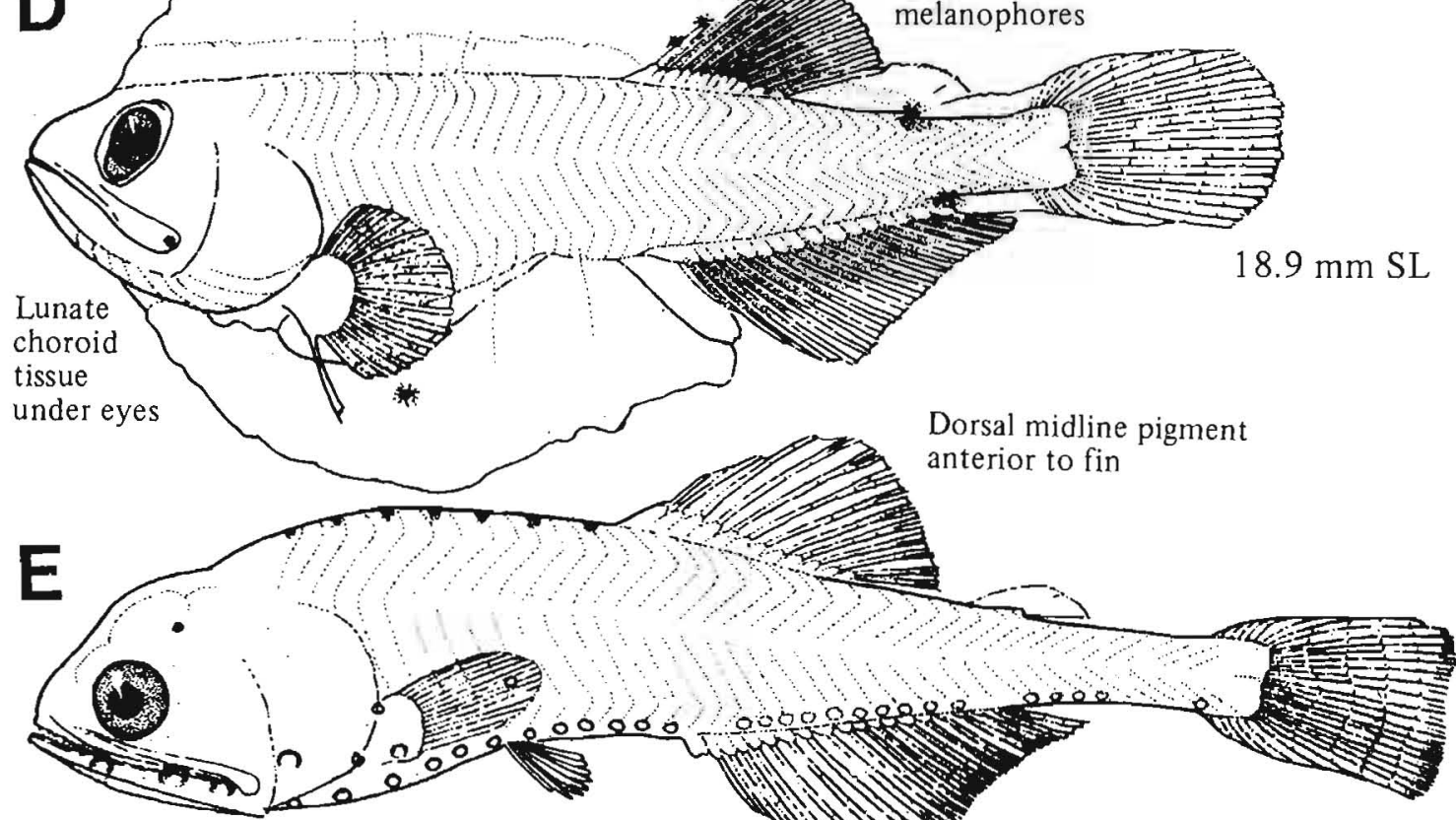

Figures A-E, Moser and AhIstrom 1970.

$21.7 \mathrm{~mm} \mathrm{SL}$ 


\section{MERISTICS}

Vertebrae $^{\mathbf{a}}$

Total: $35-36-38$

Precaudal: $\mathrm{X}-\mathrm{X}-\mathrm{X}$

Caudal: $\mathrm{X}-\mathrm{X}-\mathrm{X}$

Branchiostegal rays

Caudal fin

Pelvic fin

$8-\mathrm{X}-12$

$6,10+9,6-7$

Abdominal

R: 8-X-9

Dorsal fin

$R: 13-14-15$

Pectoral fin

$\mathrm{R}: 12-\mathrm{X}-14$

Anal fin ${ }^{\mathrm{a}}$

R: $13-\mathrm{X}-14$

Gill rakers ${ }^{\mathrm{a}}$

\section{EARLY LIFE HISTORY DESCRIPTION}

\section{EGGS}

Diameter

No. of oil globules

Oil globule diameter

Yolk

Envelope

Hatch size

Incubation time/temp.

Pigment

Diagnostic characters

\section{LARVAE}

Preanal length

$46 \%$ SL, with development $58-61 \% \mathrm{SL}$

Length at flexion

6.2-7.0 mm SL

Length at transformation $16.6-21.0 \mathrm{~mm}$ SL (may be larger)

Sequence of fin Caudal, pectorals, anal, development dorsal, pelvics

Pigment

- Generally light pigment

- Pair of melanophores at each side of gut at point of divergence of the free terminal section (retained throughout but becoming embedded with development)

- Series $(\sim 11)$ of postanal ventral melanophores in early larvae, with development usually coalescing to a single spot in postflexion larvae

\section{Diagnostic characters}

- Photophores: Br2 (7.0 mm SL); Vn (7.8 mm SL); PLO $(8.7 \mathrm{~mm} \mathrm{SL}$; develop and remain at pectoral fin base throughout larval period but at transformation they migrate dorsally to just below the lateral line); PO5 (9.0 mm SL); during transformation the remaining photophores develop sequentially

Distinguished from other myctophids with round eyes by

- Eye elliptical in preflexion larvae (although not perfectly round, the eye is easily differentiated from the narrow eyes of larval Myctophinae)

- Single spot at anus

- Preflexion larvae with $\sim 11$ postanal ventral melanophores

\footnotetext{
${ }^{a}$ Peden and Hughes (1986) report counts for the following meristic characters: Precaudal vertebrae 16 Caudal vertebrae Anal fin rays Gill rakers 20 $6-7+1+15$

${ }^{\text {b}}$ H.G. Moser, NMFS Southwest Fish. Cent., P.O. Box 271, La Jolla, CA 92038 pers. commun., 9 Jan. 1984. Ilustrations of small larvae are unavailable. A figure of a preflexion larva of $C$. warmingi is included for comparison.
} 


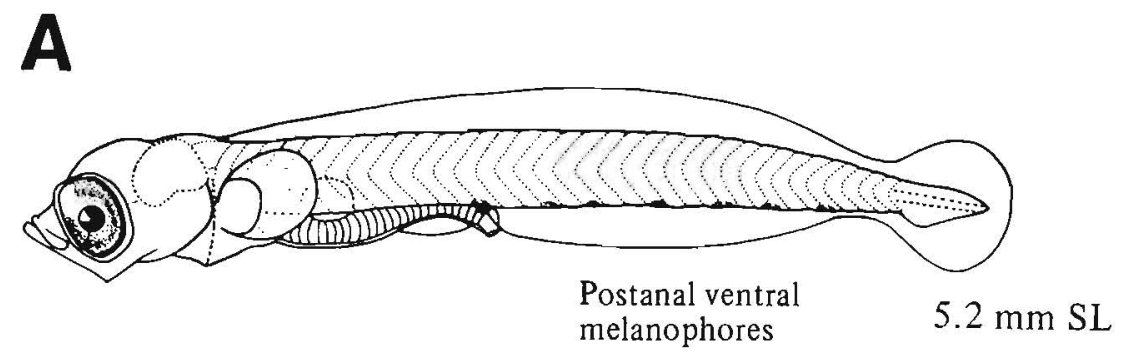

(Ceratoscopelus warmingi)

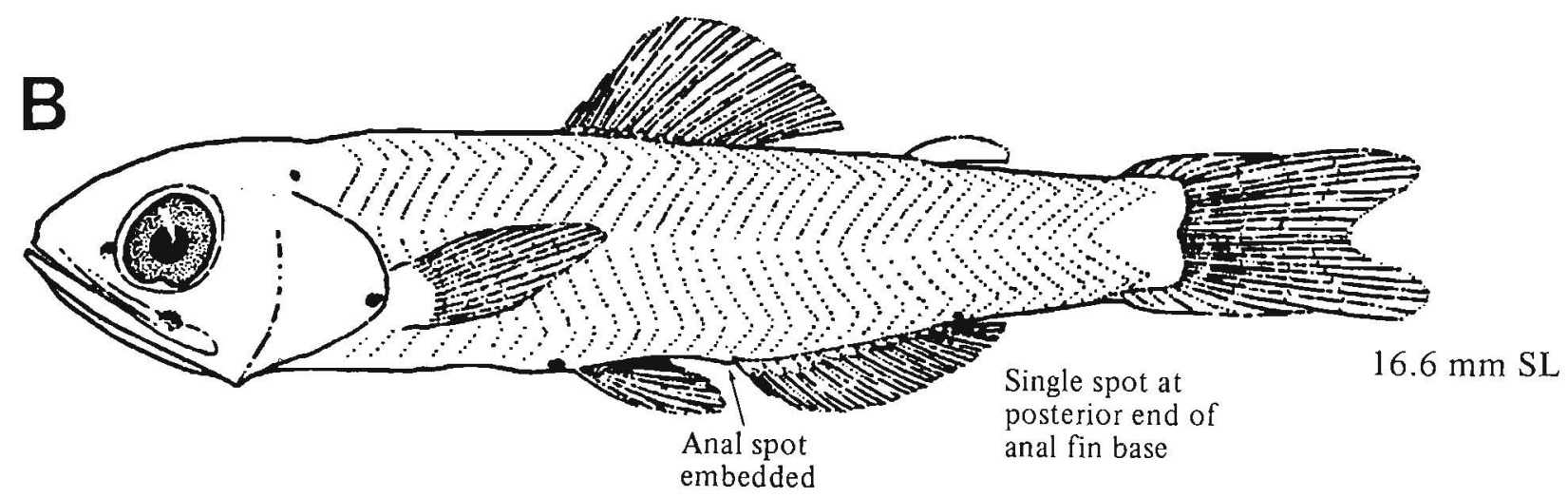

Figure A, Miller et al. 1979 (Hawaiian specimen); B, Moser and Ahlstrom 1974. 


\section{MERISTICS}

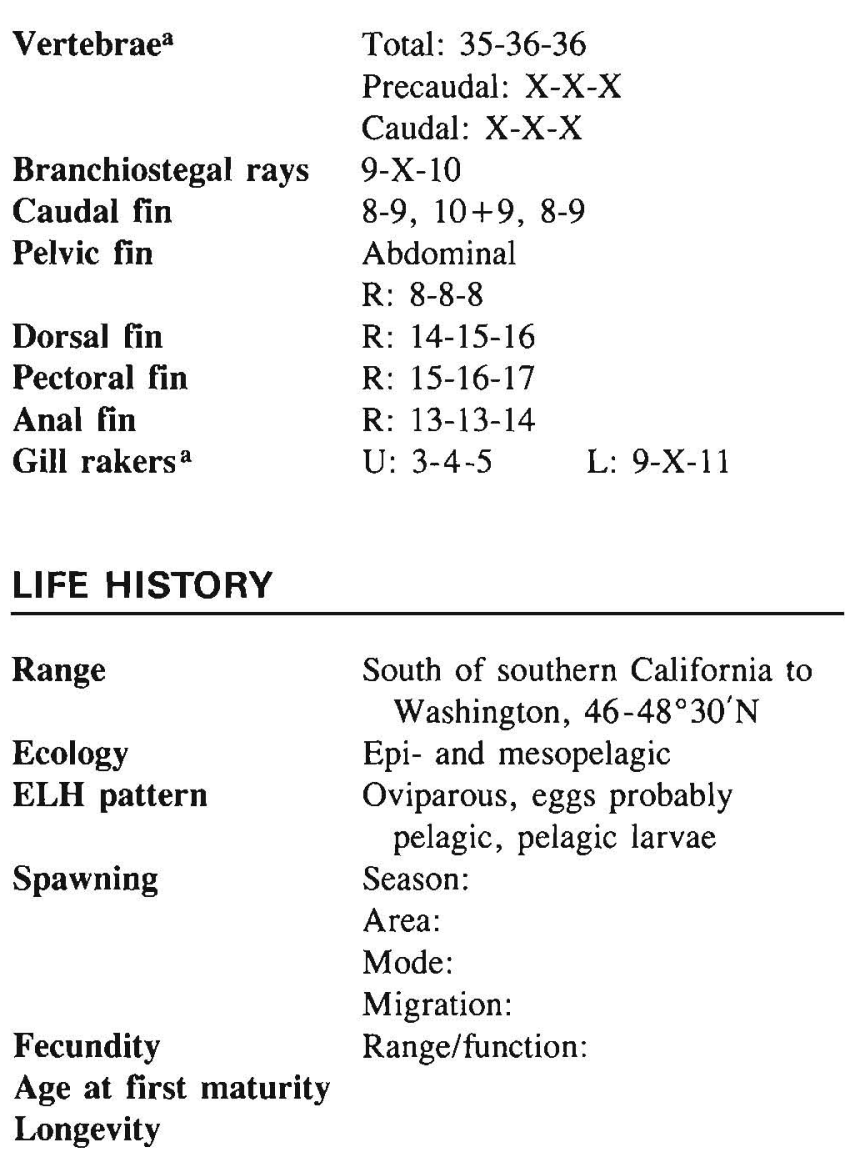

\section{EARLY LIFE HISTORY DESCRIPTION}

\section{EGGS}

Diameter

No. of oil globules

Oil globule diameter

Yolk

Envelope

Hatch size

Incubation time/temp.

Pigment

\section{Diagnostic characters}

\section{LARVAE}

\section{Preanal length $\quad 50 \% \mathrm{SL}$ \\ Length at flexion By 5-6 mm SL \\ Length at transformation $19.6 \mathrm{~mm} \mathrm{SL}$ \\ Sequence of fin Caudal, pectorals, anal, development}

Pigment - Genus ${ }^{b}$

(Pigment for $L$. urophaos more restricted to dorsal and ventral midline)

- Brain, nape, gut, swimbladder

- Double row of large melanophores along dorsal midline with an opposing double row of postanal ventral midline melanophores

- Some with more numerous dorsal and ventral melanophores and internal pigment above the notochord

\section{Diagnostic characters}

- Photophores: Br2, PLO, PO5, and Vn+PO1 develop during larval period

Distinguished from other myctophids with round eyes by

- Dorsal midline pigment anterior to anus

\footnotetext{
${ }^{a}$ Peden and Hughes (1986) report counts of the following meristic characters: Total vertebrae $\quad 38$

Precaudal vertebrae 17 Caudal vertebrae 21 Gill rakers $\quad 5+1+12$

${ }^{b}$ Discussion of pigment is based on genus, since illustrations of small larvae of $L$. urophaos are not available. Figures of a preflexion larva of $L$. luminosa are included for comparison.
}

Ref: Moser and Ahlstrom 1972; Moser et al. 1984a; H.G. Moser, NMFS Southwest Fish. Cent., P.O. Box 271, La Jolla, CA 92038, unpubl. 
A

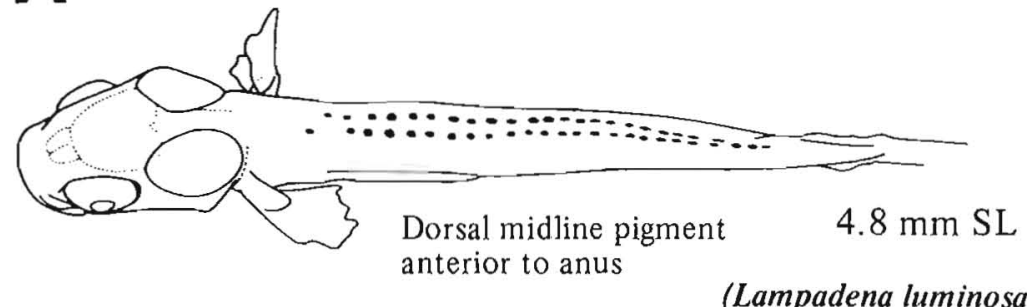

B

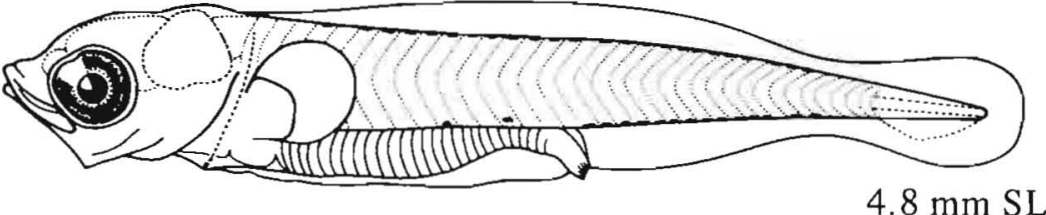

(Lampadena luminosa)
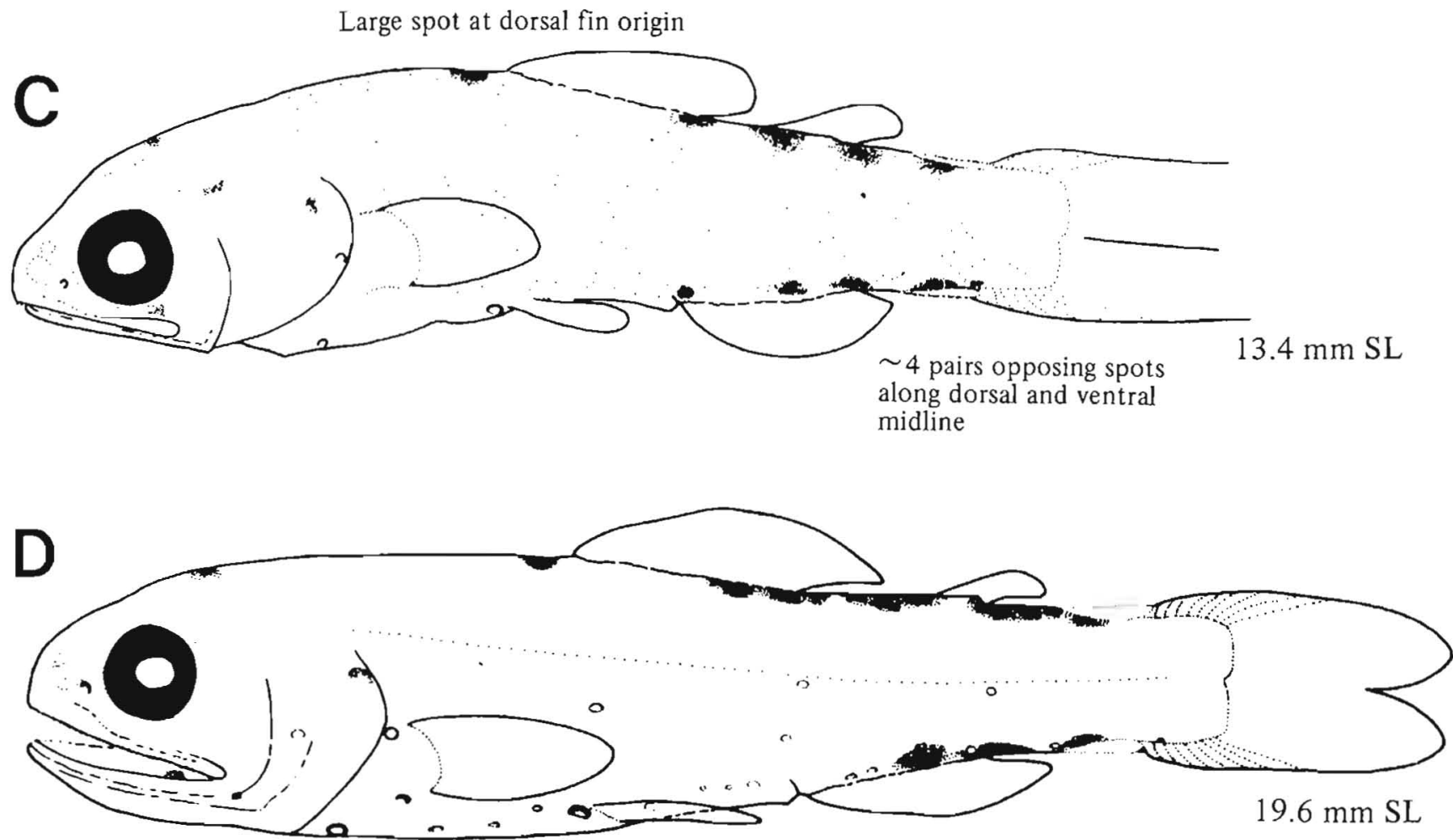

Figures A-B (A, dorsal view), Miller et al. 1979 (Hawaiian specimens); C-D, Moser and Ahlstrom 1972. 

Of the four Lampanyctus species found in the study area, we can identify larvae of only L. regalis and $L$. ritteri. Generic characters include:

- Pigment above brain

- Myoseptal trunk pigment increasing during postflexion to cover most of anterior trunk at transformation

- Slender body; deepens and becomes robust in some species

- Deep head

- Gut short in early preflexion larvae; with development lengthens to midbody

The following meristic information may aid in identifying the four Lampanyctus species.

\begin{tabular}{llccccc} 
& & & \multicolumn{4}{c}{ Fins } \\
\cline { 5 - 6 } Species & \multicolumn{1}{c}{ Distribution } & Vertebrae & Dorsal & Anal & Pectoral & Pelvic \\
\hline Lampanyctus fernae & N. Calif. - Oregon & $36-38$ & $12-14$ & $16-18$ & $12-14$ & 8 \\
Lampanyctus jordani & S. Calif. - Bering Sea & $38-40$ & $10-12$ & $17-20$ & $14-17$ & 8 \\
Lampanyctus regalis & SSC - Bering Sea & $36-39$ & $14-16$ & $17-19$ & $12-14$ & 8 \\
Lampanyctus ritteri & SSC - Bering Sea & $35-38$ & $12-15$ & $16-19$ & $10-13$ & $8-9$ \\
\hline
\end{tabular}




\section{MERISTICS}

\begin{tabular}{ll}
\hline Vertebrae & Total: 36-X-39 \\
& $\begin{array}{l}\text { Precaudal: X-X-X } \\
\text { Caudal: X-X-X }\end{array}$ \\
Branchiostegal rays & $9-9-9$ \\
Caudal fin & $6-8,10+9,6-8$ \\
Pelvic fin & Abdominal \\
& R: $8-8-8$ \\
Dorsal fin & R: $14-15-16$ \\
Pectoral fin & R: $12-13-14$ \\
Anal fin & R: $17-17-19$ \\
Gill rakers & U: $4-4-4$ \\
& \\
LIFE HISTORY & \\
\hline Range & \\
Ecology & South of southern California to \\
ELH pattern & Bering Sea, $54-66^{\circ} \mathrm{N}$ \\
Epi- and mesopelagic \\
Spawning & Oviparous, eggs probably \\
& pelagic, pelagic larvae \\
& Season: \\
Fecundity & Area: \\
Age at first maturity & Mode: \\
Longevity & Rigration: \\
&
\end{tabular}

\section{EARLY LIFE HISTORY DESCRIPTION}

\section{EGGS}

Diameter

No. of oil globules

Oil globule diameter

Yolk

Envelope

Hatch size

Incubation time/temp.

Pigment

Diagnostic characters

\section{LARVAE}

Preanal length

Length at flexion

Length at transformation

Sequence of fin development
$38 \%$ SL preflexion, $54 \%$ SL at flexion, $67 \% \mathrm{SL}$ postflexion

$>4 \mathrm{~mm} \mathrm{SL},<7 \mathrm{~mm} \mathrm{SL}$ $>10 \mathrm{~mm} \mathrm{SL},<28 \mathrm{~mm} \mathrm{SL}$ Caudal, pectorals, anal, dorsal, pelvics (pelvics may form early)

Pigment

- Preflexion larvae are unpigmented except for spots on lower jaw tip and snout

- Head: Jaw tips, internal along snout, postorbital, opercle

- Pectoral and pelvic fins

- Large spot at adipose fin

Diagnostic characters

- Photophores: Only $\mathrm{Br} 2$ develops during larval period (7.8 mm SL); remaining photophores develop synchronously at end of transformation period

Distinguished from other myctophids with round eyes by

- Morphology: Initially slender with a small head; with development a deep broad head and body with elongate snout develops; pectorals moderately large

- Jaws large with teeth

- Pigment: Large spot at adipose fin

${ }^{a}$ H.G. Moser, NMFS Southwest Fish. Cent., P.O. Box 271, La Jolla, CA 92038 pers. commun., 9 Jan. 1984.

Ref: Moser et al. 1984a; Moser, unpubl. 
A Body slender and head small

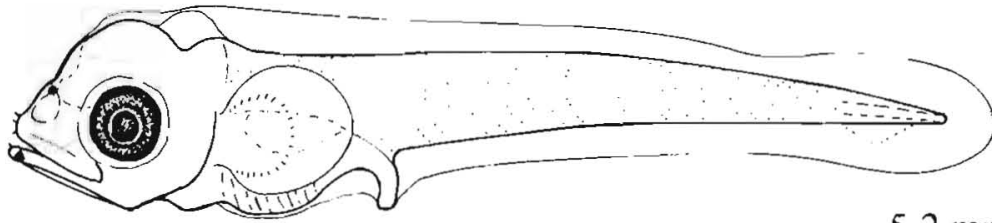

Several spots

$5.2 \mathrm{~mm} \mathrm{SL}$

on head

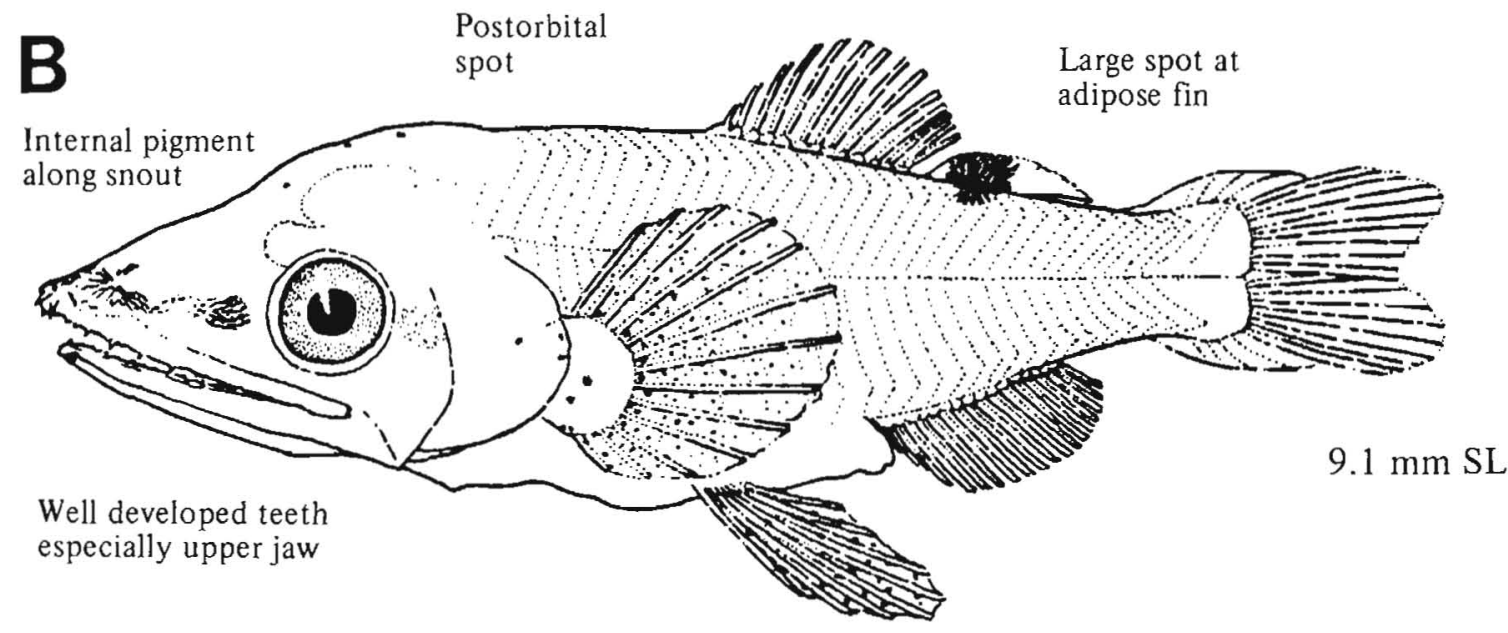

Figure A, NWAFC original (B. Vinter); B, Moser and Ahlstrom 1974. 


\section{MERISTICS}

Vertebrae

Total: $35-36-38$

Precaudal: X-X-X

Caudal: $\mathrm{X}-\mathrm{X}-\mathrm{X}$

Branchiostegal rays 9-9-9

Caudal fin

Pelvic fin

$7-8,10+9,7-8$

Abdominal

R: $8-\mathrm{X}-9$

R: $12-13-15$

Pectoral fin

$\mathrm{R}: 10-11-13$

Anal fin

R: $16-18-19$

Gill rakers

$\mathrm{U}:$ 4-4-4

L: 9-10-11
Dorsal fin

\section{EARLY LIFE HISTORY DESCRIPTION}

\section{EGGS}

Diameter

No. of oil globules

Oil globule diameter

Yolk

Envelope

Hatch size

Incubation time/temp.

Pigment

Diagnostic characters

\section{LARVAE}

Preanal length

Length at flexion

Length at transformation

Sequence of fin development

$<50 \%$ SL in preflexion, with development $50 \% \mathrm{SL}$ Bering Sea, $54-66^{\circ} \mathrm{N}$

Ecology

Epi-, meso-, and bathypelagic, 20-1098 m

ELH pattern

Oviparous, eggs probably pelagic, pelagic larvae

Spawning Season:

Area:

Mode:

Migration:

Fecundity

Range/function:

Age at first maturity

Longevity

\section{Pigment}

- Head: Snout, lower jaw, opercle; several spots anterodorsal to eye persist throughout development

- Dorsal and ventral midline on tail

- Anteroventral to liver

Diagnostic characters

- See $L$. regalis (p. 166)

- Photophores: Only $\mathrm{Br} 2$ develops during larval period; remaining photophores develop synchronously at end of transformation period

Distinguished from other myctophids with round eyes by

- See L. regalis

Distinguished from $L$. regalis by

- Morphology: Body and head moderately deep; jaws, teeth, and pectorals moderate in size

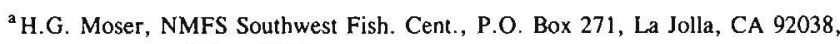
pers, commun,, 9 Jan. 1984. 


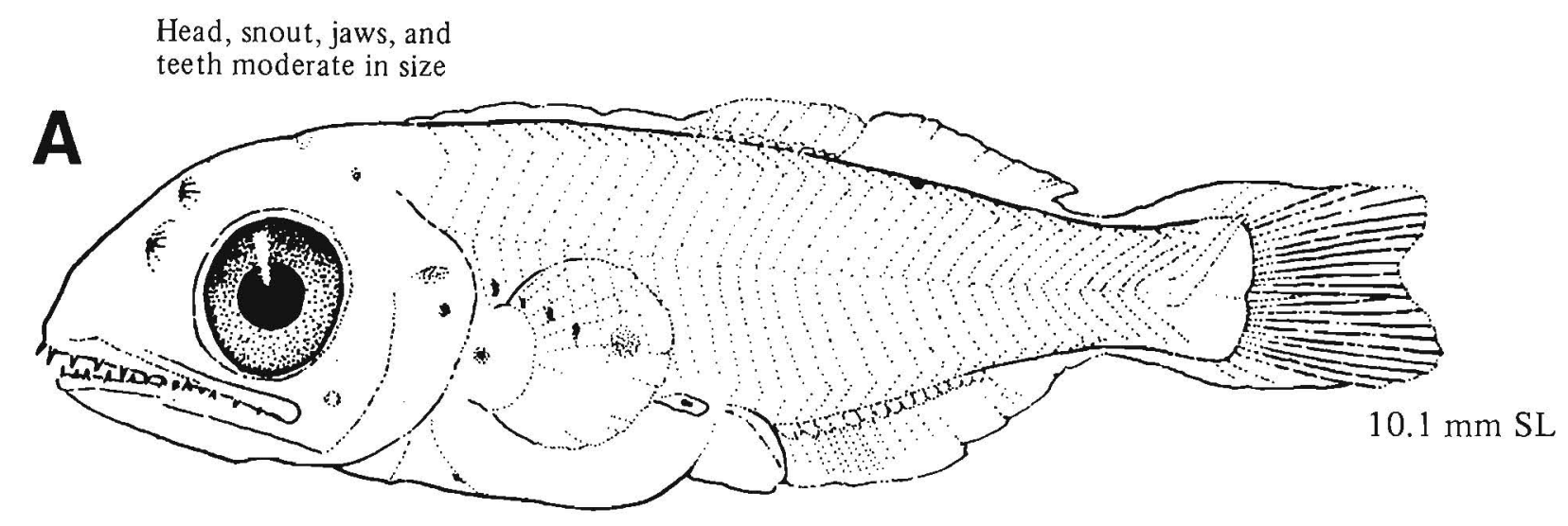

Figure A, Moser and Ahlstrom 1974. 


\section{MERISTICS}

\begin{tabular}{|c|c|}
\hline Vertebrae & $\begin{array}{l}\text { Total: } 36-37-38 \\
\text { Precaudal: } \mathrm{X}-\mathrm{X}-\mathrm{X} \\
\text { Caudal: } \mathrm{X}-\mathrm{X}-\mathrm{X}\end{array}$ \\
\hline Branchiostegal rays & $8-X-12$ \\
\hline Caudal fin & $8,10+9,8$ \\
\hline Pelvic fin & $\begin{array}{l}\text { Abdominal } \\
\mathrm{R}: 8-8-8\end{array}$ \\
\hline Dorsal fin & $\mathrm{R}: 14-\mathrm{X}-17$ \\
\hline Pectoral fin & $\mathrm{R}: 10-\mathrm{X}-13$ \\
\hline Anal fin & $\mathrm{R}: 15-\mathrm{X}-18$ \\
\hline Gill rakers & L: $12-13-15$ \\
\hline \multicolumn{2}{|l|}{ LIFE HISTORY } \\
\hline Range & $\begin{array}{l}\text { South of southern California to } \\
\text { Oregon, } 42-46^{\circ} \mathrm{N}\end{array}$ \\
\hline Ecology & Epi- and mesopelagic \\
\hline ELH pattern & $\begin{array}{l}\text { Oviparous, eggs probably } \\
\text { pelagic, pelagic larvae }\end{array}$ \\
\hline \multirow[t]{4}{*}{ Spawning } & Season: \\
\hline & Area: \\
\hline & Mode: \\
\hline & Migration: \\
\hline Fecundity & Range/function: \\
\hline Age at first maturity & \\
\hline
\end{tabular}

Ref: Moser and Ahlstrom 1974; Moser et al. 1984a; H.G. Moser, NMFS Southwest Fish. Cent., P.O. Box 271, La Jolla, CA 92038, unpubl.

\section{EARLY LIFE HISTORY DESCRIPTION}

\section{EGGS}

Diameter

No. of oil globules

Oil globule diameter

Yolk

Envelope

Hatch size

Incubation time/temp.

Pigment

Diagnostic characters

\section{LARVAE}

Preanal length

Length at flexion

Length at transformation

Sequence of fin development

$<50 \%$ SL, with development increasing to $50 \% \mathrm{SL}$

Pigment

- Early preflexion larvae probably unpigmented with pigment developing by flexion stage

- Postflexion larvae have pigment above brain, internally above otic region, lateral to cleithrum, and in anteroventral region of liver

- Postanal body with 1-2 dorsal midline melanophores and 1 ventral midline melanophore at caudal peduncle

Diagnostic characters

- Photophores: Only $\mathrm{Br} 2$ forms during the larval period

Distinguished from other myctophids with round eyes by

- Dorsal midline melanophores restricted to 1-2 spots posterior to adipose fin

- Head and eyes large with the body tapered 


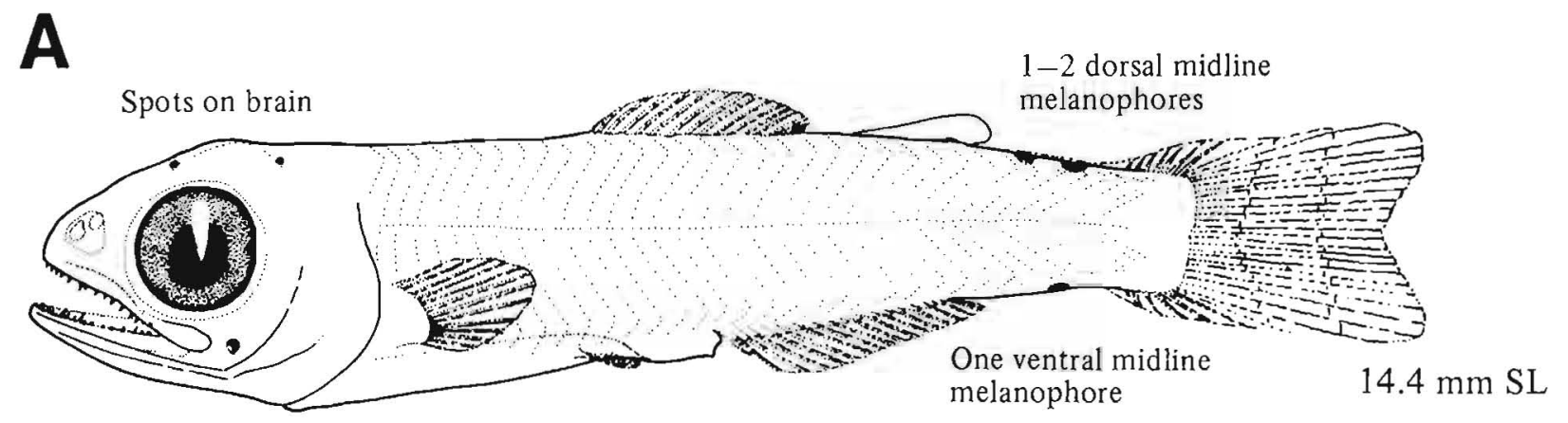

Figure A, Moser and Ahlstrom 1974. 


\section{MERISTICS}

\begin{tabular}{|c|c|}
\hline Vertebrae & $\begin{array}{l}\text { Total: } 36-37-38 \\
\text { Precaudal: } 14-15-16 \\
\text { Caudal: } 20-21-22\end{array}$ \\
\hline Branchiostegal rays & $8-\mathrm{X}-12$ \\
\hline Caudal fin & $6-8,10+9,7-8$ \\
\hline Pelvic fin & $\begin{array}{l}\text { Abdominal } \\
\text { R: } 8-8-8\end{array}$ \\
\hline Dorsal fin & $\mathrm{R}: 12-\mathrm{X}-14$ \\
\hline Pectoral fin & $\mathrm{R}: 9-\mathrm{X}-11$ \\
\hline Anal fin & $R: 14-15-16$ \\
\hline Gill rakers & L: $12-13-14$ \\
\hline \multicolumn{2}{|l|}{ LIFE HISTORY } \\
\hline Range & $\begin{array}{l}\text { South of southern California to } \\
\text { Bering Sea, } 54-66^{\circ} \mathrm{N}\end{array}$ \\
\hline Ecology & $\begin{array}{l}\text { Epi-, meso-, and bathypelagic, } \\
0-2896 \mathrm{~m}\end{array}$ \\
\hline ELH pattern & $\begin{array}{l}\text { Oviparous, eggs probably } \\
\text { pelagic, pelagic larvae }\end{array}$ \\
\hline \multirow[t]{4}{*}{ Spawning } & 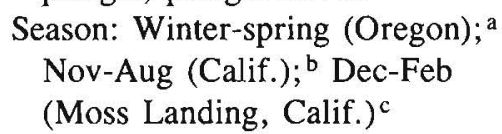 \\
\hline & Area: \\
\hline & Mode: \\
\hline & Migration: \\
\hline Fecundity & Range/function: \\
\hline Age at first maturity & 3 yr (California) ${ }^{b}$ \\
\hline & $4 \mathrm{yr}\left(\right.$ Oregon) ${ }^{\mathrm{a}}$ \\
\hline Longevity & $3-4 y r^{b}$ \\
\hline
\end{tabular}

Smoker and Pearcy 1970

${ }^{\mathrm{b}}$ Fast 1960

${ }^{\mathrm{c}}$ Wang 1981

Ref: Ahlstrom 1965, 1972; Moser and Ahlstrom 1974.

\section{EARLY LIFE HISTORY DESCRIPTION}

\section{EGGS}

Diameter

No. of oil globules

Oil globule diameter

Yolk

Envelope

Hatch size

Incubation time/temp.

Pigment

Diagnostic characters

\section{LARVAE}

Preanal length $\quad<50 \%$ SL

Length at flexion $\quad>5.3 \mathrm{~mm} \mathrm{SL}, \sim 6-7 \mathrm{~mm} \mathrm{SL}$

Length at transformation $>12.5 \mathrm{~mm} \mathrm{SL}$; 10-19 $\mathrm{mm} \mathrm{SL}^{\mathrm{b}}$

\section{Sequence of fin Caudal, pectorals, anal,} development dorsal, pelvics

Pigment

- Gut melanophores: Early preflexion, lateral spots at pectoral fin base, midgut, and anus

- Series of postanal ventral melanophores $(>15)$ in preflexion larvae which coalesce to 2 or 3 spots in postflexion larvae

- Postflexion: Above brain, nape, and embedded in trunk myosepta on each side of dorsal midline (not shown)

\section{Diagnostic characters}

- Photophores: Only Br2 forms in the larval period

Distinguished from other myctophids with round eyes by

- Gut pigment: Three spots in preflexion larvae (pectoral fin base, midgut, and anus); with development spots are retained, although their position shifts posteriorly

- Series of postanal ventral melanophores $(>15)$ in preflexion larvae

Distinguished from Diaphus theta (p. 176) by

- Lack of pigment at caudal base

- Number of postanal ventral melanophores $(>15)$ : Preflexion larvae usually have $>20$; postanal ventral midline melanophores appear more embedded in D. theta (see figure)

S. nannochir larvae are unknown. The following meristic information may aid in identification.

Total vertebrae $\quad 36-38$

Dorsal fin rays 13-14

Anal fin rays 14-16

Pectoral fin rays $9-10$

Pelvic fin rays 8 


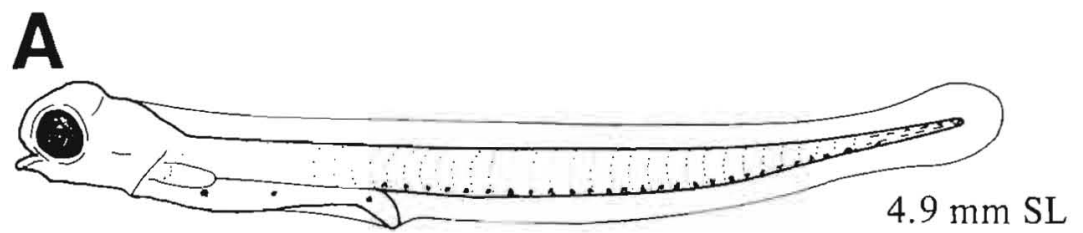

Gut melanophores Postanal ventral melanophores (usually $>15$ )

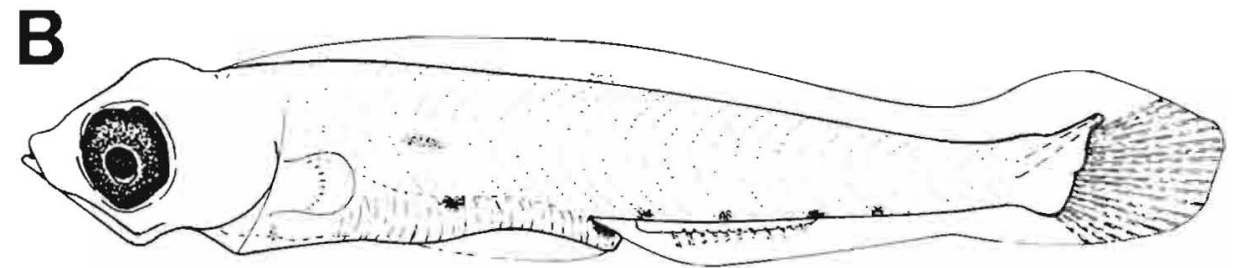

$6.3 \mathrm{~mm} \mathrm{SL}$

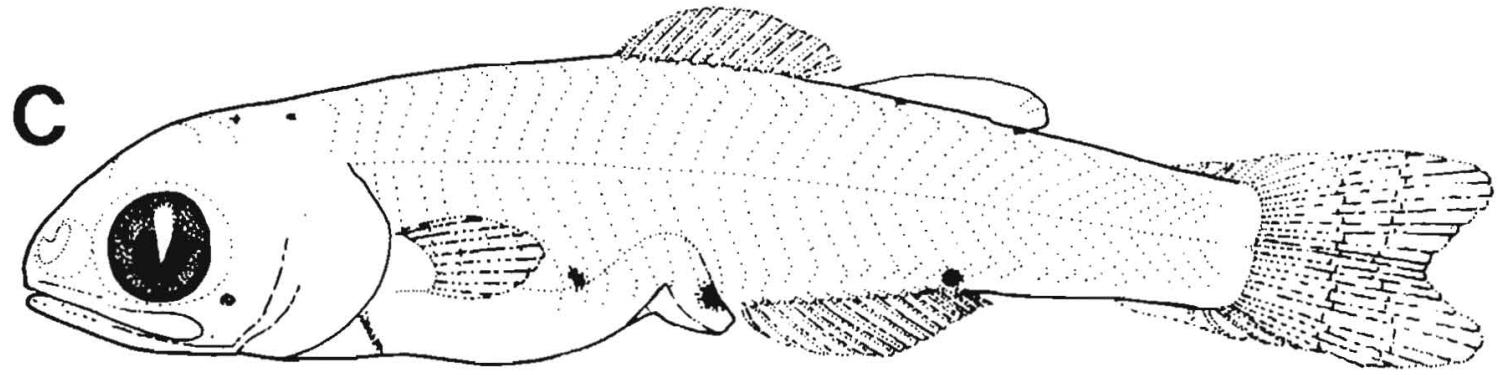

$10.4 \mathrm{~mm} \mathrm{SL}$

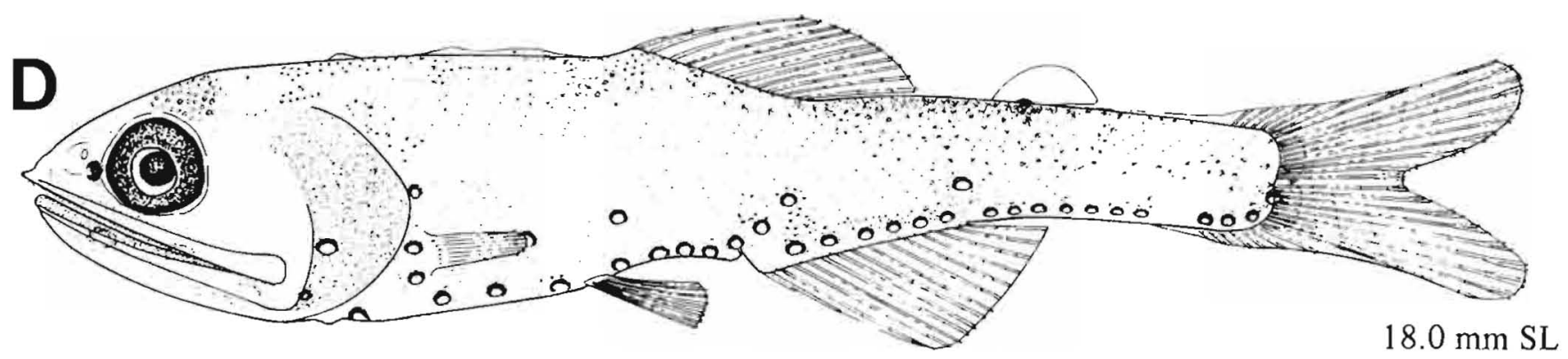

Figures A-B, D, NWAFC originals (B. Vinter); C, Moser and Ahlstrom 1974. 


\section{MERISTICS}

\begin{tabular}{|c|c|}
\hline Vertebrae & $\begin{array}{l}\text { Total: } 34-X-36 \\
\text { Precaudal: } X-X-X \\
\text { Caudal: } X-X-X\end{array}$ \\
\hline Branchiostegal rays & $8-X-12$ \\
\hline Caudal fin & $X, 10+9, X$ \\
\hline Pelvic fin & $\begin{array}{l}\text { Abdominal } \\
\text { R: } 8-8-8\end{array}$ \\
\hline Dorsal fin & $\mathrm{R}: 11-\mathrm{X}-14$ \\
\hline Pectoral fin & R: $12-X-14$ \\
\hline Anal fin & $\mathrm{R}: 12-13-14$ \\
\hline Gill rakers & $\mathrm{L}: 6-\mathrm{X}-10$ \\
\hline \multicolumn{2}{|l|}{ LIFE HISTORY } \\
\hline Range & $\begin{array}{l}\text { Cent. California, } 34-38^{\circ} \mathrm{N},{ }^{\mathrm{a}} \text { to } \\
\text { Oregon, } 42-46^{\circ} \mathrm{N}\end{array}$ \\
\hline Ecology & Meso- and bathypelagic \\
\hline ELH pattern & $\begin{array}{l}\text { Oviparous, eggs probably } \\
\text { pelagic, pelagic larvae }\end{array}$ \\
\hline \multirow{4}{*}{ Spawning } & Season: \\
\hline & Area: \\
\hline & Mode: \\
\hline & Migration: \\
\hline $\begin{array}{l}\text { Fecundity } \\
\text { Age at first maturity } \\
\text { Longevity }\end{array}$ & Range/function: \\
\hline
\end{tabular}

\footnotetext{
a Wisner 1974

${ }^{b}$ Information on $T$, minimus larvae is provided for comparison since $T$. bathyphilus larvae are unknown.
}

Ref: Moser et al. 1984a; H.G. Moser, NMFS Southwest Fish. Cent., P.O. Box 271, La Jolla, CA 92038, unpubl.

\section{EARLY LIFE HISTORY DESCRIPTION}

\section{EGGS}

Diameter

No. of oil globules

Oil globule diameter

Yolk

Envelope

Hatch size

Incubation time/temp.

Pigment

Diagnostic characters

\section{LARVAE}

Preanal length

Length at flexion

Length at transformation

Sequence of fin development
Caudal, pectorals, anal, dorsal, pelvics

Pigment - Genus ${ }^{b}$

- Above brain, otic region

- One to several opposing melanophores at postanal dorsal and ventral midline

- Late postflexion larvae may develop melanophores along each side of dorsal midline

- Base of caudal rays

- Series of embedded melanophores above spinal column

Diagnostic characters - Genus

- No photophores in larvae

Distinguished from other myctophids with round eyes by

- Morphology: Body slender, lower jaw projects beyond upper jaw

- Embedded melanophores above spinal column

- Opposing dorsal and ventral melanophores on caudal peduncle 


\section{Taaningichthys minimus}
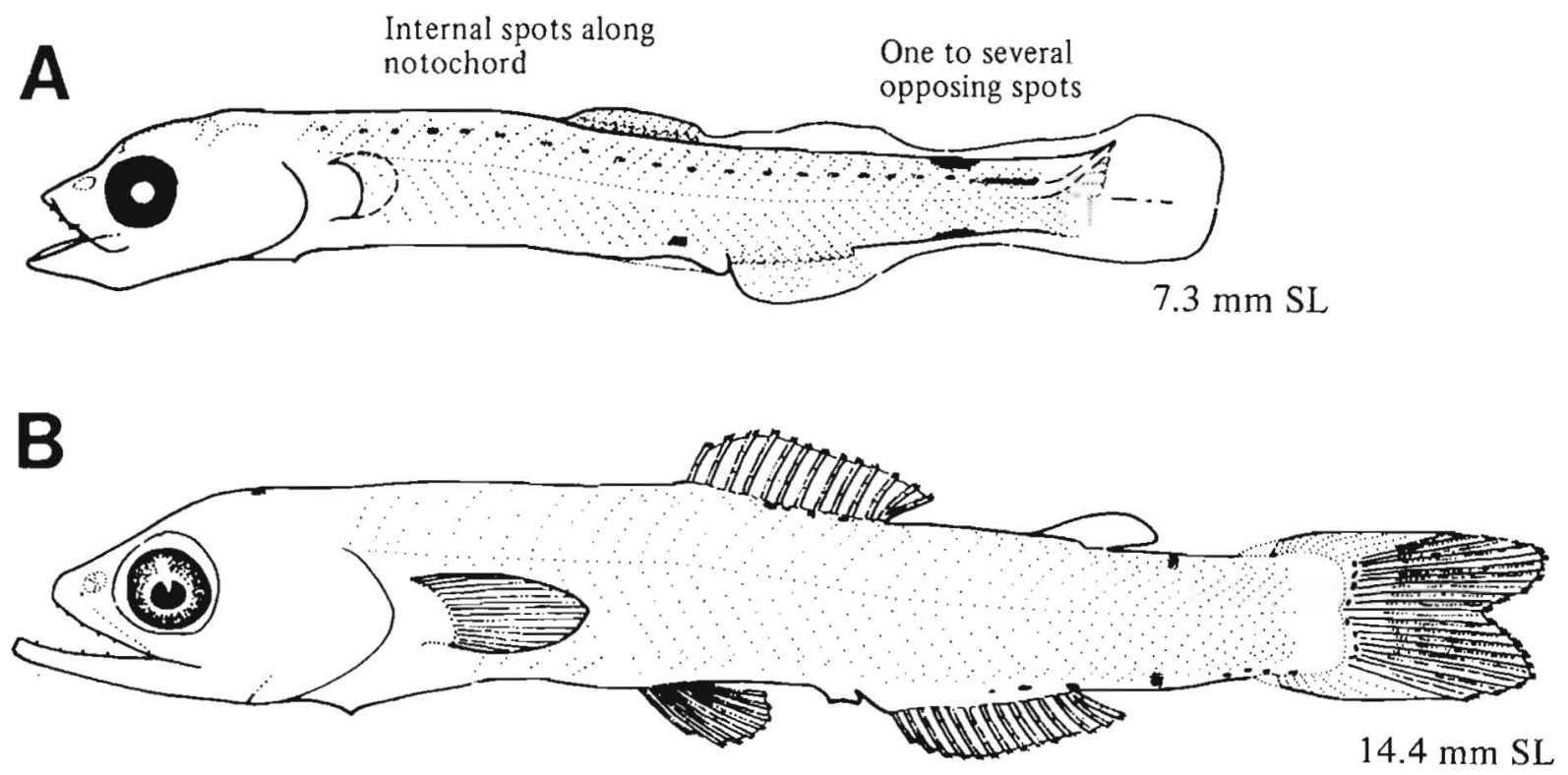


\section{MERISTICS}

Vertebrae

Total: $34-35-36$

Precaudal: $15-16-16$

Caudal: $18-19-20$

Branchiostegal rays

Pelvic fin

9-9-9

$6-8,10+9,6-8$

Abdominal

R: $7-\mathrm{X}-8$

Dorsal fin

R: $11-13-15$

Anal fin

$\mathrm{R}: 10-\mathrm{X}-12$

$\mathrm{R}: 12-\mathrm{X}-14$

Gill rakers
Caudal fin

Pectoral fin

\section{EARLY LIFE HISTORY DESCRIPTION}

\section{EGGS}

Diameter

No. of oil globules

Oil globule diameter

Yolk

Envelope

Hatch size

Incubation time/temp.

Pigment

Diagnostic characters

\section{LARVAE}

Preanal length

Length at flexion $\quad<6.9 \mathrm{~mm} \mathrm{SL}$

Length at transformation

Sequence of fin development

Caudal, pectorals, anal, dorsal, pelvics

Pigment

- Head unpigmented

- Anteroventral surface of liver, midgut, base of caudal fin rays, swimbladder

- Postanal ventral melanophores in preflexion: Numerous $(<15)$

Age at first maturity

Longevity
Season:

Area:

Mode:

Migration:

Range/function:

${ }^{3}$ Sequence of formation of photophores: Br2, PO5, PO1 [VO1, PO2, OP2, VO5,

$\mathrm{PO} 3, \mathrm{PO}, \mathrm{VLO}$ (photophores in brackets appear in late larval period).

Ref: Moser and Ahlstrom 1974. 
A

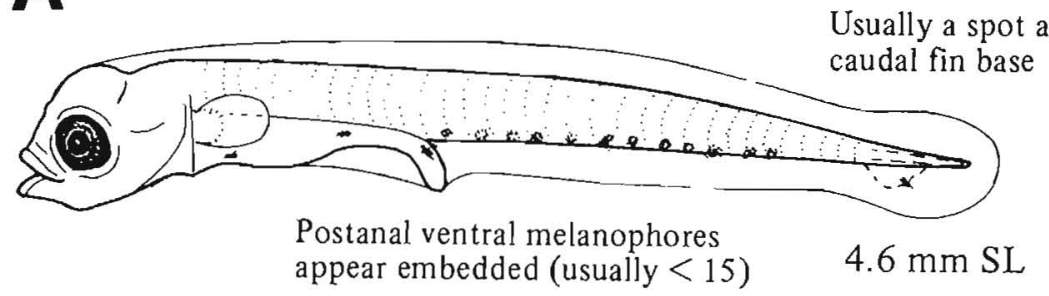

B

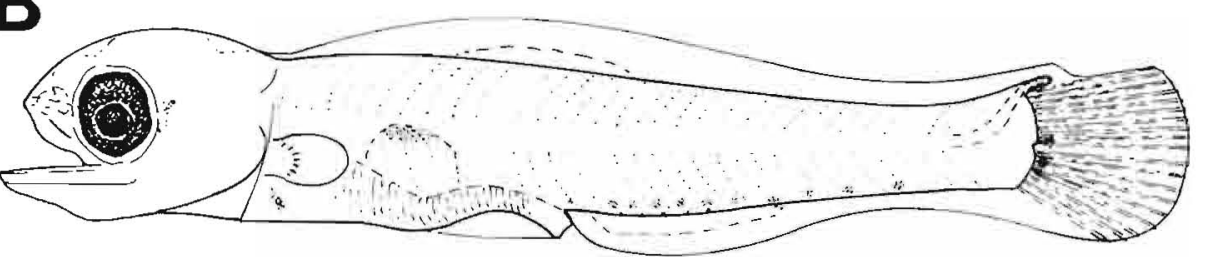

$6.3 \mathrm{~mm} \mathrm{SL}$

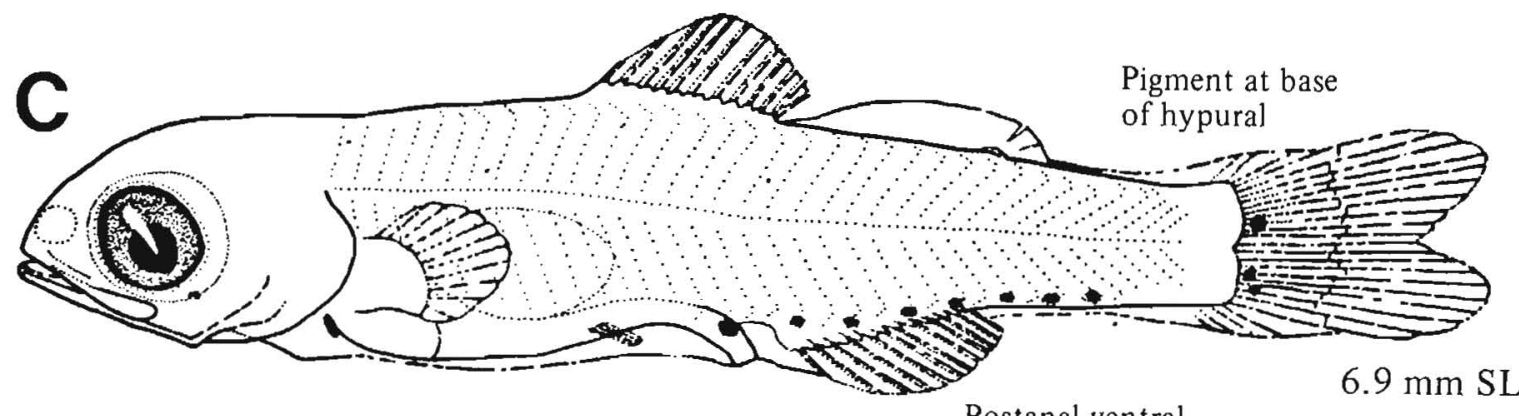

Postanal ventra

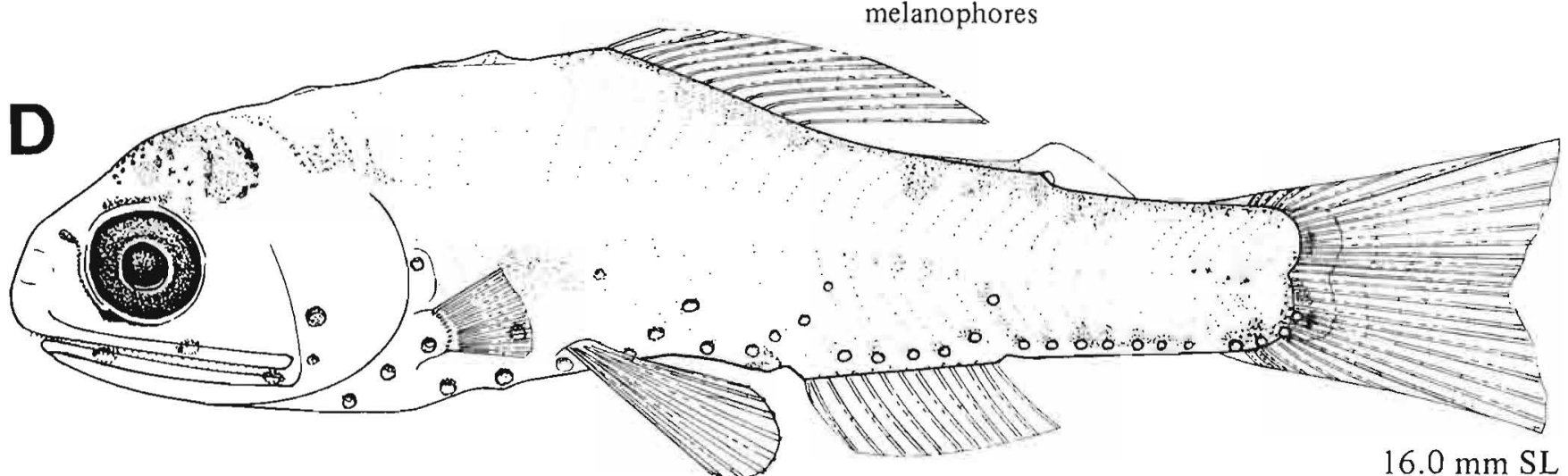

Figures A-B, D, NWAFC originals (B. Vinter); C, Moser and Ahlstrom 1974. 


\section{MERISTICS}

\begin{tabular}{|c|c|}
\hline \multirow[t]{3}{*}{ Vertebrae } & Total: $35-37-38$ \\
\hline & Precaudal: $\mathrm{X}-\mathrm{X}-\mathrm{X}$ \\
\hline & Caudal: X-X-X \\
\hline Branchiostegal rays & $9-9-9$ \\
\hline Caudal fin & $11-14,10+9,10-14$ \\
\hline \multirow[t]{2}{*}{ Pelvic fin } & Abdominal \\
\hline & $\mathrm{R}: 8-\mathrm{X}-9$ \\
\hline Dorsal fin & $\mathrm{R}: 21-22-23$ \\
\hline Pectoral fin & $\mathrm{R}: 11-12-13$ \\
\hline Anal fin & $\mathrm{R}: 18-19-20$ \\
\hline Gill rakers & $\mathrm{U}: 6-6-7 \quad \mathrm{~L}: 13-14-15$ \\
\hline \multicolumn{2}{|l|}{ LIFE HISTORY } \\
\hline Range & $\begin{array}{c}\text { South of southern California to } \\
\text { Brit. Col., } 48^{\circ} 30^{\prime}-55^{\circ} \mathrm{N}\end{array}$ \\
\hline Ecology & Epi- and mesopelagic \\
\hline ELH pattern & $\begin{array}{l}\text { Oviparous, pelagic eggs, } \\
\text { pelagic larvae }\end{array}$ \\
\hline \multirow{4}{*}{ Spawning } & Season: \\
\hline & Area: \\
\hline & Mode: \\
\hline & Migration: \\
\hline Fecundity & Range/function: \\
\hline $\begin{array}{l}\text { Age at first maturity } \\
\text { Longevity }\end{array}$ & \\
\hline
\end{tabular}

According to Peden and Hughes (1986) northern records of $N$. resplendens are probably $N$. japonicus.

${ }^{b}$ H.G. Moser, NMFS Southwest Fish. Cent., P.O. Box 271, La Jolla, CA 92038, pers. commun., 9 Jan. 1984.

${ }^{c}$ Both $N$. resplendens and $N$. japonicus are described and illustrated in Ozawa (1986d). Specimens from the eastern Atlantic are figured here, but, according to Ozawa (1986d), they may differ in pigmentation and in photophore development from specimens found in the Pacific.

Ref: Badcock and Merrett 1976; Moser and Ahlstrom 1972; Ozawa 1986d; Moser, unpubl.

\section{EARLY LIFE HISTORY DESCRIPTION}

EGGS - Genus

Diameter $\quad 0.26 \mathrm{~mm}$

No. of oil globules

Oil globule diameter

Yolk

Envelope

Hatch size

Incubation time/temp.

Pigment

Diagnostic characters

\section{LARVAE ${ }^{\mathrm{b}, \mathrm{c}}$}

Preanal length

Length at flexion

Length at transformation

Sequence of fin

development

$<50 \%$ SL preflexion, with development $50 \% \mathrm{SL}$

Caudal, pectorals, anal, dorsal, pelvics

Pigment

- Preflexion: Jaw tips, above brain and swimbladder, lateral cleithral

- Postflexion: Lower jaw, hindbrain, nape, double row of melanophores along dorsal midline beginning at midbody, series along anal fin base, caudal fin base, pelvic and anal fin, internal notochord pigment on trunk

Diagnostic characters

- Photophores: Br2, PO5, Vn, and PLO develop during larval period

Distinguished from other myctophids with round eyes by

- Morphology: Body moderately deep, head and eyes large

- Midlateral and dorsal fin base pigment in postflexion larvae

- Highest dorsal fin ray count among myctophids in study area (21-23)

- Head pigment: Jaw tips, snout, opercle and brain 

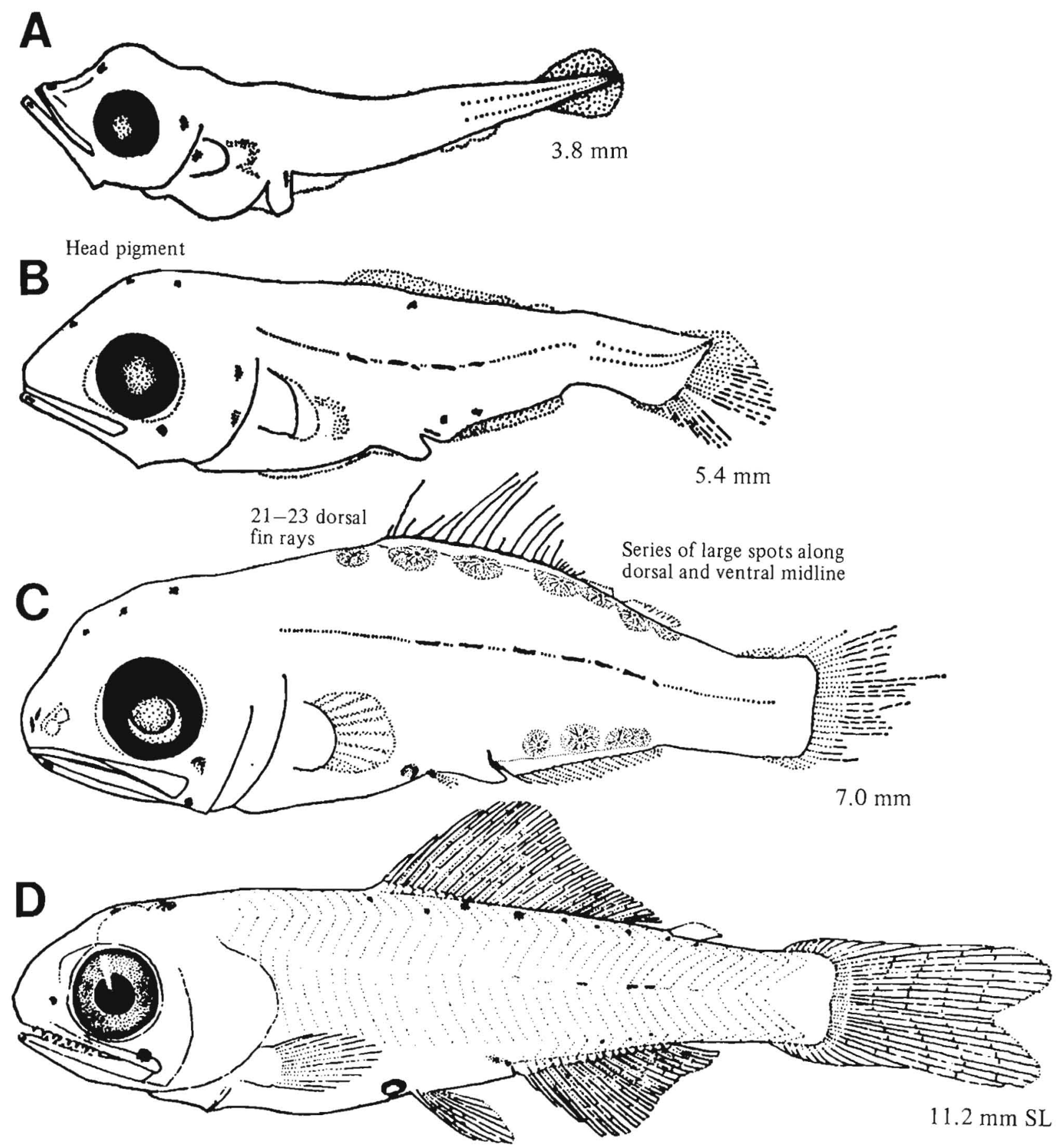

Figures A-C, Badcock and Merrett 1976 (eastern Atlantic specimens); D, Ahlstrom and Moser 1974. 



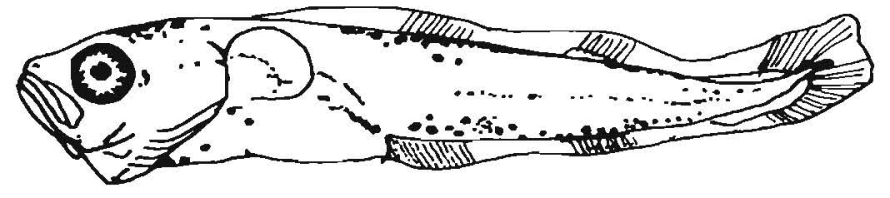

\section{Gadiformes}

Gadiform fishes include some of the most commercially important coldwater marine species in the world: the codfishes and their relatives. The order contains between 7 and 13 families, depending on the source, and well over 400 species. Fishes of this order are found worldwide and are generally bathypelagic (except for one freshwater species). Most species are elongate with some having long dorsal and/or anal fins. Pelvic fins are thoracic or jugular, and chin barbels are present in many. Although they are easily recognized and well studied, evolutionary relationships are poorly understood and most recent authors now propose a multiple origin for at least the Gadidae (Markle 1989). The early-life-history stages are well known, especially for the gadids and merlucciids, and have been studied for well over 100 years (Fahay and Markle 1984). Most eggs are pelagic, spherical, and have a single oil globule (lacking in some gadids). Larvae are commonly identified by their distinctive pigment patterns, coiled gut, and general tadpole shape. Morids, melanonids, and macrourids are less well known.

Families in study area: Moridae

Melanonidae

Merlucciidae

Gadidae

Macrouridae 


\section{MORIDAE}

Morids are benthopelagic fishes distributed throughout the world's oceans and are represented by two species in two genera in the study area. Very little is known of the early life history of the codlings. For the few species described, eggs are $0.52-1.16 \mathrm{~mm}$ in diameter, have one oil globule, and a smooth chorion (Fahay and Markle 1984). The scarcity of small fish in bottom trawls indicates the very young, and perhaps eggs, are pelagic (Iwamoto 1975). Most morids have precocious pelvic fin ray development. Usually the pelvic fin rays are elongate and probably undergo some ontogenetic reduction. Figures of larvae of Physiculus nematopus and Laemonema sp. are provided for comparison, since larvae of species in our area are unknown.

Table 21

Meristic characters of family Moridae.

\begin{tabular}{|c|c|c|c|c|c|c|c|c|c|c|c|c|}
\hline \multirow{3}{*}{$\frac{\text { Taxon }}{\text { Antimora* }}$} & \multirow[b]{2}{*}{ Distribution } & \multicolumn{2}{|c|}{ Vertebrae } & \multicolumn{6}{|c|}{ Fins } & \multicolumn{2}{|c|}{ Gill rakers } & \multirow[b]{2}{*}{ Branchiostegals } \\
\hline & & \multicolumn{2}{|c|}{$\begin{array}{c}\text { Precaudal Caudal } \\
\text { (Total) }\end{array}$} & \multirow{2}{*}{$\begin{array}{c}\begin{array}{c}\text { First } \\
\text { Dorsal }\end{array} \\
4-7\end{array}$} & $\begin{array}{c}\begin{array}{c}\text { Second } \\
\text { Dorsal }\end{array} \\
48-56\end{array}$ & \multirow{2}{*}{$\frac{\text { Anal }}{36-49}$} & $\begin{array}{c}\text { Pectoral } \\
17-25\end{array}$ & \multirow{2}{*}{$\begin{array}{c}\text { Pelvic } \\
5-7\end{array}$} & $\begin{array}{l}\text { Caudal } \\
\text { (Total) }\end{array}$ & Upper & Lower & \\
\hline & & $\begin{array}{l}24-25 \\
(57-8\end{array}$ & 1) & & & & $17-25$ & & & & & \\
\hline Antimora microlepis & SSC - Bering Sea & $24-25$ & 33 & $4-5$ & $50-55$ & $37-42$ & 20 & $6-7$ & $14,5+4,12$ & 5 & 15 & 7 \\
\hline Laemonema* & & $\begin{array}{l}15-17 \\
(50-\end{array}$ & $3)^{42-45}$ & $5-6$ & $48-75$ & $45-72$ & $15-26$ & $\begin{array}{c}1-3 \\
\text { (usually 2) }\end{array}$ & & & & \\
\hline Laemonema longipes & Bering Sea & & 1) & 6 & $50-52$ & $45-50$ & $15-17$ & $2-3$ & $(22-25)$ & $5-7$ & $19-20$ & 6 \\
\hline
\end{tabular}



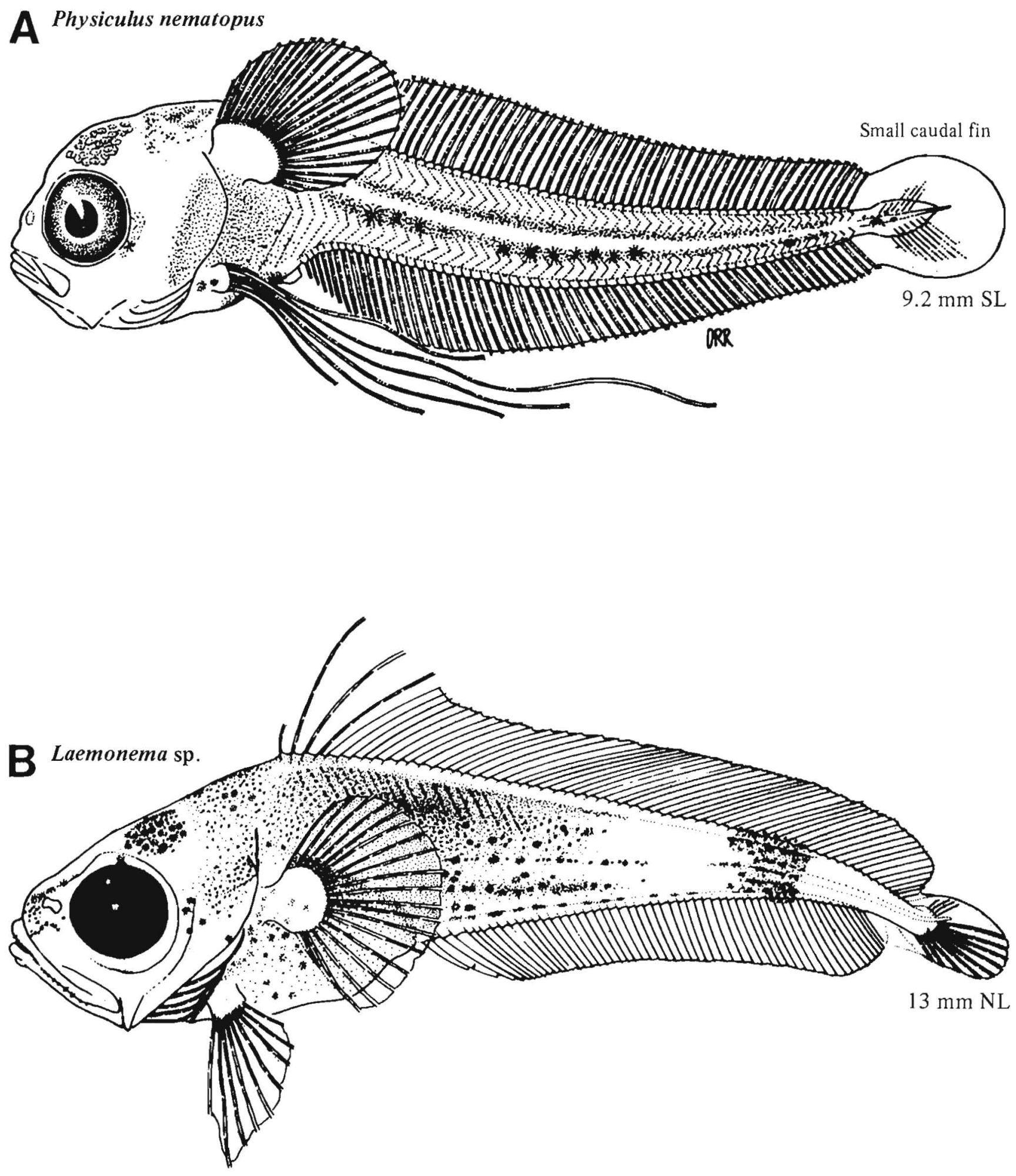

Figure A, Fahay and Markle 1984; B, Fahay 1983 (North Atlantic specimen; identification is tentative). 


\section{MERISTICS}

\begin{tabular}{|c|c|}
\hline \multirow[t]{3}{*}{ Vertebrae } & Total: $58-X-62$ \\
\hline & Precaudal: 13-13-13 \\
\hline & Caudal: $47-47-47$ \\
\hline Branchiostegal rays & $7-7-7$ \\
\hline Caudal fin & $23-25,6+3,22-25$ \\
\hline \multirow[t]{2}{*}{ Pelvic fin } & Thoracic \\
\hline & $\mathrm{R}: 5-\mathrm{X}-7$ \\
\hline Dorsal fin & $\mathrm{R}: 67-\mathrm{X}-80^{\mathrm{a}}$ \\
\hline Pectoral fin & $R: 12-X-16$ \\
\hline Anal fin & $\mathrm{R}: 52-\mathrm{X}-61^{\mathrm{a}}$ \\
\hline Gill rakers & $\mathrm{L}: 6-\mathrm{X}-11$ \\
\hline \multicolumn{2}{|l|}{ LIFE HISTORY } \\
\hline Range & $\begin{array}{l}\text { South of southern California to } \\
\text { Brit. Col., } 48^{\circ} 30^{\prime}-55^{\circ} \mathrm{N}\end{array}$ \\
\hline Ecology & Mesopelagic, $200-1000 \mathrm{~m}$ \\
\hline ELH pattern & $\begin{array}{l}\text { Parity and eggs unknown, } \\
\text { pelagic larvae }\end{array}$ \\
\hline Spawning & Season: \\
\hline & Area: \\
\hline & Mode: \\
\hline & Migration: \\
\hline Fecundity & Range/function: \\
\hline $\begin{array}{l}\text { Age at first maturity } \\
\text { Longevity }\end{array}$ & \\
\hline
\end{tabular}

\section{EARLY LIFE HISTORY DESCRIPTION}

\section{EGGS}

Diameter

No. of oil globules

Oil globule diameter

Yolk

Envelope

Hatch size

Incubation time/temp.

Pigment

Diagnostic characters

\section{LARVAE}

Preanal length

Length at flexion

Length at transformation

Sequence of fin development

Pigment

\section{Diagnostic characters}

- Large number of secondary caudal fin rays (23-25 upper and 22-25 lower) aD. Markle, Dep. Fish. Wildl., Oregon State Univ., Corvallis, OR 97331-3803,
pers. commun., 8 May 1986.

${ }^{\circ}$ Specimens $<17 \mathrm{~mm} \mathrm{SL}$ have not been identified.

Ref: Fahay and Markle 1984. 

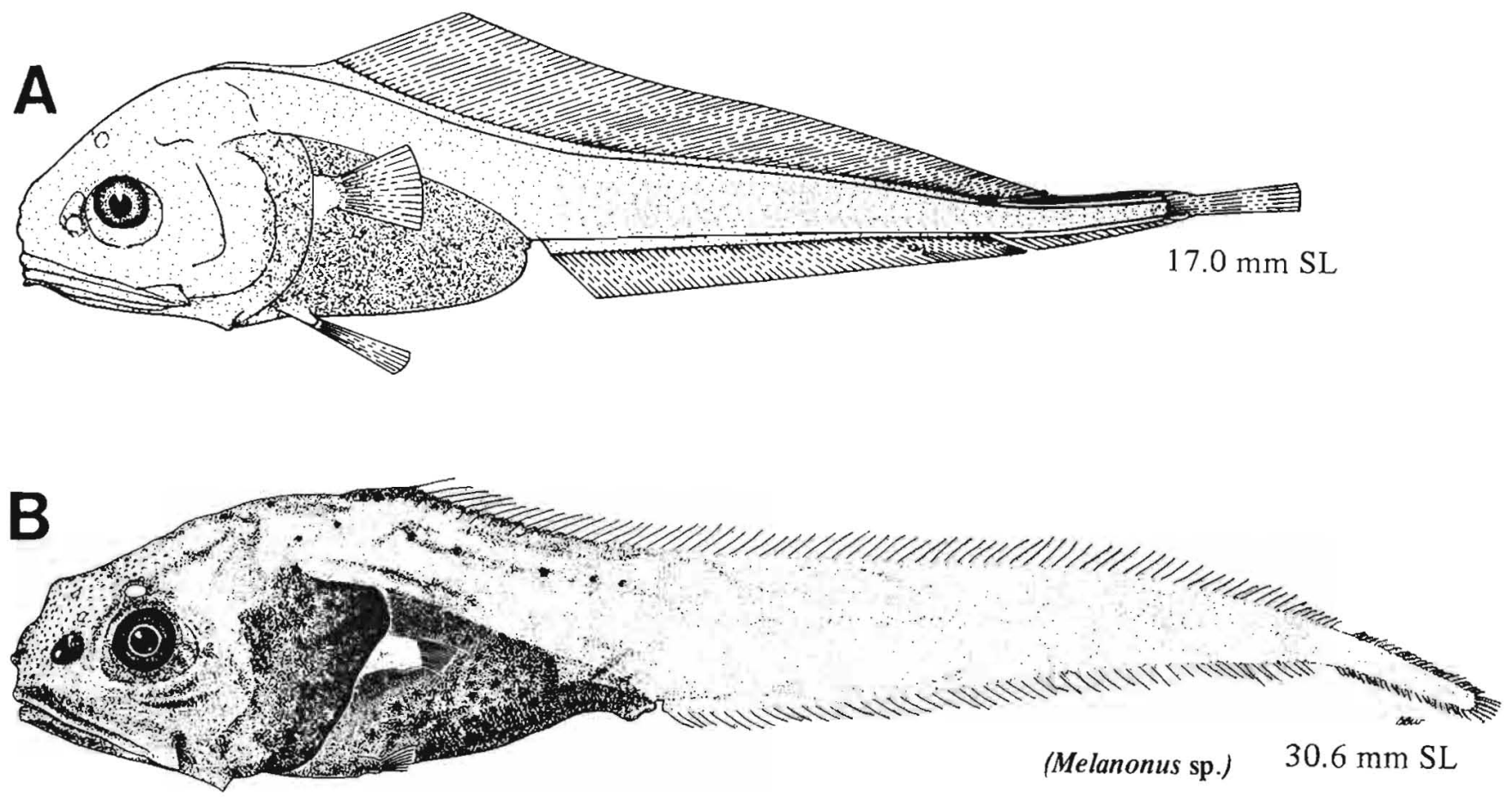

Figure A, NWAFC original (B. Vinter, Atlantic specimen); B, Fahay and Markle 1984 (South Atlantic specimen). 


\section{MERISTICS}

\begin{tabular}{|c|c|}
\hline \multirow[t]{3}{*}{ Vertebrae } & Total: $52-53-55$ \\
\hline & Precaudal: $23-24-25$ \\
\hline & Caudal: $29-29-30$ \\
\hline Branchiostegal rays & $7-7-7$ \\
\hline Caudal fin & $16-18,6+2-3,14-17$ \\
\hline Pelvic fin & Thoracic \\
\hline & $\mathrm{R}: 6-\mathrm{X}-8$ \\
\hline Dorsal fin & 1st: $10-11-13 \quad 2$ nd: $37-40-44$ \\
\hline Pectoral fin & R: $14-16-16$ \\
\hline Anal fin & R: $37-40-44$ \\
\hline Gill rakers & L: $13-16-18$ \\
\hline
\end{tabular}

\section{LIFE HISTORY}

$\begin{array}{ll}\text { Range } & \begin{array}{c}\text { South of southern California to } \\ \text { Bering Sea, } 54-66^{\circ} \mathrm{N}\end{array} \\ \text { Ecology } & \text { Epi- and mesopelagic, 0-914 m } \\ \text { ELH pattern } & \text { Oviparous, pelagic eggs, } \\ & \text { pelagic larvae } \\ \text { Spawning } & \text { Season: Jan-June } \\ & \text { Area: Offshore pelagic; }{ }^{\mathrm{b}} \\ & 130-500 \mathrm{~m}^{\mathrm{c}} \\ & \text { Mode: Schools } \\ & \text { Migration: South and offshore to } \\ & \text { spawn (southern subpopula- } \\ & \text { tion); within Puget Sound and } \\ & \text { Straits of Georgia (northern } \\ \text { Fecundity } & \text { subpopulation) } \\ \text { Age at first maturity } & 2 \mathrm{yr}^{\mathrm{d}}-4 \mathrm{yr}^{\mathrm{g}} \\ \text { Longevity } & 17 \mathrm{yr}^{\mathrm{h}}\end{array}$

\footnotetext{
${ }^{a} \mathrm{Hart} 1973$

${ }^{b}$ The main offshore population spawns offshore off southern California (Francis and Bailey 1983). Disjunct populations in the Puget Sound-Straits of Georgia area spawn in restricted areas within these regions.

${ }^{c}$ Bailey et al. 1982

${ }^{\mathrm{d}}$ Kimura and Miliken 1977

${ }^{e}$ MacGregor 1971

' MacGregor 1966

${ }^{8}$ Best 1963

${ }^{h}$ Beamish 1979
}

Ref: Ahlstrom and Counts 1955, Fahay and Markle 1984.

\section{EARLY LIFE HISTORY DESCRIPTION}

\section{EGGS}

\author{
Diameter \\ No. of oil globules \\ $1.07-1.18 \mathrm{~mm}(1.12 \mathrm{~mm})$ \\ Oil globule diameter \\ One \\ Yolk \\ Envelope \\ $0.27-0.34 \mathrm{~mm}$ \\ May appear granular \\ Hatch size \\ Clear, smooth \\ Incubation time/temp. $\quad 3.1 \mathrm{~d} / 16.6^{\circ} \mathrm{C}$ \\ Pigment \\ - On yolk \\ - Characteristic late-stage patches on embryo \\ Diagnostic characters \\ - Pigment pattern \\ - Presence of oil globule \\ - Eye forms early during embryonic development
}

\section{LARVAE}

Preanal length
Length at flexion
Length at transformation
Sequence of fin
development

Pigment
$<50 \%$ SL

$\sim 10 \mathrm{~mm} \mathrm{SL}$

$\sim 30-35 \mathrm{~mm}$ SL

Caudal, pelvics, 1 st dorsal, 2nd dorsal and anal, pectorals
- Preflexion larvae: One postanal bar, one large dendritic spot several myomeres posterior to anus; with development, bar becomes diffuse, postanal body pigment increases, especially dorsolaterally

- On head, crown, and snout; increasing with development

- Dorsolaterally on gut

\section{Diagnostic characters}

- Gadoid shape but absence of $>1$ distinct postanal pigment bar

- Two dorsal fins and one anal fin

- Specimens from Puget Sound and Straits of Georgia have pigmented pectoral rays 
B

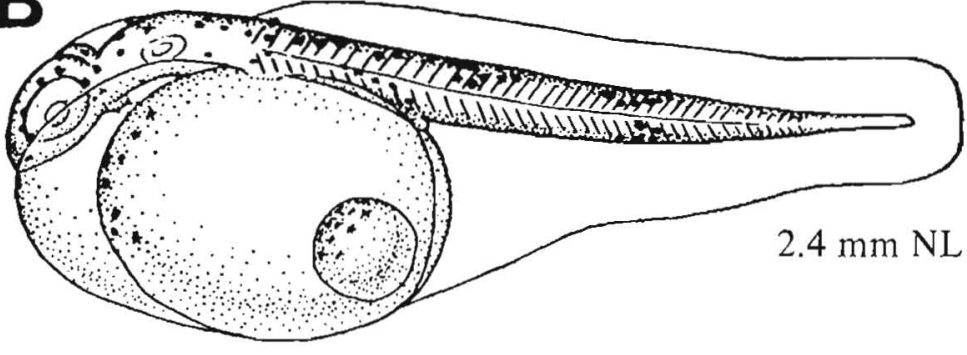

C
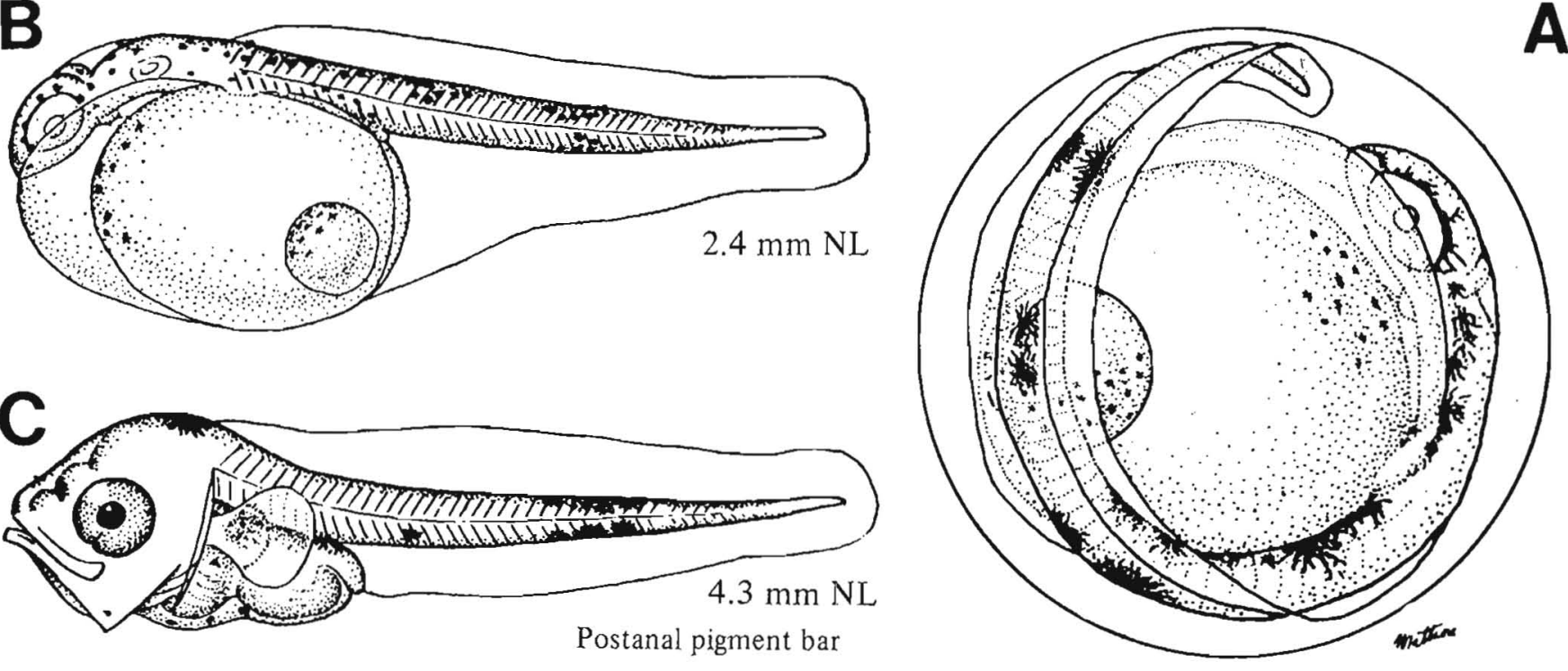

$1.07-1.18 \mathrm{~mm}$

D
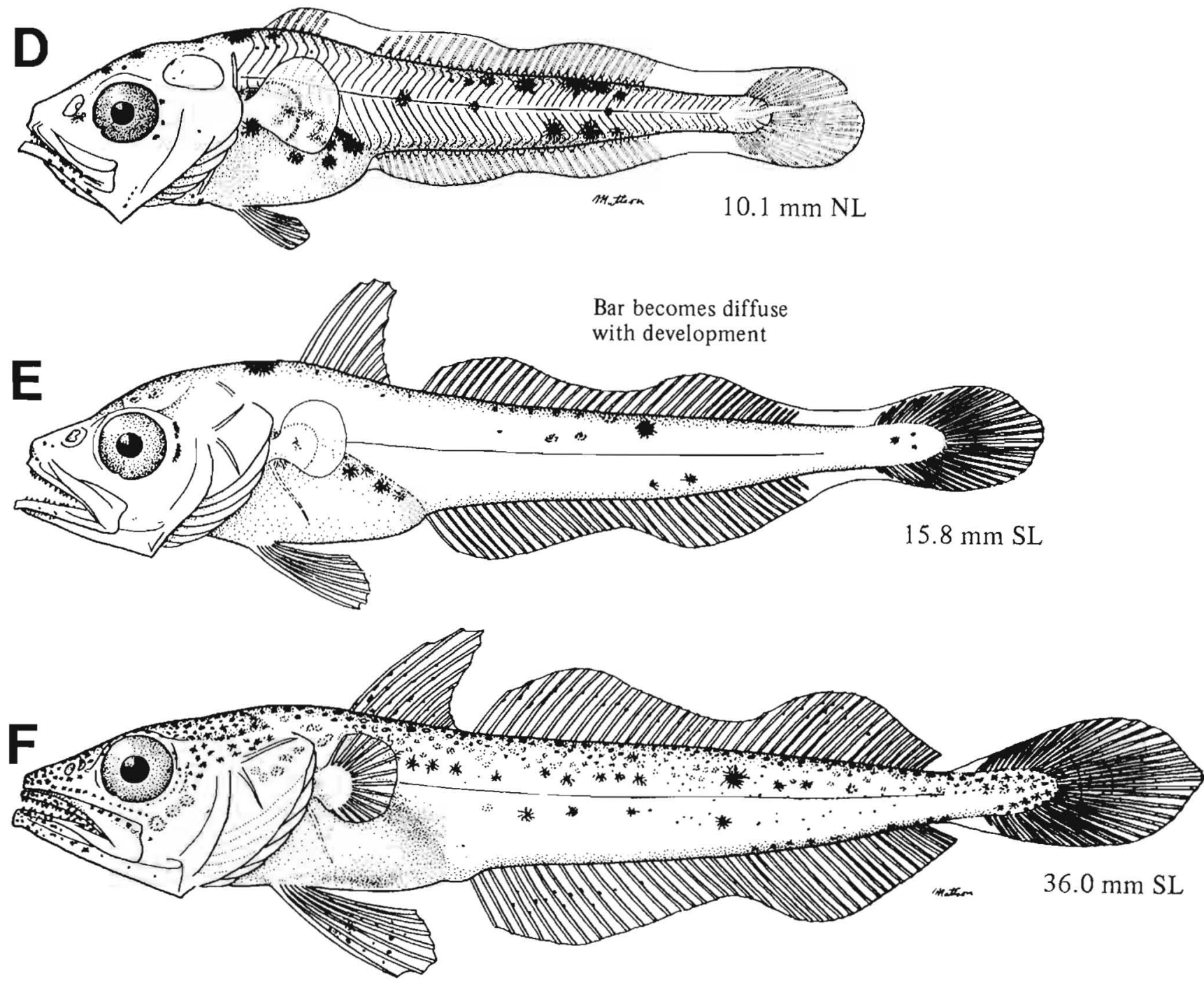

Figures A-F, Ahlstrom and Counts 1955 . 

The family Gadidae is represented in the Northeast Pacific Ocean and Bering Sea by five species: Walleye pollock, Theragra chalcogramma; Pacific cod, Gadus macrocephalus; Pacific tomcod, Microgadus proximus; saffron cod, Eleginus gracilis; and Arctic cod, Boreogadus saida. Identification of gadid larvae prior to 1980 was not possible. Gadid larvae collected during ichthyoplankton studies were routinely reported as "Gadidae." Recent taxonomic studies have continued since 1980 and have allowed the specific identification of all five species in the area (e.g., Matarese et al. 1981 and Dunn and Vinter 1984). Identification is based primarily on differences in pigmentation patterns and meristic characters. The available knowledge of the general early life histories of these five species was recently reviewed by Dunn and Matarese (1987).

\section{Table 22}

Characters useful in separating larvae of Eleginus gracilis, Boreogadus saida, Gadus macrocephalus, Theragra chalcogramma, and Microgadus proximus at specific size ranges (Dunn and Vinter 1984, in part).

\begin{tabular}{|c|c|c|c|c|c|c|}
\hline Character & $\begin{array}{l}\text { Size range } \\
(\mathrm{mm} \mathrm{SL})\end{array}$ & E. gracilis & B. saida & G. macrocephalus & T. chalcogramma & M. proximus \\
\hline \multicolumn{7}{|l|}{ Pigment } \\
\hline \multicolumn{7}{|l|}{ Preanal region } \\
\hline Ventral gut & $4.4-13.5$ & $\begin{array}{l}\text { Double row of small } \\
\text { melanophores }\end{array}$ & $\begin{array}{l}\text { Absent in larvae } \\
<10 \mathrm{~mm} \text {; spots pres- } \\
\text { ent anterior to pec- } \\
\text { toral fins in larvae } \\
>10 \mathrm{~mm}\end{array}$ & $\begin{array}{l}\text { Large melanophores } \\
\text { medially }\end{array}$ & $\begin{array}{l}\text { Relatively few scat- } \\
\text { tered spots, more } \\
\text { anterior than } \\
\text { posterior }\end{array}$ & $\begin{array}{l}\text { Large melanophores } \\
\text { present, more } \\
\text { anterior than } \\
\text { posterior }\end{array}$ \\
\hline Line of lateral pigment & $10-18$ & $\begin{array}{l}\text { Begins anterior to } \\
\text { anus }\end{array}$ & $\begin{array}{l}\text { Begins under second } \\
\text { dorsal fin }\end{array}$ & $\begin{array}{l}\text { Begins anterior to } \\
\text { anus }\end{array}$ & $\begin{array}{l}\text { Begins under second } \\
\text { dorsal fin }\end{array}$ & $\begin{array}{l}\text { Begins just anterior } \\
\text { to anus }\end{array}$ \\
\hline \multicolumn{7}{|l|}{ Postanal region } \\
\hline $\begin{array}{l}\text { Length of ventral } \\
\text { stripes (based on no. } \\
\text { of melanophores) }\end{array}$ & $4-6$ & $\begin{array}{l}\text { Both longer than } \\
\text { dorsal stripes }\end{array}$ & $\begin{array}{l}\text { Both shorter than } \\
\text { dorsal stripes }\end{array}$ & $\begin{array}{l}\text { Anterior stripe } \\
\text { longer than dorsal } \\
\text { stripe }(<5.3 \mathrm{~mm})\end{array}$ & $\begin{array}{l}\text { Posterior stripe } \\
\text { longer than dorsal } \\
\text { stripe }\end{array}$ & $\begin{array}{l}\text { Anterior stripe } \\
\text { longer than dorsal } \\
\text { stripe }(<5 \mathrm{~mm})\end{array}$ \\
\hline $\begin{array}{l}\text { No. of myomeres } \\
\text { from vertical end of } \\
\text { anus to anterior end } \\
\text { of first ventral pig- } \\
\text { ment stripe }\end{array}$ & $4-6$ & $4-6$ & $5-7$ & $\begin{array}{l}1-3 \text { (reaches vent by } \\
\sim 5.3 \mathrm{~mm} \text { ) }\end{array}$ & $4-5$ & $\begin{array}{l}1-3 \text { (reaches vent by } \\
\sim 5.0 \mathrm{~mm})\end{array}$ \\
\hline $\begin{array}{l}\text { Length at which dor- } \\
\text { sal pigment forms a } \\
\text { continuous line }\end{array}$ & $4-15$ & $\sim 10 \mathrm{~mm}$ & $\sim 7 \mathrm{~mm}$ & $\sim 5-6 \mathrm{~mm}$ & $\sim 13 \mathrm{~mm}$ & $\sim 13 \mathrm{~mm}$ \\
\hline $\begin{array}{l}\text { Length at which ven- } \\
\text { tral pigment forms a } \\
\text { continuous line }\end{array}$ & $4-10$ & $\sim 7 \mathrm{~mm}$ & $\sim 10 \mathrm{~mm}$ & $\sim 5-6 \mathrm{~mm}$ & Never merge & $\sim 5-6 \mathrm{~mm}$ \\
\hline $\begin{array}{l}\text { On ventral margin of } \\
\text { body }\end{array}$ & $10-15$ & $\begin{array}{l}\text { In double row on } \\
\text { each side of midline }\end{array}$ & $\begin{array}{l}\text { Pigment on midline } \\
\text { and scattered on } \\
\text { each side }\end{array}$ & $\begin{array}{l}\text { Single row on each } \\
\text { side of midtine }\end{array}$ & $\begin{array}{l}\text { Pigment on midline } \\
\text { and a single row on } \\
\text { each side }\end{array}$ & $\begin{array}{l}\text { Single row on each } \\
\text { side of midline } \\
\text { anteriorly, single } \\
\text { row on midline } \\
\text { posteriorly }\end{array}$ \\
\hline \multicolumn{7}{|l|}{ Morphologic } \\
\hline $\begin{array}{l}\text { Position of vent } \\
\text { relative to dorsal fins }\end{array}$ & $15-20$ & Under second dorsal & Under second dorsal & Under second dorsal & $\begin{array}{l}\text { Between first and } \\
\text { second dorsal }\end{array}$ & $\begin{array}{l}\text { Between first and } \\
\text { and second dorsal } \\
\text { (ultimately under } \\
\text { first dorsal) }\end{array}$ \\
\hline \multicolumn{7}{|l|}{ Meristic } \\
\hline $\begin{array}{l}\text { No. of rays on } \\
\text { superior hypural }\end{array}$ & $>13$ & 5 & 4 & 4 & 4 & 5 \\
\hline
\end{tabular}




\section{MERISTICS}

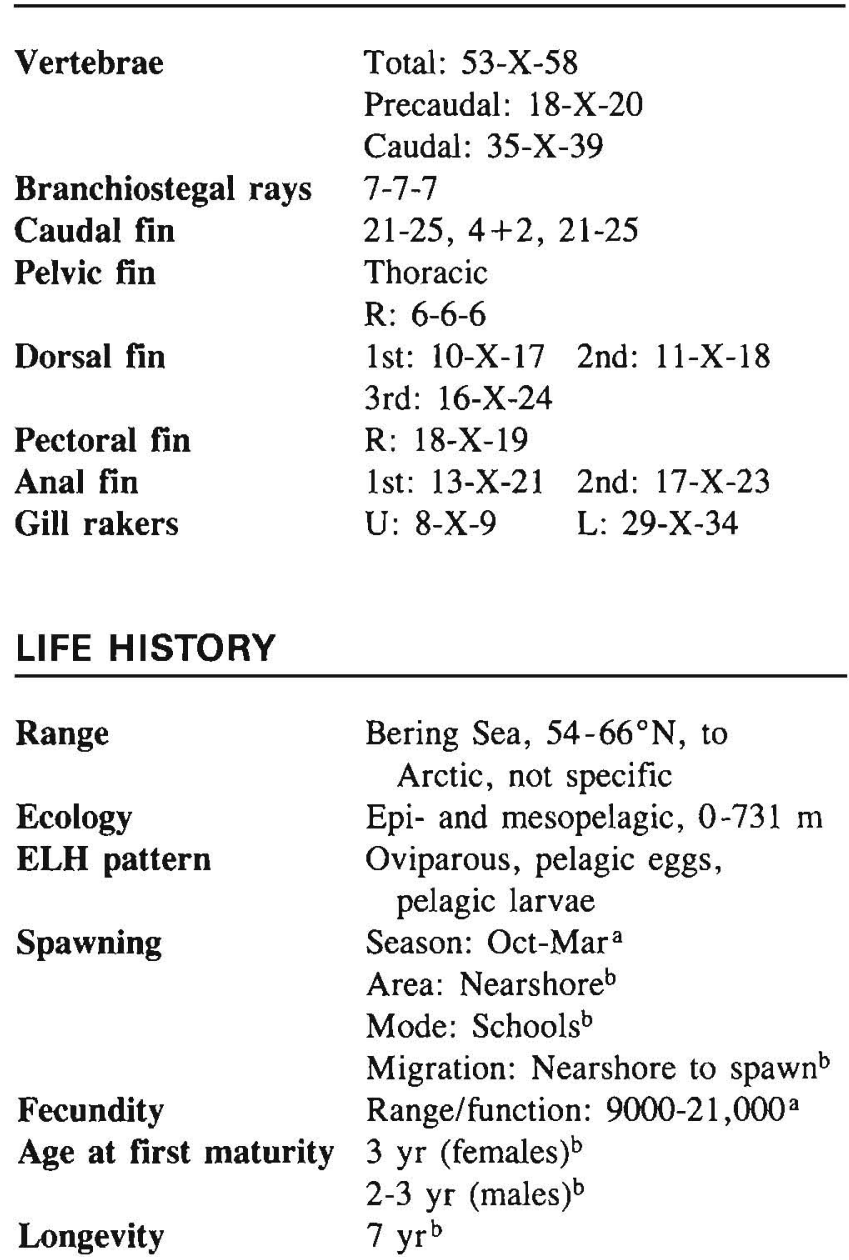

\section{EARLY LIFE HISTORY DESCRIPTION}

\section{EGGS}

Diameter

No. of oil globules

Oil globule diameter

Yolk

Envelope

$1.53-1.90 \mathrm{~mm}$

None

Homogeneous

Smooth

Hatch size

$6 \mathrm{~mm} \mathrm{SL}$

Incubation time/temp.

Pigment

Diagnostic characters

\section{LARVAE}

Preanal length

$<50 \%$ SL

Length at flexion

11-17 mm SL

Length at transformation

$17-30 \mathrm{~mm}$, pelagic until $30-45 \mathrm{~mm} \mathrm{SL}$

Sequence of fin development

Caudal, dorsals and anals, pectorals, pelvics

Pigment

- Presence of bars

- Shorter ventral stripes

- Mediolateral pigment

Diagnostic characters (see Table 22)

- Ventral pigment on midline and scattered on each side

- Rays on superior hypural $=4$

Distinguished from Theragra chalcogramma by

- Ventral pigment on midline and scattered on each side. In $T$. chalcogramma, ventral pigment consists of a row along midline and a single row on each side.

\footnotetext{
${ }^{a}$ Bain and Sekerak 1978
}

${ }^{b} \mathrm{Craig}$ et al. 1982

Ref: Dunn and Matarese 1984, Dunn and Vinter 1984. 


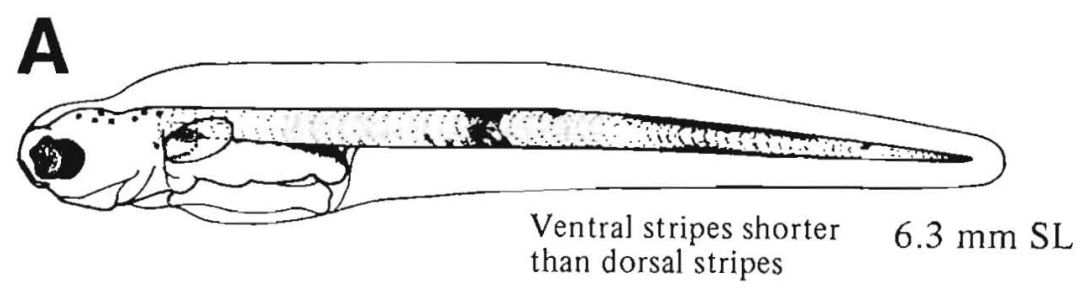

B
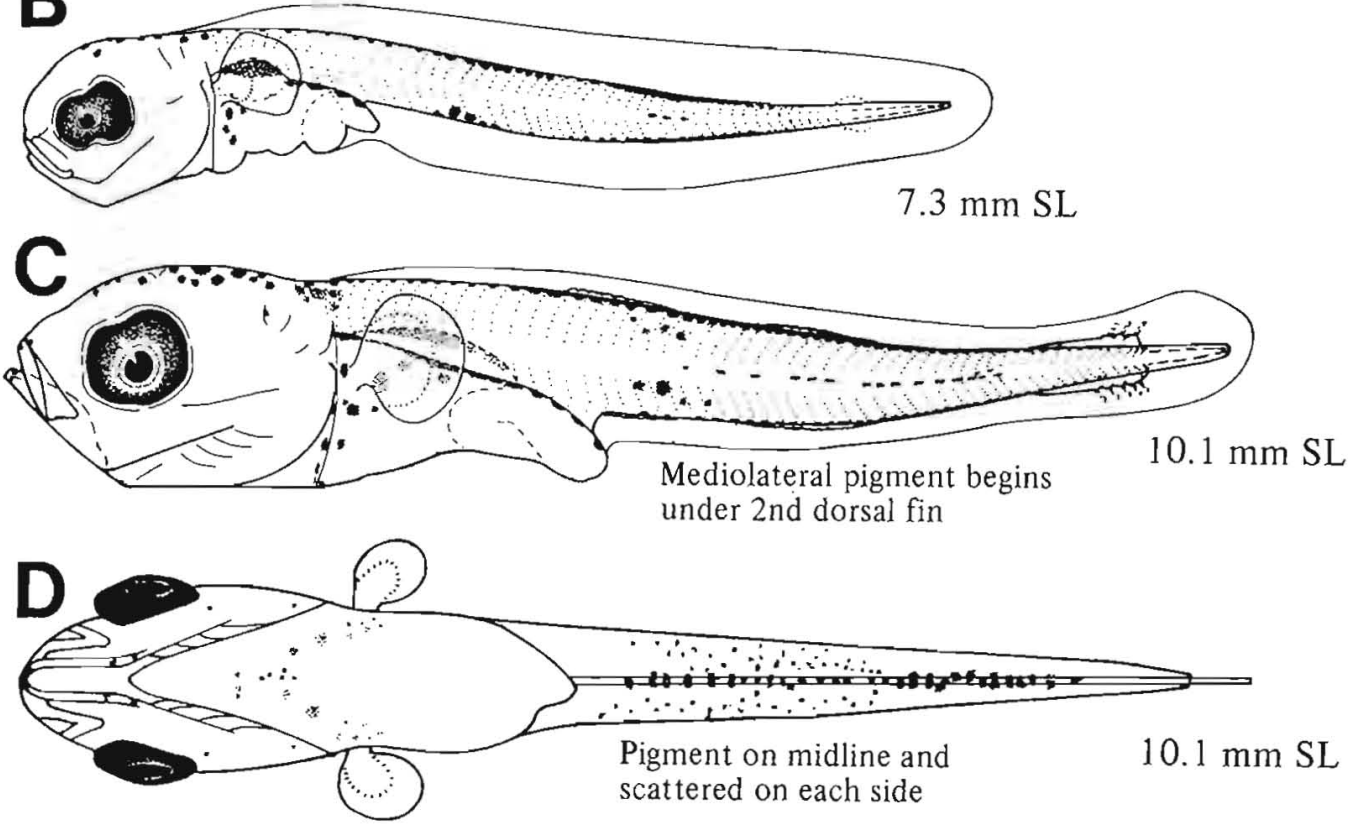

$>10 \mathrm{~mm} \mathrm{SL}$, pigment appears on anteroventral surface of gut

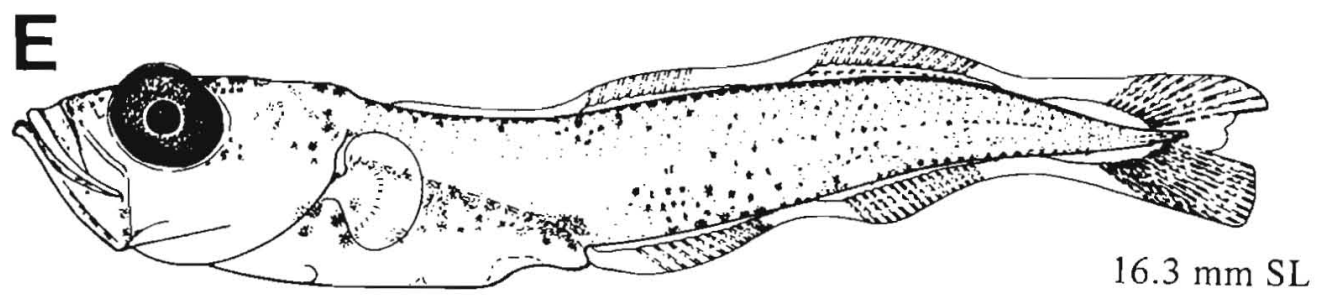

Figures A-D (D, ventral view), Dunn and Vinter 1984; E, NWAFC original (B. Vinter). 


\section{MERISTICS}

Vertebrae

Total: 57-62-64

Precaudal: 21-X-24

Caudal: $37-\mathrm{X}-41$

Branchiostegal rays

Caudal fin

Pelvic fin

7-7-7

$22-25,5+2,23-26$

Thoracic

R: 6-6-6

Dorsal fin

1st: $11-\mathrm{X}-16$ 2nd: $15-\mathrm{X}-23$

3rd: $18-X-21$

Pectoral fin R: $18-\mathrm{X}-21$

Anal fin

1st: $20-X-24$ 2nd: $19-X-22$

Gill rakers

$\mathrm{U}: 2-\mathrm{X}-3$

L: $17-\mathrm{X}-20$

LIFE HISTORY

$\begin{array}{ll}\text { Range } & \text { Gulf of Alaska, } 54-60^{\circ} \mathrm{N}, \text { to } \\ & \text { Arctic, not specific } \\ \text { Ecology } & \text { Nearshore shelf pelagic, 2-75 m } \\ \text { ELH pattern } & \text { Oviparous, demersal eggs, } \\ & \text { pelagic larvae } \\ \text { Spawning } & \text { Season: Winter } \\ & \text { Area: Shallow nearshore } \\ & \left(2-10 \mathrm{~m}^{\mathrm{a}}\right. \\ & \text { Mode: } \\ & \text { Migration: To shallow water for } \\ & \text { spawning } \\ \text { Fecundity } & \text { Range/function: } 28,900-190,700^{\mathrm{a}} \\ \text { Age at first maturity } & 2 \mathrm{yr}^{\mathrm{a}} \\ \text { Longevity } & 9 \mathrm{yr}^{\mathrm{a}}\end{array}$

\section{EARLY LIFE HISTORY DESCRIPTION}

\section{EGGS}

Diameter

No. of oil globules

Oil globule diameter

Yolk

Envelope

Hatch size

$1.0-1.7 \mathrm{~mm}(1.3-1.7 \mathrm{~mm})$

None

Homogeneous, dense

Smooth, thick

$3.5 \mathrm{~mm} \mathrm{SL}$

Incubation time/temp.

Pigment

Diagnostic characters

\section{LARVAE}

Preanal length

Length at flexion

Length at transformation

Sequence of fin development

Pigment

- Presence of bars

- Ventral stripes longer than dorsal stripes

- Ventral pigment in double row on each side of midline

- Mediolateral pigment begins anterior to anus

- Double row of melanophores along ventral surface of gut

Diagnostic characters (see Table 22)

- Pigment

-Double row of melanophores along ventral surface of gut

- Double row of ventral pigment on each side of midline

- Rays on superior hypural $=5$

${ }^{a}$ Wolotira 1985

Ref: Dunn and Matarese 1984, Dunn and Vinter 1984 


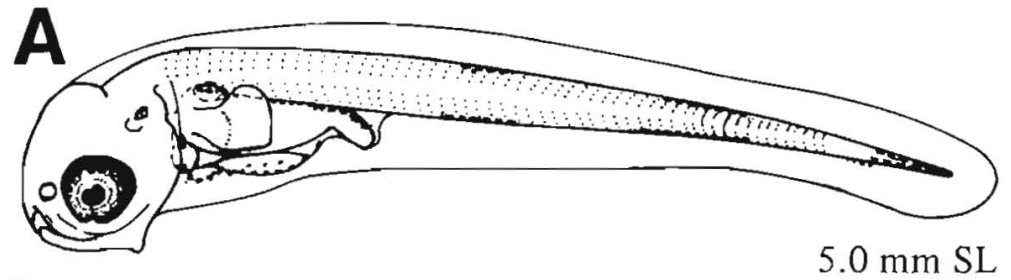

B

\section{$5.0 \mathrm{~mm} \mathrm{SL}$}
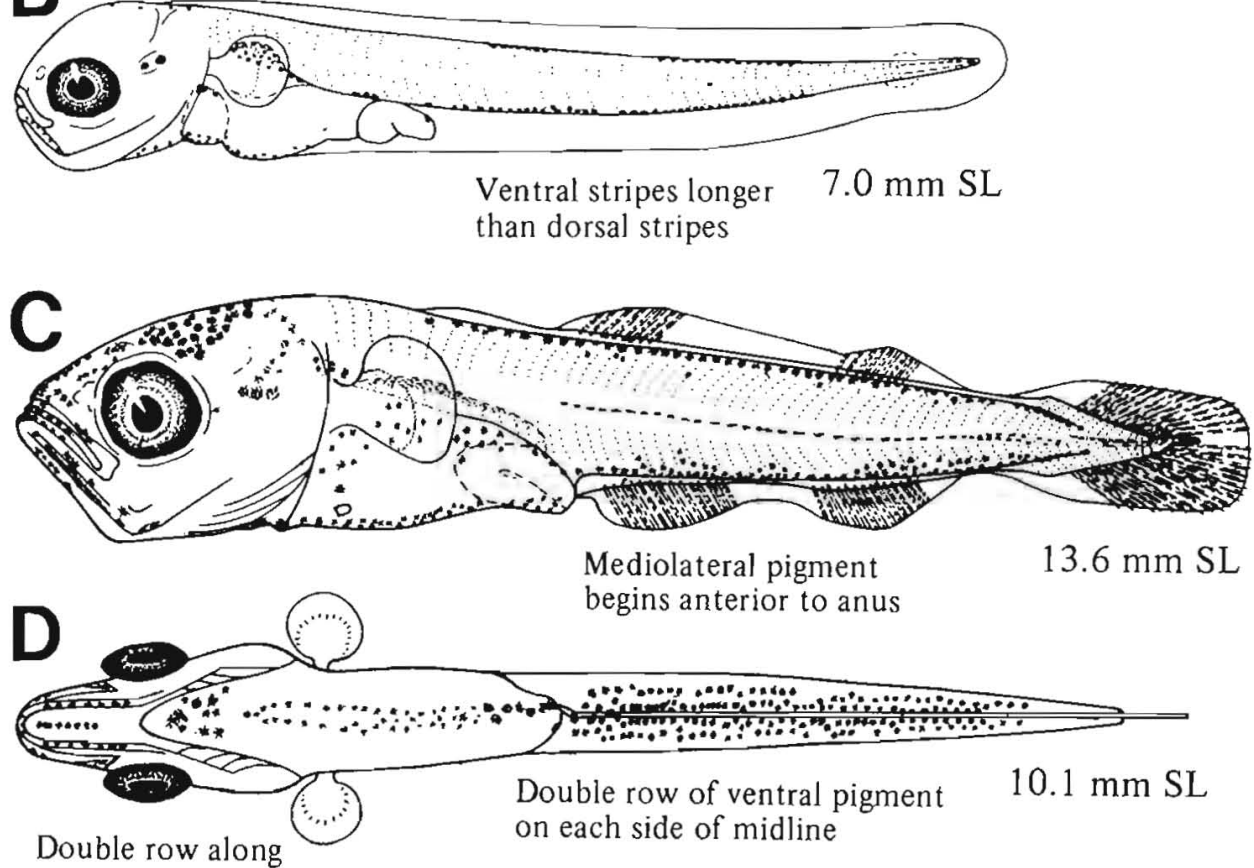

ventral surface of gut

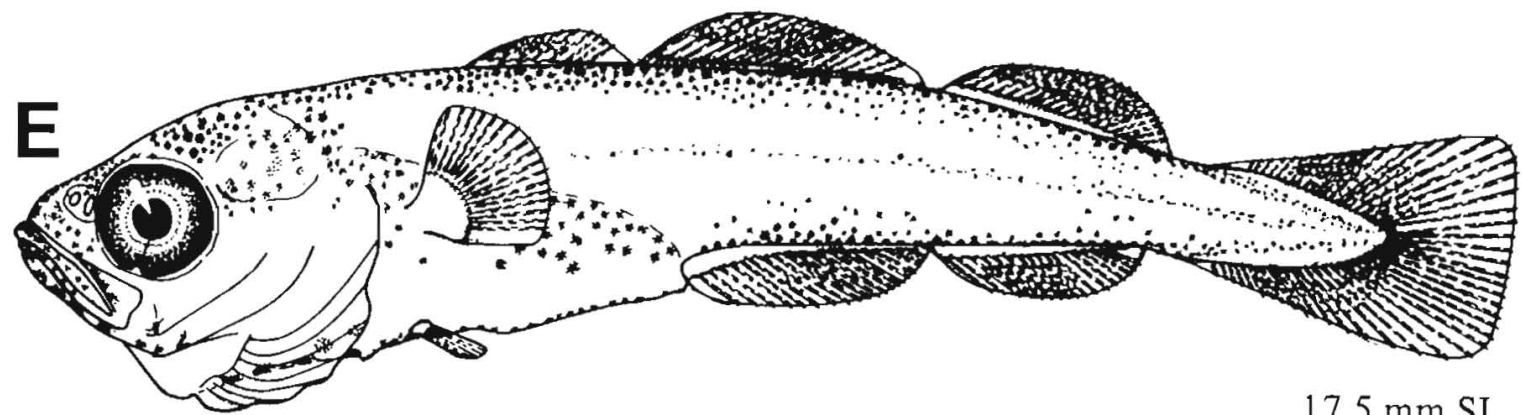

$17.5 \mathrm{~mm} \mathrm{SL}$

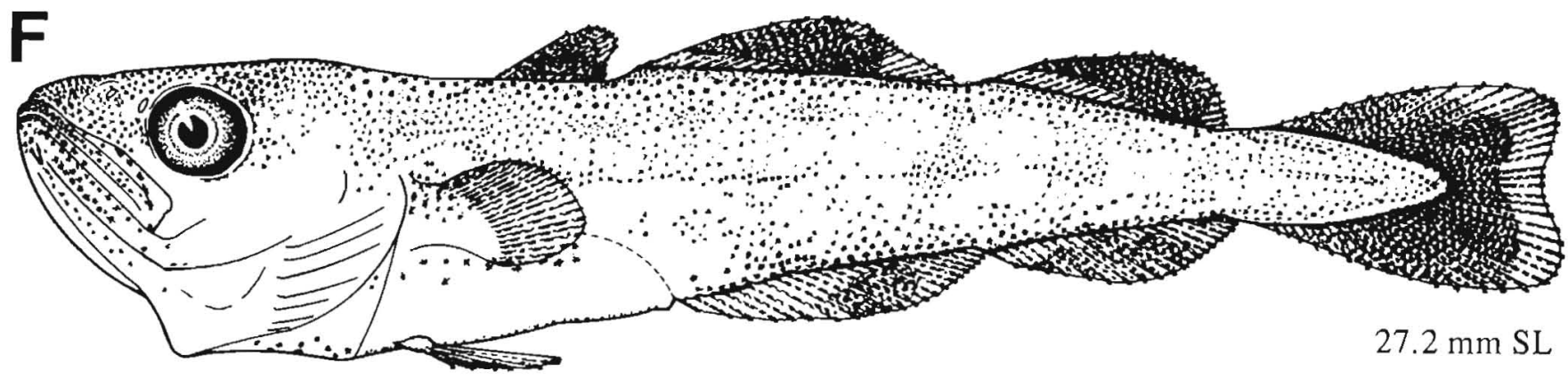

Figures $A-F(D$, ventral view), Dunn and Vinter 1984. 


\section{MERISTICS}

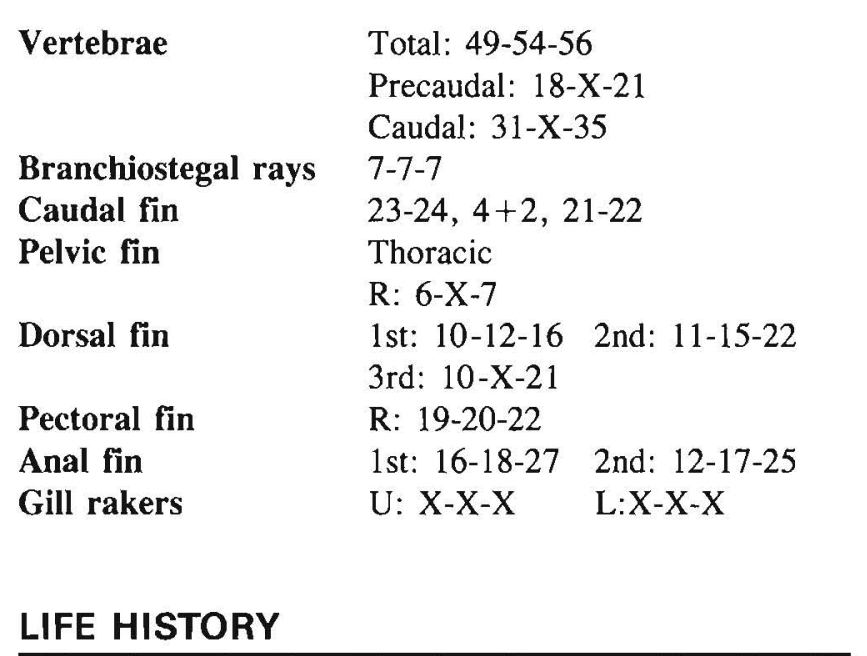

$\begin{array}{ll}\text { Range } & \text { S. California, } 32-34^{\circ} \mathrm{N} \text {, to } \\ \text { Bering Sea, } 54-66^{\circ} \mathrm{N} \\ \text { Ecology } & \text { Epi-, meso-, and bathybenthal } \\ \text { ELH pattern } & \text { Oviparous, demersal eggs, } \\ \text { pelagic larvae (small larvae } & \text { demersal) }^{\mathrm{a}} \\ \text { Spawning } & \text { Season: } \mathrm{Jan}^{\mathrm{b}}-\mathrm{July}^{\mathrm{c}} \\ & \text { Area: Semi-demersal } \\ & (73-265 \mathrm{~m})^{\mathrm{c}} \\ & \text { Mode: Schools } \\ & \text { Migration: To deepwater } \\ \text { Fecundity } & \text { Range/function: } \\ & 228,000^{\mathrm{e}}-3 \text { million } \\ & \mathrm{F}=12.024 \times \mathrm{L}^{\mathrm{f} / .959} \mathrm{~g} \\ \text { Age at first maturity } & 2 \mathrm{yr}^{\mathrm{d}} \\ \text { Longevity } & 13 \mathrm{yr}^{\mathrm{f}}\end{array}$

${ }^{a}$ Walters (1984) reported small larvae are demersal whereas Rugen and Matarese (1988) reported newly hatched larvae quickly rise to above $50 \mathrm{~m}$.

'Miller et al. 1978

${ }^{\circ}$ Hirschberger and Smith 1983

${ }^{d}$ Ketchen 1961

'Thompson 1962

${ }^{\mathrm{f}}$ Andriashev 1954

${ }^{8}$ Karp 1982

Ref: Dunn and Matarese 1984, 1987; Dunn and Vinter 1984; Matarese et al. 1981; Walters 1984

\section{EARLY LIFE HISTORY DESCRIPTION}

\section{EGGS}

Diameter

No. of oil globules

Oil globule diameter

Yolk

Envelope

Hatch size

$0.98-1.08 \mathrm{~mm}(1.02 \mathrm{~mm})$

None

Homogeneous, dense

Smooth, thick

3-4 $\mathrm{mm} \mathrm{SL}$

Incubation time/temp.

Pigment

Diagnostic characters

LARVAE

Preanal length

Length at flexion

Sequence of fin

development

Pigment

- Presence of bars

- Ventral gut with large melanophores (in larvae $>20 \mathrm{~mm} \mathrm{SL}$, small spots occur in two rows along ventral surface of gut)

- Stripe continuity ( 5-6 mm SL)

- Mediolateral pigment begins anterior to anus ( 5-6 mm SL)

Diagnostic characters (see Table 22)

- Pigment

- Large melanophores medially along length of gut

- Single irregular row of pigment on each side of ventral midline

-Ventral pigment begins at anus after yolksac absorption

- Rays on superior hypural $=4$

Distinguished from Theragra chalcogramma at yolksac stage by

- Less lateral pigment within bars

- Posterior bar longer, extending closer to tail

- Presence of about 2-6 spots in the ventral caudal region

- More pigment in snout area and on mouth

Distinguished from $T$. chalcogramma at later stages by

- Generally more pigmented, especially on head and gut 

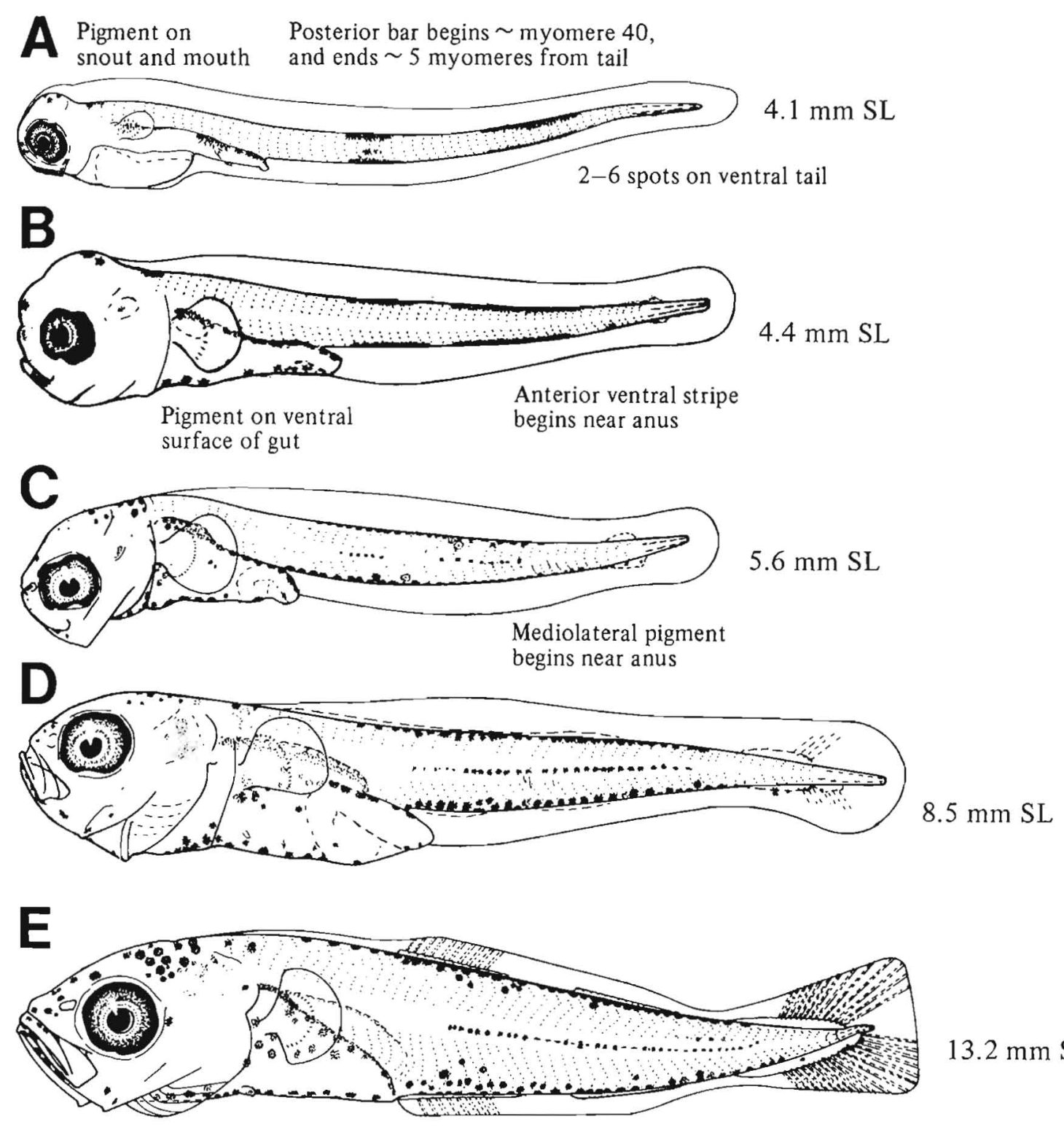

$13.2 \mathrm{~mm} \mathrm{SL}$

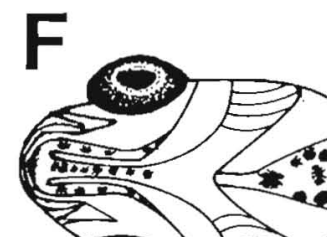

$\infty$

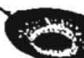

i.

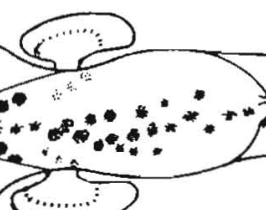

Large melanophores medially along length of gut
Pon

Single irregular row of melanophores on each side of ventral midline

Figure A, NWAFC original (B. Vinter); B, E-F (F, ventral view), Dunn and Vinter 1984; C-D, Matarese et al. 1981. 


\section{MERISTICS}

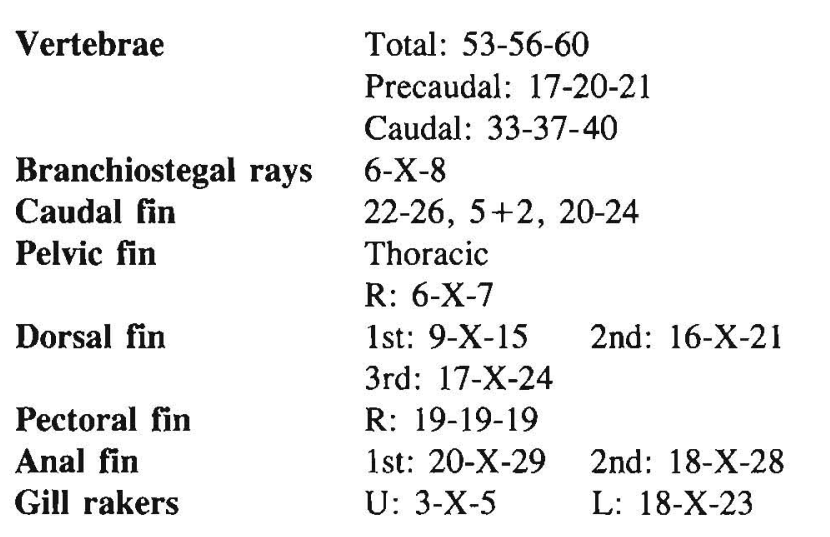

\section{LIFE HISTORY}

\begin{tabular}{|c|c|}
\hline Range & $\begin{array}{l}\text { Cent. California, } 34-38^{\circ} \mathrm{N} \text {, to } \\
\text { Bering Sea, } 54-66^{\circ} \mathrm{N}^{\mathrm{a}}\end{array}$ \\
\hline Ecology & Epi- and mesobenthal, 0-275 m \\
\hline ELH pattern & $\begin{array}{l}\text { Oviparous, demersal eggs, } \\
\text { pelagic larvae }\end{array}$ \\
\hline Spawning & $\begin{array}{l}\text { Season: Winter-spring } \\
\text { Area: } \\
\text { Mode: } \\
\text { Migration: }\end{array}$ \\
\hline $\begin{array}{l}\text { Fecundity } \\
\text { Age at first maturity } \\
\text { Longevity }\end{array}$ & Range/function: \\
\hline
\end{tabular}

\section{EARLY LIFE HISTORY DESCRIPTION}

\section{EGGS}

Diameter

No. of oil globules

Oil globule diameter

Yolk

Envelope

Hatch size

Probably none

Homogeneous

$3 \mathrm{~mm} \mathrm{SL}$

Incubation time/temp.

Pigment

Diagnostic characters

\section{LARVAE}

Preanal length

$<50 \%$ SL

Length at flexion

$8-15 \mathrm{~mm} \mathrm{SL}$

Length at transformation $22-28 \mathrm{~mm}$, pelagic from 28-45 mm SL

Sequence of fin Caudal; 1st anal; 2nd anal; development 3rd, 2nd, and 1st dorsal (nearly simultaneously); pelvics; pectorals

\section{Pigment}

- Presence of bars

- Single row on each side of ventral midline anteriorly and a single row along ventral midline posteriorly

- Large melanophores scattered on ventral surface of gut

Diagnostic characters (see Table 22)

- Pigment

- Single row on each side of ventral midline anteriorly and a single row along ventral midline posteriorly

-Large melanophores scattered on ventral surface of gut

- Anterior placement of bars

- Rays on superior hypural $=5$

Distinguished from Gadus macrocephalus by

- Dorsal pigment separated in specimens $<13 \mathrm{~mm} \mathrm{SL}$, bars not continuous

Distinguished from Theragra chalcogramma by

- Anterior bar begins closer to anus

\footnotetext{
${ }^{a}$ The presence of $M$. proximus larvae in the Bering Sea remains a question.

${ }^{\mathrm{b}}$ Richardson 1977

Ref: Dunn and Vinter 1984, Matarese et al. 1981.
} 


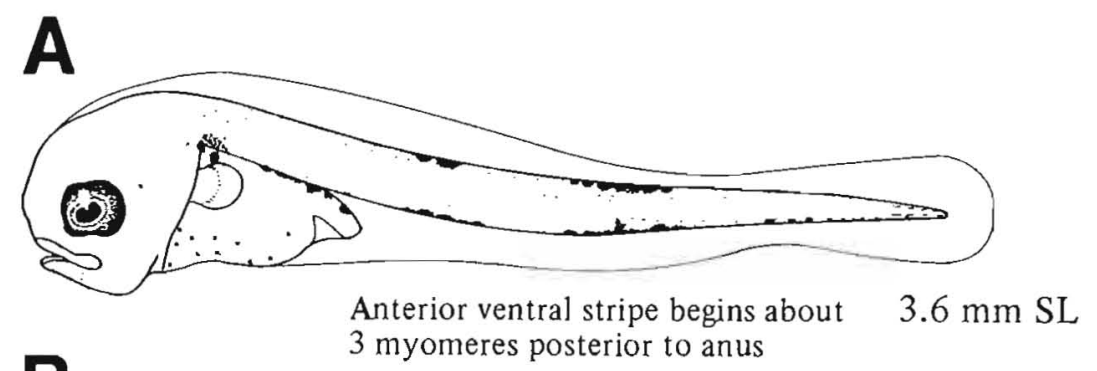

B

3 myomeres posterior to anus
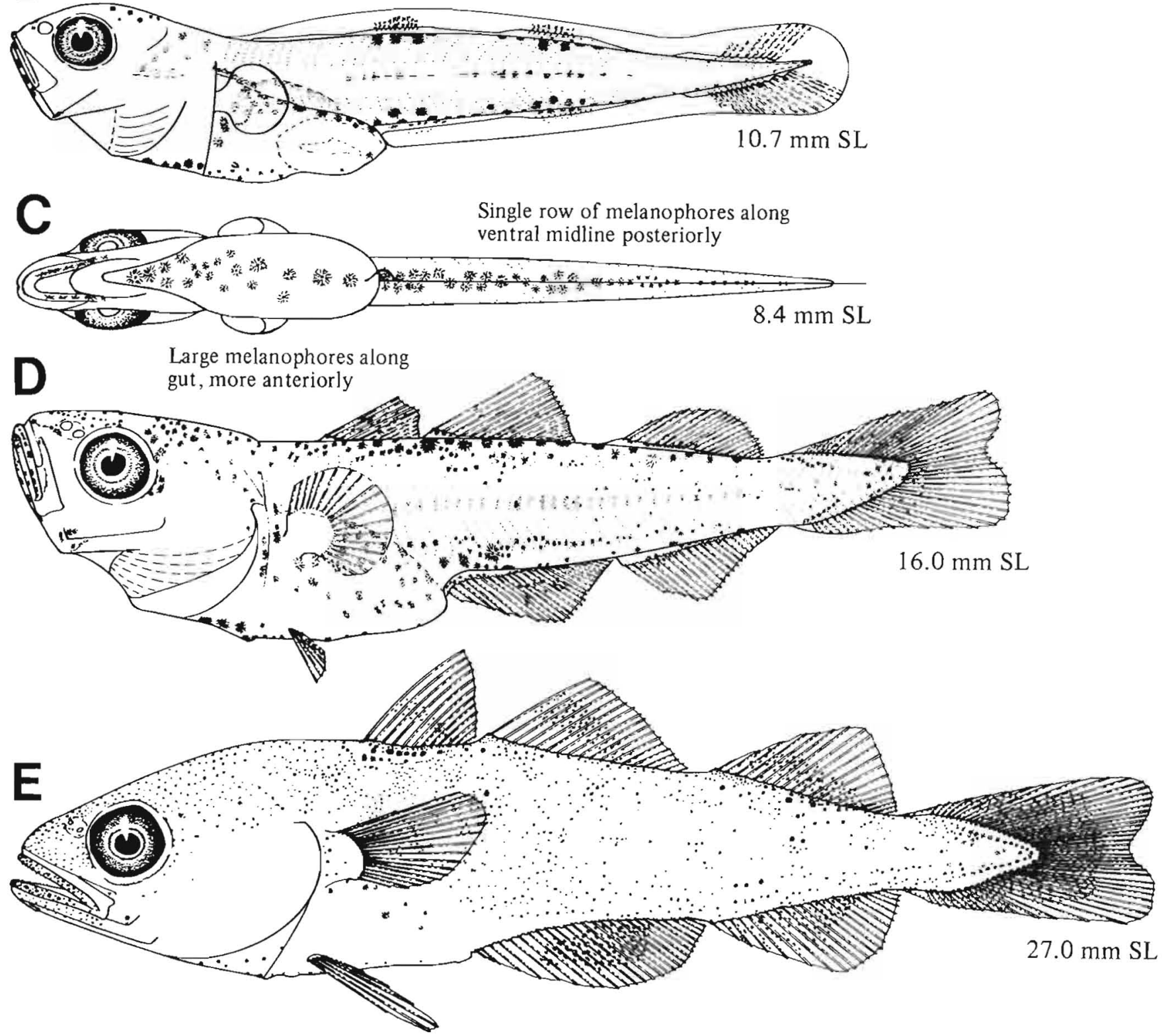

Figures A-E (C, ventral view), Matarese et al. 1981. 


\section{MERISTICS}

\begin{tabular}{|c|c|}
\hline \multirow{3}{*}{ Vertebrae } & Total: $48-51-53$ \\
\hline & Precaudal: $18-\mathrm{X}-20$ \\
\hline & Caudal: $31-X-34$ \\
\hline Branchiostegal rays & $6-X-8$ \\
\hline Caudal fin & $21-24,4+2,19-22$ \\
\hline Pelvic fin & Thoracic \\
\hline & $R: 6-X-7$ \\
\hline Dorsal fin & $\begin{array}{l}\text { 1st: } 10-12-14 \text { 2nd: } 12-14-18 \\
\text { 3rd: } 14-17-21\end{array}$ \\
\hline Pectoral fin & $\mathrm{R}: 17-20-22$ \\
\hline Anal fin & 1st: $15-18-22$ 2nd: $15-18-23$ \\
\hline Gill rakers ${ }^{\mathrm{a}}$ & $\mathrm{L}: 25-\mathrm{X}-34$ \\
\hline
\end{tabular}

\section{LIFE HISTORY}

\begin{tabular}{|c|c|}
\hline Range & $\begin{array}{l}\text { Cent. California, } 34-38^{\circ} \mathrm{N} \text {, to } \\
\text { Chukchi Sea, north of } 66^{\circ} \mathrm{N}\end{array}$ \\
\hline Ecology & $\begin{array}{l}\text { Epi-, meso-, and bathypelagic, } \\
0-975 \mathrm{~m}\end{array}$ \\
\hline ELH pattern & $\begin{array}{l}\text { Oviparous, pelagic eggs, } \\
\text { pelagic larvae }\end{array}$ \\
\hline \multirow[t]{4}{*}{ Spawning } & Season: Feb-Aug ${ }^{a}$ \\
\hline & Area: Pelagic $(50-460 \mathrm{~m})^{\mathrm{a}}$ \\
\hline & Mode: Schools ${ }^{b}$ \\
\hline & $\begin{array}{l}\text { Migration: Bering Sea, offshore } \\
\text { to outer and upper slope; } \\
\text { Gulf of Alaska to Shelikof } \\
\text { Strait }^{d}\end{array}$ \\
\hline \multirow[t]{7}{*}{ Fecundity } & Range/function: \\
\hline & $91,633-1,200,000 /$ \\
\hline & $\mathrm{F}=0.1719 \times \mathrm{L}^{3.6046}$ \\
\hline & $\mathrm{L}=\mathrm{FL} \mathrm{cm} ;{ }^{\mathrm{e}}$ \\
\hline & $96,216-1,079,540 /$ \\
\hline & $F=1.2604 \times L^{3.2169}$ \\
\hline & $\mathrm{L}=\mathrm{FL} \mathrm{cm}^{\mathrm{f}}$ \\
\hline Age at maturity & $3-4 \mathrm{yr}^{\mathrm{g}}$ \\
\hline Longevity & $17 \mathrm{yr}^{\mathrm{g}}$ \\
\hline
\end{tabular}

\section{EARLY LIFE HISTORY DESCRIPTION}

\section{EGGS}

Diameter

No. of oil globules

Oil globule diameter

Yolk

Envelope

Hatch size

$1.35-1.45 \mathrm{~mm}(1.2-1.8)$

None

Homogeneous

Smooth, clear

3-4 mm SL

Incubation time/temp. $\quad 15 \mathrm{~d} / 5^{\circ} \mathrm{C}$

Pigment

- Late-stage embryo develops bar pattern

Diagnostic characters

- Late-stage embryo with pigment

\section{LARVAE}

Preanal length $\quad<50 \%$ SL

Length at flexion $\quad 10-17 \mathrm{~mm} \mathrm{SL}$

Length at transformation $30-40 \mathrm{~mm} \mathrm{SL}$

Sequence of fin development

Caudal, 1st anal, 2nd anal, 3 rd dorsal, 2nd dorsal, 1st dorsal, pelvics, pectorals

Pigment

- Presence of bars

- A few melanophores scattered on ventral surface of gut

- Pigment along ventral midline and a single row on each side

Diagnostic characters (see Table 22)

- See Gadus macrocephalus (p. 194)

- Pigment

-A few melanophores scattered on ventral surface of gut

- Pigment along ventral midline and a single row on each side

- Rays on superior hypural $=4$

\footnotetext{
${ }^{a}$ Hirschberger and Smith 1983

b Takahura 1954

${ }^{c}$ Serobaba 1968

dDunn and Matarese 1987

${ }^{\mathrm{e}}$ Hinckley 1986 (Bering Sea specimens only)

${ }^{f}$ Miller et al. 1986 (Shelikof Strait specimens only)

${ }^{8}$ Salveson and Alton 1976a
}

Ref: Dunn and Matarese 1984, 1987; Dunn and Vinter 1984. 
Posterior bar begins $\sim$ myomere 35

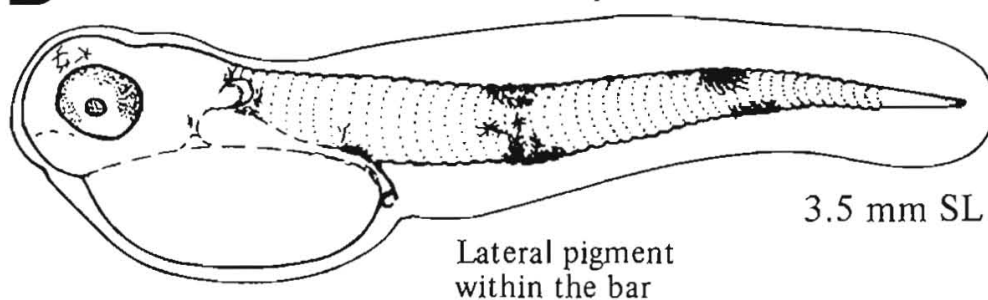

C

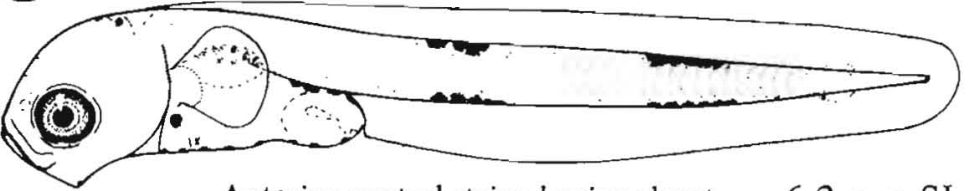

Anterior ventral stripe begins about 4-5 myomeres posterior to anus

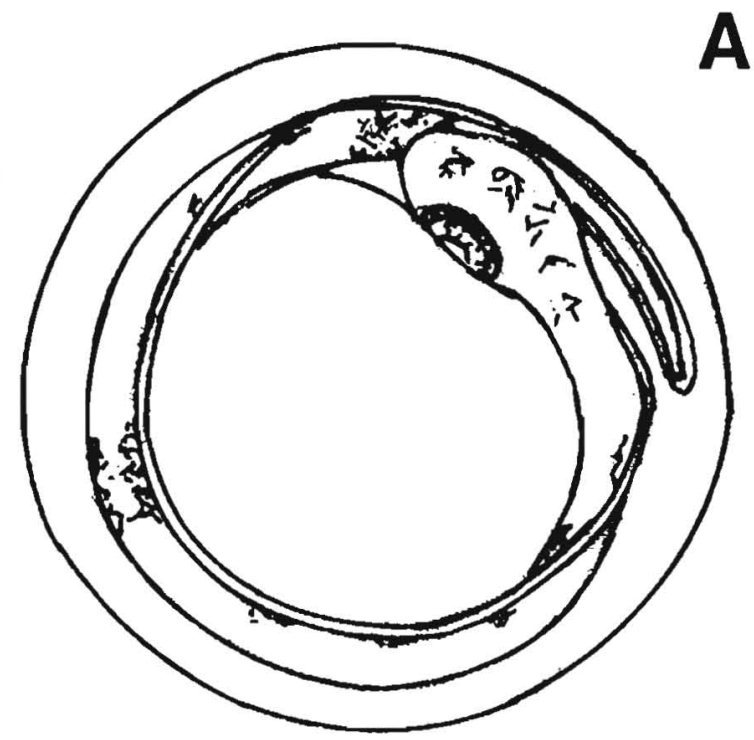

$1.2-1.8 \mathrm{~mm}$
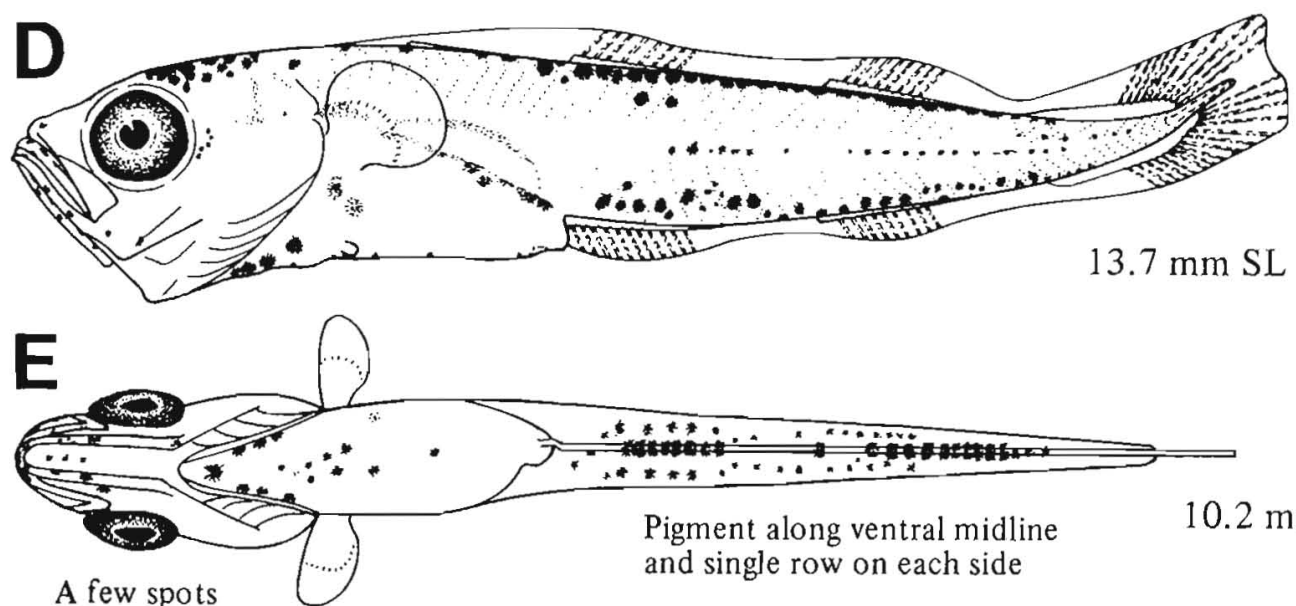
and single row on each side

$10.2 \mathrm{~mm} \mathrm{SL}$ anteriorly on gut

E

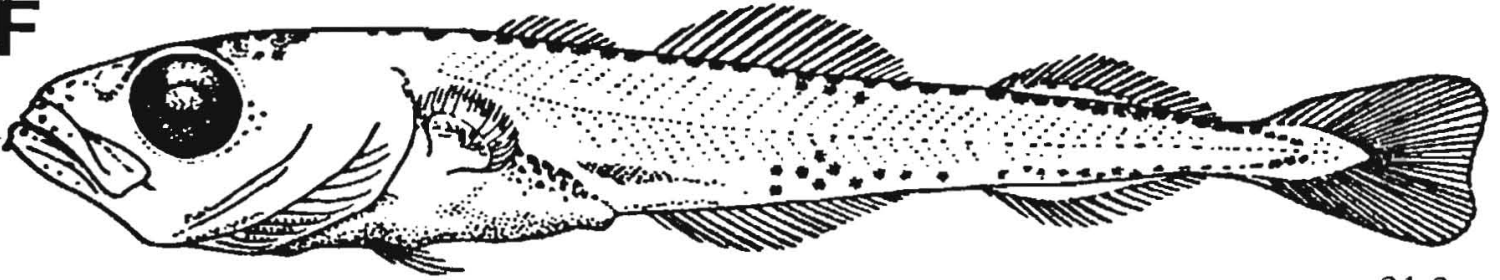

$21.3 \mathrm{~mm} \mathrm{SL}$

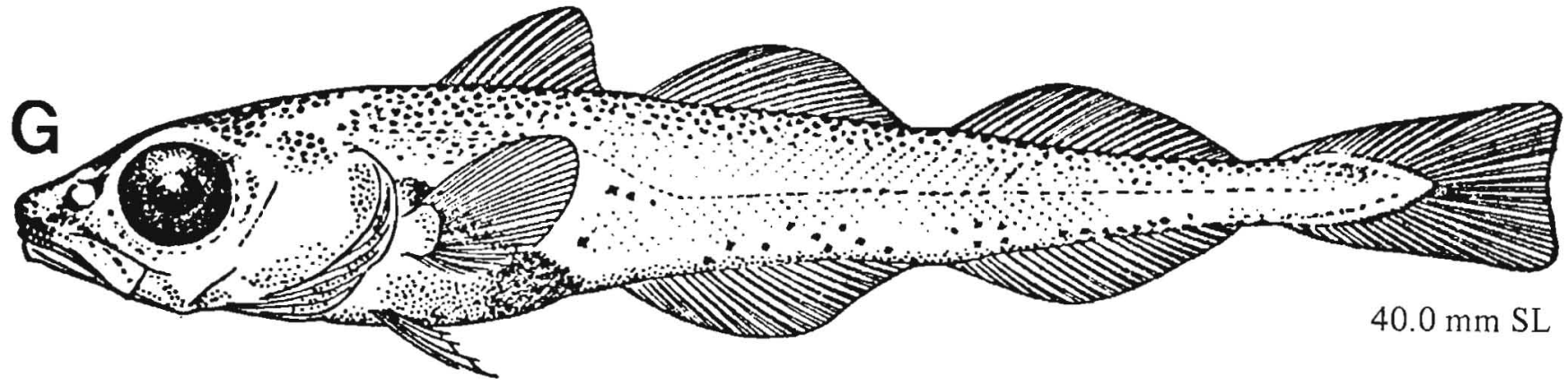

Figures A-B, NWAFC originals (B. Vinter); C-E (E, ventral view), Dunn and Vinter 1984; F-G, Gorbunova 1954. 
Among the most common of all deep-sea bottom-living fishes, grenadiers are found in all the world's oceans. They have long, tapering bodies and elongate dorsal and anal fins continuous with the tail. A chin barbel is usually present. In the Northeast Pacific there are ten species in three genera. Adults have been found in water as shallow as $100 \mathrm{~m}$ but are more commonly collected at depths greater than $300 \mathrm{~m}$ (Fitch and Lavenberg 1968). Little is known of their early life history. Macrourid eggs are generally 1-2 mm, have a single oil globule, and some species have honeycomb ornamentation on the chorion. Larvae are characterized by an elongate tail, lack of caudal fin, and an elongate pectoral fin peduncle. The transition from larva to juvenile is rapid. The most important morphological change is the loss of the pectoral fin peduncle. Other morphological differences occurring at transformation include small changes in head length, mouth orientation changing from oblique to horizontal, snout becoming more distinct, and the stomach (gut) becoming reduced in prominence (decreasing depth of posterior trunk). The occurrence of juveniles of increasing size at increasing depth suggests an ontogenetic migration of subadults to a benthic existence (Stein 1980a). Some species remain pelagic throughout a prolonged juvenile period (Hubbs and Iwamoto 1977).

\begin{tabular}{|c|c|c|c|c|c|c|c|c|c|c|}
\hline \multirow[b]{3}{*}{ Taxon } & \multirow[b]{3}{*}{ Distribution } & \multicolumn{5}{|c|}{$\begin{array}{l}\text { Table } 23 \\
\text { Meristic characters of family Macrouridae. }\end{array}$} & & & & \multirow[b]{3}{*}{ Branchiostegals } \\
\hline & & \multicolumn{2}{|c|}{ Vertebrae } & \multicolumn{5}{|c|}{ Fins } & Gill rakers & \\
\hline & & $\begin{array}{l}\text { Precaudal } \\
\text { (Total) }\end{array}$ & Caudal & $\begin{array}{l}\text { First* } \\
\text { Dorsal }\end{array}$ & $\begin{array}{l}\text { Second } \\
\text { Dorsal }\end{array}$ & Anal & Pectoral & Pelvic & $\begin{array}{l}\text { Upper Lower } \\
\text { (Total) }\end{array}$ & \\
\hline Albatrossia pectoralis & SSC - Bering Sea & $13-14$ & & $9-11$ & 126 & 131 & $16-21$ & $6-8$ & $(5-7)^{5-7}$ & 6 \\
\hline Coryphaenoides acrolepis & SSC - Bering Sea & $13-16$ & 70 & $10-13$ & $138-153$ & $123-135$ & $19-22$ & $7-9$ & $(5-7)^{5-7}$ & 6 \\
\hline Coryphaenoides armatus & SSC - Bering Sea & $13-15$ & & $10-12$ & & 77 & $18-22$ & $10-12$ & $1_{(7-9)^{6-9}}^{6}$ & 6 \\
\hline Coryphaenoides cinereus & Oregon - Bering Sea & $13-14$ & & $12-16$ & & & $17-23$ & $8-10$ & ${ }^{1}(9-12)^{9-11}$ & 6 \\
\hline Coryphaenoides filifer & S. Calif. - Bering Sea & (84) & & $13-16$ & & & $18-23$ & $9-10$ & $(8-10)$ & 6 \\
\hline Coryphaenoides Leprolepis & SSC - SE Alaska & 12 & & $10-12$ & & & $18-22$ & $9-11$ & ${ }^{1} \frac{8-11)^{8-10}}{}$ & 6 \\
\hline Coryphaenoides liocephalus & Brit. Col. & (84) & & 11 & 114 & 113 & 20 & 10 & & 6 \\
\hline Coryphaenoides longifilis & Aleutian Is. - Bering Sea & $14-15$ & & $14-16$ & & & $15-19$ & $9-10$ & $12-13$ & 6 \\
\hline Coryphaenoides yaquinae & Cent. Calif. - Oregon & & & $9-12$ & & & $16-22$ & $8-11$ & $(11-12)$ & \\
\hline Nezumia stelgidolepis & SSC - Brit. Col. & 13 & $73-77$ & $10-13$ & & & $20-26$ & $8-11$ & $(8-12)$ & 7 \\
\hline
\end{tabular}


Table 24

Morphological characters useful in identifying macrourid larvae likely to occur off the coast of Oregon (Stein 1980a).

\begin{tabular}{|c|c|c|c|c|c|c|}
\hline & \multirow{2}{*}{$\begin{array}{l}\text { Albarrossia } \\
\text { pectoralis }\end{array}$} & \multicolumn{5}{|c|}{ Coryphaenoides } \\
\hline & & C. acrolepis & C. armatus & C. cinereus & C. filifer & C. leptolepis \\
\hline Larval body pigment & Unknown & $\begin{array}{l}\text { Melanophores on dorsum } \\
\text { and venter, absent on last } \\
20 \% \text { of tail and on } \\
\text { midline }\end{array}$ & Unknown & Unknown & $\begin{array}{l}\text { Melanophores widely scat- } \\
\text { tered on dorsum around } \\
\text { dorsal fin only }\end{array}$ & $\begin{array}{l}\text { Melanophores on trunk } \\
\text { and head, closely spaced, } \\
\text { not posterior to anal fin } \\
\sim 10 \text { th ray }\end{array}$ \\
\hline First dorsal fin rays & $7-9$ & 9.11 & $8-10$ & $10-12$ & $11-15$ & $8-10$ \\
\hline Pelvic fin rays & $6-8$ & $8-9$ & $10-11$ & $8-10$ & $9-10$ & $9-10$ \\
\hline Pyloric caeca & $12-16$ & $12-14$ & $10-13$ & $5-7$ & $8-12$ & 11 \\
\hline Rostral scutes ${ }^{\mathrm{a}}$ & Absent & Strong & Absent & Strong & Strong & Absent \\
\hline $\begin{array}{l}\text { Size at which pectoral fin } \\
\text { peduncle is reduced }\end{array}$ & - & $9.4-9.8 \mathrm{~mm} \mathrm{HL}^{\mathrm{b}}$ & $18.2 \mathrm{~mm} \mathrm{HL}$ & - & $14.1-14.6 \mathrm{~mm} \mathrm{HL}$ & $6.2-15.0 \mathrm{~mm} \mathrm{HL}$ \\
\hline Precaudal vertebrae & $13-14$ & $14-15$ & $13-15$ & $13-14$ & - & 12 \\
\hline Gas glands $\mathrm{s}^{\mathrm{a}, \mathrm{c}}$ & 2 & 4 & $5-6$ & 4 & 4 & 6 \\
\hline $\operatorname{Retia}^{\mathbf{a}, c}$ & 2 & 4 & $5-6$ & 4 & 4 & 6 \\
\hline
\end{tabular}

${ }^{a}$ Aduit/juvenile characters.

${ }^{b}$ Since macrourid larvae often have damaged tails, body lengths are generally described by head lengths (HL).

${ }^{c}$ The swimbladder in postlarvae and juveniles can be examined by making an incision in the side of the abdominal cavity. 


\section{MERISTICS}

Vertebrae

Total: $86-86-86$

Precaudal: $14-X-15$

Caudal: $70-70-70$

Branchiostegal rays

6-6-6

Caudal fin

Pelvic fin

Absent

Thoracic

\section{$\mathrm{R}$ : $8-\mathrm{X}-9$}

Dorsal fin ${ }^{\mathrm{a}}$

1st: $9-\mathrm{X}-11$ 2nd: $138-\mathrm{X}-153$

Pectoral fin

Anal fin

R: $19-20-22$

$\mathrm{R}: 123-\mathrm{X}-135$

Gill rakers

\section{EARLY LIFE HISTORY DESCRIPTION}

\section{EGGS}

Diameter

No. of oil globules

Oil globule diameter

Yolk

Envelope

Hatch size

Incubation time/temp.

Pigment

Diagnostic characters

\section{LARVAE}

Preanal length

Length at flexion

Length at transformation

Sequence of fin development

Pigment

- Melanophores on dorsal and ventral body surface

- Lateral pigment absent on posterior $20 \%$ of body
Mode:

Migration:

Fecundity

Range/function:

Age at first maturity

Longevity

Bering Sea, $54-66^{\circ} \mathrm{N}$

Oviparous, eggs probably pelagic, pelagic larvae Season:

Spawning
Diagnostic characters (see Table 24)

- Number of first dorsal fin rays (9-11)

- Pelvic fin rays (8-9)

- Number of gas glands (four)

- Presence of rostral scutes in juveniles $>16 \mathrm{~mm}$ HL

${ }^{a}$ Range of counts for the first dorsal does not include "spines."

Ref: Stein 1980a. 


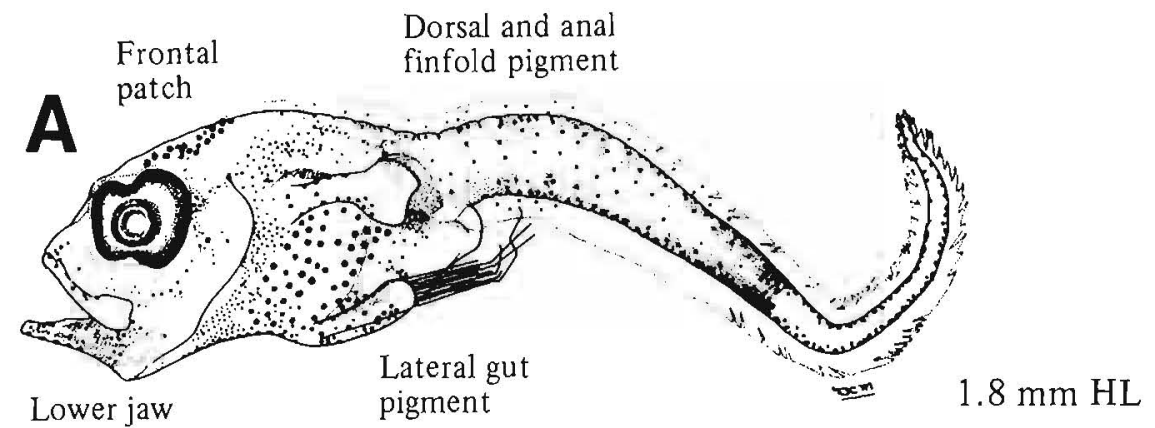

pigment
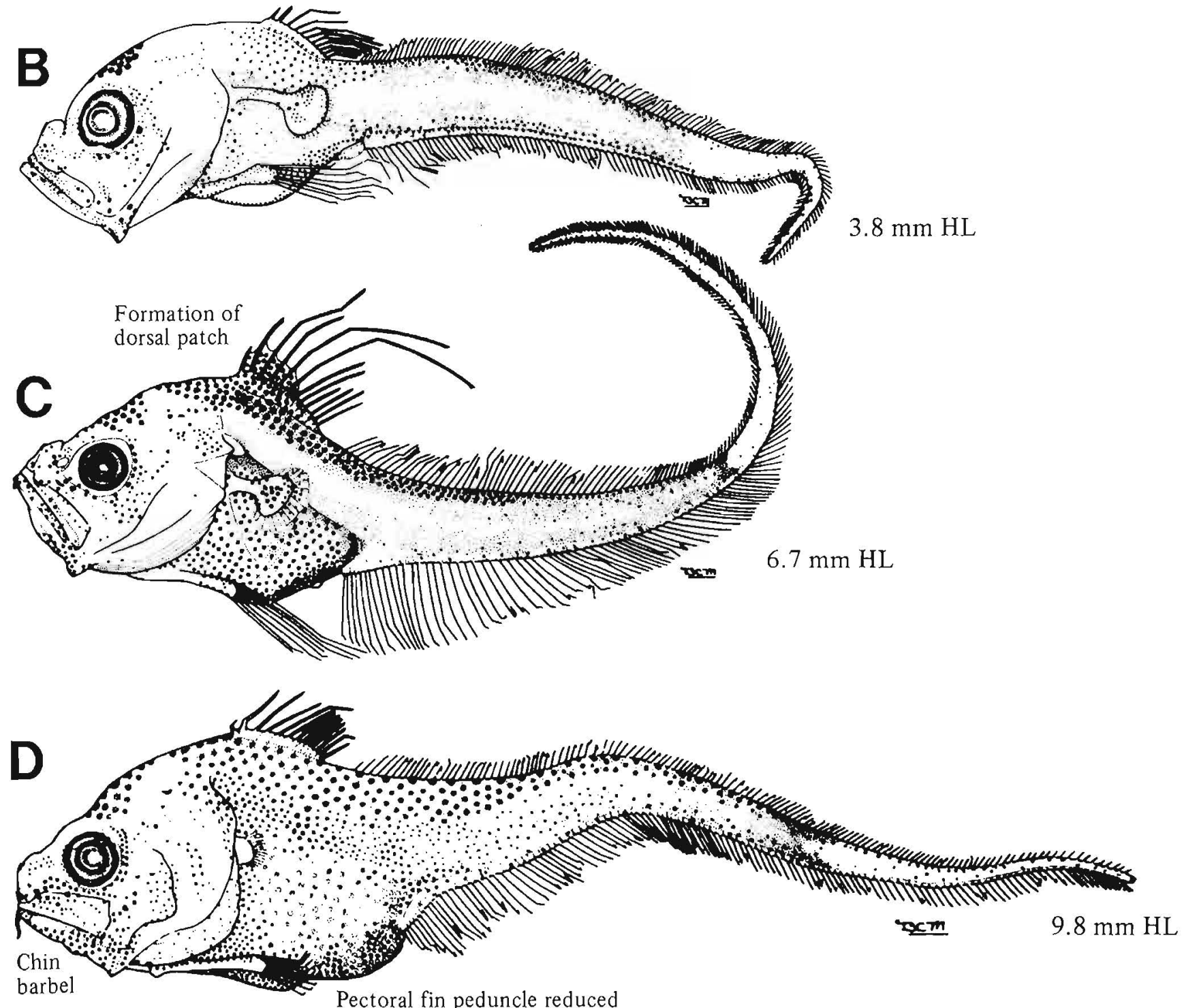

Figures A-D, Stein 1980 . 


\section{MERISTICS}

Vertebrae

Total: $84-84-84$

Precaudal: X-X-X

Caudal: $\mathrm{X}-\mathrm{X}-\mathrm{X}$

Branchiostegal rays

Caudal fin

Pelvic fin

6-6-6

Absent

Thoracic

R: 9-9-10

Dorsal fin ${ }^{\mathrm{a}}$

1st: $11-\mathrm{X}-15$ 2nd: X-X-X

Pectoral fin

R: $18-22-23$

Anal fin

Gill rakers

R: $X-X-X$

$\mathrm{U}: \mathrm{X}-\mathrm{X}-\mathrm{X} \quad \mathrm{L}: \mathrm{X}-\mathrm{X}-\mathrm{X}$

\section{LIFE HISTORY}

$\begin{array}{ll}\text { Range } & \begin{array}{c}\text { S. California, } 32-34^{\circ} \mathrm{N}, \text { to } \\ \text { Bering Sea, } 54-66^{\circ} \mathrm{N} \\ \text { Bathybenthal }\end{array} \\ \text { Ecology } & \begin{array}{c}\text { Oviparous, eggs probably } \\ \text { ELH pattern }\end{array} \\ \text { pelagic, pelagic larvae } \\ \text { Spawning } & \text { Season: } \\ & \text { Area: } \\ & \text { Mode: } \\ \text { Fecundity } & \text { Migration: } \\ \text { Age at first maturity } & \text { Range/function: } \\ \text { Longevity } & \end{array}$

\section{EARLY LIFE HISTORY DESCRIPTION}

\section{EGGS}

Diameter

No. of oil globules

Oil globule diameter

Yolk

Envelope

Hatch size

Incubation time/temp.

Pigment

Diagnostic characters

\section{LARVAE}

Preanal length

Length at flexion

Length at transformation

Sequence of fin

development

Pigment

- Melanophores widely scattered on upper dorsal body around dorsal fin

Diagnostic characters (see Table 24)

- Number of first dorsal fin rays (11-15)

- Pelvic fin rays (9-10)

- Number of gas glands (four)

- Pigment pattern: Fine pigment spots under first dorsal fin

\footnotetext{
a Range of counts for the first dorsal does not include "spines."
}

Ref: Stein 1980a. 


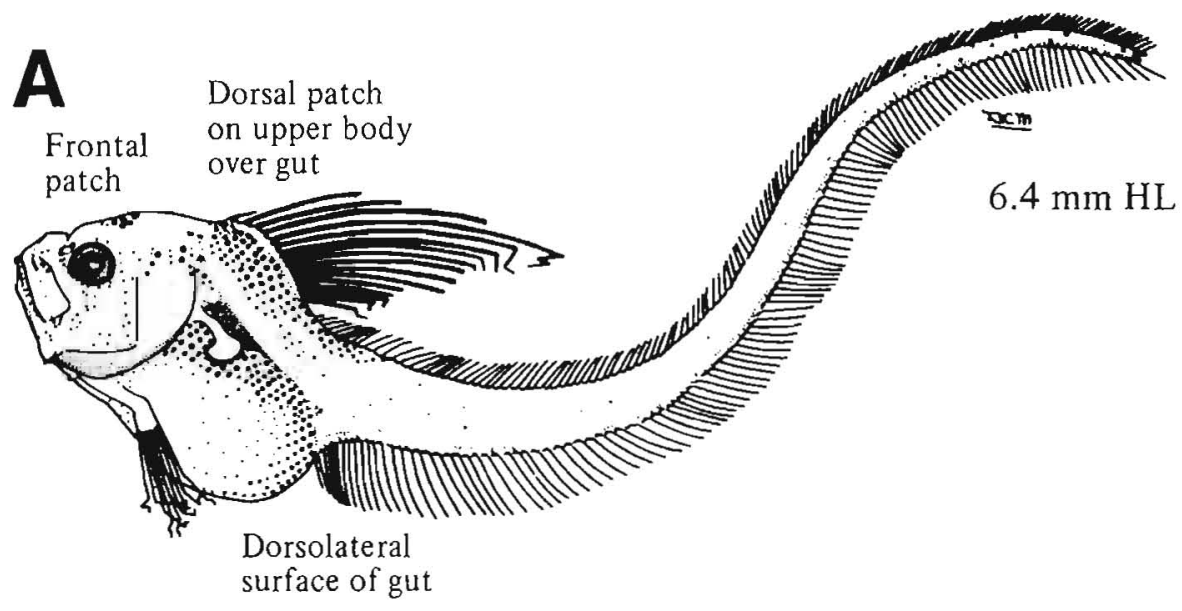

B
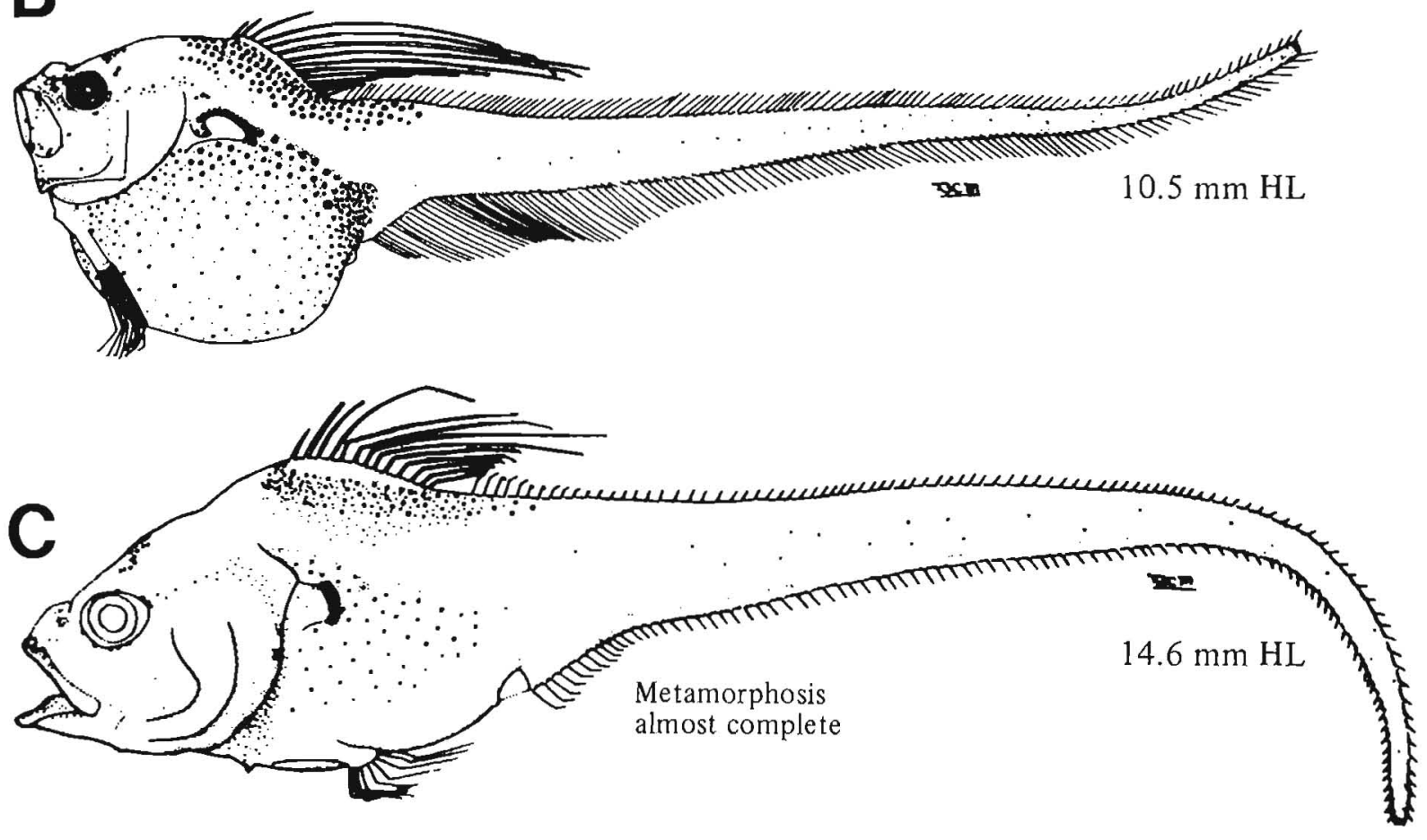

Figures A-C, Stein 1980. Figure $\mathrm{C}$ is a composite drawn from two specimens. The head has been reconstructed. 


\section{MERISTICS}

\begin{tabular}{|c|c|}
\hline \multirow[t]{3}{*}{ Vertebrae } & Total: X-X-X \\
\hline & Precaudal: $12-12-12$ \\
\hline & Caudal: X-X-X \\
\hline Branchiostegal rays & $6-6-6$ \\
\hline Caudal fin & Absent \\
\hline \multirow[t]{2}{*}{ Pelvic fin } & Thoracic \\
\hline & $\mathrm{R}: 9-\mathrm{X}-10$ \\
\hline Dorsal fin ${ }^{a}$ & 1st: $8-X-10$ 2nd: $X-X-X$ \\
\hline Pectoral fin & $\mathrm{R}: 18-19-22$ \\
\hline Anal fin & $\mathrm{R}: \mathrm{X}-\mathrm{X}-\mathrm{X}$ \\
\hline Gill rakers & $\mathrm{U}: 1-1-1 \quad \mathrm{~L}: 8-\mathrm{X}-10$ \\
\hline \multicolumn{2}{|l|}{ LIFE HISTORY } \\
\hline Range & $\begin{array}{l}\text { South of southern California to } \\
\text { SE Alaska, } 55-59^{\circ} \mathrm{N}\end{array}$ \\
\hline Ecology & Bathybenthal \\
\hline ELH pattern & $\begin{array}{c}\text { Oviparous, eggs probably } \\
\text { pelagic, pelagic larvae }\end{array}$ \\
\hline \multirow[t]{4}{*}{ Spawning } & Season: \\
\hline & Area: \\
\hline & Mode: \\
\hline & Migration: \\
\hline Fecundity & Range/function: \\
\hline $\begin{array}{l}\text { Age at first maturity } \\
\text { Longevity }\end{array}$ & \\
\hline
\end{tabular}

\section{EARLY LIFE HISTORY DESCRIPTION}

\section{EGGS}

Diameter

No. of oil globules

Oil globule diameter

Yolk

Envelope

Hatch size

Incubation time/temp.

Pigment

Diagnostic characters

\section{LARVAE}

Preanal length

Length at flexion

Length at transformation

Sequence of fin development

Pigment

- Melanophores on trunk, gut, and head dense, but not present posterior to anal fin $(\sim 10 \text { th ray })^{b}$

Diagnostic characters (see Table 24)

- Pigment pattern: Pigment to 10th anal fin ray

- Number of gas glands (six)

- Number of first dorsal fin rays $(8-10)$

\footnotetext{
${ }^{a}$ Range of counts for the first dorsal does not include "spines."

${ }^{b}$ According to Stein (1980a), this pattern may be similar in C. armatus.

Ref: Stein 1980a.
} 


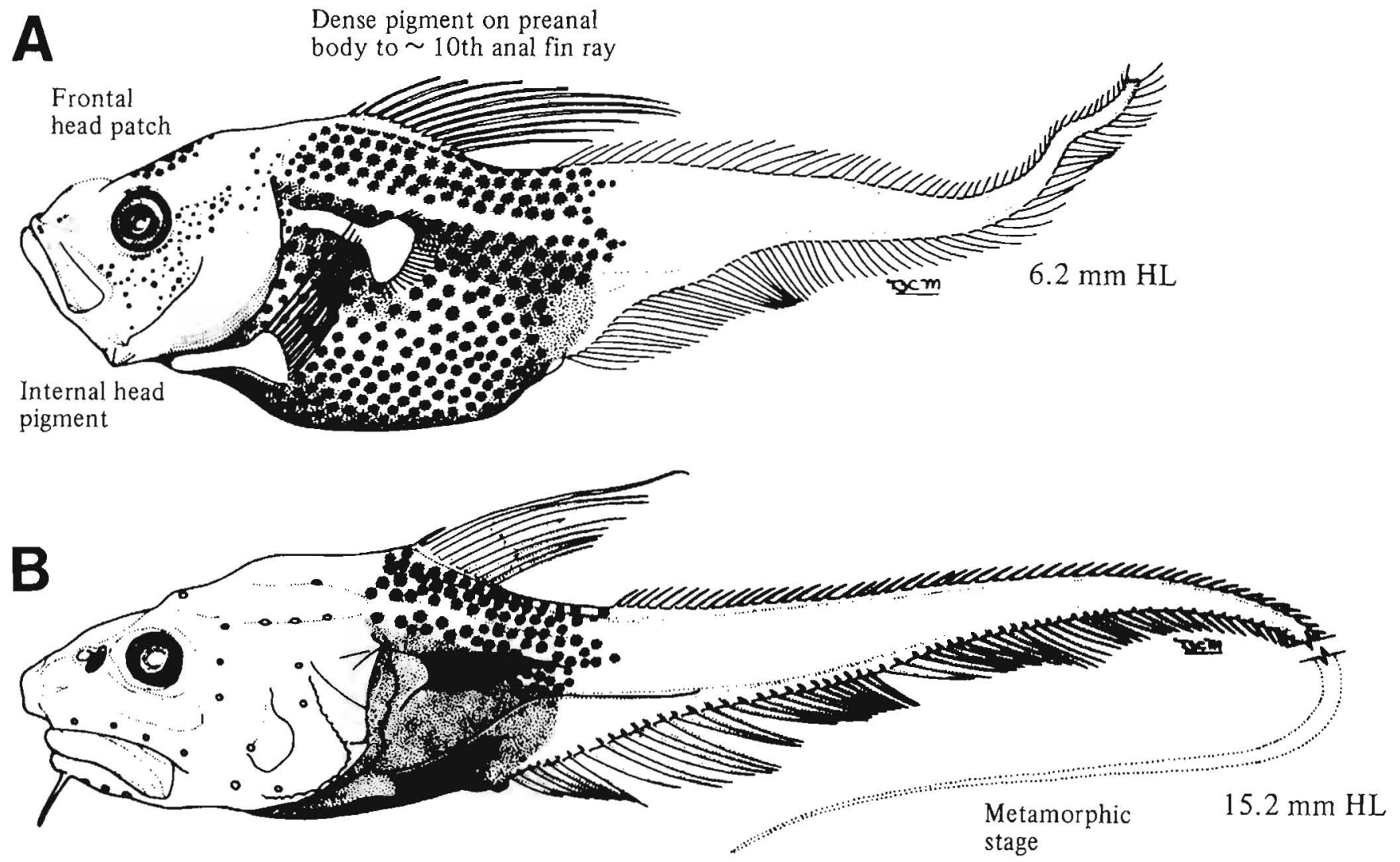

Figures A-B, Stein 1980 


\section{MERISTICS}

Vertebrae

Branchiostegal rays

Caudal fin

Pelvic fin

Dorsal fin

Pectoral fin

Anal fin

Gill rakers
Total: $86-\mathrm{X}-90$

Precaudal: 13-13-13

Caudal: $73-\mathrm{X}-77$

7-7-7

Absent

Thoracic

R: $8-\mathrm{X}-11$

S: $10-\mathrm{X}-13 \quad \mathrm{R}: \mathrm{X}-\mathrm{X}-\mathrm{X}$

R: $20-X-26$

$\mathrm{R}: \mathrm{X}-\mathrm{X}-\mathrm{X}$

$\mathrm{U}: \mathrm{X}-\mathrm{X}-\mathrm{X} \quad \mathrm{L}: \mathrm{X}-\mathrm{X}-\mathrm{X}$

\section{EARLY LIFE HISTORY DESCRIPTION}

\section{EGGS}

Diameter

No. of oil globules

Oil globule diameter

Yolk

Envelope

Hatch size

Incubation time/temp.

Pigment

Diagnostic characters

\section{LIFE HISTORY}

$\begin{array}{ll}\text { Range } & \begin{array}{c}\text { South of southern California to } \\ \text { Brit. Col., } 48^{\circ} 30^{\prime}-55^{\circ} \mathrm{N}\end{array} \\ \text { Ecology } & \text { Epi- and mesobenthal } \\ \text { ELH pattern } & \text { Oviparous, eggs unknown, } \\ & \text { pelagic larvae } \\ \text { Spawning } & \text { Season: } \\ & \text { Area: } \\ & \text { Mode: } \\ & \text { Migration: } \\ \text { Fecundity } & \text { Range/function: }\end{array}$

Age at first maturity

Longevity

\section{LARVAE - Genus}

Preanal length

Length at flexion

Length at transformation

Sequence of fin development

Pigment

- Frontal patch

- First dorsal fin patch

- Heavy over entire gut

- Ventral midline series and double lateral spots approximately first half of postanal body

\section{Diagnostic characters}

- Lateral pigment on postanal body appears to be distinctive perhaps at generic level, but whether it appears in other macrourine larvae cannot be determined at this time 


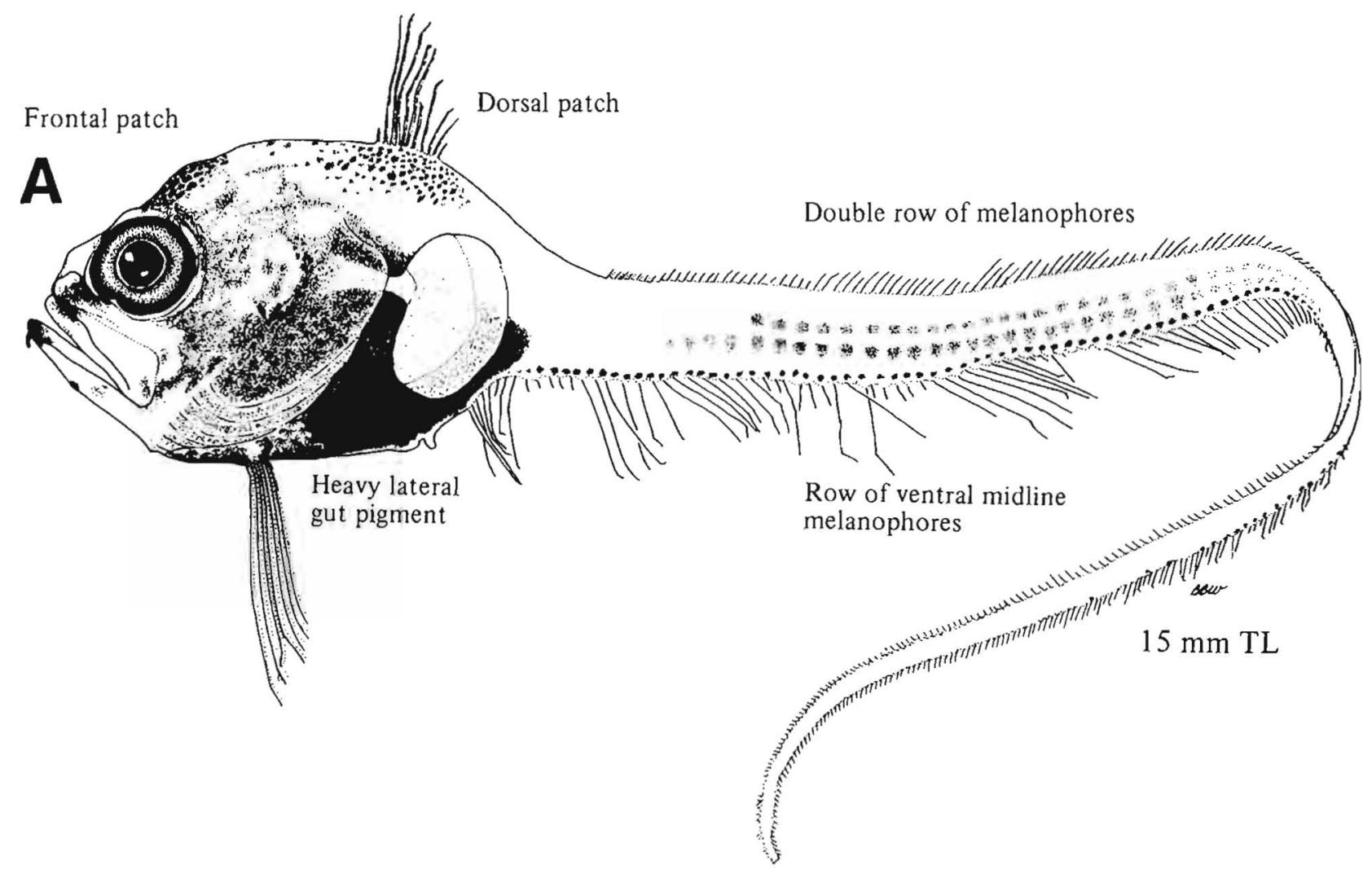

Figure A, Fahay and Markle 1984. North Atlantic specimen tentatively identified as Nezumia. 



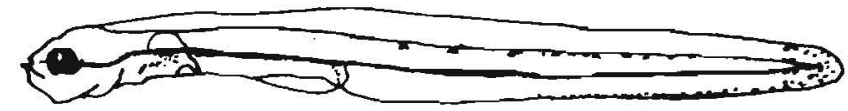

\section{Ophidiiformes}

The ophidiiforms occupy mostly benthic habitats ranging from the shallow tropics to abyssal depths and subarctic locations. Adults have long tapering bodies, with or without a caudal fin, and long dorsal and anal fins. The order comprises 4 families, 86 genera, and 294 species (J. Nelson 1984). The two suborders are defined according to mode of reproduction: Ophidioidei (Ophidiidae) are oviparous and Bythitoidei (Aphyonidae and Bythitidae) are viviparous (Cohen and Nielsen 1978). Ophidiiform eggs of oviparous forms are pelagic, may have one oil globule, and are spherical or ellipsoidal (Gordon et al. 1984). Larvae are not well known; of the eight species found in the study area only two larval series have been identified and are presented here. A third series of an unidentified ophidiid has been tentatively identified as Spectrunculus grandis.

Families in study area: Ophidiidae

Aphyonidae

Bythitidae 
Fishes of this group are found primarily in tropical and temperate waters, although a few occur in subarctic locations. In the northeastern Pacific there are six species within five genera in the family. Larvae are known only for Chilara taylori, but a second series has been tentatively identified as Spectrunculus grandis. These larvae occur in samples collected off northern California, Oregon, and Washington. Since meristic characters, especially vertebral counts, are unavailable for several species in the area, only a tentative identification can be made. Available meristics, however, match those of Spectrunculus grandis. These larvae resemble those of the pleuronectid, Embassichthys bathybius, and have been routinely mixed with them in samples. They can be distinguished from $E$. bathybius (p. 580) larvae by number of myomeres ( $>75$ vs. 65$)$, less finfold pigment, and a less pronounced loop in the gut.

Table 25

Meristic characters of family Ophidiidae.

\begin{tabular}{|c|c|c|c|c|c|c|c|c|c|c|}
\hline \multirow[b]{2}{*}{ Taxon } & \multirow[b]{2}{*}{ Distribution } & \multicolumn{2}{|c|}{ Vertebrae } & \multicolumn{4}{|c|}{ Fins } & \multicolumn{2}{|c|}{ Gill rakers } & \multirow[b]{2}{*}{ Branchiostegals } \\
\hline & & Precaudal & Caudal & Dorsal & Anal & Pectoral & Pelvic & Upper & Lower & \\
\hline Bassozetus sp. & Oregon & & & & & & & & & \\
\hline Chilara taylori & SSC - Oregon & $18-19$ & $68-72$ & $198-216$ & $156-170$ & & 1,1 & $1-4$ & $5-9$ & 7 \\
\hline Dicrolene filamentasa & SSC - Oregon & & & $100-104$ & $84-90$ & $23-29$ & 1,2 & 5 & 17 & 8 \\
\hline Holcomycreronus profundissimus & Oregon & $18-21$ & & $107-118$ & $80-95$ & $15-17$ & $\mathrm{I}, 2$ & & & 8 \\
\hline Spectrunculus grandis* & Cent. Calif. - Gulf of Alaska & 23 & 56 & $103-140$ & $73-106$ & $26-30$ & $\mathrm{I}, 2$ & $3-4$ & $8-9$ & 8 \\
\hline Spectrunculus radcliffei & Gulf of Alaska & & & & & & & & & \\
\hline
\end{tabular}

*Aboussouan and Rasonarivo (1986) described a larva as $S$. grandis from the Indian Ocean. The counts are the following:

$\begin{array}{lr}\text { precaudal vertebrae } & 18-19 \\ \text { caudal vertebrae } & 53 \\ \text { dorsal fin } & 118 \\ \text { anal fin } & 96 \\ \text { pectoral fin } & 23 / 24 \\ \text { pelvic fin } & 1,2 \\ \text { caudal fin } & 4+4 / 5\end{array}$




\section{Ophidiidae (tentatively Spectrunculus grandis)}
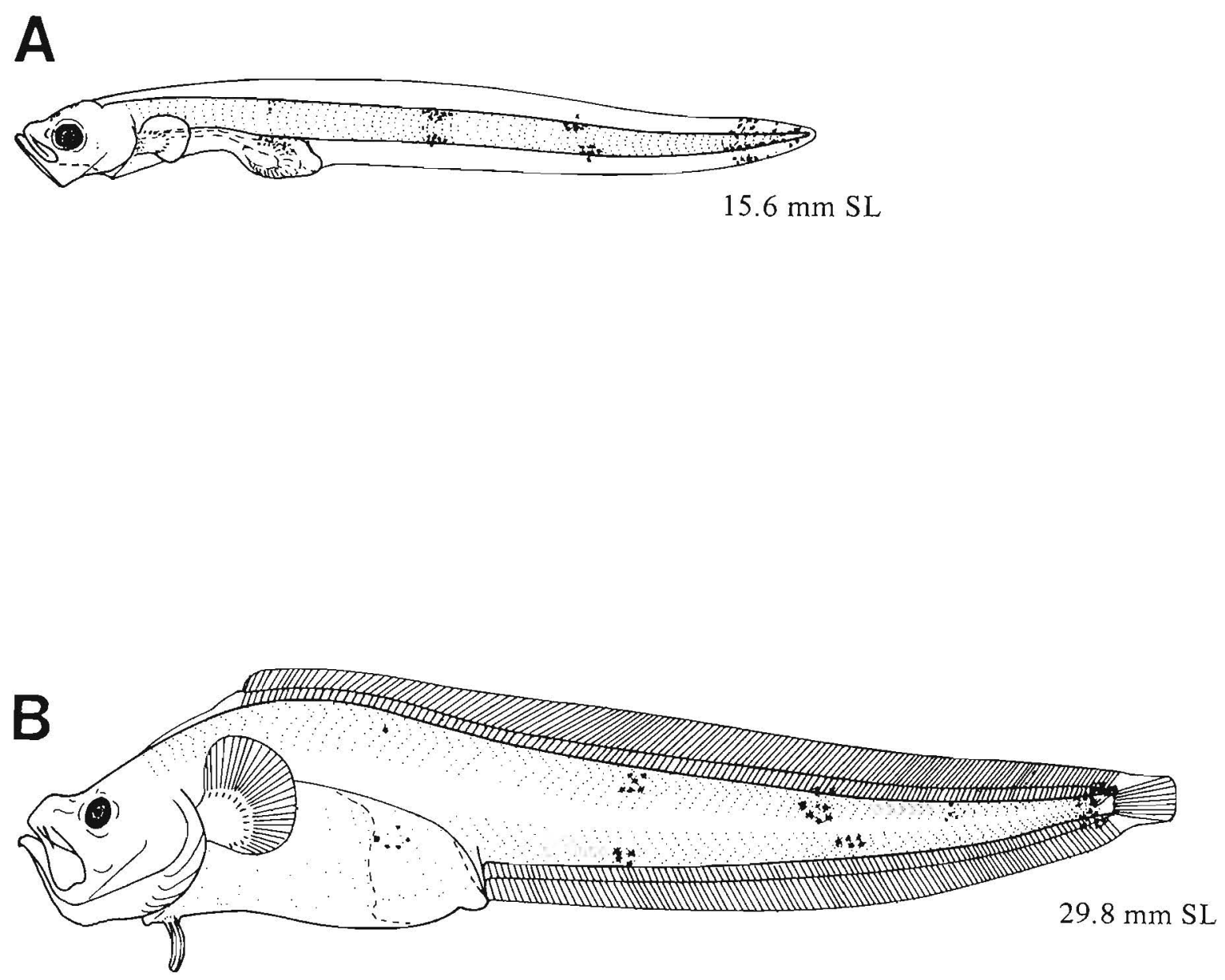

Figures A-B, NWAFC originals (B. Vinter). 


\section{MERISTICS}

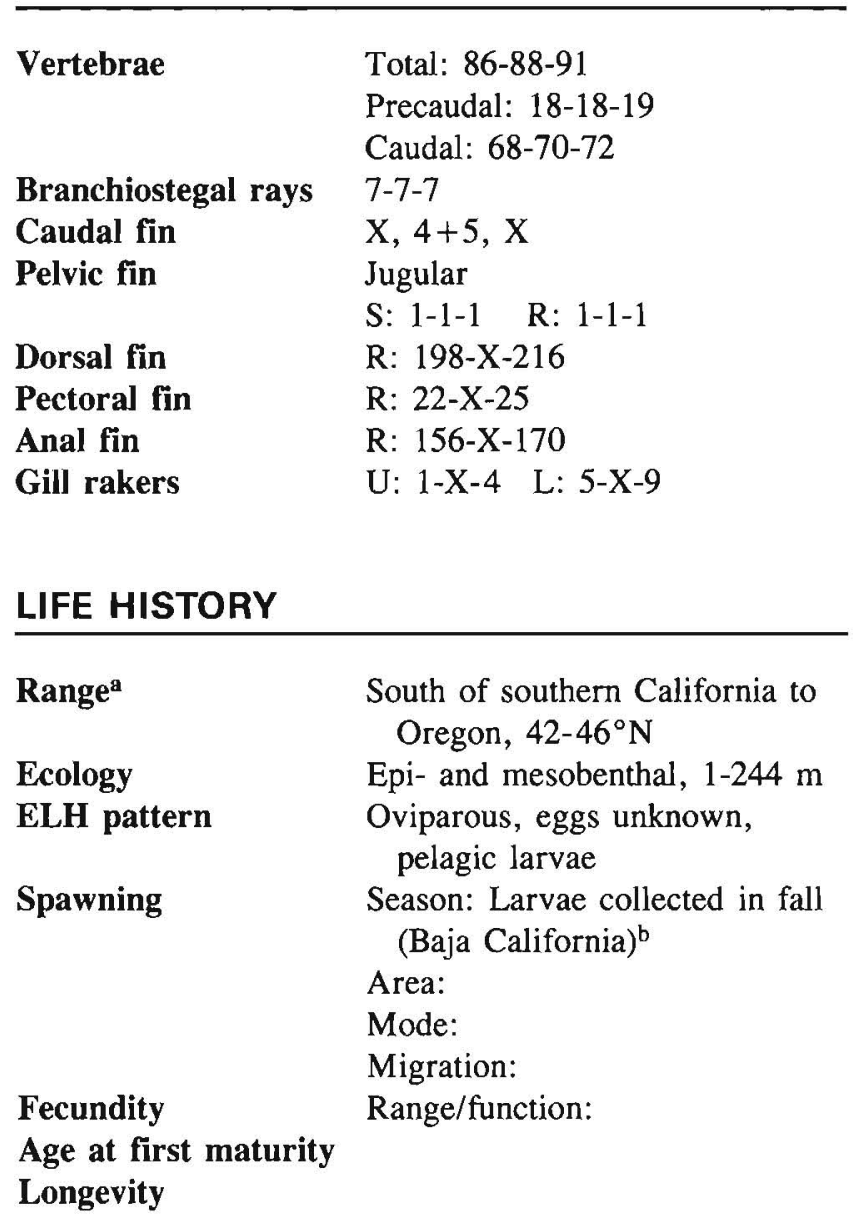

\section{EARLY LIFE HISTORY DESCRIPTION}

\section{EGGS}

Diameter

No. of oil globules

Oil globule diameter

Yolk

Envelope

Hatch size

Incubation time/temp.

Pigment

Diagnostic characters

\section{LARVAE}

Preanal length $\quad<50 \%$ SL

Length at flexion

Length at transformation $22-30 \mathrm{~mm} \mathrm{SL}$

Sequence of fin development

Pigment

- Caudal fin pigment

- Ventral (double series) with development, extends along base of brain and onto snout

- Ventral gut

Diagnostic characters

- Gut loops form at $14 \mathrm{~mm} \mathrm{SL}$

- Distinctive pigment pattern

- High number of myomeres (86-91)

${ }^{a}$ Center of distribution is Pt. Eugenia, Baja California.

${ }^{\circ}$ Ambrose et al. 1983

Ref: Ambrose et al. 1983. 
A
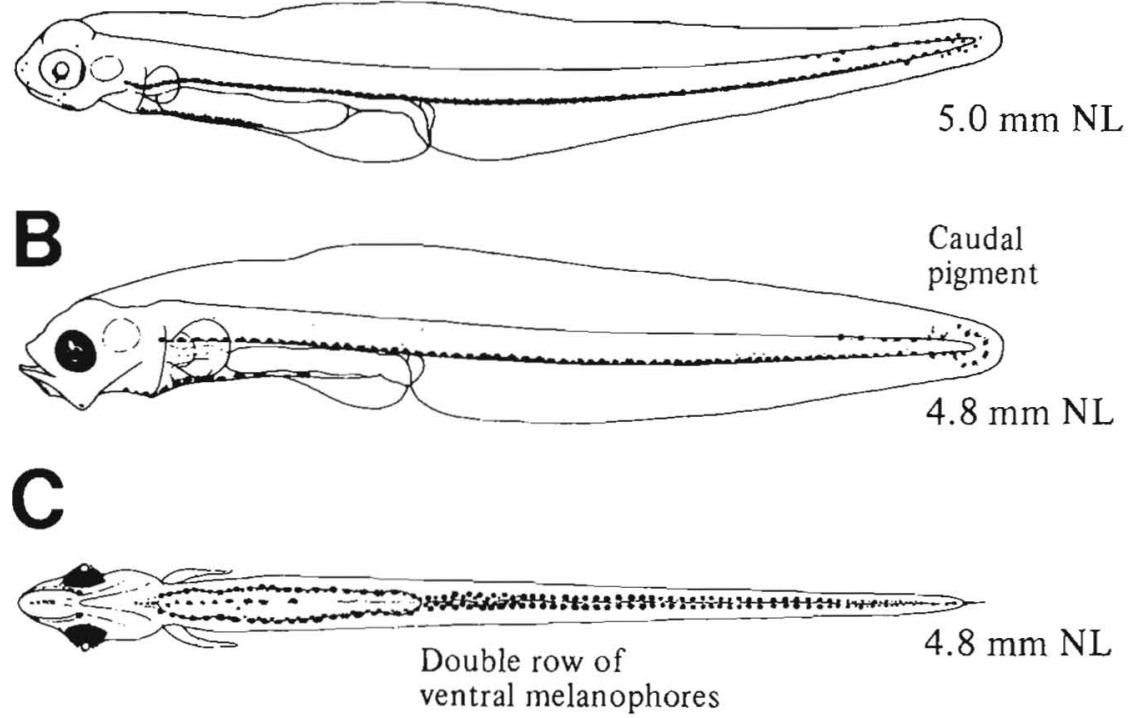

D

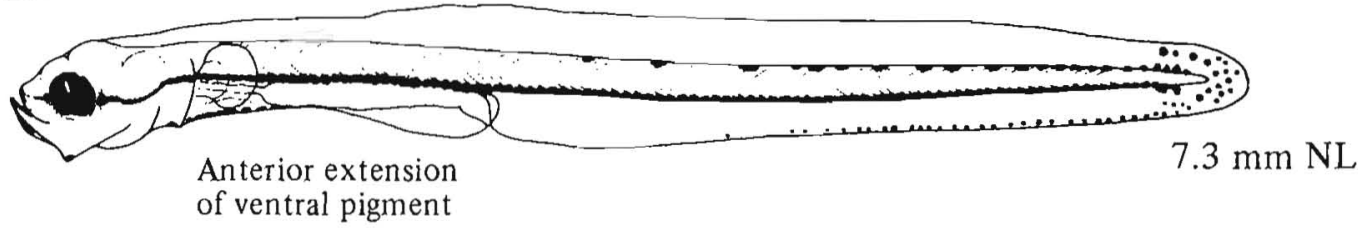

E

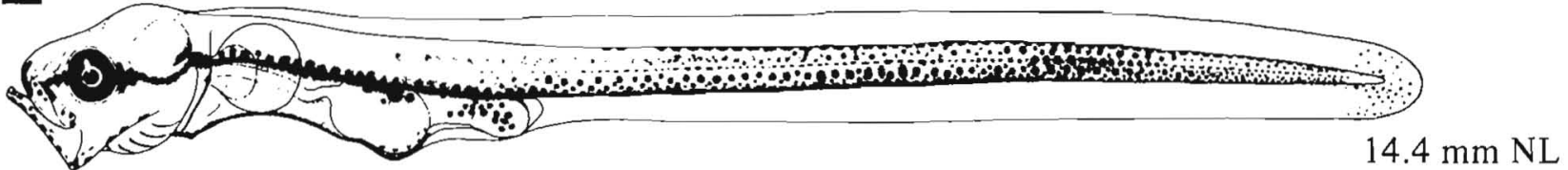



Fishes of this group are found in tropical and temperate waters, freshwater, and at abyssal depths. The families Aphyonidae and Bythitidae, which form the suborder, are represented in the Northeast Pacific by four species within four genera. Only larvae of Brosmophycis marginata are known. All bythitoid males possess an intromittent organ, some with pseudoclaspers.

\begin{tabular}{|c|c|c|c|c|c|c|c|c|c|}
\hline \multirow[b]{4}{*}{ Taxon } & \multicolumn{8}{|c|}{$\begin{array}{c}\text { Table } 26 \\
\text { Meristic characters of suborder Bythitoidei. }\end{array}$} & \multirow[b]{4}{*}{ Branchiostegal } \\
\hline & \multirow[b]{3}{*}{ Distribution } & \multicolumn{2}{|c|}{ Vertebrae } & \multicolumn{4}{|c|}{ Fins } & Gill rakers & \\
\hline & & \multirow{2}{*}{$\begin{array}{l}\text { Precaudal } \\
\text { (Total) }\end{array}$} & \multirow{2}{*}{ Caudal } & \multirow[b]{2}{*}{ Dorsal } & & \multirow{2}{*}{ Pectoral } & \multirow[b]{2}{*}{ Pelvic } & \multirow{2}{*}{$\begin{array}{l}\text { Upper Lower } \\
\text { (Total) }\end{array}$} & \\
\hline & & & & & Anal & & & & \\
\hline \multicolumn{10}{|l|}{ Aphyonidae } \\
\hline Barathronus pacificus & N. Calif. - Oregon & $37-38$ & $46-51$ & $71-75$ & $62-69$ & $25-26$ & $\mathbf{I}, 1$ & $26-28$ & \\
\hline Sciadonus pedicellaris & SSC - Oregon & $43-47$ & $36-39$ & $91-93$ & $42-47$ & $11-14$ & $\mathrm{I}, 1$ & $(14-15)$ & $9-10$ \\
\hline \multicolumn{10}{|l|}{ Bythitidae } \\
\hline Brosmophycis marginata & SSC - SE Alaska & $16-17$ & $47-49$ & $99-110$ & $72-81$ & $20-26$ & $\mathrm{I}, 2$ & & 7 \\
\hline Cataetyx nubrirostris & S. Calif. - Oregon & \multicolumn{2}{|l|}{$(60-62)$} & $102-109$ & $76-82$ & & $\mathrm{I}, 1$ & 3 & 8 \\
\hline
\end{tabular}




\section{MERISTICS}

\begin{tabular}{|c|c|}
\hline Vertebrae & $\begin{array}{l}\text { Total: } 63-64-65 \\
\text { Precaudal: } 16-16-17 \\
\text { Caudal: } 47-48-49\end{array}$ \\
\hline $\begin{array}{l}\text { Branchiostegal rays } \\
\text { Caudal fin }\end{array}$ & $7-7-7$ \\
\hline Pelvic fin & $\begin{array}{l}\text { Jugular } \\
S: 1-1-1 \quad \text { R: } 2-2-2\end{array}$ \\
\hline $\begin{array}{l}\text { Dorsal fin } \\
\text { Pectoral fin } \\
\text { Anal fin } \\
\text { Gill rakers }\end{array}$ & $\begin{array}{l}\text { R: } 99-101-110 \\
\text { R: } 20-X-26 \\
\text { R: } 72-75-81 \\
\text { U: X-X-X L: X-X-X }\end{array}$ \\
\hline \multicolumn{2}{|l|}{ LIFE HISTORY } \\
\hline Range & $\begin{array}{l}\text { South of southern California to } \\
\text { SE Alaska, } 55-59^{\circ} \mathrm{N}\end{array}$ \\
\hline Ecology & Epi- and mesobenthal, 3-256 m \\
\hline ELH pattern & $\begin{array}{l}\text { Probably ovoviviparous, pelagic } \\
\text { larvae }\end{array}$ \\
\hline Spawning & $\begin{array}{l}\text { Season: Spring } \\
\text { Area: } \\
\text { Mode: } \\
\text { Migration: }\end{array}$ \\
\hline $\begin{array}{l}\text { Fecundity } \\
\text { Age at first maturity } \\
\text { Longevity }\end{array}$ & Range/function: $12,000-30,000^{\mathrm{a}}$ \\
\hline
\end{tabular}

\section{EARLY LIFE HISTORY DESCRIPTION}

\section{EGGS}

Diameter

No. of oil globules

Oil globule diameter

Yolk

Envelope

Hatch size

$9.0 \mathrm{~mm} \mathrm{NL}$

Incubation time/temp.

Pigment

Diagnostic characters

\section{LARVAE}

Preanal length $\quad<50 \%$ SL

Length at flexion $\quad>13.5-18.0 \mathrm{~mm} \mathrm{SL}$

Length at transformation

Sequence of fin development

Pigment

- Newly hatched larvae have pigment on lower jaw

- Alternating dorsal/ventral spots

- Above/below anterior portion of tail tip (more pronounced in specimens $<12.5 \mathrm{~mm} \mathrm{SL}$ )

- Swimbladder and on gut

Diagnostic characters

- Pigment pattern: Dorsal/ventral spots 

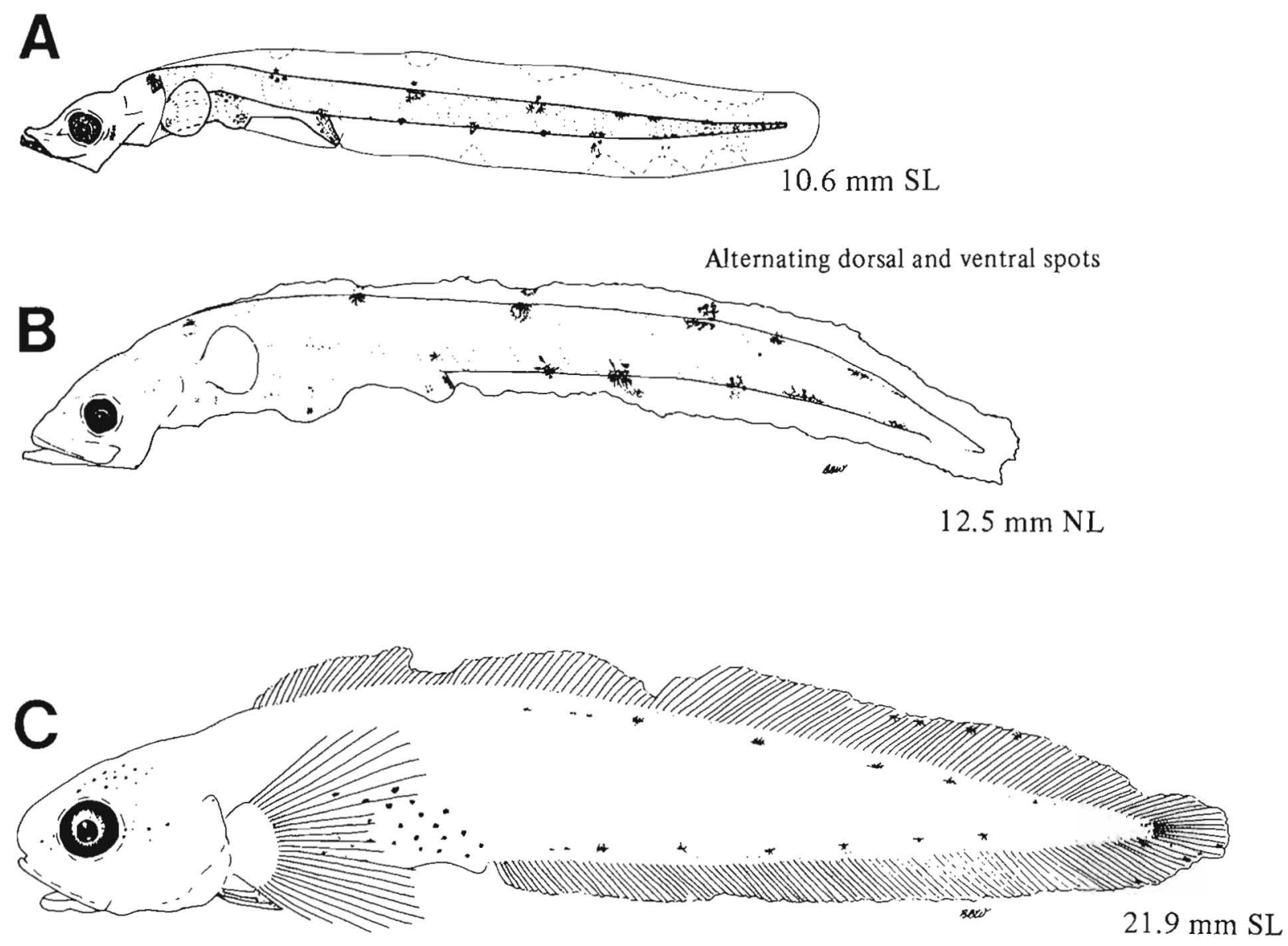

(Unidentified bythitid)

Figure A, NWAFC original (B. Vinter); B-C, Gordon et al. 1984 (C, North Atlantic specimen). 



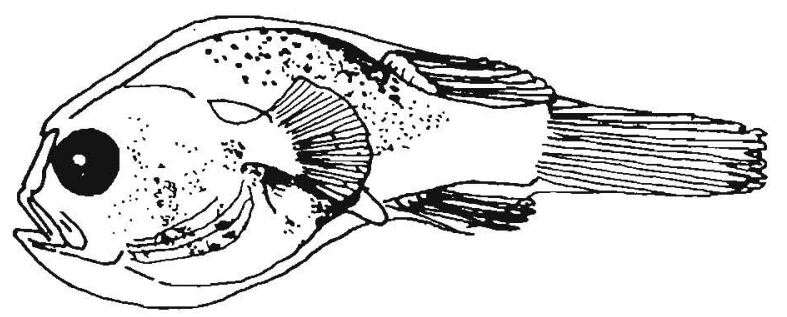

\section{Lophiiformes}

The order Lophiiformes includes some of the most unusual fishes known. Adults exhibit several forms: Dorsoventrally flattened and ventrally compressed forms found in shallow-water benthic habitat, and globose, flabby-bodied forms found at meso- and bathypelagic depths. Worldwide there are 18 families, 63 genera, and 262 species (Pietsch 1984). All families are characterized by having a luring apparatus (illicium) bearing a terminal bait (esca) originating from three modified anterior dorsal spines and a narrow tube-like gill opening located near the pectoral fin. Extreme sexual dimorphism is common among the fishes within the suborder Ceratioidei. Males are one-third the size of females or smaller and sometimes are permanently attached (parasitic) to them. Eggs of most lophiiforms are spawned in gelatinous "veils" which may be up to $1.5 \mathrm{~m}$ wide and $12 \mathrm{~m}$ long (Pietsch 1984). Several species have eggs attached to the adult (Pietsch and Grobecker 1987). Larvae are pelagic and many have large heads and an envelope of highly inflated skin. Neither eggs nor larvae have been collected in the study area.

Families in study area: Ceratiidae

Oneirodidae 
The suborder Ceratioidei is represented within the study area by members of the families Oneirodidae and Ceratiidae. Ceratioids differ from other members of the Lophiiformes in being meso- and bathypelagic, lacking pelvic fins (except larval Caulophrynidae), and in having extreme sexual dimorphism.

\section{Oneirodidae}

Found worldwide, oneirodids (dreamers) are represented by five species within three genera (Oneirodes, Chaenophryne, and Bertella) in the Northeast Pacific. Reproduction is accomplished by facultative or non-parasitic attachment of the male onto the body of the female (Pietsch 1976). Eggs and larvae are pelagic. Eggs are probably released in gelatinous veils which might break up in plankton nets. Eggs are presumably small, generally $0.5-0.8 \mathrm{~mm}$, and hatching occurs between 2.5 and $3.5 \mathrm{~mm}$ SL (Bertelsen 1984). Larvae have a transparent envelope of gelatin, under colorless skin, which may serve as an aid to flotation (Idyll 1964, Pietsch 1984).

According to Bertelsen (1984), larval characters for oneirodids include the following:

- Presence in larval males of a rudiment of illical bone

- Moderately elongate body shape (body depth up to $80-90 \%$ in most ceratioids but less pronounced in oneirodids)

- Larvae surrounded by inflated transparent skin (similar to other ceratioids)

- Head length usually about $45 \%$ SL

- Pigment: Ceratioids possess four main pigment areas (peritoneal, dorsal, caudal, and opercular). In oneirodids, the opercular pigment is dense and occurs in different patterns

At metamorphosis (usually between 8 and $10 \mathrm{~mm} \mathrm{SL}$ ), larvae descend into deeper waters.

\section{Ceratiidae}

Recently, two ceratiids (seadevils) have been identified from the Bering Sea, Ceratias holboelli and Ceratias sp. (Pietsch 1986). Larval ceratiids are distinguished from other ceratioid families in having the following combination of characters (Pietsch 1986):

- Body "hump-backed," mouth subvertical

- Female with caruncles on dorsal surface of trunk

- 4-5 dorsal fin rays, 4 anal fin rays

- Pectoral fins not reaching beyond dorsal and anal fins

- Pelvic fins absent 


\begin{tabular}{|c|c|c|c|c|c|c|c|}
\hline \multicolumn{8}{|c|}{$\begin{array}{c}\text { Table } 27 \\
\text { Meristic characters of Ceratioidei. }\end{array}$} \\
\hline \multirow[b]{3}{*}{ Taxon } & \multirow[b]{3}{*}{ Distribution } & \multicolumn{2}{|c|}{ Vertebrae } & \multirow{2}{*}{\multicolumn{3}{|c|}{ Fins }} & \multirow[b]{3}{*}{ Branchiostegals } \\
\hline & & \multirow{2}{*}{ Precaudal } & \multirow{2}{*}{ Caudal } & & & & \\
\hline & & & & Dorsal & Anal & Pectoral & \\
\hline \multicolumn{8}{|l|}{ Ceratiidae } \\
\hline Ceratias holboelli & Bering Sea & & & $4-5$ & 4 & & \\
\hline Ceratias sp. & Bering Sea & & & $4-5$ & 4 & & \\
\hline \multicolumn{8}{|l|}{ Oneirodidae } \\
\hline Bentella idiomorpha & SSC - Gulf of Alaska & 5 & 15 & $5-6$ & $4-5$ & $17-21$ & 6 \\
\hline Chaenophryne longiceps & SSC - Oregon & \multicolumn{2}{|c|}{ (21) } & $6-7$ & $5-6$ & $17-22$ & 6 \\
\hline Chaenophryne melanorhabdus & SSC - Brit. Col. & 9 & 10 & $6-8$ & $5-6$ & $16-17$ & 6 \\
\hline Oneirodes bulbosus & Oregon - Bering Sea & 4 & 16 & $6-7$ & 4 & $15-18$ & 6 \\
\hline Oneirodes thompsoni & N. Calif. - Bering Sea & 4 & 16 & $5-6$ & 4 & $14-17$ & 6 \\
\hline
\end{tabular}




\section{MERISTICS}

\begin{tabular}{|c|c|}
\hline Vertebrae & $\begin{array}{l}\text { Total: } 21-21-21 \\
\text { Precaudal: } \mathrm{X}-\mathrm{X}-\mathrm{X} \\
\text { Caudal: } \mathrm{X}-\mathrm{X}-\mathrm{X}\end{array}$ \\
\hline $\begin{array}{l}\text { Branchiostegal rays } \\
\text { Caudal fin }\end{array}$ & $6-6-6$ \\
\hline Pelvic fin & Absent \\
\hline Dorsal fin & R: $6-7-7$ \\
\hline Pectoral fin & R: $17-19-22$ \\
\hline Anal fin & R: $5-5-6$ \\
\hline Gill rakers & $\mathrm{U}: \mathrm{X}-\mathrm{X}-\mathrm{X}$ \\
\hline
\end{tabular}

LIFE HISTORY

\begin{tabular}{ll}
\hline Range & $\begin{array}{c}\text { South of southern California to } \\
\text { Oregon, } 42-46^{\circ} \mathrm{N}\end{array}$ \\
$\begin{array}{l}\text { Mesopelagic, } 200-1000 \mathrm{~m} \\
\text { ELH pattern }\end{array}$ & $\begin{array}{l}\text { Oviparous, pelagic eggs, } \\
\text { pelagic larvae }\end{array}$ \\
Spawning & $\begin{array}{l}\text { Season: } \\
\text { Area: } \\
\text { Mode: Males attach to body of } \\
\text { female }\end{array}$ \\
& $\begin{array}{l}\text { Migration: } \\
\text { Range/function: }\end{array}$ \\
$\begin{array}{l}\text { Fecundity } \\
\text { Age at first maturity } \\
\text { Longevity }\end{array}$ &
\end{tabular}

\footnotetext{
${ }^{a}$ Pietsch 1976

${ }^{b}$ Because of the possibility of involvement of other species in the description, and geographic variation, specimens in our area could differ.
}

Ref: Bertelsen 1984.

\section{EARLY LIFE HISTORY DESCRIPTION}

\section{EGGS}

Diameter

No. of oil globules

Oil globule diameter

Yolk

Envelope

Hatch size

Incubation time/temp.

Pigment

Diagnostic characters

\section{LARVAE $^{\text {b }}$}

Preanal length

Length at flexion

Length at transformation

Sequence of fin

development

Pigment

- Pigment appears in all four pigment areas -Peritoneal

-Anterior body, begins as a small dorsal patch

-Opercular

-Caudal, initially a few spots

Diagnostic characters

- Generally more lightly pigmented than Oneirodes spp. 

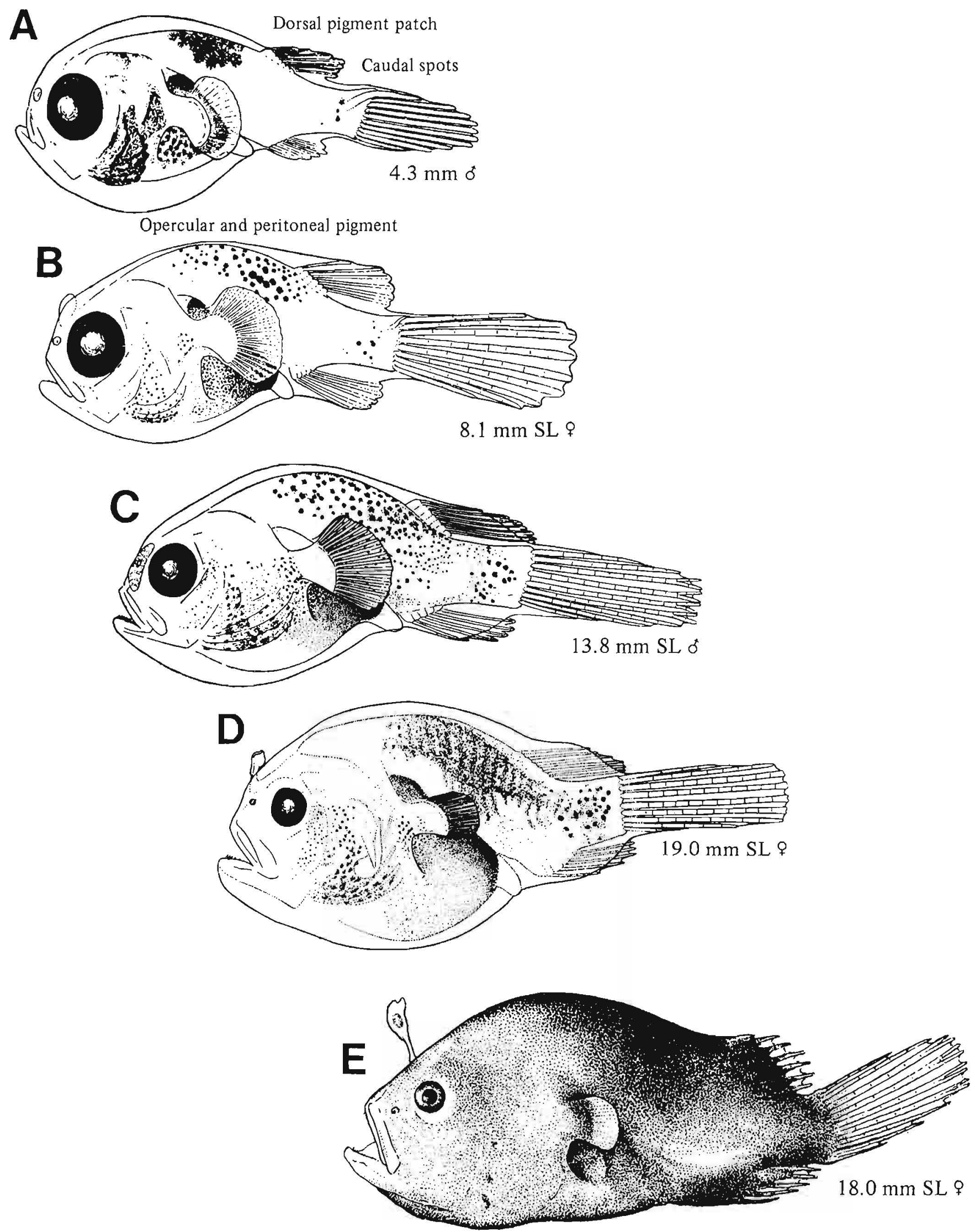

Figures A-E, Bertelsen 1951 (North Atlantic specimens). 


\section{MERISTICS}

\begin{tabular}{|c|c|}
\hline Vertebrae & $\begin{array}{l}\text { Total: } 20-20-20 \\
\text { Precaudal: } 4-4-4 \\
\text { Caudal: } 16-16-16\end{array}$ \\
\hline $\begin{array}{l}\text { Branchiostegal rays } \\
\text { Caudal fin }\end{array}$ & $6-6-6$ \\
\hline Pelvic fin & Absent \\
\hline Dorsal fin & $\mathrm{R}: 6-\mathrm{X}-7$ \\
\hline Pectoral fin & R: $15-16-18$ \\
\hline Anal fin & $R: 4-4-4$ \\
\hline Gill rakers & U: X-X-X \\
\hline
\end{tabular}

\section{LIFE HISTORY}

$\begin{array}{ll}\text { Range } & \text { Oregon, } 42-46^{\circ} \mathrm{N}, \text { to } \\ & \text { Bering Sea, } 54-66^{\circ} \mathrm{N} \\ \text { Ecology } & \text { Mesopelagic, } 200-1000 \mathrm{~m} \\ \text { ELH pattern } & \text { Oviparous, pelagic eggs, } \\ & \text { pelagic larvae } \\ \text { Spawning } & \text { Season: } \\ & \text { Area: } \\ & \text { Mode: Males attach to body of } \\ & \text { female } \\ & \text { Migration: } \\ \text { Fecundity } & \text { Range/function: } \\ \text { Age at first maturity } & \\ \text { Longevity } & \end{array}$

\section{EARLY LIFE HISTORY DESCRIPTION}

\section{EGGS}

Diameter

No. of oil globules

Oil globule diameter

Yolk

Envelope

Hatch size

Incubation time/temp.

Pigment

Diagnostic characters

\section{LARVAE \\ Preanal length \\ Length at flexion \\ Length at transformation \\ Sequence of fin development \\ Pigment - Species group \\ - Pigment in three out of the four main pigment areas -Peritoneal \\ - Anterior body \\ -Opercular \\ - Caudal pigment is light and appears late in development}

Diagnostic characters

\footnotetext{
a Pietsch 1976

${ }^{b}$ Figures of Oneirodes eschrichti group (Bertelsen 195I) are presented for comparison only. Because of the possibility of involvement of other species in the description, and geographic variation, specimens of Oneirodes in our area could differ.

Ref: Bertelsen 1984.
} 

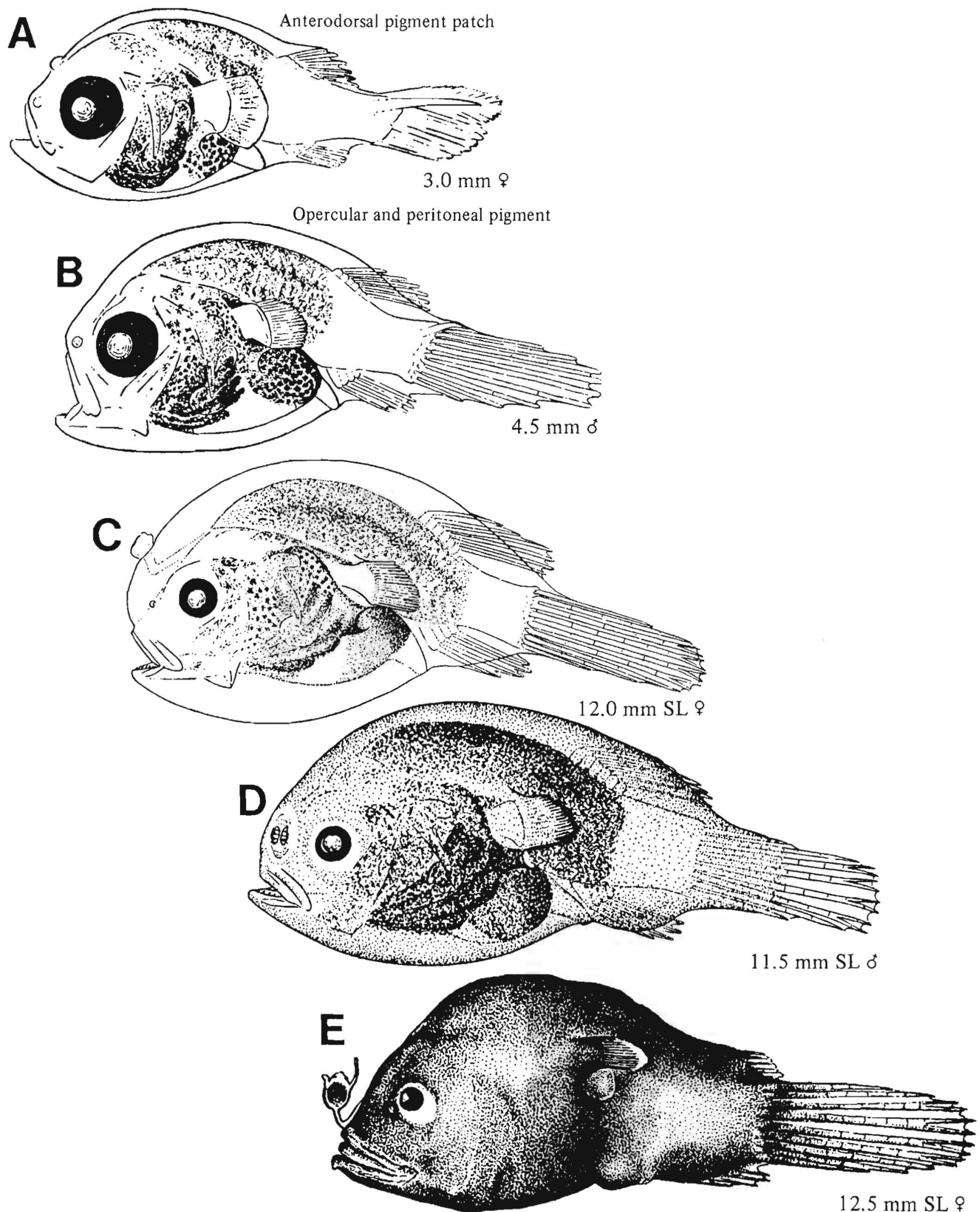

Figures A-E, Bertelsen 1951 (North Atlantic specimens from the O. eschrichti group). 



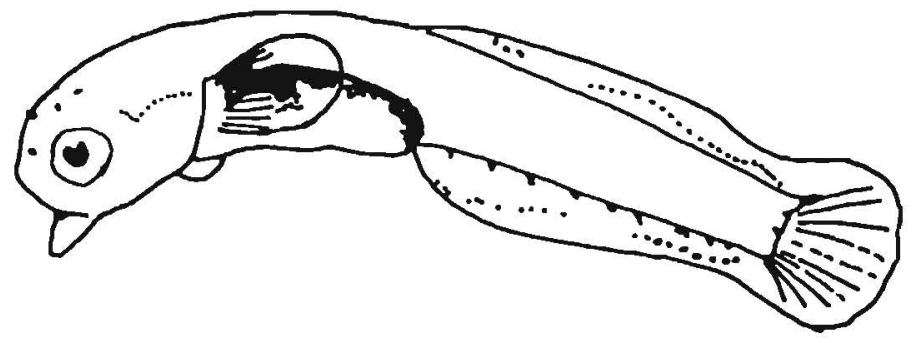

\section{Gobiesociformes}

The gobiesociforms (clingfishes) are mostly small, inshore bottom-dwelling species occurring in tropical and temperate seas. Noted primarily for having the pelvic fins modified into a sucking disc, the order has 2 families, 36 genera, and 114 species (J. Nelson 1984). All but one genus and four species are members of Gobiesocidae. Eggs are demersal, attached to substrate or kelp, ovate to ellipsoidal, and 0.7-1.9 mm. Oil globules (1-100) coalesce to one during development (Allen 1984). Larvae are well developed at hatching and may have a fully formed pelvic disc at this time (Marliave 1975a). Most are heavily pigmented and have long guts (50-70\% SL) (Allen 1984).

Family in study area: Gobiesocidae 


\section{MERISTICS}

$\begin{array}{ll}\text { Vertebrae } & \text { Total: } 32-33-34 \\ & \text { Precaudal: } 12-14-14 \\ & \text { Caudal: } 19-19-20 \\ \text { Branchiostegal rays } & 6-6-6 \\ \text { Caudal fin } & \text { Total rays }=11-13 \\ \text { Pelvic fin } & \text { Disc } \\ & \text { S: } 1-1-1 \quad \text { R: } 4-4-4 \\ \text { Dorsal fin } & \text { R: } 13-16-16 \\ \text { Pectoral fin } & \text { R: } 21-22-23 \\ \text { Anal fin } & \text { R: } 13-14-15 \\ \text { Gill rakers } & \text { U: X-X-X L: X-X-X } \\ & \\ \text { LIFE HISTORY } & \end{array}$

Range

Ecology

ELH pattern

Spawning

Fecundity

Age at first maturity Longevity
South of southern California to SE Alaska, $55-59^{\circ} \mathrm{N}$

Intertidal, nearshore, 0-8 m Oviparous; demersal, attached, guarded eggs; pelagic larvae Season: Winter-spring (British Columbia) ${ }^{\mathrm{a}}$

Area: Demersal, on underside of rocks, usually intertidal ${ }^{a}$

Mode: Polygamous males guard eggs (laid in monolayer) ${ }^{\mathrm{a}}$

Migration:

Range/function: 194-382 per female ${ }^{b}$

\section{EARLY LIFE HISTORY DESCRIPTION}

\section{EGGS}

Diameter

$1.68-1.92 \mathrm{~mm}(1.78)$

No. of oil globules

Oil globule diameter

Yolk

Envelope

Hatch size

Incubation time/temp. $\quad 30 \mathrm{~d} / 9-11^{\circ} \mathrm{C}$

Pigment

Bilobed in embryo and yolksac larva

$5.7 \mathrm{~mm} \mathrm{SL}$, yolksac present until $6.2 \mathrm{~mm} \mathrm{SL}$

Diagnostic characters

- Adhesive, hemispherical, flat at point of attachment

\section{LARVAE}

Head length

21-35\% SL; gut length (preanal minus head length) $25 \% \mathrm{SL}$

Length at flexion $7 \mathrm{~mm} \mathrm{SL}$

Length at transformation

Sequence of fin development

$10-13 \mathrm{~mm} \mathrm{SL}$

Pectoral fin buds form at $7.0 \mathrm{~mm} \mathrm{SL}$; pelvic disc is formed by $7.3 \mathrm{~mm} \mathrm{SL}$ and fully functioning by $9.1 \mathrm{~mm} \mathrm{SL}$

Pigment

- Dorsally on gut

- With development, on snout and dorsal region of head

Diagnostic characters

- Large melanophores on dorsal surface of gut, lack of pigment on lateral surface

- Forms pelvic disc

- Number of myomeres (32-34) 

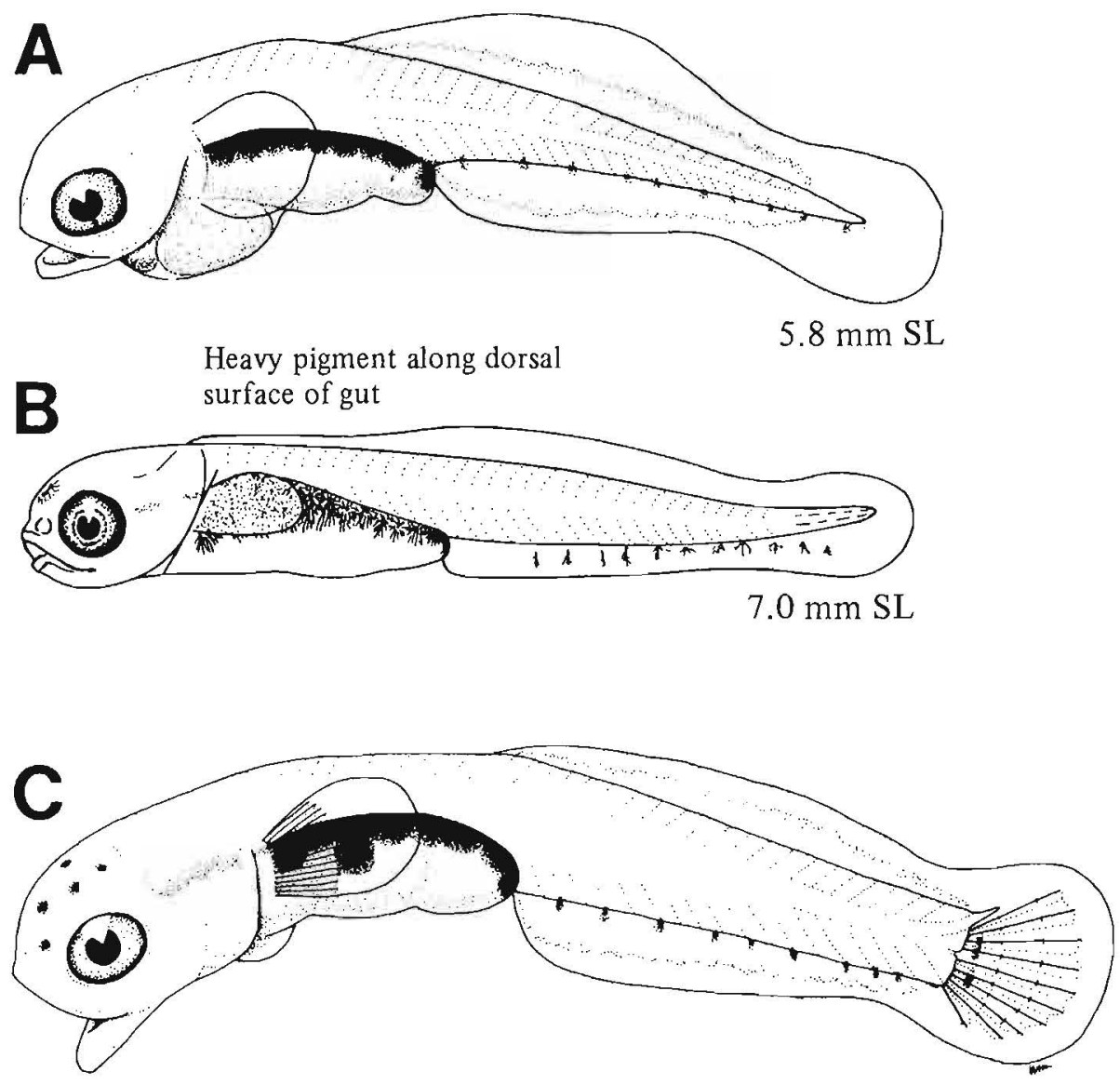

$7.3 \mathrm{~mm} \mathrm{SL}$

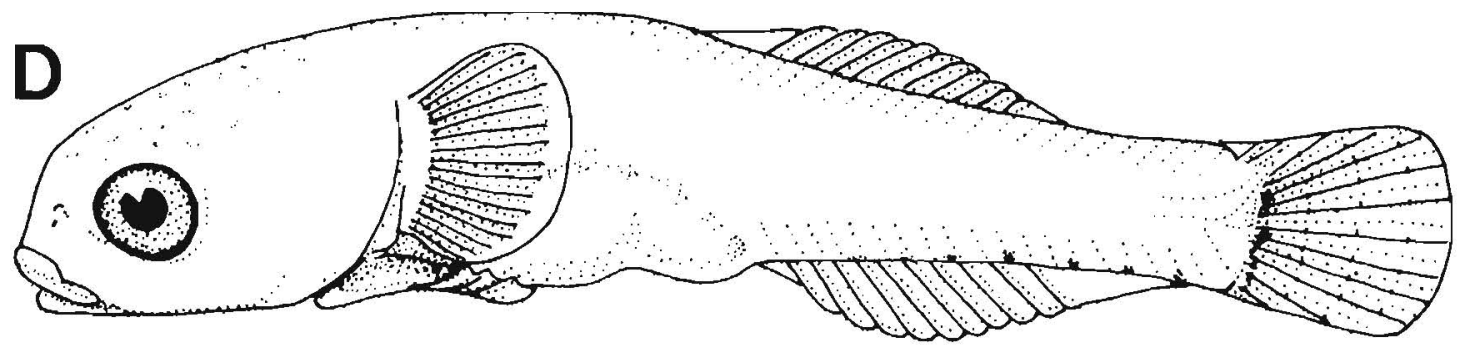

$9.8 \mathrm{~mm} \mathrm{SL}$

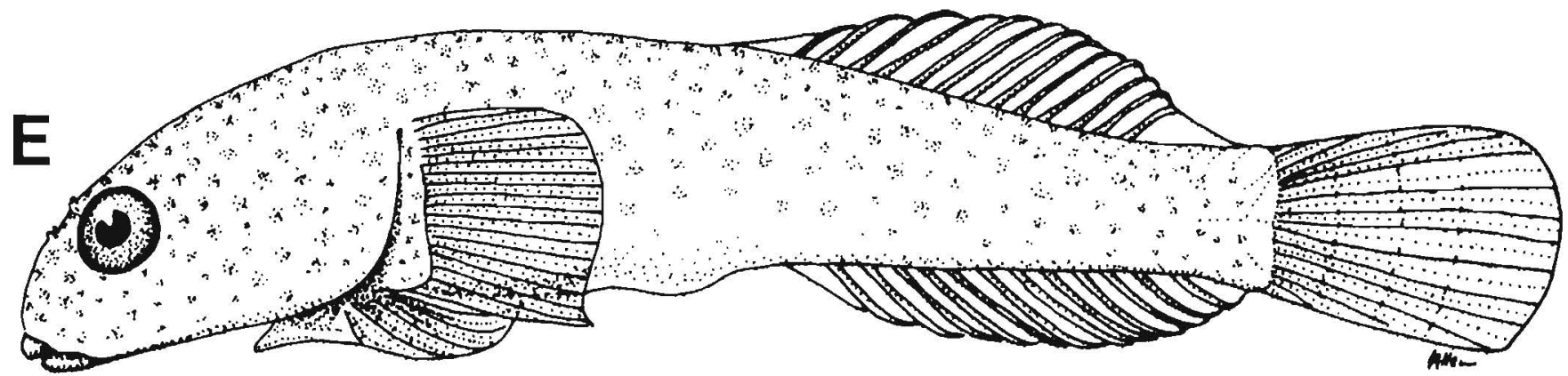

Figures A, C-E, Allen and Ilg 1983; B, NWAFC original (B. Vinter). 


\section{MERISTICS}

\begin{tabular}{ll}
\hline Vertebrae & Total: $35-\mathrm{X}-36$ \\
& $\begin{array}{l}\text { Precaudal: X-X-X } \\
\text { Caudal: X-X-X }\end{array}$ \\
Branchiostegal rays & $5-\mathrm{X}-7$ \\
Caudal fin & Total rays $=8$ \\
Pelvic fin & Disc \\
& S: $1-1-1 \quad$ R: $4-4-4$ \\
Dorsal fin & R: $6-7-8$ \\
Pectoral fin & R: $14-15-17$ \\
Anal fin & R: $6-7-8$ \\
Gill rakers & U: X-X-X L: X-X-X \\
& \\
& \\
LIFE HISTORY & \\
\hline Range & South of southern California to \\
& Brit. Col., $48^{\circ} 30^{\prime}-55^{\circ} \mathrm{N}$ \\
Ecology & Intertidal, nearshore \\
ELH pattern & Oviparous; demersal, attached \\
& eggs; pelagic larvae \\
Spawning & Season: \\
& Area: Kelp beds, on blades ${ }^{\text {a }}$ \\
& Mode: \\
Fecundity & Migration: \\
Age at first maturity & Range/function: \\
Longevity &
\end{tabular}

\section{EARLY LIFE HISTORY DESCRIPTION}

\section{EGGS}

Diameter

No. of oil globules

$1.3 \mathrm{~mm}$

Oil globule diameter

Yolk

Envelope

Hatch size

Incubation time/temp.

Pigment

Diagnostic characters

\section{LARVAE \\ Preanal length \\ Length at flexion \\ Length at transformation \\ Sequence of fin development \\ Pigment (based on figure only) \\ - Lateral melanophores (40-50) \\ - No postanal ventral melanophores \\ - No hypural pigment \\ Diagnostic characters \\ - Pelvic disc at hatching \\ - Number of myomeres (35-36)}

Note: Marliave (1975a) reared Rimicola muscarum in the laboratory. Eggs (1.3 $\mathrm{mm}$ in diameter) had a single oil globule, and late-stage embryos developed a pelvic disc. Hatching occurred when embryos reached $4.0 \mathrm{~mm}$ SL. He described them as identical to Gobiesox maeandricus larvae except that they were markedly smaller, without nasal pigment, and with the pelvic disc at hatching. According to Marliave (Vancouver Public Aquarium, P.O. Box 3232, Vancouver, B.C., Canada V6B 3X8, pers. commun., 16 Oct. $1986), R$. muscarum probably lacks a pelagic stage and the specimen illustrated here from Allen (1979) is misidentified.

Allen 1984

Ref: Allen 1979, Marliave 1975a. 


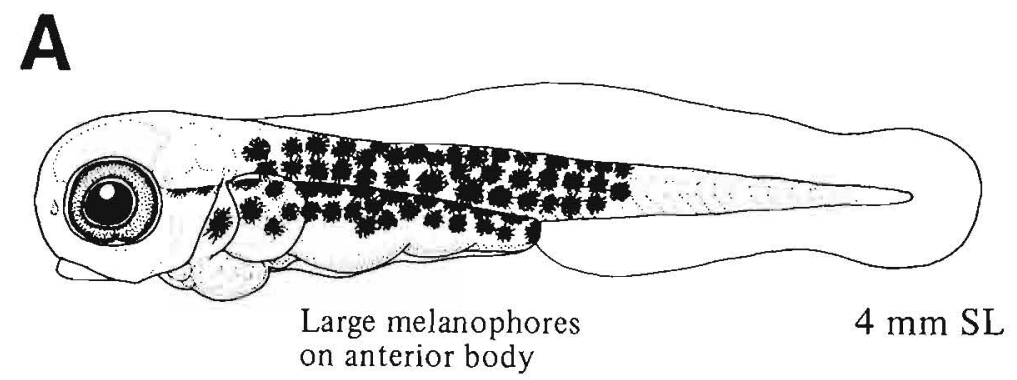

Figure A, Allen 1979. 



\section{Atherinomorpha: Beloniformes Atheriniformes}

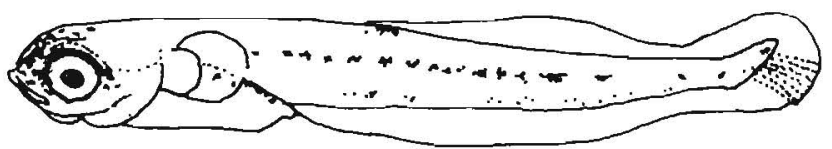

The Beloniformes and Atheriniformes together comprise a group of fishes found in freshwater and marine habitats within tropical and temperate areas. The Beloniformes (not always accorded ordinal status [J. Nelson 1984]), consists of 5 families, 37 genera, and 180 species (Collette et al. 1984a). Marine forms of this order are mostly epipelagic, the best known being exocoetids, or flying fish. There are 6 families (White et al. 1984), 49 genera, and 235 species of atheriniforms (J. Nelson 1984). The two orders belong to the superorder Atherinomorpha and share the development of large demersal eggs with filaments and oil globules that coalesce at the vegetal pole (Collette 1984). Filaments may be short or long, grouped or evenly scattered, and adhesive or non-adhesive. Beloniform larvae are well formed at hatching and many have a preanal finfold and beak-like jaws (Collette et al. 1984a). Atheriniform larvae have direct development (i.e., no specialized larval or juvenile stages), a preanal finfold, and a single row of melanophores on the dorsal midline (White et al. 1984).

Families in study area: Scomberesocidae Atherinidae 


\section{MERISTICS}

\section{Vertebrae}

Total: $62-65-69$

Precaudal: $37-38-40$

Caudal: 24-27-29a

Branchiostegal rays

Caudal fin

Pelvic fin

$14-\mathrm{X}-15$

$\mathrm{X}, 7+8, \mathrm{X}$

Abdominal

R: 6-6-6

Dorsal fin

$\mathrm{R}: 14-\mathrm{X}-18^{\mathrm{a}}$

Pectoral fin

R: $12-13-15$

Anal fin

Gill rakers

$\mathrm{R}: 18-\mathrm{X}-21^{\mathrm{a}}$

$\mathrm{U}: \mathrm{X}-\mathrm{X}-\mathrm{X}$

$\mathrm{L}: \mathrm{X}-\mathrm{X}-\mathrm{X}$

\section{LIFE HISTORY}

$\begin{array}{ll}\text { Range } & \begin{array}{c}\text { South of southern California to } \\ \text { Bering Sea, } 54-66^{\circ} \mathrm{N}\end{array} \\ \text { Ecology } & \text { Epipelagic, 0-200 m } \\ \text { Oviparous, eggs attach to } \\ \text { flotsam (kelp) with adhesive } \\ \text { filaments, pelagic larvae } \\ \text { Season: Winter-fall; }{ }^{\mathrm{b}} \text { peaks } \\ \text { Feb-July }\end{array}$

\section{EARLY LIFE HISTORY DESCRIPTION}

\section{EGGS}

Diameter

No. of oil globules

Oil globule diameter

Yolk

Envelope

Hatch size

Incubation time/temp

$1.58(1.68 \mathrm{~mm}) \times 2.13 \mathrm{~mm}$ None

Homogeneous

Adhesive filaments, 12-15

in polar cluster and

1 lateral

6.0-8.5 mm SL (family)

Pigment

Diagnostic characters

- Adhesive filaments in polar cluster

- Single lateral filament

- Slightly ovoid

\section{LARVAE}

Preanal length

Length at flexion

Length at transformation

Sequence of fin development

Pigment

- Dense body pigment

Diagnostic characters (see Table 3)

- Persistent preanal finfold

- Unpigmented area on ventrolateral region of caudal peduncle

- At 20-40 mm SL, upper and lower jaw are slightly elongate but do not form prominent beak; with growth a slight beak develops

Collette et al. 1984

${ }^{b}$ Fitch and Lavenberg 1971

${ }^{c}$ Wang 1981

Ref: Collette et al. 1984a, Fitch and Lavenberg 1971, Uchida et al. 1958 
B

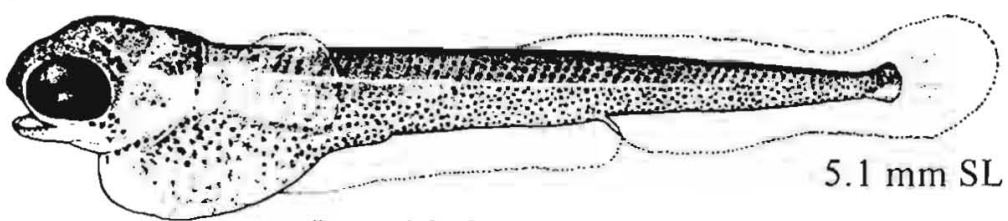

Preanal finfold persists through development

C
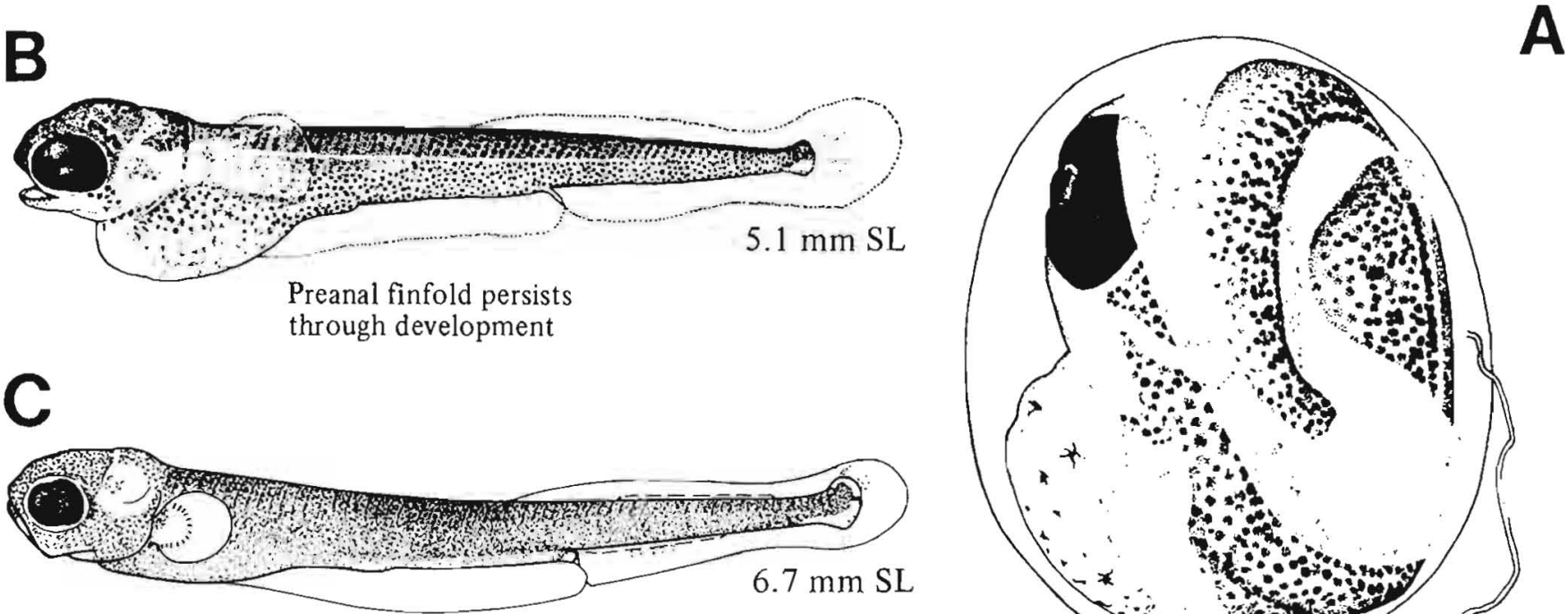

D

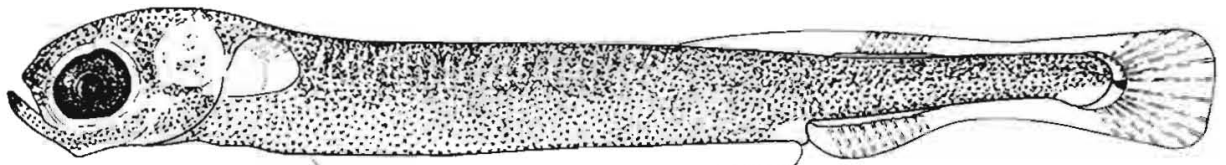

$7.4 \mathrm{~mm} \mathrm{SL}$

$1.7 \times 1.9 \mathrm{~mm}$

E

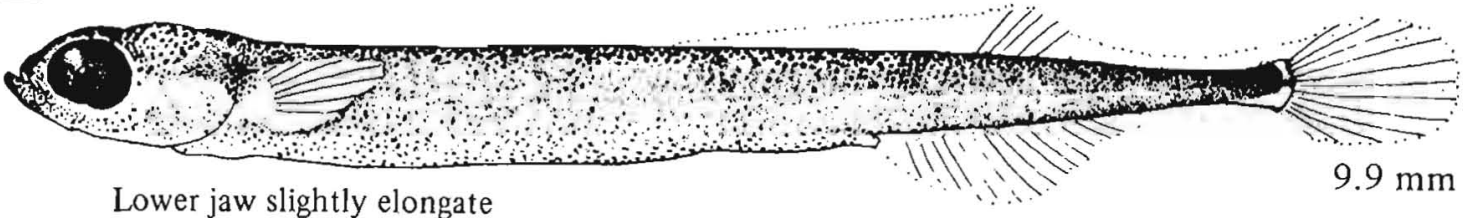

Lower jaw slightly elongate

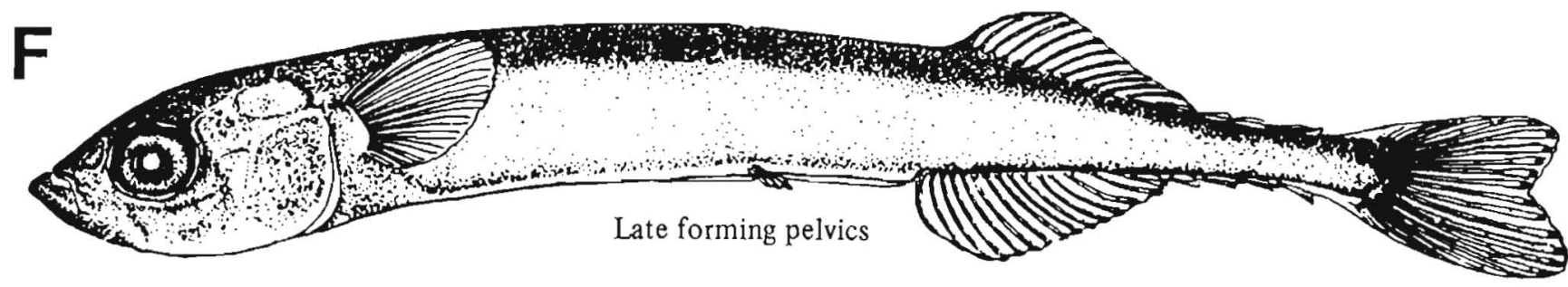

$23.0 \mathrm{~mm} \mathrm{TL}$

Figure A, Matarese and Sandknop 1984; B, Collette et al. 1984a; C-D, NWAFC originals (B. Vinter); E, Moser 1981; F, Uchida et al. 1958. 


\section{MERISTICS ${ }^{a}$}

$\begin{array}{lll}\text { Vertebrae } & \begin{array}{l}\text { Total: } 44-48-52 \\ \text { Precaudal: } 32-34-37\end{array} \\ & \text { Caudal: } 11-13-15 \\ \begin{array}{l}\text { Branchiostegal rays } \\ \text { Caudal fin }\end{array} & 5-X-6 & \\ \begin{array}{lll}\text { Pelvic fin } & & \\ & \text { Abdominal } & \\ \text { Dorsal fin } & \text { S: } 1-1-1 & \text { R: } 5-5-5 \\ \text { Pectoral fin } & \text { S: } 6-X-10 & \text { R: } 8-X-14 \\ \text { Anal fin } & \text { R: } 13-13-13 & \\ \text { Gill rakers } & \text { S: } 1-1-1 & \text { R: } 19-X-25 \\ & \text { U: } 4-X-8 & \text { L: } 21-X-34\end{array}\end{array}$

\section{LIFE HISTORY}

$\begin{array}{ll}\text { Range } & \begin{array}{c}\text { South of southern California to } \\ \text { Brit. Col., } 48^{\circ} 30^{\prime}-55^{\circ} \mathrm{N}\end{array} \\ \text { Ecology } & \text { Nearshore shelf pelagic } \\ \text { ELH pattern } & \text { Oviparous, eggs with adhesive } \\ & \text { filaments, pelagic larvae } \\ \text { Spawning } & \text { Season: May-July } \\ & \text { Area: Mudflats, eelgrass } \\ & \text { Mode: Schools } \\ & \text { Migration: To mudflats in } \\ & \text { sloughs } \\ \text { Fecundity } & \text { Range/function: } \\ \text { Age at first maturity } & 2-3 \mathrm{yr}^{\mathrm{c}} \\ \text { Longevity } & 6-7 \mathrm{yr}^{\mathrm{b}}\end{array}$

\footnotetext{
${ }^{a}$ Meristic information from White et al. (1984) is very different from the information in our database. Due to confusion in the literature over the definition of spines and rays, only total elements are reported by White et al. (1984) and they are as follows: Vertebrae 43-49 Pectoral fin 12-15 First dorsal fin 3-7 Anal fin 9-14 Second dorsal fin 10-14 Gill rakers $14-27$ ${ }^{b}$ Hart 1973

${ }^{\mathrm{c}}$ Fitch and Lavenberg 1975

${ }^{d}$ Additional unpubl. data provided by $W$. Watson, H.J. Walker, and R. Davis (W. Watson, Marine Ecological Consultants, 531 Encinitas Blyd., Suite 110, Encinitas, CA 92024, pers. commun., 3 Nov. 1986).

${ }^{e}$ Wang 1981

'According to White et al. (1984), two egg types are reported for the species, one with 40-78 filaments attached at both ends and the other with about 6 filaments attached only at one end.
}

Ref: Wang 1981, White et al. 1984.

\section{EARLY LIFE HISTORY DESCRIPTION ${ }^{\mathrm{d}}$}

\section{EGGS}

Diameter

No. of oil globules

Oil globule diameter

Yolk

Envelope

Hatch size

Incubation time/temp.

Pigment

- Dorsal and lateral spots on head, few anterior spots on yolksac

Diagnostic characters

- Narrow perivitelline space

\section{LARVAE}

Preanal length

Length at flexion

Length at transformation

Sequence of fin development

Pigment

- Dorsal midline from snout to caudal peduncle

- Mediolateral beginning above gut

- Dorsal and ventral surface of gut

- Ventrally on tail

\section{Diagnostic characters}

Distinguished from Atherinopsis californiensis by

- Presence of melanophores along ventral body midline and ventral gut 


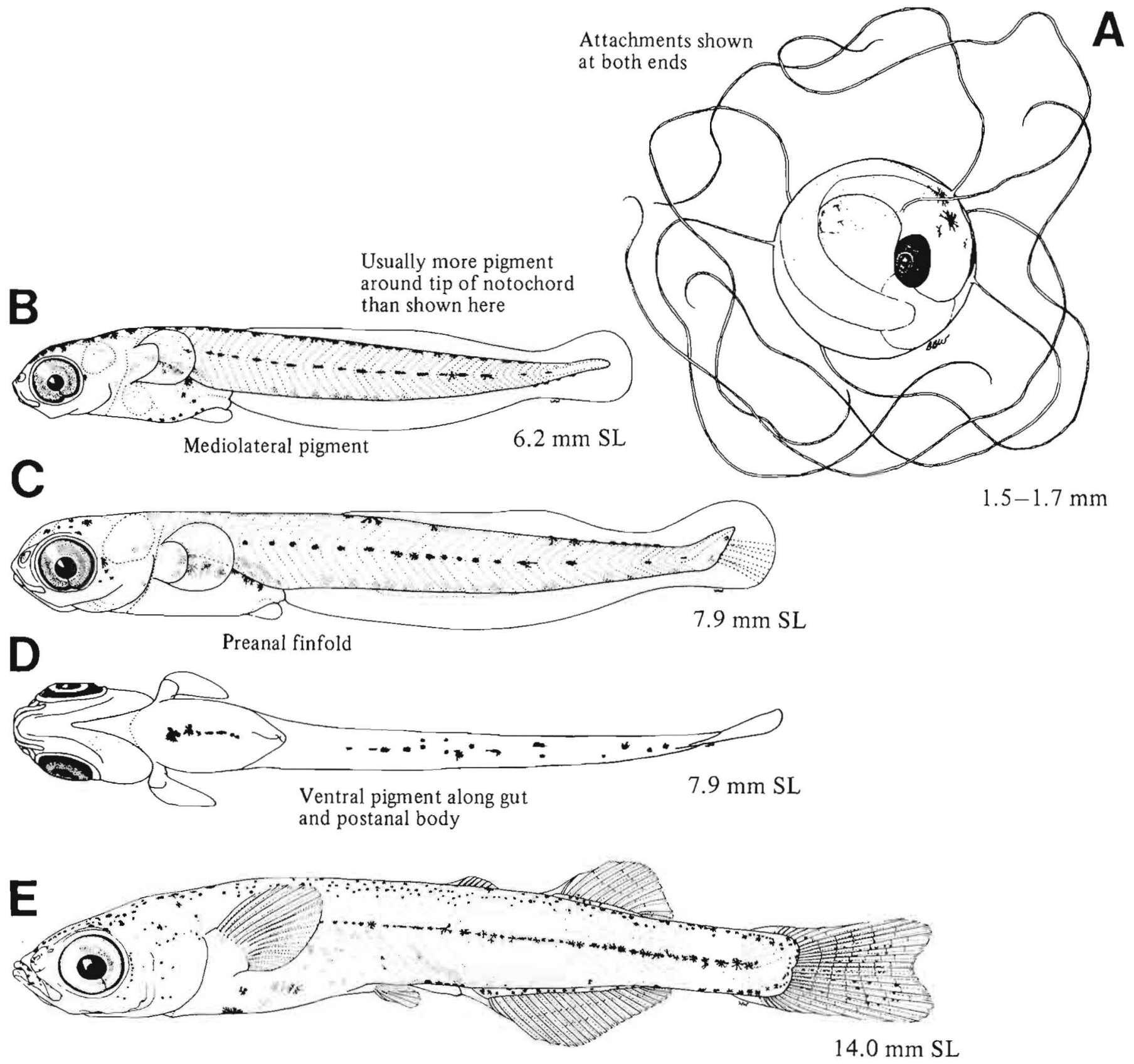

Figure A, White et al. 1984; B-E (D, ventral view), Watson and McGowen, unpubl. 
MERISTICSa

\begin{tabular}{|c|c|c|}
\hline \multirow{3}{*}{$\begin{array}{l}\text { Vertebrae } \\
\text { Branchiostegal rays } \\
\text { Caudal fin } \\
\text { Pelvic fin }\end{array}$} & \multicolumn{2}{|c|}{$\begin{array}{l}\text { Total: } 50-51-52 \\
\text { Precaudal: } 9-12-14 \\
\text { Caudal: } 37-39-41\end{array}$} \\
\hline & $5-X-6$ & \\
\hline & $\begin{array}{l}\text { Abdominal } \\
\text { S: } 1-1-1\end{array}$ & R: 6-6-6 \\
\hline $\begin{array}{l}\text { Dorsal fin } \\
\text { Pectoral fin }\end{array}$ & $\begin{array}{l}\text { S: } 6-X-10 \\
\text { R: } 15-15-15\end{array}$ & $\mathrm{R}: 11-\mathrm{X}-14$ \\
\hline $\begin{array}{l}\text { Anal fin } \\
\text { Gill rakers }\end{array}$ & $\begin{array}{l}\text { S: } 1-1-1 \\
\text { U: } X-X-X\end{array}$ & $\begin{array}{l}\text { R: } 21-X-26 \\
\text { L: X-X-X }\end{array}$ \\
\hline LIFE HISTORY & & \\
\hline Range & \multicolumn{2}{|c|}{$\begin{array}{l}\text { South of southern California to } \\
\text { Oregon, } 42-46^{\circ} \mathrm{N}\end{array}$} \\
\hline Ecology & \multicolumn{2}{|c|}{ Nearshore shelf pelagic } \\
\hline ELH pattern & \multicolumn{2}{|c|}{$\begin{array}{l}\text { Oviparous, eggs with filaments } \\
\text { in a mass or attached to } \\
\text { substrate, pelagic larvae }\end{array}$} \\
\hline Spawning & \multicolumn{2}{|c|}{$\begin{array}{l}\text { Season: Fall-spring }{ }^{b} \\
\text { Area: On algae, eelgrass }{ }^{b} \\
\text { Mode: } \\
\text { Migration: }\end{array}$} \\
\hline $\begin{array}{l}\text { Fecundity } \\
\text { Age at first maturity } \\
\text { Longevity }\end{array}$ & \multicolumn{2}{|c|}{ Range/function: } \\
\hline
\end{tabular}

\footnotetext{
${ }^{a}$ Meristics from White et al. (1984) are as follows:

Vertebrae $46-53$

First dorsal fin $\quad 4-9$

Second dorsal fin $10-15$

Pectoral fin 14-17

Anal fin 20-29

Gill rakers $\quad 18-44$

${ }^{\circ}$ Wang 1981

${ }^{c}$ Additional unpubl. data provided by W. Watson, H.J. Walker, and R. Davis (W. Watson, Marine Ecological Consultants, 531 Encinitas Blvd., Suite 110, Encinitas, CA 92024 , pers. commun., 3 Nov. 1986).
}

Ref: Wang 1981, White et al. 1984.

\section{EARLY LIFE HISTORY DESCRIPTION ${ }^{\mathrm{C}}$}

\section{EGGS}

Diameter

No. of oil globules

Oil globule diameter

Yolk

Envelope

Hatch size

Incubation time/temp.

Pigment

- Dorsal spots along nape

Diagnostic characters

- Narrow perivitelline space

- 1-2 cm filaments

\section{LARVAE}

\section{Preanal length \\ Length at flexion \\ Length at transformation \\ Sequence of fin development}

\section{$1.9-2.5 \mathrm{~mm}^{b}$}

Many (23-44) which coalesce to one or few

0.4-0.6 mm (consolidated)

Yellow-orange, granular

13-19 filaments scattered on surface

7.5-8.6 mm SL; yolk absorbed by $10 \mathrm{~mm} \mathrm{SL}$

$17-19 \mathrm{~d} / 12-16^{\circ} \mathrm{C}$

\section{Pigment}

- Dorsal midline from snout to caudal peduncle, sometimes with a break in nape area

- Mediolateral usually begins posterior to gut during preflexion stage

\section{Diagnostic characters}

Distinguished from Atherinops affinis by

- First 5-6 postanal myomeres always lack ventral pigment through the larval and early juvenile stages

- Lack of ventral midline melanophores until midflexion

- Mediolateral pigment originates further posterior 


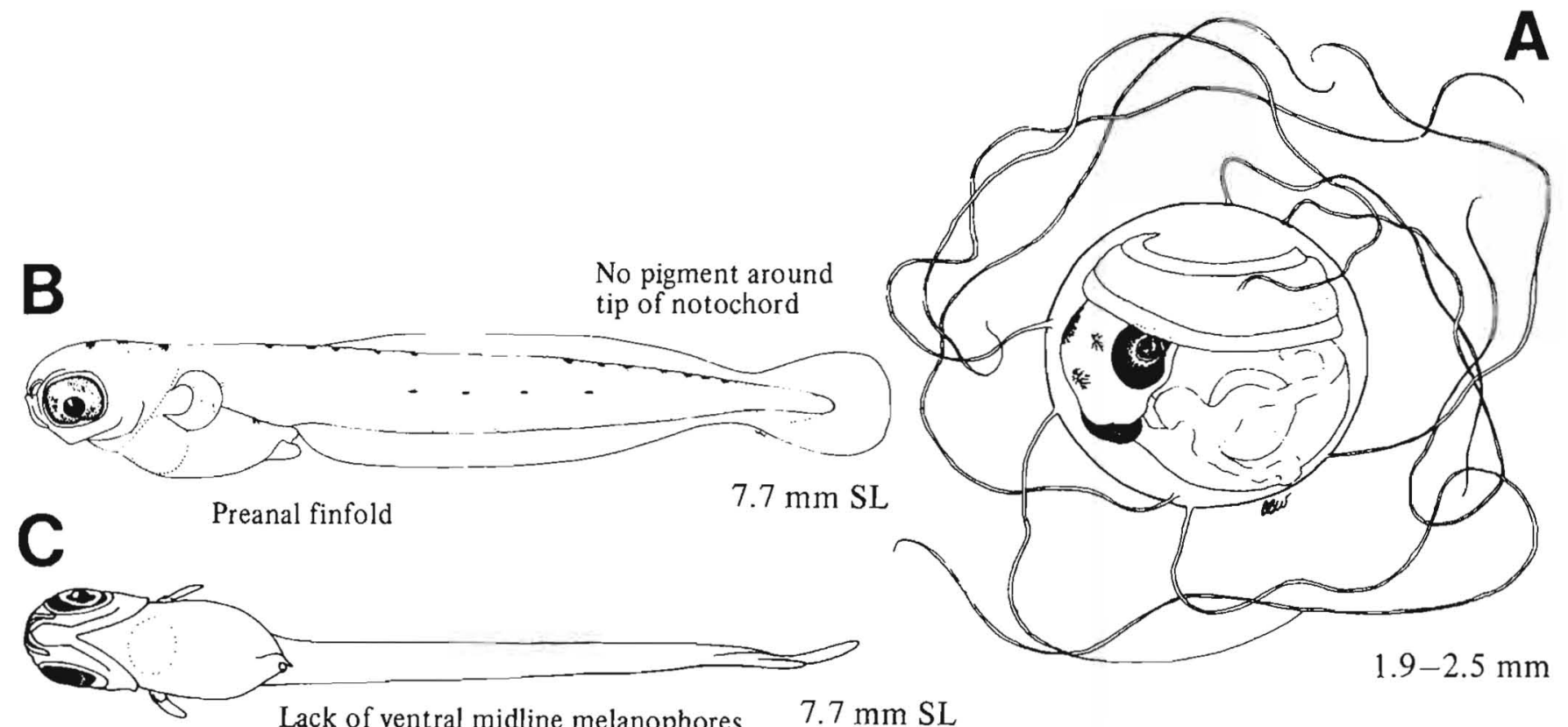

D

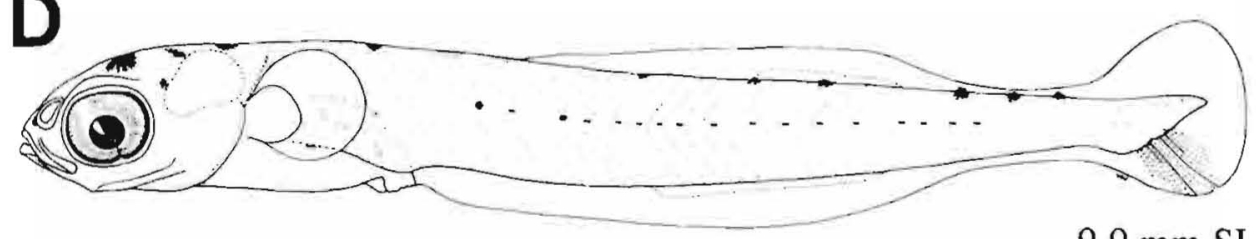

$9.9 \mathrm{~mm} \mathrm{SL}$

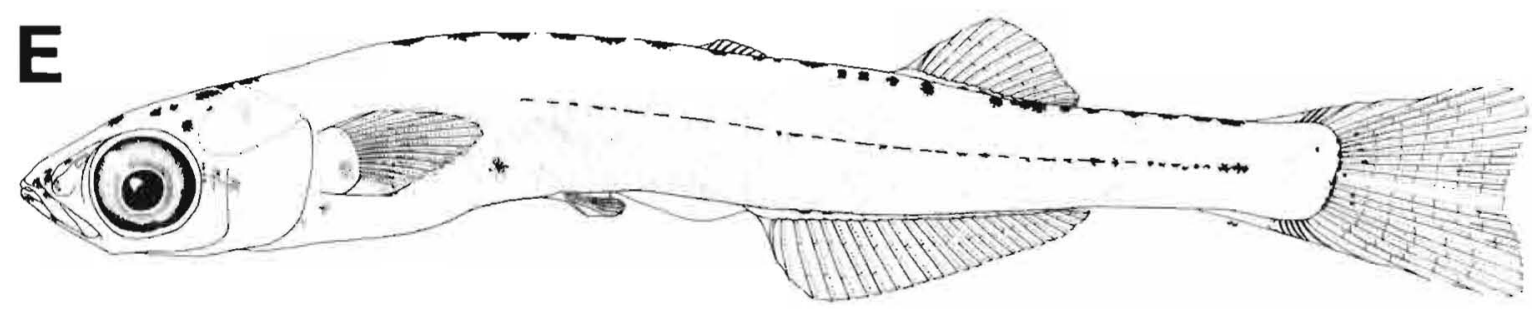

$18.3 \mathrm{~mm} \mathrm{SL}$

Figure A, White et al. 1984; B-E (C, ventral view), Watson and McGowen, unpubl. 



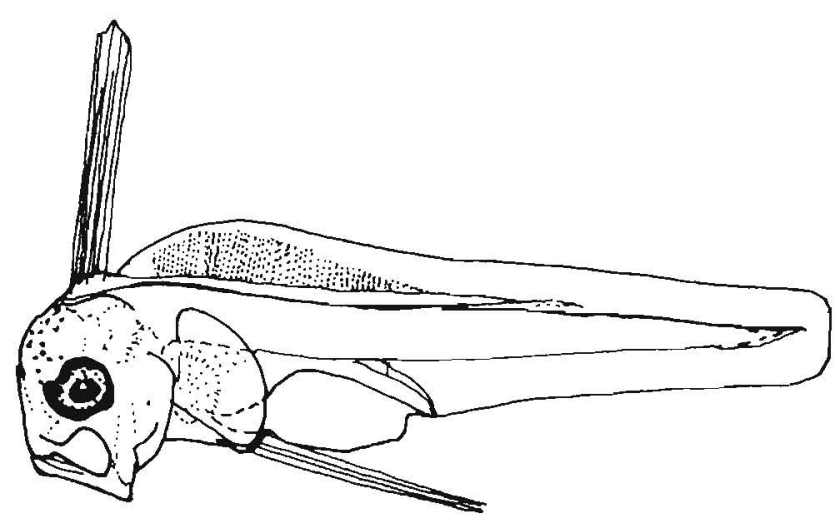

\section{Lampriformes}

Fishes of the order Lampriformes are extremely diverse. Occupying meso- and epipelagic habitats, some are deep-bodied while others are long and ribbon-like. Extreme specialization and unique body shapes have caused conflicting proposals for evolutionary relationships within the order (Olney 1984). According to Olney (1984), there are 7 families, 12 genera, and 21 families worldwide (not found in polar seas). Lampriforms share the unique arrangement of protrusible premaxilla and maxilla, or specializations thereof. Early-life-history information is available for only four genera. Eggs are large (1.7-4.0 mm) with thick resilient chorions, pelagic, and may be shaded with amber, pink, or red hues. Advanced stages of eggs of some forms are easily recognized due to precocious development of anterior dorsal and pelvic rays and distinctive pigment patterns. Newly hatched lampriforms are identified by their well-developed protrusible jaws and elongate anterior dorsal and pelvic fin elements which are often ornamented with highly pigmented serial or terminal swellings (Olney 1984).

Families in study area: Lampridae

Trachipteridae 
MERISTICS

\begin{tabular}{ll}
\hline Vertebrae & Total: $43-\mathrm{X}-46$ \\
& Precaudal: $19-\mathrm{X}-21$ \\
& Caudal: $23-24-25$ \\
Branchiostegal rays & $6-\mathrm{X}-7$ \\
Caudal fin & Total rays=30-32 \\
Pelvic fin & Abdominal \\
& R: $13-\mathrm{X}-17$ \\
Dorsal fin & R: $48-\mathrm{X}-52$ \\
Pectoral fin & R: $21-\mathrm{X}-24$ \\
Anal fin & R: $33-\mathrm{X}-42 \quad$ \\
Gill rakers & U: $2-\mathrm{X}-3 \quad$ L: $13-\mathrm{X}-14$
\end{tabular}

\section{LIFE HISTORY}

\begin{tabular}{|c|c|}
\hline Range & $\begin{array}{l}\text { South of southern California to } \\
\text { Gulf of Alaska, } 54-60^{\circ} \mathrm{N}\end{array}$ \\
\hline Ecology & Epi- and mesopelagic \\
\hline ELH pattern & $\begin{array}{l}\text { Oviparous, pelagic eggs, } \\
\text { pelagic larvae }\end{array}$ \\
\hline Spawning & $\begin{array}{l}\text { Season: Spring } \\
\text { Area: } \\
\text { Mode: } \\
\text { Migration: }\end{array}$ \\
\hline $\begin{array}{l}\text { Fecundity } \\
\text { Age at first maturity } \\
\text { Longevity }\end{array}$ & Range/function: \\
\hline
\end{tabular}

\section{EARLY LIFE HISTORY DESCRIPTION}

EGGS - Family

Diameter Large, $1.7-4.0 \mathrm{~mm}$

No. of oil globules

Oil globule diameter

Yolk

Homogeneous in known forms

Envelope Thick, resilient

Hatch size

Incubation time/temp. $\quad \sim 3$ weeks

Pigment

Diagnostic characters

- Precocious development of fins

- Ovarian eggs have thick chorion with amber tint

LARVAE

Preanal length

$<50 \% \mathrm{SL}$, increasing with

Length at flexion development to $\geqslant 50 \%$ SL

Length at transformation

Sequence of fin development

Anterior dorsal and pelvics precocious; dorsal, anal, and caudal; pectorals

Pigment

- Initially on crown and dorsal surface of gut; with development, increases along entire body except for tail region and above anal fin

Diagnostic characters

Distinguished from Trachipterus altivelis (p. 246) by

- Lack of ornamentation on dorsal and pelvic fins (when fins are intact)

- Rapid change in body form from slender at hatching to deep-bodied by $10.6 \mathrm{~mm} \mathrm{SL}$

Lampriform characters

- Well-developed protrusible jaws

- Differentiated guts with open lumen and little yolk

- Elongate anterior dorsal elements (may be ornamented) and well-developed pelvic elements (may be ornamented with lengths to $40-60 \%$ NL)

Data from Olney (1984) in part, only total elements reported.

${ }^{b}$ Fitch and Lavenberg 1971

Ref: Olney 1984 


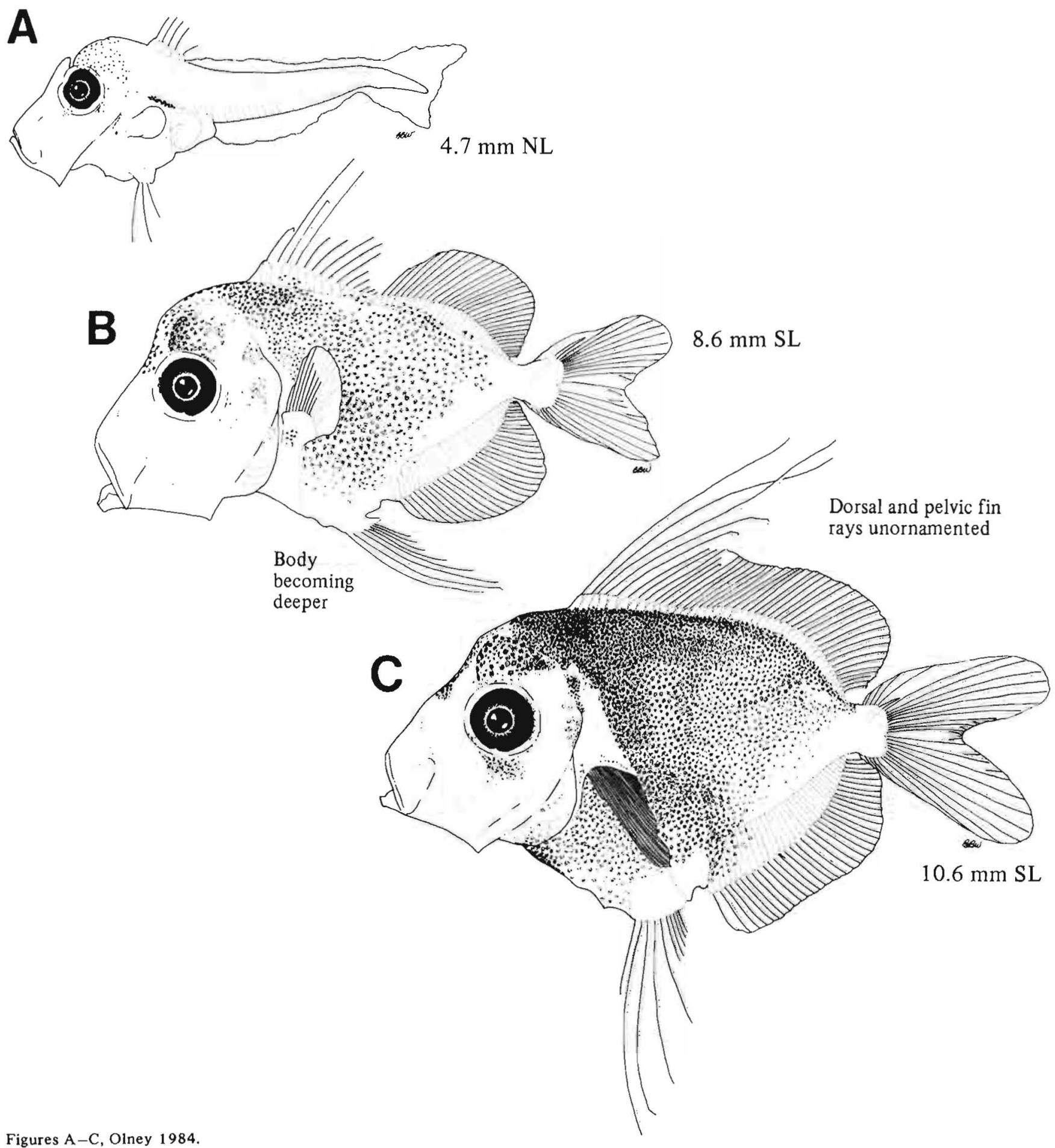




\section{MERISTICS}

Vertebrae

Total: $90-92-94$

Precaudal: 35-35-39

Caudal: $53-59-59$

Branchiostegal rays $\quad 6-\mathrm{X}-7$

Caudal fin

Pelvic fin

Thoracic

R: $6-\mathrm{X}-7$

Dorsal fin

R: $160-X-191$

Pectoral fin

Anal fin

Gill rakers
R: $10-X-11$

Absent

U: $3-\mathrm{X}-5$

L: $9-\mathrm{X}-11$

\section{EARLY LIFE HISTORY DESCRIPTION}

\section{EGGS}

Diameter

No. of oil globules

Oil globule diameter

Yolk

Envelope

Hatch size

Incubation time/temp.

Pigment

- Head and gut

- Finfold

- Ornamentation on elongate rays

Diagnostic characters

- Precocious development of elongate rays in anterior dorsal fin and pelvic fins

$\begin{array}{ll}\text { Range } & \begin{array}{c}\text { South of southern California to } \\ \text { Gulf of Alaska, 54-60 }\end{array} \\ \text { Ecology } & \text { Epi- and mesopelagic, 0-900 m } \\ \text { ELH pattern } & \text { Oviparous, pelagic eggs, } \\ & \text { pelagic larvae } \\ \text { Spawning } & \text { Season: } \\ & \text { Area: } \\ & \text { Mode: } \\ & \text { Migration: } \\ \text { Fecundity } & \text { Range/function: }\end{array}$

Age at first maturity

Longevity
Gulf of Alaska, $54-60^{\circ} \mathrm{N}$

Epi- and mesopelagic, 0-900 m

Season:

Mode:

Range/function:

\section{LARVAE}

Preanal length

Length at flexion

Length at transformation

Sequence of fin development
2.6-3.7 mm

None

Homogeneous

Clear, smooth, thick

\section{Pigment}

- Initially only on head and anterior/dorsal gut; with development pigment appears on lateral surface of gut, along body over gut, and in a series above notochord along 3/4 BL

- Several spots develop above and below notochord in caudal region

Diagnostic characters

- See Lampris guttatus (p. 244) 

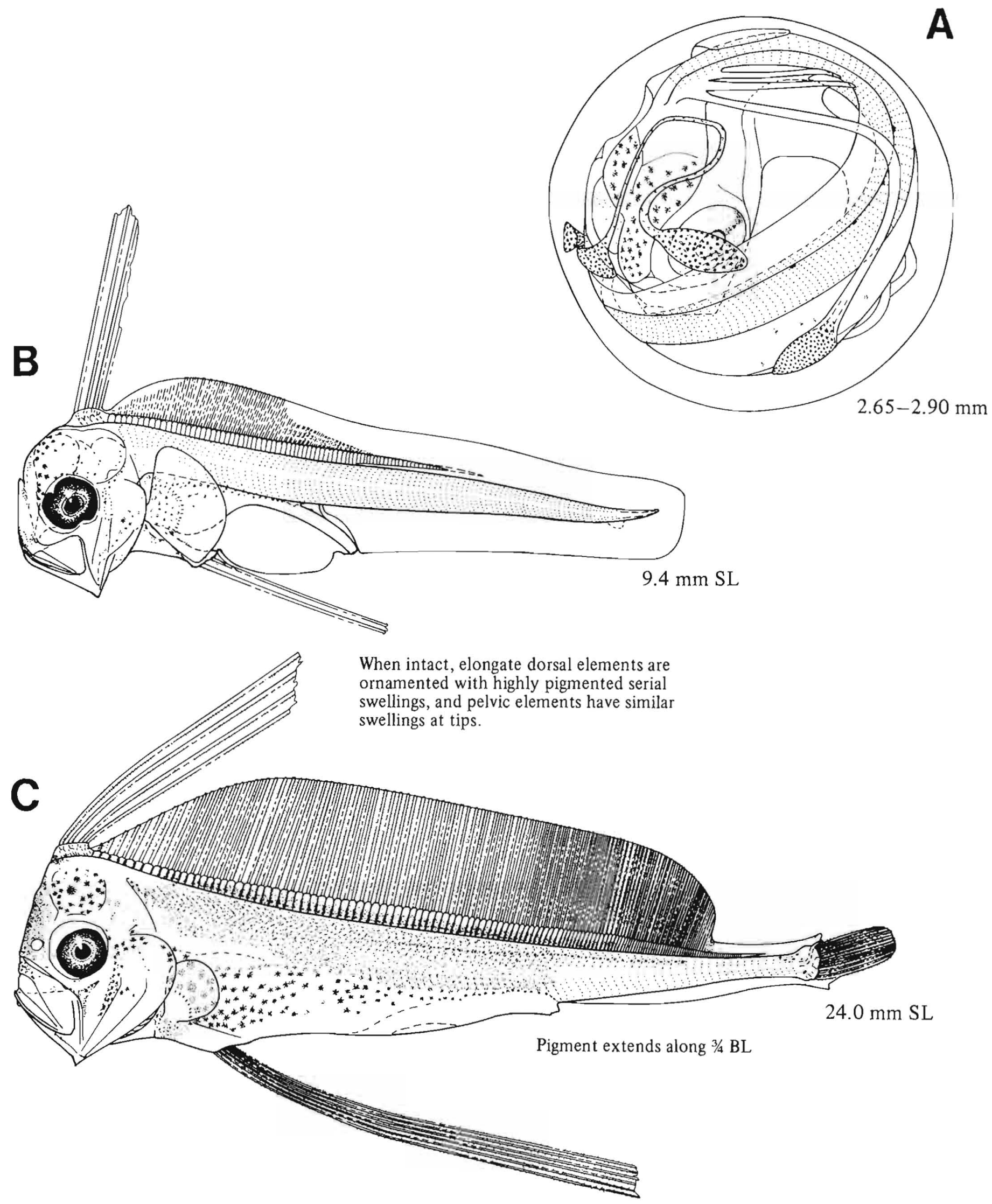

Figure A, Matarese and Sandknop 1984; B-C, NWAFC originals (B. Vinter). 



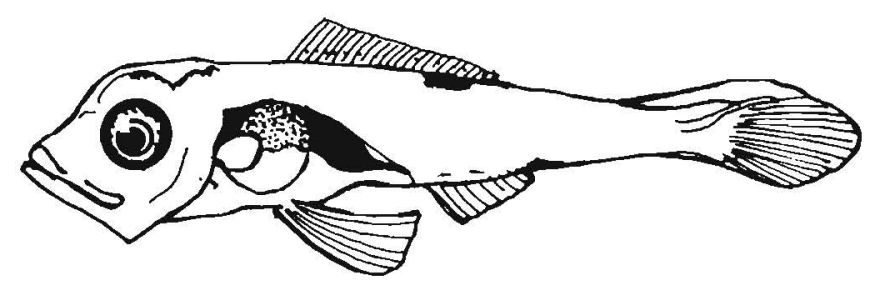

\section{Beryciformes}

Fishes of the order Beryciformes are cosmopolitan in distribution. The composition of the order is subject to much variation, but according to Keene and Tighe (1984), it consists of 16 families, 42 genera, and 155 species. Although several characters define the beryciforms (e.g., a high number of pelvic and caudal fin rays and the presence of dorsal, anal, and pelvic fin spines), none are unique to the order. No information is available on eggs, and other early-life-history stages are known for only six families. In the Northeast Pacific, larvae are known for only two families, Anoplogastridae and Melamphaidae. Anoplogastrids have large heads with short deep bodies, and well-developed preopercular, rostral, and cranial spines. Larvae of the best known family, Melamphaidae, are longer-bodied and more slender with spination generally restricted to the preopercle.

A summary of meristic characters is provided for members of the suborder Cetomimoidei (Barbourisiidae, Cetomimidae, Rondeletiidae), as larvae are unknown.

Families in study area: Anoplogastridae

Melamphaidae

Barbourisiidae

Cetomimidae

Rondeletiidae 


\section{MERISTICS}

Vertebrae

Total: 25-X-28

Precaudal: $12-12-12$

Caudal: $16-16-16$

Branchiostegal rays $8-\mathrm{X}-9$

Caudal fin $\quad X, 17, X$

Pelvic fin Thoracic
S: $1-1-1$
$\mathrm{R}:$ 6-6-6

Dorsal fin

R: $17-18-19$

Pectoral fin

Anal fin

R: $14-15-16$

R: $8-X-9$

Gill rakers

U: $7-X-11$

L: $7-\mathrm{X}-11$

\section{LIFE HISTORY}

\section{Range}

Ecology

\section{ELH pattern}

Spawning

Fecundity

Age at first maturity

Longevity
South of southern California to Oregon, $42-46^{\circ} \mathrm{N}$

Epi- and mesopelagic, below $610 \mathrm{~m}$

Parity and eggs unknown, pelagic larvae

Season: Summer (California) $)^{\mathrm{a}}$

Area:

Mode:

Migration:

Range/function:

\section{EARLY LIFE HISTORY DESCRIPTION}

\section{EGGS}

Diameter

No. of oil globules

Oil globule diameter

Yolk

Envelope

Hatch size

Incubation time/temp.

Pigment

Diagnostic characters

\section{LARVAE}

Preanal length

Length at flexion

4.5-6.0 mm SL

Length at transformation

Sequence of fin development

Pigment

- At $6 \mathrm{~mm}$ SL, lateral surface of body to caudal peduncle, gut, and pectoral fin base pigmented

- Pigment increases with development

Diagnostic characters

- Spination

- Serrate frontal ridge terminates in a short stout supraocular spine

-Long serrate parietal spine

-Long serrate preopercular spine directed posteroventrad

Juveniles

- Increase in dark pigment

- Reduction of spines

${ }^{\mathrm{a}}$ Fitch and Lavenberg 1968 Ref: Keene and Tighe 1984. 


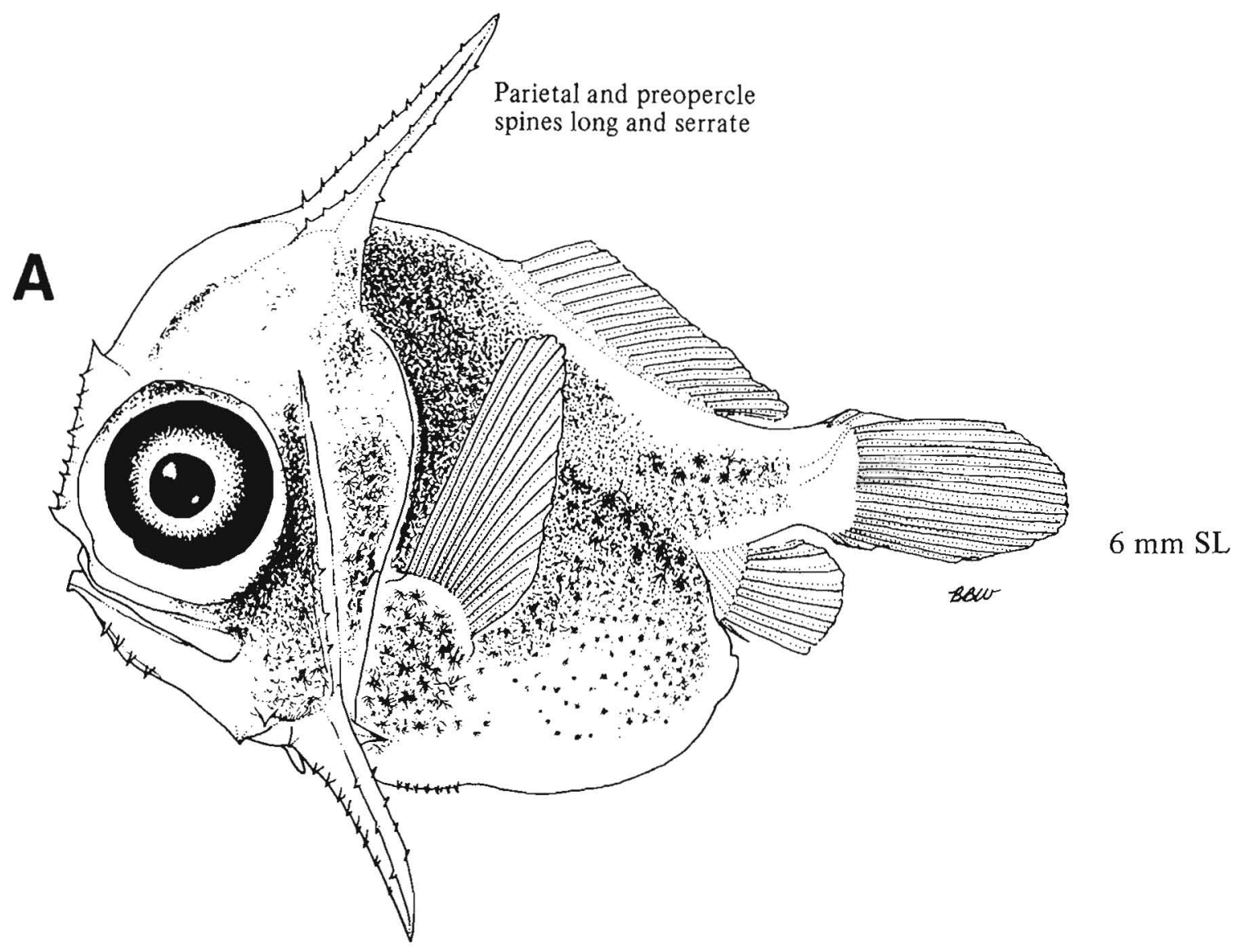

Figure A, Keene and Tighe 1984. 
Melamphaids, or bigscales, are small $(15 \mathrm{~cm})$, darkly colored, bathypelagic fish found in most oceans. They are distinguished by exceptionally thin skull bones, sometimes with ridges or crests. They are represented in the study area by five species within four genera: Melamphaes, Poromitra, Scopeloberyx, and Scopelogadus. A complete descriptive series is known only for Melamphaes lugubris. Generally, small melamphaid larvae $(2-10 \mathrm{~mm})$ are relatively elongate and slender as compared with later stages, and possess early and rapidly developing pelvic fins which are long and darkly pigmented. Melamphaes, Scopeloberyx, and Scopelogadus larvae usually have two spots near the posterior end of the dorsal and anal fin margin; with development these spots spread both anteriorly and posteriorly along the dorsal and ventral midlines (Keene and Tighe 1984). Other pigment occurs on the cranium, peritoneum, and along the caudal peduncle. Although usually damaged, the second or third dorsal fin ray is elongate in larvae of these three genera. Larger larvae (5-10 to $20 \mathrm{~mm})$ can generally be distinguished by body shape, presence of preopercular spines (e.g., Poromitra), and meristic characters. The following generic meristic characters are based on Ebeling (1962) and E.H. Ahlstrom notes, in part.

\begin{tabular}{lcrccl} 
& & \multicolumn{5}{c}{ Fins } \\
\cline { 3 - 6 } Genus & Vertebrae & \multicolumn{1}{c}{ Dorsal } & Anal & Pectoral & Pelvic \\
\hline Melamphaes & $27-31$ & III, 13-16 & I,7-9 & $14-17$ & I,7 \\
Poromitra & $24-29$ & III, 9-14 & I,8-11 & $13-15$ & I,7-8 \\
Scopeloberyx & $23-27$ & II-III, 10-13 & I,7-9 & $12-14$ & I,7-8 \\
Scopelogadus & $24-26$ & II, 10-12 & I,7-9 & $13-15$ & I,7-8 \\
\hline
\end{tabular}




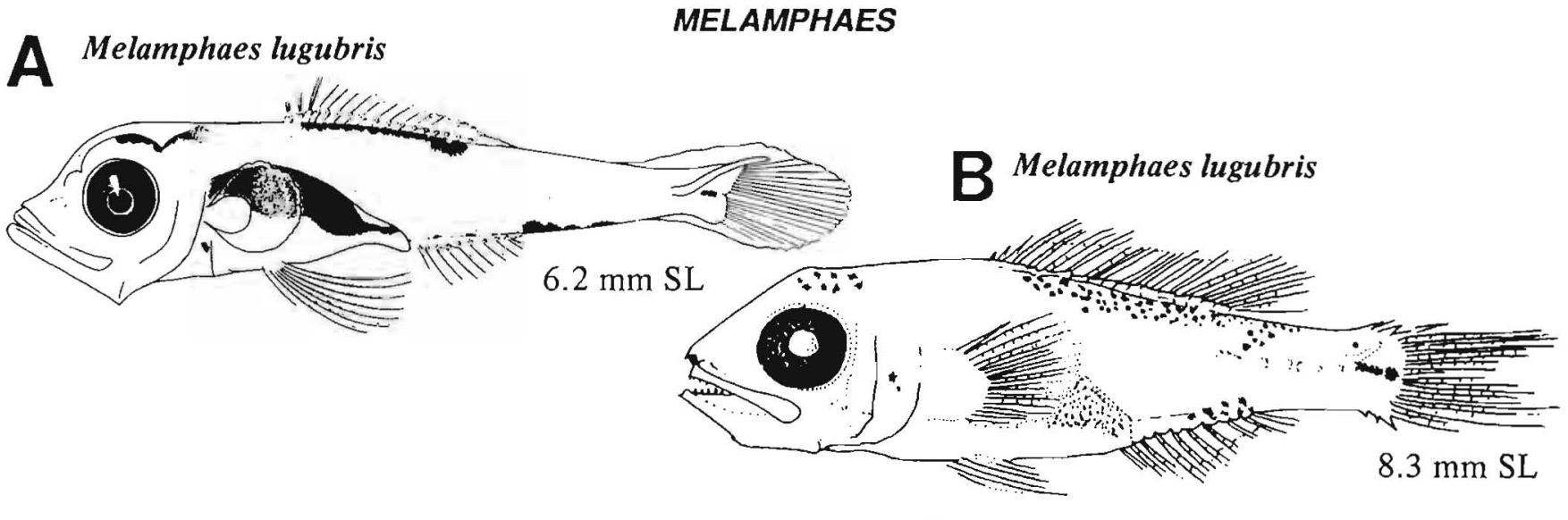

C Poromitra crassiceps

\section{POROMITRA}
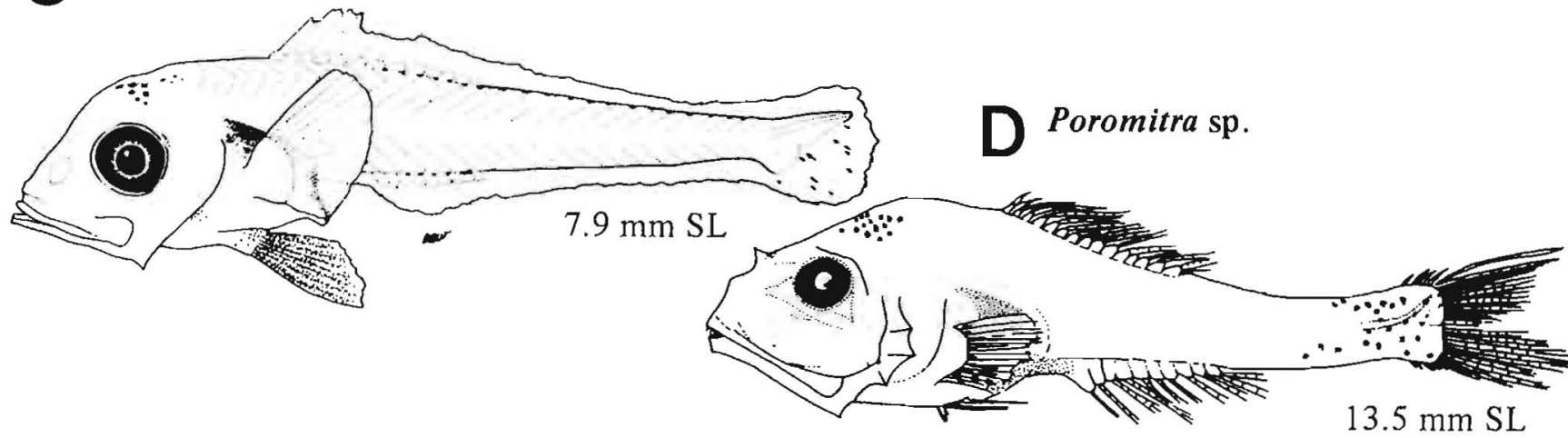

\section{E Scopeloberyx robustus}

SCOPELOBERYX

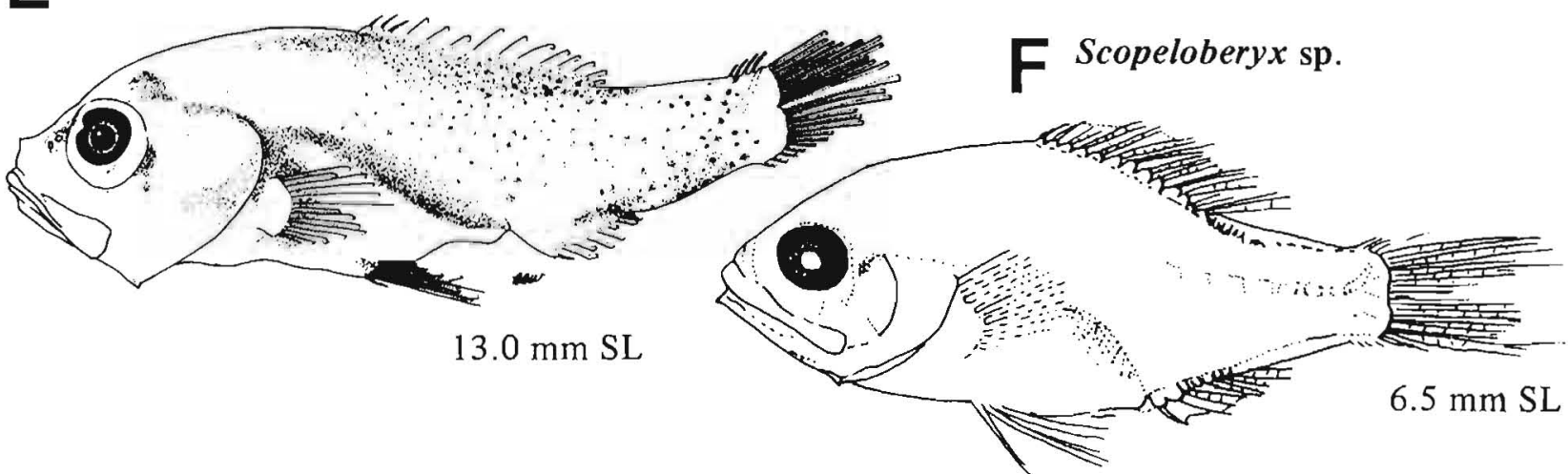

G Scopelogadus bispinosus

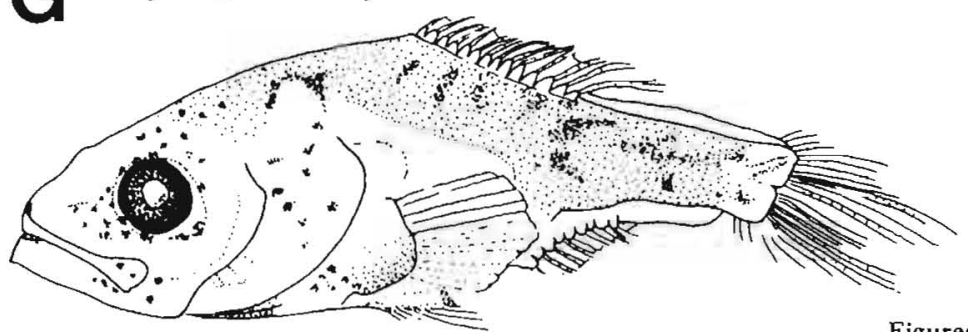

$8.0 \mathrm{~mm} \mathrm{SL}$

Figures $A-G$, Keene and Tighe 1984 (B-G, North Atlantic specimens). 
MERISTICS

\begin{tabular}{|c|c|}
\hline Vertebrae & $\begin{array}{l}\text { Total: } 28-\mathrm{X}-31 \\
\text { Precaudal: } 11-12-12 \\
\text { Caudal: } 16-\mathrm{X}-19\end{array}$ \\
\hline Branchiostegal rays & $8-8-8$ \\
\hline Caudal fin & $X, 10+9, X$ \\
\hline \multirow{2}{*}{ Pelvic fïn } & Thoracic \\
\hline & S: $1-1-1$ \\
\hline Dorsal fin & S: $3-3-3 \quad R: 14-15-16$ \\
\hline Pectoral fin & $\mathrm{R}: 15-16-17$ \\
\hline Anal fin & S: $1-1-1 \quad R: 7-8-9$ \\
\hline Gill rakers & U: $5-X-6 \quad$ L: $15-X-18$ \\
\hline \multicolumn{2}{|l|}{ LIFE HISTORY } \\
\hline Range & $\begin{array}{l}\text { South of southern California to } \\
\text { Bering Sea, } 54-66^{\circ} \mathrm{N}\end{array}$ \\
\hline Ecology & Mesopelagic, 200-1000 m \\
\hline ELH pattern & $\begin{array}{l}\text { Oviparous, pelagic eggs, } \\
\text { pelagic larvae }\end{array}$ \\
\hline Spawning & Season: \\
\hline & Area: \\
\hline & Mode: \\
\hline No & Migration: \\
\hline $\begin{array}{l}\text { Fecundity } \\
\text { Age at first maturity } \\
\text { Longevity }\end{array}$ & Range/function: \\
\hline
\end{tabular}

\section{EARLY LIFE HISTORY DESCRIPTION}

EGGS - Family

\begin{tabular}{ll}
$\begin{array}{ll}\text { Diameter } \\
\text { No. of oil globules } \\
\text { Oil globule diameter }\end{array}$ & $0.81-0.96 \mathrm{~mm} \mathrm{SL}$ \\
Yolk & One to many \\
Envelope & Segmented \\
Hatch size & Clear, spherical \\
\hline
\end{tabular}

Incubation time/temp.

Pigment

Diagnostic characters

\section{LARVAE}

$\begin{array}{lc}\text { Preanal length } & 45-55 \% \text { SL } \\ \text { Length at flexion } & \sim 6 \mathrm{~mm} \mathrm{SL} \\ \begin{array}{l}\text { Length at transformation } \\ \begin{array}{l}\text { Sequence of fin } \\ \text { development }\end{array}\end{array} & \begin{array}{c}\text { Pelvics, dorsal and caudal, } \\ \text { anal, pectorals }\end{array}\end{array}$

Pigment

- Dorsally on top of head

- Peritoneum

- Postanal: Two spots near posterior of dorsal and anal anlagen which spread anteriorly and posteriorly along dorsal and ventral midline

- Spot on caudal peduncle

\section{Diagnostic characters}

- Pigment (see above): Patches, bands

- 2nd or 3rd dorsal ray elongate (until 5-10 mm SL)

- Pelvics: (2-10 mm SL) develop rapidly and are long, fragile, and pigmented

Distinguished from Sebastolobus spp. (p. 336) at sizes $<5 \mathrm{~mm}$ SL by

- Postanal pigment band more anterior, beginning at myomere 15

- Precocious development of pelvic fin

$M$. parvus larvae are unknown. The following meristic information may aid in identification.

$\begin{array}{lc}\text { Total vertebrae } & 27-29 \\ \text { Precaudal vertebrae } & 11-12 \\ \text { Caudal vertebrae } & 16-17 \\ \text { Dorsal fin rays } & \text { III, } 13-15 \\ \text { Anal fin rays } & \text { I, } 7-9 \\ \text { Pectoral fin rays } & 14-15 \\ \text { Pelvic fin rays } & \text { I, } 7\end{array}$




\section{A}

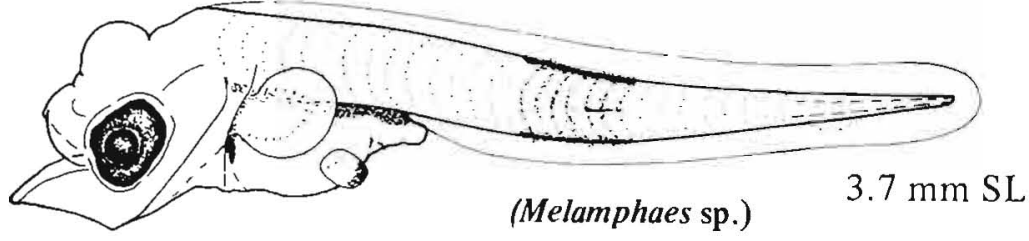

B $1-2$ dorsal spots

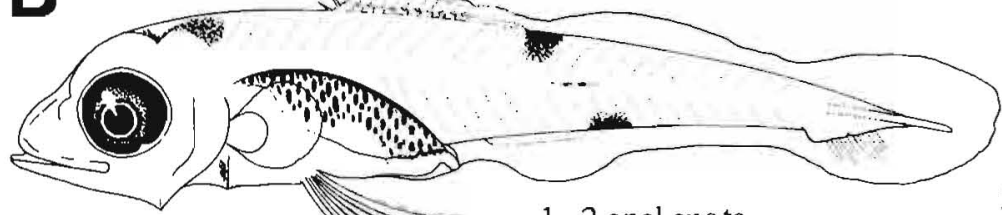

$1-2$ anal spots

$5.3 \mathrm{~mm} \mathrm{NL}$
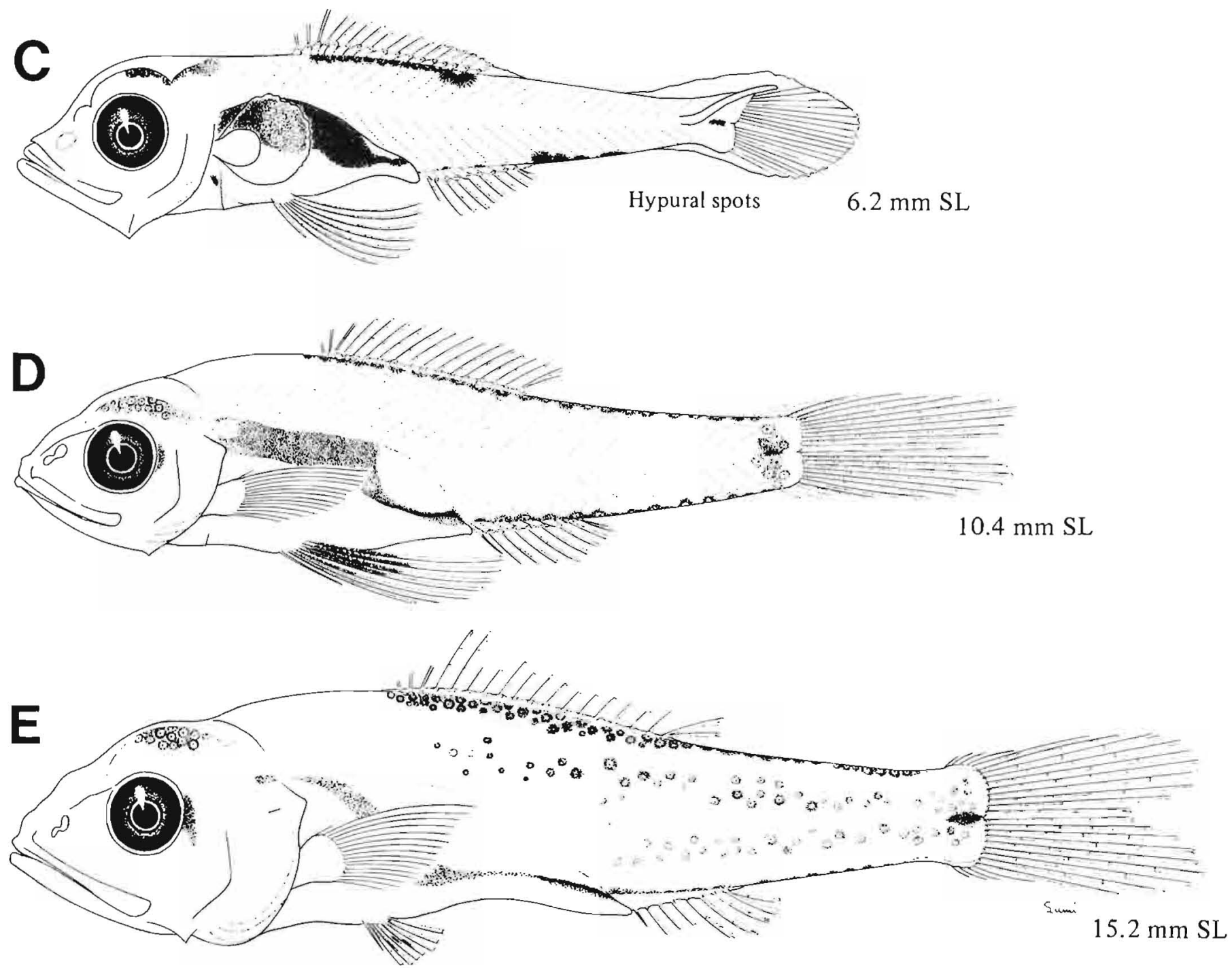

Figure A, NWAFC original (B. Vinter); B-E, Keene and Tighe 1984. 
Fishes of the suborder Cetomimoidei are bathypelagic with whale-shaped bodies, large mouths, highly distensible stomachs, and luminous tissue on the body. Eyes may be reduced or rudimentary. Whalefishes are divided into three families: Cetomimidae, Rondeletiidae, and Barbourisiidae. Worldwide in distribution, the suborder consists of 8 genera and 18 species. Of these, five species in five genera have been collected in the northeastern Pacific. Adults are taken by deep tows generally in excess of $400 \mathrm{~m}$, and then only rarely. No information on life history, eggs, or larvae is available (Keene and Tighe 1984).

Table 28

Meristic characters of suborder Cetomimoidei.

\begin{tabular}{|c|c|c|c|c|c|c|c|c|c|c|}
\hline \multirow[b]{3}{*}{ Taxon } & \multirow[b]{3}{*}{ Distribution } & \multicolumn{2}{|l|}{ Vertebrae } & \multirow{2}{*}{\multicolumn{4}{|c|}{ Fins }} & \multirow{2}{*}{\multicolumn{2}{|c|}{ Gill rakers }} & \multirow[b]{3}{*}{ Branchiostegal } \\
\hline & & \multirow{2}{*}{$\begin{array}{l}\text { Precaudal } \\
\text { (Total) }\end{array}$} & \multirow{2}{*}{ Caudal } & & & & & & & \\
\hline & & & & Dorsal & Anal & Pectoral & Pelvic & Upper & Lower & \\
\hline Barbourisiidae & & $(42-44)$ & & & & & & & & \\
\hline Barbourisia rufa & Cent. Calif. - Arctic & $\frac{17-18}{(42-44)}$ & 25 & $20-22$ & $16-18$ & $13-14$ & 6 & $4-6$ & $14-16$ & $6-7$ \\
\hline Cetomimidae & & $(51-52)$ & & & & & & & & \\
\hline Cetomimus sp. & Bering Sea & & & & & & & & & \\
\hline Cetostoma regani & Cent. Calif. - Oregon & & & 30 & 27 & 14 & Absent & & & 8 \\
\hline Gyrinomimus sp.* & N. Calif. - Brit. Col. & & & & & & & & & \\
\hline Rondeletiidae & & $(24-27)$ & & & & & & & & \\
\hline Rondeletia loricata & S. Calif. - Oregon & $\begin{array}{l}10 \\
(24-26)\end{array}$ & 16 & $13-16$ & $13-14$ & $9-11$ & 5 & $4-6$ & $14-16$ & 8 \\
\hline
\end{tabular}




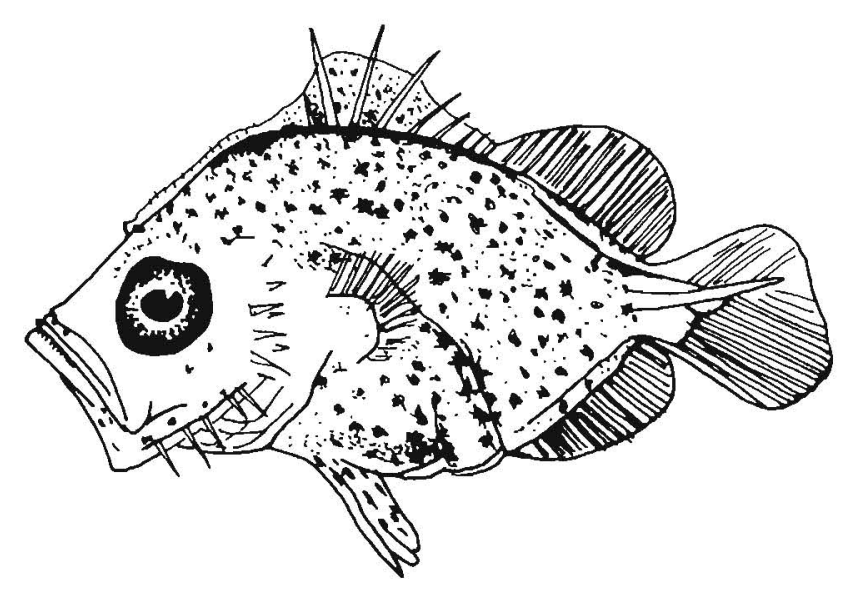

\section{Zeiformes}

The zeiforms occur in tropic and temperate areas of all oceans, in benthic and pelagic habitats from shallow to deep-water areas; little else is known of the life history of most fish in this group. Adults are generally deep-bodied with large eyes, greatly distensible jaws, and dorsal, anal and/or pelvic fin spines. The order is loosely organized according to a number of characters, none of which are unique. Presently, there are 6 families, 21 genera, and about 36 species throughout their distribution (J. Nelson 1984). The order is represented in the study area by one taxon, Allocyttus sp., a member of the Oreosomatidae. Eggs of only three species are known. Sizes range from 1.0 to $2.25 \mathrm{~mm}$ in diameter; all have a smooth chorion, homogeneous yolk, and a single oil globule (Tighe and Keene 1984). Larvae are generally deep-bodied and may be heavily pigmented. Forms exhibit a variety of armaments including serrated cranial ridges and spines, preopercular spines, and hardened cones or scaley knobs.

Family in study area: Oreosomatidae 


\section{MERISTICS}

Vertebrae

Total: $39-39-40$

Precaudal: $13-\mathrm{X}-14$

Caudal: 26-26-26

Branchiostegal rays

Caudal fin

Pelvic fin

7-7-7

Total rays $=12-18$

Thoracic

S: $1-1-1 \quad R:$ 6-6-6

Dorsal fin

S: $6-\mathrm{X}-7$

$\mathrm{R}: 30-\mathrm{X}-36$

Pectoral fin

R: $19-X-21$

Anal fin

S: $2-X-3$

R: $28-X-33$

Gill rakers

L: $18-X-21$

\section{LIFE HISTORY}

$\begin{array}{ll}\text { Range } & \begin{array}{c}\text { Cent. California, } 34-38^{\circ} \mathrm{N}, \text { to } \\ \text { Bering Sea, } 54-66^{\circ} \mathrm{N}\end{array} \\ \text { Ecology } & \text { Meso- and bathybenthal, } \\ & 366-732 \mathrm{~m} \\ \text { ELH pattern } & \text { Eggs and larvae unknown } \\ \text { Spawning } & \text { Season: } \\ & \text { Area: } \\ & \text { Mode: } \\ & \text { Migration: } \\ \text { Fecundity } & \text { Range/function: } \\ \text { Age at first maturity } & \\ \text { Longevity } & \end{array}$

\footnotetext{
allocyttus follesti and A. verrucosus may occur in the study area, but identification to species is not possible. Kobayashi et al. (1968) suggested $A$. folletii should be a synonym of $A$. verrucosus.

${ }^{b}$ Early-life-history stages of Allocytus spp. are unknown. An ilustration of a prejuvenile of Oreosoma atlanticum is presented for comparison only. A photograph of an Allocytrus verrucosus juvenile (95 $\mathrm{mm} \mathrm{TL}$ ) is presented in Kobayashi et al. (1968).
}

Ref: Kobayashi et al. 1968, Tighe and Keene 1984.

\section{EARLY LIFE HISTORY DESCRIPTION}

\section{EGGS}

Diameter

No. of oil globules

Oil globule diameter

Yolk

Envelope

Hatch size

Incubation time/temp.

Pigment

Diagnostic characters

\author{
LARVAE $^{\text {b }}$ \\ Preanal length \\ Length at flexion \\ Length at transformation \\ Sequence of fin \\ development \\ Pigment
}

Diagnostic characters - Family

- Limited data on prejuveniles only; pelagic prejuveniles are oval in outline and possess a leathery skin with distinct hardened cones or scaly knobs laterally and ventrally 


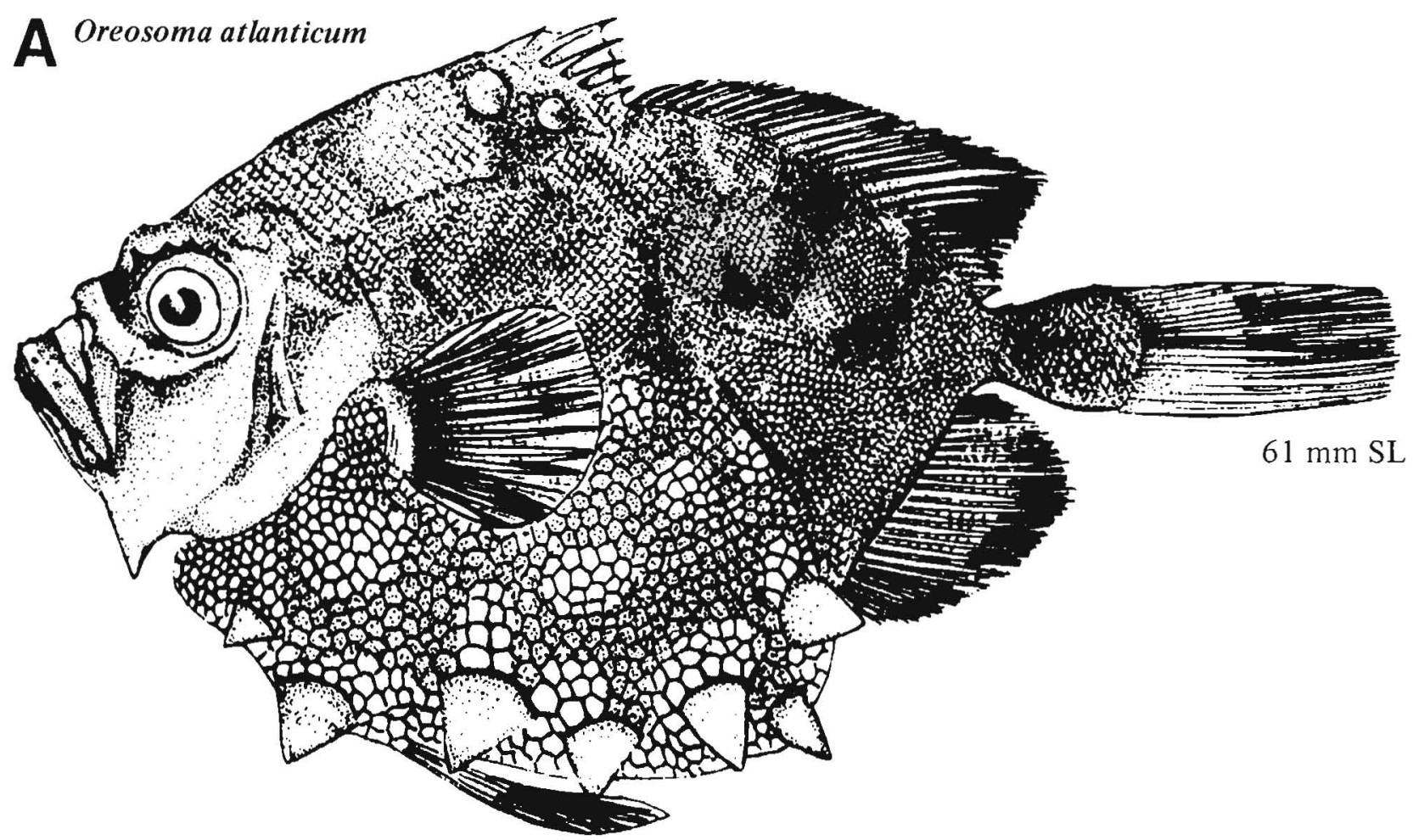

Figure A, Abe and Kaji 1972 (Tasman Sea specimen). 


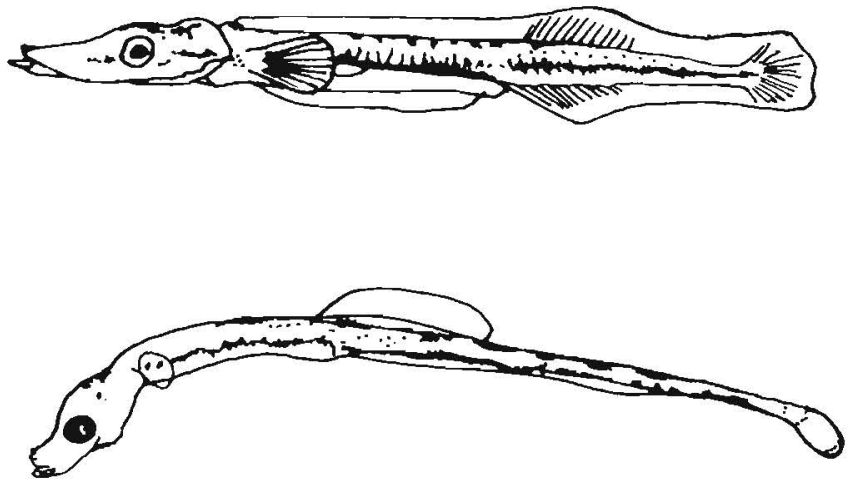

\section{Gasterosteiformes}

The order Gasterosteiformes is made up of small, cryptically colored fish found in freshwater or marine habitats in tropical and temperate areas. Most species have small mouths at the end of tubular snouts and dermal plates covering the body. Historically divided into two or three orders, the group, as supported by Pietsch (1978) and Fritzsche (1984), contains 10 families with well over 200 species. Eggs are broadcast or deposited in nests or on algae, or for syngnathids, in male brood pouches. Eggs of families other than Syngnathidae are 1.5-2.1 mm in diameter, may have one or more oil globules, and have thick chorions. Most gasterosteiform larvae hatching from externally incubated eggs (except gasterosteids) have the same distinctive characters (i.e., elongate snout and small mouth, dermal plates) as the adults. Although adult gasterosteids are sometimes collected in plankton samples, early-life-history stages are unlikely to occur and are not treated here.

Families in study area: Aulorhynchidae

Gasterosteidae

Syngnathidae 


\section{MERISTICS}

\begin{tabular}{|c|c|c|}
\hline Vertebrae & \multicolumn{2}{|c|}{$\begin{array}{l}\text { Total: } 54-54-56 \\
\text { Precaudal: } 24-25-26 \\
\text { Caudal: } 29-29-31\end{array}$} \\
\hline $\begin{array}{l}\text { Branchiostegal rays } \\
\text { Caudal fin }\end{array}$ & $4-4-4$ & \\
\hline Pelvic fin & $\begin{array}{l}\text { Thoracic } \\
\text { S: } 1-1-1\end{array}$ & R: $4-4-4$ \\
\hline Dorsal fin & S: $23-X-27$ & R: $9-\mathrm{X}-11$ \\
\hline Pectoral fin & R: X-X-X & \\
\hline Anal fin & S: $1-1-1$ & R: $9-\mathrm{X}-10$ \\
\hline Gill rakers & U: X-X-X & L: X-X-X \\
\hline
\end{tabular}

\section{LIFE HISTORY}

$\begin{array}{lc}\text { Range } & \text { South of southern California to } \\ & \text { SE Alaska, } 55-59^{\circ} \mathrm{N} \\ \text { Ecology } & \text { Nearshore shelf pelagic } \\ \text { ELH pattern } & \text { Oviparous; demersal, adhesive, } \\ & \text { guarded eggs; larvae briefly } \\ & \text { pelagic } \\ \text { Spawning } & \text { Season: Late spring-summer } \\ & \text { (Brit. Col.); }{ }^{2} \text { Feb-July (Calif.); } \\ & \text { year-round (Calif. }{ }^{\mathrm{c}} \\ & \text { Area: Demersal, on algae or } \\ & \text { marine plants } \\ & \text { Mode: Nest building; males } \\ & \text { territorial, polygamous }{ }^{\mathrm{c}} \\ \text { Fecundity } & \text { Migration: } \\ \text { Age at first maturity } & \text { Range/function: } 150-600 / \mathrm{yr}^{\mathrm{c}} \\ \text { Longevity } & 9 \mathrm{yr}^{\mathrm{b}}\end{array}$

\section{EARLY LIFE HISTORY DESCRIPTION}

\section{EGGS}

Diameter

No. of oil globules

Oil globule diameter

Yolk

Envelope

Hatch size

$2.0-2.1 \mathrm{~mm}$

One

Yellow to yellow-green

Thick

$8 \mathrm{~mm}$ TL

Incubation time/temp. $\quad 2-6 \mathrm{wk} / 10^{\circ} \mathrm{C}$

Pigment

Diagnostic characters

- Protracted hatch always occurs, embryos develop asynchronously

\section{LARVAE}

$\begin{array}{ll}\begin{array}{l}\text { Preanal length } \\ \text { Length at flexion }\end{array} & 60-65 \% \text { SL } \\ \begin{array}{l}\text { Length at transformation } \\ \text { Sequence of fin } \\ \text { development }\end{array} & \begin{array}{l}\text { Pectorals; dorsal rays, anal, } \\ \text { and caudal; dorsal spines; } \\ \text { pelvics }\end{array} \\ & \end{array}$

Pigment

- Gut: Along dorsal surface and ventral midline

- Postanal body: Dorsal and ventral midline to tail; along notochord initially over anal fin but increasing anteriorly

- Head: Dorsal and posterior to eye

Diagnostic characters

- Pigment generally heavier than on syngnathids

- Bony plates develop by notochord flexion

- Elongated snout

${ }^{a}$ Marliave 1975a

${ }^{b}$ Fitch and Lavenberg 1975

${ }^{c}$ Limbaugh 1962

Ref: Fritzsche 1984, Ida 1976, Limbaugh 1962, Marliave 1976. 

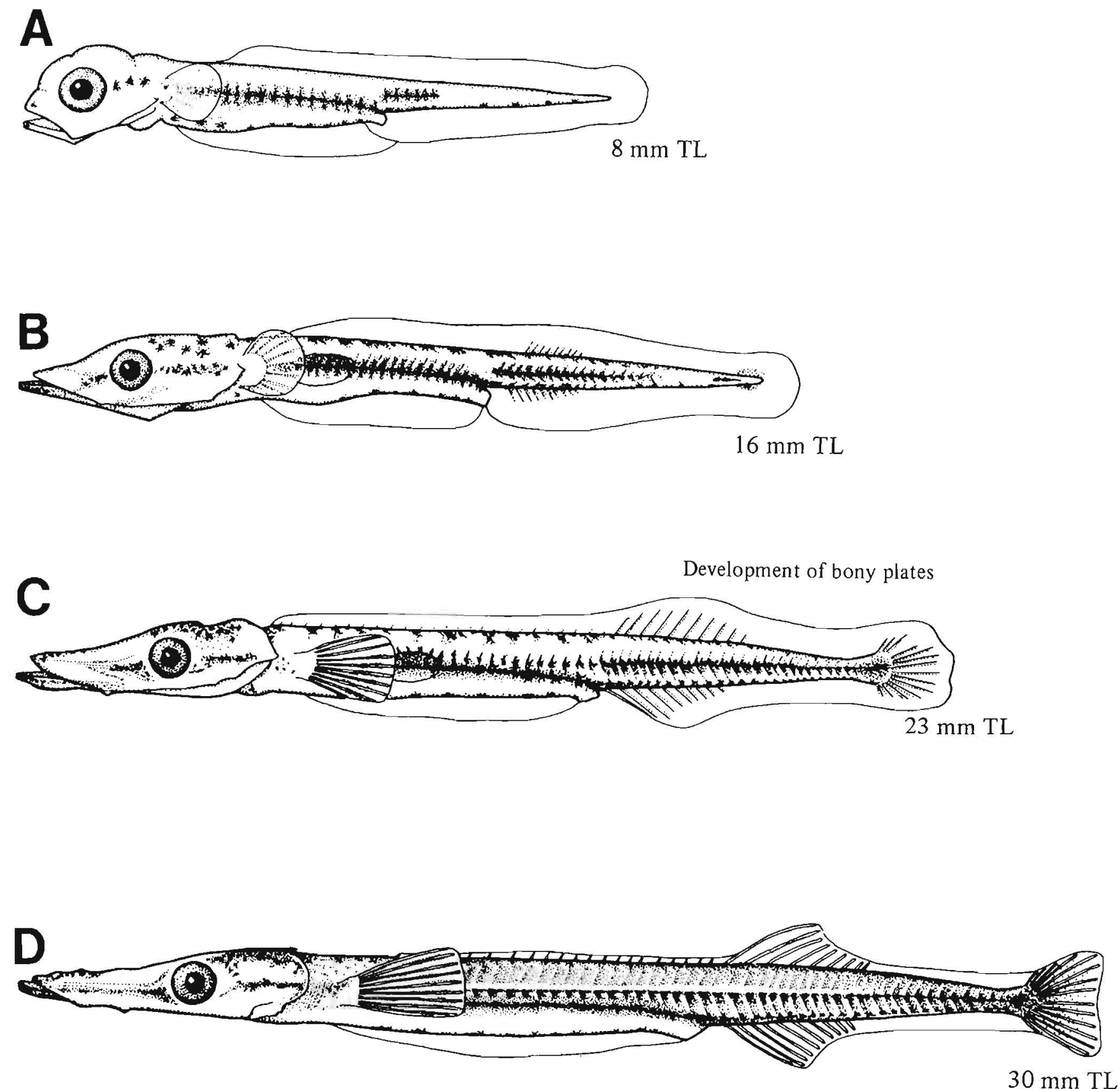

Figures A-D, Marliave 1976 . 


\section{MERISTICS}

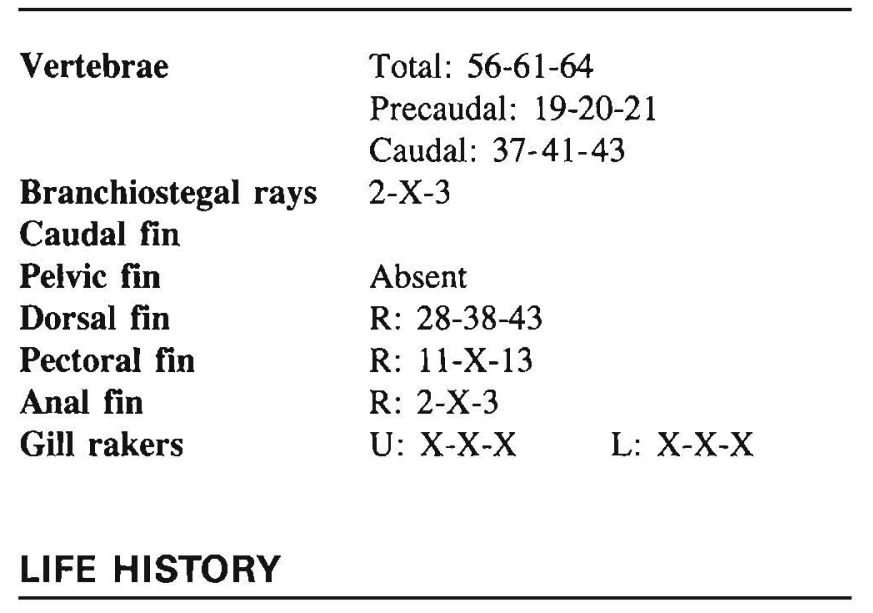

\section{Range}

Ecology

ELH pattern

Spawning

Fecundity

Age at first maturity

Longevity
South of southern California to

$$
\text { SE Alaska, } 55-59^{\circ} \mathrm{N}
$$

Intertidal, nearshore

Oviparous, eggs and larvae develop in brood pouch

Season: Spring-summer ${ }^{\text {a }}$

Area: Inshore protected areas ${ }^{b}$

Mode: Males retain eggs and developing larvae until the young have reached a juvenile stage of development ${ }^{b}$

Migration:

Range/function: $225^{\mathrm{a}}$

\section{EARLY LIFE HISTORY DESCRIPTION}

\section{EGGS}

Diameter

No. of oil globules

$1.0-1.5 \mathrm{~mm}$

Oil globule diameter

Yolk

Envelope

Hatch size

One or more

$0.4-0.5 \mathrm{~mm}$

Bright yellow

Incubation time/temp.

Pigment

4.5-5.0 mm TL

Diagnostic characters

\section{LARVAE $^{\mathrm{C}}$}

Preanal length $35-40 \%$ TL

Length at flexion

Length at transformation

Sequence of fin development

Pigment

- Sparse at hatching, developing to form vertical blotches covering the head and body

Diagnostic characters

\footnotetext{
${ }^{a}$ Wang 1981

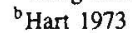

${ }^{c}$ Larval illustrations of Syngnathus Leptorhynchus are unavailable. Ilustrations of earlylife-history stages of other members of the genus are presented for comparison and may differ from early-life-history stages of $S$. leptorhynchus.

Ref: Wang 1981.
} 
A Syngnathus fuscus
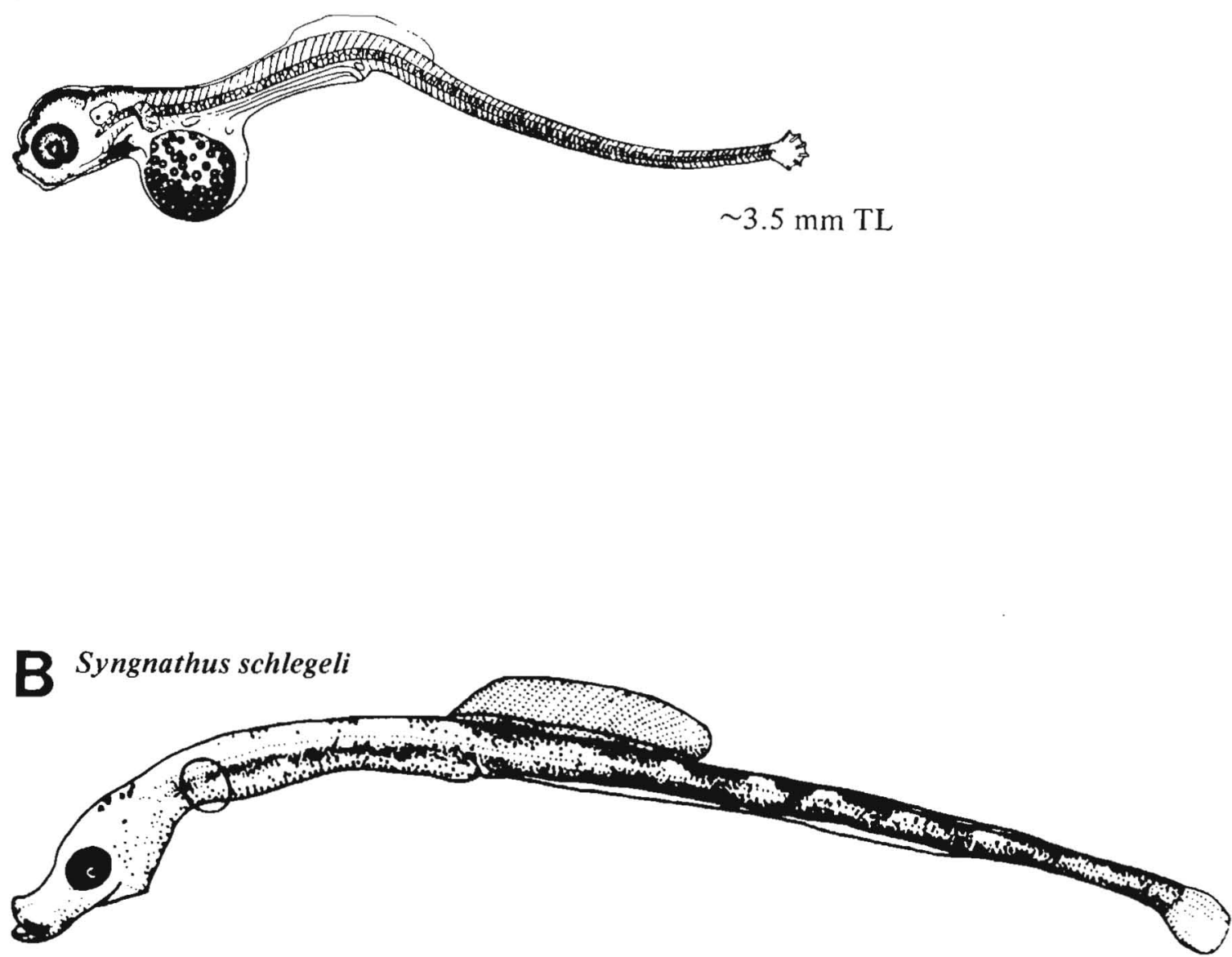

Figure A, Ryder 1887 (reversed, Atlantic specimen from brooding pouch); B, Chung 1977 (western Pacific specimen). 


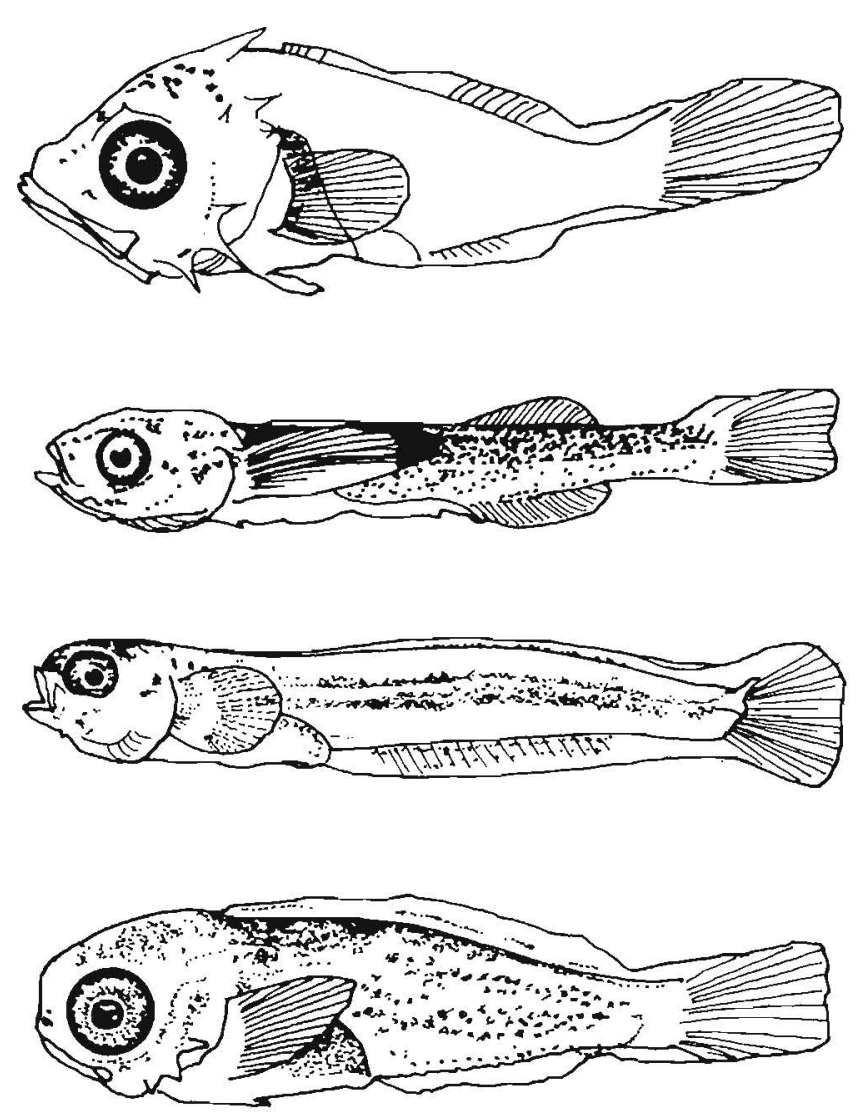

\section{Scorpaeniformes}

Most scorpaeniforms are bottom-oriented and have large heads, large rounded pectoral fins, rounded caudal fins, and many have head spines or bony plates (or combinations thereof). One character unites the group: a bony suborbital stay extending from the third suborbital bone to the opercle. Within the Scorpaeniformes, there are 20 families, 269 genera, and about 1160 species (J. Nelson 1984). The order is usually divided into four suborders: Scorpaenoidei, Anoplopomatoidei, Hexagrammoidei, and Cottoidei. Although members of the order are distributed worldwide, more than 250 species occur in the North Pacific Ocean and Bering Sea of which 190 are endemic.

Reproduction is oviparous except in Sebastes where eggs are internally fertilized and live feeding young are extruded. Larvae are extremely diverse. Head spines are common in larval scorpaenoids and cottoids.

Families in study area: Scorpaenidae

Anoplopomatidae

Hexagrammidae

Cottidae

Agonidae

Cyclopteridae 


\section{Scorpaenidae}

In the study area this speciose family consists of Sebastes, with about 40 species, and Sebastolobus with 3 species. The larvae can be identified to genus, but identification is presently possible for only a few of the species of Sebastes. Information is included here which will aid in identifying species for which early-life-history series are known, and also characters are presented which may prove useful in identifying species whose larvae are not yet known. Full text pages are provided for those species for which an illustration is available of a postflexion larva and at least one other stage. Species with illustrations of only an extrusion larva or pelagic juvenile are treated in the comparative sections. For Sebastes species accounts, a section on head spines has been substituted for the egg section, and counts of lateral line pores and total gill rakers have been added to the meristic section.

\section{Sebastinae}

Among the 70 or so species of Sebastes in the Northeast Pacific, 40 species are known to occur in the study area (Table 29). Meristic information on the following pages has been updated from recent sources (Chen 1986; Laroche, in prep.). Sebastes are ovoviviparous (or viviparous ${ }^{1}$ ) and give birth to live young which are similar in stage of development to firstfeeding larvae of oviparous scorpaenids. Eggs, generally between 0.75 and $1.9 \mathrm{~mm}$ diameter, are retained in the lumen of the ovary after ovulation and internal fertilization. Eggs possess a homogeneous yolk, narrow perivitelline space, fragile smooth chorion, and one to many oil globules. Hatching precedes extrusion with newborn larvae, 3.8-7.5 mm SL, having already utilized most of their yolk.

Newly extruded larvae have functional eyes, jaws, and pectoral fins. Notochord flexion occurs at about 6-12 mm SL and transformation (acquisition of adult complement of fin rays) occurs at about 15-25 mm SL. Many species have a distinct pelagic juvenile stage that may last until about $60 \mathrm{~mm}$ SL. Preflexion larvae have relatively slender bodies and compact guts (13-23\% SL). Snout-to-anus length may increase from $40-50 \%$ SL to $>60 \%$ SL in some species during the larval period. Preflexion larvae usually have pigment over the gut and a series of postanal ventral melanophores. Some species may have pigment along the dorsal midline which develops gradually. Other pigment may occur on the brain, jaws, opercle, fins (especially pectorals), and caudal peduncle.

A prominent feature in Sebastes larvae is head spination. Pterotic, parietal, and preopercular spines form before larvae undergo flexion, and other spines appear gradually thereafter. The full complement of head spines generally develops during the larval period, and some spines that develop in the larval period (e.g., pterotic, anterior preopercles, and lower posttemporal, and others in some) are overgrown during the juvenile stage (Washington et al. 1984b).

Larval series of 16 species of Sebastes occurring in our study area are known. Extrusion or yolk-exhaustion larvae have been illustrated for 32 species, and pelagic juveniles have been illustrated for 33 species. Although Sebastes larvae are often abundant in plankton samples from the Northeast Pacific in winter and spring, the specific identity of most cannot presently be determined.

\section{Sebastolobinae}

Sebastolobus is represented by three species in the study area. Eggs are extruded in bilobed gelatinous masses which float at the surface. The eggs are slightly elliptical and have homogeneous yolk, a narrow perivitelline space, and a smooth chorion. A single oil globule (0.18-0.20 mm diameter) is present (Pearcy 1962, Moser 1974).

Besides differences in meristic characters, Sebastolobus larvae can be distinguished from those of Sebastes in that preflexion larvae have a postanal band of pigment instead of the ventral and possibly dorsal midline melanophores in Sebastes. A parietal ridge develops in Sebastolobus and terminates in a double spine, the parietal and nuchal. In Sebastes the parietal spine develops singly, usually with a smaller nuchal spine immediately posterior to it. 
Table 29

Distribution, parturition season, and meristic characters of genus Sebastes. Range of counts followed by mode in parentheses. All have modal counts of 13 dorsal spines (except $S$. polyspinis with 14") and 3 anal spines (Chen 1986).

\begin{tabular}{|c|c|c|c|c|c|c|c|}
\hline \multirow{3}{*}{$\frac{\text { Taxon }}{\text { S. aleutianus }}$} & \multirow{3}{*}{$\begin{array}{c}\text { Distribution } \\
\text { S. Calif.-Bering Sea }\end{array}$} & \multirow{3}{*}{$\frac{\text { Parturition }}{r^{b}}$} & \multicolumn{3}{|c|}{ Fin rays } & \multirow{2}{*}{$\begin{array}{l}\text { Total gill rakers } \\
\text { on first arch }\end{array}$} & \multirow{2}{*}{$\begin{array}{l}\text { No. of lateral } \\
\text { line pores }\end{array}$} \\
\hline & & & \multirow{2}{*}{$\begin{array}{c}\text { Dorsal } \\
13-15(14)\end{array}$} & Anal & \multirow{2}{*}{$\begin{array}{c}\text { Pectoral } \\
17-19(18)\end{array}$} & & \\
\hline & & & & $7-8 \quad(7)$ & & $28-33(3 \mathrm{I})$ & $30-33$ \\
\hline S. alutus & S. Calif.-Bering Sea & Jan-May ${ }^{c}$ & $13-16(15)$ & $7-9 \quad(8)$ & $17-19(18)$ & $34-39(35 / 38)$ & $46-55$ \\
\hline S. auriculatus & SSC-SE Alaska & Mar-June $^{d}$ & $12-14(13)$ & $6-7 \quad(7)$ & $16-19(18)$ & $25-29(27)$ & $42-50$ \\
\hline S. aurora & S. Calif.-Brit. Col. & Mar-May ${ }^{e}$ & $12-13(13)$ & $5-7 \quad(6)$ & $17-19(17)$ & $24-28(27)$ & $28-31$ \\
\hline S. babcocki & S. Calif.-Bering Sea & Apr-May ${ }^{b}$ & $13-15(14)$ & $6-8 \quad(7)$ & $17-20(19)$ & $29-33(31)$ & $42-51$ \\
\hline S. borealis & Cent. Calif.-Bering Sea & $\mathrm{Apr}^{\mathrm{c}}$ & $12-15(13)$ & $6-8 \quad(7)$ & $17-20(19)$ & $27-31(30)$ & $28-32$ \\
\hline S. brevispinis & SSC-Bering Sea & June $^{b}$, June-July $^{c}$ & $13-15(14)$ & $7-7 \quad(7)$ & $17-18(18)$ & $33-36(33)$ & $46-51$ \\
\hline S. caurinus & SSC-Gulf of Alaska & $\mathrm{Apr}^{\mathrm{b}}$ & $11-14(13)$ & $5-7 \quad(6)$ & $16-18(17)$ & $26-31(28)$ & $39-47$ \\
\hline S. chlorosticrus & SSC-Wash. & July ${ }^{f}$ & $11-13(12)$ & $5-7 \quad(6)$ & $16-18(17)$ & $31-36(33)$ & $35-43$ \\
\hline S. ciliatus & Brit. Col.-Bering Sea & & $14-16(15)$ & $6-9 \quad(8)$ & $17-19(18)$ & $32-35(35)$ & $42-53$ \\
\hline S. crameri & S. Calif.-Bering Sea & $\mathrm{Feb}^{\mathrm{c}}$ & $13-15(13)$ & $7-7 \quad(7)$ & $18-20(19)$ & $30-34(32)$ & $40-51$ \\
\hline S. diploproa & SSC-Gulf of Alaska & May-June $^{b}$, July $^{c}$ & $11-14(12)$ & $5-7 \quad(7)$ & $17-18(18)$ & $32-37(34)$ & $32-42$ \\
\hline S. elongatus & SSC-Gulf of Alaska & May-June ${ }^{b}$ & $12-14(13)$ & $6-7 \quad(6)$ & $16-18(17)$ & $29-33(31)$ & $37-47$ \\
\hline S. emphaeus & N. Calif.-Gulf of Alaska & Aug-Sept ${ }^{8}$ & $13-15(14)$ & $6-7 \quad(7)$ & $16-18(17)$ & $37-41(40)$ & $40-46$ \\
\hline S. entomelas & SSC-Gulf of Alaska & $\mathrm{Apr}^{\mathrm{c}}$, Nov-Mar ${ }^{\mathrm{h}}$ & $14-16(15)$ & $7-9 \quad(8)$ & $18-19(18)$ & $34-38(36)$ & $54-60$ \\
\hline S. flavidus & S. Calif.-Gulf of Alaska & $\mathrm{Mar}^{c}$, Nov-Feb & $14-15(14)$ & $7-8 \quad(8)$ & $17-18(18)$ & $34-37(36)$ & $49-55$ \\
\hline S. glaucus & Bering Sea & & $14-17(15)$ & $7-9 \quad(8)$ & $18-20(19)$ & $35-41(38)$ & \\
\hline S. goodei & SSC-Brit. Col. & Dec-Mar ${ }^{h}$ & $13-14(14)$ & $8-9 \quad(8)$ & $16-18(17)$ & $34-39(36)$ & $50-57$ \\
\hline S. helvomaculatus & SSC-Gulf of Alaska & June $^{c}$ & $12-14(13)$ & $6-7 \quad(6)$ & $15-17(16)$ & $28-33(30)$ & $34-45$ \\
\hline S. jordani & SSC-Gulf of Alaska & $\operatorname{Mar}^{c}$, Nov-Mar ${ }^{h}$ & $13-16(14)$ & $9-10(9)$ & $19-22(20)$ & $40-47(44)$ & $53-64$ \\
\hline S. maliger & Cent. Calif.-Gulf of Alaska & $A p r^{c}$ & $11-13(13)$ & $6-7 \quad(7)$ & $16-18(17)$ & $29-33(31)$ & $34-45$ \\
\hline S. melanops & Cent. Calif.-Aleutian Is. & $A p r^{b}$ & $14-15(15)$ & $7-9 \quad(8)$ & $18-19(19)$ & $34-38(37)$ & $47-55$ \\
\hline S. melanostictus & Bering Sea & & $13-14(14)$ & $7-7 \quad(7)$ & $18-18(18)$ & $32-35(33)$ & \\
\hline S. melanostomus & SSC-Wash. & & $12-14(13)$ & $6-8 \quad(7)$ & $18-20(19)$ & $30-34(31)$ & $28-33$ \\
\hline S. miniatus & SSC-Brit. Col. & Nov-Mar ${ }^{h}$ & $13-15(14)$ & $6-7 \quad(7)$ & $17-18(18)$ & $36-42(41)$ & $41-48$ \\
\hline S. mystinus & SSC-Aleutian Is. & Nov-Jan ${ }^{i}$ & $15-17(\mathrm{I} 6)$ & $8-10(9)$ & $17-19(18)$ & $32-38(35)$ & $47-53$ \\
\hline S. nebulosus & S. Calif.-SE Alaska & & $12-14(13)$ & $6-7 \quad(7)$ & $17-18(18)$ & $27-31(28)$ & $38-43$ \\
\hline S. nigrocinctus & Cent. Calif.-SE Alaska & May $^{c}$ & $12-15(14)$ & $6-7 \quad(7)$ & $18-19(19)$ & $28-32(28)$ & $36-46^{k}$ \\
\hline S. paucispinis & SSC-Gulf of Alaska & Jan-Feb ${ }^{c}$, Nov-Apr ${ }^{j}$ & $13-15(14)$ & $8-10(9)$ & $14-16(15)$ & $27-32(28)$ & $51-62$ \\
\hline S. pinniger & SSC-Gulf of Alaska & Jan-Mar ${ }^{c}$, Nov-Mar ${ }^{\prime}$ & $13-15(14)$ & $7-7 \quad(7)$ & $16-18(17)$ & $41-44(43)$ & $40-47$ \\
\hline S. polyspinis & Brit. Col.-Bering Sea & & $13-16(15)$ & $7-9 \quad(8)$ & $17-19(18)$ & $35-39(36)$ & $43-53$ \\
\hline$S$. proriger & S. Calif.-Bering Sea & July ${ }^{c}$ & $14-15(15)$ & $7-7 \quad(7)$ & $16-17(17)$ & $36-41(38)$ & $48-55$ \\
\hline$S$. rastrelliger & SSC-Oregon & & $12-13(13)$ & $6-6 \quad(6)$ & $18-20(19)$ & $17-25(23)$ & $40-49$ \\
\hline S. reedi & N. Calif.-SE Alaska & $A p r^{c}$ & $13-15(14)$ & $7-8 \quad(7)$ & $18-20(19)$ & $30-36(34)$ & $47-55$ \\
\hline S. ruberrimus & SSC-Gulf of Alaska & June $^{b}$, July ${ }^{c}$ & $14-16(15)$ & $7-7 \quad(7)$ & $18-19(19)$ & $26-30(29 / 30)$ & $39-45$ \\
\hline S. rufus & SSC-Oregon & Dec-May ${ }^{e}$ & $13-16(15)$ & $8-9$ & $17-19(18)$ & $32-37(35)$ & $49-56$ \\
\hline S. saxicola & SSC-Gulf of Alaska & $\mathrm{Feb}^{\mathrm{c}}$, Nov-Mar ${ }^{h}$ & $11-13(12)$ & $6-7 \quad(7)$ & $16-18(16)$ & $30-35(32)$ & $35-43$ \\
\hline S. variegatus & Brit. Col.-Gulf of Alaska & $J_{u n e}{ }^{c}$ & $14-15(14)$ & $6-7 \quad(7)$ & $17-19(18)$ & $37-41(38)$ & $43-52$ \\
\hline S. wilsoni & S. Calif.-Gulf of Alaska & June $^{c}$ & $13-15(14)$ & $5-6 \quad(6)$ & $16-18(17)$ & $37-42(39)$ & $37-46$ \\
\hline S. zacentrus & S. Calif.-Gulf of Alaska & July ${ }^{c}$ & $13-15(14)$ & $7-8 \quad(7)$ & $16-18(17)$ & $33-37(35)$ & $39-47$ \\
\hline 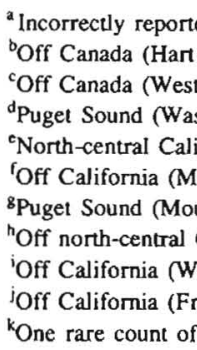 & $\begin{array}{l}\text { s } 13 \text { instead of } 14 \text { in Chen (19 } \\
\text { 3). } \\
\text { im 1975). } \\
\text { gton et al. 1978). } \\
\text { ia (Wyllie Echeverria 1987). } \\
\text { 1967). } \\
\text { 1975). } \\
\text { fornia (Phillips 1964). } \\
\text { 1952). } \\
\text { 1971). }\end{array}$ & & & & & & \\
\hline
\end{tabular}




\section{Head spines}

There are differences in terminology for Sebastes head spines between the two major sources of early-life-history descriptions (i.e., Richardson and Laroche 1979, Moser and Ahlstrom 1978). Most of the differences are semantic; however, the numbering of anterior preopercular spines is a substantive difference (Table 30). Among the species studied to date, posterior preopercular spine 1 has not had an anterior spine associated with it. Therefore, Richardson and Laroche (1979) gave the number 1 to the anterior preopercular spine associated with the second posterior preopercular spine. Moser and Ahlstrom (1978) gave the same numbers to the anterior and posterior preopercular spines. Thus the same anterior preopercular spine is numbered one less by Richardson and Laroche (1979) than it is by Moser and Ahlstrom (1978). Succeeding descriptions by these and other authors perpetuate these differences (e.g., Washington et al. 1984b). Here we use the system of Moser and Ahlstrom (1978).

There are also differences between the Sebastes head spine terminology used for adults and that used for larvae (Table 30). This situation resulted from the difficulty of determining spine/bone associations by external examination of adults and is complicated by the overgrowth of some spines during development (Richardson and Laroche 1979). The major points of confusion are that the supracleithral of larvae is called the upper posttemporal spine of adults, and the cleithral spine of larvae is called the supracleithral spine of adults. Head spine patterns which may help identify adult Sebastes are listed in Table 31 .

Table 30

Head spine terminology in Sebastes, listed by sequence in which the spines develop in S. melanostomus (Moser and Ahlstrom 1978). Names used by Richardson and Laroche (1979) are listed only when they differ from those used by Moser and Ahlstrom (1978).

\begin{tabular}{|c|c|c|c|c|}
\hline Abbreviation & Moser and Ahlstrom (1978) & Richardson and Laroche (1979) & Bone of origin & Adult spine \\
\hline PA & Parietal & & Parietal & Parietal \\
\hline NU & Nuchal & & Parietal & Nuchal \\
\hline PSO & Postocular & & Frontal & Postocular \\
\hline $\mathbf{P T}$ & Pterotic & & Pterotic & (Overgrown) \\
\hline LPST & Lower posttemporal & Inferior posttemporal & Posttemporal & (Overgrown) \\
\hline UOP & Upper opercular & Superior opercular & Opercle & Opercular \\
\hline U1O-1 & lst upper infraorbital & Superior infraorbutal series, lst & Infraorbital 1 & (Overgrown) \\
\hline LIO-1 & Ist lower infraorbital & Inferior infraorbital series, lst & Infraorbital 1 & Lachrymal projection (suborbital spine) \\
\hline APO-2 & 2nd anterior preopercular & Anterior preopercular series, Ist & Preopercle & (Overgrown) \\
\hline PPO-2 & 2nd posterior preopercular & Posterior preopercular series, 2 nd & Preopercle & Preopercular \\
\hline APO-3 & 3rd anterior preopercular & Anterior preopercular series, 2 nd & Preopercle & (Overgrown) \\
\hline PPO-3 & 3 rd posterior preopercular & Posterior preopercular series, 3 rd & Preopercle & Preopercular \\
\hline $\mathrm{APO}-4$ & 4th anterior preopercular & Anterior preopercular series, 3rd & Preopercle & (Overgrown) \\
\hline PPO-4 & 4th posterior preopercular & Posterior preopercular series, 4 th & Preopercle & Preopercular \\
\hline PPO-1 & lst posterior preopercular & Posterior preopercular series, 1st & Preopercle & Preopercular \\
\hline PPO-5 & 5th posterior preopercular & Posterior preopercular series, 5th & Preopercle & Preopercular \\
\hline чло-4 & 4th upper infraorbital & Superior infraorbital series, $4 \mathrm{th}$ & Infraorbital 3 & (Overgrown) \\
\hline SC & Supracleithral & & Supracleithrum & Cleithral \\
\hline LIO-2 & 2nd lower infraorbital & Inferior infraorbital series, 2nd & Infraorbital 1 & Lachrymal projection (suborbital spine) \\
\hline LIO-3 & & Inferior infraorbital series, 3rd & Infraorbital 1 & (Overgrown) \\
\hline LOP & Lower opercular & Inferior opercular & Opercle & Opercular \\
\hline UIO-2 & 2nd upper infraorbital & Superior infraorbital series, 2nd & Infraorbital 1 & (Overgrown) \\
\hline UPST & Upper posttemporal & Superior posttemporal & Posttemporal & Supracleithral \\
\hline SPO & Supraocular & & Frontal & Supraocular \\
\hline U10-3 & 3rd upper infraorbital & Superior infraorbital series, 3rd & Infraotbital 2 & (Overgrown) \\
\hline NA & Nasal & & Nasal & Nasal \\
\hline PRO & Preocular & & Lateral ethmoid (prefrontal) & Preocular \\
\hline CL & Cleithral & & Cleithrum & (Overgrown) \\
\hline IOP & Interopercular & & Interopercular (interopercle) & Gill cover spine \\
\hline TM & Tympanic & & Frontal & Tympanic \\
\hline SOP & Subopercular & & Subopercular (subopercle) & Gill cover spine \\
\hline $\mathrm{CO}$ & Coronal & & Frontal & Coronal \\
\hline
\end{tabular}




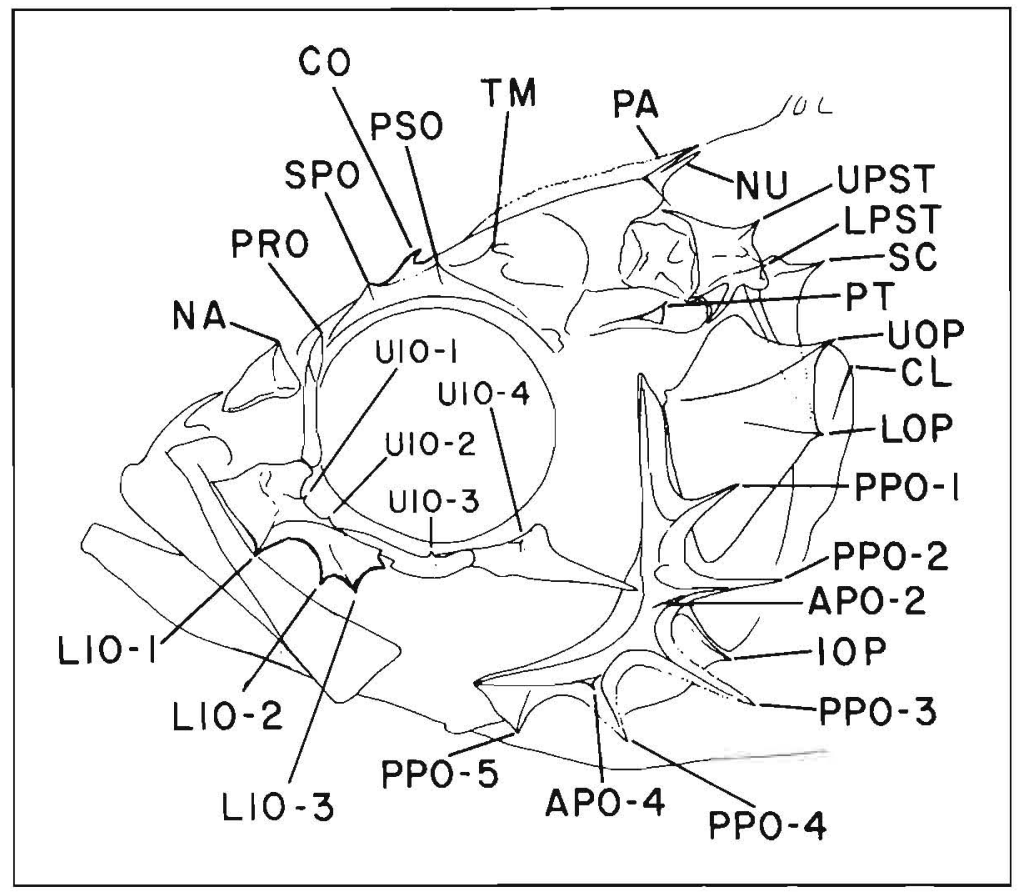

Positions and abbreviations of larval head spines in larval Sebastes. Based on a 16.0-mm stained larva of Sebastes melanostomus augmented to show the position of the coronal (CO) and 3rd lower infraorbital (LIO-3) spines. From Moser and Ablstrom (1978) and Laroche (in prep.).

\begin{tabular}{|c|c|c|c|c|c|c|c|c|c|c|c|}
\hline \multicolumn{12}{|c|}{$\begin{array}{l}\text { Table } 31 \\
\text { Presence and absence of head spines which are diagnostic for adults of members of the genus Sebastes } \\
(+ \text { indicates presence, } 0 \text { indicates absence, blank indicates unknown character state). }\end{array}$} \\
\hline & $\begin{array}{l}\text { Pre- } \\
\text { ocular }\end{array}$ & $\begin{array}{l}\text { Supra- } \\
\text { ocular }\end{array}$ & $\begin{array}{l}\text { Post- } \\
\text { ocular }\end{array}$ & Tympanic & Coronal & & $\begin{array}{c}\text { Pre- } \\
\text { ocular }\end{array}$ & $\begin{array}{l}\text { Supra- } \\
\text { ocular }\end{array}$ & $\begin{array}{l}\text { Post- } \\
\text { ocular }\end{array}$ & Tympanic & Coronal \\
\hline S. aleutianus & + & + & + & + & + & S. maliger & + & 0 & + & + & 0 \\
\hline S. alutus & + & + & + & + & 0 & S. melanops & 0 & 0 & + & + & 0 \\
\hline S. auriculatus & + & 0 & + & + & + & S. melanostictus & & & + & + & \\
\hline S. aurora & + & + & + & + & 0 & S. melanostomus & + & + & + & + & 0 \\
\hline S. babcocki & + & 0 & + & + & 0 & S. miniatus & + & + & + & + & 0 \\
\hline S. borealis & + & + & + & + & + & S. mystinus & + & + & + & + & 0 \\
\hline S. brevispinis & 0 & 0 & + & + & 0 & S. nebulosus & + & 0 & + & + & 0 \\
\hline S. caurinus & + & 0 & + & + & 0 & $S$. nigrocinctus & + & 0 & + & + & + \\
\hline S. chlorosticrus & + & + & + & + & 0 & $S$, paucispinis & + & 0 & 0 & + & 0 \\
\hline S. ciliatus & 0 & + & + & + & 0 & $S$. pinniger & + & + & + & + & 0 \\
\hline S. crameri & + & + & + & + & 0 & S. polyspinis & 0 & 0 & + & + & 0 \\
\hline S. diploproa & + & 0 & + & + & 0 & $S$. proriger & + & 0 & + & + & 0 \\
\hline S. elongatus & + & 0 & + & + & 0 & S. rastrelliger & + & 0 & + & + & 0 \\
\hline S. emphaeus & + & 0 & + & + & 0 & S. reedi & + & + & + & + & 0 \\
\hline S. entomelas & + & + & + & + & 0 & S. ruberrimus & + & + & + & + & 0 \\
\hline S. flavidus & 0 & 0 & + & + & 0 & S. rufus & & & + & + & 0 \\
\hline S. glaucus & & & + & + & 0 & S. saxicola & + & 0 & + & + & 0 \\
\hline$S$. goodei & 0 & 0 & + & 0 & 0 & S. variegatus & + & 0 & + & + & 0 \\
\hline S. helvomaculatus & + & + & + & + & 0 & S. wilsoni & + & 0 & + & + & 0 \\
\hline S. jordani & + & 0 & + & + & 0 & S. zacentrus & + & 0 & + & + & 0 \\
\hline
\end{tabular}




\section{Extrusion or Yolk-Exhaustion Larvae}

Various authors have illustrated and described larvae which have been extruded from pregnant female Sebastes. In some cases, the larvae have been maintained in seawater for several days after extrusion, and in a few cases (e.g., Stahl-Johnson 1985, Moser and Butler 1987) the larvae were fed and reared. With handling, pregnant Sebastes will release larvae that are not full-term, and such larvae may be represented in some illustrations. Furthermore, some illustrations were drawn by adding observed pigmentation for a particular species to a general outline of an extrusion Sebastes larva (c.f. Westrheim et al. 1968a). The generalized outline used by Westrheim et al. (e.g., 1968a) and DeLacy et al. (1964) lacked pectoral fins, apparently the result of an oversight. Several illustrations of extrusion larvae have been published for some species, and there are notable differences among some of these illustrations. One of the illustrations of an extrusion larva purported to be Sebastes zacentrus by Harling et al. (1971) was obviously misidentified, as discussed by Laroche and Richardson (1981). With these problems in mind, pages 273-280 present all of the published, and some previously unpublished, illustrations of extrusion larvae of Sebastes spp. which occur in the study area, not so much for use in species identifications but as reference for further research. 


\section{EXTRUSION OR YOLK-EXHAUSTION LARVAE}

\section{S. aleutianus}

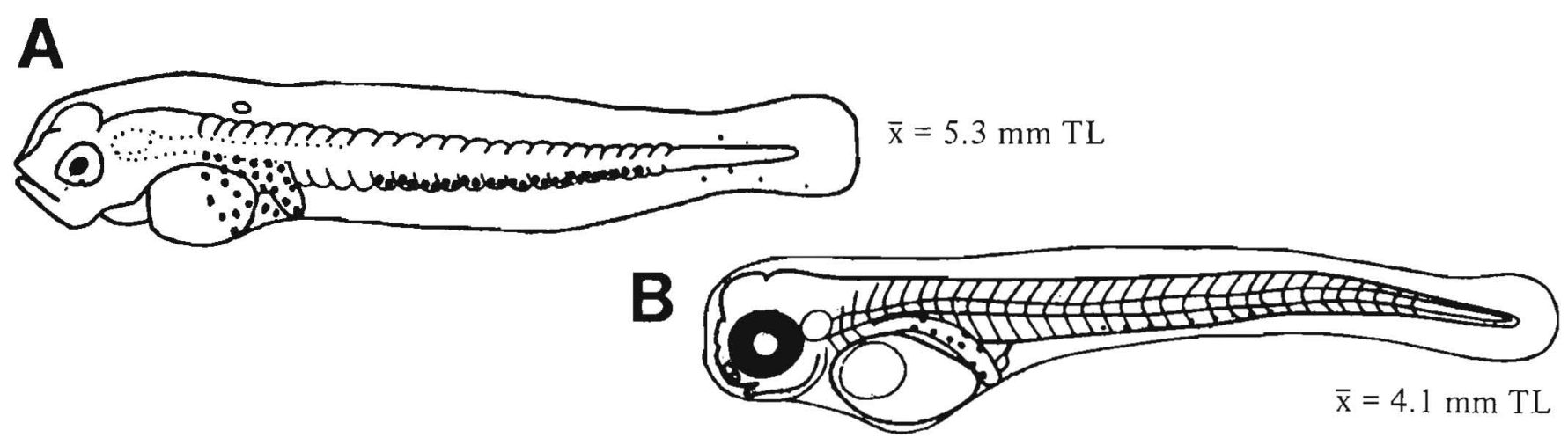

\section{S. alutus}

C

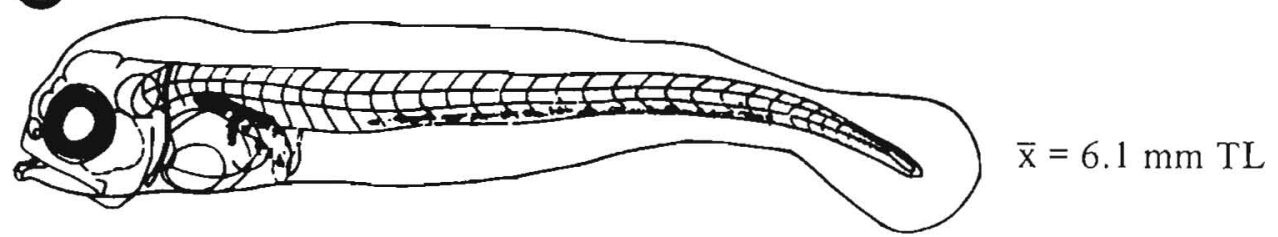

D

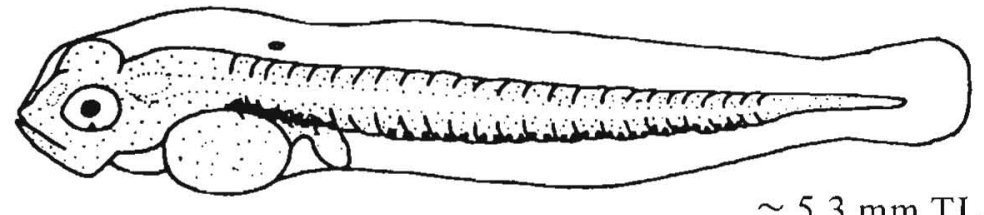

E

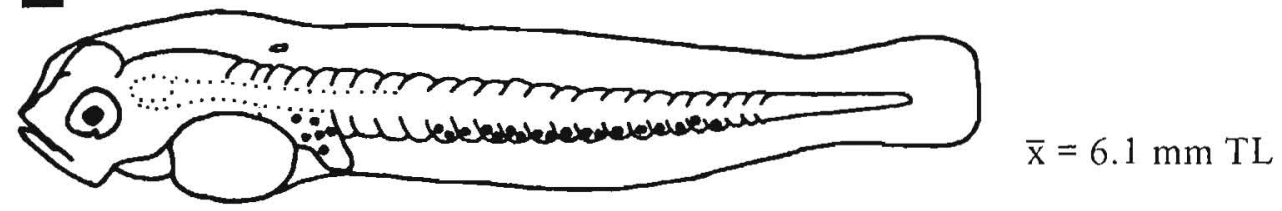

S. auriculatus

드

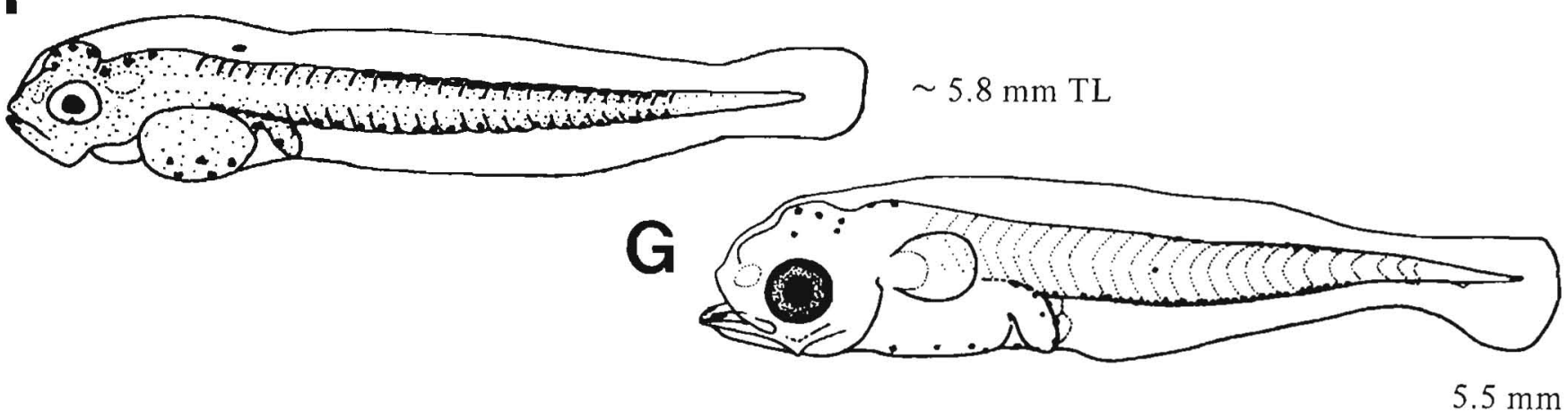

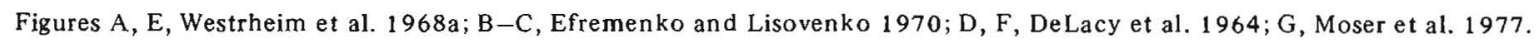




\section{A S. aurora}

\section{EXTRUSION OR YOLK-EXHAUSTION LARVAE}

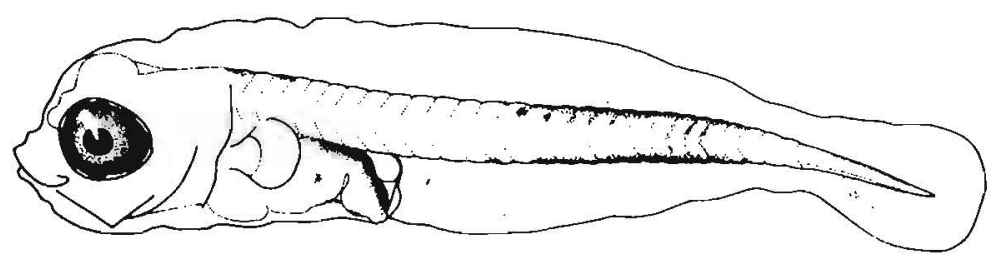

\section{S. babcocki}

\section{B}
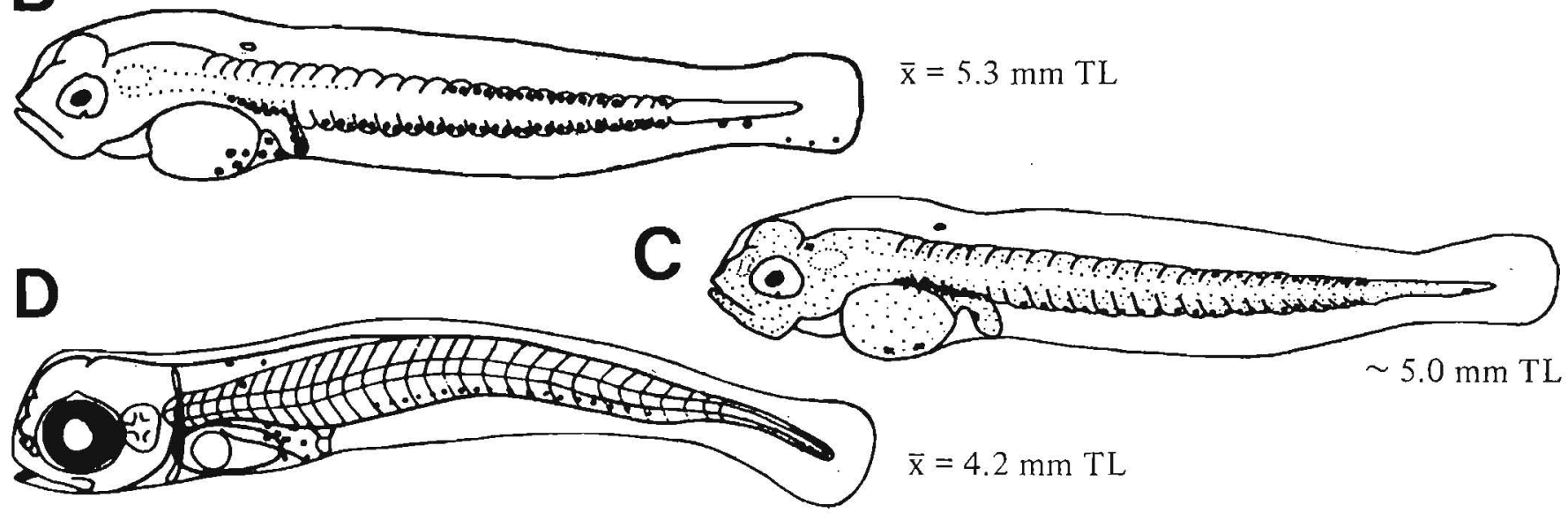

\section{S. brevispinis}

E.
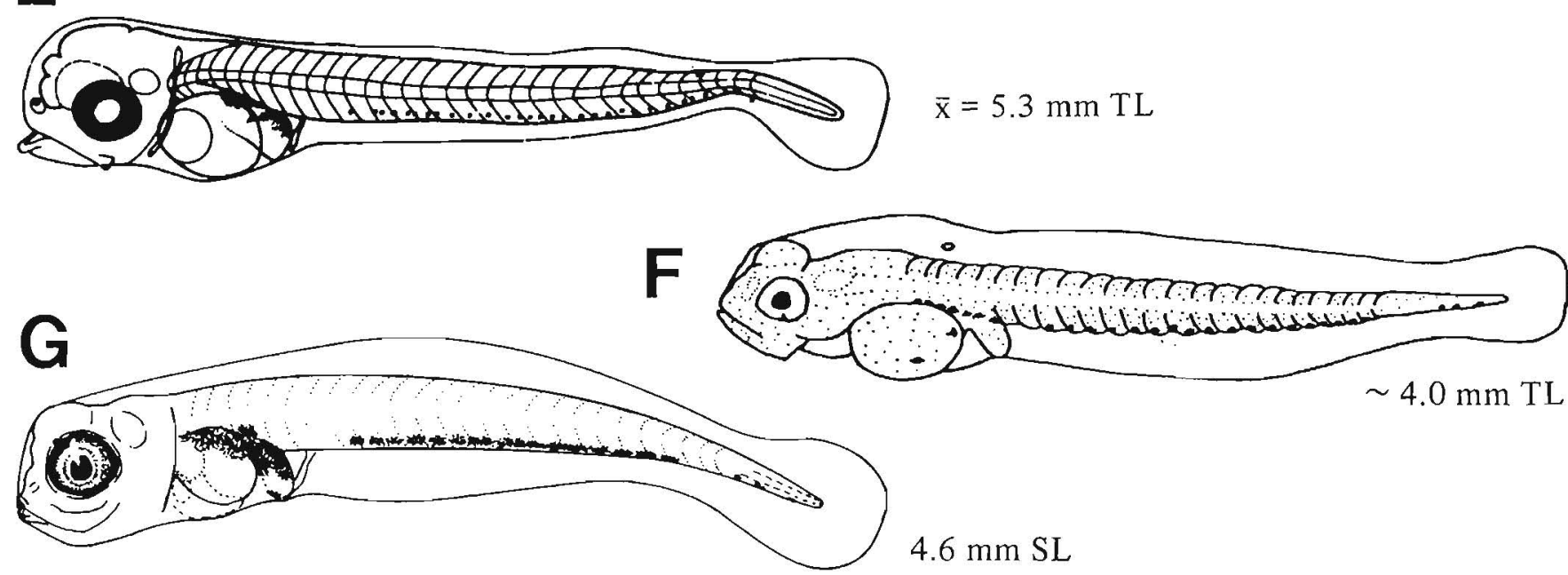


\section{EXTRUSION OR YOLK-EXHAUSTION LARVAE}

\section{S. caurinus}
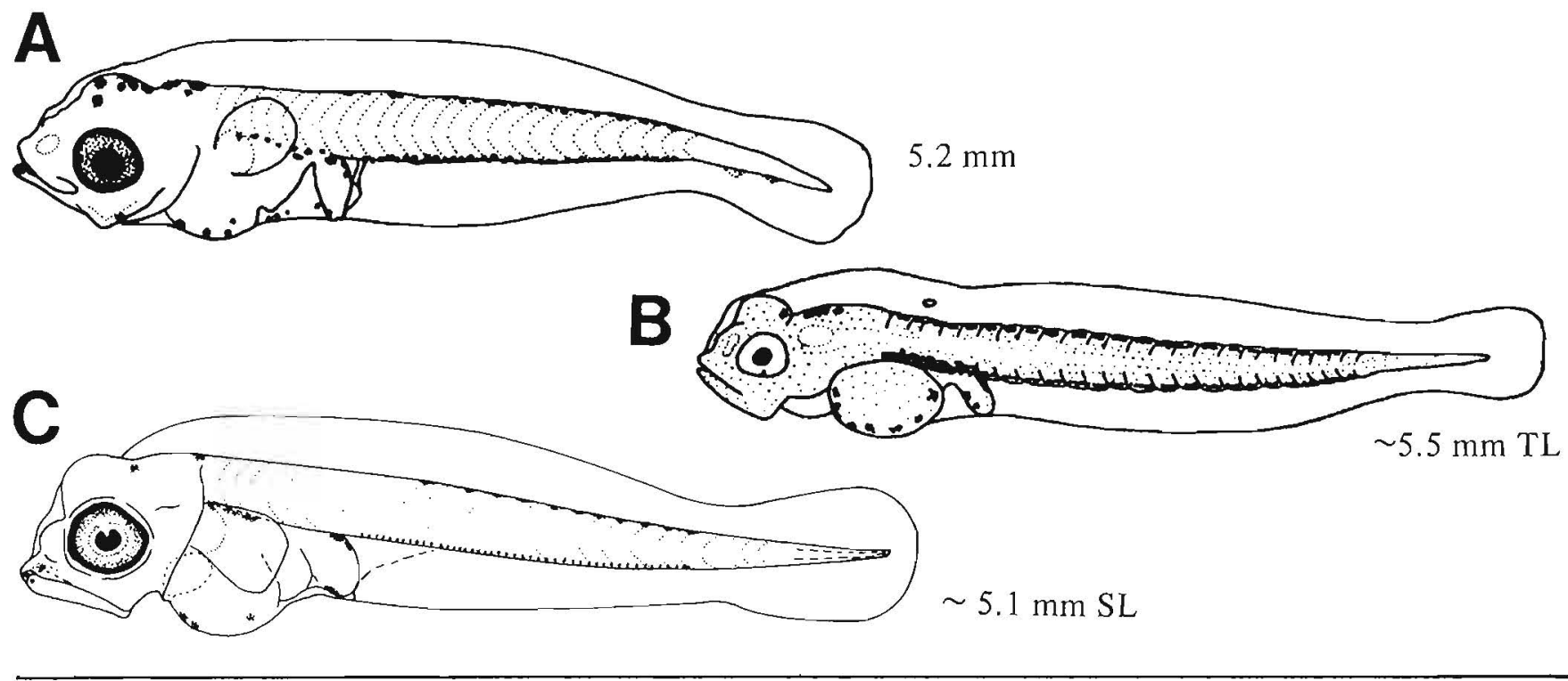

S. chlorostictus

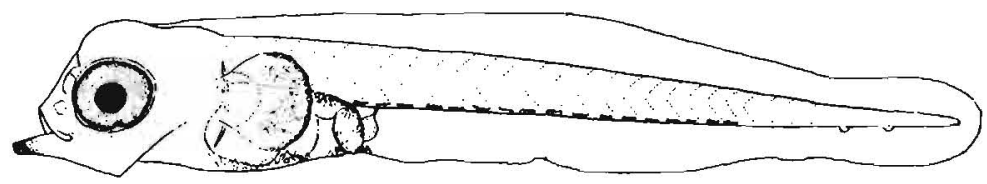

$4.2 \mathrm{~mm}$

\section{S. ciliatus}

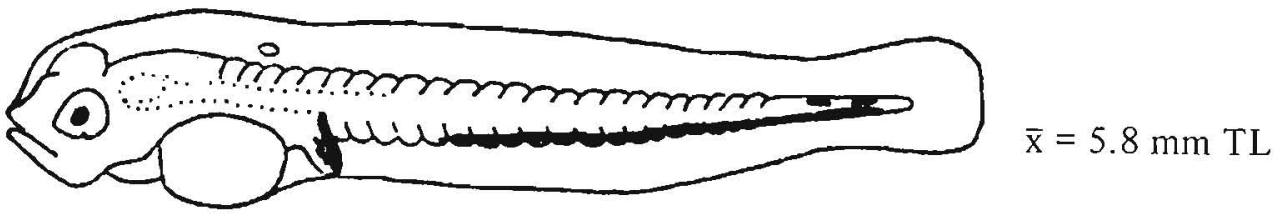

\section{S. crameri}

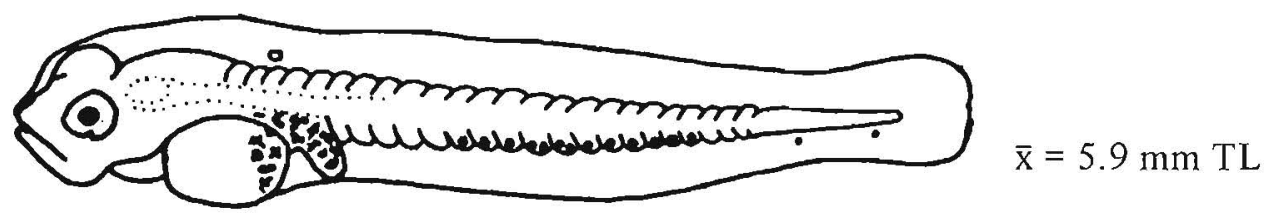




\section{EXTRUSION OR YOLK-EXHAUSTION LARVAE}

\section{S. diploproa}

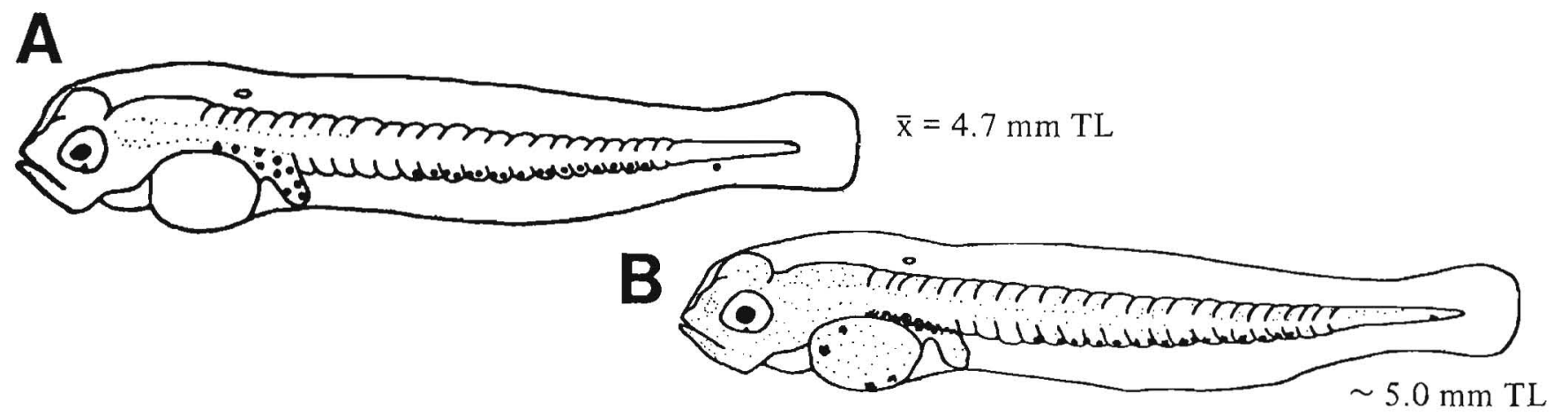

\section{S. elongatus}

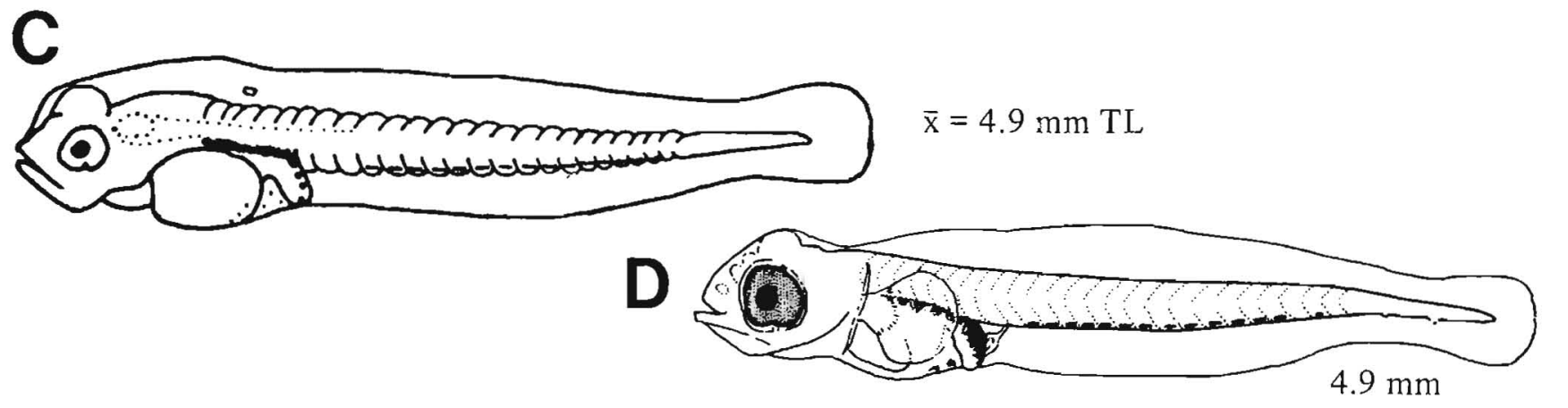

\section{S. entomelas}

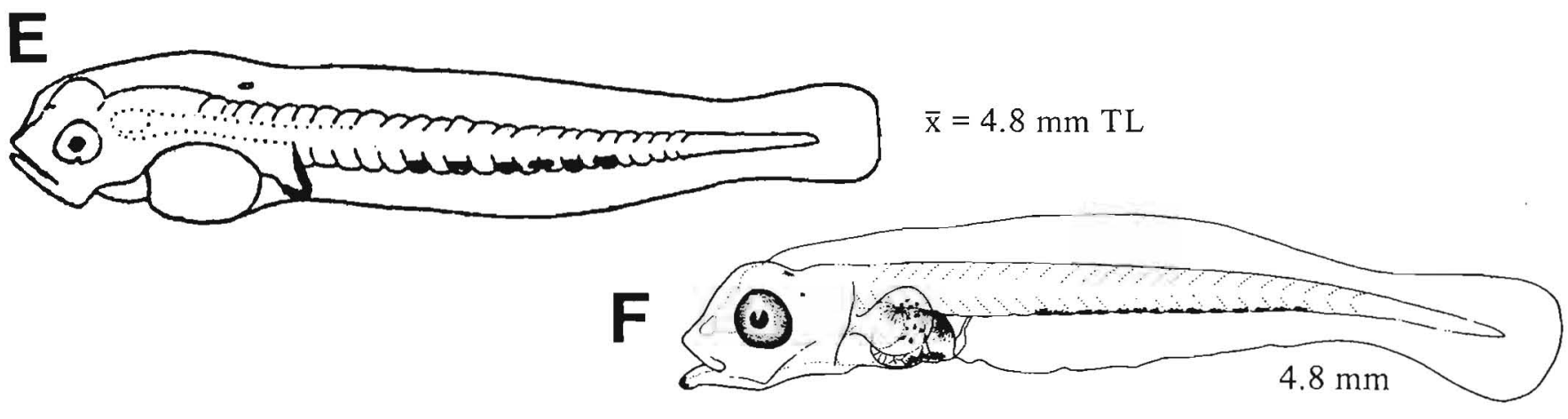

\section{S. flavidus}

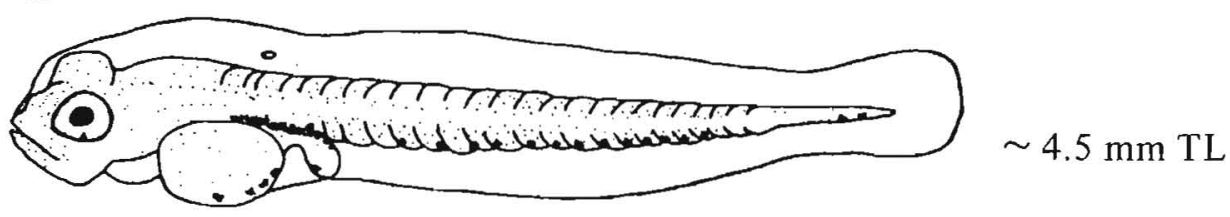




\section{EXTRUSION OR YOLK-EXHAUSTION LARVAE}

\section{A S. goodei}

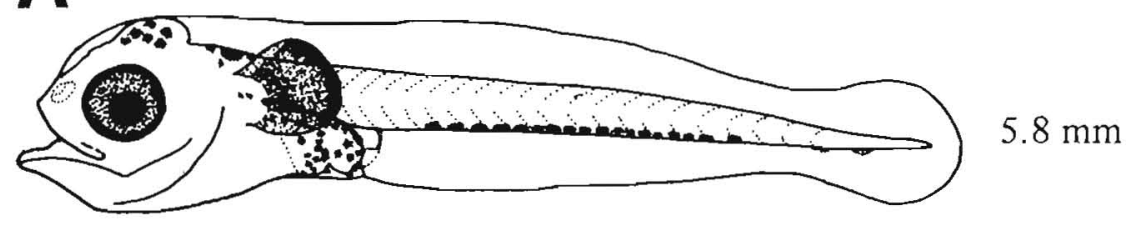

\section{S. helvomaculatus}

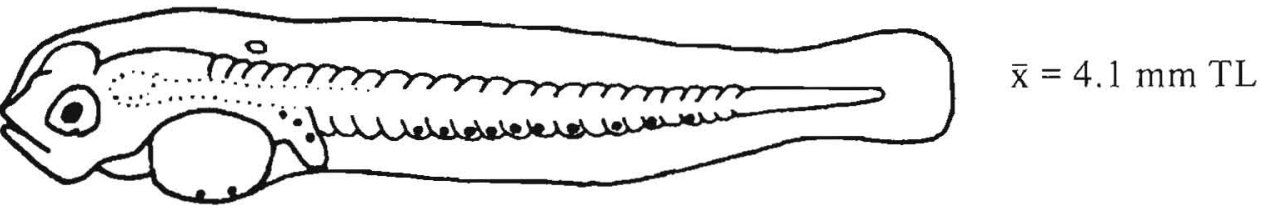

$C^{\text {S. jordani }}$
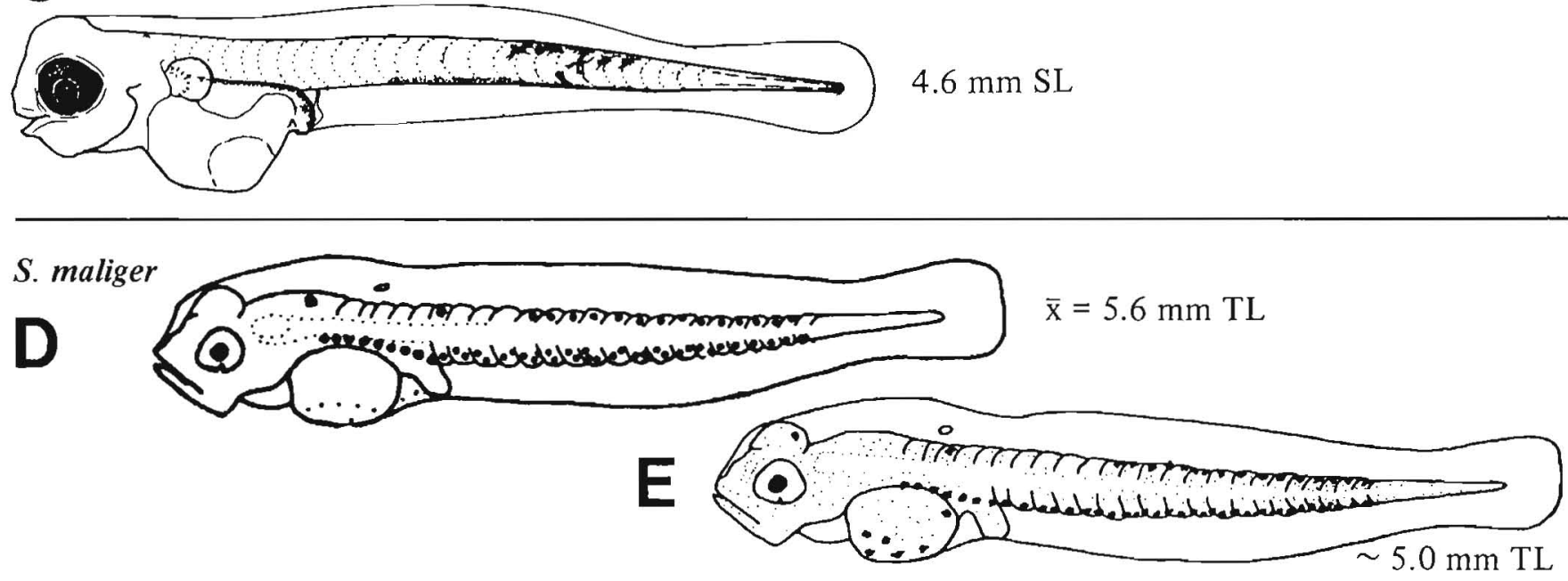

F S. melanops

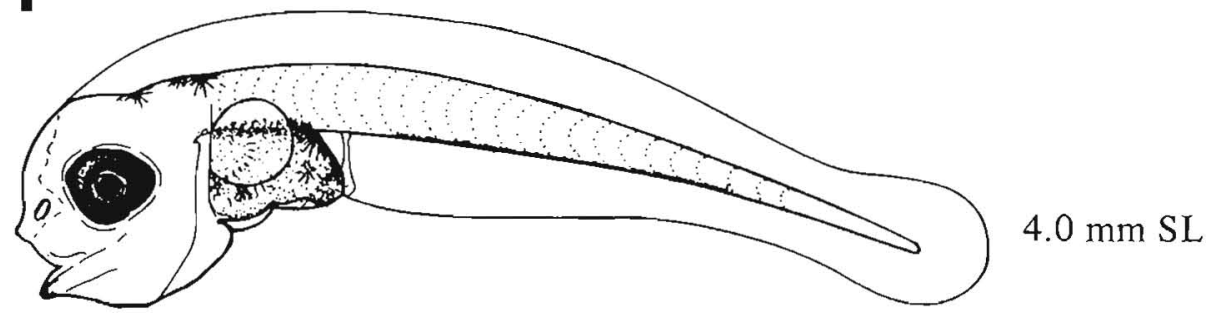

S. melanostomus

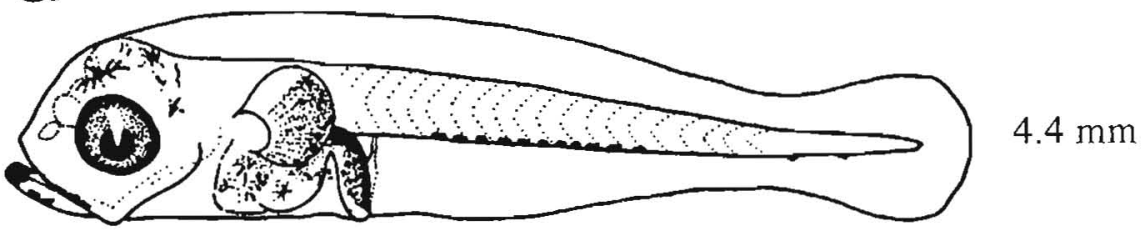

Figures A, G, Moser et al. 1977; B, D, Westrheim et al. 1968b; C, F, NWAFC originals (B. Vinter); E, DeLacy et al. 1964. 
$A^{\text {S. miniatus }}$

\section{EXTRUSION OR YOLK-EXHAUSTION LARVAE}

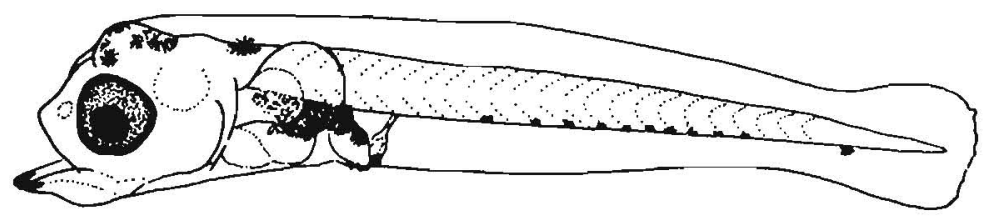

$4.3 \mathrm{~mm}$

\section{S. mystinus}

$803.8 \mathrm{~mm} \mathrm{NL}$

C S.paucispinis

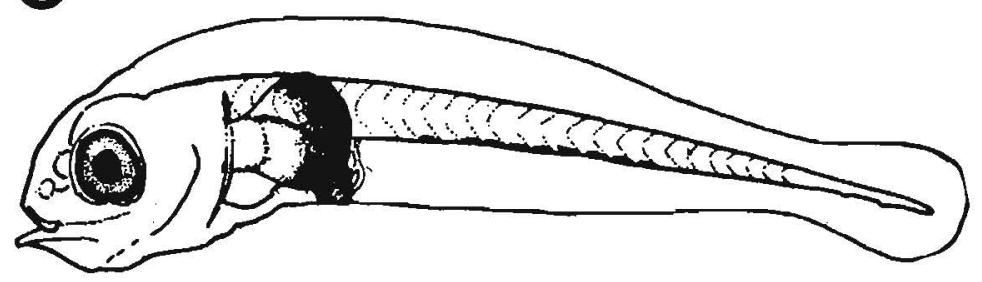

$6.1 \mathrm{~mm}$

D S. pinniger

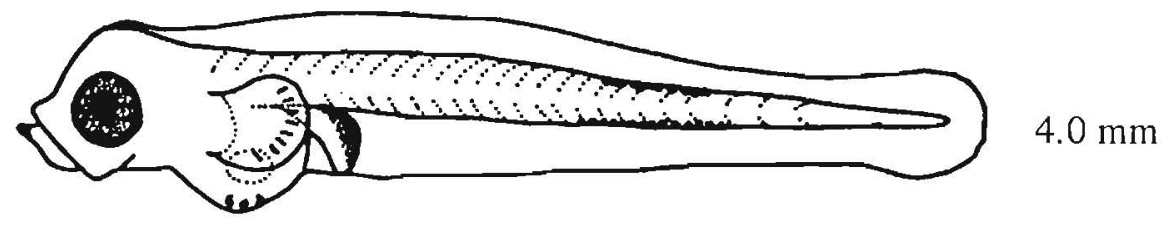

E S. polyspinis

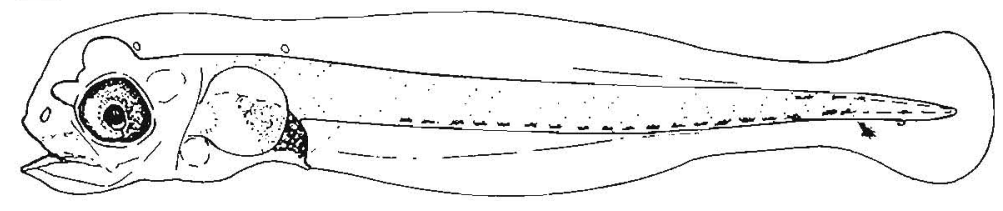

$6.1 \mathrm{~mm} \mathrm{SL}$

E S.proriger

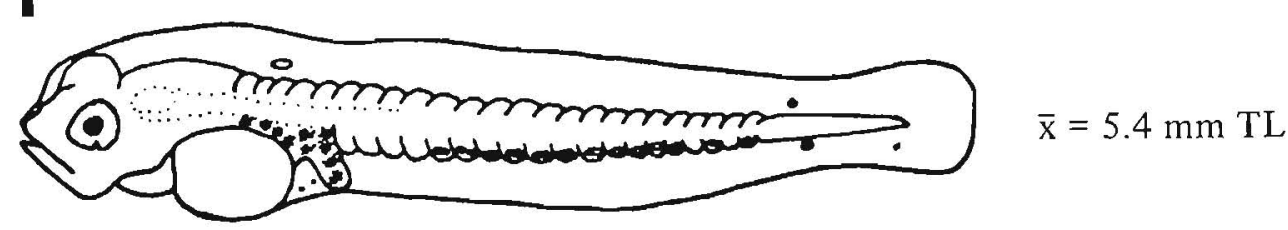

Figures A, C-D, Moser et al. 1977; B, Wold, unpubl.; E, NWA FC original (B. Vinter); F, Westrheim et al. 1968 b. 


\section{EXTRUSION OR YOLK-EXHAUSTION LARVAE}

A S. reedi

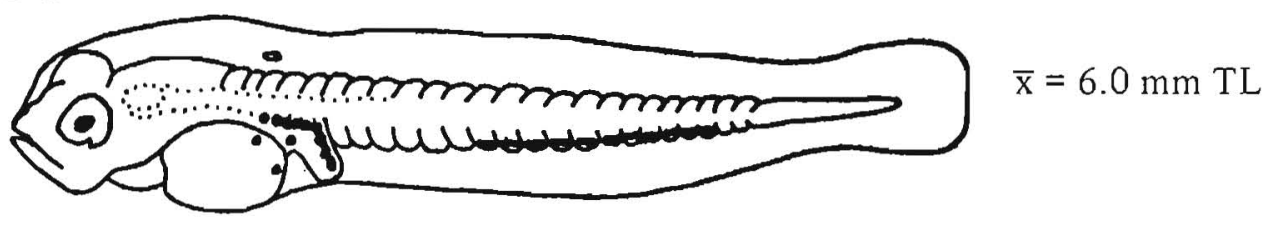

S. ruberrimus

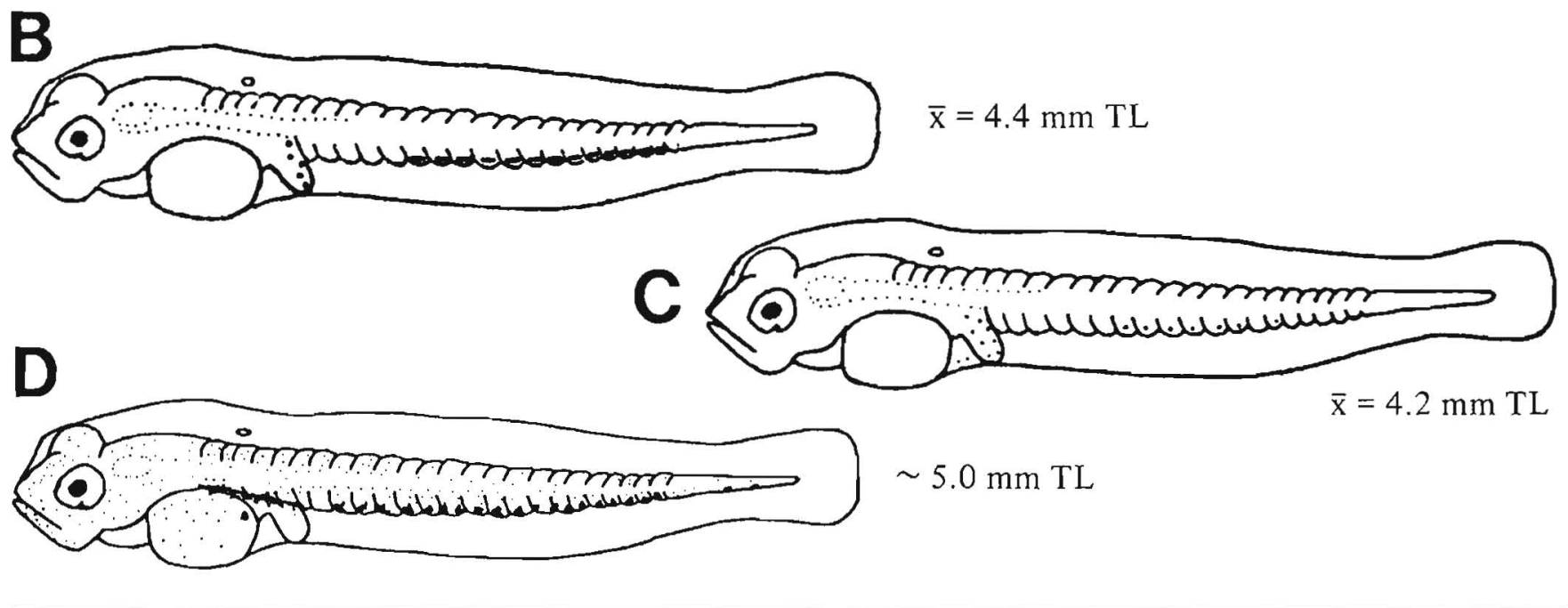

E S. rufus

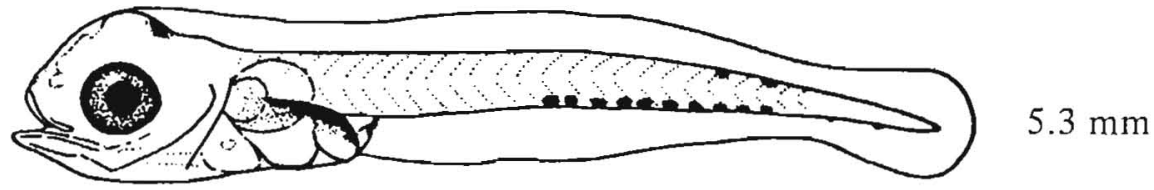

S. saxicola

E
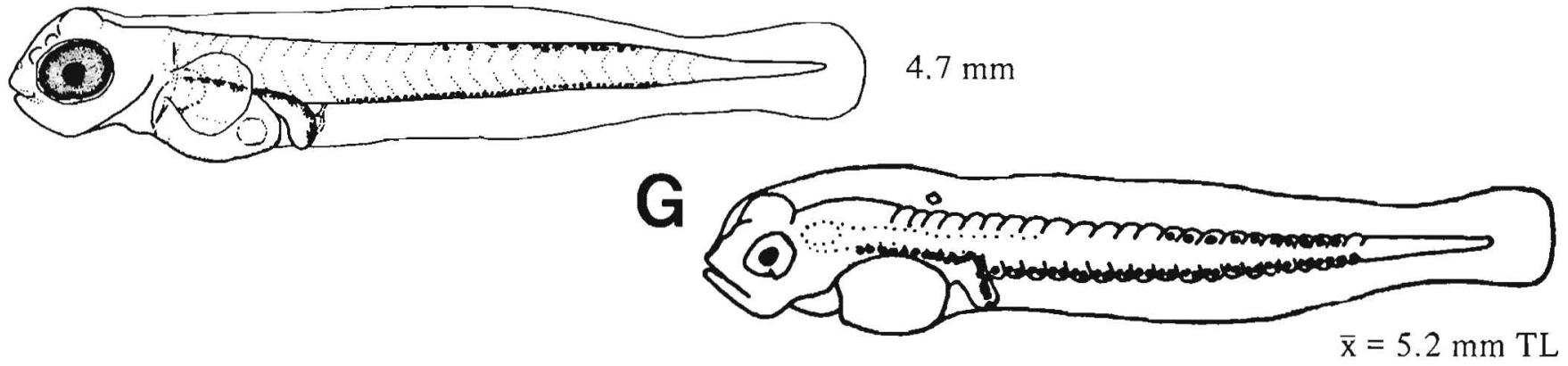

Figures A, G, Westrheim et al. 1968a; B, Harling et al. 1971; C, Westrheim et al. 1968b; D, DeLacy et al. $1964 ;$ E-F, Moser et al. 1977. 


\section{EXTRUSION OR YOLK-EXHAUSTION LARVAE}

\section{S. variegatus}

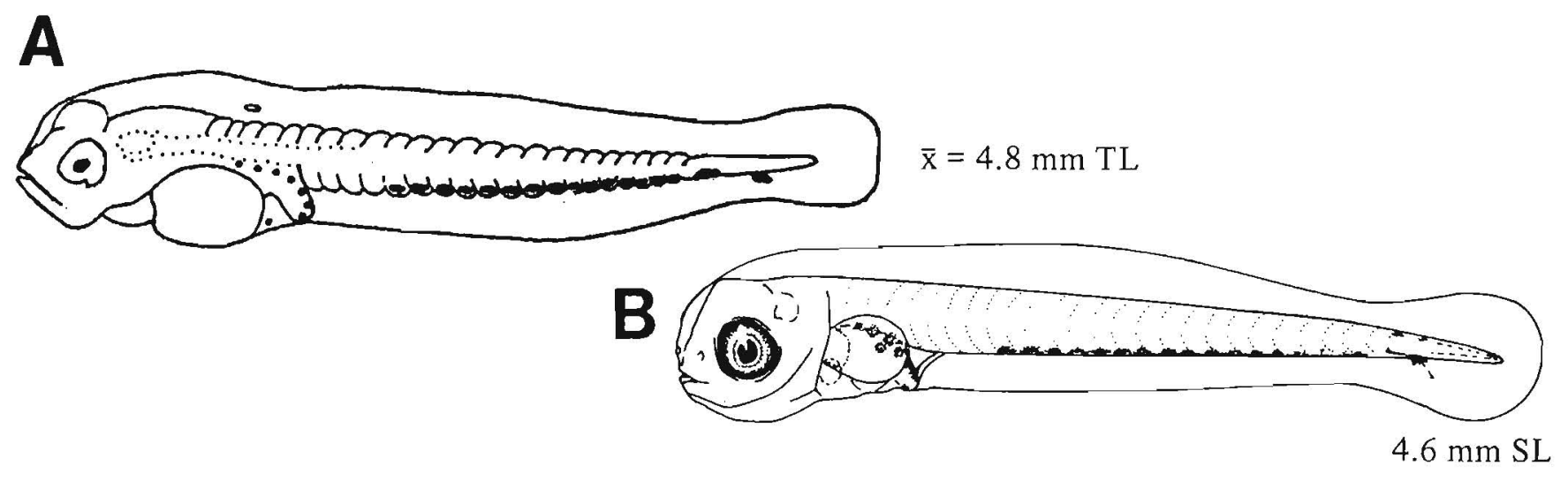

S. zacentrus

C

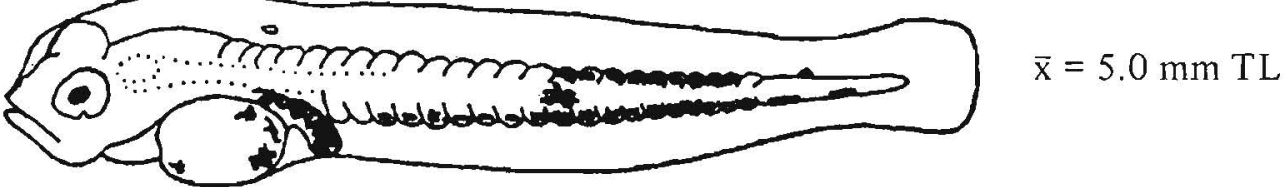

(Misidentified, see Laroche and Richardson 1981)
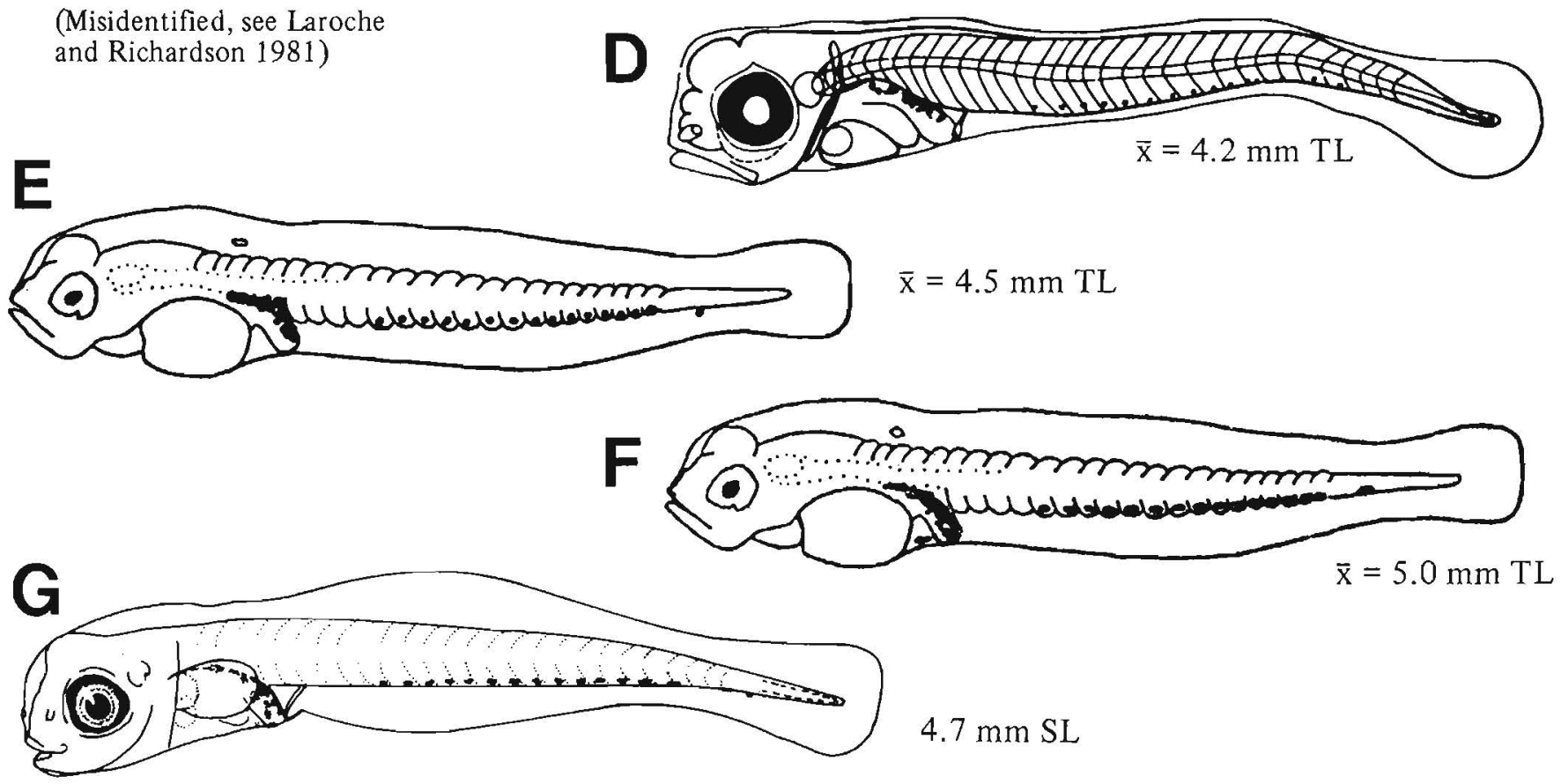


\section{Sebastes Pelagic Juveniles}

The following six pages present illustrations of pelagic juveniles of 33 of the 40 species of Sebastes occurring in the study area. Pelagic juveniles of the remaining species are unknown or have not yet been illustrated. Illustrations are grouped to form a pictorial key based primarily on pigment characters of the juveniles as derived from Laroche (in prep.). Species are grouped on the pages according to predominant pigment characters; within these groups, species are separated according to other pigment characters. Several species possess identical states for these characters. Identification of these will require use of additional characters such as meristics, body shape, head spination, time and location of collection, and other pigment characters. These characters should also be checked on fish separated based on the pictorial key to verify their identity. Variation in pigment can be expected due to size and other differences in specimens of a species. Also, pigment characters of species for which pelagic juveniles are unknown may closely resemble those species illustrated here, which would lead to misidentification unless other characters were examined. 


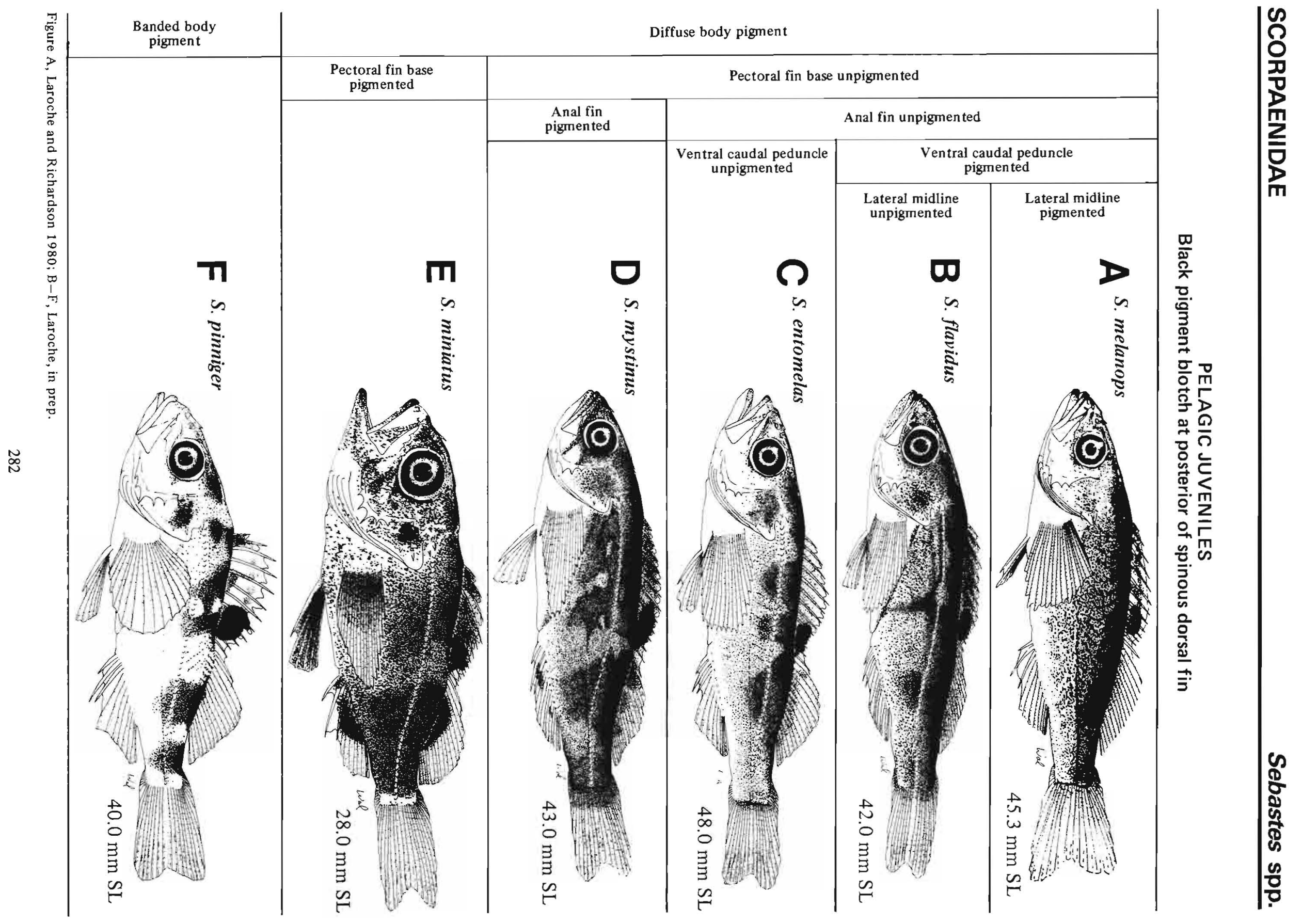


PELAGIC JUVENILES

Fringe of pigment on spinous dorsal fin

Body pigment diffuse

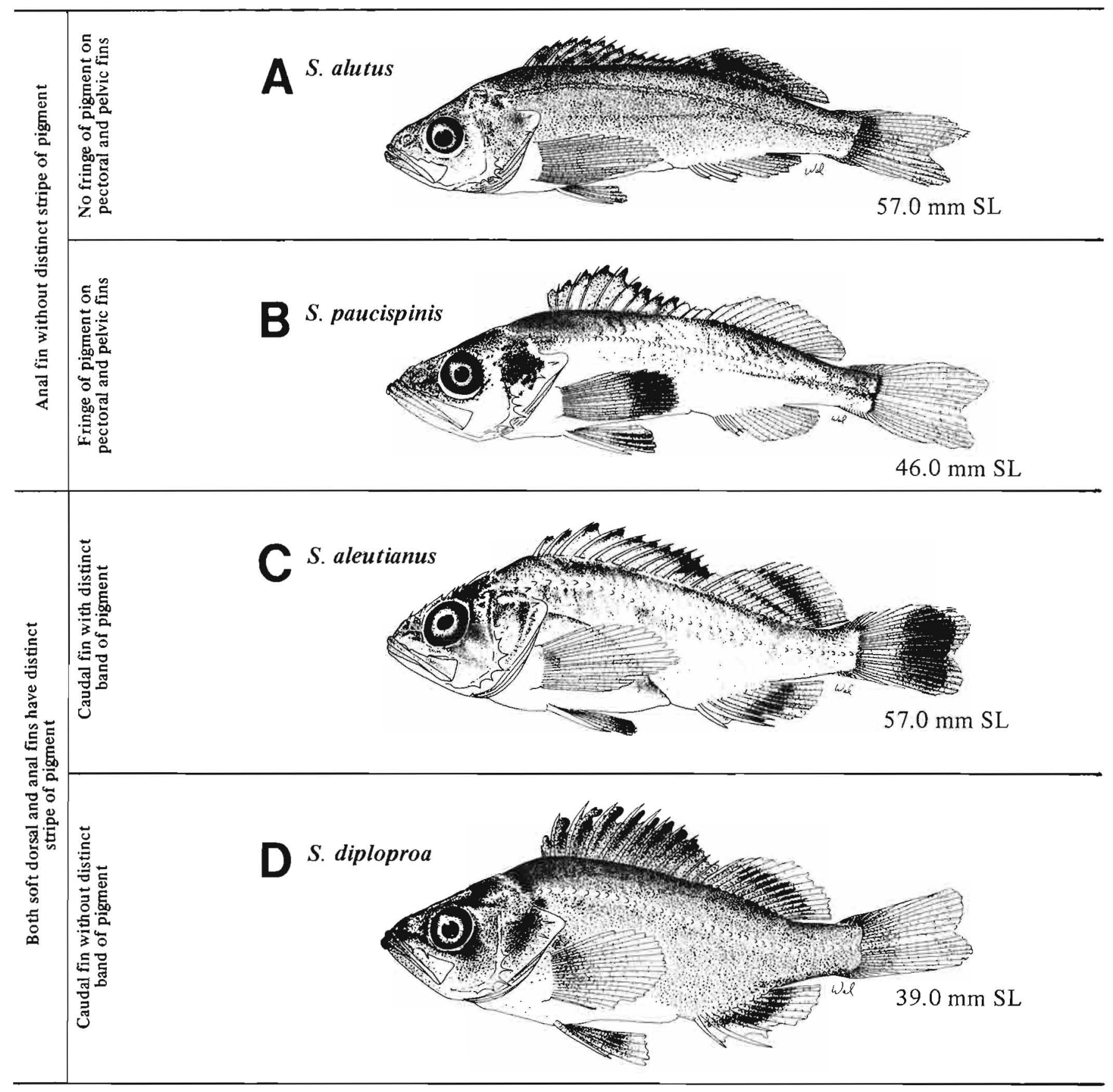

Figures A-D, Laroche, in prep. 


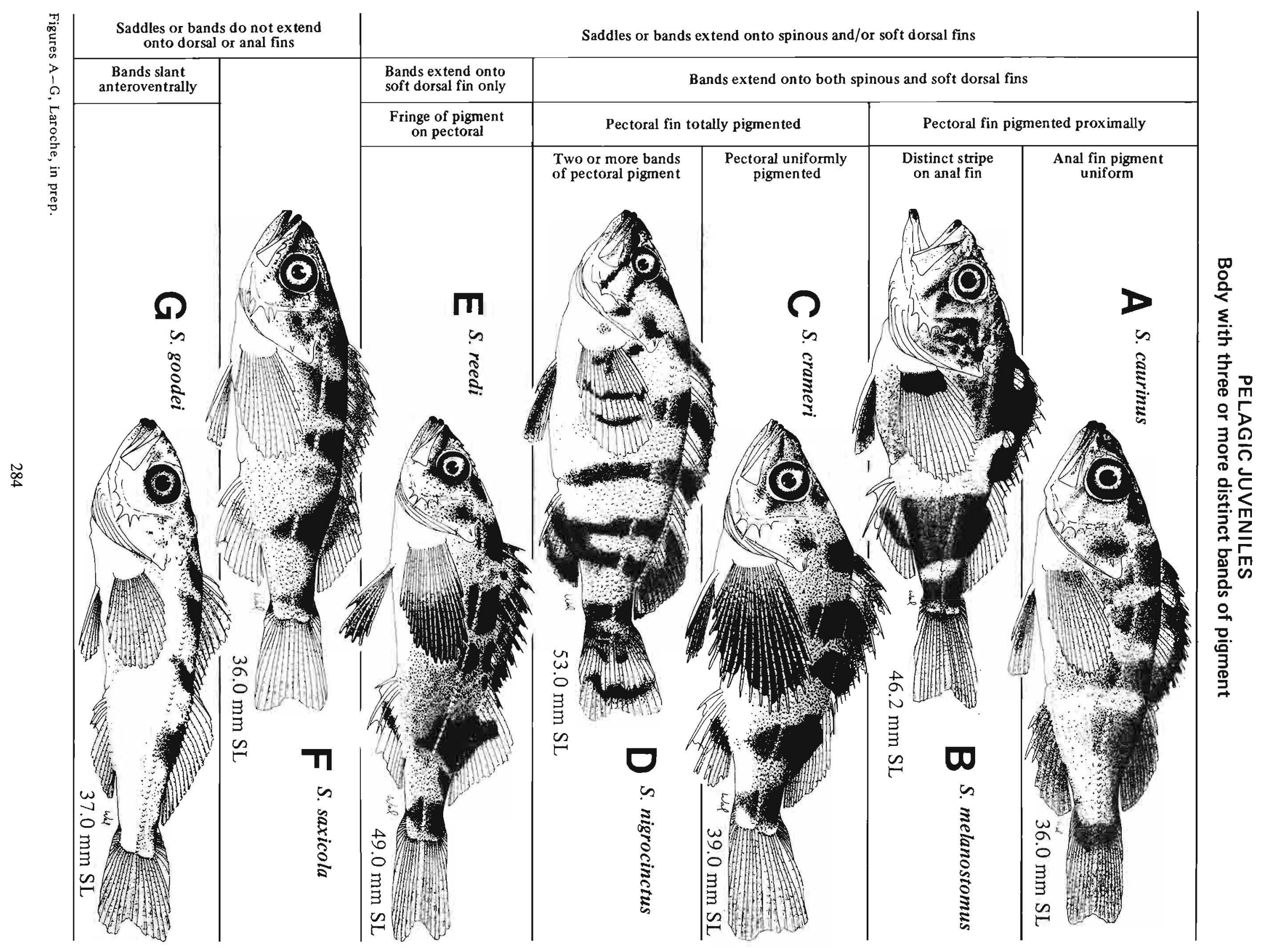


PELAGIC JUVENILES

Diffuse body and dorsal fin pigment

Ventral caudal peduncle pigmented

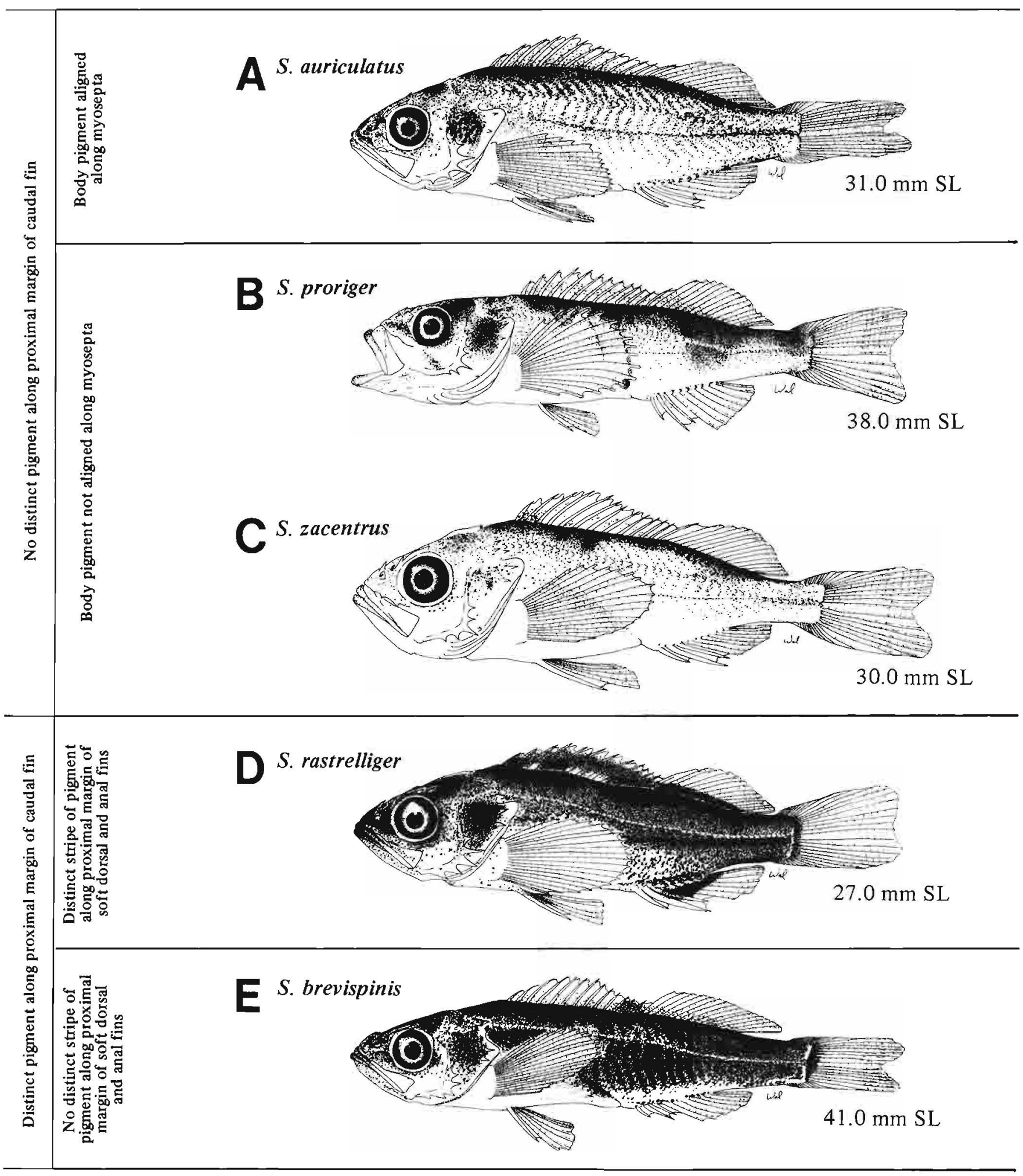




\section{PELAGIC JUVENILES}

Diffuse body and dorsal fin pigment

Ventral caudal peduncle unpigmented

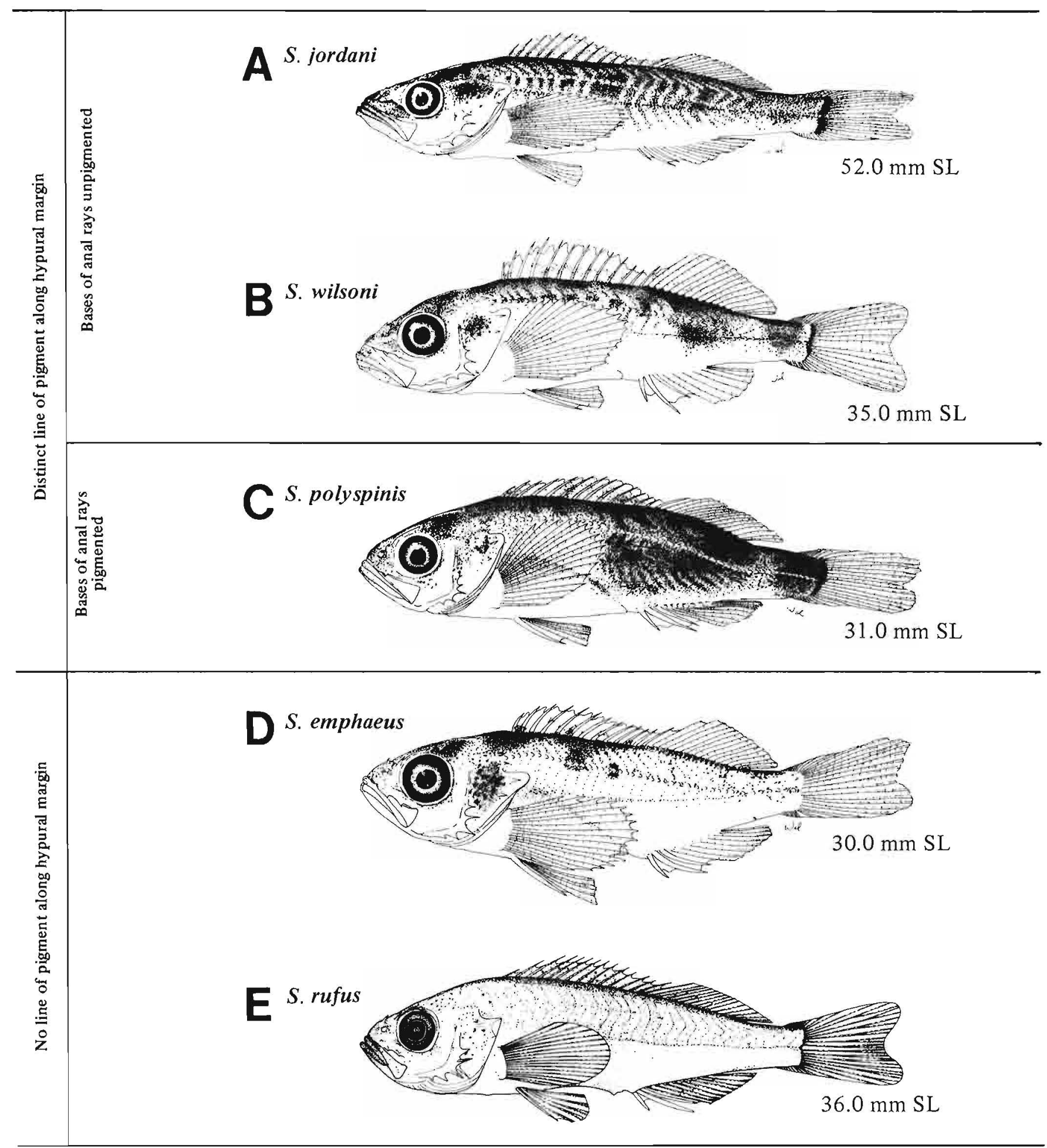

Figures $A-D$, Laroche, in prep.; E, NWAFC original (B. Vinter). 


\section{PELAGIC JUVENILES}

Body with fewer than three pigment bands, with stripes of pigment, or with a patch of pigment on the caudal peduncle

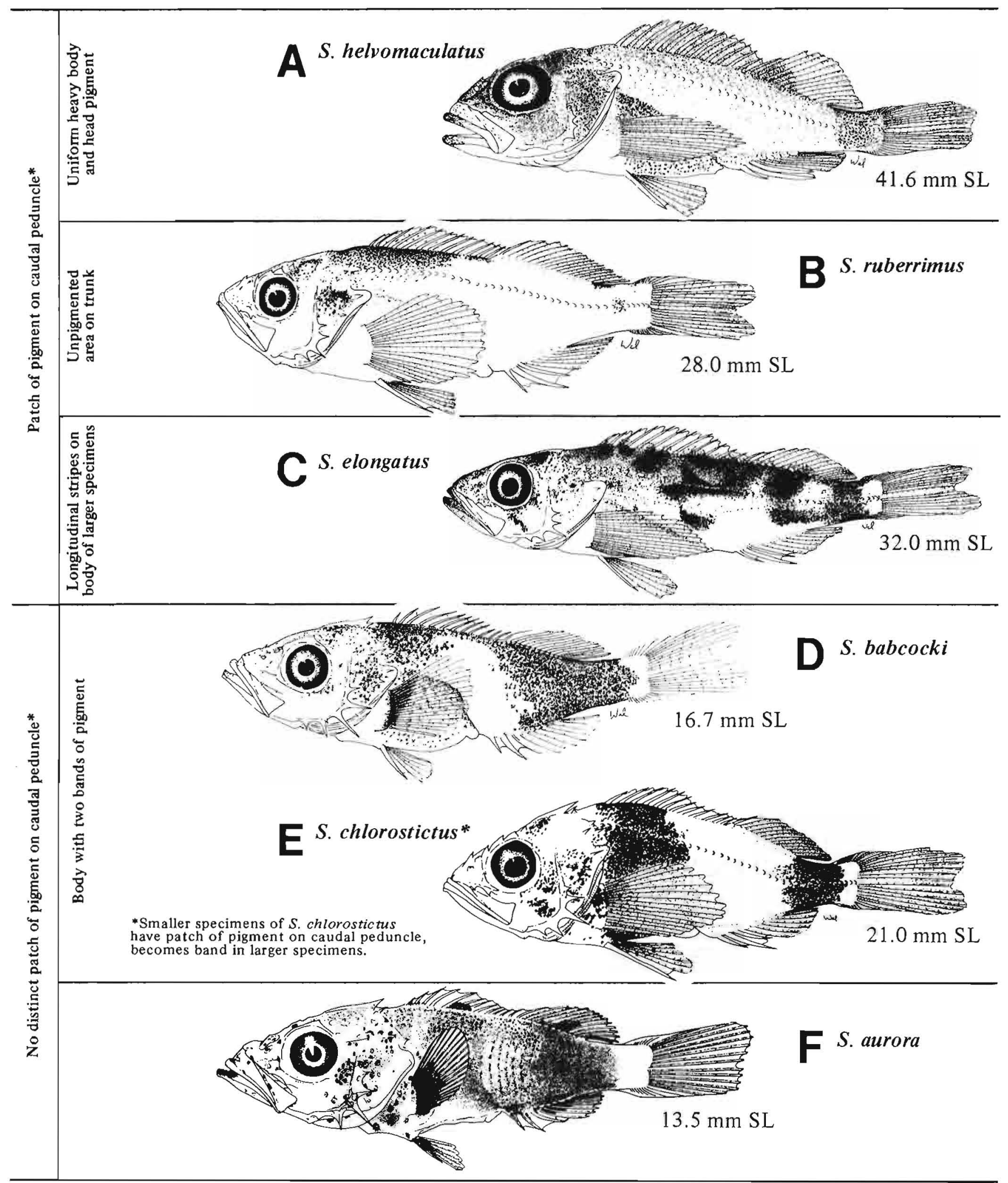

Figures A-E, Laroche, in prep.; F, Moser et al. 1985. 
MERISTICS

Vertebrae

Total: $27-27-27$

Precaudal: $\mathrm{X}-\mathrm{X}-\mathrm{X}$

Caudal: $\mathrm{X}-\mathrm{X}-\mathrm{X}$

Branchiostegal rays $\quad 7-7-7$

Caudal fin

Pelvic fin

$\mathrm{X}, 8+7, \mathrm{X}$

Thoracic

S: $1-1-1 \quad$ R: $5-5-5$

Dorsal fin

S: $13-13-14$ R: $13-14-15$

Pectoral fin

R: $17-18-19$

S: $3-3-3$

R: $7-7-8$

U: $8-9-11$

L: $20-22-24$

Gill rakers

Lateral line pores

$30-33$

\section{LIFE HISTORY}

\begin{tabular}{|c|c|}
\hline Range & $\begin{array}{l}\text { S. California, } 32-34^{\circ} \mathrm{N} \text {, to } \\
\text { Bering Sea, } 54-66^{\circ} \mathrm{N}\end{array}$ \\
\hline Ecology & $\begin{array}{l}\text { Meso- and bathybenthal, } \\
183-732 \mathrm{~m}\end{array}$ \\
\hline ELH pattern & Ovoviviparous, pelagic larvae \\
\hline \multirow[t]{4}{*}{ Spawning } & Season: Apr $^{\mathrm{a}}$ \\
\hline & Area: \\
\hline & Mode: \\
\hline & Migration: \\
\hline Fecundity & Range/function: \\
\hline Age at first maturity & \\
\hline Longevity & \\
\hline
\end{tabular}

\section{HEAD SPINES}

$\begin{array}{ll}\text { Preocular } & + \\ \text { Postocular } & + \\ \text { Coronal } & + \\ \text { Supraocular } & + \\ \text { Tympanic } & + \\ \text { Inferior infraorbital } & 2\end{array}$

\section{EARLY LIFE HISTORY DESCRIPTION}

\section{LARVAE}

Hatch size $4.1-5.3 \mathrm{~mm}$ TL

Preanal length

Length at flexion

Length at transformation

Sequence of fin development

Pigment

- Extrusion larvae: Series along ventral body

- Postflexion larvae >19.3 mm SL: Pigment covering entire body except on lower cheek, pectoral fin base, and tail tip

Diagnostic characters

\section{PELAGIC JUVENILES}

- Body pigment light, diffuse

- Banded pigment on median fins

${ }^{\circledR} \mathrm{Hart} 1973$

Ref: Laroche, in prep. 

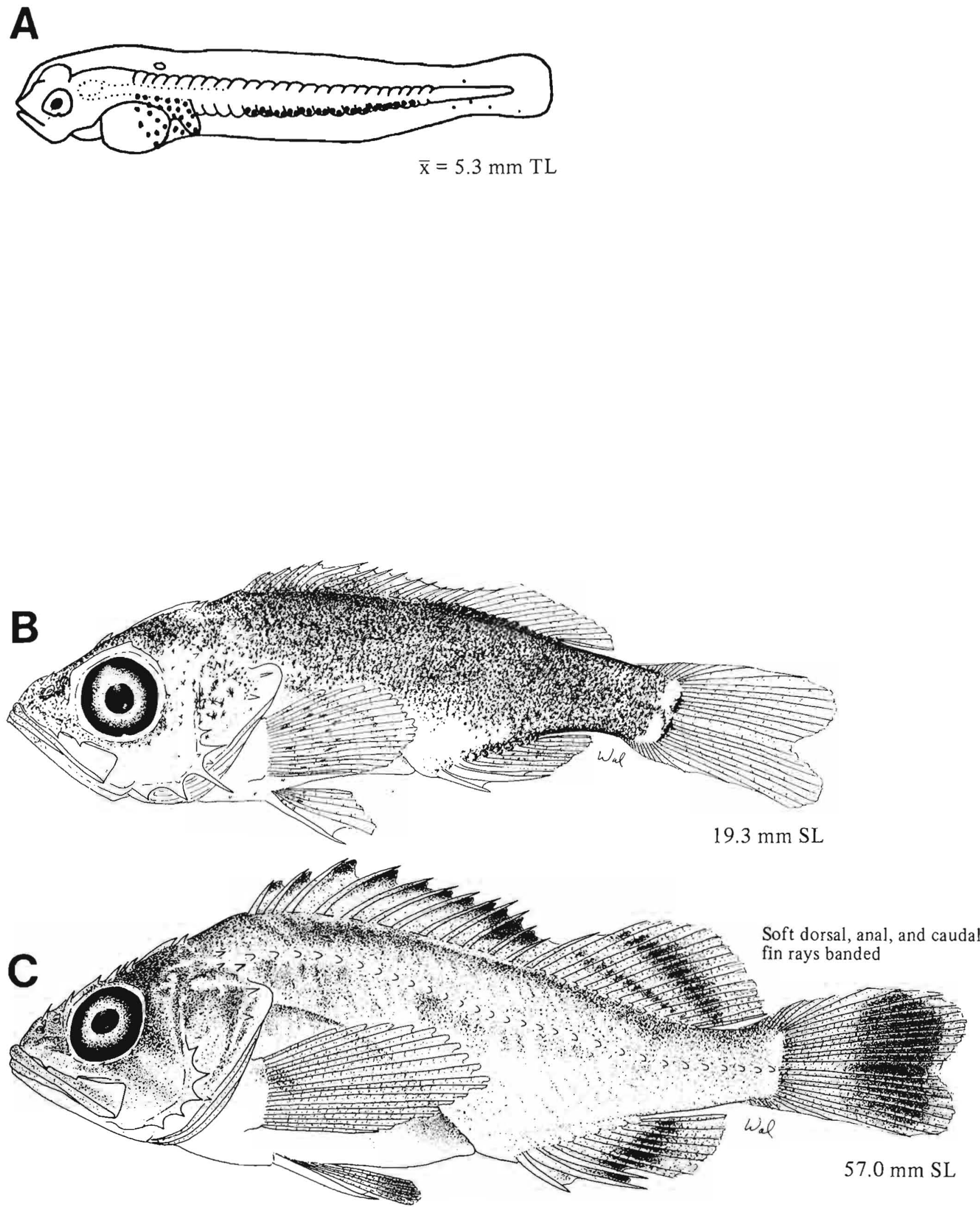

Figure A, Westrheim et al. 1968a; B-C, Laroche, in prep. 


\section{MERISTICS}

Vertebrae

Total: 26-26-27

Precaudal: X-X-X

Caudal: $\mathrm{X}-\mathrm{X}-\mathrm{X}$

Branchiostegal rays

Caudal fin

7-7-7

Pelvic fin

9-11, 8+7, 9-11

Thoracic
S: $1-1-1$
$\mathrm{R}: 5-5-5$
S: 13-13-13 R: 12-13-14
R: $16-18-19$
S: $3-3-3$
R: $6-7-7$
U: $8-8-10$
L: $18-20-21$

Dorsal fin

Pectoral fin

Gill rakers

Lateral line pores $\quad 42-50$
Anal fin

\section{HEAD SPINES}

$\begin{array}{lc}\text { Preocular } & + \\ \text { Postocular } & + \\ \text { Coronal } & + \\ \text { Supraocular } & 0 \\ \text { Tympanic } & + \\ \text { Inferior infraorbital } & 2\end{array}$

\section{EARLY LIFE HISTORY DESCRIPTION}

\section{LARVAE}

Hatch size

Preanal length

Length at flexion

\section{7-6.7 mm SL} $<50 \% \mathrm{SL}$

Between 7.4 and $9.0 \mathrm{~mm} \mathrm{SL}$

Length at transformation

Sequence of fin development

Caudal and pectorals, pelvics, dorsal and anal

Pigment $^{\mathrm{d}}$

- Mostly on dorsal head, with development covering entire head

Nearshore shelf demersal

Ovoviviparous, pelagic larvae

ELH pattern

Season: Mar-June ${ }^{a}$

Area:

Mode:

Migration:

Fecundity

Range/function: $52,000-339,000^{\mathrm{b}}$

Age at first maturity $5 \mathrm{yr}$ (females) $^{\mathrm{c}}$

Longevity
- Nape

- Gut: Dorsal and ventrolateral surface, increasing laterally with development

- Dorsal and ventral midline melanophores begin posterior to anus; with development, dorsal melanophores extend to nape

- Increase in lateral pigment on posterior half of body and to hypural region

- Pectoral fins

Diagnostic characters

Distinguished from $S$. caurinus (p. 296) by

- Dorsal midline melanophores postanally at birth, gradually spread forward to nape

- More pigment on opercular than in S. caurinus

\section{PELAGIC JUVENILES}

- Diffuse body pigment

- Opercular pigment blotch present

- Lateral midline pigmented

${ }^{a}$ Washington et al. 1978

${ }^{\mathrm{B}} \mathrm{Hart} 1973$

${ }^{c}$ Wyllie Echeverria 1987

${ }^{d}$ Description of pigment and illustrations based on laboratory-reared specimens which may be more melanistic than wild-caught specimens.

Ref: Laroche, in prep.; Stahl-Johnson (1985), who noted a mistake in Moser et al. (1977); yolk-depleted $S$. auriculatus figure reversed with $S$. caurinus. 


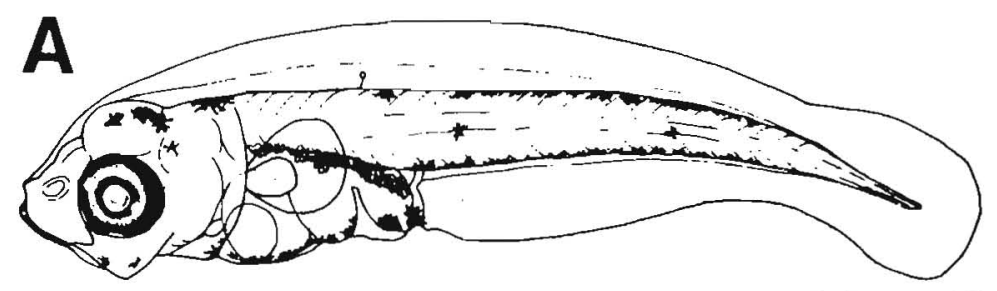

Unpigmented area dorsally,

$5.5 \mathrm{~mm} \mathrm{SL}$
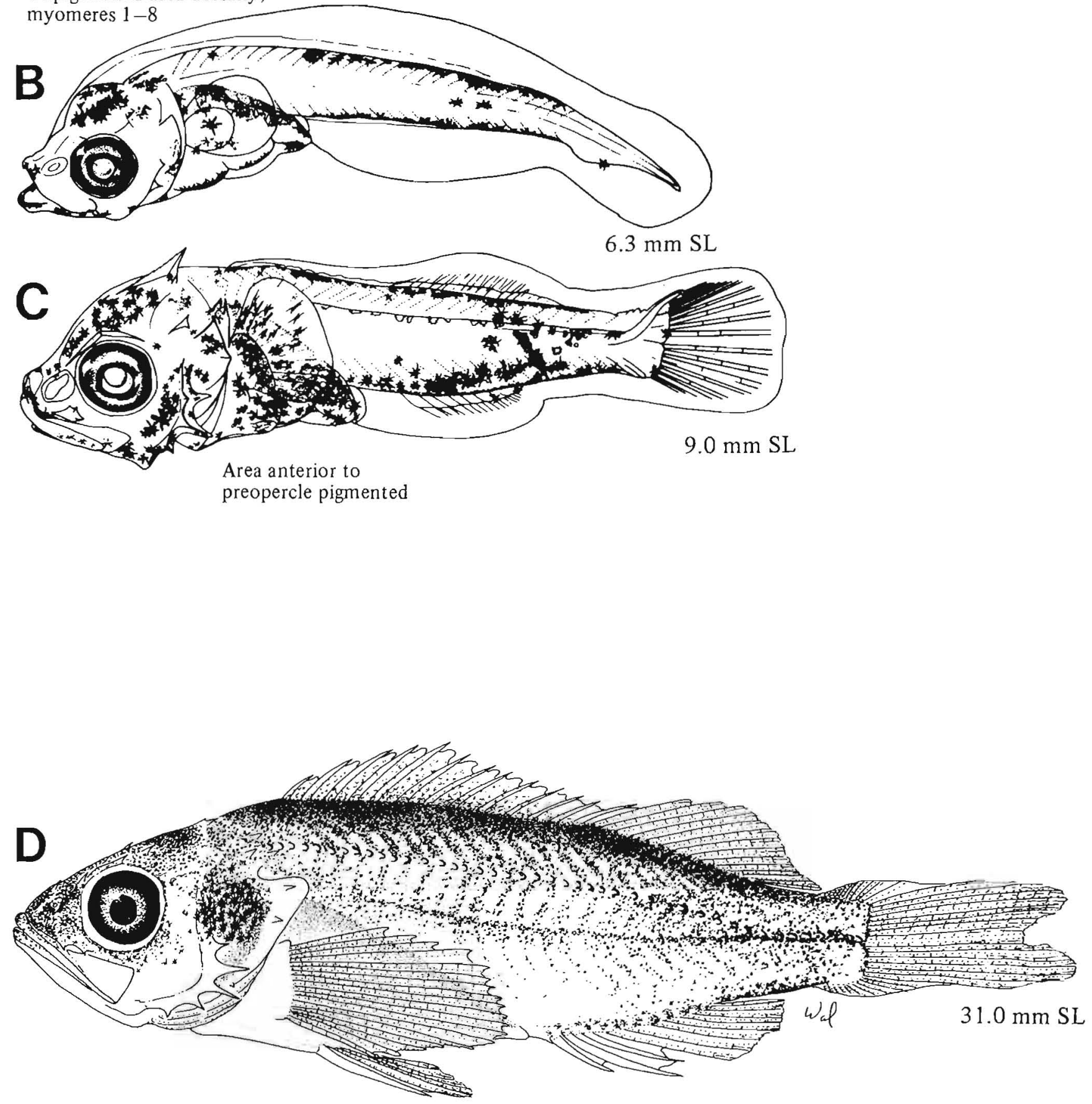

Figures A-C, Stahl-Johnson 1985 (reared); D, Laroche, in prep. 


\section{MERISTICS}

Vertebrae

Total: $26-26-26$

Precaudal: $10-10-10$

Caudal: $16-16-16$

Branchiostegal rays

7-7-7

Caudal fin

$10,8+7,9-10$

Pelvic fin

Thoracic

$$
\text { S: } 1-1-1 \quad R: 5-5-5
$$

Dorsal fin

$S: 13-13-13 \quad R: 12-13-13$

Pectoral fin

Anal fin

R: $17-17-19$

S: 3-3-3 R: 5-6-7

Gill rakers

$\mathrm{U}: 7-8-8$

L: $18-19-20$

(T: 24-27-28)

Lateral line pores $\quad 28-31$

\section{LIFE HISTORY}

$\begin{array}{ll}\text { Range } & \text { South of southern California to } \\ & \text { Brit. Col., } 48^{\circ} 30^{\prime}-55^{\circ} \mathrm{N} \\ \text { Ecology } & \text { Epi-, meso-, and bathybenthal, } \\ & 125-768 \mathrm{~m} \\ \text { ELH pattern } & \text { Ovoviviparous, pelagic larvae } \\ \text { Spawning } & \text { Season: Mar-May (north-central } \\ & \text { California) } \\ & \text { Area: } \\ & \text { Mode: } \\ & \text { Migration: } \\ \text { Fecundity } & \text { Range/function: }\end{array}$

Age at first maturity

Longevity

\section{HEAD SPINES}

$\begin{array}{ll}\text { Preocularb } & + \\ \text { Postocular } & + \\ \text { Coronal } & 0 \\ \text { Supraocular } & + \\ \text { Tympanic } & + \\ \text { Inferior infraorbital } & 2\end{array}$

\section{EARLY LIFE HISTORY DESCRIPTION}

\section{LARVAE}

Hatch size

Preanal length

Length at flexion

Length at transformation

Sequence of fin development

Pigment

- Head: Dorsal spots spreading with development to opercle then to entire head

- Snout and jaws pigmented

- Gut: Posterior pigment increasing dorsolaterally with development

- Postanal body: Dorsal and ventral midline over body (between postanal myomeres 4 and 15); lateral pigment increases with development to form a band

- Pectoral fin first, other fins with development

\section{Diagnostic characters}

- Morphology: Snout-to-anus length greater than in other species studied

- Pigment: Snout, postanal band

\section{PELAGIC JUVENILES ${ }^{c}$}

- Low total gill raker count (24-28) and lateral line pore count (28-31) are diagnostic for the species

- Body pigment faintly banded with unpigmented caudal peduncle

- Pectoral rays heavily pigmented medially

\footnotetext{
${ }^{a}$ Wyllie Echeverria 1987

${ }^{b}$ According to Moser et al. (1985), the preocular spine develops by $10.5 \mathrm{~mm}$ SL but is not visible on their $13.5-\mathrm{mm}$ SL specimen shown here.

' Largest pelagic juvenile collected is $34.4 \mathrm{~mm}$ SL.

Ref: Moser et al. 1985.
} 
A

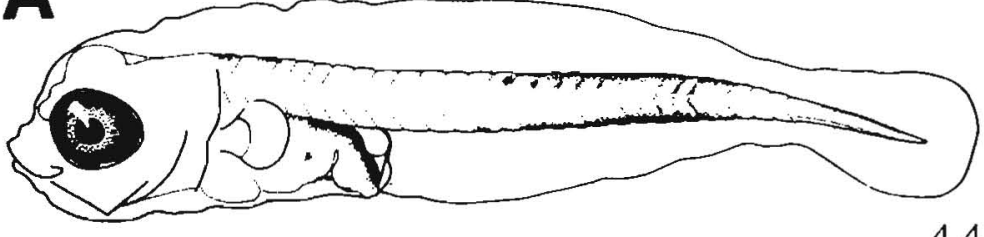

$4.4 \mathrm{~mm}$

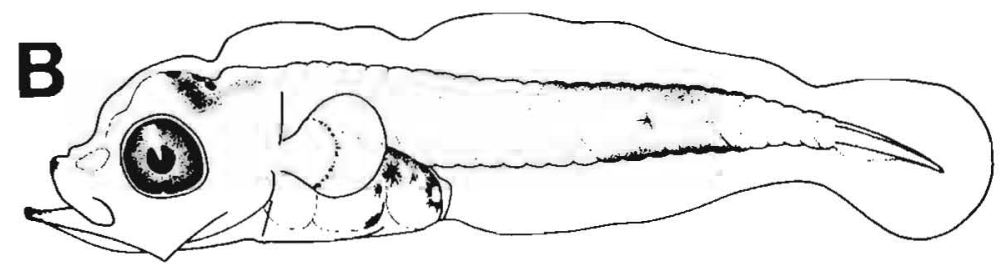

$4.8 \mathrm{~mm}$

C

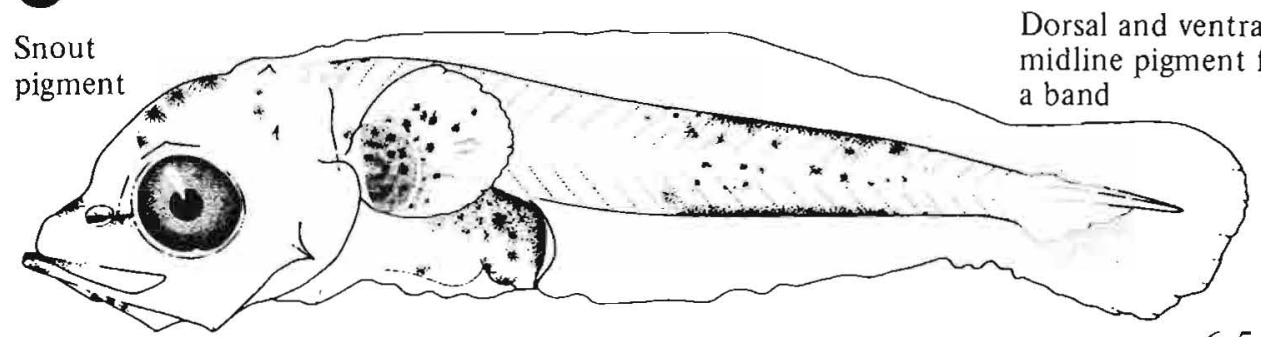

$6.5 \mathrm{~mm}$
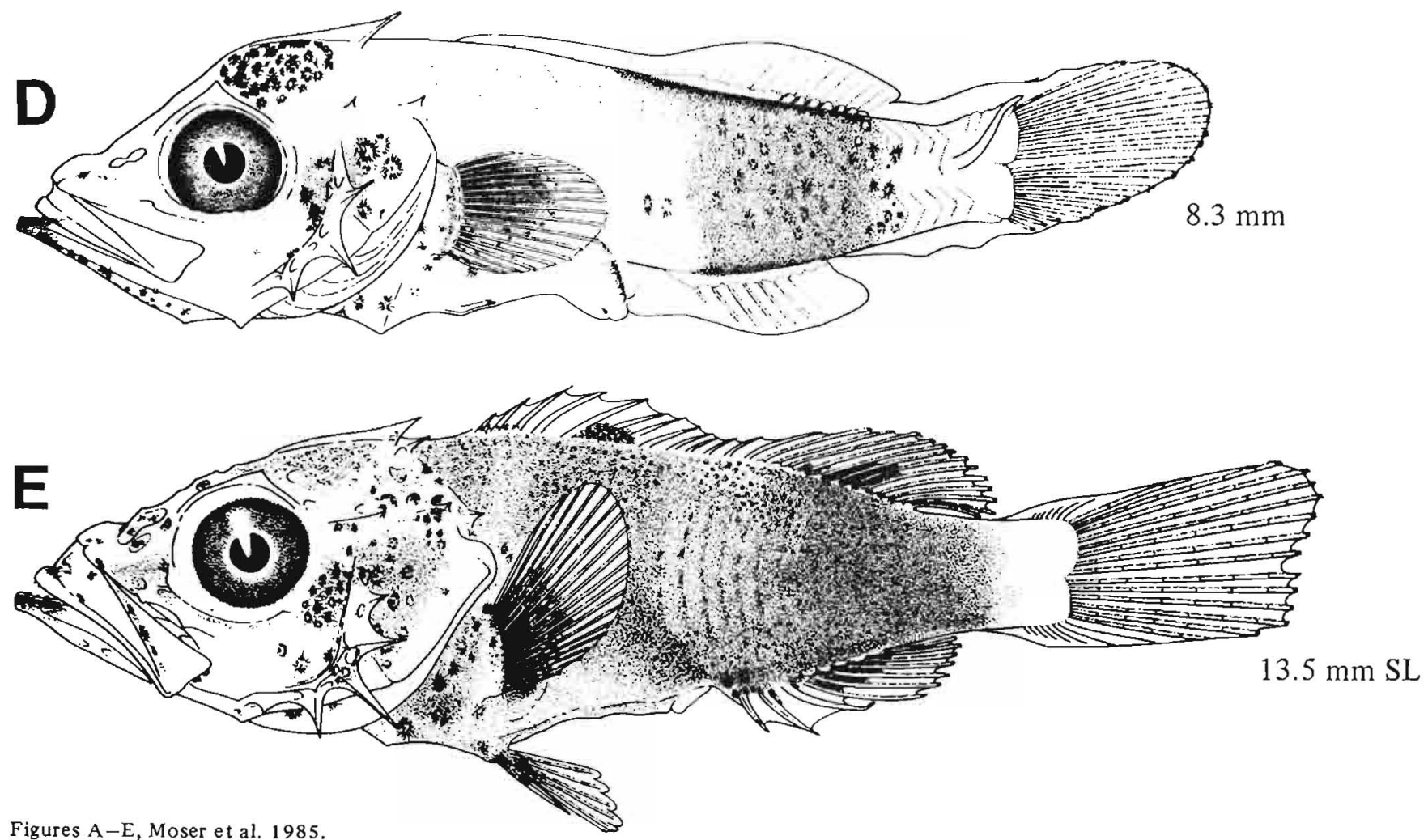


\section{MERISTICS}

\begin{tabular}{|c|c|c|}
\hline \multirow[t]{3}{*}{ Vertebrae } & \multicolumn{2}{|c|}{ Total: $26-26-26$} \\
\hline & \multicolumn{2}{|c|}{ Precaudal: $\mathrm{X}-\mathrm{X}-\mathrm{X}$} \\
\hline & \multicolumn{2}{|c|}{ Caudal: X-X-X } \\
\hline Branchiostegal rays & \multicolumn{2}{|l|}{$7-7-7$} \\
\hline Caudal fin & \multicolumn{2}{|l|}{$X, 8+7, X$} \\
\hline Pelvic fin & \multicolumn{2}{|l|}{ Thoracic } \\
\hline & $S: 1-1-1$ & $R: 5-5-5$ \\
\hline Dorsal fin & S: $13-13-13$ & R: $13-14-15$ \\
\hline Pectoral fin & $\mathrm{R}: 17-19-20$ & \\
\hline Anal fin & S: $3-3-3$ & $\mathrm{R}: 6-7-8$ \\
\hline Gill rakers & $\begin{array}{r}\mathrm{U}: \mathrm{X}-\mathrm{X}-\mathrm{X} \\
(\mathrm{T}: 29\end{array}$ & $\begin{array}{l}\mathrm{L}: \mathrm{X}-\mathrm{X}-\mathrm{X} \\
31-33)\end{array}$ \\
\hline Lateral line pores & $42-51$ & \\
\hline
\end{tabular}

\section{Range}

S. California, $32-34^{\circ} \mathrm{N}$, to

Bering Sea, $54-66^{\circ} \mathrm{N}$

Ecology

ELH pattern

Spawning

Age at first maturity

Longevity

\section{HEAD SPINES}

$\begin{array}{ll}\text { Preocular } & + \\ \text { Postocular } & + \\ \text { Coronal } & 0 \\ \text { Supraocular } & 0 \\ \text { Tympanic } & + \\ \text { Inferior infraorbital } & 2\end{array}$

\section{EARLY LIFE HISTORY DESCRIPTION}

\section{LARVAE}

Hatch size $4.2-5.3 \mathrm{~mm}$

Preanal length

Length at flexion

Length at transformation

Sequence of fin

development

Pigment

- Extrusion larvae

-Series along ventral body

- Shorter series along posterior dorsal midline

- Postflexion larvae $>14.6 \mathrm{~mm}$ SL

-Wide postanal band

- Light pigment dorsally on head and laterally on gut

Diagnostic characters

\section{PELAGIC JUVENILES}

- Body pigment in two bands

- Little pigment on median fins 

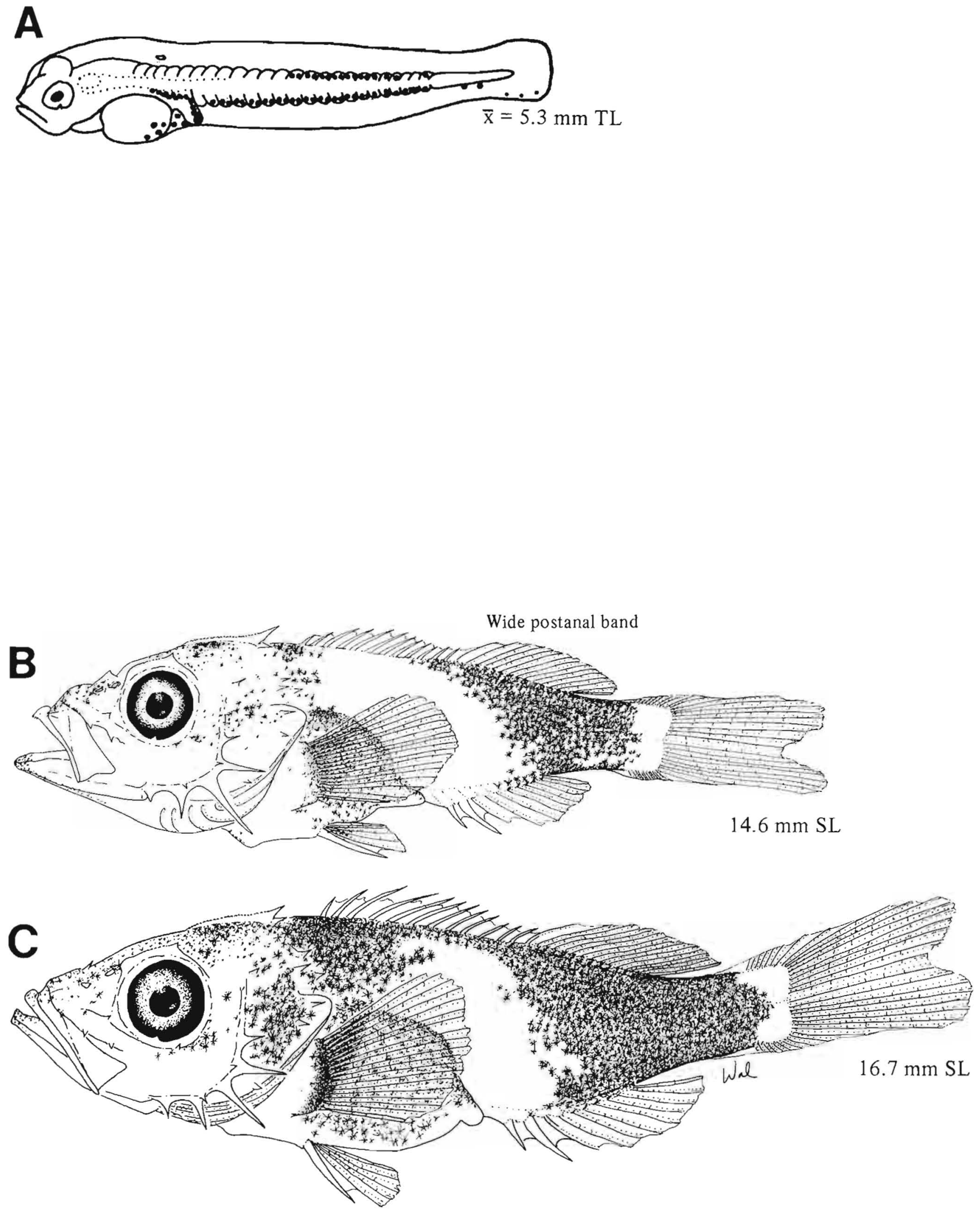

Figure A, Westrheim et al. 1968a; B-C, Laroche, in prep. 


\section{MERISTICS}

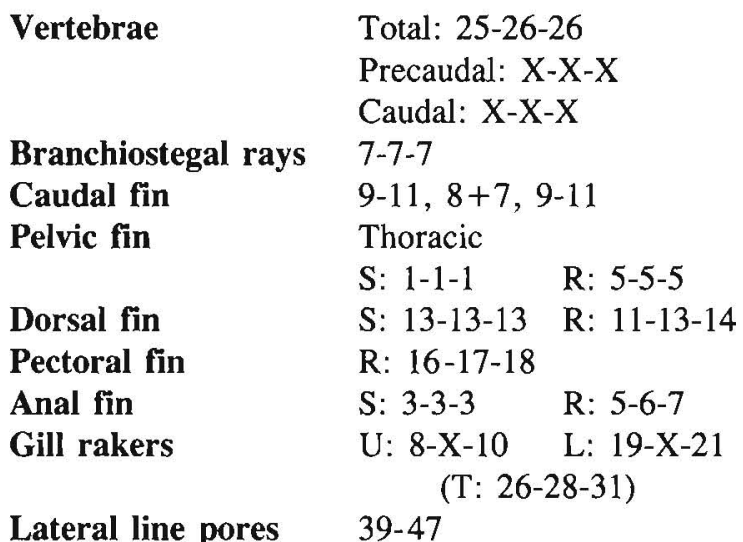

\section{LIFE HISTORY}

\begin{tabular}{|c|c|}
\hline Range & $\begin{array}{l}\text { South of southern California to } \\
\text { Gulf of Alaska, } 54-60^{\circ} \mathrm{N}\end{array}$ \\
\hline Ecology & $\begin{array}{l}\text { Nearshore shelf demersal, } \\
0-183 \mathrm{~m}\end{array}$ \\
\hline ELH pattern & Ovoviviparous, pelagic larvae \\
\hline Spawning & Season: Apr $^{\text {a }}$ \\
\hline & Area: \\
\hline & Mode: \\
\hline & Migration: \\
\hline Fecundity & $\begin{array}{l}\text { Range/function: } 20,000-640,000^{a} \\
\begin{aligned} F & =0.000000027404 \times \mathrm{L}^{4.956 ?} \\
\mathrm{~L} & =\mathrm{TL} \mathrm{mm} ; \mathrm{b} \\
\mathrm{F} & =0.0000000034554 \times \mathrm{L}^{5.30011} \\
\mathrm{~L} & =\mathrm{TL} \mathrm{mm}^{\mathrm{c}}\end{aligned}\end{array}$ \\
\hline Age at first maturity & $\begin{array}{l}3-4 y^{d} \\
6 y r(f e m a l e s) e\end{array}$ \\
\hline nge & \\
\hline
\end{tabular}

\section{HEAD SPINES}

$\begin{array}{ll}\text { Preocular } & + \\ \text { Postocular } & + \\ \text { Coronal } & 0 \\ \text { Supraocular } & 0 \\ \text { Tympanic } & + \\ \text { Inferior infraorbital } & 2\end{array}$

\section{EARLY LIFE HISTORY DESCRIPTION}

\section{LARVAE}

Hatch size

Preanal length

Length at flexion

Length at transformation

Sequence of fin development
$+$
0
2

$+$

0

$+$

Pigment

- Head: Dorsal and nape spreading, with development, to entire head

- Gut: Dorsal and ventrolateral surface, increasing laterally with development

- Dorsal midline melanophores extend from head to just anterior to tail with no break over gut

- Ventral midline melanophores, with development increasing ventrolaterally and in hypural region

Diagnostic characters

- See notes on S. auriculatus (p. 290) about reared specimens

Distinguished from $S$. auriculatus by

- Until midflexion, more dorsal midline pigment anterior to anus

- Less pigment in the opercular region (flexion and postflexion)

\section{PELAGIC JUVENILES}

- Four pigment bands on body

- Fins pigmented

\footnotetext{
'Har 1973

${ }^{b}$ Washington et al. 1978

'DeLacy et al. 1964

${ }^{d}$ Patten 1973

'Wyllie Echeverria 1987

Ref: Laroche, in prep.; Stahl-Johnson 1985.
} 

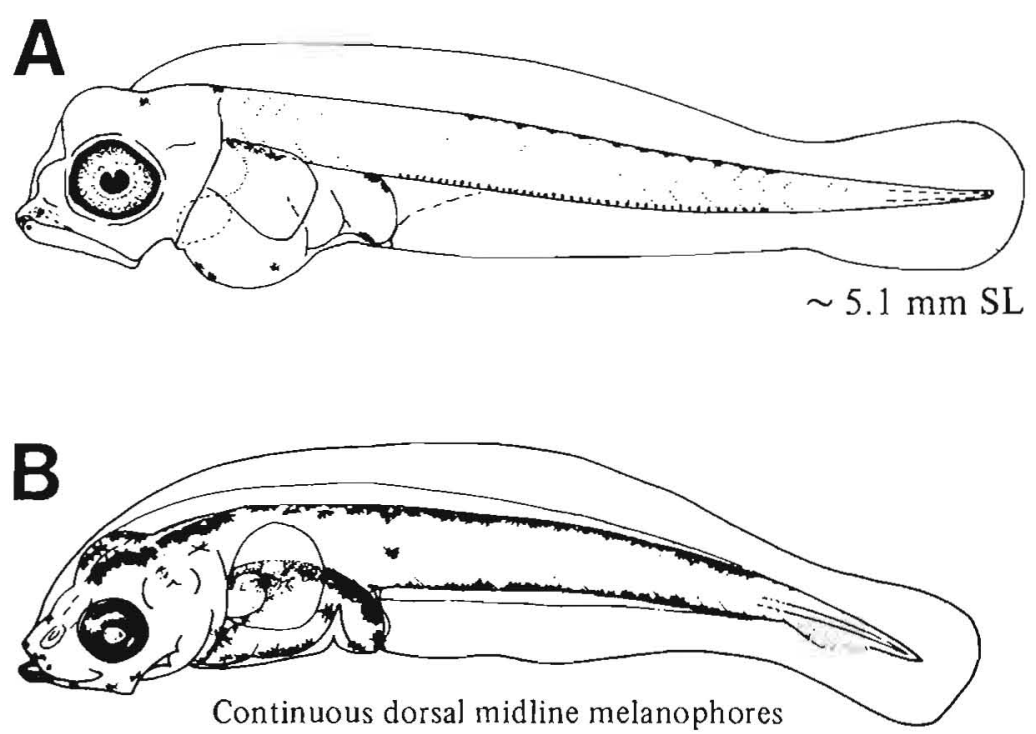

$6.3 \mathrm{~mm} \mathrm{SL}$

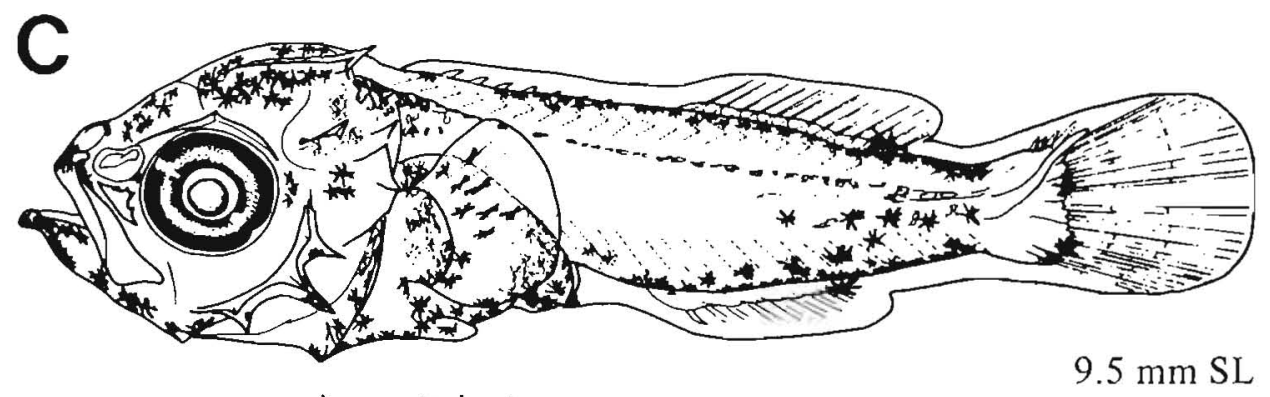

Area anterior to

preopercle unpigmented

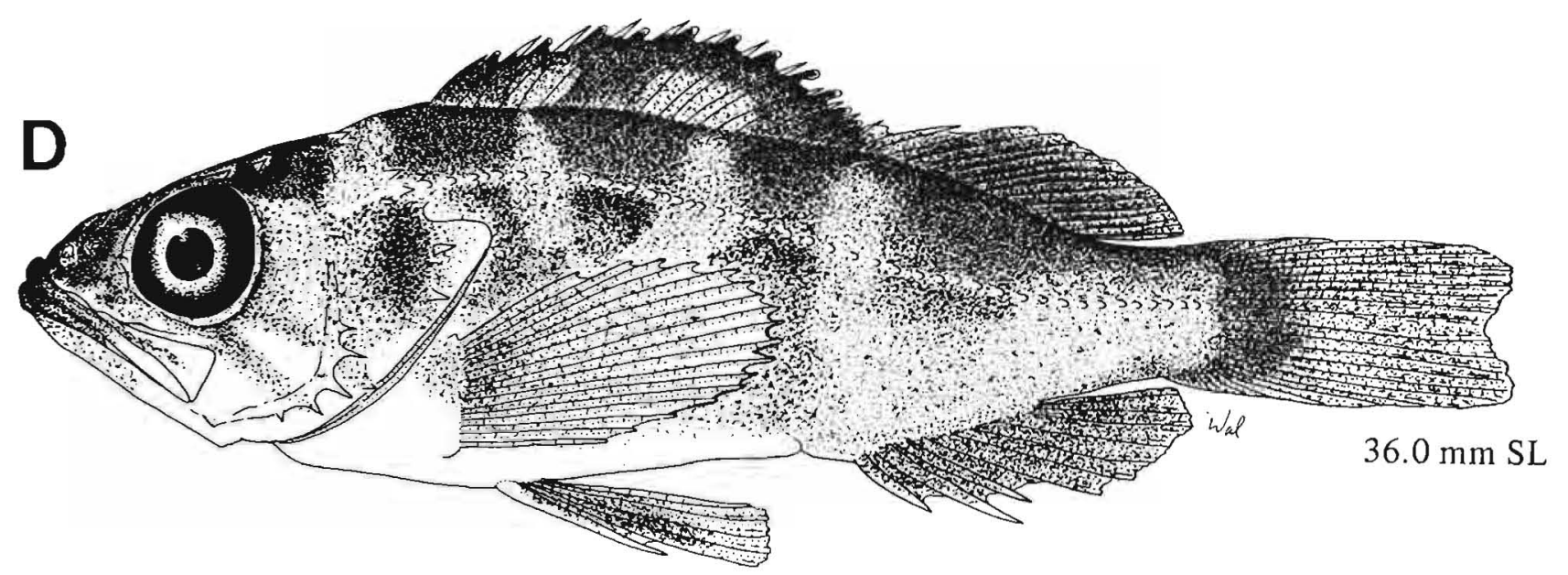

Figure A, NWA FC original (B. Vinter); B-C, Stahl-Johnson 1985 (reared); D, Laroche, in prep. 


\section{MERISTICS}

\begin{tabular}{|c|c|c|}
\hline \multirow[t]{3}{*}{ Vertebrae } & \multicolumn{2}{|c|}{ Total: $26-26-26$} \\
\hline & \multicolumn{2}{|c|}{ Precaudal: X-X-X } \\
\hline & \multicolumn{2}{|c|}{ Caudal: X-X-X } \\
\hline Branchiostegal rays & \multicolumn{2}{|l|}{$7-7-7$} \\
\hline Caudal fin & \multicolumn{2}{|l|}{$X, 8+7, x$} \\
\hline Pelvic fin & \multicolumn{2}{|l|}{ Thoracic } \\
\hline & $S: 1-1-1$ & $\mathrm{R}: 5-5-5$ \\
\hline Dorsal fin & S: $13-13-13$ & $\mathrm{R}: 13-13-15$ \\
\hline Pectoral fin & $\mathrm{R}: 17-18-19$ & \\
\hline Anal fin & $S: 3-3-3$ & $\mathrm{R}: 7-7-7$ \\
\hline Gill rakers & $\mathrm{U}: 8-9-10$ & L: $21-23-25$ \\
\hline & \multicolumn{2}{|c|}{ (T: $30-32-34)$} \\
\hline Lateral line pores & $40-51$ & \\
\hline
\end{tabular}

\begin{tabular}{|c|c|}
\hline Range & $\begin{array}{l}\text { S. California, } 32-34^{\circ} \mathrm{N} \text {, to } \\
\text { Bering Sea, } 54-66^{\circ} \mathrm{N}\end{array}$ \\
\hline Ecology & Epi- and mesobenthal, $29-549 \mathrm{~m}$ \\
\hline ELH pattern & Ovoviviparous, pelagic larvae \\
\hline \multirow[t]{4}{*}{ Spawning } & $\begin{array}{l}\text { Season: Feb (Brit. Col. }{ }^{\mathrm{a}} \text {-Oregon }{ }^{\mathrm{b}} \text { ); } \\
\text { Nov-Mar in southern part } \\
\text { of range }^{\mathrm{c}}\end{array}$ \\
\hline & Area: \\
\hline & Mode: \\
\hline & Migration: \\
\hline Fecundity & Range/function: $50,000-609,800^{c}$ \\
\hline \multirow[t]{2}{*}{ Age at first maturity } & $5-6 \mathrm{yr}^{\mathrm{c}}$ \\
\hline & 4 yr (females) ${ }^{d}$ \\
\hline Longevity & $30 \mathrm{yr}^{\mathrm{c}}$ \\
\hline
\end{tabular}

\section{HEAD SPINES}

$\begin{array}{lc}\text { Preocular } & + \\ \text { Postocular } & + \\ \text { Coronal } & 0 \\ \text { Supraocular } & + \\ \text { Tympanic } & + \\ \text { Inferior infraorbital } & 2\end{array}$

\section{EARLY LIFE HISTORY DESCRIPTION}

\section{LARVAE}

Hatch size

$5.7 \mathrm{~mm} \mathrm{SL}$

Preanal length $54-65 \%$ SL

Length at flexion $8.0-9.3 \mathrm{~mm} \mathrm{SL}$

Length at transformation $16-21 \mathrm{~mm} \mathrm{SL}^{\mathrm{e}}$

Sequence of fin development

Caudal and pectorals, pelvics, dorsal and anal

Pigment

- Extrusion larvae: Series along ventral body

- Pigmented pectorals and pelvics

- Heavy nape and top of head

- No dorsal midline

- 11 ventral midline melanophores reducing to $\sim 4$ on caudal peduncle

- Spinous dorsal fin

- Tip of lower jaw

Diagnostic characters

- Heavy nape and paired fin pigment

- Pigment on spinous dorsal fin

\section{PELAGIC JUVENILES}

- Five pigment bands on body, four extending onto dorsal fin

- Paired fins heavily pigmented

${ }^{a} \mathrm{Hart} 1973$

${ }^{\mathrm{b}} \mathrm{Hitz} 1962$

${ }^{c}$ Phillips 1964

¿Wyllie Echeverria 1987

'Juveniles become benthic at $40-60 \mathrm{~mm} \mathrm{SL}$. 

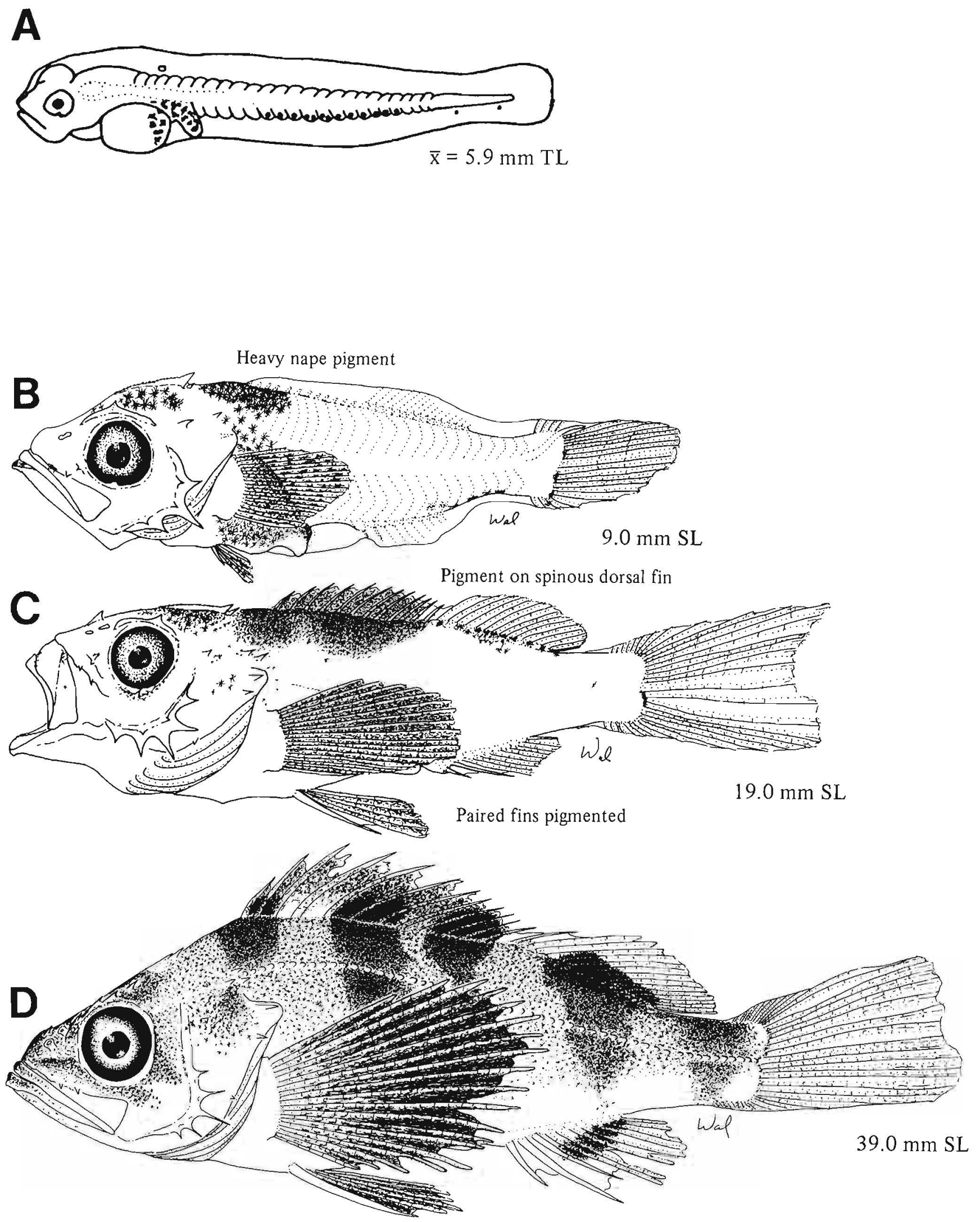

Figure A, Westrheim et al. 1968a; B-C, Richardson and Laroche 1979; D, Laroche, in prep. 


\section{MERISTICS}

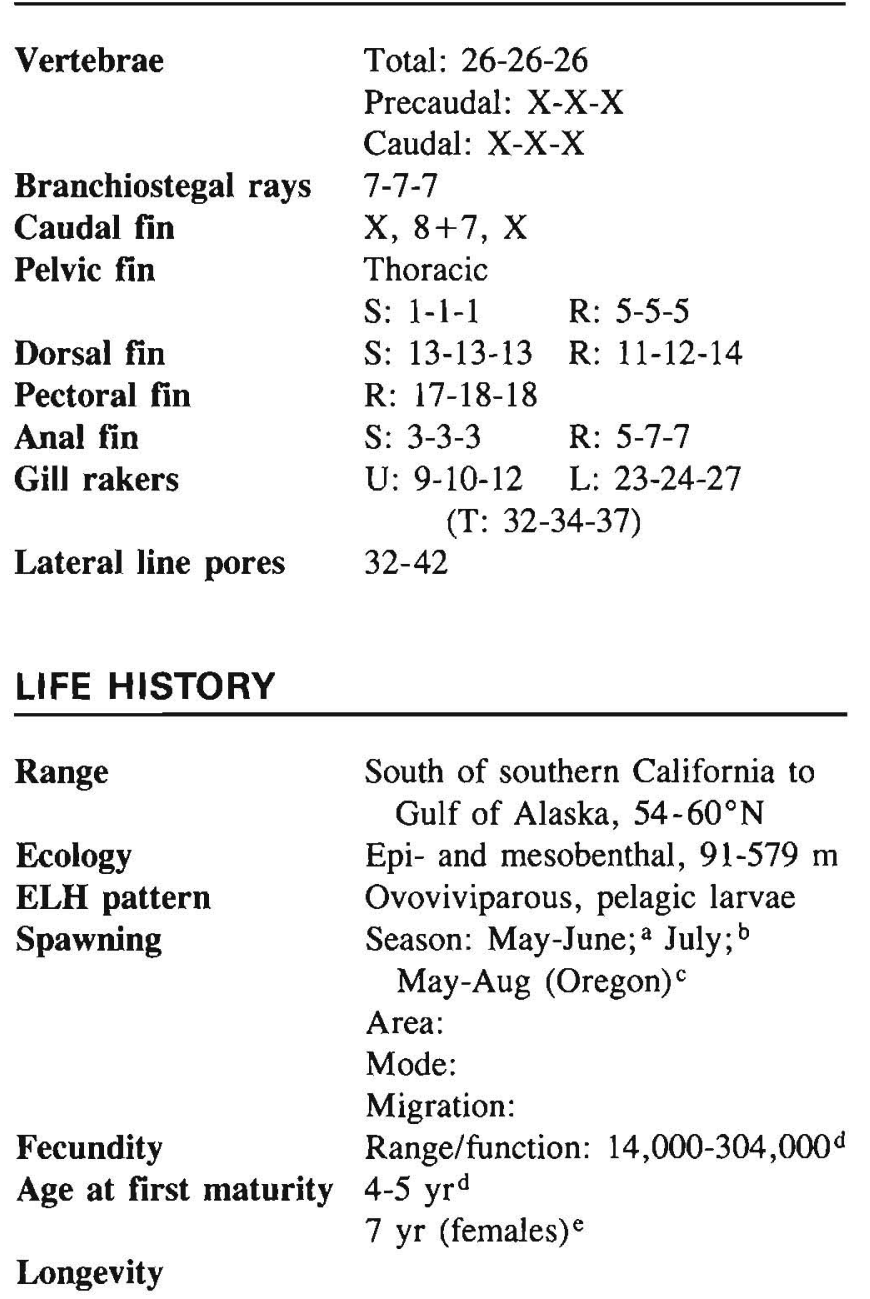

\section{HEAD SPINES}

$\begin{array}{lc}\text { Preocular } & + \\ \text { Postocular } & + \\ \text { Coronal } & 0 \\ \text { Supraocular } & 0 \\ \text { Tympanic } & + \\ \text { Inferior infraorbital } & 2\end{array}$

\section{EARLY LIFE HISTORY DESCRIPTION}

\section{LARVAE}

\author{
Hatch size $\quad 5.2 \mathrm{~mm}$ \\ Preanal length \\ Length at flexion \\ Length at transformation \\ Sequence of fin \\ development \\ Pigment \\ - Extrusion larvae: Series along ventral body \\ - Postflexion larvae $>8.7 \mathrm{~mm}$ SL \\ -Pigment at base of parietal spine, increasing with \\ development to head and nape \\ -Internal spots along anal fin pterygiophores \\ -Along hypural margin
}

Diagnostic characters

\section{PELAGIC JUVENILES}

- Body and fins (except distal portions) pigmented

- Spinous dorsal fin with fringe of pigment

- Distinct band of pigment on soft dorsal and anal fins

\footnotetext{
${ }^{a}$ Hart 1973

${ }^{b}$ Westrheim 1975

${ }^{c}$ Hitz 1962

${ }^{d}$ Phillips 1964

eWyllie Echeverria 1987

Ref: Laroche, in prep.
} 

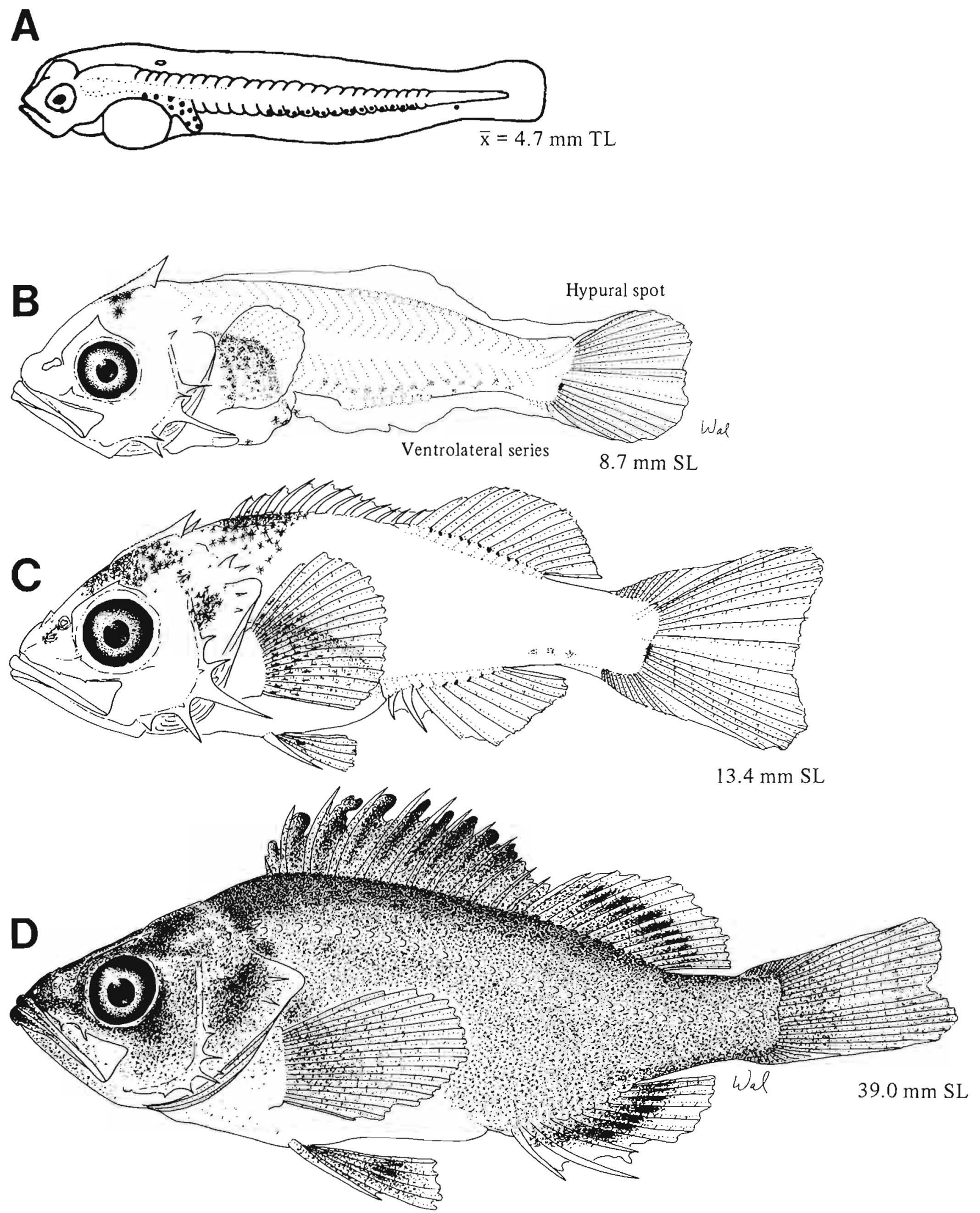

Figure A, Westrheim et al. 1968b; B-D, Laroche, in prep. 


\section{MERISTICS}

Vertebrae

Total: $26-26-26$

Precaudal: X-X-X

Caudal: $\mathrm{X}-\mathrm{X}-\mathrm{X}$

Branchiostegal rays $\quad 7-7-7$

Caudal fin

Pelvic fin

$\mathrm{X}, 8+7, \mathrm{X}$

Thoracic

S: $1-1-1 \quad$ R: $5-5-5$

Dorsal fin

Pectoral fin

Anal fin

Gill rakers

S: $13-13-13$

R: $12-13-14$

R: $16-17-18$

S: $3-3-3$

R: 6-6-7

U: $8-9-10$

L: $20-22-23$

(T: 29-31-33)

Lateral line pores

$37-47$

\section{LIFE HISTORY}

$\begin{array}{ll}\text { Range } & \begin{array}{c}\text { South of southern California to } \\ \text { Gulf of Alaska, } 54-60^{\circ} \mathrm{N}\end{array} \\ & \text { Epi- and mesobenthal, 61-402 m } \\ \text { Ecology } & \text { Ovoviviparous, pelagic larvae } \\ \text { ELH pattern } & \text { Season: May-June } \\ \text { Spawning } & \text { Area: } \\ & \text { Mode: } \\ & \text { Migration: } \\ \text { Fecundity } & \text { Range/function: } \\ \text { Age at first maturity } & 7 \mathrm{yr} \text { (females) }^{\mathrm{b}} \\ \text { Longevity } & \end{array}$

\section{HEAD SPINES}

$\begin{array}{ll}\text { Preocular } & + \\ \text { Postocular } & + \\ \text { Coronal } & 0 \\ \text { Supraocular } & 0 \\ \text { Tympanic } & + \\ \text { Inferior infraorbital } & 2\end{array}$

\section{EARLY LIFE HISTORY DESCRIPTION}

\section{LARVAE}

\author{
Hatch size $\sim 5 \mathrm{~mm}$ \\ Preanal length \\ Length at flexion \\ Length at transformation \\ Sequence of fin \\ development \\ Pigment \\ - Extrusion larvae: Series along ventral body \\ - Postflexion larvae >17.4 mm SL \\ -Along dorsal body margin \\ -Internal row of spots along lateral midline \\ - Above and below notochord along caudal peduncle \\ -A few spots along ventral body margin
}

Diagnostic characters

\section{PELAGIC JUVENILES}

- Body pigment blotchy, longitudinal stripe on larger specimens

- Fins lightly pigmented, or unpigmented

\footnotetext{
${ }^{a}$ Hart 1973

${ }^{b}$ Wyllie Echeverria 1987

Ref: Laroche, in prep.
} 
A
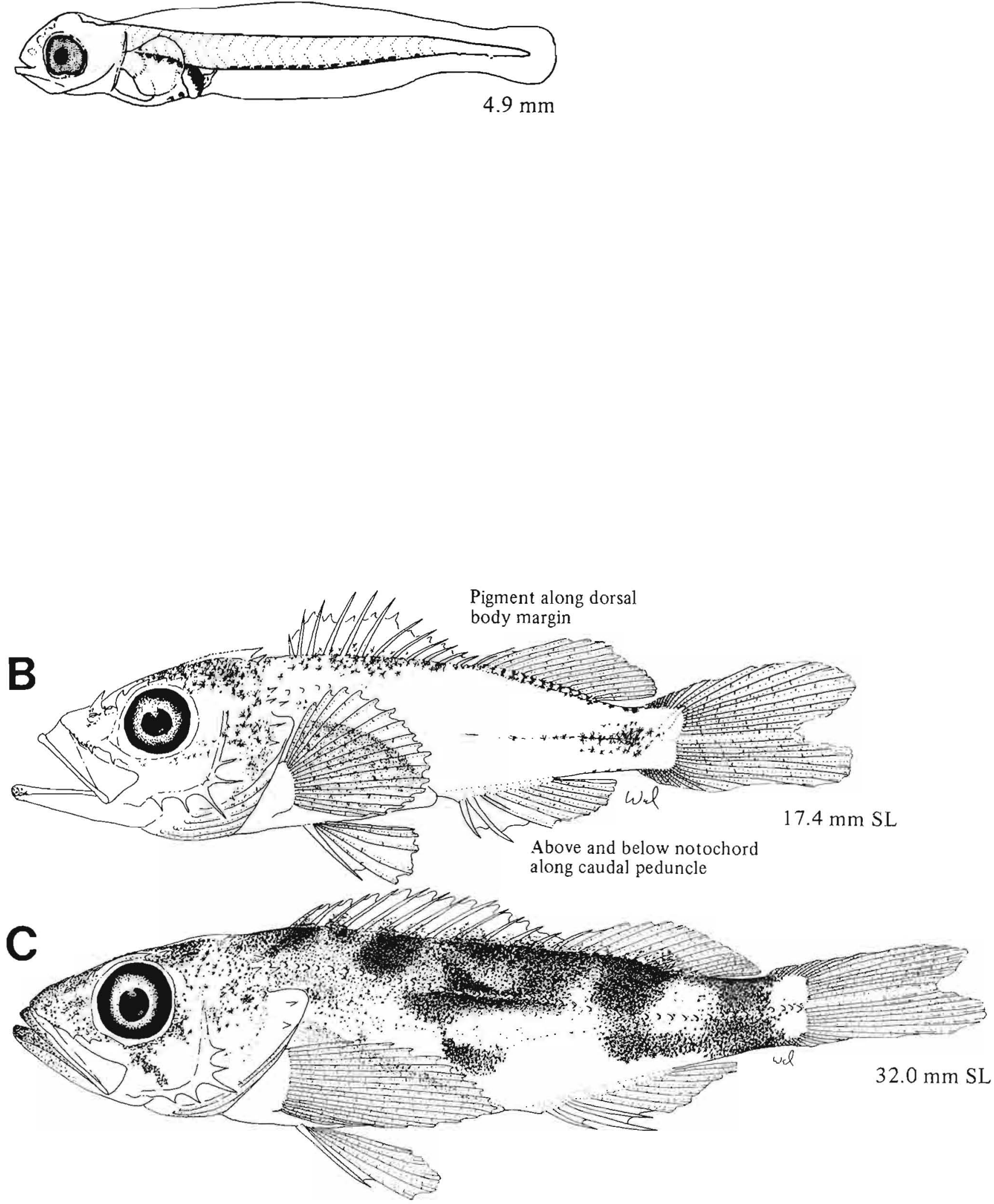

Figure A, Moser et al. 1977; B--C, Laroche, in prep. 


\section{MERISTICS}

Vertebrae

Total: $27-27-28$

Precaudal: $\mathrm{X}-\mathrm{X}-\mathrm{X}$

Caudal: $\mathrm{X}-\mathrm{X}-\mathrm{X}$

Branchiostegal rays $7-7-7$

Caudal fin

Pelvic fin

$\mathrm{X}, 8+7, \mathrm{X}$

Thoracic

S: $1-1-1 \quad$ R: 5-5-5

Dorsal fin

S: $13-13-13$ R: $13-14-15$

Pectoral fin

Anal fin

Gill rakers

R: $16-17-18$

S: $3-3-3$

R: $6-7-7$

U: $11-11-13$ L: $28-30-31$

(T: $37-40-41$ )

Lateral line pores

40-46

\section{LIFE HISTORY}

$\begin{array}{ll}\text { Range } & \text { N. California, } 38-42^{\circ} \mathrm{N}, \text { to } \\ & \text { Gulf of Alaska, 54-60 } \\ \text { Ecology } & \text { Epi- and mesobenthal, 10-366 m } \\ \text { ELH pattern } & \text { Ovoviviparous, pelagic larvae } \\ \text { Spawning } & \text { Season: Aug-Sept } \\ & \text { Area: } \\ & \text { Mode: } \\ & \text { Migration: } \\ \text { Fecundity } & \text { Range/function: } \\ \text { Age at first maturity } & 2-3 \mathrm{yr}^{\mathrm{a}} \\ \text { Longevity } & \end{array}$

\section{HEAD SPINES}

$\begin{array}{ll}\text { Preocular } & + \\ \text { Postocular } & + \\ \text { Coronal } & 0 \\ \text { Supraocular } & 0 \\ \text { Tympanic } & 0 \\ \text { Inferior infraorbital } & 2\end{array}$

\section{EARLY LIFE HISTORY DESCRIPTION}

\section{LARVAE}

Hatch size

Preanal length

Length at flexion

Length at transformation

Sequence of fin development

Pigment

- Postflexion larvae $>16.4 \mathrm{~mm} \mathrm{SL}$

-Along base of soft dorsal fin rays

- Along base of parietal spine

-Along lateral line in caudal peduncle area

Diagnostic characters

\section{PELAGIC JUVENILES}

- Body pigment mainly dorsal to midline

- Opercular blotch present

- Lateral midline pigmented

- Little fin pigment 


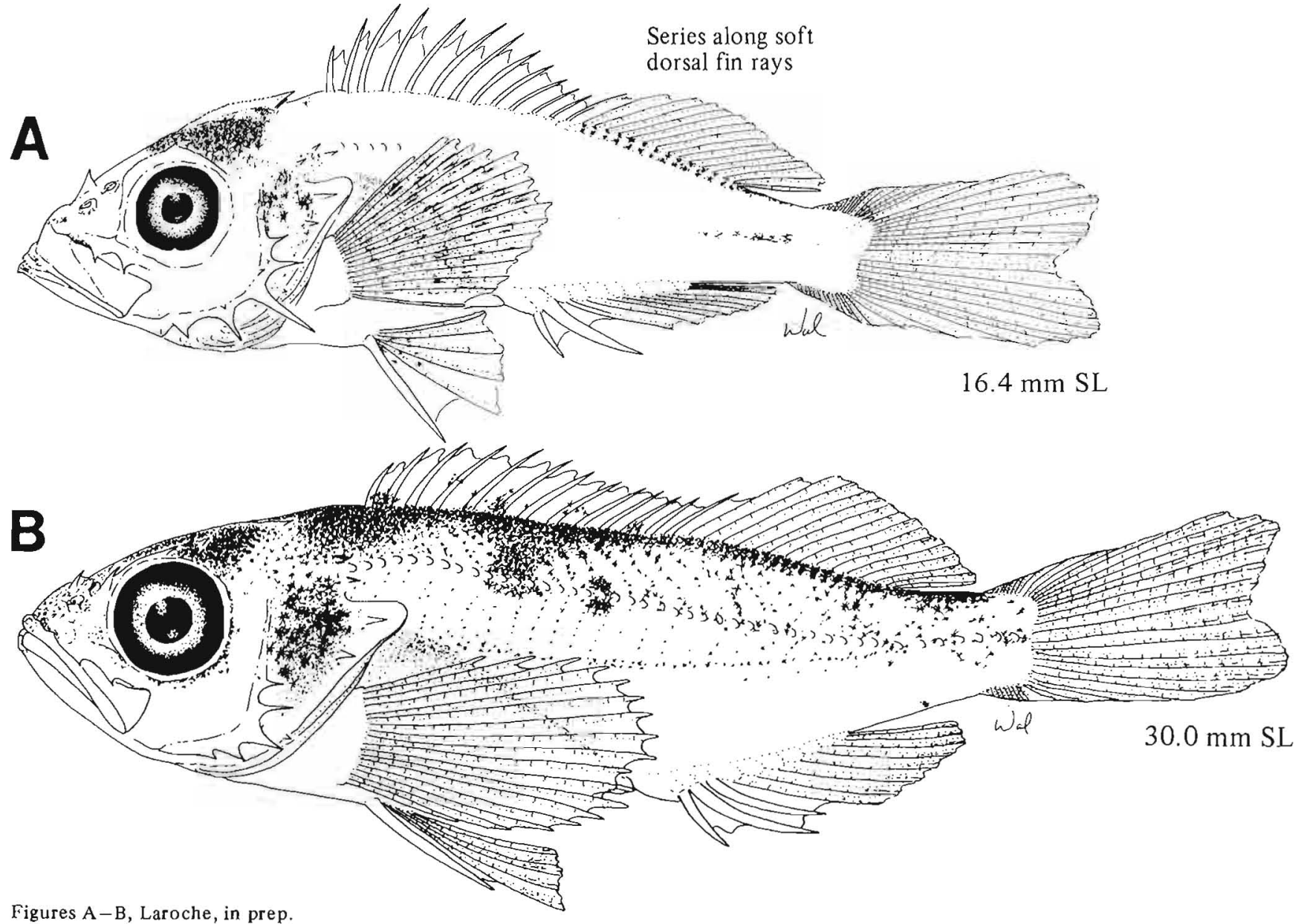




\section{MERISTICS}

Vertebrae

Total: $26-26-27$

Precaudal: X-X-X

Caudal: $\mathrm{X}-\mathrm{X}-\mathrm{X}$

Branchiostegal rays

\section{7-7-7}

Caudal fin

Pelvic fin

$\mathrm{X}, 8+7, \mathrm{X}$

Thoracic

S: $1-1-1$

$\mathrm{R}: 5-5-5$

Dorsal fin

$S: 13-13-13 \quad R: 14-15-16$

Pectoral fin

R: $18-18-19$

Anal fin

S: $3-3-3$

R: $7-8-9$

Gill rakers

U: $9-10-11$

L: $24-26-28$

(T: 34-36-38)

Lateral line pores $\quad 54-60$

LIFE HISTORY

Range
Ecology
ELH pattern
Spawning

South of southern California to

Gulf of Alaska, $54-60^{\circ} \mathrm{N}$

Epi- and mesobenthal, 0-375 m

Ovoviviparous, pelagic larvae

Season: Nov-Mar; ${ }^{a}$

Apr (British Columbia) ${ }^{b}$

Area:

Mode:

Migration:

Fecundity

Range/function: $55,600-915,200^{\mathrm{a}}$

Age at first maturity 3-4 yr (California) ${ }^{\mathrm{a}}$

5 yr (females) ${ }^{c}$

Longevity
$28 \mathrm{yr}^{\mathrm{d}}$

$56 \mathrm{yr}$ (females) $^{\mathrm{e}}$

$57 \mathrm{yr}$ (males) ${ }^{\mathrm{e}}$
HEAD SPINES

$\begin{array}{lc}\text { Preocular } & + \\ \text { Postocular } & + \\ \text { Coronal } & 0 \\ \text { Supraocular } & + \\ \text { Tympanic } & + \\ \text { Inferior infraorbital } & 3\end{array}$

\section{EARLY LIFE HISTORY DESCRIPTION}

\section{LARVAE}

Hatch size

Preanal length

Length at flexion

Length at transformation

Sequence of fin development

Pigment

- Extrusion larvae: Short series midway along ventral body

- Larvae >10 mm SL

-Beneath dorsal fin

-Above and below notochord at tail

-Dorsal and ventral margin of caudal peduncle

-Moderate on paired fins

Diagnostic characters

- Pigment along dorsal fin base

- Relatively slender body

- No pigment at anal ray bases

- Pigment at tip of notochord

\section{PELAGIC JUVENILES}

- Pigment blotch on last few dorsal fin spines

- Body heavily pigmented

- Pectoral fin base, anal fin, and ventral caudal peduncle unpigmented

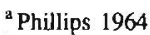

${ }^{b}$ Westrheim 1975

${ }^{c}$ Wyllie Echeverria 1987

Toole 1982

${ }^{\mathrm{C}}$ R. Mandapat, Wash. Dep. Fish., 7600 Sand Point Way N.E., Seattle, WA $98115-$

0070 , pers. commun., 1 June 1987.

${ }^{i}$ Juveniles are pelagic at $55-75 \mathrm{~mm}$ SL.

Ref: Laroche, in prep.; Laroche and Richardson 1981; Moser and Butler 1987; Washington et al. $1984 \mathrm{~b}$. 


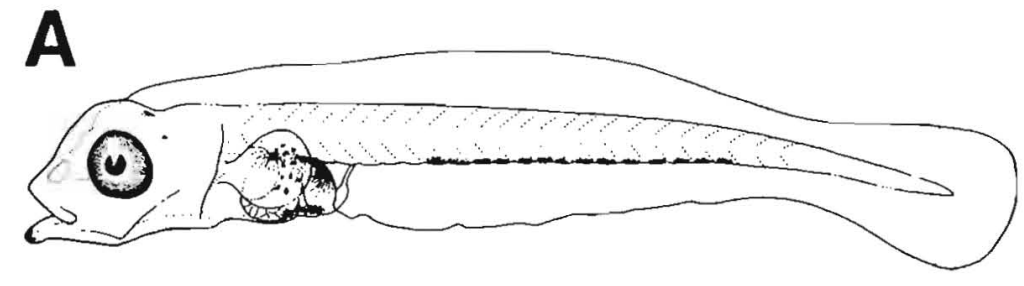

$4.8 \mathrm{~mm}$
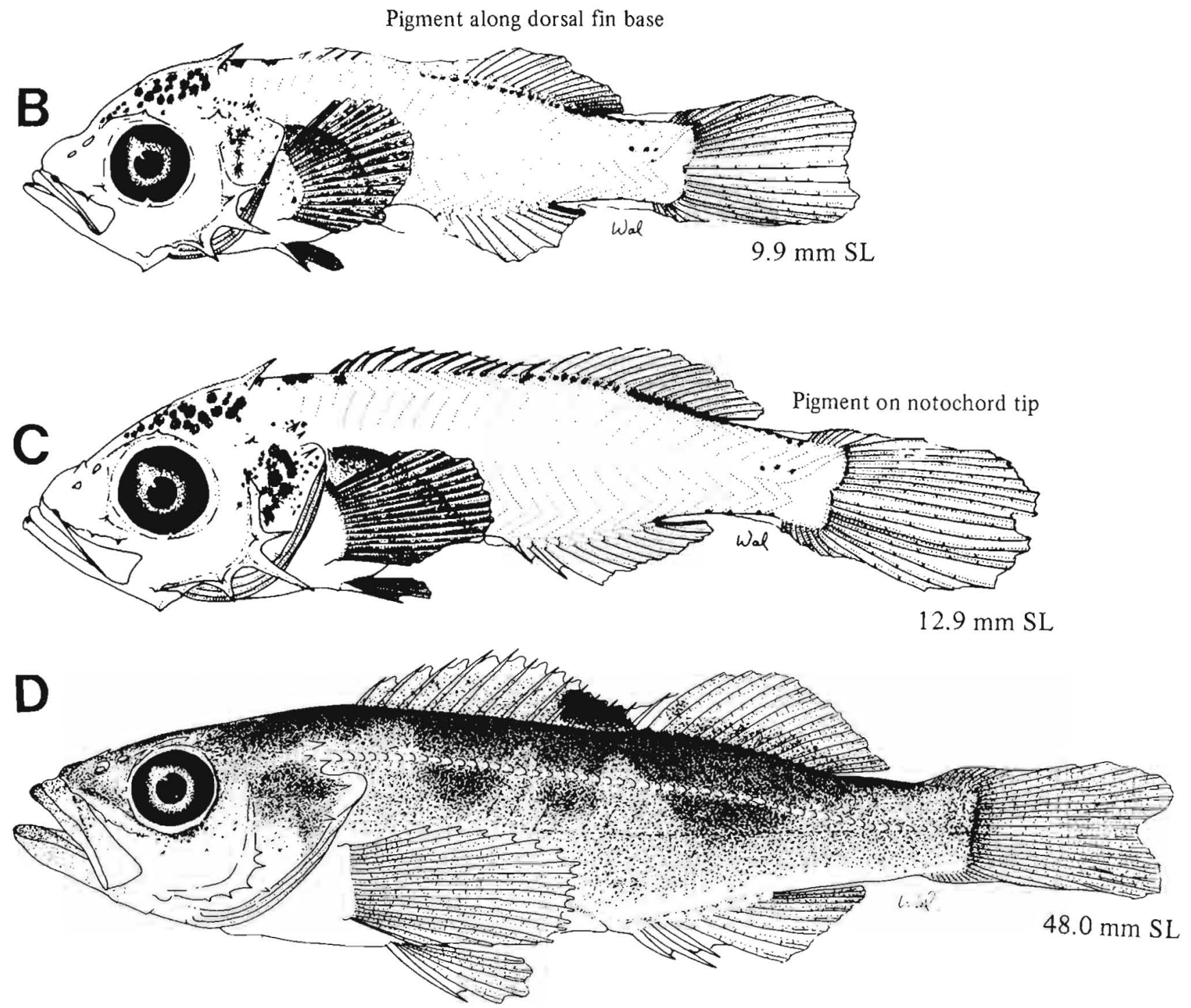

Figure A, Moser and Butler 1987; B-C, Laroche and Richardson 1981; D, Laroche, in prep. 


\section{MERISTICS}

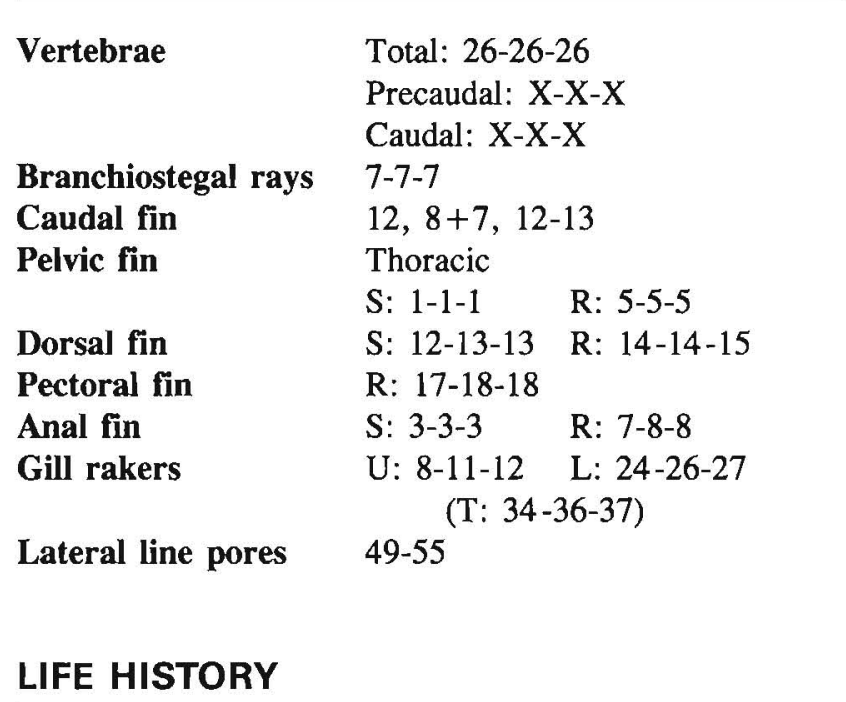

$\begin{array}{ll}\text { Range } & \begin{array}{l}\text { S. California, } 32-34^{\circ} \mathrm{N}, \text { to } \\ \text { Gulf of Alaska, } 54-60^{\circ} \mathrm{N}\end{array} \\ \text { Ecology } & \text { Epi- and mesobenthal, 0-549 m } \\ \text { ELH pattern } & \text { Ovoviviparous, pelagic larvae } \\ \text { Spawning } & \text { Season: Nov-Feb; }{ }^{\mathrm{a}} \mathrm{Mar}^{\mathrm{b}} \\ & \text { Area: } \\ & \text { Mode: } \\ & \text { Migration: } \\ \text { Fecundity } & \text { Range/function: } 48,000-632,800 / \\ & \mathrm{F}=82721.8 \times \mathrm{L}-323516^{\mathrm{c}} \\ \text { Age at first maturity } & 3-5 \mathrm{yr}^{\mathrm{a}} \\ & 7 \mathrm{yr} \text { (females) }^{\mathrm{d}} \\ \text { Longevity } & \end{array}$

a Phillips 1964

${ }^{b}$ Westrheim 1975

${ }^{c}$ Gunderson et al. 1980

${ }^{d}$ Wyllie Echeverria 1987

¿ Juveniles are pelagic at $40-50 \mathrm{~mm} \mathrm{SL}$.

Ref: DeLacy et al. 1964; Laroche, in prep.; Laroche and Richaroson 1980.

\section{HEAD SPINES}

$\begin{array}{ll}\text { Preocular } & 0 \\ \text { Postocular } & + \\ \text { Coronal } & 0 \\ \text { Supraocular } & 0 \\ \text { Tympanic } & + \\ \text { Inferior infraorbital } & 3\end{array}$

\section{EARLY LIFE HISTORY DESCRIPTION}

\section{LARVAE}

\section{Hatch size \\ Preanal length \\ $4.5 \mathrm{~mm}$ SL (pre-extrusion) \\ Length at flexion $50-75 \%$ SL \\ Length at transformation \\ Sequence of fin development \\ Complete at $10.1 \mathrm{~mm} \mathrm{SL}$ \\ 23-27 mm SL \\ Caudal and pectorals, pelvics, dorsal and anal}

Pigment

- Extrusion larvae: Series along ventral body

- See also S. melanops (p. 314)

- Moderate on paired fins

- Over tip of notochord

- Along base of second dorsal fin, developing all along dorsal midline

- Dorsal and ventral margin of caudal peduncle

- At bases of some dorsal and anal fin rays

Diagnostic characters

- See also S. melanops

- Number of pectoral fin rays (18)

- Lateral line pores $49-55$, usually $>50$

- Slender caudal peduncle

- Heavy pigment develops along entire dorsal midline

- Pigment at bases of some dorsal and anal fin rays

\section{PELAGIC JUVENILES}

- Pigment blotch on last few dorsal fin spines

- Body heavily pigmented, diffuse

- Ventral caudal peduncle pigmented 

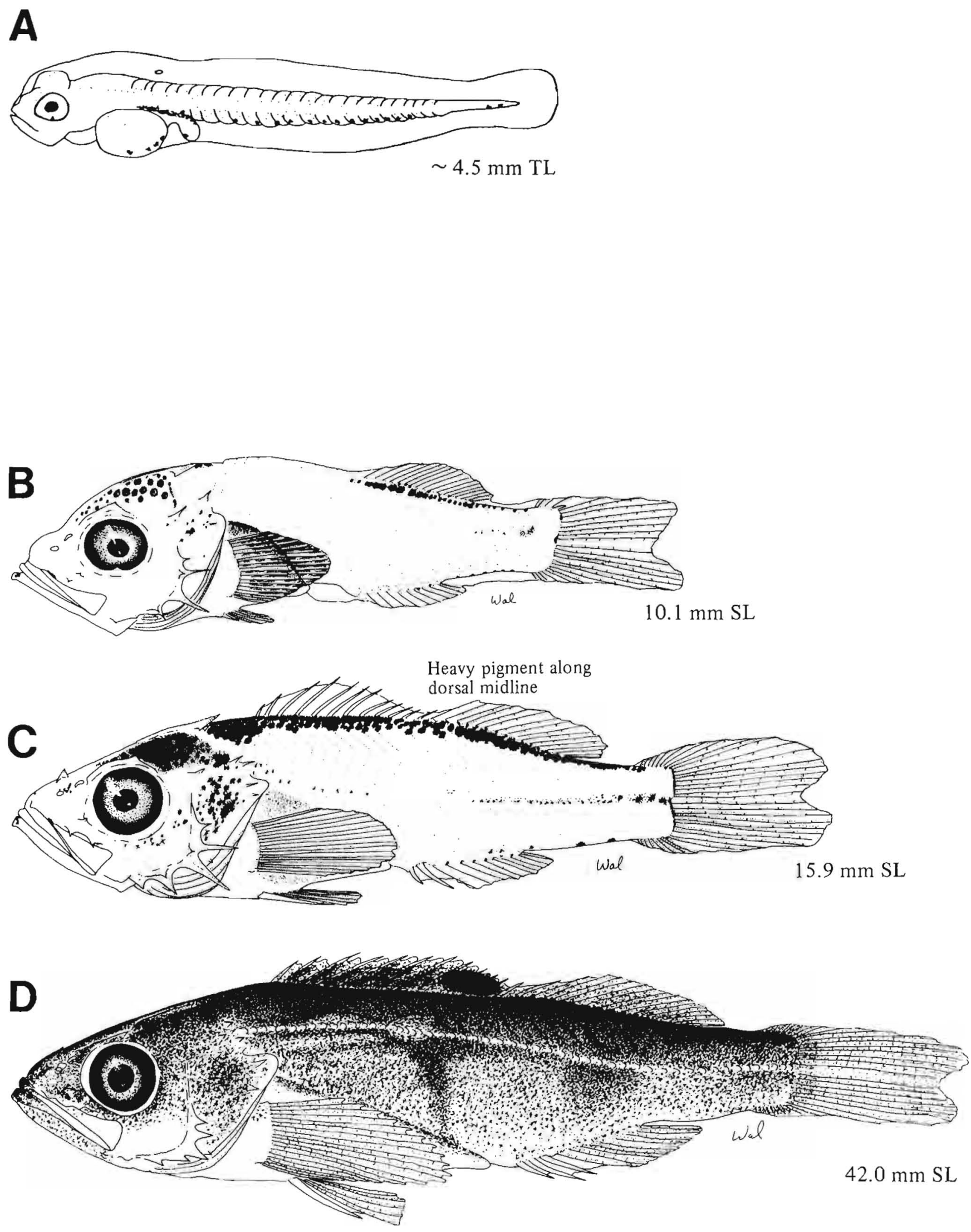

Figure A, DeLacy et al. 1964; B-C, Laroche and Richardson 1980; D, Laroche, in prep. 


\section{MERISTICS}

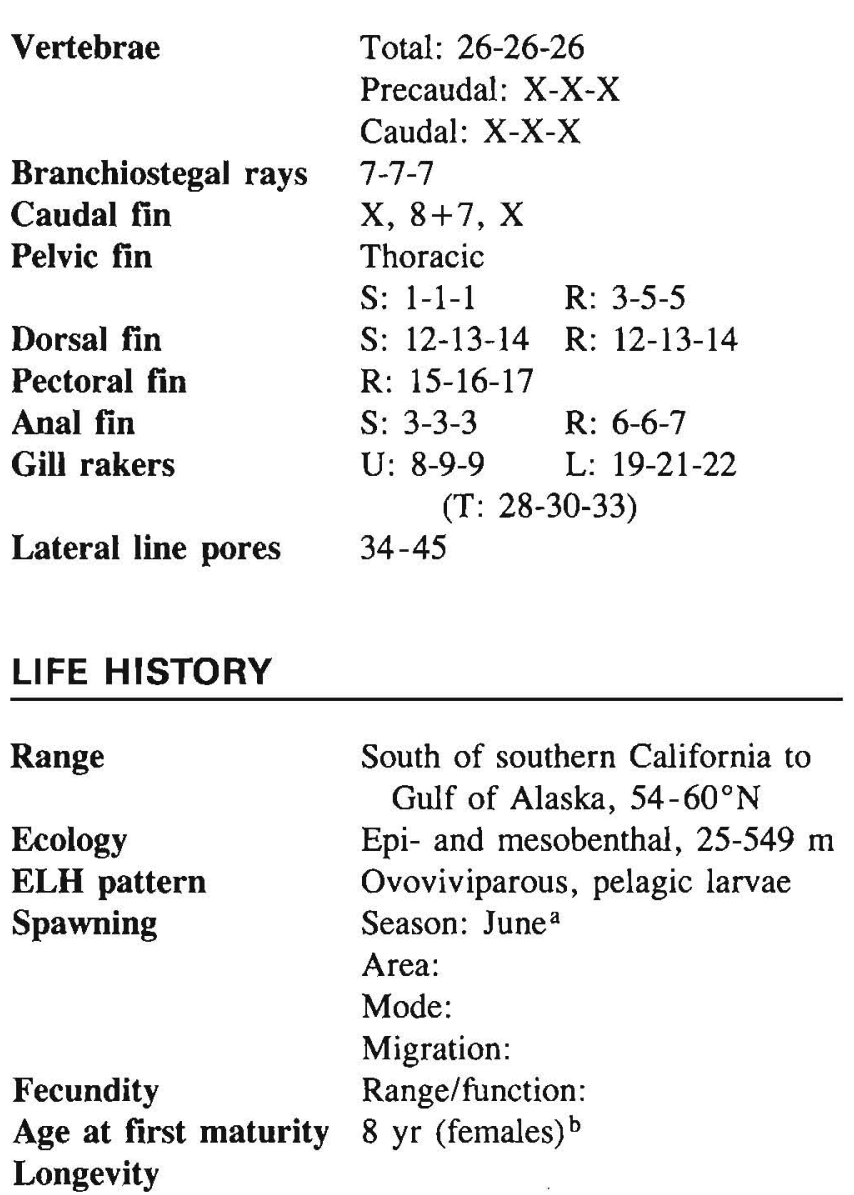

\section{HEAD SPINES}

$\begin{array}{ll}\text { Preocular } & + \\ \text { Postocular } & + \\ \text { Coronal } & 0 \\ \text { Supraocular } & + \\ \text { Tympanic } & + \\ \text { Inferior infraorbital } & 2\end{array}$

\section{EARLY LIFE HISTORY DESCRIPTION}

\section{LARVAE}

Hatch size

Preanal length

Length at flexion

$+$

$+$

0

$+$

$+$

Length at transformation

Sequence of fin development

Pigment

- Extrusion larvae: Series along ventral body

- Lack of body pigment, with development caudal peduncle patch

- Pigmented area at base of long parietal spine

- Pigmented fringes of pectoral and pelvic fins

Diagnostic characters

- Pigmented fringes of pectoral and pelvic fins

- Lack of body pigment

- Long serrate parietal and middle posterior preopercular spines

\section{PELAGIC JUVENILES}

- Body pigment diffuse, heavier over gut and on caudal peduncle

\footnotetext{
${ }^{a}$ Westrheim 1975

${ }^{b}$ Wyllie Echeverria 1987

' Juveniles are pelagic to $60 \mathrm{~mm}$ SL.

Ref: Laroche, in prep.; Richardson and Laroche 1979; Washington et al. 1984b; Westrheim 1975
} 

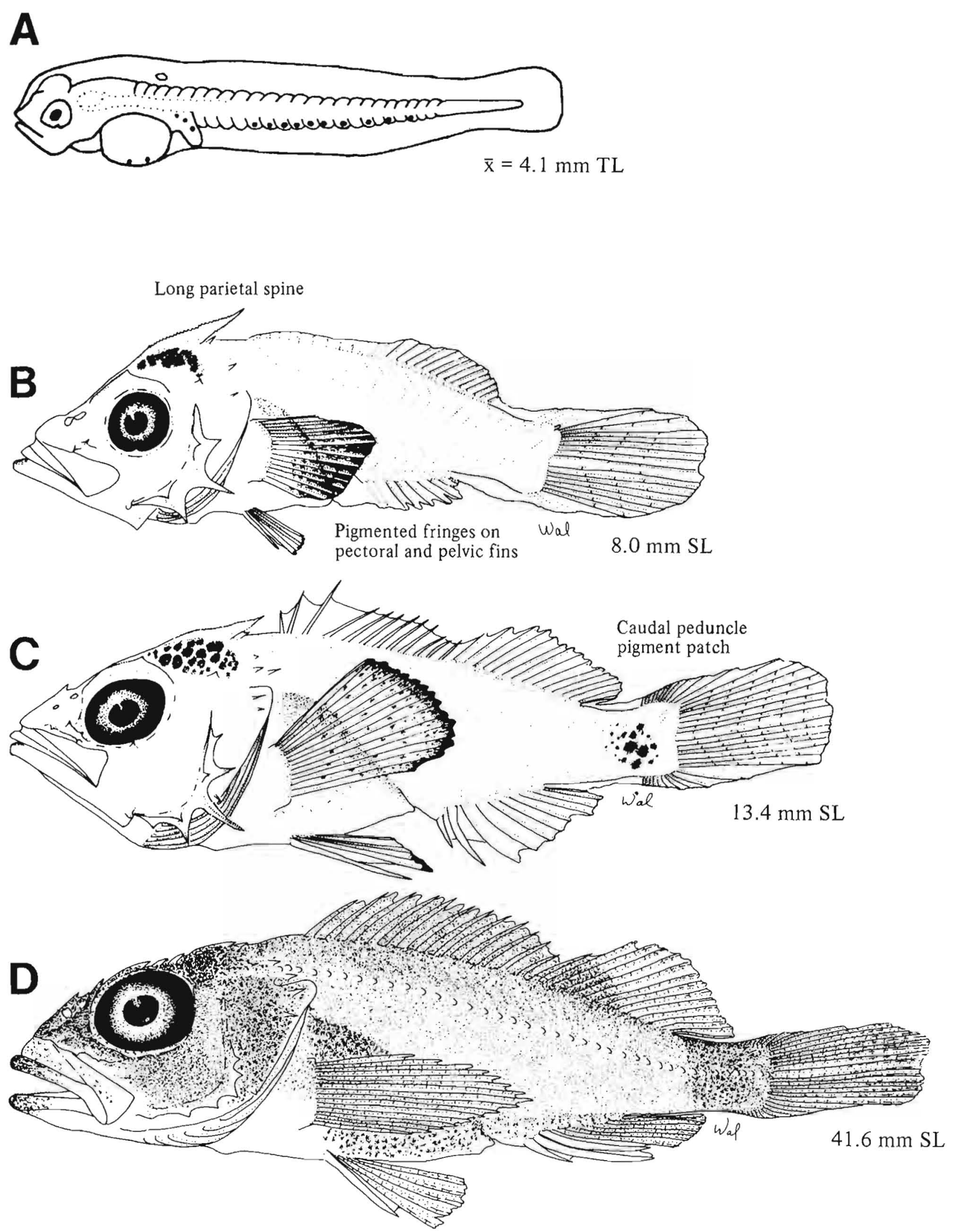

Figure A, Westrheim et al. 1968a; B-C, Richardson and Laroche 1979; D, Laroche, in prep. 


\section{MERISTICS}

\begin{tabular}{|c|c|}
\hline \multirow[t]{3}{*}{ Vertebrae } & Total: $26-26-26$ \\
\hline & Precaudal: $\mathrm{X}-\mathrm{X}-\mathrm{X}$ \\
\hline & Caudal: $\mathrm{X}-\mathrm{X}-\mathrm{X}$ \\
\hline Branchiostegal rays & 7-7-7 \\
\hline Caudal fin & $X, 8+7, x$ \\
\hline Pelvic fin & Thoracic \\
\hline & $R: 5-5-5$ \\
\hline Dorsal fin & S: $13-13-13$ R: $13-14-16$ \\
\hline Pectoral fin & R: $19-20-22$ \\
\hline Anal fin & R: $9-9-10$ \\
\hline Gill rakers & $\begin{array}{c}\mathrm{U}: 12-12-12 \text { L: } 31-31-31 \\
\text { (T: } 40-44-47)\end{array}$ \\
\hline Lateral line pores & $53-64$ \\
\hline
\end{tabular}

$\begin{array}{ll}\text { Range } & \begin{array}{l}\text { South of southern California to } \\ \text { Gulf of Alaska, 54-60 }\end{array} \\ \text { Ecology } & \text { Epi- and mesobenthal, 0-350 m } \\ \text { ELH pattern } & \text { Ovoviviparous, pelagic larvae } \\ \text { Spawning } & \text { Season: Nov-Mar; }{ }^{\mathrm{a}} \mathrm{Mar}^{\mathrm{b}} \\ & \text { Area: } \\ & \text { Mode: } \\ & \text { Migration: } \\ \text { Fecundity } & \text { Range/function: 7000-50,000 } \\ \text { Age at first maturity } & 3 \mathrm{yr} \text { (females) }^{\mathrm{d}} \\ \text { Longevity } & \end{array}$

\section{HEAD SPINES}

$\begin{array}{ll}\text { Preocular } & + \\ \text { Postocular } & + \\ \text { Coronal } & 0 \\ \text { Supraocular } & 0 \\ \text { Tympanic } & + \\ \text { Inferior infraorbital } & 2\end{array}$

\section{EARLY LIFE HISTORY DESCRIPTION}

\section{LARVAE}

\section{Hatch size}

Preanal length

Length at flexion

Length at transformation

Sequence of fin development

Pigment

- Brain (dorsal head on crown)

- Pigment over dorsolateral surface of gut

- Dorsal and ventral postanal midline (see illustrations)

\section{Diagnostic characters}

- Most slender Sebastes sp. larvae described to date (body depth $<25 \%$ SL)

- Large larvae (5.4 mm SL at hatching, 8-10 mm SL at flexion)

- Opposing dorsal and ventral postanal midline pigment

- Anterior placement of anus in juveniles/adults

- Large gap between anus and origin of anal fin

\section{PELAGIC JUVENILES}

- Elongate shape, large gap between anus and origin of anal fin

- Body pigment diffuse, but myosepta pattern evident

\footnotetext{
Phillips 1964

${ }^{\mathrm{b}}$ Westrheim 1975

${ }^{\mathrm{c}}$ Hart 1973

${ }^{d}$ Wyllie Echeverria 1987

'Juveniles are pelagic at $30-63 \mathrm{~mm} \mathrm{SL}$.

Ref: Laroche, in prep.; Moser et al. 1977; Washington et al. 1984b.
} 
A
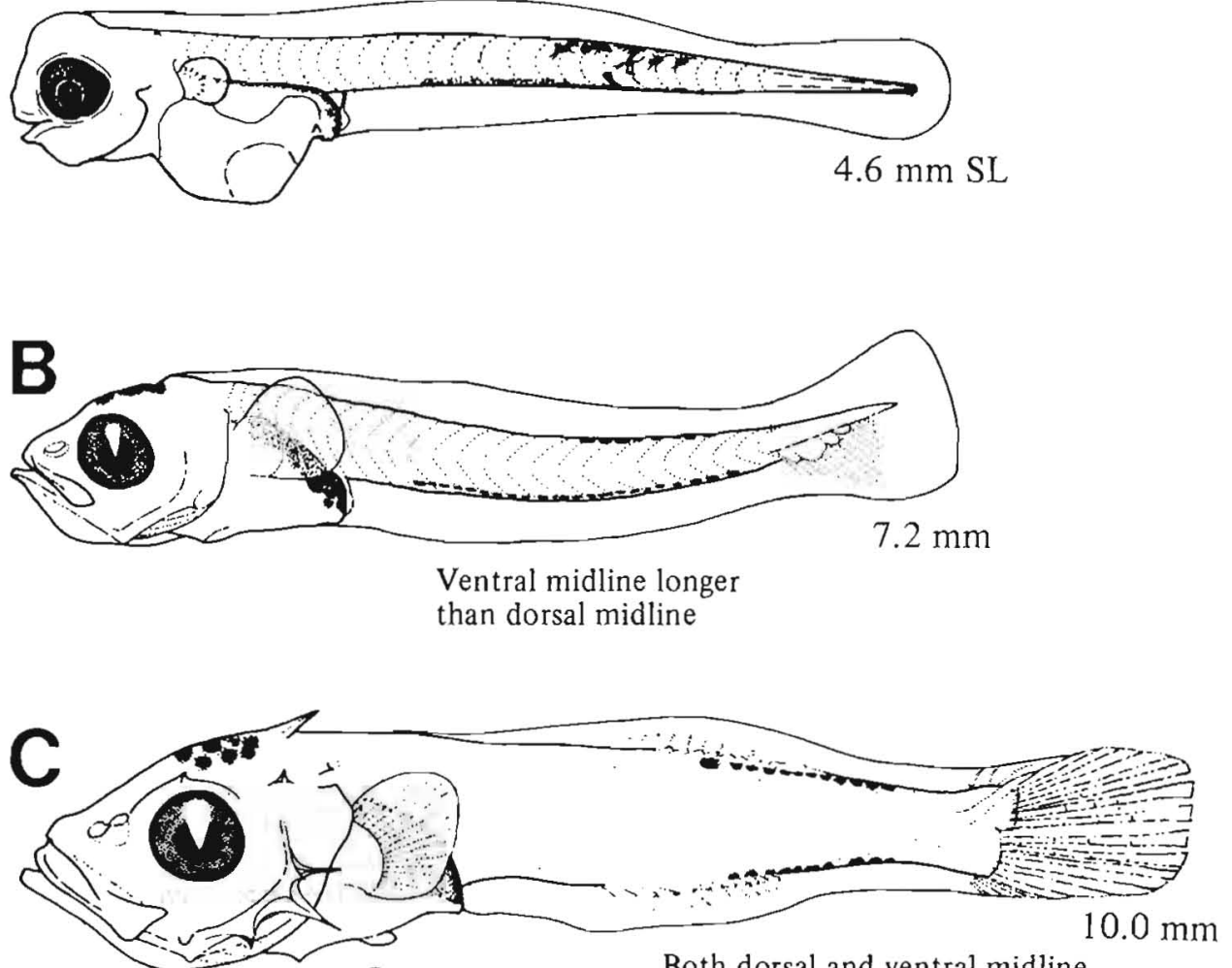

Gap between anus and anal fin origin

Both dorsal and ventral midline equal in length, extending from midbody to just anterior to caudal peduncle
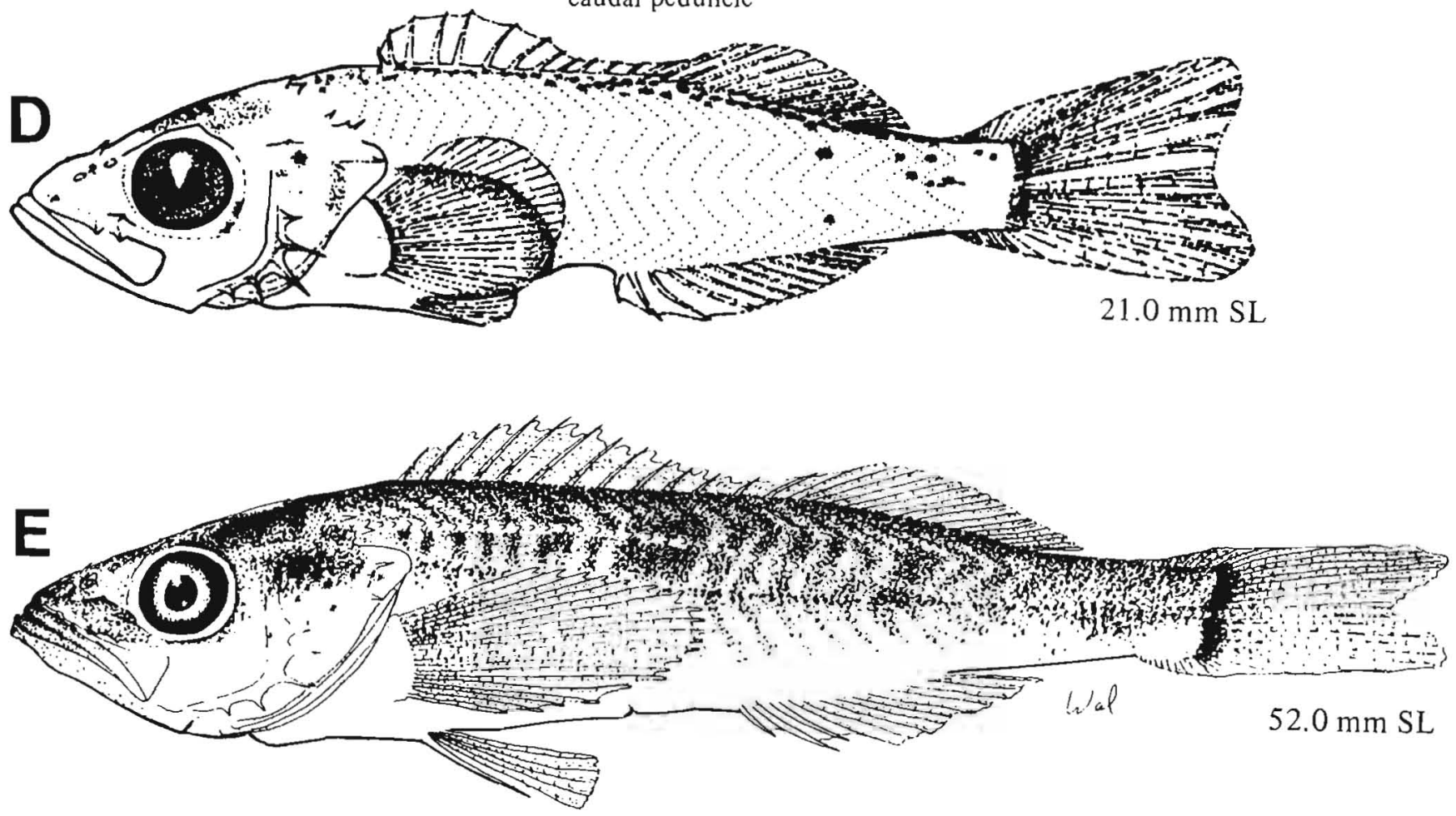

Figure A, NWAFC original (B. Vinter); B-D, Moser et al. 1977; E, Laroche, in prep. 
MERISTICS

\begin{tabular}{|c|c|}
\hline \multirow[t]{3}{*}{ Vertebrae } & Total: $26-26-26$ \\
\hline & Precaudal: X-X-X \\
\hline & Caudal: X-X-X \\
\hline Branchiostegal rays & $7-7-7$ \\
\hline Caudal fin & $12,8+7,12-13$ \\
\hline \multirow[t]{2}{*}{ Pelvic fin } & Thoracic \\
\hline & S: $1-1-1 \quad R: 5-5-5$ \\
\hline Dorsal fin & S: $13-13-14$ R: $14-15-15$ \\
\hline Pectoral fin & $\mathrm{R}: 18-19-19$ \\
\hline Anal fin & $S: 3-3-3 \quad R: 7-8-9$ \\
\hline Gill rakers & $\begin{array}{c}\mathrm{U}: 9-11-13 \quad \mathrm{~L}: 23-26-28 \\
\text { (T: } 34-37-38)\end{array}$ \\
\hline Lateral line pores & $47-55$ \\
\hline \multicolumn{2}{|l|}{ LIFE HISTORY } \\
\hline Range & $\begin{array}{l}\text { Cent. California, } 34-38^{\circ} \mathrm{N} \text {, to } \\
\text { Aleutian Is., } 51-55^{\circ} \mathrm{N}\end{array}$ \\
\hline Ecology & \multirow{3}{*}{$\begin{array}{l}\text { Epi- and mesobenthal, 0-366 m } \\
\text { Ovoviviparous, pelagic larvae } \\
\text { Season: Jan; }{ }^{a} \text { Feb-Apr }{ }^{b}\end{array}$} \\
\hline ELH pattern & \\
\hline Spawning & \\
\hline & Area: \\
\hline & Mode: \\
\hline & Migration: \\
\hline Fecundity & Range/function: \\
\hline $\begin{array}{l}\text { Age at first maturity } \\
\text { Longevity }\end{array}$ & 7 yr (females) ${ }^{c}$ \\
\hline
\end{tabular}

\section{HEAD SPINES}

$\begin{array}{lc}\text { Preocular } & 0 \\ \text { Postocular } & + \\ \text { Coronal } & 0 \\ \text { Supraocular } & 0 \\ \text { Tympanic } & + \\ \text { Inferior infraorbital } & 3\end{array}$

\section{EARLY LIFE HISTORY DESCRIPTION}

\section{LARVAE}

\section{Hatch size}

Preanal length

$50-75 \% \mathrm{SL}$

Length at flexion

Complete at $10.6 \mathrm{~mm} \mathrm{SL}$

Length at transformation $24-33 \mathrm{~mm} \mathrm{SL}^{\mathrm{d}}$

Sequence of fin development

Caudal and pectorals, pelvics, dorsal and anal

Pigment

- Extrusion larvae: Series along ventral body

- Larvae >10.6 mm SL

-Head, on crown and opercle

- Pigment over dorsolateral surface of gut

- Dorsal midline heavier than ventral midline

- Hypural margin

-Along posterior lateral line, extends anteriorly along notochord with development

-Paired fin blades moderately pigmented

-Discrete melanophores at the articulation of several dorsal and anal fin rays

\section{Diagnostic characters}

Distinguished from $S$. flavidus (p. 308) by

- High pectoral fin ray count (usually 19)

- Fewer lateral line pores (usually $<50$ )

- Deeper, shorter caudal peduncle

\section{PELAGIC JUVENILES}

- Body pigment heavy, diffuse

- Pigment blotch on last few dorsal fin spines

- Lateral midline pigmented

\footnotetext{
Westrheim 1975

bHart 1973

'Wyllie Echeverria 1987

${ }^{d}$ Juveniles are pelagic at $40-50 \mathrm{~mm}$ SL.
}

Ref: Laroche and Richardson 1980 

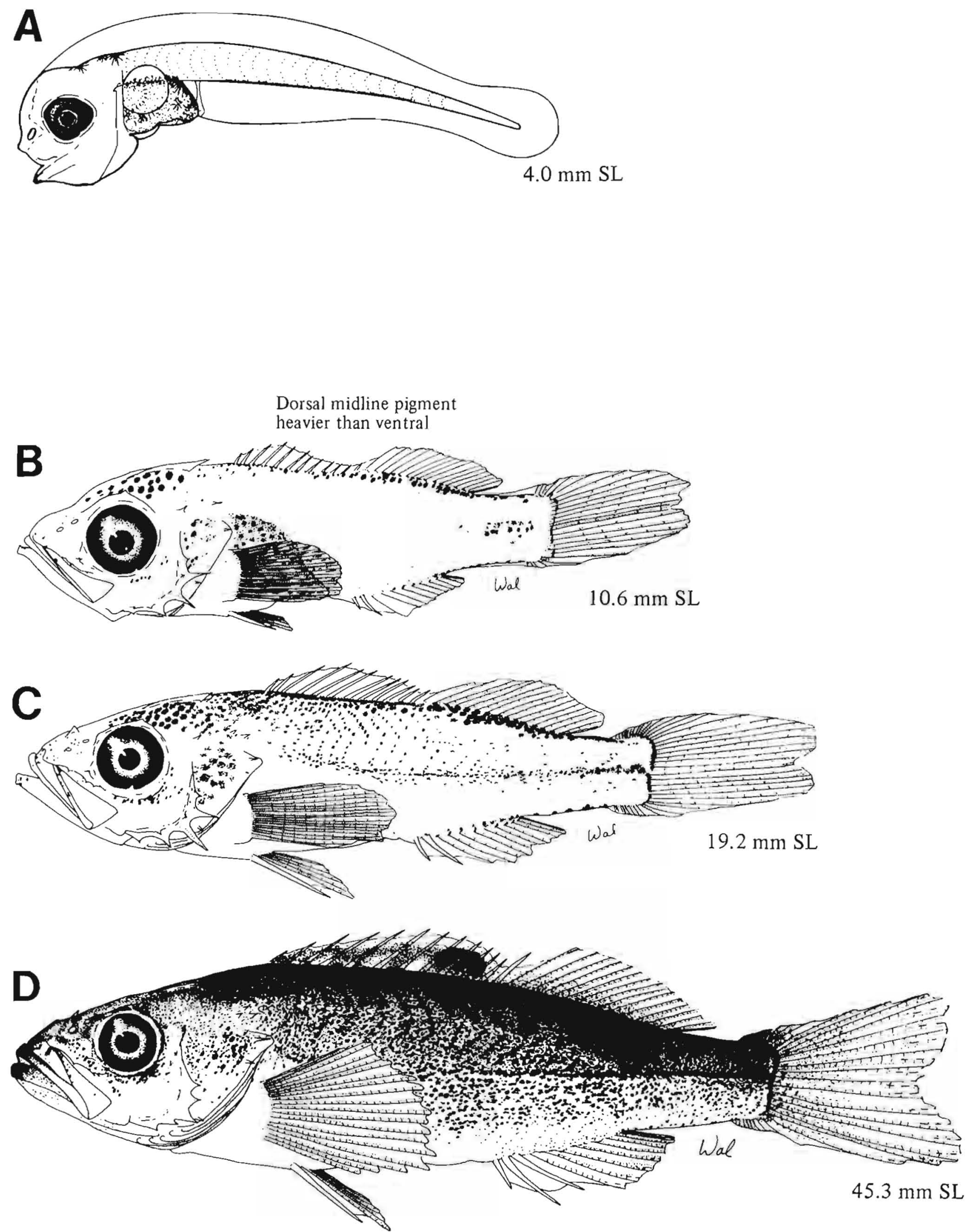

Figure A, NWAFC original (B. Vinter); B-D, Laroche and Richardson 1980. 


\section{MERISTICS}

\begin{tabular}{|c|c|c|}
\hline Vertebrae & $\begin{array}{l}\text { Total: } 26-26 \\
\text { Precaudal: X } \\
\text { Caudal: X-X }\end{array}$ & $\begin{array}{l}26 \\
X-X \\
X\end{array}$ \\
\hline Branchiostegal rays & $7-7-7$ & \\
\hline Caudal fin & $9-10,8+7$, & -11 \\
\hline Pelvic fin & Thoracic & \\
\hline & S: $1-1-1$ & $\mathrm{R}: 5-5-5$ \\
\hline Dorsal fin & S: $13-13-13$ & R: $12-13-14$ \\
\hline Pectoral fin & $\mathrm{R}: 18-19-20$ & \\
\hline Anal fin & $S: 3-3-3$ & $R: 6-7-8$ \\
\hline Gill rakers & $\mathrm{U}: 8-\mathrm{X}-9$ & $\mathrm{~L}: 22-22-22$ \\
\hline & (T: 3 & $31-34)$ \\
\hline
\end{tabular}

\section{LIFE HISTORY}

$\begin{array}{ll}\text { Range } & \begin{array}{c}\text { South of southern California to } \\ \text { Washington, } 46-48^{\circ} 30^{\prime} \mathrm{N} \\ \text { Epi-, meso-, and bathybenthal, } \\ \text { Ecology }\end{array} \\ \begin{array}{ll}\text { Ovoviviparous, pelagic larvae } \\ \text { ELH pattern }\end{array} & \begin{array}{l}\text { Season: Abundance of newborn } \\ \text { larvae peaks in February off }\end{array} \\ & \text { S. California }{ }^{\mathrm{a}} \\ & \text { Area: } \\ & \text { Mode: } \\ & \text { Migration: } \\ \text { Fecundity } & \text { Range/function: } \\ \text { Age at first maturity } & 8 \mathrm{yr} \text { (females) }^{\mathrm{b}} \\ \text { Longevity } & \end{array}$

${ }^{a}$ Moser and Ahlstrom 1978

${ }^{\mathrm{b}}$ Wyllie Echeverria 1987

${ }^{c}$ Largest pelagic juvenile collected is $46.2 \mathrm{~mm} \mathrm{SL}$; smallest demersal juvenile collected is $36.0 \mathrm{~mm}$ SL.

Ref: Laroche, in prep.; Moser and Ahlstrom 1978.

\section{HEAD SPINES}

$\begin{array}{ll}\text { Preocular } & + \\ \text { Postocular } & + \\ \text { Coronal } & 0 \\ \text { Supraocular } & + \\ \text { Tympanic } & + \\ \text { Inferior infraorbital } & 2\end{array}$

\section{EARLY LIFE HISTORY DESCRIPTION}

\section{LARVAE}

$\begin{array}{ll}\text { Hatch size } & 4.5 \mathrm{~mm} \mathrm{SL} \\ \text { Preanal length } & \begin{array}{c}37 \% \text { SL increasing with } \\ \text { development to } 62 \% \mathrm{SL}\end{array} \\ & 6.2-7.2 \mathrm{~mm} \mathrm{SL} \\ \begin{array}{l}\text { Length at flexion } \\ \text { Length at transformation }\end{array} & \sim 16 \mathrm{~mm} \mathrm{SLc} \\ \begin{array}{c}\text { Sequence of fin } \\ \text { development }\end{array} & \begin{array}{c}\text { Caudal and pectorals, } \\ \text { pelvics, dorsal and anal }\end{array}\end{array}$

Pigment

- Preflexion larvae

- Single row postanal ventral midline ( $~ 8$ spots)

-Brain

-Lower jaw, streak along upper jaw

-Dorsolaterally on gut

-Pectoral fin

- Flexion larvae: Opercle blotch and upper jaw streak

- Postflexion and pelagic juveniles: Anterior bar; with development, two additional bars form, under second dorsal and on caudal peduncle

\section{Diagnostic characters}

- Pigment

-Jaw, opercle blotch, postanal bars

-Blades of paired fins heavily pigmented

-Band of pigment on body centered at about dorsal spine 5-7

- Head spination (see p. 271)

-Large serrate parietal spine

-Large weakly serrate preopercle angle spine

- Deep bodied $(>35 \% \mathrm{SL})$

\section{PELAGIC JUVENILES}

- Banded body pigment extends onto dorsal and anal fins

- Pectoral fin, but not base, pigmented 

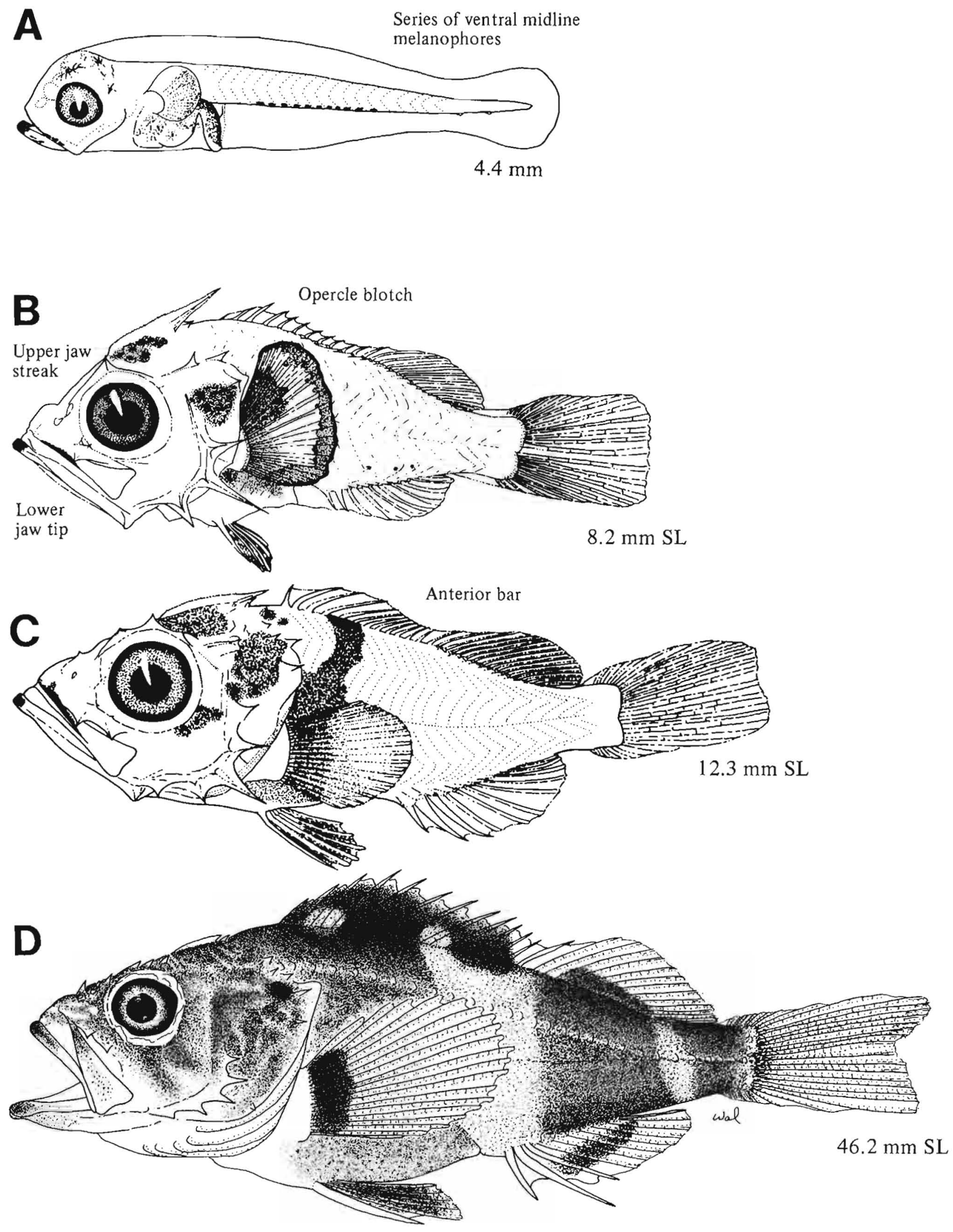

Figure A, Moser et al. 1977; B-C, Moser and Ahlstrom 1978; D, Laroche, in prep. 


\section{MERISTICS}

Vertebrae

Caudal fin

Pelvic fin

Dorsal fin

Pectoral fin

Anal fin

Gill rakers

Lateral line pores

$41-48$

\section{LIFE HISTORY}

$\begin{array}{ll}\text { Range } & \begin{array}{c}\text { South of southern California to } \\ \text { Brit. Col., } 48^{\circ} 30^{\prime}-55^{\circ} \mathrm{N}\end{array} \\ \text { Ecology } & \text { Epi- and mesobenthal, 0-274 m } \\ \text { ELH pattern } & \text { Ovoviviparous, pelagic larvae } \\ \text { Spawning } & \text { Season: Nov-Mar }{ }^{\mathrm{a}} \\ & \text { Area: } \\ & \text { Mode: } \\ & \text { Migration: } \\ \text { Fecundity } & \text { Range/function: } \\ \text { Age at first maturity } & 5 \mathrm{yr} \text { (females) }^{\mathrm{b}} \\ \text { Longevity } & \end{array}$

Total: $26-26-26$

Precaudal: X-X-X

Caudal: $\mathrm{X}-\mathrm{X}-\mathrm{X}$

\section{7-7-7}

$\mathrm{X}, 8+7, \mathrm{X}$

Thoracic
S: $1-1-1$
R: $5-5-5$

S: $13-13-13$ R: $13-14-15$

R: $17-18-18$

S: $3-3-3$

R: $6-7-7$

$\mathrm{U}: \mathrm{X}-\mathrm{X}-\mathrm{X}$

L: X-X-X

$$
\text { (T: } 36-41-42)
$$

HEAD SPINES

$\begin{array}{lc}\text { Preocular } & + \\ \text { Postocular } & + \\ \text { Coronal } & 0 \\ \text { Supraocular } & + \\ \text { Tympanic } & + \\ \text { Inferior infraorbital } & 2\end{array}$

\section{EARLY LIFE HISTORY DESCRIPTION}

\section{LARVAE}

Hatch size $4.3 \mathrm{~mm}$

Preanal length

Length at flexion

Length at transformation

Sequence of fin development

Pigment

- Extrusion larvae: Short series midway along ventral body

- Postflexion larvae $>12.3 \mathrm{~mm} \mathrm{SL}$ -Dorsolateral patch under spinous dorsal -Along dorsal and anal fin ray bases

- Series along ventral midline toward caudal, becoming internal

-Along hypural margin

Diagnostic characters

\section{PELAGIC JUVENILES}

- Body and head pigment diffuse, heavy

- Pigment blotch on last few dorsal fin spines

- Paired fins pigmented 

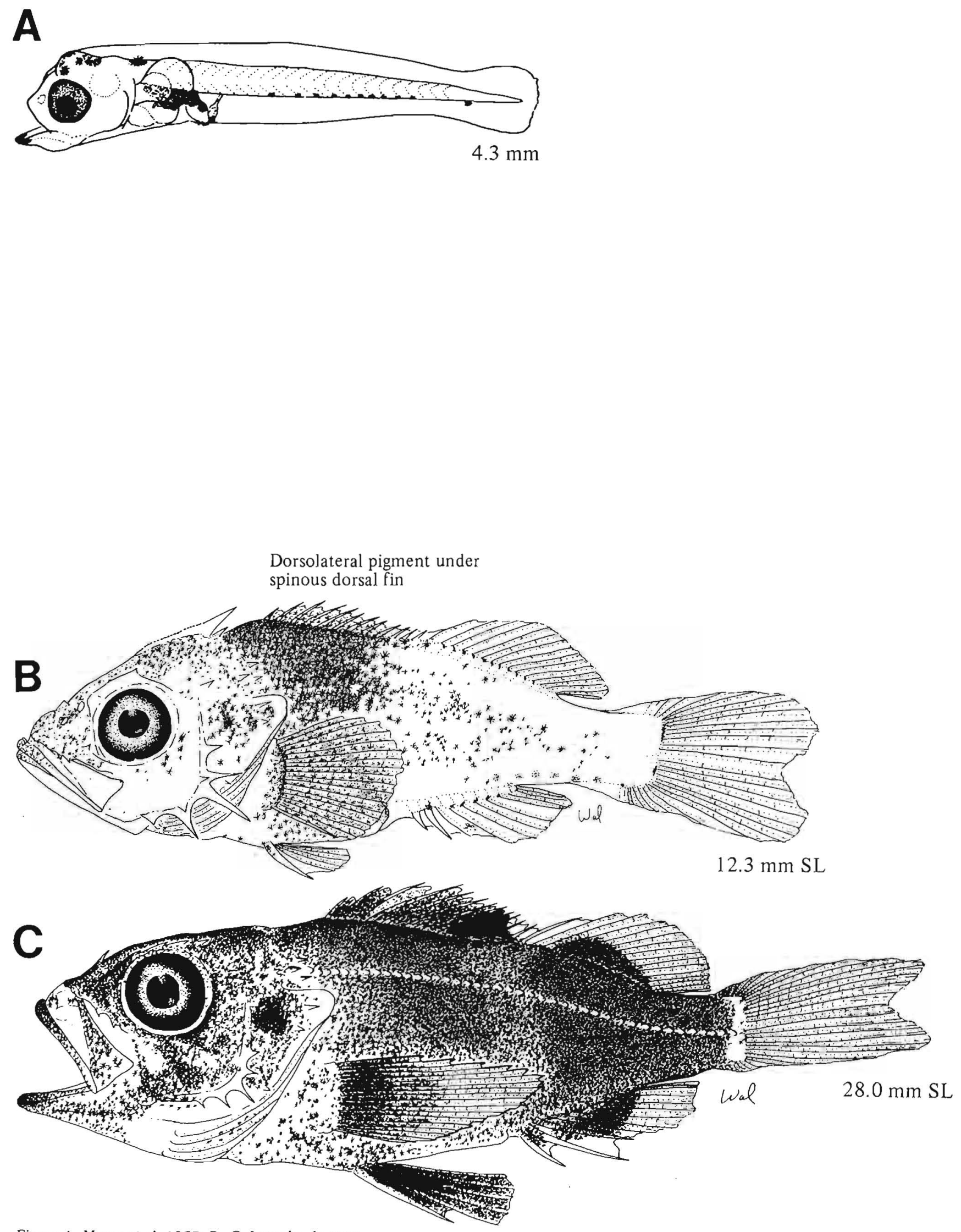

Figure A, Moser et al. 1977; B-C, Laroche, in prep. 


\section{MERISTICS}

\begin{tabular}{|c|c|c|}
\hline \multirow[t]{3}{*}{ Vertebrae } & \multicolumn{2}{|c|}{ Total: $26-26-27$} \\
\hline & \multicolumn{2}{|c|}{ Precaudal: $\mathrm{X}-\mathrm{X}-\mathrm{X}$} \\
\hline & \multicolumn{2}{|c|}{ Caudal: X-X-X } \\
\hline Branchiostegal rays & \multicolumn{2}{|l|}{$7-7-7$} \\
\hline Caudal fin & \multicolumn{2}{|l|}{$X, 8+7, X$} \\
\hline Pelvic fin & \multicolumn{2}{|l|}{ Thoracic } \\
\hline & S: $1-1-1$ & $R: 5-5-5$ \\
\hline Dorsal fin & S: $13-13-13$ & $\mathrm{R}: 15-16-17$ \\
\hline Pectoral fin & \multicolumn{2}{|l|}{$\mathrm{R}: 17-18-19$} \\
\hline Anal fin & $S: 3-3-3$ & $\mathrm{R}: 8-9-10$ \\
\hline Gill rakers & $\begin{array}{r}\mathrm{U}: 9-10-12 \\
(\mathrm{~T}: 32-\end{array}$ & $(\mathrm{T}: 32-35-38)$ \\
\hline Lateral line pores & $47-53$ & \\
\hline
\end{tabular}

$\begin{array}{ll}\text { Range } & \begin{array}{r}\text { South of southern California to } \\ \text { Aleutian Is., } 51-55^{\circ} \mathrm{N}\end{array} \\ & \text { Epi- and mesopelagic, 0-549 m } \\ \text { Ecology } & \text { Ovoviviparous, pelagic larvae } \\ \text { ELH pattern } & \text { Season: Nov-Jan } \\ \text { Spawning } & \text { Area: } \\ & \text { Mode: } \\ & \text { Migration: } \\ \text { Fecundity } & \text { Range/function: } \\ \text { Age at first maturity } & 6 \text { yr (females) } \\ \text { Longevity } & \end{array}$

a Wales 1952

${ }^{b}$ Wyllie Echeverria 1987

${ }^{c}$ L. Wold and G. Moreno, Moss Landing Mar. Lab., Moss Landing, CA 95035-0450, pers. commun., 26 July 1988. Drawing (Fig. A) by L. McMasters. Based on results from California Sea Grant Project R/F 115.

Ref: Efremenko and Lisovenko 1970; Laroche, in prep.

\section{HEAD SPINES}

\begin{tabular}{lc} 
Preocular & + \\
Postocular & + \\
Coronal & 0 \\
Supraocular & + \\
Tympanic & + \\
Inferior infraorbital & 3 \\
& \\
EARLY LIFE HISTORY DESCRIPTION \\
\hline
\end{tabular}

\section{LARVAE}

Hatch size $5.2 \mathrm{~mm} ; 3.8 \mathrm{~mm} \mathrm{SL}^{\mathrm{c}}$

\section{Preanal length}

Length at flexion

Length at transformation

Sequence of fin development

Pigment

- Extrusion larvae: Short series along ventral body ${ }^{\mathrm{c}}$

- Preflexion larvae

-Spots develop at tip of lower jaw and on pectoral fin blade ${ }^{c}$

-Spots form at junction of cleithra

- Postflexion larvae $>12.8 \mathrm{~mm} \mathrm{SL}$

-Along body margins beneath soft dorsal fin and posterior to anal fin

- Hypural margin

-Internal and external spots along lateral line

Diagnostic characters

\section{PELAGIC JUVENILES}

- Body and head pigment diffuse, heavy

- Pigment blotch on last few dorsal fin spines 
A
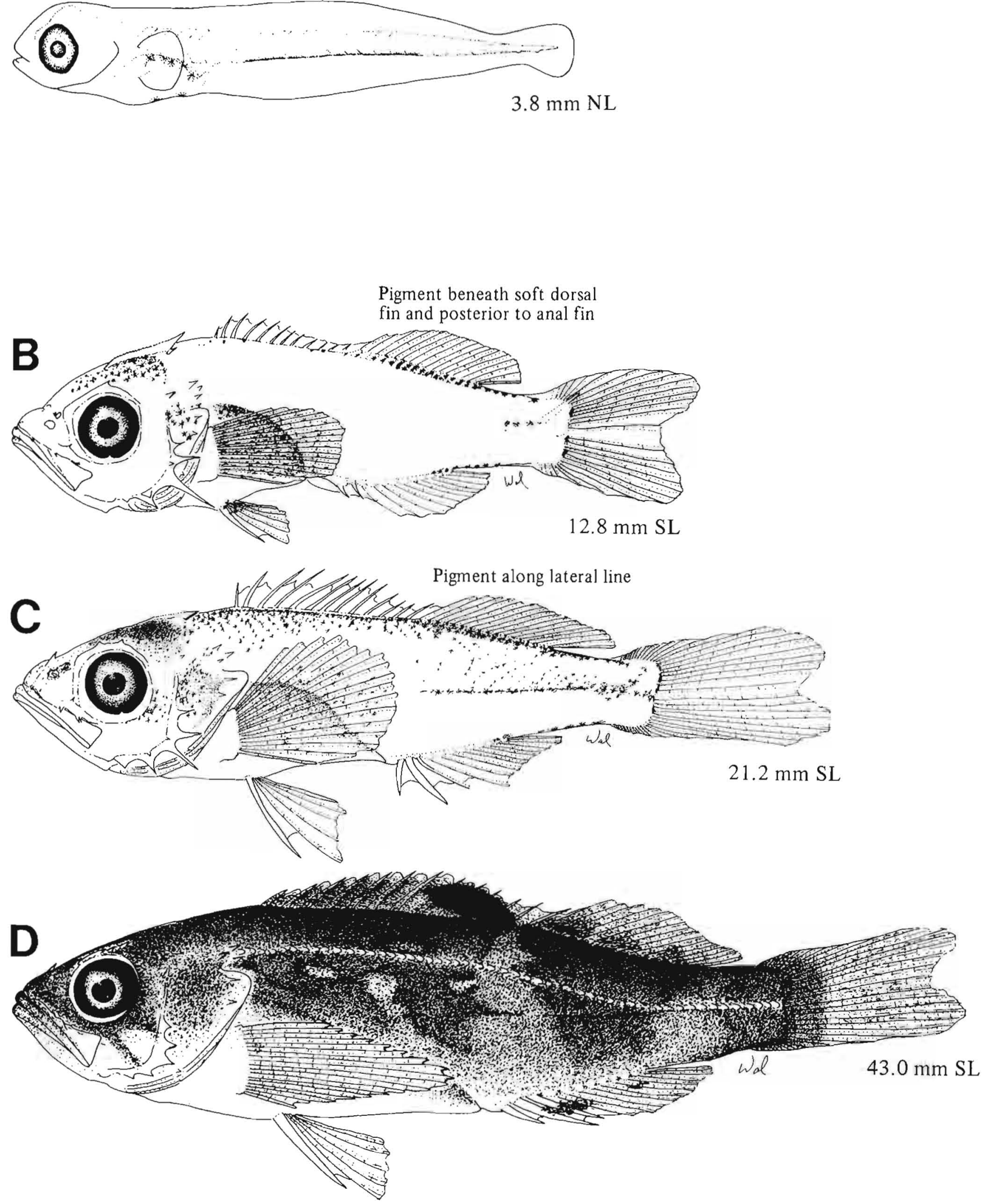

Figure A, Wold, unpubl.; B-D, Laroche, in prep. 


\section{MERISTICS}

\begin{tabular}{|c|c|c|}
\hline Vertebrae & \multicolumn{2}{|c|}{$\begin{array}{l}\text { Total: } 26-26-26 \\
\text { Precaudal: X-X-X } \\
\text { Caudal: X-X-X }\end{array}$} \\
\hline Branchiostegal rays & $7-7-7$ & \\
\hline Caudal fin & $X, 8+7, X$ & \\
\hline Pelvic fin & $\begin{array}{l}\text { Thoracic } \\
\text { S: } 1-1-1\end{array}$ & $R: 5-5-5$ \\
\hline Dorsal fin & S: $13-13-15$ & $\mathrm{R}: 13-14-15$ \\
\hline Pectoral fin & $R: 14-15-16$ & \\
\hline Anal fin & $S: 3-3-3$ & $\mathrm{R}: 8-9-10$ \\
\hline Gill rakers & 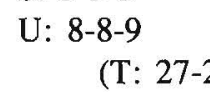 & $\begin{array}{l}\text { L: } 20-21-21 \\
28-32)\end{array}$ \\
\hline Lateral line pores & $51-62$ & \\
\hline
\end{tabular}

$\begin{array}{ll}\text { Range } & \begin{array}{c}\text { South of southern California to } \\ \text { Gulf of Alaska, } 54-60^{\circ} \mathrm{N}\end{array} \\ \text { Ecology } & \text { Epi- and mesobenthal, 0-475 m } \\ \text { ELH pattern } & \text { Ovoviviparous, pelagic larvae } \\ \text { Spawning } & \text { Season: Jan-Feb; }{ }^{\mathrm{a}} \text { Nov-Apr } \\ & \text { Area: Semi-demersal }(25-305 \mathrm{~m})^{\mathrm{c}} \\ & \text { Mode: } \\ & \text { Migration: } \\ \text { Fecundity } & \text { Range/function: 20,000 d- } \\ & 2,440,000^{\mathrm{e}} \text { (may mature more } \\ & \text { than one brood per year) } \\ \text { Age at first maturity } & 3-4 \mathrm{yr}^{\mathrm{d}} \\ & 4 \mathrm{yr}^{\mathrm{d}} \text { females) }^{\mathrm{f}} \\ \text { Longevity } & 30 \mathrm{yr}^{\mathrm{g}} \\ & 60 \mathrm{yr}^{\mathrm{h}}\end{array}$

\footnotetext{
${ }^{a}$ Westrheim 1975

${ }^{\circ}$ Frey 1971

${ }^{c}$ Moser 1967

${ }^{d}$ Phillips 1964

${ }^{\mathrm{e}}$ Gunderson et al. 1980

'Wyllie Echeverria 1987

${ }^{8}$ Hart 1973

${ }^{\text {h}}$ Beamish 1979

Ref: Laroche, in prep.; Moser 1967; Moser et al. 1977.
}

\section{HEAD SPINES}

$\begin{array}{lc}\text { Preocular } & + \\ \text { Postocular } & 0 \\ \text { Coronal } & 0 \\ \text { Supraocular } & 0 \\ \text { Tympanic } & + \\ \text { Inferior infraorbital } & 3-4\end{array}$

\section{EARLY LIFE HISTORY DESCRIPTION}

\section{LARVAE}

Hatch size

Preanal length

4-5 $\mathrm{mm} \mathrm{SL}$

$<50 \%$, with development $50-75 \%$ SL

Length at flexion $\quad 7.2-9.7 \mathrm{~mm} \mathrm{SL}$

Length at transformation $\sim 15 \mathrm{~mm} \mathrm{SL}$

Sequence of fin development

Caudal and pectorals, pelvics, dorsal and anal

Pigment

- Fringed margins of pectoral and pelvic fins

- Few (6-14) ventral midline melanophores migrate with development to form large patch on caudal peduncle

Diagnostic characters

- Early development of elongate pigmented paired fins

- Midlateral pigment blotch on caudal peduncle

- Gap between anus and anal fin origin

\section{PELAGIC JUVENILES}

- Body pigment diffuse, light

- Paired and first dorsal fins heavily pigmented distally 

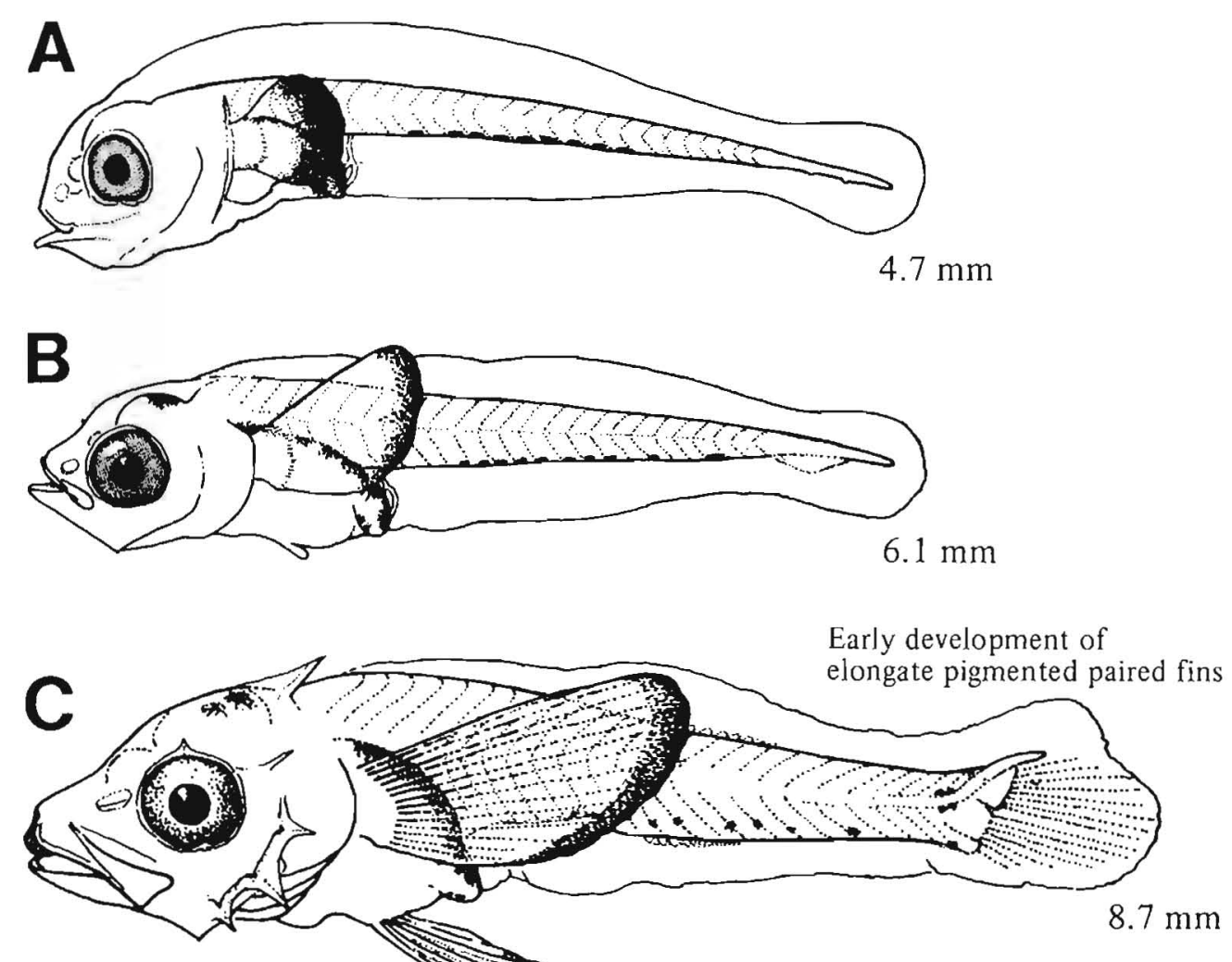

Early development of

elongate pigmented paired fins

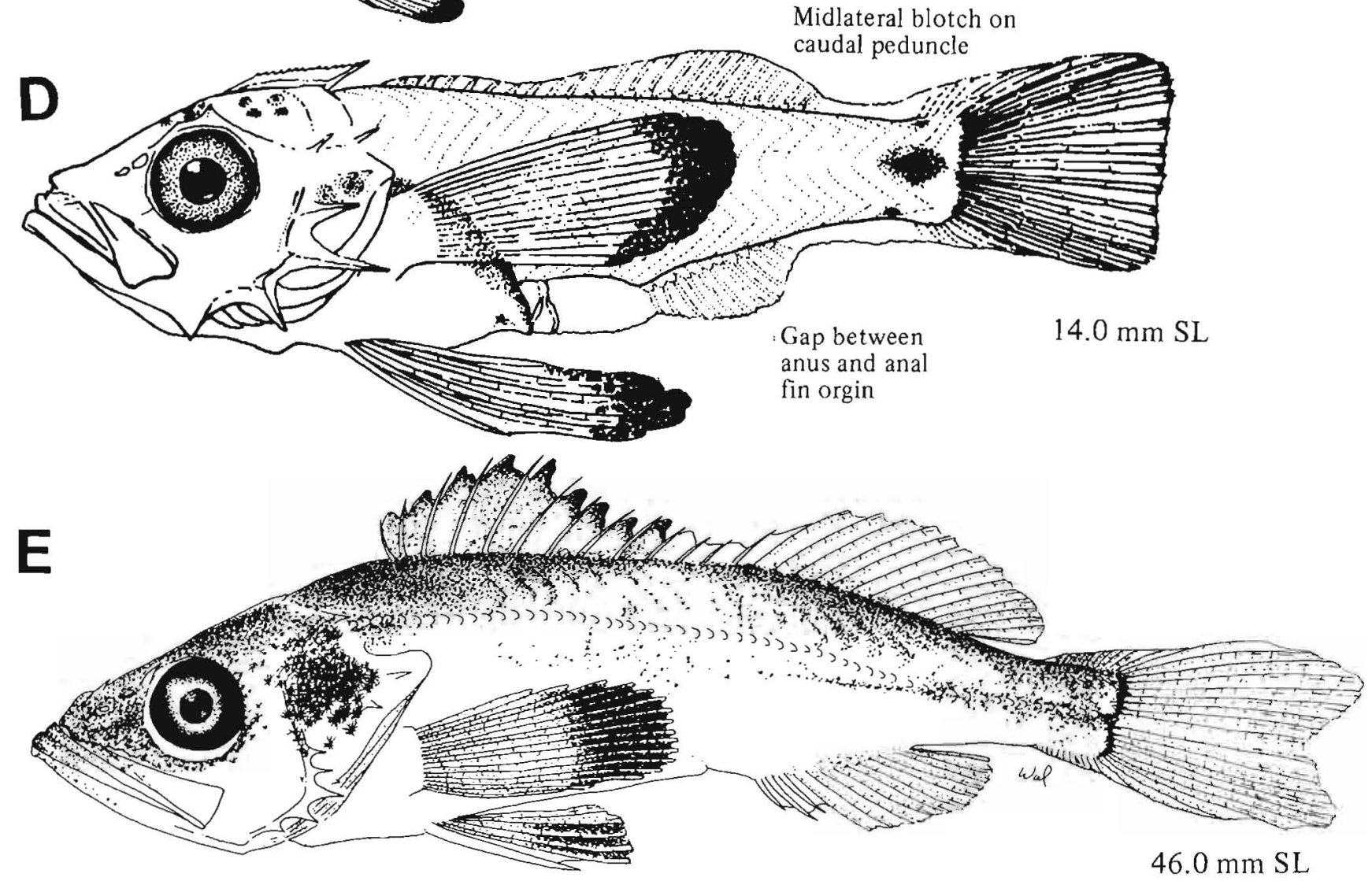

Figures A-D, Moser et al. 1977; E, Laroche, in prep. 


\section{MERISTICS}

\begin{tabular}{|c|c|}
\hline \multirow{3}{*}{ Vertebrae } & Total: $26-26-26$ \\
\hline & Precaudal: $\mathrm{X}-\mathrm{X}-\mathrm{X}$ \\
\hline & Caudal: X-X-X \\
\hline Branchiostegal rays & $7-7-7$ \\
\hline Caudal fin & $X, 8+7, X$ \\
\hline \multirow[t]{2}{*}{ Pelvic fin } & Thoracic \\
\hline & $\mathrm{R}: 5-5-5$ \\
\hline Dorsal fin & S: $13-13-13$ R: $13-14-15$ \\
\hline Pectoral fin & $\mathrm{R}: 16-17-18$ \\
\hline Anal fin & S: $3-3-3 \quad R: 7-7-7$ \\
\hline Gill rakers & $\begin{array}{c}\mathrm{U}: 12-14-15 \quad \mathrm{~L}: 26-28-31 \\
\text { (T: } 41-43-44)\end{array}$ \\
\hline Lateral line pores & $40-47$ \\
\hline \multicolumn{2}{|l|}{ LIFE HISTORY } \\
\hline Range & $\begin{array}{l}\text { South of southern California to } \\
\text { Gulf of Alaska, } 54-60^{\circ} \mathrm{N}\end{array}$ \\
\hline Ecology & Epi- and mesobenthal, $0-425 \mathrm{~m}$ \\
\hline ELH pattern & Ovoviviparous, pelagic larvae \\
\hline Spawning & $\begin{array}{l}\text { Season: Jan-Mar (Brit. Col.); }{ }^{a} \\
\text { Nov-Mar (California) }^{b}\end{array}$ \\
\hline & Area: \\
\hline & Mode: \\
\hline & Migration: \\
\hline Fecundity & $\begin{array}{l}\text { Range/function: } \\
260,000-1,897,600^{c /} \\
F=64221.3 \times L-2330029^{d}\end{array}$ \\
\hline Age at first maturity & $3-5 \mathrm{yr}^{\mathrm{c}}$ \\
\hline Longevity & \\
\hline
\end{tabular}

\footnotetext{
a Westrheim 1975

${ }^{b}$ Moser 1967

${ }^{c}$ Phillips 1964

${ }^{d}$ Gunderson et al. 1980

${ }^{e}$ Wyllie Echeverria 1987

' Larvae are pelagic to $50 \mathrm{~mm} \mathrm{SL}$

Ref: Laroche, in prep.; Moser et al. 1977; Richardson and Laroche 1979; Waldron 1968; Washington et al. 1984b
}

\section{HEAD SPINES}

$\begin{array}{ll}\text { Preocular } & + \\ \text { Postocular } & + \\ \text { Coronal } & 0 \\ \text { Supraocular } & + \\ \text { Tympanic } & + \\ \text { Inferior infraorbital } & 2\end{array}$

\section{EARLY LIFE HISTORY DESCRIPTION}

\section{LARVAE}

Hatch size

Preanal length

Length at flexion

3.6-4.0 mm SL

Length at transformation $12.8-18.4 \mathrm{~mm} \mathrm{SL}^{\mathrm{f}}$

Sequence of fin development

Caudal and pectorals, pelvics, dorsal and anal

Pigment

- Initially: Lower jaw, ventral surface of gut and posterior gut, short dorsal and ventral midline series at $\sim 3 / 4 \mathrm{BL}$ between myomeres 18 and 24

- Short dorsal and ventral midline series on caudal peduncle

- Lightly pigmented paired fins

- Head: Dorsal surface; with development, patch also on upper part of opercle

- Paired fins have large melanophores on blades

\section{Diagnostic characters}

- Pigment: Opercular spot, spots on nape

- Morphology: Deep bodied ( $40 \%$ SL)

- Spines: Large serrate parietal and third posterior preopercular spine

\section{PELAGIC JUVEINILES}

- Body pigment banded, mainly above lateral midline

- Pigment blotch on last few dorsal fin spines, little pigment on rest of fins 

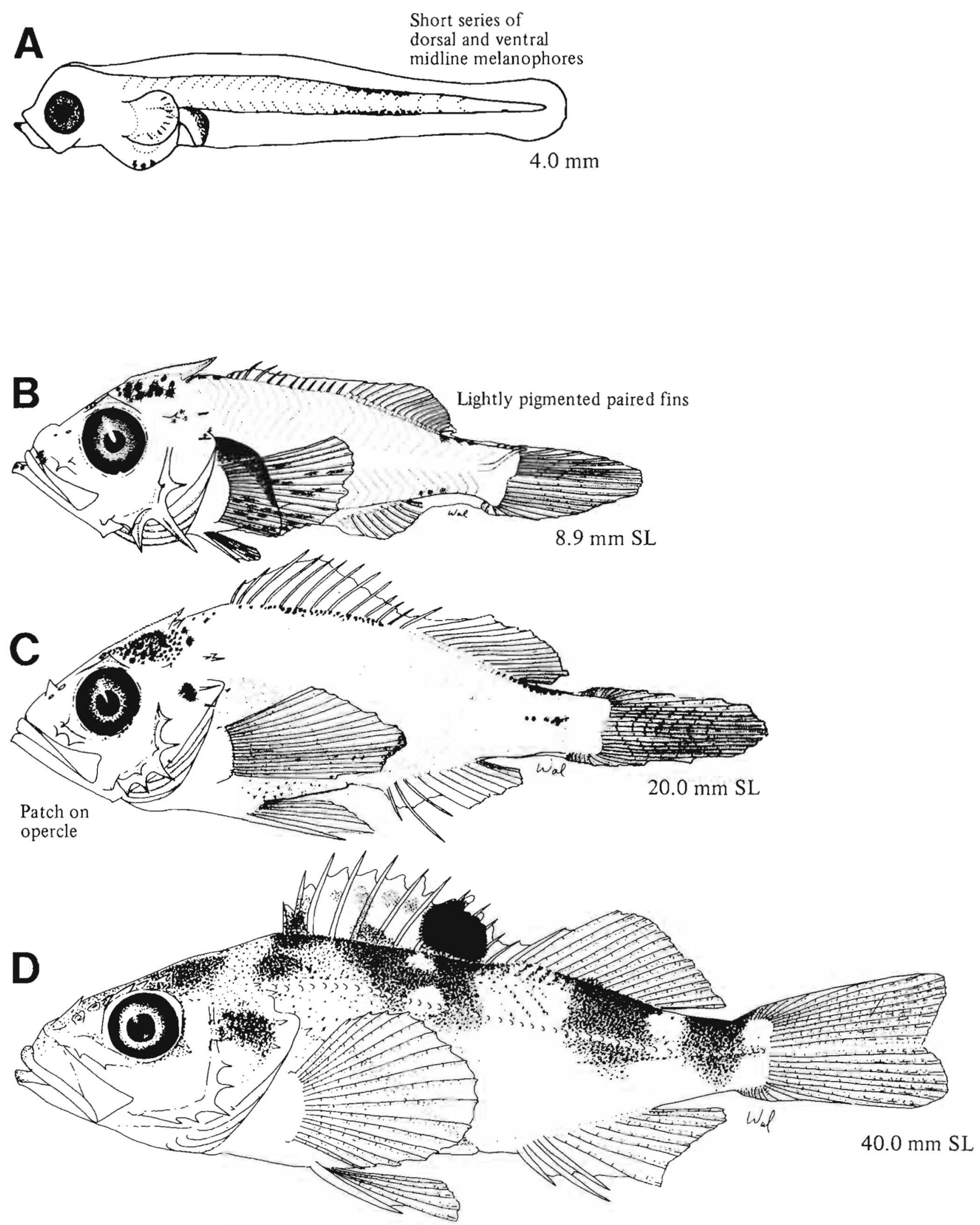

Figure A, Moser et al. 1977; B-C, Richardson and Laroche 1979; D, Laroche, in prep. 


\section{MERISTICS}

\begin{tabular}{|c|c|c|}
\hline \multirow{3}{*}{ Vertebrae } & \multicolumn{2}{|c|}{ Total: $27-27-27$} \\
\hline & \multicolumn{2}{|c|}{ Precaudal: X-X-X } \\
\hline & \multicolumn{2}{|c|}{ Caudal: X-X-X } \\
\hline Branchiostegal rays & \multicolumn{2}{|c|}{$7-7-7$} \\
\hline Caudal fin & \multicolumn{2}{|l|}{$X, 8+7, X$} \\
\hline Pelvic fin & \multicolumn{2}{|l|}{ Thoracic } \\
\hline & S: $1-1-1$ & $\mathrm{R}: 5-5-5$ \\
\hline Dorsal fin & S: $13-13-13$ & $\mathrm{R}: 14-15-15$ \\
\hline Pectoral fin & R: $16-17-17$ & \\
\hline Anal fin & S: $3-3-3$ & $\mathrm{R}: 7-7-7$ \\
\hline Gill rakers & 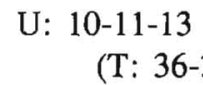 & $\begin{array}{l}\text { L: } 26-27-30 \\
38-41)\end{array}$ \\
\hline Lateral line pores & $48-55$ & \\
\hline
\end{tabular}

$\begin{array}{ll}\text { Range } & \text { S. California, } 32-34^{\circ} \mathrm{N}, \text { to } \\ & \text { Bering Sea, 54-66 } \mathrm{N} \\ \text { Ecology } & \text { Epi- and mesobenthal, 12-274 m } \\ \text { ELH pattern } & \text { Ovoviviparous, pelagic larvae } \\ \text { Spawning } & \text { Season: July } \\ & \text { Area: } \\ & \text { Mode: } \\ & \text { Migration: } \\ \text { Fecundity } & \text { Range/function: } \\ \text { Age at first maturity } & \\ \text { Longevity } & \end{array}$

\section{HEAD SPINES}

$\begin{array}{lc}\text { Preocular } & + \\ \text { Postocular } & + \\ \text { Coronal } & 0 \\ \text { Supraocular } & 0 \\ \text { Tympanic } & + \\ \text { Inferior infraorbital } & 2\end{array}$

\section{EARLY LIFE HISTORY DESCRIPTION}

\author{
LARVAE \\ Hatch size \\ Preanal length \\ Length at flexion \\ Length at transformation \\ Sequence of fin \\ development \\ Pigment \\ - Extrusion larvae: Series along ventral body \\ - Postflexion larvae $>16.8 \mathrm{~mm}$ SL \\ -On head dorsally \\ - Along dorsal body margin and anal fin ray base \\ -Internal and external spots along lateral midline \\ -Hypural margin
}

Diagnostic characters

\section{PELAGIC JUVENILES}

- Body elongate, pigment diffuse

- Little fin pigment

\footnotetext{
${ }^{\mathrm{a}}$ Westrheim 1975

Ref: Laroche, in prep.
} 

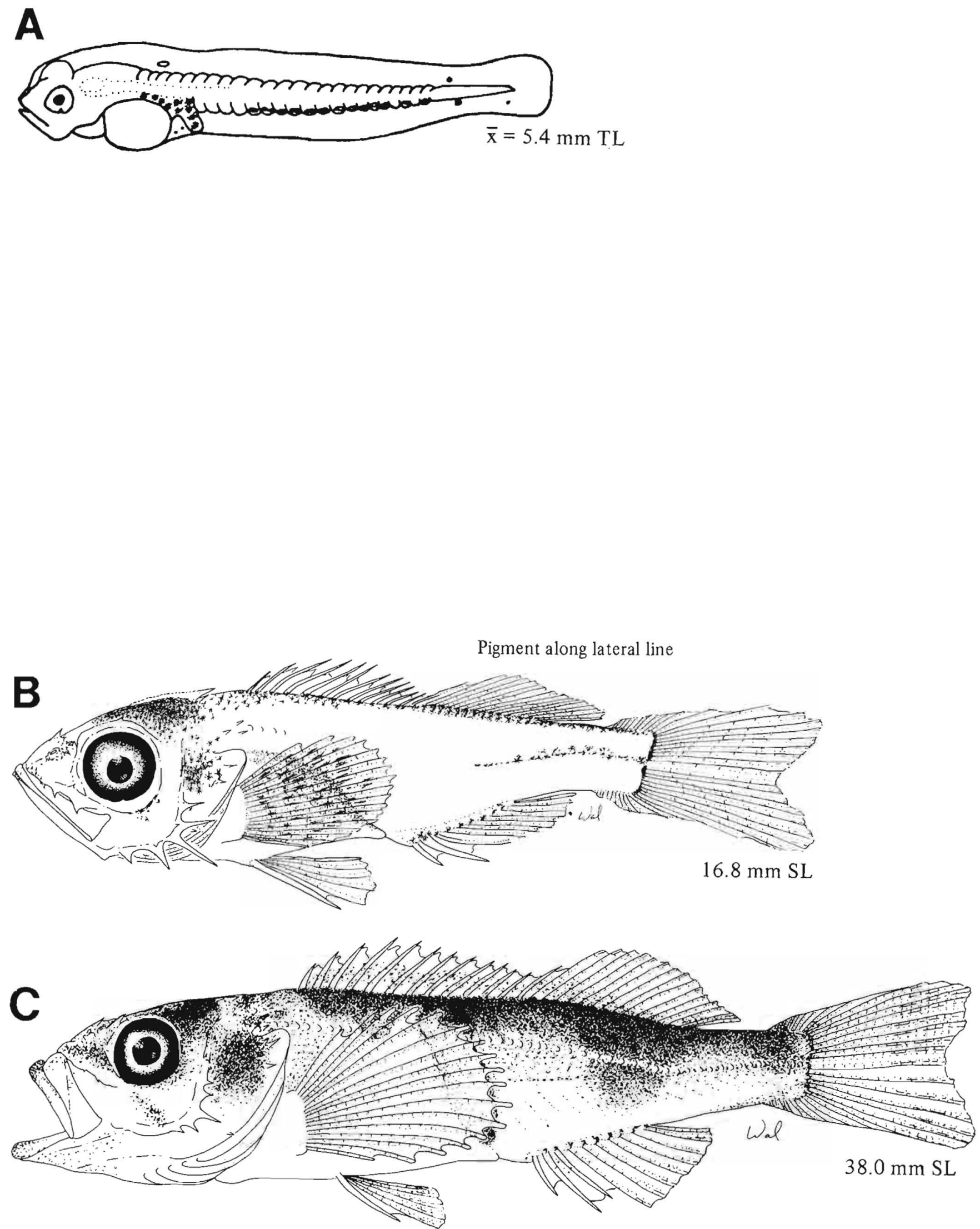

Figure A, Westrheim et al. 1968b; B-C, Laroche, in prep. 


\section{MERISTICS}

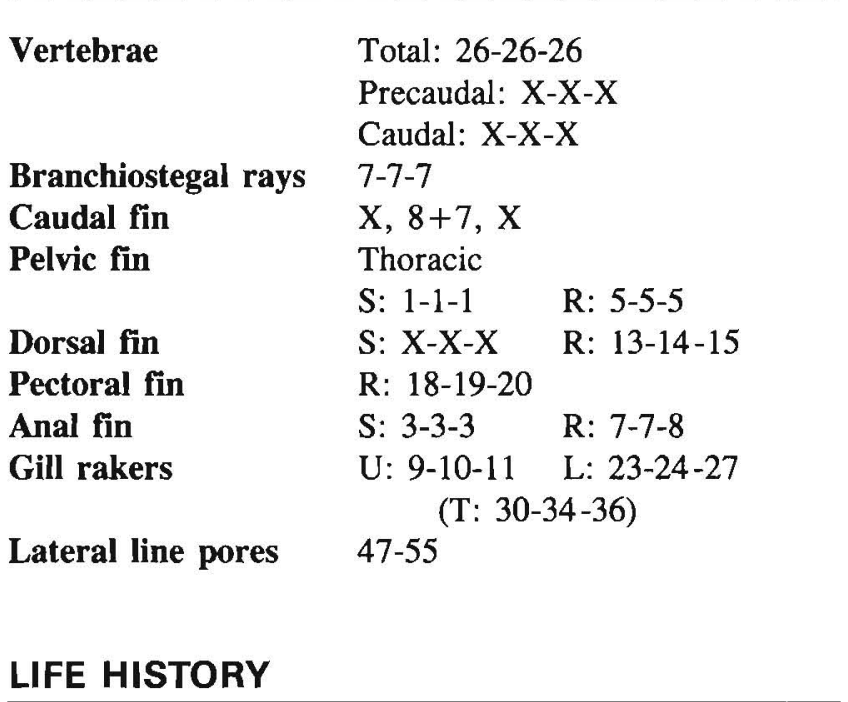

Range

N. California, $38-42^{\circ} \mathrm{N}$, to SE Alaska, $55-59^{\circ} \mathrm{N}$

Ecology

ELH pattern Spawning

Fecundity Age at first maturity Longevity
HEAD SPINES

$\begin{array}{lc}\text { Preocular } & + \\ \text { Postocular } & + \\ \text { Coronal } & 0 \\ \text { Supraocular } & + \\ \text { Tympanic } & + \\ \text { Inferior infraorbital } & 2\end{array}$

\section{EARLY LIFE HISTORY DESCRIPTION}

\section{LARVAE}

Hatch size

Preanal length

Length at flexion

Length at transformation

Sequence of fin development

Pigment

- Extrusion larvae: Short series along ventral body

- Postflexion larvae $>18.5 \mathrm{~mm} \mathrm{SL}$ -Dorsally on head -Along dorsal body margin - Hypural margin

Diagnostic characters

\section{PELAGIC JUVENILES}

- Body pigment banded, heavy

- Fins heavily pigmented 

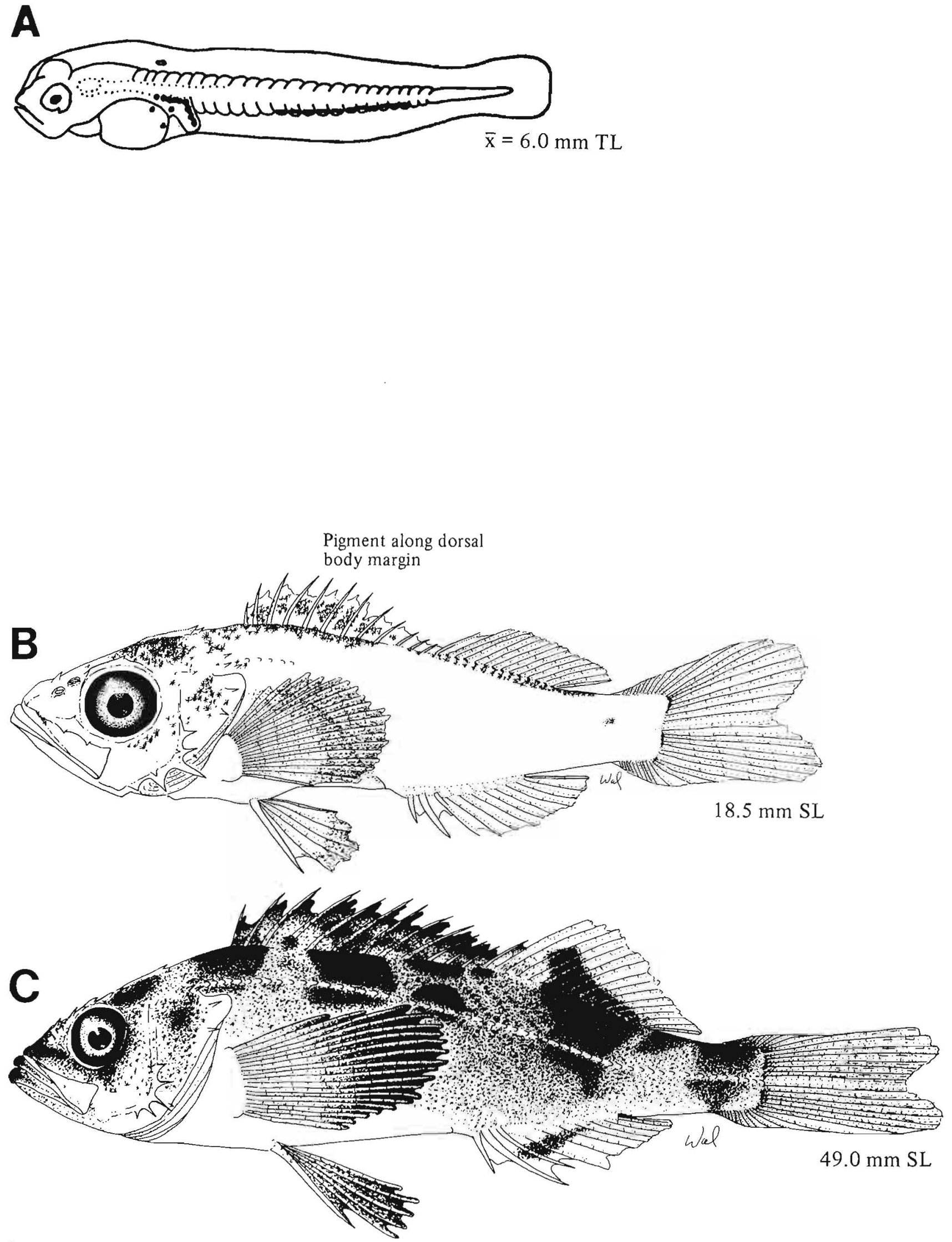

Figure A, Westrheim et al. 1968a; B-C, Laroche, in prep. 


\section{MERISTICS}

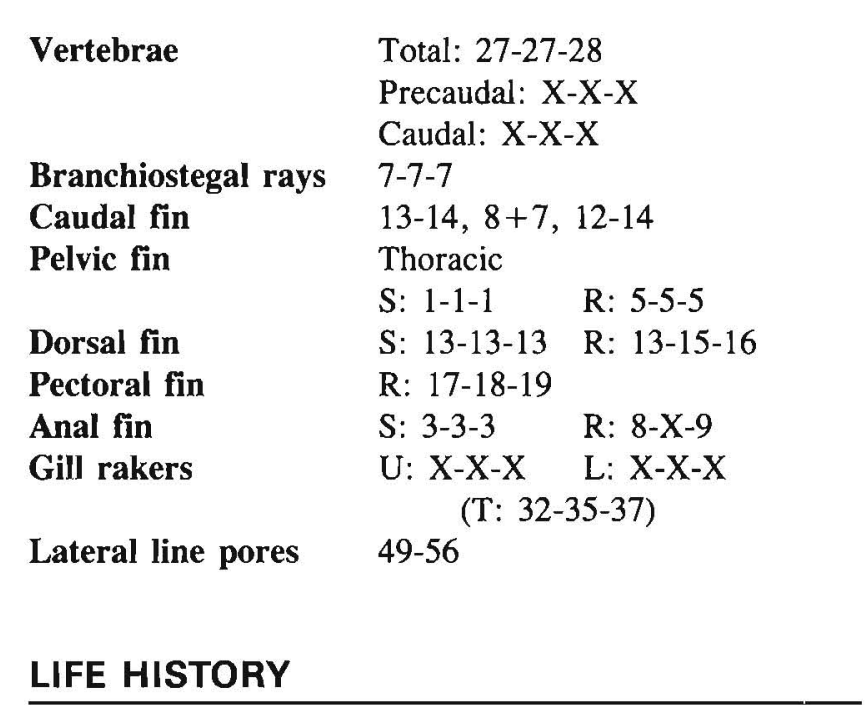

$\begin{array}{ll}\text { Range } & \begin{array}{c}\text { South of southern California to } \\ \text { Oregon, } 42-46^{\circ} \mathrm{N}^{\mathrm{a}} \\ \text { Epi- and mesobenthal }\end{array} \\ \begin{array}{l}\text { Ecology } \\ \text { ELH pattern }\end{array} & \begin{array}{l}\text { Ovoviviparous, pelagic larvae } \\ \text { Spawning }\end{array} \\ & \text { Season: Dec-May (north-central } \\ & \text { California) } \\ & \text { Mode: } \\ & \text { Migration: } \\ \text { Fecundity } & \text { Range/function: } \\ \text { Age at first maturity } & 3 \text { yr (females, California) } \\ \text { Longevity } & \end{array}$

HEAD SPINES

$\begin{array}{lc}\text { Preocular } & + \\ \text { Postocular } & + \\ \text { Coronal } & 0 \\ \text { Supraocular } & + \\ \text { Tympanic } & + \\ \text { Inferior infraorbital } & 2\end{array}$

\section{EARLY LIFE HISTORY DESCRIPTION}

\section{LARVAE}

Hatch size

Preanal length

4.3-4.8 $\mathrm{mm} \mathrm{BL}$

Length at flexion $38-43 \% \mathrm{BL}$

Length at transformation 7.2-7.6 mm BL

Sequence of fin development

Pigment

- Increases from extrusion through flexion

- Tip of lower jaw

- Line develops on maxillaries

- Above brain and on nape

- Develops on opercular region

- Short ventral midline series

- Dorsal midline series develops

- Some internal pigment posteriorly above notochord

- Base and blade of pectoral fin

Diagnostic characters

\section{PELAGIC JUVENILES}

- Relatively light, uniform pigment

- Midlateral line pigmented

- Myosepta outlined with pigment 
A
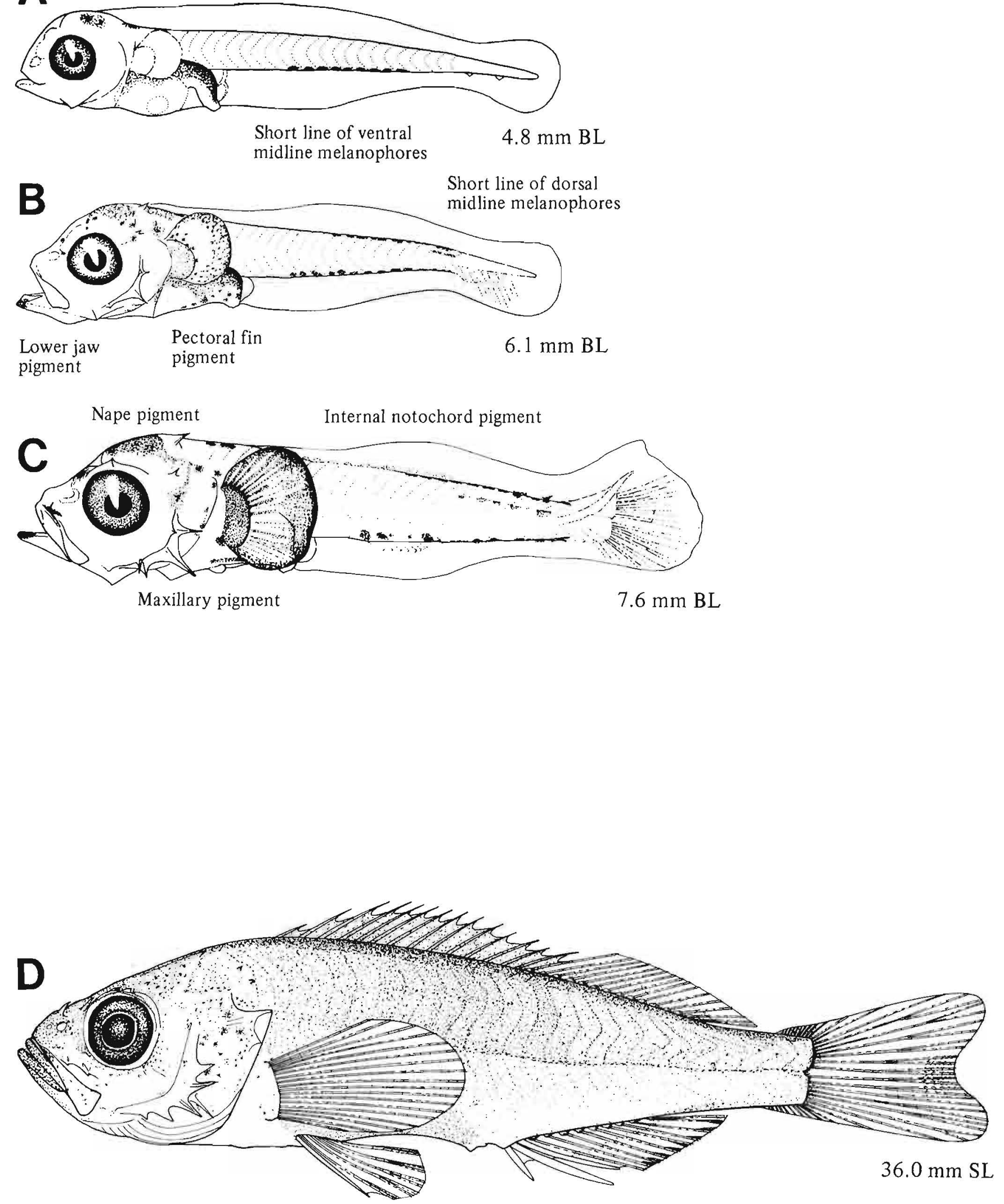

Figures A-C, Moser and Butler 1987; D, NWAFC original (B. Vinter). 


\section{MERISTICS}

Vertebrae

Total: 26-26-26

Precaudal: X-X-X

Caudal: $\mathrm{X}-\mathrm{X}-\mathrm{X}$

Branchiostegal rays

\section{7-7-7}

Caudal fin

Pelvic fin

$\mathrm{X}, 8+7, \mathrm{X}$

Thoracic

S: $1-1-1$

S: $13-13-13 \quad R: 11-12-13$

Dorsal fin

Pectoral fin

Anal fin

R: $16-16-18$

S: $3-3-3$

R: $6-7-7$

Gill rakers

U: $8-10-10$

L: $22-23-26$

(T: $30-32-35)$

Lateral line pores $\quad 35-43$

\section{LIFE HISTORY}

\begin{tabular}{|c|c|}
\hline Range & $\begin{array}{c}\text { South of southern California to } \\
\text { Gulf of Alaska, } 54-60^{\circ} \mathrm{N}\end{array}$ \\
\hline Ecology & Epi- and mesobenthal, $46-421 \mathrm{~m}$ \\
\hline ELH pattern & Ovoviviparous, pelagic larvae \\
\hline \multirow[t]{4}{*}{ Spawning } & Season: Dec-Mar; ${ }^{\mathrm{a}} \mathrm{Feb}^{\mathrm{b}}$ \\
\hline & Area: \\
\hline & Mode: \\
\hline & Migration: \\
\hline Fecundity & Range/function: $13,500-230,000^{2}$ \\
\hline \multirow[t]{2}{*}{ Age at first maturity } & $2-4 y^{a}$ \\
\hline & $2 \mathrm{yr}^{(\text {females })^{\mathrm{c}}}$ \\
\hline
\end{tabular}

\section{HEAD SPINES}

$\begin{array}{lc}\text { Preocular } & + \\ \text { Postocular } & + \\ \text { Coronal } & 0 \\ \text { Supraocular } & 0 \\ \text { Tympanic } & + \\ \text { Inferior infraorbital } & 2\end{array}$

\section{EARLY LIFE HISTORY DESCRIPTION}

\section{LARVAE}

Hatch size

Preanal length

Length at flexion

Length at transformation

Sequence of fin development

Pigment

- Extrusion larvae: Series along ventral body, shorter series along posterior dorsal midline

- Postflexion larvae $>17 \mathrm{~mm}$ SL

-Pigment aligned along epaxial musculature

-Internal and external spots along lateral midline

Diagnostic characters

\section{PELAGIC JUVENILES}

- Body pigment banded

- Median fins pigmented 

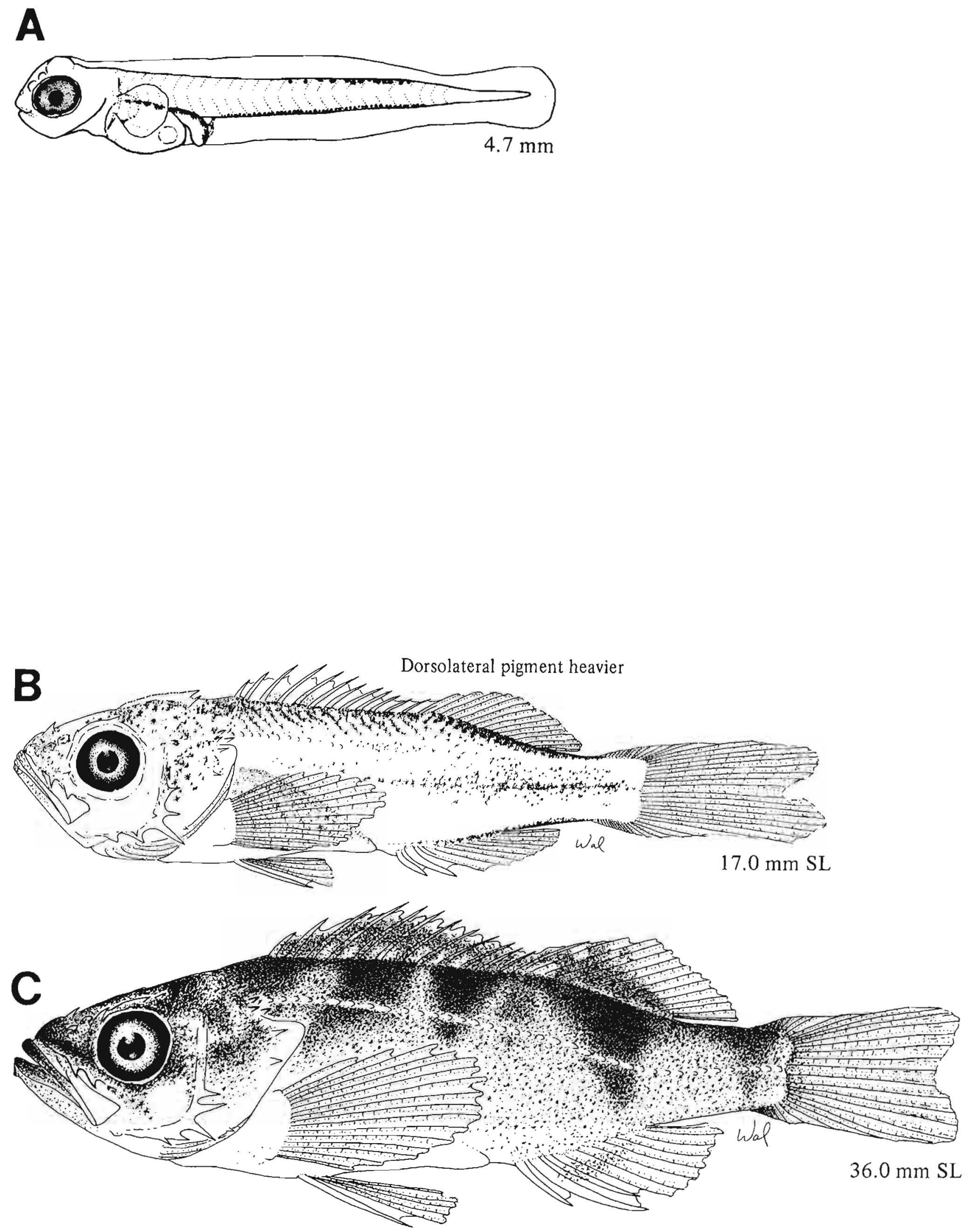

Figure A, Moser et al. 1977; B-C, Laroche, in prep. 


\section{MERISTICS}

\section{Vertebrae}

Total: $27-27-27$

Precaudal: X-X-X

Caudal: $\mathrm{X}-\mathrm{X}-\mathrm{X}$

Branchiostegal rays $\quad 7-7-7$

Caudal fin

Pelvic fin

$\mathrm{X}, 8+7, \mathrm{X}$

Thoracic
S: $1-1-1$
R: 5-5-5

Dorsal fin

S: $13-13-13$

$R: 13-14-15$

Pectoral fin

Anal fin

Gill rakers

R: $16-17-18$

S: $3-3-3 \quad R: 7-7-8$

U: $9-10-11 \quad$ L: $23-25-27$

(T: 33-35-37)

Lateral line pores $\quad 39-47$

\section{LIFE HISTORY}

$\begin{array}{ll}\text { Range } & \begin{array}{l}\text { South of southern California to } \\ \text { Gulf of Alaska, 54-60 }\end{array} \\ \text { Ecology } & \text { Epi- and mesobenthal, 25-475 m } \\ \text { ELH pattern } & \text { Ovoviviparous, pelagic larvae } \\ \text { Spawning } & \text { Season: July (British Columbia) } \\ & \text { Area: } \\ & \text { Mode: } \\ & \text { Migration: } \\ \text { Fecundity } & \text { Range/function: }\end{array}$

Age at first maturity

Longevity

\section{HEAD SPINES}

$\begin{array}{lc}\text { Preocular } & + \\ \text { Postocular } & + \\ \text { Coronal } & 0 \\ \text { Supraocular } & 0 \\ \text { Tympanic } & + \\ \text { Inferior infraorbital } & 2\end{array}$

\section{EARLY LIFE HISTORY DESCRIPTION}

\section{LARVAE}

Hatch size

Preanal length

Length at flexion

$4.3 \mathrm{~mm} \mathrm{SL}$

$50-75 \%$ SL

7.4-9.0 mm SL

Length at transformation

13.5-20.0 mm SL;

$13.7-19.6 \mathrm{~mm}^{\mathrm{b}, \mathrm{c}}$

Sequence of fin

Caudal and pectorals, development pelvics, dorsal and anal

Pigment

- Extrusion larvae: Posterior gut, along ventral midline, and on head

- Larvae >7.4 mm SL: A few melanophores along ventral midline, head, and dorsal gut

- Postflexion larvae $>12.7 \mathrm{~mm}$ SL: Along dorsal midline and fin base, on caudal peduncle

Diagnostic characters

- Moderately pigmented pectorals and pelvics

- Lack of body pigment

- Long, deeply serrate parietal spine and posterior preopercular spine

- Little ventral postanal midline pigment

- Development of dorsal midline pigment $(>10 \mathrm{~mm} \mathrm{SL})$

- Large head (>40\% SL), deep body (>30\% SL)

\section{PELAGIC JUVENILES}

- Body pigment diffuse, lateral midline pigmented

- Little fin pigment

\footnotetext{
Westrheim 1975

${ }^{b}$ Washington et al. $1984 \mathrm{~b}$

' Juveniles are pelagic to $65 \mathrm{~mm} \mathrm{SL}$, although they may become demersal at $35 \mathrm{~mm} \mathrm{SL}$.
}

Ref: Laroche, in prep.; Laroche and Richardson 1981; Westrheim 1975. 


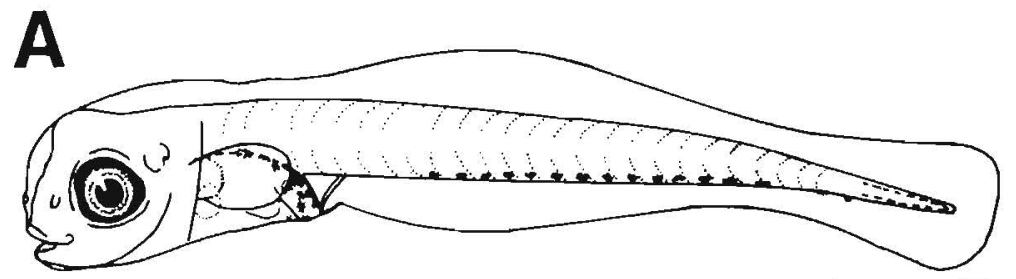

$4.7 \mathrm{~mm} \mathrm{SL}$
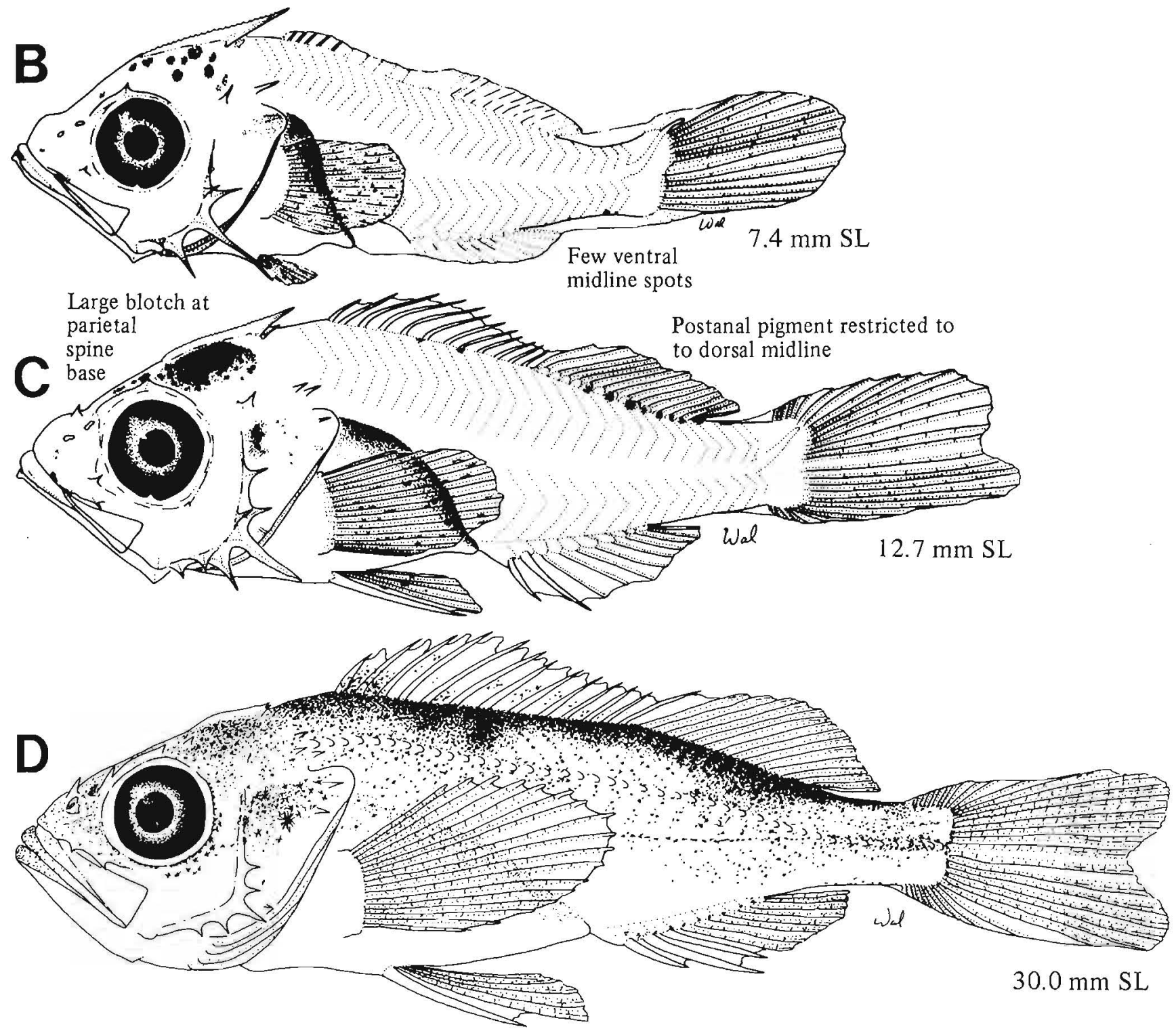

Figure A, NWAFC original (B. Vinter); B-C, Laroche and Richardson 1981; D, Laroche, in prep. 


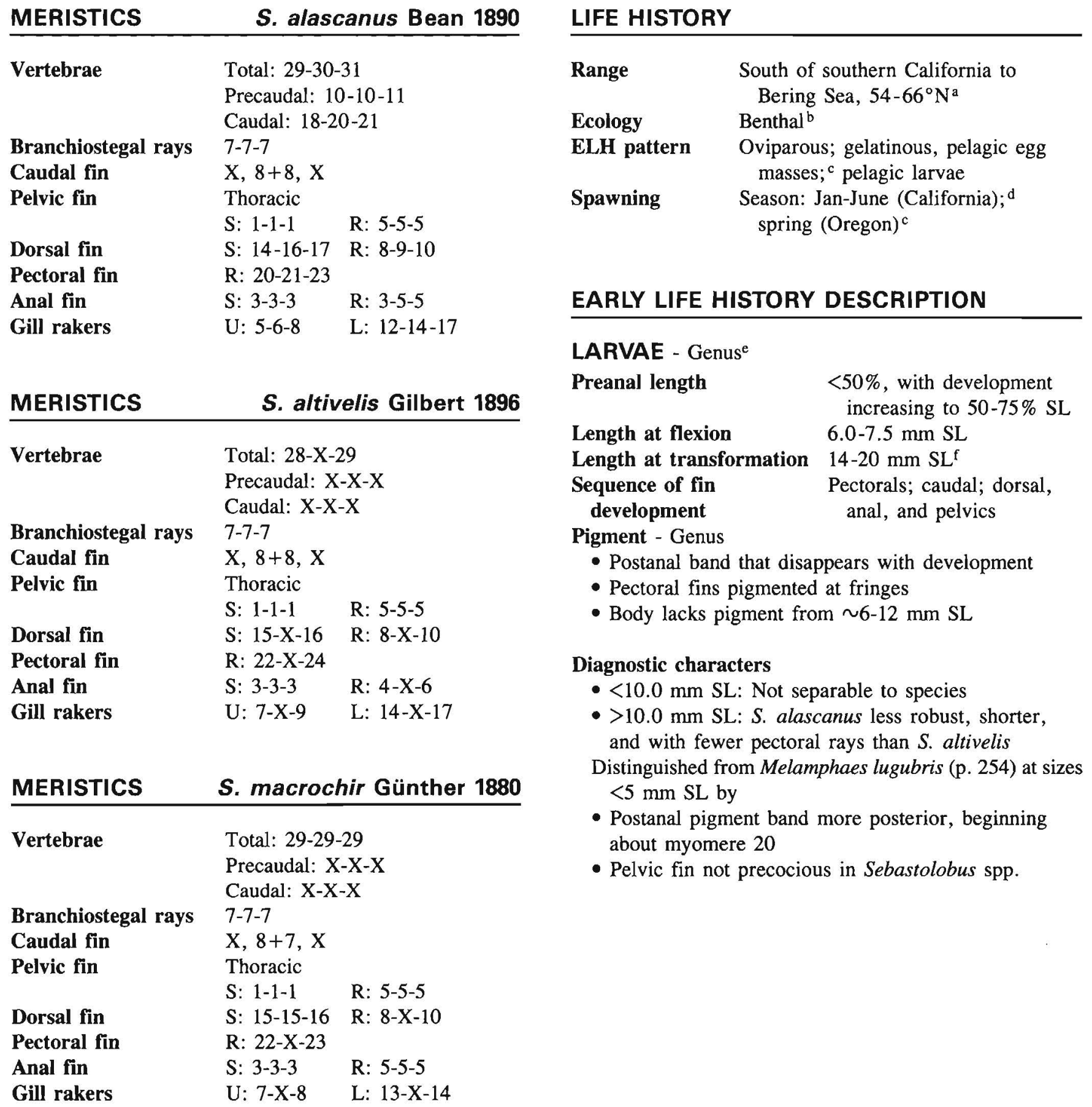

'S. macrochir found only in the Bering Sea.

${ }^{b} S$. alascanus epi-, meso-, and bathybenthal (26-1524 m); S. altivelis meso- and bathybenthal (305-1524 m); $S$. macrochir mesobenthal.

c Pearcy 1962

dMoser 1974

'Larvae of $S$. macrochir are unknown.

f Juveniles are pelagic to $42-56 \mathrm{~mm} \mathrm{SL}$.

Ref: Moser 1974, Moser et al. 1977, Washington et al. 1984b. 

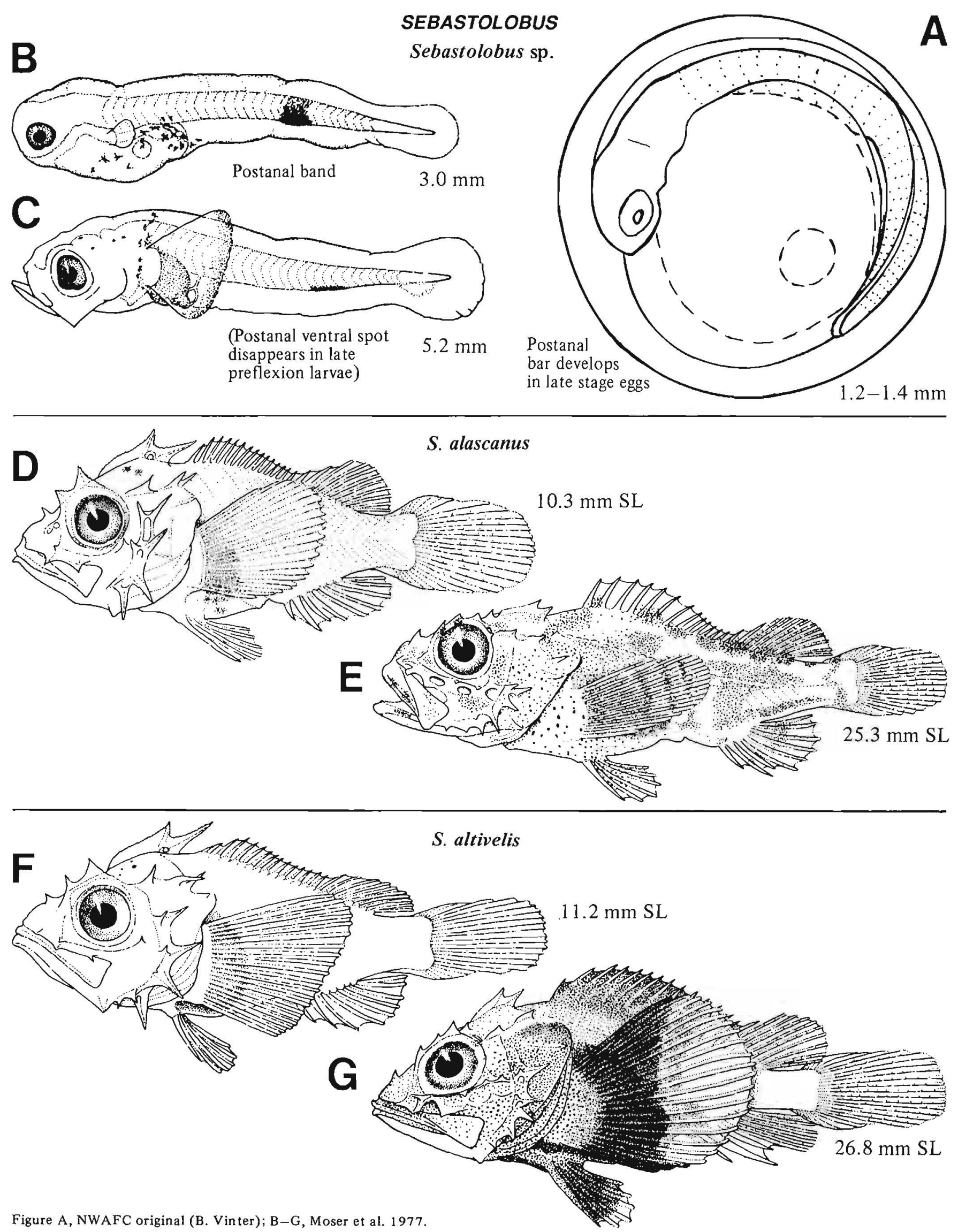


\section{MERISTICS}

$\begin{array}{lll}\text { Vertebrae } & \text { Total: } 61-63-66 \\ & \text { Precaudal: } 29-31-33 \\ & \text { Caudal: } 31-33-34 \\ \text { Branchiostegal rays } & 6-6-6 & \\ \text { Caudal fin } & \text { X, } 7+7, X & \\ \text { Pelvic fin } & \text { Thoracic } & \\ & \text { S: } 1-1-1 & \text { R: } 5-5-5 \\ \text { Dorsal fin } & \text { S: } 17-22-30 & \text { R: } 16-18-21 \\ \text { Pectoral fin } & \text { R: } 14-14-14 & \\ \text { Anal fin } & \text { S: } 2-X-3 & \text { R: } 15-19-23 \\ \text { Gill rakers } & \text { U: }: \text { X-X } & \text { L: } X-X-X\end{array}$

\section{LIFE HISTORY}

\begin{tabular}{|c|c|}
\hline Range & $\begin{array}{l}\text { South of southern California to } \\
\text { Bering Sea, } 54-66^{\circ} \mathrm{N}\end{array}$ \\
\hline Ecology & $\begin{array}{l}\text { Epi-, meso-, and bathybenthal, } \\
0 \text { (juveniles) }-2740 \mathrm{~m}\end{array}$ \\
\hline ELH pattern & $\begin{array}{l}\text { Oviparous, pelagic eggs, pelagic } \\
\text { larvae }\end{array}$ \\
\hline Spawning & $\begin{array}{l}\text { Season: Dec-Apr (California); }{ }^{\mathrm{a}} \\
\text { Sept-Apr (Oregon-Brit.Col.); } \\
\text { fall-summer (Bering Sea) } \\
\text { Area: Pelagic (175-1450 m) } \\
\text { Mode: } \\
\text { Migration: To deeper water }{ }^{\mathrm{b}}\end{array}$ \\
\hline Fecundity & $\begin{array}{l}\text { Range/function: } \\
\qquad 100,000^{\mathrm{a}}-1,300,000^{\mathrm{d}}\end{array}$ \\
\hline Age at first maturity & $5-7 \mathrm{yr}^{\mathrm{d}}$ \\
\hline Longevity & $55 \mathrm{yr}^{\mathrm{e}}$ \\
\hline
\end{tabular}

\section{EARLY LIFE HISTORY DESCRIPTION}

\section{EGGS}

Diameter

No. of oil globules

2.05-2.10 mm

Yolk

Envelope

Hatch size

None

Homogeneous

Smooth, unsculptured $6 \mathrm{~mm} \mathrm{SL}$

Incubation time/temp.

Pigment

- Unpigmented embryo

Diagnostic characters

- Narrow perivitelline space

- Unpigmented embryo with high myomere count and long gut

- No oil globule

- Large size (2.05-2.10 mm)

\section{LARVAE}

Preanal length

Length at flexion

Length at transformation

Sequence of fin development

Pigment

- Initially unpigmented

- Preflexion larvae develop dorsal and ventral midline pigment

- Flexion and later larvae are heavily and uniformly pigmented

Diagnostic characters (see Table 3)

- Slender, heavily pigmented larvae (flexion)

- Long preanal length (50-60\% SL)

- >15 mm SL: long pigmented pectoral fins

- Separate dorsal fins (larger larvae)

Larvae of Erilepis zonifer (skilfish), an anoplopomatid from the study area, are unknown. The following meristics distinguish them from $A$. fimbria.

$\begin{array}{lr}\text { Total vertebrae } & 45-46 \\ \text { Dorsal fin rays } & \text { XII-XVI, } 15-19 \\ \text { Pectoral fin rays } & 16-19 \\ \text { Anal fin rays } & \text { III, 11-14 }\end{array}$

\footnotetext{
a Phillips and Imamura 1954

${ }^{b}$ Mason et al. 1983

'Kendall and Matarese 1987

${ }^{d}$ Alton and Webber 1976

eMcFarlane and Beamish 1983
}

Ref: Ahlstrom and Stevens 1976, Kendall and Matarese 1987. 


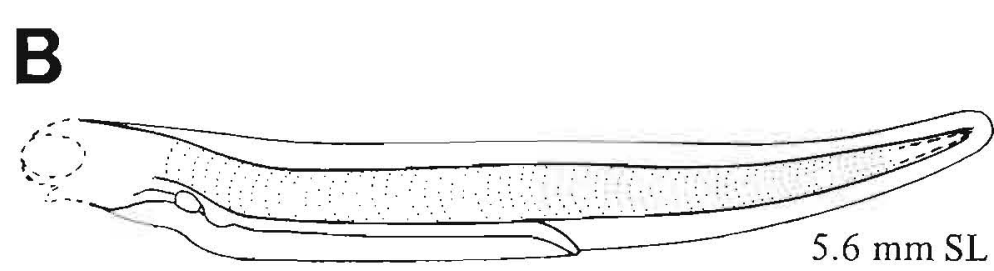

Unpigmented

C

(19)

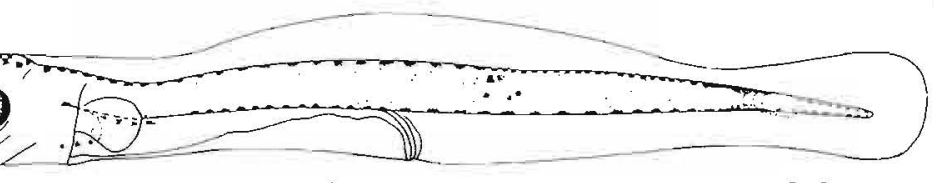

Anus curves

ventrad

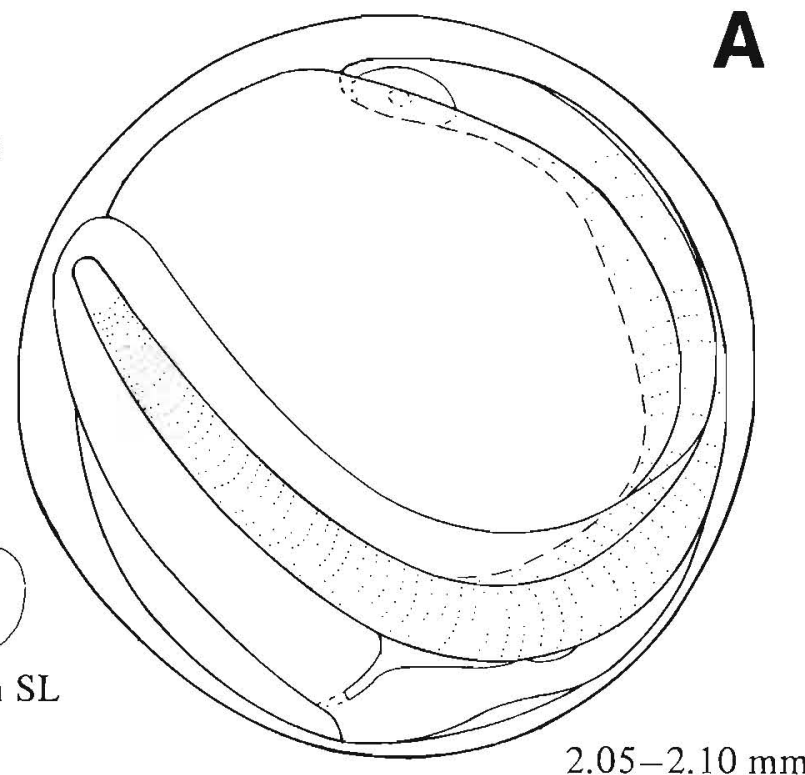

D

40

(6)

$8.8 \mathrm{~mm} \mathrm{SL}$

$2.05-2.10 \mathrm{~mm}$

E

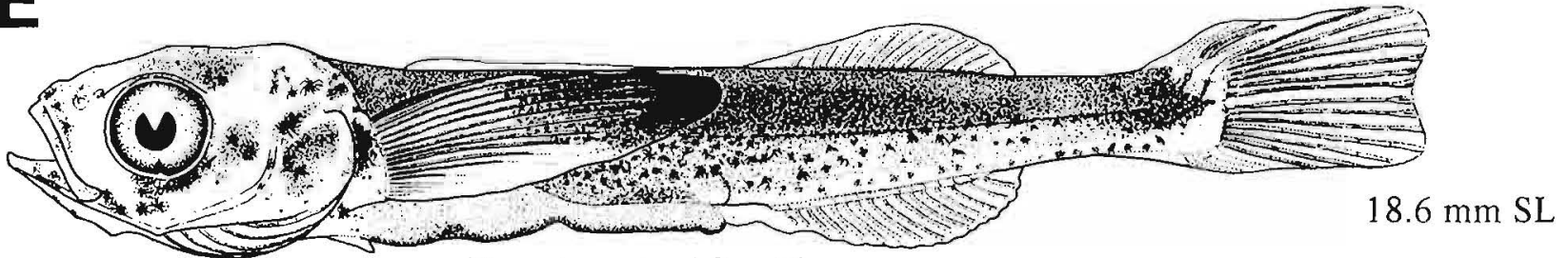

Elongate pectoral fin with

pigmented tips

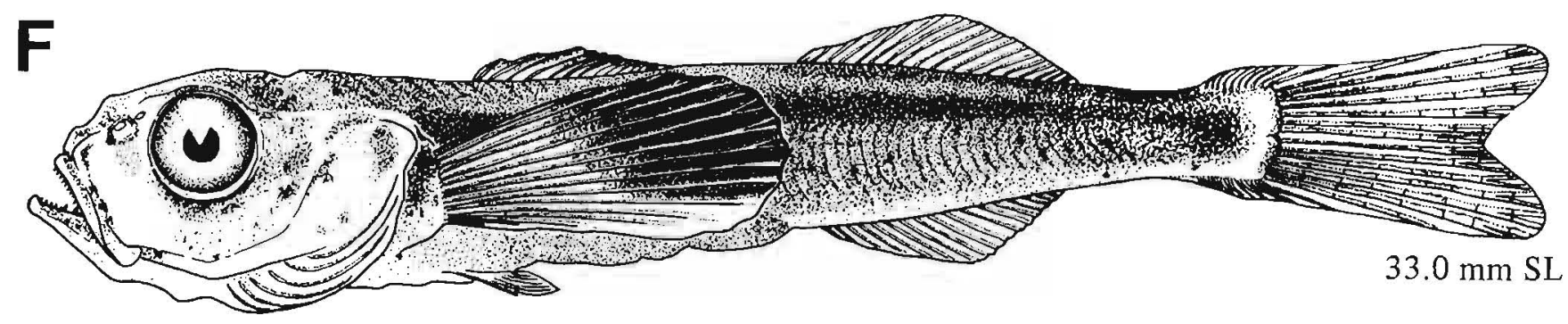

Figures A-D, Kendall and Matarese 1987; E-F, AhIstrom and Stevens 1976. 



\section{Hexagrammidae}

The greenlings, endemic to the North Pacific Ocean, are composed in our area of nine species in five genera. Most adult greenlings are demersal and occur nearshore; however, larvae commonly occur off the continental shelf. Eggs are demersal and are usually guarded in nests. Development from hatching to juvenile is direct without any marked transformation. An epipelagic juvenile stage occurs in most species. Larvae are heavily pigmented with scattered melanophores over most of the body, especially dorsally. Characters that will distinguish hexagrammids from other heavily pigmented larvae are presented in Table 3. Identification is accomplished using a combination of pigment and meristic characters along with geographic occurrence. Oxylebius pictus and Zaniolepis spp. larvae have lower vertebral counts, larger pectoral fins with heavier pigment, and differ from other genera in a number of morphological features (e.g., larger heads, longer preanal length, and deeper bodies). Ophiodon elongatus larvae can be separated by the presence of a pointed snout and a large terminal mouth. Pleurogrammus monopterygius larvae lack pigment on the snout which serves to distinguish them from larvae of Hexagrammos spp. Differences among the larvae of the various species of Hexagrammos spp. are discussed in Tables 34-35 and illustrated on page 351.

Table 32

Meristic characters of Northeast Pacific hexagrammids. For all species the normal count for branchiostegal rays is 6 and for pelvic fin rays is $I, 5$ (Kendall and Vinter 1984).

\begin{tabular}{|c|c|c|c|c|c|c|c|c|c|c|c|c|}
\hline \multirow[b]{4}{*}{ Species } & & & & \multicolumn{8}{|c|}{ Fin rays } & \multirow{4}{*}{$\begin{array}{l}\text { Gill rakers } \\
\text { First arch }\end{array}$} \\
\hline & & & & \multirow{2}{*}{\multicolumn{2}{|c|}{ Dorsal }} & \multirow{3}{*}{$\begin{array}{l}\text { Total } \\
\text { anal* }\end{array}$} & \multirow[b]{3}{*}{ Pectoral } & \multicolumn{4}{|c|}{ Caudal } & \\
\hline & \multicolumn{3}{|c|}{ Vertebrae } & & & & & \multicolumn{2}{|c|}{ Dorsal } & \multicolumn{2}{|c|}{ Ventral } & \\
\hline & Precaudal & Caudal & Total & (spines) & (soft rays) & & & Secondary & Principal & Principal & Secondary & \\
\hline Oxylebius pictus & $13-15$ & $23-25$ & $36-40$ & $15-17$ & $13-16$ & $14-17$ & 14-17 & 9 & 7 & 6 & 9 & $11-14$ \\
\hline Zaniolepis frenata & $14-15$ & $26-28$ & $40-43$ & 21 & 12 & $18-19$ & & & & & & \\
\hline Zaniolepis latipinnis & 14 & 28 & 42 & $21-22$ & $11-12$ & $18-20$ & 14 & 6-8 & 7 & 6 & $7-9$ & $11-12$ \\
\hline Ophiodon elongatus & $23-24$ & $33-35$ & $56-59$ & $25-28$ & $19-21$ & $21-25$ & $16-18$ & $13-15$ & 7 & 7 & $12-14$ & $19-28$ \\
\hline Pleurogrammus monopterygius & $26-28$ & $32-35$ & $58-63$ & $21-24$ & $24-30$ & $23-28$ & $23-28$ & $16-19$ & 8 & 11 & $16-20$ & $22-27$ \\
\hline Hexagrammos decagrammus & $20-22$ & $33-35$ & $52-57$ & $21-23$ & $22-26$ & $23-26$ & $18-20$ & $12-16$ & 7 & 9 & $12-14$ & $15-20$ \\
\hline Hexagrammos lagocephalus & $20-23$ & $32-34$ & $52-57$ & $20-23$ & $20-25$ & $21-24$ & $18-21$ & $17-22$ & 7 & 10 & $15-19$ & $14-18$ \\
\hline Hexagrammos octogrammus & $18-19$ & $32-35$ & $50-54$ & $18-20$ & $22-25$ & $23-26$ & $18-19$ & $15-17$ & 7 & 8 & $14-15$ & 14-17 \\
\hline Hexagrammos stelleri & $20-22$ & $31-34$ & $51-56$ & $22-25$ & $18-22$ & $22-25$ & $18-20$ & $16-17$ & 7 & 8 & $14-15$ & $16-20$ \\
\hline
\end{tabular}

*Anal spines are very weak in Pleurogrammus and Hexagrammos, therefore only total anal fin elements are given for these taxa. Counts for anal spines in specific taxa are given on the individual text pages.

Table 33

Larval characters that allow distinction between Oxylebius pictus and Zaniolepis spp. (Kendall and Vinter 1984).

\begin{tabular}{lll}
\hline & Oxylebius pictus & Zaniolepis spp. \\
\hline 3-7 mm SL & & \\
Presence of pigment & No & \\
On isthmus & Less & Yes \\
Laterally above gut & More & More \\
On ventral midline of trunk & No & Less \\
On tip of snout and onto tip of palate & Yes & Yes \\
On internal surface of pectoral fin base & No & No \\
On lower lip & Smaller $(<20 \%$ head length) & Sometimes \\
Morphology & No & Larger $(>30 \%$ head length) \\
Eye size & Reaches anus by $8.0 \mathrm{~mm}$ & Yes \\
Preanal finfold $(5$ to $8 \mathrm{~mm})$ & $7-9 \mathrm{~mm}$ & Reaches anus by $5.5 \mathrm{~mm}$ \\
Pectoral fin length & No & $5-7$ mm \\
Notochord flexion & & Yes \\
Dorsal indentation on eye & No & \\
$>7$ mm SL & Stout $(<30 \% \mathrm{SL})$ & Yes \\
Spiny scales cover body & & Slender $(<25 \% \mathrm{SL})$ \\
Body depth & &
\end{tabular}




\section{MERISTICS}

$\begin{array}{lll}\text { Vertebrae } & \text { Total: } 36-X-40 \\ & \text { Precaudal: } 13-13-15 \\ & \text { Caudal: } 23-24-25 \\ \text { Branchiostegal rays } & 6-6-6 & \\ \text { Caudal fin } & 9,7+6,9 & \\ \text { Pelvic fin } & \text { Thoracic } & \\ & \text { S: } 1-1-1 & \text { R: } 5-5-5 \\ \text { Dorsal fin } & \text { S: } 15-X-17 & \text { R: } 13-X-17 \\ \text { Pectoral fin } & \text { R: } 14-X-17 & \\ \text { Anal fin } & \text { S: } 3-X-4 & \text { R: } 12-X-14 \\ \text { Gill rakers } & \text { U: } 2-X-5 & \text { L: } 7-X-8\end{array}$

LIFE HISTORY

\begin{tabular}{|c|c|}
\hline Range & $\begin{array}{l}\text { South of southern California to } \\
\text { Gulf of Alaska, } 54-60^{\circ} \mathrm{N}\end{array}$ \\
\hline Ecology & $\begin{array}{l}\text { Nearshore shelf demersal, } \\
\text { intertidal to } 49 \mathrm{~m}\end{array}$ \\
\hline ELH pattern & $\begin{array}{l}\text { Oviparous; demersal, adhesive } \\
\text { eggs; pelagic larvae }\end{array}$ \\
\hline \multirow[t]{4}{*}{ Spawning } & $\begin{array}{l}\text { Season: May-Aug (Puget } \\
\text { Sound); }{ }^{a} \text { Oct-July (Calif.) }\end{array}$ \\
\hline & $\begin{array}{l}\text { Area: Demersal, on or near } \\
\text { rocks }^{b}\end{array}$ \\
\hline & $\begin{array}{l}\text { Mode: Eggs guarded by male; } \\
\text { may have three breeding } \\
\text { cycles/season }^{b}\end{array}$ \\
\hline & Migration: \\
\hline Fecundity & $\begin{array}{l}\text { Range/function: } 12,000-28,000 / \\
\begin{array}{l}F=0.0338 \times \mathrm{L}^{2.114} \\
\mathrm{~L}=\mathrm{TL} \mathrm{mm} \text { (Shilshole break- } \\
\text { water, Washington); } \\
\mathrm{F}=0.003 \times \mathrm{L}^{2.628}, \\
\mathrm{~L}=\mathrm{TL} \mathrm{mm} \text { (Monterey, Calif.) }^{\mathrm{b}}\end{array}\end{array}$ \\
\hline \multirow[t]{2}{*}{ Age at first maturity } & 3 yr (females) ${ }^{b}$ \\
\hline & $2 \mathrm{yr}$ (males) $)^{b}$ \\
\hline Longevity & $>8 \mathrm{yr}^{\mathrm{c}}$ \\
\hline
\end{tabular}

\section{EARLY LIFE HISTORY DESCRIPTION}

\section{EGGS}

Diameter

No. of oil globules

Oil globule diameter

Yolk

Envelope

Hatch size 4-5 mm SL

Incubation time/temp.

Pigment

Diagnostic characters

\section{LARVAE}

Preanal length 54-64\% SL

Length at flexion $7-9 \mathrm{~mm} \mathrm{SL}$

Length at transformation $16-20 \mathrm{~mm} \mathrm{SL}$

Sequence of fin development

Pigment

- Pectoral fin

- Ventral midline

- Dorsolateral on gut

- Flexion larvae with increased pigment on anterior body

\section{Diagnostic characters}

- See Table 33 for characters that allow separation from Zaniolepis spp.

- Large pigmented pectoral fins

' Fitcb and Lavenberg 1975 


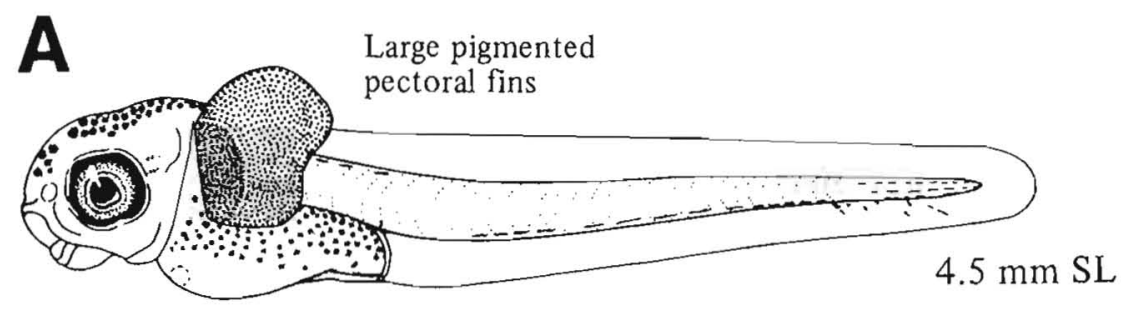

B
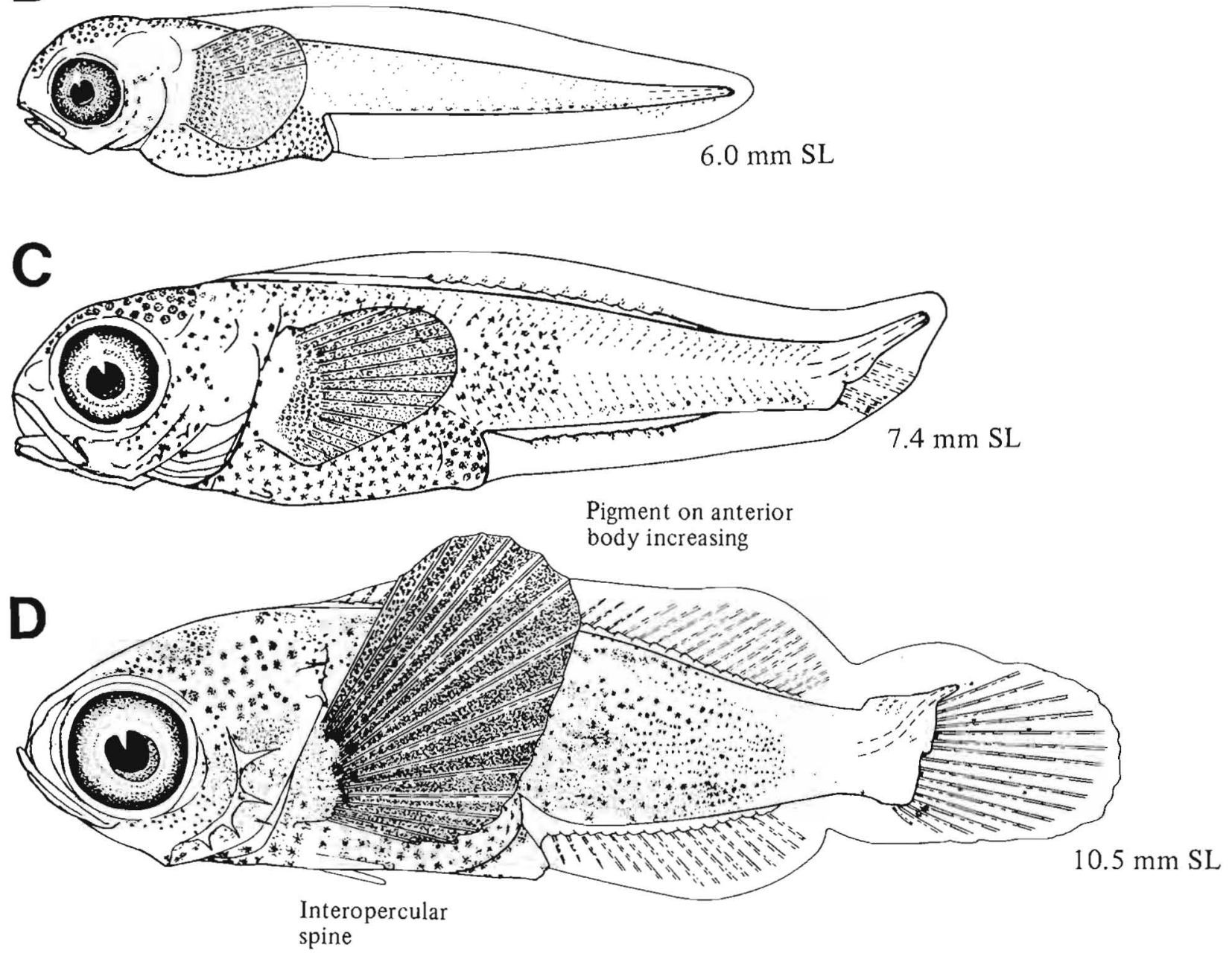

Figures A-D, Kendall and Vinter 1984. 


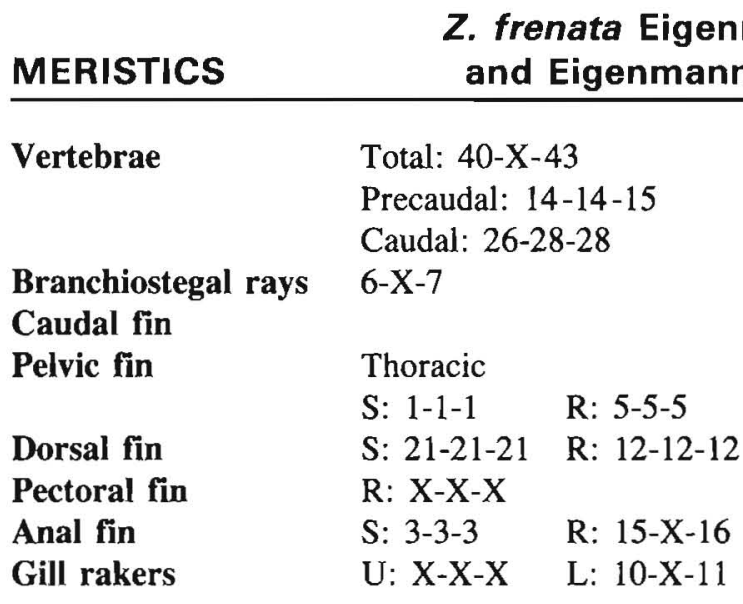

MERISTICS

Z. latipinnis Girard 1857

Vertebrae

Total: $42-42-42$

Precaudal: 14-14-14

Caudal: 28-28-28

Branchiostegal rays

$6-\mathrm{X}-7$

Caudal fin

$6-8,7+6,7-9$

Pelvic fin

Thoracic
S: $1-1-1$
$\mathrm{R}: 5-5-5$
S: $21-\mathrm{X}-22$
R: $11-\mathrm{X}-12$
R: $14-14-14$
S: $3-3-3$
R: $15-\mathrm{X}-17$
U: $3-3-3$
L: $8-X-9$

Dorsal fin

Pectoral fin

Anal fin

Gill rakers

\section{LIFE HISTORY}

$\begin{array}{ll}\text { Range } & \begin{array}{c}\text { South of southern California to } \\ \text { Brit. Col., } 48^{\circ} 30^{\prime}-55^{\circ} \mathrm{N}^{\mathrm{a}}\end{array} \\ \begin{array}{l}\text { Ecology } \\ \text { ELH pattern }\end{array} & \begin{array}{l}\text { Benthal } \\ \text { Oviparous, demersal eggs, } \\ \text { pelagic larvae }\end{array} \\ \text { Spawning } & \text { Season: Fall-winter (California) } \\ & \text { Area: } \\ & \text { Mode: } \\ & \text { Migration: } \\ \text { Fecundity } & \text { Range/function: } 350-6530 \text { (may } \\ & \text { produce three clutches/seasen) }\end{array}$

Age at first maturity

Longevity

\section{EARLY LIFE HISTORY DESCRIPTION}

\section{EGGS}

Diameter

No. of oil globules

Oil globule diameter

Yolk

Envelope

Hatch size

$1.40-1.56 \mathrm{~mm}^{\mathrm{d}}$

$30-50$ (red, orange) $^{\mathrm{d}}$

$0.04-0.12 \mathrm{~mm}^{\mathrm{d}}$

Incubation time/temp.

Pigment

Finely striated $^{\mathrm{d}}$

$2.5 \mathrm{~mm} \mathrm{SL}$

Diagnostic characters

\section{LARVAE}

Preanal length

Length at flexion

Length at transformation

Sequence of fin development

Pigment

- Laterally on gut

- Ventral midline

- Flexion larvae with increased pigment on body

Diagnostic characters

- See Table 33 for characters that allow separation from Oxylebius pictus

- Spiny scales

${ }^{a} Z$. frenata only to Oregon, $42-46^{\circ} \mathrm{N}$

${ }^{\mathrm{b}} Z$. frenata epi- and mesobenthal $(55-244 \mathrm{~m}) ; Z$. latipinnis nearshore shelf $(37-201 \mathrm{~m})$.

${ }^{\mathrm{c}} Z$. latipinnis only (Goldberg 1980a).

${ }^{\mathrm{d}}$ Data are from unfertilized, hydrated eggs of $Z$. frenata (W. Watson, Marine Ecological Consultants, 531 Encinitas Blvd., Suite 110, Encinitas, CA 92024, pers. commun., 6 Feb. 1988)

Ref: Kendall and Vinter 1984. 

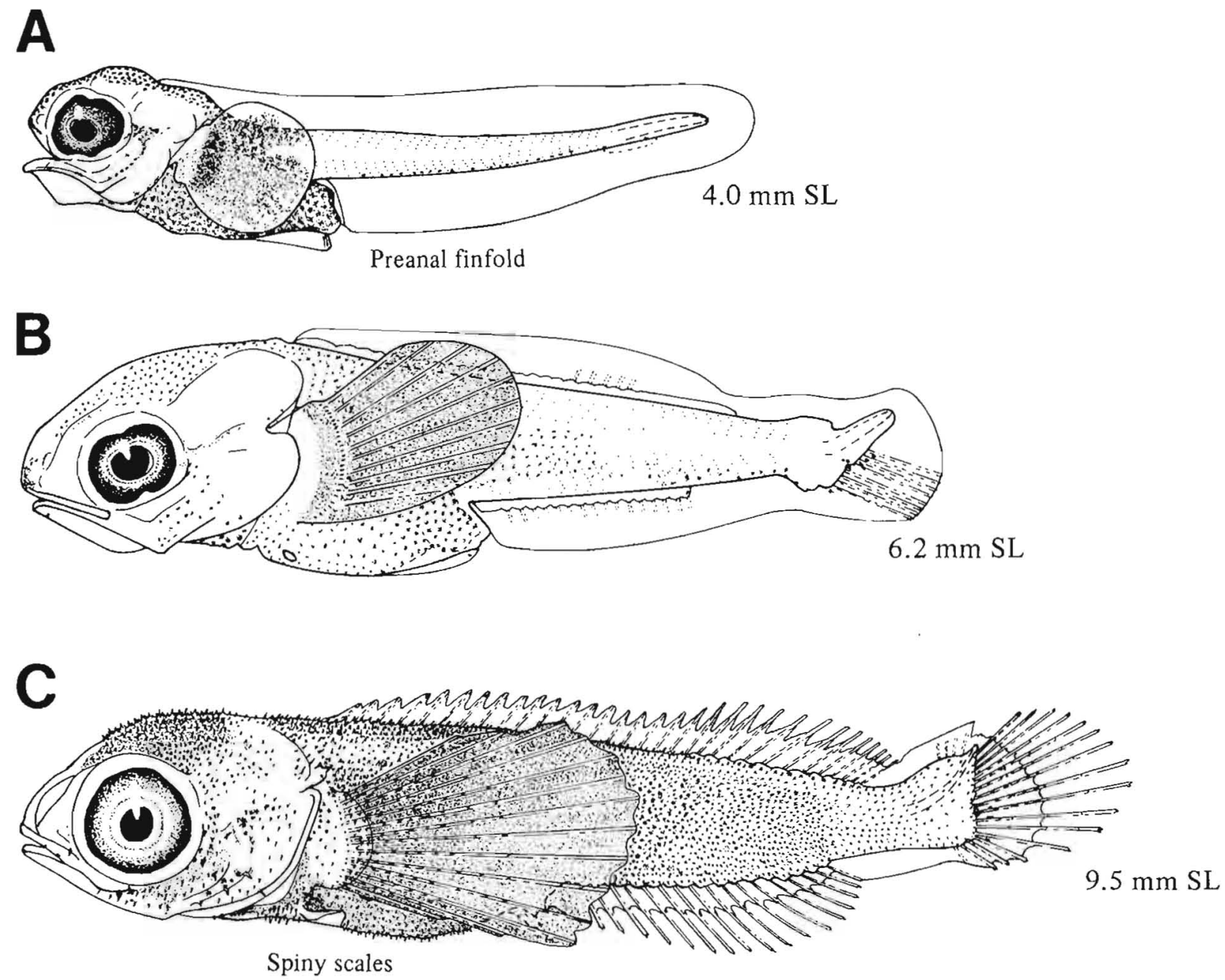

Figures A-C, Kendall and Vinter 1984. 


\section{MERISTICS}

Vertebrae

Total: $56-\mathrm{X}-59$

Precaudal: 23-23-24

Caudal: $33-34-35$

Branchiostegal rays $6-\mathrm{X}-7$

Caudal fin

Pelvic fin

Dorsal fin

Pectoral fin

Anal fin

Gill rakers

\section{LIFE HISTORY}

$13-15,7+7,12-14$

Thoracic
S: $1-1-1$
$\mathrm{R}: 5-5-5$

S: $25-\mathrm{X}-28$

R: $16-X-18$

S: $3-3-3$

R: $19-X-21$

U: $5-\mathrm{X}-8$

$\mathrm{R}: 21-\mathrm{X}-25$

L: $16-X-19$
South of southern California to Gulf of Alaska, $54-60^{\circ} \mathrm{N}$

Ecology

ELH pattern

Spawning

Fecundity

Age at first maturity $2-3 \mathrm{yr}^{\mathrm{g}}$

Longevity $20 \mathrm{yr}^{\mathrm{h}}$

Migration: To shallow water

$$
\mathrm{N}=0.0002824 \times \mathrm{L}^{3.001 \mathrm{f}}
$$

\section{EARLY LIFE HISTORY DESCRIPTION}

\section{EGGS}

Diameter

No. of oil globules

2.24-3.23 mm

Oil globule diameter

Yolk

Envelope

Hatch size

One

Incubation time/temp.

Pigment

Diagnostic characters

\section{LARVAE}

Preanal length

$\sim 43-55 \%$ SL

Length at flexion

11-15 mm SL

Length at transformation $30 \mathrm{~mm} \mathrm{SL}$; larvae remain pelagic to $52 \mathrm{~mm} \mathrm{SL}$

Sequence of fin Caudal and pectorals, 2nd development dorsal (rays) and anal, 1st dorsal (spines), pelvics

\section{Pigment}

- Head and snout

- Laterally on surface of gut

Range/function: $60,000-500,000^{\circ} /$

- Along dorsal midline and ventrolateral body

- Rather uniformly pigmented (flexion)

Diagnostic characters (see Table 3)

Distinguished from other hexagrammids by

- Pointed snout, protruding lower jaw

- Large terminal mouth with gape directed upward

- Longer gut

- Distribution of pigment on tail
${ }^{a}$ LaRiviere et al. 1981

${ }^{\mathrm{b}}$ Giorgi 1981

${ }^{c} J$ Jewell 1968

${ }^{d}$ Miller and Geibel 1973

'Phillips 1959

${ }^{\prime}$ Hart 1967; $N=$ egg number.

gFrey 1971

${ }^{\mathrm{h}}$ Fitch and Lavenberg 1971

Ref: Kendall and Vinter 1984. 

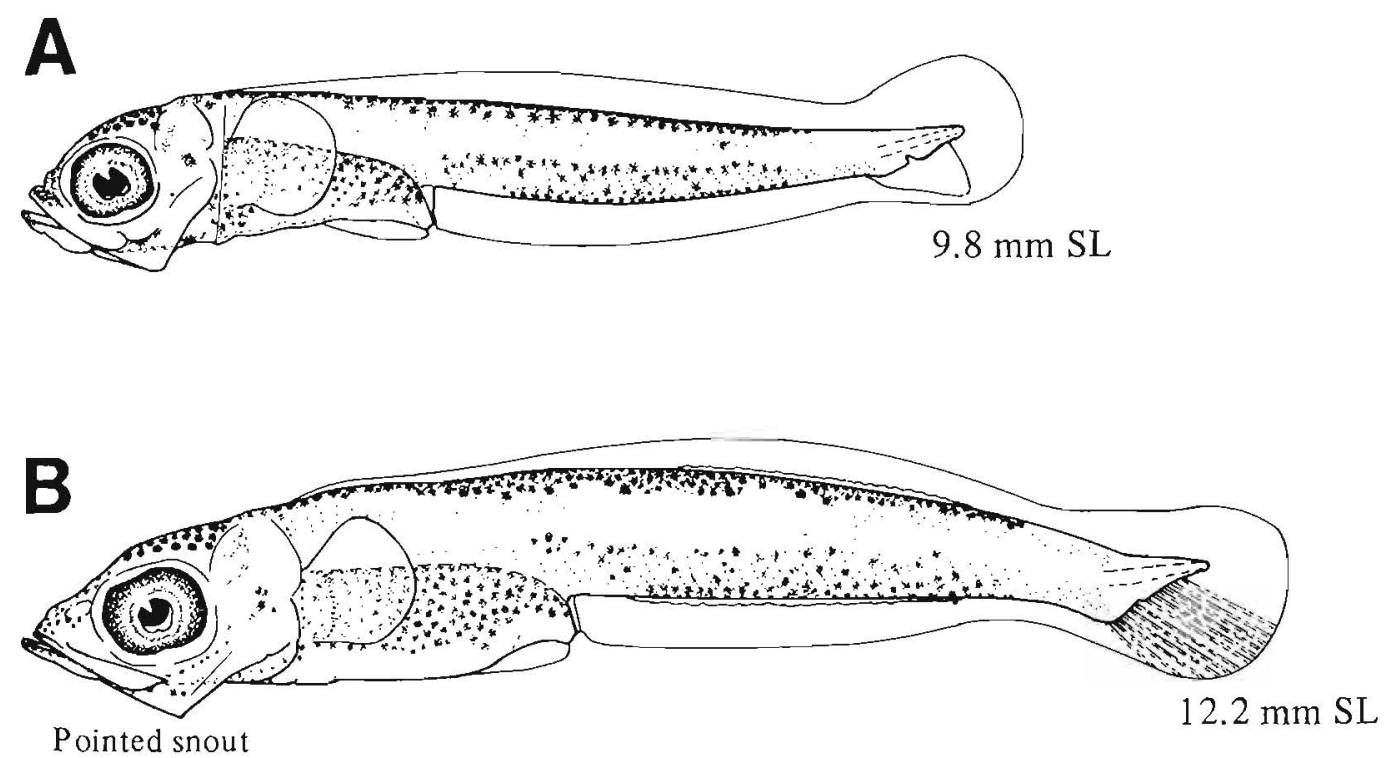

Pointed snout

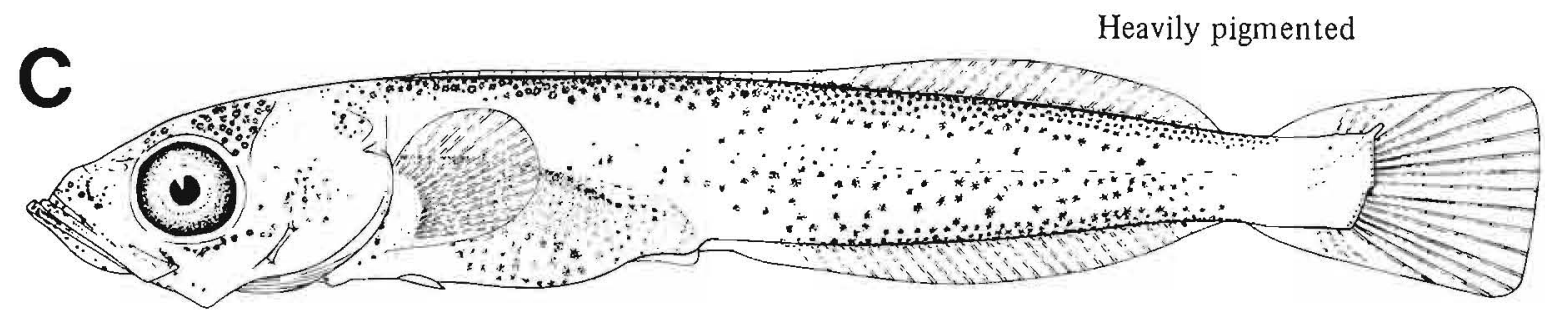

$19.0 \mathrm{~mm} \mathrm{SL}$

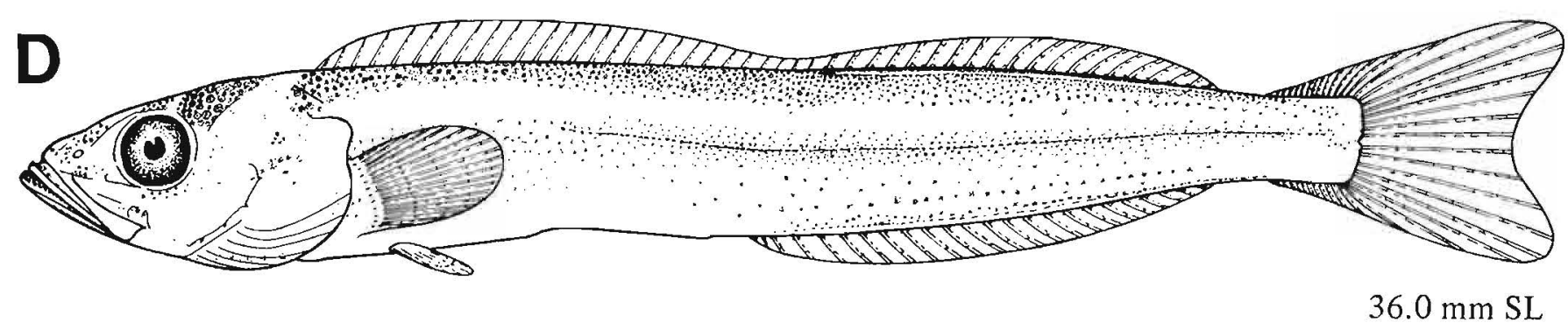

Figures A-D, Kendall and Vinter 1984. 


\section{MERISTICS}

Vertebrae

Total: $58-X-63$

Precaudal: $26-X-28$

Caudal: $32-\mathrm{X}-35$

Branchiostegal rays

$5-\mathrm{X}-7$

Caudal fin

Pelvic fin

$16-19,8+11,16-20$

Thoracic
S: $1-1-1$
$\mathrm{R}: 5-5-5$

Dorsal fin

S: $21-\mathrm{X}-24$

Pectoral fin

R: $23-X-28$

R: $23-X-28$

$\mathrm{U}: 6-\mathrm{X}-8$

L: $16-X-19$

Gill rakers

$\mathrm{R}: 24-\mathrm{X}-30$

\section{EARLY LIFE HISTORY DESCRIPTION}

\section{EGGS}

Diameter

No. of oil globules

Oil globule diameter

Yolk

Envelope

Hatch size

2.5-2.8 mm

Many in group

$1.38-1.40 \mathrm{~mm}$

Smooth

$\sim 8 \mathrm{~mm} \mathrm{SL}$

Incubation time/temp.

Pigment

Diagnostic characters

\section{LIFE HISTORY}

$\begin{array}{ll}\text { Range } & \begin{array}{l}\text { S. California, } 32-34^{\circ} \mathrm{N} \text {, to } \\ \text { Bering Sea, } 54-66^{\circ} \mathrm{N}\end{array} \\ \text { Ecology } & \begin{array}{l}\text { E- and mesobenthal, intertidal } \\ \text { to } 575 \mathrm{~m} \\ \text { Oviparous; demersal, attached } \\ \text { eggs; pelagic larvae }\end{array} \\ \text { ELH pattern } & \text { Season: June-Sept (Bering Sea) } \\ & \text { Area: } \\ \text { Spawning } & \text { Mode: Nests guarded by males } \\ & \text { Migration: } \\ & \text { Range/function: } 3653-18,694 ;^{\mathrm{c}} \\ \text { Fecundity } & 43,000^{\mathrm{a}} \\ & 3-4 \mathrm{yr}^{\mathrm{a}} \\ \text { Age at first maturity } & 11 \mathrm{yr}^{\mathrm{a}}\end{array}$

\section{LARVAE}

$\begin{array}{ll}\text { Preanal length } & 33-50 \% \text { SL } \\ \begin{array}{l}\text { Length at flexion } \\ \text { Length at transformation }\end{array} & 14-19 \mathrm{~mm} \mathrm{SL} \\ \begin{array}{l}\text { Sequence of fin } \\ \text { development }\end{array} & \begin{array}{c}\text { Caudal, pectorals, dorsal } \\ \text { and anal, pelvics }\end{array}\end{array}$

Pigment

- Midbrain and internally on hindbrain

- Dorsolateral surface of gut

- Postanal: Dorsal midline along body length to last myomere; ventral midline extends only from midbody to last myomere

- Internal above and below notochord

Diagnostic characters (see Table 3)

Distinguished from other hexagrammids by

- Pigment pattern (unpigmented snout)

- Morphology: Larger eye, shorter snout

${ }^{c}$ Lee 1985

Ref: Kendall and Vinter 1984. 


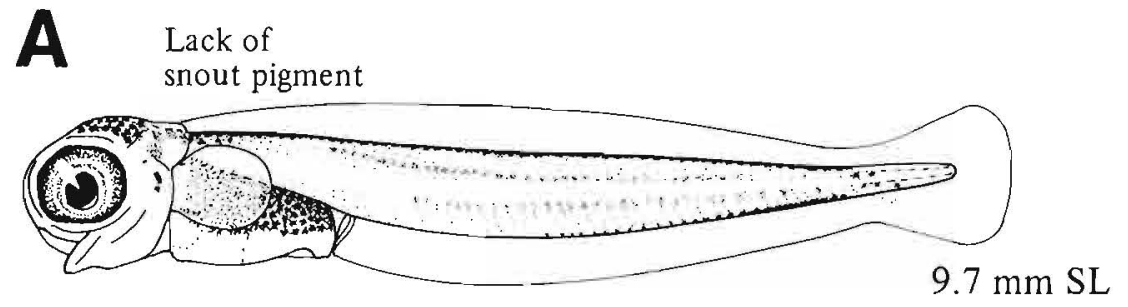

B

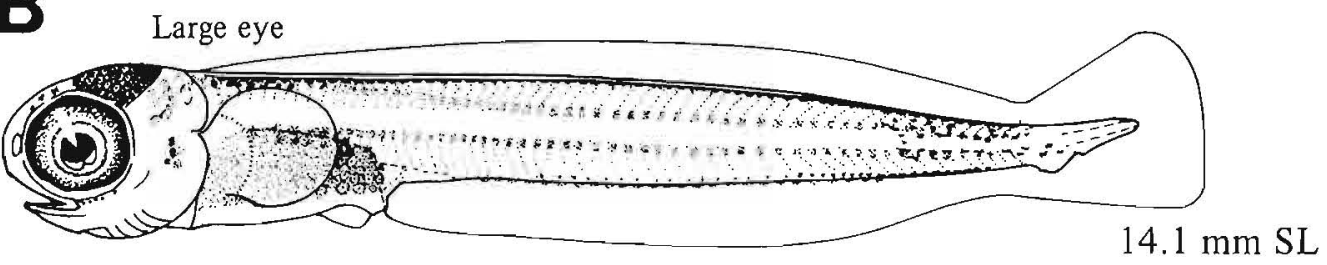

C

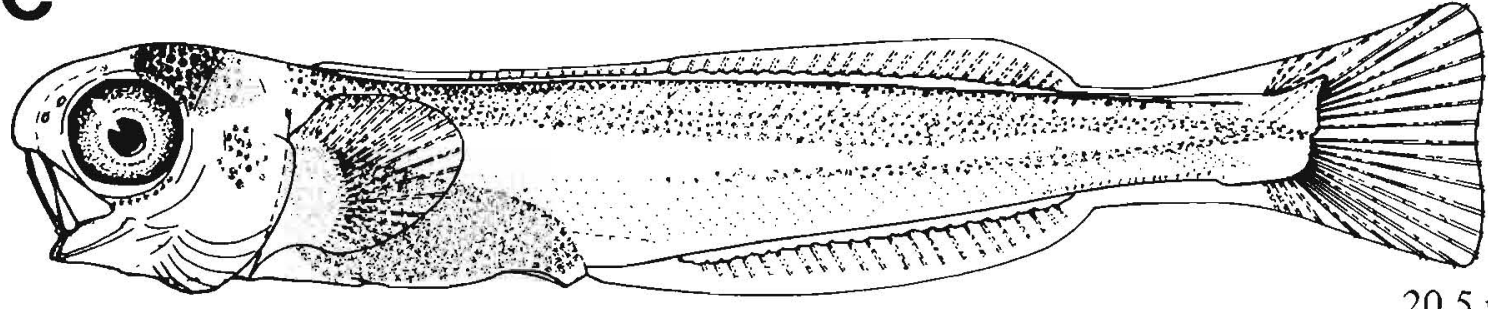

$20.5 \mathrm{~mm} \mathrm{SL}$

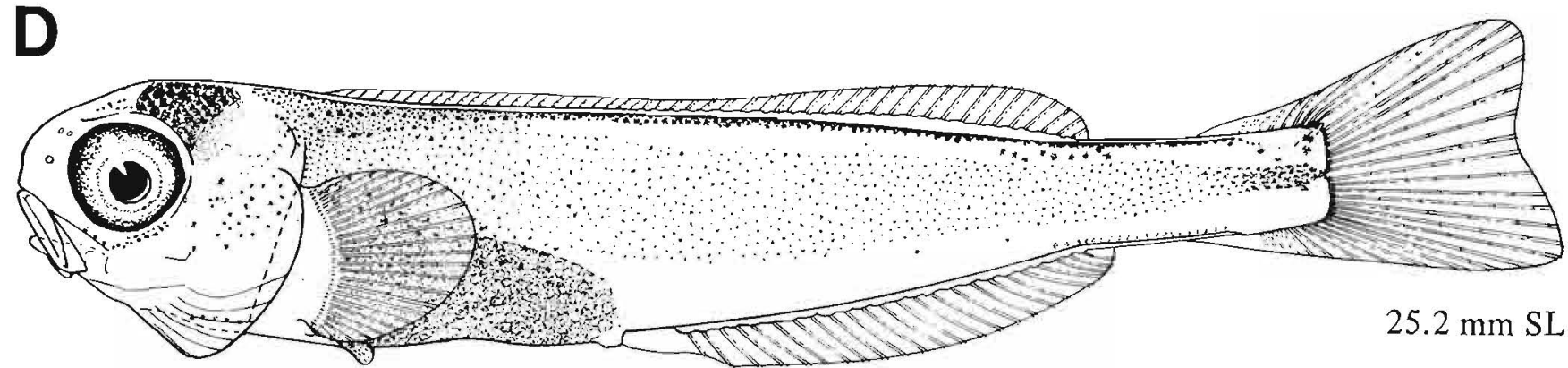

Figures $A-D$, Kendall and Vinter 1984. 


\section{Table 34}

Guide for identifying Northeast Pacific species of Hexagrammos based on meristic characters (Kendall and Vinter 1984).

\begin{tabular}{|c|c|}
\hline Based on p & fin \\
\hline $\begin{array}{l}\text { Principal ventral caudal rays } \\
\text { precaudal vertebrae } \\
\text { precaudal vertebrae }\end{array}$ & $\begin{array}{l}8 \\
20-22=H . \text { steller } \\
18-19=H . \text { octogrammus }\end{array}$ \\
\hline Principal ventral & $=H$. decagrammus \\
\hline Principal ventral caudal rays & $10=H$. lagocephalus \\
\hline ased on extremes of merist & ic characters \\
\hline Dorsal fin spinous rays & $\begin{array}{l}18-19=H . \text { octogrammus } \\
20-23=\text { indeterminate } \\
24-25=H . \text { stelleri }\end{array}$ \\
\hline Dorsal fin soft rays & $\begin{aligned} 18-19 & =H . \text { sielleri } \\
20-25 & =\text { indeterminate } \\
26 & =H . \text { decagrammus }\end{aligned}$ \\
\hline Total anal fin elements & $\begin{aligned} 21 & =H . \text { lagocephalus } \\
22-26 & =\text { indeterminate }\end{aligned}$ \\
\hline Precaudal vert & $\begin{array}{l}18-19=H . \text { octogrammus } \\
20-23=\text { indeterminate }\end{array}$ \\
\hline Caudal vertebrae & $\begin{aligned} 31 & =H . \text { stelleri } \\
32-35 & =\text { indeterminate }\end{aligned}$ \\
\hline Tot & $\begin{aligned} 50 & =H . \text { octogrammus } \\
51-57 & =\text { indeterminate }\end{aligned}$ \\
\hline
\end{tabular}

*Prior to completion of caudal fin development, the principal ventral caudal fin ray count can be one less in larvae.

\begin{tabular}{|c|c|c|c|c|}
\hline \multirow[b]{3}{*}{ Species } & tation characteristics that distinguis & $\begin{array}{l}\text { Table } 35 \\
\text { larvae }<\mathbf{3 0} \mathrm{mm} \text { of the four species } \\
\text { Kendall and Vinter } 1984 \text {, in part). }\end{array}$ & f Hexagrammos in the North & st Pacific \\
\hline & \multicolumn{4}{|c|}{ Pigment area } \\
\hline & Postanal ventral midline & Isthmus & Notochord tip & $\begin{array}{c}\text { Internal pectoral } \\
\text { fin base }{ }^{b}(>17 \mathrm{~mm})\end{array}$ \\
\hline H. stelleri & $\begin{array}{l}\text { Absent until } \sim 15 \mathrm{~mm} \text {, then starts to } \\
\text { form posteriorly (on caudal peduncle), } \\
\text { later }(>23 \mathrm{~mm} \text { ) forms along entire base } \\
\text { of anal fin. }\end{array}$ & $\begin{array}{l}\text { Absent at hatching. Anterior half } \\
\text { gradualy becomes pigmented starting at } \\
10 \mathrm{~mm} \text {. }\end{array}$ & Absent & Present \\
\hline H. decagrammus & $\begin{array}{l}\text { Absent until } \sim 13 \mathrm{~mm} \text {, then starts to } \\
\text { form along anal fin base near its origin. } \\
\text { By } 18 \mathrm{~mm} \text { along anal fin base to caudal } \\
\text { peduncle. Spots more numerous and } \\
\text { smaller than on } H \text {. lagocephalus. }\end{array}$ & $\begin{array}{l}\text { About four equal-sized and -spaced } \\
\text { spots form on each side making a "V." } \\
\text { Present from } \sim 9 \mathrm{~mm} \text { on. }\end{array}$ & $\begin{array}{l}\text { Absent at hatching but forms by } \\
\sim 10 \mathrm{~mm} \text {. }\end{array}$ & Inconsistently present \\
\hline H. lagocephalus & $\begin{array}{l}\text { Present throughout development. } \\
\text { Particularly dense on caudal peduncle. } \\
\text { Spots along anal fin base tend not to } \\
\text { touch each other. Spots uneven in } \\
\text { spacing and size to create irregular } \\
\text { line. Few spots anterior to origin of } \\
\text { anal fin. }\end{array}$ & $\begin{array}{l}\text { An anterior medial spot and about five } \\
\text { equal-sized and -spaced spots present } \\
\text { from } \sim 9 \mathrm{~mm} \text { on. }\end{array}$ & Present & Absent \\
\hline H. octogrammus & $\begin{array}{l}\text { Present throughout development. } \\
\text { Extends from anus to base of caudal } \\
\text { fin. Dense on caudal peduncle. Spots } \\
\text { tend to touch each other to create a } \\
\text { continuous straight line. }\end{array}$ & $\begin{array}{l}\text { Present; anteriormost and posteriormost } \\
\text { spots la:ger than others. Anterior } \\
\text { medial spot seen at } 10 \mathrm{~mm} \text {, up to five } \\
\text { on each side of isthmus seen at } 12 \mathrm{~mm} \text {. } \\
\text { Spacing uneven. }\end{array}$ & Present & Present \\
\hline
\end{tabular}


VENTRAL VIEW OF HEXAGRAMMOS SPP. LARVAE (16-18 mm SL) DEPICTING PIGMENT ON THE LOWER JAW, ISTHMUS, AND POSTANAL VENTRAL MIDLINE

A Hexagrammos stelleri
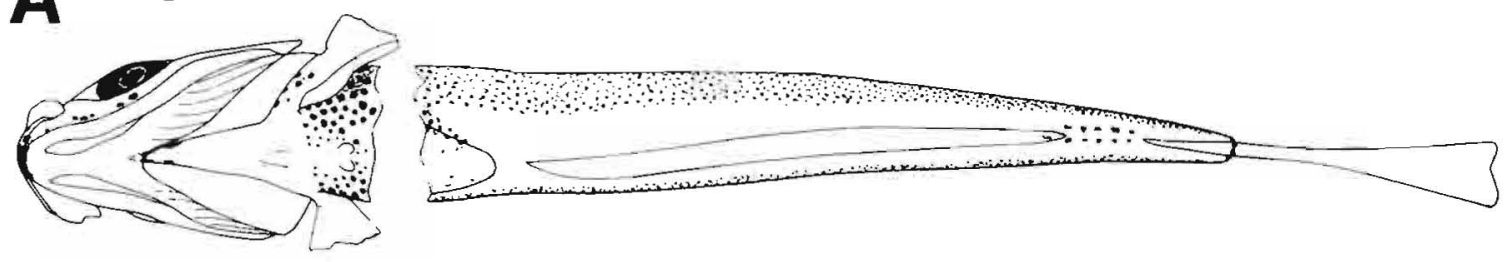

8 Hexagrammos decagrammus
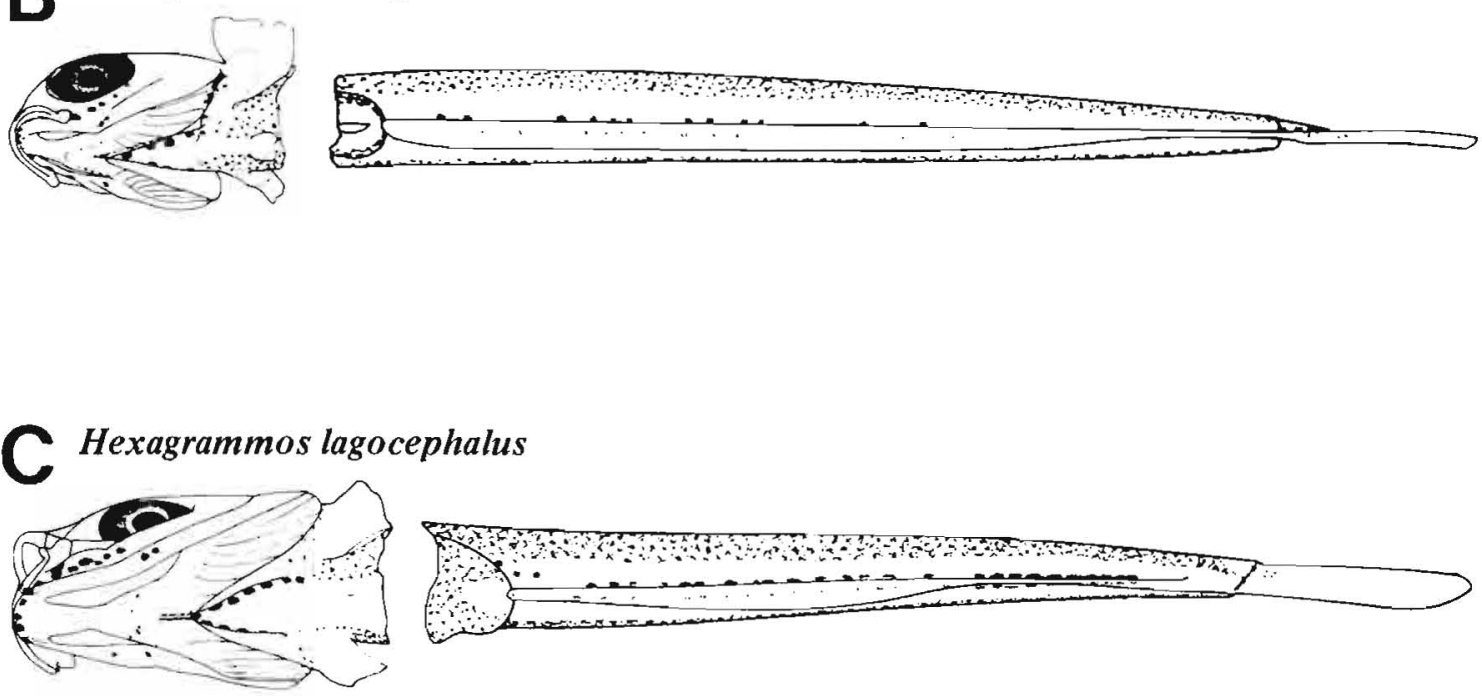

D Hexagrammos octogrammus
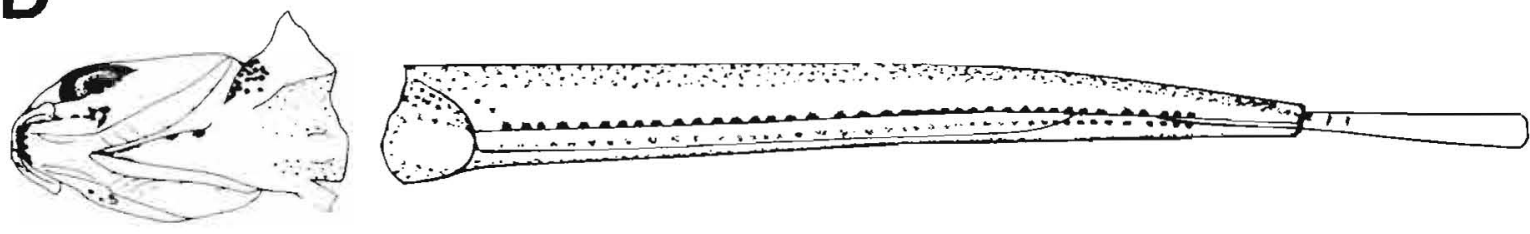

Figures A-D, Kendall and Vinter 1984. 
MERISTICS

Vertebrae

Caudal fin

Pelvic fin

Dorsal fin

Pectoral fin

Anal fin

Gill rakers
Total: $52-\mathrm{X}-57$

Precaudal: 20-21-22

Caudal: $33-\mathrm{X}-35$

6-6-6

$12-16,7+9,12-14$

Thoracic
S: $1-1-1$
R: $5-5-5$

S: $21-\mathrm{X}-23$

R: $18-X-20$

R: $23-X-26$

$\mathrm{U}: 3-\mathrm{X}-5$

R: $22-X-26$

L: $9-\mathrm{X}-14$

\section{EARLY LIFE HISTORY DESCRIPTION}

\section{EGGS}

Diameter

No. of oil globules

Oil globule diameter

Yolk

Envelope

Hatch size

7-9 mm SL

Incubation time/temp.

Pigment

Diagnostic characters

\section{LIFE HISTORY}

$\begin{array}{ll}\text { Range } & \begin{array}{c}\text { S. California, } 32-34^{\circ} \mathrm{N}, \text { to } \\ \text { Bering Sea, } 54-66^{\circ} \mathrm{N}\end{array} \\ \text { Ecology } & \text { Intertidal, nearshore, 0-46 m } \\ \text { ELH pattern } & \text { Oviparous; demersal, attached } \\ & \text { eggs; pelagic larvae } \\ \text { Spawning } & \text { Season: Oct-Nov }{ }^{\mathrm{a}} \\ & \text { Area: } \\ & \text { Mode: Egg masses on rocks }{ }^{\mathrm{a}} \\ & \text { Migration: } \\ \text { Fecundity } & \text { Range/function: } \\ \text { Age at first maturity } & \\ \text { Longevity } & \end{array}$

\section{LARVAE}

Preanal length $\quad$ Much $<50 \%$ before flexion

Length at flexion

Length at transformation

Sequence of fin

development

Pigment

- Heavily pigmented

- Postanal ventral midline absent until $\sim 13 \mathrm{~mm} \mathrm{SL}$, then starts near anal fin origin; reaches caudal peduncle by $18 \mathrm{~mm} \mathrm{SL}$

Diagnostic characters (see Table 3 and tables this section)

- Principal caudal fin ray count in juveniles $(7+9)$

${ }^{a}$ Marliave 1975a

Ref: Kendall and Vinter 1984. 
A

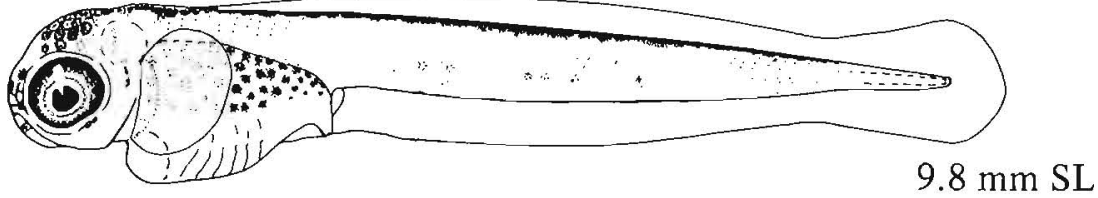

B

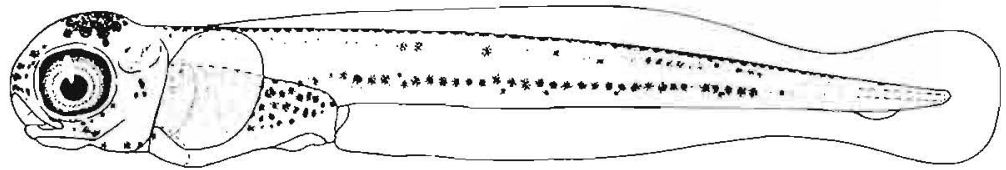

$10.6 \mathrm{~mm} \mathrm{SL}$

C

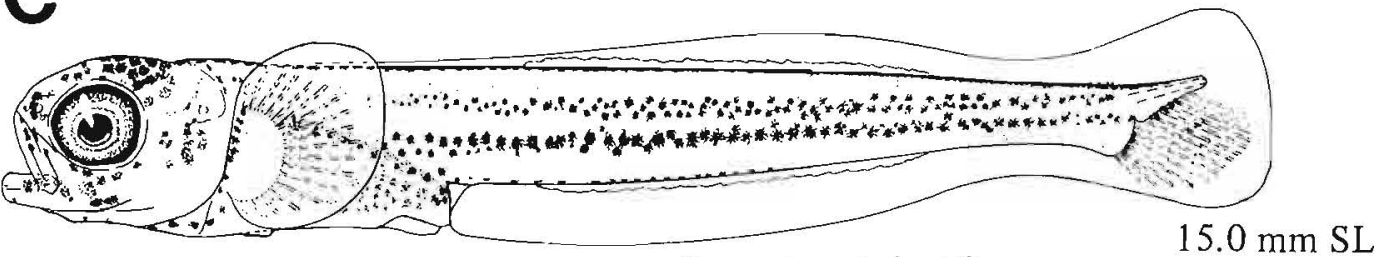

Postanal ventral midline

$15.0 \mathrm{~mm} \mathrm{SL}$ series appears

D

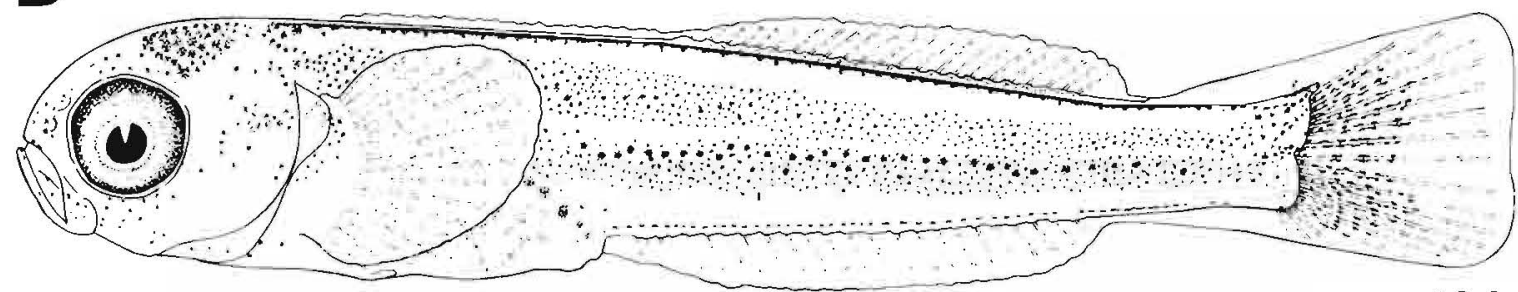

$18.2 \mathrm{~mm} \mathrm{SL}$

E

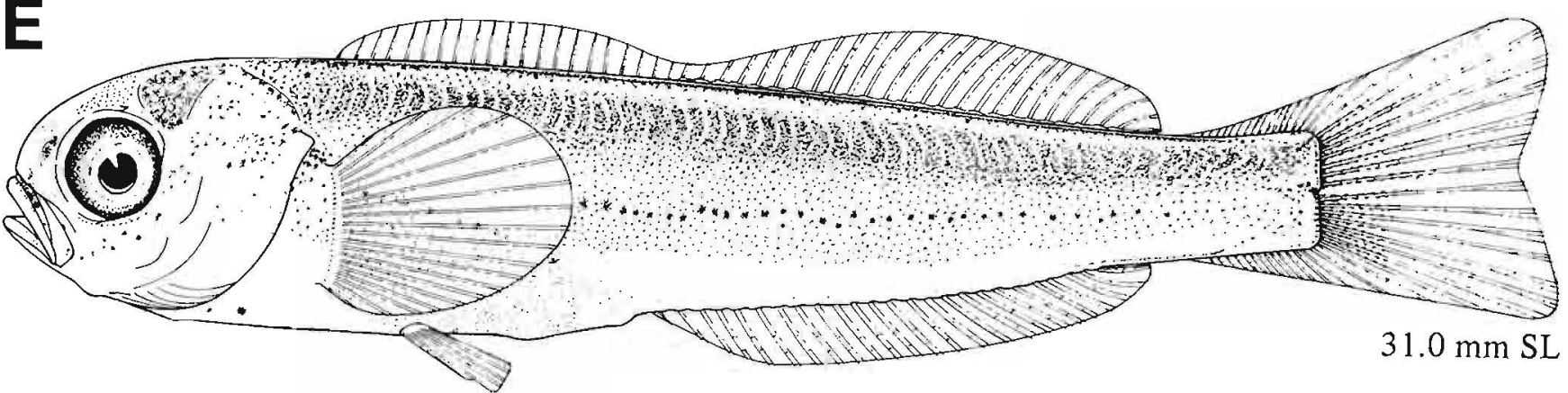

Figures A-E, Kendall and Vinter 1984. 


\section{MERISTICS}

\begin{tabular}{|c|c|c|}
\hline \multirow[t]{3}{*}{ Vertebrae } & \multicolumn{2}{|c|}{ Total: $52-X-57$} \\
\hline & \multicolumn{2}{|c|}{ Precaudal: $20-20-23$} \\
\hline & \multicolumn{2}{|c|}{ Caudal: 32-33-34 } \\
\hline Branchiostegal rays & \multicolumn{2}{|l|}{$6-6-6$} \\
\hline Caudal fin & \multicolumn{2}{|c|}{$17-22,7+10,15-19$} \\
\hline Pelvic fin & \multicolumn{2}{|l|}{ Thoracic } \\
\hline & S: $1-1-1$ & $R: 5-5-5$ \\
\hline Dorsal fin & S: $20-X-23$ & $\mathrm{R}: 20-\mathrm{X}-25$ \\
\hline Pectoral fin & $\mathrm{R}: 18-\mathrm{X}-21$ & \\
\hline Anal fin & $\mathrm{R}: 21-\mathrm{X}-24$ & \\
\hline Gill rakers & U: $4-X-5$ & L: 9-X-11 \\
\hline
\end{tabular}

\section{LIFE HISTORY}

\begin{tabular}{|c|c|}
\hline Range & $\begin{array}{l}\text { Cent. California, } 34-38^{\circ} \mathrm{N} \text {, to } \\
\text { Bering Sea, } 54-66^{\circ} \mathrm{N}\end{array}$ \\
\hline Ecology & Intertidal, nearshore \\
\hline ELH pattern & $\begin{array}{l}\text { Oviparous; demersal, adhesive, } \\
\text { attached eggs; pelagic larvae }\end{array}$ \\
\hline \multirow[t]{4}{*}{ Spawning } & $\begin{array}{l}\text { Season: June-Aug (Aleutian Is.); } \\
\text { June-Sept (west. Pacific) }\end{array}$ \\
\hline & $\begin{array}{l}\text { Area: On rocks or algal holdfasts } \\
\text { in areas of strong currents }{ }^{\mathrm{a}}\end{array}$ \\
\hline & $\begin{array}{l}\text { Mode: Intermittent, males guard } \\
\text { nests }^{c}\end{array}$ \\
\hline & Migration: \\
\hline Fecundity & Range/function: $14,400-103,000^{b}$ \\
\hline $\begin{array}{l}\text { Age at first maturity } \\
\text { Longevity }\end{array}$ & $3-4 y^{b}$ \\
\hline
\end{tabular}

\section{EARLY LIFE HISTORY DESCRIPTION}

\section{EGGS}

Diameter

No. of oil globules

Oil globule diameter

Yolk

Envelope

Hatch size $\quad 7-9 \mathrm{~mm} \mathrm{SL}$

Incubation time/temp. $\quad 30 \mathrm{~d} / 6-10^{\circ} \mathrm{C}$

Pigment

Diagnostic characters

\section{LARVAE \\ Preanal length \\ Length at flexion \\ Much $<50 \%$ before flexion \\ Length at transformation \\ Sequence of fin \\ development \\ $\sim 16 \mathrm{~mm} \mathrm{SL}$ \\ Caudal, pectorals, 2nd dorsal (rays) and anal, 1st dorsal (spines), pelvics}

Pigment

- Heavily pigmented

- Postanal ventral midline present throughout development, particularly heavy on caudal peduncle; spots create irregular line

Diagnostic characters (see Table 3 and tables this section)

- Principal caudal fin ray count in juveniles $(7+10)$

\footnotetext{
${ }^{a}$ Simenstad 1971

${ }^{b}$ Gorbunova 1962

${ }^{\mathrm{c}}$ Kendall and Vinter 1984
}

Ref: Kendall and Vinter 1984. 

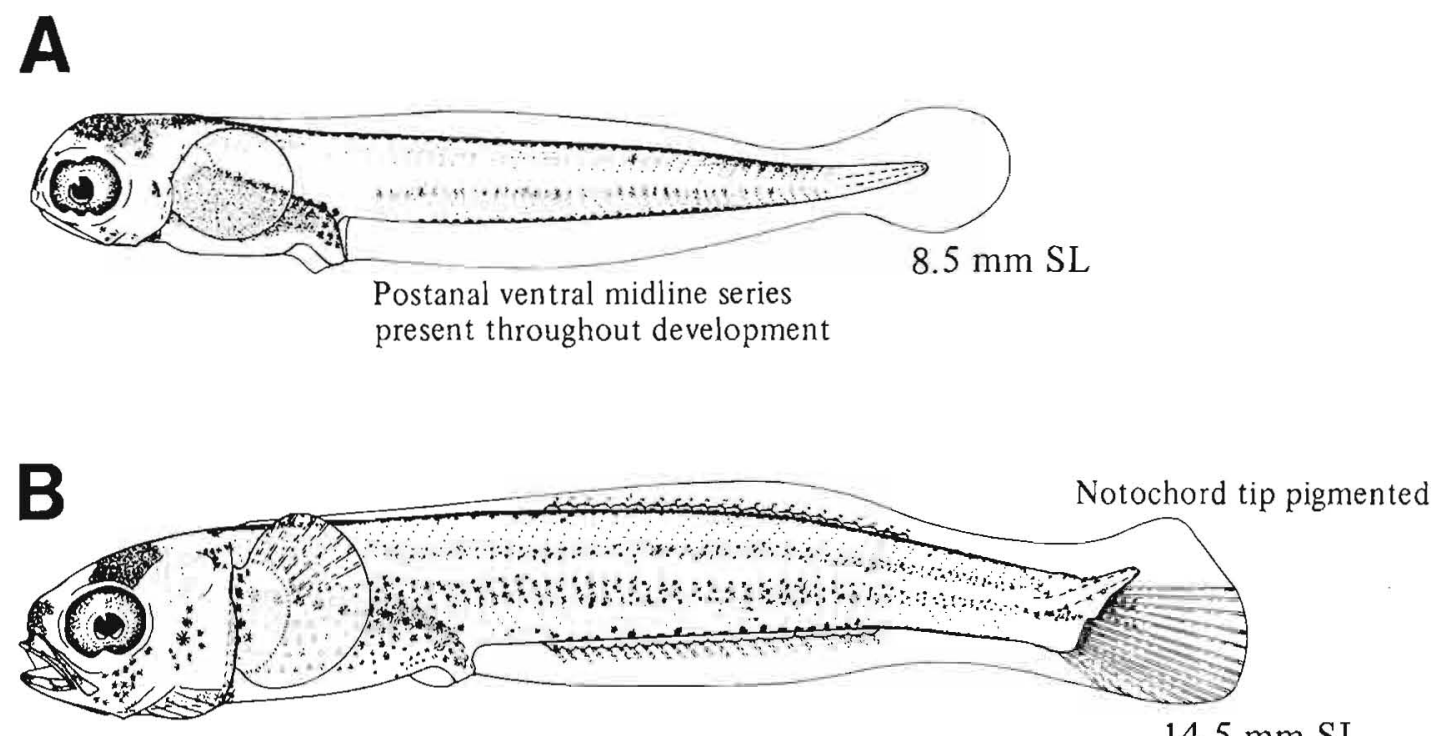

$14.5 \mathrm{~mm} \mathrm{SL}$
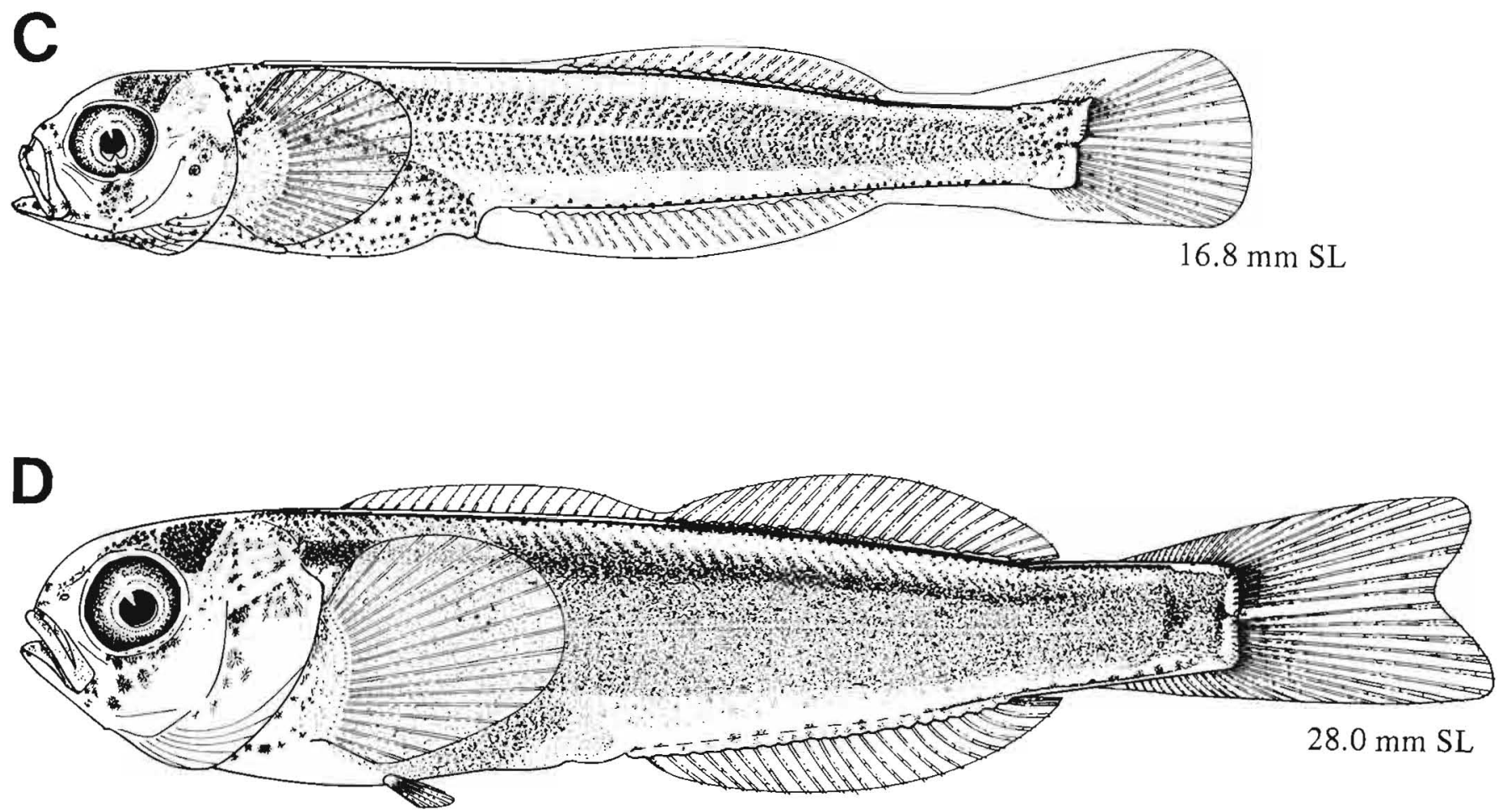

Figures A-D, Kendall and Vinter 1984. 
MERISTICS

$\begin{array}{lll}\text { Vertebrae } & \text { Total: } 50-\mathrm{X}-54 \\ & \begin{array}{l}\text { Precaudal: } 18-\mathrm{X}-19 \\ \text { Caudal: } 32-\mathrm{X}-35\end{array} \\ & \begin{array}{l}\text { Branchiostegal rays } \\ \text { Caudal fin }\end{array} & 6-6-6 \\ \text { Pelvic fin } & 15-17,7+8,14-15 \\ & \text { Thoracic } & \\ \text { Dorsal fin } & \text { S: } 1-1-1 & \text { R: } 5-5-5 \\ \text { Pectoral fin } & \text { S: } 18-\mathrm{X}-20 & \text { R: } 22-\mathrm{X}-25 \\ \text { Anal fin } & \text { R: } 18-\mathrm{X}-19 & \\ \text { Gill rakers } & \text { R: } 23-\mathrm{X}-26 & \\ & \text { U: } 4-\mathrm{X}-5 & \text { L: } 11-\mathrm{X}-12\end{array}$

\section{LIFE HISTORY}

$\begin{array}{ll}\text { Range } & \text { Brit. Col., } 48^{\circ} 30^{\prime}-55^{\circ} \mathrm{N} \text {, to } \\ & \text { Bering Sea, } 54-66^{\circ} \mathrm{N} \\ \text { Ecology } & \text { Nearshore shelf demersal } \\ \text { ELH pattern } & \text { Oviparous; demersal, attached } \\ & \text { eggs; pelagic larvae } \\ \text { Spawning } & \text { Season: } \\ & \text { Area: } \\ & \text { Mode: Nests guarded by males }{ }^{\text {a }} \\ & \text { Migration: } \\ \text { Fecundity } & \text { Range/function: } \\ \text { Age at first maturity } & \\ \text { Longevity } & \end{array}$

\section{EARLY LIFE HISTORY DESCRIPTION}

\section{EGGS $^{b}$}

Diameter

No. of oil globules

Oil globule diameter

Yolk

Envelope

Hatch size

$1.75-2.10 \mathrm{~mm}$

Many

$0.8 \mathrm{~mm}$

$7-9 \mathrm{~mm} \mathrm{SL}$

Pigment

Diagnostic characters

\section{LARVAE}

Preanal length $\quad$ Much $<50 \%$ before flexion

Length at flexion $\sim 16 \mathrm{~mm} \mathrm{SL}$

Length at transformation

Sequence of fin development

Caudal, pectorals, 2nd dorsal (rays) and anal, 1st dorsal (spines), pelvics

\section{Pigment}

- Most heavily pigmented Hexagrammos sp.

- Postanal ventral midline present throughout development, forms continuous line from anus to base of caudal fin

Diagnostic characters (see Table 3 and tables this section)

- Principal caudal fin ray count in juveniles $(7+8)$

\footnotetext{
${ }^{2}$ Kendall and Vinter 1984

${ }^{b}$ Data are from Gorbunova (1962) as cited in Washington et al. (1984b).

Ref: Kendall and Vinter 1984.
} 
A

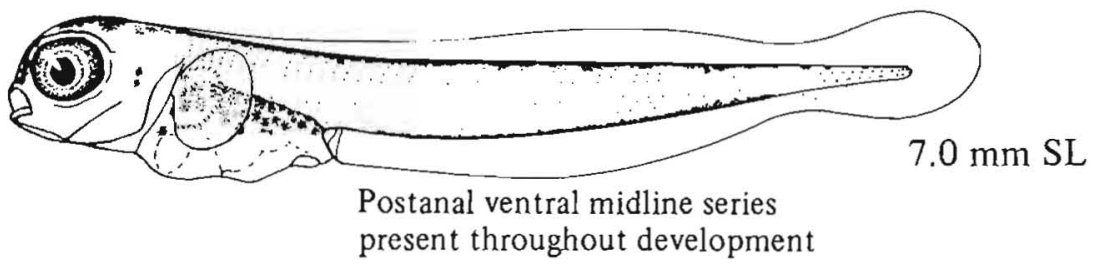

B

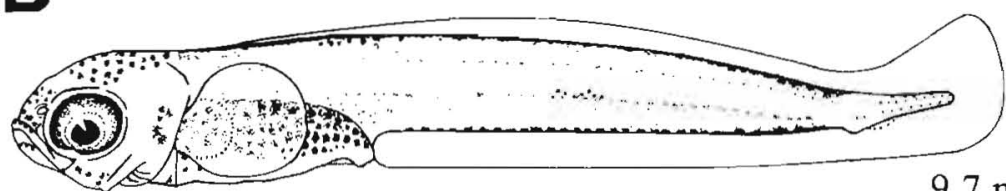

$9.7 \mathrm{~mm} \mathrm{SL}$

C

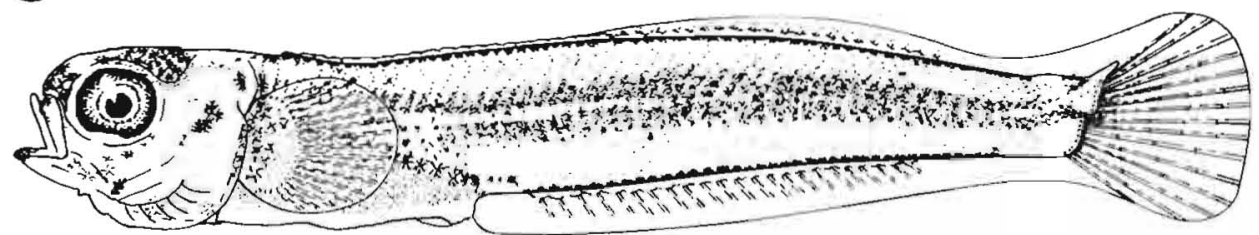

$15.2 \mathrm{~mm} \mathrm{SL}$

D

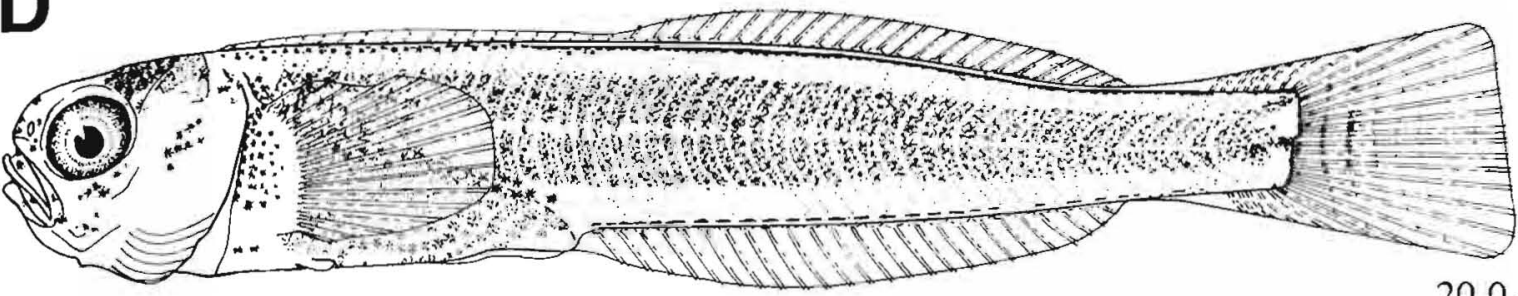

$20.0 \mathrm{~mm} \mathrm{SL}$

E

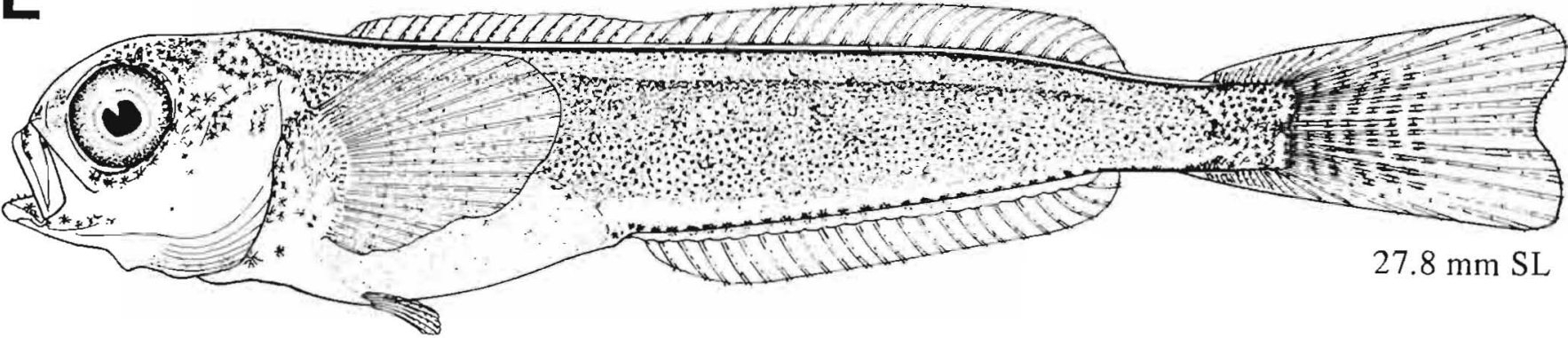

Figures A-E, Kendall and Vinter 1984. 


\section{MERISTICS}

$\begin{array}{lll}\text { Vertebrae } & \text { Total: } 51-\mathrm{X}-56 \\ & \begin{array}{l}\text { Precaudal: } 20-\mathrm{X}-22 \\ \text { Caudal: } 31-\mathrm{X}-34\end{array} \\ \text { Branchiostegal rays } & 6-6-6 \\ \text { Caudal fin } & 16-17,7+8,14-15 \\ \text { Pelvic fin } & \text { Thoracic } & \\ & \text { S: } 1-1-1 & \text { R: } 5-5-5 \\ \text { Dorsal fin } & \text { S: } 22-\mathrm{X}-25 & \text { R: } 18-\mathrm{X}-22 \\ \text { Pectoral fin } & \text { R: } 18-\mathrm{X}-20 & \\ \text { Anal fin } & \text { R: } 22-\mathrm{X}-25 & \\ \text { Gill rakers } & \text { U: } X-X-X & \text { L: } X-X-X\end{array}$

\section{LIFE HISTORY}

$\begin{array}{ll}\text { Range } & \text { Oregon, } 42-46^{\circ} \mathrm{N} \text {, to } \\ & \text { Chukchi Sea, north of } 66^{\circ} \mathrm{N} \\ \text { Nearshore shelf demersal, } & \text { intertidal to } 175 \mathrm{~m} \\ \text { Oviparous; demersal, attached } & \text { eggs; pelagic larvae } \\ \text { ELH pattern } & \text { Season: Feb }{ }^{\mathrm{a}} \text { or Apr }{ }^{\mathrm{b}} \text { (British } \\ \text { Spawning } & \text { Columbia); reported summer } \\ & \text { to fall at extremes of range } \\ & \text { in Pacific Oceanc } \\ \text { Area: } & \\ \text { Mode: May be deposited on } \\ \text { rocks }\end{array}$

Age at first maturity Longevity

\section{EARLY LIFE HISTORY DESCRIPTION}

\section{EGGS}

Diameter

No. of oil globules

Oil globule diameter

Yolk

Envelope

Hatch size $\quad$ 7-9 mm SL

Incubation time/temp.

Pigment

Diagnostic characters

\section{LARVAE}

Preanal length Much $<50 \%$ before flexion Length at flexion $\quad \sim 15 \mathrm{~mm} \mathrm{SL}$

Length at transformation

Sequence of fin development

Caudal, pectorals, 2nd dorsal (rays) and anal, 1st dorsal (spines), pelvics

\section{Pigment}

- Most lightly pigmented Hexagrammos sp.

- Postanal ventral midline absent until $\sim 15 \mathrm{~mm}$ SL, then starts on caudal peduncle; by $\sim 23 \mathrm{~mm}$ SL all along anal fin base

Diagnostic characters (see Table 3 and tables this section)

- Principal caudal fin ray count in juveniles $(7+8)$

${ }^{3}$ Clemens and Wilby 1961

bHart 1973

'Gorbunova 1962

Ref: Kendall and Vinter 1984. 


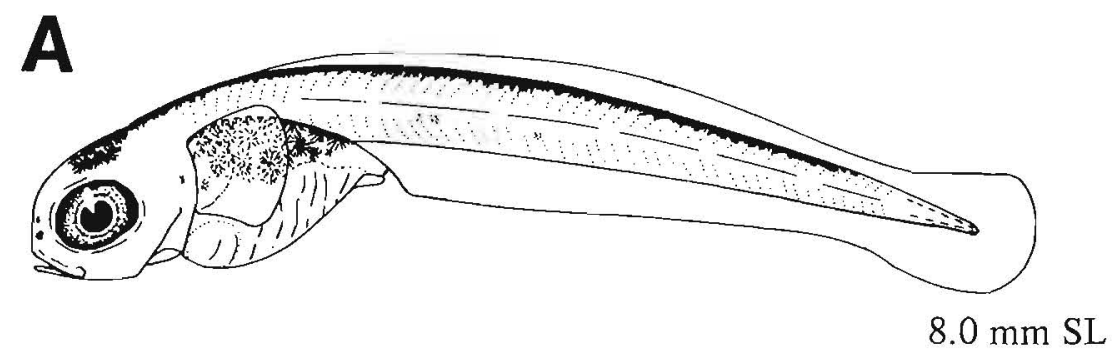

Absence of postanal ventral

B midline series

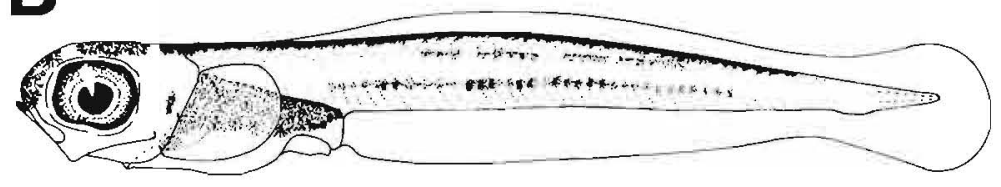

\section{$9.2 \mathrm{~mm} \mathrm{SL}$}

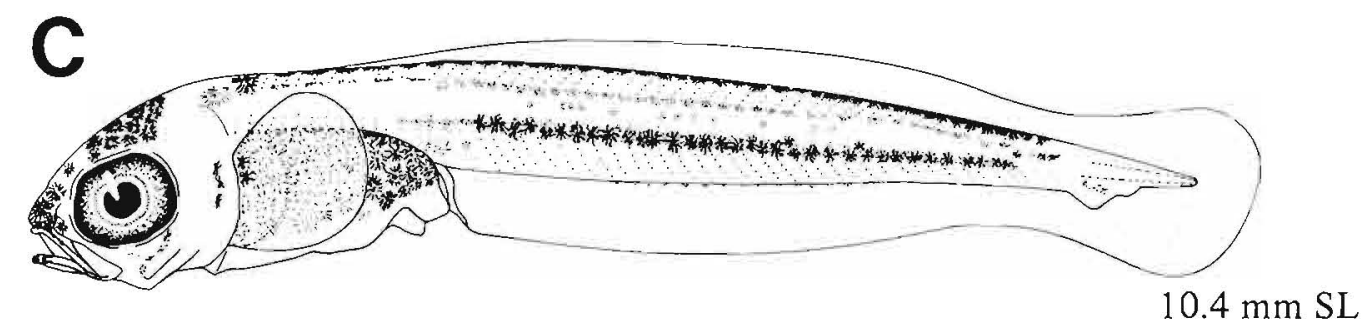

D
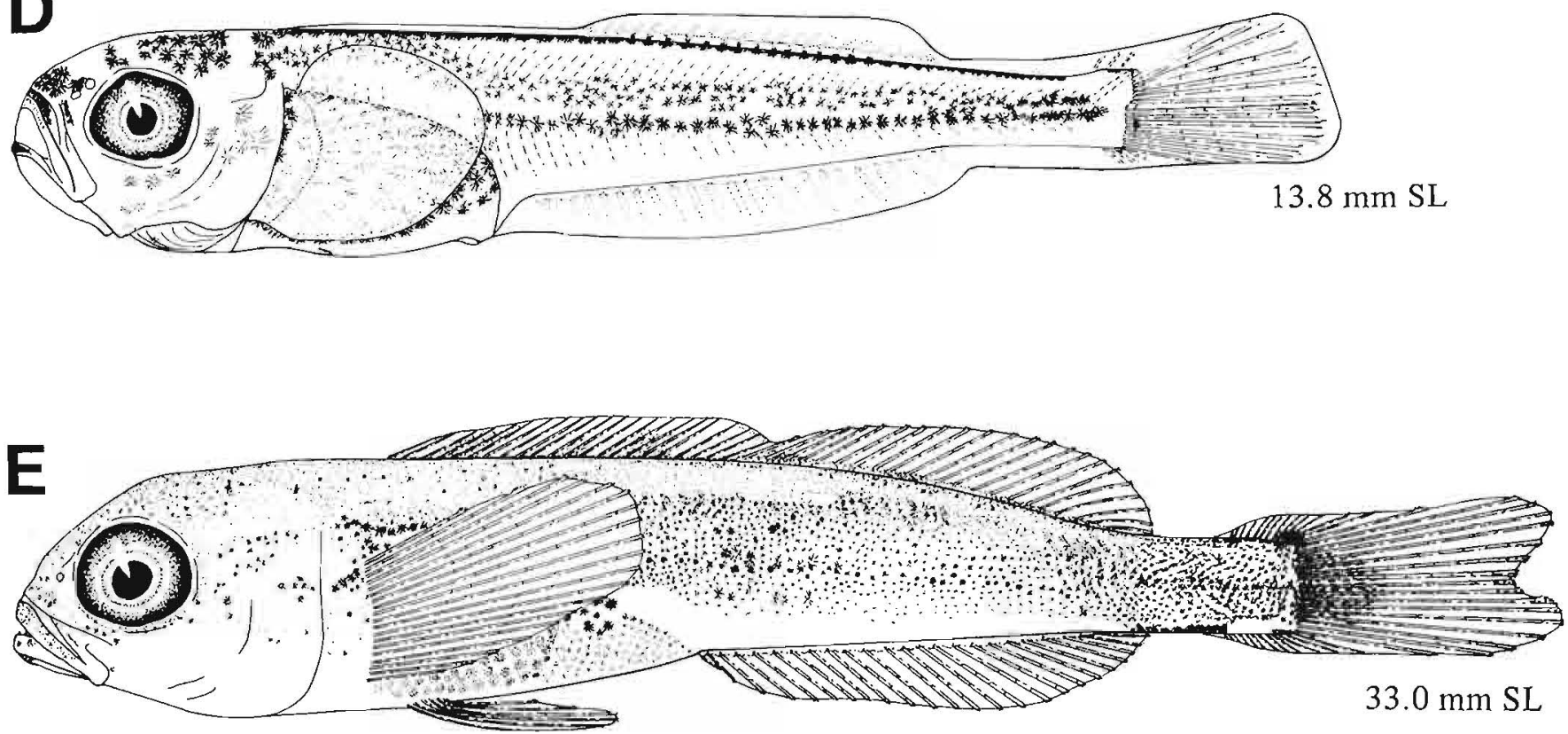

Figures A-E, Kendall and Vinter 1984. 


\section{COTTIDAE}

The sculpins are represented by over 100 species within 45 genera in the study area. Most species are benthic as juveniles and adults and planktonic as larvae. Adults commonly occur in nearshore and intertidal regions throughout the study area. Cottids generally produce demersal eggs that are guarded in shallow water. Although larval cottids are commonly collected during ichthyoplankton surveys, the early life histories of most species are poorly known and larval descriptions are available for fewer than half the species. The cottid section is arranged according to the phenetic groups first described by Richardson (1981a) and recently updated by Washington et al. (1984a). ${ }^{1}$ Preceding the taxonomic sections, the groups are briefly described, representative taxa from each group are illustrated, and a table of meristic characters is provided (Table 36). Betsy Washington provided vertebral counts for several genera based on her osteological studies of the cottids. ${ }^{2}$ In most cases (except for Clinocottus acuticeps where $n=3$ ), counts were taken from one specimen.

\footnotetext{
'Psychrolutidae (sensu J. Nelson 1984) is included within Cottidae.
}

${ }^{2}$ B.B. Washington, NMFS Natl. Systematics Lab., Natl. Mus. Nat. Hist., Wash., D.C. 20560, pers. commun., 8 Dec. 1986. 


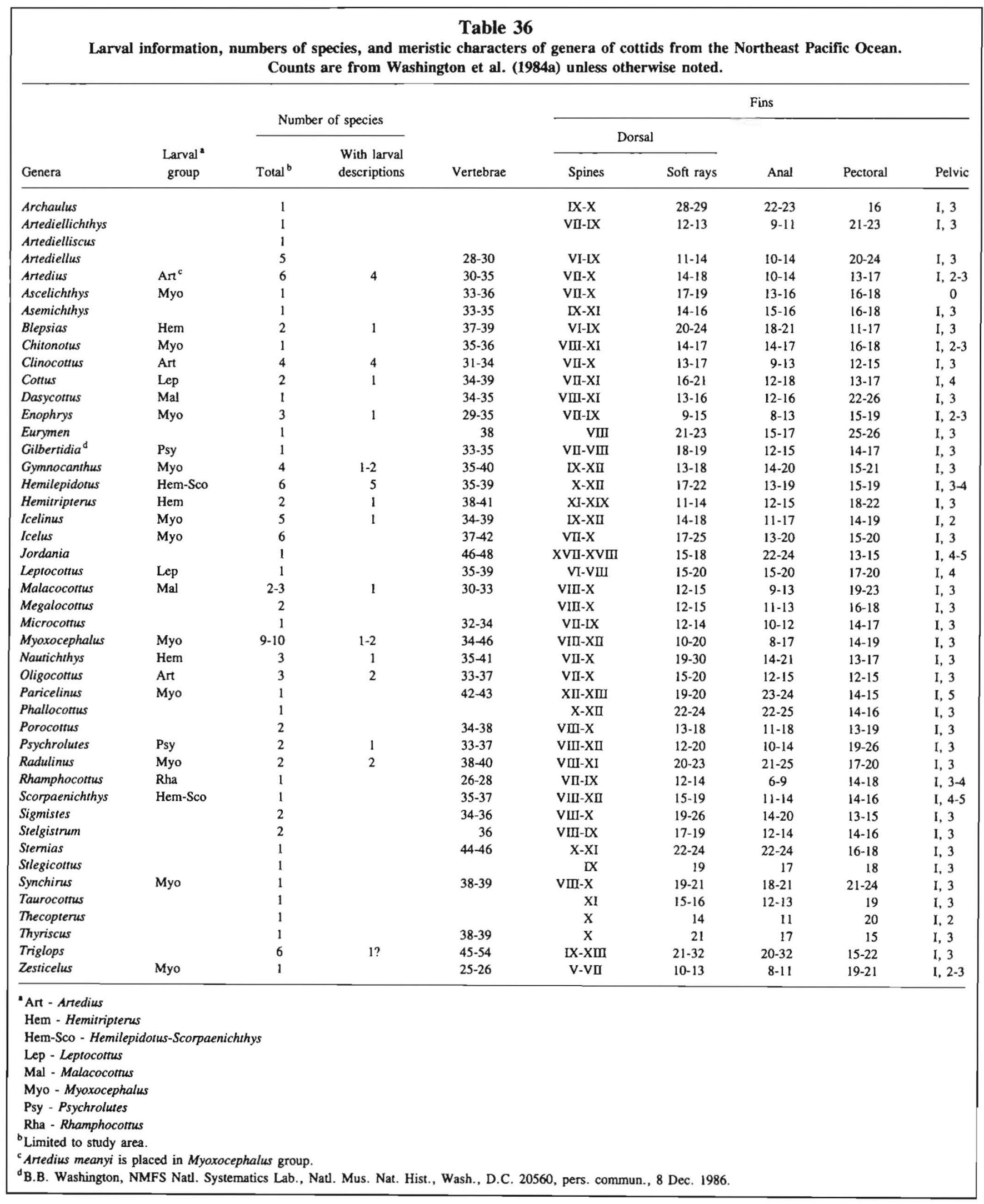




\section{PHENETIC GROUPS}

Rhamphocottus (Rhamphocottus) Larvae are extremely deep-bodied with a long snout-anus length. Melanophores develop uniformly over the body except on the caudal peduncle and ventral gut surface. Small prickles develop all over the body by $9-10 \mathrm{~mm}$, and only one preopercular spine develops.

Hemilepidotus-Scorpaenichthys (Hemilepidotus, Scorpaenichthys) Larvae are relatively long and slender at hatching with moderately long guts (40-60\% SL) and rounded snouts. They become increasingly deep-bodied. Larvae are relatively heavily pigmented. Four prominent preopercular spines develop.

Myoxocephalus (Artedius meanyi, Ascelichthys, Chitonotus, Enophrys, Gymnocanthus, Icelinus, Icelus, Myoxocephalus, Paricelinus, Radulinus, Synchirus, Triglops) Larvae are generally slender-bodied with pointed snouts. Pigment is variable, but most members have heavy pigment on dorsal surface of the gut, nape, and along the postanal ventral midline. Larvae develop four preopercular spines and a distinct bony preopercular shelf. Parietal, nuchal, supracleithral, posttemporal, and occasionally postocular spines develop.

Artedius (Artedius other than A. meanyi; Clinocottus, Oligocottus) Larvae are stubby-bodied with a slightly humped appearance at the nape. Snouts are rounded and guts trail distinctively below the ventral body midline. Some species have gut diverticula. Pigment is relatively light, occurring on the nape, over the gut, and along the postanal ventral midline. Larvae develop a unique preopercular spine pattern with 6-24 spines (enlarged on illustrations). 


\section{PHENETIC GROUPS}

A Rhamphocottus richardsoni

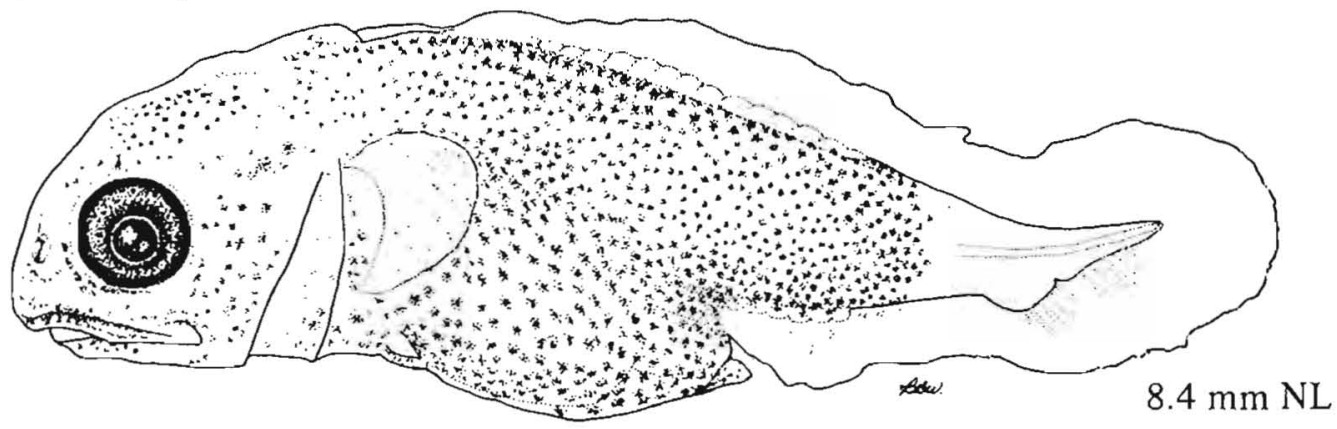

\section{B Hemilepidotus spinosus}

\section{HEMILEPIDOTUS - SCORPAENICHTHYS}

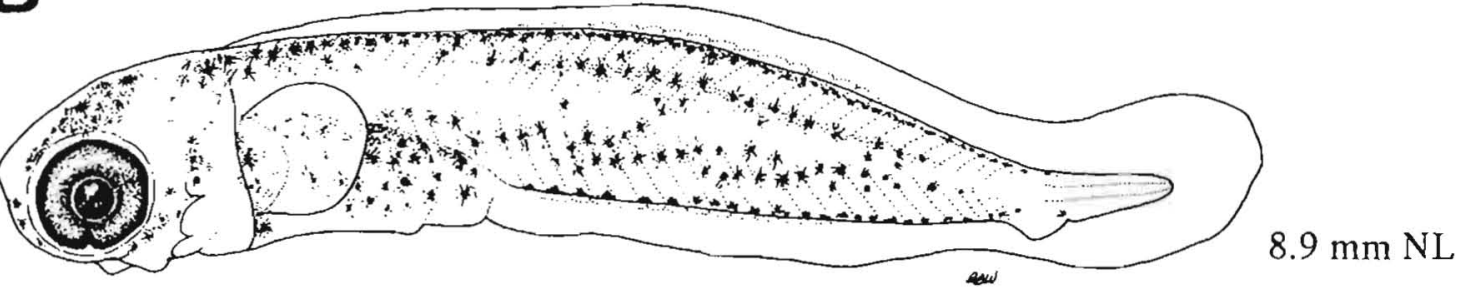

C Ascelichthys rhodorus

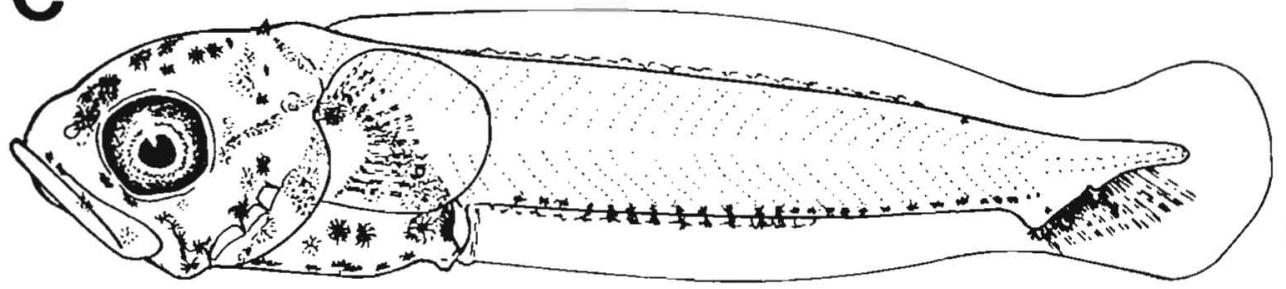

$9.0 \mathrm{~mm} \mathrm{SL}$

D Myoxocephalus G

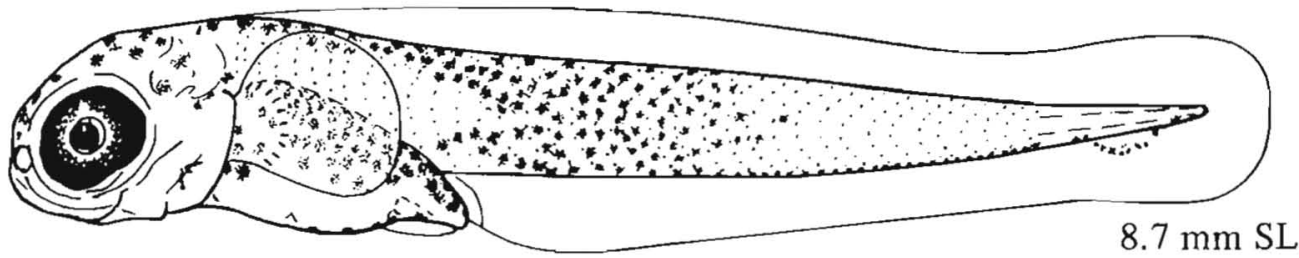

\section{Artedius harringtoni}

\section{ARTEDIUS}

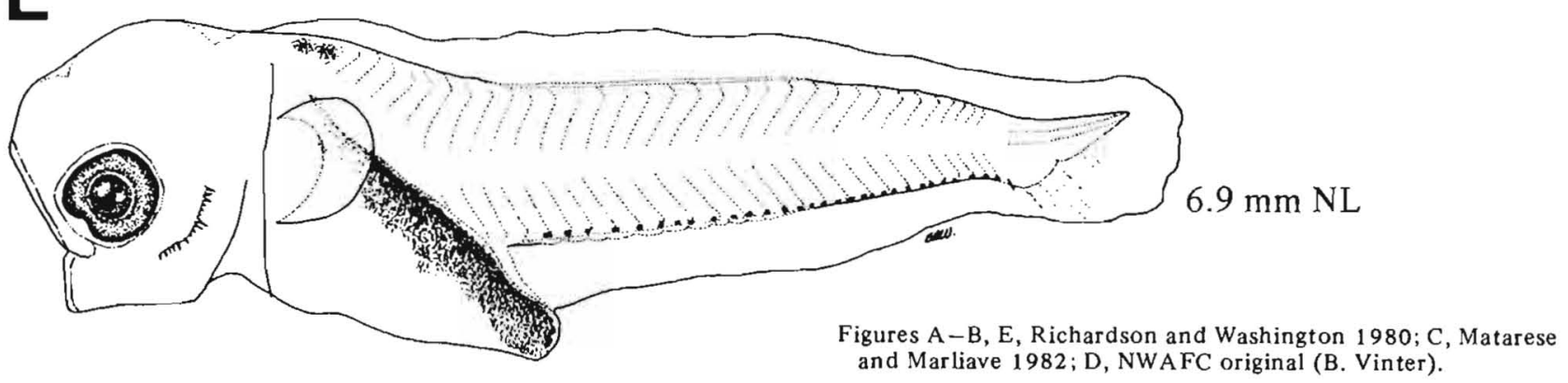


Psychrolutes (Gilbertidia, Psychrolutes) Larvae are tadpole-shaped with large rounded heads tapering toward the tail. They possess an outer layer of flabby skin. The head, nape, gut, and pectoral fins are pigmented. No postanal ventral midline melanophores are present, but pigment is added laterally with development. Head and preopercular spines are absent.

Malacocottus (Dasycottus, Malacocottus) Similar to Psychrolutes group but larvae develop 4-5 preopercular spines.

Leptocottus (Cottus, Leptocottus) Larvae are relatively slender with rounded snouts and moderately short guts. Pigment is light on the postanal body. Other pigment occurs on nape, over gut, and widely spaced along the postanal ventral midline. Four weak preopercular spines develop but other head spines are lacking.

Hemitripterus (Blepsias, Hemitripterus, Nautichthys) Newly hatched larvae are elongate and slender, becoming deeper with development. Nautichthys larvae have long pigmented pectoral fins. Pigmentation is heavy, covering the body except for the caudal peduncle. Pigment extends into the dorsal and ventral finfolds. Larvae develop four prominent preopercular spines. 


\section{PHENETIC GROUPS}

\section{A Psychrolutes paradoxus
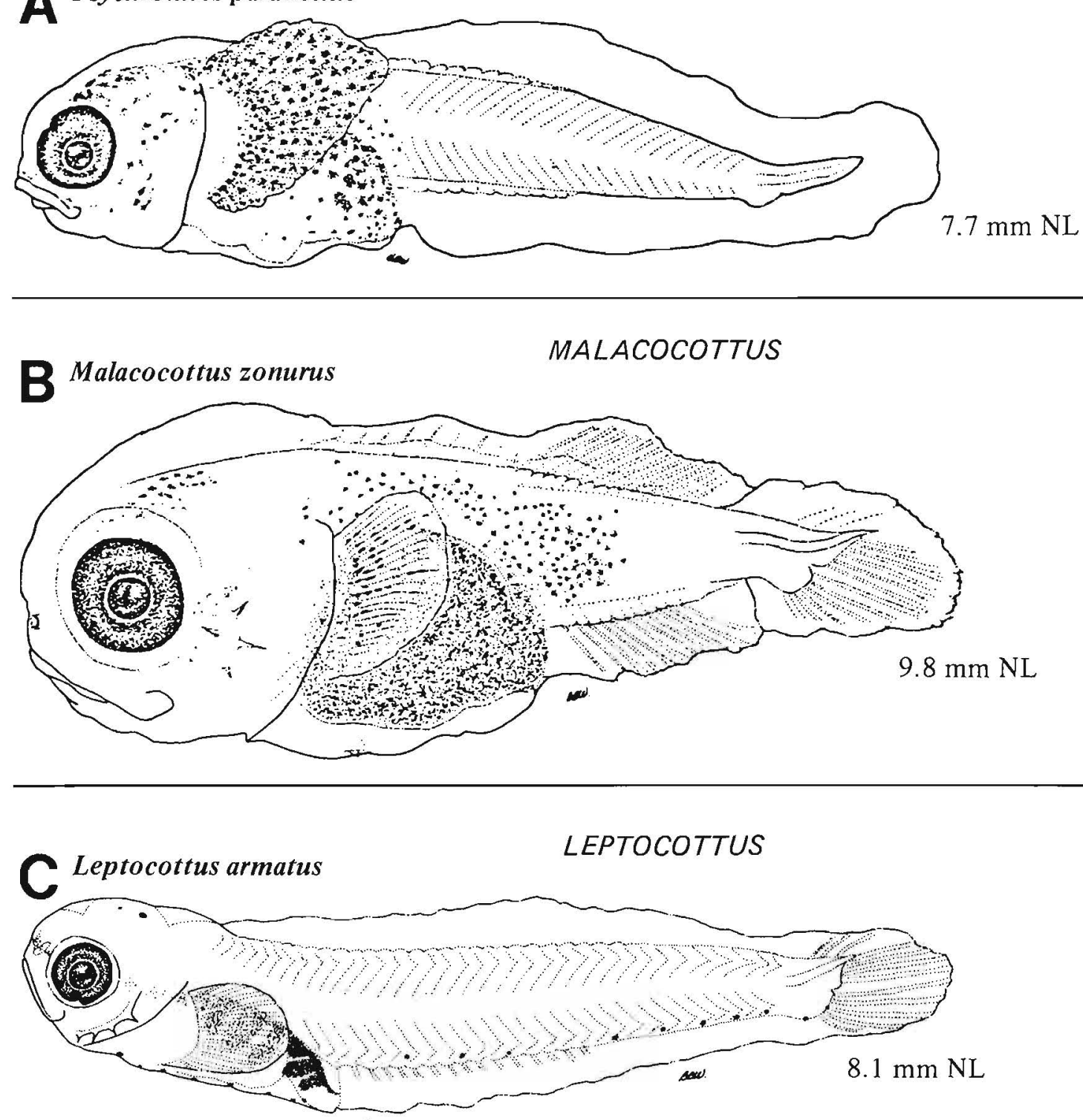

\section{Demitripterus villosus}

\section{HEMITRIPTERUS}

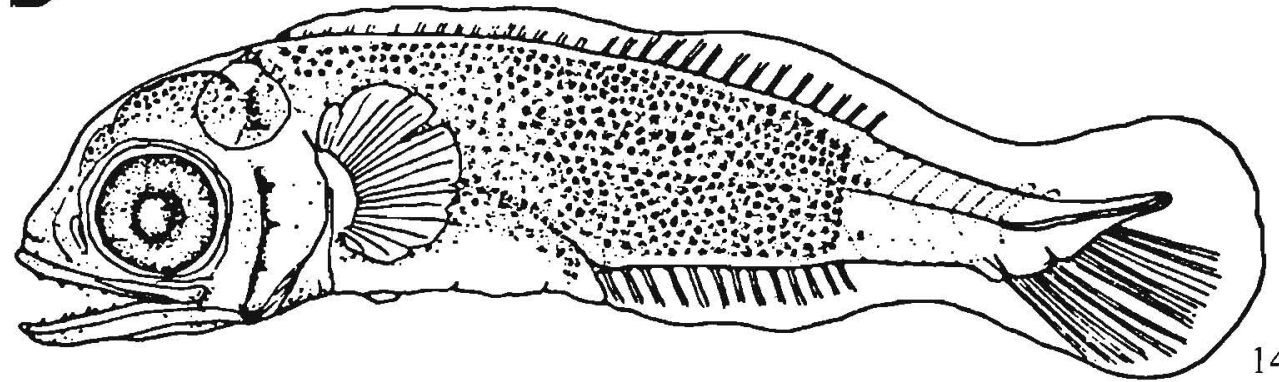

$14.4 \mathrm{~mm} \mathrm{SL}$

Figures A-B, Richardson and Bond 1980; C, Richardson and Washington 1980; D, Okiyama and Sando 1976. 
MERISTICS

\begin{tabular}{|c|c|c|}
\hline \multirow[t]{3}{*}{ Vertebrae } & \multicolumn{2}{|c|}{ Total: $26-26-28$} \\
\hline & \multicolumn{2}{|c|}{ Precaudal: $12-12-12^{\mathrm{a}}$} \\
\hline & \multicolumn{2}{|c|}{ Caudal: $15-15-15^{\mathrm{a}}$} \\
\hline Branchiostegal rays & \multicolumn{2}{|l|}{$6-6-6$} \\
\hline Caudal fin & & \\
\hline \multirow[t]{2}{*}{ Pelvic fin } & Thoracic & \\
\hline & S: $1-1-1$ & $\mathrm{R}: 3-3-4$ \\
\hline Dorsal fin & S: $7-8-9$ & $\mathrm{R}: 12-12-14$ \\
\hline Pectoral fin & R: $14-15-18$ & \\
\hline Anal fin & $\mathrm{R}: 6-7-9$ & \\
\hline Gill rakers & $\mathrm{U}: \mathrm{X}-\mathrm{X}-\mathrm{X}$ & $\mathrm{L}: \mathrm{X}-\mathrm{X}-\mathrm{X}$ \\
\hline
\end{tabular}

\section{LIFE HISTORY}

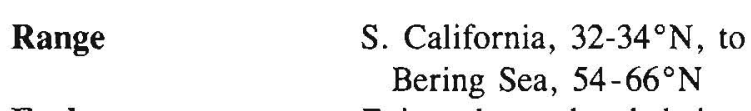

Ecology

ELH pattern

Epi- and mesobenthal, intertidal to $274 \mathrm{~m}$

Spawning

Fecundity

Age at first maturity

Longevity pelagic larvae

Season: Aug-Oct (California); ${ }^{b}$ winter (British Columbia) ${ }^{c}$

Area: Nearshore $(20 \mathrm{~m})^{\mathrm{d}}$

Mode: Egg masses guarded by females $^{\text {d }}$

Migration:
EARLY LIFE HISTORY DESCRIPTION

\section{EGGS}

Diameter

$2.5-2.8 \mathrm{~mm}$

No. of oil globules

Oil globule diameter

Yolk

Envelope

Hatch size

Orange; ${ }^{\mathrm{c}}$ white ${ }^{\mathrm{d}}$

Incubation time/temp.

Pigment

6-7 mm SL

Diagnostic characters

\author{
LARVAE \\ Preanal length \\ $>50 \%$ SL \\ Length at flexion \\ 6.9-10.0 mm NL \\ Length at transformation \\ Sequence of fin \\ 14-15 mm SL \\ development \\ Dorsal, anal, and pectorals \\ followed by caudal and \\ pelvics \\ Pigment \\ - Larvae are uniformly covered with melanophores \\ except for caudal peduncle and the ventral surface \\ of gut \\ Diagnostic characters (see Table 3) \\ - Morphology: Deep-bodied, very long snout-to-anus \\ length \\ - Uniformily heavily pigmented \\ - Presence of pigmented preanal finfold \\ - Prickles develop over most of body by 9-10 mm SL \\ - Spines: Only one preopercular (small spiny projec- \\ tions appear along the preopercular margin); others \\ include parietal, nuchal, supracleithral, posttem- \\ poral, and postocular
}

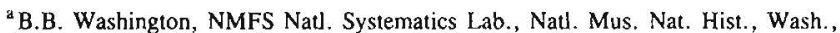
D.C. 20560 , unpubl.

${ }^{b}$ Fitch and Lavenberg 1975

${ }^{c}$ Hart 1973

${ }^{d}$ Garrison and Miller 1982

Ref: Blackburn 1973, Marliave 1975a, Richardson and Washington 1980, Saruwatari et al. 1987, Washington et al. 1984b.
} 

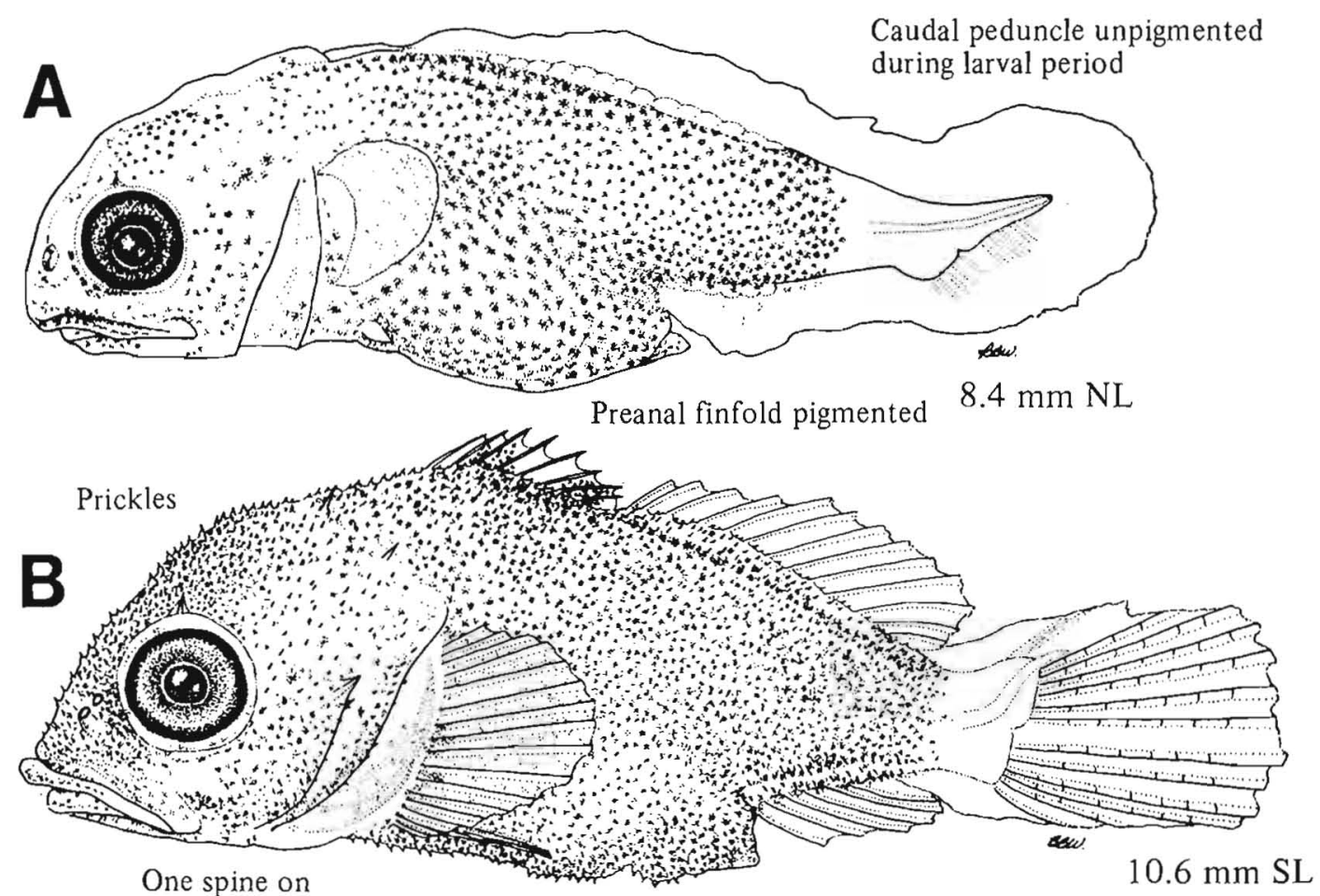

dorsal margin

of preopercle

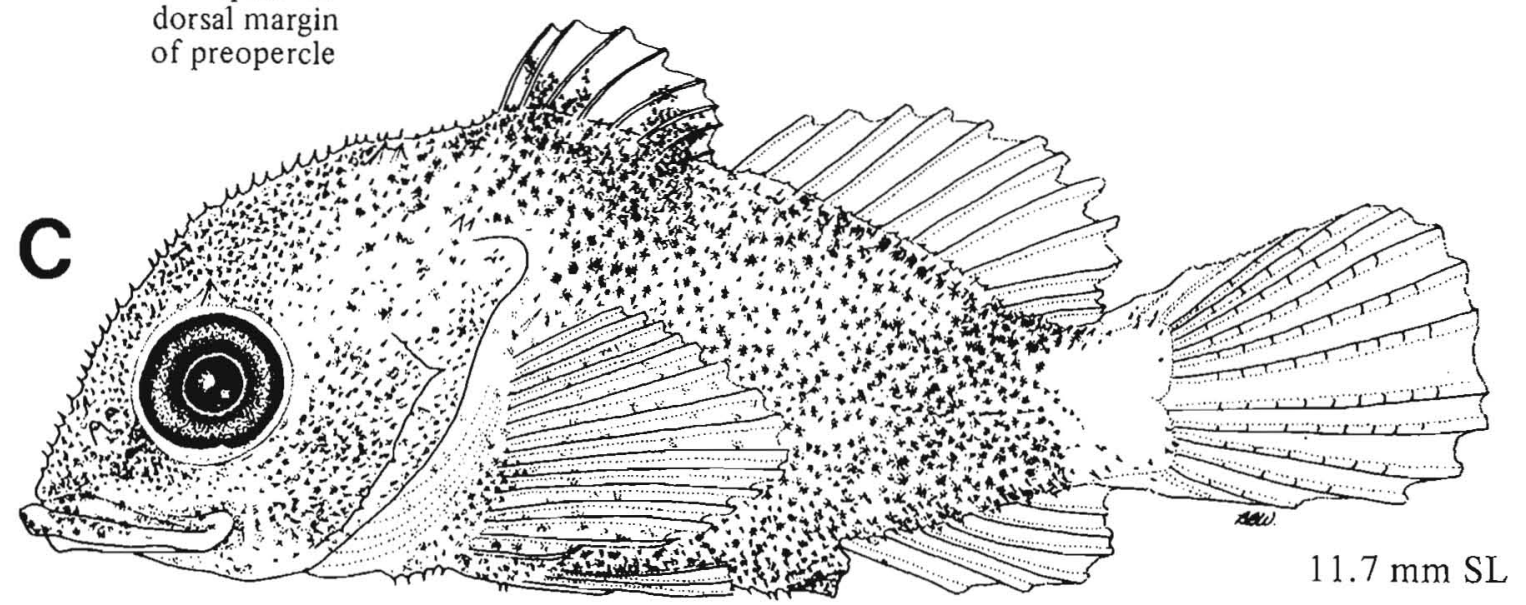

Figures A-C, Richardson and Washington 1980. 
Table 37

Selected postanal pigmentation characters useful in distinguishing preflexion and flexion Hemilepidotus larvae (Matarese and Vinter 1985). Larvae of $H$. papilio are unknown. All lengths are mm SL.

\begin{tabular}{|c|c|c|c|c|c|c|c|c|}
\hline \multirow[b]{2}{*}{ Taxon } & \multicolumn{7}{|c|}{ Pigmentation characters } & \multirow[b]{2}{*}{ Diagnostic } \\
\hline & Dorsal midline & $\begin{array}{l}\text { Dorso- } \\
\text { lateral }\end{array}$ & $\begin{array}{c}\text { Above } \\
\text { notochord } \\
\text { (internal) }\end{array}$ & $\begin{array}{c}\text { Below } \\
\text { notochord } \\
\text { (internal) }\end{array}$ & $\begin{array}{l}\text { Ventro- } \\
\text { lateral }\end{array}$ & $\begin{array}{l}\text { Ventral } \\
\text { midline }\end{array}$ & $\begin{array}{l}\text { Caudal } \\
\text { region }\end{array}$ & \\
\hline $\begin{array}{l}\text { Hemilepidotus } \\
\text { spinosus }\end{array}$ & $\begin{array}{l}\text { By } 5 \mathrm{~mm} \text {, a con- } \\
\text { tinuous line from } \\
\text { head to posterior- } \\
\text { most myomere, } \\
\text { becoming heavier }\end{array}$ & $\begin{array}{l}\text { By } 6 \mathrm{~mm} \text {, } \\
\text { becoming } \\
\text { heavier }\end{array}$ & $\begin{array}{l}\text { By } 8-9 \mathrm{~mm} \text {, } \\
\text { along length } \\
\text { of body }\end{array}$ & Not obvious & $\begin{array}{l}\text { By } 6 \mathrm{~mm} \text {, } \\
\text { becoming } \\
\text { heavier }\end{array}$ & $\begin{array}{l}\text { From anus to pos- } \\
\text { teriormost myomere, } \\
>15 \text { melanophores }\end{array}$ & $\begin{array}{l}\text { Sparse, ventral } \\
\text { midline continuous }\end{array}$ & $\begin{array}{l}\text { Lateral, ventral } \\
\text { midline }\end{array}$ \\
\hline H. hemilepidotus & $\begin{array}{l}\text { Until } 7 \mathrm{~mm} \text {, unpig- } \\
\text { mented area be- } \\
\text { tween myomeres } \\
4-11 \text { becoming } \\
\text { moderately heavy }\end{array}$ & $\begin{array}{l}\text { By } 11 \mathrm{~mm} \text {, } \\
\text { moderate }\end{array}$ & $\begin{array}{l}\text { By } 6-7 \mathrm{~mm} \text {, } \\
\text { along length } \\
\text { of body }\end{array}$ & $\begin{array}{l}\text { By } 8-9 \mathrm{~mm} \text {, } \\
\text { begins pos- } \\
\text { terior to } \\
\text { anus }\end{array}$ & $\begin{array}{l}\text { By } 11 \mathrm{~mm} \text {, } \\
\text { moderate }\end{array}$ & $\begin{array}{l}\text { Begins } 9 \text { myomeres } \\
\text { after anus, }<15 \\
\text { melanophores }\end{array}$ & None & $\begin{array}{l}\text { Lateral, lack of } \\
\text { caudal pigment }\end{array}$ \\
\hline H. jordani $i^{\mathbf{a}}$ & $\begin{array}{l}\text { Similar to } H \text {. hemi- } \\
\text { lepidotus but not as } \\
\text { heavy }\end{array}$ & None & $\begin{array}{l}\text { Similar to } \\
\text { H. hemi- } \\
\text { lepidotus }\end{array}$ & $\begin{array}{l}\text { Similar to } \\
\text { H. hemi- } \\
\text { lepidotus }\end{array}$ & $\begin{array}{l}\text { Some } \\
\text { internal } \\
\text { only }\end{array}$ & $\begin{array}{l}\text { By flexion, a } \\
\text { few internal } \\
\text { melanophores }\end{array}$ & None & $\begin{array}{l}\text { Lack of lateral and } \\
\text { caudal pigment }\end{array}$ \\
\hline H. zapus & $\begin{array}{l}\text { Similar to } H \text {. hemi- } \\
\text { lepidotus but not as } \\
\text { heavy }\end{array}$ & None & $\begin{array}{l}\text { By } 6 \mathrm{~mm} \text {, } \\
\text { along length } \\
\text { of body }\end{array}$ & $\begin{array}{l}\text { By } 8 \mathrm{~mm} \text {, } \\
\text { incomplete; } \\
\text { begins pos- } \\
\text { terior to } \\
\text { anus }\end{array}$ & $\begin{array}{l}\text { Some } \\
\text { internal } \\
\text { only }\end{array}$ & $\begin{array}{l}\text { Begins } 11 \text { myomeres } \\
\text { after anus, }<15 \\
\text { melanophores }\end{array}$ & $\begin{array}{l}\text { Ventral midline } \\
\text { continuous, above } \\
\text { and below urostyle }\end{array}$ & Urostyle \\
\hline H. gilberi $i^{b}$ & $\begin{array}{l}\text { Pigment begins } 8-9 \\
\text { myomeres after } \\
\text { anus }\end{array}$ & None & None & None & None & $\begin{array}{l}\text { Begins } 10 \text { myomeres } \\
\text { after anus, }<15 \\
\text { melanophores }\end{array}$ & None & $\begin{array}{l}\text { Unpigmented area } \\
\text { along dorsal } \\
\text { midline, lack of } \\
\text { lateral pigment }\end{array}$ \\
\hline
\end{tabular}




\section{POSTANAL PIGMENT PATTERNS OF HEMILEPIDOTUS SPP. LARVAE}

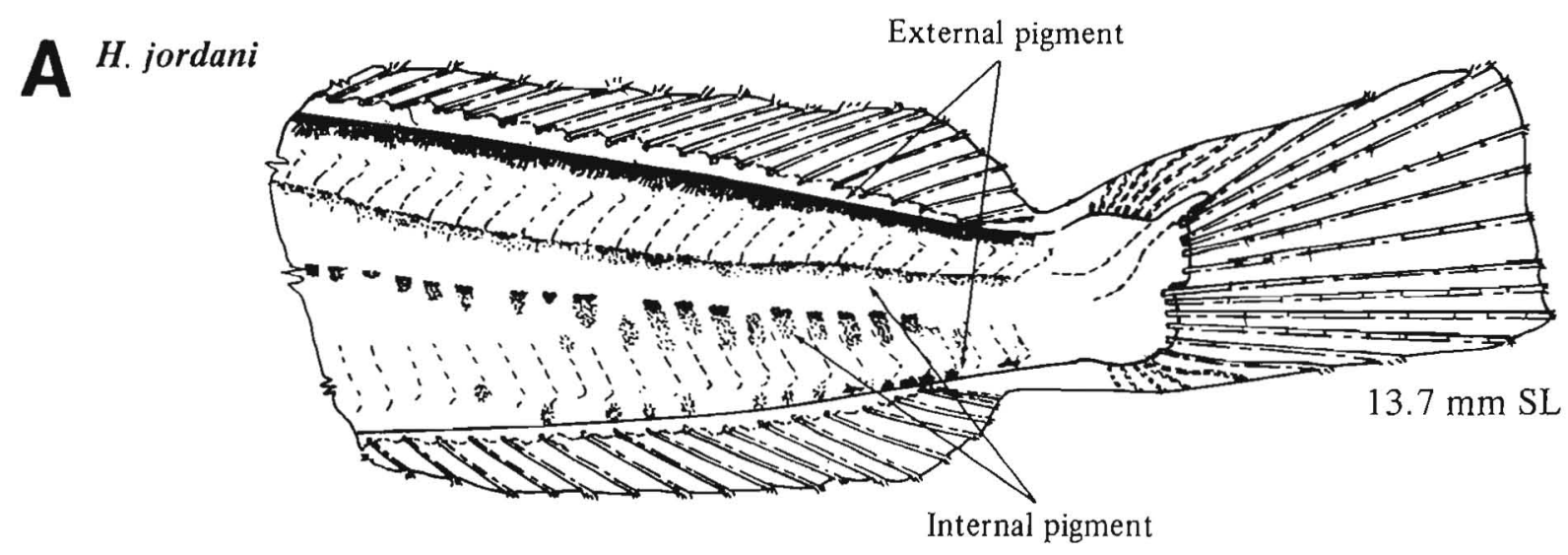

B
H. hemilepidotus
External pigment

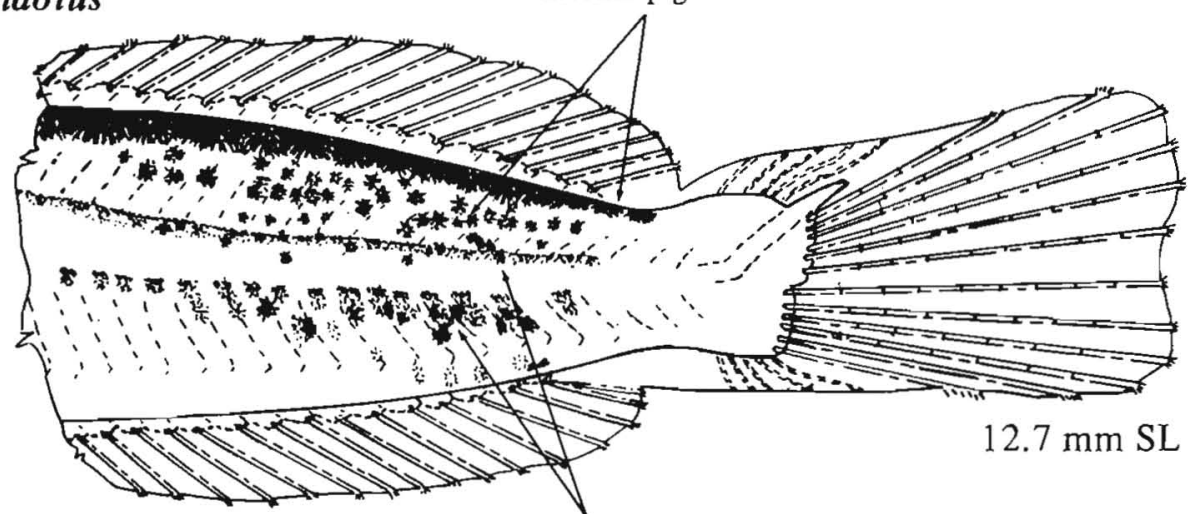

Internal pigment
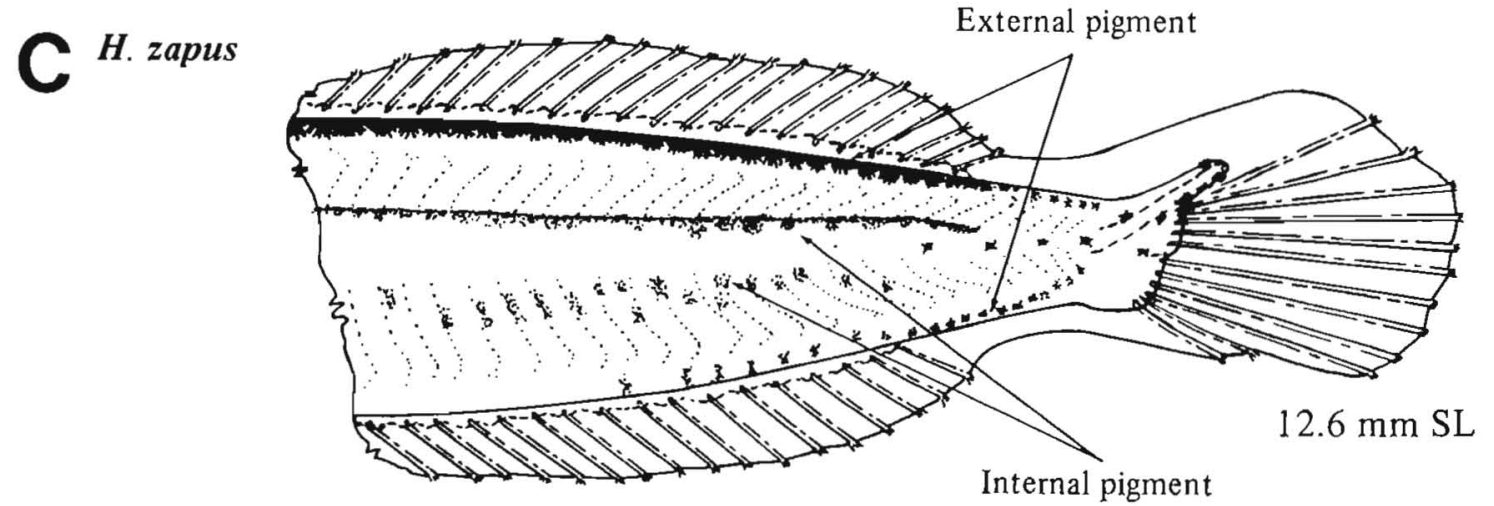

Figures $\mathrm{A}-\mathrm{C}$, Matarese and Vinter 1985. 
Table 38

Characters useful in distinguishing pelagic juveniles of Hemilepidotus spp. (Peden 1978, in part). Specimens of H. papilio and $\boldsymbol{H}$. gilberti have not been collected from the study area and are not included here.

\begin{tabular}{lllll}
\hline Character & H. hemilepidotus & H. jordani & H. spinosus & H. zapus \\
\hline Pectoral fin rays & $16(15-17)$ & $18(17-19)$ & $15-16(14-16)$ & $16(15-17)$ \\
$\begin{array}{l}\text { Total sof fin rays; dorsal, anal, and both } \\
\text { pectoral fins }\end{array}$ & $63-68$ & 7178 & $63-66$ & $67-76$ \\
Lateral line pores & $65(59-68)$ & $64(59-68)$ & $63(57-67)$ & $52(47-58)$ \\
Vertebrae & $35-37$ & $37-39$ & 36 & $37-38$ \\
Horizontal rows in ventral scale band & 6 or 7 & $\sim 8$ & $\sim 4$ & 8 or 9 \\
$\begin{array}{l}\text { Gill membranes fused to isthmus so as to form a } \\
\text { free fold posteriorly }\end{array}$ & Yes & Yes & No free fold & Yes \\
Horizontal rows in dorsal scale band & $\leqslant 5$ & $\leqslant 5$ & $\geqslant 6$ & $\leqslant 5$
\end{tabular}




\section{PELAGIC JUVENILES}

\section{A H. hemilepidotus}
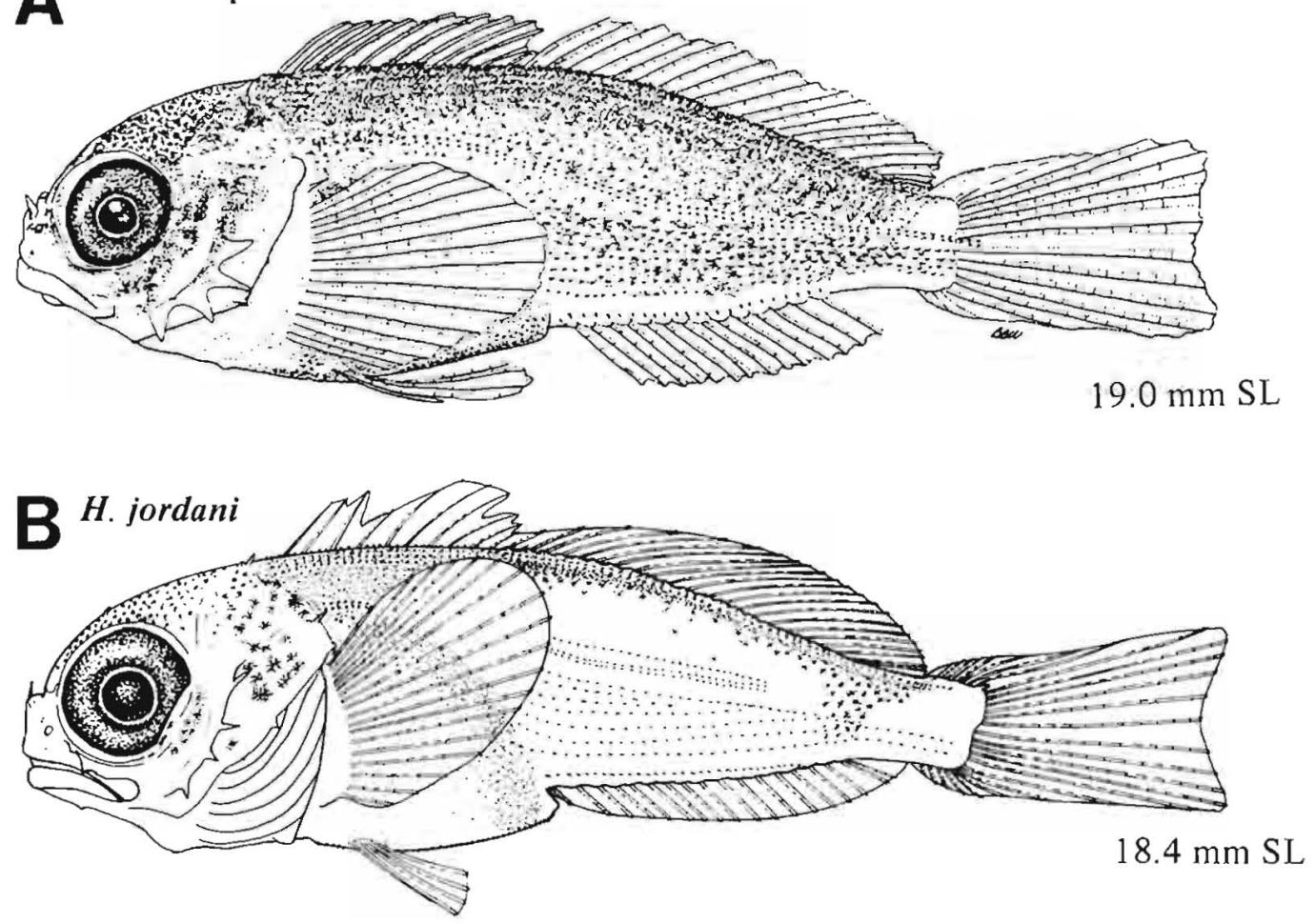

\section{CH. spinosus}
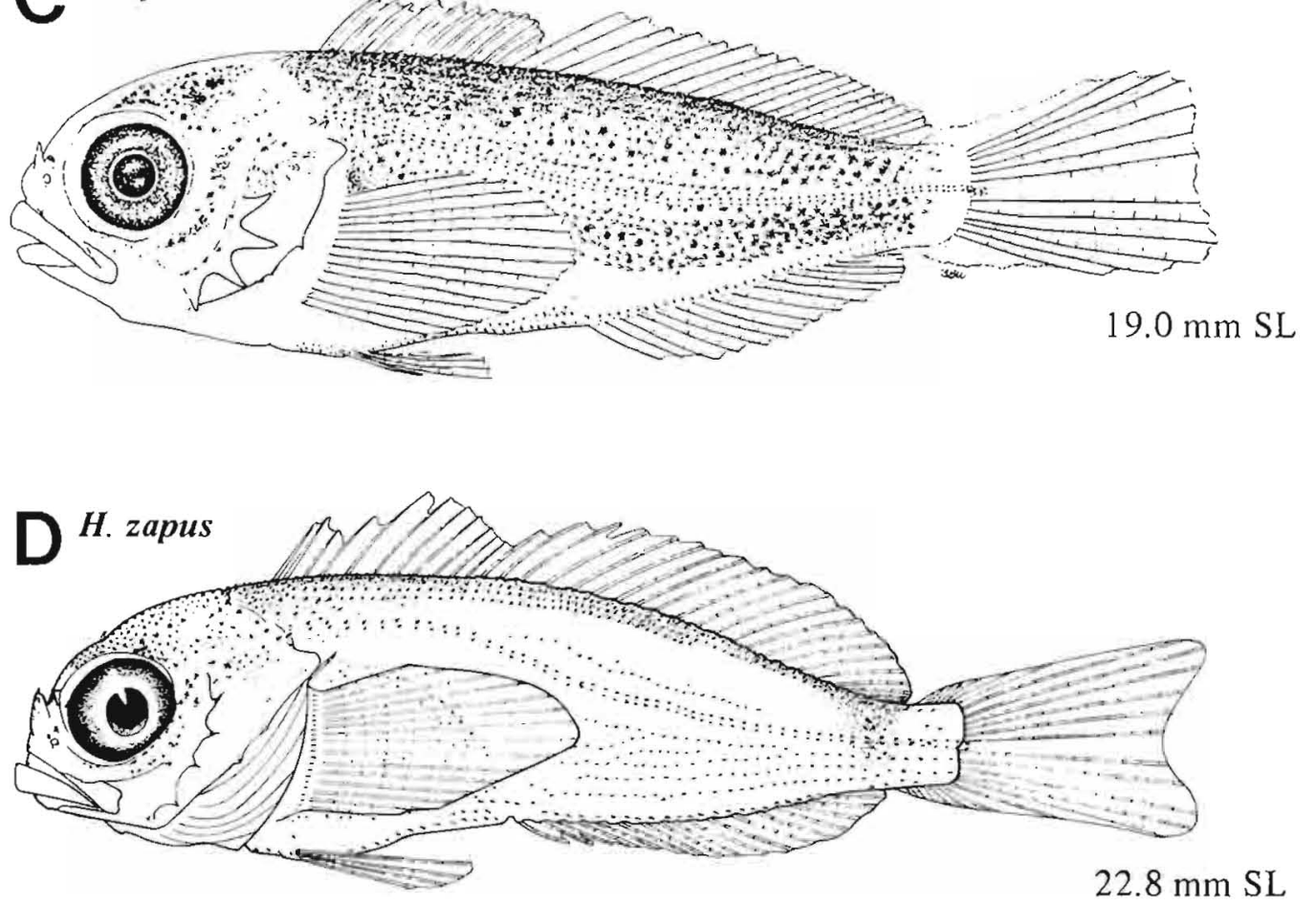

Figure A, C, Richardson and Washington 1980; B, NWAFC original (B. Vinter); D, Matarese and Vinter 1985. 


\section{MERISTICS}

\begin{tabular}{|c|c|c|}
\hline \multirow[t]{3}{*}{ Vertebrae } & \multicolumn{2}{|c|}{ Total: $36-\mathrm{X}-38$} \\
\hline & \multicolumn{2}{|c|}{ Precaudal: X-X-X } \\
\hline & \multicolumn{2}{|c|}{ Caudal: X-X-X } \\
\hline Branchiostegal rays & \multicolumn{2}{|l|}{$6-6-6$} \\
\hline \multirow{2}{*}{ Pelvic fin } & Thoracic & \\
\hline & $S: 1-1-1$ & $\mathrm{R}: 4-4-4$ \\
\hline Dorsal fin & $S: 11-X-12$ & $\mathrm{R}: 17-\mathrm{X}-22$ \\
\hline Pectoral fin & $\mathrm{R}: 15-\mathrm{X}-17$ & \\
\hline Anal fin & $R: 14-X-19$ & \\
\hline Gill rakers & $\mathrm{U}: \mathrm{X}-\mathrm{X}-\mathrm{X}$ & $\mathrm{L}: \mathrm{X}-\mathrm{X}-\mathrm{X}$ \\
\hline
\end{tabular}

\section{LIFE HISTORY}

Range
Ecology
ELH pattern
Spawning
Fecundity
Age at first maturity
Longevity

Bering Sea, 54-66 $\mathrm{N}$

Nearshore shelf demersal

Oviparous, eggs probably demersal, pelagic larvae Season:

Area:

Mode:

Migration:

Range/function:

\section{EARLY LIFE HISTORY DESCRIPTION}

\section{EGGS}

Diameter

No. of oil globules

Oil globule diameter

Yolk

Envelope

Hatch size

Incubation time/temp.

Pigment

Diagnostic characters 


\section{A}

Pigment
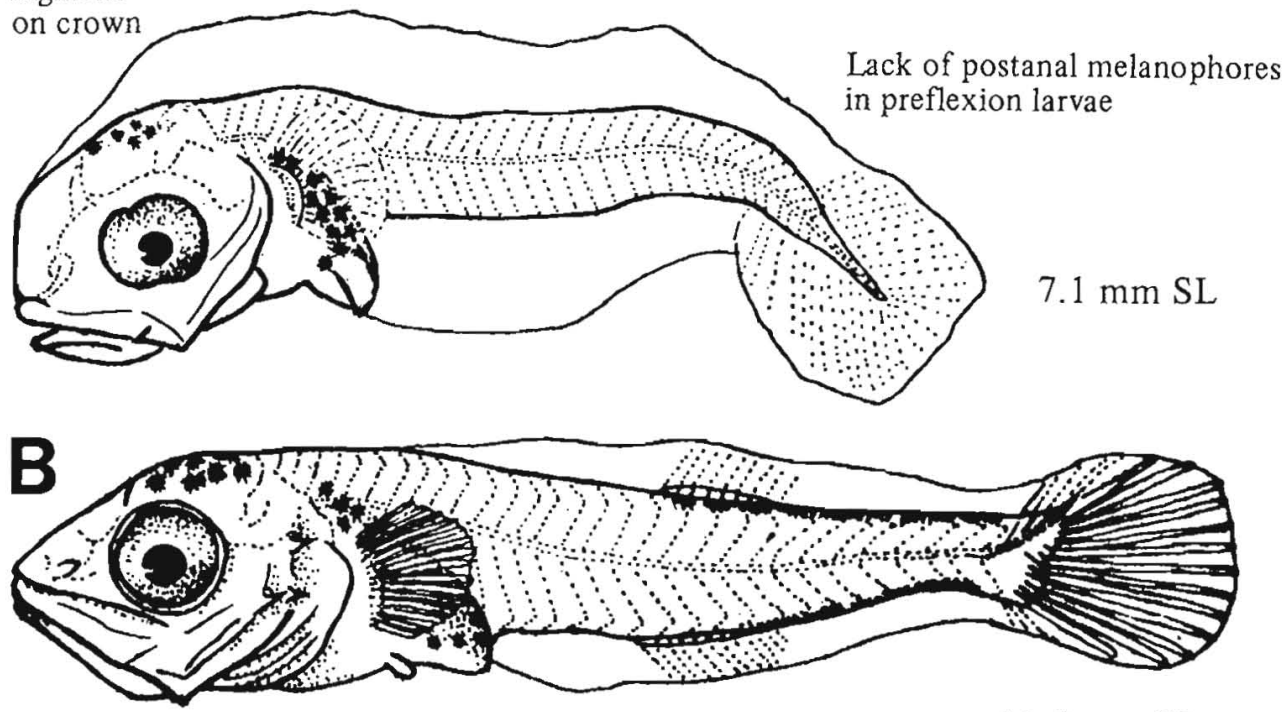

$11.6 \mathrm{~mm} \mathrm{SL}$

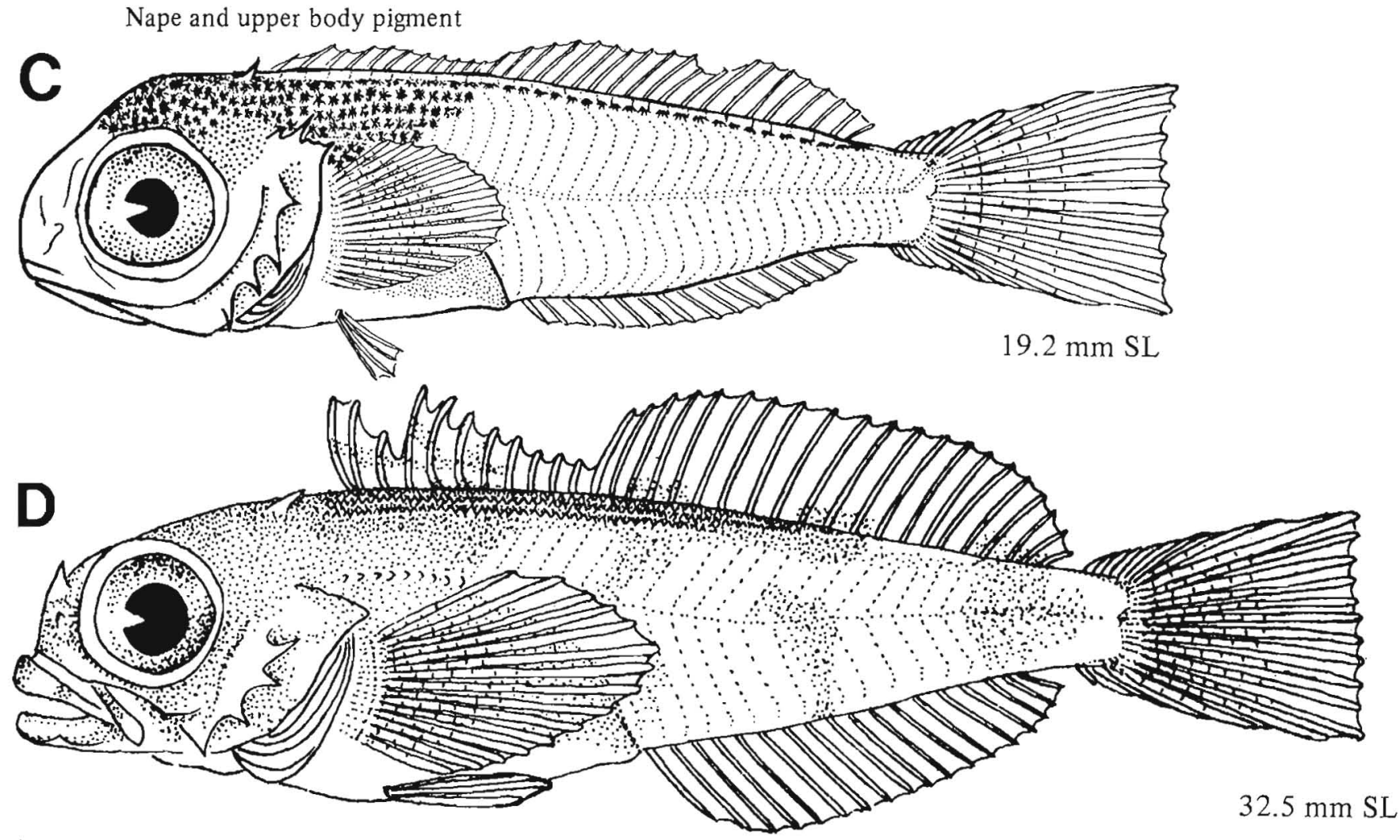

Figures A-D, Hattori 1964. Identification of Figure B is questionable as only 30 myomeres are illustrated. 


\section{MERISTICS}

\begin{tabular}{|c|c|c|}
\hline Vertebrae & \multicolumn{2}{|c|}{$\begin{array}{l}\text { Total: } 35-36-37 \\
\text { Precaudal: } 12-12-12^{a} \\
\text { Caudal: } 24-24-24^{\mathrm{a}}\end{array}$} \\
\hline $\begin{array}{l}\text { Branchiostegal rays } \\
\text { Caudal fin }\end{array}$ & $6-6-6$ & \\
\hline Pelvic fin & $\begin{array}{l}\text { Thoracic } \\
\text { S: } 1-1-1\end{array}$ & R: $3-4-4$ \\
\hline Dorsal fin & S: $10-11-13$ & $\mathrm{R}: 18-19-20$ \\
\hline Pectoral fin & R: $15-16-17$ & \\
\hline Anal fin & $R: 13-15-16$ & \\
\hline Gill rakers & U: X-X-X & L: X-X-X \\
\hline
\end{tabular}

\section{LIFE HISTORY}

$\begin{array}{ll}\text { Range } & \text { Cent. California, } 34-38^{\circ} \mathrm{N} \text {, to } \\ \text { Bering Sea, } 54-66^{\circ} \mathrm{N} \\ \text { Ecology } & \text { Epi- and mesobenthal, intertidal } \\ & \text { to } 275 \mathrm{~m} \\ \text { ELH pattern } & \text { Oviparous; demersal, adhesive, } \\ & \text { guarded eggs; pelagic larvae } \\ \text { Spawning } & \begin{array}{l}\text { Season: Oct-Jan } \\ \text { Area: Shallow water on rocks or } \\ \text { pilings in areas with high } \\ \text { current velocities }\end{array} \\ & \begin{array}{l}\text { Mode: Guarded by female, male, } \\ \text { or both }\end{array} \\ & \begin{array}{l}\text { Migration: } \\ \text { Range/function: } 59,000-126,000^{\mathrm{b}}\end{array} \\ \text { Fecundity } & 4 \mathrm{yr}^{\mathrm{b}} \\ \text { Age at first maturity } \\ \text { Longevity }\end{array}$

${ }^{a}$ B.B. Washington, NMFS Systematics Lab., Natl. Mus. Nat. Hist., Wash., D.C. 20560, unpubl.

${ }^{\mathrm{b}}$ Garrison and Miller 1982

Ref: Richardson and Washington 1980, Washington et al. $1984 \mathrm{~b}$.

\section{EARLY LIFE HISTORY DESCRIPTION}

\section{EGGS}

Diameter

No. of oil globules

$1.5-1.6 \mathrm{~mm}$

Oil globule diameter

One

Yolk

Envelope

Hatch size

0.31-0.56 mm

Incubation time/temp.

Pigment

Diagnostic characters

\section{LARVAE}

Preanal length

Length at flexion

$34 \%$ SL increasing with development to $>50 \%$ SL

Length at transformation $>19-23 \mathrm{~mm}$ SL

Sequence of fin development

Pigment

- Dorsal pigment on head and gut

- Break in dorsal midline pigment above gut (preflexion)

- Short posteriorly placed ventral midline series

- Lateral pigment on postanal body

Diagnostic characters (see Tables 3 and 37-38)

- Pigment

- Lack of pigment around urostyle

-Presence of dorso- and ventrolateral pigment 
A

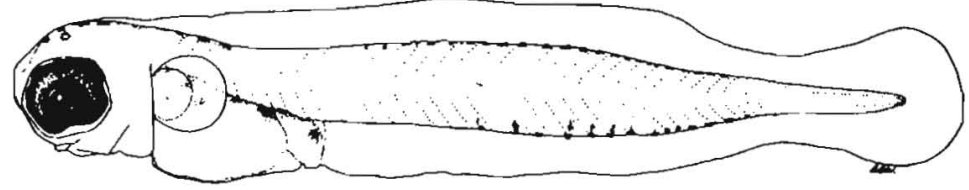

$5.8 \mathrm{~mm} \mathrm{NL}$

B
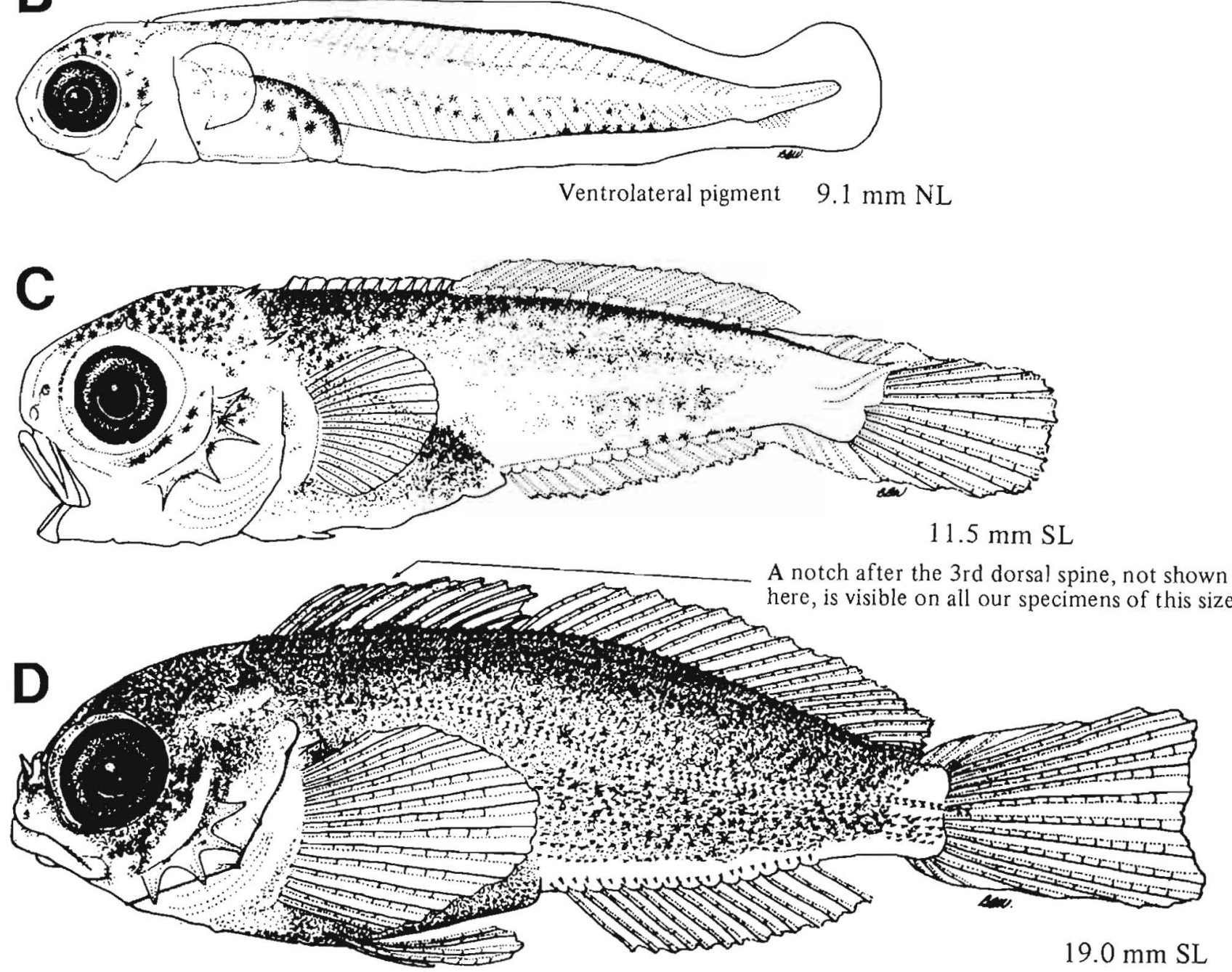

Figures $A-D$, Richardson and Washington 1980 . 


\section{MERISTICS}

\begin{tabular}{|c|c|c|}
\hline \multirow[t]{3}{*}{ Vertebrae } & \multicolumn{2}{|c|}{ Total: $37-38-39$} \\
\hline & \multicolumn{2}{|c|}{ Precaudal: $\mathrm{X}-\mathrm{X}-\mathrm{X}$} \\
\hline & \multicolumn{2}{|c|}{ Caudal: X-X-X } \\
\hline Branchiostegal rays & \multicolumn{2}{|l|}{$6-6-6$} \\
\hline \multicolumn{3}{|l|}{ Caudal fin } \\
\hline \multirow[t]{2}{*}{ Pelvic fin } & Thoracic & \\
\hline & $S: 1-1-1$ & R: $3-4-4$ \\
\hline Dorsal fin & S: $10-11-12$ & R: $17-21-22$ \\
\hline Pectoral fin & R: $17-18-19$ & \\
\hline Anal fin & R: $16-17-18$ & \\
\hline Gill rakers & $\mathrm{U}: \mathrm{X}-\mathrm{X}-\mathrm{X}$ & $\mathrm{L}: \mathrm{X}-\mathrm{X}-\mathrm{X}$ \\
\hline
\end{tabular}

\section{LIFE HISTORY}

\begin{tabular}{|c|c|}
\hline Range & $\begin{array}{l}\text { SE Alaska, } 55-59^{\circ} \mathrm{N} \text {, to } \\
\text { Bering Sea, } 54-66^{\circ} \mathrm{N}\end{array}$ \\
\hline Ecology & Epi- and mesobenthal, $25-525 \mathrm{~m}$ \\
\hline ELH pattern & $\begin{array}{l}\text { Oviparous, eggs probably } \\
\text { demersal, pelagic larvae }\end{array}$ \\
\hline Spawning & $\begin{array}{l}\text { Season: } \\
\text { Area: } \\
\text { Mode: } \\
\text { Migration: }\end{array}$ \\
\hline $\begin{array}{l}\text { Fecundity } \\
\text { Age at first maturity } \\
\text { Longevity }\end{array}$ & Range/function: \\
\hline
\end{tabular}

${ }^{\text {a }}$ Preflexion larvae of $H$. hemilepidotus and $H$. jordani cannot presently be separated in samples from areas where they co-occur.

Ref: Matarese and Vinter 1985.

\section{EARLY LIFE HISTORY DESCRIPTION}

\section{EGGS}

Diameter

No. of oil globules

Oil globule diameter

Yolk

Envelope

Hatch size

Incubation time/temp.

Pigment

Diagnostic characters

\section{LARVAE}

Preanal length

Length at flexion

Length at transformation

Sequence of fin development

\section{Pigment}

- Similar to $H$. hemilepidotus except generally less pigmented (see p. 374)

- Ventrolateral pigment internal only

Diagnostic characters (see Tables 3 and 37-38)

- Lack of lateral and caudal pigment

Distinguished from $H$. hemilepidotus postflexion larvae by

- Less dorso- and ventrolateral pigment (see figure, p. 371)

- Delayed development of postocular and parietal spines 
Postocular and parietal spines
not developed by this size
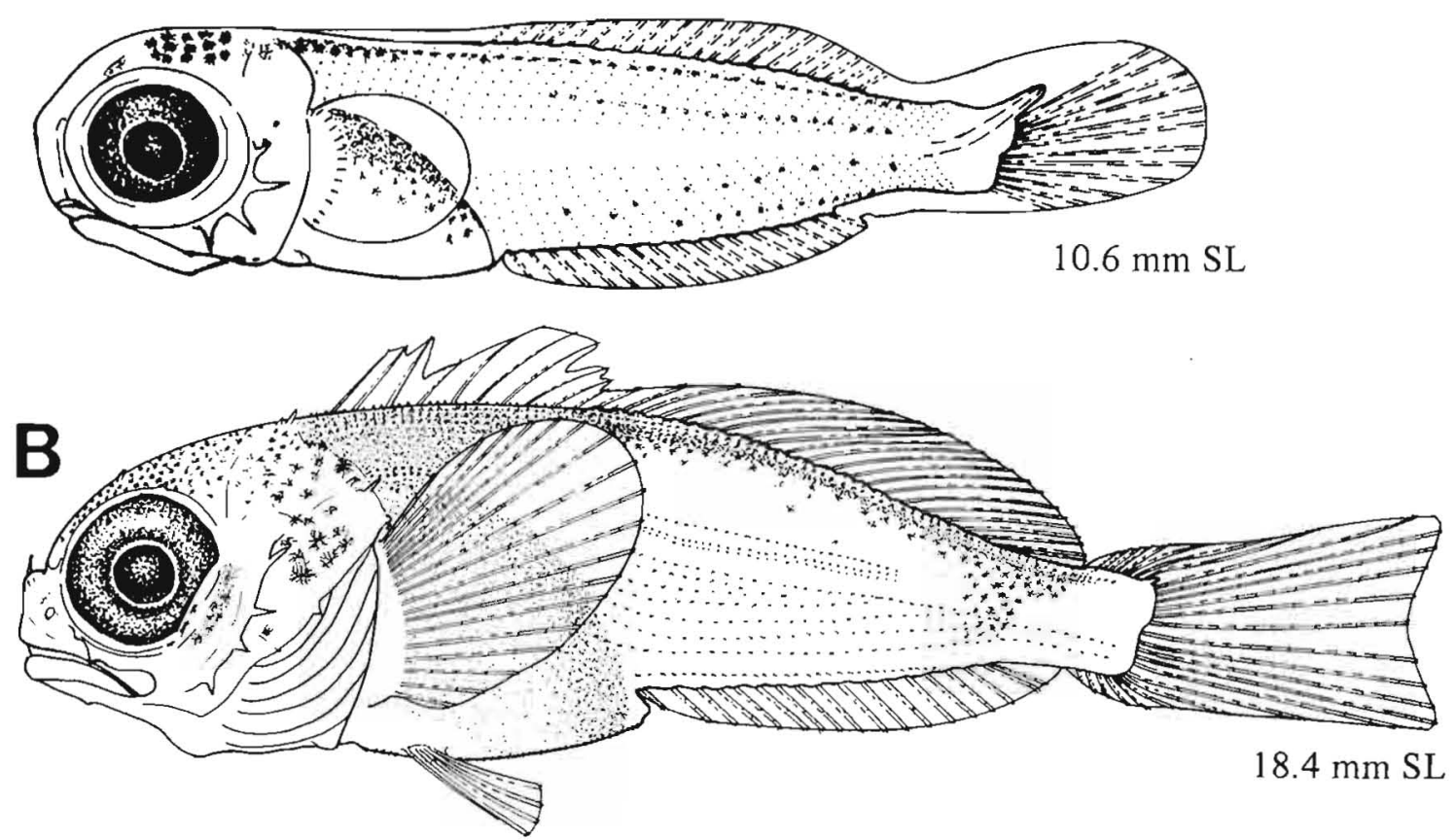

Figures A-B, NWAFC originals (B. Vinter). 


\section{MERISTICS}

\begin{tabular}{|c|c|c|}
\hline \multirow[t]{3}{*}{ Vertebrae } & \multicolumn{2}{|c|}{ Total: $35-36-37$} \\
\hline & \multicolumn{2}{|c|}{ Precaudal: $12-12-12$} \\
\hline & \multicolumn{2}{|c|}{ Caudal: $23-24-25$} \\
\hline Branchiostegal rays & \multicolumn{2}{|l|}{$6-6-6$} \\
\hline \multicolumn{3}{|l|}{ Caudal fin } \\
\hline Pelvic fin & Thoracic & \\
\hline & S: $1-1-1$ & R: $4-4-4$ \\
\hline Dorsal fin & S: $10-11-11$ & R: $18-20-20$ \\
\hline Pectoral fin & R: $14-15-16$ & \\
\hline Anal fin & R: $14-15-16$ & \\
\hline Gill rakers & U: $2-2-2$ & $L: 5-X-8$ \\
\hline
\end{tabular}

\section{LIFE HISTORY}

\begin{tabular}{|c|c|}
\hline Range & $\begin{array}{l}\text { S. California, } 32-34^{\circ} \mathrm{N} \text {, to } \\
\text { SE Alaska, } 55-59^{\circ} \mathrm{N}\end{array}$ \\
\hline Ecology & $\begin{array}{l}\text { Nearshore shelf demersal, } \\
\text { intertidal to } 97 \mathrm{~m}\end{array}$ \\
\hline ELH pattern & $\begin{array}{l}\text { Oviparous, eggs probably } \\
\text { demersal, pelagic larvae }\end{array}$ \\
\hline Spawning & $\begin{array}{l}\text { Season: } \\
\text { Area: } \\
\text { Mode: } \\
\text { Migration: }\end{array}$ \\
\hline $\begin{array}{l}\text { Fecundity } \\
\text { Age at first maturity } \\
\text { Longevity }\end{array}$ & Range/function: \\
\hline
\end{tabular}

\section{EARLY LIFE HISTORY DESCRIPTION}

\section{EGGS}

Diameter

No. of oil globules

Oil globule diameter

Yolk

Envelope

Hatch size $\sim 5 \mathrm{~mm} \mathrm{SL}$

Incubation time/temp.

Pigment

Diagnostic characters

LARVAE

Preanal length

Length at flexion

$38 \%$ SL increasing with development to $>50 \% \mathrm{SL}$

Length at transformation $19 \mathrm{~mm} \mathrm{SL}$

Sequence of fin development

Pigment

- Dorso- and ventrolateral at $6 \mathrm{~mm} \mathrm{SL}$ becoming heavier with development

- Preflexion larvae (>5 mm SL) have continuous line of dorsal midline pigment extending from head to posteriormost myomere

Diagnostic characters (see Tables 3 and 37-38)

- Pigment: Lateral, ventral midline

- Preflexion: Continuous pigment along dorsal and ventral body midline

- Flexion and larger: Heavy concentration of lateral pigment

Ref: Richardson and Washington 1980, Washington et al. 1984b. 

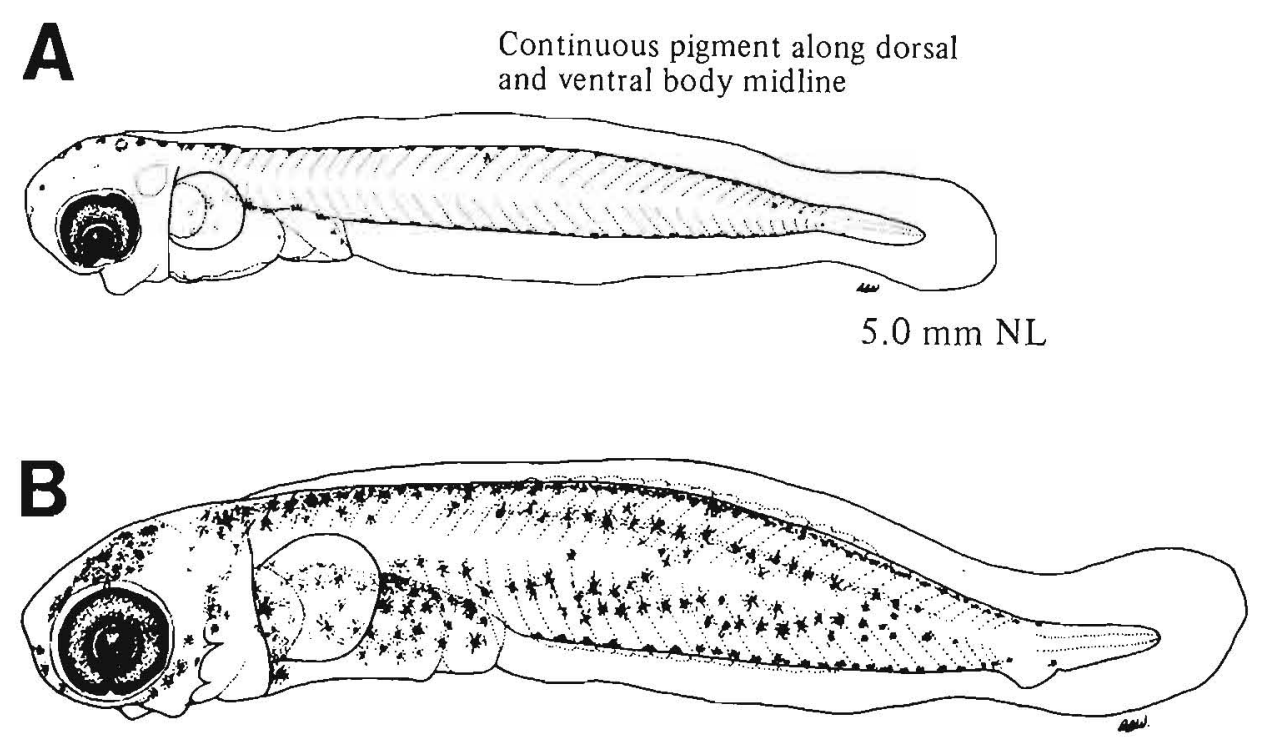

$8.9 \mathrm{~mm} \mathrm{NL}$

Heavy dorso- and ventrolateral pigment
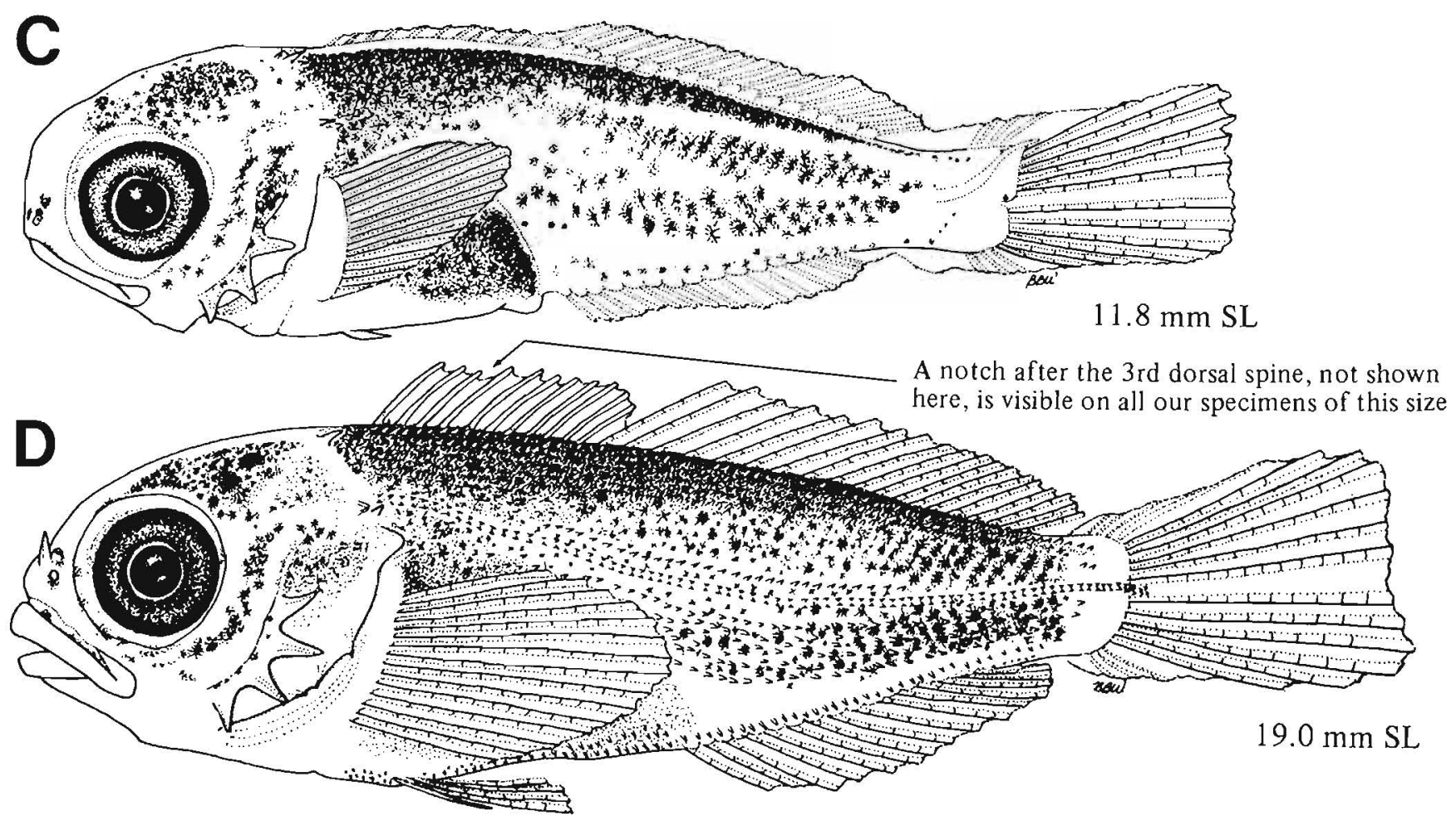

Figures A-D, Richardson and Washington 1980. 


\section{MERISTICS}

\begin{tabular}{|c|c|}
\hline Vertebrae & $\begin{array}{l}\text { Total: } 37-38-38 \\
\text { Precaudal: } \mathrm{X}-\mathrm{X}-\mathrm{X} \\
\text { Caudal: } \mathrm{X}-\mathrm{X}-\mathrm{X}\end{array}$ \\
\hline Branchiostegal rays & $6-6-6$ \\
\hline Caudal fin & $X, 6+6, x$ \\
\hline Pelvic fin & $\begin{array}{l}\text { Thoracic } \\
\text { S: } 1-1-1 \quad \text { R: } 4-4-4\end{array}$ \\
\hline Dorsal fin & S: $11-11-12$ R: $18-20-22$ \\
\hline Pectoral fin & $\mathrm{R}: 15-15-17$ \\
\hline Anal fin & $\mathrm{R}: 16-17-17$ \\
\hline Gill rakers & $\mathrm{U}: \mathrm{X}-\mathrm{X}-\mathrm{X} \quad \mathrm{L}: \mathrm{X}-\mathrm{X}-\mathrm{X}$ \\
\hline \multicolumn{2}{|l|}{ LIFE HISTORY } \\
\hline Range & $\begin{array}{l}\text { Aleutian Is., } 51-55^{\circ} \mathrm{N} \text {, to } \\
\text { Chukchi Sea, north of } 66^{\circ} \mathrm{N}\end{array}$ \\
\hline Ecology & Nearshore shelf demersal \\
\hline ELH pattern & $\begin{array}{l}\text { Oviparous, eggs probably } \\
\text { demersal, pelagic larvae }\end{array}$ \\
\hline Spawning & $\begin{array}{l}\text { Season: } \\
\text { Area: } \\
\text { Mode: } \\
\text { Migration: }\end{array}$ \\
\hline $\begin{array}{l}\text { Fecundity } \\
\text { Age at first maturity } \\
\text { Longevity }\end{array}$ & Range/function: \\
\hline
\end{tabular}

\section{EARLY LIFE HISTORY DESCRIPTION}

\section{EGGS}

Diameter

No. of oil globules

Oil globule diameter

Yolk

Envelope

Hatch size

$\sim 4.3 \mathrm{~mm} \mathrm{SL}$

Incubation time/temp.

Pigment

Diagnostic characters

\section{LARVAE}

Preanal length $<50 \%$ SL

Length at flexion Complete at $13 \mathrm{~mm} \mathrm{SL}$

Length at transformation $>22.8 \mathrm{~mm} \mathrm{SL}$

Sequence of fin

development

Pigment

- Similar to $H$. hemilepidotus except generally less pigmented (see p. 374)

- Preflexion larvae have pigment above and below urostyle

Diagnostic characters (see Tables 3 and 37-38)

- Pigment above and below urostyle

See also pelagic juvenile figure of $H$. hemilepidotus and $H$. jordani (p. 371) 


\section{A}

Urostyle pigment
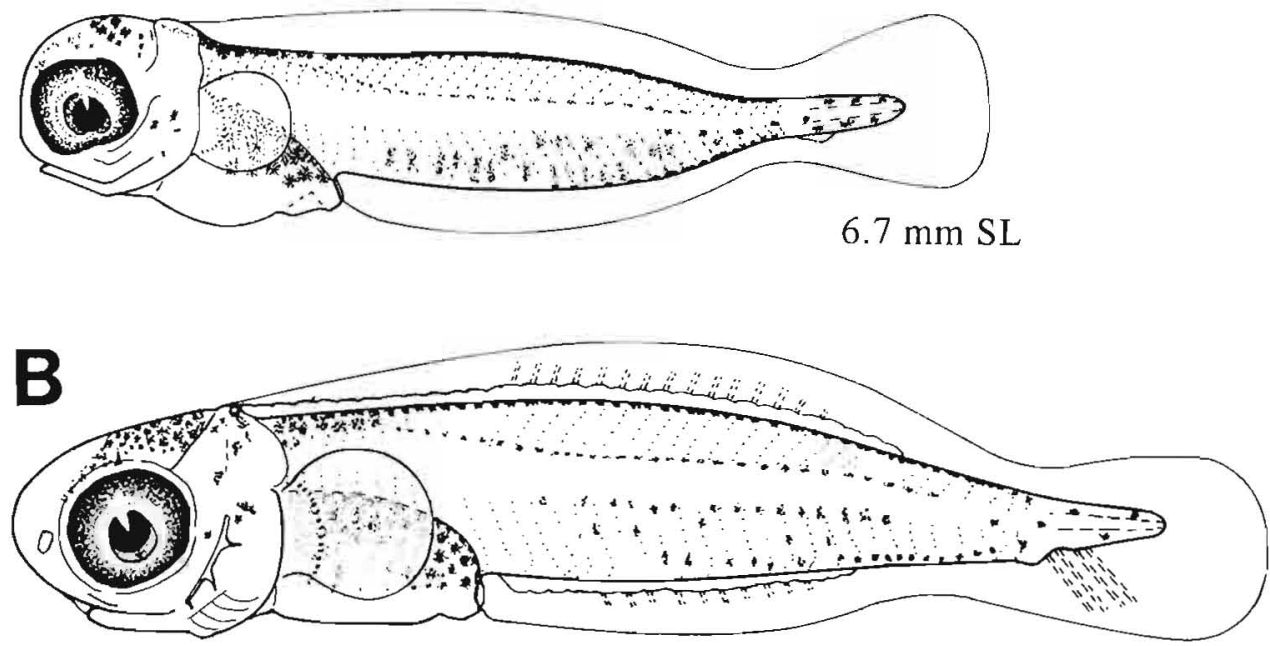

$11.0 \mathrm{~mm} \mathrm{SL}$
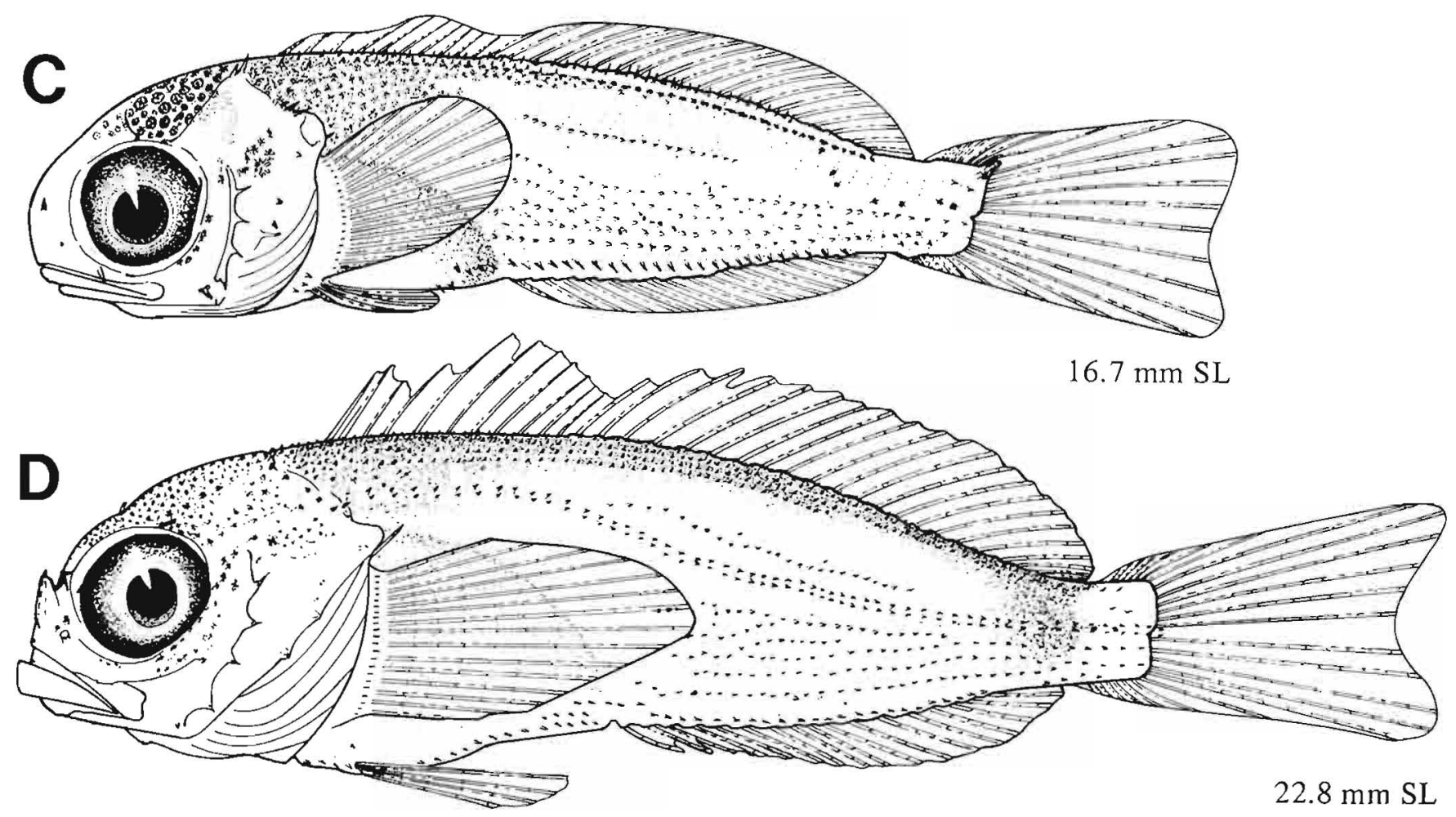

Figures A-D, Matarese and Vinter 1985 . 


\section{MERISTICS}

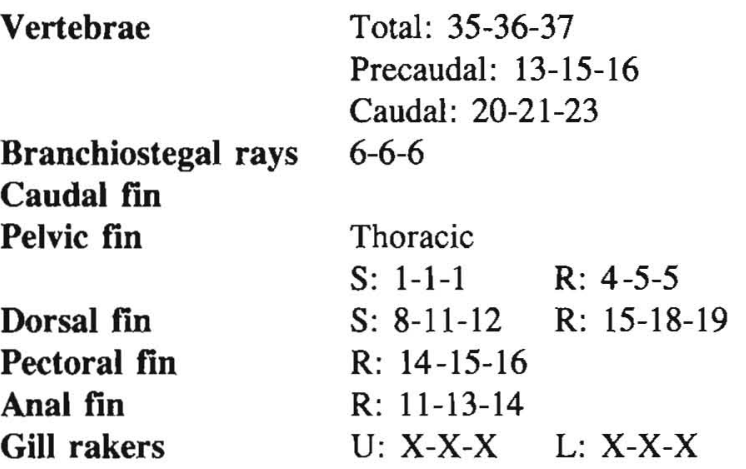

\section{LIFE HISTORY}

\begin{tabular}{|c|c|}
\hline Range & $\begin{array}{l}\text { South of southern California to } \\
\text { SE Alaska, } 55-59^{\circ} \mathrm{N}\end{array}$ \\
\hline Ecology & $\begin{array}{l}\text { Nearshore shelf demersal, } \\
\text { intertidal to } 76 \mathrm{~m}\end{array}$ \\
\hline ELH pattern & $\begin{array}{l}\text { Oviparous; demersal, adhesive, } \\
\text { attached eggs; pelagic larvae }\end{array}$ \\
\hline \multirow[t]{4}{*}{ Spawning } & $\begin{array}{c}\text { Season: Nov-Mar (California); }{ }^{a}{ }^{\text {Jan-May (British Columbia) }}{ }^{b}\end{array}$ \\
\hline & $\begin{array}{l}\text { Area: In rocky crevices }{ }^{c} \text { or } \\
\text { on algae }^{\mathrm{d}}\end{array}$ \\
\hline & Mode: \\
\hline & Migration: \\
\hline Fecundity & $\begin{array}{l}\text { Range/function: } 49,000-98,000 \\
\text { (may spawn twice in one } \\
\text { season) }^{\mathrm{a}}\end{array}$ \\
\hline \multirow{2}{*}{ Age at first maturity } & $3-4$ yr (females) ${ }^{a}$ \\
\hline & $2-3$ yr (males) ${ }^{a}$ \\
\hline Longevity & $13 y^{a}$ \\
\hline
\end{tabular}

a O'Connell 1953

${ }^{b}$ Pillsbury 1957

'Feder et al. 1974

${ }^{d}$ Burge and Schultz 1973

'Wang 1981

'Richardson and Washington 1980

Ref: O'Connell 1953, Richardson and Washington 1980, Wang 1981, Washington et al. 1984b.

\section{EARLY LIFE HISTORY DESCRIPTION}

\section{EGGS}

Diameter
No. of oil globules
Oil globule diameter
Yolk
Envelope
Hatch size
Incubation time/temp.
Pigment

Diameter

Pigment

Diagnostic characters

\author{
$1.4-1.9 \mathrm{~mm}$ \\ One large with 1-4 smaller \\ ones
}

$0.2-0.3 \mathrm{~mm}^{\mathrm{e}}$

Homogeneous

Thick, translucent

4-6 mm SL; ${ }^{2}$

3.1-4.8 mm SLe (yolk absorbed by $6.5 \mathrm{~mm} \mathrm{SL}$ )

\section{LARVAE}

Preanal length

Length at flexion

Length at transformation

Sequence of fin development

Pigment

- Dense pigment covering body except for caudal peduncle

Diagnostic characters (see Table 3)

- Develops bony bumps on areas where head spines occur in Hemilepidotus spp.

- Becomes increasingly deep-bodied with development

- Uniformity of pigment
$45-50 \% \mathrm{SL}$ $\sim 7.5-8.7 \mathrm{~mm} \mathrm{SL}$

$14 \mathrm{~mm} \mathrm{SL}$, but remains pelagic until $35 \mathrm{~mm} \mathrm{SL}$ 
A

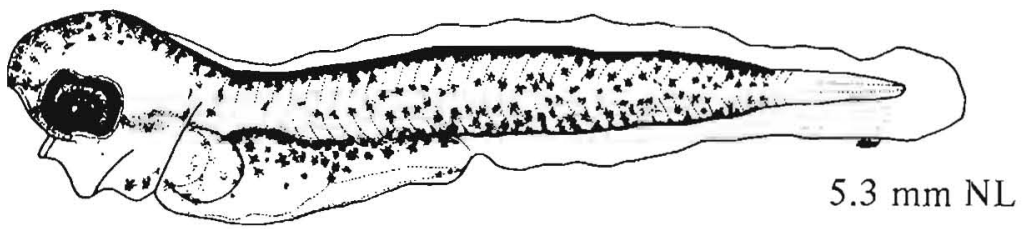

B

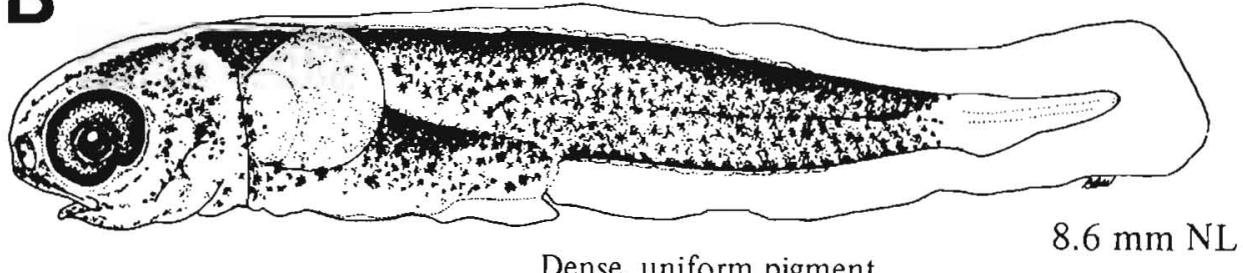

Dense, uniform pigment except at caudal peduncle

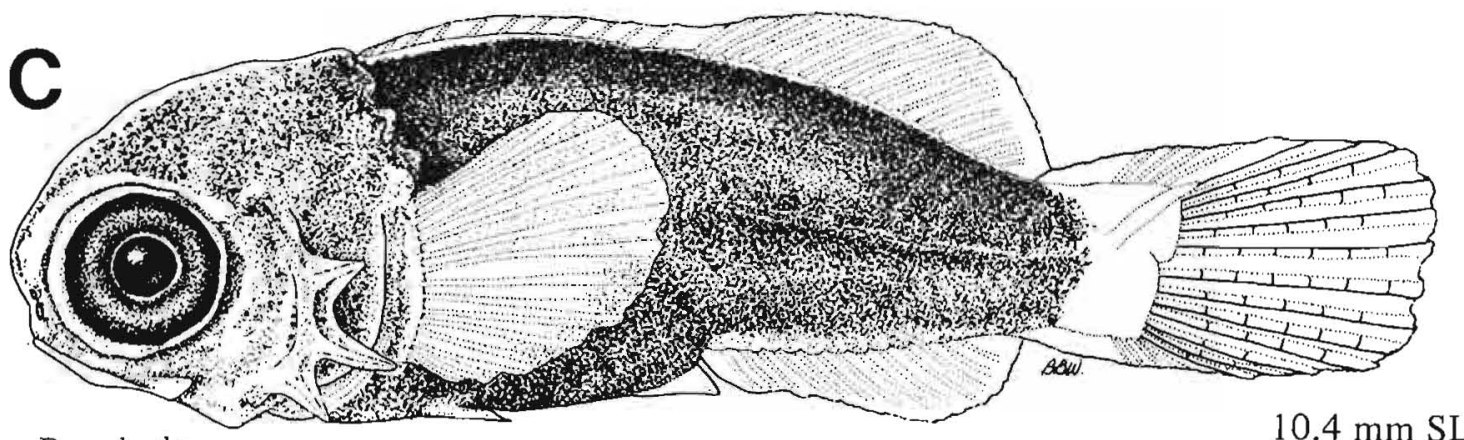

Deep body

$10.4 \mathrm{~mm} \mathrm{SL}$

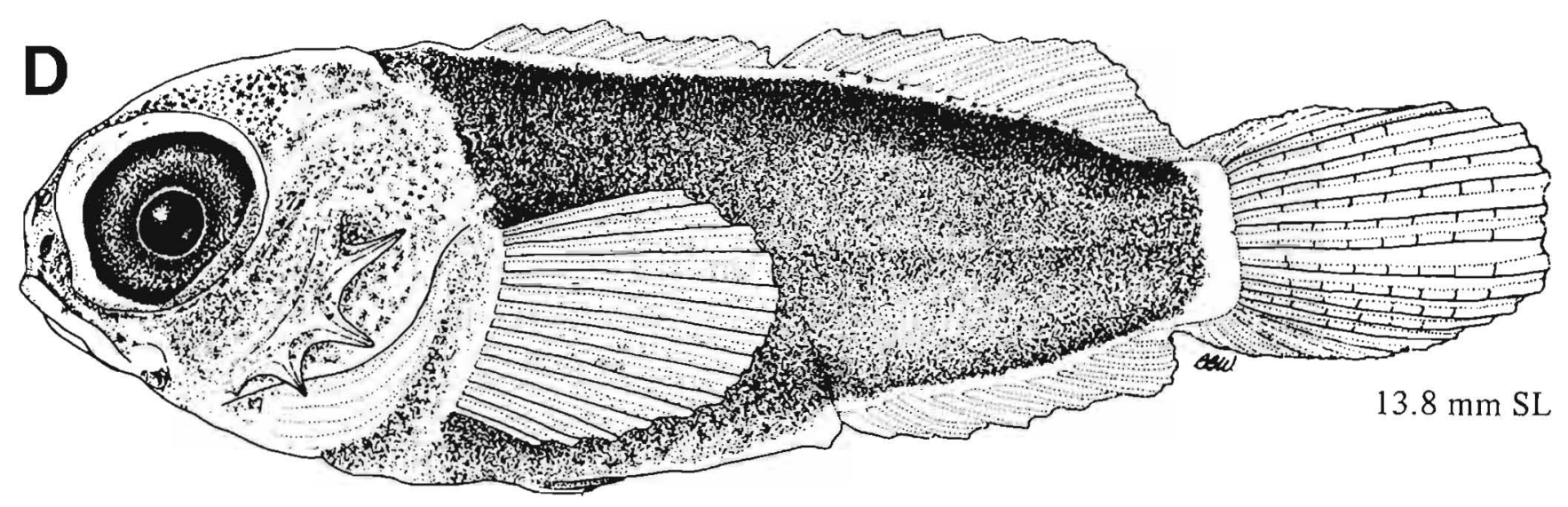

Figures A-D, Richardson and Washington 1980 . 


\section{MERISTICS}

\begin{tabular}{|c|c|c|}
\hline Vertebrae & $\begin{array}{l}\text { Total: } 33-33- \\
\text { Precaudal: } 11 \\
\text { Caudal: } 24-2\end{array}$ & $\begin{array}{l}35^{\mathrm{a}} \\
-11-11^{\mathrm{b}} \\
4-24^{\mathrm{b}}\end{array}$ \\
\hline $\begin{array}{l}\text { Branchiostegal rays } \\
\text { Caudal fin }\end{array}$ & $6-6-6$ & \\
\hline Pelvic fin & $\begin{array}{l}\text { Thoracic } \\
\text { S: } 1-1-1\end{array}$ & $\mathrm{R}: 2-3-3^{\mathrm{c}}$ \\
\hline Dorsal fin & S: $9-10-10$ & R: $14-16-17$ \\
\hline Pectoral fin & $\mathrm{R}: 14-15-16$ & \\
\hline Anal fin & $\mathrm{R}: 10-12-12$ & \\
\hline Gill rakers & U: X-X-X & L: X-X-X \\
\hline
\end{tabular}

\begin{tabular}{|c|c|}
\hline Range & $\begin{array}{l}\text { N. California, } 38-42^{\circ} \mathrm{N} \text {, to } \\
\text { SE Alaska, } 55-59^{\circ} \mathrm{N}\end{array}$ \\
\hline Ecology & $\begin{array}{l}\text { Nearshore shelf demersal, } \\
\text { intertidal to } 82 \mathrm{~m}\end{array}$ \\
\hline ELH pattern & $\begin{array}{l}\text { Parity and eggs unknown, } \\
\text { pelagic larvae }\end{array}$ \\
\hline \multirow[t]{4}{*}{ Spawning } & Season: \\
\hline & Area: \\
\hline & Mode: \\
\hline & Migration: \\
\hline Fecundity & Range/function: \\
\hline
\end{tabular}

Age at first maturity

Longevity

\section{EARLY LIFE HISTORY DESCRIPTION}

\section{EGGS}

Diameter

No. of oil globules

Oil globule diameter

Yolk

Envelope

Hatch size

$\sim 3 \mathrm{~mm} \mathrm{SL}$

Incubation time/temp.

Pigment

Diagnostic characters

\section{LARVAE}

Preanal length $<50 \%$ SL

Length at flexion $\quad 6.2-9.4 \mathrm{~mm} \mathrm{SL}$

Length at transformation $13-19 \mathrm{~mm} \mathrm{SL}$

Sequence of fin

development

Caudal, 2nd dorsal (rays) and anal, 1st dorsal (spines) and pectorals, pelvics (pectorals and pelvics formed by 11.5 $\mathrm{mm} \mathrm{SL)}$

Pigment

- Low number $(<15)$ ventral midline melanophores

- Dorsal and anal finfolds

- Base of cleithrum

Diagnostic characters

- Short gut (33\% SL)

- Pointed snout

- Pigment (see above)

- Four preopercular spines, other spines (as for Myoxocephalus group)

- Pelvic fin ray count I,2; visible in late-stage larvae and juveniles

Total myomere count from Figures A and $\mathbf{B}=36$.

${ }^{b}$ B.B. Washington, NMFS Systematics Lab., Natl. Mus. Nat. Hist., Wash., D.C. 20560, unpubl.

' $\mathrm{I}, 2$ is the most common count. The first ray is greatly thickened and broadly branched distally, probably leading to the erroneous counts of 1,3 in the literature (B.B. Washington, pers. commun., 8 Dec. 1986).

Ref: Richardson and Washington 1980 (as Icelinus spp.), Washington 1986, Washington et al. $1984 \mathrm{~b}$. 
B

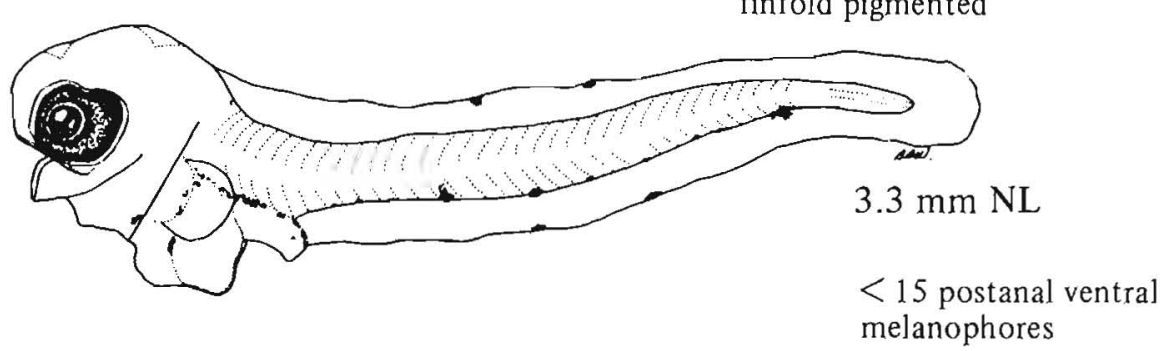

C

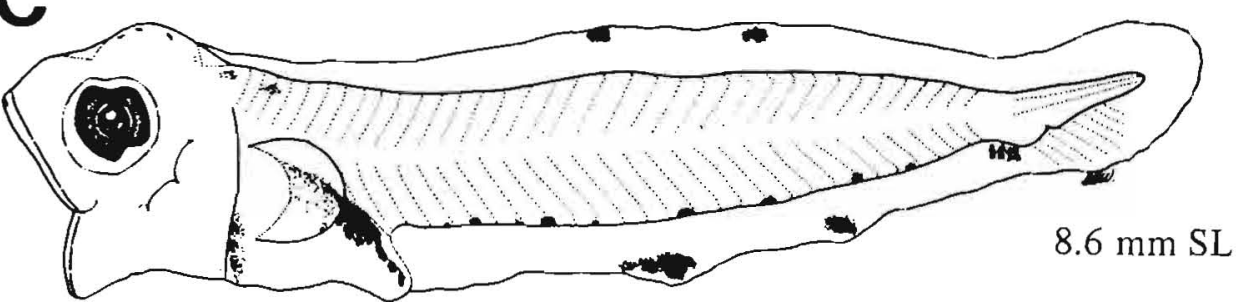

D
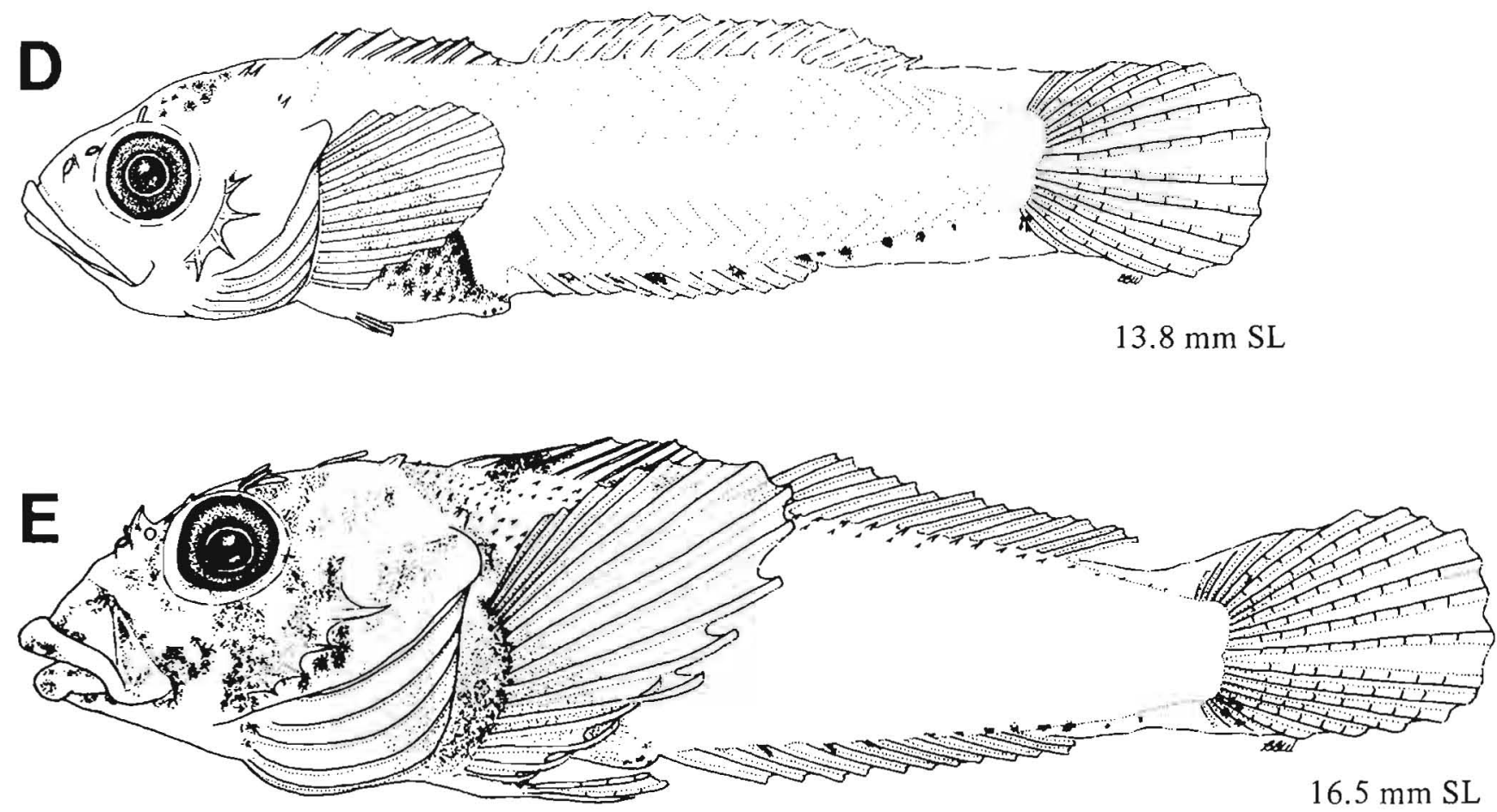

Figure A, Washington 1986 ; B-E, Richardson and Washington 1980 . Total myomere counts for Figures B and C are 36 . 
COTTIDAE

\section{MERISTICS}

Vertebrae

Total: $33-36-36$

Precaudal: $10-11-12$

Caudal: $24-25-25$

Branchiostegal rays

6-6-7

Caudal fin

Pelvic fin

$10-13,6+7,8-11$

Absent

Dorsal fin

S: $7-9-10$

R: $17-18-19$

Pectoral fin

R: $16-17-18$

Anal fin

R: $13-15-16$

Gill rakers

L: $3-\mathrm{X}-5$

\section{EARLY LIFE HISTORY DESCRIPTION}

\section{EGGS}

Diameter

1.7-2.0 mm

No. of oil globules

Oil globule diameter

Yolk

Envelope

Hatch size

None

Homogeneous, transparent blue developing to purple

Smooth

$6 \mathrm{~mm} \mathrm{SL}$

Incubation time/temp. $\quad 24 \mathrm{~d} / 10^{\circ} \mathrm{C}$

Pigment

Diagnostic characters

\section{LARVAE}

Preanal length

$<50 \%$ SL

Length at flexion

$8.8-11.0 \mathrm{~mm} \mathrm{SL}$

Spawning

eggs; pelagic larvae

Season: Mar (British Columbia) ${ }^{\text {a }}$

Area: Cobble beach, under boulders ${ }^{a}$

Mode:

Migration:

Fecundity Range/function:

Age at first maturity

Longevity

\section{Length at transformation $12-13 \mathrm{~mm} \mathrm{SL}$}

Sequence of fin development

Pigment

- Heavy pigment on head and gut

- 20-30 ventral midline melanophores prior to flexion, 15-20 in postflexion larvae

Diagnostic characters

- Moderately slender form

- Pointed snout

- Four preopercular spines

- Ventral midline melanophores (the number at various developmental stages)

${ }^{a}$ Matarese and Marliave 1982

Ref: Matarese and Marliave 1982. 
A
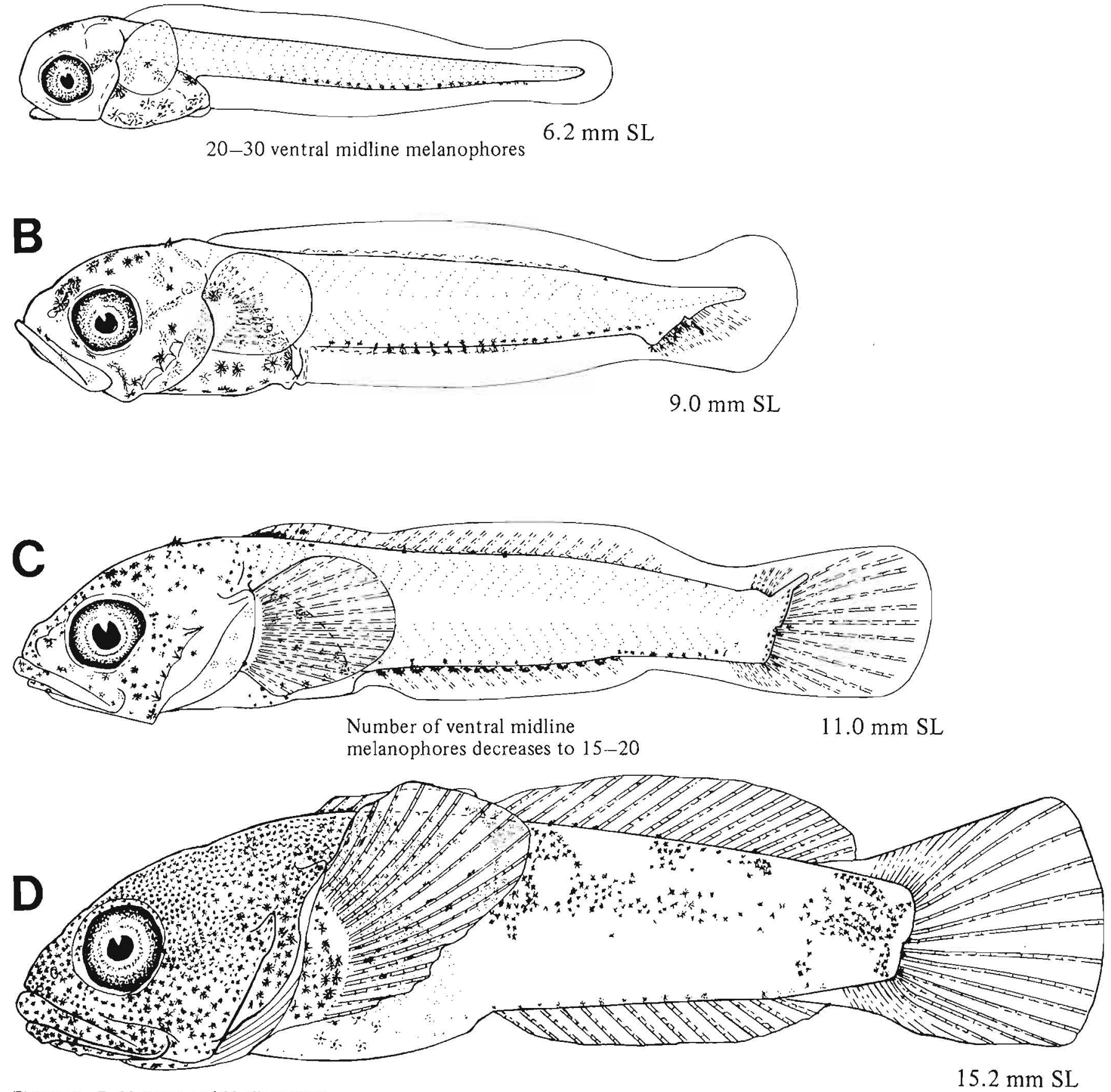

Figures A-D, Matarese and Marliave 1982. 
MERISTICS

\begin{tabular}{|c|c|c|}
\hline \multirow{3}{*}{ Vertebrae } & \multicolumn{2}{|c|}{ Total: $35-35-36$} \\
\hline & \multicolumn{2}{|c|}{ Precaudal: $10-11-11$} \\
\hline & \multicolumn{2}{|c|}{ Caudal: $24-25-25$} \\
\hline Branchiostegal rays & \multicolumn{2}{|c|}{$6-6-6$} \\
\hline Caudal fin & \multicolumn{2}{|l|}{$x, 6+6, x$} \\
\hline Pelvic fin & \multicolumn{2}{|l|}{ Thoracic } \\
\hline & $S: 1-1-1$ & $\mathrm{R}: 2-3-3$ \\
\hline Dorsal fin & S: $8-10-11$ & $\mathrm{R}: 14-16-17$ \\
\hline Pectoral fin & R: $16-17-18$ & \\
\hline Anal fin & R: $14-16-17$ & \\
\hline Gill rakers & $\mathrm{U}: \mathrm{X}-\mathrm{X}-\mathrm{X}$ & L: X-X-X \\
\hline
\end{tabular}

LIFE HISTORY

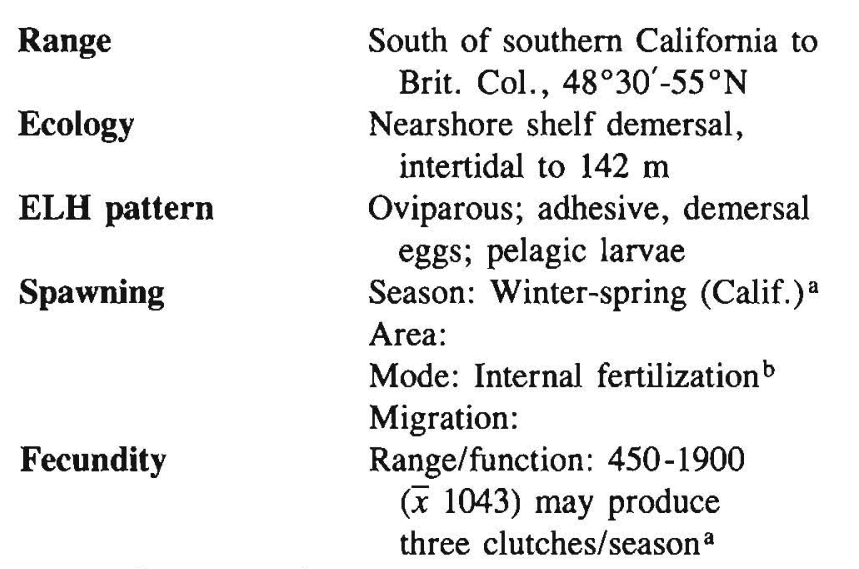

Age at first maturity Longevity

\section{EARLY LIFE HISTORY DESCRIPTION}

\section{EGGS}

Diameter

No. of oil globules

Oil globule diameter

Yolk

Envelope

Hatch size

1.02-1.05 mm

One large, 5-8 small

$0.3 \mathrm{~mm}$ (large)

Salmon-colored

Incubation time/temp.

Pigment

Diagnostic characters

\section{LARVAE}

Preanal length $\quad<50 \%$ SL

Length at flexion

Length at transformation Begins $\sim 16.6 \mathrm{~mm} \mathrm{SL}$

Sequence of fin development

Caudal first, pelvics last

Pigment

- Dorsolateral gut pigment generally light according to Richardson and Washington (1980), ${ }^{\mathrm{c}}$ but specimens we collect have heavier pigment (compare Figures $A$ and $B$ with $C$ and D)

- Our specimens have heavy crown pigment

\section{Diagnostic characters}

- Number of ventral midline melanophores $>40$ in preflexion larvae $(<6 \mathrm{~mm} \mathrm{SL})$ and $>20$ in others

- Upper two preopercular spines larger than lower two

\footnotetext{
a Goldberg 1980b

${ }^{\text {b }}$ Misitano 1980

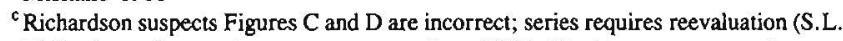
Richardson, deceased, pers. commun., Oct. 1984). Washington suspects they are Icelinus, probably $I$. quadriseriatus, which occurs outside our study area (B.B. Washington, NMFS Systematics Lab., Natl. Mus. Nat. Hist., Wash., D.C. 20560 , pers. commun, 8 Dec. 1986).
}

Ref: Misitano 1980, Richardson and Washington 1980, Washington et al. 1984b. 
A
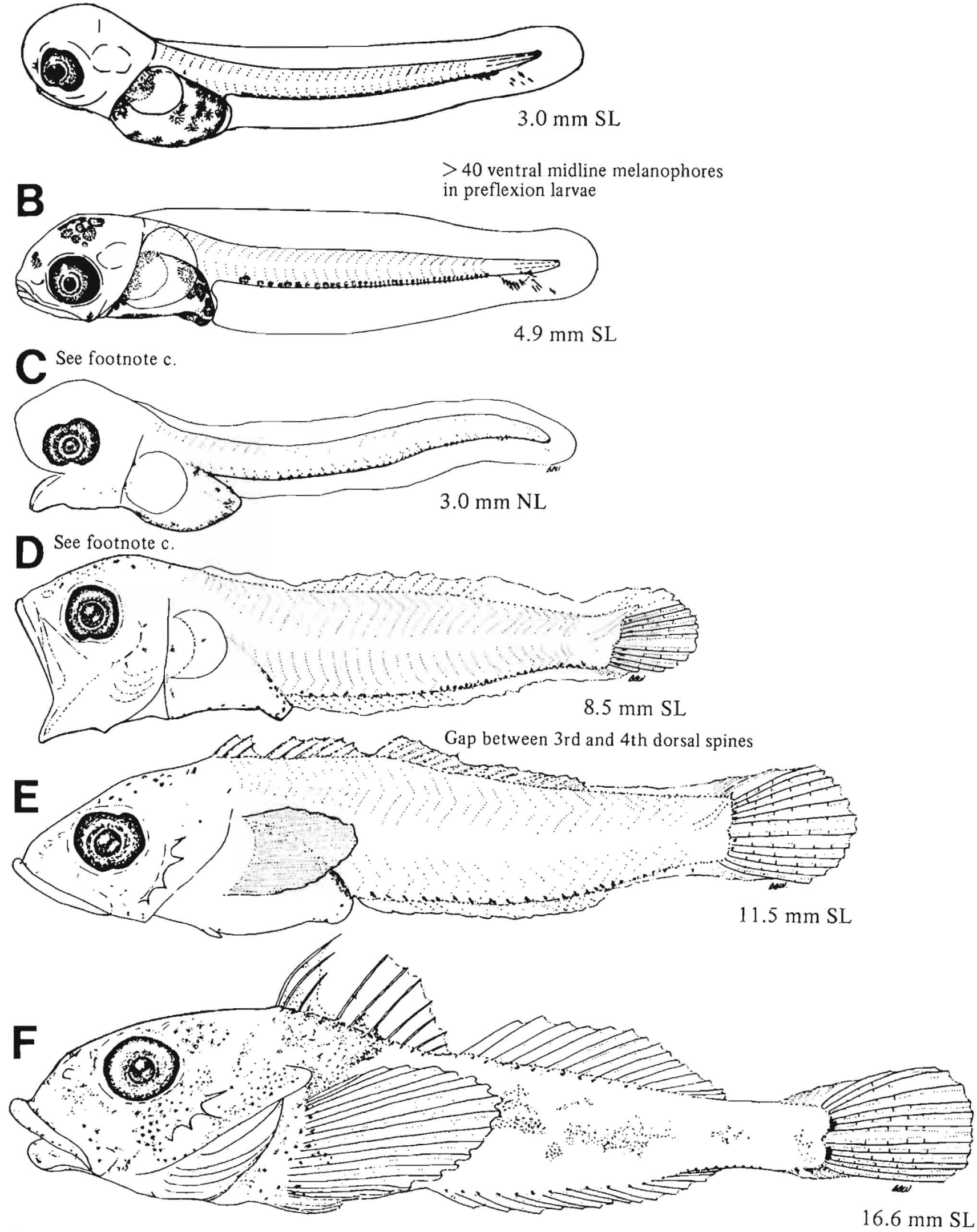

Figures A-B, NWAFC originals (B. Vinter); C-F, Richardson and Washington 1980. 


\section{MERISTICS}

$\begin{array}{lll}\text { Vertebrae } & \begin{array}{l}\text { Total: } 29-31-31 \\ \text { Precaudal: } 11-11-12\end{array} \\ & \begin{array}{l}\text { Caudal: } 18-20-20 \\ \text { Branchiostegal rays }\end{array} & 6-6-6 \\ \begin{array}{l}\text { Caudal fin } \\ \text { Pelvic fin }\end{array} & \text { Thoracic } & \\ & \text { S: } 1-1-1 & \text { R: } 3-3-3 \\ \text { Dorsal fin } & \text { S: } 7-8-9 \quad \text { R: } 9-12-13 \\ \begin{array}{l}\text { Pectoral fin } \\ \text { Anal fin }\end{array} & \text { R: } 15-16-17 & \\ \text { Gill rakers } & \text { R: } 8-9-10 & \\ & \text { U: } 0-X-1 & \text { L: } 4-X-6\end{array}$

LIFE HISTORY

$\begin{array}{ll}\text { Range } & \text { Cent. California, } 34-38^{\circ} \mathrm{N}, \text { to } \\ \text { Gcology } & \text { Gulf of Alaska, } 54-60^{\circ} \mathrm{N} \\ \text { ELH pattern } & \text { Nearshore shelf demersal, } 0-20 \mathrm{~m} \\ & \text { Oviparous; adhesive, demersal, } \\ \text { Spawning } & \text { Searded eggs; pelagic larvae } \\ & \begin{array}{l}\text { Season: Feb-May (Puget Sound) } \\ \text { Area: On rocks or pilings } \\ \text { nearshore }\end{array} \\ & \text { Mode: Internal fertilization } \\ & \text { likely, polygamous male } \\ & \text { guards nests } \\ & \text { Migration: } \\ \text { Range/function: } 18,800-31,900 \\ \text { (spawn twice each season) }\end{array}$

Age at first maturity Longevity

\section{EARLY LIFE HISTORY DESCRIPTION}

\section{EGGS}

Diameter

No. of oil globules

$1.7-2.0 \mathrm{~mm}$

Oil globule diameter

Yolk

Envelope

Hatch size

One

$0.36 \mathrm{~mm}$

Homogeneous, orange

Clear, orange-brown

Incubation time/temp.

Pigment

4.9-5.2 $\mathrm{mm} \mathrm{SL} \mathrm{(5} \mathrm{mm} \mathrm{SL)}$

Diagnostic characters

\section{LARVAE}

Preanal length

Length at flexion

Length at transformation

Sequence of fin development

Pigment

- Dorsal surface of gut

- Ventral midline melanophores $(\leqslant 15)$

- Nape

Diagnostic characters

- Only stout-bodied member of Myoxocephalus group

- Ventral midline: $\leqslant 15$ melanophores beginning several myomeres after anus

- Four preopercular spines

- Head spines prominent (e.g., parietal)

- Low vertebral count (29-31)

Eggs and larvae of $E$. diceraus and $E$. lucasi are unknown. The following information may aid in their identification.

Total vertebrae

Dorsal fin spines

E. diceraus

31-35

E. lucasi

Dorsal fin rays

Anal fin rays

Pectoral fin rays

Pelvic fin rays

Range
$7-8$

$13-15$

11-13

15-19

2-3

SE Alaska-

Arctic
32-34

7-8

12-14

9-11

15-18

2-3

Brit. Col.-

Bering Sea

\footnotetext{
${ }^{a}$ DeMartini 1978
}

${ }^{b}$ Andriashev 1954

${ }^{\mathrm{c}}$ Misitano 1978

Ref: Misitano 1978, Richardson and Washington 1980, Washington et al. 1984b. 


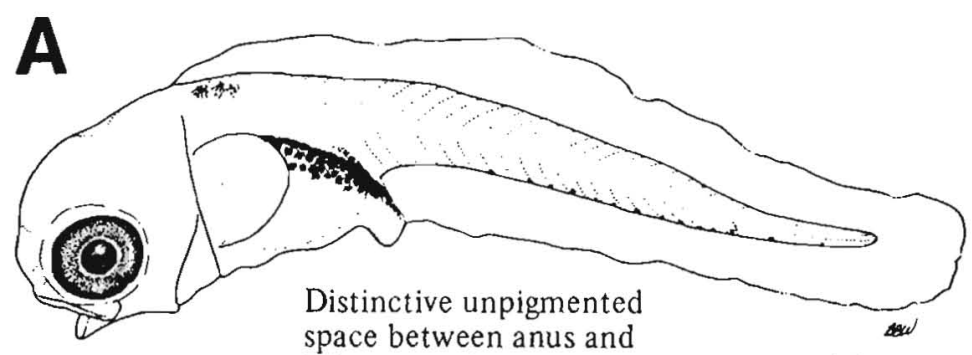
space between anus and

$4.8 \mathrm{~mm} \mathrm{NL}$
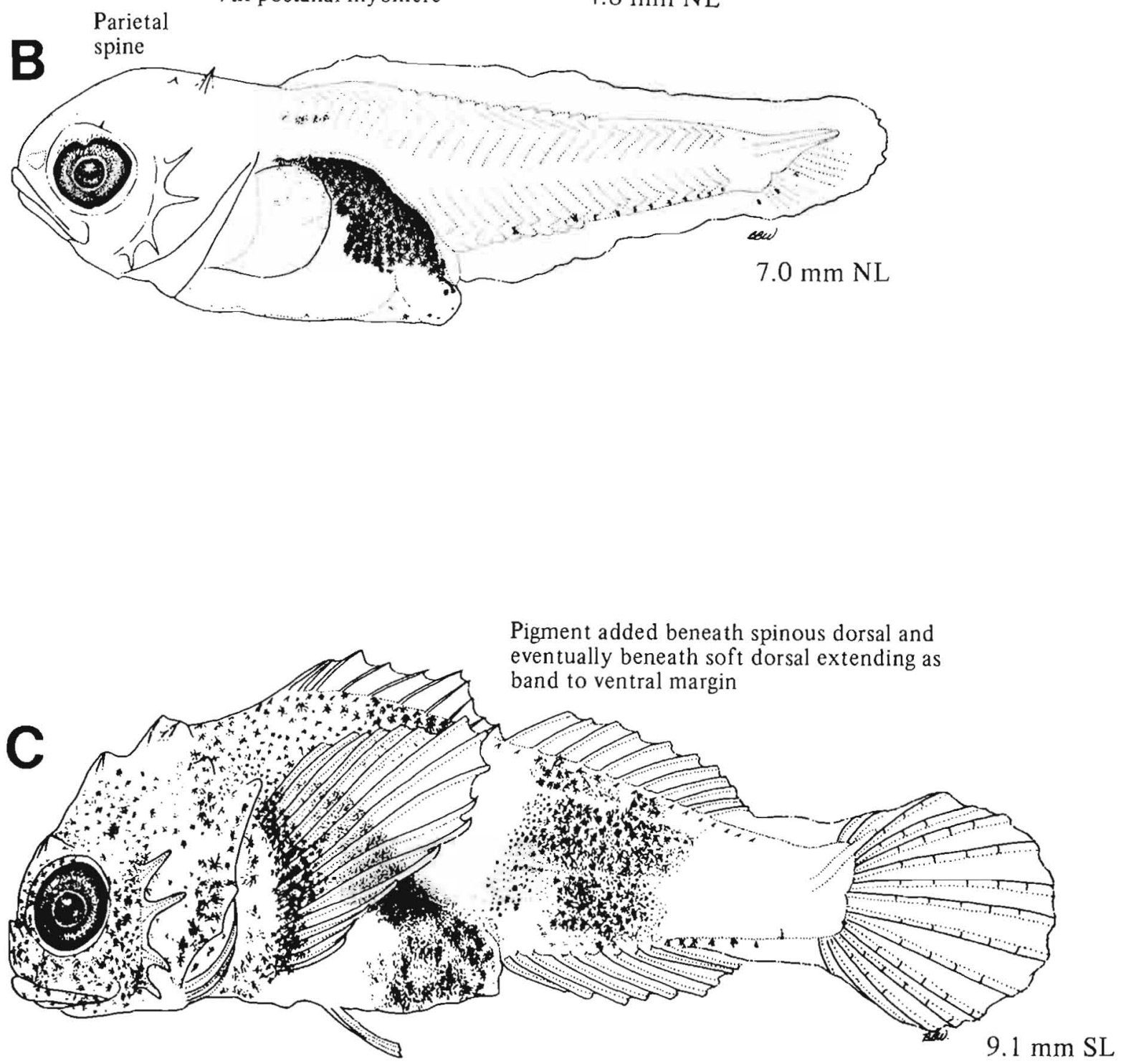

Figures A-C, Richardson and Washington 1980. 


\section{COTTIDAE}

Presently we cannot identify to species Gymnocanthus larvae from samples collected in our study area. We identify one type, Gymnocanthus A, and include illustrations of $G$. hertzensteini for comparison. Generic characters include:

- Patch of pigment on crown

- Heavy dorsal pigment on gut, increasing dorsolaterally with development

- Numerous, closely spaced, ventral midline melanophores in preflexion larvae

The following meristic information may aid in their identification.

Fins

\begin{tabular}{|c|c|c|c|c|c|c|c|}
\hline \multirow[b]{3}{*}{ Species } & \multirow[b]{3}{*}{ Distribution } & \multirow[b]{3}{*}{ Vertebrae } & \multirow{2}{*}{\multicolumn{2}{|c|}{ Dorsal }} & & & \\
\hline & & & & & \multirow[b]{2}{*}{ Anal rays } & \multirow[b]{2}{*}{ Pectoral rays } & \multirow[b]{2}{*}{ Pelvic rays } \\
\hline & & & Spines & Rays & & & \\
\hline Gymnocanthus detriscus & Bering Sea & $37-39$ & IX-XI & $15-18$ & $15-19$ & $19-20$ & $\mathrm{I}, 3$ \\
\hline Gymnocanthus galeatus & Brit. Col. - Bering Sea & $37-40$ & $\mathrm{X}-\mathrm{XII}$ & $14-17$ & $17-20$ & $19-21$ & $\mathrm{I}, 3$ \\
\hline Gymnocanthus pistilliger & SE Alaska - Bering Sea & $35-38$ & IX-XI & $13-16$ & 14-18 & $15-20$ & $\mathrm{I}, 3$ \\
\hline Gymnocanthus tricuspis & Bering Sea - Arctic & $36-40$ & $\mathrm{X}-\mathrm{XII}$ & $15-17$ & $15-18$ & $17-20$ & $\mathrm{I}, 3$ \\
\hline
\end{tabular}




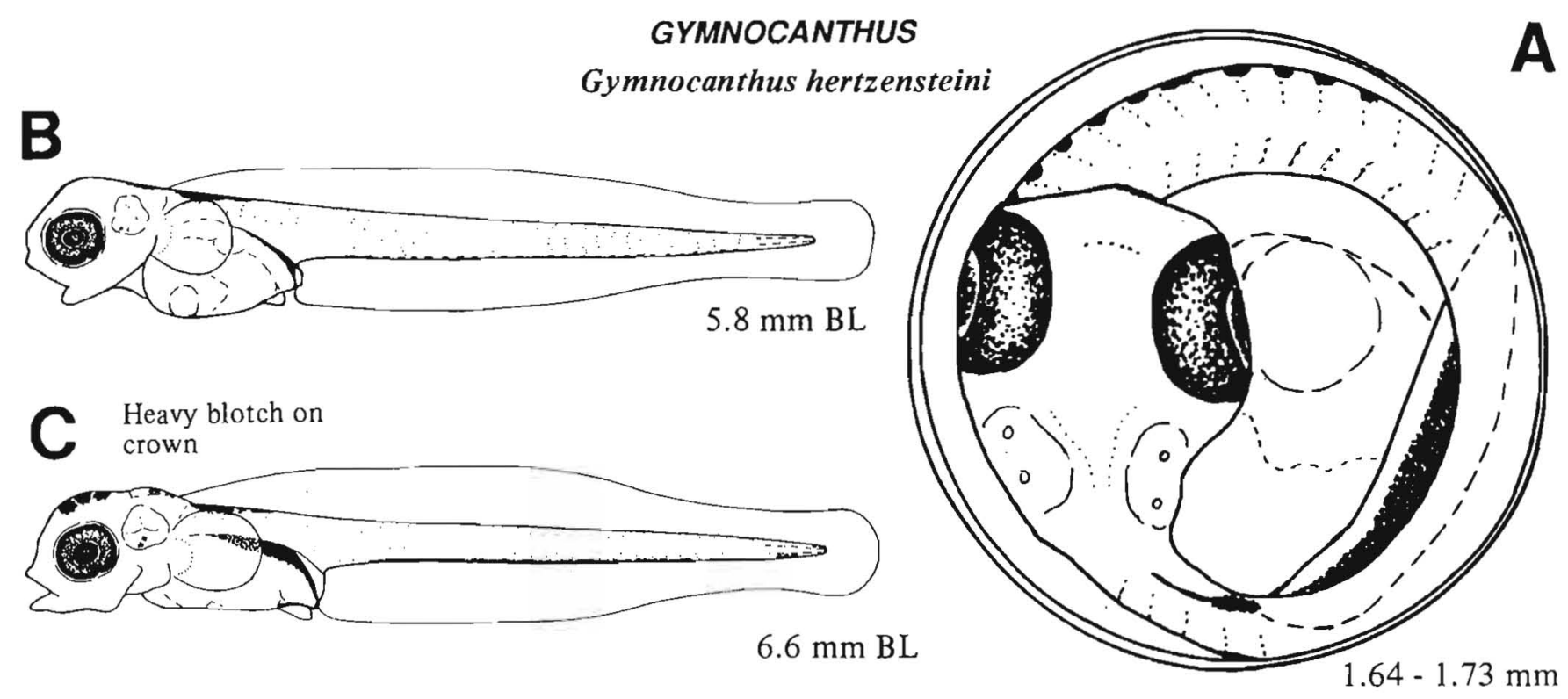

Heavy pigment on dorsolateral gut surface

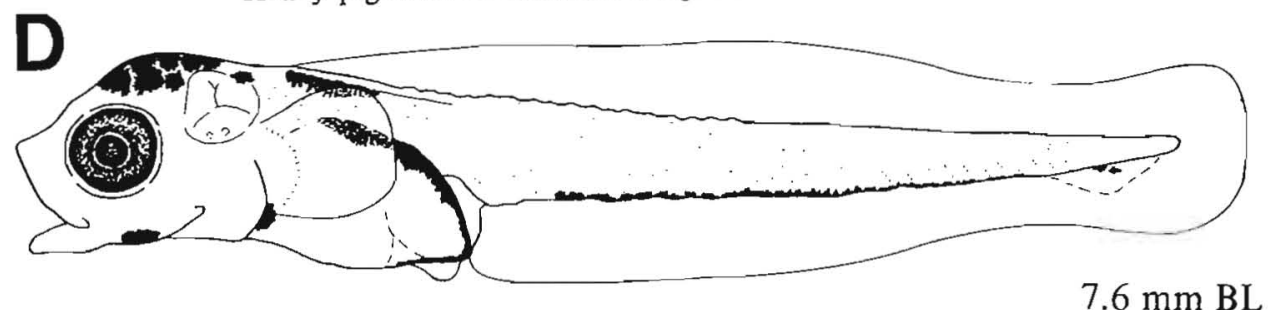

\section{Gymnocanthus A}

E

$7.6 \mathrm{~mm} \mathrm{BL}$

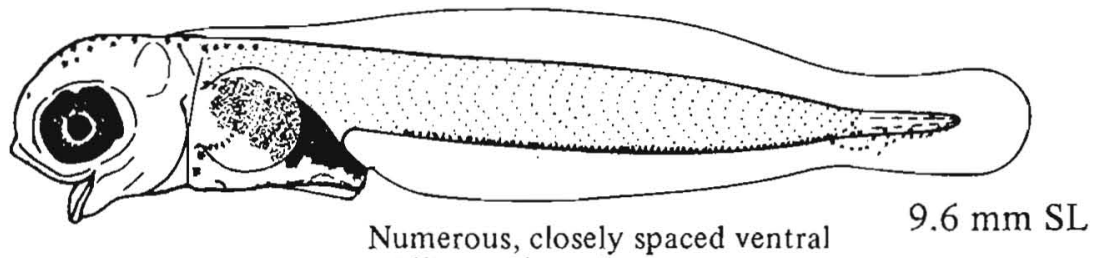

midline melanophores

Heavy pigment on dorsolateral gut surface

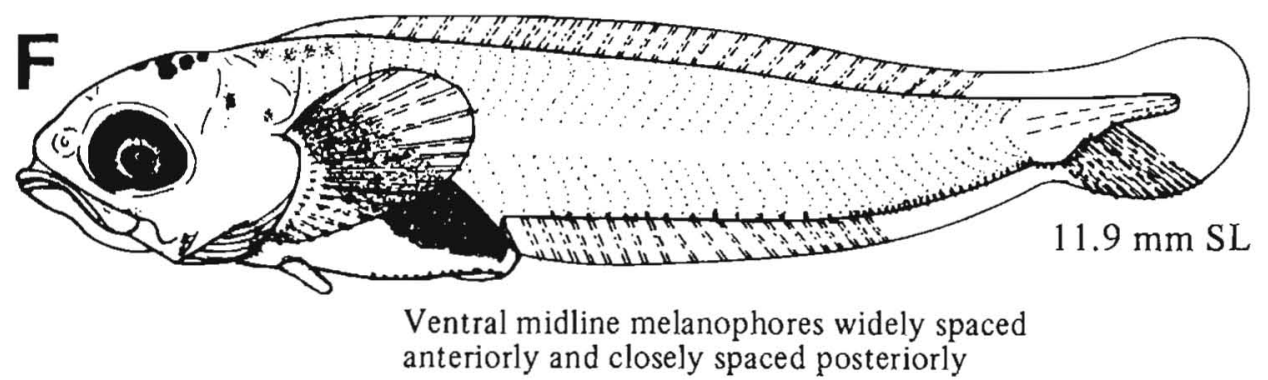

Figures A-D, Kyushin 1970 (reared from specimens collected near Hokkaido, Japan; B-D, redrawn); E-F, NWAFC originals (B. Vinter). 


\section{MERISTICS}

\begin{tabular}{|c|c|c|}
\hline Vertebrae & \multicolumn{2}{|c|}{$\begin{array}{l}\text { Total: } 36-38-40 \\
\text { Precaudal: X-X-X } \\
\text { Caudal: } X-X-X\end{array}$} \\
\hline $\begin{array}{l}\text { Branchiostegal rays } \\
\text { Caudal fin }\end{array}$ & $6-6-6$ & \\
\hline Pelvic fin & $\begin{array}{l}\text { Thoracic } \\
\text { S: } 1-1-1\end{array}$ & R: $3-3-3$ \\
\hline Dorsal fin & S: $10-11-12$ & $\mathrm{R}: 15-15-17$ \\
\hline Pectoral fin & R: $17-18-20$ & \\
\hline Anal fin & R: $15-17-18$ & \\
\hline Gill rakers & U: X-X-X & L: X-X-X \\
\hline
\end{tabular}

$\begin{array}{ll}\text { Range } & \begin{array}{c}\text { Bering Sea, } 54-66^{\circ} \mathrm{N}, \text { to Arctic } \\ \text { (throughout) } \\ \text { Epi- and mesobenthal, } 0-240 \mathrm{~m}\end{array} \\ \begin{array}{l}\text { Ecology } \\ \text { Oviparous, demersal eggs, } \\ \text { pelagic larvae }\end{array} \\ \begin{array}{l}\text { Season: Winter-spring } \\ \text { Spawning } \\ \text { Area: } \\ \text { Mode: Internal fertilization } \\ \text { likely } \\ \text { Migration: }\end{array} \\ \begin{array}{l}\text { Range/function: } 2000-3500 \\ (117-158 \mathrm{~mm} \text { specimens) }\end{array} \\ \begin{array}{l}\text { Fecundity } \\ \text { Age at first maturity } \\ \text { Longevity }\end{array}\end{array}$

andriashev 1954

${ }^{\mathrm{b}}$ Specimens have not been collected from our study area. Wlustrations of out-of-area specimens are presented for comparison and may differ.

Ref: Andriashev 1954, Khan 1972, Washington et al. $1984 \mathrm{~b}$.

\section{EARLY LIFE HISTORY DESCRIPTION}

\section{EGGS}

Diameter

$2 \mathrm{~mm}$

No. of oil globules

Oil globule diameter

Yolk

Envelope

Hatch size

Incubation time/temp.

Pigment

Diagnostic characters

\section{LARVAE}

Preanal length

Length at flexion

Length at transformation

Sequence of fin

development

Pigment $^{b}$

- Heavy pigment on crown and dorsolateral surface of gut

- Larvae $>12.2 \mathrm{~mm}$ SL have pigment along dorsal midline, and larvae $>13.9 \mathrm{~mm}$ SL develop pigment along lateral line

- Ventral midline melanophores are widely spaced anteriorly and closely spaced posteriorly

Diagnostic characters

- Specimens of other species of Gymnocanthus at comparable developmental stages have not been described 

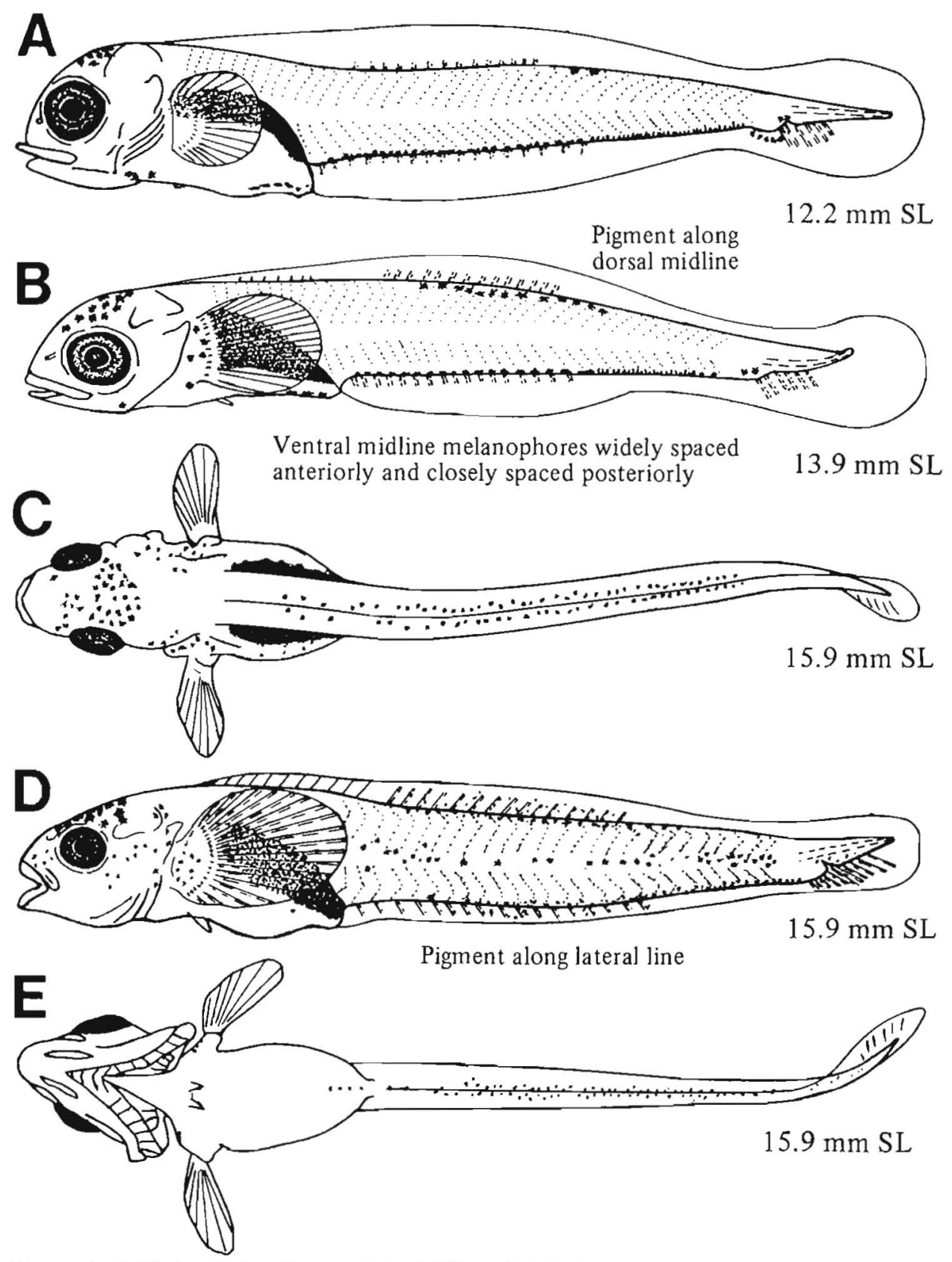

Figures A-E (C, dorsal view; E, ventral view), Khan 1972 (Atlantic specimens, redrawn). 
Eggs and larvae of Icelinus spp. from the study area are unknown. Illustrations of Icelinus A and B, both tentatively identified as $I$. borealis by Washington and Richardson (unpubl.), ${ }^{1}$ are presented here.

The following meristic characters may aid in their identification.

\begin{tabular}{|c|c|c|c|c|c|c|c|}
\hline \multirow[b]{3}{*}{ Species } & \multirow[b]{3}{*}{ Distribution } & \multirow[b]{3}{*}{ Vertebrae } & \multicolumn{5}{|c|}{ Fins } \\
\hline & & & \multicolumn{2}{|c|}{ Dorsal } & \multirow[b]{2}{*}{ Anal rays } & \multirow[b]{2}{*}{ Pectoral rays } & \multirow[b]{2}{*}{ Pelvic rays } \\
\hline & & & Spines & Rays & & & \\
\hline Icelinus borealis & Wash. - Bering Sea & $35-36$ & IX-XI & $14-17$ & $11-14$ & $14-17$ & $\mathrm{I}, 2$ \\
\hline Icelinus burchami & S. Calif. - SE Alaska & $35-37$ & $\mathrm{X}-\mathrm{XI}$ & $16-18$ & $12-14$ & $16-19$ & 1,2 \\
\hline Icelinus filamentosus & S. Calif. - Gulf of Alaska & $34-37$ & $\mathrm{X}-\mathrm{XII}$ & $15-17$ & $13-16$ & $16-18$ & $\mathrm{I}, 2$ \\
\hline Icelinus fimbriatus & S. Calif. - Brit. Col. & 37 & $\mathrm{XI}$ & $15-17$ & $13-14$ & 17 & 1,2 \\
\hline Icelinus tenuis* & SSC - Brit. Col. & $37-39$ & LX-XI & $16-18$ & $14-17$ & $15-17$ & $\mathrm{I}, 2$ \\
\hline
\end{tabular}

*A partial series of $I$. tenuis larvae has been identified at Los Angeles County Museum (R. Feeney, Los Ang. Cty. Mus. Nat. Hist., 900 Exposition Blvd., Los Angeles, CA 90007, pers. commun., Oct. 1986).

'B.B. Washington, NMFS Systematics Lab., Natl. Mus. Nat. Hist., Wash., D.C. 20560. 


\section{ICELINUS}

Icelinus A

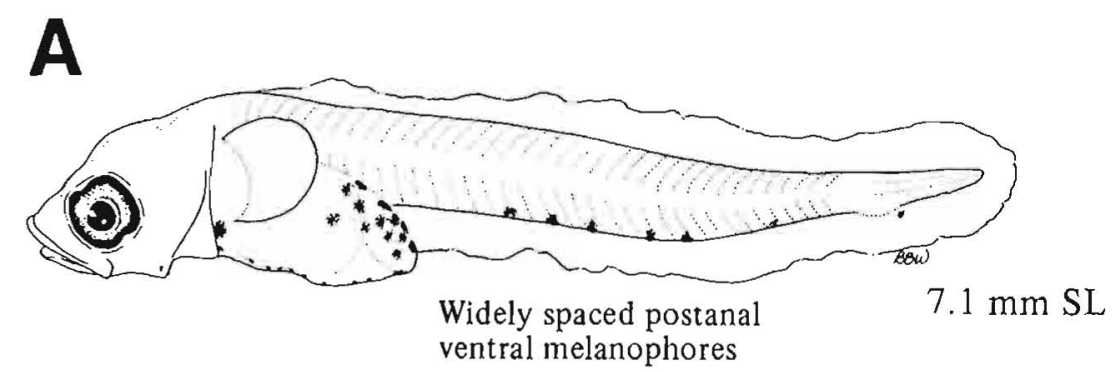

\section{Icelinus B}

B
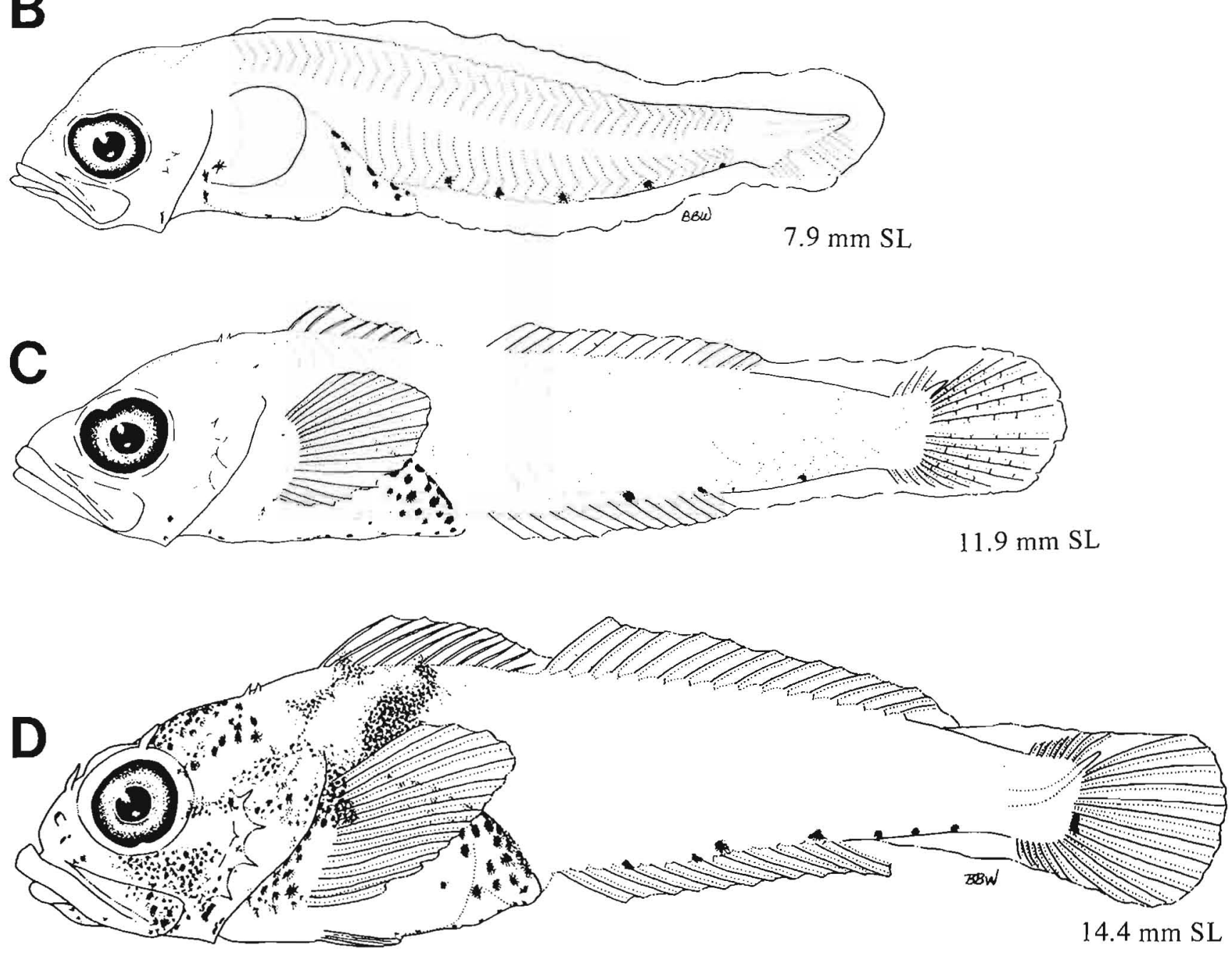

Figures A-D, Washington and Richardson, unpubl. 
Eggs and larvae of Icelus spp. from our study area are unknown. The following meristic characters may aid in their identification.

\begin{tabular}{|c|c|c|c|c|c|c|c|}
\hline \multirow[b]{3}{*}{ Species } & \multirow[b]{3}{*}{ Distribution } & \multirow[b]{3}{*}{ Vertebrae } & \multicolumn{5}{|c|}{ Fins } \\
\hline & & & \multicolumn{2}{|c|}{ Dorsal } & \multirow[b]{2}{*}{ Anal rays } & \multirow[b]{2}{*}{ Pectoral rays } & \multirow[b]{2}{*}{ Pelvic rays } \\
\hline & & & Spines & Rays & & & \\
\hline Icelus canaliculatus & Gulf of Alaska - Bering Sea & 41 & VII-VIII & $22-25$ & $18-20$ & $15-19$ & $\mathrm{I}, 3$ \\
\hline Icelus euryops & Gulf of Alaska - Bering Sea & & VIII-X & $20-23$ & $15-19$ & $16-18$ & $\mathrm{I}, 3$ \\
\hline Icelus scutiger* & Gulf of Alaska - Bering Sea & $39-41$ & IX-X & $18-21$ & $17-19$ & $17-19$ & $\mathrm{I}, 3$ \\
\hline Icelus spatula & Gulf of Alaska - Arctic & $39-41$ & VII-X & $17-22$ & $13-18$ & $16-20$ & $\mathrm{I}, 3$ \\
\hline Icelus spiniger & Brit. Col. - Bering Sea & $40-42$ & VIII-X & $19-24$ & $15-20$ & $17-20$ & $I, 3$ \\
\hline Icelus uncinalis & Bering Sea & $37-40$ & IX-X & $18-20$ & $14-16$ & $17-18$ & $\mathrm{I}, 3$ \\
\hline
\end{tabular}

*Placed in the genus Rastrinus by D.W. Nelson (1984). 
Presently we cannot identify to species Myoxocephalus larvae from samples we collect in our study area. We collect at least two types based on the following pigmentation characters:

Myoxocephalus B Ventral midline melanophore series ( $>40$ spots), dorsal pigment on gut, and crown pigment; with development, dorsolateral pigment appears covering about $2 / 3$ body length, small ventrolateral patch appears at midbody. Ventrolateral body from over gut to about six myomeres after anus is unpigmented.

Myoxocephalus G (probably M. polyacanthocephalus) Preflexion pigment pattern similar to flexion pattern of the other Myoxocephalus type; crown, gut, and ventral midline series with dorsolateral and lateral pigment covering $2 / 3$ body length. Body pigment becomes more intense and crown pigment covers entire head with development. No unpigmented area on lateral body over gut and anus.

The following meristic characters may aid in their identification.

\begin{tabular}{|c|c|c|c|c|c|c|c|}
\hline \multirow[b]{3}{*}{ Species } & \multirow[b]{3}{*}{ Distribution } & \multirow[b]{3}{*}{ Vertebrae } & \multicolumn{5}{|c|}{ Fins } \\
\hline & & & \multicolumn{2}{|c|}{ Dorsal } & \multirow[b]{2}{*}{ Anal rays } & \multirow{2}{*}{$\begin{array}{l}\text { Pectoral } \\
\text { rays }\end{array}$} & \multirow{2}{*}{$\begin{array}{l}\text { Pelvic } \\
\text { rays }\end{array}$} \\
\hline & & & Spines & Rays & & & \\
\hline Myoxocephalus axillaris* & Bering Sea - Arctic & & VIII-X & $15-17$ & $11-13$ & $14-16$ & $\mathrm{I}, 3$ \\
\hline Myoxocephalus brandti & Bering Sea & & IX & 15 & $12-13$ & $16-17$ & $\mathrm{I}, 3$ \\
\hline Myoxocephalus jaok & Gulf of Alaska - Chukchi Sea & $35-38$ & VIII-XI & $13-17$ & $12-16$ & $17-19$ & 1,3 \\
\hline Myoxocephalus niger & Bering Sea & $36-39$ & VIII-X & $14-18$ & $10-12$ & $16-18$ & $\mathrm{I}, 3$ \\
\hline Myoxocephalus polyacanthocephalus & Wash. - Bering Sea & 34-37 & IX-X & $10-15$ & $8-13$ & $16-19$ & $\mathrm{I}, 3$ \\
\hline Myoxocephalus quadricornis & Bering Sea - Arctic & $38-42$ & VII-X & $12-16$ & $13-17$ & $14-18$ & $\mathrm{I}, 3$ \\
\hline Myoxocephalus scorpioides* & Bering Sea - Arctic & $35-38$ & VIII-X & $13-18$ & $10-14$ & $14-17$ & $\mathrm{I}, 3$ \\
\hline Myoxocephalus scorpius* & SE Alaska - Arctic & $38-46$ & VIII-XII & $12-20$ & $10-16$ & $16-19$ & $\mathrm{I}, 3$ \\
\hline Myoxocephalus stelleri & Bering Sea - Chukchi Sea & & VIII-X & $15-16$ & $11-14$ & $16-18$ & $\mathrm{I}, 3$ \\
\hline Myoxocephalus verrucosus* & Brit. Col. - Arctic & & IX-XII & $15-18$ & $13-15$ & $16-19$ & $\mathrm{I}, 3$ \\
\hline
\end{tabular}

*According to Neelov (1979), M. scorpioides $=M$. axillaris, and $M$. scorpius in the study area is $M$. verrucosus. 


\section{MYOXOCEPHALUS}

Myoxocephalus B

\section{A}

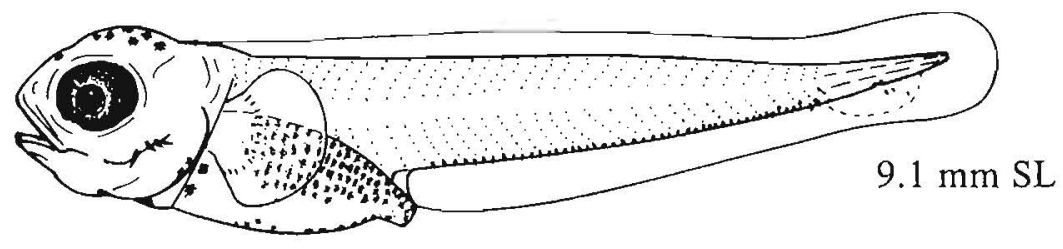

B

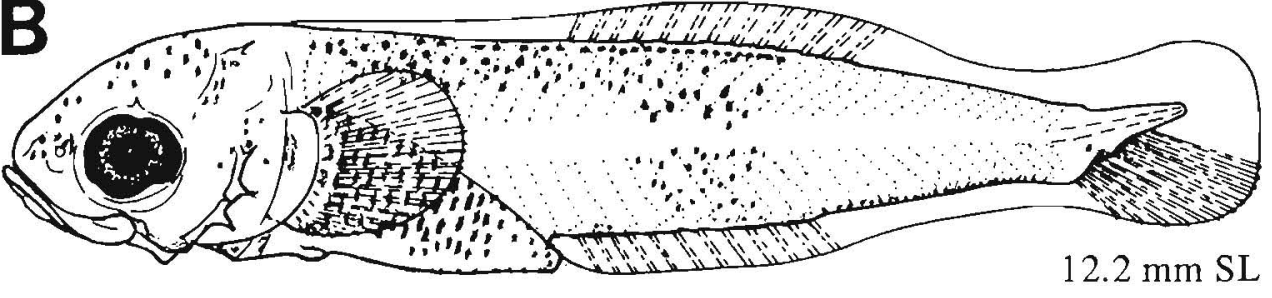

\section{Myoxocephalus G}
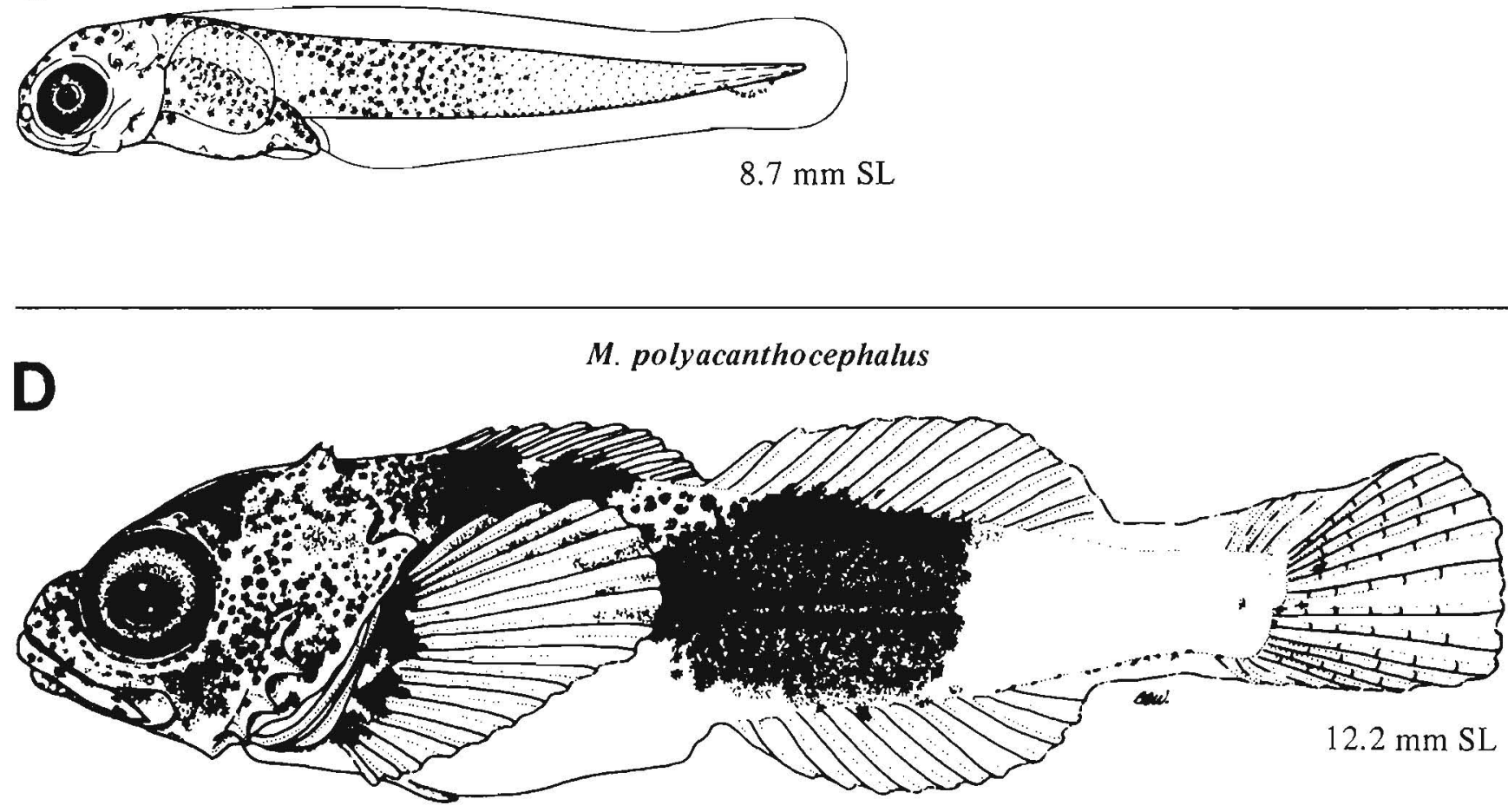

Figures A-C, NWAFC originals (B. Vinter); D, Richardson 1981 a. 


\section{MERISTICS}

\begin{tabular}{|c|c|c|}
\hline \multirow[t]{3}{*}{ Vertebrae } & \multicolumn{2}{|c|}{ Total: $38-X-46$} \\
\hline & \multicolumn{2}{|c|}{ Precaudal: X-X-X } \\
\hline & \multicolumn{2}{|c|}{ Caudal: $\mathrm{X}-\mathrm{X}-\mathrm{X}$} \\
\hline Branchiostegal rays & \multicolumn{2}{|l|}{$6-6-6$} \\
\hline \multicolumn{3}{|l|}{ Caudal fin } \\
\hline \multirow[t]{2}{*}{ Pelvic fin } & Thoracic & \\
\hline & $S: 1-1-1$ & R: $3-3-3$ \\
\hline Dorsal fin & S: $8-X-12$ & $\mathrm{R}: 12-\mathrm{X}-20$ \\
\hline Pectoral fin & $\mathrm{R}: 16-X-19$ & \\
\hline Anal fin & $\mathrm{R}: 10-\mathrm{X}-16$ & \\
\hline Gill rakers & $\mathrm{U}: \mathrm{X}-\mathrm{X}-\mathrm{X}$ & $\mathrm{L}: \mathrm{X}-\mathrm{X}-\mathrm{X}$ \\
\hline \multicolumn{3}{|l|}{ LIFE HISTORY } \\
\hline Range & \multicolumn{2}{|c|}{$\begin{array}{l}\text { Brit. Col., } 48^{\circ} 30^{\prime}-55^{\circ} \mathrm{N} \text {, to } \\
\text { Arctic, not specific }\end{array}$} \\
\hline Ecology & \multicolumn{2}{|c|}{$\begin{array}{l}\text { Epi-, meso-, and bathybenthal, } \\
0-550 \mathrm{~m}\end{array}$} \\
\hline ELH pattern & \multicolumn{2}{|c|}{$\begin{array}{l}\text { Oviparous, pelagic eggs, } \\
\text { pelagic larvae }\end{array}$} \\
\hline Spawning & \multicolumn{2}{|c|}{ Season: Winter ${ }^{b}$} \\
\hline & \multicolumn{2}{|l|}{ Area: } \\
\hline & \multirow{2}{*}{\multicolumn{2}{|c|}{$\begin{array}{l}\text { Mode: Eggs guarded by males } \\
\text { Migration: }\end{array}$}} \\
\hline & & \\
\hline Fecundity & \multicolumn{2}{|c|}{ Range/function: $2700^{\mathrm{b}}$} \\
\hline $\begin{array}{l}\text { Age at first maturity } \\
\text { Longevity }\end{array}$ & \multicolumn{2}{|l|}{$3-4 \mathrm{yr}^{\mathrm{b}}$} \\
\hline
\end{tabular}

\section{EARLY LIFE HISTORY DESCRIPTION}

\section{EGGS}

Diameter

No. of oil globules

Oil globule diameter

Yolk

Envelope

Hatch size

$2.0-2.5 \mathrm{~mm} ;{ }^{\text {b }} 1.8-2.5 \mathrm{~mm}^{\mathrm{c}}$ Several

$0.4-0.5 \mathrm{~mm}$

Incubation time/temp.

Pigment

Diagnostic characters

\section{LARVAE}

Preanal length

Length at flexion $\quad 9-15 \mathrm{~mm} \mathrm{SL}$

Length at transformation $17-20 \mathrm{~mm}$ SL

Sequence of fin

development

Pigment

- Crown, dorsolateral gut surface, ventral midline starting at myomere 16

- Develops midbody patch at flexion with dorsal pigment extending anteriorly

Diagnostic characters

\footnotetext{
${ }^{a}$ According to Neelov (1979), specimens from our area are $M$. verrucosus. Illustrations of Atlantic specimens of $M$. scorpius are presented for comparison. Specimens

from our area may differ.

${ }^{\mathrm{b}}$ Andriashev 1954

${ }^{c}$ Washington et al. $1984 \mathrm{~b}$
}

Ref: Andriashev 1954, Washington et al. $1984 \mathrm{~b}$. 

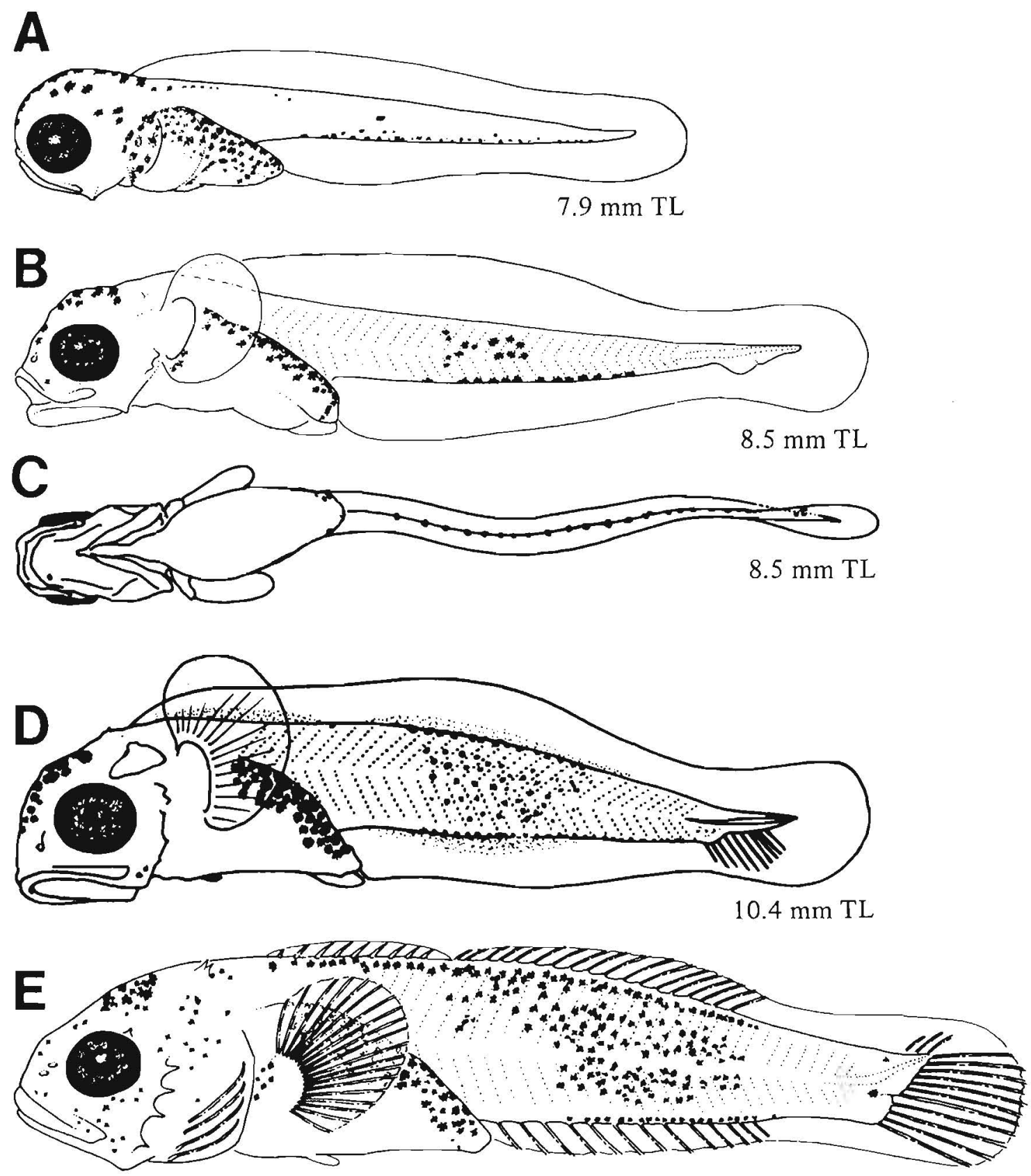

$17.4 \mathrm{~mm} \mathrm{TL}$

Figures A-B, E, Fahay 1983 (A, after Rass 1949; B, E, after Khan 1972); C-D (C, ventral view), Khan 1972 (B-E, Gulf of St. Lawrence specimens). 


\section{MERISTICS}

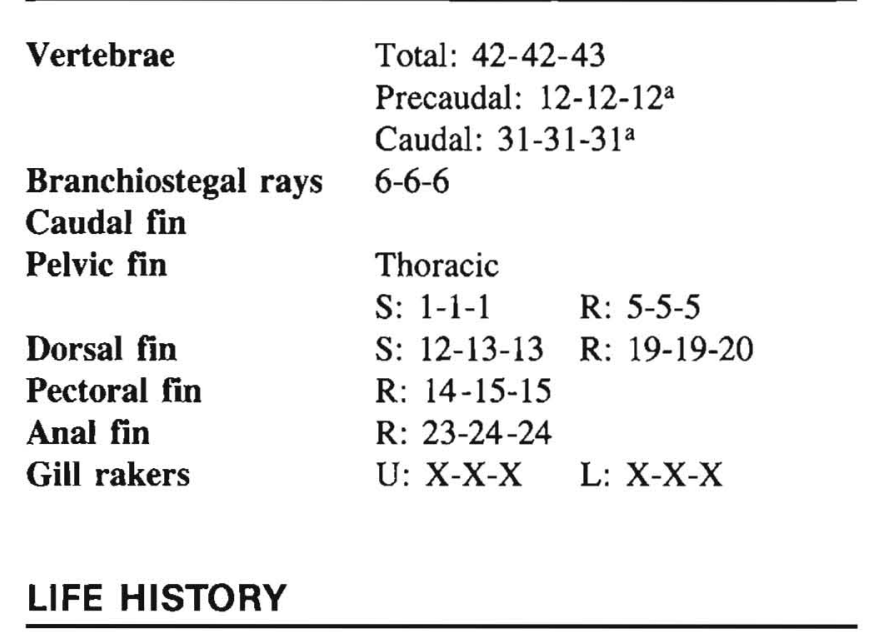

$\begin{array}{ll}\text { Range } & \text { S. California, } 32-34^{\circ} \mathrm{N}, \text { to } \\ & \text { Brit. Col., } 48^{\circ} 30^{\prime}-55^{\circ} \mathrm{N} \\ \text { Ecology } & \text { Nearshore shelf demersal, } \\ & 0-183 \mathrm{~m} \\ & \text { Parity and eggs unknown, } \\ \text { ELH pattern } & \text { pelagic larvae } \\ \text { Spawning } & \text { Area: } \\ & \text { Mode: } \\ & \text { Migration: } \\ \text { Fecundity } & \text { Range/function: } \\ \text { Age at first maturity } & \\ \text { Longevity } & \end{array}$

\section{EARLY LIFE HISTORY DESCRIPTION}

\section{EGGS}

Diameter

No. of oil globules

Oil globule diameter

Yolk

Envelope

Hatch size $\quad<5.6 \mathrm{~mm} \mathrm{SL}$

Incubation time/temp.

Pigment

Diagnostic characters

\author{
LARVAE \\ Preanal length \\ $35-36 \%$ SL, increasing \\ with development \\ Length at flexion \\ Length at transformation $\sim 25 \mathrm{~mm}$ SL \\ Sequence of fin \\ development \\ Pigment \\ - Gut pigmented all over \\ - Ventral midline melanophores $>30$, decreasing to \\ 15-20 by postflexion stage \\ - Melanophores near tail tip \\ Diagnostic characters \\ - Pigment pattern (tail pigment) \\ - Pointed snout and slender body \\ - Four preopercular spines \\ - Myomeres (42-43) \\ - Spiny scales develop in postflexion larvae
}

${ }^{a}$ B.B. Washington, NMFS Systematics Lab., Natl. Mus. Nat. Hist., Wash., D.C. 20560, unpubl.

Ref: Richardson and Washington 1980. 

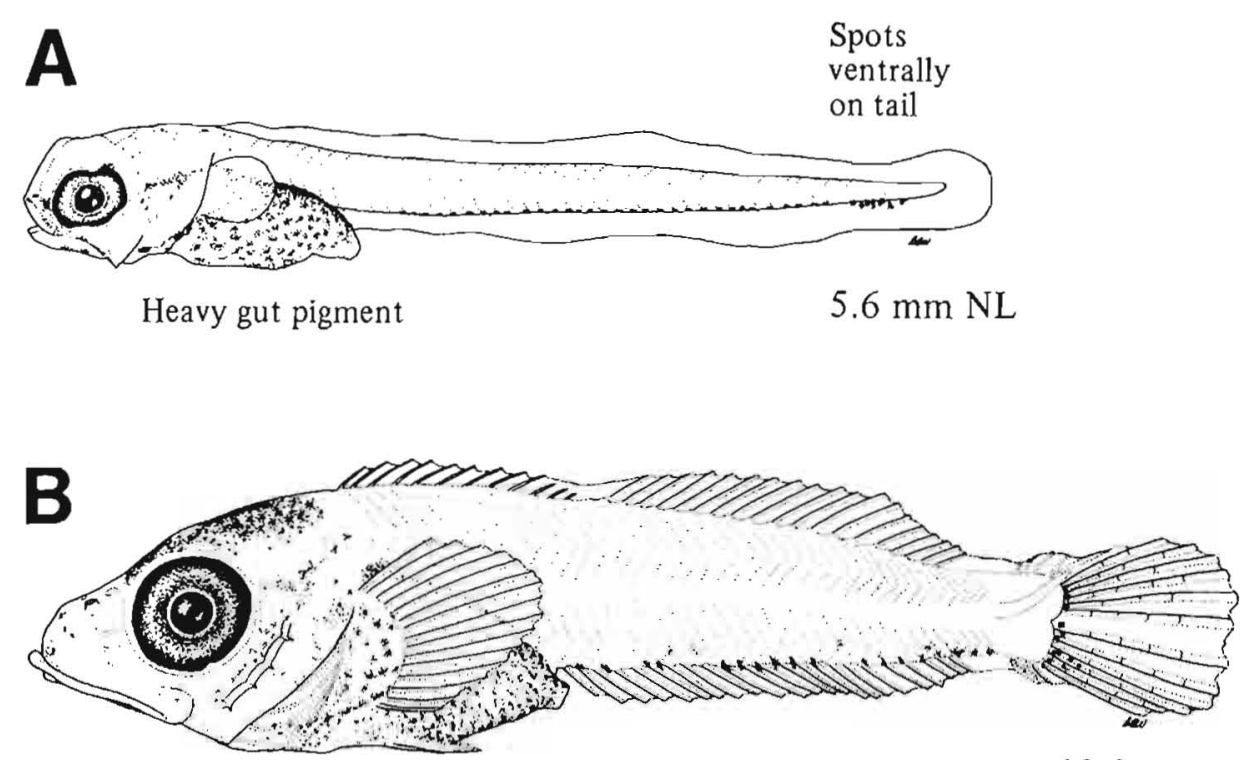

$13.8 \mathrm{~mm} \mathrm{NL}$

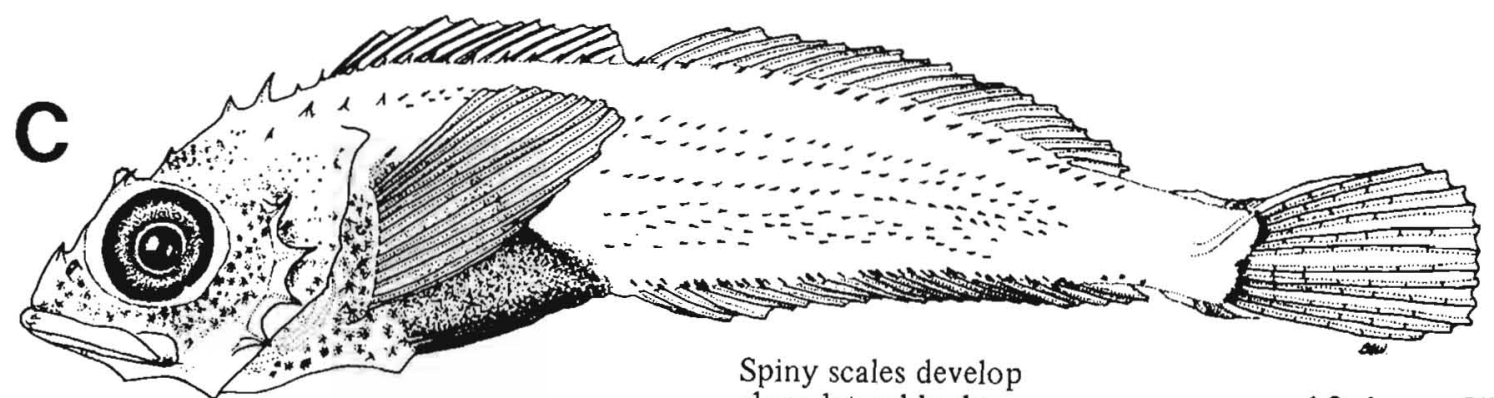
along lateral body

$18.6 \mathrm{~mm} \mathrm{SL}$

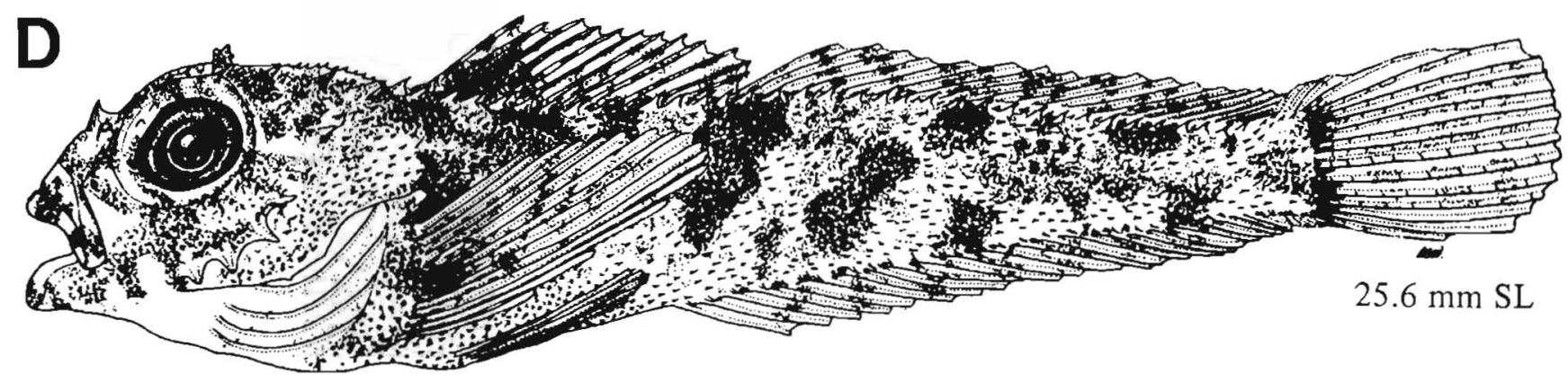

Figures $\mathrm{A}-\mathrm{D}$, Richardson and Washington 1980 . 


\section{MERISTICS}

Vertebrae

$\begin{array}{lll} & \begin{array}{l}\text { Precaudal: } 12-\mathrm{X}-13 \\ \text { Caudal: } 27-27-27\end{array} \\ \begin{array}{lll}\text { Branchiostegal rays } \\ \begin{array}{l}\text { Caudal fin } \\ \text { Pelvic fin }\end{array} & \text { 6-6-6 } & \\ & & \\ & \text { Thoracic } & \\ \text { Dorsal fin } & \text { S: } 1-1-1 & \text { R: } 3-3-3 \\ \text { Pectoral fin } & \text { S: } 8-9-10 & \text { R: } 20-22-23 \\ \text { Anal fin } & \text { R: } 17-18-20 & \\ \text { Gill rakers } & \text { R: } 21-23-25 & \\ & \text { U: } X-X-1 & \text { L: } 7-X-8\end{array}\end{array}$

\section{Range}

Ecology

ELH pattern

Spawning

Age at first maturity

Longevity

\section{LIFE HISTORY}

\section{EARLY IIIFE HISTORY DESCRIPTION}

\section{EGGS}

Diameter

No. of oil globules

Oil globule diameter

Yolk

Homogeneous

Envelope

Hatch size

$\leqslant 4.7 \mathrm{~mm} \mathrm{NL}$
Pigment

Diagnostic characters

\section{LARVAE}

Preanal length

44-54\% SL

Length at flexion $7.2-10.9 \mathrm{~mm} \mathrm{NL}$

Length at transformation $\geqslant 14-15 \mathrm{~mm} \mathrm{SL}$

Sequence of fin development

Pigment

- Heavy over body except dorsolaterally above body midline over gut and dorsally and laterally on tail tip

Diagnostic characters (see Table 3)

- Heavy pigmentation

- Preopercular spines not prominent

- Gut distinctively coiled

Distinguished from Scorpaenichthys marmoratus (p. 382) by

- Series of melanophores along lateral line 


\section{A}

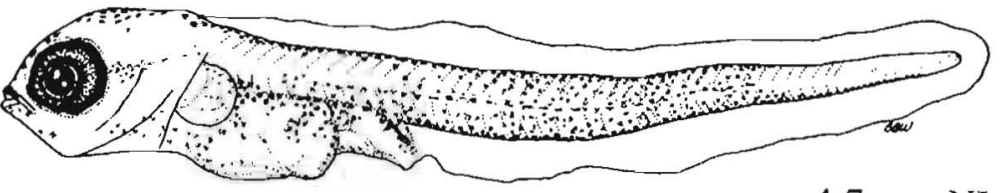

\section{$4.7 \mathrm{~mm} \mathrm{NL}$}

B

Mediolateral series

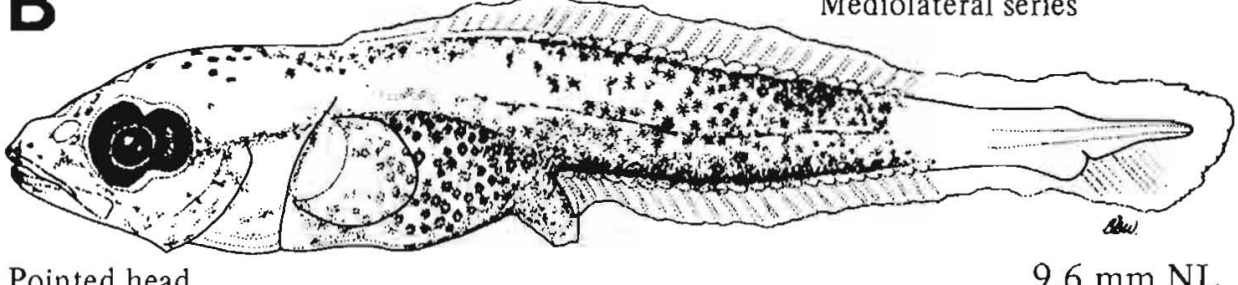

Pointed head

$9.6 \mathrm{~mm} \mathrm{NL}$

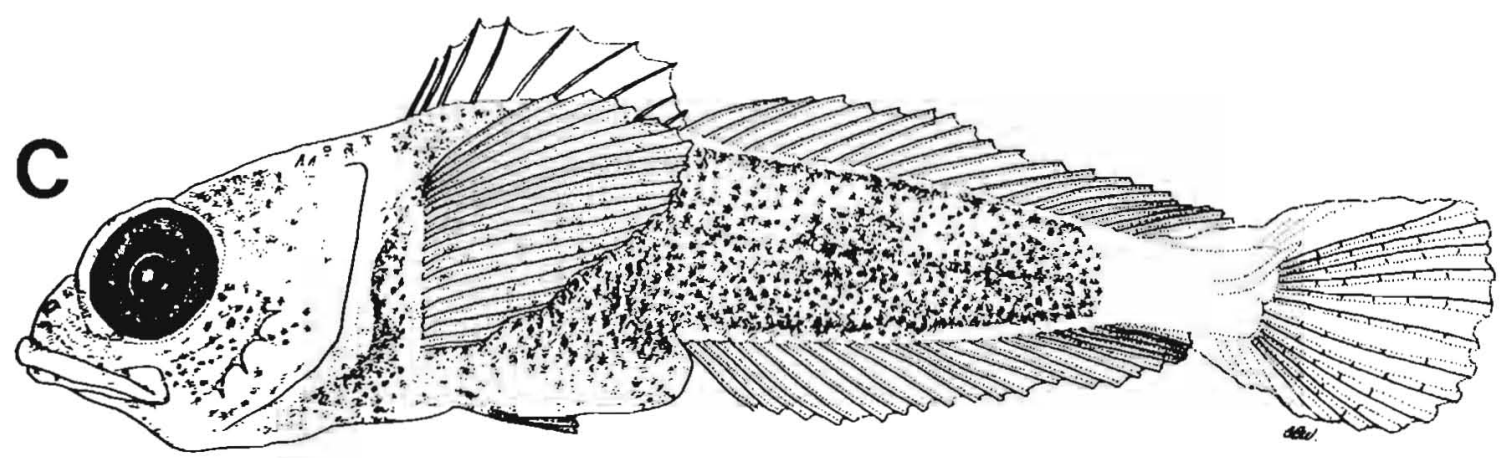

$12.6 \mathrm{~mm} \mathrm{SL}$

D

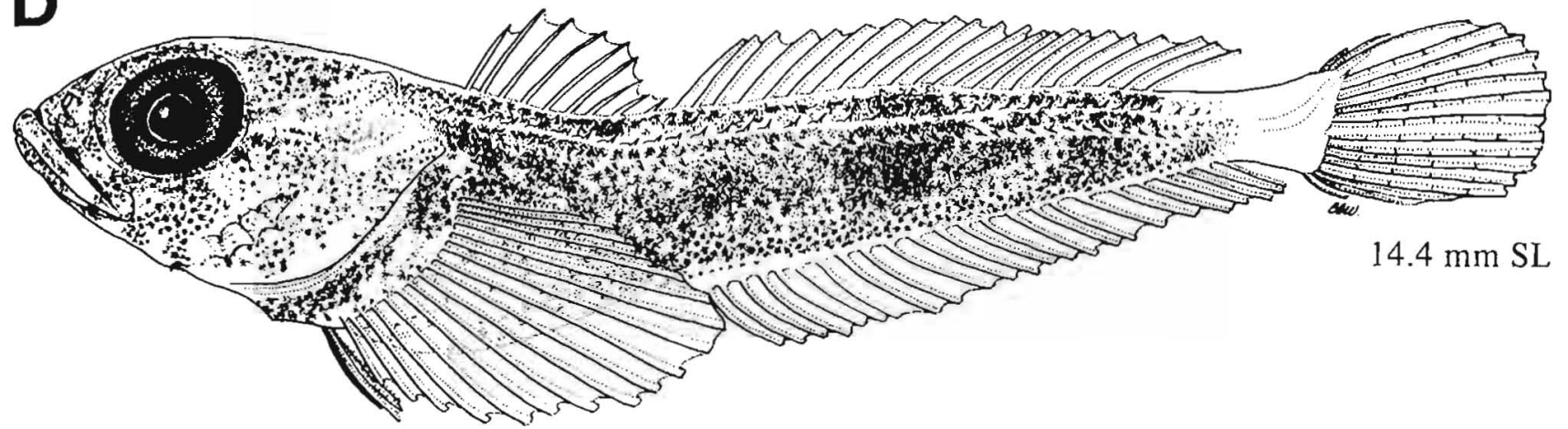

Figures A-D, Richardson and Washington 1980. 


\section{MERISTICS}

\begin{tabular}{|c|c|c|}
\hline \multirow{3}{*}{ Vertebrae } & \multicolumn{2}{|c|}{ Total: $39-40-40$} \\
\hline & \multicolumn{2}{|c|}{ Precaudal: X-X-X } \\
\hline & \multicolumn{2}{|c|}{ Caudal: X-X-X } \\
\hline Branchiostegal rays & $6-6-6$ & \\
\hline Caudal fin & $x, 6+6, x$ & \\
\hline Pelvic fin & Thoracic & \\
\hline & S: $1-1-1$ & R: $3-3-3$ \\
\hline Dorsal fin & S: $8-9-11$ & R: $20-20-22$ \\
\hline Pectoral fin & R: $18-19-20$ & \\
\hline Anal fin & $\mathrm{R}: 21-22-23$ & \\
\hline Gill rakers & $\mathrm{U}: \mathrm{X}-\mathrm{X}-\mathrm{X}$ & L: X-X-X \\
\hline
\end{tabular}

Range

Ecology

ELH pattern

Spawning

Fecundity

Age at first maturity

Longevity
S. California, $32-34^{\circ} \mathrm{N}$, to Brit. Col., $48^{\circ} 30^{\prime}-55^{\circ} \mathrm{N}$

Nearshore shelf demersal, 15-146 m

Oviparous, eggs probably demersal, pelagic larvae Season:

Area:

Mode:

Migration:

Range/function:

\section{EARLY LIFE HISTORY DESCRIPTION}

\section{EGGS}

Diameter

No. of oil globules

Oil globule diameter

Yolk

Homogeneous

Envelope

Hatch size

Incubation time/temp.

Pigment

Diagnostic characters

\author{
LARVAE \\ Preanal length $\quad \sim 58 \%$ SL \\ Length at flexion $\quad \sim 8.7 \mathrm{~mm} \mathrm{SL}$ \\ Length at transformation \\ Sequence of fin \\ development \\ Pigment \\ - Generally heavily pigmented over anterior $3 / 4$ body \\ Diagnostic characters (see Table 3) \\ Distinguished from $R$. asprellus by \\ - Greater portion of tail unpigmented \\ - Morphological differences \\ -Preanal length longer \\ -Greater body depth
}

Ref: Richardson and Washington 1980. 


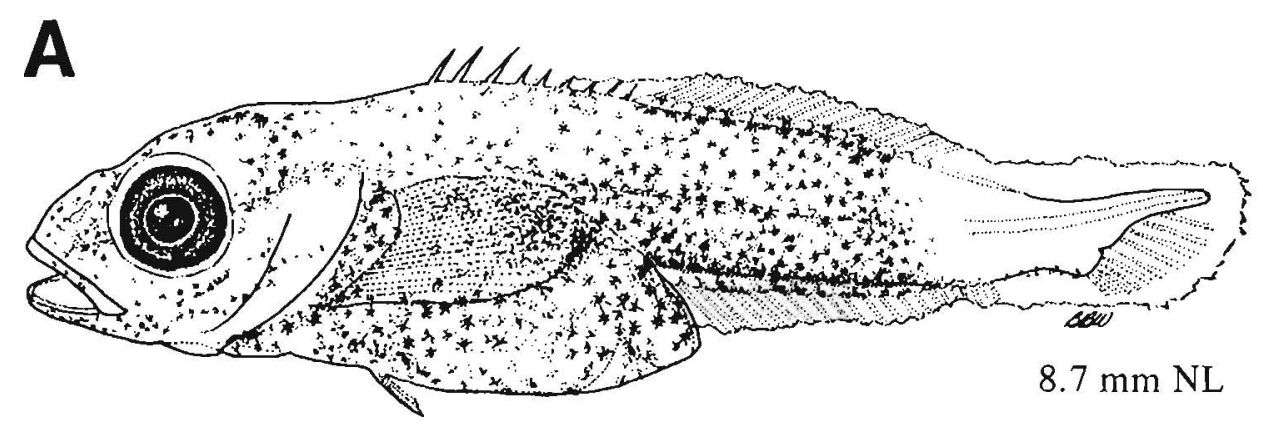

Figure A, Richardson and Washington 1980 . 


\section{MERISTICS}

$\begin{array}{ll}\text { Vertebrae } & \text { Total: } 38-39-39 \\ & \text { Precaudal: } 12-12-12 \\ & \text { Caudal: } 26-26-26 \\ \text { Branchiostegal rays } & 6-6-6^{\mathrm{a}} \\ \text { Caudal fin } & 8-9,6+5,7-8 \\ \text { Pelvic fin } & \text { Thoracic } \\ & \text { S: } 1-1-1 \quad \text { R: } 3-3-3 \\ \text { Dorsal fin } & \text { S: } 8-10-10 \quad \text { R: } 19-20-21 \\ \text { Pectoral fin } & \text { R: } 21-22-24 \\ \text { Anal fin } & \text { R: } 18-20-21 \quad \\ \text { Gill rakers } & \text { U: } X-X-X \quad \text { L: } X-X-X\end{array}$

LIFE HISTORY

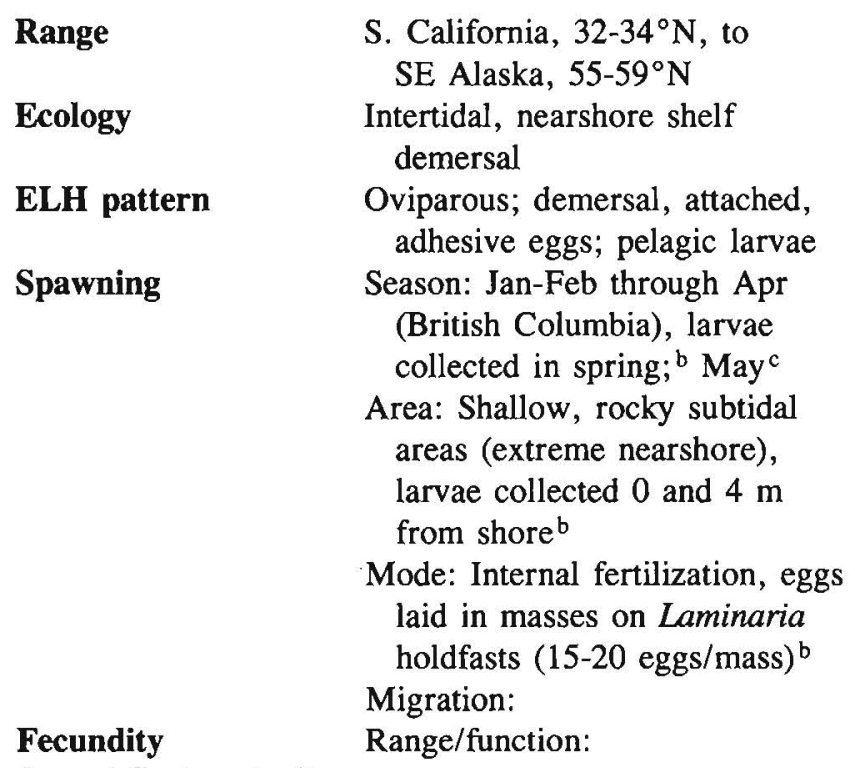

Age at first maturity

Longevity

\section{EARLY LIFE HISTORY DESCRIPTION}

\section{EGGS}

Diameter

No. of oil globules

Oil globule diameter

Yolk

Envelope

Homogeneous, pink (wild)

Hatch size

Incubation time/temp.

Pigment

Diagnostic characters

\author{
LARVAE $^{\mathrm{d}}$ \\ Preanal length \\ $37-42 \%$ SL \\ Length at flexion \\ 6.5-8.5 mm SL \\ Length at transformation \\ Sequence of fin \\ development \\ Pectorals and caudal, dorsal \\ and anal, pelvics \\ Pigment \\ - Gut pattern \\ - High number of postanal ventral melanophores \\ ( $\bar{x} 51$ in preflexion, 47 in flexion) \\ Diagnostic characters \\ - Pigment on gut \\ - Accessory or inner-shelf spines associated with \\ preopercular spines in preflexion larvae (not present \\ in other members of Myoxocephalus group) \\ - Pectoral fin rays develop toward each other \\ ventrally \\ - Spines: Nasal spines develop at 12-14 mm SL
}

'According to Marliave et al. (1985), branchiostegal rays $=7$.

${ }^{b}$ Marliave et al. 1985

${ }^{c}$ Hart 1973

Tentative placement in Myoxocephalus group.

Ref: Marliave et al. 1985. 

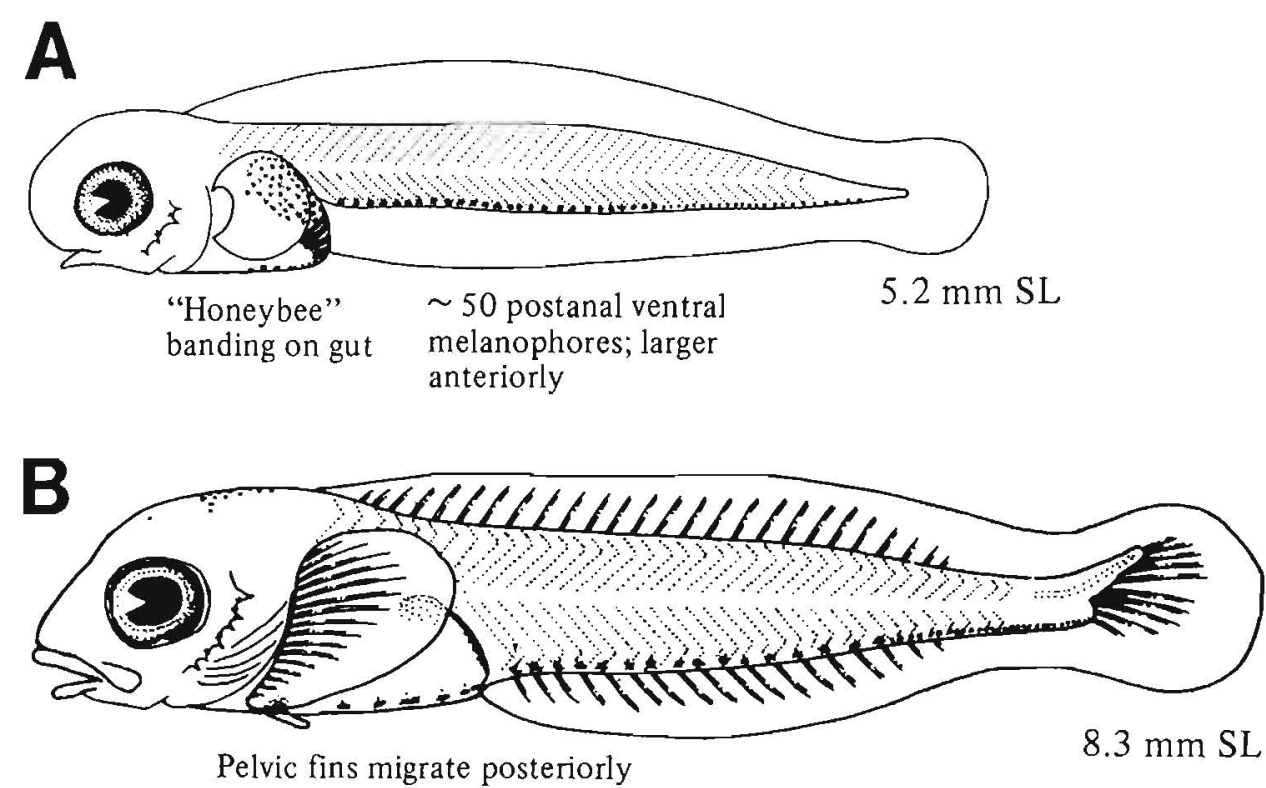

as rays develop
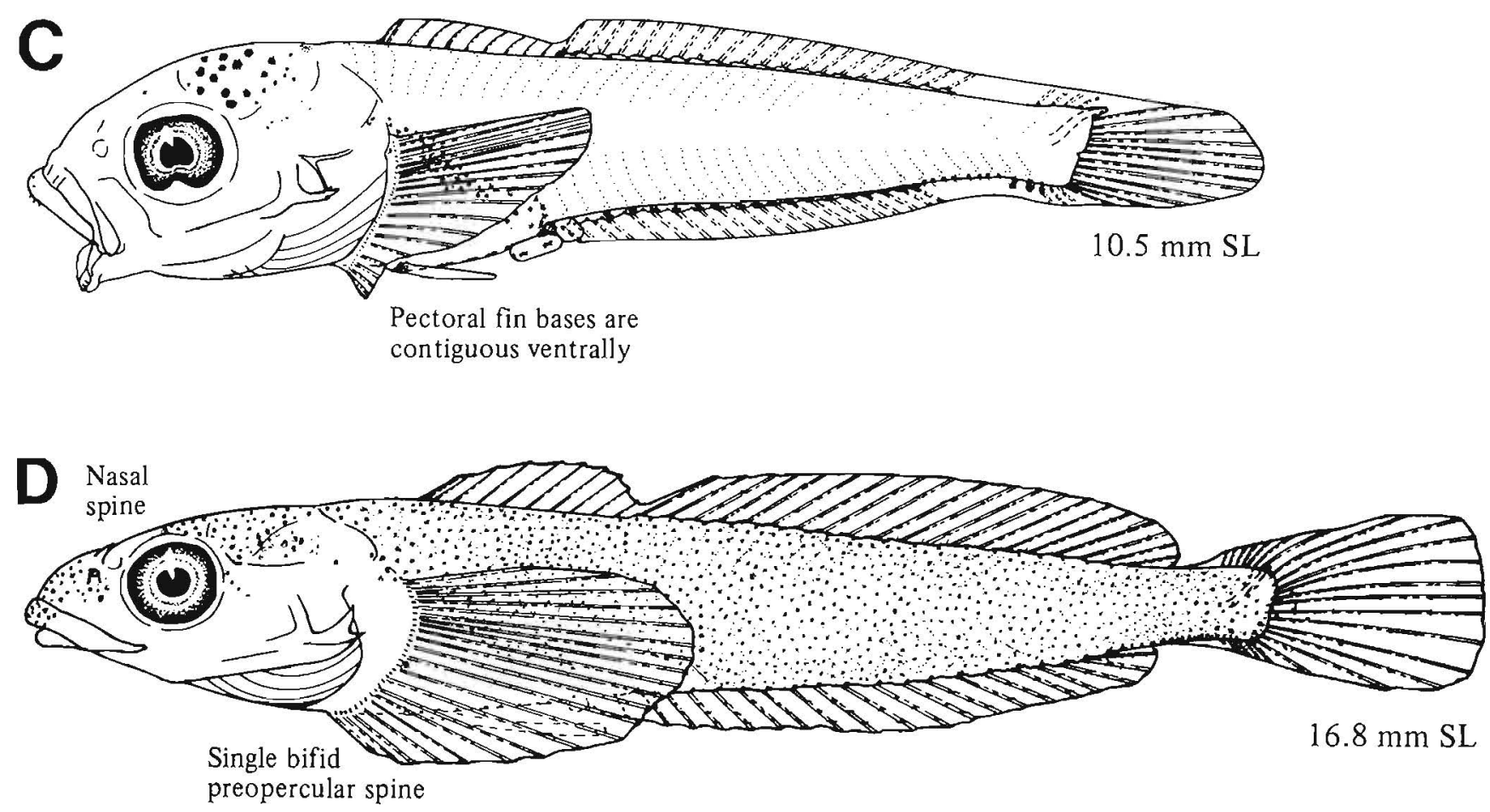

Figures A-B, Marliave et al. 1985 ; C-D, NWAFC originals (B. Vinter). 
Presently we cannot identify to species Triglops spp. larvae from samples we collect in our study area. Illustrations of Triglops sp. (designated as B here) (Richardson and Washington 1980) and Triglops A (Washington and Richardson, unpubl. ${ }^{1}$ ) are presented here. Generic characters include:

- High myomere count $(>45)$

- Pointed snout

- Heavy dorsolateral pigmentation on gut

- Postanal ventral midline melanophores, probably becoming embedded in postflexion larvae (except Triglops B as figured by Richardson and Washington [1980]).

The following meristic information may aid in their identification.

\begin{tabular}{|c|c|c|c|c|c|c|c|}
\hline \multirow[b]{3}{*}{ Species } & \multirow[b]{3}{*}{ Distribution } & \multirow[b]{3}{*}{ Vertebrae } & \multicolumn{5}{|c|}{ Fins } \\
\hline & & & \multicolumn{2}{|c|}{ Dorsal } & \multirow[b]{2}{*}{ Anal rays } & \multirow[b]{2}{*}{ Pectoral rays } & \multirow[b]{2}{*}{ Pelvic rays } \\
\hline & & & Spines & Rays & & & \\
\hline Triglops forficata & Gulf of Alaska - Bering Sea & $52-54$ & IX-XI & $27-32$ & $27-32$ & $20-22$ & $\mathrm{I}, 3$ \\
\hline Triglops jordani & Bering Sea & & IX-XI & $24-28$ & $23-29$ & $19-21$ & $\mathrm{I}, 3$ \\
\hline Triglops macellus & Wash. - Bering Sea & 51 & $\mathrm{X}-\mathrm{XI}$ & $27-31$ & $27-31$ & $15-17$ & $\mathrm{I}, 3$ \\
\hline Triglops metopias & SE Alaska - Bering Sea & $48-49$ & $X-X I$ & $23-27$ & $22-27$ & $18-22$ & $\mathrm{I}, 3$ \\
\hline Triglops pingeli & Wash. - Arctic & $46-48$ & IX-XIII & $22-28$ & $20-28$ & $16-19$ & $\mathrm{I}, 3$ \\
\hline Triglops scepticus & SE Alaska - Bering Sea & $45-46$ & $\mathrm{X}-\mathrm{XII}$ & $21-23$ & $22-24$ & $17-19$ & $\mathrm{I}, 3$ \\
\hline
\end{tabular}

'B.B. Washington, NMFS Systematics Lab., Natl. Mus. Nat. Hist., Wash., D.C. 20560. 


\section{TRIGLOPS}

Triglops A (possibly $T$. scepticus based on counts)

A

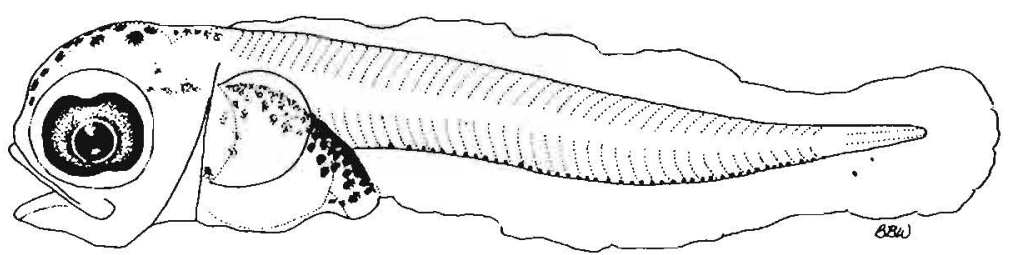

Ventral midline melanophores $\quad 6.5 \mathrm{~mm} \mathrm{SL}$

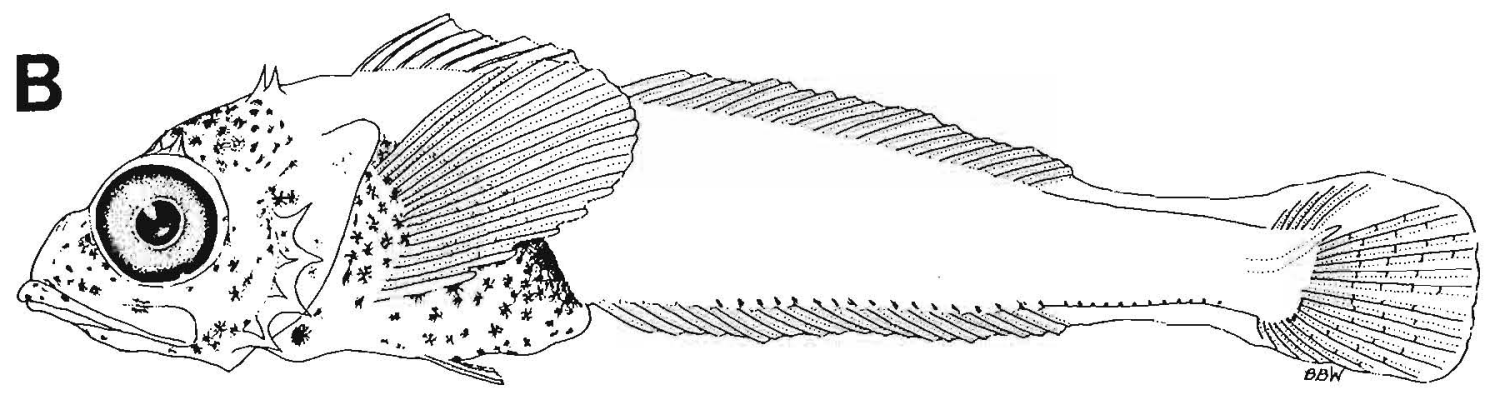

$16.9 \mathrm{~mm} \mathrm{SL}$

\section{Triglops B}

C

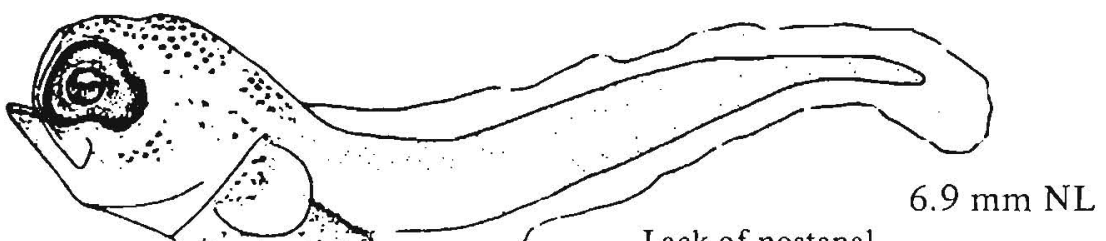

Lack of postanal body pigment

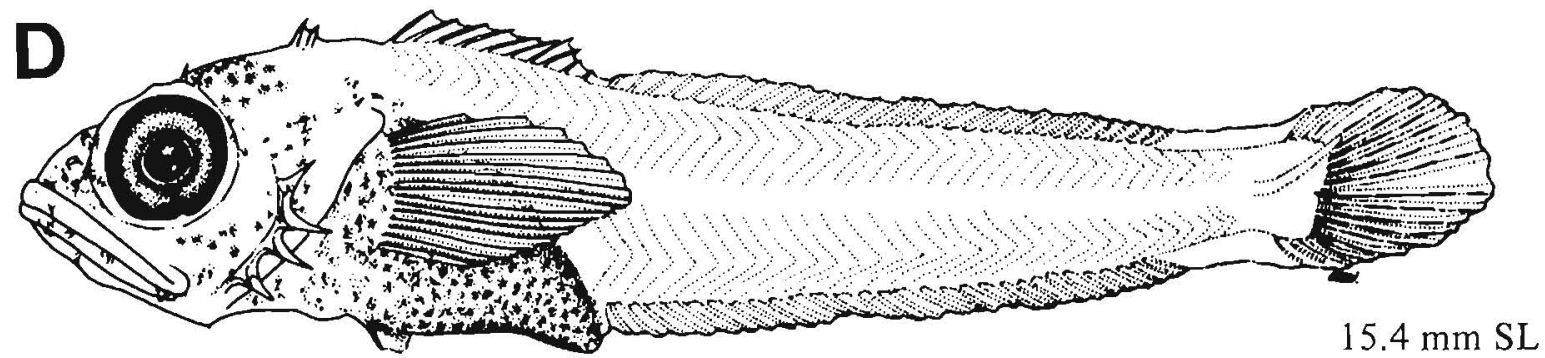

Figures $\mathrm{A}-\mathrm{B}$, Washington, unpubl, $\mathrm{C}-\mathrm{D}, \mathrm{R}$ ichardson and Washington 1980. 


\section{MERISTICS}

\begin{tabular}{|c|c|c|}
\hline \multirow[t]{3}{*}{ Vertebrae } & \multicolumn{2}{|c|}{ Total: $32-33-35$} \\
\hline & \multicolumn{2}{|c|}{ Precaudal: $11-\mathrm{X}-12^{\mathrm{a}}$} \\
\hline & \multicolumn{2}{|c|}{ Caudal: $22^{a}-X-23$} \\
\hline Branchiostegal rays & \multicolumn{2}{|l|}{$6-6-6$} \\
\hline Caudal fin & \multicolumn{2}{|l|}{$x, 6+6, x$} \\
\hline Pelvic fin & \multicolumn{2}{|l|}{ Thoracic } \\
\hline & S: $1-1-1$ & $\mathrm{R}: 3-3-3$ \\
\hline Dorsal fin & S: $8-9-10$ & R: $16-17-18$ \\
\hline Pectoral fin & $R: 14-15-16$ & \\
\hline Anal fin & R: $12-13-14$ & \\
\hline Gill rakers & U: $1-1-1$ & L: $4-X-5$ \\
\hline
\end{tabular}

\section{LIFE HISTORY}

\begin{tabular}{|c|c|}
\hline Range & $\begin{array}{l}\text { Cent. California, } 34-38^{\circ} \mathrm{N} \text {, to } \\
\text { Aleutian Is., } 51-55^{\circ} \mathrm{N}\end{array}$ \\
\hline Ecology & $\begin{array}{l}\text { Nearshore shelf demersal, } \\
\text { intertidal to } 55 \mathrm{~m}\end{array}$ \\
\hline ELH pattern & $\begin{array}{l}\text { Oviparous; demersal, adhesive, } \\
\text { attached eggs; pelagic larvae }\end{array}$ \\
\hline \multirow{4}{*}{ Spawning } & Season: Feb-May ${ }^{b}$ \\
\hline & Area: Intertidal, under rocks ${ }^{b}$ \\
\hline & $\begin{array}{l}\text { Mode: Polygamous males, eggs } \\
\text { laid in nests }{ }^{b}\end{array}$ \\
\hline & Migration: \\
\hline Fecundity & Range/function: \\
\hline Age at first maturity & \\
\hline
\end{tabular}

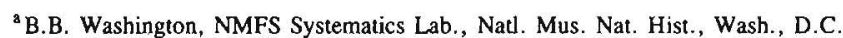
20560, unpubl.

${ }^{\mathrm{b}}$ Marliave 1975a

${ }^{c}$ Pigment patterns within the genus Artedius appear to be highly variable. Reared specimens vary between hatches and stages (J. Marliave, Vancouver Public Aquarium, P.O. Box 3232, Vancouver, B.C., Canada V6B 3X8, pers. commun., 16 Oct. 1986). Wild specimens collected from Yaquina Bay, Oregon, show variations from the pigment patterns described in Washington (1986) (B. Mundy, NMFS Southwest Fish. Cent., Honolulu Lab., 2570 Dole St., Honolulu, HI 96822, pers. commun., 1 Oct. 1986).
}

Ref: Marliave 1975a; Richardson and Washington 1980 (see Aredius 2); Washington 1981,1986

\section{EARLY LIFE HISTORY DESCRIPTION}

\section{EGGS}

Diameter

No. of oil globules

Oil globule diameter

Yolk

Envelope

Hatch size

Homogeneous; blue, purple, or gray

Incubation time/temp.

Pigment

Diagnostic characters

\section{LARVAE}

$\begin{array}{ll}\text { Preanal length } & <50 \% \mathrm{SL} \\ \text { Length at flexion } & 5.9-6.8 \mathrm{~mm} \mathrm{NL} \\ \begin{array}{l}\text { Length at transformation } \\ \text { Sequence of fin } \\ \text { development }\end{array} & \begin{array}{l}\text { Caudal, dorsal and anal } \\ \text { rays, dorsal spines, } \\ \text { pectorals, pelvics }\end{array}\end{array}$

\section{Pigment ${ }^{\mathrm{c}}$}

- Lack of head pigment

- Usually relatively light nape pigment present

- 13-19 postanal ventral midline melanophores

\section{Diagnostic characters}

Distinguished from other cottids by

- Body shape stubby, humped

- Presence of gut diverticula

- Preopercular spines (postflexion, 18-22)

- Parietal spines (postflexion)

Distinguished from other Artedius spp. with gut diverticula by

\begin{tabular}{|c|c|c|c|c|}
\hline & \multicolumn{2}{|c|}{ Preflexion } & \multicolumn{2}{|c|}{ Flexion } \\
\hline & $\begin{array}{c}\text { No. of } \\
\text { postanal ventral } \\
\text { melanophores }\end{array}$ & $\begin{array}{l}\text { Presence of } \\
\text { nape } \\
\text { pigment }\end{array}$ & $\begin{array}{l}\text { Presence of } \\
\text { brain } \\
\text { pigment }\end{array}$ & $\begin{array}{c}\text { No. of } \\
\text { preopercular } \\
\text { spines }\end{array}$ \\
\hline A. fenestralis & $13-19$ & Irreg. & No & $18-22$ \\
\hline A. lateralis & $22-32$ & No & Yes & $14-16$ \\
\hline Artedius 3 & $9-13$ & Yes & No & $22-24$ \\
\hline
\end{tabular}


B

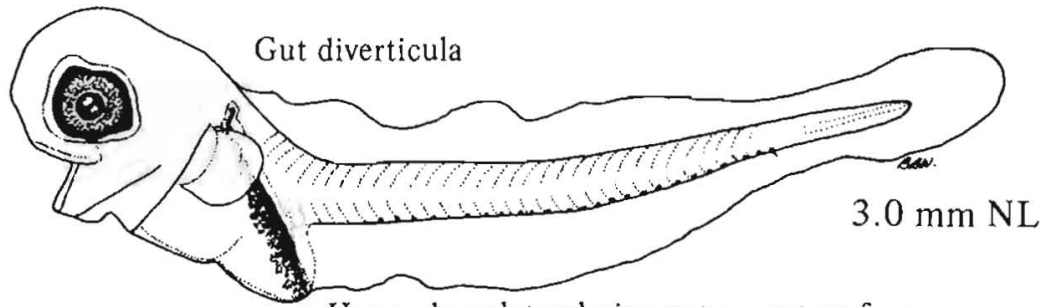

Heavy dorsolateral pigment on gut surface
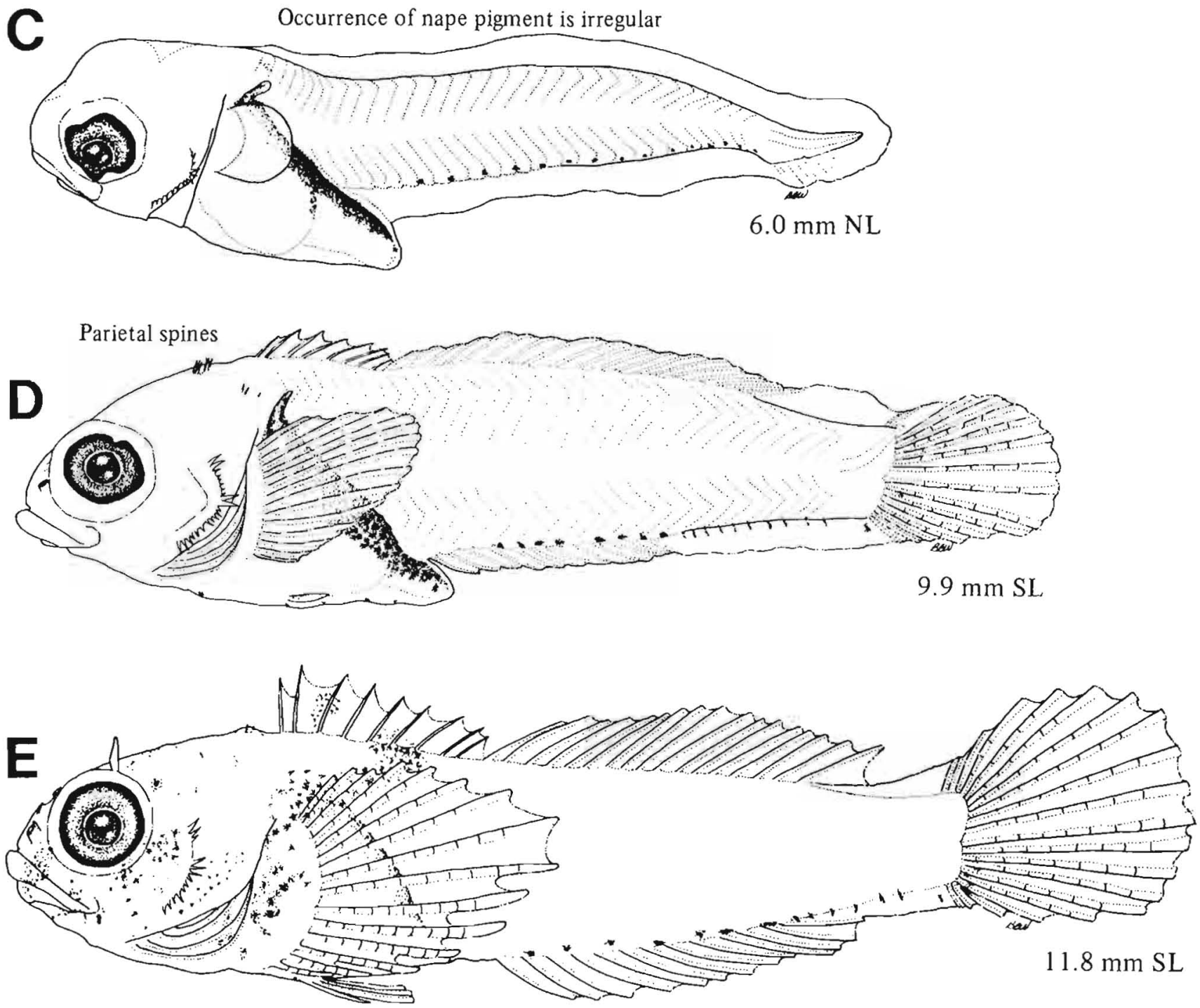

Figure A, Washington 1986; B-E, Richardson and Washington 1980. 
COTTIDAE

\section{MERISTICS}

Vertebrae

Total: $32-33-34$

Precaudal: $11-\mathrm{X}-12$

Caudal: $22-X-23$

Branchiostegal rays

Caudal fin

Pelvic fin

7-7-7

$\mathrm{X}, 6+6, \mathrm{x}$

Thoracic

\section{S: 1-1-1 R: 3-3-3}

Dorsal fin

S: $9-9-9$

R: $15-17-18$

Pectoral fin

R: $13-14-15$

Anal fin

R: $10-13-14$

Gill rakers

$\mathrm{U}: \mathrm{X}-\mathrm{X}-\mathrm{X}$

$\mathrm{L}: \mathrm{X}-\mathrm{X}-\mathrm{X}$

\section{EARLY LIFE HISTORY DESCRIPTION}

\section{EGGS}

Diameter

No. of oil globules

Oil globule diameter

Yolk

Homogeneous

Envelope

Hatch size $<3 \mathrm{~mm} \mathrm{SL}$

Incubation time/temp.

Pigment

Diagnostic characters

\section{LIFE HISTORY}

Range

Ecology

ELH pattern

Spawning

Fecundity

Age at first maturity

Longevity
S. California, $32-34^{\circ} \mathrm{N}$, to Aleutian Is., $51-55^{\circ} \mathrm{N}$

Nearshore shelf demersal, intertidal to $21 \mathrm{~m}$

Oviparous, eggs unknown, pelagic larvae

Season: Possibly spring $(\text { California })^{a}$

Area:

Mode: Internal fertilization likelyb

Migration:

Range/function:

\section{LARVAE}

Preanal length

Length at flexion

Length at transformation

Sequence of fin development

pectorals, pelvics

igment (see $A$. fenestralis, p. 414 , footnote c)

- Lack of head pigment

- Presence of nape pigment

- 21-33 postanal ventral midline melanophores

Diagnostic characters

Distinguished from other Artedius spp. (A. fenestralis, A. lateralis, and Artedius 3) by

- Pigment (see above)

- Absence of gut diverticula

- Humped appearance in nape region

- Preopercular spines: 18-22 (late flexion, postflexion)

- Seven branchiostegal rays

\footnotetext{
${ }^{a}$ Burge and Schultz 1973
}

'NWAFC unpubl.

Ref: Richardson and Washington 1980; Washington 1981, 1986 
B

C
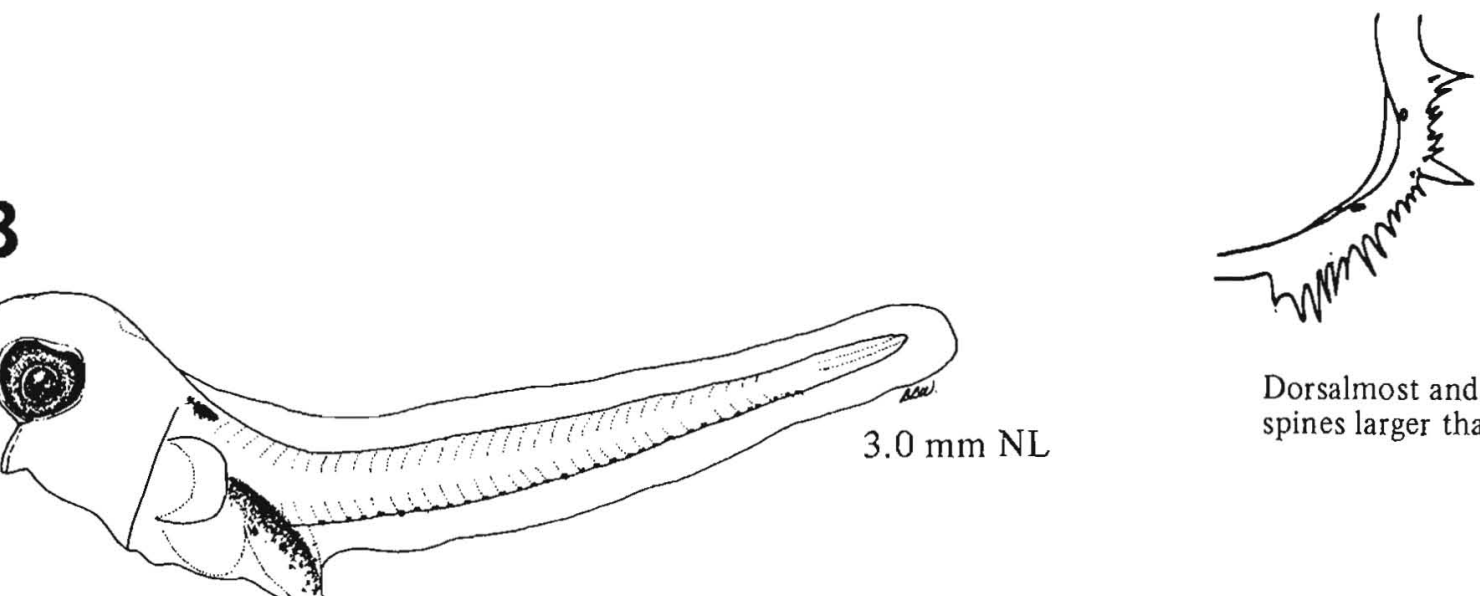

Dorsalmost and middle spines larger than rest

Nape pigment
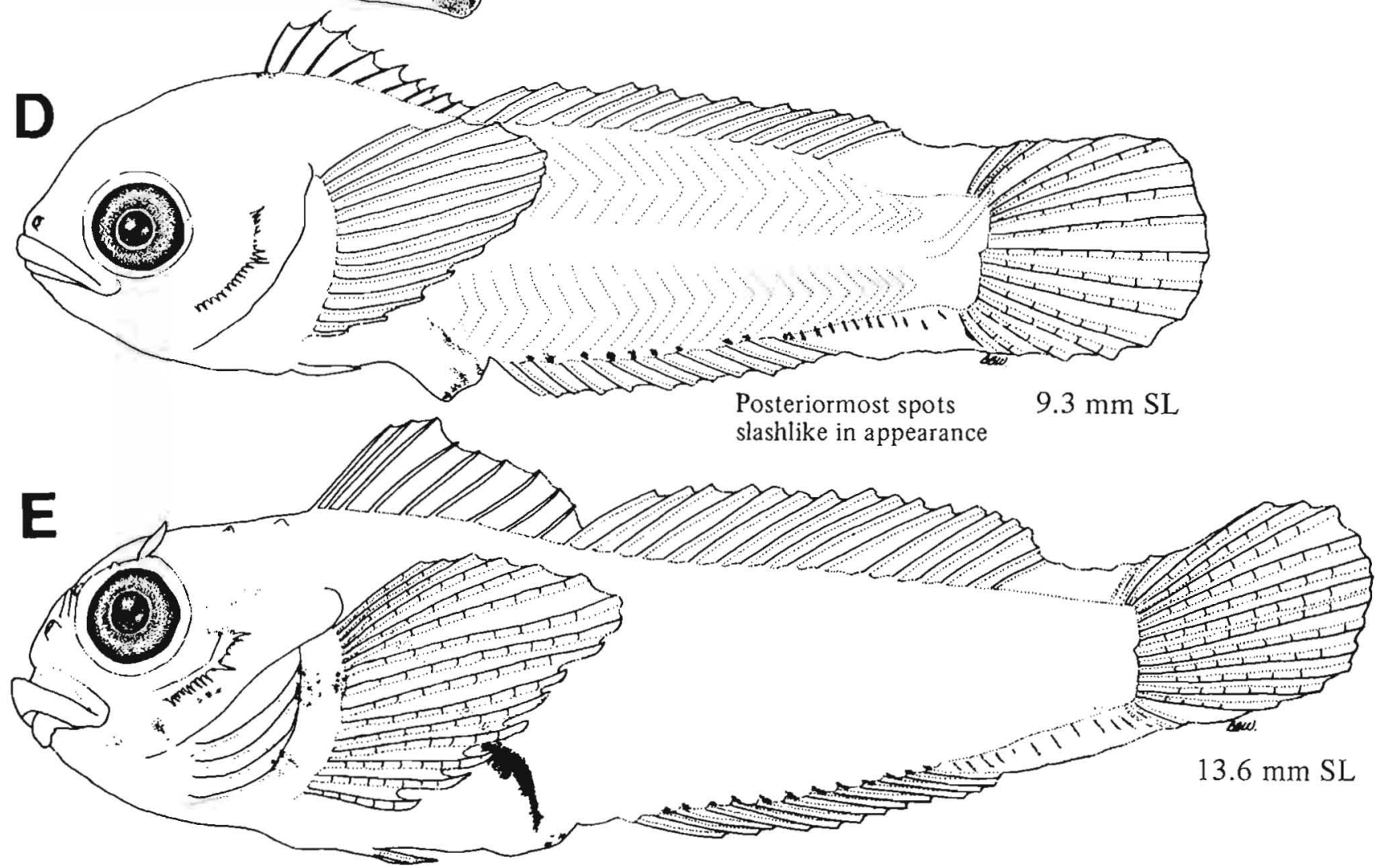

Figure A, Washington 1986; B-E, Richardson and Washington 1980. 


\section{MERISTICS}

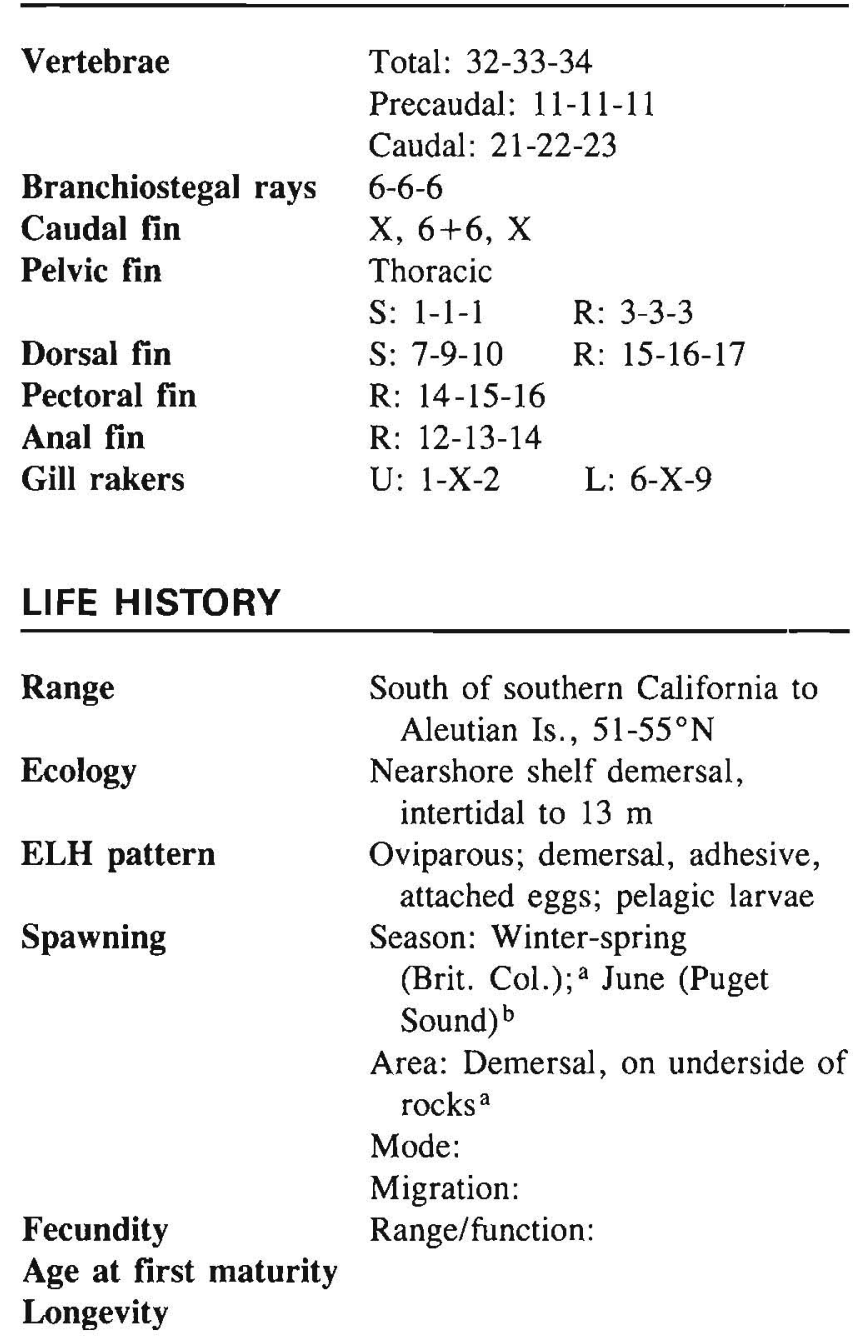

\section{EARLY LIFE HISTORY DESCRIPTION}

\section{EGGS}

\section{Diameter}

No. of oil globules

Oil globule diameter

Yolk

Envelope

Hatch size

Incubation time/temp.

Pigment

\section{Diagnostic characters}

\section{LARVAE}

Preanal length Length at flexion $<50 \%$ SL 5.0-6.3 mm SL Length at transformation $8-10 \mathrm{~mm} \mathrm{SL}$ Sequence of fin Caudal, dorsal and anal development rays, dorsal spines, pectorals, pelvics

Pigment (see A. fenestralis, p. 414, footnote c)

- Lack of head and nape pigment is preflexion larvae

- Number (22-32) and size variation of postanal ventral midline melanophores

- Postflexion larvae have a marked increase in head pigment

Diagnostic characters

- Presence of gut diverticula

- Pigment (see above)

Distinguished from other Artedius spp. with gut diverticula (A. fenestralis and Artedius 3 ) by

- See A. fenestralis

\footnotetext{
${ }^{a}$ Marliave 1977
}

${ }^{b}$ NWAFC, unpubl.

Ref: Marliave 1975a; Richardson and Washington 1980; Washington 1981, 1986; Washington et al. 1984b. 
$B$ Lack of head and nape pigment
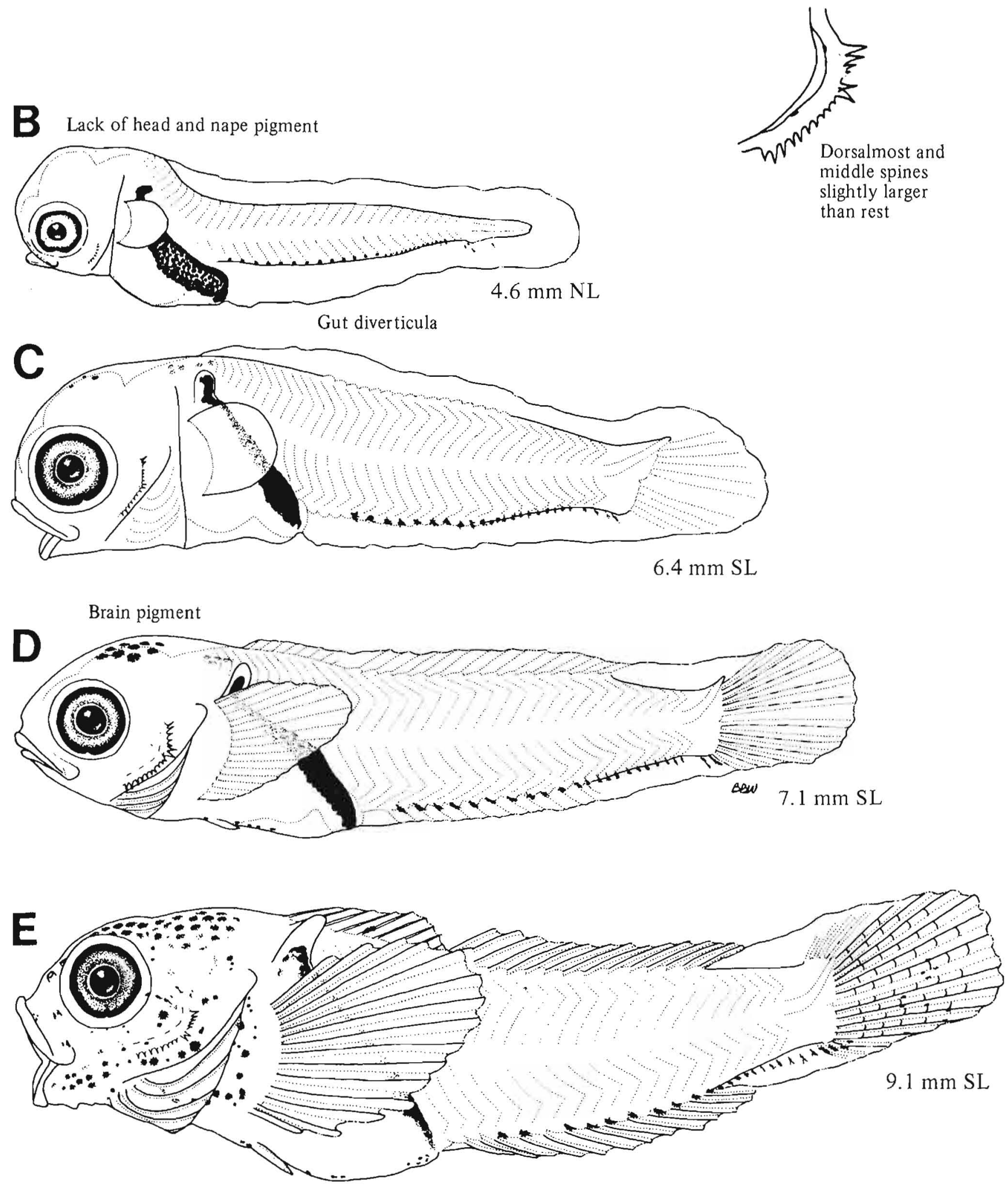

Figures A-E, Washington 1986 . 
COTTIDAE

MERISTICS

A. corallinus (Hubbs 1926)

$\begin{array}{lll}\text { Vertebrae } & \text { Total: } 31-32-33 \\ & \text { Precaudal: X-X-X } \\ & \text { Caudal: } 41-\mathrm{X}-43 \\ \text { Branchiostegal rays } & 6-6-6 & \\ \text { Caudal fin } & \text { X, } 6+6, \mathrm{X} & \\ \text { Pelvic fin } & \text { Thoracic } & \\ & \text { S: } 1-1-1 & \text { R: } 3-3-3 \\ \text { Dorsal fin } & \text { S: } 8-9-9 & \text { R: } 15-16-16 \\ \text { Pectoral fin } & \text { R: } 14-15-16 & \\ \text { Anal fin } & \text { R: } 12-13-13 & \\ \text { Gill rakers } & \text { U: X-X-X } & \text { L: X-X-X }\end{array}$

MERISTICS

A. notospilotus Girard 1856

$\begin{array}{lll}\text { Vertebrae } & \text { Total: } 32-33-34 \\ & \text { Precaudal: } 12-12-12 \\ & \text { Caudal: } 21-21-21 \\ \text { Branchiostegal rays } & 6-6-6 & \\ \text { Caudal fin } & \text { X, } 6+6, \mathrm{X} & \\ \text { Pelvic fin } & \text { Thoracic } & \\ & \text { S: } 1-1-1 & \text { R: } 3-3-3 \\ \text { Dorsal fin } & \text { S: } 9-9-10 \quad \text { R: } 14-15-16 \\ \text { Pectoral fin } & \text { R: } 14-16-17 & \\ \text { Anal fin } & \text { R: } 11-12-13 & \\ \text { Gill rakers } & \text { U: } 2-2-2 & \text { L: } 8-X-10\end{array}$

\section{LIFE HISTORY}

$\begin{array}{ll}\text { Range } & \begin{array}{c}\text { South of southern California to } \\ \text { Washington, 46-48 } 30^{\prime} \mathrm{N} \\ \text { Intertidal, nearshore shelf } \\ \text { demersal } \\ \text { Parity and eggs unknown, } \\ \text { pelagic larvae }\end{array} \\ \text { ELH pattern } & \begin{array}{l}\text { Season: } \\ \text { Area: }\end{array} \\ \text { Spawning } & \text { Mode: } \\ & \text { Migration: } \\ \text { Fecundity } & \text { Range/function: } \\ \text { Age at first maturity } & \\ \text { Longevity } & \end{array}$

\section{EARLY LIFE HISTORY DESCRIPTION}

\section{EGGS}

Diameter

No. of oil globules

Oil globule diameter

Yolk

Envelope

Hatch size

$\leqslant 2.9 \mathrm{~mm} \mathrm{NL}$

Incubation time/temp.

Pigment

Diagnostic characters
LARVAE (Artedius 3)

Preanal length

Length at flexion

Length at transformation

Sequence of fin development
$45 \% \mathrm{SL}$

5.6-6.9 mm NL

Caudal, dorsal and anal rays, dorsal spines and pectorals, pelvics
Pigment (see $A$. fenestralis, p. 414 , footnote c)

- Nape pigment

- 9-13 evenly spaced postanal ventral midline melanophores; begin about 3-4 myomeres posterior to anus

\section{Diagnostic characters}

Distinguished from other Artedius spp. with gut diverticula ( $A$. fenestralis and $A$. lateralis) by

- Low number of postanal ventral midline melanophores in small larvae (9-13)

- Pigment: Lack of head pigment and presence of nape pigment

- Preopercular spines form earlier than other Artedius spp. $(<4.1 \mathrm{~mm} \mathrm{NL})$

- Number of preopercular spines higher than in other Artedius spp.

\footnotetext{
"Artedius 3 larvae are either $A$. corallinus or $A$. notospilotus. For a complete description see Washington (1986).

Ref: Washington 1986.
} 
B

Gut diverticula and

8 nape pigment
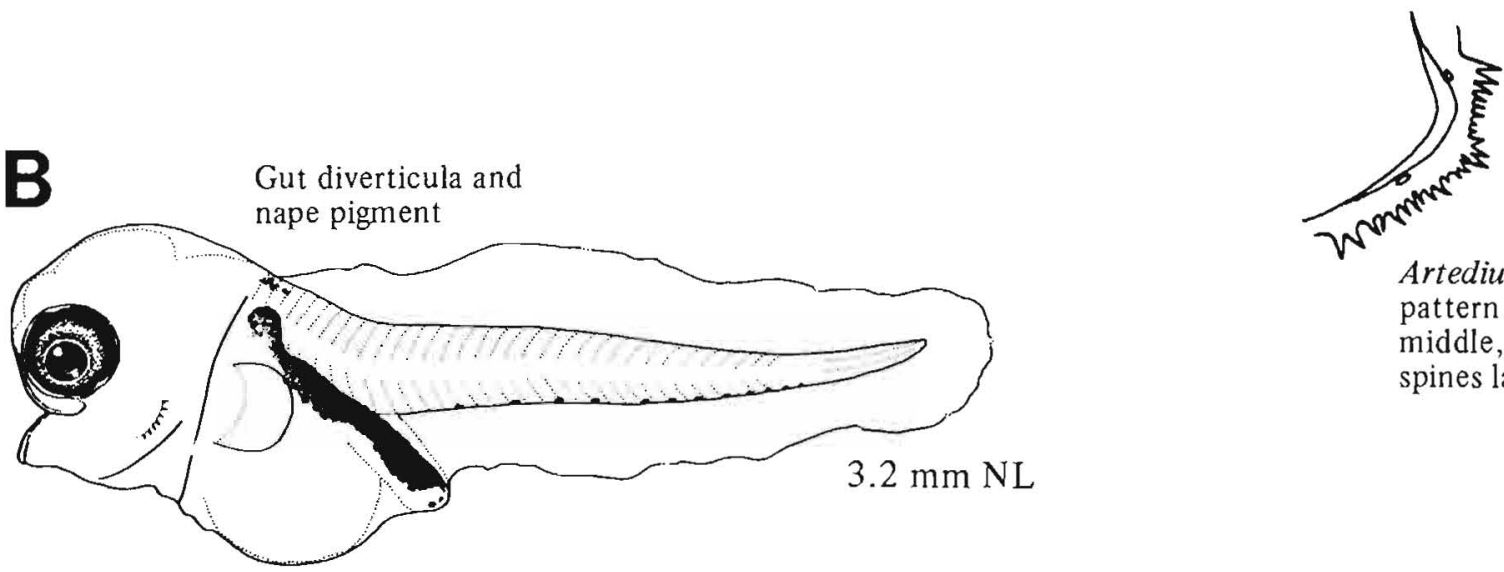

Artedius type spine

pattern: dorsalmost, middle, and ventralmost spines larger than rest
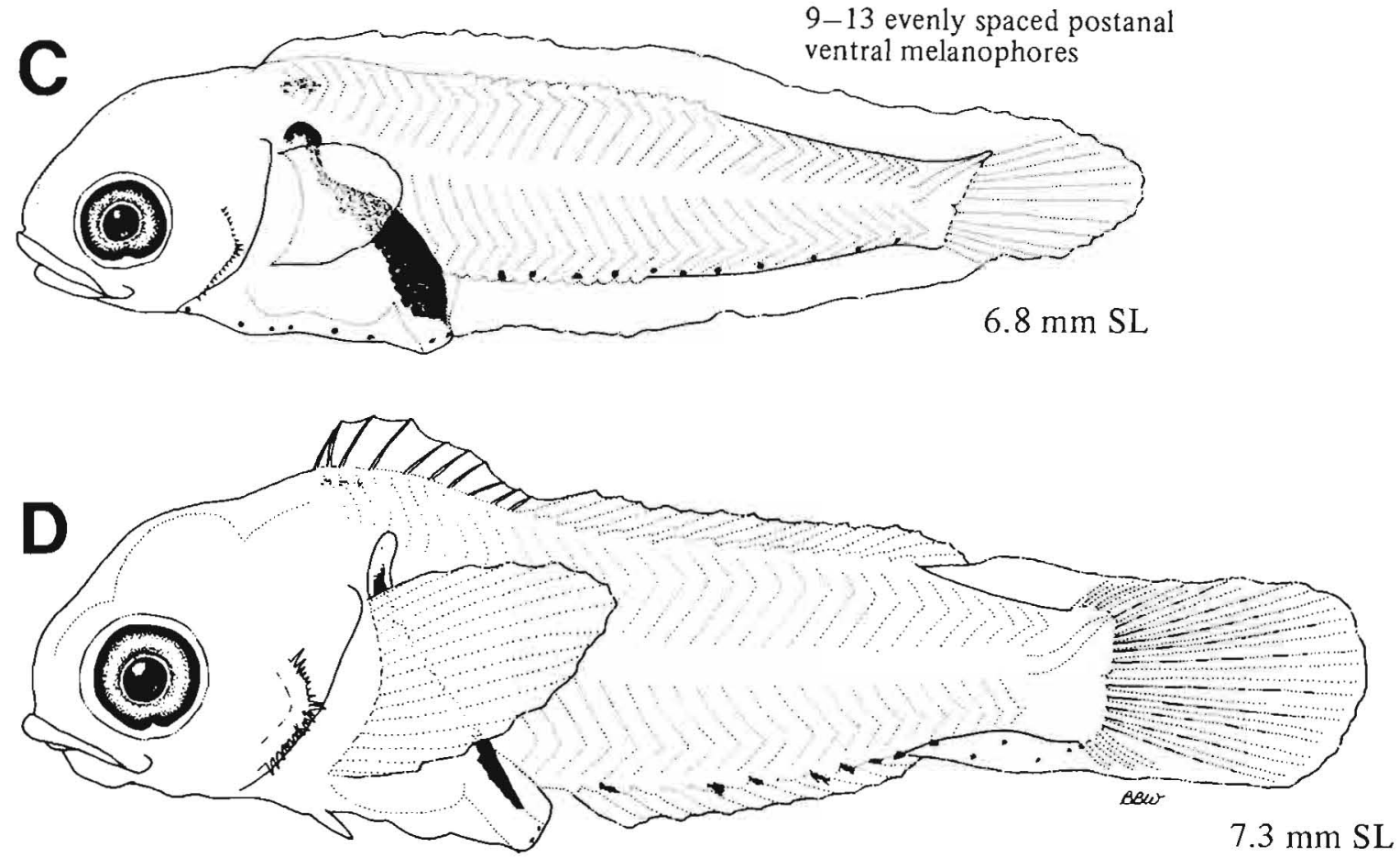


\section{MERISTICS}

\begin{tabular}{|c|c|}
\hline Vertebrae & $\begin{array}{l}\text { Total: } 31-32-33 \\
\text { Precaudal: } 10-\mathrm{X}-11^{\mathrm{a}} \\
\text { Caudal: } 21-22-23\end{array}$ \\
\hline $\begin{array}{l}\text { Branchiostegal rays } \\
\text { Caudal fin }\end{array}$ & $6-6-6$ \\
\hline Pelvic fin & $\begin{array}{l}\text { Thoracic } \\
S: 1-1-1\end{array}$ \\
\hline Dorsal fin & R: $13-15-17$ \\
\hline Pectoral fin & R: $13-14-15$ \\
\hline Anal fin & R: 9-12-13 \\
\hline Gill rakers & U: X-X-X L: X-X-X \\
\hline \multicolumn{2}{|l|}{ LIFE HISTORY } \\
\hline Range & $\begin{array}{l}\text { Cent. California, } 34-38^{\circ} \mathrm{N} \text {, to } \\
\text { Bering Sea, } 54-66^{\circ} \mathrm{N}\end{array}$ \\
\hline Ecology & $\begin{array}{l}\text { Intertidal, nearshore shelf } \\
\text { demersal }\end{array}$ \\
\hline ELH pattern & $\begin{array}{l}\text { Oviparous, demersal eggs, } \\
\text { pelagic larvae }\end{array}$ \\
\hline Spawning & Season: Spring \\
\hline & $\begin{array}{l}\text { Area: Vertical rock surfaces } \\
\text { under Fucus in upper intertidal } \\
\text { zone (laid in monolayer) }\end{array}$ \\
\hline & \multirow{2}{*}{$\begin{array}{l}\text { Mode: Internal fertilization } \\
\text { likely }{ }^{c} \\
\text { Migration: }\end{array}$} \\
\hline & \\
\hline $\begin{array}{l}\text { Fecundity } \\
\text { Age at first maturity } \\
\text { Longevity }\end{array}$ & Range/function: \\
\hline
\end{tabular}

\section{EARLY LIFE HISTORY DESCRIPTION}

\section{EGGS}

\section{Diameter}

No. of oil globules

Oil globule diameter

Yolk

\section{Envelope \\ Hatch size}

Incubation time/temp.

$$
1.0-1.2 \mathrm{~mm}
$$

Homogeneous; brown or purple

3-4 mm NL

(3.1-3.3 $\mathrm{mm} \mathrm{NL})$

Pigment

Diagnostic characters

\section{LARVAE}

Preanal length

Length at flexion

Length at transformation

Sequence of fin development $\sim 63 \% \mathrm{SL}$

5.5-7.3 mm NL

13-14 mm SL

Caudal, dorsal and anal rays, dorsal spines, pectorals, pelvics

\section{Pigment}

- Dorsal gut surface

- Relatively few (4-10) ventral midline melanophores

\section{Diagnostic characters}

Distinguished from all other cottids by

- Long gut with hindgut diverticula

- Flabby appearance

- Outer bubble of skin

\footnotetext{
a B.B. Washington, NMFS Systematics Lab., Natl. Mus. Nat. Hist., Wash., D.C. 20560, unpubl.

b Marliave 1981a

${ }^{c}$ Andriashev 1954

Ref: Blackburn 1973; Richardson and Washington 1980; Washington 1981, 1986; Washington et al. 1984b.
} 
B

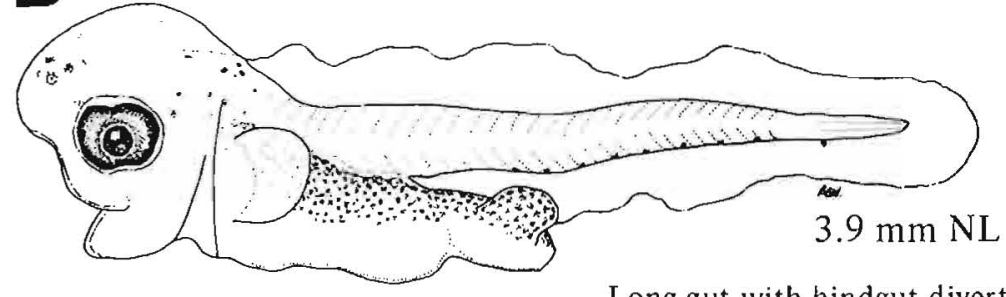

C

Long gut with hindgut diverticula

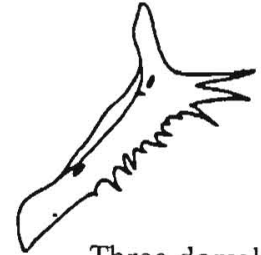

Three dorsalmost spines larger than rest
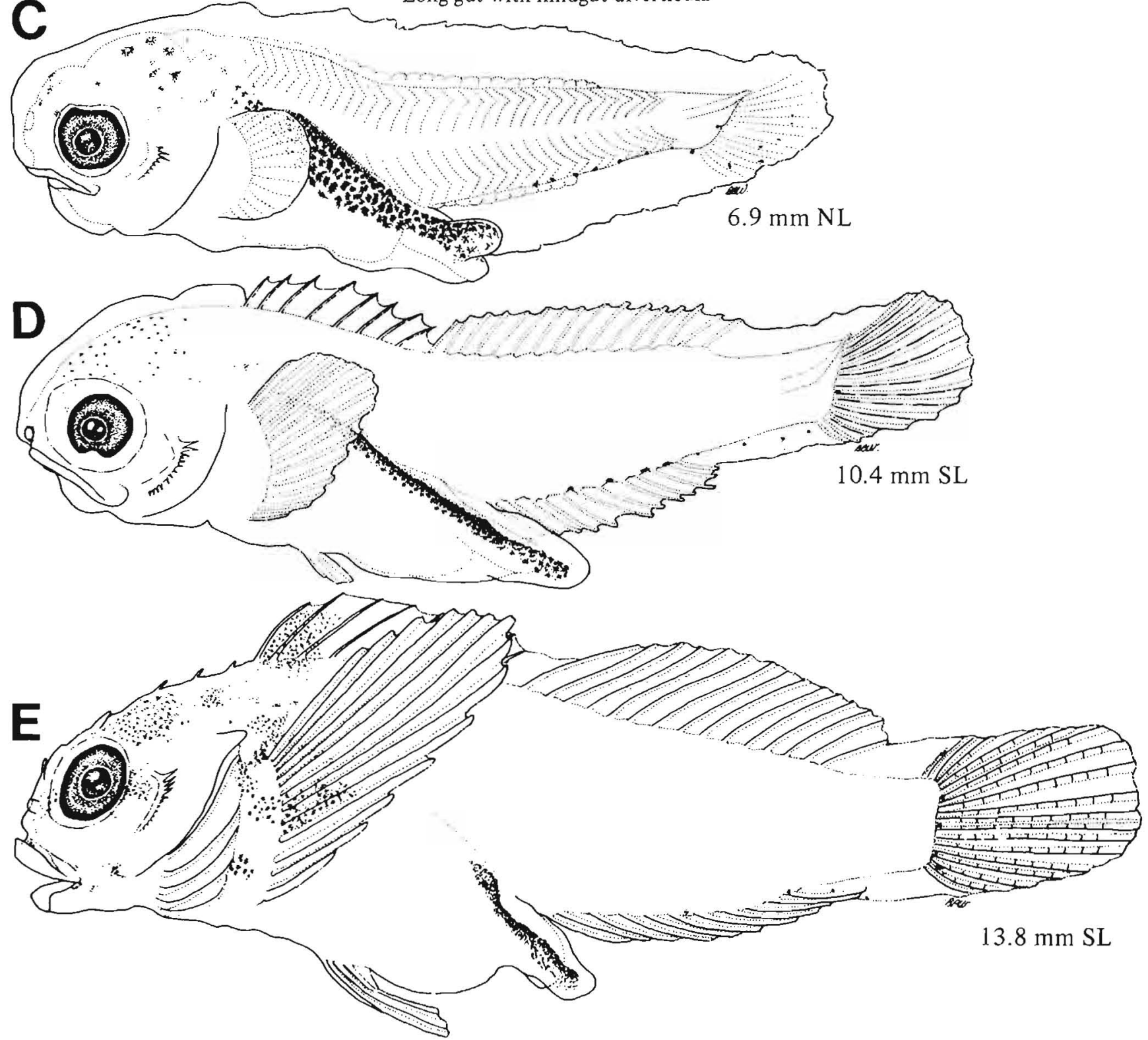

$13.8 \mathrm{~mm} \mathrm{SL}$

Figure A, Washington 1986; B-E, Richardson and Washington 1980. 


\section{MERISTICS}

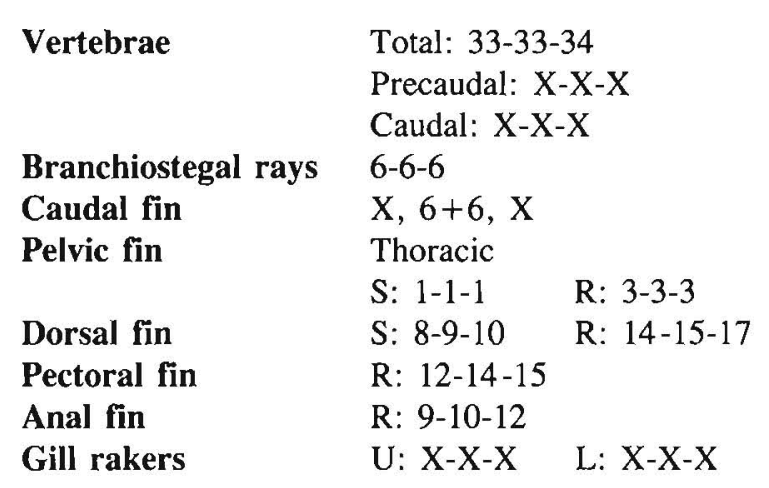

\section{LIFE HISTORY}

\begin{tabular}{|c|c|}
\hline Range & $\begin{array}{l}\text { South of southern California to } \\
\text { Bering Sea, } 54-66^{\circ} \mathrm{N}\end{array}$ \\
\hline Ecology & Intertidal \\
\hline ELH pattern & $\begin{array}{l}\text { Oviparous, demersal eggs, } \\
\text { pelagic larvae }\end{array}$ \\
\hline \multirow[t]{4}{*}{ Spawning } & Season: \\
\hline & Area: \\
\hline & $\begin{array}{l}\text { Mode: Internal fertilization } \\
\text { likely }^{\mathrm{a}}\end{array}$ \\
\hline & Migration: \\
\hline Fecundity & Range/function: \\
\hline Age at first maturity & \\
\hline Longevity & \\
\hline
\end{tabular}

${ }^{a}$ Andriashev 1954

Ref: Richardson and Washington 1980 (see Cottidae 2); Washington 1981, 1996.

\section{EARLY LIFE HISTORY DESCRIPTION}

\section{EGGS}

Diameter

No. of oil globules

Oil globule diameter

Yolk

Homogeneous

Envelope

Hatch size

$\sim 4 \mathrm{~mm} \mathrm{SL}$

Pigment

Diagnostic characters

\section{LARVAE}

Preanal length

Length at flexion

Length at transformation

Sequence of fin development

\section{Pigment}

- Presence of head pigment is variable during development

- Light pigment on gut

- High number ventral midline melanophores (15-21)

Diagnostic characters

- Usually lacks head pigment; although variable, other Clinocottus spp. have head and snout pigment

- Long trailing gut

- Absence of hindgut diverticula

- 11-14 preopercular spines, tiny parietal spine 
B

Usually lacks head

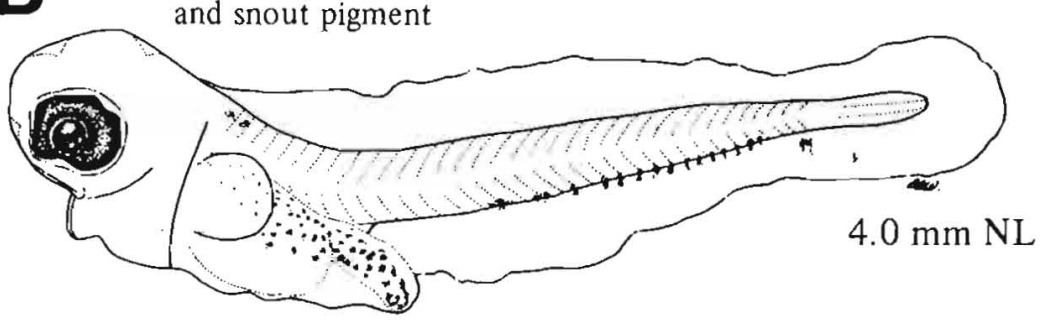

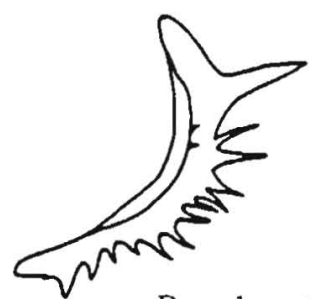

Dorsalmost spines larger than rest

C

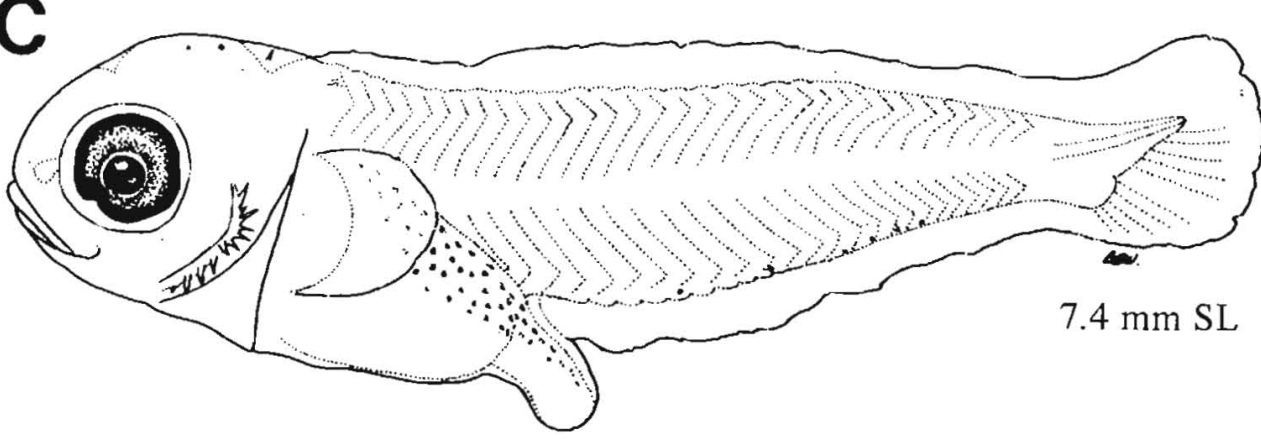

D
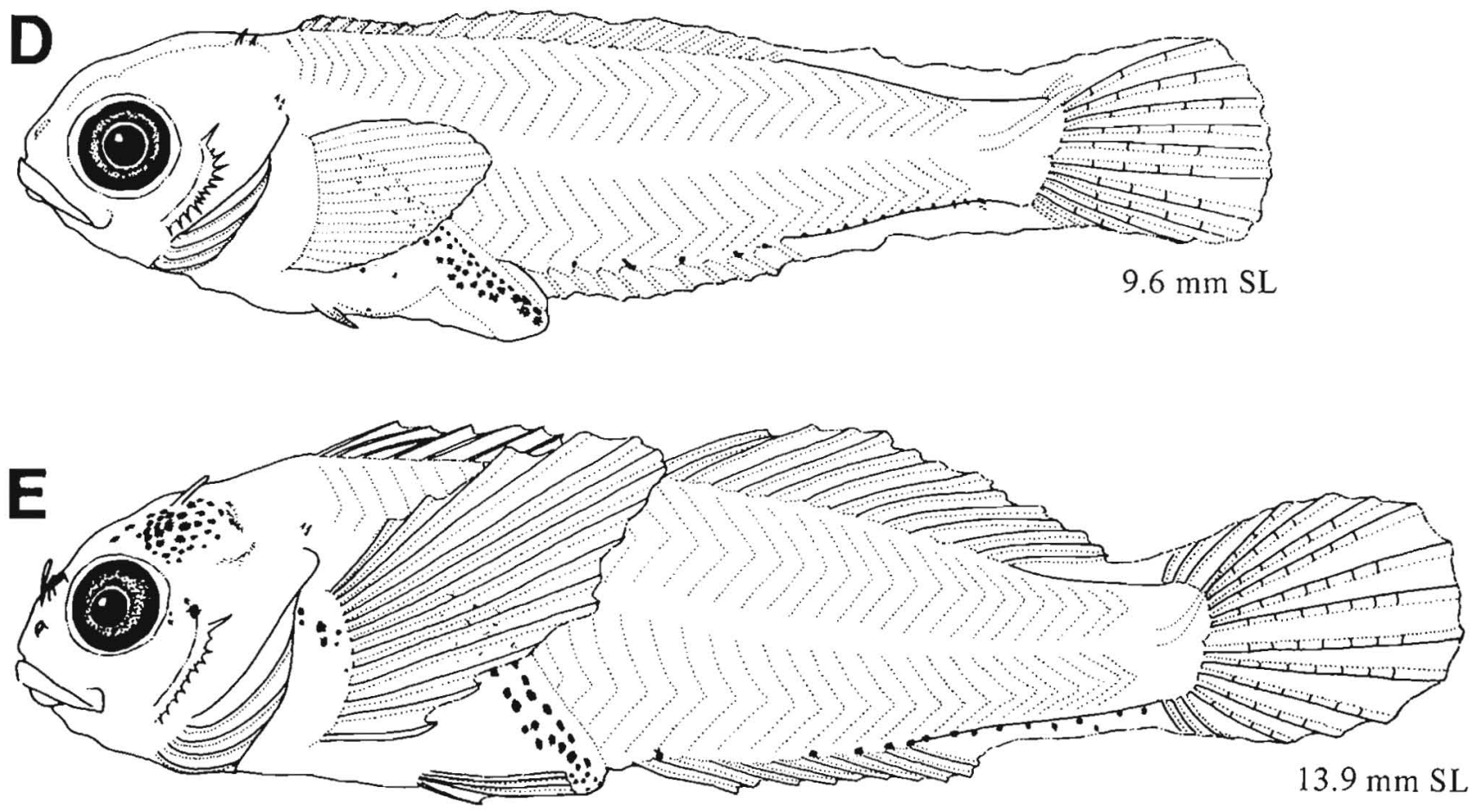

Figures A, D-E, Washington 1986; B-C, Richardson and Washington 1980. 


\section{MERISTICS}

\begin{tabular}{|c|c|c|}
\hline \multirow[t]{3}{*}{ Vertebrae } & \multicolumn{2}{|c|}{ Total: $32-33-34$} \\
\hline & \multicolumn{2}{|c|}{ Precaudal: 11-12-12 } \\
\hline & \multicolumn{2}{|c|}{ Caudal: $20-21-23$} \\
\hline Branchiostegal rays & \multicolumn{2}{|c|}{ 6-6-6 (occasionally 7) } \\
\hline Caudal fin & \multicolumn{2}{|l|}{$X, 6+6, X$} \\
\hline \multirow[t]{2}{*}{ Pelvic fin } & Thoracic & \\
\hline & S: $1-1-1$ & $\mathrm{R}: 3-3-3$ \\
\hline Dorsal fin & S: $8-9-10$ & R: $13-16-17$ \\
\hline Pectoral fin & R: $13-14-15$ & \\
\hline Anal fin & R: $11-11-12$ & \\
\hline Gill rakers & $\mathrm{U}: 1-1-1$ & $L: 5-5-5$ \\
\hline LIFE HISTORY & & \\
\hline
\end{tabular}

$\begin{array}{ll}\text { Range } & \begin{array}{c}\text { S. California, } 32-34^{\circ} \mathrm{N}, \text { to } \\ \text { Gulf of Alaska, } 54-60^{\circ} \mathrm{N} \\ \text { Intertidal } \\ \text { Ecology }\end{array} \\ \begin{array}{l}\text { Oviparous, demersal eggs, } \\ \text { pelagic larvae }\end{array} \\ \text { Spawning } & \begin{array}{l}\text { Season: } \\ \text { Area: } \\ \text { Mode: Internal fertilization } \\ \text { likely }\end{array} \\ & \begin{array}{l}\text { Migration: } \\ \text { Fecundity }\end{array} \\ \text { Age at first maturity } \\ \text { Longevity }\end{array}$

\section{EARLY LIFE HISTORY DESCRIPTION}

\section{EGGS}

Diameter

No. of oil globules

Oil globule diameter

Yolk

Envelope

Hatch size

$1.5-2.0 \mathrm{~mm}$

Incubation time/temp.

Pigment

Homogeneous

5.1-5.4 mm SL

Diagnostic characters

\section{LARVAE}

Preanal length

Length at flexion

Length at transformation

Sequence of fin development

Pigment

- Heavy on head, nape, dorsolateral surface of gut

- Ventral midline melanophores along posteriormost 10 myomeres

Diagnostic characters

- Late flexion: 15-20 preopercular spines and a cluster of spines in parietal region

Distinguished from other preflexion Clinocottus spp. by

- Heavy pigment on head, nape, and gut

Distinguished from $C$. recalvus by

- 4-8 ventral midline melanophores

\footnotetext{
${ }^{a}$ Andriashev 1954
} 
B

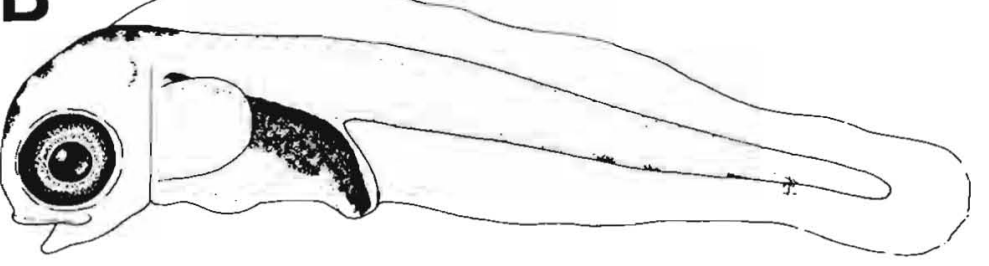

4-8 discrete spots beginning $5.0 \mathrm{~mm} \mathrm{NL}$

$\sim 10$ myomeres from tail

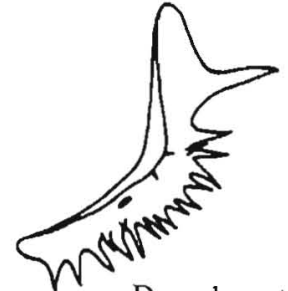

Dorsalmost spines larger than rest

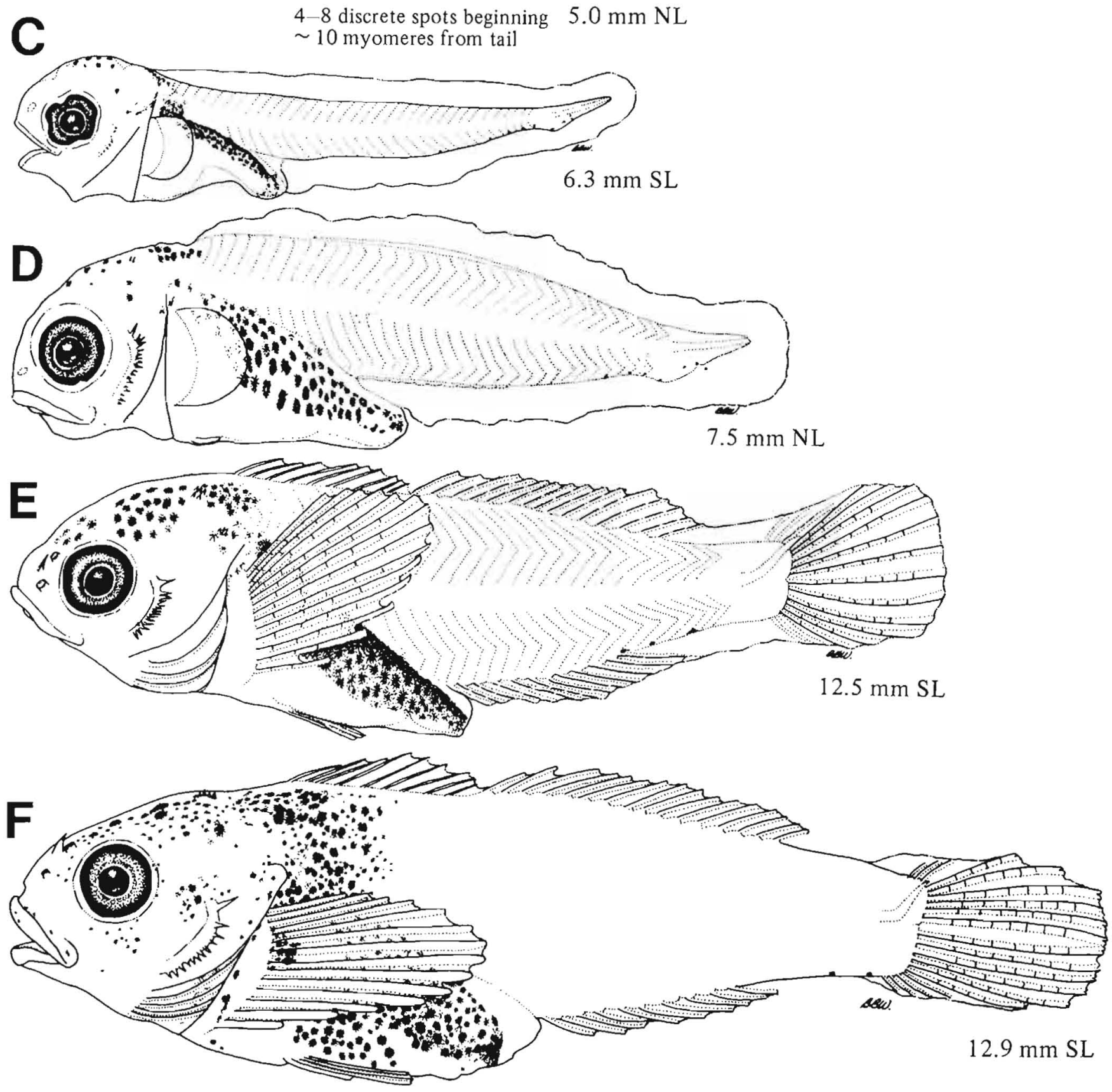

Figures A-B, F, Washington 1986; C-E, Richardson and Washington 1980. 
MERISTICS

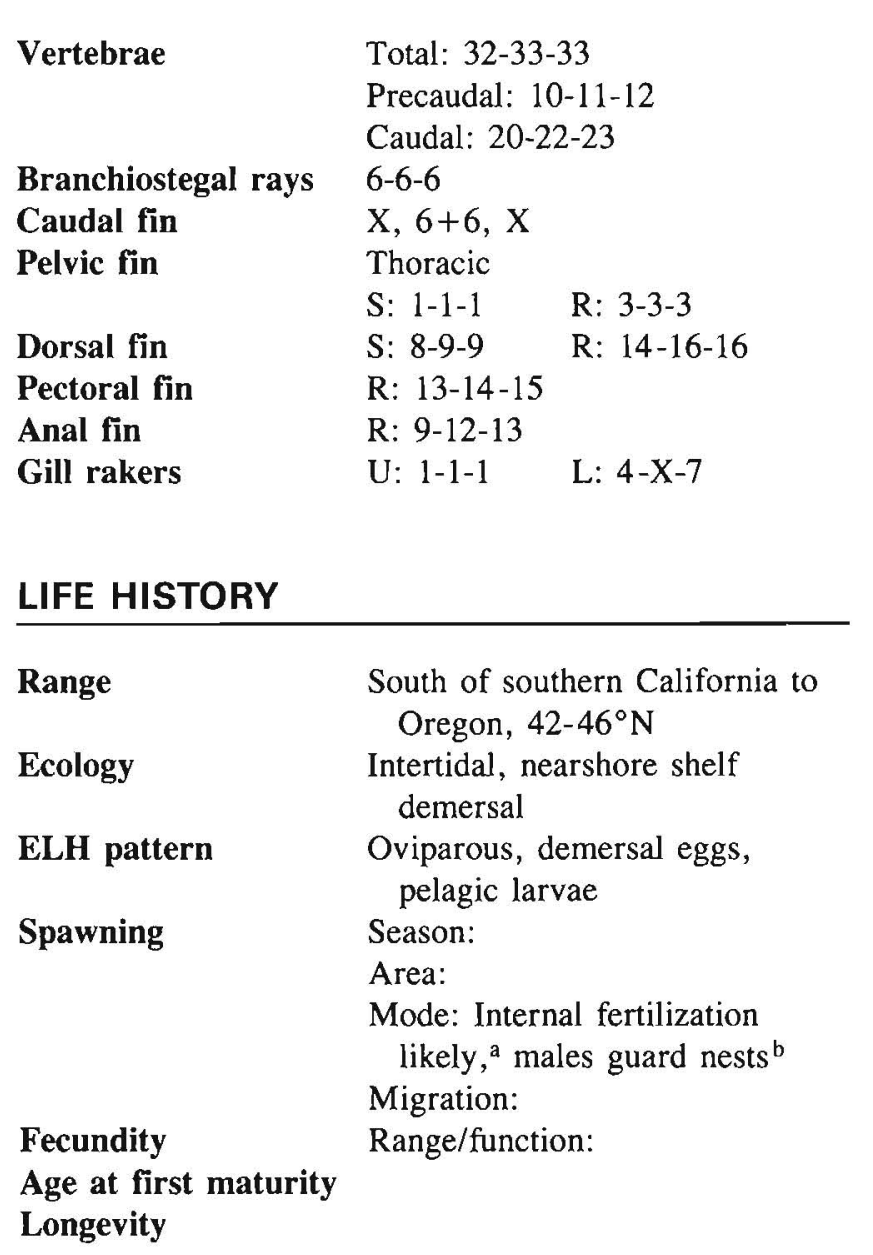

\section{EARLY LIFE HISTORY DESCRIPTION}

\section{EGGS}

Diameter

No. of oil globules

Oil globule diameter

Yolk

Envelope

Hatch size $\quad \sim 4.6 \mathrm{~mm} \mathrm{SL}$

Incubation time/temp.

Pigment
$1.25-1.35 \mathrm{~mm}(1.3 \mathrm{~mm})$

Several

Homogeneous

\section{Diagnostic characters}

\author{
LARVAE \\ Preanal length \\ Length at flexion \\ Length at transformation $9-11 \mathrm{~mm} \mathrm{SL}$ \\ Sequence of fin \\ development \\ Pigment \\ - Heavy on dorsolateral surface of gut \\ - Series of ventral midline melanophores extending \\ into finfold \\ - Heavy pigment on snout, crown, and nape
}

\section{Diagnostic characters}

- Multiple preopercular spines

Distinguished from other Clinocottus spp. by

- Minimal trailing of hindgut

\footnotetext{
${ }^{\text {a }}$ Andriashev 1954

${ }^{\text {b} M o r r i s ~} 1951$

Ref: Morris 1951.
} 

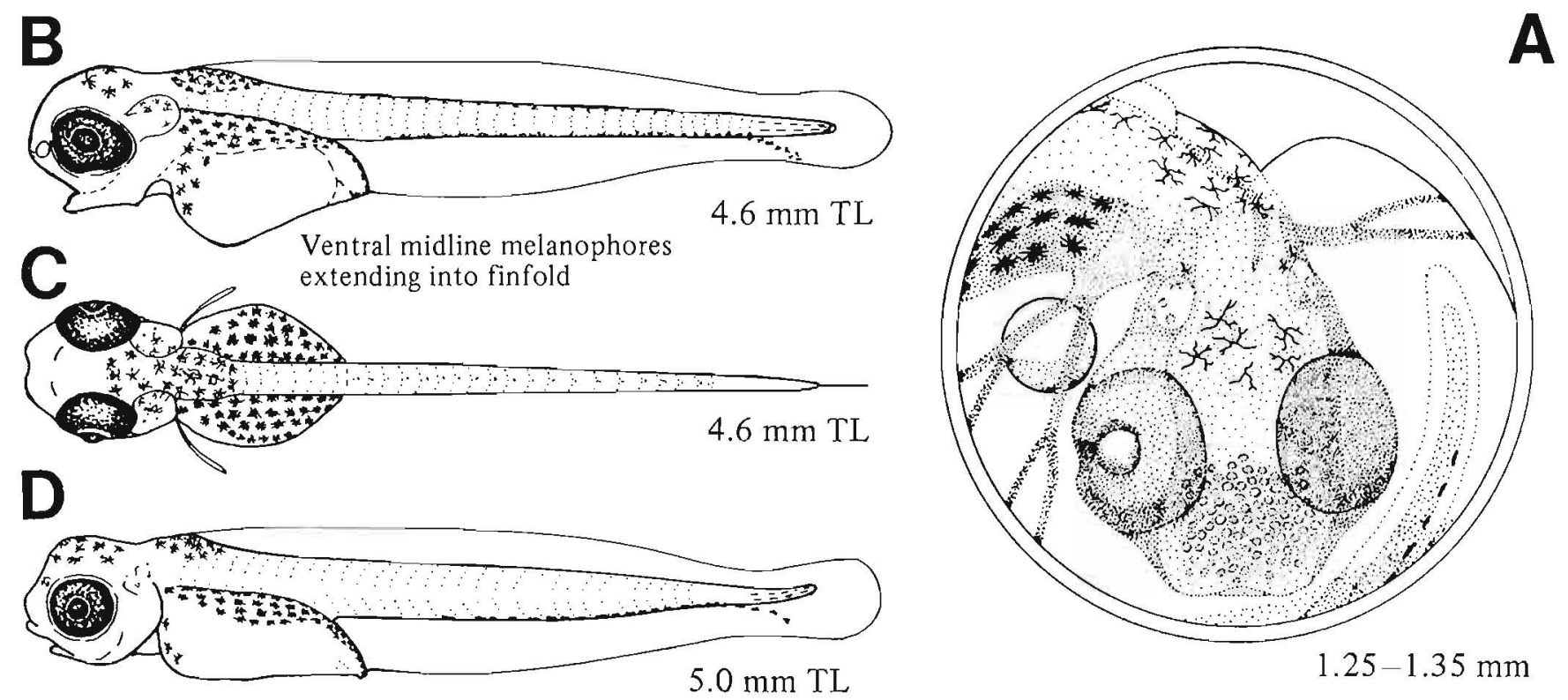

E
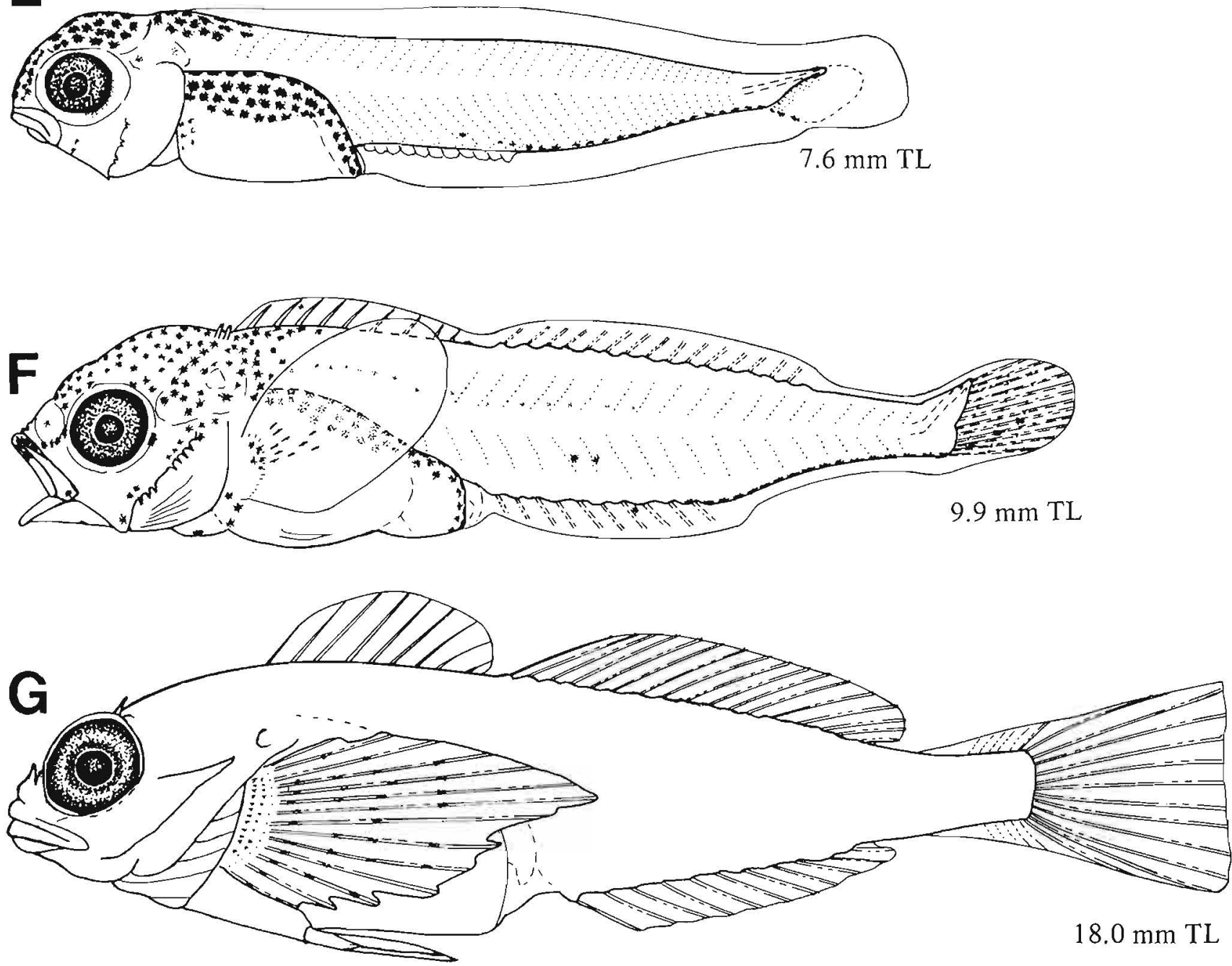

Figures $A-G$ (C, dorsal view), Morris 1951 (B-G, redrawn). Redrawn figures are based only on distribution of melanophores. Other pigment cells shown on the original figures are deleted. 
COTTIDAE

\section{MERISTICS}

\begin{tabular}{|c|c|c|}
\hline Vertebrae & $\begin{array}{l}\text { Total: } 33-34 \\
\text { Precaudal: } 1 \\
\text { Caudal: } 21-2\end{array}$ & $\begin{array}{l}-34 \\
1-12-12 \\
2-23\end{array}$ \\
\hline $\begin{array}{l}\text { Branchiostegal rays } \\
\text { Caudal fin }\end{array}$ & $6-6-6$ & \\
\hline Pelvic fin & $\begin{array}{l}\text { Thoracic } \\
\text { S: } 1-1-1\end{array}$ & R: $3-3-3$ \\
\hline Dorsal fin & S: $8-8-9$ & R: $15-17-18$ \\
\hline Pectoral fin & R: $12-14-15$ & \\
\hline Anal fin & R: $12-13-14$ & \\
\hline Gill rakers & $\mathrm{U}: 1-1-1$ & L: $4-X-5$ \\
\hline
\end{tabular}

LIFE HISTORY

$\begin{array}{ll}\text { Range } & \text { S. California, } 32-34^{\circ} \mathrm{N} \text {, to } \\ \text { Bering Sea, } 54-66^{\circ} \mathrm{N} \\ \text { Ecology } & \text { Intertidal } \\ \text { ELH pattern } & \text { Oviparous; demersal, adhesive } \\ & \text { eggs; pelagic larvae } \\ \text { Spawning } & \text { Season: Apr-July (Puget Sound) } \\ & \text { Area: Intertidal, between rocks, } \\ & \text { barnacles, or bay mussels } \\ & \text { (not attached to substrate) } \\ & \text { Mode: Internal fertilization, } \\ & \text { multiple spawning (three) } \\ & \text { Migration: } \\ \text { Fecundity } & \text { Range/function: } \\ \text { Age at first maturity } & 1 \text { yr }{ }^{\mathrm{a}} \\ \text { Longevity } & \end{array}$

atkinson 1939

${ }^{6}$ J. Marliave, Vancouver Public Aquarium, P.O. Box 3232, Vancouver, B.C., Canada V6B 3X8; pers. commun., 25 Oct. 1988.

"Specimens reared in the laboratory have shown a high degree of variability in pigmentation. Postanal ventral melanophores range from 10 to $>50$. Other characters appear to vary between geographical area, e.g., presence of ventral or lateral gut pigment and the nape bubble (J. Marliave, pers. commun., 25 Oct. 1988).

Ref: Stein 1973; Washington 1981, 1986; Washington et al. 1984b.

\section{EARLY LIFE HISTORY DESCRIPTION}

\section{EGGS}

\section{Diameter}

No. of oil globules

Oil globule diameter

Yolk

Envelope

Hatch size

1.3-1.5 mm

Incubation time/temp.

One large, many small

Green, brown, or red ${ }^{b}$

$4.2-4.5 \mathrm{~mm} \mathrm{NL}$

\section{Diagnostic characters}

\section{LARVAE}

Preanal length

Length at flexion

Length at transformation

Sequence of fin development

Pigment $^{\mathrm{c}}$

- Head and nape

- Dorsolateral surface of gut

- Postanal ventral midline series, with development becoming more closely spaced posteriorly

Diagnostic characters

- Bubble of skin anterior to origin of dorsal finfold

Distinguished from $O$. snyderi by

- Ventral midline melanophores $(>15)$

- Bubble of skin pigmented

Eggs and larvae of $O$. rimensis are unknown. The following information will aid in identification.

$\begin{array}{lr}\text { Total vertebrae } & 34-37 \\ \text { Precaudal } & 11-13 \\ \text { Caudal } & 22-25 \\ \text { Dorsal fin spines } & 8-10 \\ \text { Dorsal fin rays } & 16-19 \\ \text { Anal fin rays } & 13-15 \\ \text { Pectoral fin rays } & 13-15 \\ \text { Pelvic fin rays } & 3\end{array}$

Range

SSC-SE Alaska 

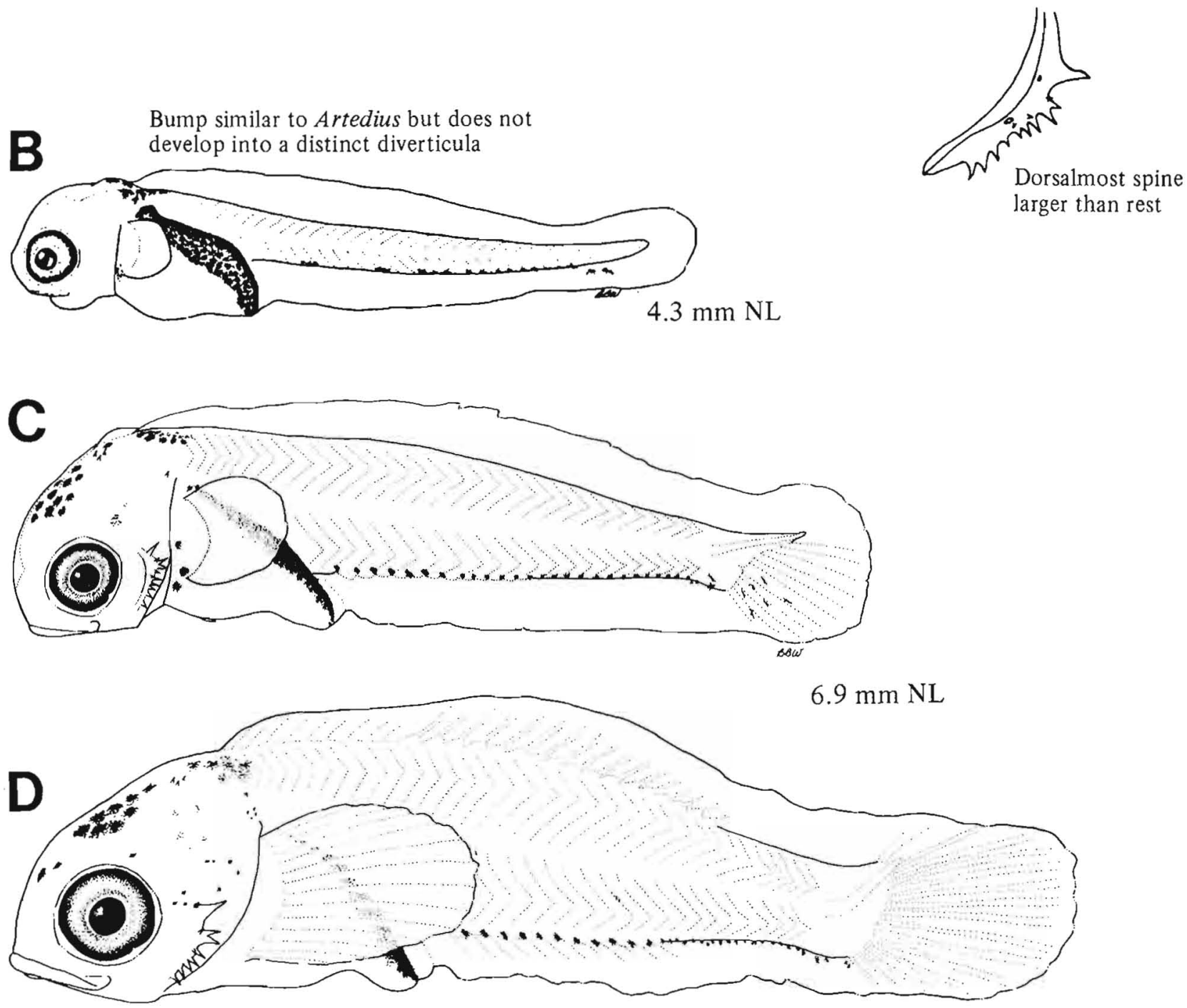

$7.8 \mathrm{~mm} \mathrm{SL}$ 
COTTIDAE

\section{MERISTICS}

$\begin{array}{lll}\text { Vertebrae } & \begin{array}{l}\text { Total: } 34-35-37 \\ \text { Precaudal: } 10-11-12\end{array} \\ & \begin{array}{l}\text { Caudal: } 23-24-25 \\ \text { Branchiostegal rays }\end{array} & 6-6-6 \\ \begin{array}{l}\text { Caudal fin } \\ \text { Pelvic fin }\end{array} & & \\ & \text { Thoracic } & \\ \text { Dorsal fin } & \text { S: } 1-1-1 & \text { R: } 3-3-3 \\ \text { Pectoral fin } & \text { S: } 7-8-9 & \text { R: } 17-19-20 \\ \text { Anal fin } & \text { R: } 12-14-15 & \\ \text { Gill rakers } & \text { R: } 12-14-15 & \\ & \text { U: X-X-X } & \text { L: X-X-X }\end{array}$

\section{LIFE HISTORY}

Range

Ecology

ELH pattern

Spawning

Age at first maturity

Longevity
South of southern California to SE Alaska, $55-59^{\circ} \mathrm{N}$

Intertidal, nearshore shelf demersal

Oviparous, demersal eggs, pelagic larvae

Season: Winter-spring; fall in more northerly populations ${ }^{a}$

Area:

Mode: Internal fertilization (spawn twice/season) $^{a}$

Migration:

Range/function:

$<1 \mathrm{yr}^{\mathrm{a}}$

$>2 \mathrm{yr}^{\mathrm{a}}$

\section{EARLY LIFE HISTORY DESCRIPTION}

\section{EGGS}

Diameter

$1.2-1.3 \mathrm{~mm}$

No. of oil globules

Oil globule diameter

Yolk

Envelope

Hatch size

$4.47 \mathrm{~mm} \mathrm{SL}$

Pigment

Diagnostic characters

\section{LARVAE}

Preanal length

Length at flexion

Length at transformation

Sequence of fin development

Pigment

- Head and nape very lightly pigmented

- Dorsolateral surface of gut

- Ventral midline series with $<10$ evenly spaced melanophores

Diagnostic characters

- Spines

-Patch of parietal spines

-10-12 spines develop along preopercular margin

-8-10 accessory spines form anteriorly at the bases of the preopercular spines

Distinguished from $O$. maculosus by

- Bubble of skin anterior to origin of dorsal finfold unpigmented and less obvious than $O$. maculosus

- Ventral midline melanophores $(<10)$

\footnotetext{
"Grossman and DeVlaming 1984
} 
B
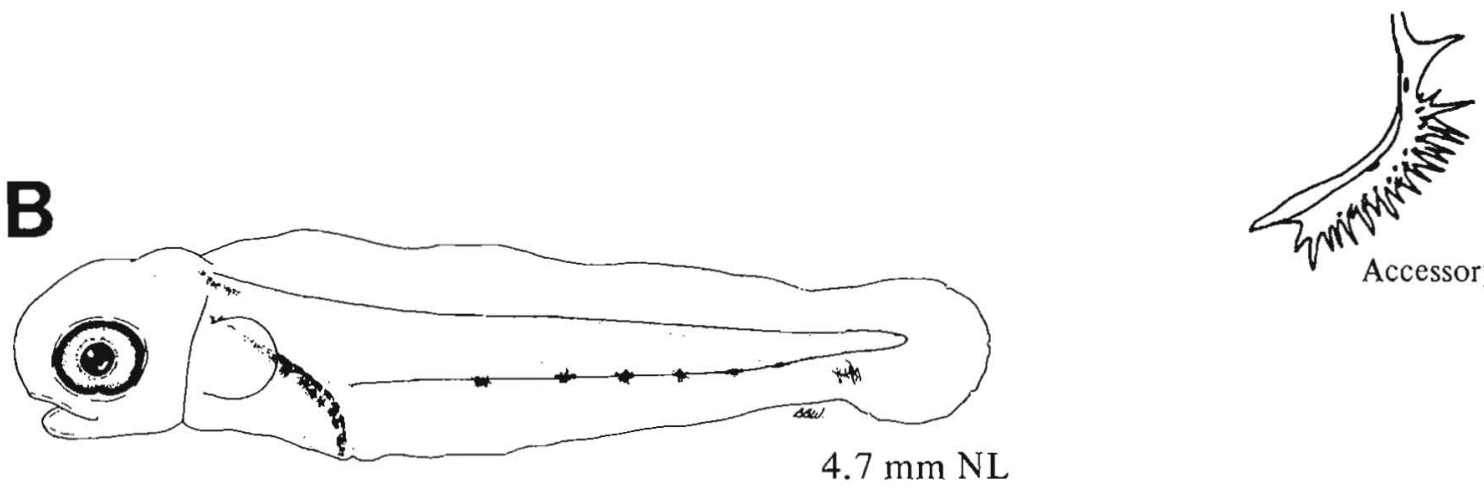

Accessory spines
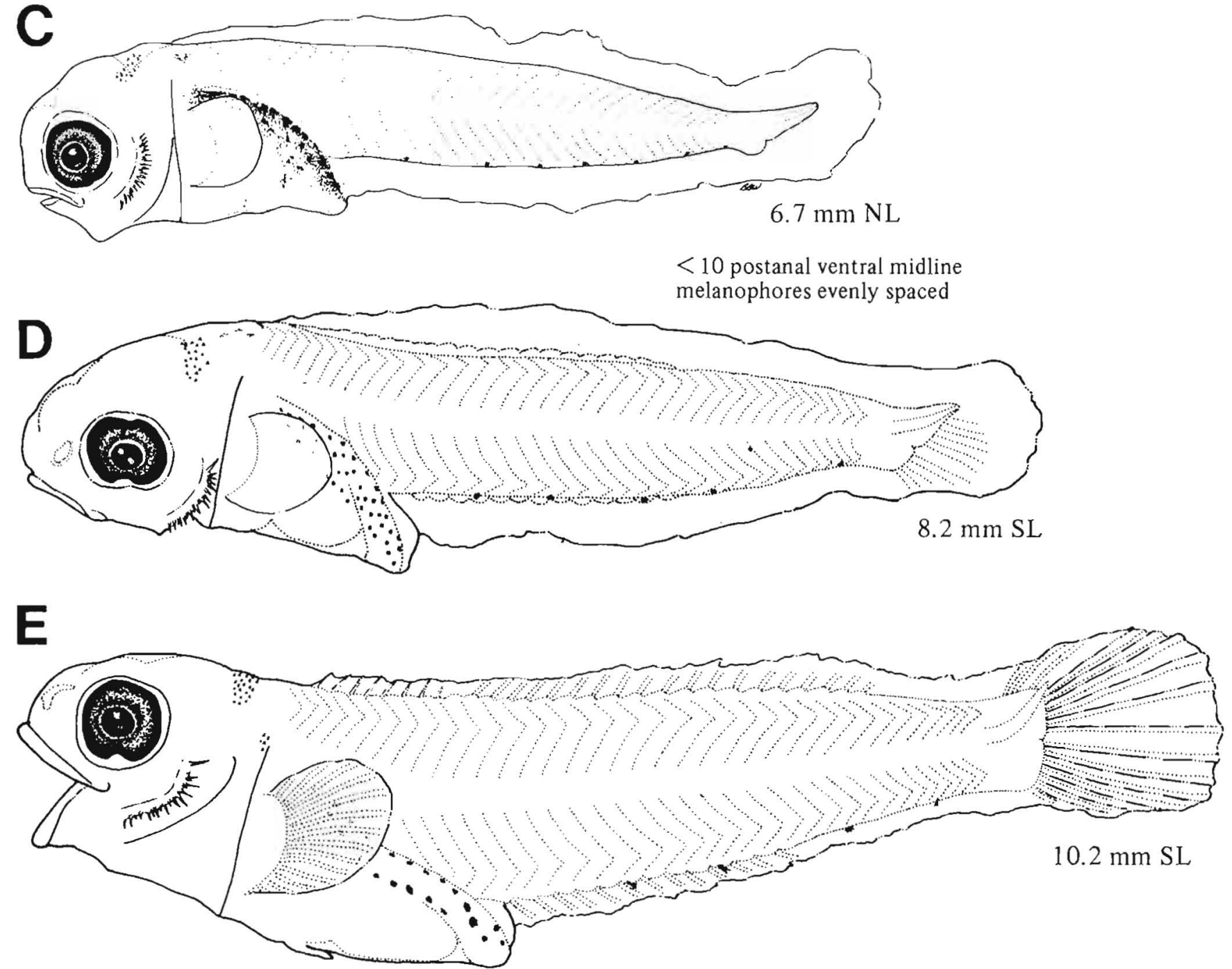

Figures $A-B, D-E$, Washington 1986; C, Richardson and Washington 1980 (B-C, reared). 


\section{MERISTICS}

\section{Vertebrae}

Total: $33-34-35$

Precaudal: $13-13-13^{\text {a }}$

Caudal: $20-20-20^{\text {a }}$

Branchiostegal rays
Caudal fin

Pelvic fin

7-7-7

Thoracic

\section{S: $1-1-1$}

Dorsal fin

S: $7-X-8$

R: $3-3-3$

Pectoral fin

Anal fin

R: $14-16-17$

R: $12-14-15$

Gill rakers
R: $18-X-19$

L: X-X-X

\section{LIFE HISTORY}

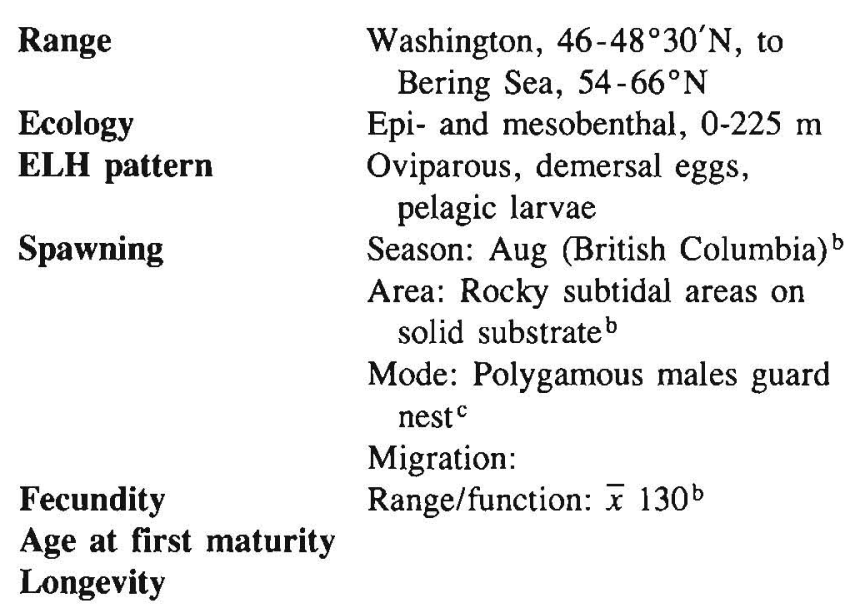

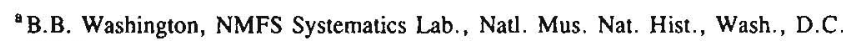
20560, unpubl.

${ }^{\mathrm{b}}$ Marliave 1975a

'J. Marliave, Vancouver Public Aquarium, P.O. Box 3232, Vancouver, B.C., Canada

V6B $3 \times 8$, pers. commun., 16 Oct. 1986.

dJuveniles have a tendency to reenter the water column for feeding, producing a protracted period of ambivalence about settlement (Marliave 1981b).

Ref: Marliave 1975a, Washington et al. $1984 \mathrm{~b}$.

\section{EARLY LIFE HISTORY DESCRIPTION}

\section{EGGS}

Diameter

No. of oil globules

Oil globule diameter

Yolk

Envelope

Hatch size

$\sim 2.3 \mathrm{~mm}$

Incubation time/temp.

Pigment

Diagnostic characters
Homogeneous; pink

White

$\sim 6-7 \mathrm{~mm} \mathrm{SL}$

\section{LARVAE}

Preanal length

Length at flexion

Length at transformation $18-20 \mathrm{~mm} \mathrm{SL}$ (settle) ${ }^{\mathrm{d}}$

Sequence of fin

development

Pigment (Psychrolutes Group)

- Head, nape, gut, and pectoral fins

- Lacks postanal ventral melanophores

\section{Diagnostic characters}

- Morphology

-Tadpole shape

-Large head

- Outer layer of loose flabby skin

- Large pigmented pectoral fins

- No head or preopercular spines

Distinguished from Psychrolutes paradoxus (p. 436) by

- Pectoral fin ray count (14-17)

- Less body pigment 


\section{A}
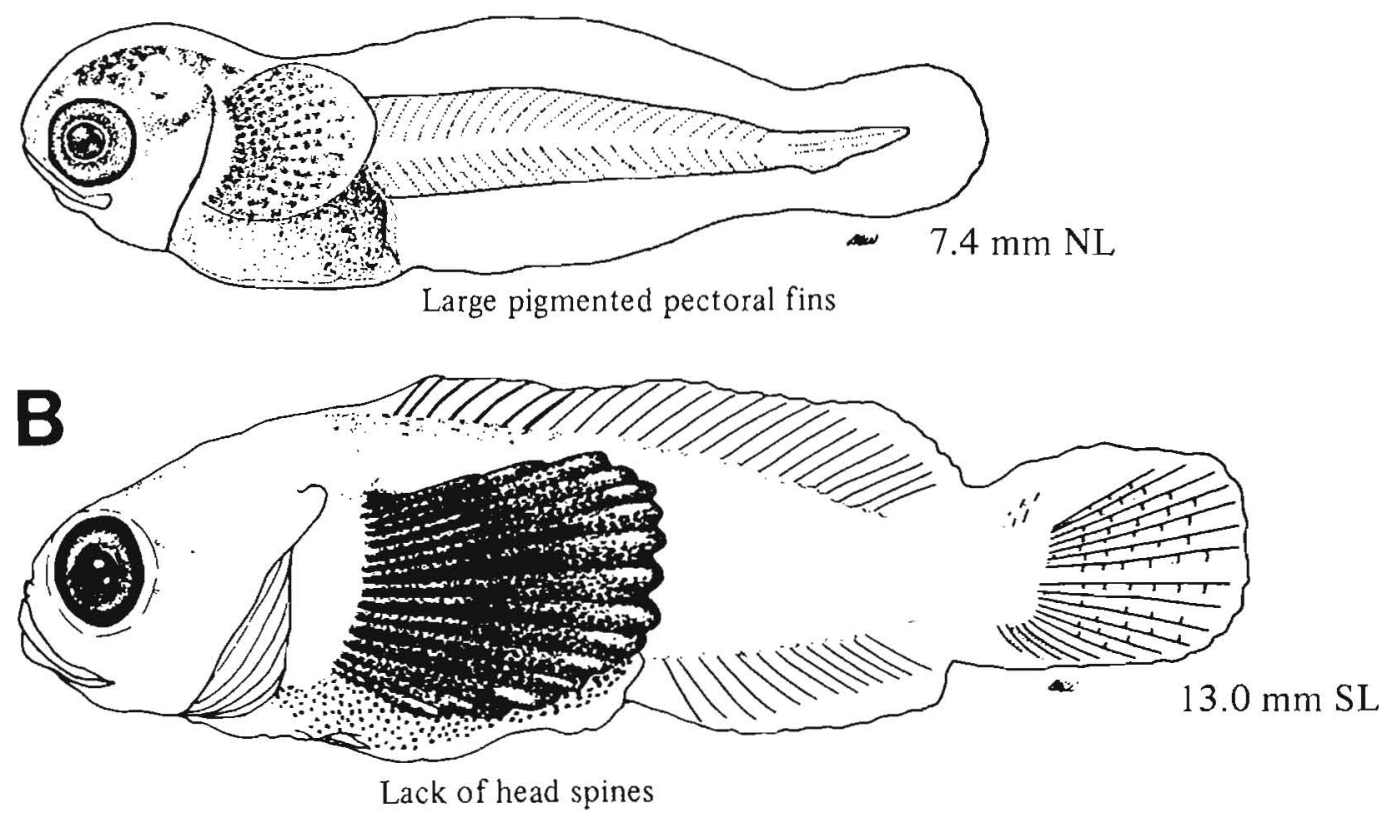

Figure A, Richardson and Bond 1978; B, Richardson $1981 \mathrm{a}$. 
COTTIDAE

\section{MERISTICS}

\begin{tabular}{|c|c|c|}
\hline Vertebrae & \multicolumn{2}{|c|}{$\begin{array}{l}\text { Total: } 36-36-37 \\
\text { Precaudal: } 11-11-11^{\mathrm{a}} \\
\text { Caudal: } 21-21-21^{\mathrm{a}}\end{array}$} \\
\hline $\begin{array}{l}\text { Branchiostegal rays } \\
\text { Caudal fin }\end{array}$ & $7-7-7$ & \\
\hline Pelvic fin & $\begin{array}{l}\text { Thoracic } \\
\text { S: } 1-1-1\end{array}$ & R: $3-3-3$ \\
\hline $\begin{array}{l}\text { Dorsal fin } \\
\text { Pectoral fin } \\
\text { Anal fin }\end{array}$ & $\begin{array}{l}\text { S: } 9-11-12 \\
\text { R: } 19-21-23 \\
\text { R: } 10-12-14\end{array}$ & R: $12-14-17$ \\
\hline $\begin{array}{l}\text { Anal fin } \\
\text { Gill rakers }\end{array}$ & $\begin{array}{l}\text { R: } 10-12-14 \\
\text { U: } X-X-X\end{array}$ & L: X-X-X \\
\hline
\end{tabular}

\section{LIFE HISTORY}

$\begin{array}{ll}\text { Range } & \text { Washington, 46-48 } 30^{\circ} \mathrm{N}, \text { to } \\ \text { Bering Sea, 54-66 } & \mathrm{N} \\ \text { Ecology } & \text { Epi- and mesobenthal, 9-219 m } \\ \text { ELH pattern } & \text { Oviparous, demersal eggs, } \\ & \text { pelagic larvae } \\ \text { Spawning } & \text { Season: Winter-spring (British } \\ & \text { Columbia) } \\ & \text { Area: } \\ & \text { Mode: } \\ & \text { Migration: } \\ \text { Fecundity } & \text { Range/function: } \\ \text { Age at first maturity } & \\ \text { Longevity } & \end{array}$

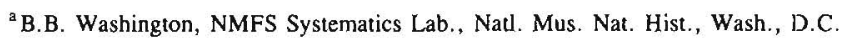
20560, unpubl.

${ }^{\mathrm{b}}$ Marliave $1975 \mathrm{a}$

Ref: Blackburn 1973, Marliave 1975a.

\section{EARLY LIFE HISTORY DESCRIPTION}

\section{EGGS}

Diameter $\sim 1.4 \mathrm{~mm}$

No. of oil globules

Oil globule diameter

Yolk

Envelope

Hatch size

Homogeneous

Incubation time/temp.

$\sim 6-7 \mathrm{~mm} \mathrm{SL}$

Diagnostic characters

\section{LARVAE}

Preanal length

Length at flexion

Length at transformation $\sim 13-14 \mathrm{~mm} \mathrm{SL}$

Sequence of fin development

Pigment

- Initially restricted to head, gut, and upper body (including pectoral fins); with development, increasing to $3 / 4$ body

\section{Diagnostic characters}

- Morphology

-Tadpole shape

- Large head

-Outer layer of loose flabby skin

- Large pigmented pectoral fins

- No head or preopercular spines

Distinguished from Gilbertidia sigalutes (p. 434) by

- More lateral pigment on head and body with development

- Pectoral fin ray count (19-23)

Eggs and larvae of $P$. phrictus are unknown (see Cottoid A, p. 438). The following information will aid in identification.

$\begin{array}{lc}\text { Total vertebrae } & 33-35 \\ \text { Dorsal fin spines } & 8-9 \\ \text { Dorsal fin rays } & 19-20 \\ \text { Anal fin rays } & 12-14 \\ \text { Pectoral fin rays } & 22-26 \\ \text { Pelvic fin rays } & 3\end{array}$

Range Cent. Calif.-Bering Sea 


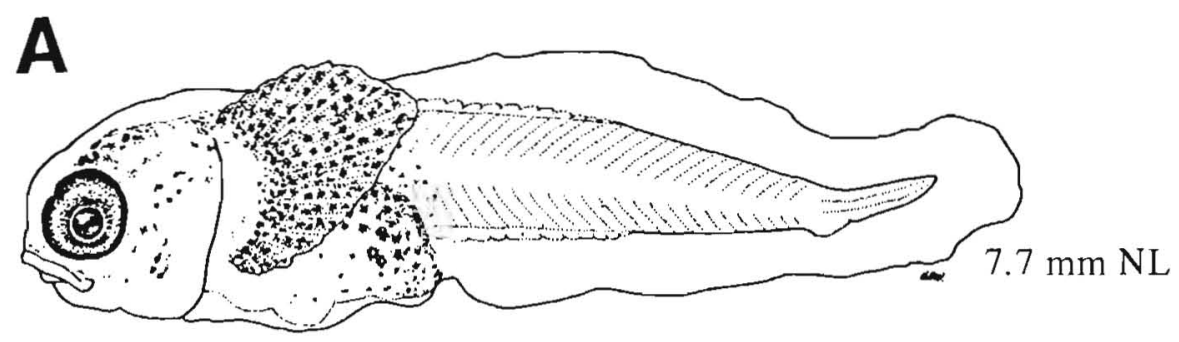

B
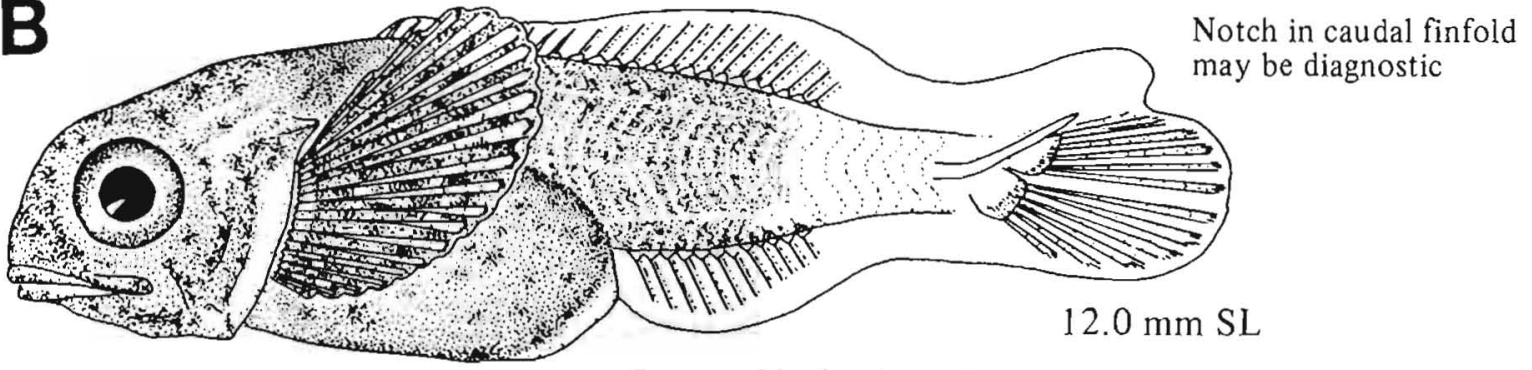

Increased body pigment
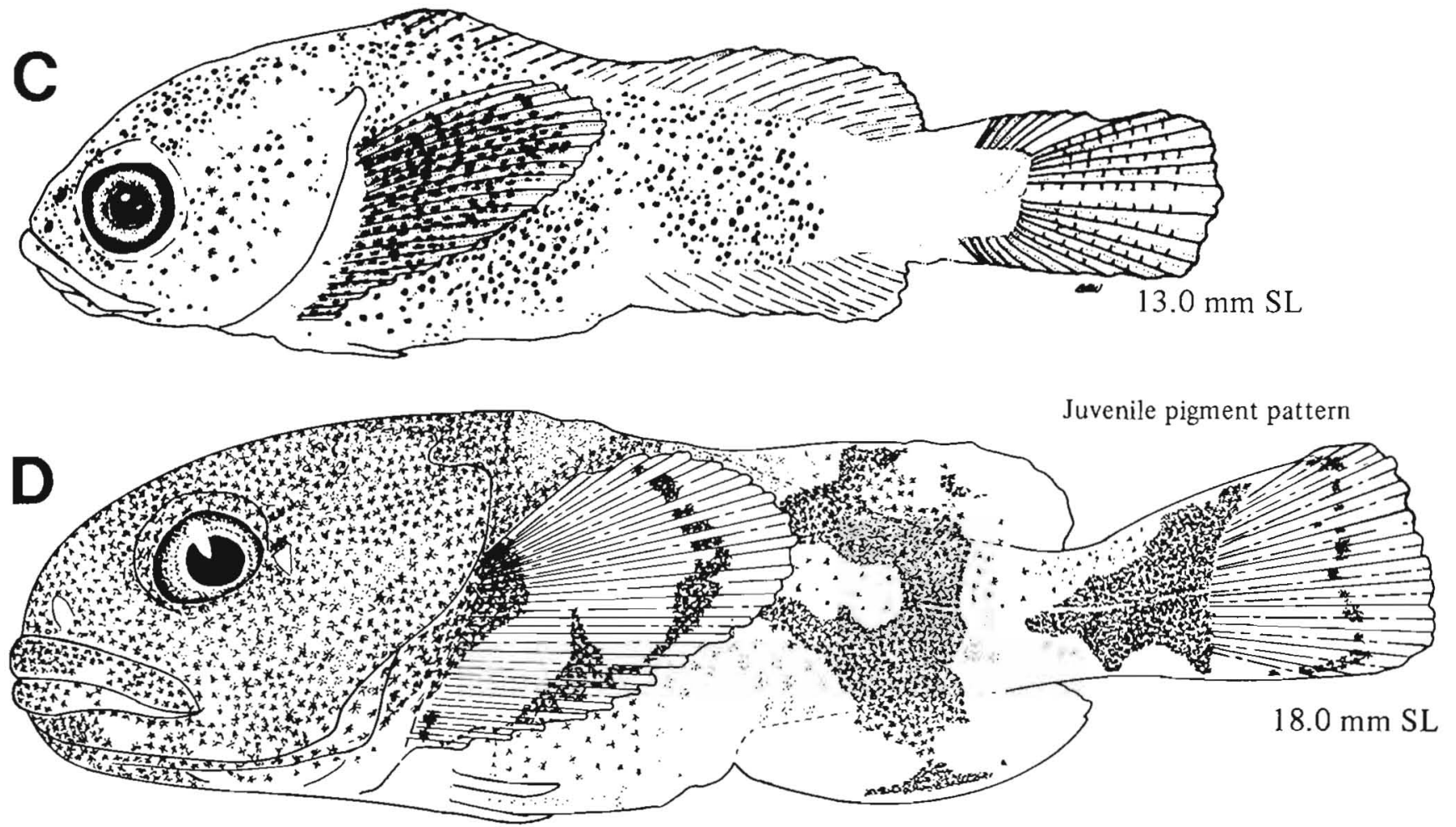

Figure A, Richardson and Bond 1978; B, Marliave 1975a (redrawing provided by Marliave); C, Richardson 1981a; D, NWAFC original (B. Vinter). 
COTTIDAE

\section{MERISTICS}

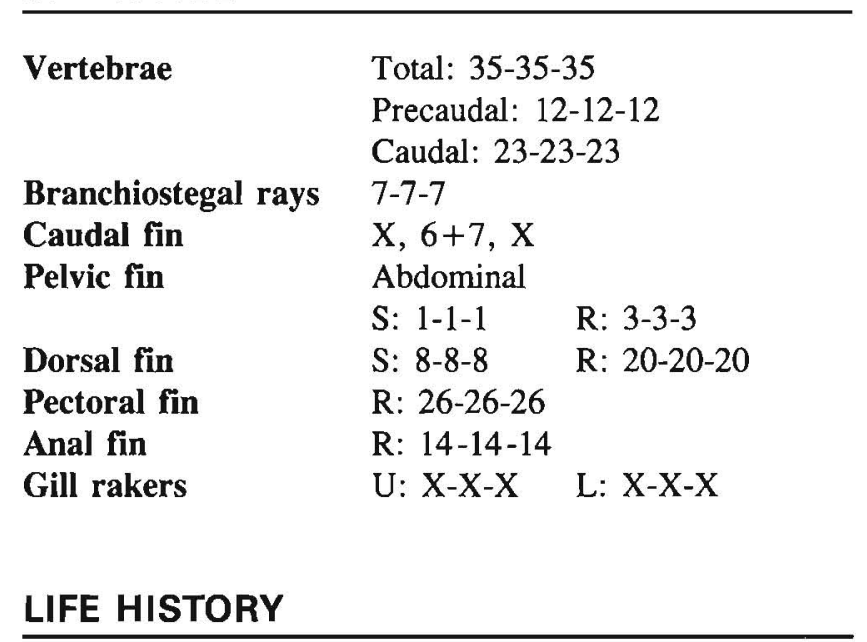

\begin{tabular}{|c|c|}
\hline Range & Oregon, $42-46^{\circ} \mathrm{N}$ \\
\hline Ecology & Unknown \\
\hline ELH pattern & $\begin{array}{l}\text { Parity and eggs unknown, } \\
\text { pelagic larvae }\end{array}$ \\
\hline \multirow[t]{4}{*}{ Spawning } & Season: \\
\hline & Area: \\
\hline & Mode: \\
\hline & Migration: \\
\hline Fecundity & Range/function: \\
\hline Age at first maturity & \\
\hline Longevity & \\
\hline
\end{tabular}

\footnotetext{
a Based on three specimens $(9.8,12.8,13.4 \mathrm{~mm} \mathrm{SL})$ collected off Oregon.

bentative placement in Psychrolutes group.

Ref: Richardson and Washington 1980.
}

\section{EARLY LIFE HISTORY DESCRIPTION}

\section{EGGS}

Diameter

No. of oil globules

Oil globule diameter

Yolk

Envelope

Hatch size

Incubation time/temp.

Pigment

Diagnostic characters
$58 \%$ SL, increasing with development to $79 \%$ SL

Length at flexion $\sim 9.8 \mathrm{~mm} \mathrm{SL}$

Length at transformation $>13.4 \mathrm{~mm} \mathrm{SL}$

Sequence of fin development

Pigment

- Larvae are pigmented over entire body except at the tail tip; dorsal and anal finfolds and the distal portion of the pectoral fin are unpigmented

Diagnostic characters

- Lack of head spines

- Prickles over body

- Globose morphology unlike any other cottid

- Loose outer skin

- Pelvic fin appears to be inserted in pockets of skin with only the tips exposed

Meristic characters of Cottoid A specimens agree with those of Psychrolutes phrictus (pectoral rays $=26$, branchiostegals $=7$ ), but identification is tentative until additional material is available (see discussion in Richardson and Washington [1980]). 


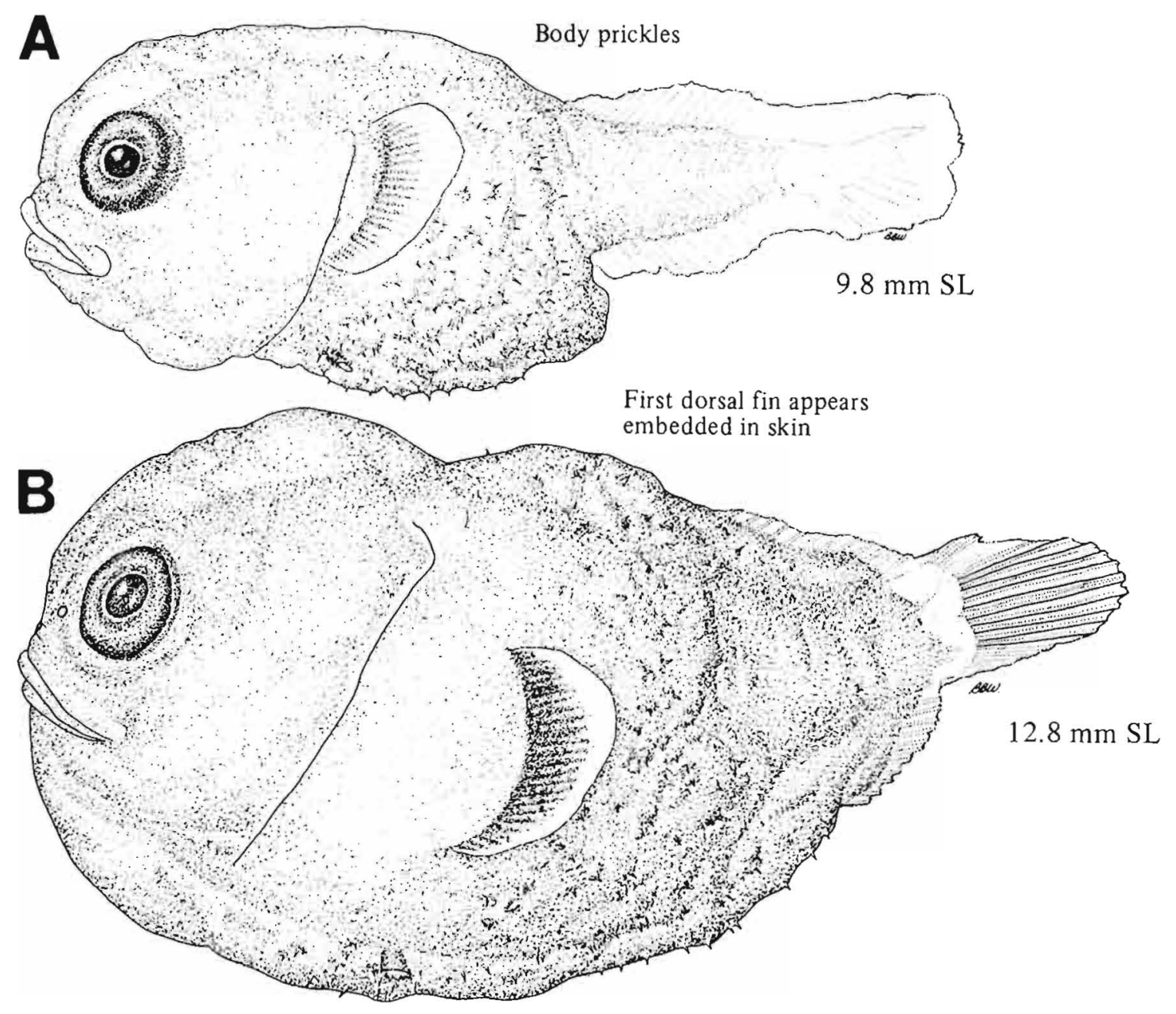

Pelvic fin inserted in pocket 


\section{MERISTICS}

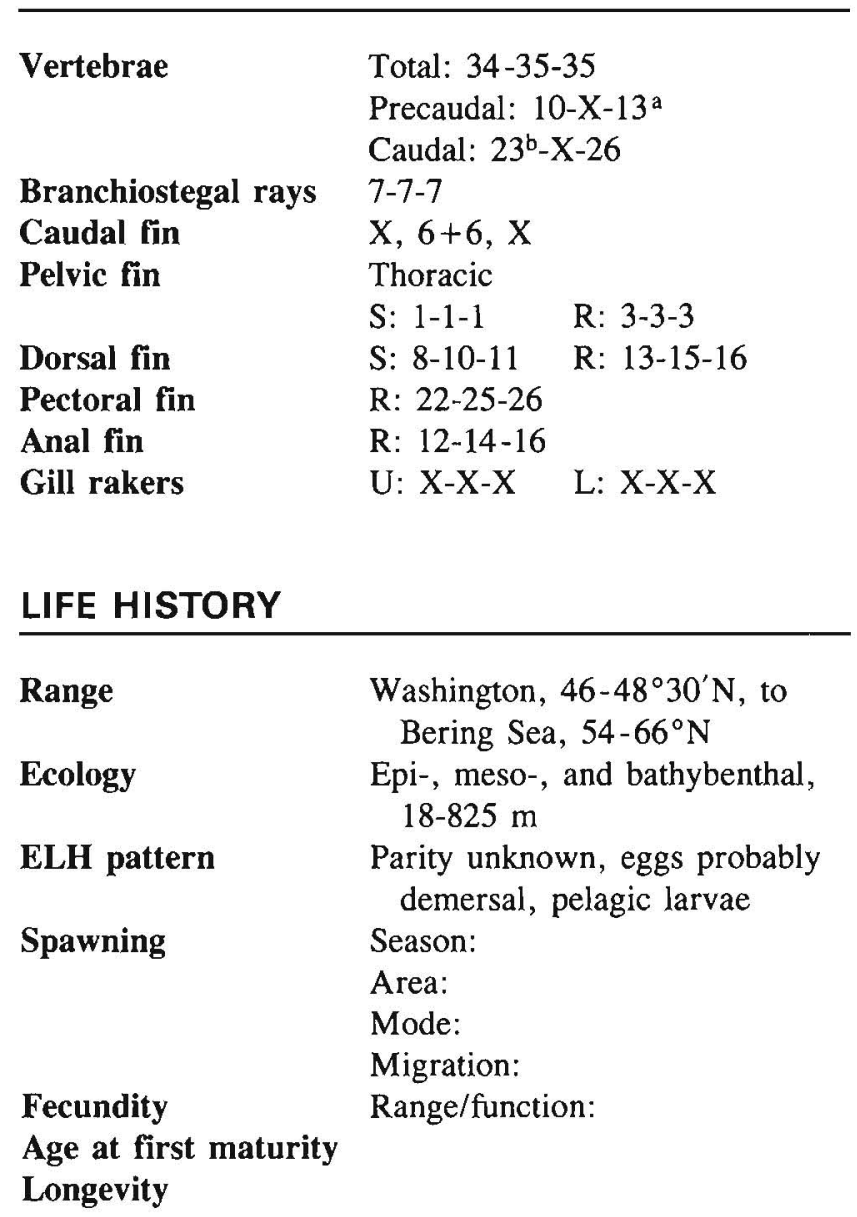

\section{EARLY LIFE HISTORY DESCRIPTIOIN}

\section{EGGS}

Diameter

No. of oil globules

Oil globule diameter

Yolk

Envelope

Hatch size

Homogeneous

Incubation time/temp.

$\sim 7.4 \mathrm{~mm} \mathrm{SL}$

Pigment

\section{Diagnostic characters}

\section{LARVAE}

Preanal length

Length at flexion

Length at transformation

Sequence of fin development

$<50 \%$ SL

Fins complete at $12 \mathrm{~mm} \mathrm{SL}$

\section{Pigment}

- Heavy spots on head and gut

- Double row of small pigment spots occurs along the midline of the ventral surface of gut; more pronounced in specimens $>8 \mathrm{~mm} \mathrm{SL}$

- Pectoral fin pigmented at base

\section{Diagnostic characters}

- Similar to Psychrolutes group but with four preopercular spines

\footnotetext{
${ }^{a}$ One specimen with precaudal vertebrae $=13$ (B.B. Washington, NMFS Systematics

Lab., Natl. Mus. Nat. Hist., Wash., D.C. 20560, unpubl.).

${ }^{b}$ B.B. Washington, unpubl.

Ref: Blackburn 1973.
} 


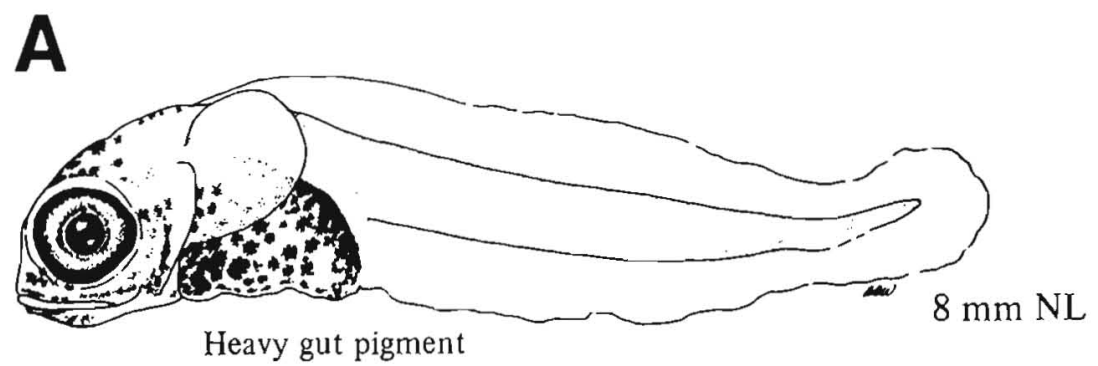

Four preopercular spines

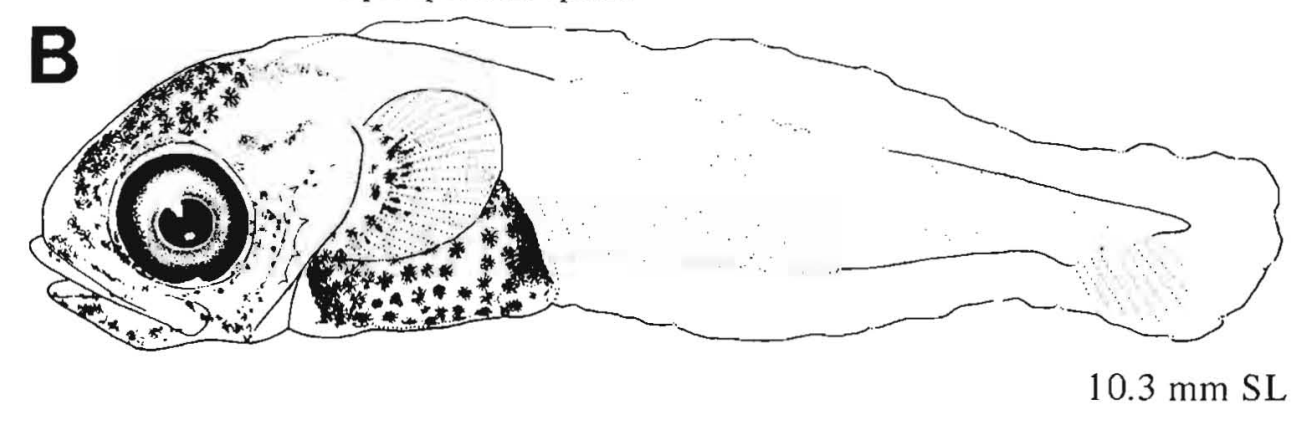

Figure A, Richardson 1981a; B, Washington et al. 1984a. 


\section{MERISTICS}

\begin{tabular}{|c|c|c|}
\hline Vertebrae & \multicolumn{2}{|c|}{$\begin{array}{l}\text { Total: } 30-32-33 \\
\text { Precaudal: X-X-X } \\
\text { Caudal: X-X-X }\end{array}$} \\
\hline $\begin{array}{l}\text { Branchiostegal rays } \\
\text { Caudal fin }\end{array}$ & $7-7-7$ & \\
\hline Pelvic fin & $\begin{array}{l}\text { Thoracic } \\
\text { S: } 1-1-1\end{array}$ & $\mathrm{R}: 3-3-3$ \\
\hline Dorsal fin & S: $8-9-9$ & $\mathrm{R}: 12-14-15$ \\
\hline Pectoral fin & R: $19-20-23$ & \\
\hline Anal fin & R: 9-11-12 & \\
\hline Gill rakers & $\mathrm{U}: \mathrm{X}-\mathrm{X}-\mathrm{X}$ & $\mathrm{L}: \mathrm{X}-\mathrm{X}-\mathrm{X}$ \\
\hline
\end{tabular}

$\begin{array}{ll}\text { Range } & \begin{array}{c}\text { Washington, } 46-48^{\circ} 30^{\prime} \mathrm{N}, \text { to } \\ \text { Bering Sea, } 54-66^{\circ} \mathrm{N}\end{array} \\ \text { Ecology } & \begin{array}{c}\text { Epi-, meso-, and bathybenthal, } \\ 75-1980 \mathrm{~m}\end{array} \\ \text { ELH pattern } & \begin{array}{c}\text { Parity and eggs unknown, } \\ \text { pelagic larvae }\end{array} \\ \text { Spawning } & \text { Season: } \\ & \text { Area: } \\ & \text { Mode: } \\ \text { Fecundity } & \text { Migration: } \\ \text { Age at first maturity } & \text { Range/function: } \\ \text { Longevity } & \end{array}$

\section{EARLY LIFE HISTORY DESCRIPTION}

\section{EGGS}

Diameter

No. of oil globules

Oil globule diameter

Yolk

Envelope

Hatch size

Incubation time/temp.

Pigment

Diagnostic characters

\section{LARVAE}

Preanal length

Length at flexion

Length at transformation

Sequence of fin development

\section{Pigment}

- Head, nape, entire gut

- Laterally over $1 / 4$ body, with development increasing to $3 / 4$ body

Diagnostic characters

- Fifth accessory preopercular spine (sometimes difficult to see)

- Outer layer of loose skin more pronounced than in other genera (genus)

Eggs and larvae of $M$. kincaidi are unknown. The following information may aid in identification.

Total vertebrae

$31-33$

Dorsal fin spines $\quad 8-10$

Dorsal fin rays $\quad 13-15$

Anal fin rays $\quad 10-13$

Pectoral fin rays 19-21

Ref: Richardson and Bond 1978. 

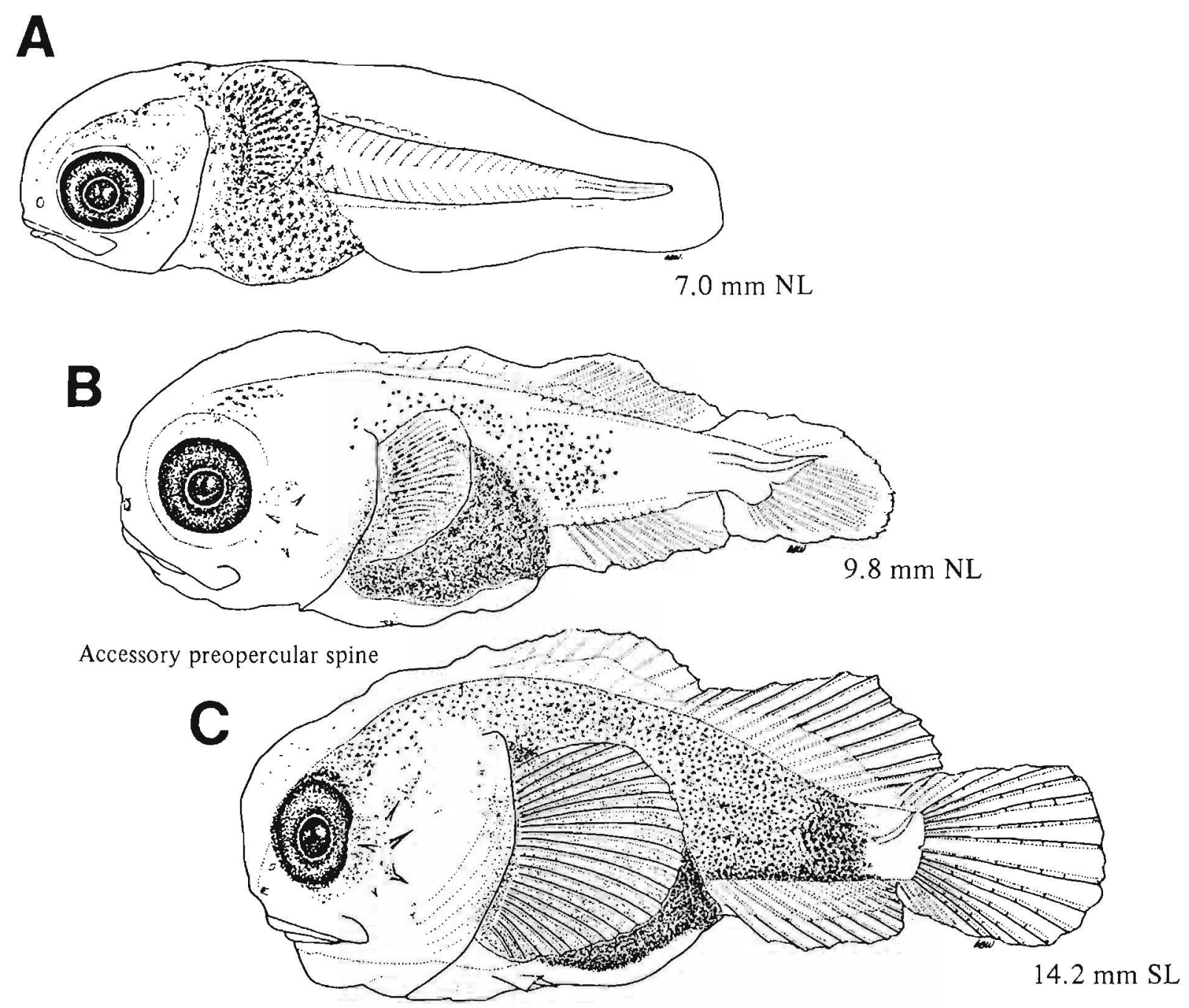

Figures A-D, Richardson and Bond 1978.

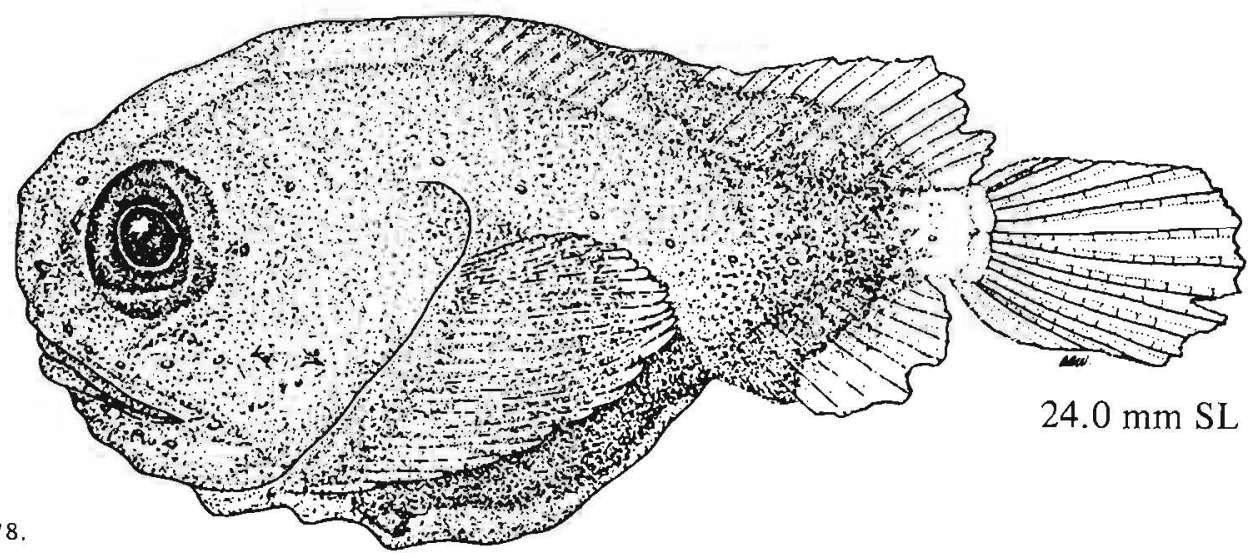




\section{COTTIDAE}

\section{MERISTICS}

\begin{tabular}{|c|c|}
\hline \multirow[t]{3}{*}{ Vertebrae } & Total: $34-37-39$ \\
\hline & Precaudal: $\mathrm{X}-\mathrm{X}-\mathrm{X}$ \\
\hline & Caudal: $X-X-X$ \\
\hline Branchiostegal rays & $6-6-6$ \\
\hline Caudal fin & $X, 6+6, X$ \\
\hline \multirow[t]{2}{*}{ Pelvic fin } & Thoracic \\
\hline & $R: 4-4-4$ \\
\hline Dorsal fin & S: 7-9-11 R: $18-20-21$ \\
\hline Pectoral fin & $\mathrm{R}: 14-15-17$ \\
\hline Anal fin & $\mathrm{R}: 14-17-18$ \\
\hline Gill rakers & $\mathrm{U}: \mathrm{X}-\mathrm{X}-\mathrm{X} \quad \mathrm{L}: \mathrm{X}-\mathrm{X}-\mathrm{X}$ \\
\hline \multicolumn{2}{|l|}{ LIFE HISTORY } \\
\hline Range & $\begin{array}{l}\text { S. California, } 32-34^{\circ} \mathrm{N} \text {, to } \\
\text { Gulf of Alaska, } 54-60^{\circ} \mathrm{N}\end{array}$ \\
\hline Ecology & FW or anadromous type \\
\hline ELH pattern & $\begin{array}{l}\text { Oviparous; demersal, adhesive } \\
\text { eggs; pelagic larvae }\end{array}$ \\
\hline \multirow[t]{4}{*}{ Spawning } & Season: Jan-Apr; ${ }^{a}$ Feb-July ${ }^{b}$ \\
\hline & Area: Under rocks ${ }^{b}$ \\
\hline & $\begin{array}{l}\text { Mode: Polygamous males, eggs } \\
\text { guarded }^{\mathrm{b}}\end{array}$ \\
\hline & $\begin{array}{l}\text { Migration: Downstream in spring } \\
\text { to spawn }{ }^{b}\end{array}$ \\
\hline Fecundity & Range/function: $280-10,980^{a}$ \\
\hline \multicolumn{2}{|l|}{ Age at first maturity } \\
\hline Longevity & $>7 \mathrm{yr}^{b}$ \\
\hline
\end{tabular}

\section{EARLY LIFE HISTORY DESCRIPTION}

\section{EGGS}

Diameter

No. of oil globules

Oil globule diameter

Yolk

Envelope

Hatch size

Incubation time/temp.

Pigment

Diagnostic characters

\section{LARVAE}

Preanal length

Length at flexion

$40 \%$ SL

Length at transformation

Sequence of fin development

$\sim 7 \mathrm{~mm} \mathrm{SL}$

$10 \mathrm{~mm} \mathrm{SL}$

Pectorals and pelvics by $10 \mathrm{~mm} \mathrm{SL}$

\section{Pigment}

- Dorsal and ventral gut surface

- Postanal ventral midline series decreasing in number and becoming more evenly spaced with development

Diagnostic characters

- Morphology: Slender, round snout

- Spines: Four preopercular, without other head spines

- Gut shape unique, posteriorly forked

Larvae of $C$. aleuticus are unknown. Spawning takes place primarily in freshwater and larvae may not occur in coastal marine plankton.

\footnotetext{
${ }^{a}$ Wang 1981

${ }^{\mathrm{b}}$ Morrow 1980

${ }^{c}$ Richardson and Washington 1980

Ref: Morrow 1980, Stein 1972, Richardson and Washington 1980, Wang 1981, Washington et al. $1984 \mathrm{~b}$.
} 

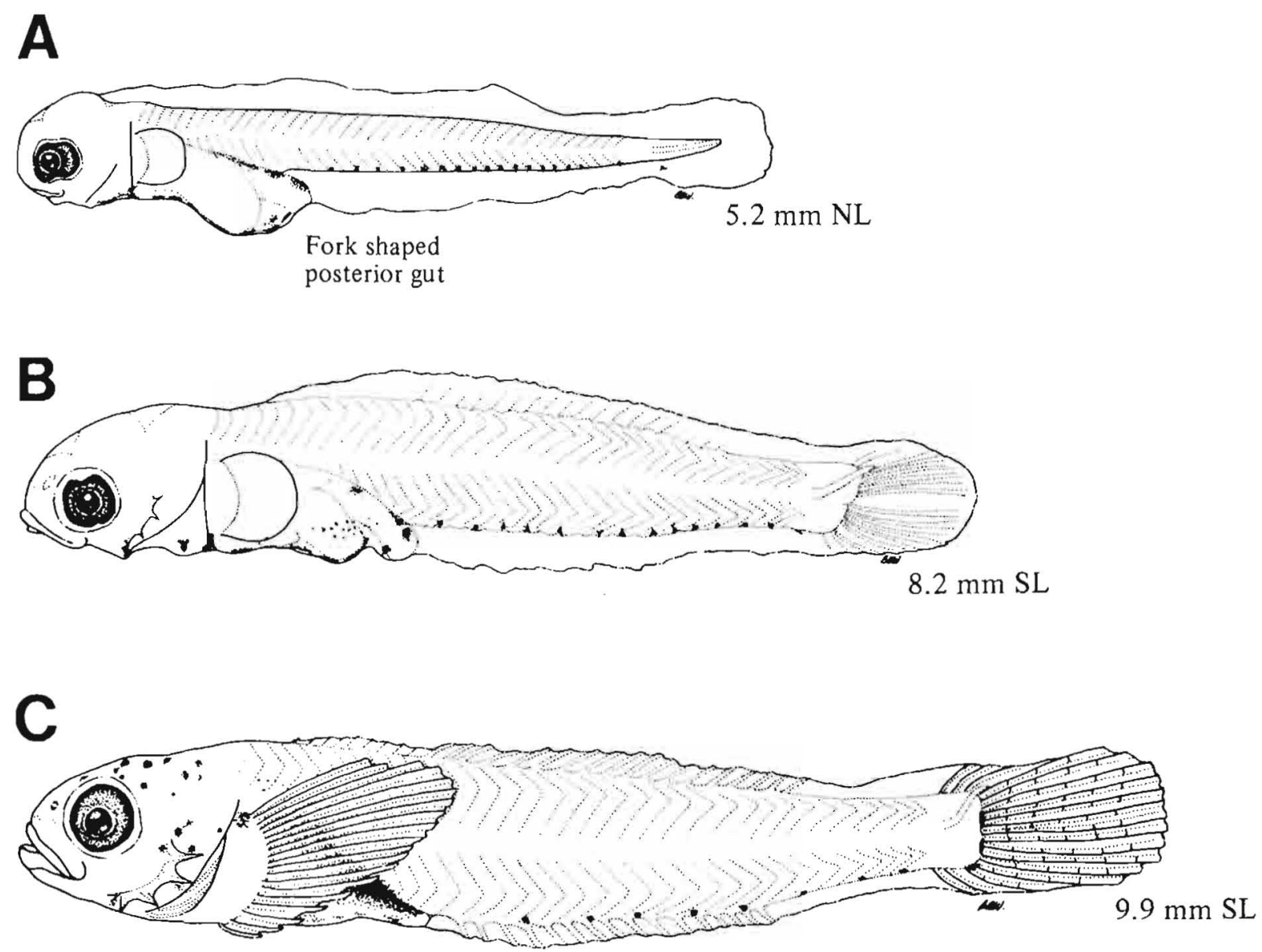
MERISTICS

\begin{tabular}{|c|c|c|}
\hline \multirow{3}{*}{$\begin{array}{l}\text { Vertebrae } \\
\text { Branchiostegal rays } \\
\text { Caudal fin } \\
\text { Pelvic fin }\end{array}$} & \multicolumn{2}{|c|}{$\begin{array}{l}\text { Total: } 35-36-39 \\
\text { Precaudal: } 10-11-12 \\
\text { Caudal: } 24-25-27 \\
6-6-6\end{array}$} \\
\hline & $6-6-6$ & \\
\hline & $\begin{array}{l}\text { Thoracic } \\
S: 1-1-1\end{array}$ & $\mathrm{R}: 4-4-4$ \\
\hline Dorsal fin & $S: 6-7-8$ & $\mathrm{R}: 15-18-20$ \\
\hline Pectoral fin & R: $17-19-20$ & \\
\hline Anal fin & R: $15-16-20$ & \\
\hline Gill rakers & $\mathrm{U}: 1-\mathrm{X}-\mathbf{3}$ & L: $8-X-10$ \\
\hline \multicolumn{3}{|l|}{ LIFE HISTORY } \\
\hline Range & \multicolumn{2}{|c|}{$\begin{array}{l}\text { South of southern California to } \\
\text { Bering Sea, } 54-66^{\circ} \mathrm{N}\end{array}$} \\
\hline Ecology & \multicolumn{2}{|c|}{$\begin{array}{l}\text { Nearshore shelf demersal, } \\
\text { intertidal to } 91 \mathrm{~m}\end{array}$} \\
\hline ELH pattern & \multicolumn{2}{|c|}{$\begin{array}{l}\text { Oviparous; demersal, adhesive } \\
\text { eggs; pelagic larvae }\end{array}$} \\
\hline Spawning & \multicolumn{2}{|c|}{ Season: Oct-Mar (California) ${ }^{\mathrm{a}}$} \\
\hline & \multicolumn{2}{|l|}{ Area: } \\
\hline & \multicolumn{2}{|l|}{ Mode: } \\
\hline Fecundity & \multirow{2}{*}{\multicolumn{2}{|c|}{$\begin{array}{l}\text { Range/function: } 2000-11,000 / \mathrm{b} \\
\mathrm{N}=0.355 \times \mathrm{L}^{1.84} \\
\mathrm{~N}=\text { no. maturing eggs, } \\
\mathrm{L}=\mathrm{TL} \mathrm{mm}^{\mathrm{a}}\end{array}$}} \\
\hline & & \\
\hline Age at first maturity & \multicolumn{2}{|l|}{$1 \mathrm{yr}^{\mathrm{a}}$} \\
\hline Longevity & \multicolumn{2}{|l|}{$5 \mathrm{yr}^{\mathrm{c}}$} \\
\hline
\end{tabular}

\section{EARLY LIFE HISTORY DESCRIPTION}

\section{EGGS}

Diameter

No. of oil globules

Oil globule diameter

Yolk

Envelope

Hatch size

1.4-1.5 mm (1.43)

One, smaller ones

$0.3 \mathrm{~mm}$

Thick, transparent, bumpy

3.8-5.0 mm SL;

3.9-4.8 $\mathrm{TL}^{\mathrm{a}}$

Incubation time/temp. $\quad 9-14 \mathrm{~d} / 15^{\circ} \mathrm{C}$

Diagnostic characters

\section{LARVAE}

Preanal length $\quad 37-40 \%$ SL

Length at flexion $\quad \sim 8 \mathrm{~mm} \mathrm{SL}$

Length at transformation $15-20 \mathrm{~mm} \mathrm{SL}$

Sequence of fin development

Pigment

- Internal snout pigment

- 6-8 bars of pigment on dorsolateral surface of gut

- Postanal ventral midline series

\section{Diagnostic characters}

- Gut pigment appearing as 6-8 bars

- Internal snout pigment

${ }^{c}$ Fitch and Lavenberg 1975

Ref: Jones 1962, Wang 1981, Washington et al. $1984 \mathrm{~b}$. 
A

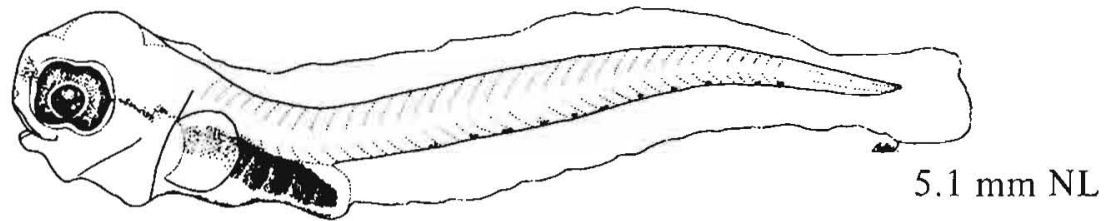

B

Snout pigment internal

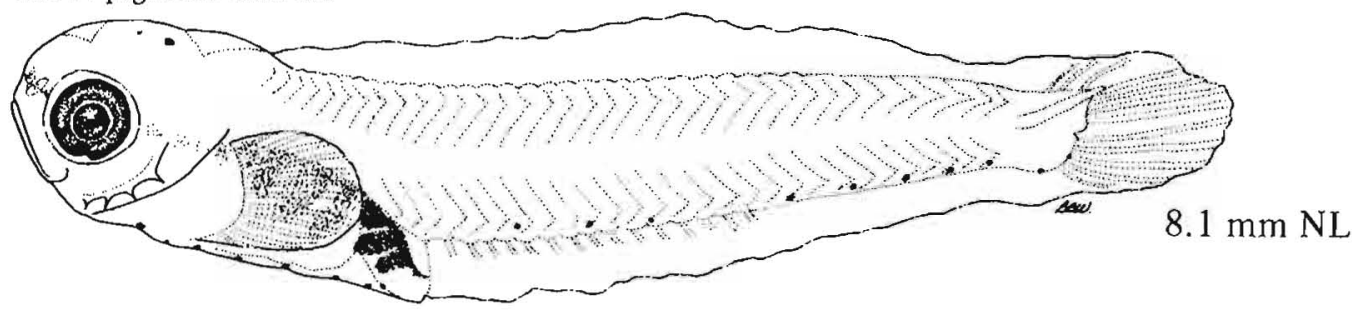

Bar pattern on gut

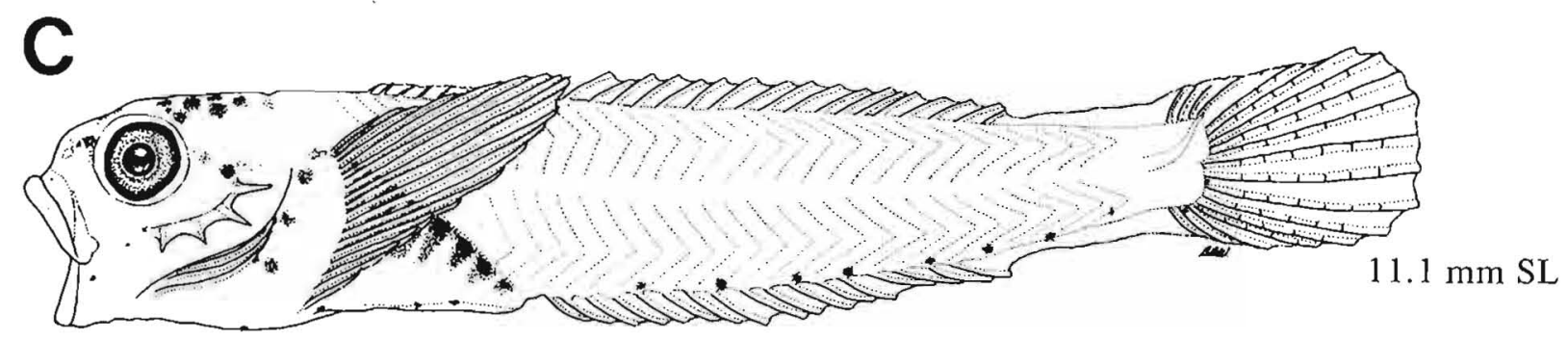


MERISTICS

\begin{tabular}{|c|c|c|}
\hline Vertebrae & \multicolumn{2}{|c|}{$\begin{array}{l}\text { Total: } 37-38-38 \\
\text { Precaudal: X-X-X } \\
\text { Caudal: X-X-X }\end{array}$} \\
\hline $\begin{array}{l}\text { Branchiostegal rays } \\
\text { Caudal fin }\end{array}$ & $6-6-6$ & \\
\hline Pelvic fin & $\begin{array}{l}\text { Thoracic } \\
\text { S: } 1-1-1\end{array}$ & R: $3-3-3$ \\
\hline Dorsal fin & S: 7-8-9 & $\mathrm{R}: 20-21-22$ \\
\hline Pectoral fin & R: $15-16-17$ & \\
\hline Anal fin & R: $18-19-20$ & \\
\hline Gill rakers & $\mathrm{U}: \mathrm{X}-\mathrm{X}-\mathrm{X}$ & L: X-X-X \\
\hline
\end{tabular}

$\begin{array}{ll}\text { Range } & \text { Brit. Col., } 48^{\circ} 30^{\prime}-55^{\circ} \mathrm{N} \text {, to } \\ & \text { Bering Sea, } 54-66^{\circ} \mathrm{N} \\ \text { Ecology } & \text { Nearshore shelf demersal } \\ \text { ELH pattern } & \begin{array}{c}\text { Probably oviparous, eggs } \\ \text { probably demersal, pelagic } \\ \text { larvae }\end{array} \\ \text { Spawning } & \text { Season: } \\ & \text { Area: } \\ & \text { Mode: } \\ & \text { Migration: } \\ \text { Fecundity } & \text { Range/function: } \\ \text { Age at first maturity } & \\ \text { Longevity } & \end{array}$

\section{EARLY LIFE HISTORY DESCRIPTION}

\section{EGGS}

Diameter

No. of oil globules

Oil globule diameter

Yolk

Envelope

Hatch size

Incubation time/temp.

Pigment

Diagnostic characters

\section{LARVAE}

Preanal length

Length at flexion $\leqslant 12 \mathrm{~mm} \mathrm{SL}$

Length at transformation

Sequence of fin development

Pigment

- Entire body heavily pigmented with small, densely packed melanophores except for caudal peduncle, opercle area, and ventrolateral gut surface

Diagnostic characters (see Table 3)

- Genus

-Elongate body becoming deeper with development

- Pigment relatively heavy

- Strong frontoparietal ridge

Distinguished from $B$. cirrhosus by

- More pectoral fin rays (15-17)

- Pigment

- Larger area of caudal peduncle unpigmented

- Smaller, denser melanophores

- Lack of pigment on underside of mouth 
Small, densely packed melanophores
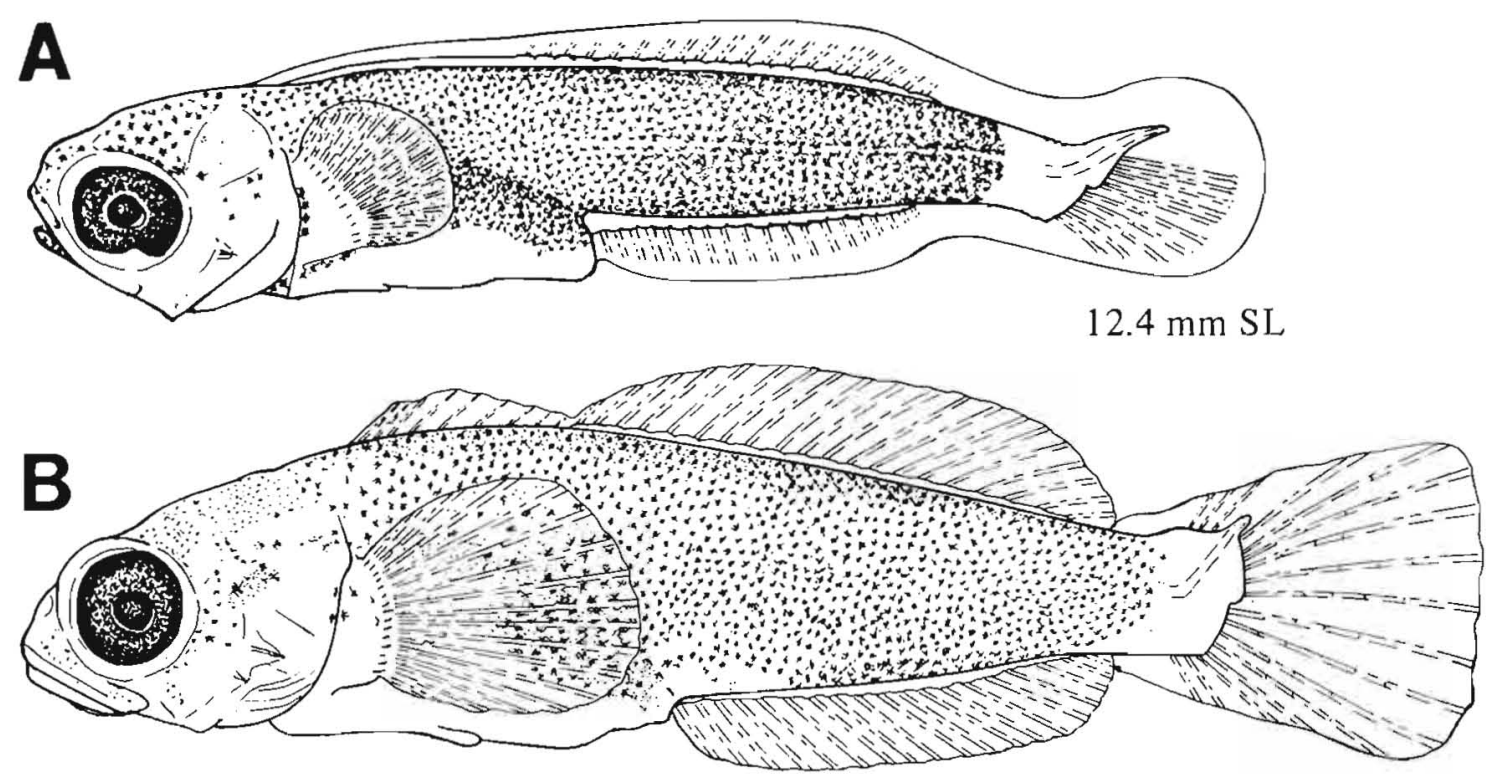

\section{5-17 pectoral fin rays}

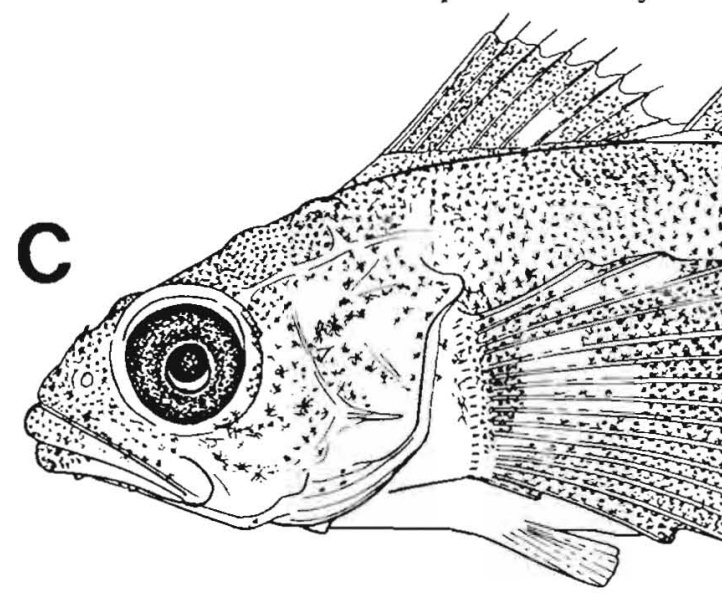

Figures A-C, NWAFC originals (B. Vinter).

$16.7 \mathrm{~mm} \mathrm{SL}$ 


\section{MERISTICS}

\begin{tabular}{|c|c|}
\hline Vertebrae & $\begin{array}{l}\text { Total: } 37-37-39^{a} \\
\text { Precaudal: } 13-13-13^{a} \\
\text { Caudal: } 26-26-26^{a}\end{array}$ \\
\hline Branchiostegal rays & $6-6-6$ \\
\hline Caudal fin & $X, 6+6, x$ \\
\hline \multirow[t]{2}{*}{ Pelvic fin } & Thoracic \\
\hline & $S: 1-1-1$ \\
\hline Dorsal fin & S: $6-8-8 \quad R: 20-23-24$ \\
\hline Pectoral fin & R: $11-12-13$ \\
\hline Anal fin & R: $18-19-21$ \\
\hline Gill rakers & U: X-X-X $\quad L: X-X-X$ \\
\hline \multicolumn{2}{|l|}{ LIFE HISTORY } \\
\hline Range & $\begin{array}{l}\text { Cent. California, } 34-38^{\circ} \mathrm{N} \text {, to } \\
\text { Bering Sea, } 54-66^{\circ} \mathrm{N}\end{array}$ \\
\hline Ecology & $\begin{array}{l}\text { Nearshore shelf demersal, } \\
\text { intertidal to } 37 \mathrm{~m}\end{array}$ \\
\hline ELH pattern & $\begin{array}{l}\text { Oviparous, demersal eggs, } \\
\text { pelagic larvae }\end{array}$ \\
\hline Spawning & $\begin{array}{l}\text { Season: Late winter (British } \\
\text { Columbia) }\end{array}$ \\
\hline & $\begin{array}{l}\text { Area: Demersal, on rocks } \\
\text { Mode: } \\
\text { Migration: }\end{array}$ \\
\hline $\begin{array}{l}\text { Fecundity } \\
\text { Age at first maturity } \\
\text { Longevity }\end{array}$ & Range/function: \\
\hline
\end{tabular}

\section{EARLY LIFE HISTORY DESCRIPTION}

\section{EGGS}

Diameter

No. of oil globules

Oil globule diameter

Yolk

Envelope

Homogeneous

Hatch size

Incubation time/temp.

Pigment

Diagnostic characters

\section{LARVAE}

Preanal length $\quad<50 \% \mathrm{SL}$

Length at flexion $\quad \leqslant 11 \mathrm{~mm} \mathrm{SL}$

Length at transformation

Sequence of fin

development

Pigment

- Entire body heavily pigmented except for caudal peduncle, opercle area, and ventrolateral gut surface

- Pigment along underside of mouth between dentary bones (chin)

Diagnostic characters (see Table 3)

Distinguished from $B$. bilobus by

- Low number of pectoral fin rays (11-13)

- Shorter area of caudal peduncle unpigmented

- Pigment on underside of mouth

- See B. bilobus for generic characters (p. 448)

\footnotetext{
a.B. Washington, NMFS Systematics Lab., Natl. Mus. Nat. Hist., Wash., D.C 20560, unpubl.

${ }^{b}$ Marliave 1975a

${ }^{\mathrm{c}}$ Clemens and Wilby 1961
}

Ref: Marliave 1975a, Washington et al, $1984 \mathrm{~b}$. 

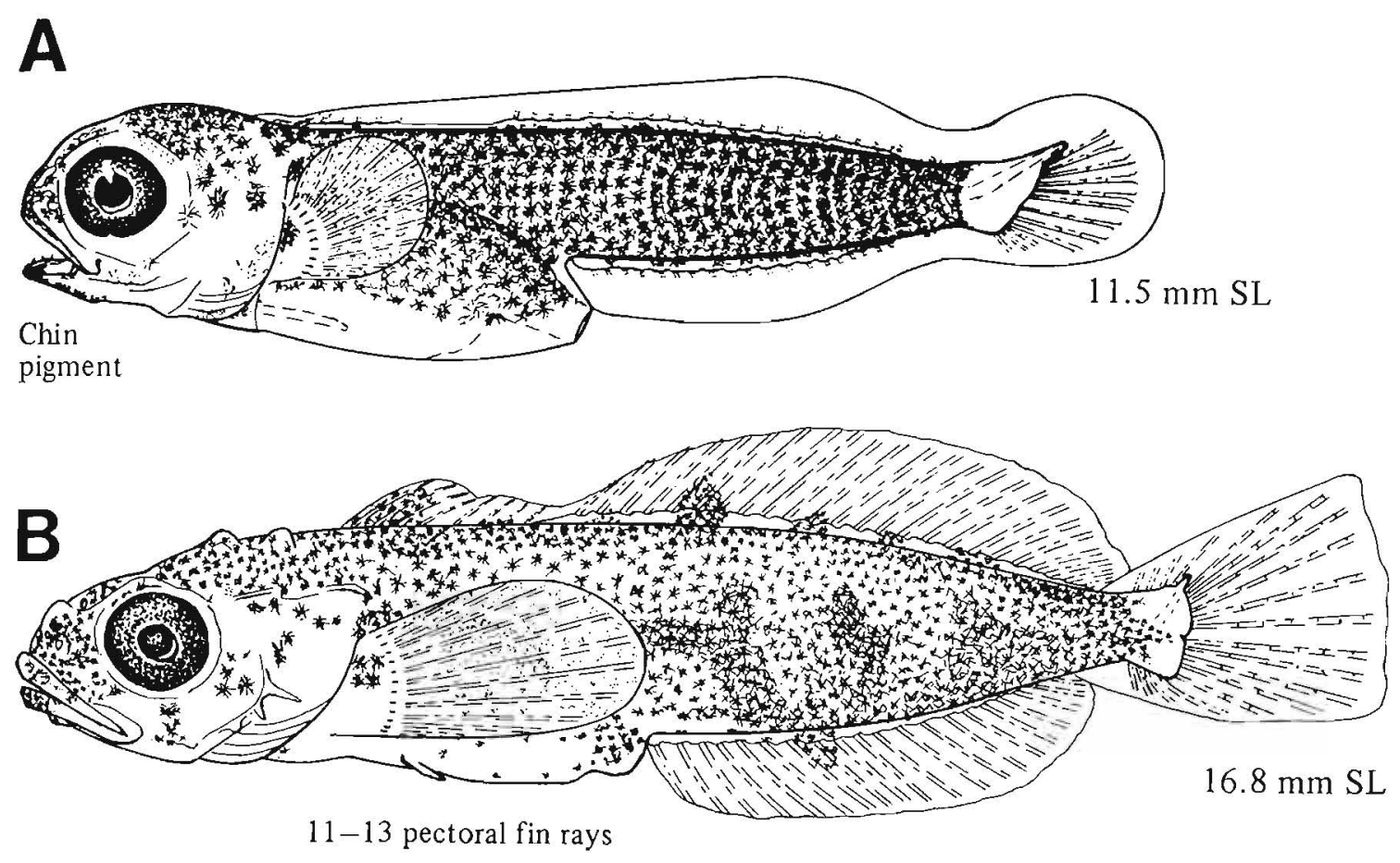

Figures A-B, NWAFC originals (B. Vinter). 


\section{MERISTICS}

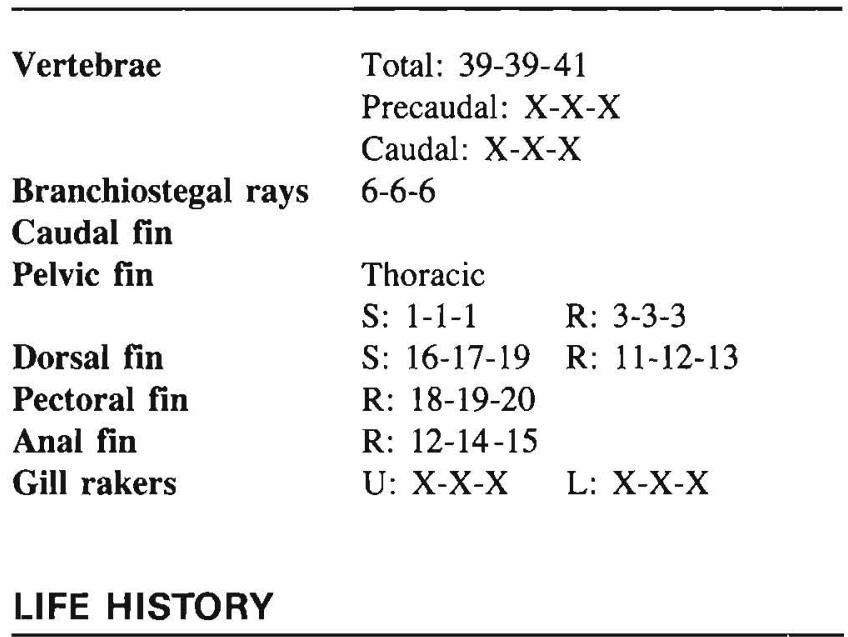

$\begin{array}{ll}\text { Range } & \text { Gulf of Alaska, } 54-60^{\circ} \mathrm{N} \text {, to } \\ & \text { Bering Sea, } 54-66^{\circ} \mathrm{N} \\ \text { Ecology } & \text { Nearshore shelf demersal } \\ \text { ELH pattern } & \text { Oviparous; demersal, adhesive } \\ & \text { eggs; pelagic larvae } \\ \text { Spawning } & \text { Season: Fall (Funka Bay, Japan) } \\ & \text { Area: Rocky sea bottom, } \\ & 10-30 \mathrm{~m}^{\mathrm{a}} \\ & \text { Mode: } \\ & \text { Migration: } \\ \text { Fecundity } & \text { Range/function: } 2250-11,170 / \\ & \text { E }=0.00002147 \times \mathrm{L}^{3.374}, \\ & \mathrm{E}=\text { ovarian eggs, } \\ & \mathrm{L}=\mathrm{BL} \mathrm{mm}^{\mathrm{a}}\end{array}$

Age at first maturity Longevity

\section{EARLY LIFE HISTORY DESCRIPTION}

\section{EGGS}

Diameter

No. of oil globules

Oil globule diameter

Yolk

Envelope

Hatch size

\subsection{1-4.61 mm} Multiple (120-160)

0.05-0.26 mm

Yellow/orange

Thick, white

14.1-15.2 mm SL; ${ }^{\mathrm{a}}$

10.9-11.6 $\mathrm{mm} \mathrm{SL}^{\mathrm{b}}$

Incubation time/temp. $\quad 100 \mathrm{~d} / 12^{\circ} \mathrm{C}$

Pigment

- Light on yolksac

Diagnostic characters

- Oil globules coalesce to one by late embryonic development

\section{LARVAE}

Preanal length

Length at flexion

$\sim 50 \%$ SL

$\leqslant 14.4 \mathrm{~mm} \mathrm{SL}$ (close to hatching)

Length at transformation $\sim 20 \mathrm{~mm} \mathrm{SL}$

Sequence of fin development

Pigment

- Heavily pigmented at hatching (head, $3 / 4 \mathrm{BL}$, and dorsolateral surface of gut)

- Distinctive finfold pigment

Diagnostic characters (see Table 3)

- Hemitripterus group characters

- Finfold pigment

- Prickles, scales

- Newly hatched larvae large and well developed

Eggs and larvae of $H$. bolini are unknown. The following information will aid in identification.

Total vertebrae

Dorsal fin spines

Dorsal fin rays

Anal fin rays

Pectoral fin rays

20-22

Pelvic fin rays 3

${ }^{a}$ Kyushin 1968

${ }^{b}$ Okiyama and Sando 1976

${ }^{c}$ Lea and Quirollo 1986

Ref: Kyushin 1968, Okiyama and Sando 1976. 

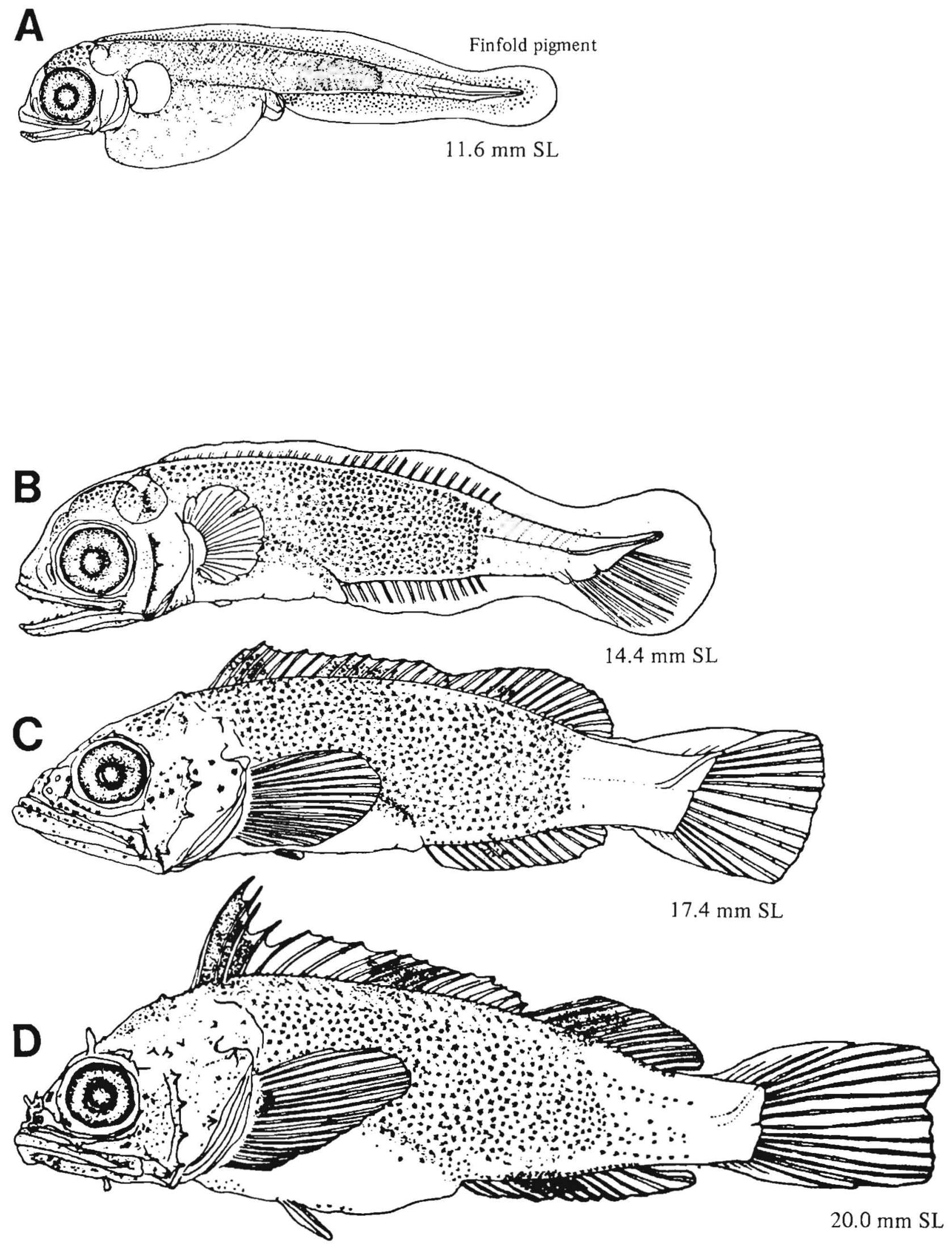

Figures A-D, Okiyama and Sando 1976 (reared from specimens collected near Hokkaido, Japan). 


\section{MERISTICS}

\begin{tabular}{|c|c|}
\hline \multirow[t]{3}{*}{ Vertebrae } & Total: $40-41-41$ \\
\hline & Precaudal: X-X-X \\
\hline & Caudal: X-X-X \\
\hline Branchiostegal rays & $6-6-6$ \\
\hline \multicolumn{2}{|l|}{ Caudal fin } \\
\hline \multirow[t]{2}{*}{ Pelvic fin } & Thoracic \\
\hline & S: $1-1-1$ \\
\hline Dorsal fin & R: $27-29-30$ \\
\hline Pectoral fin & R: $13-14-14$ \\
\hline Anal fin & $\mathrm{R}: 16-19-21$ \\
\hline Gill rakers & $\mathrm{U}: \mathrm{X}-\mathrm{X}-\mathrm{X} \quad \mathrm{L}: \mathrm{X}-\mathrm{X}-\mathrm{X}$ \\
\hline \multicolumn{2}{|l|}{ LIFE HISTORY } \\
\hline Range & $\begin{array}{l}\text { Cent. California, } 34-38^{\circ} \mathrm{N} \text {, to } \\
\text { Bering Sea, } 54-66^{\circ} \mathrm{N}\end{array}$ \\
\hline Ecology & $\begin{array}{l}\text { Nearshore shelf demersal, } \\
0-110 \mathrm{~m}\end{array}$ \\
\hline ELH pattern & $\begin{array}{l}\text { Oviparous; demersal, adhesive, } \\
\text { attached eggs; pelagic larvae }\end{array}$ \\
\hline \multirow[t]{3}{*}{ Spawning } & Season: Fall-spring ${ }^{a}$ \\
\hline & Area: Intertidal, in mussel zone \\
\hline & $\begin{array}{l}\text { Migration: Females move from } \\
\text { subtidal area to mussel zone } \\
\text { to deposit eggs a }\end{array}$ \\
\hline Fecundity & Range/function: \\
\hline $\begin{array}{l}\text { Age at first maturity } \\
\text { Longevity }\end{array}$ & $1 \mathrm{yr}^{\mathrm{b}}$ \\
\hline
\end{tabular}

J. Marliave, Vancouver Public Aquarium, P.O. Box 3232, Vancouver, B.C., Canada V6B $3 \times 8$, pers. commun., 16 Oct. 1986.

${ }^{\mathrm{b}}$ Fitch and Lavenberg 1975

${ }^{c}$ Andriashev 1954; eggs $2.5-2.7 \mathrm{~mm}$.

Ref: Blackburn 1973, Marliave 1975a, Richardson and Washington 1980, Washington et al. $1984 \mathrm{~b}$.

\section{EARLY LIFE HISTORY DESCRIPTION}

\section{EGGS}

Diameter

No. of oil globules

2.0-2.5 mm

Oil globule diameter

Yolk

Envelope

Hatch size

Incubation time/temp.

One

Orange

$\sim 9 \mathrm{~mm}$ TL (possibly as small as $7 \mathrm{~mm} \mathrm{TL}$ )

\section{Pigment}

\section{Diagnostic characters}

- Wild: Only egg with bright orange yolk laid in Mytilus beds ${ }^{\mathrm{a}}$

\section{LARVAE}

Preanal length $\quad \sim 50 \%$ SL

Length at flexion $\sim 9-11 \mathrm{~mm} \mathrm{SL}$

Length at transformation $\sim 26 \mathrm{~mm} \mathrm{SL}$ (largest pelagic specimen caught $=16-17$ $\mathrm{mm} \mathrm{SL)}$

\section{Sequence of fin development}

Pigment

- Finfold pigment

- Distinctive pigment over lateral surface of trunk

- Pectorals pigmented in band posteriorly

Diagnostic characters (see Table 3)

- Long pigmented pectorals (of sample size $=2$, length of pectorals ranged from 42 to $59 \% \mathrm{SL}$ )

- Bumps and parietal ridge

The following information will aid in identification of $N$. pribilovius (larvae unknown) ${ }^{c}$ and $N$. robustus (eggs and larvae unknown).

Total vertebrae

Dorsal fin spines

$$
\begin{array}{cc}
\text { N. pribilovius } & \text { N. robustus } \\
36-37 & 35 \\
7-10 & 7-8 \\
22-26 & 19-21 \\
15-20 & 14-15 \\
15-17 & 14-16 \\
3 & 3 \\
\text { SE Alaska - } & \text { Wash. - } \\
\text { Chukchi Sea } & \text { Bering Sea }
\end{array}
$$

Dorsal fin rays

Anal fin rays
Pectoral fin rays

Pelvic fin rays

Range 

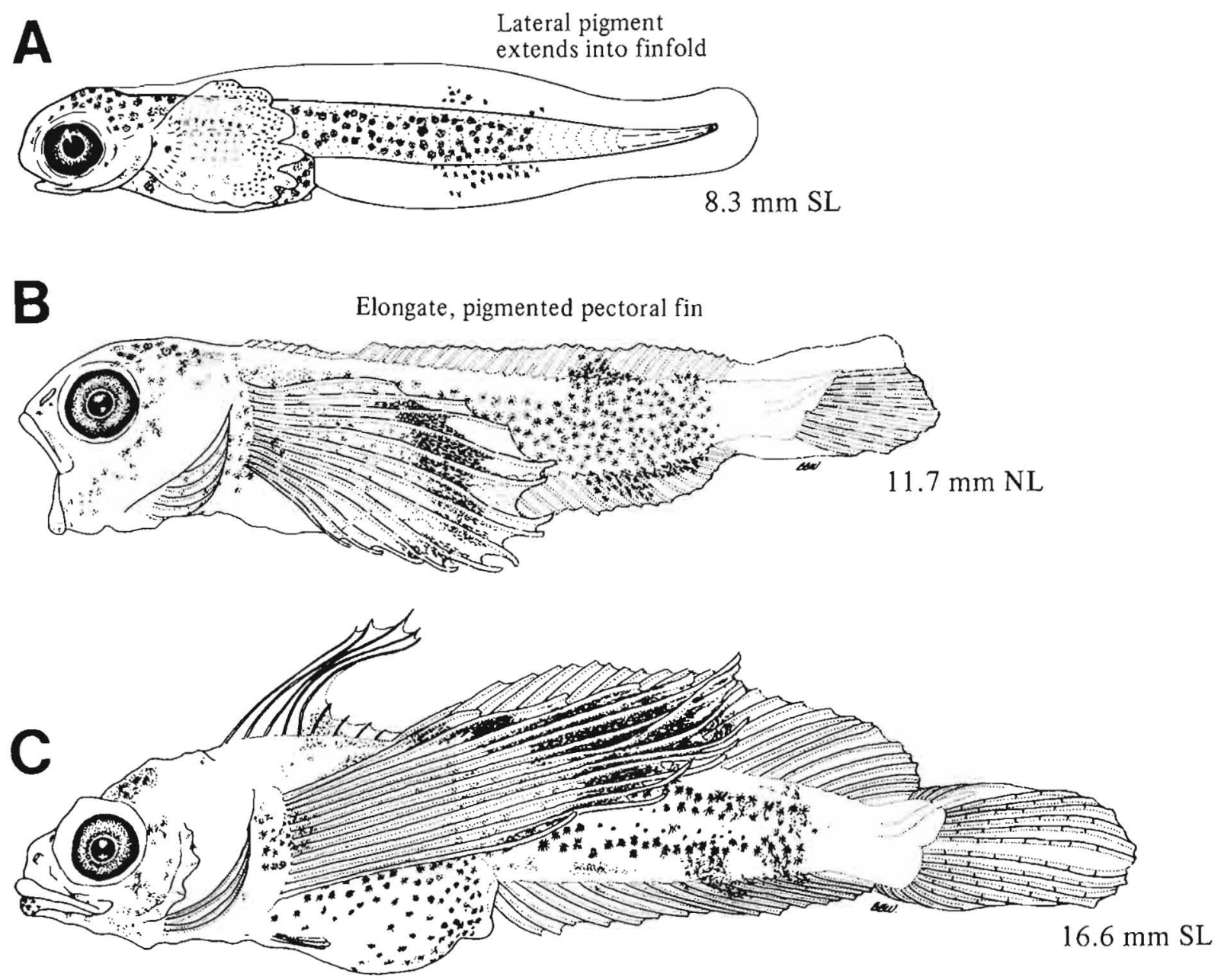

Figure A, NWA FC original (B. Vinter); B-C, Richardson and Washington 1980. 
Poachers and alligatorfishes range from Baja California to the Bering Sea, with the center of abundance in the North Pacific. They are mostly elongate with large fused bony plates covering the body. Pectoral fins are fanlike. Adults are demersal and occur from moderate depths to $1250 \mathrm{~m}$. Some have also been found in tidepools (Hart 1973, Garrison and Miller 1982). The family is represented by 25 species and 15 genera within the study area. Among the few species studied, demersal adhesive eggs are attached to holdfasts of laminarians (Breder and Rosen 1966, Garrison and Miller 1982). Larvae are pelagic and are occasionally taken in plankton nets near the surface. Bony plates, characteristic of agonids, first appear in the larvae as spines. Settlement in some species may occur at approximately 2 months after hatching (Marliave 1975a). Descriptions of larvae of three species are available for inclusion here (Agonomalus mozinoi, Bothragonus swani, and Xeneretmus latifrons). Illustrations of single specimens are provided for Stellerina, Hypsagonus, Ocella, Aspidophoroides (species outside study area), and an unidentified agonid. Two illustrations are provided for Agonidae A, another unidentified agonid from the study area.

Table 39

Meristic characters of family Agonidae. All have pelvic counts of 1,2 and 6 branchiostegal rays.

\begin{tabular}{|c|c|c|c|c|c|c|c|c|}
\hline \multirow[b]{3}{*}{ Taxon } & \multirow[b]{3}{*}{ Distribution } & \multicolumn{2}{|c|}{ Vertebrae } & \multicolumn{3}{|c|}{ Fins } & \multicolumn{2}{|c|}{ Gill rakers } \\
\hline & & \multirow{2}{*}{ Precauda } & \multirow{2}{*}{ Caudal } & \multirow[b]{2}{*}{ Dorsal } & Fins & \multirow[b]{2}{*}{ Pectoral } & Upper & Lower \\
\hline & & & & & Anal & & \multicolumn{2}{|c|}{ (Total) } \\
\hline Agonomalus mozinoi & Cent. Calif.-Brit. Col. & & & VIII-IX,6-8 & $11-12$ & $11-12$ & & \\
\hline Agonopsis vulsa & SSC-Gulf of Alaska & \multicolumn{2}{|c|}{$(39-42)$} & VIII-X,7-9 & $10-12$ & $13-15$ & & \\
\hline Agonus acipenserinus ${ }^{\mathrm{a}}$ & N. Calif.-Chukchi Sea & \multicolumn{2}{|c|}{$(39-41)$} & VII-X,6-9 & $6-9$ & $16-19$ & & \\
\hline Agonus decagonus ${ }^{\mathrm{a}}$ & Bering Sea-Arctic & \multicolumn{2}{|c|}{$(47-48)^{b}$} & V-VII,5-8 & $6-8$ & $13-16$ & & \\
\hline Anoplagonus inermis & N. Calif.-Aleutian Is. & \multicolumn{2}{|c|}{$(41-45)$} & $4-6$ & $4-5$ & $8-10$ & & \\
\hline Aspidophoroides bartoni & Gulf of Alaska-Arctic & \multicolumn{2}{|c|}{$(51-53)$} & $4-6$ & $4-6$ & $9-10$ & & \\
\hline Aspidophoroides olriki & Bering Sea-Arctic & \multicolumn{2}{|c|}{$(38-40)$} & 5-7 & $5-7$ & $13-16$ & & \\
\hline Bathyagonus alascanus & N. Calif.-Bering Sea & \multicolumn{2}{|c|}{$(39-41)$} & V-VIII,5-8 & $6-8$ & 15 & & \\
\hline Bathyagonus infraspinatus & N. Calif.-Bering Sea & \multicolumn{2}{|c|}{$(38-39)$} & V-VIII,5-8 & $5-8$ & $15-16$ & & \\
\hline Bathyagonus nigripinnis & N. Calif.-Bering Sea & \multicolumn{2}{|c|}{$(44-45)$} & VI-VIII,6-7 & $7-9$ & $15-16$ & & \\
\hline Bathyagonus pentacanthus & S. Calif.-Bering Sea & \multicolumn{2}{|c|}{$(40-46)$} & V-VIII,5-8 & 6-9 & $14-16$ & & \\
\hline Bothragonus swani & Cent. Calif.-Gulf of Alaska & \multicolumn{2}{|c|}{$(29-31)$} & $\Pi-\mathrm{V}, 4-6$ & $4-5$ & $10-12$ & & \\
\hline Hypsagonus quadricornus & Wash.-Bering Sea & \multicolumn{2}{|c|}{ (36) } & IX-XI,5-7 & $9-11$ & $12-14$ & & \\
\hline Ocella dodecaedron & Aleutian Is.-Chukchi Sea & \multicolumn{2}{|c|}{$(38-39)$} & LX-XI,7-8 & $14-16$ & $14-15$ & & \\
\hline Ocella impi ${ }^{c}$ & Brit. Col. & \multicolumn{2}{|c|}{$(37)^{\mathrm{b}}$} & $\mathrm{IX}, 6$ & 9 & 18 & & \\
\hline Ocella verrucosa & Cent. Calif.-Bering Sea & $13-14$ & $21-24$ & VII-IX,6-9 & 7-12 & $14-15$ & $1-2$ & $8-12$ \\
\hline Odontopyxis trispinosa & SSC-SE Alaska & $10-12$ & $27-30$ & III-VI,5-7 & $5-7$ & $13-15$ & & 7 \\
\hline Pallasina barbata & Cent. Calif.-Bering Sea & \multicolumn{2}{|c|}{$(45-47)$} & V-LX, $6-7$ & $9-14$ & $10-13$ & & \\
\hline Percis japonicus & Bering Sea & \multicolumn{2}{|c|}{ (42) } & V-VII,6 & $7-9$ & 12 & & \\
\hline Sarritor frenatus & Brit. Col.-Bering Sea & \multicolumn{2}{|c|}{$(46-48)$} & VI-VIU, 6-8 & $6-7$ & $15-17$ & & \\
\hline Sarritor leptorhynchus & Gulf of Alaska-Bering Sea & \multicolumn{2}{|c|}{$(42-44)$} & VI-LX,5-8 & $6-8$ & $13-15$ & & \\
\hline Stellerina xyosterna & SSC-Brit. Col. & \multirow{2}{*}{\multicolumn{2}{|c|}{$(34-37)$}} & VI-VIII,5-7 & $8-9$ & $17-19$ & $1-2$ & $8-12$ \\
\hline & & & & & & & & 1) \\
\hline Xeneretmus lauifrons & SSC-Brit. Col. & $11-13$ & $28-30$ & VI-VII, 6-8 & $6-9$ & $13-15$ & & $10-11$ \\
\hline Xeneretmus leiops & S. Calif.-SE Alaska & & & VI-VII,6-8 & $5-8$ & $13-15$ & & \\
\hline Xeneretmus triacanthus & SSC-Brit. Col. & 12 & $29-30$ & V-VII,6-7 & 5-7 & $12-14$ & 1 & $8-13$ \\
\hline
\end{tabular}

a Placement in the genus Agonus is questionable (Lea and Dempster 1982).

${ }^{b}$ W.A. Laroche, 24 Maple Park, Box 216, Enosburg Falls, VT 05450, pers. commun., 10 Dec. 1986.

${ }^{c}$ Ocella impi may not be a valid species, rather it may be the juvenile of Stellerina xyosterna (A.E. Peden, Brit. Col. Prov. Mus., Victoria, B.C., Canada V8V 1 X4, pers. commun., 22 Jan. 1987). 


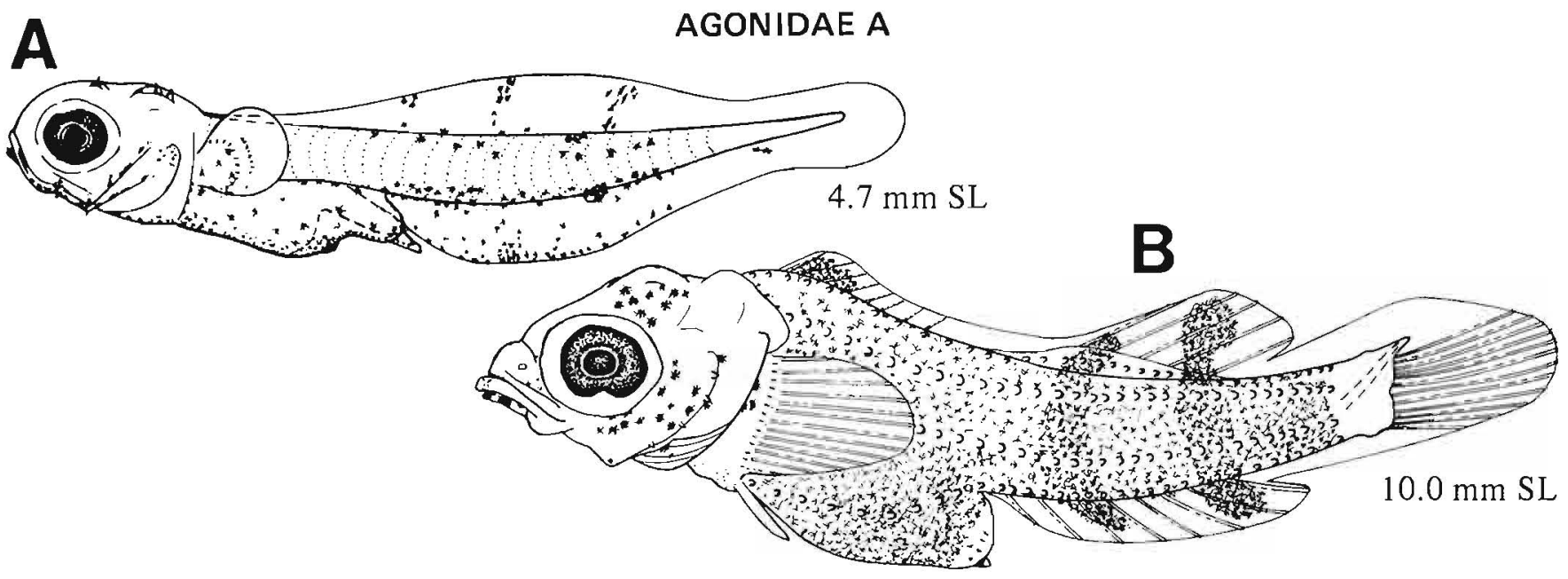

\section{OTHER AGONIDS}

C Unidentified agonid

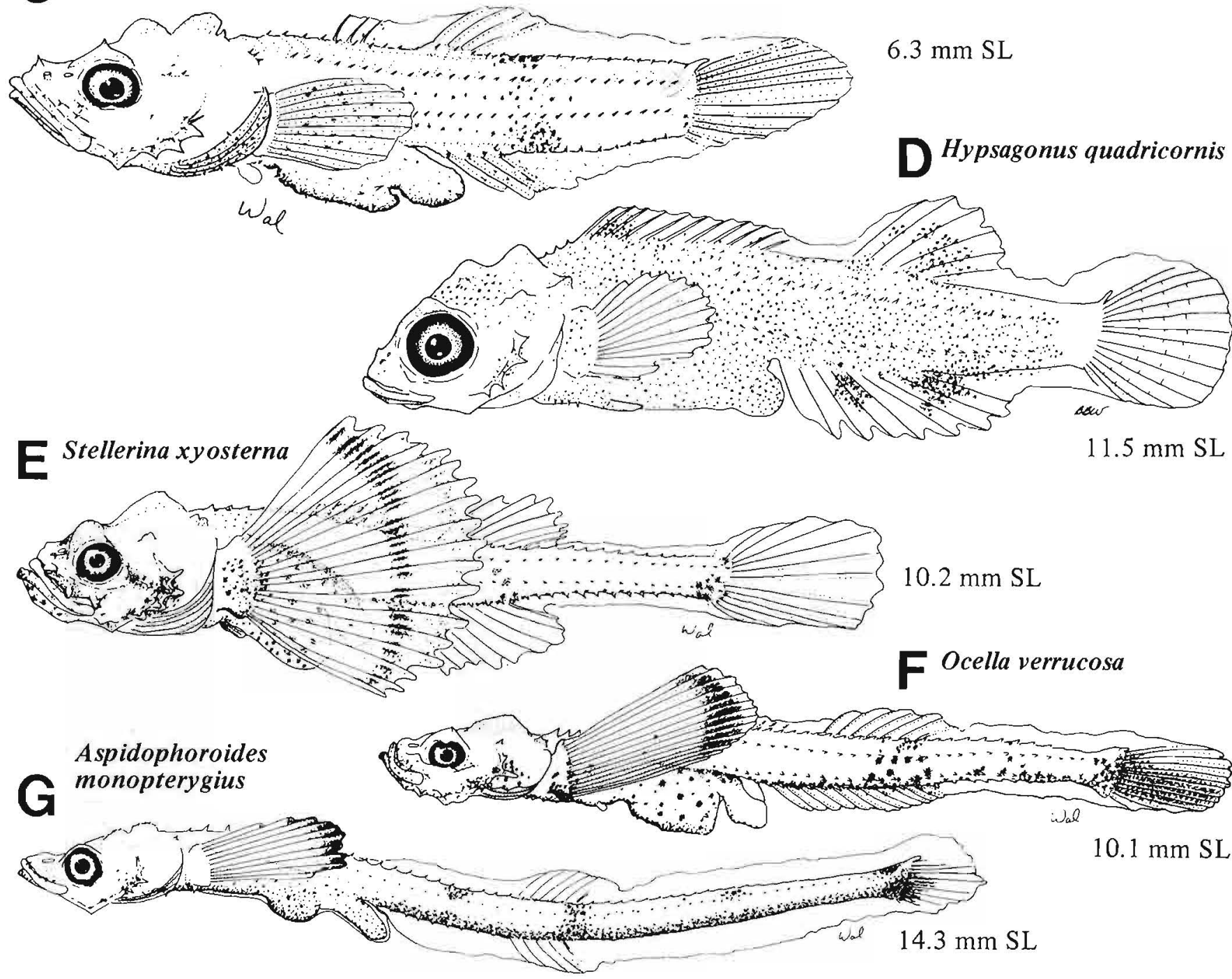

Figures A-B, NWAFC originals (B. Vinter); $\mathrm{C}-\mathrm{G}$, Washington et al. 1984b (Figure C was misidentified in Washington et al. (1984b) as Bothragonus swani; G, Atlantic specimen). 
MERISTICS

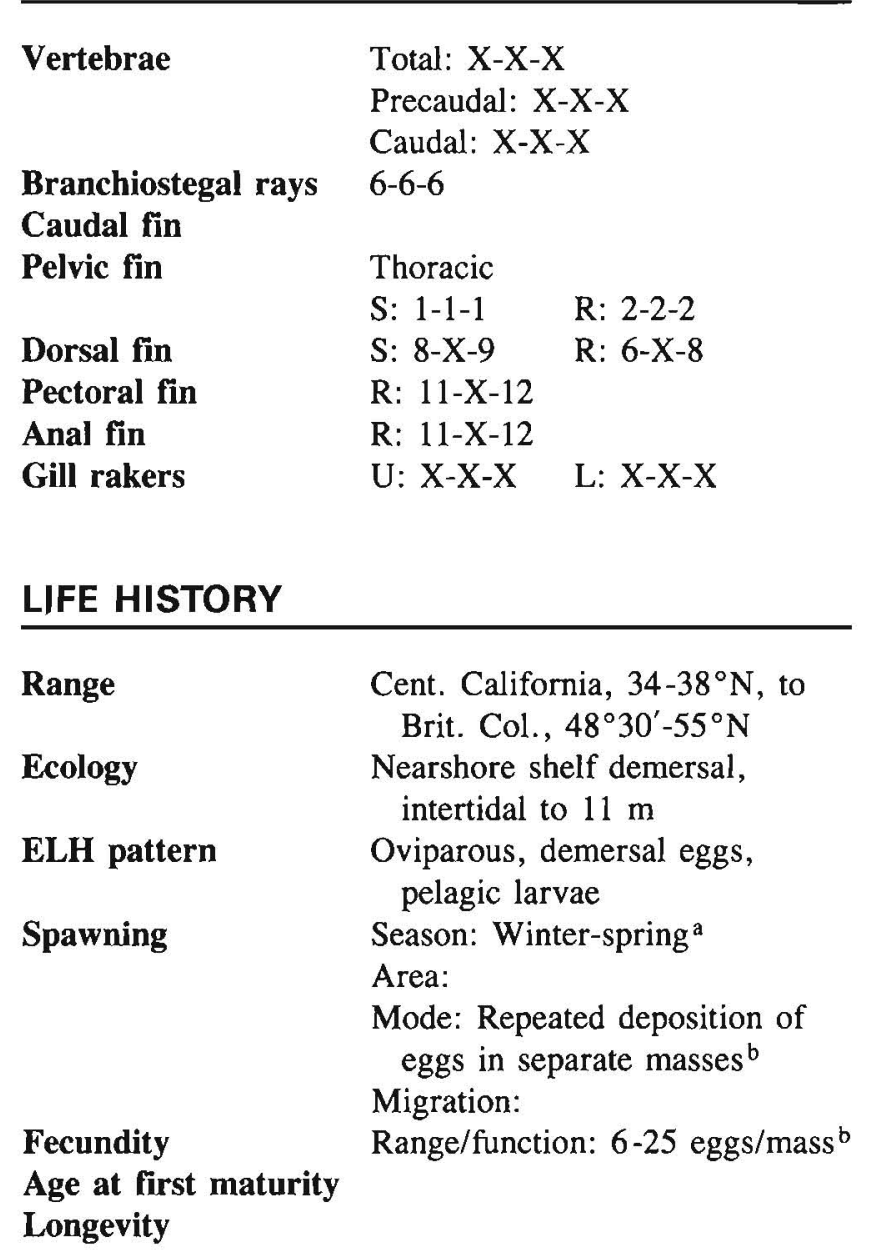

\section{EARLY LIFE HISTORY DESCRIPTION}

\section{EGGS}

Diameter

No. of oil globules

Oil globule diameter

Yolk

Envelope

Hatch size $\quad 5.5 \mathrm{~mm} \mathrm{SL}$

Incubation time/temp.

Pigment

Diagnostic characters

\author{
LARVAE \\ Preanal length \\ Length at flexion \\ Length at transformation \\ Sequence of fin \\ development \\ Pigment \\ - Dorsal and ventral finfolds \\ - Heavily pigmented body, pigment increasing with \\ development \\ Diagnostic characters \\ - Based on one specimen (8.2 mm SL), they are \\ superficially similar to cottid Hemitripterus group \\ (p. 448)
}

\footnotetext{
a.A. Laroche, 24 Maple Park, Box 216, Enosburg Falls, VT 05450, pers. commun., 10 Dec. 1986.

bJ. Marliave, Vancouver Public Aquarium, P.O. Box 3232, Vancouver, B.C., Canada V6B 3X8, pers. commun., 16 Oct. 1986.

'Incomplete series.

Ref: Washington et al. 1984a,b.
} 

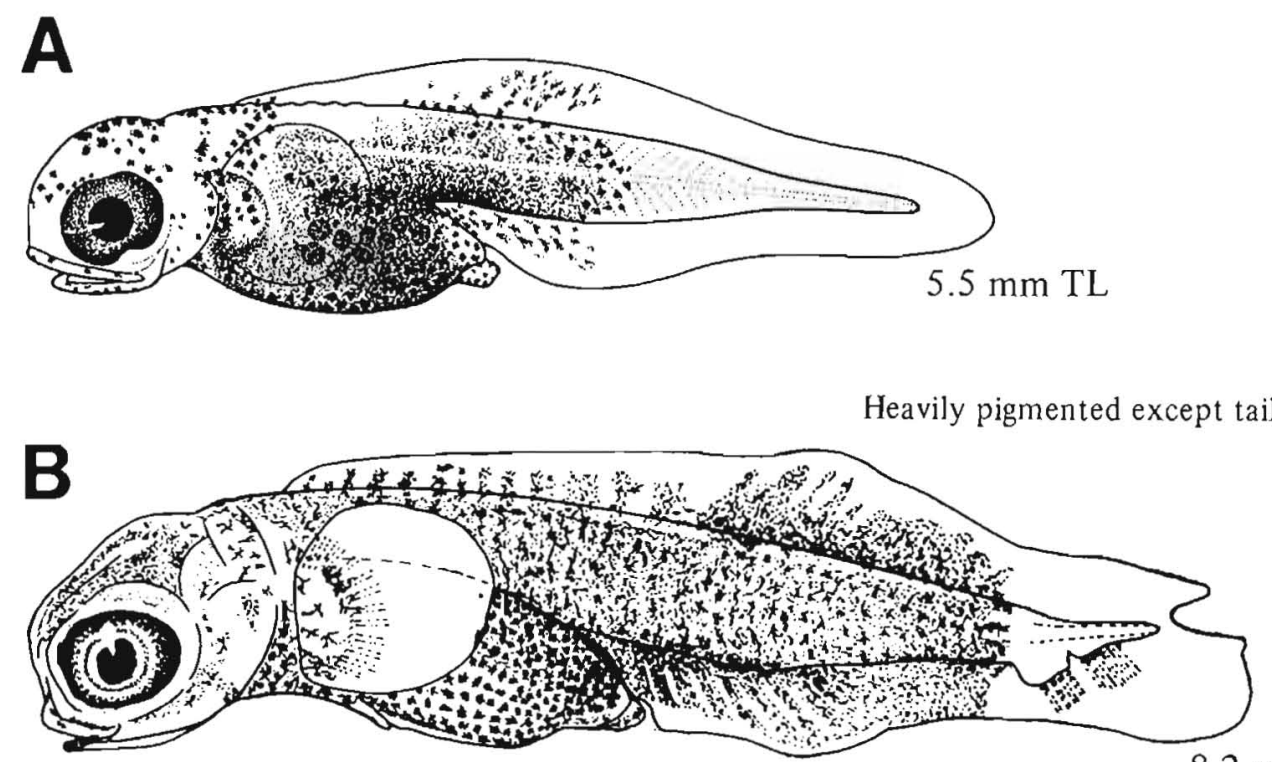

$8.2 \mathrm{~mm} \mathrm{SL}$

Figure A, Marliave 1978; B, NWAFC original (B. Vinter). 


\section{MERISTICS}

$\begin{array}{lll}\text { Vertebrae } & \begin{array}{l}\text { Total: } 29-30-31 \\ \text { Precaudal: X-X-X } \\ \text { Caudal: X-X-X }\end{array} \\ \begin{array}{lll}\text { Branchiostegal rays } & 6-6-6 \\ \begin{array}{l}\text { Caudal fin } \\ \text { Pelvic fin }\end{array} & & \\ & \text { Thoracic } & \\ \text { Dorsal fin } & \text { S: } 1-1-1 & \text { R: } 2-2-2 \\ \text { Pectoral fin } & \text { S: } 2-3-5 & \text { R: } 4-5-6 \\ \text { Anal fin } & \text { R: } 10-12-12 & \\ \text { Gill rakers } & \text { R: } 4-4-5 & \\ & \text { U: } X-X-X & \text { L: } X-X-X\end{array}\end{array}$

\section{LIFE HISTORY}

$\begin{array}{ll}\text { Range } & \begin{array}{c}\text { Cent. California, } 34-38^{\circ} \mathrm{N}, \text { to } \\ \text { Gulf of Alaska, } 54-60^{\circ} \mathrm{N}\end{array} \\ \text { Ecology } & \begin{array}{l}\text { Nearshore shelf demersal, } \\ \text { intertidal to } 18 \mathrm{~m}\end{array} \\ \text { ELH pattern } & \text { Oviparous; demersal, attached } \\ \text { eggs; pelagic larvae } & \text { Season: Winter-spring } \\ \text { Spawning } & \text { Area: On kelp holdfasts } \\ & \text { Mode: } \\ & \text { Migration: } \\ \text { Fecundity } & \text { Range/function: } \\ \text { Age at first maturity } & \\ \text { Longevity } & \end{array}$

\section{EARLY LIFE HISTORY DESCRIPTION}

\section{EGGS}

$7.5 \mathrm{~mm} \mathrm{TL}$
Diameter

No. of oil globules

Oil globule diameter

Yolk

Envelope

Hatch size

Incubation time/temp.

Pigment

$2 \mathrm{~mm}$

Diagnostic characters

\section{LARVAE}

Preanal length

Length at flexion $\quad \sim 10-12 \mathrm{~mm}$ TL

Length at transformation $>16 \mathrm{~mm} \mathrm{TL}$

Sequence of fin development

Pigment

- Upper and lower jaw

- Dorsal head

- Anterior gut and body

- Outer edge of pectoral fin

- Flexion and postflexion larvae develop several vertical bars on body

Diagnostic characters

- Large pigmented pectoral fins

- Body spines develop in flexion larvae

- Body short and stocky compared with Xeneretmus latifrons

${ }^{a}$ W.A. Laroche, 24 Maple Park, Box 216, Enosburg Falls, VT 05450, pers. commun., 10 Dec. 1986.

${ }^{\mathrm{b}}$ Marliave 1975a

Ref: Marliave 1975a, Washington et al. 1984b. 

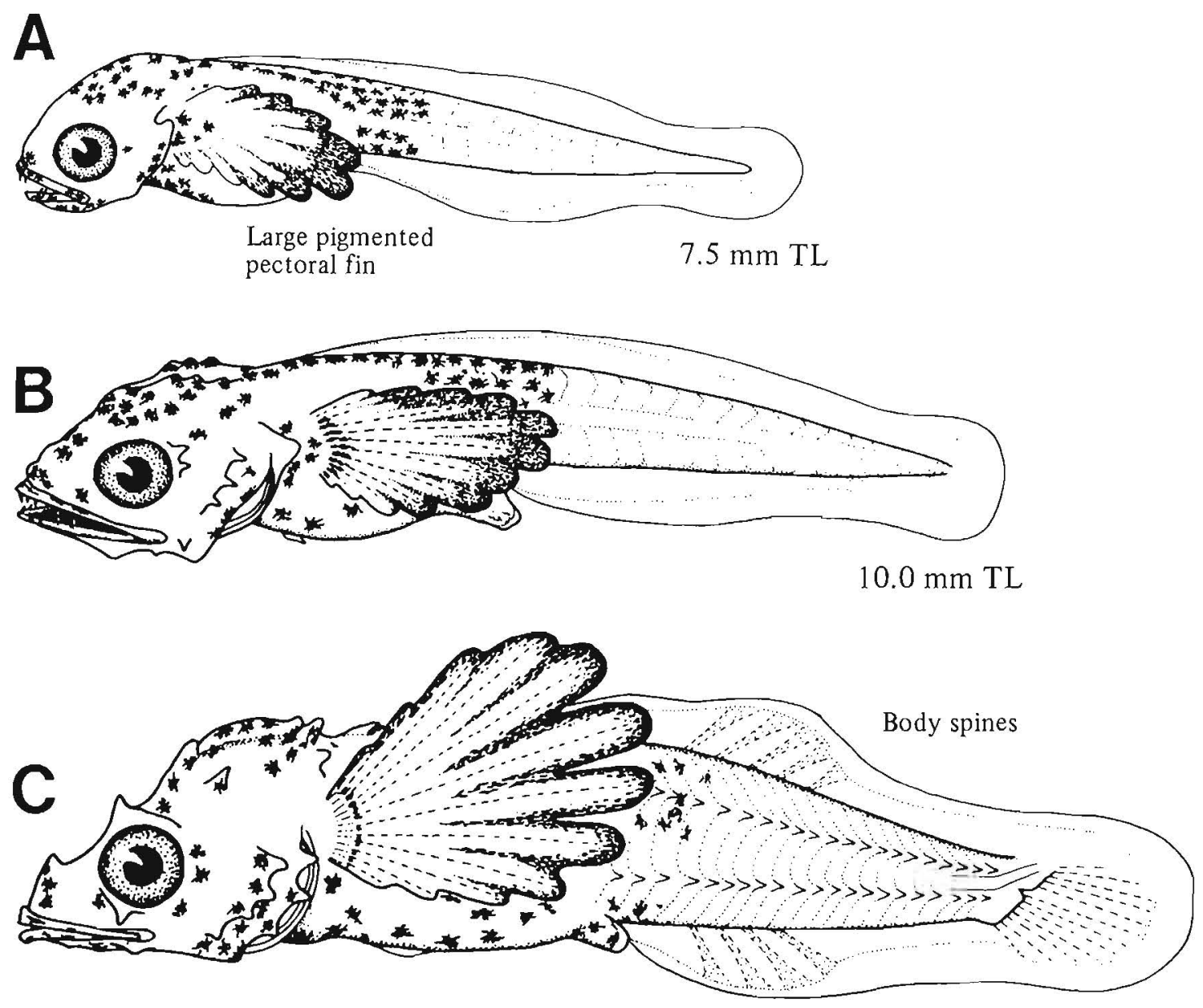

$12.0 \mathrm{~mm} \mathrm{TL}$

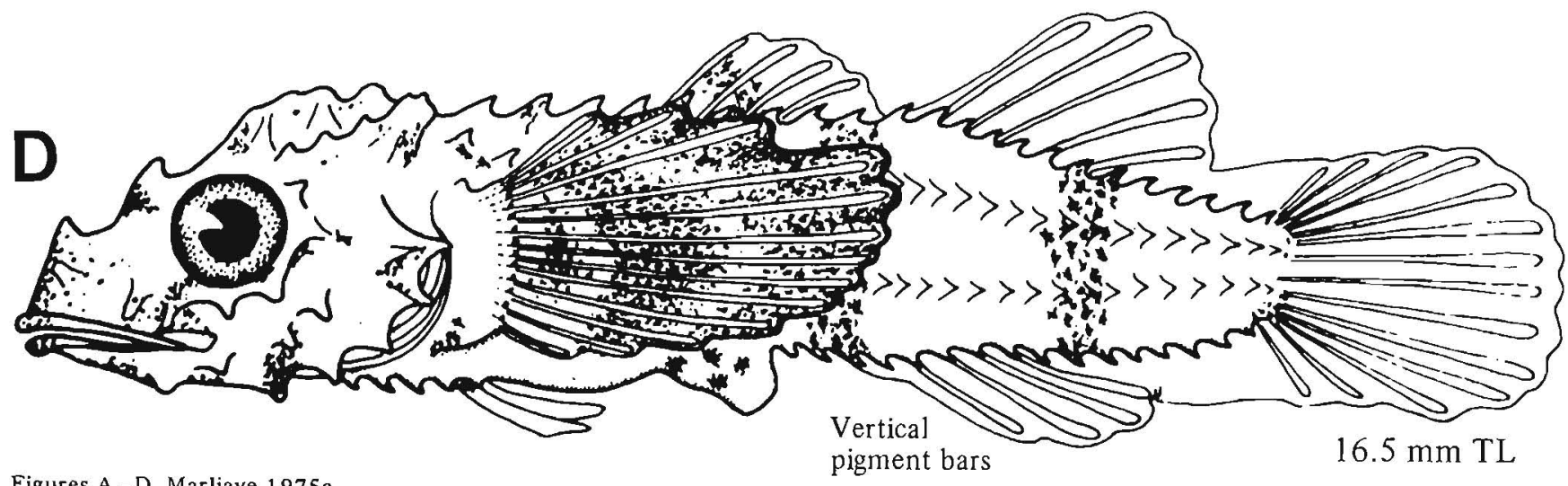

Figures A-D, Marliave 1975a. 


\section{MERISTICS}

Vertebrae

Total: $39-41-42$

Precaudal: 11-12-13

Caudal: $28-29-30$

Branchiostegal rays

6-6-6

Caudal fin

Pelvic fin

Thoracic

S: $1-1-1$

S: $6-7-8$

R: $2-2-2$

Dorsal fin

R: $13-14-15$

Pectoral fin

Anal fin

R: $6-7-9$

Gill rakers

$\mathrm{U}: \mathrm{X}-\mathrm{X}-\mathrm{X}$

L: $10-X-11$

\section{EARLY LIFE HISTORY DESCRIPTION}

\section{EGGS}

Diameter

No. of oil globules

Oil globule diameter

Yolk

Envelope

Hatch size $\quad \sim 7 \mathrm{~mm}$ TL

Incubation time/temp.

Pigment

Diagnostic characters

\section{LARVAE}

Preanal length

Length at flexion $\quad \sim 10 \mathrm{~mm}$ TL

Length at transformation

Sequence of fin development

Pigment $^{c}$

- Body pigment extending into finfold in four vertical bars: Over posterior gut, midbody, at $3 / 4$ body length, and tail region

- Preflexion larvae have pigment on jaws

Diagnostic characters

- Four vertical pigment bars extending into finfolds

- Body spines develop in postflexion larvae

- Long slender body compared with Bothragonus swani

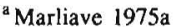

${ }^{\mathrm{b}}$ Fitch and Lavenberg 1968

${ }^{c}$ J. Marliave, Vancouver Public Aquarium, P.O. Box 3232, Vancouver, B.C., Canada

V6B $3 \times 8$, pers. commun., 16 Oct. 1986.

Ref: Marliave 1975a, Washington et al. 1984b.
} 

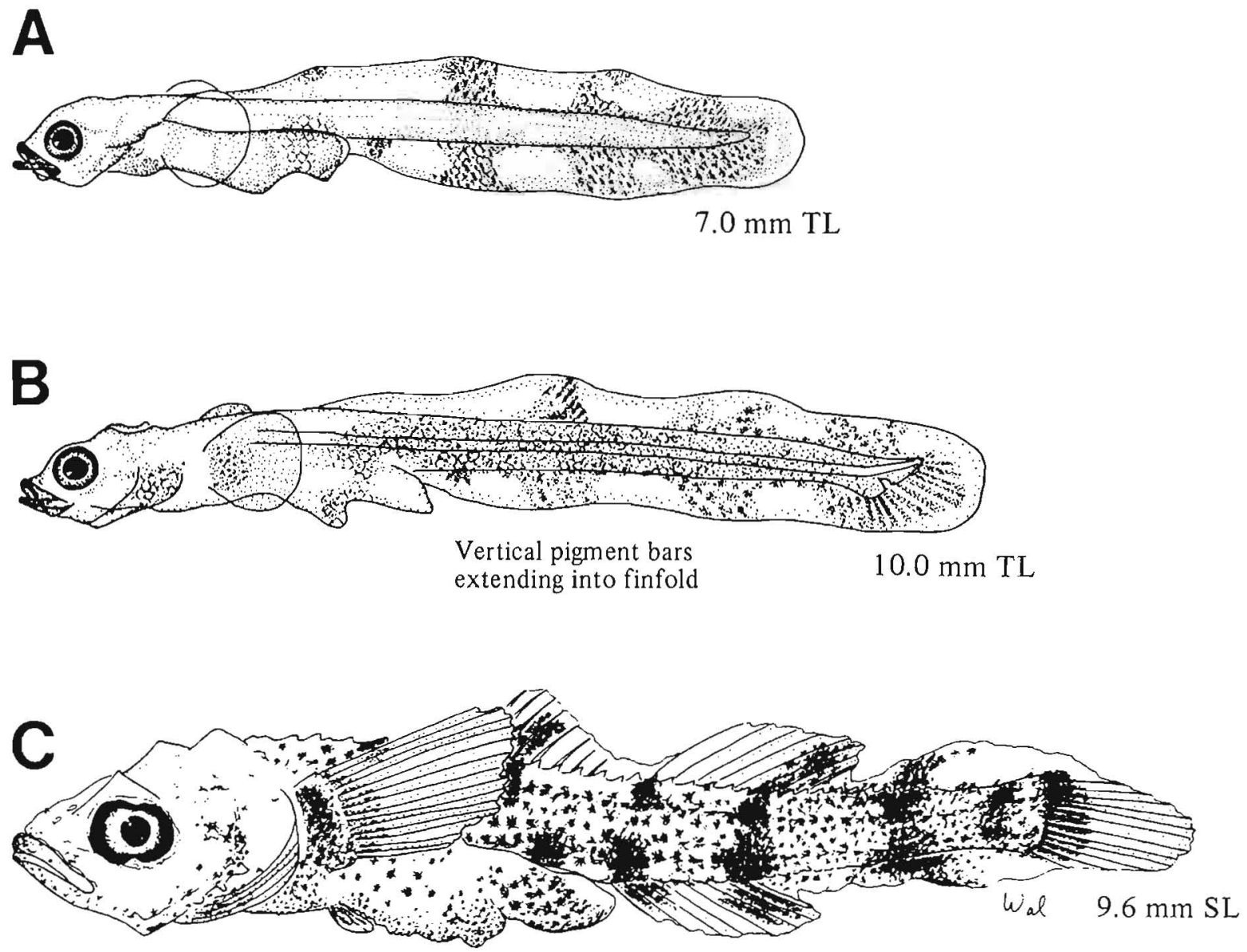

D

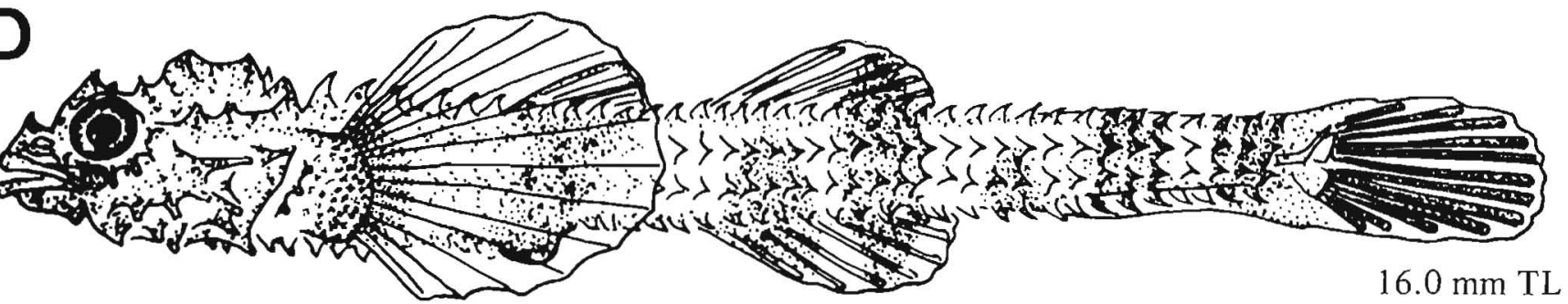

Figures A-B, D, Marliave 1975a (D, length in Marliave 1975a is given as $21.0 \mathrm{~mm}$ TL [footnote c]) ; C, Washington et al. $1984 \mathrm{~b}$. 

The family Cyclopteridae is composed of two subfamilies: the Cyclopterinae (lumpsuckers) and the Liparidinae (snailfishes). Although both subfamilies possess a ventral sucking disc, many differences distinguish the two groups (e.g., lumpsuckers have 2 dorsal fins and about 23-29 vertebrae, and snailfishes have a single dorsal fin and about 38-86 vertebrae).

\section{Cyclopterinae}

Cyclopterines are found exclusively in the cooler waters of the Northern Hemisphere. The subfamily is represented by eleven species in five genera in the Northeast Pacific and Bering Sea. Mostly benthic, adults may be found clinging to rocks in tidal zones or in rocky habitat as deep as $225 \mathrm{~m}$ (Hart 1973). Eggs, which are adhesive, 1.9-5.0 $\mathrm{mm}$ in diameter, and may contain one or more oil globules, are laid in nest sites within rocky crevices or shells and are guarded during incubation (Able et al. 1984). Newly hatched larvae may be identified as cyclopterines by their stout body shape, heavy pigmentation, large sucking disc on the ventral surface, and advanced state of development. They are found attached to substrate and prefer areas with current (A.C. Matarese and S.F. Borton, unpubl.). Larvae of only two species can be identified in our area, Aptocyclus ventricosus and Eumicrotremus orbis.

Table 40

Meristic characters of subfamily Cyclopterinae. All have pelvic discs and six branchiostegal rays.

\begin{tabular}{|c|c|c|c|c|c|c|c|c|}
\hline \multirow[b]{2}{*}{ Taxon } & \multirow[b]{2}{*}{ Distribution } & \multicolumn{2}{|c|}{ Vertebrae } & \multicolumn{3}{|c|}{ Fins } & \multicolumn{2}{|c|}{ Gill rakers } \\
\hline & & Precaudal & Caudal & Dorsal & Anal & Pectoral & Upper & Lower \\
\hline Aptocyclus ventricosus & Brit. Col.-Bering Sea & 14 & $13-15$ & $V, 8-11$ & $6-9$ & $19-22$ & & $5-7$ \\
\hline Cyclopteropsis phrynoides & Gulf of Alaska-Bering Sea & & & Vח,11 & & 25 & & \\
\hline Eumicrotremus andriashevi & Bering Sea-Chukchi Sea & & & VI-VII, 10-12 & $10-11$ & $23 \cdot 27$ & & \\
\hline Eumicrotremus barbatus & Aleutian Is. & 11 & 16 & VII, 11 & 10 & 23 & & \\
\hline Eumicrotremus birulai & Gulf of Alaska-Bering Sea & 11 & 17 & VI-VII,9-12 & $9-11$ & $25-29$ & & $6-9$ \\
\hline Eumicrotremus gyrinops & Aleutian Is.-Bering Sea & & & VIII,9 & 9 & 24 & & \\
\hline Eumicrotremus orbis & Wash.-Bering Sea & $10-11$ & $17-18$ & V-VII,9-11 & $9-11$ & $19-27$ & & $5-6$ \\
\hline Eumicrotremus soldatovi & Bering Sea & 11 & 18 & VI, $11-12$ & 10 & 24 & & \\
\hline Eumicrotremus taranetzi & Bering Sea & 11 & 16 & V-VI,9-10 & $9-10$ & $24-26$ & & $6-7$ \\
\hline Lethotremus muticus & Aleutian Is.-Bering Sea & & & VII, II & 10 & 23 & & \\
\hline Pelagocyclus vitiazi & Bering Sea & & & IV-V,9-10 & $8-9$ & $19-21$ & & \\
\hline
\end{tabular}




\section{MERISTICS}

\begin{tabular}{|c|c|c|}
\hline Vertebrae & \multicolumn{2}{|c|}{$\begin{array}{l}\text { Total: } 27-X-29 \\
\text { Precaudal: } 14-14-14 \\
\text { Caudal: } 13-X-15\end{array}$} \\
\hline $\begin{array}{l}\text { Branchiostegal rays } \\
\text { Caudal fin }\end{array}$ & $6-6-6$ & \\
\hline Pelvic fin & $\begin{array}{l}\text { Disc } \\
\text { S: X-X-X }\end{array}$ & R: X-X-X \\
\hline Dorsal fin & $S: 5-5-5$ & $R: 8-10-11$ \\
\hline Pectoral fin & R: $19-20-22$ & \\
\hline Anal fin & R: $6-8-9$ & \\
\hline Gill rakers & $\mathrm{U}: \mathrm{X}-\mathrm{X}-\mathrm{X}$ & L: $5-X-7$ \\
\hline
\end{tabular}

\section{LIFE HISTORY}

$\begin{array}{ll}\text { Range } & \text { Brit. Col., } 48^{\circ} 30^{\prime}-55^{\circ} \mathrm{N} \text {, to } \\ \text { Bering Sea, } 54-66^{\circ} \mathrm{N} \\ \text { Ecology } & \text { Epi-, meso-, and bathypelagic, } \\ & 0-1500 \mathrm{~m} \\ \text { ELH pattern } & \text { Oviparous; demersal, adhesive } \\ & \text { eggs; demersal larvae } \\ \text { Spawning } & \text { Season: } \\ & \text { Area: } \\ & \text { Mode: Males guard eggs }{ }^{\mathrm{a}} \\ & \text { Migration: } \\ \text { Fecundity } & \text { Range/function: } 3800^{\mathrm{a}} \\ \text { Age at first maturity } & \\ \text { Longevity } & \end{array}$

\section{EARLY LIFE HISTORY DESCRIPTION}

\section{EGGS}

\section{Diameter}

No. of oil globules Oil globule diameter

2.3-2.4 mm

(Present)

Yolk

Envelope

Hatch size

6.5-7.0 $\mathrm{mm} \mathrm{TL}$
Incubation time/temp.

Pigment

Diagnostic characters

\section{LARVAE}

Preanal length

Length at flexion

Length at transformation

Sequence of fin development

Pigment

- Initially, body is lightly pigmented but with development pigment increases to cover entire body

Diagnostic characters

- Smooth body (lack of spines)

Distinguished from other cyclopterines by

- Distribution

- Meristic characters:

Total vertebrae

Dorsal fin spines

Dorsal fin rays

Anal fin rays
$33 \%$ SL

6.5-7.0 mm TL

Pectorals and pelvic disc form before hatching

\footnotetext{
${ }^{a}$ Able et al. 1984
}

Ref: Able et al. 1984, Kobayashi 1962 

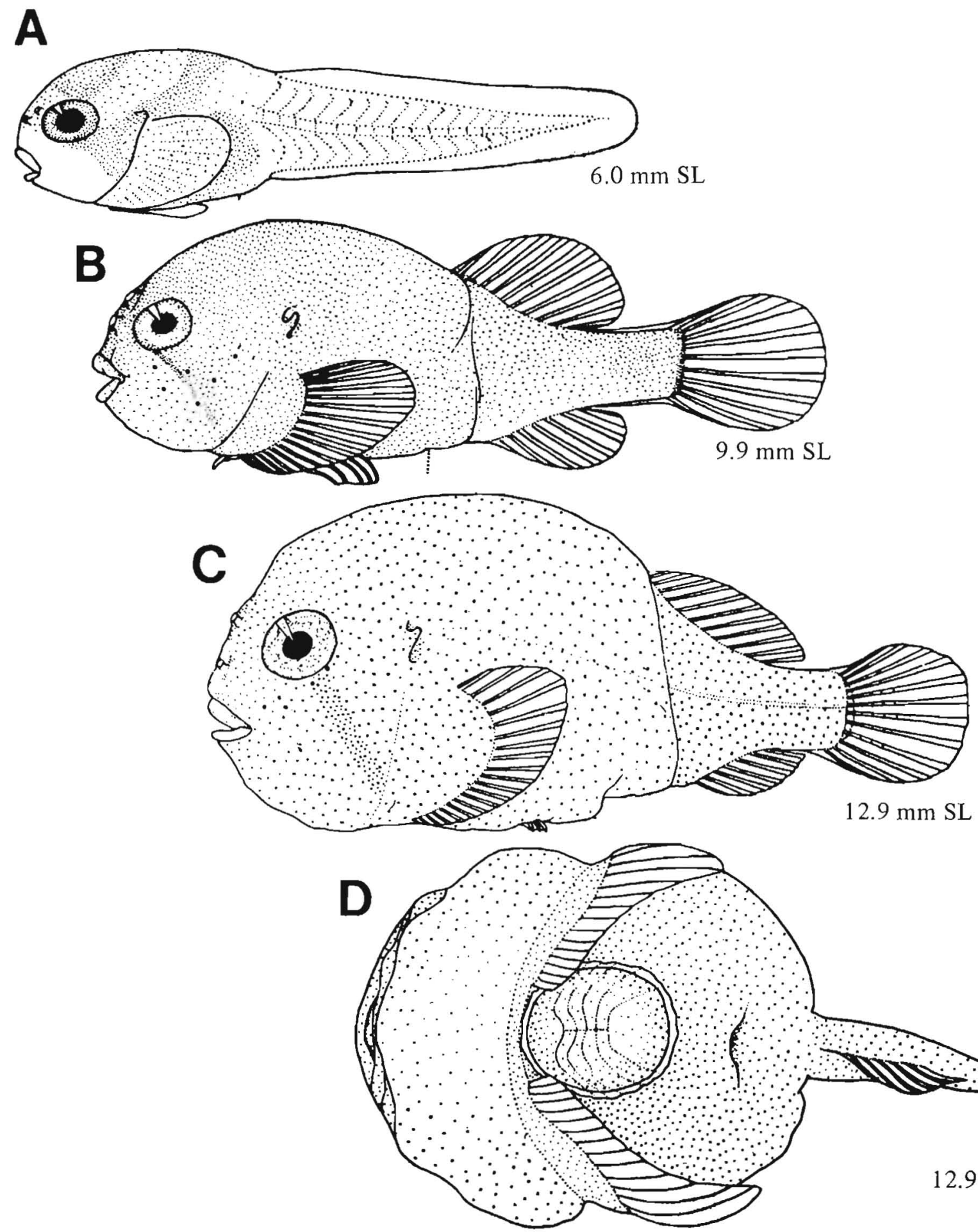

$12.9 \mathrm{rnm} \mathrm{SL}$

Figures A-D (D, ventral view), Kobayashi 1962 (A, reared from specimens collected near Hokkaido, Japan). 


\section{MERISTICS}

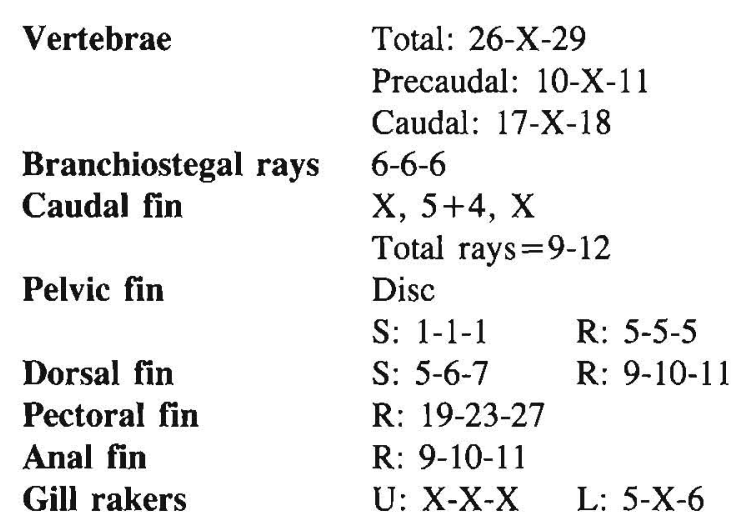

LIFE HISTORY

$\begin{array}{ll}\text { Range } & \text { Washington, } 46-48^{\circ} 30^{\prime} \mathrm{N}, \text { to } \\ & \text { Chukchi Sea, north of } 66^{\circ} \mathrm{N} \\ \text { Ecology } & \text { Epi-, meso-, and bathybenthal, } \\ & 0-575 \mathrm{~m} \\ \text { ELH pattern } & \text { Oviparous; demersal, attached, } \\ & \text { adhesive eggs; demersal larvae } \\ \text { Spawning } & \text { Season: Fall-winter (Brit. Col.) }{ }^{\mathrm{a}} \\ & \text { Area: } \\ & \text { Mode: Males guard eggs laid in } \\ & \text { nests }{ }^{\mathrm{b}} \\ & \text { Migration: } \\ \text { Recundity } & \text { Range/function: } 305-1590 \\ & (737 \text { at } 52.5 \mathrm{~mm}) / \\ & \mathrm{F}=47.67 \times \mathrm{L}-1766.86, \\ & \mathrm{~L}=\mathrm{TL} \mathrm{mm} \mathrm{m}^{\mathrm{b}}\end{array}$

Age at first maturity $1 \mathrm{yr}$ (males) ${ }^{\mathrm{b}}$ Longevity

\section{EARLY LIFE HISTORY DESCRIPTION}

\section{EGGS}

Diameter

No. of oil globules

Oil globule diameter

Yolk

Envelope

$1.9-2.2 \mathrm{~mm}$

Hatch size

One to multiple

Incubation time/temp. $\quad 26 \mathrm{~d} / 9.7-12.0^{\circ} \mathrm{C}$

Pigment

Clear, homogeneous

Translucent

4.5-4.7 SL

Diagnostic characters

- Degree of body pigment

- Precocious fin development

\section{LARVAE}

Preanal length

50-75\% SL

Length at flexion

Prior to hatch

Length at transformation

Sequence of fin development

\section{9-8.7 mm SL}

Pelvics and caudal (prior to hatch), dorsal spines, dorsal rays, pectorals, anal (although caudal development is initiated prior to hatch, it is the last to complete development)

\section{Pigment}

- Initially, heavily pigmented except caudal peduncle, area around anus, first three anal rays, and snout

\section{Diagnostic characters}

- Pigment: Small melanophores covering most of body and dorsal fin

- Morphology: Globular shape, spines

- Precocious fin development

a Arita 1969

${ }^{b}$ A.C. Matarese and S.F. Borton, unpubl.

Ref: Matarese and Borton, unpubl. 
B
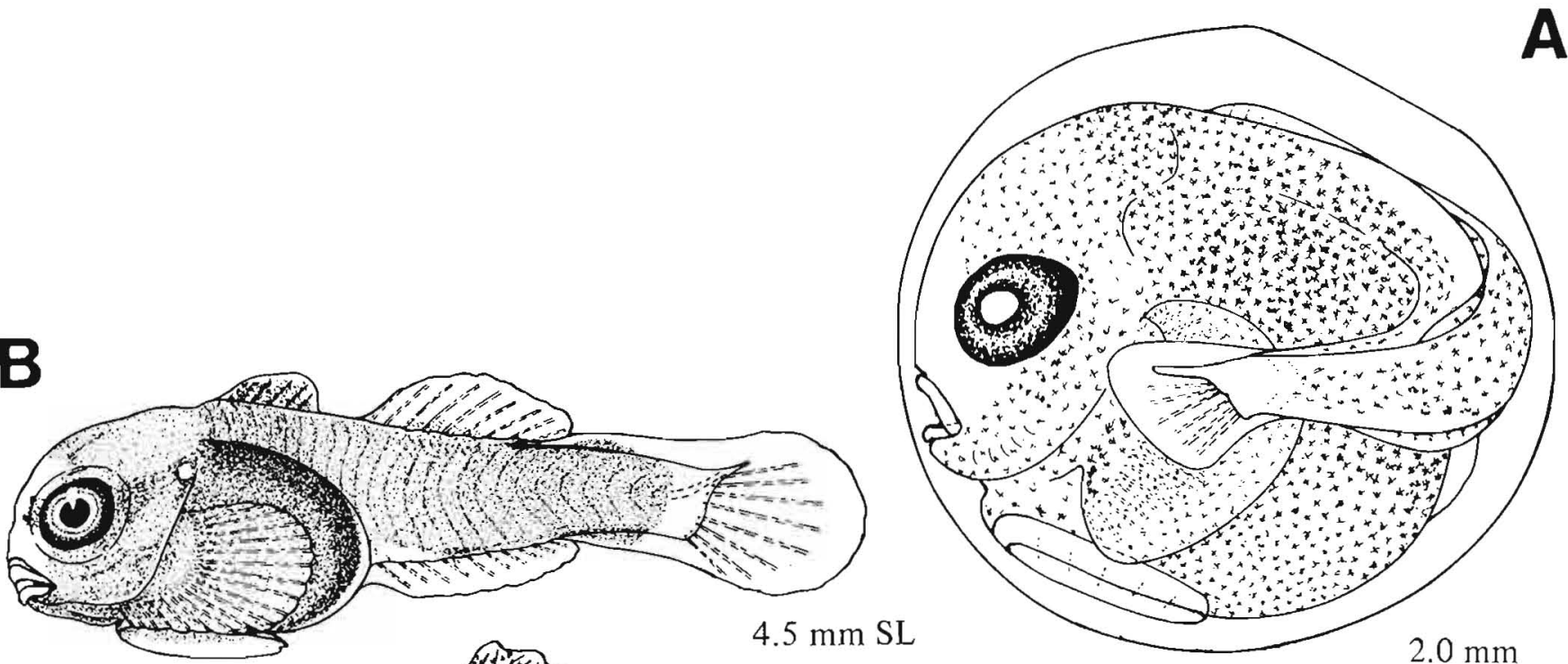

a

$4.5 \mathrm{~mm} \mathrm{SL}$

$2.0 \mathrm{~mm}$

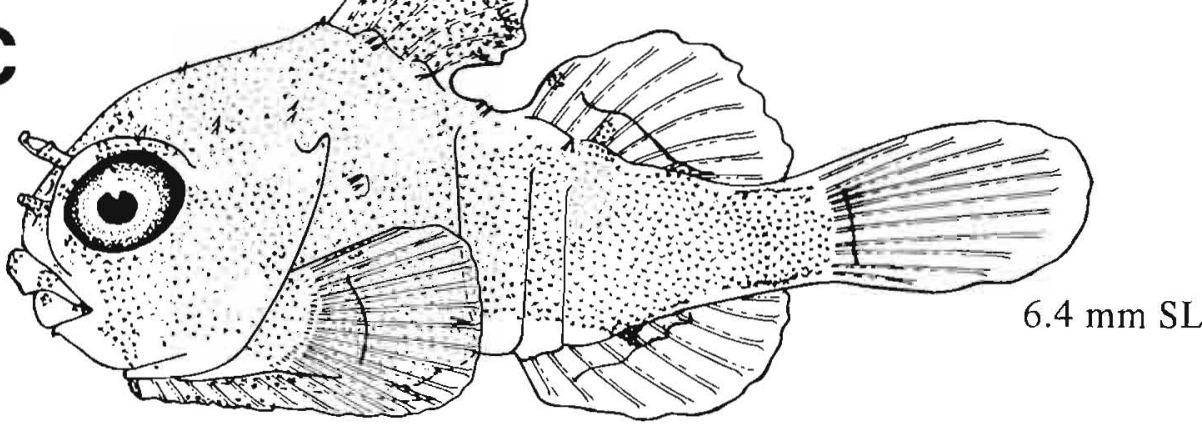

D
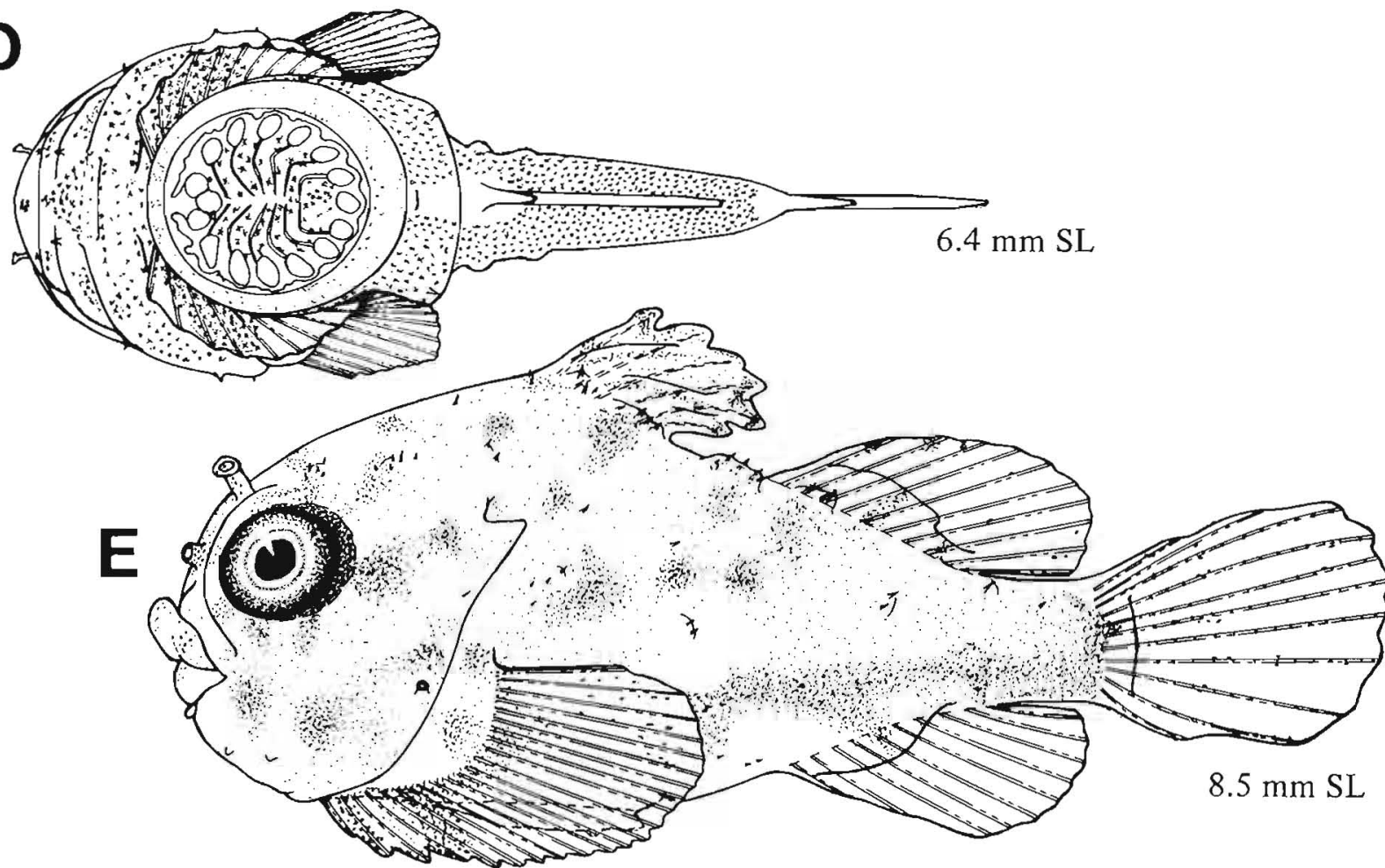

$8.5 \mathrm{~mm} \mathrm{SL}$

Figures $A-E(D$, ventral view), Matarese and Borton, unpubl. 


\section{CYCLOPTERIDAE}

\section{Liparidinae}

This large subfamily is circumpolar about both poles in distribution. Within the study area there are 74 described species within 14 genera. Adults are demersal or pelagic and have been found from tidepools to depths of $7000 \mathrm{~m}$ (Hart 1973, Able et al. 1984). Spawning habits are diverse. Adhesive clumps of eggs $(1-8 \mathrm{~mm})$ have been seen attached to algae, mollusc shells, and tubeworms; eggs and larvae of Careproctus spp. have been found in gill cavities of lithodid crabs (Anderson and Cailliet 1974). Parental care of eggs (i.e., hiding, paternal guarding, or both) is exhibited in most taxa studied. Most species studied, especially deep-water forms, hatch at an advanced stage of development (Able et al. 1984). Larvae are planktonic or benthic. Only one complete larval series is available from our area, Rhinoliparis barbulifer. Illustrations of two unidentified larvae are provided since each of them is relatively common in our collection. The small larva $(4.0 \mathrm{~mm} \mathrm{SL})$ is common in our Gulf of Alaska collections whereas the larger larva $(5.8 \mathrm{~mm} \mathrm{SL})$ is routinely collected in our surveys along the Pacific Coast and represents one of the ten most abundant groups of ichthyoplankton collected off Oregon (Mundy, pers. commun.) ${ }^{1}$. Representatives of specimens from other areas (Atlantic, Arctic) are presented for three genera: Careproctus, Paraliparis, and Liparis. Early juvenile specimens of Paraliparis sp. (probably $P$. deani) are occasionally collected in plankton nets. Illustrations of a tentatively identified partial series of Nectoliparis pelagicus are also provided.

${ }^{1}$ B. Mundy, NMFS Southwest Fish. Cent., Honolulu Lab., 2570 Dole St., Honolulu, HI 96822-2396, pers. commun., 1 Oct. 1986. 


\section{Unidentified Liparidinae}
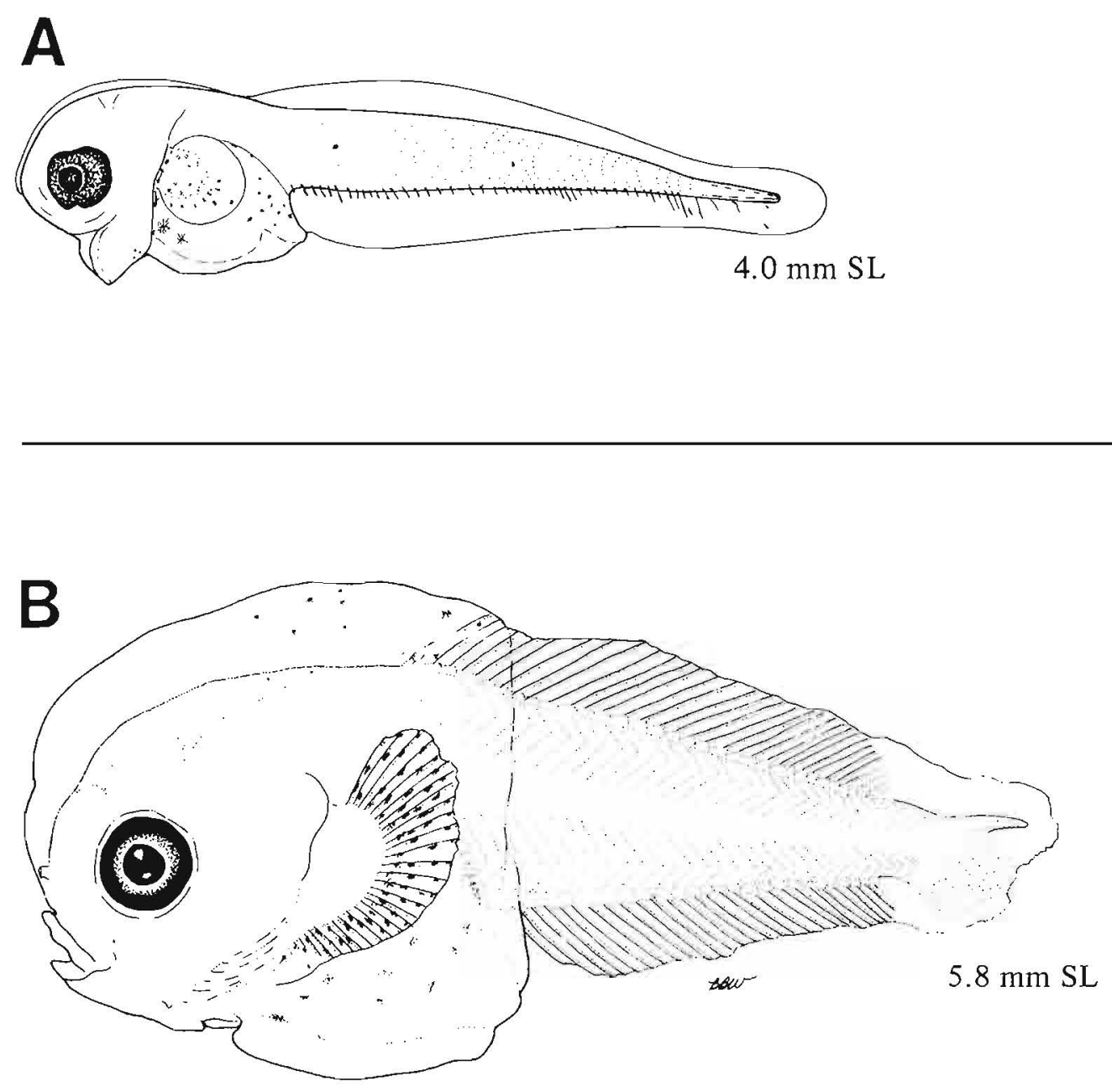

Figure A, NWAFC original (B. Vinter); B, Able et al. 1984. 


\section{LIPARIS}

\section{L. fabricii}

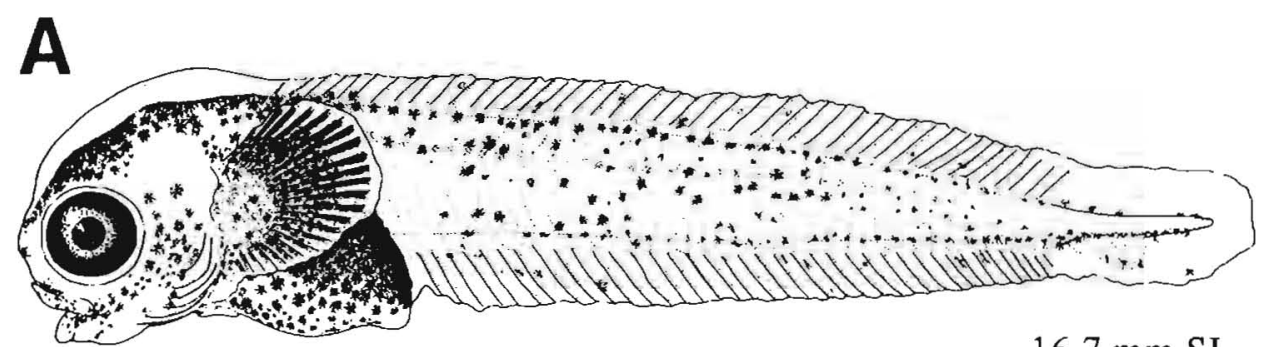

B

$16.7 \mathrm{~mm} \mathrm{SL}$
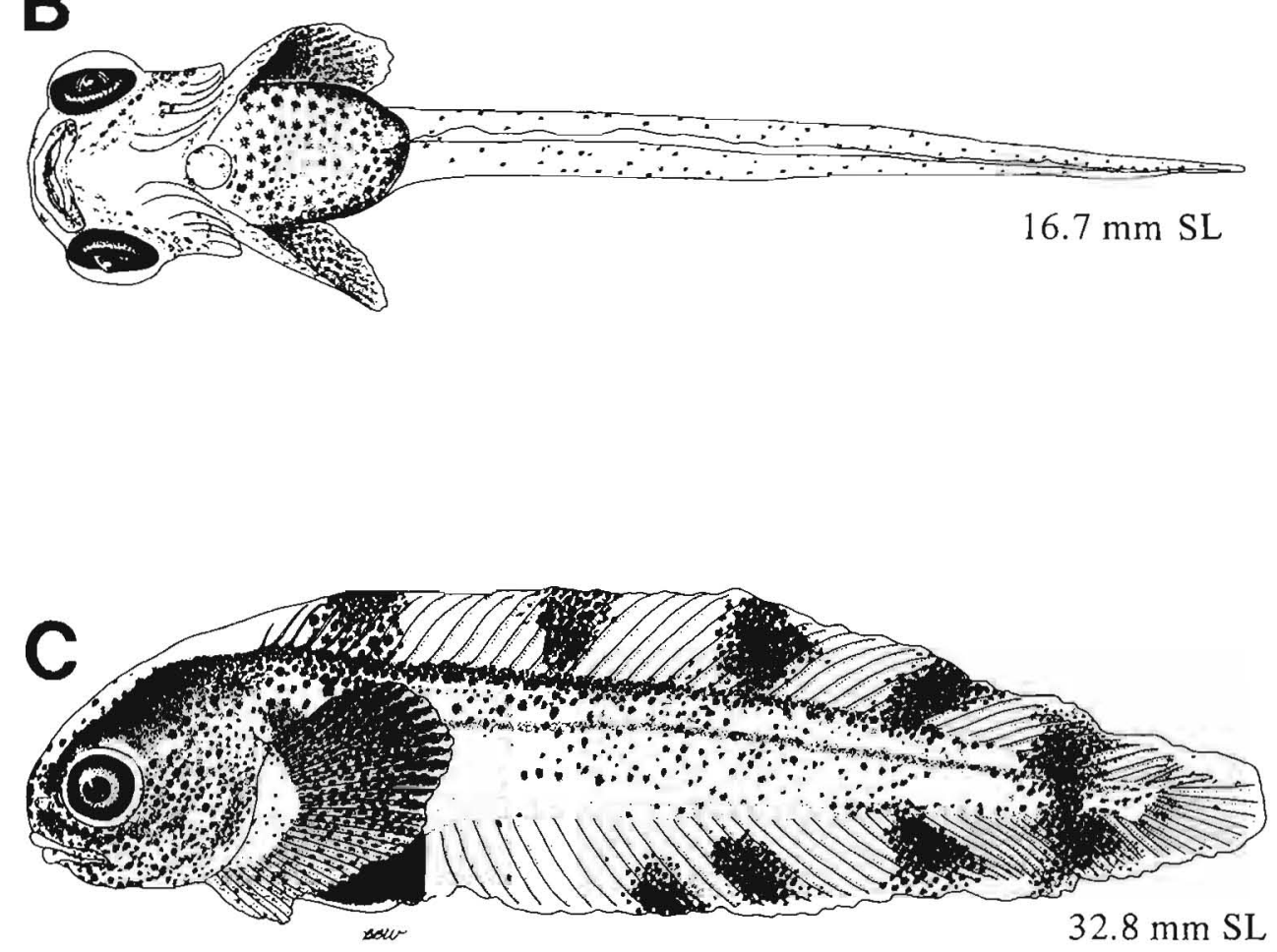

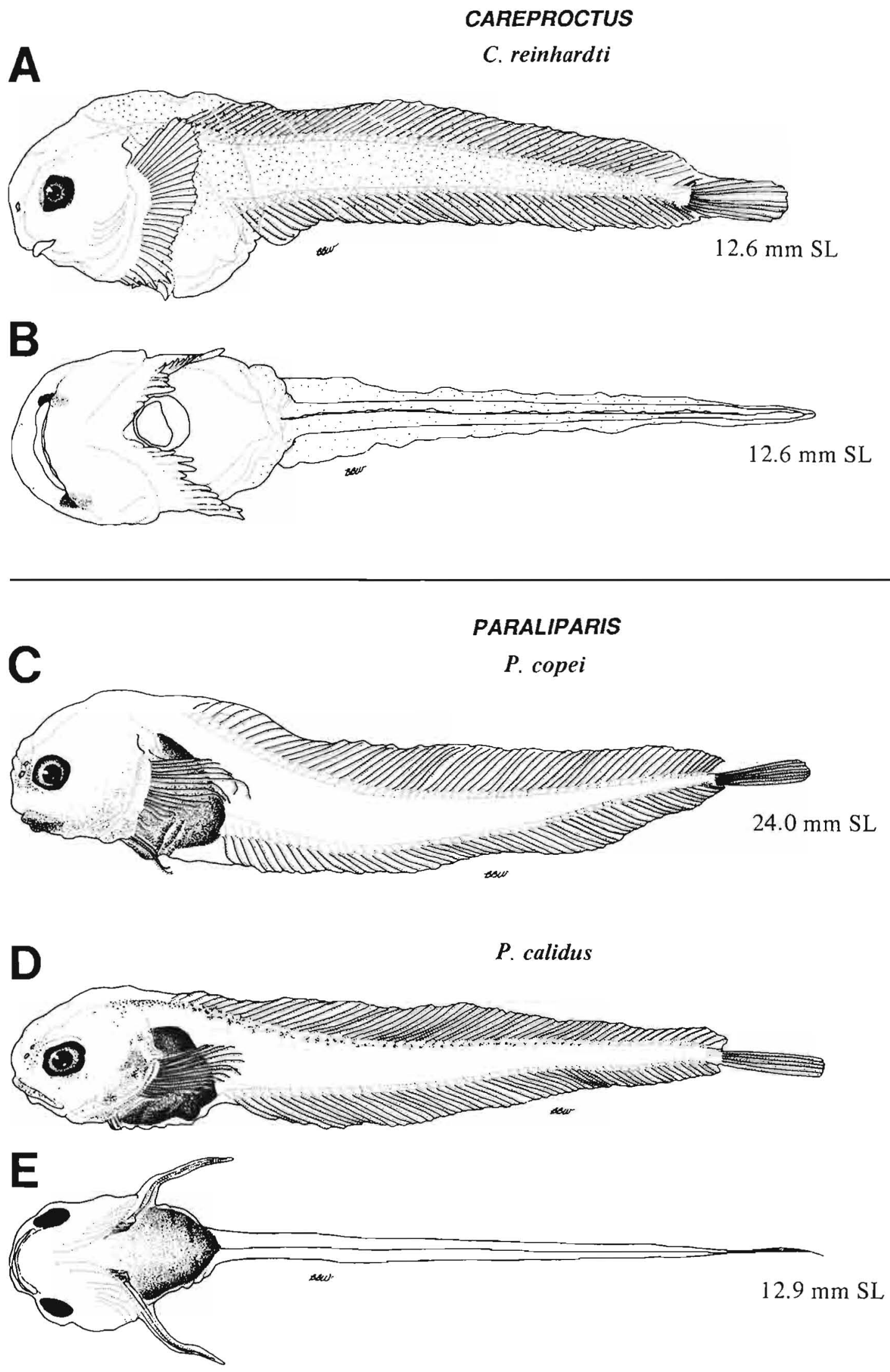

Figures A-E (B, E, ventral views), Able et al. 1984 (Atlantic specimens). 
Table 41

Meristic characters of subfamily Liparidinae."

\begin{tabular}{|c|c|c|c|c|c|c|c|c|c|c|}
\hline \multirow[b]{2}{*}{ Taxon } & \multirow[b]{2}{*}{ Distribution } & \multicolumn{2}{|c|}{ Vertebrae } & \multicolumn{4}{|c|}{ Fins } & \multicolumn{2}{|c|}{ Gill rakers } & \multirow[b]{2}{*}{ Branchiostegals } \\
\hline & & \multicolumn{2}{|c|}{$\begin{array}{c}\text { Precaudal Caudal } \\
\text { (Total) }\end{array}$} & \multirow[t]{2}{*}{ Dorsal } & \multirow[t]{2}{*}{ Anal } & \multirow[t]{2}{*}{ Pectoral } & Pelvic & \multicolumn{2}{|c|}{$\begin{array}{l}\text { Upper Lower } \\
\text { (Total) }\end{array}$} & \\
\hline Acantholiparis ${ }^{\mathrm{b}}$ & Bering Sea & & & & & & Absent & & & 6 \\
\hline Acantholiparis caecus & Oregon & $8-10$ & $43-46$ & $48-52$ & $43-45$ & 21 & Absent & $(10-11)$ & & $6-7$ \\
\hline Acantholiparis opercularis & Calif.-Bering Sea & 9 & 41 & $45-52$ & $38-47$ & $20-24$ & Absent & $(8-10)$ & & 6 \\
\hline Careproctus abbreviatus & SE Alaska-Bering Sea & & & 39 & 32 & 21 & Disc & & & 6 \\
\hline Careproctus attenuatus & Bering Sea & & & 48 & 40 & 34 & Disc & & & 6 \\
\hline Careproctus bowersianus & Bering Sea & $9-10$ & $47-50$ & $51-54$ & $46-48$ & $34-38$ & Disc & & & 6 \\
\hline Careproctus cameliae & Bering Sea & & & 50 & 45 & 28 & Disc & & & 6 \\
\hline Careproctus canus & Aleutian Is. & $\stackrel{11-12}{(55}$ & 8) & $51-53$ & $43-46$ & $33-36$ & Disc & & & 6 \\
\hline Careproctus colletii & Aleutian Is.-Bering Sea & $(59$ & & $52-58$ & $47-52$ & $25-31$ & Disc & & & 6 \\
\hline Careproctus cypselurus & Oregon-Bering Sea & ${ }^{8}$ & 0) $57-59$ & $58-64$ & $52-58$ & $32-37$ & Disc & & & 6 \\
\hline Careproctus ectenes & Aleutian Is.-Bering Sea & & & 48 & 44 & $30-32$ & Disc & & & 6 \\
\hline Careproctus filamentosus & Oregon & $\begin{array}{r}9-10 \\
\quad(63\end{array}$ & $8)^{54-58}$ & $58-63$ & $51-55$ & $21-24$ & Disc & & & 6 \\
\hline Careproctus furcellus & Bering Sea & ${ }_{(66}^{10-11}$ & 1) $56-61$ & $60-65$ & $54-59$ & $32-37$ & Disc & & & 6 \\
\hline Careproctus gilberti & Cent. Calif.-Bering Sea & ${ }^{8-9}(55$ & 8) $47-49$ & $45-55$ & $41-48$ & $30-33$ & Disc & & & 6 \\
\hline Careproctus Longifilis & SSC-Oregon & $\begin{array}{r}7.10 \\
\quad(55\end{array}$ & ${ }^{4)} 46-50$ & $50-54$ & $44-48$ & $17-23$ & Disc & & & 6 \\
\hline Careproctus melanurus & SSC-Bering Sea & $\begin{array}{r}8-11 \\
\quad(57\end{array}$ & 3) & $53-58$ & $47-51$ & $27-33$ & Disc & & & 6 \\
\hline Careproctus microstomus & Oregon & $\begin{array}{r}9-10 \\
\quad(67\end{array}$ & 9) $58-59$ & $61-67$ & $54-60$ & $22-27$ & Disc & & & 6 \\
\hline Careproctus mollis & Bering Sea & & & 51 & 47 & 35 & Disc & & & 6 \\
\hline Careproctus opisthotremus & Aleutian Is.-Bering Sea & & & 46 & 36 & 32 & Disc & & & 6 \\
\hline Careproctus oregonensis & Oregon & $\begin{array}{r}8-10 \\
(65\end{array}$ & 9) $57-60$ & $61-67$ & $55-57$ & $19-23$ & Disc & & & 6 \\
\hline Careproctus ostentum & Aleutian Is.-Bering Sea & & & 54 & 47 & 32 & Disc & & & 6 \\
\hline Careproctus ovigerum & Oregon-Brit. Col. & $\stackrel{10-12}{(47}$ & 9) & $43-45$ & $34-37$ & $31-34$ & Disc & & & 6 \\
\hline Careproctus pellucidus & SE Alaska & & & $52-55$ & $45-48$ & $33-35$ & Disc & & & 6 \\
\hline Careproctus phasma & Bering Sea & & & 53 & 45 & 34 & Disc & & & 6 \\
\hline Careproctus pycnosoma & Bering Sea & $\begin{array}{l}10 \\
(46\end{array}$ & 9) $36-39$ & $42-45$ & $36-39$ & $38-39$ & Disc & & & \\
\hline Careproctus rastrinus & SE Alaska-Bering Sea & $\begin{array}{l}8-9 \\
(59\end{array}$ & 4) $50-52$ & $55-59$ & $49-52$ & $33-37$ & Disc & & & 6 \\
\hline Careproctus scottae & SE Alaska-Bering Sea & & & $52-56$ & $47-51$ & $32-34$ & Disc & 2 & 10 & 6 \\
\hline Careproctus simus & Aleutian Is.-Bering Sea & $10-11$ & $48-52$ & $54-58$ & $47-51$ & $31-37$ & Disc & & & 6 \\
\hline Careproctus spectrum & SE Alaska-Bering Sea & & & 52 & 47 & 32 & Disc & & & 6 \\
\hline Careproctus zachirus & Aleutian Is. & $\stackrel{12-13}{(56}$ & )$^{44-46}$ & $51-58$ & $43-45$ & $28-31$ & Disc & & & \\
\hline Crystallichthys cyclospilus & Gulf of Alaska-Bering Sea & ${ }^{11}$ & 3) 42 & $48-50$ & $42-43$ & $33-36$ & $?$ & & & 6 \\
\hline Crystallichthys mirabilis & Bering Sea & & & 53 & 44 & $30-33$ & $?$ & & & 6 \\
\hline Elassodiscus caudaus & Cent. Calif.-SE Alaska & $9-11$ & $46-51$ & $49-55$ & $41-50$ & $27-29$ & Disc & & & 6 \\
\hline Elassodiscus tremebundus & Bering Sea & $\begin{array}{r}9-12 \\
(62\end{array}$ & 4) $52-63$ & $55-67$ & $49-60$ & $25-33$ & Disc & & & 6 \\
\hline Gyrinichthys minytremus & Aleutian Is.-Bering Sea & & & 25 & 14 & 25 & Disc & & & 6 \\
\hline Liparis bristolensis & Bering Sea-Chukchi Sea & & 9) & $38-40$ & $30-35$ & $33-37$ & Disc & & & 6 \\
\hline Liparis callyodon & Wash.-Bering Sea & & & 33-35 & $25-27$ & $28-31$ & Disc & & & 6 \\
\hline
\end{tabular}




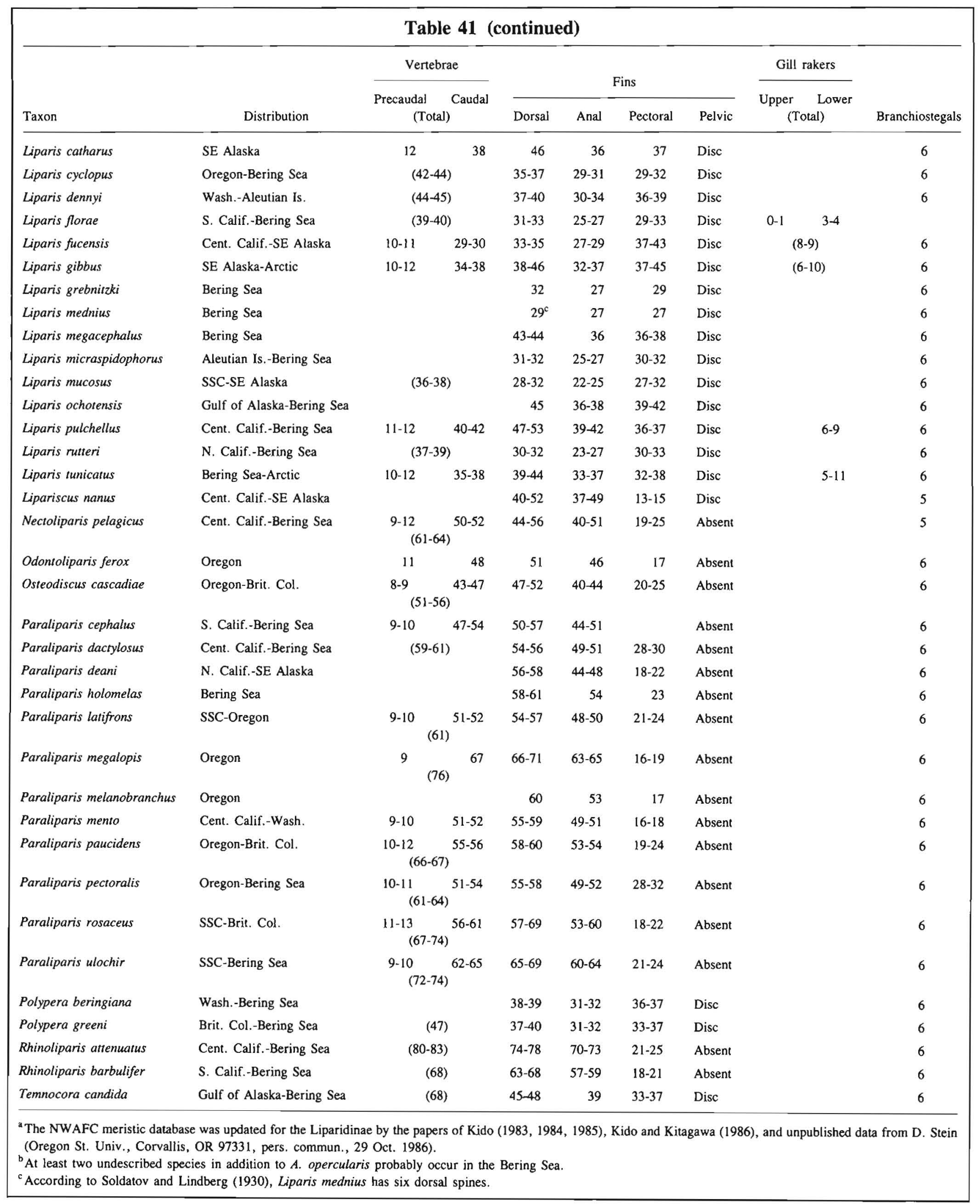




\section{MERISTICS}

\begin{tabular}{|c|c|}
\hline Vertebrae & $\begin{array}{l}\text { Total: } 61-X-64 \\
\text { Precaudal: } 9-X-12 \\
\text { Caudal: } 50-X-52\end{array}$ \\
\hline $\begin{array}{l}\text { Branchiostegal rays } \\
\text { Caudal fin }\end{array}$ & $5-5-5$ \\
\hline Pelvic fin & Absent \\
\hline Dorsal fin & $\mathrm{R}: 44-\mathrm{X}-56$ \\
\hline Pectoral fin & R: $19-20-25$ \\
\hline Anal fin & R: $40-X-51$ \\
\hline Gill rakers & $\mathrm{U}: \mathrm{X}-\mathrm{X}-\mathrm{X}$ \\
\hline \multicolumn{2}{|l|}{ LIFE HISTORY } \\
\hline Range & $\begin{array}{l}\text { Cent. California, } 34-38^{\circ} \mathrm{N} \text {, to } \\
\text { Bering Sea, } 54-66^{\circ} \mathrm{N}\end{array}$ \\
\hline Ecology & Nearshore shelf demersal \\
\hline ELH pattern & $\begin{array}{l}\text { Oviparous, eggs probably } \\
\text { demersal, pelagic larvae }\end{array}$ \\
\hline \multirow[t]{4}{*}{ Spawning } & Season: \\
\hline & Area: \\
\hline & Mode: \\
\hline & Migration: \\
\hline $\begin{array}{l}\text { Fecundity } \\
\text { Age at first maturity } \\
\text { Longevity }\end{array}$ & Range/function: \\
\hline
\end{tabular}

\section{EARLY LIFE HISTORY DESCRIPTION}

\section{EGGS}

Diameter

No. of oil globules

Oil globule diameter

Yolk

Envelope

Hatch size

Incubation time/temp.

Pigment

Diagnostic characters

\section{LARVAE}

Preanal length Changes with development

Length at flexion

Length at transformation

Sequence of fin development

Pigment

- Darkly pigmented peritoneum

- Ventral half of head pigmented, appearing bearded

Diagnostic characters

- Presence of two separate pectoral fin lobes

- General tadpole shape

- Position of anus: Apparently moves forward with development; in adults anus is directly under eye

- Absence of disc

- Number of branchiostegal rays (five)

${ }^{2}$ Identification of small larvae prior to pectoral fin development and movement of anus forward is tentative. A cleared and stained 7.9-mm SL specimen has five branchiostegal rays.

Ref: Har 1973. 

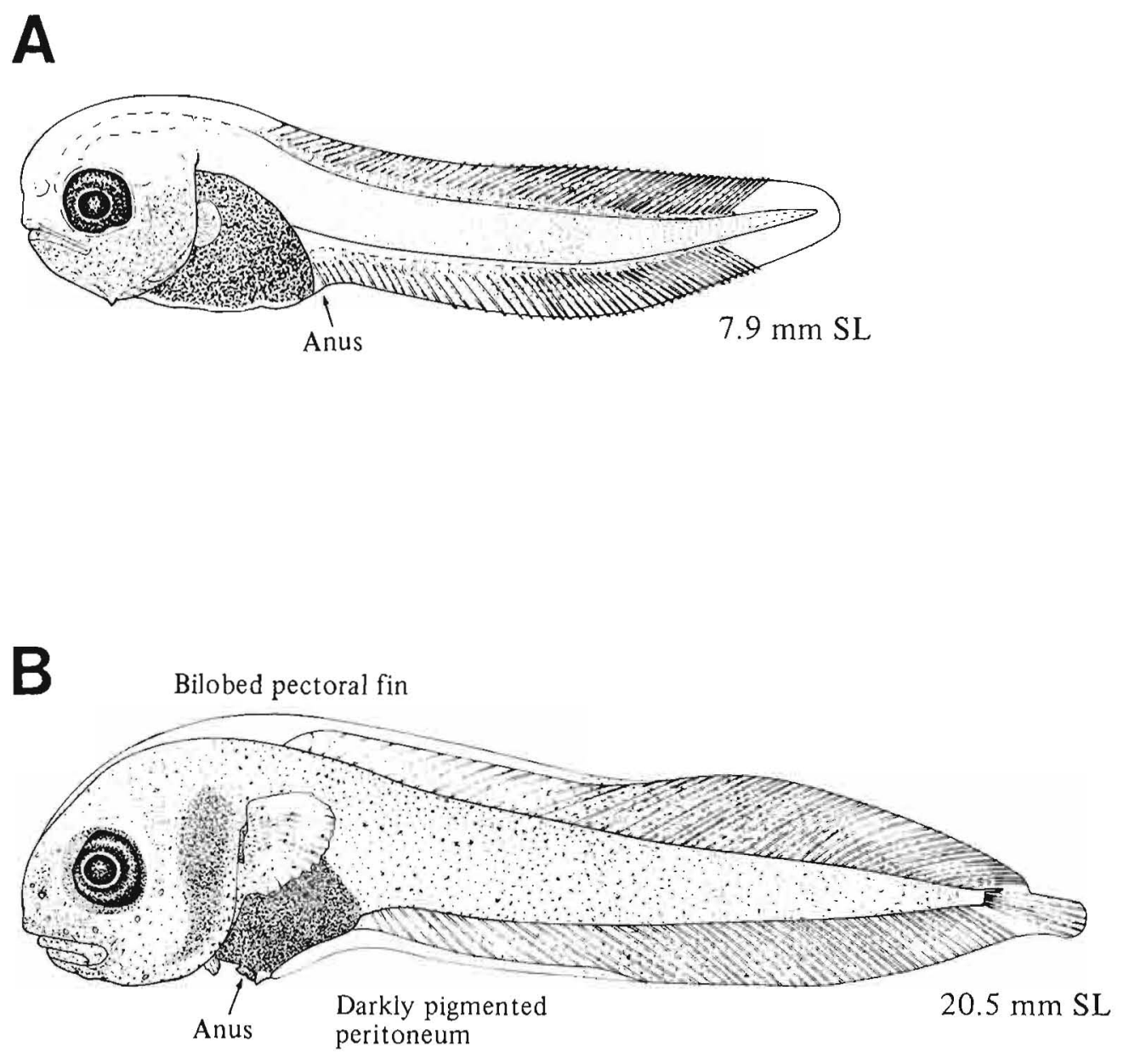

Figures $A-B, N W A F C$ originals (B. Vinter). 


\section{MERISTICS}

Vertebrae

Branchiostegal rays

Caudal fin

Pelvic fin

Dorsal fin

Pectoral fin

Anal fin

Gill rakers
Total: X-X-X

Precaudal: $\mathrm{X}-\mathrm{X}-\mathrm{X}$

Caudal: X-X-X

6-6-6

Absent

R: $56-X-58$

R: $18-X-22$

R: $44-X-48$

$\mathrm{U}: \mathrm{X}-\mathrm{X}-\mathrm{X}$

$\mathrm{L}: \mathrm{X}-\mathrm{X}-\mathrm{X}$

\section{EARLY LIFE HISTORY DESCRIPTION}

\section{EGGS}

Diameter

$\sim 2 \mathrm{~mm}$

No. of oil globules

Oil globule diameter

Yolk

Envelope

Hatch size

Incubation time/temp.

Pigment

Diagnostic characters

\section{LARVAE}

Preanal length

Length at flexion

Length at transformation

Sequence of fin

development

Pigment

Diagnostic characters

- Characteristic notch in pectoral fin

- General body shape

- Absence of disc

Fecundity

\section{Range/function:}

Age at first maturity

Longevity 


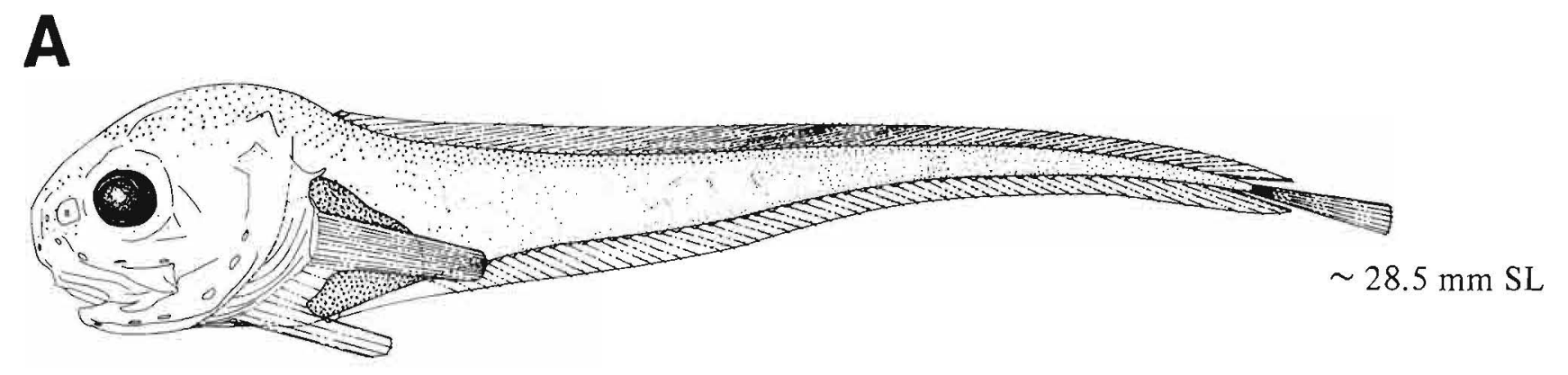


MERISTICS

Vertebrae

Total: 68-68-68

Caudal: $\mathrm{X}-\mathrm{X}-\mathrm{X}$

Branchiostegal rays 6-6-6

Caudal fin

Pelvic fin

$\mathrm{X}, 3, \mathrm{X}$

Absent

Dorsal fin
S: $63-\mathrm{X}-65$
$\mathrm{R}: \mathrm{X}-\mathrm{X}-\mathrm{X}$
R: $18-\mathrm{X}-20$
S: $57-X-59$
$\mathrm{R}: \mathrm{X}-\mathrm{X}-\mathrm{X}$
$\mathrm{U}: \mathrm{X}-\mathrm{X}-\mathrm{X}$
L: X-X-X

Precaudal: $\mathrm{X}-\mathrm{X}-\mathrm{X}$

\section{EARLY LIFE HISTORY DESCRIPTION}

\section{EGGS}

Diameter

$2.5 \mathrm{~mm}$

No. of oil globules

Oil globule diameter

Yolk

Envelope

Hatch size

$\leqslant 9.1 \mathrm{~mm} \mathrm{SL}$

Incubation time/temp.

Pigment

- Unpigmented

Diagnostic characters

- Yolk absorbed by $11.7 \mathrm{~mm} \mathrm{SL}$

- Flexion and fin ray development occurs prior to hatching

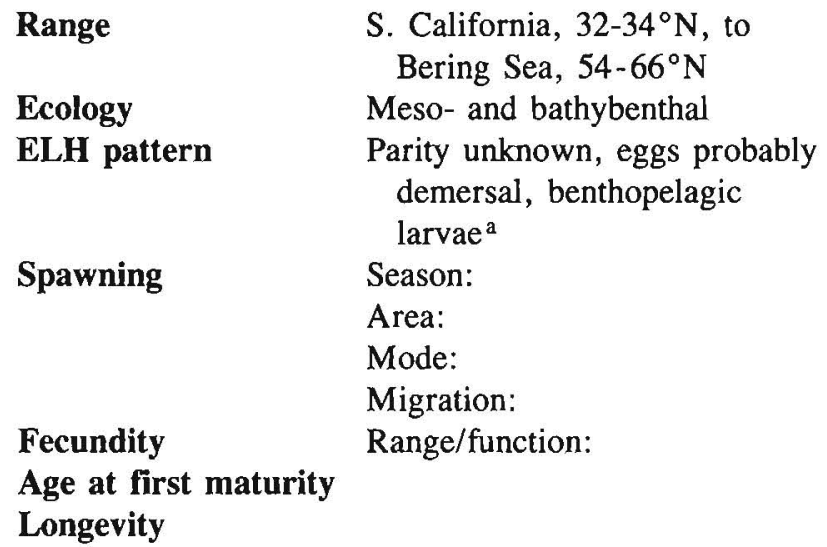

LARVAE (postflexion and juvenile)

Preanal length $<50 \%$ SL

Length at flexion $\quad<9.1 \mathrm{~mm} \mathrm{SL}$

Length at transformation

Sequence of fin development

Dorsal, anal, and caudal; pectoral

\section{Pigment}

- Larvae $<11.0 \mathrm{~mm}$ SL have no pigment

- Nape, peritoneum, and lateral body pigmented by $12.5 \mathrm{~mm} \mathrm{SL}$

- Pigment on the head and body increases in larger specimens

Diagnostic characters

- Loose skin over body

- Morphology

-Depressed head

-Large snout

-Slender tapering body

- Absence of disc

${ }^{a}$ Kido and Kitagawa 1986

Ref: Able et al. 1984, Kido and Kitagawa 1986. 

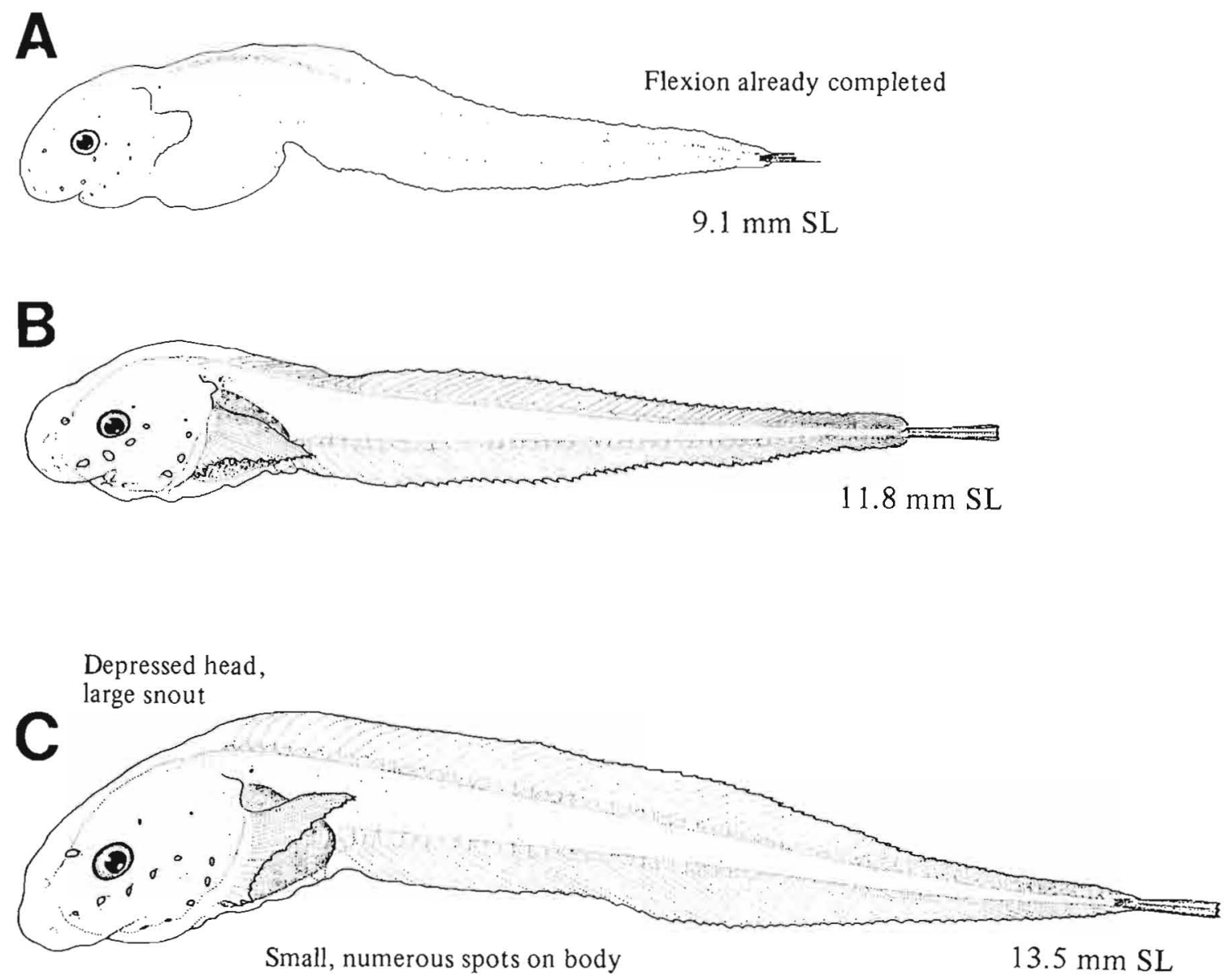

Figures A-C (A, yolksac larva; B-C, juveniles), Kido and Kitagawa 1986 (specimens from Iwate Prefecture, Honshu, Japan). 


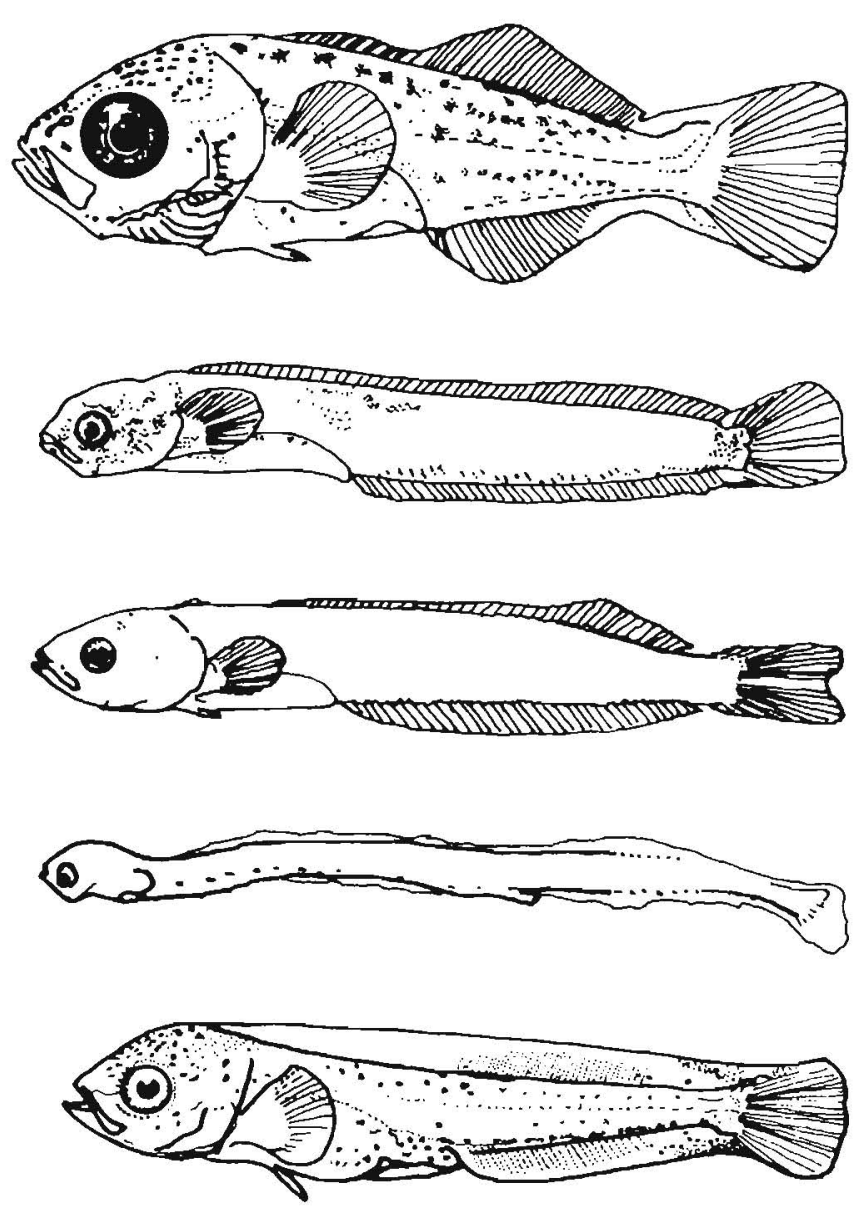

\section{Perciformes}

The most diversified of all fish orders is the Perciformes. Although the order encompasses the entire range of fish forms and behavior, most species are adapted for life as predators in shallow or surface waters. More than a dozen characters (many secondarily lost) define this group of spiny-rayed fishes. Most obvious of these are fin spines, two dorsal fins, scales generally ctenoid, pelvic fin inserted forward of the abdomen, and vertical insertion of the pectoral fin. There are 22 suborders, 150 families, 1,367 genera, and about 7,800 species within the order (J. Nelson 1984). The most speciose groups are the percoids and zoarcoids. Within the study area, nine suborders are represented; four of them are composed of a single species. Most species are members of the families Stichaeidae and Zoarcidae.

Some early-life-history stages are known for most families within our area except for Zoarcidae and Scytalinidae. When describing the sequence of fin ray development for percoids, we used the patterns A-F summarized in G.D. Johnson (1984) (see also Introduction, p. 29). A summary of meristic characters of zoarcids is provided to assist in identification.

Families in study area: Carangidae Bramidae

Caristiidae

Sciaenidae

Pentacerotidae

Bathymasteridae

Zoarcidae

Stichaeidae

Cryptacanthodidae

Pholididae

Anarhichantidae

Ptilichthyidae

\author{
Zaproridae \\ Scytalinidae \\ Trichodontidae \\ Clinidae \\ Icosteidae \\ Ammodytidae \\ Gobiidae \\ Trichiuridae \\ Scombridae \\ Luvaridae \\ Stromateidae \\ Centrolophidae \\ Tetragonuridae
}




\section{MERISTICS}

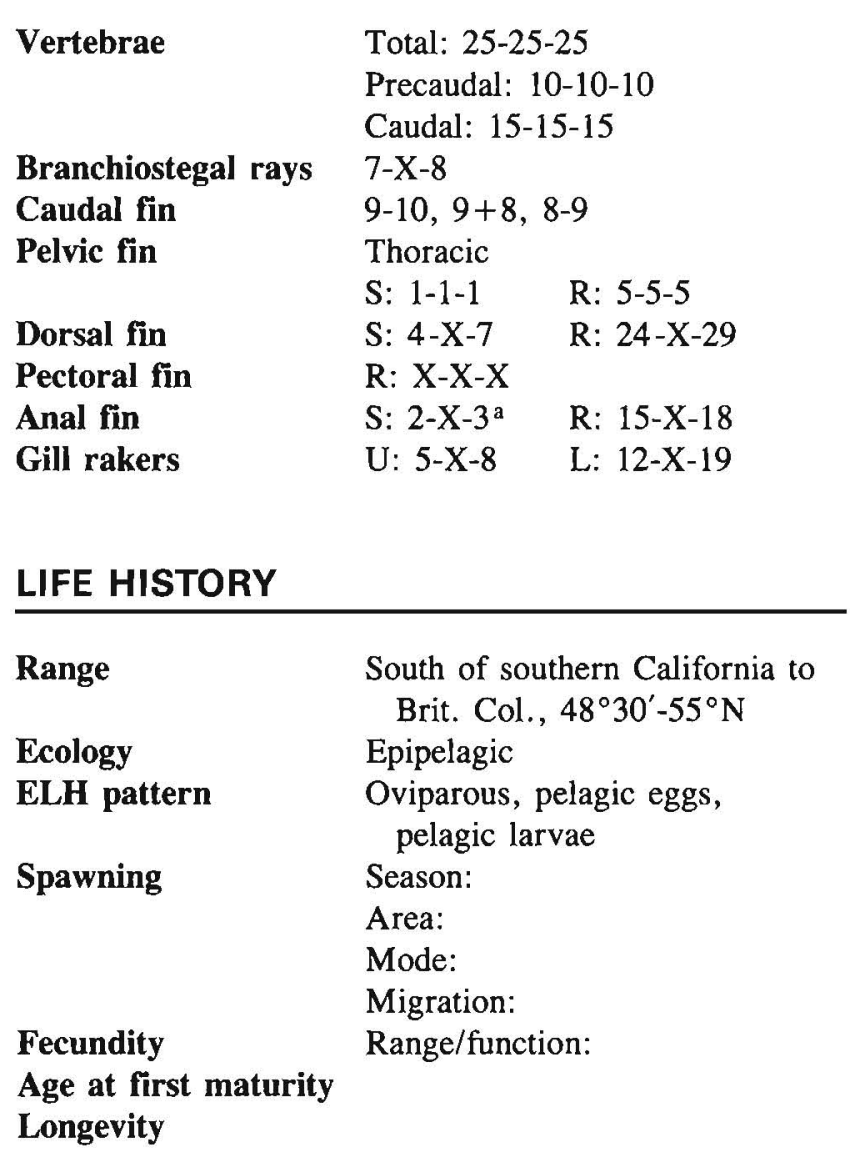

\section{EARLY LIFE HISTORY DESCRIPTION}

\section{EGGS}

Diameter

No. of oil globules

Oil globule diameter

Yolk

Envelope Smooth

Hatch size

Incubation time/temp. $\quad 24-48 \mathrm{hr} / 18-30^{\circ} \mathrm{C}$

Pigment

Diagnostic characters

\section{LARVAE}

Preanal length $\quad \sim 50-75 \%$ SL

Length at flexion $>4.1 \mathrm{~mm} \mathrm{SL}$

Length at transformation

Sequence of fin "A" pattern: ${ }^{b}$ 2nd dorsal development

(rays), anal, and caudal simultaneously followed by 1st dorsal (spines), pelvics, and pectorals (some carangids have precocious pelvics)

\section{Pigment}

- Uniform heavy pigmentation

- Body: Dorsolateral, lateral midline, and scattered on ventrolateral

- Vomer and branchiostegal membrane

- Antimedial rows on dorsal body margin present or absent $^{\mathrm{c}}$

\section{Diagnostic characters}

- Relatively deep bodied

- Uniform heavy pigment except in caudal region

- Pigment on branchiostegal membrane

- 25 myomeres

- Flexion and postflexion

-Large posttemporal and supracleithral, supraocular and preopercular spines (not serrate)

-No supraoccipital crest

\footnotetext{
a The first two spines are physically separated from the third, shorter spine which is associated with the anal fin soft rays.

${ }^{\circ}$ G.D. Johnson 1984

'Laroche et al. 1984

Ref: Laroche et al. 1984, Sanzo 1931 b.
} 


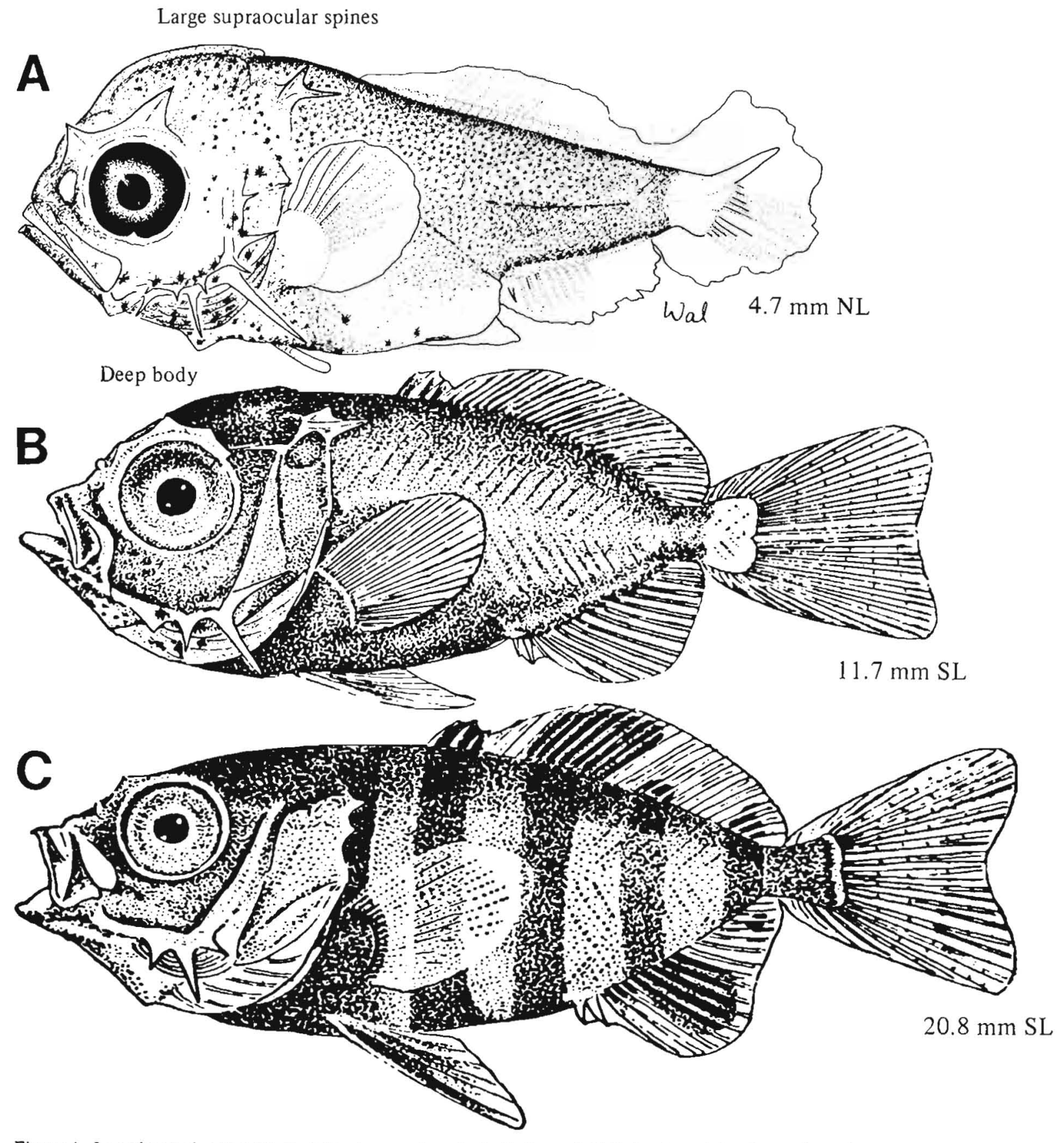

Figure A, Laroche et al. 1984 (Gulf of Mexico specimen); B-C, Sanzo 1931 b (eastern Atlantic specimens). 


\section{MERISTICS}

$\begin{array}{lll}\text { Vertebrae } & \text { Total: } 23-24-25 \\ & \text { Precaudal: } 10-10-10 \\ & \text { Caudal: } 14-14-14 \\ \text { Branchiostegal rays } & 7-X-8 & \\ \text { Caudal fin } & 9-10,9+8,9-10 \\ \text { Pelvic fin } & \text { Thoracic } & \\ & \text { S: } 1-1-1 & \text { R: } 5-5-5 \\ \text { Dorsal fin } & \text { S: } 8-X-9 & \text { R: } 28-X-38 \\ \text { Pectoral fin } & \text { R: } X-X-X & \\ \text { Anal fin } & \text { S: } 2-X-3^{\text {a }} & \text { R: } 22-X-33 \\ \text { Gill rakers } & \text { U: } 7-X-15 & \text { L: } 25-X-42\end{array}$

\section{LIFE HISTORY}

$\begin{array}{ll}\text { Range } & \begin{array}{c}\text { South of southern California to } \\ \text { Gulf of Alaska, } 54-60^{\circ} \mathrm{N}\end{array} \\ \text { Epi- and mesopelagic, } 0-403 \mathrm{~m}^{\mathrm{b}} \\ \begin{array}{l}\text { Ecology } \\ \text { ELH pattern }\end{array} & \begin{array}{l}\text { Oviparous, pelagic eggs, } \\ \text { pelagic larvae } \\ \text { Season: Jan-Nov; }\end{array} \\ \text { Spawning } & \text { (California) }^{\mathrm{d}} \\ & \text { Area: Epipelagic, offshore } \\ & \text { Mode: } \\ & \text { Migration: } \\ \text { Fecundity } & \text { Range/function: } 53,000^{\mathrm{b}} \\ \text { Age at first maturity } & 2-3 \mathrm{yr}^{\mathrm{e}} \\ \text { Longevity } & >30 \mathrm{yr}^{\mathrm{e}}\end{array}$

\footnotetext{
${ }^{a}$ The first two spines are physically separated from the third, shorter spine which is associated with the anal fin soft rays.

${ }^{\mathrm{b}} \mathrm{Hart} 1973$

${ }^{\mathrm{c}}$ Frey 1971

${ }^{d}$ MacCall and Stauffer 1983

'Fitch and Lavenberg 1971

${ }^{f}$ Remains within this range from 2.2 to $50.0 \mathrm{~mm} \mathrm{SL}$

${ }^{8} \mathrm{G}$.D. Johnson 1984

Ref: Ahlstrom and Ball 1954, Laroche et al. 1984.
}

\section{EARLY LIFE HISTORY DESCRIPTION}

\section{EGGS}

$\begin{array}{ll}\text { Diameter } & 0.90-1.08 \mathrm{~mm} \\ \text { No. of oil globules } & \text { One } \\ \text { Oil globule diameter } & 0.25 \mathrm{~mm} \\ \text { Yolk } & \text { Segmented } \\ \text { Envelope } & \text { Smooth, clear } \\ \text { Hatch size } & 1.91-2.38 \mathrm{~mm} \mathrm{SL} \\ \text { Incubation time/temp. } & 24-48 \mathrm{hr} / 18-30^{\circ} \mathrm{C}\end{array}$

Pigment

- Oil globule

- Dorsal and ventral melanophores

\section{Diagnostic characters}

- Anterior position of oil globule in yolksac larvae

- Pigment

- Lack of yolk pigment distinguishes eggs from Merluccius productus (p. 186) and Scomber japonicus (p. 554)

\section{LARVAE}

Preanal length $52-61 \% \mathrm{SL}^{\mathrm{f}}$

Length at flexion 8-11 mm SL

Length at transformation $16 \mathrm{~mm} \mathrm{SL}$

Sequence of fin development (rays), anal, and caudal simultaneously followed by 1 st dorsal (spines), pelvics, and pectorals (some carangids have precocious pelvics)

Pigment

- Crown, dorsal and ventral midline

- Mediolateral streak

- Some superficial lateral pigment develops

Diagnostic characters

- Low myomere count (usually 24)

- Dorsal body margin pigment; antimedial rows absent with median rows only

- Flexion and postflexion

— 9 preopercular spines (not serrate)

-Supraoccipital crest present 

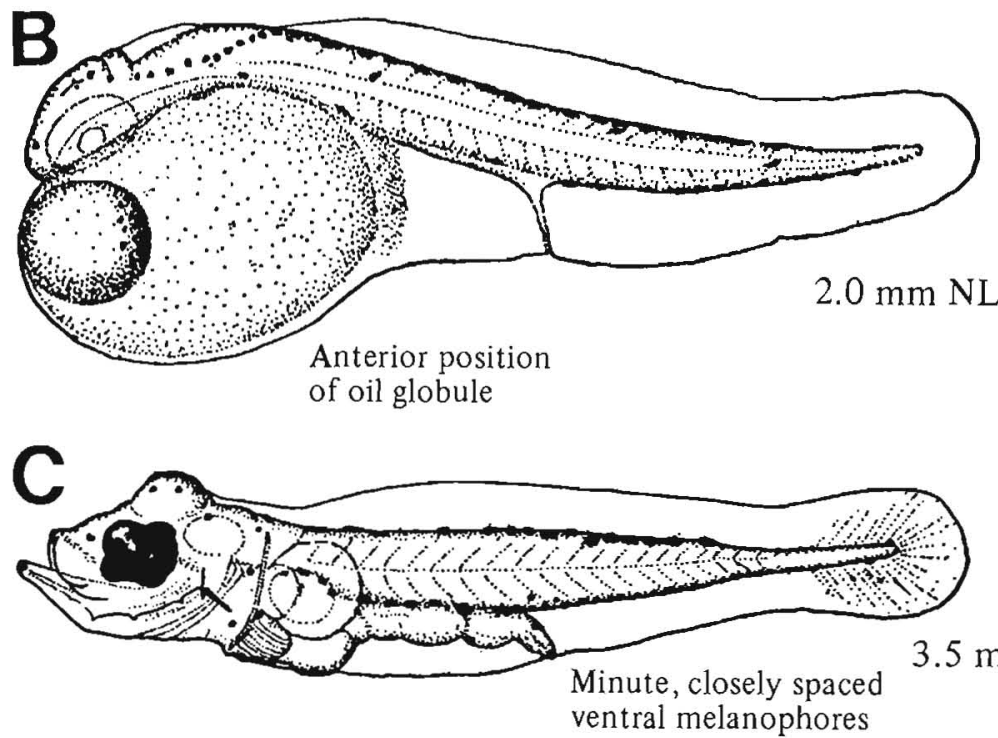
Crown pigment
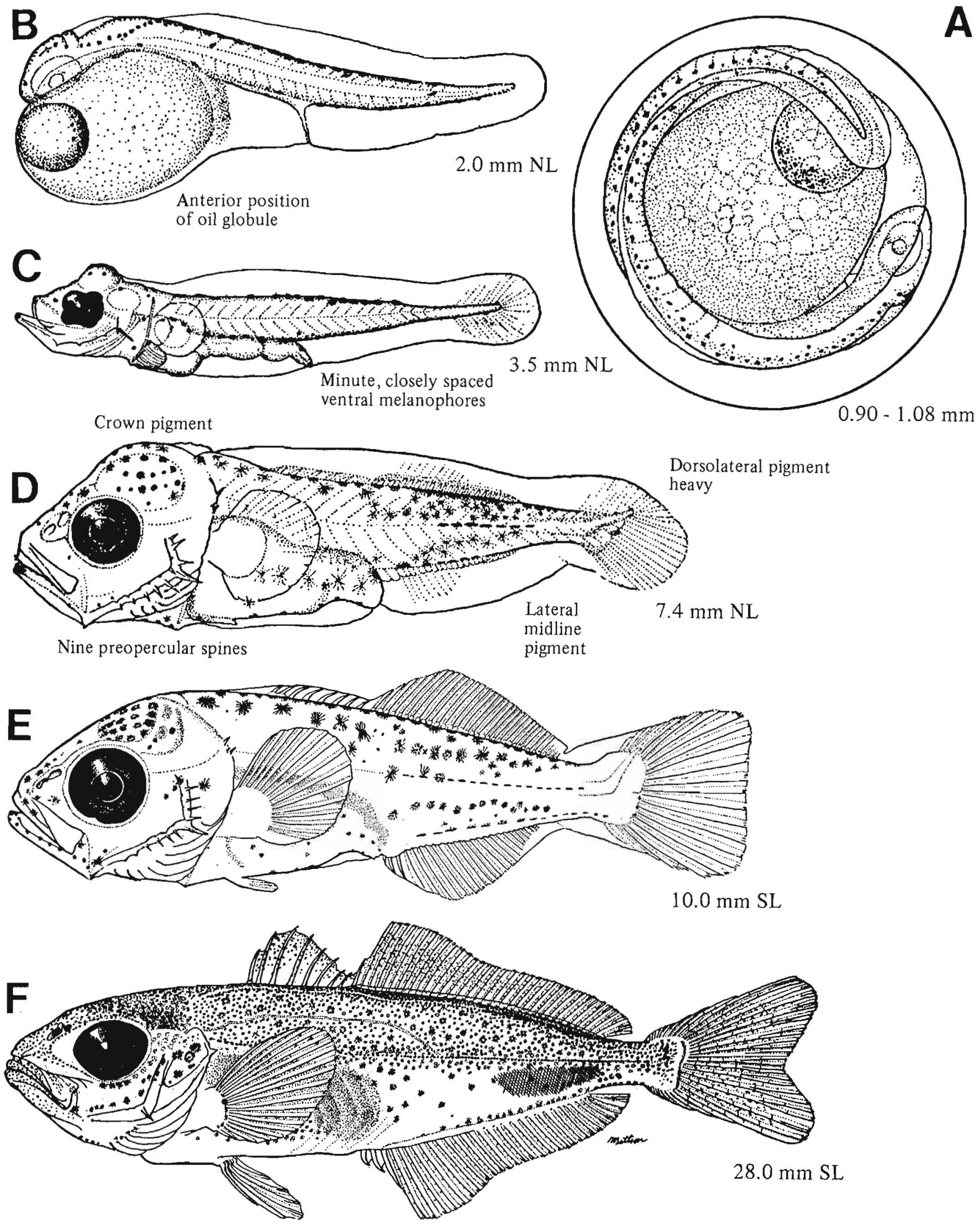

Figures A-F, Ahlstrom and Ball 1954. 


\section{MERISTICS}

Vertebrae

Total: $39-40-41$

Precaudal: 15-16-17

Caudal: 23-24-26

Branchiostegal rays $7-\mathrm{X}-8$

Caudal fin

Pelvic fin

$8,9+8,7$

Thoracic

S: $1-1-1$

$\mathrm{R}: 5-5-5$

Dorsal fin ${ }^{\mathrm{a}}$

S: $3-3-5$

R: $30-X-35$

$\mathrm{D} 1+\mathrm{D} 2=33-36$

Pectoral fin

R: $21-22-23$

Anal fina

S: $2-\mathrm{X}-3$

R: $25-X-29$

$\mathrm{A} 1+\mathrm{A} 2=27-30$

Gill rakers

$\mathrm{U}: \mathrm{X}-\mathrm{X}-\mathrm{X} \quad \mathrm{L}$ : $\mathrm{X}-\mathrm{X}-\mathrm{X}$

LIFE HISTORY

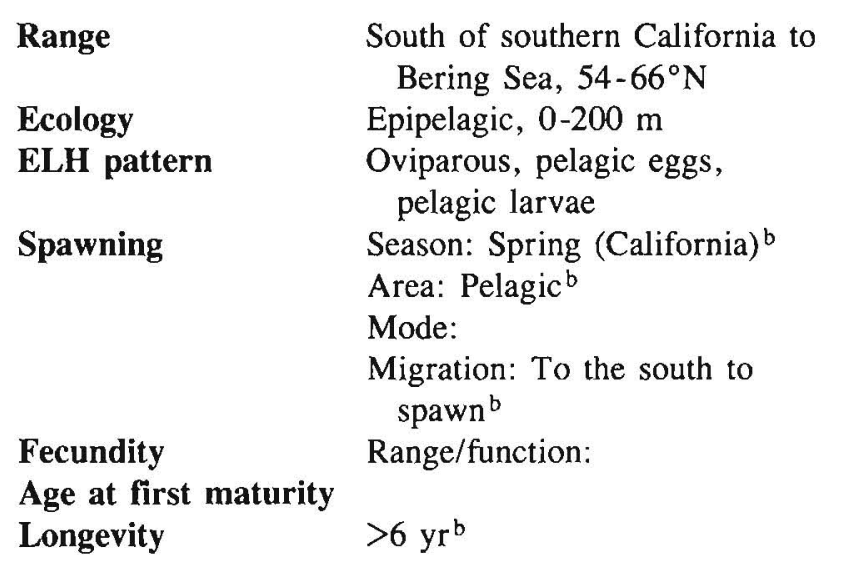

"Since spines are weak or lacking in fins, there has been much confusion in the literature. According to data collected by G.D. Johnson (Natl. Mus. Nat. Hist., Wash., D.C., 20560, pers. commun., 7 Nov. 1986), all dorsal and anal elements are soft rays. Total counts from Mead (1972) are therefore more useful and are presented here.

${ }^{6}$ Fitch and Lavenberg 1971

${ }^{c}$ G.D. Johnson 1984

${ }^{d}$ G.D. Johnson, pers. commun., 7 Nov. 1986.

Ref: Mead 1972.

\section{EARLY LIFE HISTORY DESCRIPTION}

\section{EGGS}

Diameter

No. of oil globules

$1.56-1.60 \mathrm{~mm}$

Oil globule diameter

One

Yolk

Envelope

Hatch size

Incubation time/temp.

Pigment

Diagnostic characters

\section{LARVAE}

Preanal length

Length at flexion

Length at transformation

Sequence of fin development

Pigment

- Gut and anterior body

- Crown

- With development along hypural margin

- Pectoral fin fringes

Diagnostic characters

- Pigment covers anterior body

- Body depth: Deep upper body

- Large pectoral fin

- Patterns of preopercular spines are useful diagnostic characters for bramid larvae

Taractes asper larvae are unknown. The following information may aid in their identification.

$\begin{array}{lc}\text { Total vertebrae } & 41-42 \\ \text { Caudal } & 17-18 \\ \text { Precaudal } & 23-24 \\ \text { Dorsal fin (D1+D2) } & 31-34 \\ \text { Anal fin (A1+A2) } & 23-26 \\ \text { Pectoral fin rays } & 18-20 \\ \text { Pelvic fin } & \text { I,5 } \\ \text { Range } & \text { Cent. Calif.- } \\ & \text { Gulf of Alaska }\end{array}$



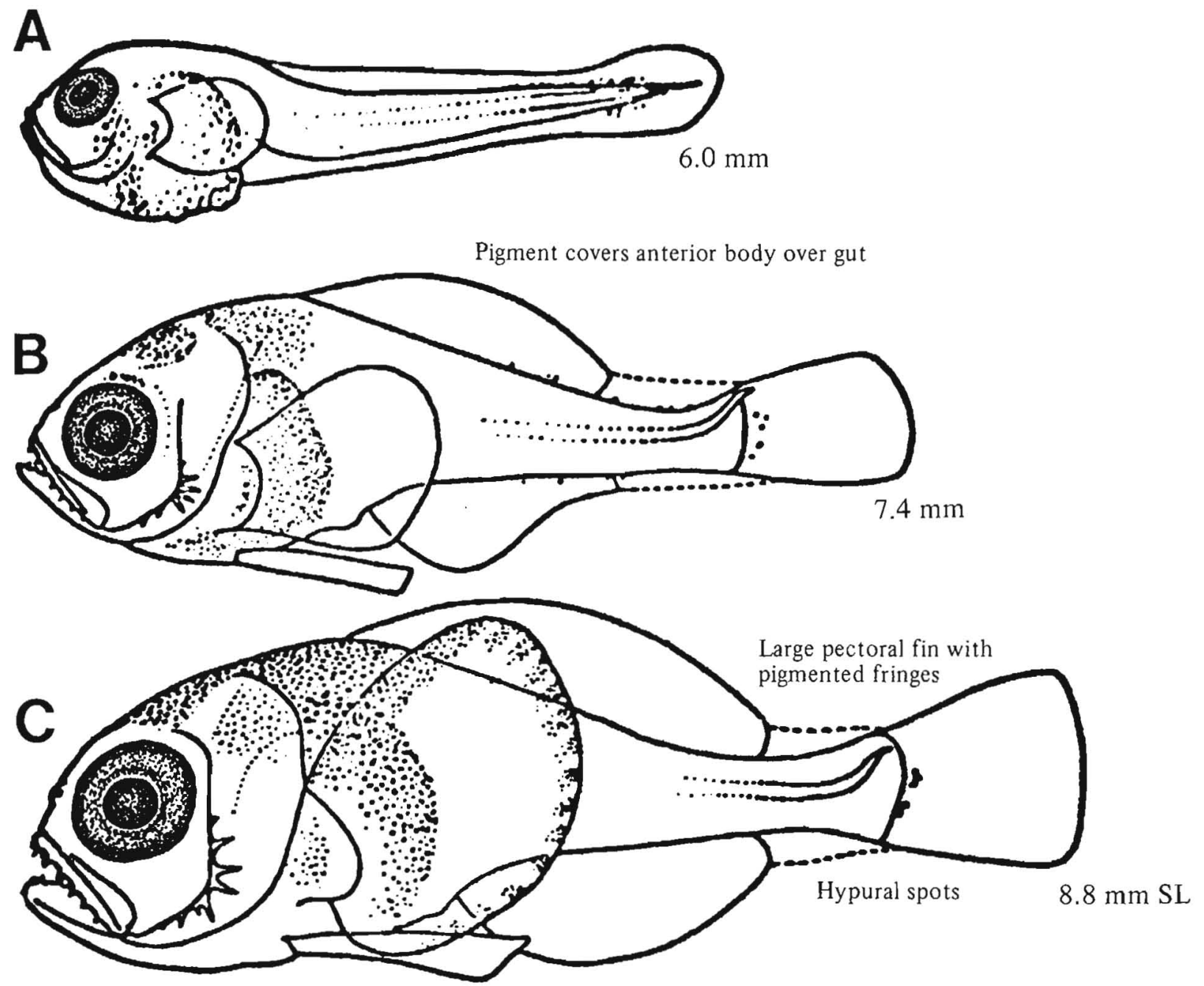

Distinctive preopercular spine pattern 


\section{MERISTICS}

\begin{tabular}{|c|c|}
\hline \multirow{3}{*}{ Vertebrae } & Total: $37-38-40$ \\
\hline & Precaudal: 16-16-16 \\
\hline & Caudal: 19-19-19 \\
\hline Branchiostegal rays & $7-7-7$ \\
\hline Caudal fin & $6-7,9+8,6$ \\
\hline \multirow{2}{*}{ Pelvic fin } & Thoracic \\
\hline & $S: 1-1-1 \quad R: 5-5-5$ \\
\hline Dorsal fina & $\mathrm{D} 1+\mathrm{D} 2=32-34$ \\
\hline Pectoral fin & $\mathrm{R}: 14-\mathrm{X}-19$ \\
\hline Anal fin ${ }^{a}$ & $A 1+A 2=20-22$ \\
\hline Gill rakers & $\mathrm{U}: 6-6-6 \quad \mathrm{~L}: 16-16-16$ \\
\hline \multicolumn{2}{|l|}{ LIFE HISTORY } \\
\hline Range & $\begin{array}{c}\text { South of southern California to } \\
\text { Brit. Col., } 48^{\circ} 30^{\prime}-55^{\circ} \mathrm{N}\end{array}$ \\
\hline Ecology & Mesopelagic, $305-610 \mathrm{~m}$ \\
\hline ELH pattern & $\begin{array}{l}\text { Oviparous, pelagic eggs, } \\
\text { pelagic larvae }\end{array}$ \\
\hline Spawning & Season: \\
\hline & Area: \\
\hline & Mode: \\
\hline & Migration: \\
\hline $\begin{array}{l}\text { Fecundity } \\
\text { Age at first maturity } \\
\text { Longevity }\end{array}$ & Range/function: \\
\hline
\end{tabular}

\section{EARLY LIFE HISTORY DESCRIPTION}

\section{EGGS}

Diameter

No. of oil globules

Oil globule diameter

Yolk

Envelope

Hatch size

Incubation time/temp.

Pigment

Diagnostic characters

LARVAE

Preanal length

Length at flexion

Between 5.8 and 10.1 $\mathrm{mm} \mathrm{SL}$

Length at transformation

Sequence of fin development

"A" pattern: b 2nd dorsal (rays), anal, and caudal simultaneousiy followed by 1st dorsal (spines), pelvics, and pectorals

Pigment

- Postanal bar pattern

- Head and mediolateral gut

Diagnostic characters

- Myomeres (>35)

- Distinct pigment pattern with bars

- Head and body shape

\footnotetext{
${ }^{a}$ There is confusion in the literature whether spines are present in the dorsal (1-3) and anal (1) fins. According to data collected by G.D. Johnson, all dorsal and anal elements are soft rays (Natl. Mus. Nat. Hist., Wash., D.C. 20560, pers. commun., 7 Nov. 1986). Total counts are therefore more useful and are presented here.

${ }^{b}$ G.D. Johnson 1984

Ref: G.D. Johnson 1984.
} 


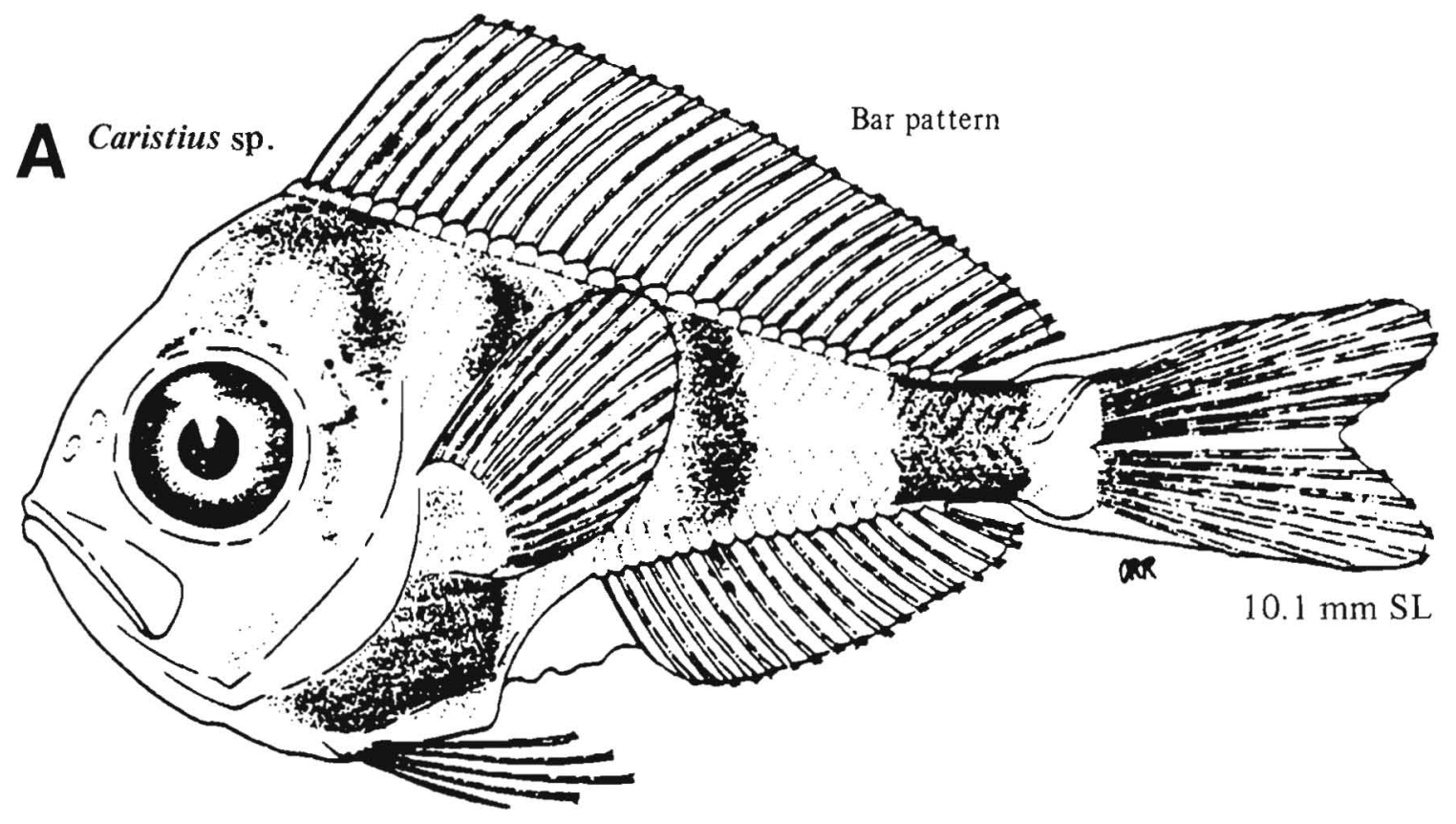




\section{MERISTICS}

\begin{tabular}{|c|c|c|}
\hline \multirow[t]{3}{*}{ Vertebrae } & \multicolumn{2}{|c|}{ Total: $26-26-26$} \\
\hline & \multicolumn{2}{|c|}{ Precaudal: $10-11-12$} \\
\hline & \multicolumn{2}{|c|}{ Caudal: 14-15-16 } \\
\hline Branchiostegal rays & \multicolumn{2}{|l|}{$7-X-8$} \\
\hline Caudal fin & \multicolumn{2}{|c|}{$X, 9+8, X(15-17$ secondary $)$} \\
\hline Pelvic fin & \multicolumn{2}{|l|}{ Thoracic } \\
\hline & $S: 1-1-1$ & $R: 5-5-5$ \\
\hline Dorsal fin & S: $13-X-16$ & $\mathrm{R}: 18-22-25$ \\
\hline Pectoral fin & R: $16-17-19$ & \\
\hline Anal fin & $S: 2-2-2$ & $\mathrm{R}: 10-11-12$ \\
\hline Gill rakers & $\mathrm{U}: 9-\mathrm{X}-12$ & L: $17-X-21$ \\
\hline \multicolumn{3}{|l|}{ LIFE HISTORY } \\
\hline Range & \multicolumn{2}{|c|}{$\begin{array}{l}\text { South of southern California to } \\
\text { Brit. Col., } 48^{\circ} 30^{\prime}-55^{\circ} \mathrm{N}\end{array}$} \\
\hline Ecology & \multicolumn{2}{|c|}{ Nearshore shelf pelagic } \\
\hline ELH pattern & \multicolumn{2}{|c|}{$\begin{array}{l}\text { Oviparous, pelagic eggs, } \\
\text { pelagic larvae }\end{array}$} \\
\hline Spawning & \multicolumn{2}{|c|}{ Season: Winter-spring a } \\
\hline & \multicolumn{2}{|c|}{ Area: Pelagic ${ }^{b}$} \\
\hline & \multicolumn{2}{|l|}{ Mode: } \\
\hline & \multicolumn{2}{|c|}{ Migration: } \\
\hline Fecundity & \multirow{3}{*}{\multicolumn{2}{|c|}{ Range/function: }} \\
\hline Age at first maturity & & \\
\hline Longevity & & \\
\hline
\end{tabular}

\section{EARLY LIFE HISTORY DESCRIPTION}

\section{EGGS}

Diameter

No. of oil globules

Oil globule diameter

Yolk

\section{Envelope}

Hatch size

Incubation time/temp.

Pigment

- Yolk and oil globule

- Embryonic pigment increases on head and trunk (dorsally and dorsolaterally)

Diagnostic characters

\section{LARVAE}

Prenanal length

Length at flexion

$38-53 \%$ SL

Length at transformation

Sequence of fin development

\section{4-6.4 mm SL}

$>12.7 \mathrm{~mm} \mathrm{SL}$

2nd dorsal (rays), anal, 1 st dorsal (spines), pelvics

\section{Pigment}

- Presence of nape melanophore

- Melanophore above hindgut absent or small

- 2-21 ventral melanophores; number increasing with development

\section{Diagnostic characters}

Among the fish larvae occurring in the study area, G. lineatus most closely resembles Scomber japonicus (p. 554). The myomere counts will usually separate them.
G. lineatus
26
S. japonicus
30-31

\footnotetext{
"Hart 1973

bWatson 1982

Ref: Watson 1982
} 
B

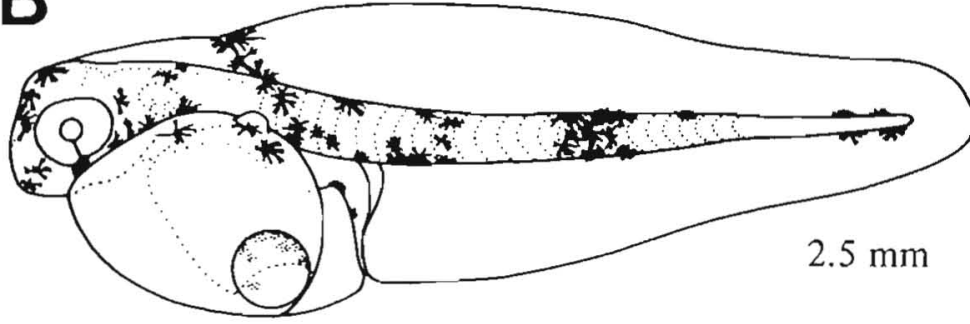

C

Spot on nape
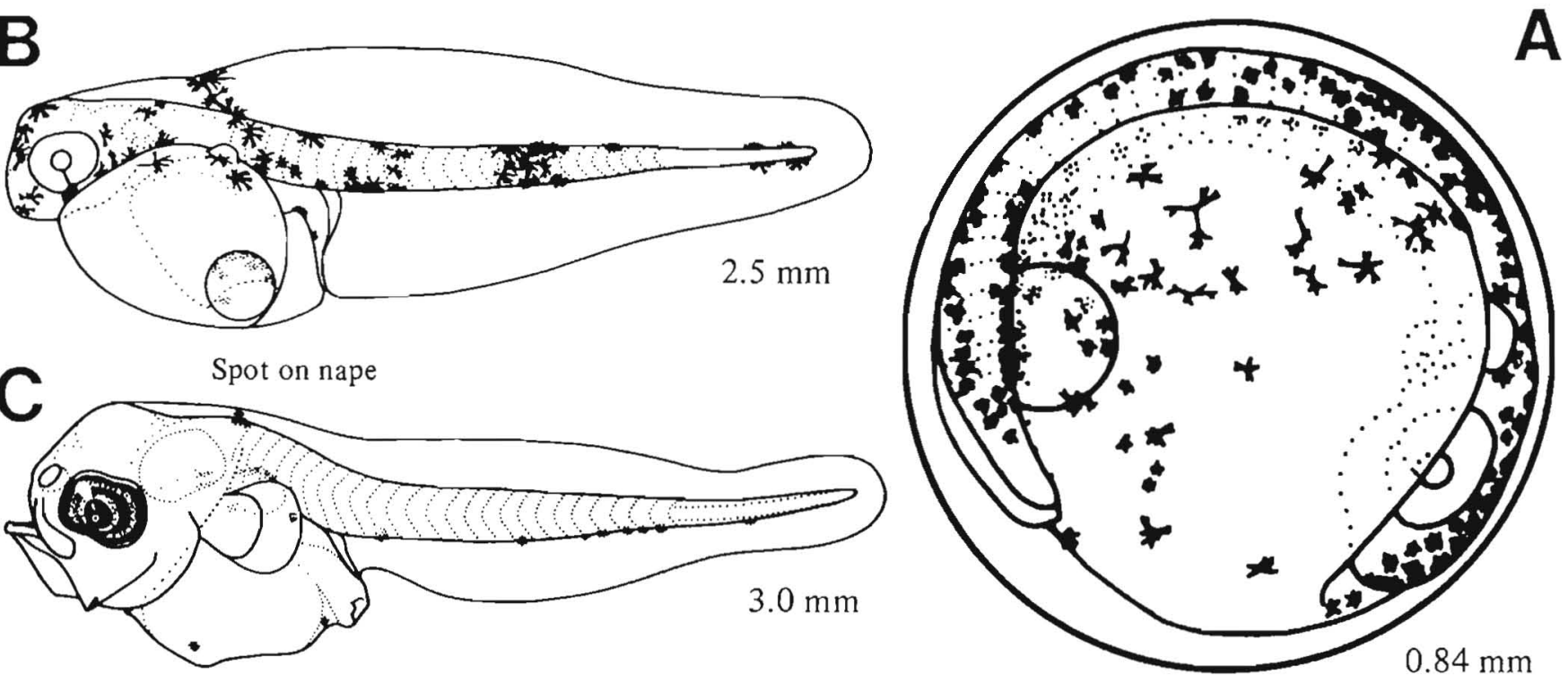

D

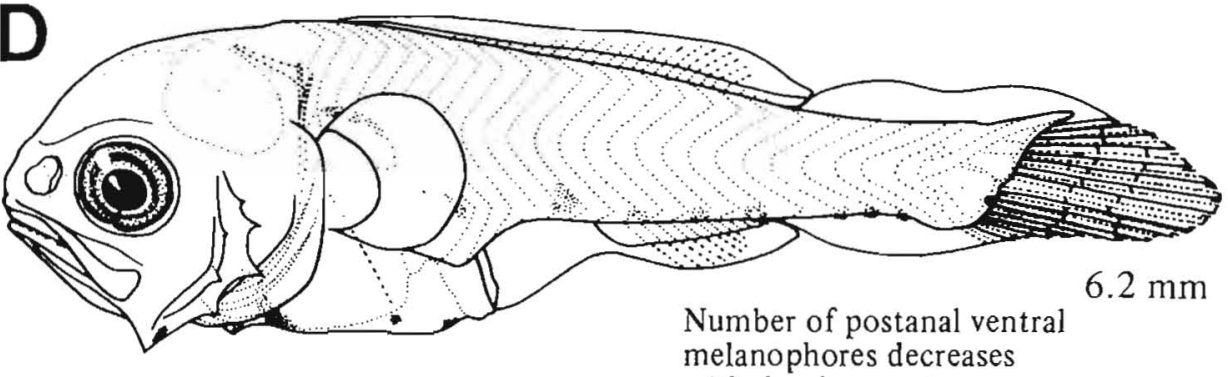

melanophores decreases
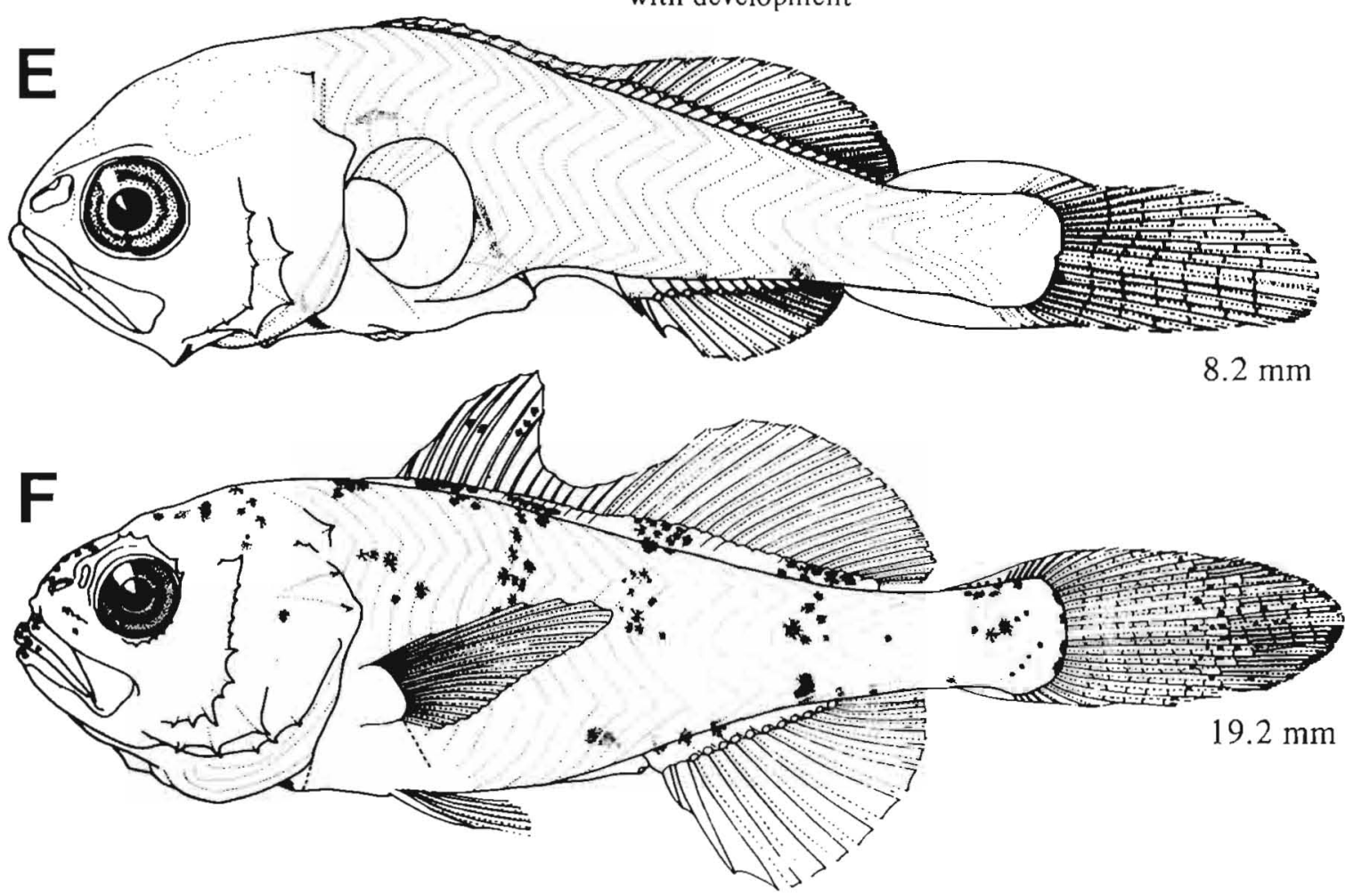

Figures A-F, Watson 1982. 


\section{MERISTICS $^{\mathrm{b}}$}

Vertebrae

Total: $24-\mathrm{X}-25$

Precaudal: 12-X-13

Caudal: $13-13-13$

Branchiostegal rays

Caudal fin

Pelvic fin

7-7-7

$7,9+8,5-6$

Thoracic

S: $1-1-1$

Dorsal fin

S: $13-\mathrm{X}-15$

Pectoral fin

R: $16-X-19$

Anal fin

S: $3-4-5$

U: $5-\mathrm{X}-8$
$\mathrm{R}: 5-5-5$

R: $8-X-10$

R: $6-X-9$

L: $14-\mathrm{X}-20$

\section{EARLY LIFE HISTORY DESCRIPTION}

\section{EGGS}

Diameter

No. of oil globules

Oil globule diameter

Yolk

Envelope

Hatch size

Incubation time/temp.

Pigment

\section{Diagnostic characters}

\section{LARVAE}

Preanal length

Length at flexion $\quad<9 \mathrm{~mm} \mathrm{SL}$

Length at transformation

Sequence of fin development

Pigment (early juvenile $P$. richardsoni)

- Entire body heavily pigmented with intense patches along dorsal body midline and on spinous dorsal, anal, and pelvic fins

Diagnostic characters (early juvenile $P$. richardsoni)

- Pigment pattern

- Bony cranial projections, spines by $10-16 \mathrm{~mm} \mathrm{SL}$; most prominent are the following: median supraoccipital; posttemporal; lateral expansions which develop over orbit, becoming highly serrated along the edge with development; preopercular

- Anteriorly serrated pelvic spine

- With development there is a reduction in spination

\footnotetext{
${ }^{a}$ Pentaceros $=$ Pseudopentaceros (Hardy 1983). J. Nelson (1984) does not recognize the genus Pseudopentaceros and cites Smith (1964) who included Pseudopentaceros in the synonomy of Pentaceros. Hardy (1983) removed Pseudopentaceros from synonomy but his work is not cited in J. Nelson (1984). The genus Pseudopentaceros may include up to three species:

Pseudopentaceros richardsoni-Restricted to Southern Hemisphere

$P$. wheeleri-North Pacific Ocean from Japan to Hawaii

$P$. pectoralis-North Pacific, Hawaii to Aleutian Is. (overlaps with $P$. wheeleri in central Pacific)

Hardy (1983) describes $P$. wheeleri as the most slender form. He suggests they are the "slender forms" referred to by Zama et al. (1977a) in their description of Pentaceros richardsoni. Hardy does not synonomize their "high-bodied" forms with $P$. wheeleri. The juvenile form described by Zama et al. (1977a) may include $P$. wheeleri in part. Hardy (1983) names this species as the one targeted on the Hawaiian ridge for intense commercial fishing. Pseudopentaceros wheeleri and $P$. pectoralis are probably the same species at different ontogenetic stages. Most likely two species occur worldwide (Humphreys et al. 1989).

${ }^{b}$ Hardy 1983; includes meristic data for Pseudopentaceros richardsoni, P. pectoralis, and $P$. wheeleri.

${ }^{c}$ Juveniles collected at surface to at least $260 \mathrm{~mm}$ FL; the shift from pelagic to bottom life may be ambivalent, occurring between 260 and $300 \mathrm{~mm}$ FL.
}

Ref: Hardy 1983, Humphreys et al. 1989, G.D. Johnson 1984, Zama et al. 1977b. 


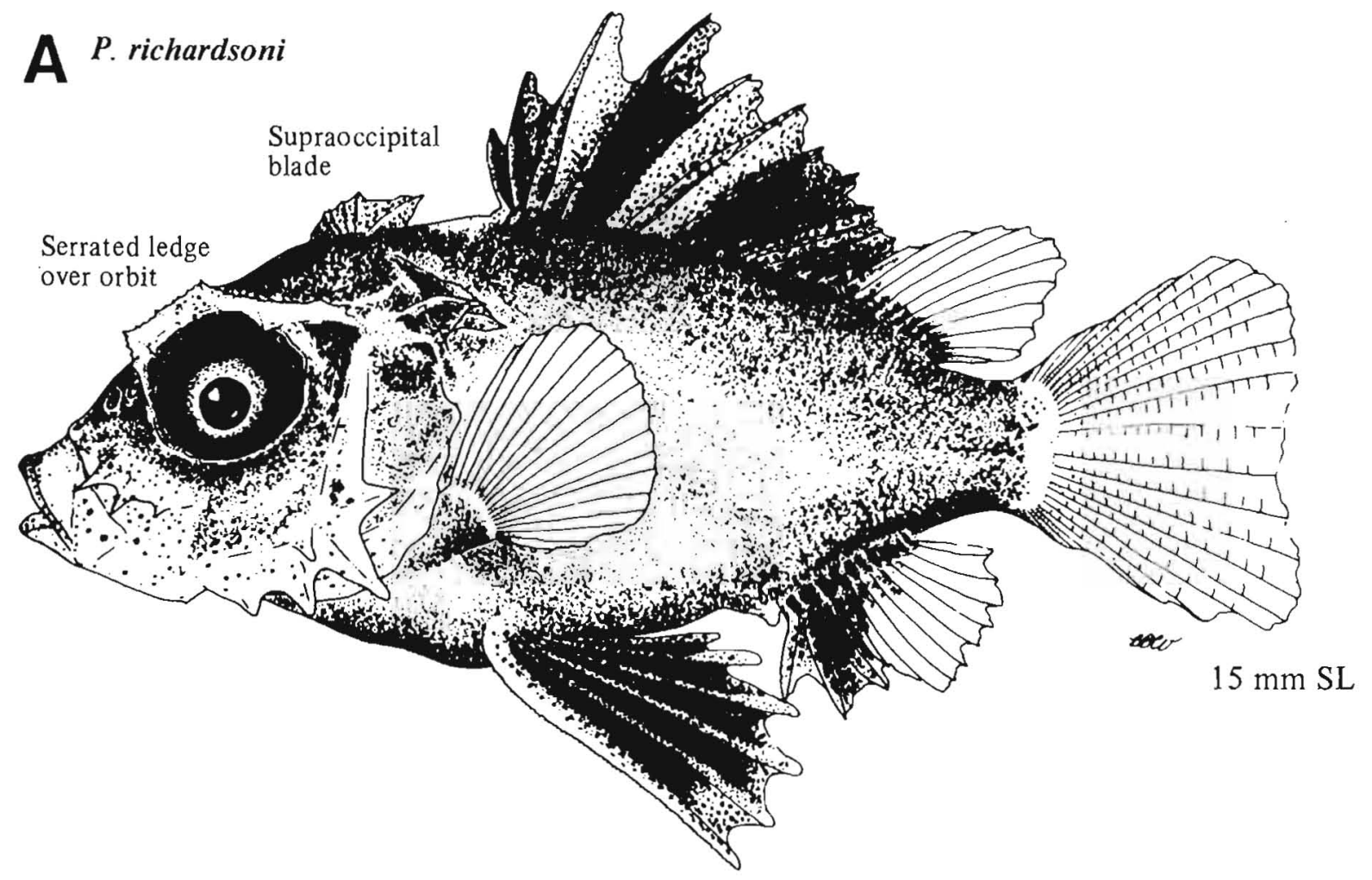

Figure A, G.D. Johnson 1984 (southern hemisphere specimen). 
This endemic North Pacific family occurs throughout the study area where four species from two genera are found. Ronquils are elongate with long dorsal and anal fins composed almost entirely of soft rays. Little is known of the adult habits in the family except for some members of the genus Rathbunella, occurring from south of Point Conception to northern California, which inhabit rocky areas 10-92 m deep (and are occasionally taken with trawl nets). Courtship of Rathbunella is paired and may result in spawning occurring over a protracted period of time. Demersal eggs, which measure 0.9-1.1 mm and contain one oil globule, are deposited in a nonadhesive mass that is guarded by the male (Fitch and Lavenberg 1975, NWAFC unpubl.).

Pelagic larvae of Ronquilus jordani are collected in ichthyoplankton surveys conducted off Alaska and along the Pacific coast to northern California. Bathymaster spp. (6-40 mm SL) are routinely collected in the Gulf of Alaska and in the Bering Sea. Ronquilus larvae can be separated from Bathymaster larvae by their lack of pigment around the urostyle. Bathymaster spp. larvae are presently not identifiable to species. Meristic characters offer some potential for larger larvae (e.g., B. signatus usually has higher counts, especially vertebrae and total caudal fin rays). Before their fin rays develop, bathymasterid larvae may often be confused with stichaeid larvae. Most stichaeids (except members of the Stichaeini tribe) have a myomere count $>55$, whereas bathymasterids have a count $<55$. Ronquilus jordani larvae (myomeres $49-50$ ) most closely resemble Stichaeus punctatus larvae (myomeres 51-55) due to the presence of distinctive lateral pigment along the hypaxial myomeres. Bathymaster spp. larvae (myomeres 49-55) most closely resemble those of Bryozoichthys-Chirolophis (myomeres about 60-75) due to the presence of pigment around the urostyle.

\begin{tabular}{|c|c|c|c|c|c|c|c|c|c|c|}
\hline \multirow[t]{4}{*}{ eristic characters } & \multicolumn{3}{|r|}{$\begin{array}{l}\text { Table } 4 \\
\text { fic fin cou }\end{array}$} & ts of $1,5,7$ & 7 principal & caudal fin & s, and & branchi & tegal $\mathbf{r}$ & \\
\hline & \multirow[b]{3}{*}{ Distribution } & \multirow{2}{*}{\multicolumn{2}{|c|}{ Vertebrae }} & \multicolumn{5}{|c|}{ Fins } & \multirow{2}{*}{\multicolumn{2}{|c|}{ Gill rakers }} \\
\hline & & & & \multirow[b]{2}{*}{ Dorsal $^{b}$} & & \multirow[b]{2}{*}{ Pectoral } & \multicolumn{2}{|c|}{ Caudal } & & \\
\hline & & $\begin{array}{r}\text { Precaudal } \\
\text { (To }\end{array}$ & $\begin{array}{l}\text { Caudal } \\
\text { al) }\end{array}$ & & & & Upper & Lower & Upper & Lower \\
\hline Bathymaster caeruleofasciatus & Brit. Col.-Bering Sea & $\begin{array}{r}14-15-16 \\
(50-5\end{array}$ & $\begin{array}{l}35-38-39 \\
-53)\end{array}$ & $44-47-48$ & $33-35-36$ & $16-18-19$ & & & 5 & $12-14$ \\
\hline Bathymaster leurolepis & Gulf of Alaska-Bering Sea & $\begin{array}{r}14-15-15 \\
(49-5\end{array}$ & $\begin{array}{l}35-36-37 \\
-52)\end{array}$ & $45-46-47$ & $32-34-34$ & $17-18-19$ & 10 & 10 & $4-5$ & $12-14$ \\
\hline Bathymaster signatus & Wash.-Arctic & $\begin{array}{r}15-16-16 \\
(54-5\end{array}$ & $\begin{array}{l}34-37-39 \\
-55)\end{array}$ & $46-48-49$ & $33-34-36$ & $19-20-21$ & $10-12$ & $9-11$ & & $15-18$ \\
\hline Ronquilus jordani & N. Calif.-Bering Sea & $\begin{array}{r}13-14-15 \\
(49-5\end{array}$ & $\begin{array}{l}34-36-37 \\
-50)\end{array}$ & $44-45-46$ & $33-35-35$ & $17-17-19$ & $5-7$ & $5-7$ & & \\
\hline
\end{tabular}




\section{Bathymaster A}
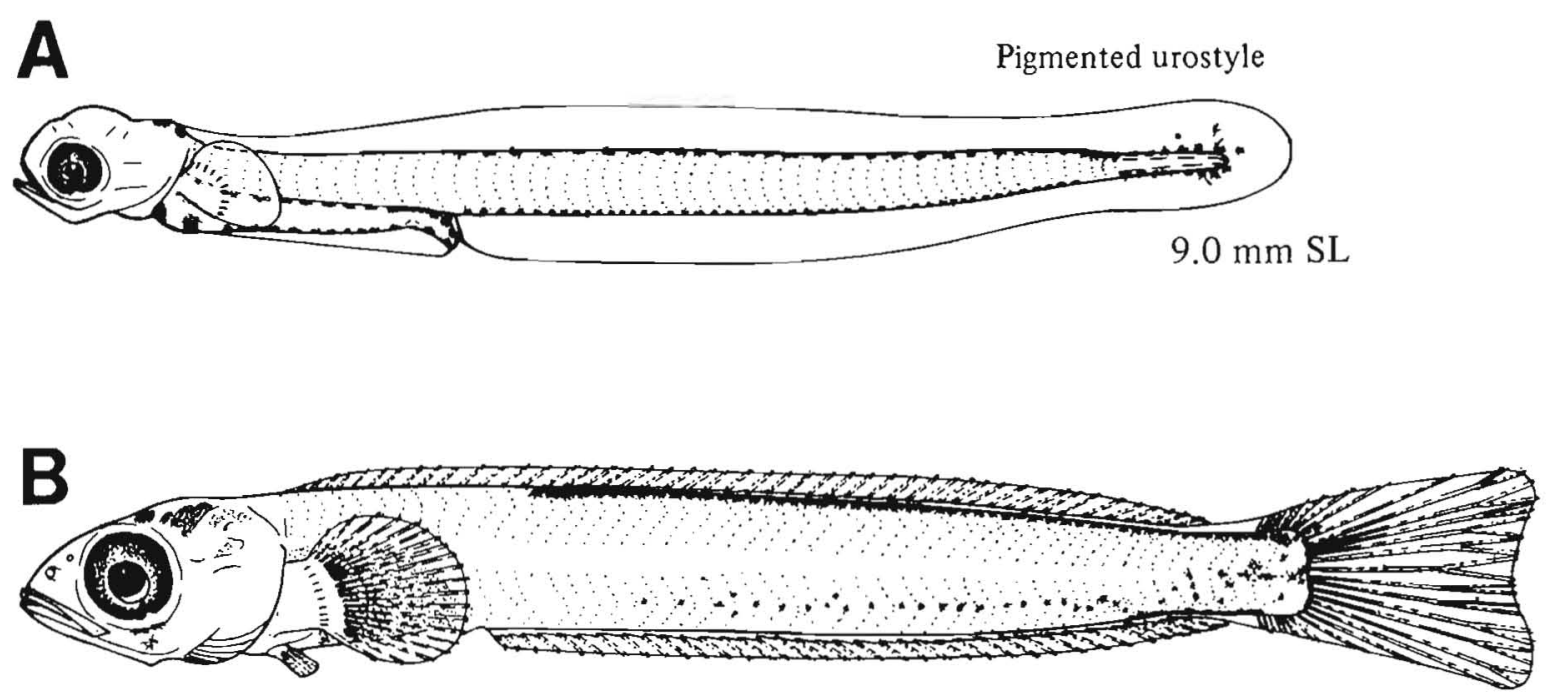

$29.6 \mathrm{~mm} \mathrm{SL}$

\section{Ronquilus jordani}

C

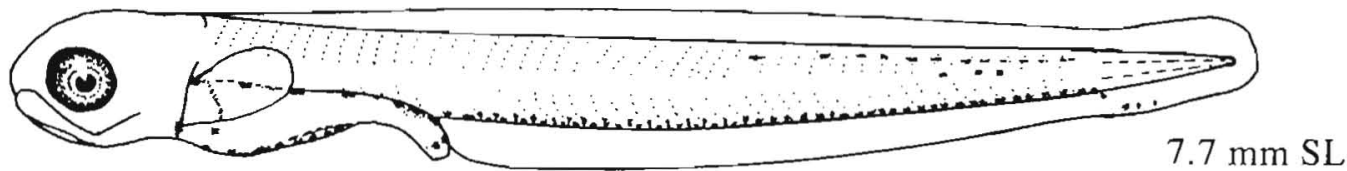

D

Figures A-D, NWAFC originals (B. Vinter). 
Eelpouts are found chiefly in colder marine waters of the Northern Hemisphere. They have elongate, tapered bodies with long dorsal and anal fins confluent with the caudal fin. The pelvic fins are small and jugular when present. The head is large and the mouth is often big with thick lips. Within the study area there are 48 species within 14 genera. Adults are found from the intertidal zone to depths of more than $1900 \mathrm{~m}$ (Hart 1973). Although members of the genus Zoarces are viviparous, all species in our area, where reproductive mode is known, are oviparous. Oviparous species have been observed guarding their eggs by wrapping themselves around the egg mass. Demersal eggs are adhesive, spherical, and possess one oil globule. Diameters range from 1.7 to $9.0 \mathrm{~mm}$. Newly hatched larvae are quite advanced and strongly resemble adult zoarcids (Anderson 1984b). Larvae of only five taxa have been illustrated; four are from outside the study area. Larvae probably become demersal or semidemersal soon after hatching because they are virtually never collected in plankton nets.

\begin{tabular}{|c|c|c|c|c|c|c|c|c|c|c|}
\hline \multirow[b]{4}{*}{ Taxon } & \multicolumn{9}{|c|}{ Table 43} & \multirow[b]{4}{*}{ Branchiostegals } \\
\hline & \multirow[b]{3}{*}{ Distribution } & \multicolumn{2}{|c|}{ Vertebrae } & \multirow{2}{*}{\multicolumn{4}{|c|}{ Fins }} & \multicolumn{2}{|c|}{ Gill rakers } & \\
\hline & & \multirow{2}{*}{\multicolumn{2}{|c|}{$\begin{array}{c}\text { Precaudal Caudal } \\
\text { (Total) }\end{array}$}} & & & & & Upper & Lower & \\
\hline & & & & \multirow{2}{*}{$\begin{array}{c}\text { Dorsal } \\
107-112\end{array}$} & \multirow{2}{*}{$\begin{array}{c}\text { Anal } \\
92-96\end{array}$} & \multirow{2}{*}{$\frac{\text { Pectoral }}{14-17}$} & \multirow{2}{*}{$\frac{\text { Pelvic }^{a}}{a b}$} & \multicolumn{2}{|c|}{ (Total) } & \\
\hline Bothrocara brunneum & SSC-Bering Sea & 22 & 94 & & & & & $3-5$ & $14-15$ & \\
\hline Bothrocara hollandi & Bering Sea & \multirow{2}{*}{\multicolumn{2}{|c|}{$(120)$}} & $114-117$ & $94-99$ & $15-17$ & $a b$ & 4 & 11 & 6 \\
\hline Bothrocara molle & SSC-Bering Sea & & & $100-112$ & $89-101$ & $13-14$ & $a b$ & & 2) & \\
\hline Bothrocara pusillum & SE Alaska-Brit. Col. & $18-20$ & $95-101$ & $113-121$ & $100-107$ & $14-17$ & $a b$ & & & \\
\hline Bothrocara remigerum & Cent. Calif.-Wash. & 23 & 16) 96 & $107-117$ & $93-94$ & $13-16$ & & & & \\
\hline Derepodichthys alepidotus & SSC-Brit. Col. & $22-26$ & $92-98$ & $110-116$ & $94-101$ & $10-11$ & 3 & $0-1$ & $11-12$ & 6 \\
\hline Gymnelis hemifasciatus & Gulf of Alaska-Arctic & $\begin{array}{r}18-21 \\
(85\end{array}$ & 5) $65-77$ & $80-92^{b}$ & & & & & & \\
\hline Gymnelis popovi & Gulf of Alaska-Bering Sea & & & 101 & 89 & & $a b$ & 3 & 12 & \\
\hline Gymnelis viridis & Aleutian Is.-Arctic & $(87$ & & $92-93$ & 74 & $10-12$ & $a b$ & & & \\
\hline Krusenstemiella pavlovskii & Bering Sea & $20-21$ & $88-89$ & $70-75$ & & 12 & $a b$ & & & \\
\hline Lycenchelys altus & Aleutian Is. & 21 & 8) 67 & 83 & 68 & 18 & & 1 & 7 & \\
\hline Lycenchelys camchaticus & SSC-Bering Sea & $21-24$ & $97-103$ & $112-117$ & $98-105$ & $13-17$ & & ${ }^{2}(14$ & $13-16$ & 6 \\
\hline Lycenchelys crotalinus & S. Calif.-Bering Sea & $22-24$ & $98-107$ & $113-123$ & $99-109$ & $14-17$ & & 1 & $14-18$ & 6 \\
\hline Lycenchelys hippopotamus & Bering Sea & $23-24$ & $109-113$ & & & $13-17$ & & 3 & $12-14$ & 6 \\
\hline Lycenchelys jordani & Calif.-SE Alaska & $22-24$ & $100-109$ & 116 & 93 & $15-17$ & & 1 & $15-18$ & 6 \\
\hline Lycenchelys longirostris & Bering Sea & $\begin{array}{r}21-22 \\
(113\end{array}$ & $\begin{array}{l}92-93 \\
15)^{9}\end{array}$ & $108-109$ & $93-95$ & $15-16$ & & & & \\
\hline Lycenchelys microporus & Bering Sea & 29 & 94 & & & 18 & & 2 & 10 & 6 \\
\hline Lycenchelys pliciferus & Bering Sea & $28-29$ & $96-97$ & & & 15 & & & & 6 \\
\hline Lycenchelys rassi & Bering Sea & $23-25$ & $98-103$ & 119 & 104 & $15-16$ & & $1-2$ & $8-11$ & 6 \\
\hline Lycenchelys ratmanovi & Bering Sea & $22-23$ & $88-89$ & & & $16-19$ & & $1-2$ & $7-9$ & 6 \\
\hline Lycenchelys roseus & Aleutian Is. & 28 & $118-119$ & $130-133$ & $114-115$ & $14-15$ & & $1-2$ & $9-10$ & \\
\hline Lycenchelys volki & Bering Sea & 30 & 96 & & & 17 & & 2 & 14 & 6 \\
\hline Lycodapus derjugini & Bering Sea & $14-15$ & $56-59$ & $70-79$ & $57-68$ & $6-7$ & $a b$ & & & 6 \\
\hline Lycodapus dermatinus & SSC-SE Alaska & $13-15$ & $62-68$ & $70-75$ & $62-66$ & $6-7$ & $\mathrm{ab}$ & & & 6 \\
\hline Lycodapus endemoscotus & SSC-Brit. Col. & $14-17$ & $72-79$ & $84-91$ & $74-81$ & $6-8$ & $a b$ & & & 6 \\
\hline Lycodapus fierasfer & SSC-Bering Sea & $13-15$ & $69-77$ & $78-85$ & $68-74$ & $6-8$ & $\mathrm{ab}$ & & & 6 \\
\hline Lycodapus leptus & Bering Sea & $16-19$ & $78-82$ & $91-94$ & & $6-8$ & $\mathrm{ab}$ & & & 6 \\
\hline Lycodapus mandibularis & S. Calif-Bering Sea & $14-17$ & $67-80$ & $76-90$ & $65-79$ & $6-9$ & $a b$ & & & 6 \\
\hline Lycodapus pachysoma & Oregon-Brit. Col. & $14-16$ & $60-63$ & $70-74$ & $58-64$ & $7-8$ & $a b$ & & & 6 \\
\hline Lycodapus parviceps & Wash.-Bering Sea & $18-20$ & $81-85$ & $94-98$ & & $8-9$ & $a b$ & & & 6 \\
\hline Lycodapus poecilus & Bering Sea & $15-17$ & $65-72$ & $75-83$ & & $5-7$ & $a b$ & & & 6 \\
\hline Lycodapus psarosomatus & Bering Sea & $17-19$ & $77-82$ & $89-93$ & & 8 & $a b$ & & & 6 \\
\hline Lycodes brevipes & Oregon-Bering Sea & $20-22$ & $80-82$ & $85-102$ & $74-89$ & $19-21$ & 3 & $2-3$ & 11 & \\
\hline Lycodes concolor & Bering Sea & 22 & $92-93$ & $47-118$ & $98-99$ & 21 & & $0-2$ & 12 & \\
\hline Lycodes cortezianus & S. Calif.-SE Alaska & $22-24$ & $83-90$ & $112-114$ & $95-97$ & $18-21$ & 3 & 2 & 11 & 6 \\
\hline Lycodes diapterus & S. Calif,-Bering Sea & $21-23$ & 100 & $90-124$ & $94-107$ & $18-25$ & 3 & 1 & 13 & \\
\hline Lycodes mucosus & Bering Sea-Arctic & & & $88-93$ & $69-73$ & $17-18$ & & & & \\
\hline Lycodes pacifica & SSC-Gulf of Alaska & $21-23$ & $79-85$ & $90-107$ & $70-90$ & $16-19$ & 3 & $0-2$ & $8-12$ & \\
\hline Lycodes palearis & Oregon-Chukchi Sea & (96 & 05) & $94-106$ & $83-90$ & 17 & 3 & $2-3$ & 10 & \\
\hline Lycodes raridens & Bering Sea-Arctic & & & $83-93$ & $72-76$ & $18-19$ & & 3 & $10-12$ & \\
\hline Lycodes tumeri & Bering Sea-Arctic & & & $89-97$ & $69-78$ & $15-18$ & 3 & & & \\
\hline Lyconema barbatum $^{c}$ & SSC-Bering Sea & $20-21$ & $86-93$ & $101-105$ & $90-94$ & 15 & & 2 & $8-9$ & \\
\hline Melanostigma pammelas & SSC-Brit. Col. & $19-20$ & $69-72$ & $73-88$ & $64-75$ & $6-8$ & $a b$ & & & $6-7$ \\
\hline Nalbantichthys elongatus ${ }^{c}$ & Bering Sea & 25 & $119-125$ & $143-152$ & $121-127$ & 6 & $a b$ & & & 7 \\
\hline Opaeophacus acrogeneius ${ }^{c, d}$ & Bering Sea & $\begin{array}{r}25-26 \\
(144\end{array}$ & & $141-148$ & $121-124$ & $4-5$ & $a b$ & 3 & 11 & 6 \\
\hline Pachycara bulbiceps & SSC-Brit. Col. & & & 109 & 89 & 16 & $a b$ & & & 6 \\
\hline Puzanovia rubra ${ }^{\mathrm{c}}$ & Bering Sea & $22-24$ & $110-125$ & $137-147$ & $115-128$ & $10-12$ & $a b$ & $3-5$ & $9-13$ & 6 \\
\hline Taranetzella lycoderma ${ }^{\mathrm{c}}$ & Oregon-Bering Sea & $19-20$ & $69-78$ & $84-91$ & $71-79$ & 15 & 3 & 3 & 13 & \\
\hline & & & & & & & & & & \\
\hline $\begin{array}{l}{ }^{a} a b=a b s e n t . \\
{ }^{b} \text { Dorsal fin count does not ir }\end{array}$ & $\begin{array}{l}\text { 'Total } \\
\text { elonga } \\
{ }^{\mathrm{d}} \text { Bond a }\end{array}$ & $\begin{array}{l}\text { ipal cauda } \\
7-10 ; O p a \\
\text { Stein } 1984\end{array}$ & $\begin{array}{l}\text { ray cot } \\
\text { hacus a }\end{array}$ & $\begin{array}{l}\text { availab } \\
\text { eneius, }\end{array}$ & $\begin{array}{l}\text { ronly } \\
\text { Puzan }\end{array}$ & $\begin{array}{l}\text { ollowin } \\
\text { rubra, }\end{array}$ & $\begin{array}{l}\text { ecies: } \\
2 ; T a r\end{array}$ & $\begin{array}{l}\text { onema } \\
\text { tzella } l\end{array}$ & $\begin{array}{l}\text { batum, } \\
\text { erma, } 8\end{array}$ & Nalbantichthys \\
\hline
\end{tabular}


A Unidentified zoarcid

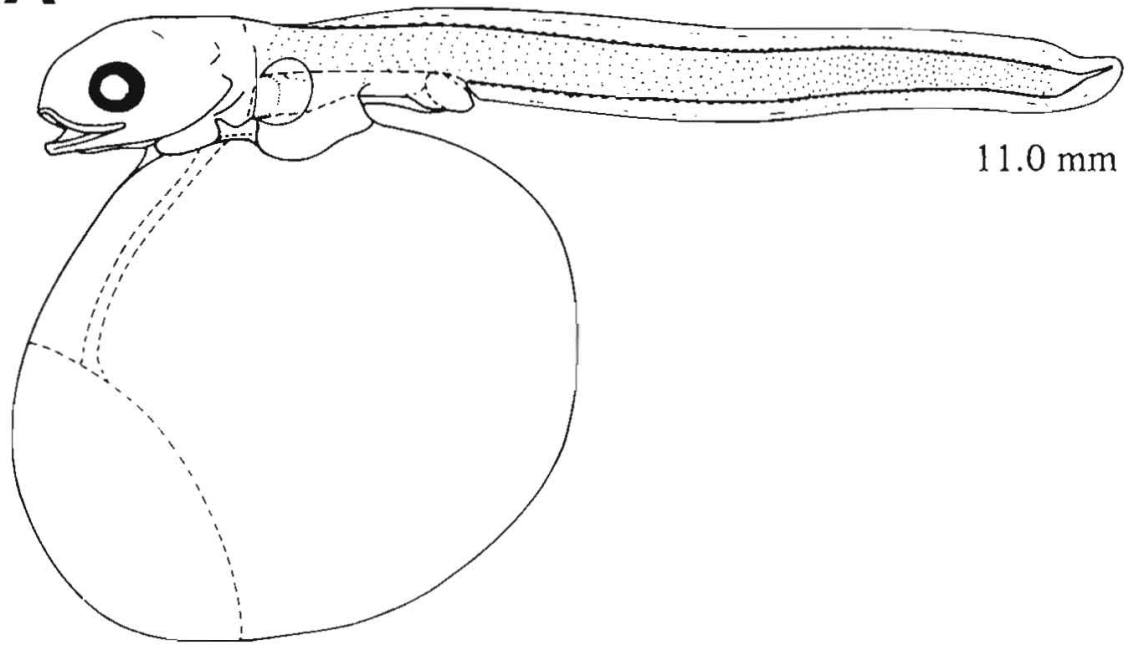

B Zoarces viviparous

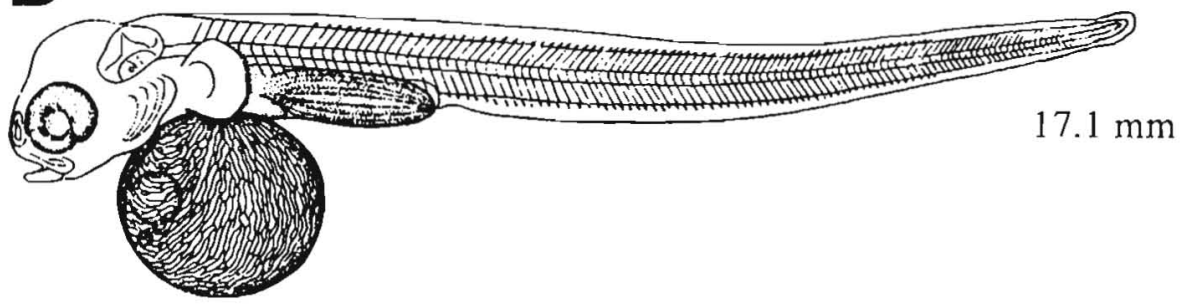

\section{Bothrocara hollandi}

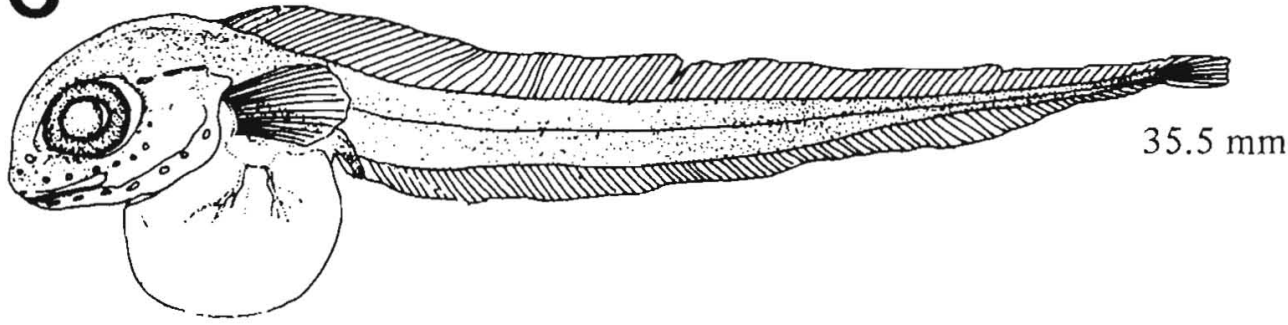

\section{Gymnelus viridis}

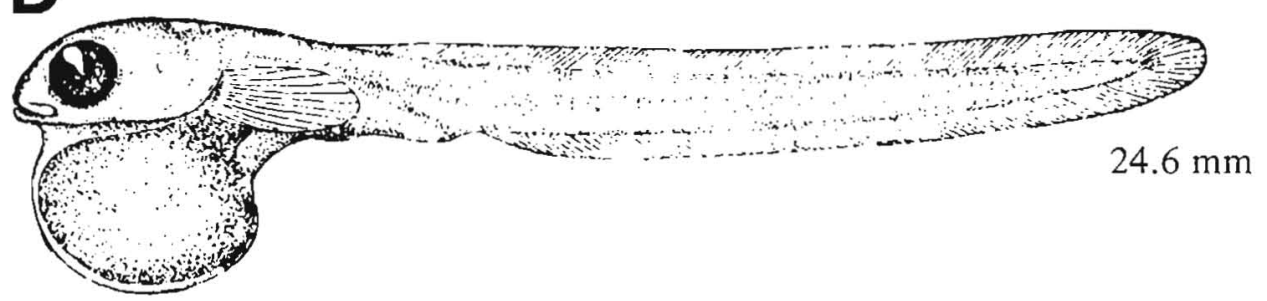

\section{E Macrozoarces americanus}

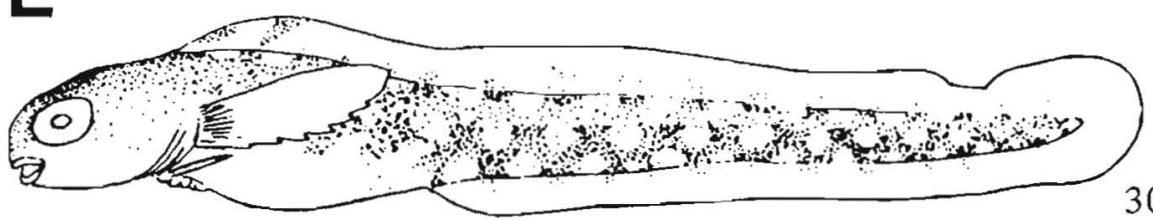

Figures A-E, Kendall et al. 1983 (B, collected from Gulf of Riga, Baltic Sea; C, Japan Sea specimen; D, Barents Sea specimen; E, collected near New Brunswick). 
Pricklebacks are found mostly in the North Pacific in inshore areas. Adults are long and somewhat eel-like with a long dorsal fin composed of all spines in most species. Seventeen genera and 26 species are found in the study area. This family is separated into eight tribes grouped within four subfamilies. Six tribes within three subfamilies occur in our area. ${ }^{1}$ Larvae are abundant in the area but very few larval series have been described. Before fin rays develop, small larvae are elongate and resemble other elongate forms, especially bathymasterids and pholidids (see Table 4). In general, bathymasterids have fewer myomeres and pholidids have a longer gut and more myomeres. Since so few complete larval series are available, general characters based on only a few species are presented for each tribe.

\section{Stichaeinae}

Adult characters include: Pelvic fins I,3-4

(Gymnoclinus I,2)

Large pectoral fins

Vertebrae $49-76$

\section{Stichaeini}

Genera found within the study area: Eumesogrammus (one species) and Stichaeus (one species). Myomere counts are low $(<55)$. Stichaeus larvae are identified by the distinctive lateral pigment along the hypaxial myomeres.

\section{Chirolophini}

Genera found within the study area: Bryozoichthys (two species), Chirolophis (four species), and Gymnoclinus (one species). Myomere counts are $>55$. Larvae are generally more pigmented than Stichaeini. Melanophores occur along the dorsal and ventral body midline, over the notochord internally, and, in some taxa, around the urostyle.

'Makushok (1958) places the genera Eulophias and Azygopterus in separate tribes under Xiphisterinae. Anderson (1984b) places them together in the Eulophiini. Other nomenclatural changes not affecting taxa in the study area are presented by Yatsu (1986).

Ref: Anderson 1984a,b; Makushok 1958. 
STICHAEINI

Stichaeus punctatus

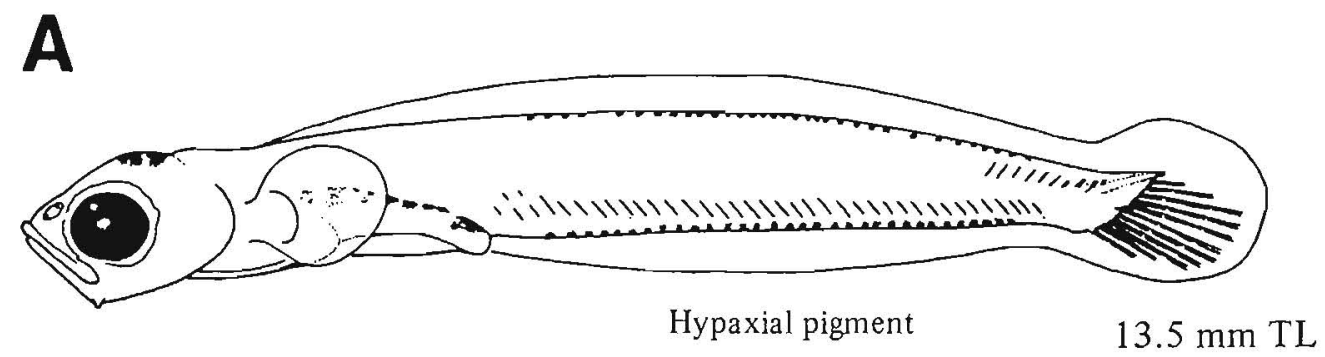

B

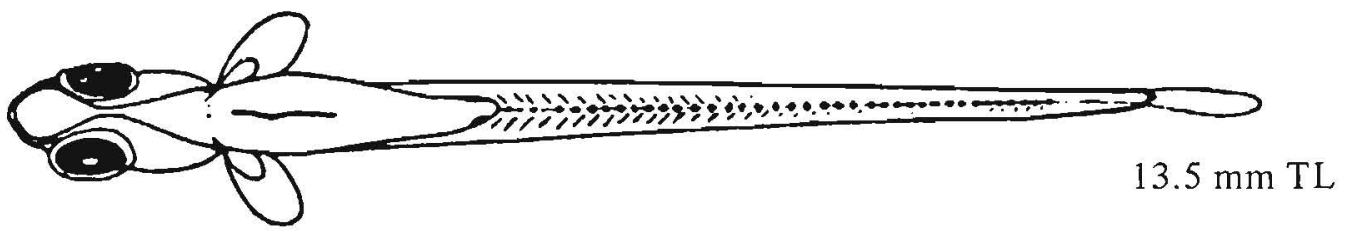

\section{CHIROLOPHINI}

Bryozoichthys_Chirolophis

C

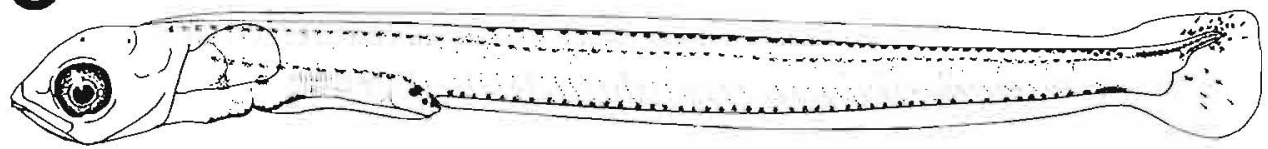

$16.5 \mathrm{~mm} \mathrm{SL}$

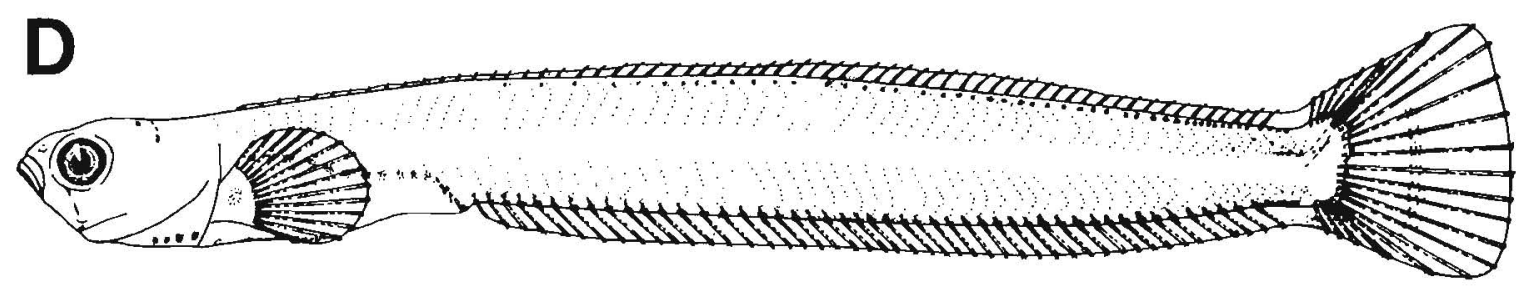

$29.0 \mathrm{~mm} \mathrm{SL}$

Figures A-B (B, ventral view), Fahay 1983 (after Faber 1976, North Atlantic specimen); C-D, NWAFC originals (B. Vinter). 


\section{Lumpeninae}

Adult characters include: Pelvic fins I,3 or absent

Large pectoral fins

Vertebrae $\quad 60-81$

\section{Lumpenini}

Genera found within the study area: Acantholumpenus (one species), Anisarchus (one species), Lumpenella (one species), Lumpenus (four species), and Poroclinus (one species). Lumpenini larvae generally lack pigment along the dorsal midline. Diagnostic pigment usually occurs over the dorsal surface of the gut and anus, along the ventral midline, and in the hypural area.

Lumpenella larvae generally have $>70$ myomeres and pigment around the urostyle, and, with development, can be identified by their distinctive snout and the presence of up to five anal spines. Lumpenus spp. larvae can be identified by meristics, number of postanal ventral melanophores, and number of melanophores on the anus. Hypural pigment is usually restricted to several spots along the posterior edge of the hypural area. Poroclinus larvae have distinctive pigment occurring above and below the notochord in the caudal peduncle area. They can also be distinguished from other Lumpenini by the presence of three anal spines. 


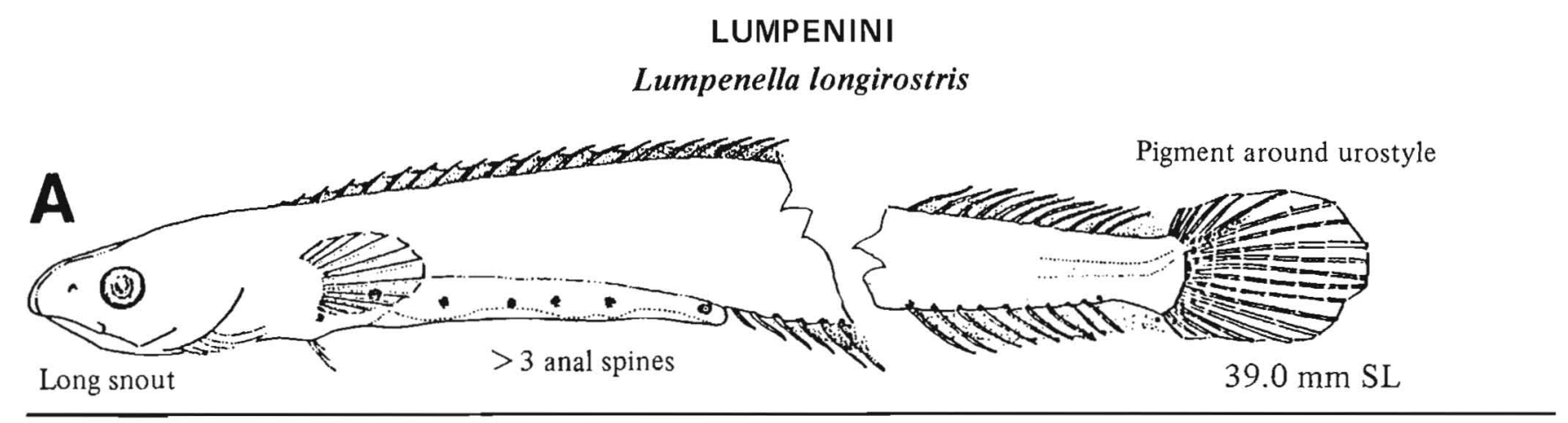

B

\section{Lumpenus maculatus}

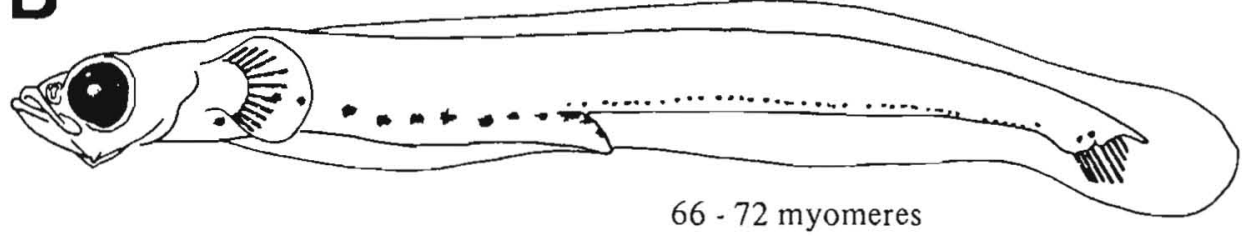

$13.5 \mathrm{~mm} \mathrm{TL}$

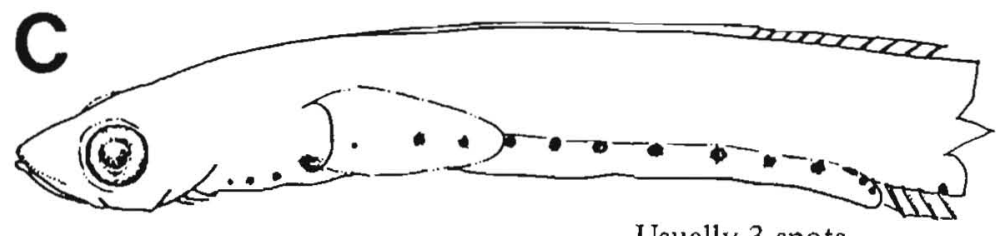

Usually 3 spots

on anus

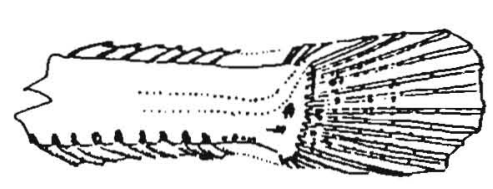

$31.3 \mathrm{~mm} \mathrm{SL}$

\section{Lumpenus sagitta}

D

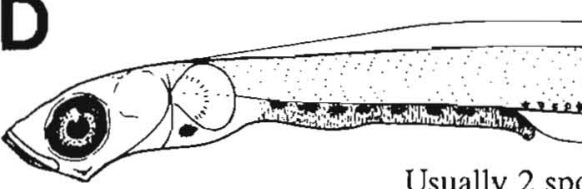

Usually 2 spots

E

72 - 82 myomeres

$$
\text { on anus }
$$

$17.3 \mathrm{~mm} \mathrm{SL}$

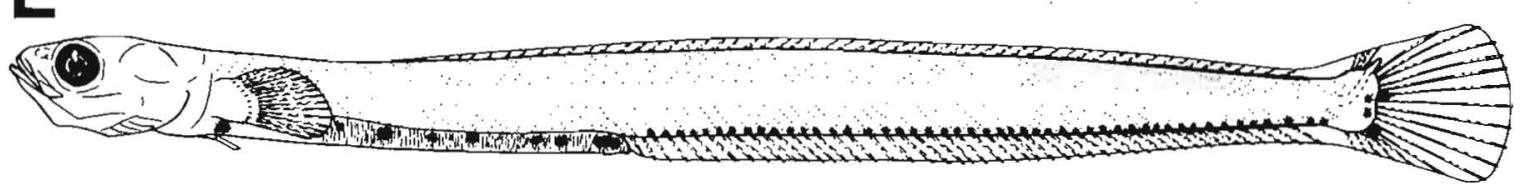

$35.1 \mathrm{~mm} \mathrm{SL}$

\section{Poroclinus rothrocki}

Pigment above and below notochord

$F$

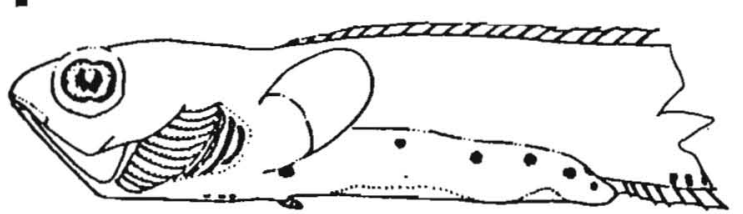

Three anal spines

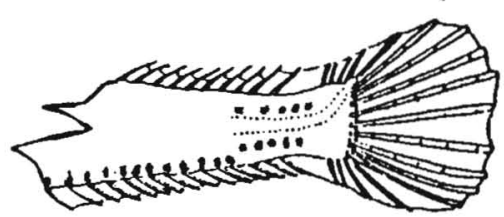

$25.4 \mathrm{~mm} \mathrm{SL}$

Figures A, C, F, Garrison, unpubl.; B, Fahay 1983 (after Faber 1976, Atlantic specimen); D-E, NWAFC originals (B. Vinter). 


\section{Opisthocentrini}

Genera found within the study area: Allolumpenus (one species), Opisthocentrus (one species), and Plectobranchus (one species). Larvae are known only for Opisthocentrus and Plectobranchus. Plectobranchus larvae have a distinctive pigment pattern quite dissimilar to Opisthocentrus (see species pages). Opisthocentrus larvae appear to resemble Alectrini larvae (e.g., similar pigment pattern and lack of pelvic fins).

\section{Xiphisterinae}

Adult characters include: Pelvic fins absent

Pectoral fins small

Vertebrae $57-84$

\section{Alectrini}

Genera found within the study area: Alectridium (one species) and Anoplarchus (two species). Only larvae of Anoplarchus purpurescens are known from our area. They have a row of postanal ventral midline melanophores and a few spots along the hypural margin. No single unique character distinguishes them from Xiphisterini larvae but usually a combination of characters allows identification. These characters include fewer myomeres (58-68 vs. generally $>70)$, smaller relative eye diameter, fewer melanophores dorsally on gut, and presence of hypural spots throughout the larval period (see species pages).

\section{Xiphisterini}

Genera found within the study area: Cebidichthys (one species), Phytichthys (one species), and Xiphister (two species). Although series of Phytichthys and Xiphister have been described, larvae are difficult to distinguish from one another and from Anoplarchus. Xiphisterini larvae usually have a row of postanal ventral melanophores and a row of internal pigment above the notochord.

Ref: Anderson 1984a,b; Makushok 1958. 


\section{OPISTHOCENTRINI}

Opisthocentrus ocellatus

A

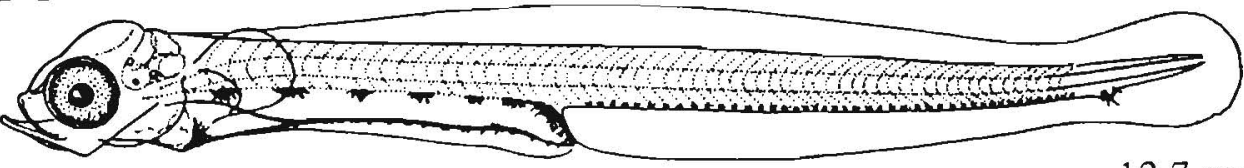

$12.7 \mathrm{~mm} \mathrm{TL}$

B

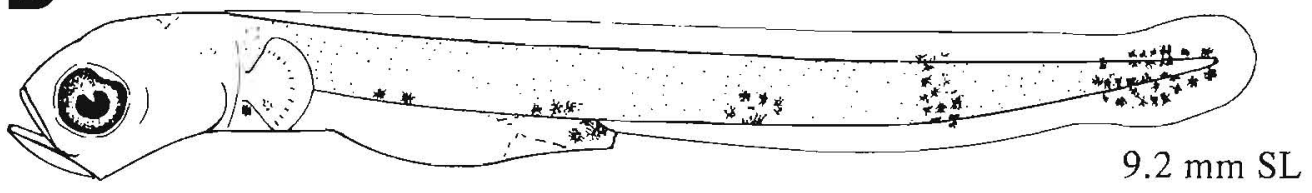

Plectobranchus evides

$9.2 \mathrm{~mm} \mathrm{SL}$

\section{ALECTRINI \\ Anoplarchus purpurescens}

C

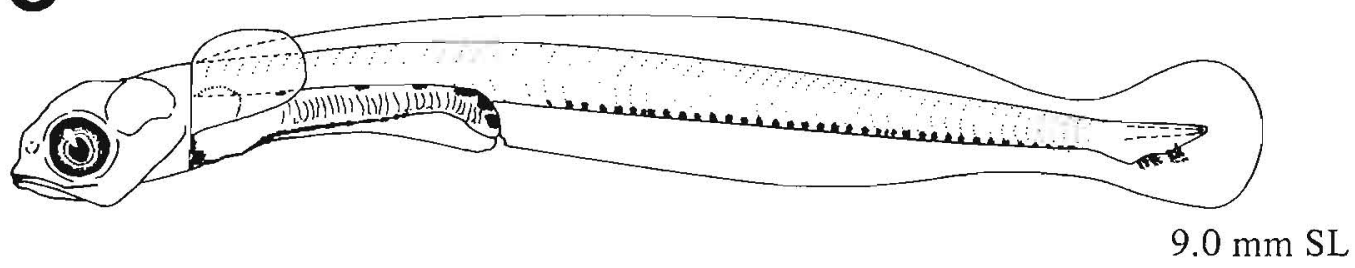

\section{XIPHISTERINI}

Xiphister atropurpureus

D

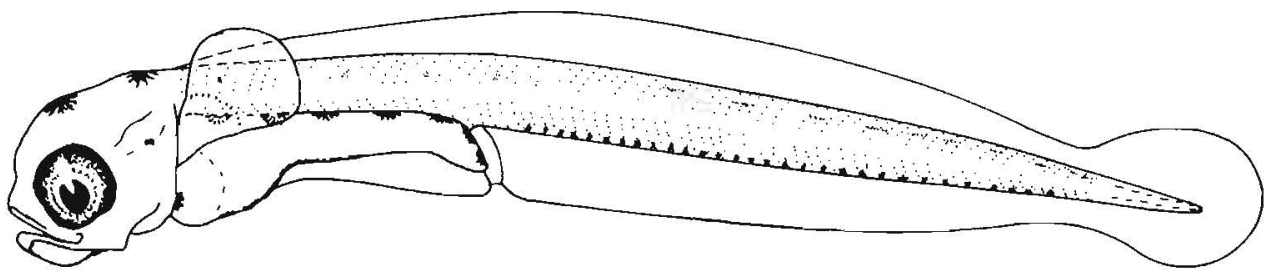

$8.0 \mathrm{~mm} \mathrm{SL}$

Figure A, Shiogaki 1982 (reared from Japanese specimens); B-D, NWAFC originals (B. Vinter). 

Table 44

Meristic characters of family Stichaeidae.

\begin{tabular}{|c|c|c|c|c|c|c|c|c|c|c|}
\hline \multirow[b]{3}{*}{ Taxon } & \multirow[b]{3}{*}{ Distribution } & \multicolumn{2}{|c|}{ Vertebrae } & \multirow{2}{*}{\multicolumn{4}{|c|}{ Fins }} & \multirow{2}{*}{\multicolumn{2}{|c|}{ Gill rakers }} & \multirow[b]{3}{*}{ Branchiostegal } \\
\hline & & \multirow{2}{*}{$\begin{array}{l}\text { Precaudal } \\
\text { (Total) }\end{array}$} & \multirow{2}{*}{$\begin{array}{l}\text { Caudal } \\
\text { d) }\end{array}$} & & & & & & & \\
\hline & & & & Dorsal & Anal & Pectoral & Pelvic ${ }^{a}$ & Upper & Lower & \\
\hline \multicolumn{11}{|l|}{ STICHAEINAE } \\
\hline \multicolumn{11}{|l|}{ Stichaeini } \\
\hline \multicolumn{11}{|c|}{$(50-52)$} \\
\hline \multirow{2}{*}{\multicolumn{11}{|c|}{$(51-55)$}} \\
\hline Chirolophini & & & & & & & & & & \\
\hline Bryozoichthys lysimus & Aleutian Is.-Bering Sea & $16-17$ & $53-54$ & LXIII-LXVI & I,49-50 & $14-15$ & 1,3 & $4-5$ & $9-11$ & 6 \\
\hline \multicolumn{11}{|c|}{$(72-75)$} \\
\hline Chirolophis decoratus & N. Calif.-Bering Sea & & & LXI-LXIII & I,44-51 & $14-15$ & 1,4 & & & 6 \\
\hline Chirolophis nugator & S. Calif.-Aleutian Is. & & & LIII-LV & I, $37-42$ & $13-14$ & $\mathrm{I}, 4$ & & & 6 \\
\hline \multicolumn{11}{|c|}{$(63-65)$} \\
\hline Chirolophis tarsodes & Brit. Col.-Bering Sea & & & LVU-LX & $1,43-45$ & $14-15$ & $1,3-4$ & & & 6 \\
\hline Gymnoclinus cristulatus & Bering Sea & & & LXI & $40-43$ & 14 & $\mathrm{I}, 1-2$ & & & 6 \\
\hline \multicolumn{11}{|l|}{$\begin{array}{l}\text { LUMPENINAE } \\
\text { Lumpenini }\end{array}$} \\
\hline \multirow{2}{*}{\multicolumn{11}{|c|}{ Acantholumpenus mackayi }} \\
\hline Anisarchus medius & & & & & & & & & & \\
\hline Lumpenella longirostris ${ }^{b}$ & Brit. Col.-Gulf of Alaska & $24-25$ & $47-49$ & LXI-LXXI & II-V, 36-42 & $13-14$ & $1,2-3$ & & & 6 \\
\hline Lumpenus fabricii ${ }^{\mathrm{b}}$ & SE Alaska-Arctic & \multicolumn{8}{|c|}{$(70-75)$} & 6 \\
\hline Lumpenus maculatus $^{b}$ & Wash.-Arctic & $\begin{array}{r}26-30 \\
(66\end{array}$ & 72) & LVII-LXIV & I-II, 34-40 & $14-16$ & $1,3-4$ & & & 6 \\
\hline \multicolumn{10}{|c|}{$(65-70)$} & \\
\hline Lumpenus saginta $^{b}$ & N. Calif.-Bering Sea & $26-28$ & $46-54$ & LXIV-LXXII & I,45-50 & $15-17$ & $1,3-4$ & & & 6 \\
\hline Poroclinus rothrocki & S. Calif.-Bering Sea & \multicolumn{2}{|c|}{$(65)$} & LVII-LXVII & III, $40-44$ & $13-15$ & $\mathrm{I}, 3^{\mathrm{c}}$ & & & 6 \\
\hline \multicolumn{11}{|l|}{ Opisthocentrini } \\
\hline Allolumpenus hypochromus & S. Calif.-Brit. Col. & & & XLIV-XLIX & 1,31 & 12 & I, 3 & & & 6 \\
\hline \multicolumn{11}{|c|}{$(63-67)$} \\
\hline Plectobranchus evides ${ }^{b}$ & S. Calif.-Brit. Col. & \multicolumn{2}{|c|}{$(60)$} & LIV-LVII & II-III, 34-36 & 15 & $\mathrm{I}, 3$ & & & $5-6$ \\
\hline \multicolumn{11}{|l|}{$\begin{array}{l}\text { XIPHISTERINAE } \\
\text { Alectrini }\end{array}$} \\
\hline \multicolumn{11}{|c|}{$\begin{array}{c}19-21 \quad 46-48 \\
(65-68)\end{array}$} \\
\hline Anoplarchus insignis & N. Calif.-Aleutian Is. & $17-19$ & $44-49$ & LVII-LXIV & $40-46$ & $9-10$ & $a b$ & & & 5 \\
\hline Anoplarchus purpurescens & S. Calif.-Bering Sea & $\begin{array}{r}17-19 \\
(58\end{array}$ & $64)^{40-46}$ & LIV-LX & $I, 36-41$ & $9-10$ & $a b$ & $3-5$ & $5-10$ & 5 \\
\hline Xiphisterini & & & & & & & & & & \\
\hline Cebidichthys violaceus & SSC-Oregon & $\begin{array}{r}23-25 \\
(65\end{array}$ & 71) $40-47$ & $\begin{array}{c}\text { XXII-XXV, } \\
40-43\end{array}$ & $I-\mathrm{L}, 39-42$ & $10-11$ & $a b$ & $3-4$ & $6-10$ & 6 \\
\hline Phytichthys chirus ${ }^{\mathrm{b}}$ & S. Calif.-Bering Sea & $\begin{array}{r}24-25 \\
\quad(75\end{array}$ & 76) & LXLX-LXXVIII & IIIIII,40-50 & 15 & $\mathrm{ab}$ & & & 6 \\
\hline Xiphister atropurpureus ${ }^{b}$ & SSC-Bering Sea & $\begin{array}{r}22-24 \\
\quad(75\end{array}$ & $80)^{51-56}$ & LXV-LXXII & $1,49-55$ & $11-12$ & $a b$ & $2-3$ & $6-10$ & 6 \\
\hline Xiphister mucosus ${ }^{b}$ & Cent. Calif.-SE Alaska & $\begin{array}{r}29-31 \\
\quad(73\end{array}$ & $83)^{44-53}$ & LXXI-LXXVIII & $1,46-50$ & 12 & $a b$ & & & 6 \\
\hline
\end{tabular}

${ }^{a} a b=$ absent.

${ }^{\mathrm{b}}$ Principal caudal fin ray counts available for only the following species: Lumpenella longirostris, $6+6-7 ;$ Lumpenus fabricii, 6+7; Lumpenus maculatus, $7+6$; Lumpenus sagitta, 6+7; Plectobranchus evides, 6+5; Phytichshys chirus, 6+7; Xiphister atropurpureus, 6+7; Xiphister mucosus, $6+7$.

${ }^{c}$ Rudimentary. 
MERISTICS

\begin{tabular}{|c|c|}
\hline \multirow[t]{3}{*}{ Vertebrae } & Total: $63-X-67$ \\
\hline & Precaudal: $22-\mathrm{X}-23$ \\
\hline & Caudal: $40-X-44$ \\
\hline Branchiostegal rays & $5-5-5$ \\
\hline \multicolumn{2}{|l|}{ Caudal fin } \\
\hline Pelvic fin & Absent \\
\hline Dorsal fin & S: $58-X-62$ \\
\hline Pectoral fin & $\mathrm{R}: 20-\mathrm{X}-21$ \\
\hline Anal fin & R: $37-X-39$ \\
\hline Gill rakers & U: X-X-X $\quad$ L: X-X-X \\
\hline \multicolumn{2}{|l|}{ LIFE HISTORY } \\
\hline Range & Bering Sea, $54-66^{\circ} \mathrm{N}$ \\
\hline Ecology & Nearshore shelf demersal \\
\hline ELH pattern & $\begin{array}{l}\text { Oviparous; demersal, adhesive } \\
\text { eggs; pelagic larvae }\end{array}$ \\
\hline \multirow[t]{4}{*}{ Spawning } & $\begin{array}{l}\text { Season: Dec-Jan (Mutsu Bay, } \\
\text { Japan) }\end{array}$ \\
\hline & $\begin{array}{l}\text { Area: Narrow cavities under } \\
\text { stones on muddy sand bottoms }\end{array}$ \\
\hline & $\begin{array}{l}\text { Mode: Eggs spawned in masses } \\
\text { and guarded by female }{ }^{\mathrm{a}}\end{array}$ \\
\hline & Migration: \\
\hline Fecundity & Range/function: $700-3300^{a}$ \\
\hline Age at first maturity & $1 \mathrm{yr}^{\mathrm{a}}$ \\
\hline Longevity & $2-3 \mathrm{yr}^{\mathrm{a}}$ \\
\hline
\end{tabular}

EARLY LIFE HISTORY DESCRIPTION

\section{EGGS}

Diameter

No. of oil globules

Oil globule diameter

Yolk

Envelope

Hatch size $\quad 9-10 \mathrm{~mm} \mathrm{SL}$

Incubation time/temp. $\quad 48 \mathrm{~d} / 5-10^{\circ} \mathrm{C}$

Pigment

Diagnostic characters

\section{LARVAE}

Preanal length $\quad<50 \%$ SL

Length at flexion

Length at transformation $33-40 \mathrm{~mm} \mathrm{SL}^{\mathrm{b}}$

Sequence of fin development

Pigment

- Dorsally and ventrally on gut

- Postanal ventral melanophores

- Hypural spot

Diagnostic characters

${ }^{\text {a }}$ Shiogaki 1982

${ }^{b}$ Collected with a small trawl net.

Ref: Shiogaki 1982. 


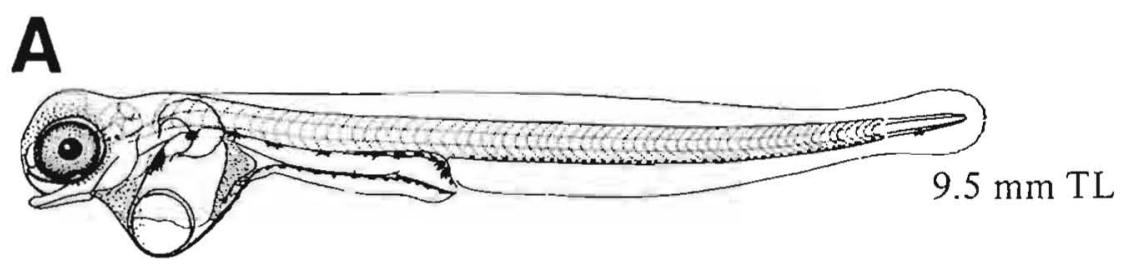

B
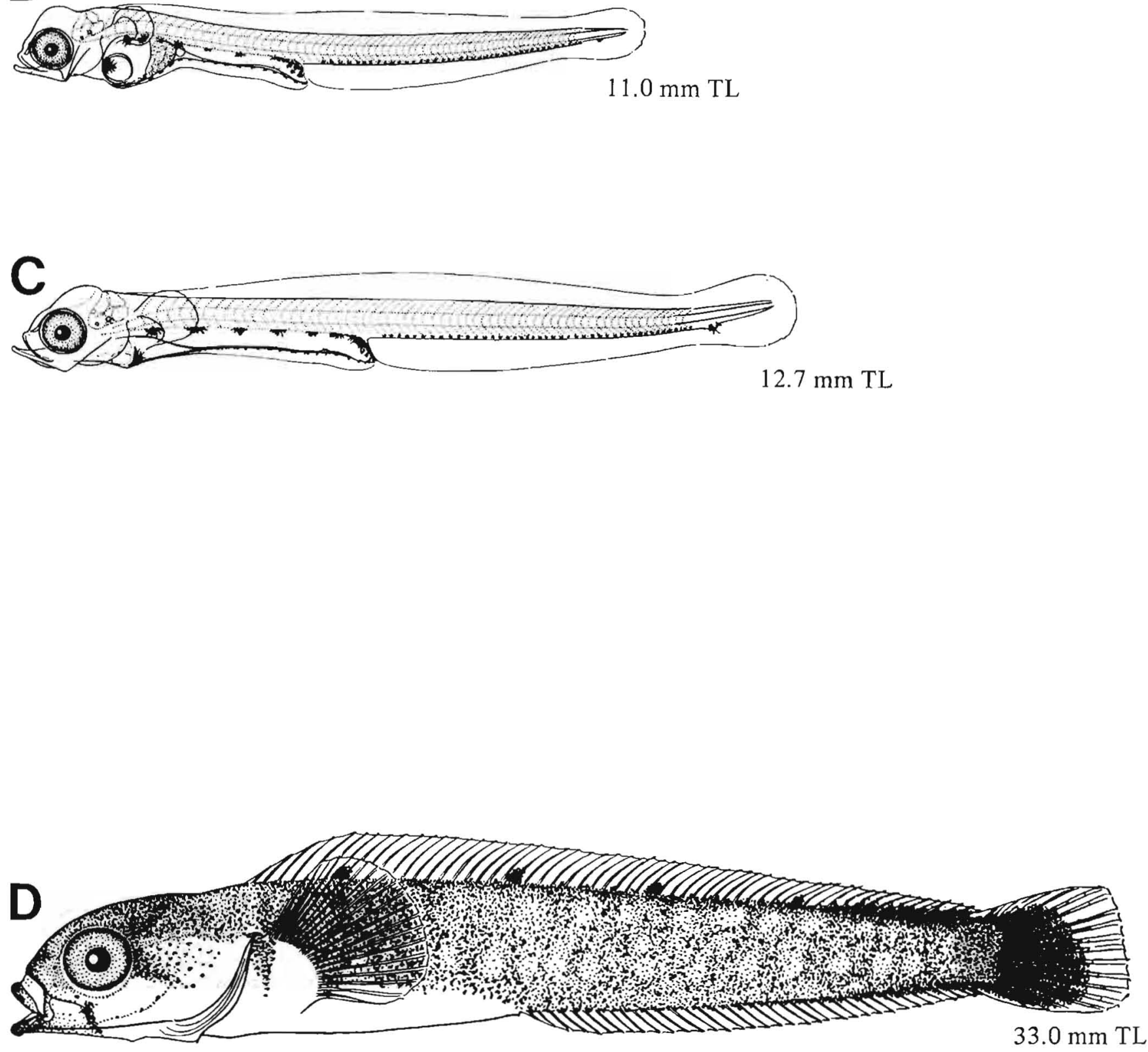

Figures A-D, Shiogaki 1982 (A-C, reared from specimens collected from Mutsu Bay, Japan; D, collected from Mutsu Bay, Japan-probably newly settled). 


\section{MERISTICS}

\begin{tabular}{|c|c|c|}
\hline \multirow{4}{*}{$\begin{array}{l}\text { Vertebrae } \\
\text { Branchiostegal rays } \\
\text { Caudal fin } \\
\text { Pelvic fin }\end{array}$} & \multicolumn{2}{|c|}{$\begin{array}{l}\text { Total: } 60-60-60 \\
\text { Precaudal: X-X-X } \\
\text { Caudal: X-X-X }\end{array}$} \\
\hline & $5-X-6$ & \\
\hline & $X, 6+5, X$ & \\
\hline & $\begin{array}{l}\text { Thoracic } \\
\text { S: } 1-1-1\end{array}$ & $\mathrm{R}: 3-3-3$ \\
\hline Dorsal fin & S: $54-X-57$ & \\
\hline Pectoral fin & $\mathrm{R}: 15-15-15$ & \\
\hline Anal fin & $S: 2-X-3$ & $\mathrm{R}: 34-\mathrm{X}-36$ \\
\hline Gill rakers & $\mathrm{U}: \mathrm{X}-\mathrm{X}-\mathrm{X}$ & L: X-X-X \\
\hline
\end{tabular}

\begin{tabular}{|c|c|}
\hline Range & $\begin{array}{l}\text { S. California, } 32-34^{\circ} \mathrm{N} \text {, to } \\
\text { Brit. Col., } 48^{\circ} 30^{\prime}-55^{\circ} \mathrm{N}\end{array}$ \\
\hline Ecology & Epi- and mesobenthal, $84-274 \mathrm{~m}$ \\
\hline ELH pattern & $\begin{array}{l}\text { Probably oviparous, eggs } \\
\text { probably demersal, pelagic } \\
\text { larvae }\end{array}$ \\
\hline \multirow[t]{4}{*}{ Spawning } & Season: \\
\hline & Area: \\
\hline & Mode: \\
\hline & Migration: \\
\hline Fecundity & Range/function: \\
\hline Age at first maturity & \\
\hline Longevity & \\
\hline
\end{tabular}

\section{EARLY LIFE HISTORY DESCRIPTION}

\section{EGGS}

Diameter

No. of oil globules

Oil globule diameter

Yolk

Envelope

Hatch size

Incubation time/temp.

Pigment

Diagnostic characters

\author{
LARVAE \\ Preanal length $\quad \sim 50 \%$ SL \\ Length at flexion Between 10 and $16 \mathrm{~mm} \mathrm{SL}$ \\ Length at transformation \\ Sequence of fin \\ development \\ Pigment \\ - Blotch medially over gut near cleithrum \\ - Blotches on ventral body at midgut, hindgut over \\ anus, and at myomeres 30 and 45 \\ - Pigment becomes more internal in larger specimens \\ - Above and below notochord, increasing with \\ development anteriorly in area where hypurals form
}

Diagnostic characters

- Pigment pattern: Five blotches along body

- Meristics: anal spines 2-3

pelvic fin $\mathrm{I}, 3$

a Larvae obtained from Bruce Mundy, formerly of Oregon State University (present 


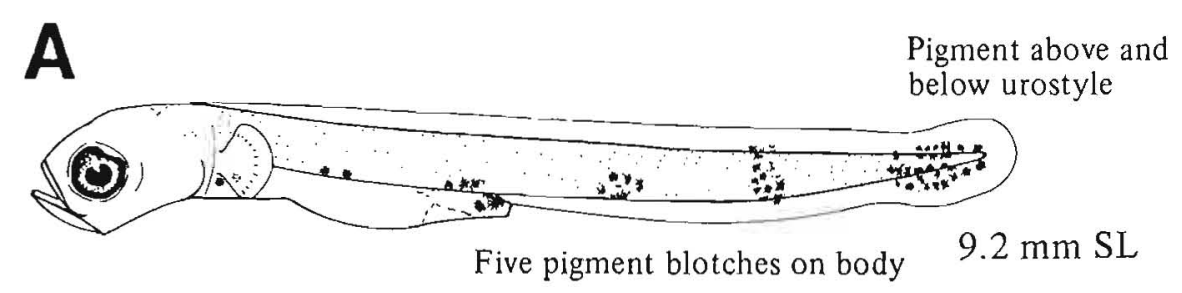

\section{B}

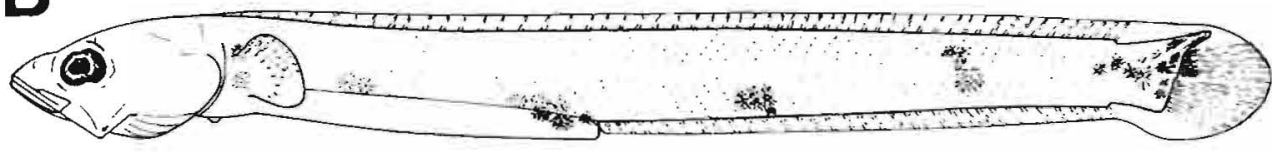

Blotches becoming more internal

$16.9 \mathrm{~mm} \mathrm{SL}$

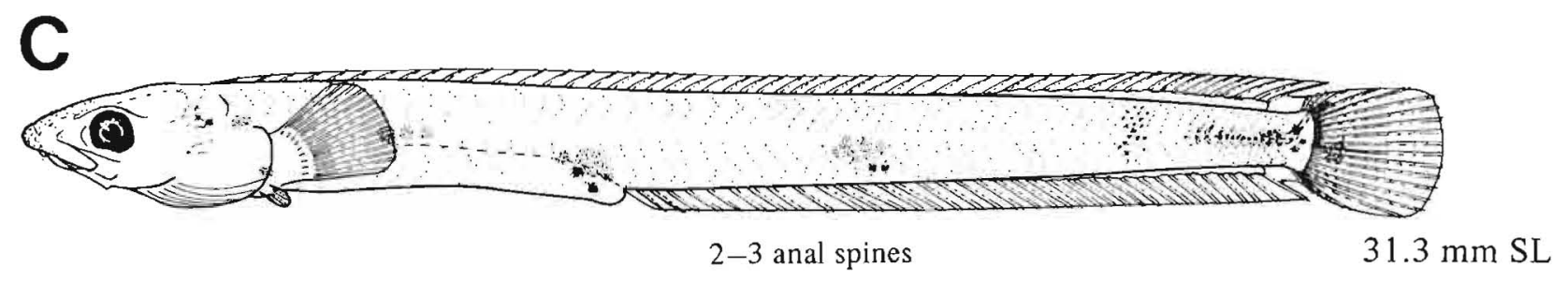

Figures A-C, NWAFC originals (B. Vinter). 


\section{MERISTICS}

Vertebrae

Total: $58-60-64$

Precaudal: $17-18-19$

Caudal: $40-43-46$

Branchiostegal rays 5-5-5

Caudal fin

Pelvic fin

Dorsal fin

Pectoral fin

Anal fin

Gill rakers

Absent

S: $54-X-60$

R: $9-\mathrm{X}-10$

S: $1-1-1$

R: $36-39-41$

$\mathrm{U}: 3-\mathrm{X}-5$

L: $5-\mathrm{X}-10$

\section{EARLY LIFE HISTORY DESCRIPTION}

\section{EGGS}

Diameter

No. of oil globules

Oil globule diameter

Yolk

Envelope

Hatch size

$1.27-1.45 \mathrm{~mm}$

1-3, with development

1 large and 1 small

Incubation time/temp.

Pigment
White

$\sim 7.5 \mathrm{~mm}$ TL

\section{LIFE HISTORY}

\begin{tabular}{|c|c|}
\hline Range & $\begin{array}{l}\text { S. California, } 32-34^{\circ} \mathrm{N} \text {, to } \\
\text { Bering Sea, } 54-66^{\circ} \mathrm{N}\end{array}$ \\
\hline Ecology & $\begin{array}{l}\text { Nearshore shelf demersal, } \\
\text { intertidal to } 30 \mathrm{~m}\end{array}$ \\
\hline ELH pattern & $\begin{array}{l}\text { Oviparous; demersal, adhesive, } \\
\text { attached eggs; pelagic larvae }\end{array}$ \\
\hline \multirow{4}{*}{ Spawning } & Season: $\operatorname{Jan}^{\mathrm{a}}-\mathrm{Mar}^{\mathrm{b}}$ \\
\hline & $\begin{array}{l}\text { Area: Demersal, under rocks or } \\
\text { on shells }\end{array}$ \\
\hline & $\begin{array}{l}\text { Mode: Pairs; eggs laid in } \\
\text { masses; }{ }^{c} \text { females guard nests }\end{array}$ \\
\hline & Migration: \\
\hline Fecundity & Range/function: $2000-3000^{d}$ \\
\hline Age at first maturity & $2-3 \mathrm{yr}^{\mathrm{d}}$ \\
\hline
\end{tabular}

Longevity

Marliave 1975a

${ }^{b}$ Schultz and DeLacy 1932

${ }^{c} J$. Marliave, Vancouver Public Aquarium, P.O. Box 3232, Vancouver, B.C., Canada

V6B $3 \times 8$, pers. commun., 16 Oct. 1986.

${ }^{\mathrm{d}}$ Peppar 1965

Ref: Marliave 1975a, Peppar 1965, Schultz and DeLacy 1932.

Diagnostic characters

- Eggs laid in pedestal-shaped mass (flattened with lateral constriction) ${ }^{c}$

\section{LARVAE}

Preanal length $\quad 40-45 \% \mathrm{SL}$
Length at flexion $\quad \sim 10 \mathrm{~mm}$ SL
Length at transformation $\sim 12 \mathrm{~mm} \mathrm{SL}$
Sequence of fin
development
Pigment
- Postanal ventral midline: A melanophore on nearly
every myoseptum, at base of each anal fin ray
- A few melanophores at caudal fin base along
- hypural margin
- Dorsal and ventral gut pigment: Dorsally about five
spots on posterior half, ventrally a continuous line
on anterior $2 / 3$ of gut
- Heavy uniform superficial body pigment on larvae
$>12$ mm SL (early juveniles)
Diagnostic characters
Distinguished from Xiphister spp. (p. 516,518 ) and
Phytichthys chirus (p. 514 ) by
- Smaller relative eye diameter
- Fewer dorsal gut melanophores $(<8)$
- Hypural spots throughout larval period




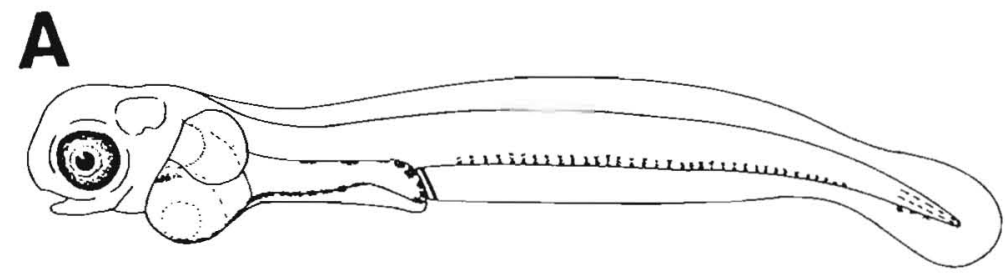

$6.1 \mathrm{~mm} \mathrm{SL}$
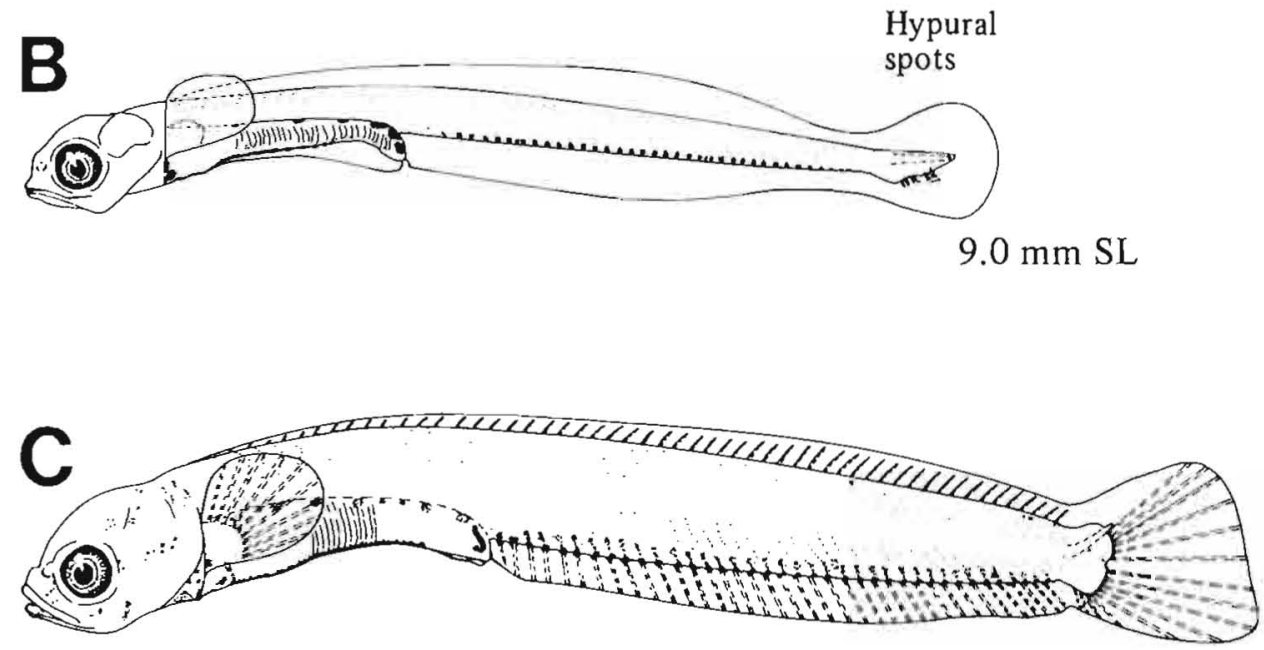

$12.0 \mathrm{~mm} \mathrm{SL}$

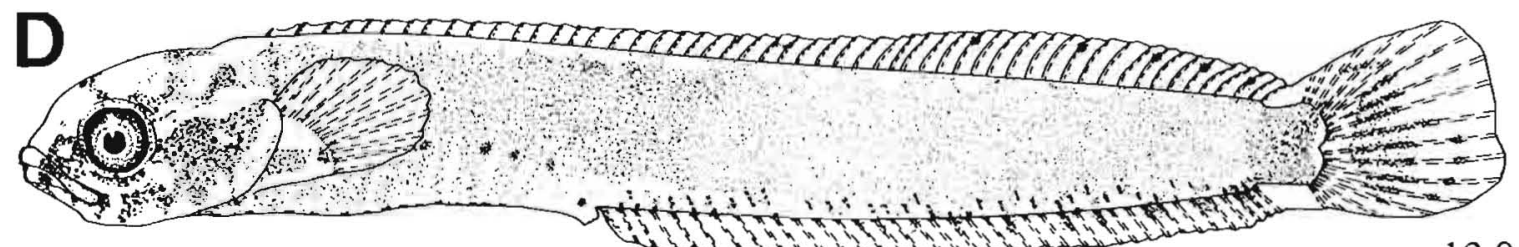

Facial pattern develops in

$12.0 \mathrm{~mm} \mathrm{SL}$ larvae approaching settlement

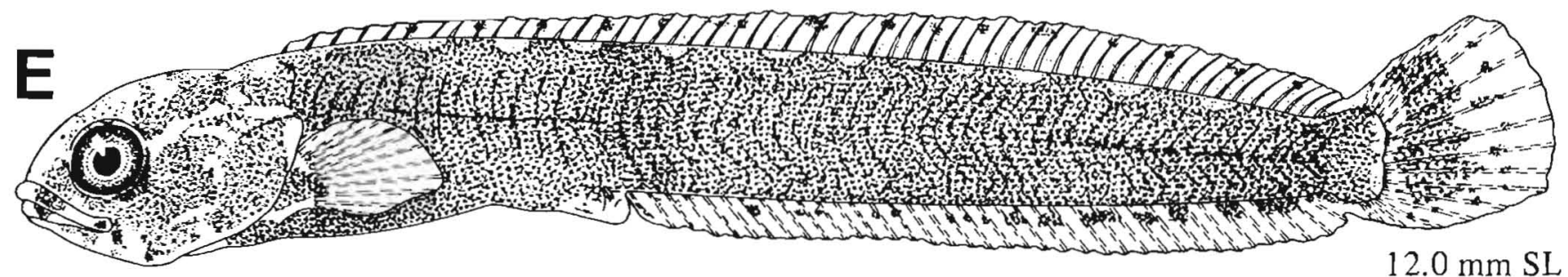

Figures A-E, NWAFC originals (B. Vinter, reared). 


\section{MERISTICS}

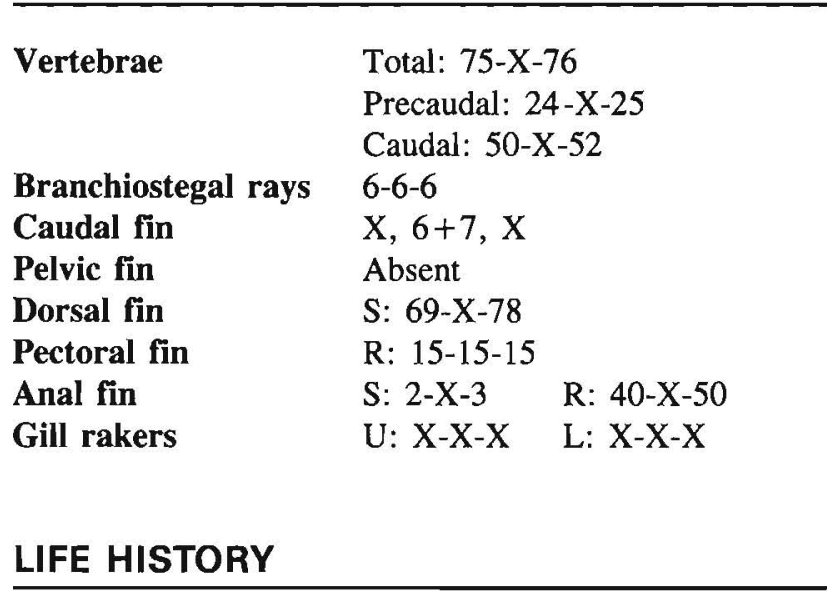

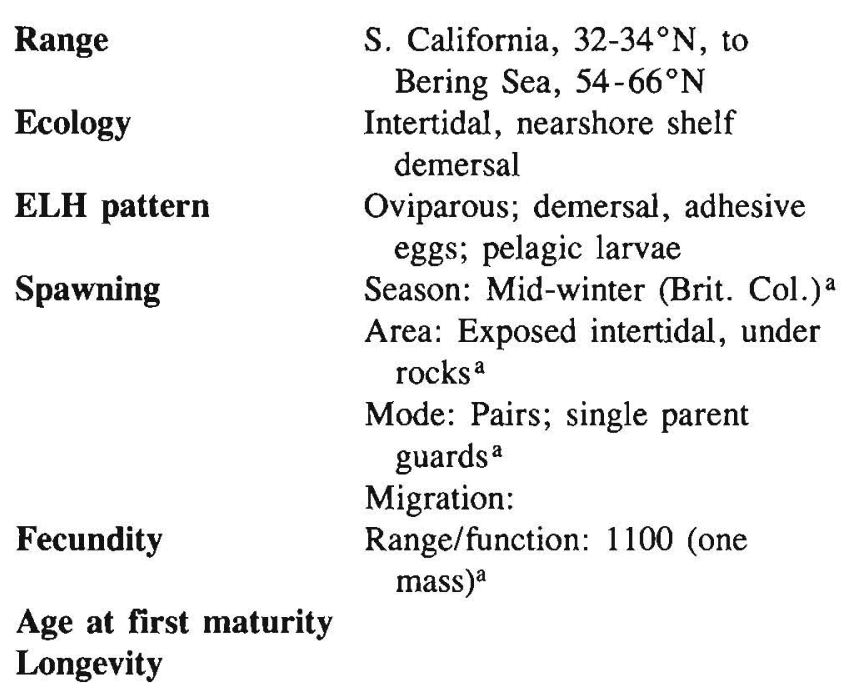

J. Marliave, Vancouver Public Aquarium, P.O. Box 3232, Vancouver, B.C., Canada V6B $3 \times 8$, pers, commun., $16 \mathrm{Oct}$. 1986. Drawings by C. Hui.

Ref: Marliave, unpubl.

\section{EARLY LIFE HISTORY DESCRIPTION ${ }^{\mathrm{a}}$}

\section{EGGS}

Diameter

$2.25 \mathrm{~mm}$

No. of oil globules

Oil globule diameter

Yolk

Envelope

White

Hatch size

$11.2 \mathrm{~mm} \mathrm{SL}$

Pigment

Diagnostic characters

- Eggs laid in conical-shaped mass

\section{LARVAE}

Preanal length

Length at flexion

$45 \% \mathrm{SL}$

Length at transformation

Sequence of fin development

$13 \mathrm{~mm} \mathrm{SL}$

18-21 mm SL

Caudal, dorsal and anal, pectorals

Pigment

- Postanal ventral midline with about 30 spots

- Dorsally about eight spots on gut; ventral pigment present (not shown on figure)

- Row of internal pigment over notochord

Diagnostic characters

Distinguished from Xiphister spp. (p. 516, 518) by

- Relatively larger at comparable stages of development (e.g., hatching occurs $>10.0 \mathrm{~mm} \mathrm{SL}$ )

- Larger relative eye diameter

- Dorsal body midline unpigmented in preflexion and flexion larvae

Distinguished from Anoplarchus purpurescens (p. 512) by

- Lack of hypural spots in preflexion and flexion larvae 

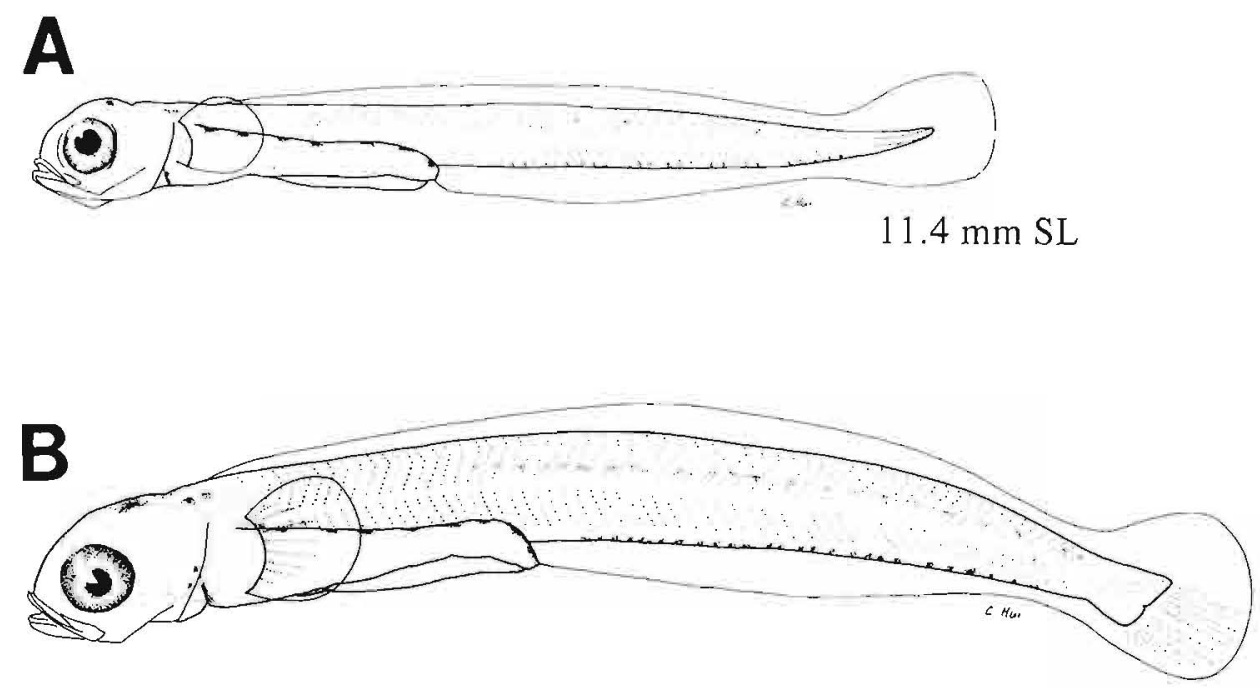

$13.2 \mathrm{~mm} \mathrm{SL}$

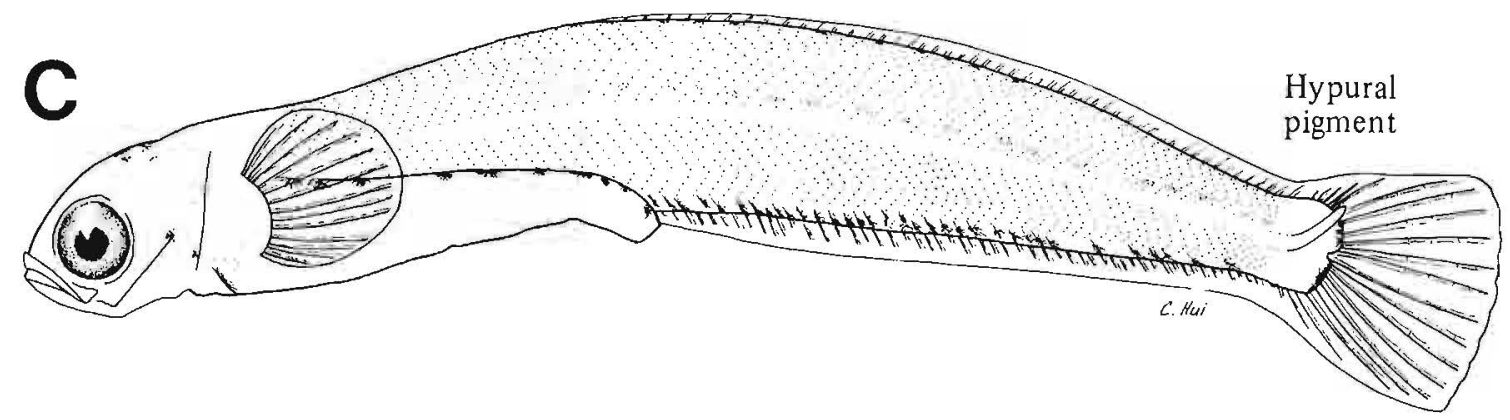

$15.7 \mathrm{~mm} \mathrm{SL}$ 


\section{MERISTICS}

$\begin{array}{lll}\text { Vertebrae } & \text { Total: } 75-75-80 \\ & \text { Precaudal: } 22-23-24 \\ & \text { Caudal: } 51-52-56 \\ \text { Branchiostegal rays } & 6-6-6 & \\ \text { Caudal fin } & \text { X, } 6+7, X & \\ \text { Pelvic fin } & \text { Absent } & \\ \text { Dorsal fin } & \text { S: } 65-X-72 & \\ \text { Pectoral fin } & \text { R: } 11-X-12 & \\ \text { Anal fin } & \text { S: } 1-1-1 & \text { R: } 49-X-55 \\ \text { Gill rakers } & \text { U: } 2-X-3 & \text { L: } 6-X-10\end{array}$

\section{LIFE HISTORY}

$\begin{array}{ll}\text { Range } & \begin{array}{c}\text { South of southern California to } \\ \text { Bering Sea, } 54-66^{\circ} \mathrm{N}\end{array} \\ \text { Ecology } & \text { Nearshore shelf demersal, } \\ & \text { intertidal to } 8 \mathrm{~m} \\ \text { Oviparous; demersal, adhesive } & \text { eggs; pelagic larvae } \\ \text { ELH pattern } & \text { Season: Late winter-early spring } \\ \text { Spawning } & \text { (British Columbia) } \\ & \text { Area: Intertidal, under rocks } \\ & \text { Progression: Early in protected } \\ & \text { waters, later on exposed } \\ & \text { shores } \\ & \text { Mode: Pairs; males guard nests } \\ & \text { Migration: } \\ & \text { Range/function: } 900-1700^{\mathrm{b}} \\ \text { Fecundity } & 2-3 \mathrm{yr}^{\mathrm{c}} \\ \text { Age at first maturity } \\ \text { Longevity }\end{array}$

\footnotetext{
a Marliave 1975b

${ }^{b}$ Marliave and DeMartini 1977

c Wingert 1974

dJ. Marliave, Vancouver Public Aquarium, P.O. Box 3232, Vancouver, B.C., Canada

V6B $3 \times 8$, pers, commun., 16 Oct. 1986. Drawings by C. Hui.

Ref: Marliave, unpubl.
}

\section{EARLY LIFE HISTORY DESCRIPTION ${ }^{d}$}

\section{EGGS}

Diameter

No. of oil globules

Oil globule diameter

Yolk

Envelope White

Hatch size $\quad 8.5 \mathrm{~mm} \mathrm{SL}$

Incubation time/temp.

Pigment

Diagnostic characters

\section{LARVAE}

$\begin{array}{ll}\text { Preanal length } & 43-45 \% \text { SL } \\ \text { Length at flexion } & 11 \mathrm{~mm} \mathrm{SL} \\ \text { Length at transformation } & 18 \mathrm{~mm} \mathrm{SL} \\ \begin{array}{c}\text { Sequence of fin } \\ \text { development }\end{array} & \begin{array}{c}\text { Caudal, anal and dorsal, } \\ \text { pectorals }\end{array}\end{array}$

\section{Pigment}

- Postanal ventral midline with about 36 spots (range 28-46)

- Dorsally about eight spots on gut; ventral pigment present (not shown on figure)

- Row of internal pigment over notochord

Diagnostic characters

- See Anoplarchus purpurescens (p. 512) and Phytichthys chirus (p. 514)

Distinguished from $X$. mucosus by

- Relatively smaller at comparable stages of development

- Internal pigment over notochord generally more prominent

- Relatively short snout-anus length (preanal myomeres $23-25$ vs. $28-30$ in $X$. mucosus) 

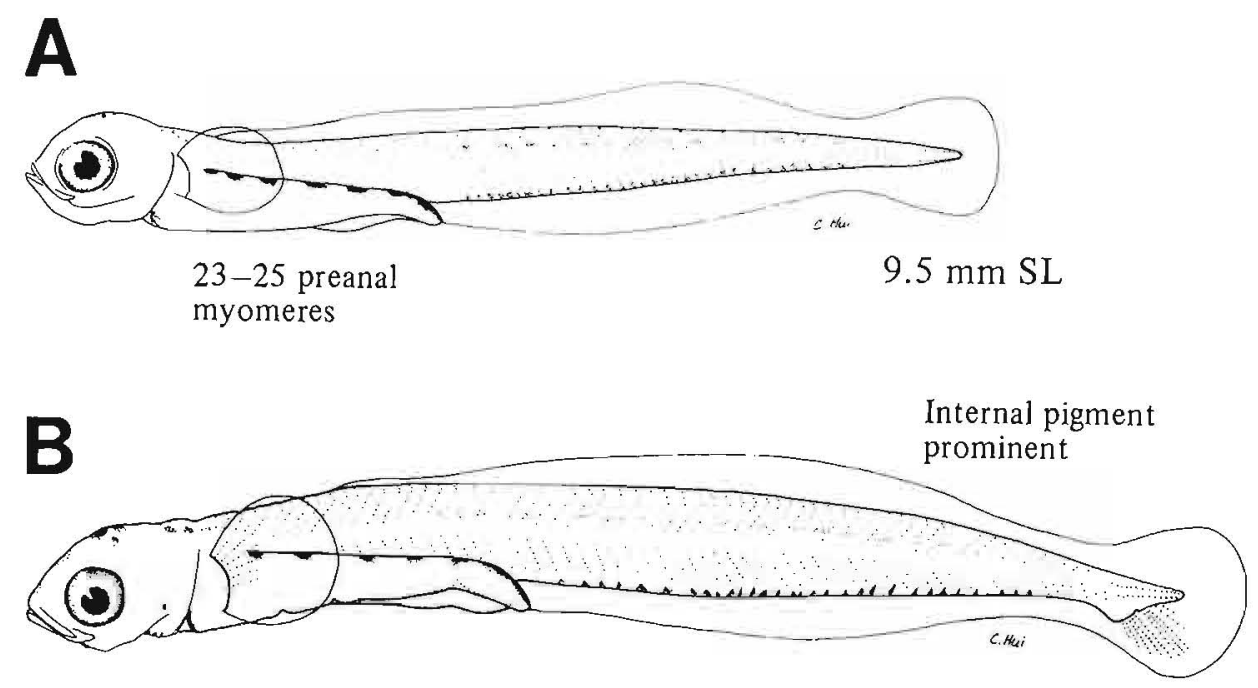

$12.5 \mathrm{~mm} \mathrm{SL}$

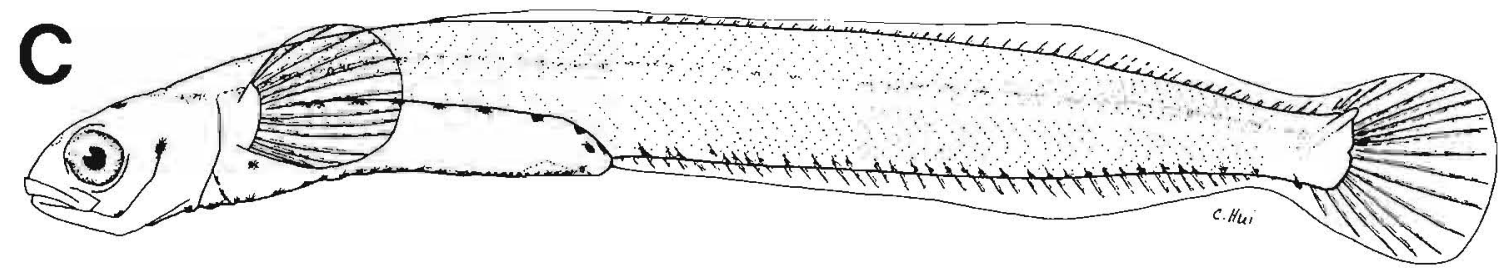

$18.8 \mathrm{~mm} \mathrm{SL}$

Figures A-C, Marliave, unpubl. 


\section{MERISTICS}

\begin{tabular}{|c|c|}
\hline Vertebrae & $\begin{array}{l}\text { Total: } 73-81-83 \\
\text { Precaudal: } 29-30-31 \\
\text { Caudal: } 44-50-53\end{array}$ \\
\hline Branchiostegal rays & $6-6-6$ \\
\hline Caudal fin & $x, 6+7, x$ \\
\hline Pelvic fin & Absent \\
\hline Dorsal fin & S: $71-X-78$ \\
\hline Pectoral fin & $\mathrm{R}: 12-12-12$ \\
\hline Anal fin & $\mathrm{R}: 46-\mathrm{X}-50$ \\
\hline Gill rakers & $\mathrm{U}: \mathrm{X}-\mathrm{X}-\mathrm{X} \quad \mathrm{L}: \mathrm{X}-\mathrm{X}-\mathrm{X}$ \\
\hline \multicolumn{2}{|l|}{ LIFE HISTORY } \\
\hline Range & $\begin{array}{l}\text { Cent. California, } 34-38^{\circ} \mathrm{N} \text {, to } \\
\text { SE Alaska, } 55-59^{\circ} \mathrm{N}\end{array}$ \\
\hline Ecology & $\begin{array}{l}\text { Nearshore shelf demersal, } \\
\text { intertidal to } 18 \mathrm{~m}\end{array}$ \\
\hline ELH pattern & $\begin{array}{l}\text { Oviparous; demersal, adhesive } \\
\text { eggs; pelagic larvae }\end{array}$ \\
\hline Spawning & $\begin{array}{l}\text { Season: Late winter-spring } \\
(\text { British Columbia })^{\mathrm{a}}\end{array}$ \\
\hline & \multirow{2}{*}{$\begin{array}{l}\text { Area: Exposed intertidal, under } \\
\text { rocks }{ }^{\mathrm{a}} \\
\text { Mode: Pairs; males guard nests } \\
\text { Migration: }\end{array}$} \\
\hline & \\
\hline Fecundity & Range/function: $5500-9500^{\mathrm{a}}$ \\
\hline $\begin{array}{l}\text { Age at first maturity } \\
\text { Longevity }\end{array}$ & $5 \mathrm{yr}^{\mathrm{c}}$ \\
\hline
\end{tabular}

\section{EARLY LIFE HISTORY DESCRIPTION ${ }^{\mathrm{d}}$}

\section{EGGS}

Diameter

No. of oil globules

Oil globule diameter

Yolk

Envelope White

Hatch size $\quad 9.5 \mathrm{~mm}$ SL

Incubation time/temp.

Pigment

Diagnostic characters

\section{LARVAE}

Preanal length $\quad 47-49 \% \mathrm{SL}$

Length at flexion $\quad 12 \mathrm{~mm} \mathrm{SL}$

Length at transformation $18 \mathrm{~mm} \mathrm{SL}$

Sequence of fin Caudal, dorsal and anal, development pectorals

Pigment

- Postanal ventral midline about 26 spots (range 18-35)

- Dorsally about eight spots on gut; ventral pigment present (not shown on figure)

- Row of internal pigment over notochord

\section{Diagnostic characters}

- See Anoplarchus purpurescens (p. 512) and Phytichthys chirus (p. 514)

Distinguished from $X$. atropurpureus by

- Generally larger at comparable stages of development

- Internal pigment over notochord generally lighter

\footnotetext{
${ }^{a}$ Marliave 1975a

${ }^{\mathrm{b}}$ Marliave and DeMartini 1977

cWingert 1974

dJ. Marliave, Vancouver Public Aquarium, P.O. Box 3232, Vancouver, B.C., Canada V6B 3X8, pers. commun., 16 Oct. 1986. Drawings by C. Hui.

Ref: Marliave 1975a; Marliave, unpubl.
} 

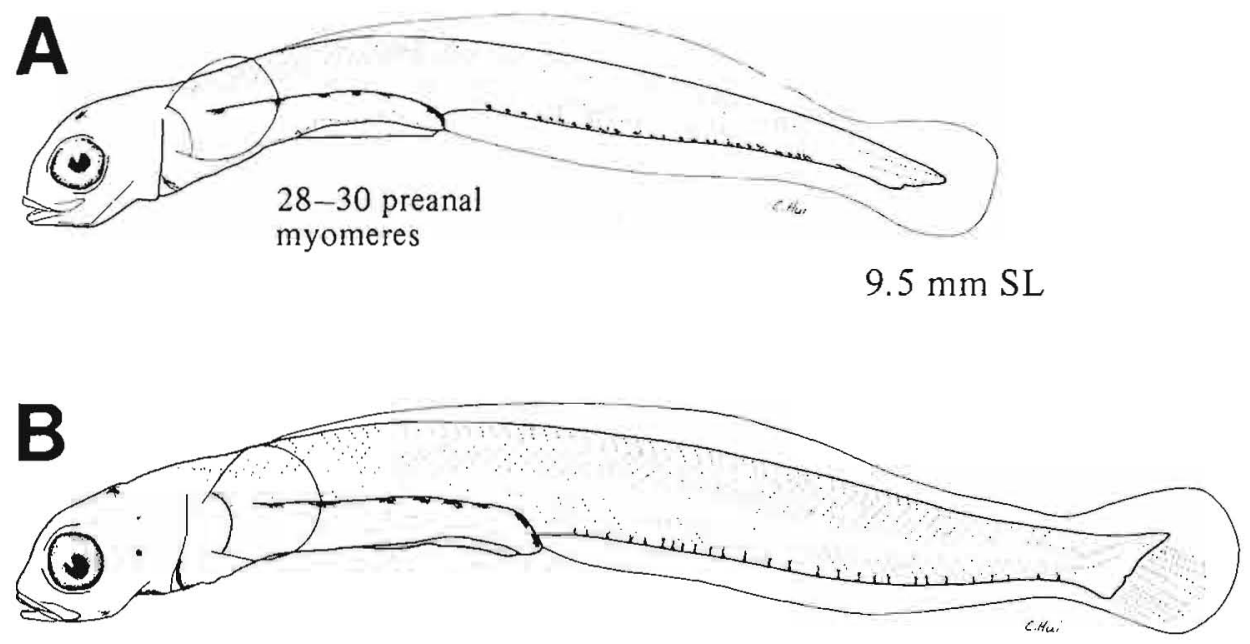

$12.7 \mathrm{~mm} \mathrm{SL}$

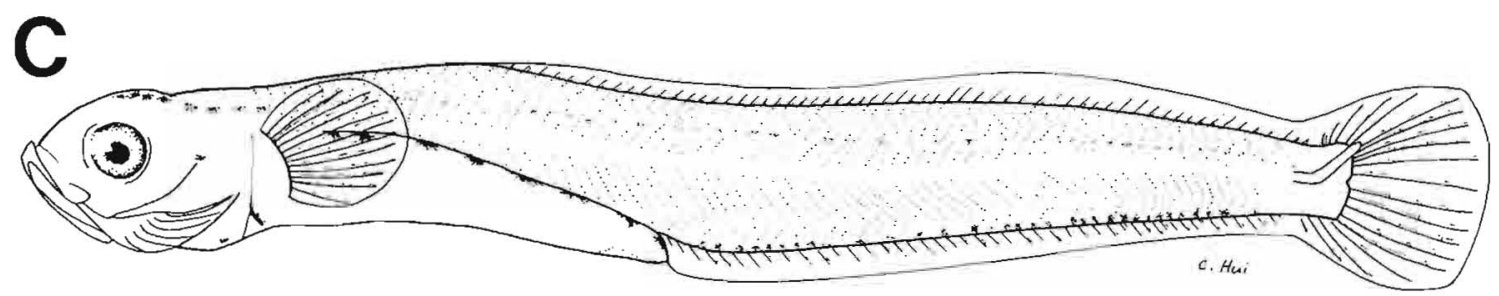

$17.3 \mathrm{~mm} \mathrm{SL}$

Figures $\mathrm{A}-\mathrm{C}$, Marliave, unpubl. 
MERISTICS Delolepis gigantea (Kittlitz 1858)

Vertebrae

Total: $81-\mathrm{X}-85$

Precaudal: $\mathrm{X}-\mathrm{X}-\mathrm{X}$

Caudal: $49-X-51$

Branchiostegal rays 6-6-6

Caudal fin

Pelvic fin

Dorsal fin

Pectoral fin

Anal fin

Gill rakers
$\mathrm{X}, 7+8, \mathrm{X}$

Absent

S: $73-X-77$

R: $13-13-13$

S: $2-2-2$

$\mathrm{U}: \mathrm{X}-\mathrm{X}-\mathrm{X}$

R: $43-X-49$

L: X-X-X

MERISTICS Lyconectes aleutensis Gilbert 1896

Vertebrae

Caudal fin

Pelvic fin

Dorsal fin

Pectoral fin

Anal fin

Gill rakers
Total: $71-\mathrm{X}-77$

Precaudal: $\mathrm{X}-\mathrm{X}-\mathrm{X}$

Caudal: $47-X-51$

6-6-6

$\mathrm{X}, 7+8, \mathrm{X}$

Absent

S: $60-\mathrm{X}-69$

R: $12-\mathrm{X}-13$

S: $2-X-3$

R: $45-X-49$

$\mathrm{U}: \mathrm{X}-\mathrm{X}-\mathrm{X}$

L: X-X-X

\section{LIFE HISTORY}

$\begin{array}{ll}\text { Range } & \text { N. California, } 38-42^{\circ} \mathrm{N} \text {, to } \\ \text { Bering Sea, } 54-66^{\circ} \mathrm{N} \\ \text { Ecology } & \text { Nearshore shelf demersal } \\ & (\text { D. gigantea, } 6-128 \mathrm{~m}) \\ & \text { epi- and mesobenthal } \\ & (\text { L. aleutensis, } 46-350 \mathrm{~m}) \\ \text { Oviparous, demersal eggs, } & \text { pelagic larvae } \\ \text { ELH pattern } & \text { Season: Spring-summer } \\ \text { Spawning } & (L . \text { aleutensis })^{\mathrm{b}} \\ & \text { Area: } \\ & \text { Mode: } \\ & \text { Migration: } \\ \text { Fecundity } & \text { Range/function: } \\ \text { Age at first maturity } & \\ \text { Longevity } & \end{array}$

a Nawojchik (1986) includes Lyconectes and Delolepis in the genus Cryptacanthodes.

${ }^{\mathrm{b}} \mathrm{Han} 1973$

Ref: Hart 1973.

\section{EARLY LIFE HISTORY DESCRIPTION}

\section{EGGS}

Diameter

4.41-4.85 mm (D. gigantea); $1.8 \mathrm{~mm}$ (L. aleutensis)

No. of oil globules

Oil globule diameter

Yolk

Envelope

Hatch size

Smooth (D. gigantea)

Larvae with yolk at 16-17 mm SL

(D. gigantea)

Incubation time/temp.

Pigment

Diagnostic characters

\section{LARVAE}

Preanal length

Length at flexion

Length at transformation

Sequence of fin

development

$\sim 50 \%$ SL $(D$. gigantea $)$
$\quad<50 \%$ SL $($ L. aleutensis $)$

Pigment

Caudal, pectorals, dorsal and anal; fin development may begin prior to hatching in $D$. gigantea

Diagnostic characters (see Table 3)

- Number of myomeres: 81-85 in D. gigantea, 71-77 in L. aleutensis

- Pigment on isthmus and over gut (absent in D. gigantea)

- Preanal length: $\sim 50 \%$ SL in D. gigantea, $<50 \%$ SL in L. aleutensis

- Size at stage of development: $D$. gigantea larvae hatch at a larger size and more advanced stage of development 


\section{A Delolepis gigantea}

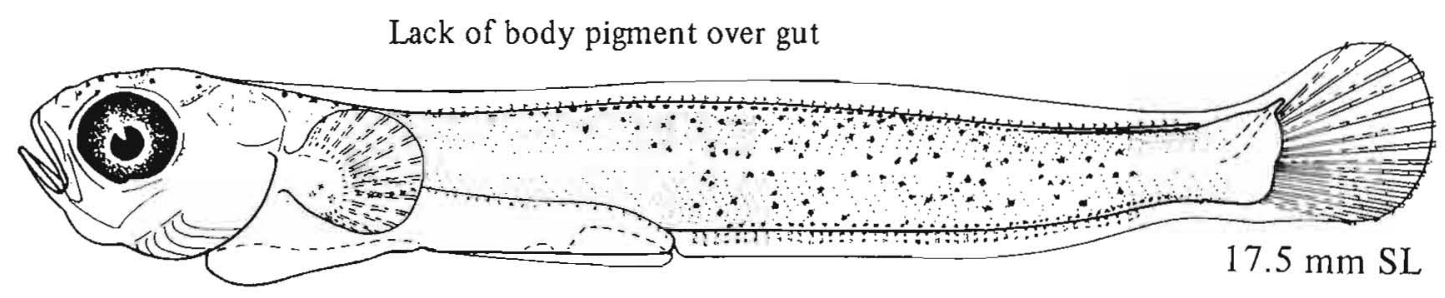

Remnant of yolk

\section{B Lyconectes aleutensis}

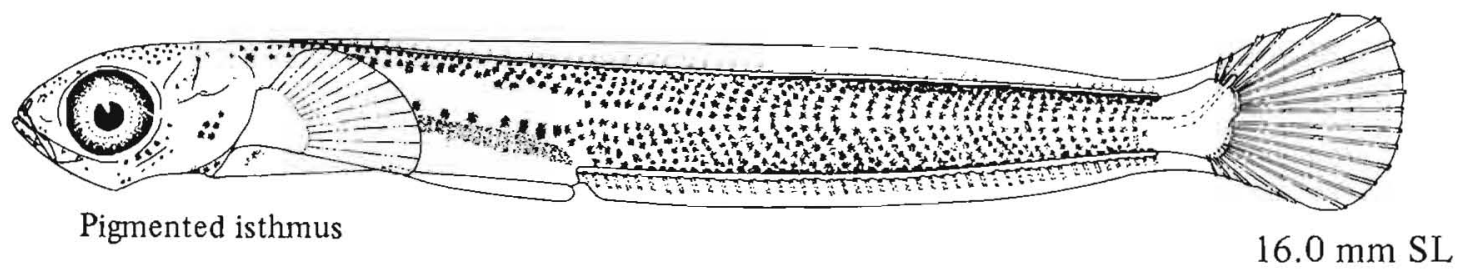

Figures A-B, NWAFC originals (B. Vinter). 
Although some species of gunnels are found in the North Atlantic, most members of this family are located in the eastern Pacific. Pholidids are eel-like with long compressed bodies, a dorsal fin extending from head to caudal fin and made up entirely of spines, and small pelvic fins $(I, 1)$ when present. There are nine species in three genera within the study area. ${ }^{1}$ Adults are demersal, occurring from as deep as $75 \mathrm{~m}$ to tidepools (Hart 1973). Pholidids are noted for paired spawning and subsequent guarding of eggs which may be 1.4-3.0 mm and have one oil globule (A.C. Matarese, unpubl.). One or both partners may guard the eggs during incubation by coiling around them (Breder and Rosen 1966). Larvae are pelagic and may settle after 50 days (Garrison and Miller 1982).

Presently, larvae of Pholis spp. cannot be identified to species in our study area. The following characters may be helpful in separating Pholis spp. larvae from those of Apodichthys flavidus.

Morphology Head and eye generally smaller in Pholis at comparable stages of development

Meristics Presence of pelvic fins and two anal spines in Pholis

Pigment Internal pigment above notochord is less pronounced and disappears sometime before larvae undergo flexion; presence of a continuous series of melanophores along ventral surface of gut; pigment spots along dorsal surface of gut are smaller and more numerous (postflexion)

Meristic characters may be useful in separating larger Pholis postflexion larvae and juveniles. Off Washington, Oregon, and California, myomere counts enable separation of $P$. clemensi (94-98) and $P$. schultzi (89-93) from other species (usually <90).

Larvae of Xererpes fucorum are inadequately known. X. fucorum can be separated from Pholis spp. by their lack of pelvic fins and from Apodichthys flavidus by the presence of one or two anal spines and a lower dorsal spine count (83-87 vs. 90-94).

${ }^{1}$ Yatsu (1985) proposes alternative generic placements for several species; according to his arrangement, nine species within four genera occur in the area.

Table 45

Meristic characters of family Pholididae.*

\begin{tabular}{|c|c|c|c|c|c|c|c|c|c|c|}
\hline \multirow[b]{3}{*}{ Taxon } & \multirow[b]{3}{*}{ Distribution } & \multicolumn{2}{|c|}{ Vertebrae } & \multirow{2}{*}{\multicolumn{4}{|c|}{ Fins }} & \multirow{2}{*}{\multicolumn{2}{|c|}{ Gill rakers }} & \multirow[b]{3}{*}{ Branchiostegals } \\
\hline & & Preoudel & Coudal & & & & & & & \\
\hline & & \multicolumn{2}{|c|}{ (Total) } & Dorsal & Anal & Pectoral & Pelvic & Upper & Lower & \\
\hline Apodichthys flavidus & S. Calif.-Gulf of Alaska & $50-51$ & 48 & $\mathrm{XC}-\mathrm{XCIV}$ & I, $36-42$ & 14 & & 3 & $10-13$ & 5 \\
\hline Pholis clemensi & N. Calif.-SE Alaska & $37-39$ & $57-59$ & LXXXVII-XCI & II, $48-53$ & $11-14$ & 1,1 & & 11 & 5 \\
\hline Pholis dolichogaster & Aleutian Is.-Bering Sea & $41-45$ & $56-57$ & XCIII-XCVI & $\mathrm{II}, 48-51$ & $13-15$ & $\mathrm{I}, 1$ & & & 5 \\
\hline Pholis fasciata & Bering Sea & $40-44$ & 50 & LXXXIU-LXXXVIII & II,41-44 & $11-13$ & $\mathrm{I}, 1$ & & & 5 \\
\hline Pholis gilli & Bering Sea & & & LXXXIV & II, 43 & & $\mathrm{I}, 1$ & & & 5 \\
\hline Pholis laeta & N. Calif.-Bering Sea & $\begin{array}{l}40-42 \\
\quad(81-8\end{array}$ & 9) $43-44$ & LXXIV-LXXXI & II, 32-38 & $11-12$ & $\mathrm{I}, \mathrm{I}$ & & & 5 \\
\hline Pholis ormata & Cent. Calif.-Brit. Col. & $(80-8$ & & LXXIV-LXXX & I, 34-38 & $11-12$ & $\mathrm{I}, \mathrm{I}$ & & & 5 \\
\hline Pholis schultzi & Cent. Calif.-Brit. Col. & $(89-9$ & & LXXX-LXXXIX & II, $40-44$ & $10-12$ & $1,1-2$ & $1-2$ & $7-10$ & \\
\hline Xererpes fucorum & SSC-Brit. Col. & 52 & 40 & LXXXII-LXXXVII & $1,29-38$ & 12 & & $1-2$ & $6-9$ & 5 \\
\hline
\end{tabular}

*Yatsu (1985) places Pholis clemensi, P. laeta, and P. schultzi in the genus Allopholis. P. dolichogaster is placed in the genus Rhodymenichthys. Xererpes fucorum is placed in the genus Apodichthys. 


\section{Apodichthys flavidus}

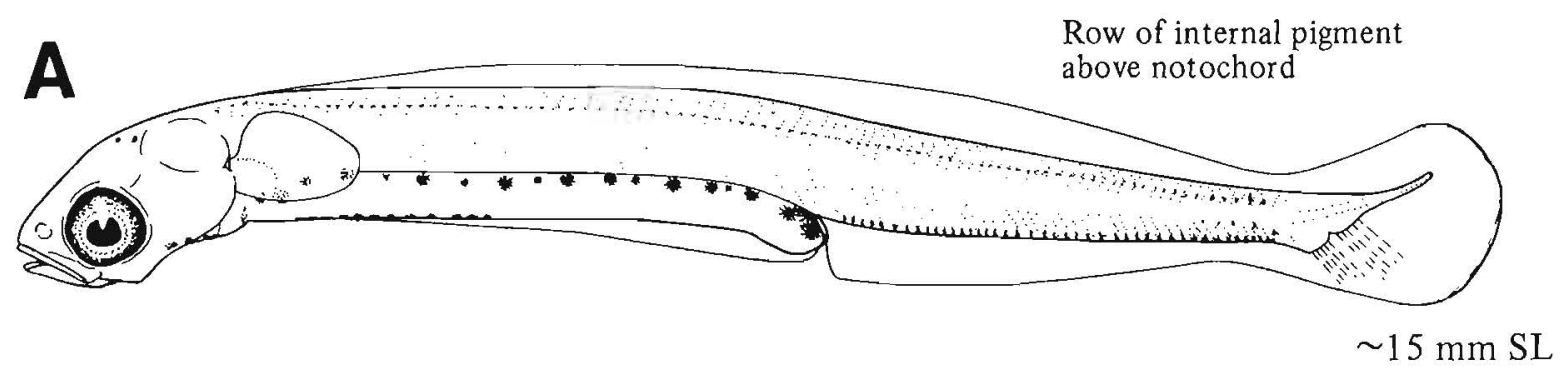

\section{Pholis sp.}

B

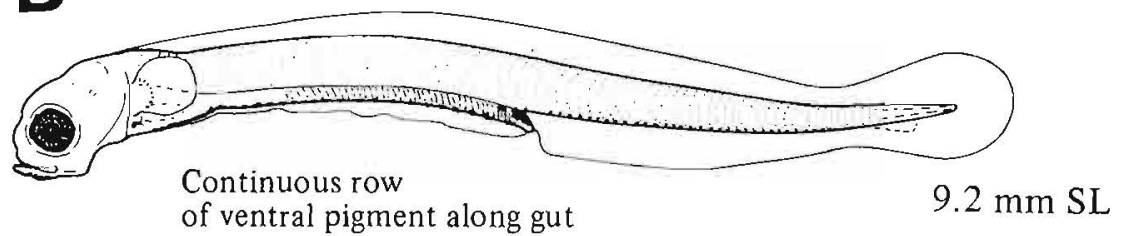

C

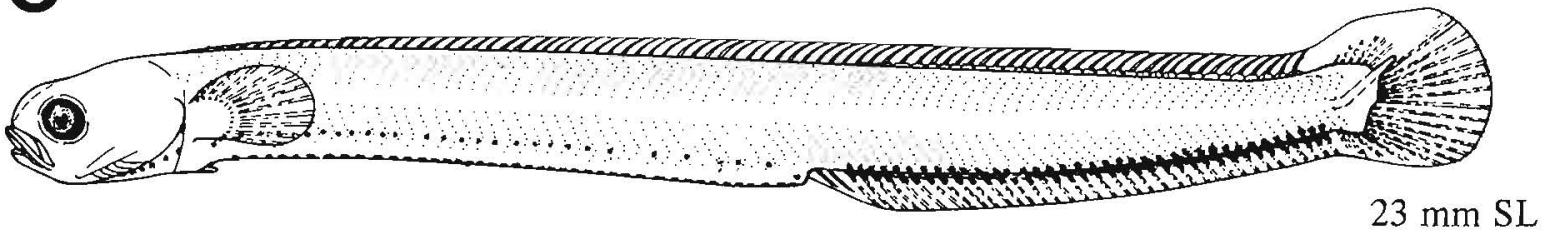

Figures A-C, NWAFC originals (B, Vinter). 


\section{MERISTICS}

Vertebrae

Caudal fin

Pelvic fin

Dorsal fin

Pectoral fin

Anal fin

Gill rakers
Total: $88-\mathrm{X}-89$

Precaudal: 29-X-31

Caudal: 57-X-59

6-X-7

Absent

S: $81-\mathrm{X}-88$

R: $20-X-22$

S: $X-X-X$

$\mathrm{U}: \mathrm{X}-\mathrm{X}-\mathrm{X}$

$\mathrm{R}: 50-\mathrm{X}-55$

L: X-X-X

\section{EARLY LIFE HISTORY DESCRIPTION}

\section{EGGS}

Diameter

No. of oil globules

4-8 mm

Oil globule diameter

Yolk

Envelope

Hatch size

One

Incubation time/temp.

Pigment

Diagnostic characters

\section{LARVAE}

Preanal length $\quad 50 \%$ SL

Length at flexion $\quad<20 \mathrm{~mm} \mathrm{SL}$

Length at transformation $\sim 40 \mathrm{~mm} \mathrm{SL}$

Sequence of fin development

Pigment (see Table 3)

- Heavily pigmented over body except ventral surface of gut, pectoral fin base, and posteriormost opercular area

Diagnostic characters

Distinguished from Anarrhichthys ocellatus by

- Morphology: Body not elongate

- Lower vertebral count (88-89 myomeres)

- Eye diameter (large)

\footnotetext{
${ }^{\mathrm{a}}$ Breder and Rosen 1966
}

Ref: Andriashev 1954, Barsukov 1959. 


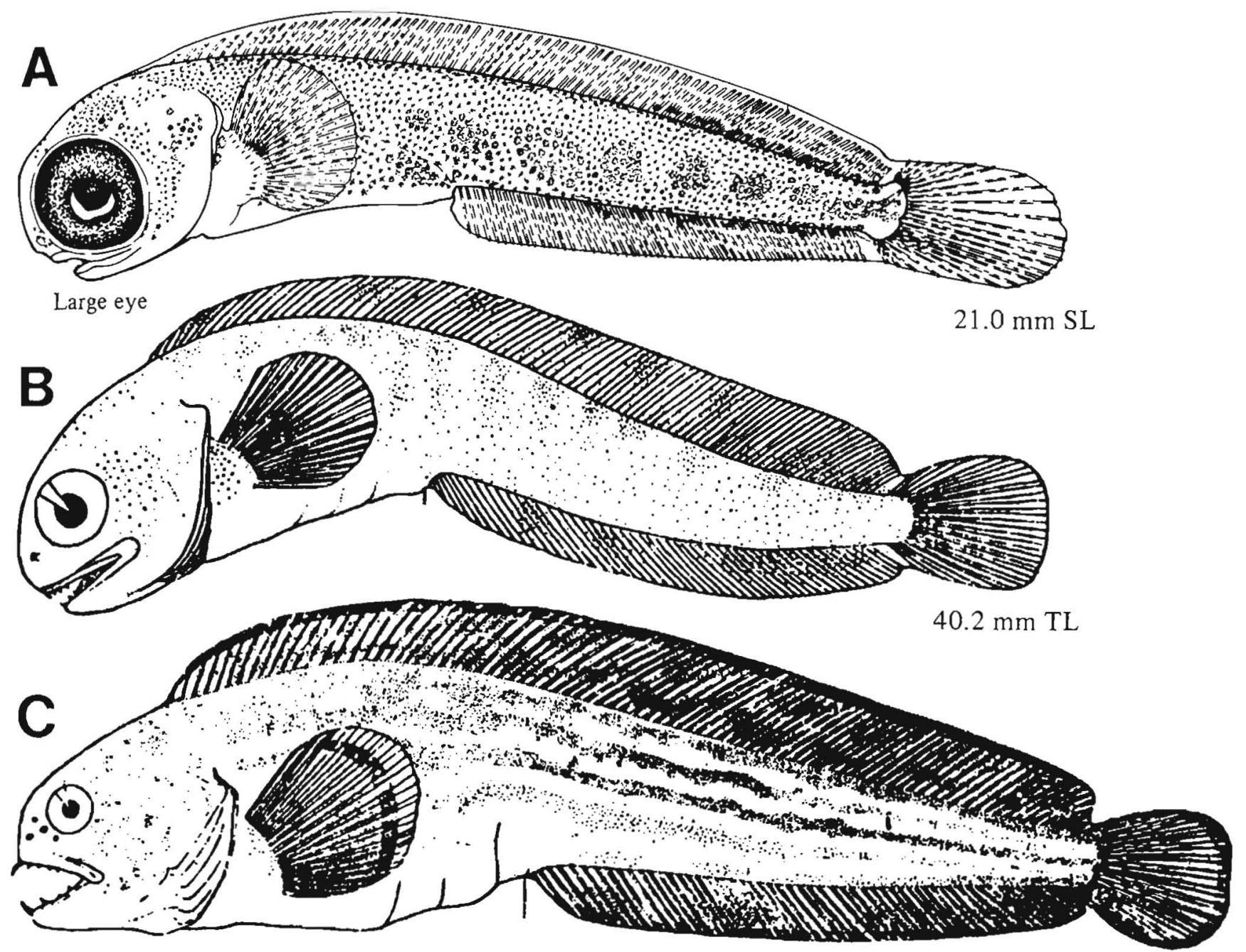

Figure A, NWAFC original (B. Vinter); B-C, Kobayashi 1961a.

$116.2 \mathrm{~mm} \mathrm{TL}$ 


\section{MERISTICS}

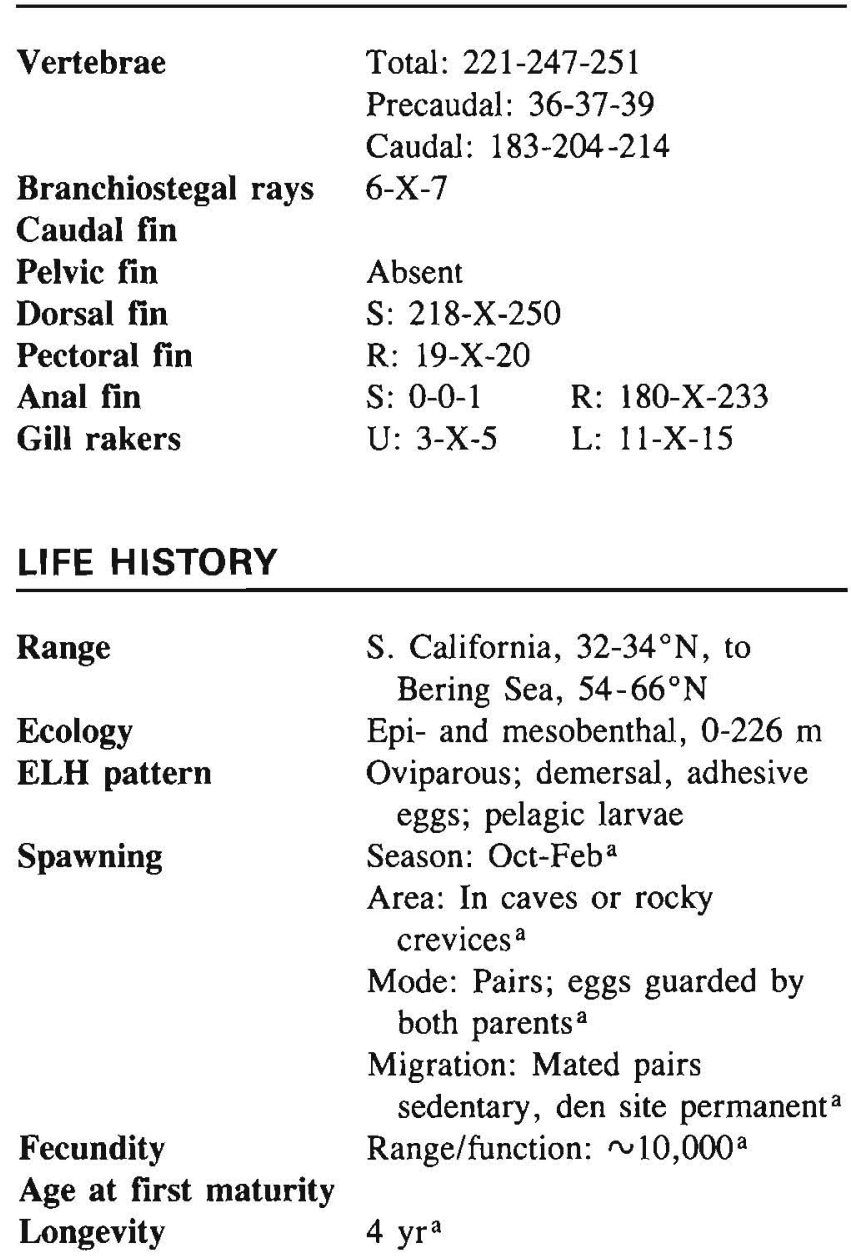

\section{EARLY LIFE HISTORY DESCRIPTION}

\section{EGGS}

Diameter

No. of oil globules

Oil globule diameter

Yolk

Envelope

Hatch size

$5.2-5.4 \mathrm{~mm}$

Reddish

Smooth; white, becoming brownish

$33.5 \mathrm{~mm} \mathrm{SL}$

Incubation time/temp. $\quad 3-4.5 \mathrm{mo}$

Pigment

\section{Diagnostic characters}

- Embyro coils around 3-3.5 times within envelope

LARVAE

Preanal length
$25 \% \mathrm{SL}$ at hatching, decreasing with develop- ment

Length at flexion

Tail fully formed at hatching

Length at transformation Hatch as juveniles

Sequence of fin development

Fins fully formed at hatching with yolk present

Pigment

- Dorsal head

- Upper body over gut

- Small concentrated melanophores along dorsal and ventral body midlines that extend onto the body laterally and into the finfolds with development

Diagnostic characters

- Elongate body shape

- High number of myomeres (221-251)

${ }^{a}$ Marliave 1987

Ref: Marliave 1975a, 1987. 


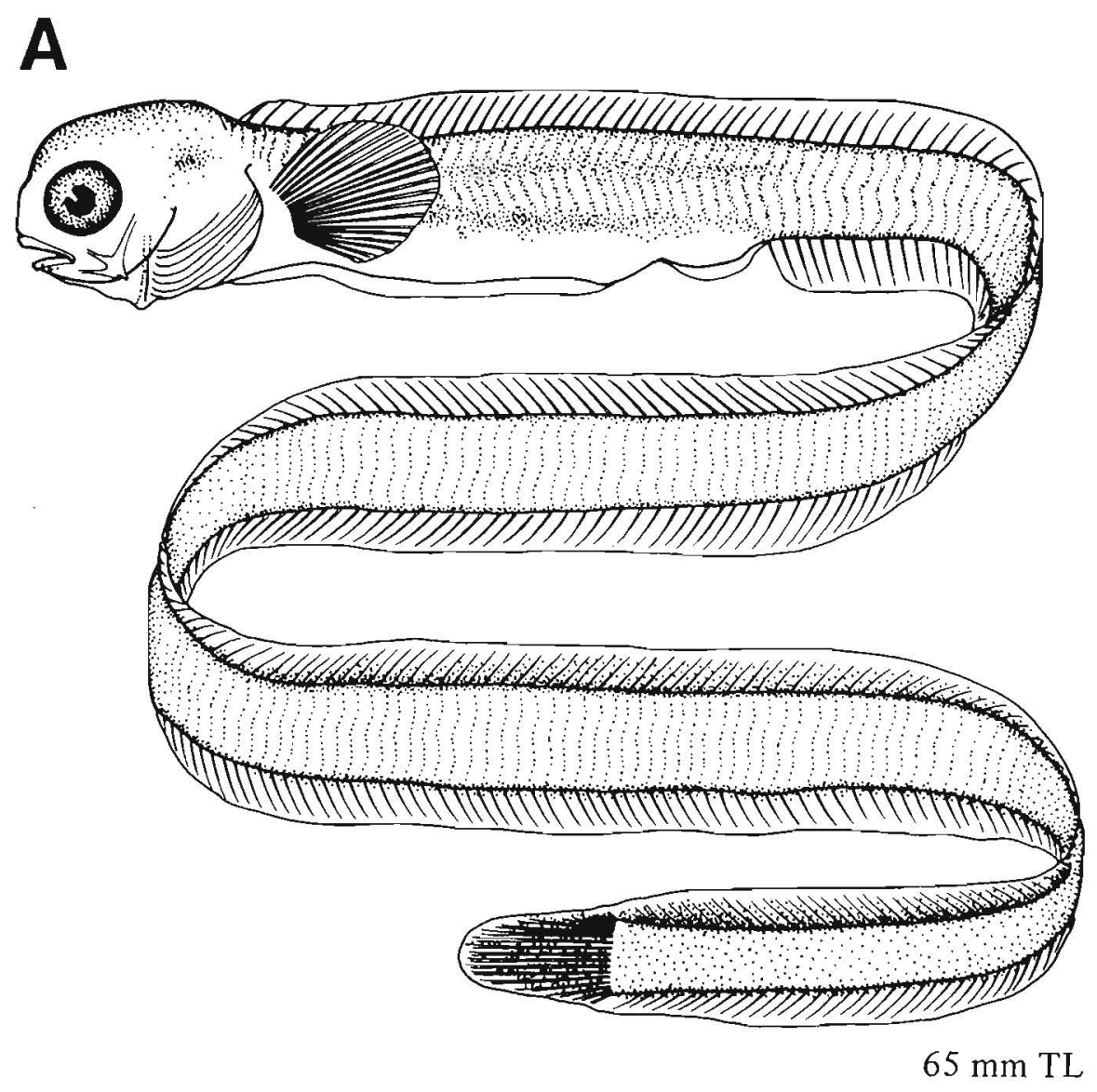

Figure A, Marliave 1975a. 
MERISTICS ${ }^{a}$

\begin{tabular}{|c|c|c|}
\hline \multirow{3}{*}{ Vertebrae } & \multicolumn{2}{|c|}{ Total: $227-\mathrm{X}-240$} \\
\hline & \multicolumn{2}{|c|}{ Precaudal: $53-X-59$} \\
\hline & \multicolumn{2}{|c|}{ Caudal: $174-X-181$} \\
\hline Branchiostegal rays & \multirow{2}{*}{\multicolumn{2}{|c|}{$5-5-5$}} \\
\hline Caudal fin & & \\
\hline Pelvic fin & Absent & \\
\hline Dorsal fin & S: $83-X-90$ & $\mathrm{R}: 137-\mathrm{X}-145$ \\
\hline Pectoral fin & R: $13-13-13$ & \\
\hline Anal fin & R: $180-X-196$ & \\
\hline Gill rake & $\mathrm{U}: \mathrm{X}-\mathrm{X}-\mathrm{X}$ & L: X-X-X \\
\hline
\end{tabular}

LIFE HISTORY

$\begin{array}{ll}\text { Range } & \begin{array}{c}\text { Oregon, } 42-46^{\circ} \mathrm{N}, \text { to } \\ \text { Bering Sea, } 54-66^{\circ} \mathrm{N}\end{array} \\ \text { Ecology } & \text { Nearshore shelf demersal, 0-80 m } \\ \text { ELH pattern } & \begin{array}{c}\text { Parity and eggs unknown, } \\ \text { pelagic larvae }\end{array} \\ \text { Spawning } & \text { Season: Spring } \\ & \text { Area: } \\ & \text { Mode: } \\ & \text { Migration: } \\ \text { Fecundity } & \text { Range/function: } \\ \text { Age at first maturity } & \\ \text { Longevity } & \end{array}$

\footnotetext{
${ }^{a}$ Kobayashi (1961b) reports slightly different meristics in specimens from Japanese waters:

Dorsal fin spines $\quad 79-83$

Dorsal fin rays 141-157

Anal fin rays $166-193$

Pectoral fin rays 11-13

Branchiostegal rays 6

Richardson and Dehart (1975) note that in four larval specimens collected off Oregon the total vertebral counts of 227 were considerably lower than counts in Bering Sea specimens (236-240).

'Larvae 20.3-36.0 mm SL collected March-May $18 \mathrm{~km}$ from coast of Oregon.
}

Ref: Richardson and Dehart 1975.

\section{EARLY LIFE HISTORY DESCRIPTION}

\section{EGGS}

Diameter

No. of oil globules

Oil globule diameter

Yolk

Envelope

Hatch size

Incubation time/temp.

Pigment

Diagnostic characters

\section{LARVAE}

Preanal length

Length at flexion

Length at transformation $\sim 114 \mathrm{~mm} \mathrm{SL}$

Sequence of fin development

Pigment (larvae 20.3-36.0 mm SL)

- Head

-Lower jaw

-Isthmus

-Internally at base of hindbrain

- Gut: Dorsal and ventral surface

- Body: Concentrated dorsally and ventrally

- Caudal: "Fleshy caudal extension" is distinctly pigmented; pigment is scattered evenly dorsally and ventrally on body and into finfolds

\section{Diagnostic characters}

- Morphology: Elongate form, gut length (40\% SL)

- Number of myomeres (>225)

- Pigment pattern: Concentrated ventrolateral spots and pigment on fleshy caudal extension 


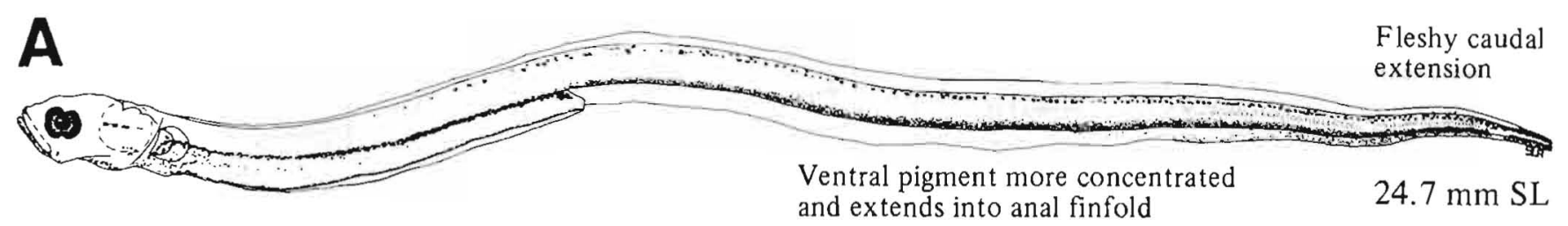

Figure A, Richardson and Dehart 1975. 


\section{MERISTICS}

\begin{tabular}{|c|c|c|}
\hline Vertebrae & $\begin{array}{l}\text { Total: } 61-\mathrm{X}- \\
\text { Precaudal: } 2 \\
\text { Caudal: X-X } \\
6-6-6\end{array}$ & $\begin{array}{l}2 \\
-X-26 \\
x\end{array}$ \\
\hline Caudal fin & & \\
\hline Pelvic fin & \multicolumn{2}{|l|}{ Absent } \\
\hline Dorsal fin & \multicolumn{2}{|l|}{ S: $54-X-57$} \\
\hline Pectoral fin & \multicolumn{2}{|l|}{$\mathrm{R}: 20-\mathrm{X}-25$} \\
\hline Anal fin & $S: 4-4-4$ & $\mathrm{R}: 24-\mathrm{X}-30$ \\
\hline Gill rakers & $\mathrm{U}: \mathbf{8 - 8 - 8}$ & L: $18-X-20$ \\
\hline \multicolumn{3}{|l|}{ LIFE HISTORY } \\
\hline Range & \multicolumn{2}{|c|}{$\begin{array}{l}\text { Cent. California, } 34-38^{\circ} \mathrm{N} \text {, to } \\
\text { Bering Sea, } 54-66^{\circ} \mathrm{N}\end{array}$} \\
\hline Ecology & \multicolumn{2}{|c|}{$\begin{array}{l}\text { Epi-, meso-, and bathybenthal, } \\
10-675 \mathrm{~m}\end{array}$} \\
\hline ELH pattern & \multicolumn{2}{|c|}{$\begin{array}{l}\text { Parity and eggs unknown, } \\
\text { pelagic larvae }\end{array}$} \\
\hline Spawning & \multicolumn{2}{|l|}{ Season: } \\
\hline & \multicolumn{2}{|l|}{ Area: } \\
\hline & \multicolumn{2}{|l|}{ Mode: } \\
\hline & \multicolumn{2}{|l|}{ Migration: } \\
\hline Fecundity & \multicolumn{2}{|c|}{ Range/function: } \\
\hline $\begin{array}{l}\text { Age at first maturity } \\
\text { Longevity }\end{array}$ & \multicolumn{2}{|l|}{ Ar (male)a } \\
\hline
\end{tabular}

\section{EARLY LIFE HISTORY DESCRIPTION}

\section{EGGS}

Diameter

No. of oil globules

Oil globule diameter

Yolk

Envelope

Hatch size

Incubation time/temp.

Pigment

Diagnostic characters

\section{LARVAE \\ Preanal length \\ Length at flexion \\ $55 \%$ SL, with development increasing to $65 \% \mathrm{SL}$ 17-24 mm BL \\ Length at transformation $\sim 30 \mathrm{~mm} \mathrm{SL}$ \\ Sequence of fin development \\ Caudal, pectorals, dorsal, anal}

Pigment

- Body, except ventral surface of gut and tip of tail, entirely pigmented with small densely concentrated melanophores throughout development

- Becomes banded in juveniles

Diagnostic characters (see Table 3)

- Small melanophores covering body

- Rounded snout

${ }^{\mathrm{a}}$ Fitch and Lavenberg 1971 

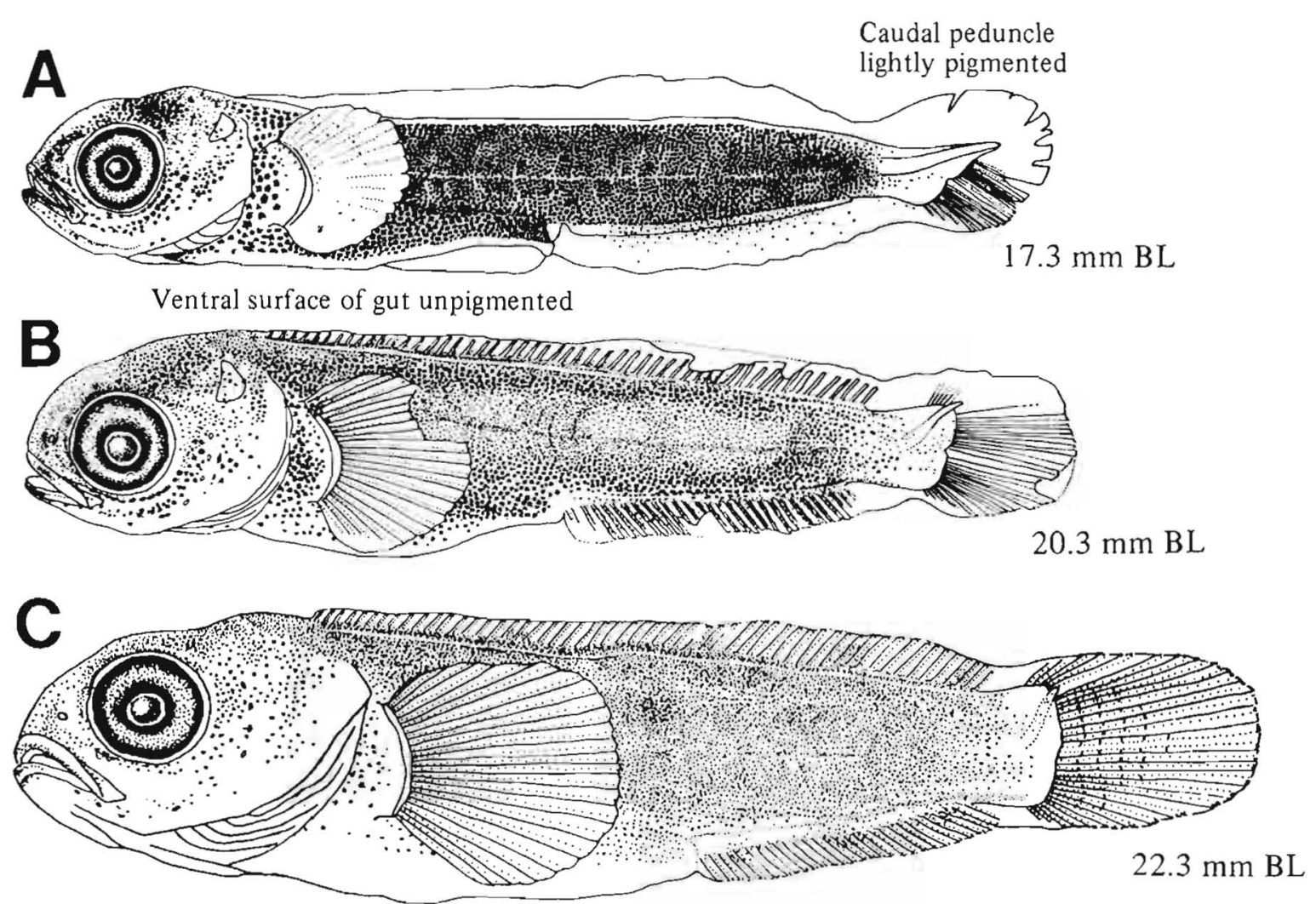

D

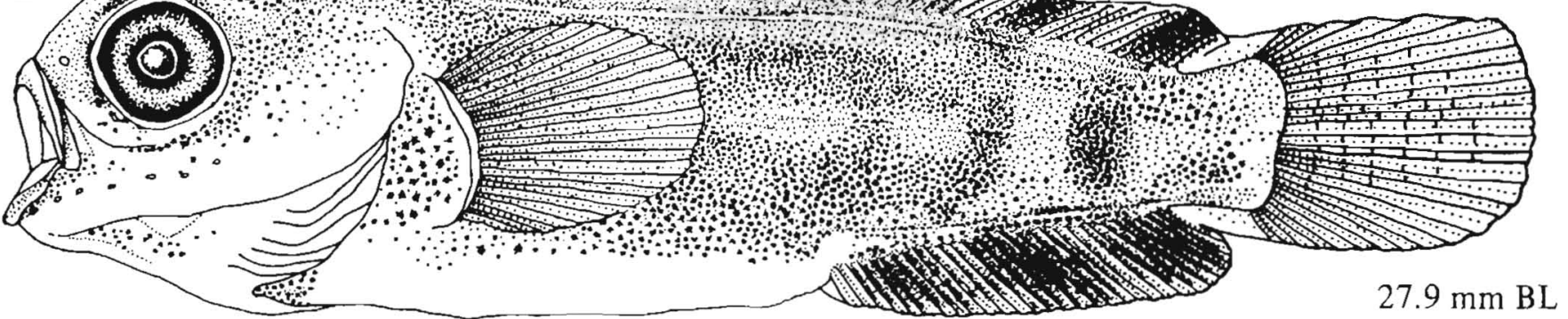

Figures A-D, Haryu and Nishiyama 1981. 


\section{MERISTICS}

\begin{tabular}{|c|c|c|}
\hline \multirow[t]{3}{*}{ Vertebrae } & \multicolumn{2}{|c|}{ Total: $44-X-47$} \\
\hline & \multicolumn{2}{|c|}{ Precaudal: $12-12-14^{a}$} \\
\hline & \multicolumn{2}{|c|}{ Caudal: $32-34-40^{a}$} \\
\hline Branchiostegal rays & \multicolumn{2}{|l|}{$6-6-6$} \\
\hline \multicolumn{3}{|l|}{ Caudal fin } \\
\hline Pelvic fin & Thoracic & \\
\hline & $S: 1-1-1$ & $\mathrm{R}: 5-5-5$ \\
\hline Dorsal fin & $S: 14-X-16$ & $\mathrm{R}: 18-\mathrm{X}-20$ \\
\hline Pectoral fin & R: $21-X-22$ & \\
\hline Anal fin & S: $1-1-1$ & $\mathrm{R}: 28-\mathrm{X}-29$ \\
\hline Gill rakers & U: X-X-X & L: X-X-X \\
\hline
\end{tabular}

${ }^{a}$ Marliave $1981 \mathrm{c}$

${ }^{\mathrm{b}}$ M. Okiyama, Univ. Tokyo, Ocean Res. Inst., 1-15-1 Minamidai, Nakano-Ku, Tokyo 164, Japan, pers. commun., 8 Nov. 1985.

Ref: Marliave 1981c.

\section{EARLY LIFE HISTORY DESCRIPTION}

\section{EGGS}

Diameter

$3.5 \mathrm{~mm}$

No. of oil globules

One

Oil globule diameter

Yolk

Envelope

Hatch size $\quad 13 \mathrm{~mm} \mathrm{SL}$ (preserved)

Incubation time/temp. $\quad \sim 1 \mathrm{yr}$

Pigment

Diagnostic characters

- Precocious caudal development

\section{LARVAE}

Preanal length

Length at flexion

Length at transformation

Sequence of fin development

Pigment

- On about every other myomere along ventral midline

- Distinct spots on hypural margin, increase in number with development to become a line

- Large melanophores cover entire surface of gut

- Several large melanophores on crown

- Anterior dorsal midline starts on nape, increases posteriorly

\section{Diagnostic characters}

- Early caudal development

- Hypural margin pigment: First forms line, then an anteriorly open bracket; similar smaller bracket develops on the caudal peduncle

- Slightly separate dorsal fins

- Anal fin longer than second dorsal fin

To distinguish from Arctoscopus japonicus (present in the Bering Sea $)^{\mathrm{b}}$

- A. japonicus larvae are less advanced at similar stages of development than those of $T$. trichodon, e.g., newly hatched $A$. japonicus larvae possess no developing fin rays and an unflexed notochord

- Dorsal margin pigment not present until juvenile stage in A. japonicus

- A. japonicus: Dorsal X-XI,13; anal 30-31 
A Crown Hypural pigment
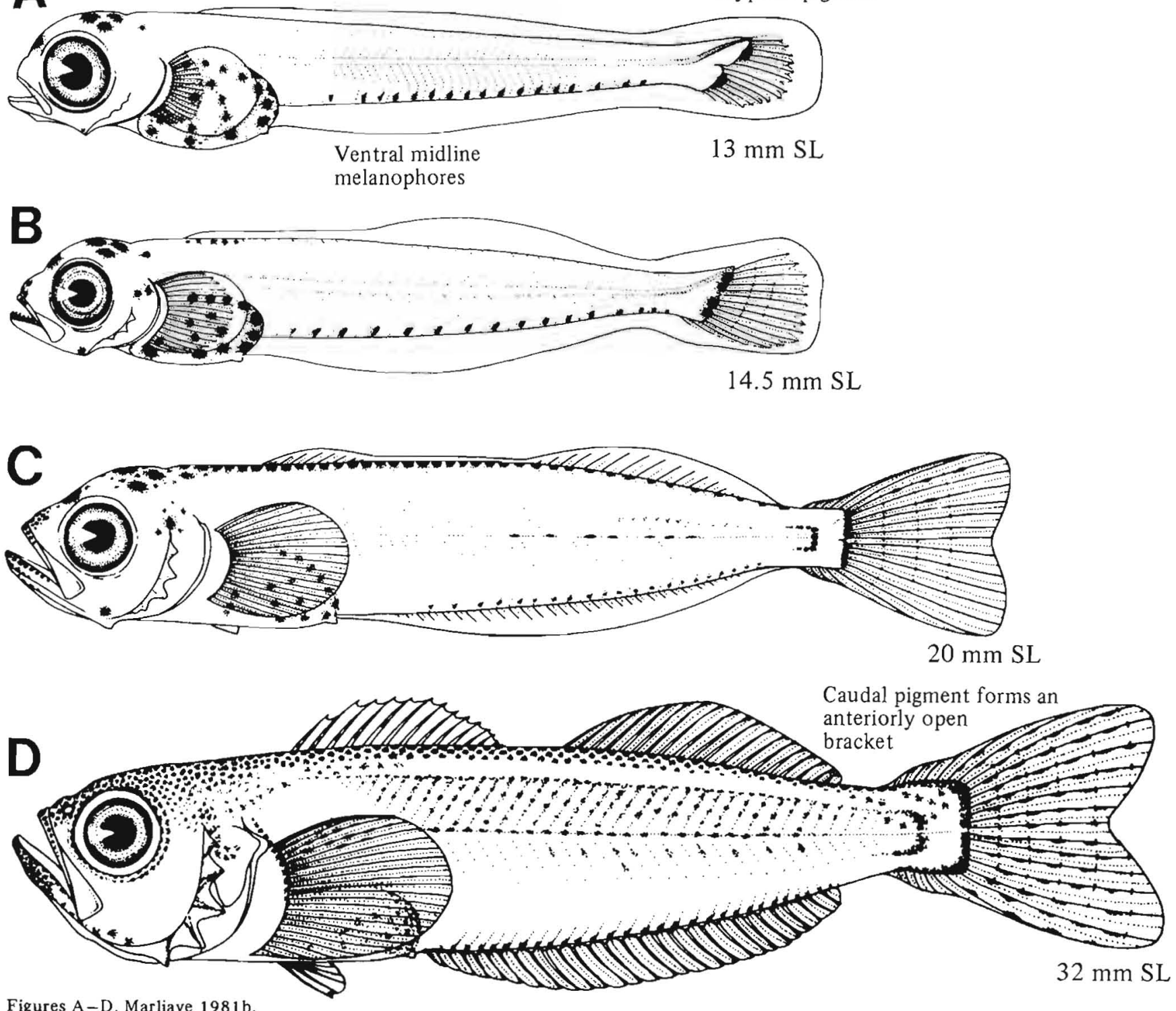

Figures A-D, Marliave $1981 \mathrm{~b}$. 

Clinids are found in both Atlantic and Pacific tropical and temperate waters. They are small (many $<7.5 \mathrm{~cm}$ ) and elongate, and somewhat deep-bodied. The dorsal fin extends from behind the head almost to the caudal fin. In the northeastern Pacific the family is represented by only three species in two genera. Adults inhabit nearshore rocky areas from intertidal zones to depths of $50 \mathrm{~m}$. Spawning behavior consists of nest building by a male in a rocky crevice, on seaweed, or in the lumen of a living sponge (Breder and Rosen 1966). Eggs, which are laid by one or more females, form clumps. The adhesive eggs $(0.85-1.7 \mathrm{~mm})$ have one or more oil globules which may be uncolored, pale yellow, or orange; sticky filaments are present on eggs of some species. Males of some tribes possess a modified anal fin or intromittent organ (Ophiclini, Clinini), and reproduction within those tribes is either viviparous or ovoviviparous. Larvae are pelagic for a brief period before settling to the bottom (Breder and Rosen 1966). The early-life-history stages of Heterostichus rostratus are presented here. Other clinids in the study area include two species of the genus Gibbonsia. In comparison to H. rostratus, members of Gibbonsia have fewer myomeres (48-54), anal fin rays (23-29), pectoral fin rays (11-13), and upper gill rakers (3-4). They undergo flexion and transformation earlier than $H$. rostratus $\left(5.0-8.1 \mathrm{~mm} \mathrm{SL}\right.$ and $\sim 19 \mathrm{~mm} \mathrm{SL}$, respectively) ${ }^{1}$ Pigment is generally limited to the ventral midline and dorsally over the swimbladder.

'W. Watson, Marine Ecological Consultants, 531 Encinitas Blvd., Suite 110, Encinitas, CA 92024, pers. commun., 1983.

\begin{tabular}{|c|c|c|c|c|c|c|c|c|c|c|}
\hline \multirow[b]{3}{*}{ Taxon } & \multirow[b]{3}{*}{ Distribution } & \multicolumn{6}{|c|}{$\begin{array}{c}\text { Table } 46 \\
\text { Meristic characters of family Clinidae. }\end{array}$} & & & \multirow[b]{3}{*}{ Branchiostegals } \\
\hline & & \multicolumn{2}{|c|}{ Vertebrae } & \multicolumn{4}{|c|}{ Fins } & \multicolumn{2}{|c|}{ Gill rakers } & \\
\hline & & Precaudal & Caudal & Dorsal & Anal & Pectoral & Pelvic & Upper & Lower & \\
\hline Gibbonsia metzi & SSC-Brit. Col. & $18-20$ & $32-34$ & $\begin{array}{c}\text { XXXIV-XXXVII } \\
7-10\end{array}$ & II, $24-29$ & $11-13$ & $\mathrm{I}, 3$ & $3-4$ & $7-8$ & $5-6$ \\
\hline Gibbonsia montereyensis & SSC-Brit. Col. & $16-17$ & $32-35$ & $\begin{array}{c}\text { XXXIV-XXXVI, } \\
5-8\end{array}$ & II, $23-28$ & $11-13$ & $\mathrm{I}, 3$ & $3-4$ & $7-12$ & 6 \\
\hline Heterostichus rostratus & SSC-Brit. Col. & $21-22$ & $34-36$ & $\begin{array}{c}\text { XXXV-XXXVII } \\
11-13\end{array}$ & u, $31-35$ & $12-14$ & $\mathrm{I}, 3$ & $5-7$ & $12-13$ & 6 \\
\hline
\end{tabular}




\section{MERISTICS}

\begin{tabular}{|c|c|c|}
\hline Vertebrae & Total: $55-57$ & \\
\hline & Precaudal: 2 & $-22-22$ \\
\hline & Caudal: $34-3$ & $5-36$ \\
\hline Branchiostegal rays & $6-6-6$ & \\
\hline Caudal fin & & \\
\hline Pelvic fin & Thoracic & \\
\hline & S: $1-1-1$ & $R: 3-3-3$ \\
\hline Dorsal fin & S: $35-37-38$ & $\mathrm{R}: 11-\mathrm{X}-13$ \\
\hline Pectoral fin & R: $12-13-14$ & \\
\hline Anal fin & $S: 2-2-2$ & $\mathrm{R}: 31-\mathrm{X}-35$ \\
\hline Gill rakers & $\mathrm{U}: 5-\mathrm{X}-7$ & $\mathrm{~L}: 12-\mathrm{X}-13$ \\
\hline
\end{tabular}

\section{LIFE HISTORY}

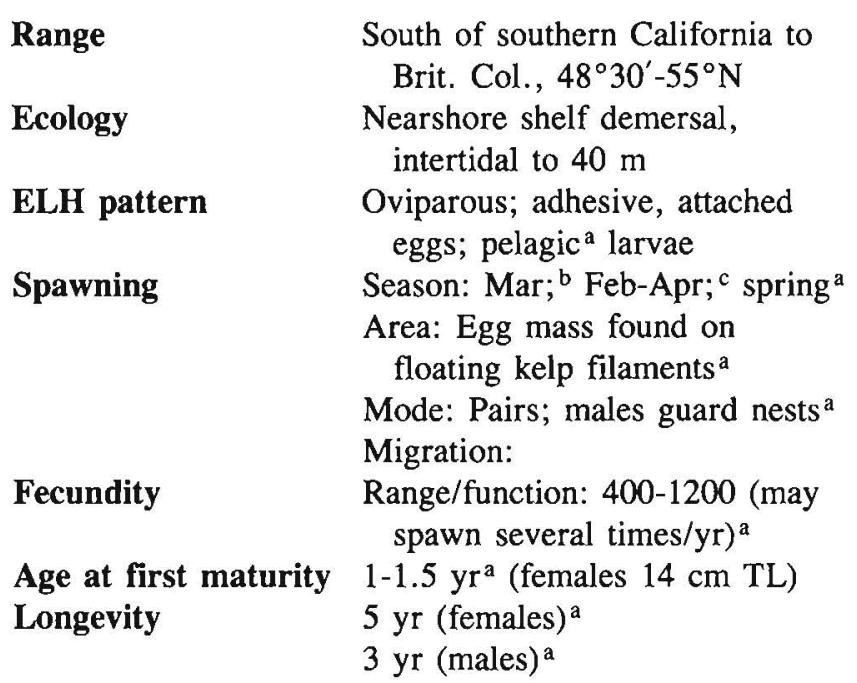

\section{EARLY LIFE HISTORY DESCRIPTION}

\section{EGGS}

Diameter

No. of oil globules

Oil globule diameter

Yolk

Envelope

Hatch size $\quad 5.5-6.2 \mathrm{~mm}$ TL $1.35 \mathrm{~mm} ; 1.4 \mathrm{~mm}^{\mathrm{a}}$

One

Red or brown

Incubation time/temp. $\quad 12-17 \mathrm{~d} / 18^{\circ} \mathrm{C}$

Pigment

- Yolk

- Ventral midline melanophores on embryo

Diagnostic characters

- Filaments

\section{LARVAE}

Preanal length

$50 \% \mathrm{SL}$

Length at flexion

$\sim 7-9 \mathrm{~mm} \mathrm{SL}$

Length at transformation By $25 \mathrm{~mm} \mathrm{SL}, 30-50 \mathrm{~mm}$ TL

Sequence of fin development

Caudal, dorsal and anal, pectorals and pelvics

Pigment

- Postanal ventral midline series: Denser posteriorly, with development becoming about one melanophore at base of each anal fin ray

- Anteriorly and dorsally on gut

Diagnostic characters

- Pigmented swimbladder (not shown on figures)

- Ventral midline melanophores

- More myomeres (56-60) than Gibbonsia spp. (47-53)

- Dorsal and anal fin development begins posteriorly

\footnotetext{
${ }^{a}$ Stepien 1986

${ }^{b}$ Wang 1981

'W. Watson, Marine Ecological Consultants, 531 Encinitas Blvd., Suite 110, Encinitas,

CA 92024, pers. commun., 3 Nov. 1986.

${ }^{d}$ Barnhart 1932

Ref: Banhart 1932, Matarese et al. 1984a, Stepien 1986
} 
B

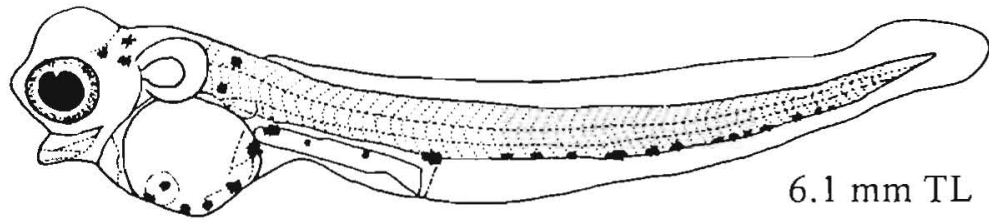

C
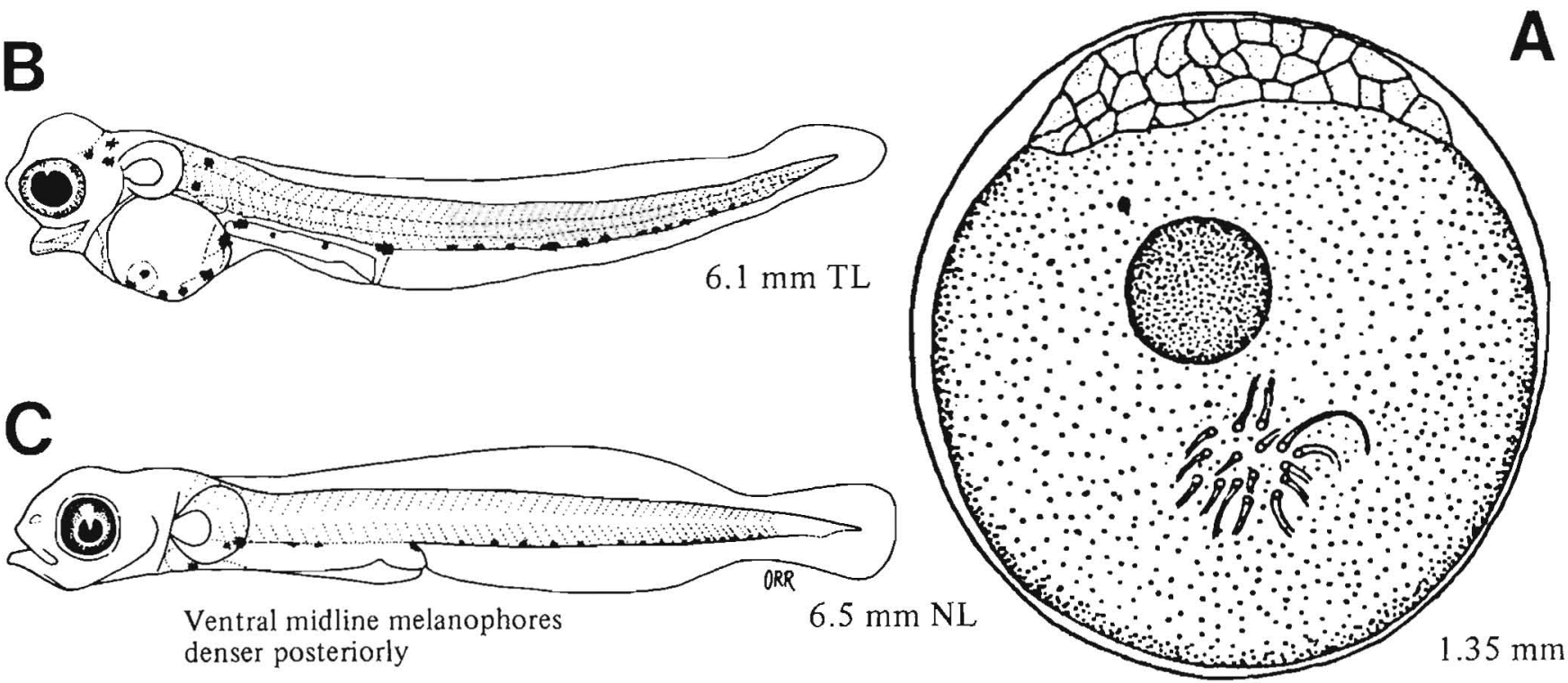

D
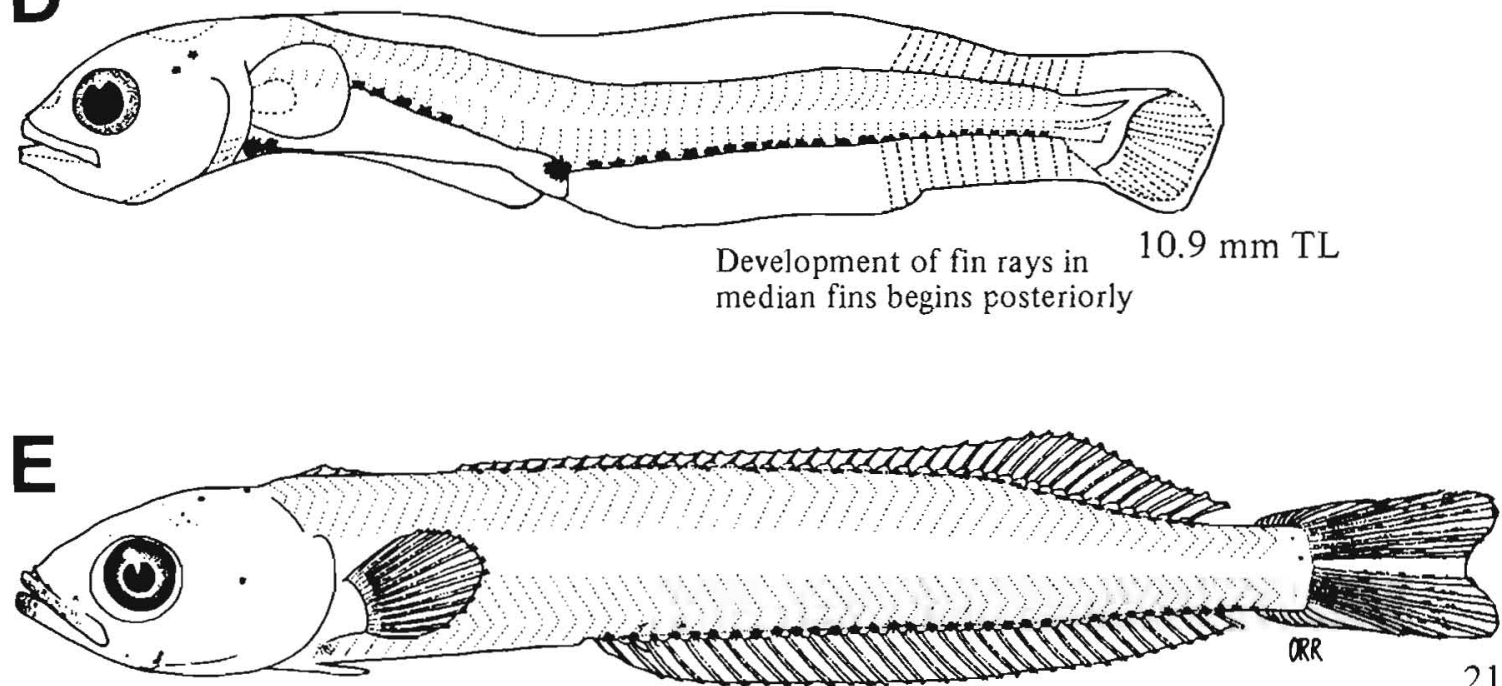

$21.2 \mathrm{~mm} \mathrm{SL}$

Figure A, Barnhart 1932; B, D, Stepien 1986 (reared); C, E, Matarese et al. 1984a. 


\section{MERISTICS}

Vertebrae

chiostegal rays

Caudal fin

Pelvic fin

$\begin{array}{lll} & \text { S: } 1-1-1 & \text { R: } 4-4-4 \\ \text { Dorsal fin } & \text { R: } 52-X-56 & \\ \text { Pectoral fin } & \text { R: } 20-X-21 & \\ \text { Anal fin } & \text { R: } 34-X-44 & \\ \text { Gill rakers } & \text { U: } 1-1-1 \quad \text { L: } 6-6-6\end{array}$

\section{LIFE HISTORY}

\begin{tabular}{|c|c|}
\hline Range & $\begin{array}{l}\text { S. California, } 32-34^{\circ} \mathrm{N} \text {, to } \\
\text { Bering Sea, } 54-66^{\circ} \mathrm{N}\end{array}$ \\
\hline Ecology & Epi- and mesopelagic \\
\hline ELH pattern & $\begin{array}{l}\text { Oviparous, pelagic eggs, } \\
\text { pelagic larvae }\end{array}$ \\
\hline Spawning & $\begin{array}{l}\text { Season: Spring-early summer, } \\
\quad \text { early fall; }{ }^{\mathrm{a}} \text { winter }^{\mathrm{b}} \\
\text { Area: Pelagic }{ }^{\mathrm{a}} \\
\text { Mode: } \\
\text { Migration: To coastal } \text { areas }^{\mathrm{b}}\end{array}$ \\
\hline Fecundity & $\begin{array}{l}\text { Range/function: } 230,000- \\
430,000^{\mathrm{b}}\end{array}$ \\
\hline $\begin{array}{l}\text { Age at first maturity } \\
\text { Longevity }\end{array}$ & $3-4 \mathrm{yr}^{\mathrm{b}}$ \\
\hline
\end{tabular}

Total: 66-X-68

Precaudal: $23-23-23$

Caudal: $45-45-45$

$6-\mathrm{X}-7$

$6-9,9+8,6-9$

Abdominal in larvae, absent in adults
S: $1-1-1$
L: $6-6-6$

\section{EARLY LIFE HISTORY DESCRIPTION}

\section{EGGS}

Diameter

No. of oil globules

Oil globule diameter

Yolk

Envelope

Hatch size

Incubation time/temp.

Pigment

- Yolk

- Oil globule

- Embryo: Finfolds, above and below tail, body

\section{Diagnostic characters}

- Large size

- Large oil globule, decreasing in size with embryonic development

\section{LARVAE}

Preanal length

$\sim 40 \%$ SL

Length at flexion

$11-17 \mathrm{~mm} \mathrm{SL}$

Length at transformation

Sequence of fin

development

Pectorals, pelvics, dorsal, anal; caudal not complete in 28.5 SL specimen

Pigment

- Head and gut covered with discrete spots

- Dorsal body margin

- Caudal finfold pigment becoming less prominent with development

- Pectoral and pelvic fin bases (postflexion)

- Three opposing blotches on dorsal and ventral finfolds in preflexion larvae

\section{Diagnostic characters}

- Preflexion pigment: Three opposing blotches in median finfold, one in caudal finfold

- Pelvic fin present in larvae, lost in adults

- Dorsal and anal fin begin to develop in finfold, have deep bases

- Morphological changes with development, from elongate to deep-bodied

- Blunt head

- Small preopercular spines

${ }^{a}$ NWAFC, unpub!.

${ }^{\mathrm{b}}$ Fitch and Lavenberg 1971

Ref: Matarese et al. 1984b; Matarese, unpubl. 

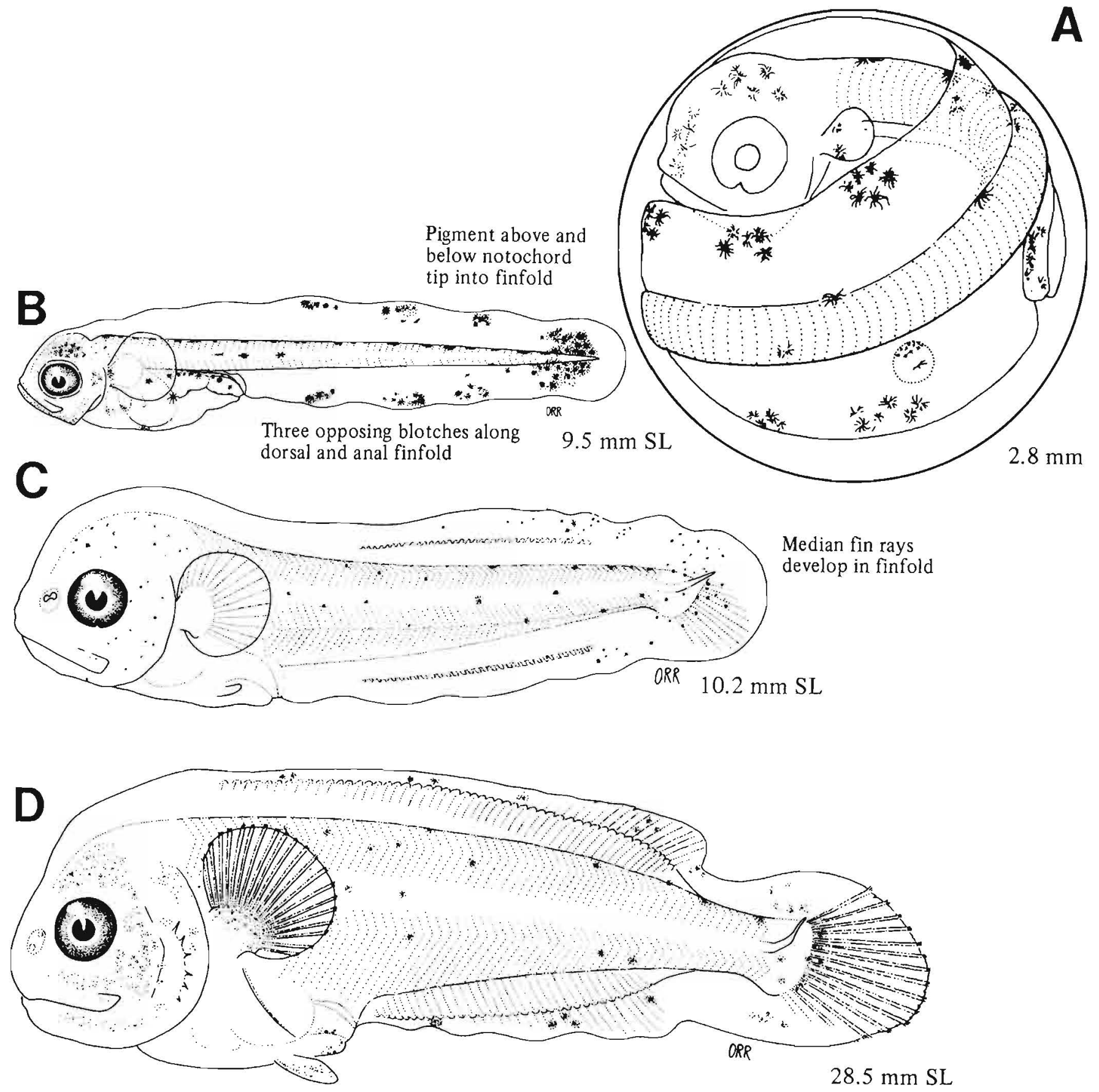

Figures A-D, Matarese et al. 1984b. 


\section{MERISTICS}

$\begin{array}{ll}\text { Vertebrae } & \text { Total: } 65-67-74 \\ & \text { Precaudal: } 40-44-47 \\ & \text { Caudal: } 23-24-25 \\ \text { Branchiostegal rays } & 6-X-8 \\ \text { Caudal fin } & \text { X, } 8+7, X \\ \text { Pelvic fin } & \text { Absent } \\ \text { Dorsal fin } & \text { R: } 54-X-63 \\ \text { Pectoral fin } & \text { R: } 13-14-15 \\ \text { Anal fin } & \text { R: } 24-X-32 \quad \\ \text { Gill rakers } & \text { U: } 3-X-6 \quad \text { L: } 16-X-22\end{array}$

\section{LIFE HISTORY}

$\begin{array}{ll}\text { Range } & \text { S. California, } 32-34^{\circ} \mathrm{N} \text {, to } \\ \text { Arctic, not specific } \\ \text { Ecology } & \text { Epi- and mesobenthal, intertidal } \\ & \text { to } 275 \mathrm{~m} \\ \text { ELH pattern } & \text { Oviparous; demersal, adhesive } \\ & \text { eggs; pelagic larvae } \\ \text { Spawning } & \text { Season: Nov-Feb } \\ & \text { Areas: In areas of strong current } \\ & \text { Mode: } \\ & \text { Migration: } \\ \text { Fecundity } & \text { Range/function: } 1000^{\mathrm{c}} \text { (A. per- } \\ & \text { sonatus, western Pacific)- } \\ & 22,100^{\mathrm{d}} \text { (southwestern Barents } \\ & \text { Sea) } \\ \text { Age at first maturity } & 1 \mathrm{yr}^{\mathrm{e}} \text { (western Pacific) }\end{array}$

Longevity

\section{EARLY LIFE HISTORY DESCRIPTION}

\section{EGGS}

Diameter

No. of oil globules

$0.67-0.91 \mathrm{~mm}(0.80 \mathrm{~mm})$

Oil globule diamete

Yolk

Envelope

Hatch size

$\sim 0.26 \mathrm{~mm}$

6-7 $\mathrm{mm} \mathrm{SL} \mathrm{(as} \mathrm{small} \mathrm{as}$ $4 \mathrm{~mm}$ )

Incubation time/temp. $\quad 2-12 \mathrm{wk}$

Pigment

- Embryo: Eyes, dorsal and ventral body

Diagnostic characters

\section{LARVAE}

Preanal length

$\sim 60 \%$ SL

Length at flexion

11-13 mm SL

Length at transformation

Sequence of fin development

$16-31 \mathrm{~mm} \mathrm{SL}$

Caudal, pectorals, dorsal and anal

Pigment

- Gut

- Double row postanal ventral pigment

Diagnostic characters (see Table 4)

- Elongate body

- Gut length ( $60 \%$ SL)

- Lightly pigmented

- Postanal ventral pigment (double row)

- Dorsal and anal fins begin development opposed to each other

- Elongate head shape in larger specimens

${ }^{a}$ Trumble 1973

${ }^{b}$ Andriashev 1954

${ }^{c}$ Inoue et al. 1967

dMacy et al. 1978

${ }^{\text {e}}$ Hamada 1966

Ref: Kobayashi 1961c, Stevens et al. 1984. 

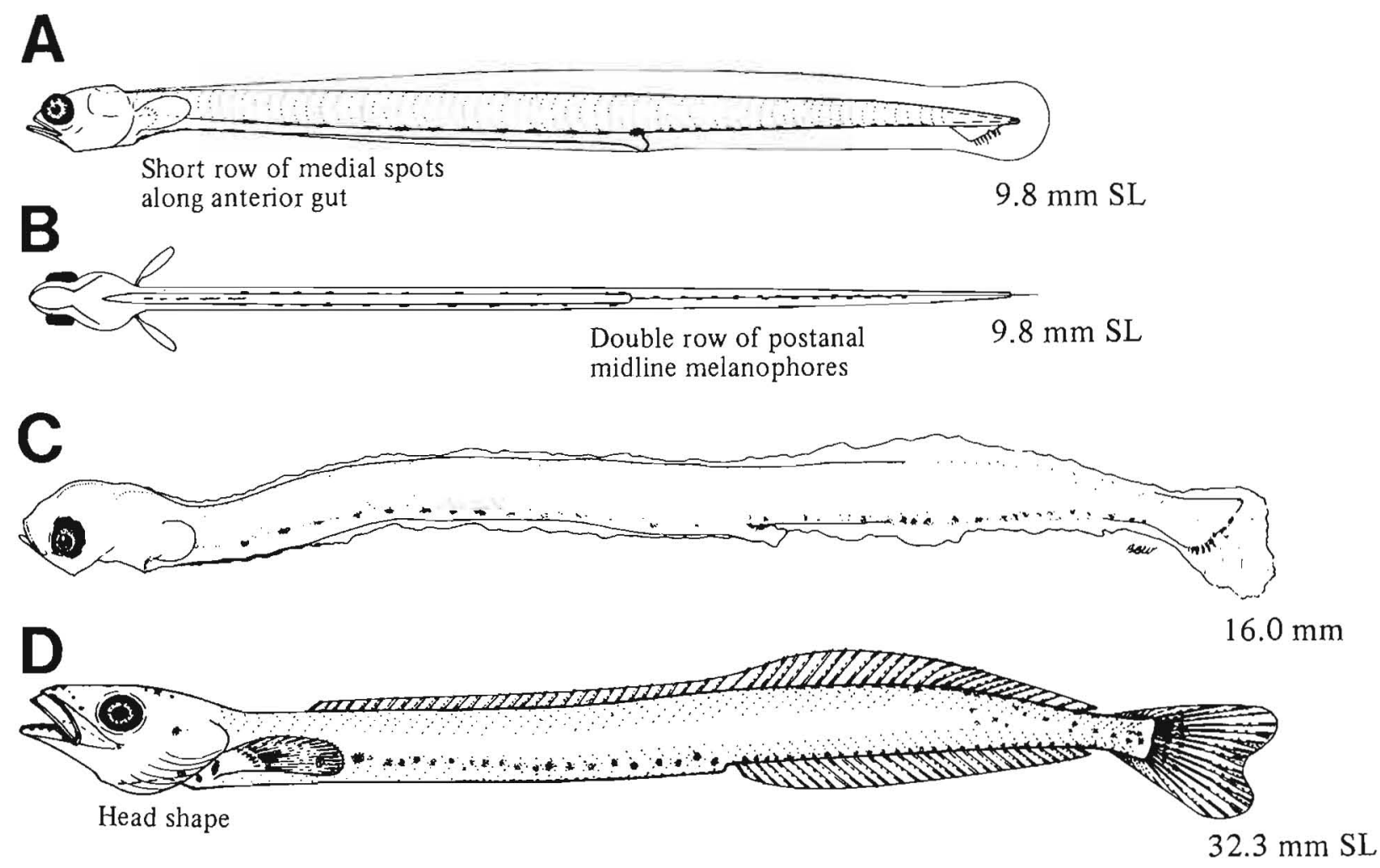

Figures A-B, D, (B, ventral view), NWAFC originals (B. Vinter); C, Stevens et al. 1984. 

Gobiidae is the most speciose family of marine fishes, although some species occur in brackish or freshwater environments. Gobies are generally small bottom-dwelling fishes with pelvic fins united to form a sucking disc. Found mainly in subtropical and tropical areas, adults inhabit shallow to moderate depths in salt and brackish water (some in freshwater). The study area includes three species: Clevelandia ios, Coryphopterus nicholsi, and Lepidogobius lepidus. Larvae are easily recognized by their conspicuously pigmented swimbladder and pigment patterns. Larvae are commonly collected inshore and in bays and estuaries. They are rare in coastal ichthyoplankton collections in the study area.

Small larvae of Clevelandia and Lepidogobius have been confused for some time in the literature. Since we have incomplete developmental series of the two species, we are presenting a consensus of opinion from researchers who have had more experience studying or collecting gobies. Gobiid larvae with three dorsal melanophores previously assigned to Clevelandia are now considered Lepidogobius (Wang 1986; W. Watson and G. McGowen, pers. commun. ${ }^{1}$ ).

${ }^{1}$ W. Watson, Marine Ecological Consultants, 531 Encinitas Blvd., Suite 110, Encinitas, CA 92024, pers. commun., 3 Nov. 1986; G. McGowen, Los Ang. Cty. Mus. Nat. Hist., 900 Exposition Blvd., Los Angeles, CA 90036, pers, commun., 31 Oct. 1986.

Table 47

Early-life-history characters of gobiid larvae from the Northeast Pacific (Wang 1981, in part).

\begin{tabular}{|c|c|c|c|}
\hline & Clevelandia ios & Coryphopterus nicholsi & Lepidogobius lepidus \\
\hline Spawning Site & Burrow & Rocky reef & Burrow \\
\hline Egg Shape & $\begin{array}{l}\text { Elliptical with narrow, blunt distal } \\
\text { end }\end{array}$ & Spindle-shaped, narrow, elongate & Elliptical \\
\hline \multicolumn{4}{|l|}{ Larvae } \\
\hline Total myomeres & $34-36$ & $25-26$ & $36-38$ \\
\hline Preanal myomeres & $16-18$ & $9-10$ & $14-17$ \\
\hline Postanal myomeres & $16-19$ & $14-17$ & $19-23$ \\
\hline Distinguishing pigmentation & $\begin{array}{l}\text { Single large melanophore along dor- } \\
\text { sal midline of body which forms a } \\
\text { band at about myomere } 26\end{array}$ & $\begin{array}{l}\text { A series of } 10-15 \text { melanophores } \\
\text { along ventral body midline and a } \\
\text { shorter series along dorsal midline } \\
\text { near caudal }\end{array}$ & $\begin{array}{l}\text { Three dorsal midline melanophores, } \\
\text { posteriormost forming a band at } \\
\text { about myomeres } 19-26\end{array}$ \\
\hline \multicolumn{4}{|l|}{ Juveniles } \\
\hline Dorsal fin & IV-VI; 0-I, 14-17 & V-VI; I-II, 9-15 & VI-IX; 0-I, 14-18 \\
\hline Anal fin & $0-I, 14-17$ & $0-I, 11-14$ & $0-I, 13-16$ \\
\hline Pectoral fin & $18-21$ & $21-24$ & $20-22$ \\
\hline Vertebrae & $35-37$ & 26 & $37-38$ \\
\hline Distribution & Seawater-polyhaline-oligohaline & Seawater-polyhaline & Seawater-polyhaline-oligohaline \\
\hline Distinguishing characteristics & $\begin{array}{l}\sim 12 \text { dark bands on dorsum; } \sim 12 \\
\text { close melanophores on lateral line }\end{array}$ & $\begin{array}{l}\text { Large black eye; black margin on } \\
\text { spinous dorsal fin; side of body } \\
\text { without dark vertical bands }\end{array}$ & $\begin{array}{l}\text { Black margin on spinous dorsal fin; } \\
\text { broad dark band at base of caudal } \\
\text { region; body pigmentation light }\end{array}$ \\
\hline
\end{tabular}




\section{MERISTICS}

$\begin{array}{lll}\text { Vertebrae } & \text { Total: } 35-36-37 \\ & \begin{array}{l}\text { Precaudal: } 15-15-15 \\ \text { Caudal: } 21-21-22\end{array} \\ \begin{array}{l}\text { Branchiostegal rays } \\ \text { Caudal fin }\end{array} & 3-X-5 \\ \begin{array}{ll}\text { Pelvic fin } \\ \text { Thoracic }\end{array} & \\ \text { Dorsal fin } & \text { R: } 5-5-5 & \\ & \text { 1st S: } 4-X-6 & \\ \text { Pectoral fin } & \text { 2nd S: } 0-X-1 & \text { R: } 14-X-17 \\ \text { Anal fin } & \text { R: } 20-20-20 & \\ \text { Gill rakers } & \text { S: } 0-X-1 & \text { R: } 14-X-17 \\ & \text { U: } 1-X-3 & \text { L: } 5-X-7\end{array}$

\section{LIFE HISTORY}

$\begin{array}{ll}\text { Range } & \begin{array}{c}\text { South of southern California to } \\ \text { Brit. Col., } 48^{\circ} 30^{\prime}-55^{\circ} \mathrm{N}\end{array} \\ \text { Ecology } & \text { Intertidal } \\ \text { ELH pattern } & \text { Oviparous; demersal, attached } \\ & \text { eggs; pelagic larvae } \\ \text { Spawning } & \text { Season: Nov-June or later } \\ & \text { Area: } \\ & \text { Mode: } \\ & \text { Migration: } \\ \text { Fecundity } & \text { Range/function: } 750-1000 \\ & \text { (may be multiple spawners) } \\ & \mathrm{F}=0.0306 \times \mathrm{L}^{2.04} \mathrm{c}\end{array}$

Age at first maturity $1 \mathrm{yr}^{\mathrm{c}}$

Longevity 2-3 $\mathrm{yr}^{\mathrm{c}}$

\section{EARLY LIFE HISTORY DESCRIPTION}

\section{EGGS}

Diameter

No. of oil globules

Oil globule diameter

Yolk

Envelope

Hatch size

0.70-0.85 mm

Many coalescing to one

Transparent; adhesive threads at one pole

Incubation time/temp. $\quad 10-12 \mathrm{~d} / 15-15.5^{\circ} \mathrm{C}$

Pigment

Diagnostic characters

- Ellipsoidal

\section{LARVAE}

Preanal length

$45-52 \%$ SL

Length at flexion

Length at transformation

Sequence of fin development

Caudal, 2nd dorsal and anal, 1st dorsal and pectorals, pelvics

Pigment

- Single large melanophore along dorsal midline of body which forms a band at about myomere 26

- Dorsal surface of swimbladder

Diagnostic characters (see Table 47)

- Conspicuously pigmented swimbladder (family)

Distinguished from Coryphopterus nicholsi by

- Total myomeres (fewer in C. nicholsi, 25-26 vs. 35-37)

Distinguished from Lepidogobius lepidus by

- Pigment pattern: No anterior melanophores along dorsal midline; present in L. lepidus

\section{Postflexion}

- Dorsal spine count (4-6), anal count (14-17), mouth size

'Brothers 1975

Ref: Prasad 1958, Ruple 1984, Wang 1981. 

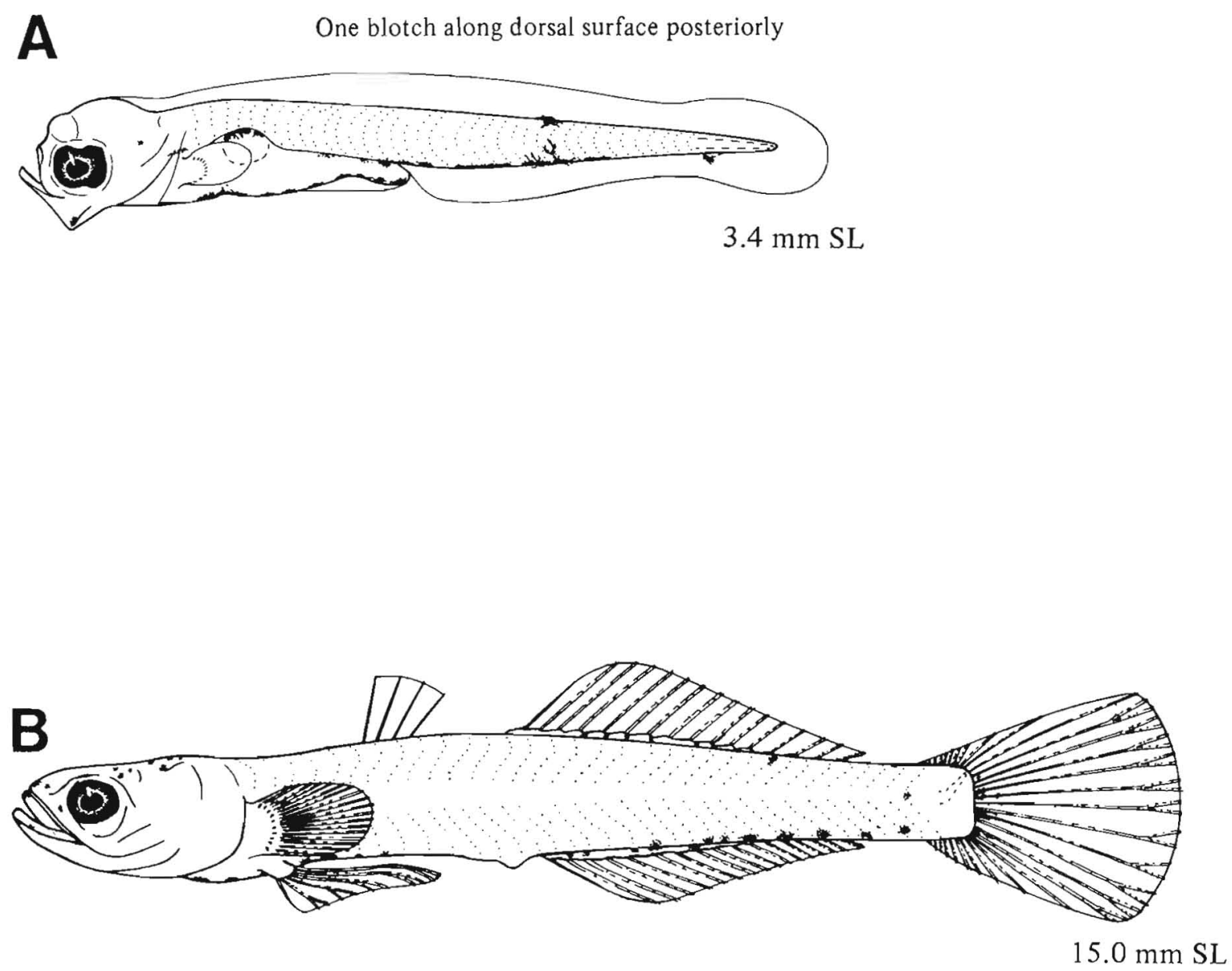


\section{MERISTICS}

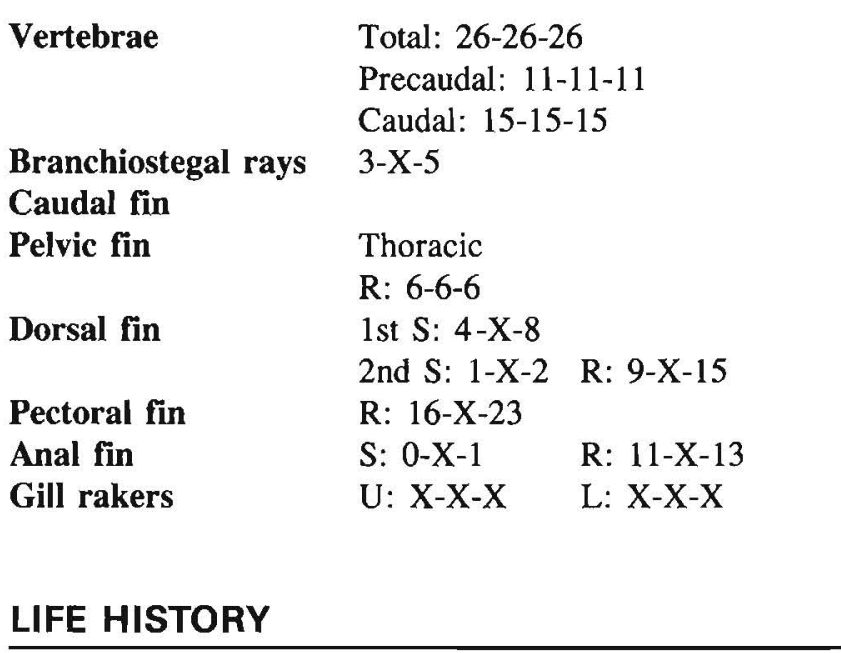

Range

Ecology

ELH pattern

Spawning

Fecundity

$\begin{array}{ll}\text { Age at first maturity } & 2-5 \mathrm{yr} \text { (females) }^{\mathrm{a}} \\ & 3-5 \mathrm{yr} \text { (males) }^{\mathrm{a}}\end{array}$

Longevity
South of southern California to Brit. Col., $48^{\circ} 30^{\prime}-55^{\circ} \mathrm{N}$

Nearshore shelf demersal, intertidal to $106 \mathrm{~m}$

Oviparous; demersal, attached eggs; pelagic larvae

Season: $\mathrm{Feb}^{\mathrm{a}}-\mathrm{Oct}^{\mathrm{b}}$ (California)

Area: Under rocks ${ }^{\mathrm{a}}$

Mode: Pairs; eggs guarded by males $^{\text {a }}$

Migration:

Range/function: $3274-4788$ (may be multiple spawners) ${ }^{\mathrm{a}}$

\section{EARLY LIFE HISTORY DESCRIPTION}

\section{EGGS}

Diameter

No. of oil globules

Oil globule diameter

Yolk

Envelope

Hatch size

$2.1 \times 0.48 \mathrm{~mm}$

Multiple

Incubation time/temp.

Pigment

Transparent, smooth

$2.94 \mathrm{~mm}$ TL

\section{Diagnostic characters}

- Ellipsoidal (pointed at each end)

- Pigmented dorsally over yolk and along ventral body midline

\section{LARVAE}

Preanal length $50 \% \mathrm{SL}$

Length at flexion

Length at transformation

Sequence of fin development

\section{Caudal, 2nd dorsal and anal, 1st dorsal and pectorals, pelvics}

Pigment

- Dorsal surface of swimbladder

- Dorsal surface of posterior gut

- Series of dorsal midline melanophores along posterior half of body

- 10-15 postanal ventral melanophores

- With development, pigment in hypural area

Diagnostic characters (see Table 47 and Clevelandia ios, p. 544)

- Myomeres (26)

${ }^{3}$ Wiley 1973

${ }^{b}$ Ebert and Turner 1962

Ref: Ruple 1984, Wang 1981 


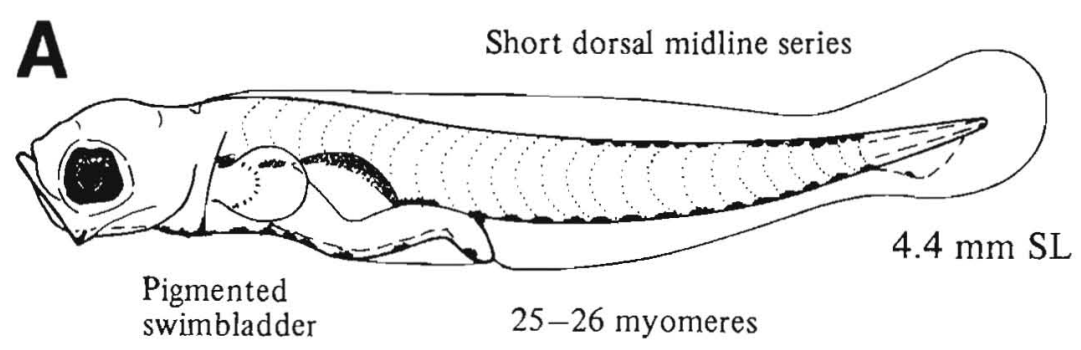

Figure A, NWAFC original (B. Vinter; specimen loaned by Bruce Frost, University of Washington, for illustration). 


\section{MERISTICS}

\section{Vertebrae}

Total: $37-37-38$

Precaudal: $15-15-15$

Caudal: $22-22-23$

Branchiostegal rays $\quad 3-\mathrm{X}-4$

Caudal fin

Pelvic fin

Thoracic

\section{R: 5-5-5}

Dorsal fin

Pectoral fin

Anal fin

Gill rakers 1st S: $6-\mathrm{X}-9$

2nd S: $0-\mathrm{X}-1 \quad \mathrm{R}: 14-\mathrm{X}-18$

R: $20-20-20$

S: $0-X-1$

$\mathrm{U}: \mathrm{X}-\mathrm{X}-\mathrm{X}$

$\mathrm{R}: 13-\mathrm{X}-16$

L: X-X-X

\section{EARLY LIFE HISTORY DESCRIPTION}

\section{EGGS}

Diameter

No. of oil globules

Oil globule diameter

Yolk

Envelope

Hatch size

Incubation time/temp.

Pigment

Diagnostic characters

\section{LARVAE}

Preanal length

Length at flexion

Length at transformation

Sequence of fin development
$1.3-1.8 \times 0.8-1.0 \mathrm{~mm}$ (unfertilized)

Many (unfertilized)

Granular, yellowish

Transparent, smooth

ELH pattern

Spawning

Season: Nov-June (California) ${ }^{\mathbf{a}}$

Area: Intertidal mudflats ${ }^{\mathbf{a}}$

Mode:

Migration:

Fecundity

Age at first maturity

Longevity

\section{Range/function:}

\section{Pigment}

\section{$42-45 \% \mathrm{TL}$}

- Dorsal surface of swimbladder

- Three dorsal midline melanophores, posteriormost forming a band at about myomeres 19-26

Diagnostic characters (see Table 47 and Clevelandia ios, (p. 544)

Distinguished from $C$. ios by

- Anterior melanophores along dorsal midline; not present in $C$. ios

Distinguished from Coryphopterus nicholsi by

- Total myomeres (fewer in C. nicholsi, $25-26$ vs. $37-38$ )

\footnotetext{
${ }^{a}$ Wang 1981

Ref: Ruple 1984, Wang 1981.
} 


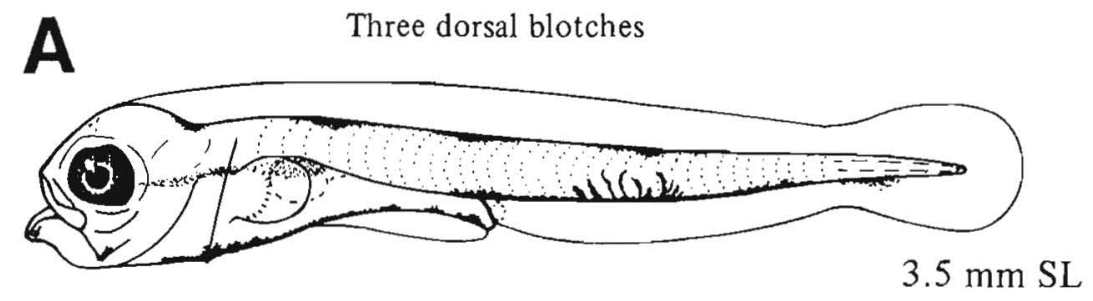

Pigment on dorsal surface of swimbladder

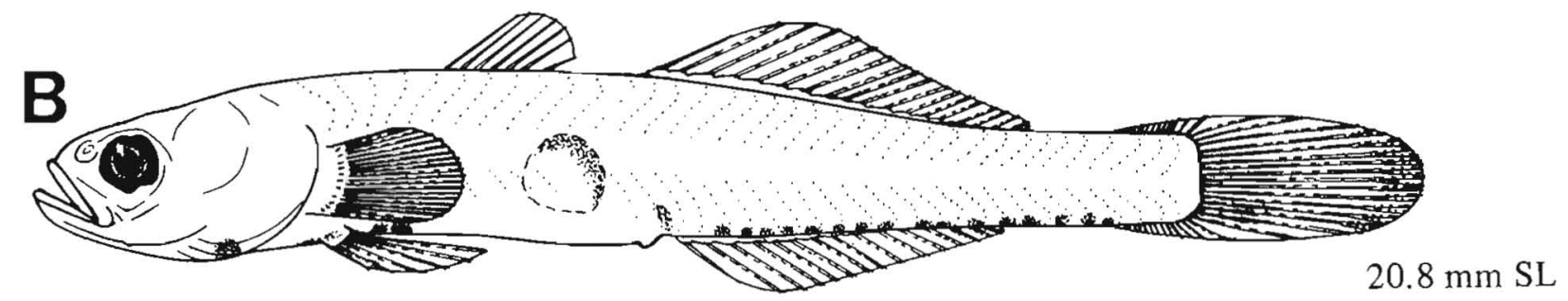

Figures A-B, NWAFC originals (B. Vinter; specimens loaned by Bruce Mundy, formerly of Oregon State University, for illustration). 

There are four species of cutlassfishes in three genera of this family in the northeastern Pacific. General body shape is elongate and ribbonlike, tapering to a small caudal fin. Voracious predators, most have a large, well-toothed mouth and pointed snout. Adult cutlassfishes are benthopelagic but have been found at the surface at night (Fritzsche 1978). Spawning occurs offshore, resulting in pelagic eggs which can be 1.6-2.5 mm and have a single reddish-yellow oil globule (Breder and Rosen 1966, Fritzsche 1978). Pelagic larvae have a high myomere count (generally $>100$ ) and develop three dorsal spines, the first of which may be elongate (Schmidt and Strubberg 1918). Larvae have a small head with a long, tapering body (Ozawa 1986e). No early-life-history stages have been collected in our area.

\begin{tabular}{|c|c|c|c|c|c|c|c|c|c|c|}
\hline \multirow[b]{4}{*}{ Taxon } & \multicolumn{9}{|c|}{$\begin{array}{c}\text { Table } 48 \\
\text { Meristic characters of family Trichiuridae. }\end{array}$} & \multirow[b]{4}{*}{ Branchiostegals } \\
\hline & \multirow[b]{3}{*}{ Distribution } & \multicolumn{2}{|c|}{ Vertebrae } & \multirow{2}{*}{\multicolumn{4}{|c|}{ Fins }} & \multirow{2}{*}{\multicolumn{2}{|c|}{ Gill rakers }} & \\
\hline & & \multirow{2}{*}{$\begin{array}{l}\text { Precaudal } \\
\text { (Tota }\end{array}$} & \multirow{2}{*}{ Caudal } & & & & & & & \\
\hline & & & & Dorsal & Anal & Pectoral & Pelvic & Upper & Lower & \\
\hline Aphanopus carbo & N. Calif.-Brit. Col. & $42-46$ & $55-59$ & $\begin{array}{c}\text { XXXVIII-XLIII, } \\
53-56\end{array}$ & I-II, 44-49 & 12 & $\mathrm{I}, \mathrm{l}$ & & & $7-8$ \\
\hline Benthodesmus elongatus & Cent. Calif.-Brit. Col. & $(148-1$ & & $\begin{array}{l}\text { XLIV-XLVI, } \\
98-102\end{array}$ & II, 91-98 & 12 & $\mathrm{I}, 1$ & 5 & 9 & 7 \\
\hline Benthodesmus tenuis & Brit. Col. & $(121-1$ & & $\begin{array}{c}\mathrm{XXXDX}-\mathrm{XLII} \\
79-88\end{array}$ & II, 69-75 & & & & & \\
\hline Lepidopus fitchi & SSC-Oregon & $\begin{array}{l}35 \\
(84-9\end{array}$ & 50 & IX, $78-86$ & II, $41-49$ & 12 & $\mathrm{I}, 1^{*}$ & 7 & 10 & 7 \\
\hline
\end{tabular}




\section{MERISTICS}

(Steindachner 1891)

Vertebrae

Branchiostegal rays

Caudal fin

Pelvic fin

Dorsal fin

Pectoral fin

Anal fin

Gill rakers
B. elongatus

Total: 148-X-153

Precaudal: $\mathrm{X}-\mathrm{X}-\mathrm{X}$

Caudal: $\mathrm{X}-\mathrm{X}-\mathrm{X}$

\section{7-7-7}

Thoracic

S: $44-44-47$ R: $98-X-102$

R: $12-12-12$

S: $2-2-2$

R: 91-93-98

U: $5-5-5$

L: 9-9-9
S: 1-1-1
R: $1-1-1$

EARLY LIFE HISTORY DESCRIPTION

EGGS - Family

Diameter

$1.7-2.0 \mathrm{~mm}$

No. of oil globules

Oil globule diameter

Yolk

Envelope

Hatch size

4.5-6.5 mm SL

Incubation time/temp.

Pigment

Diagnostic characters

\section{MERISTICS}

\begin{tabular}{|c|c|c|}
\hline \multirow{3}{*}{ Vertebrae } & \multicolumn{2}{|c|}{ Total: $121-X-131$} \\
\hline & \multicolumn{2}{|c|}{ Precaudal: X-X-X } \\
\hline & \multicolumn{2}{|c|}{ Caudal: X-X-X } \\
\hline Branchiostegal rays & \multicolumn{2}{|l|}{$7-7-7$} \\
\hline \multicolumn{3}{|l|}{ Caudal fin } \\
\hline \multirow[t]{2}{*}{ Pelvic fin } & Thoracic & \\
\hline & $S: X-X-X$ & $\mathrm{R}: \mathrm{X}-\mathrm{X}-\mathrm{X}$ \\
\hline Dorsal fin & S: $39-X-42$ & R: $79-X-88$ \\
\hline Pectoral fin & $\mathrm{R}: \mathrm{X}-\mathrm{X}-\mathrm{X}$ & \\
\hline Anal fin & $S: 2-2-2$ & $\mathrm{R}: 69-\mathrm{X}-75$ \\
\hline Gill rakers & U: X-X-X & L: X-X-X \\
\hline
\end{tabular}

\section{LIFE HISTORY}

$\begin{array}{ll}\text { Range } & \begin{array}{l}\text { Cent. California, } 34-38^{\circ} \mathrm{N},{ }^{a} \text { to } \\ \text { Brit. Col., } 48^{\circ} 30^{\prime}-55^{\circ} \mathrm{N}\end{array} \\ \text { Ecology } & \begin{array}{l}\text { Epi- and mesopelagic } \\ \text { Oviparous, pelagic eggs, } \\ \text { pLH pattern }\end{array} \\ \begin{array}{l}\text { pelagic larvae } \\ \text { Spawning }\end{array} & \text { Area: } \\ & \text { Mode: } \\ & \text { Migration: } \\ \text { Fecundity } & \text { Range/function: } \\ \text { Age at first maturity } & \\ \text { Longevity } & \end{array}$

${ }^{a} B$. tenuis range restricted to British Columbia.

bescription based on $B$. elongatus simonyi, a subspecies from the North Atlantic, and $B$. elongatus pacificus from Japan. Ozawa (1986e) describes and figures one late postflexion larva of $B$. tenuis ( $27.1 \mathrm{~mm} \mathrm{SL}$ ). Larvae of $B$. elongatus and $B$. tenuis from our area may differ.

'Ozawa 1986e

Ref: Collette et al. 1984b, Evseenko 1982, Gorbunova 1982b, Ozawa 1986e, Peden and Hughes 1986.

\section{LARVAE $^{b}$}

Preanal length

Length at flexion

Length at transformation

Sequence of fin development

\section{Pigment}

- Blotch on upper preopercle

- Crown

- Dorsal midline beginning just anterior to dorsal fin origin and running along developing fin, increasing with development

- Dorsolateral gut

- Ventrolateral blotch about mid-postanal body; with development becoming a series along ventral midline about $3 / 4$ length of anal fin

- B. elongatus pacificus has a blotch at the origin of the anal fin (not shown on figure) ${ }^{c}$

Diagnostic characters

- Family

-Serrate spines in dorsal, anal, and pelvic fins

-In some genera (e.g., Benthodesmus), the anteriormost rays of the dorsal fin are extremely elongate in the smallest larvae

- Genus

- Caudal fin development distinct

-Pelvic fin located approximately below pectoral fin

- Number of dorsal fin spines $(>30)$ 


\section{BENTHODESMUS}

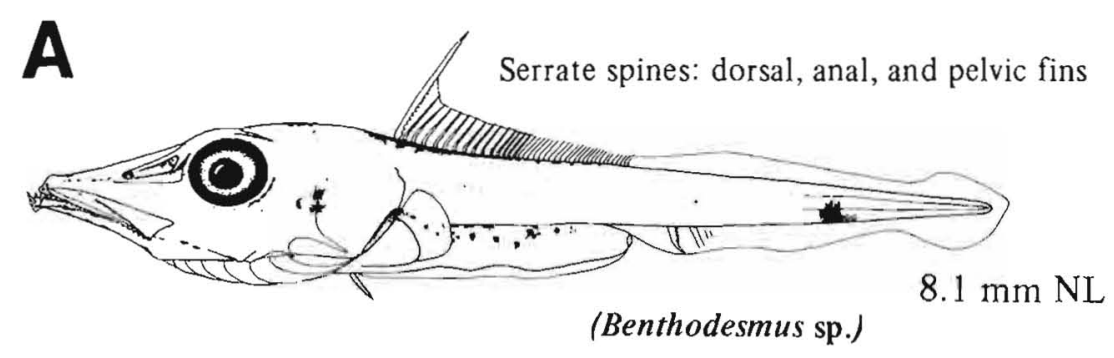

B

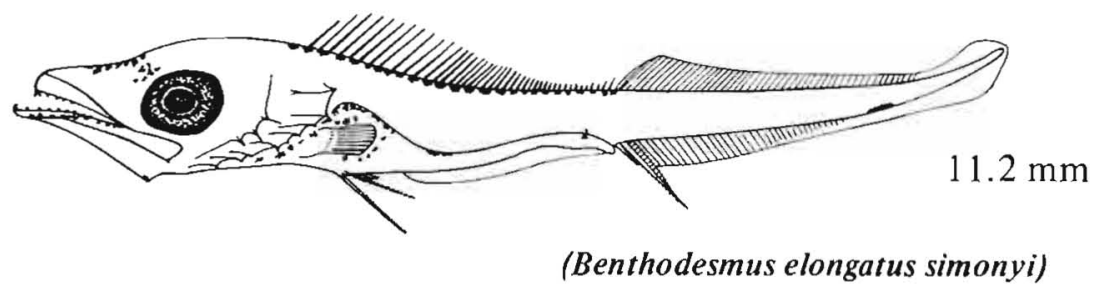

\section{C}

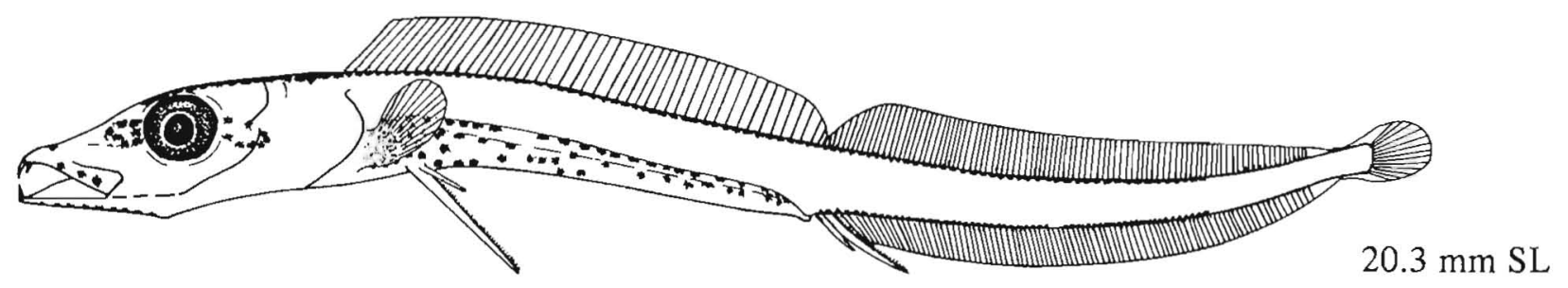

(Benthodesmus elongatus simonyi)

Figure A, Collette et al. 1984b (Gulf of Mexico specimen); B, Gorbunova 1982b; C, Evseenko 1982 (B-C, redrawn; subspecies from North Atlantic). 


\section{MERISTICS}

Vertebrae

Total: $30-31-31$

Precaudal: $14-14-15$

Caudal: $16-17-17$

Branchiostegal rays $\quad$ 7-7-7

Caudal fin $\quad X, 9+8, X$

Pelvic fin Thoracic
S: $1-1-1$
$\mathrm{R}: 5-5-5$
S: $9-10-12$
R: $20-20-20$
S: $1-1-1$
U: $11-13-14 \quad$ L: $27-28-30$
R: $11-12-12$

Dorsal fin

Pectoral fin

Anal fin

Gill rakers

\section{LIFE HISTORY}

\section{Range \\ Ecology \\ ELH pattern}

Spawning

Fecundity

Age at first maturity

Longevity
South of southern California to SE Alaska, $55-59^{\circ} \mathrm{N}$

Epi- and mesopelagic, $0-300 \mathrm{~m}^{\mathrm{a}}$

Oviparous, pelagic eggs, pelagic larvae

Season: Apr-July (California) ${ }^{b}$

Area: 0-72 m, 3-32 km from shore $^{\mathrm{c}}$

Mode:

Migration:

Range/function: $>1$ million (may spawn more than once each year $)^{b}$

$2 \mathrm{yr}^{\mathrm{b}}-6 \mathrm{yr}^{\mathrm{d}}$

$10 \mathrm{yr}^{\mathrm{b}} ; 12 \mathrm{yr}^{\mathrm{e}}$

\section{EARLY LIFE HISTORY DESCRIPTION}

\section{EGGS}

Diameter

No. of oil globules

$0.9-1.3 \mathrm{~mm}$

Oil globule diameter

Yolk

Envelope

Hatch size

One, ventral

$0.26 \mathrm{~mm}$

Homogeneous

Smooth, clear

Incubation time/temp.

Pigment

- Oil globule

- Yolk

\section{Diagnostic characters}

- Posterior position of oil globule

\section{LARVAE}

Preanal length

Length at flexion Length at transformation

Sequence of fin development

Pigment

- Pigment on crown extends anteriorly with development

- Dorsolaterally on gut

- Ventral midline

- Postflexion

-Short dorsal midline series develops under dorsal fin, spreading anteriorly

- Ventral midline becomes restricted to posterior half of body

-Mediolateral pigment

Diagnostic characters

- Large head with teeth

- Myomeres (30-31)

- Pigment pattern

${ }^{a}$ Collette and Nauen 1983

bHart 1973

${ }^{c}$ Fritzsche 1978

${ }^{\circledR}$ Schaefer 1980

${ }^{\top}$ Fitch and Lavenberg 1971

Ref: Berrien 1978, Collette et al. 1984b, G.D. Johnson 1984, Kramer 1960. 

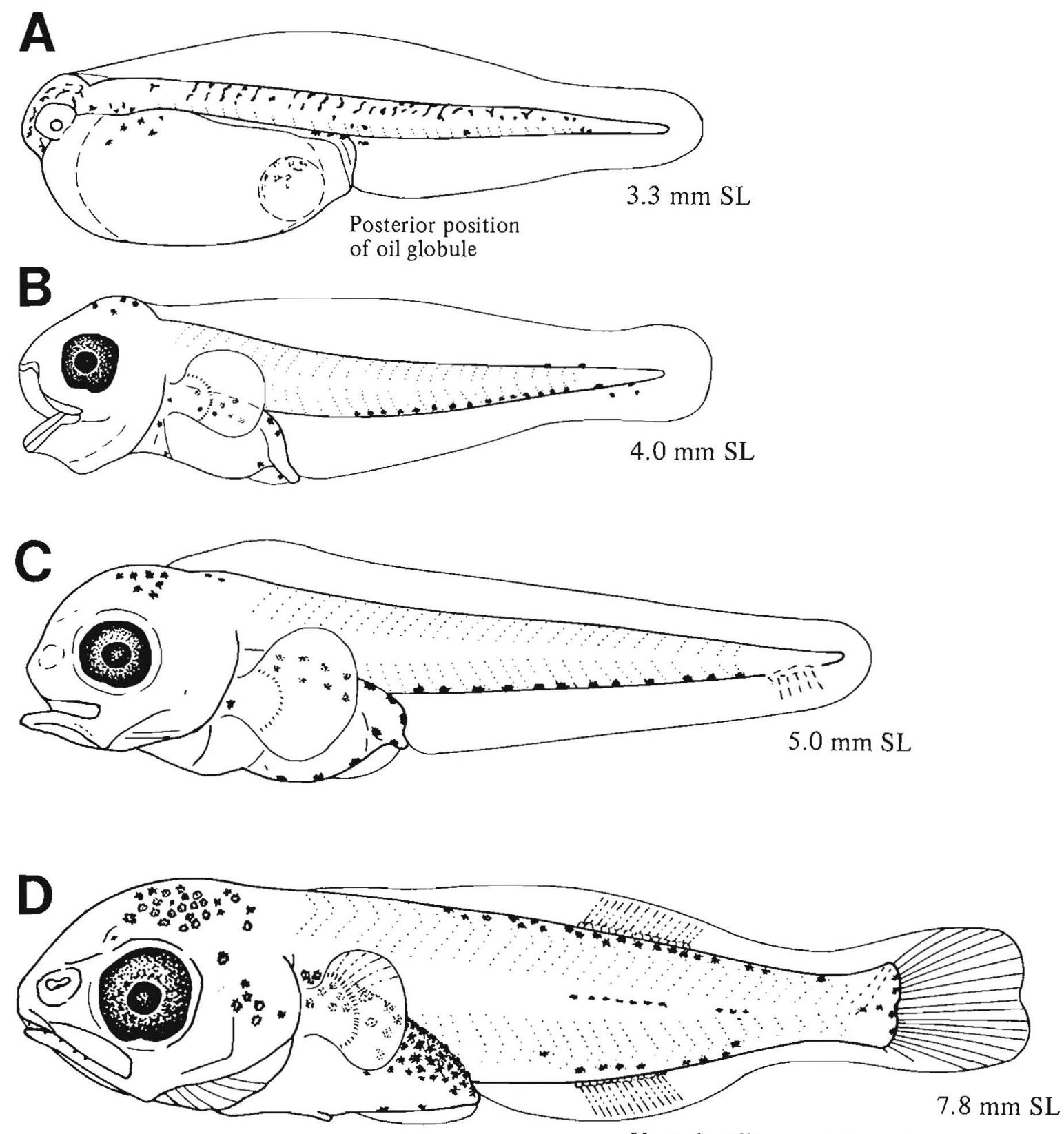

Ventral midline spots becoming restricted to posterior body

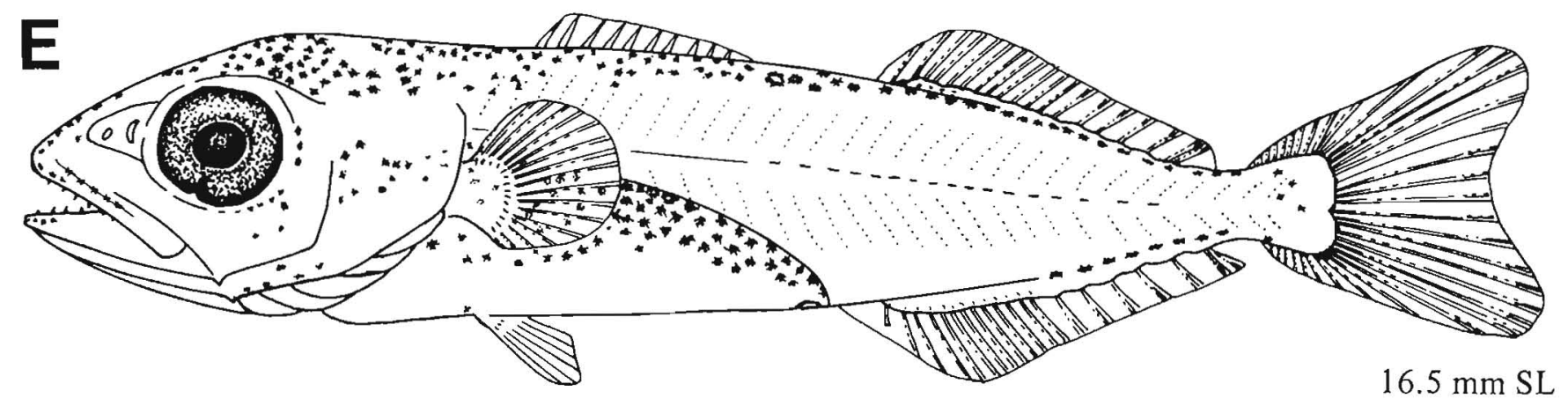

Figures A-E, Kramer 1960 (redrawn). 


\section{MERISTICS}

$\begin{array}{lll}\text { Vertebrae } & \text { Total: } 22-22-23 \\ & \text { Precaudal: } 10-10-10 \\ & \text { Caudal: } 12-12-13 \\ \text { Branchiostegal rays } & 5-\mathrm{X}-6 & \\ \text { Caudal fin } & \text { X, } 16, \mathrm{X} & \\ \text { Pelvic fin }^{\mathrm{a}} & \text { Thoracic } & \\ & \text { S: } 1-1-1 & \text { R: } 4-4-4 \\ \text { Dorsal fin }^{\mathrm{a}} & \text { S: } 2-2-2 & \text { R: } 24-24-24 \\ \text { Pectoral fin }_{\text {Anal fin }}^{\mathrm{a}} & \text { R: } 17-\mathrm{X}-20 & \\ \text { Gill rakers } & \text { S: } 14-\mathrm{X}-17 & \\ & \text { U: } 4-\mathrm{X}-6 & \text { L: } 11-\mathrm{X}-14\end{array}$

L.IFE HISTORY

$\begin{array}{ll}\text { Range } & \begin{array}{c}\text { South of southern California to } \\ \text { Washington, 46-48 } 30^{\prime} \mathrm{N}\end{array} \\ \text { Ecology } & \text { Epi- and mesopelagic } \\ \text { ELH pattern } & \begin{array}{c}\text { Parity and eggs unknown, } \\ \text { pelagic larvae }\end{array} \\ \text { Spawning } & \text { Season: Spring-early summer } \\ & \text { Area: } \\ & \text { Mode: } \\ & \text { Migration: } \\ \text { Fecundity } & \text { Range/function: } \\ \text { Age at first maturity } & \\ \text { Longevity } & \end{array}$

\footnotetext{
${ }^{a}$ Meristics for larvae and adults are different: Larvae Adults Dorsal fin rays $24 \quad 11-14$ Anal fin rays $\quad 18 \quad 13-15$ Pelvic fin rays 4 absent ${ }^{b}$ Fitch and Lavenberg 1971

${ }^{c}$ Based on illustrations only, specimens were not available.

${ }^{d}$ G.D. Johnson, Natl. Mus. Nat. Hist., Wash., D.C. 20560 , pers. commun., 7 Nov. 1986.

Ref: Leis and Richards 1984, Nishikawa 1987.
}

\section{EARLY LIFE HISTORY DESCRIPTION}

\section{EGGS}

Diameter

No. of oil globules

Oil globule diameter

Yolk

Envelope

Hatch size $\quad<3.5 \mathrm{~mm} \mathrm{SL}$

Incubation time/temp.

Pigment

Diagnostic characters

\section{LARVAE}

Preanal length

\section{Length at flexion}

Length at transformation

Sequence of fin development

$\sim 60 \%$ SL, decreasing with development

Pigment $^{\mathrm{c}}$

- Few spots on upper jaw

- With development above urostyle

- Lightly on caudal fin, hypural region with several spots

- Gut

- With development on pectoral fin rays

Diagnostic characters

- Morphology

-Deep-bodied but not as kite-shaped as acanthurids

-Large square-shaped head with small terminal mouth

-Extensive head spination

-With development, minute spines on soft rays and along body surface

-Dorsal and pelvic spines elongate, finely serrated

- Loss of meristic elements with growth

- Not shown on figured

-Spines on ascending process of premaxillary bone (important feature uniquely shared with Zanclus canescens, a closely related acanthuroid, and acanthurids)

-Small dorsal spine anterior to first dorsal spine appears later in development 

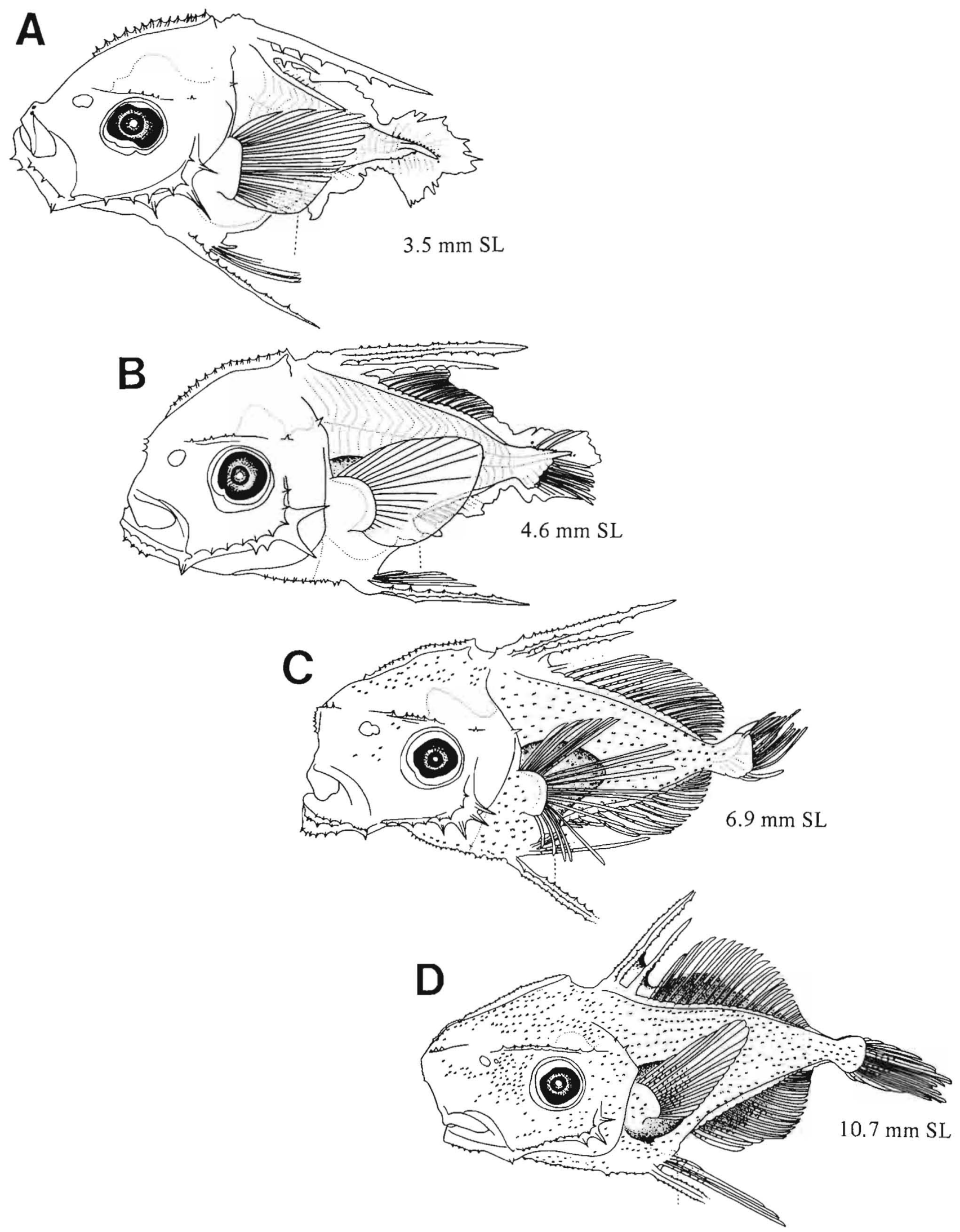

Figures A-D, Nishikawa 1987 (western Pacific specimen). 
MERISTICS

Vertebrae

Total: $56-59-62$

Precaudal: 22-24-25 (adults) ${ }^{\mathrm{a}}$

Caudal: $33-35-38$

Branchiostegal rays

7-7-7

Caudal fin

Pelvic fin

$11-14,9+8,10-13$

Thoracic
S: $1-1-1$
R: $5-5-5$

Dorsal fin

S: $3-3-3$

R: $34-39-43$

Pectoral fin

R: $18-\mathrm{X}-21$

Anal fin

S: $3-3-3$

R: $20-\mathrm{X}-29$

Gill rakers

$\mathrm{U}: 4-\mathrm{X}-6$

L: $12-\mathrm{X}-14$

\section{LIFE HISTORY}

\begin{tabular}{|c|c|}
\hline Range & $\begin{array}{l}\text { South of southern California to } \\
\text { Gulf of Alaska, } 54-60^{\circ} \mathrm{N}\end{array}$ \\
\hline Ecology & Epipelagic, 0-91 m \\
\hline ELH pattern & $\begin{array}{l}\text { Oviparous, pelagic eggs, } \\
\text { pelagic larvae }\end{array}$ \\
\hline Spawning & $\begin{array}{l}\text { Season: } \\
\text { Area: } \\
\text { Mode: } \\
\text { Migration: }\end{array}$ \\
\hline $\begin{array}{l}\text { Fecundity } \\
\text { Age at first maturity } \\
\text { Longevity }\end{array}$ & Range/function: \\
\hline
\end{tabular}

${ }^{a}$ Number of preanal myomeres appears to decrease with development (B. Sumida, NMFS Southwest Fish. Cent., P.O. Box 271, La Jolla, CA 92038, pers. commun., 16 Dec. 1986).

Ref: Ahlstrom et al. 1976, Horn 1984.

\section{EARLY LIFE HISTORY DESCRIPTION}

\section{EGGS}

Diameter

No. of oil globules

$1.52-1.80 \mathrm{~mm}$

Oil globule diameter

One

Yolk

$0.30-0.44 \mathrm{~mm}$ (large)

Envelope

Homogeneous

Clear, smooth

Hatch size

Incubation time/temp.

Pigment

- Dorsal pigment along body

- Ventral pigment from head to tip of tail

- Underside of oil globule becomes pigmented with development

\section{Diagnostic characters}

\section{LARVAE}

Preanal length

$50-58 \% \mathrm{SL}$

Length at flexion

$\sim 9.3-11.0 \mathrm{~mm} \mathrm{SL}$

Length at transformation $\sim 20 \mathrm{~mm} \mathrm{SL}$

Sequence of fin

development

Caudal, 2nd dorsal (rays), anal, pectorals, 1 st dorsal (spines), pelvics

Pigment

- Dorsal and ventral body midline pigment

- Lateral line dashes develop during flexion

- Head and tail pigment increases with development

- Become heavily, uniformly pigmented including fin membranes

Diagnostic characters

Distinguished from Tetragonurus cuvieri (p. 560) by

- High myomere counts (56-62)

- Preflexion: Dorsal body pigment over posterior half of body; only on tail in $T$. cuvieri

- Flexion: No preopercular spines

- Postflexion

-Dorsal and anal spines weak, few (three) dorsal spines

-More dorsal and anal fin rays than in $T$. cuvieri 

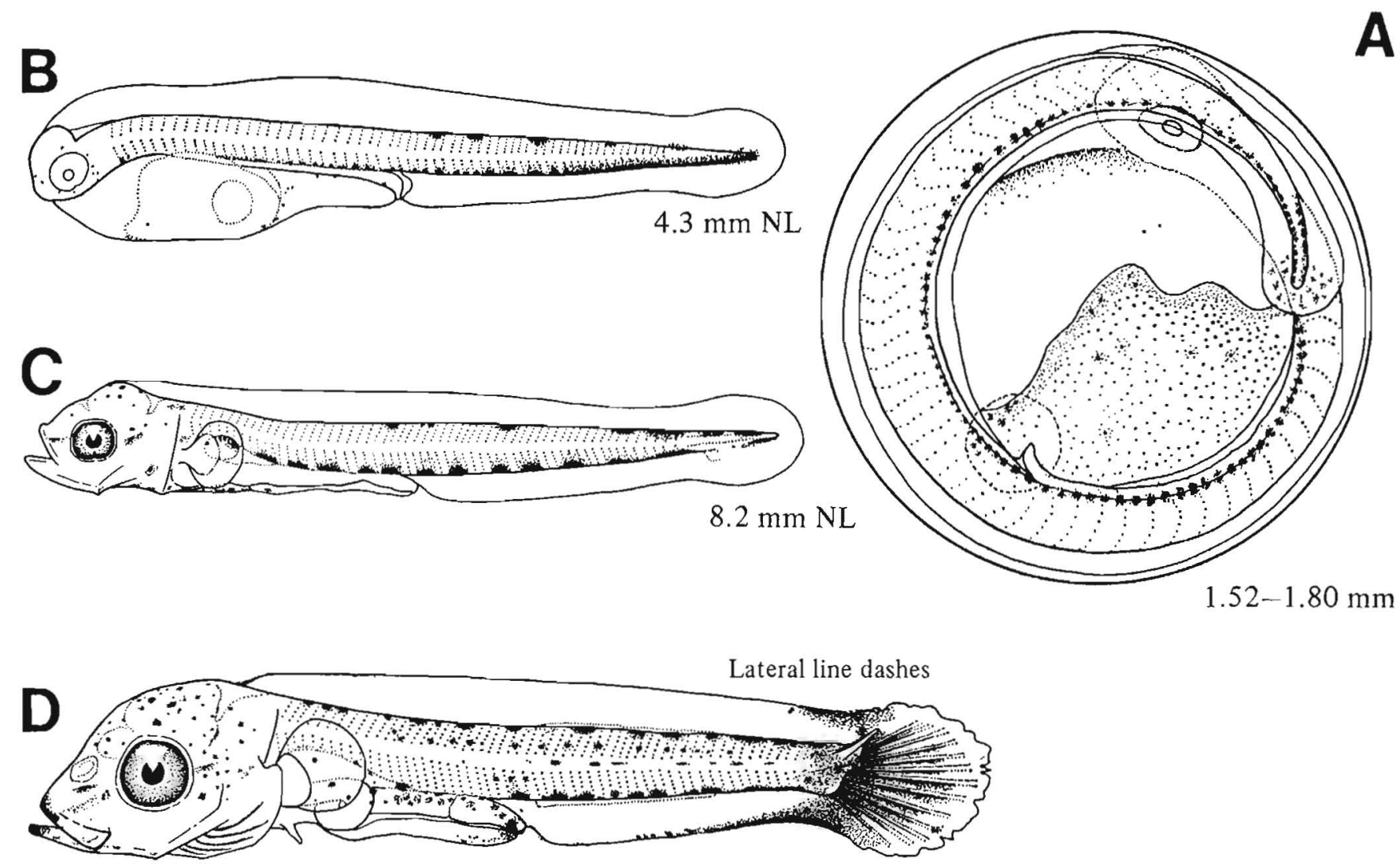

$10.4 \mathrm{~mm} \mathrm{NL}$
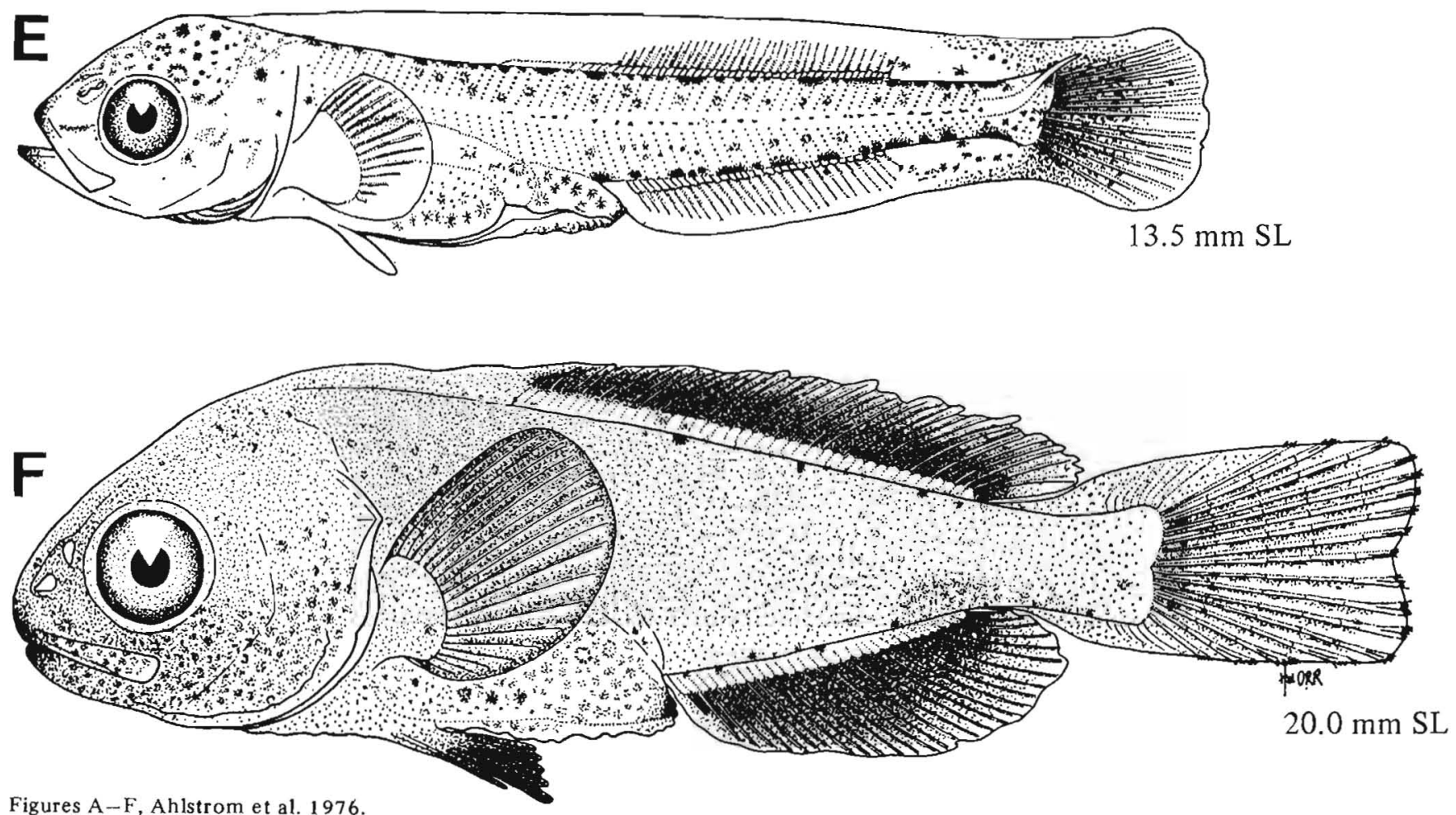


\section{MERISTICS}

Vertebrae

Total: $52-53-57$

Precaudal: 25-28-29 (adults) ${ }^{\text {a }}$

Caudal: 24-26-28

Branchiostegal rays

Caudal fin

Pelvic fin

5-6-6

$9-13,9+8,9-12$

Thoracic
S: $1-1-1$
R: 5-5-5

Dorsal fin

S: $15-17-21$

Pectoral fin

R: $14-\mathrm{X}-17$

Anal fin

S: $2-2-2$

R: $10-12-17$

Gill rakers

$\mathrm{U}:$ 6-6-6

R: $9-X-15$

L: $7-\mathrm{X}-14$

\section{LIFE HISTORY}

$\begin{array}{ll}\text { Range } & \begin{array}{c}\text { South of southern California to } \\ \text { Aleutian Is., } 51-55^{\circ} \mathrm{N} \\ \text { Epipelagic } \\ \text { Ecology }\end{array} \\ \begin{array}{l}\text { Oviparous, pelagic eggs, } \\ \text { pelagic larvae }\end{array} \\ \text { Spawning } & \begin{array}{l}\text { Season: } \\ \text { Area: }\end{array} \\ & \text { Mode: } \\ & \text { Migration: } \\ \text { Fecundity } & \text { Range/function: } \\ \text { Age at first maturity } & \\ \text { Longevity } & \end{array}$

\section{EARLY LIFE HISTORY DESCRIPTION}

\section{EGGS}

Diameter

No. of oil globules

$1.10-1.28 \mathrm{~mm}$

Oil globule diameter

Yolk

Envelope

Hatch size

One

0.25-0.30 $\mathrm{mm}$ (amorphous)

Homogeneous

Smooth, golden

Incubation time/temp.

Pigment

- Oil globule

- Distinctive pigment on embryo; double dorsal line separating prior to head, outlining brain, and extending forward to snout

- Ventral pigment above digestive tract, continuing along tail

Diagnostic characters

- Pigment

\section{LARVAE}

Preanal length $60-70 \%$ SL

Length at flexion

7.6-10.1 mm SL

Length at transformation $\sim 21.4 \mathrm{~mm} \mathrm{SL}$

Sequence of fin

Caudal; 2nd dorsal (rays), development anal, and pectorals; 1 st dorsal (spines); pelvics

Pigment

- Tail

- Lateral line notable during flexion

- Eye bar

- Dorsal midline on caudal peduncle extending anteriorly with development

Diagnostic characters

Distinguished from Icichthys lockingtoni (p. 558) by

- Myomere count (52-57)

- Pigment

- Notochord tip and base of caudal

-Ventral row over gut to tail

- Early formation of opposing 2nd dorsal and anal fins

- Slender body with long caudal peduncle

\footnotetext{
"Number of preanal myomeres appears to decrease with development (B. Sumida, NMFS Southwest Fish. Cent., P.O. Box 271, La Jolla, CA 92038, pers. commun., 16 Dec. 1986).
}

Ref: Ahlstrom et al. 1976. 
B

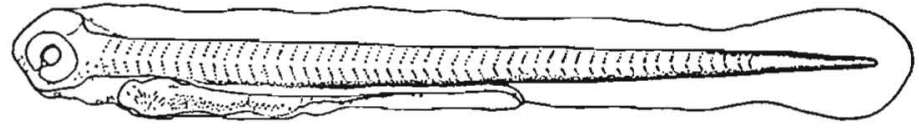

$4.1 \mathrm{~mm} \mathrm{NL}$

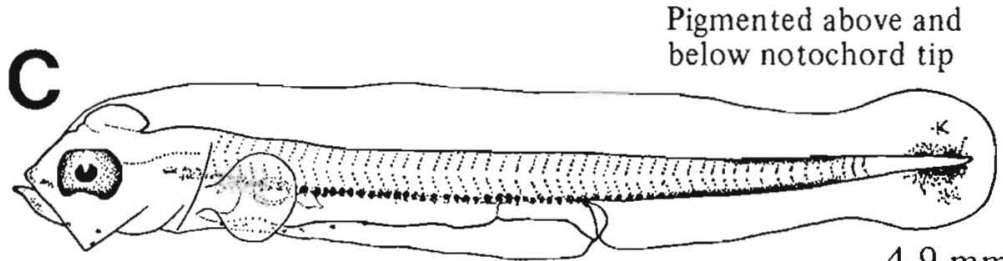

$4.9 \mathrm{~mm} \mathrm{NL}$
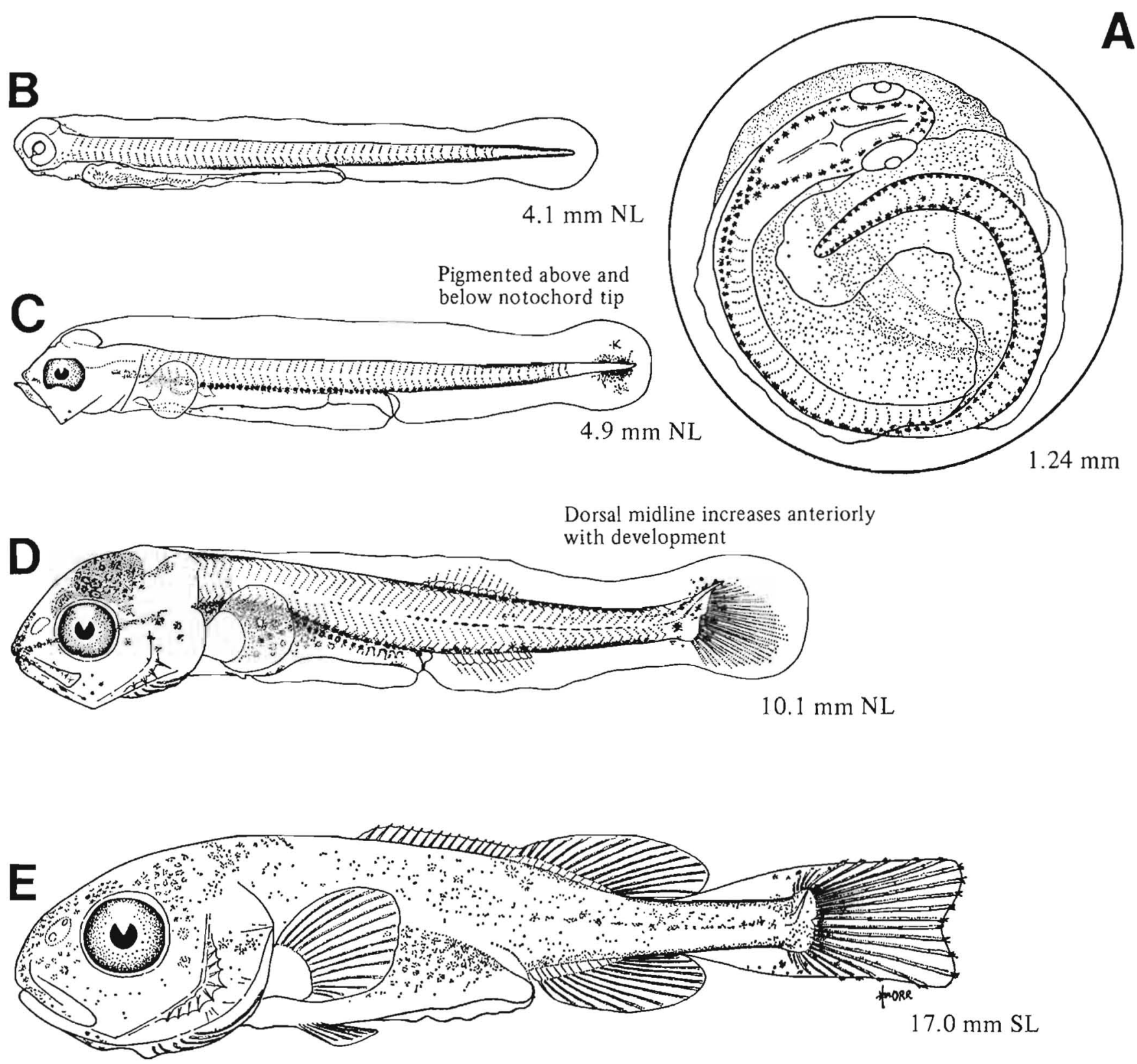

Figures A-E, Ahlstrom et al. 1976. 


\section{MERISTICS}

\begin{tabular}{|c|c|}
\hline Vertebrae & $\begin{array}{l}\text { Total: } 28-30-31 \\
\text { Precaudal: } 11-13-14 \text { (adults) }^{a} \\
\text { Caudal: } 17-17-19\end{array}$ \\
\hline Branchiostegal rays & $6-6-6$ \\
\hline Caudal fin & $7-9,9+8,6-8$ \\
\hline Pelvic fin & Absent \\
\hline Dorsal fin & S: $2-3-4 \quad R: 41-45-48$ \\
\hline Pectoral fin & $\mathrm{R}: 19-21-23$ \\
\hline Anal fin & $S: 2-3-3 \quad R: 35-39-44$ \\
\hline Gill rakers & $\mathrm{U}: 3-3-3 \quad \mathrm{~L}: 11-11-11$ \\
\hline \multicolumn{2}{|l|}{ LIFE HISTORY } \\
\hline Range & $\begin{array}{l}\text { South of southern California to } \\
\text { Brit. Col., } 48^{\circ} 30^{\prime}-55^{\circ} \mathrm{N}\end{array}$ \\
\hline Ecology & Epipelagic, 9-91 m \\
\hline ELH pattern & $\begin{array}{l}\text { Oviparous, pelagic eggs, } \\
\text { pelagic larvae }\end{array}$ \\
\hline Spawning & $\begin{array}{l}\text { Season: Spring-summer } \\
\text { (California) }^{\mathrm{b}}\end{array}$ \\
\hline & Area: \\
\hline & Mode: \\
\hline & Migration: \\
\hline $\begin{array}{l}\text { Fecundity } \\
\text { Age at first maturity } \\
\text { Longevity }\end{array}$ & Range/function: \\
\hline
\end{tabular}

\section{EARLY LIFE HISTORY DESCRIPTION}

\section{EGGS}

Diameter

No. of oil globules One

Oil globule diameter $\quad 0.2 \mathrm{~mm}$ (in yolksac larva)

Yolk

Envelope

Hatch size

$1.8-2.0 \mathrm{~mm} \mathrm{SL}$

Incubation time/temp.

Pigment

Diagnostic characters

\section{LARVAE}

Preanal length

Length at flexion

Length at transformation $\sim 20 \mathrm{~mm} \mathrm{SL}$

Sequence of fin development

Pigment

- Anterior body

- Median ventral series of melanophores from isthmus to anus

- Large melanophores on head and anterior half of body, becoming uniformly pigmented on anterior $3 / 4$ of body, later over whole body

Diagnostic characters

- Pigment

- Increase in relative body depth during ontogeny

- Absence of pelvic fins

${ }^{\text {a }}$ Number of preanal myomeres appears to decrease with development (B. Sumida, NMFS Southwest Fish. Cent., P.O. Box 271, La Jolla, CA 92038, pers. commun., 16 Dec. 1986).

${ }^{\mathrm{b}}$ Fitch and Lavenberg 1971

Ref: D'Vincent et al. 1980 

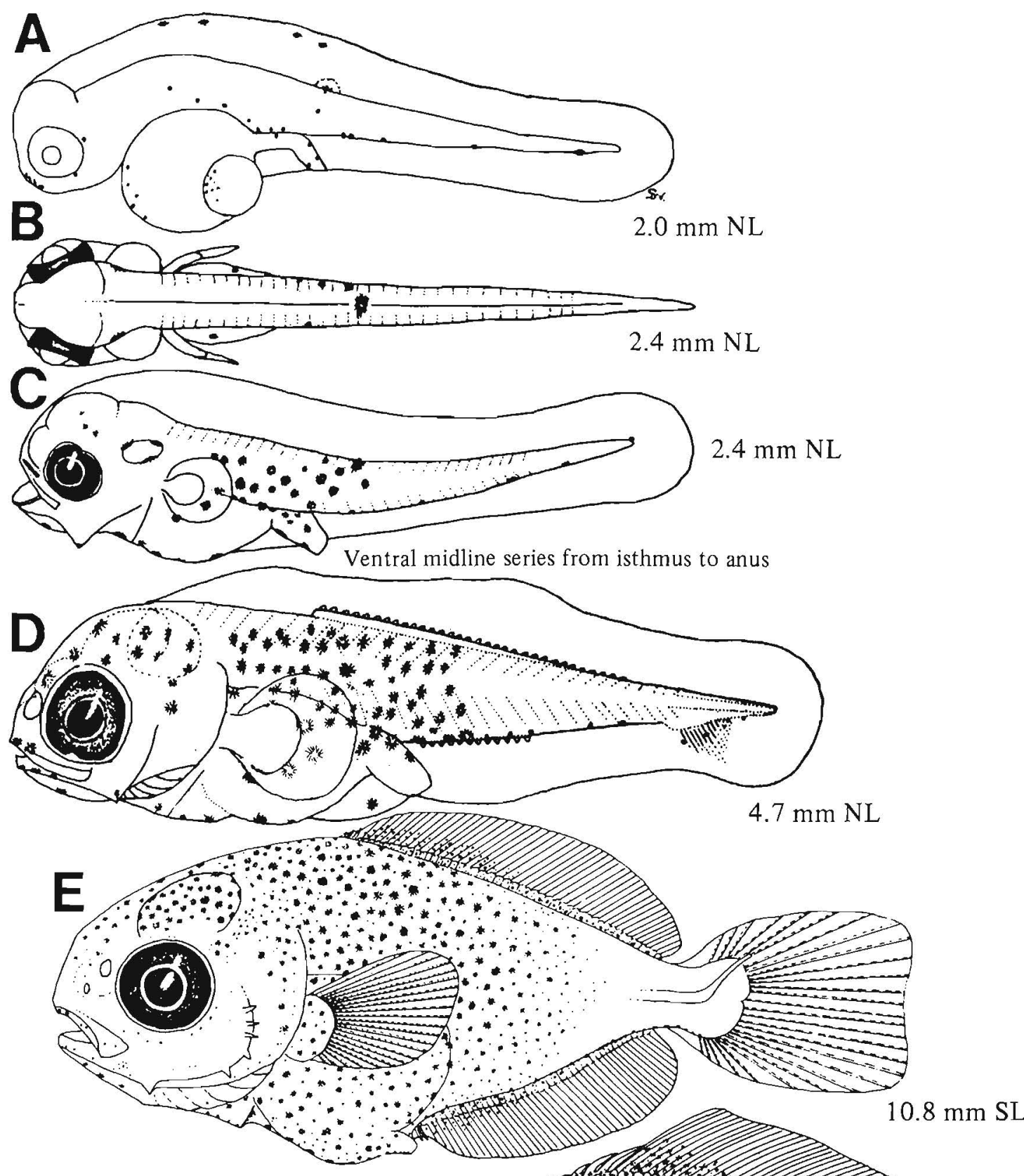

Figures $A-F$ (B, dorsal view), D'Vincent et al. 1980.

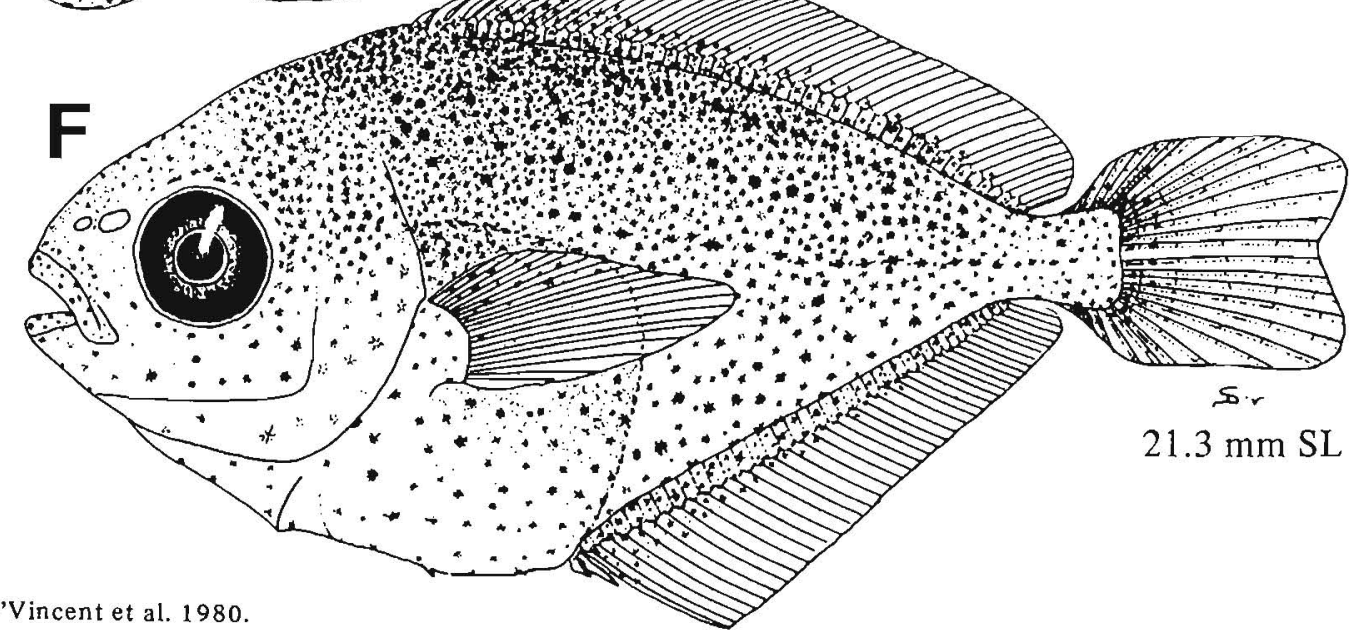





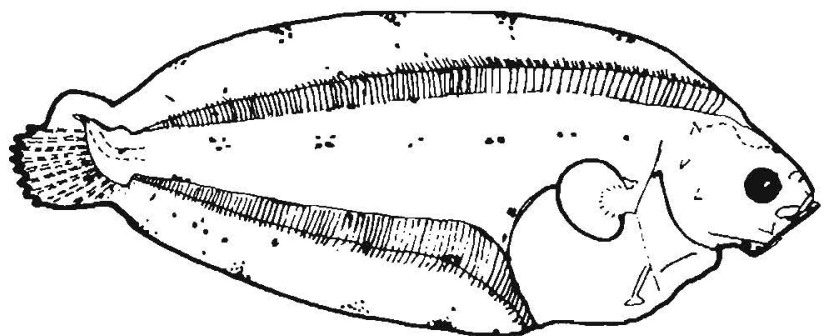

\section{Pleuronectiformes}

Pleuronectiforms are benthic fishes that are asymmetrical with both eyes on one side of the head. Asymmetry is also reflected in dentition, cranial osteology, pelvic fin placement and morphology, pigment, and squamation. Worldwide in distribution, there are 7 families, with well over 120 genera and 500 species (Ahlstrom et al. 1984a). Most families are defined as dextral (eyes on right side) or sinistral (eyes on left side), although some species are indiscriminate. A total of 3 families with 31 species occur in the study area. Much is known about the early life history of many species. Eggs of most are pelagic, spherical, and have homogeneous yolks. Chorions may be smooth, striated, or ornamented with raised polygonal patterns (Ahlstrom et al. 1984a). Early larvae are bilaterally symmetrical and swim upright. Of help in larval identification are body shape (short gut, elongate body), pigment patterns (postanal bands, pigment on the urostyle and finfolds), and meristic characters. Size at transformation and the development of spines and elongate dorsal or pelvic fin rays can be diagnostic. During transformation, the eyes migrate to their adult position and the larvae assume the distinctive flat profile unique to the order. At this point, juveniles settle and become benthic inhabitants.

Families in study area: Paralichthyidae

Pleuronectidae

Cynoglossidae 

Table 49

Characters useful in identifying pleuronectiform larvae. Size data are after Ahlstrom et al. (1984a), in part. All measurements are in millimeters NL or SL. Taxa listed alphabetically within families.

\begin{tabular}{|c|c|c|c|c|}
\hline Species $^{\mathbf{a}}$ & $\begin{array}{c}\text { Size at } \\
\text { hatching }\end{array}$ & $\begin{array}{c}\text { Size at } \\
\text { notochord flexion }\end{array}$ & $\begin{array}{c}\text { Size at } \\
\text { transformation }\end{array}$ & Total myomeres \\
\hline \multicolumn{5}{|l|}{ Paralichthyidae } \\
\hline Citharichthys sordidus & 2.0 & $10.0-11.4$ & $20.0-39.0$ & $39-40$ \\
\hline C. stigmaeus & 2.0 & $9.2-10.2$ & $24.0-36.0$ & $36-39$ \\
\hline \multicolumn{5}{|l|}{ Pleuronectidae } \\
\hline Acanthopsetta nadeshnyi & $<3.0$ & $8.4-9.9$ & $20.0-24.0$ & $39-40^{\circ}$ \\
\hline Atheresthes evermanni & $<8.4$ & $11.5-15.0$ & - & - \\
\hline A. stomias & $<10.0$ & $10.0-12.0$ & $33.0-?$ & $47-50$ \\
\hline Clidoderma asperrimum & - & - & - & $42-44^{b}$ \\
\hline Embassichthys bathybius & 9.0 & $15.4-16.2$ & $16.2-?$ & $57-65$ \\
\hline Eopsetta jordani & 2.8 & - & - & $41-45$ \\
\hline Glyptocephalus stelleri & $4.1-5.2$ & $15.0-17.0$ & $19.0-48.0$ & $52-60$ \\
\hline G. zachirus & $5.0-6.0$ & $15.3-24.0$ & $49.0-72.0$ & $63-66$ \\
\hline Hippoglossoides elassodon & $5.3-6.9$ & $9.0-10.2$ & $18.0-?$ & $43-47$ \\
\hline H. robustus & 4.0 & $11.0-?$ & $>28.6$ & 44 \\
\hline Hippoglossus stenolepis & $7.8-8.5$ & $13.6-17.8$ & $14.7-24.1$ & $49-51$ \\
\hline Inopsetta ischyra & - & - & - & 41 \\
\hline Isopsetta isolepis & $2.7-2.9$ & $9.1-14.0$ & $15.0->21.9$ & $41-42$ \\
\hline Lepidopsetta bilineara & $3.4-4.0$ & $8.4-9.9$ & $>17.7$ & $39-42$ \\
\hline Limanda aspera & $2.2-2.8$ & $7.5-9.5$ & $10.0-?$ & $40-41$ \\
\hline L. proboscidea & $<4.8$ & - & - & - \\
\hline Liopsetta glacialis & 3.7 & - & - & $37-41$ \\
\hline Lyopsetta exilis & 5.6 & $9.0-10.9$ & $15.7-24.7$ & $43-47$ \\
\hline Microstomus pacificus & 6.0 & $10.0-15.0$ & $20.0->45.0$ & $50-55$ \\
\hline Parophrys vetulus & $2.3-2.8$ & $8.8-10.5$ & 17.5-? & $42-47$ \\
\hline Platichthys stellatus & $1.9-2.1$ & $5.5-6.0$ & $8.0-?$ & $35-38$ \\
\hline Pleuronectes quadrituberculatus & 5.6 & $8.0-10.0$ & 10.0-? & $41-42$ \\
\hline Pleuronichthys coenosus & 3.9 & $6.2-8.5$ & $8.2->11.4$ & $37-39$ \\
\hline P. decurrens & $4.9-5.5$ & $7.8-11.0$ & $10.5->21.0$ & $38-41$ \\
\hline Psettichthys melanostictus & $<3.0$ & $8.0-10.0$ & $>22.6$ & $38-41$ \\
\hline Reinhardrius hippoglossoides & $10.0-16.0$ & $25.0-27.0$ & $45.0-65.0$ & $61-64$ \\
\hline \multicolumn{5}{|l|}{ Cynoglossidae } \\
\hline Symphurus atricauda & 1.9 & $9.4-10.8$ & $19.0-24.2$ & $50-52$ \\
\hline \multicolumn{5}{|c|}{${ }^{a}$ The following nomenclatural changes have been recommended by Sakamoto (1984): } \\
\hline Glyptocephalus (= Errex) zachirus & Liopsetta & nectes) & & \\
\hline Isopsetta $\quad(=$ Pleuronectes $)$ & Parophrys & nectes) & & \\
\hline Lepidopsetta (= Pleuronectes) & Lyopsetta & & & \\
\hline Limanda $\quad$ (= Pleuronectes $)$ & & & & \\
\hline
\end{tabular}




\section{MERISTICS}

$\begin{array}{ll}\text { Vertebrae } & \text { Total: } 39-\mathrm{X}-40 \\ & \text { Precaudal: } 11-11-12 \\ & \text { Caudal: } 27-28-29 \\ \text { Branchiostegal rays } & 6-\mathrm{X}-7 \\ \text { Caudal fin } & \text { X, } 7+6, \mathrm{X} \\ & \text { Total rays }=17^{\mathrm{a}} \\ & \text { Thoracic } \\ \text { Pelvic fin } & \text { R: } 6-6-6 \\ & \text { R: } 86-91-102 \\ \text { Dorsal fin } & \text { R: } 12-12-12 \\ \text { Pectoral fin } & \text { R: } 67-72-81 \\ \text { Anal fin } & \text { U: } 6-\mathrm{X}-9 \\ \text { Gill rakers } & \\ & \\ & \\ \text { LIFE HISTORY } 12-13-16\end{array}$

$\begin{array}{ll}\text { Range } & \begin{array}{c}\text { South of southern California to } \\ \text { Bering Sea, } 54-66^{\circ} \mathrm{N}\end{array} \\ \text { Ecology } & \text { Epi-, meso-, and bathybenthal, } \\ & 0-549 \mathrm{~m} \\ \text { Oviparous, pelagic eggs, } & \text { pelagic larvae } \\ \text { ELH pattern } & \text { Season: Mar-May (Puget } \\ \text { Spawning } & \text { Sound) }{ }^{\mathrm{b}} \text {; July-Sept (Calif.; } \\ & \text { may spawn twice each } \\ & \text { season) } \\ & \text { Area: } \\ & \text { Mode: } \\ & \text { Migration: } \\ \text { Fecundity } & \text { Range/function: } \\ \text { Age at first maturity } & 2-3 \mathrm{yr}^{\mathrm{b}} \\ \text { Longevity } & \end{array}$

a 11 branched rays.

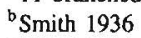

'Arora 1951

'Ahlstrom et al. 1984a

${ }^{e}$ Early preflexion larvae $(<6-7 \mathrm{~mm} \mathrm{SL})$ of $C$. sordidus and $C$. stigmaeus are not identifiable to species until the elongate dorsal fin rays form on $C$. sordidus $(\sim 7 \mathrm{~mm}$ SL). Preflexion larvae collected in our study area do not resemble the published illustrations of either species (Ahlstrom and Moser 1975); they have ventral gut pigment extending more anteriorly, a series of postanal ventral midline melanophores, no finfold pigment, and less intense caudal pigment (Fig. A). Specimens $>6.9 \mathrm{~mm}$ SL that have elongated dorsal fin rays are $C$, sordidus (Fig. B), but do not exhibit the intense caudal pigment shown by Ahlstrom and Moser (1975). Our specimens indicate a decrease in intensity of caudal pigment with development for $C$. sordidus and an increase for $C$. stigmaeus. The preflexion larva of $C$. sordidus illustrated by Ahlstrom and Moser (1975) may belong to another species probably south of our study area, most likely $C$. xanthostigma (B. Sumida, NMFS Southwest Fish. Cent., Box 271, La Jolla, CA 92038, pers. commun., 16 Dec. 1986).

Ref: Ahlstrom 1965, Ahlstrom and Moser 1975, Ahlstrom et al. 1984a.

\section{EARLY LIFE HISTORY DESCRIPTION}

\section{EGGS}

Diameter

No. of oil globules

Oil globule diameter

Yolk

Envelope

Hatch size

$0.55-0.77 \mathrm{~mm} ;{ }^{\mathrm{c}}$

Incubation time/temp.

Pigment

One

0.09-0.12 mm

Homogeneous

Smooth

$\sim 2.0 \mathrm{~mm} \mathrm{SL}$

\section{Diagnostic characters}

We cannot identify Citharichthys spp. eggs to species. We collect two types of paralichthyid eggs: the first type has an average diameter of $\sim 0.64-0.68 \mathrm{~mm}$, and the other type averages $\sim 0.78-0.88 \mathrm{~mm}$.

\section{LARVAE $^{\circ}$}

Preanal length

Length at flexion

Length at transformation

Sequence of fin development
$<50 \%$ SL $<10.0-11.4 \mathrm{~mm} \mathrm{SL}$ 20->39 mm SL

Dorsal, anal, caudal, pelvics, pectorals or caudal, dorsal and anal, pelvics, pectorals

\section{Pigment}

- Ventral body midline pigment posterior to anus, coalesces later into two melanophores

- Band at 3/4 BL

- Posteriorly on gut

- Tips of elongate dorsal and pelvic fin rays

Diagnostic characters

- Elongate dorsal and pelvic fin rays 
A

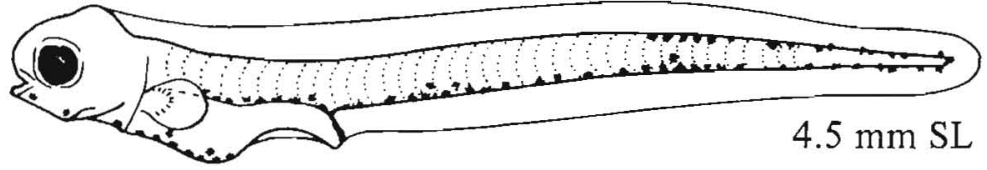

B

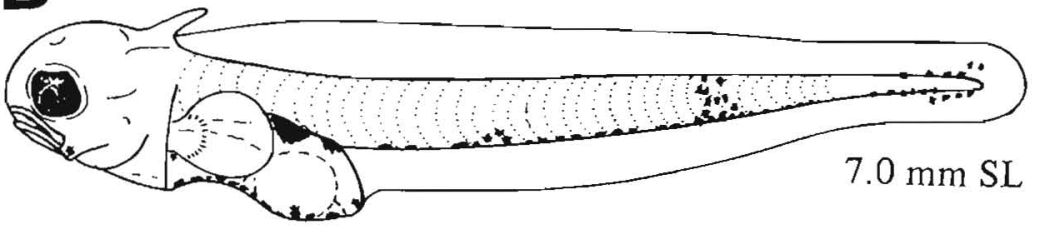

Elongate dorsal and pelvic fin rays

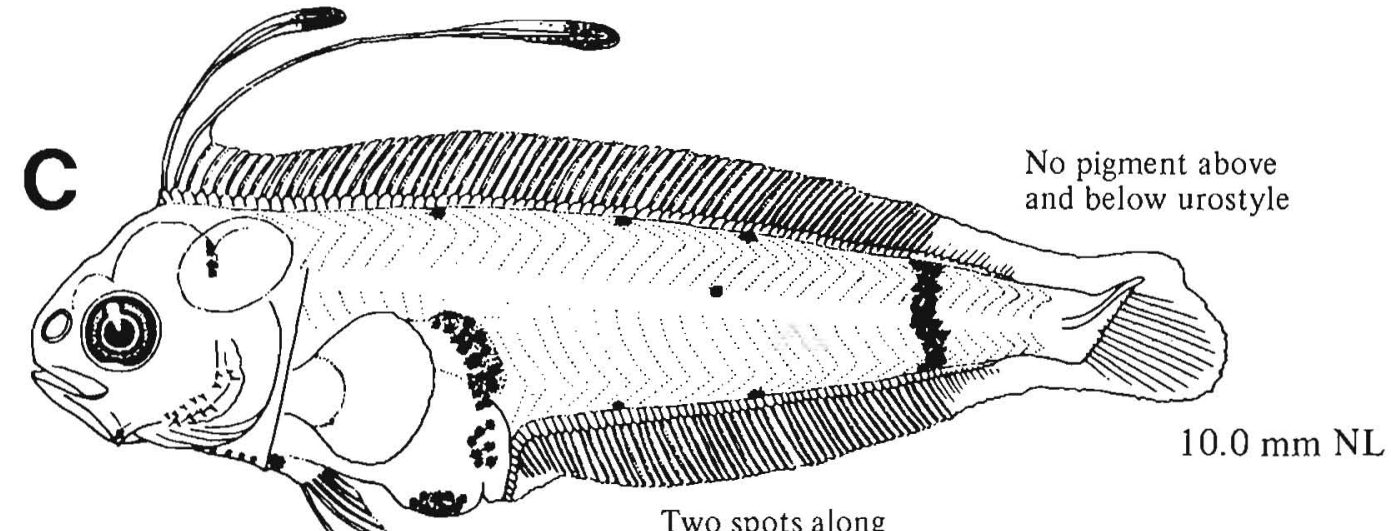

Two spots along

ventral body midline

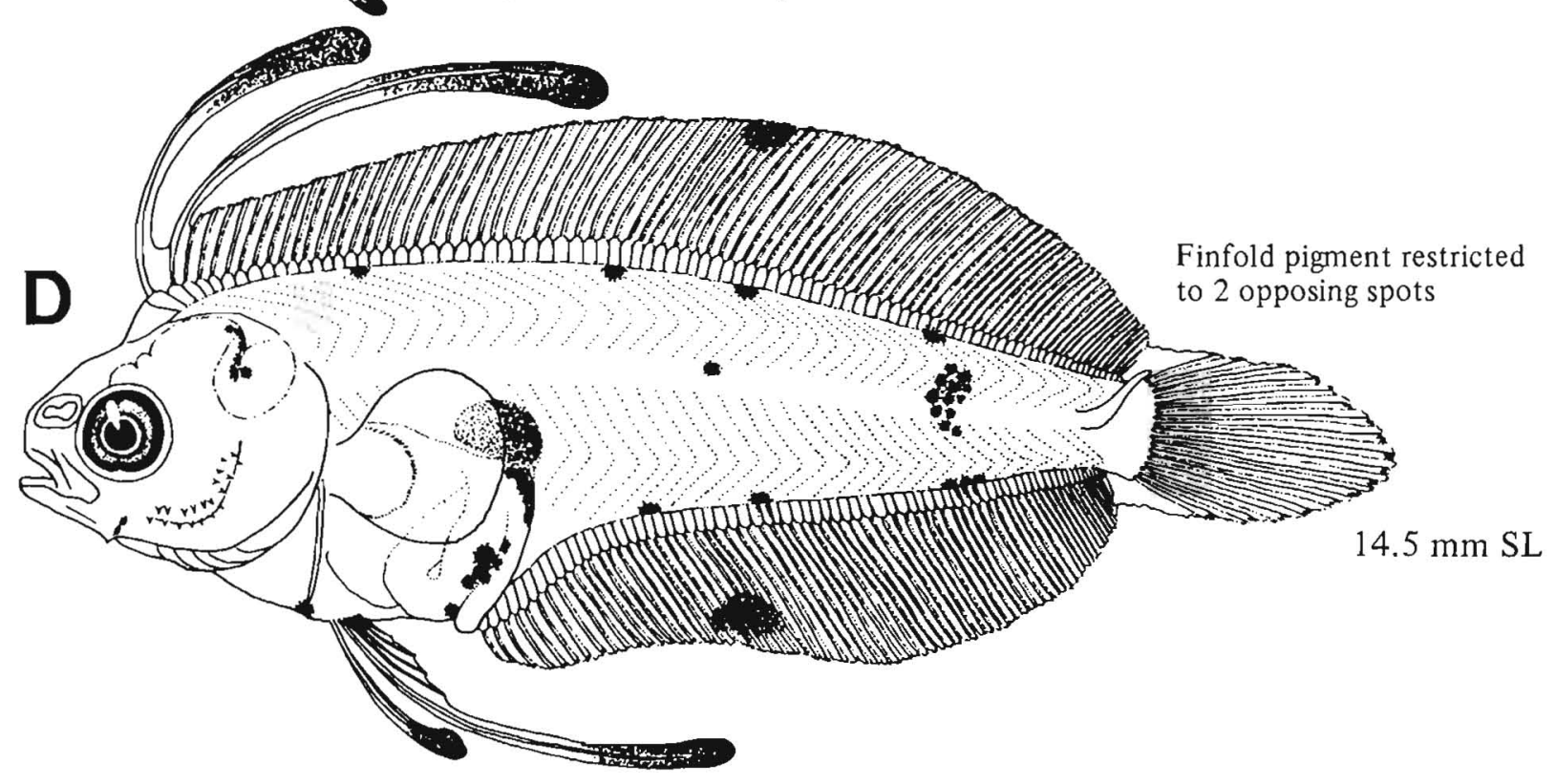

Figures A-B, NWAFC originals (B. Vinter); C-D, Ahlstrom and Moser 1975. 
MERISTICS

\begin{tabular}{|c|c|}
\hline Vertebrae & $\begin{array}{l}\text { Total: } 36-37-39 \\
\text { Precaudal: } 9-10-10 \\
\text { Caudal: } 27-28-29\end{array}$ \\
\hline Branchiostegal rays & $6-X-7$ \\
\hline Caudal fin & $\begin{array}{l}2,7+6,2 \\
\text { Total rays }=17^{\text {a }}\end{array}$ \\
\hline Pelvic fin & $\begin{array}{l}\text { Thoracic } \\
\text { R: } 6-6-6\end{array}$ \\
\hline Dorsal fin & R: $75-84-97$ \\
\hline Pectoral fin & $\mathrm{R}: 12-12-12$ \\
\hline Anal fin & R: $58-64-77$ \\
\hline $\begin{array}{l}\text { Gill rakers } \\
\text { LIFE HISTORY } \\
\end{array}$ & $\mathrm{U}: 3-\mathrm{X}-5$ \\
\hline Range & $\begin{array}{l}\text { South of southern California to } \\
\text { SE Alaska, } 55-59^{\circ} \mathrm{N}\end{array}$ \\
\hline Ecology & Epi- and mesobenthal, 0-366 m \\
\hline ELH pattern & $\begin{array}{l}\text { Oviparous, pelagic eggs, } \\
\text { pelagic larvae }\end{array}$ \\
\hline Spawning & $\begin{array}{l}\text { Season: Spring-summer (Calif.) } \\
\text { Area: } \\
\text { Mode: } \\
\text { Migration: }\end{array}$ \\
\hline $\begin{array}{l}\text { Fecundity } \\
\text { Age at first maturity } \\
\text { Longevity }\end{array}$ & Range/function: \\
\hline
\end{tabular}

\section{EARLY LIFE HISTORY DESCRIPTION}

\section{EGGS}

Diameter

No. of oil globules

$0.75-0.83 \mathrm{~mm}$

Oil globule diameter

Yolk

Envelope

Hatch size

One

0.09-0.10 mm

Homogeneous

Smooth

$2 \mathrm{~mm} \mathrm{SL}$

Incubation time/temp.

Pigment

\section{Diagnostic characters}

See notes on $C$. sordidus (p. 568)

\section{LARVAE}

Preanal length

Length at flexion

9.2-10.2 $\mathrm{mm} \mathrm{SL}$

Length at transformation $24.0-35.5 \mathrm{~mm} \mathrm{SL}$

Sequence of fin development

Dorsal, anal, caudal, pelvics, pectorals or caudal, dorsal and anal,

Pigment (see $C$. sordidus) pelvics, pectorals

- Urostyle pigment increases in intensity to flexion stage, then fades

- Small melanophores along ventral body midline posterior to anus; these migrate onto anal fin pterygiophores in postflexion larvae

- Band at 3/4 BL, lost after flexion

- Caudal fin

Diagnostic characters

- Lack of elongate fin rays

a 11 branched rays.

${ }^{b}$ Ahlstrom and Moser 1975

Ref: Ahlstrom et al. 1984a. 
No elongate fin rays
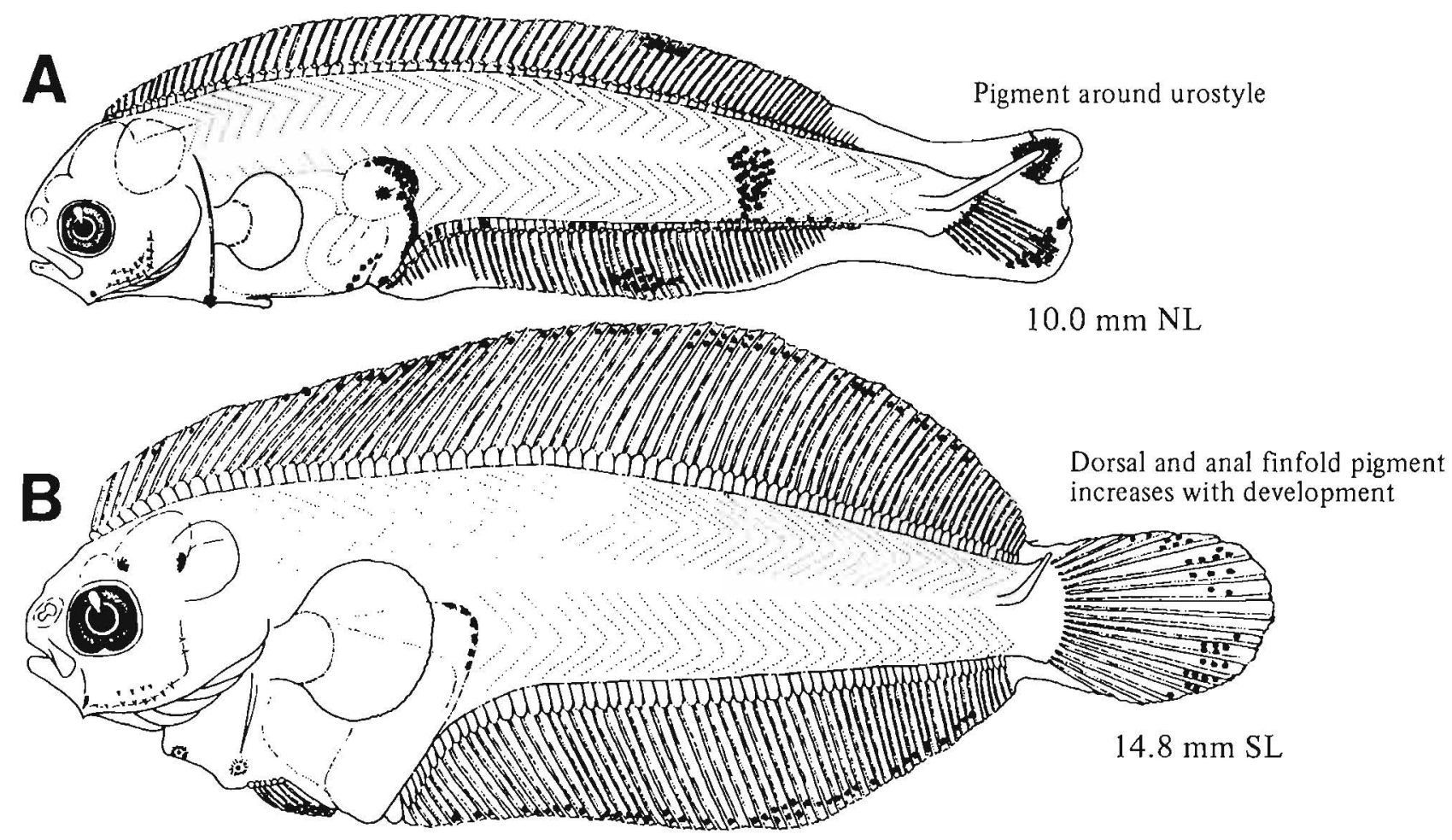

The majority of species in the order (27 out of a total of 31 ) belong to the family Pleuronectidae. Ahlstrom et al. (1984a) summarize the following ontogenetic characters for the family.

Egg size ranges from 0.66 to $4.50 \mathrm{~mm}$, yolk is homogeneous, oil globules are absent in most species, and the chorion is smooth (exceptions include Pleuronichthys ritteri and $P$. cornutus). Some light sculpturing of the chorion is seen in several species.

Larvae hatch from 1.7 to $16.0 \mathrm{~mm}$ SL. They possess no elongate dorsal or pelvic fin rays, gut is normal, and spines are limited to the preopercular, otic, and frontal regions, although absent in most.

The caudal fin ray count is not stabilized within pleuronectids, in contrast to most other taxa. Most pleuronectids have 17-19 total caudal fin rays ( 18 is most common). Within species there is variability in the total fin ray count and in the number of rays that are branched. Since variability exists, total counts, number of branched rays, and principal and secondary rays are given when available.

Table 50

Pigment characters and myomere counts that may be useful in the identification of pleuronectid larvae during the preflexion and early flexion stages.*

\begin{tabular}{|c|c|c|c|c|c|c|}
\hline \multirow[b]{3}{*}{ Genus } & \multicolumn{5}{|c|}{ Pigment characters } & \multirow[b]{3}{*}{ Total myomeres } \\
\hline & \multirow[b]{2}{*}{ Number of bands } & \multirow{2}{*}{$\begin{array}{c}\text { Slashlike } \\
\text { hypaxial pigment }\end{array}$} & \multirow[b]{2}{*}{ Urostyle } & \multicolumn{2}{|c|}{ Finfold } & \\
\hline & & & & Dorsal & Anal & \\
\hline \multicolumn{7}{|c|}{ Postanal pigment bands present } \\
\hline Embassichthys & 3 & No & Yes & Yes & Yes & $57-65$ \\
\hline Eopsetta & 2 & No & Yes & No & No & $41-45$ \\
\hline Glyptocephalus & $3-4$ & No & Yes & Yes/no & Yes/no & $52-66$ \\
\hline Hippoglossoides & $3-4$ & Yes & Yes & Yes & Yes & $44-51$ \\
\hline Isopsetta & 3 & No & Yes & No & Yes & 41.42 \\
\hline Lepidopsetta & 1 & No & No, few spots & Yes & Yes & $39-42$ \\
\hline Microstomus & 4 & No & Yes & Yes & Yes & $50-55$ \\
\hline \multicolumn{7}{|c|}{ Postanal pigment bands absent } \\
\hline Acanthopsetta & - & Yes & Yes & No & No & $39-40$ \\
\hline Atheresthes & - & No & Yes/no & No & No & $47-50$ \\
\hline Hippoglossus & - & No & Yes & Yes & Yes & $49-51$ \\
\hline Limanda & - & Yes & No & No & Yes & $40-41$ \\
\hline Liopsetta & - & No & Yes & No & No & $37-41$ \\
\hline Lyopserta & - & Yes & Yes & Yes & Yes & $43-47$ \\
\hline Parophrys & - & Yes & Yes & No & Yes & $42-47$ \\
\hline Platichshys & - & No & Yes & Yes & Yes & $35-38$ \\
\hline Pleuronectes & - & No & No & No & Yes & $41-42$ \\
\hline Pleuronichthys & - & No & Yes & Yes & Yes & $38-41$ \\
\hline Psertichthys & - & No & Yes & Yes & Yes & 38.41 \\
\hline Reinhardtius & - & No & Yes & Yes & Yes & $61-64$ \\
\hline
\end{tabular}

*Characters are discussed only for taxa where at least some early-life-bistory stages are known. Only general trends are presented since pigment may vary from specimen to specimen. In cases where actual specimens were not available, subjective decisions were made based on previously published illustrations. 


\section{MERISTICS}

$\begin{array}{ll}\text { Vertebrae } & \text { Total: } 39-\mathrm{X}-42 \\ & \text { Precaudal: } 9-\mathrm{X}-10 \\ & \text { Caudal: } 30-\mathrm{X}-31 \\ \text { Branchiostegal rays } & 7-\mathrm{X}-8 \\ \text { Caudal fin } & \text { Total rays }=18 \\ \text { Pelvic fin } & \text { Thoracic } \\ & \text { R: } 6-6-6 \\ \text { Dorsal fin } & \text { R: } 67-\mathrm{X}-78 \\ \text { Pectoral fin } & \text { R: } 9-\mathrm{X}-11 \\ \text { Anal fin } & \text { R: } 54-\mathrm{X}-62 \\ \text { Gill rakers } & \text { U: } X-X-X \quad \text { L: } 10-X-12\end{array}$

\section{LIFE HISTORY}

$\begin{array}{ll}\text { Range } & \text { Bering Sea, } 54-66^{\circ} \mathrm{N} \\ \text { Ecology } & \text { Epi- and mesobenthal } \\ \text { ELH pattern } & \text { Oviparous, pelagic eggs, } \\ & \text { pelagic larvae } \\ \text { Spawning } & \begin{array}{l}\text { Season: Summer } \\ \text { Area: Deep water }\end{array} \\ & \text { Mode: } \\ & \text { Migration: } \\ \text { Fecundity } & \text { Range/function: } \\ \text { Age at first maturity } & \\ \text { Longevity } & \end{array}$

\section{EARLY LIFE HISTORY DESCRIPTION}

\section{EGGS}

Diameter

$0.86-1.03 \mathrm{~mm}(0.92 \mathrm{~mm})$

No. of oil globules

Oil globule diameter

Yolk

Envelope Smooth

Hatch size $\quad<3 \mathrm{~mm} \mathrm{SL}$

Incubation time/temp.

Pigment

Diagnostic characters

\section{LARVAE}

Preanal length

Length at flexion

$<50 \% \mathrm{SL}$

Length at transformation

Sequence of fin development

$8.4-9.9 \mathrm{~mm} \mathrm{SL}$

$\sim 20-24 \mathrm{~mm} \mathrm{SL}$

Caudal, dorsal and anal, pelvics, pectorals

Pigment

- Series of ventral midline melanophores extending around urostyle

- Several spots above notochord, number increases with development

- During flexion, pigment develops along hypaxial myomeres

- Initially, gut pigment restricted to dorsal and anteroventral surface; with development it extends posterodorsally and along ventral surface

Diagnostic characters

- Pigment

- Pigment pattern without bands

- Presence of hypaxial pigment

-Presence of pigment around urostyle

\footnotetext{
${ }^{\text {a }}$ Pertseva-Ostroumova 1961
}

Ref: Ahlstrom et al. 1984a, Pertseva-Ostroumova 1961. 


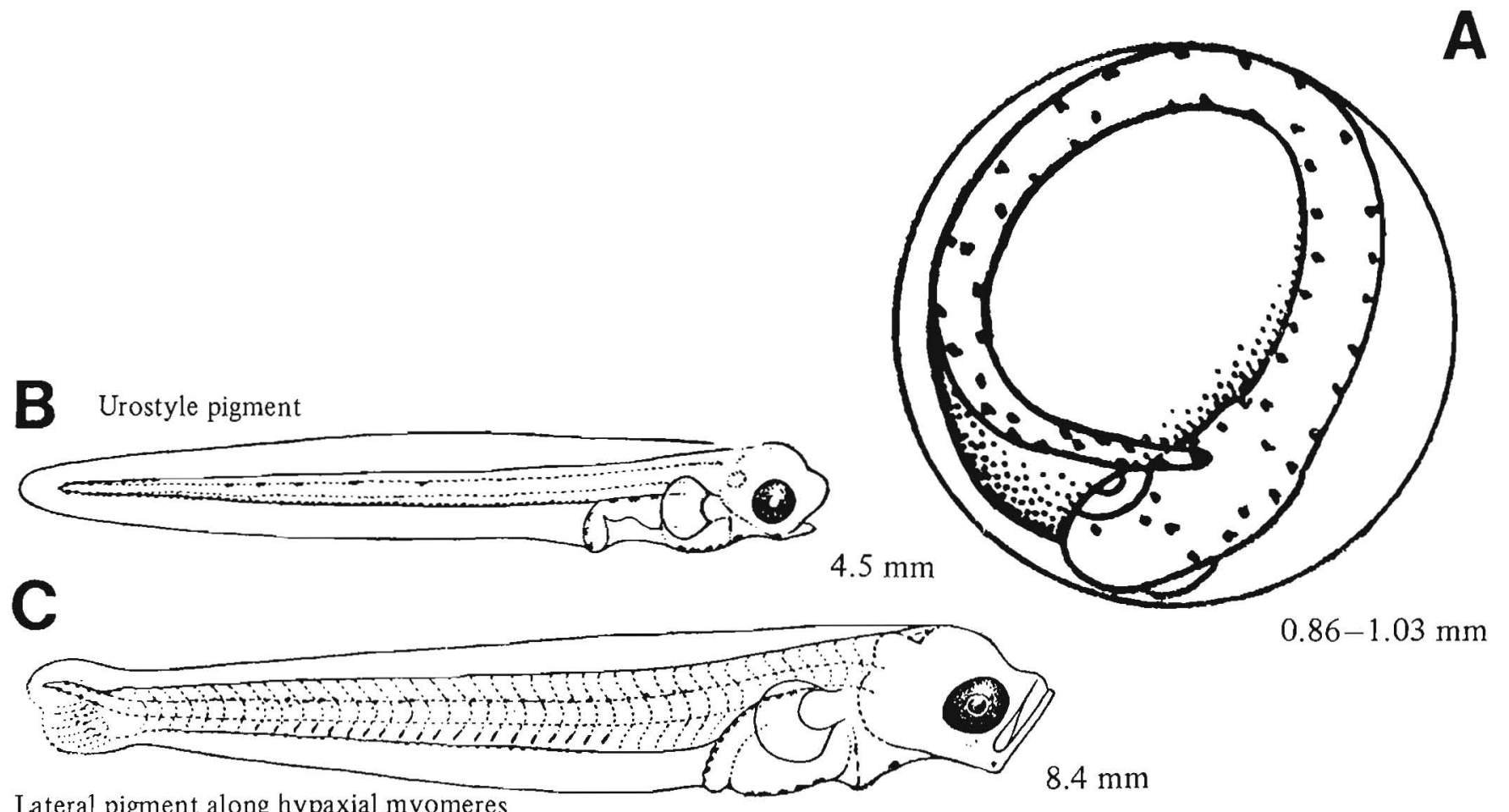

Lateral pigment along hypaxial myomeres
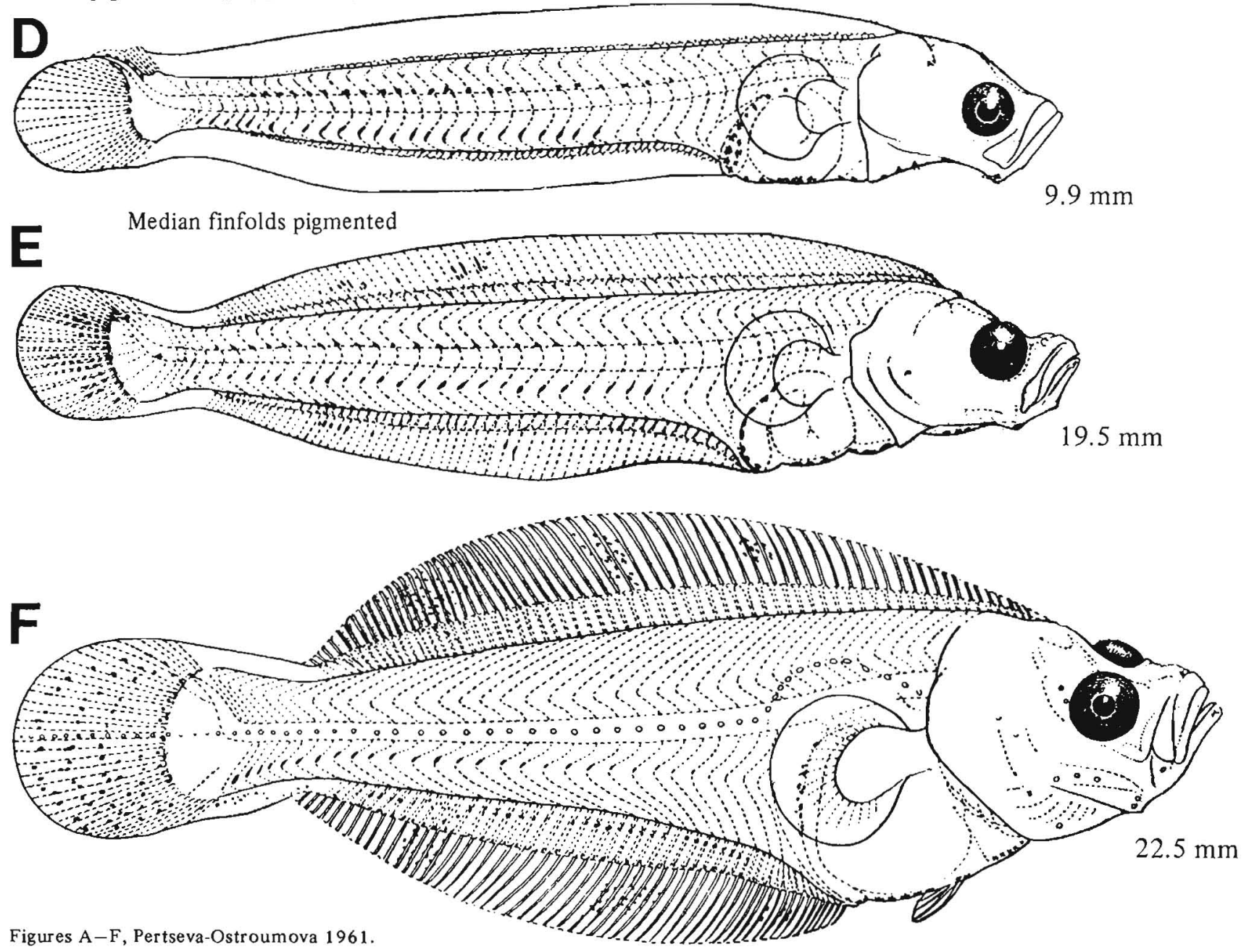


\section{MERISTICS}

\begin{tabular}{|c|c|}
\hline Vertebrae & $\begin{array}{l}\text { Total: } 49-X-52 \\
\text { Precaudal: } 10-X-11 \\
\text { Caudal: } 39-X-41\end{array}$ \\
\hline $\begin{array}{l}\text { Branchiostegal rays } \\
\text { Caudal fin }\end{array}$ & $7-X-8$ \\
\hline Pelvic fin & $\begin{array}{l}\text { Thoracic } \\
\text { R: 6-6-6 }\end{array}$ \\
\hline Dorsal fin & R: $95-107-112$ \\
\hline Pectoral fin & R: $13-X-14$ \\
\hline Anal fin & $\mathrm{R}: 75-\mathrm{X}-92$ \\
\hline Gill rakers & U: X-X-X \\
\hline LIFE HISTORY & \\
\hline Range & $\begin{array}{l}\text { Gulf of Alaska, } 54-60^{\circ} \mathrm{N} \text {, to } \\
\text { Bering Sea, } 54-66^{\circ} \mathrm{N}\end{array}$ \\
\hline Ecology & Epi- and mesobenthal \\
\hline ELH pattern & $\begin{array}{l}\text { Oviparous, bathypelagic eggs, } \\
\text { pelagic larvae }\end{array}$ \\
\hline Spawning & $\begin{array}{l}\text { Season: Fall-winter } \\
\text { Area: Demersal, deep water } \\
\text { Mode: } \\
\text { Migration: }\end{array}$ \\
\hline Fecundity & $\begin{array}{l}\text { Range/function: } 130,000- \\
500,000^{\mathrm{a}}\end{array}$ \\
\hline Age at first maturity & $\begin{array}{l}9-10 \text { yr (females) } \\
6-7 \text { yr (males) } \\
{ }^{a}\end{array}$ \\
\hline
\end{tabular}

\section{EARLY LIFE HISTORY DESCRIPTION}

\section{EGGS}

Diameter

No. of oil globules

Oil globule diameter

Yolk

Envelope

Hatch size

Incubation time/temp.

Pigment

\subsection{5-2.20 mm; $2.5-3.5 \mathrm{~mm}^{\mathrm{a}}$ None}

Greenish yellow

Thin, smooth, clear

$<8.4 \mathrm{~mm} \mathrm{SL}$

Diagnostic characters

\section{LARVAE}

Preanal length

Length at flexion

Length at transformation

Sequence of fin development

Pigment

- Two dorsolateral patches which, with development, meet and extend over the caudal peduncle

Diagnostic characters

According to figures in Pertseva-Ostroumova (1961), pigment pattern and morphology are similar to A. stomias. No larvae of $A$. evermanni have been identified from the study area.

${ }^{\text {a }}$ Pertseva-Ostroumova 1961

Ref: Ahlstrom et al. 1984a, Pertseva-Ostroumova 1961. 


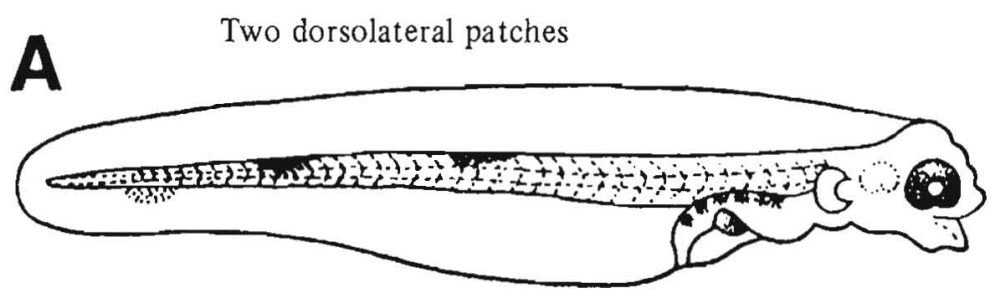

B

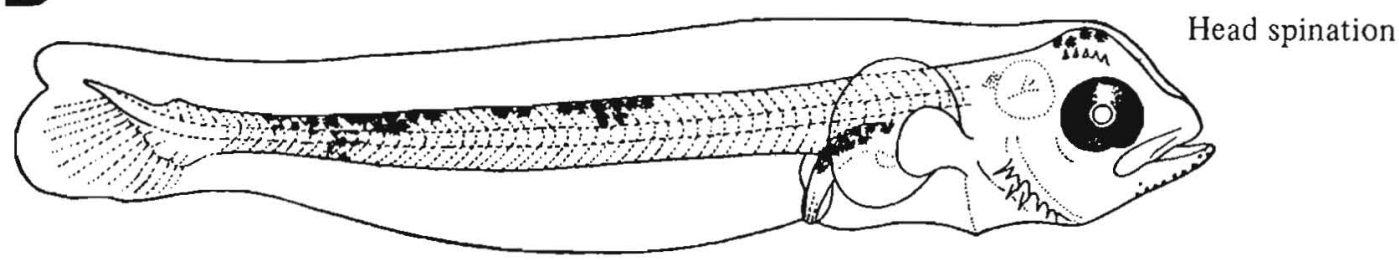




\section{MERISTICS}

\begin{tabular}{|c|c|}
\hline Vertebrae & $\begin{array}{l}\text { Total: } 47-49-50 \\
\text { Precaudal: } 12-12-12 \\
\text { Caudal: } 35-37-38\end{array}$ \\
\hline Branchiostegal rays & $7-X-8$ \\
\hline Caudal fin & Total rays $=17^{\mathrm{a}}$ \\
\hline Pelvic fin & $\begin{array}{l}\text { Thoracic } \\
\text { R: } 6-6-6\end{array}$ \\
\hline Dorsal fin & $\mathrm{R}: 92-108-115$ \\
\hline Pectoral fin & $\mathrm{R}: 14-\mathrm{X}-15$ \\
\hline Anal fin & R: $72-87-99$ \\
\hline Gill rakers & $\mathrm{L}: 11-\mathrm{X}-13$ \\
\hline \multicolumn{2}{|l|}{ LIFE HISTORY } \\
\hline Range & $\begin{array}{l}\text { Cent. California, } 34-38^{\circ} \mathrm{N} \text {, to } \\
\text { Bering Sea, } 54-66^{\circ} \mathrm{N}\end{array}$ \\
\hline Ecology & $\begin{array}{l}\text { Epi-, meso-, and bathybenthal, } \\
18-900 \mathrm{~m}\end{array}$ \\
\hline ELH pattern & $\begin{array}{l}\text { Oviparous, mesopelagic eggs, } \\
\text { mesopelagic larvae }\end{array}$ \\
\hline \multirow[t]{4}{*}{ Spawning } & $\begin{array}{l}\text { Season: Dec-Mar (Bering Sea); } \\
\quad \text { Aug (Gulf of Alaska) }\end{array}$ \\
\hline & Area: $108-360 \mathrm{~m}^{\mathrm{c}}$ \\
\hline & Mode: \\
\hline & Migration: \\
\hline Fecundity & Range/function: \\
\hline Age at first maturity & \\
\hline Longevity & $20 \mathrm{yr}^{\mathrm{d}}$ \\
\hline
\end{tabular}

\section{EARLY LIFE HISTORY DESCRIPTION}

\section{EGGS}

Diameter

No. of oil globules

Oil globule diameter

Yolk

Envelope

Hatch size

Incubation time/temp.

Pigment

Diagnostic characters

\section{LARVAE}
Preanal length $\quad<50 \% \mathrm{SL}$
Length at flexion $\quad \sim 10-12 \mathrm{~mm}$ SL
Length at transformation $25.6-33.0 \mathrm{~mm} \mathrm{SL}$
Sequence of fin
development
Caudal, dorsal and anal, pelvics, pectorals

\section{Pigment}

- Pigment on midbrain, spreading with development to dorsal surface of head, lower jaw, opercle

- Dorsally on gut, spreading laterally with development

- Two dorsolateral patches which, with development, meet and increase in size to cover caudal peduncle posteriorly

\section{Diagnostic characters}

Distinguished from other pleuronectids by

- Head spination: Early development of preopercular spines

- Pigmented supraocular crest

- Pigment pattern without bands; two dorsal patches with a lack of ventral pigment

- Head and snout blunt

Distinguished from $A$. evermanni by

- See A. evermanni (p. 576)

\footnotetext{
" 13 branched rays.

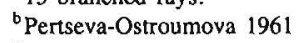

${ }^{6}$ Hirschberger and Smith 1983

${ }^{d}$ Kabata and Forrester 1974

Ref: Ahlstrom et al. 1984a, Pertseva-Ostroumova 1961.
} 

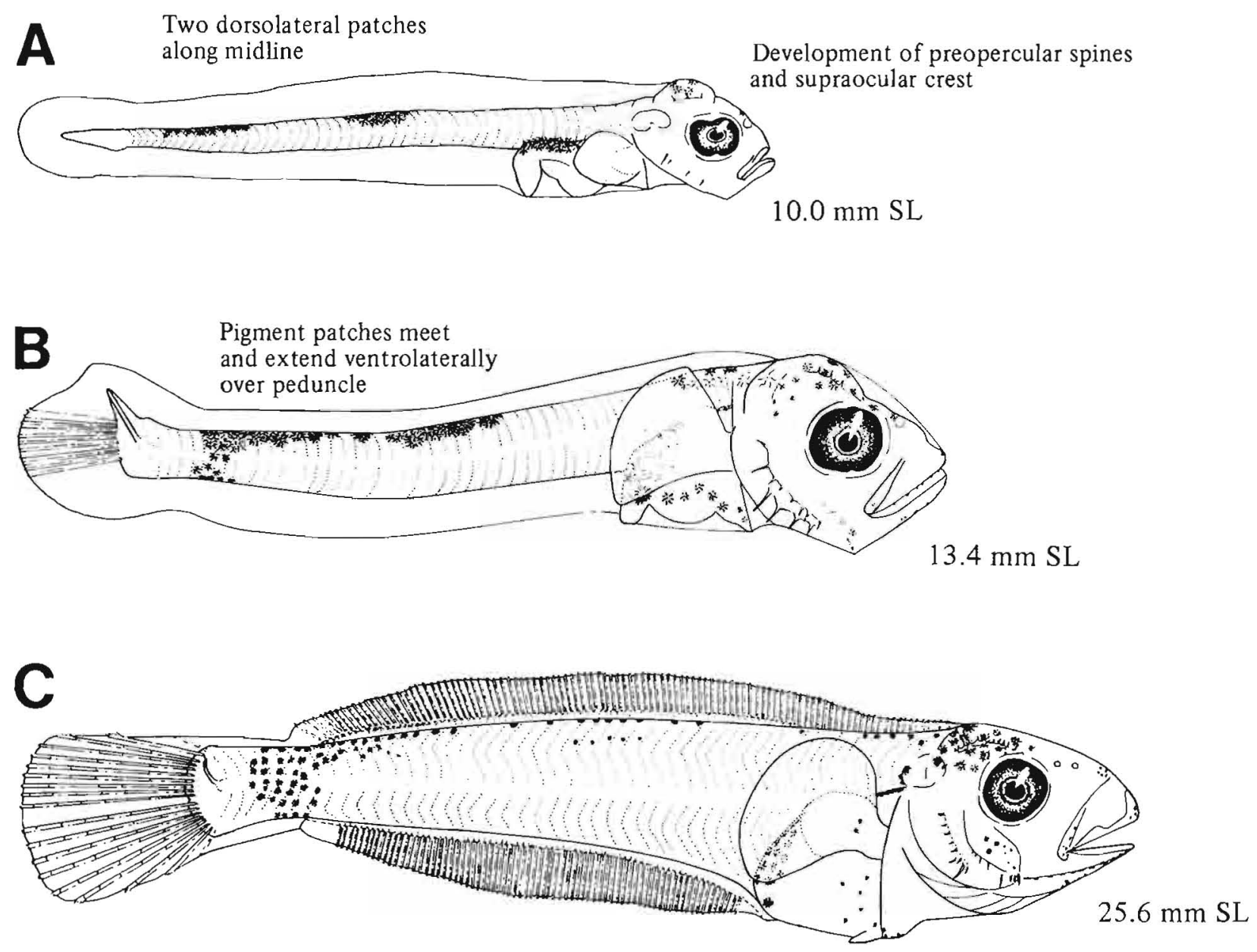

Figures A-C, NWAFC originals (B. Vinter). 
MERISTICS

\begin{tabular}{ll} 
Vertebrae & Total: $57-62-65$ \\
& Precaudal: $13-14-14$ \\
& Caudal: $44-48-51$ \\
Branchiostegal rays & $7-X-8$ \\
Caudal fin & X, $10+9, \mathrm{X}$ \\
& Total rays $=19-21^{\text {a }}$ \\
Pelvic fin & Thoracic \\
& R: $5-\mathrm{X}-6$ \\
Dorsal fin & $\mathrm{R}: 109-\mathrm{X}-117$ \\
Pectoral fin & $\mathrm{R}: 11-11-11$ \\
Anal fin & $\mathrm{R}: 94-\mathrm{X}-98$ \\
Gill rakers & $\mathrm{U}: 6-\mathrm{X}-9$ \\
& \\
& \\
LIFE HISTORY & \\
\hline
\end{tabular}

\section{Range}

S. California, $32-34^{\circ} \mathrm{N}$, to Bering Sea, $54-66^{\circ} \mathrm{N}$

Ecology

\section{ELH pattern}

Spawning Age at first maturity Longevity

$$
\text { 320-1433 m }
$$

Oviparous, pelagic eggs, pelagic larvae

Season: Spring (possibly winter-spring) ${ }^{\mathrm{b}}$

Area:

Mode:

Migration:

Range/function:

\section{EARLY LIFE HISTORY DESCRIPTION}

\section{EGGS}

Diameter

No. of oil globules

Oil globule diameter

Yolk

Envelope

Hatch size

Incubation time/temp.

Pigment

- Eye

- Hindgut extending out into yolk

- Three postanal bands

Diagnostic characters

- Size

- Pigment pattern

\section{LARVAE}

${ }^{a} 17$ branched rays.

${ }^{b}$ Richardson 1981 b

${ }^{\mathrm{c}}$ According to Figures $\mathrm{C}$ and $\mathrm{E}$ from Richardson (1981b), flexion occurs rapidly between 15.4 and $16.2 \mathrm{~mm} \mathrm{SL}$. However, the 18.5-mm NL specimen (Fig. D) illustrated by Ahlstrom et al. (1984a) does not appear fully flexed. Differences in size may be a result of preservation.

\section{Preanal length \\ $40 \%$ SL}

Length at flexion $\quad 15.4-16.2 \mathrm{~mm} \mathrm{SL}^{\mathrm{c}}$

Length at transformation $>16.2 \mathrm{~mm} \mathrm{SL}$ when eye migration commences

Sequence of fin Caudal, dorsal, and anal; development pelvics; pectorals

\section{Pigment}

- Initially, three postanal bands with pigment on the finfold in region of bands; number of dorsal and ventral spots change with size and stage of development

- Pigmentation increases with development along finfold margin ( $\sim 7$ spots along dorsal and 5 spots along anal in flexion larvae)

\section{Diagnostic characters}

- Postanal band pattern in preflexion larvae (three bands)

- High myomere count (57-65)

Distinguished from similar larvae with high myomere count by

- Presence of three postanal pigment bands and median finfold pigment

-Reinhardtius hippoglossoides (p. 622): Lightly pigmented and without bands

-Glyptocephalus spp. (p. 584, 586): Three bands (more laterally intense) in G. stelleri; in G. zachirus, caudal band, no finfold pigment

-Unidentified ophidiid larvae (p. 213): More myomeres $(>75)$, less finfold pigment, and less pronounced loop in gut 

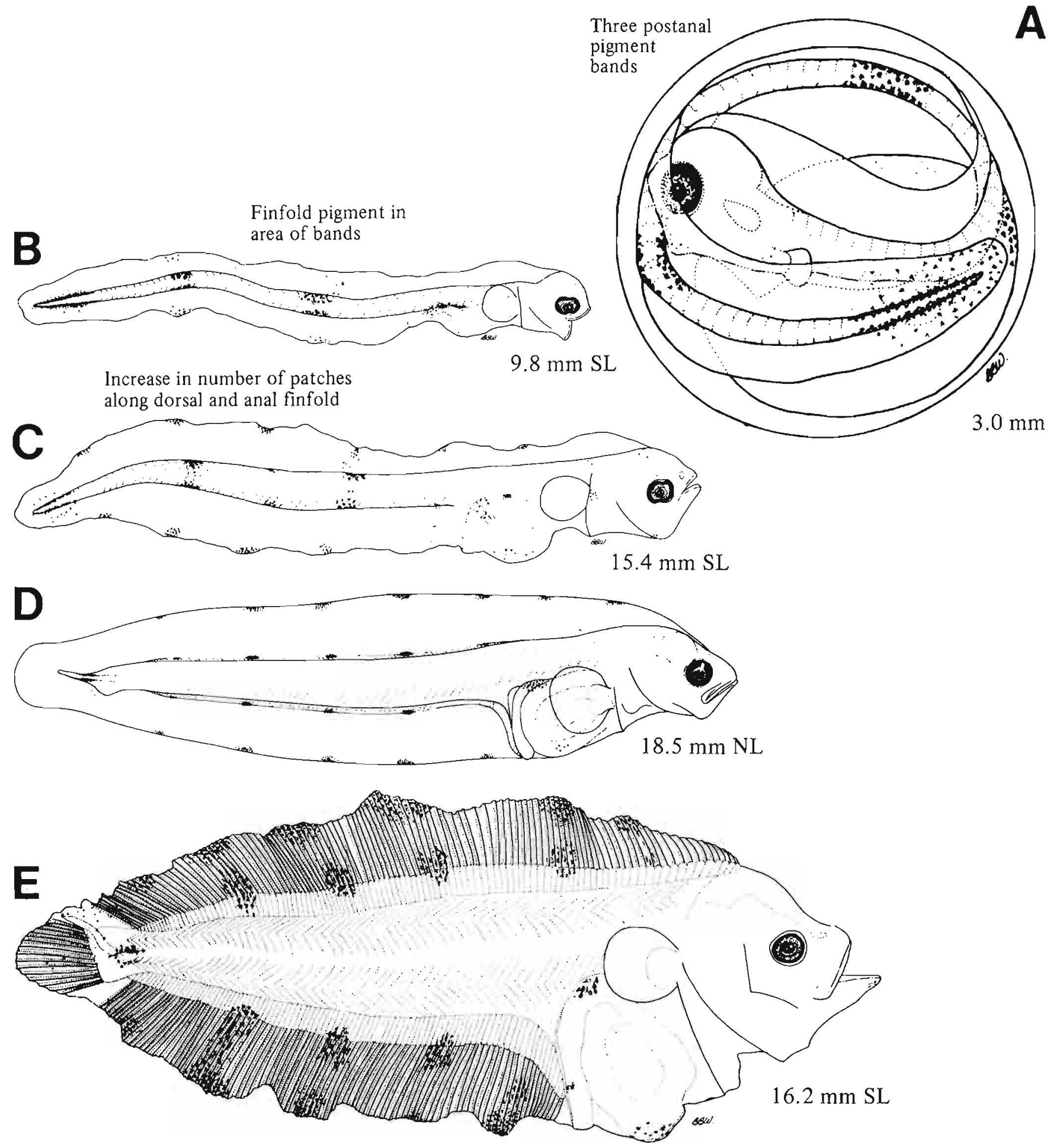

Figures A-C, E, Richardson 1981 b; D, Ahlstrom et al. 1984a. 


\section{MERISTICS}

\begin{tabular}{ll}
\hline Vertebrae & Total: $41-\mathrm{X}-45$ \\
& Precaudal: $11-11-11$ \\
& Caudal: $30-32-34$ \\
Branchiostegal rays & $7-\mathrm{X}-8$ \\
Caudal fin & $\mathrm{X}, 9+8, \mathrm{X}$ \\
& Total rays=19a \\
Pelvic fin & Thoracic \\
& R: $6-6-6$ \\
Dorsal fin & R: $82-97-103$ \\
Pectoral fin & R: $13-13-13$ \\
Anal fin & R: $67-73-79$ \\
Gill rakers & U: X-X-X $\quad$ L: $15-X-17$
\end{tabular}

\section{LIFE HISTORY}

\begin{tabular}{|c|c|}
\hline Range & $\begin{array}{l}\text { South of southern California to } \\
\text { Gulf of Alaska, } 54-60^{\circ} \mathrm{N}\end{array}$ \\
\hline Ecology & $\begin{array}{l}\text { Epi-, meso-, and bathybenthal, } \\
0-550 \mathrm{~m}\end{array}$ \\
\hline ELH pattern & $\begin{array}{l}\text { Oviparous, pelagic eggs, } \\
\text { pelagic larvae }\end{array}$ \\
\hline \multirow[t]{4}{*}{ Spawning } & Season: Dec-Apr ${ }^{b}$ \\
\hline & $\begin{array}{l}\text { Area: Off continental slope, } \\
274-366 \mathrm{~m}^{\mathrm{c}}\end{array}$ \\
\hline & Mode: \\
\hline & $\begin{array}{l}\text { Migration: To deep water for } \\
\text { spawning }{ }^{c}\end{array}$ \\
\hline Fecundity & $\begin{array}{l}\text { Range/function: } 400,000- \\
1,200,000^{d}\end{array}$ \\
\hline \multirow[t]{2}{*}{ Age at first maturity } & $3-8$ yr (females) ${ }^{c}$ \\
\hline & $4-9 \mathrm{yr}$ (males) ${ }^{\mathrm{c}}$ \\
\hline \multirow[t]{2}{*}{ Longevity } & $25 \mathrm{yr}$ (females) $^{\mathrm{c}}$ \\
\hline & $21 \mathrm{yr}^{(\text {males })^{\mathrm{c}}}$ \\
\hline
\end{tabular}

a 15 branched rays.

b Alderdice and Forrester 1971

'Frey 1971

dForrester 1969

${ }^{e}$ Larvae are rare. Since most routine ichthyoplankton surveys sample only to $200 \mathrm{~m}$, the absence of small larvae in field collections indicates larvae may occur below $200 \mathrm{~m}$.

Ref: Ahlstrom et al. 1984a, Alderdice and Forrester 1971.

\section{EARLY LIFE HISTORY DESCRIPTION}

\section{EGGS}

Diameter

No. of oil globules

$1.21-1.25 \mathrm{~mm}$

Oil globule diameter

None

Yolk

Envelope

Hatch size

$2.8 \mathrm{~mm} \mathrm{SL}$

Incubation time/temp.

Pigment

Diagnostic characters

\author{
LARVAE $^{\mathrm{e}}$ \\ Preanal length $\quad<50 \% \mathrm{SL}$ \\ Length at flexion \\ Length at transformation \\ Sequence of fin \\ development \\ Pigment \\ - Unpigmented at hatching \\ - Preflexion \\ - Midbody patch and urostyle pigment \\ - Melanophores on body over anus \\ -Posteriorly on gut and on head \\ - Flexion and early postflexion larvae are undescribed \\ Diagnostic characters \\ Distinguished from other pleuronectids by \\ - Presence of two postanal pigment bands including \\ pigment on urostyle \\ - Presence of preopercular spines
}



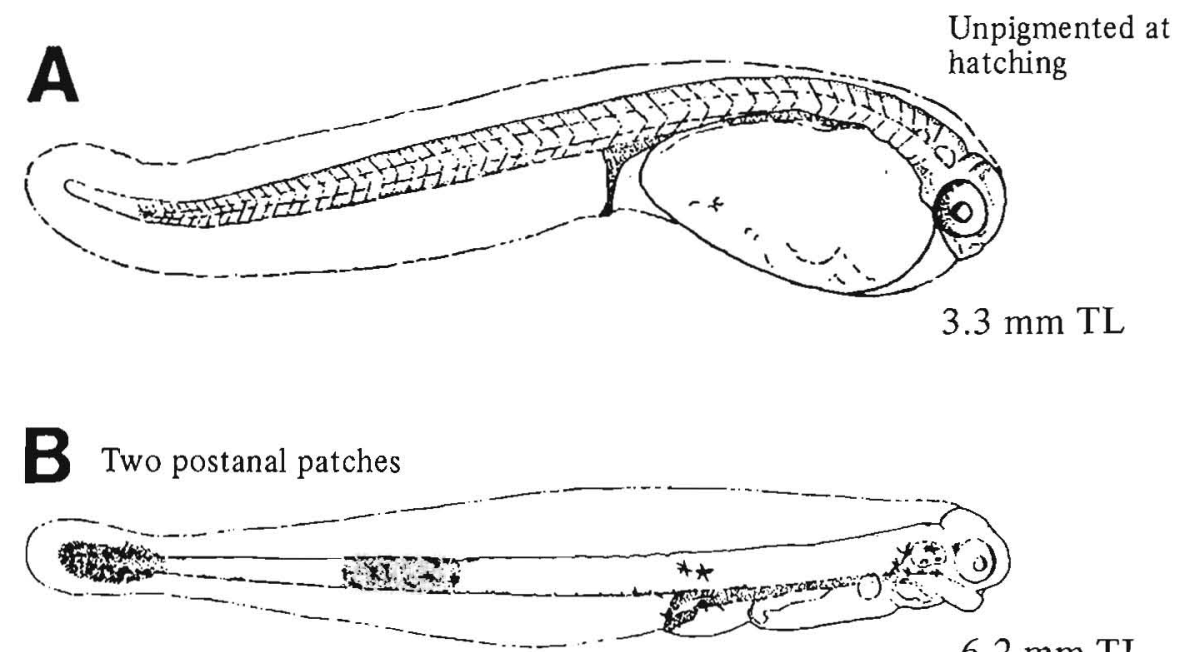

\section{$6.2 \mathrm{~mm} \mathrm{TL}$}

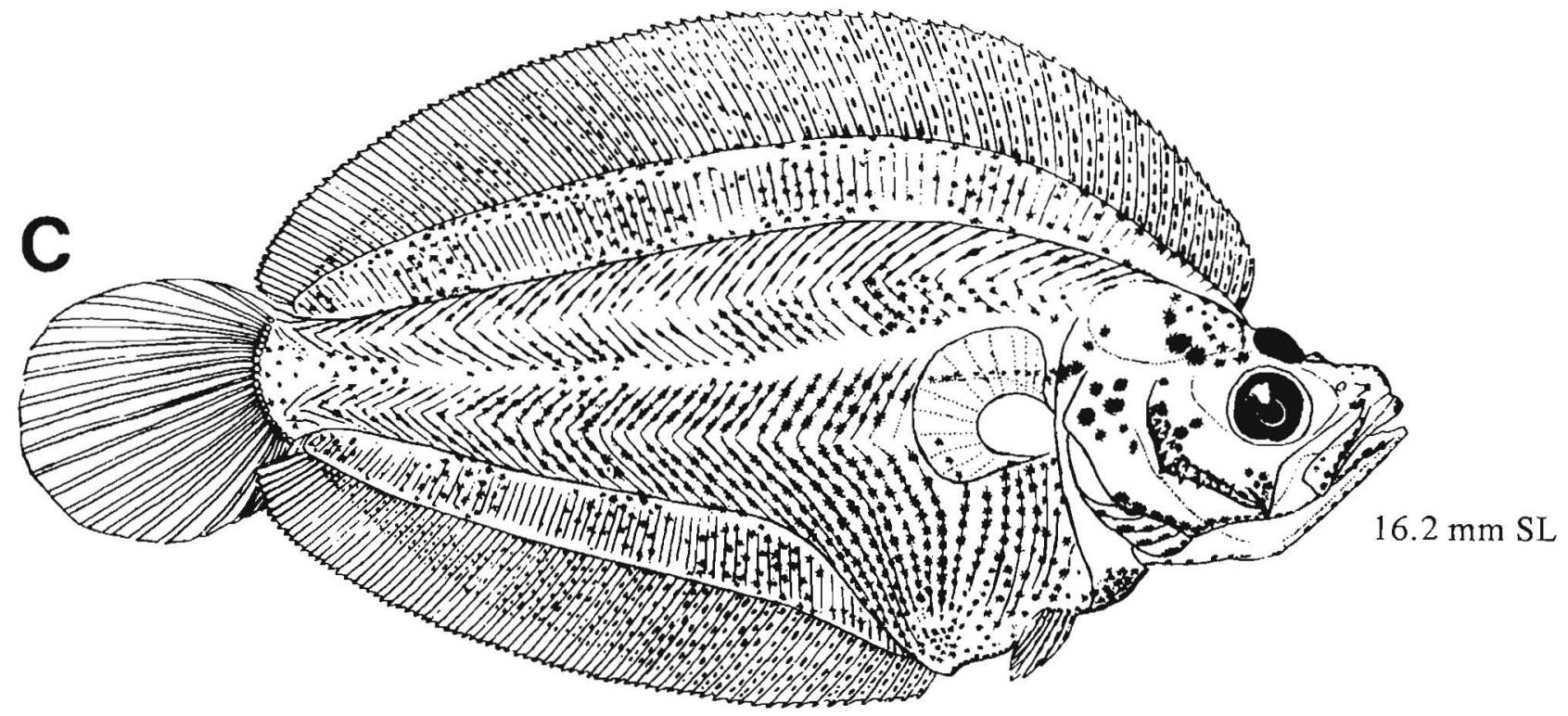




\section{MERISTICS}

$\begin{array}{ll}\text { Vertebrae } & \text { Total: } 52-\mathrm{X}-60 \\ & \begin{array}{l}\text { Precaudal: } 9-9-9 \\ \text { Caudal: } 43-43-43\end{array} \\ \text { Branchiostegal rays } & 7-7-7 \\ \begin{array}{ll}\text { Caudal fin } \\ \text { Pelvic fin }\end{array} & \begin{array}{l}\text { Thoracic } \\ \text { R: } 6-6-6\end{array} \\ \text { Dorsal fin } & \text { R: } 83-X-97 \\ \text { Pectoral fin } & \text { R: } 10-X-12 \\ \text { Anal fin } & \text { R: } 72-X-80 \\ \text { Gill rakers } & \text { U: X-X-X } \\ & \\ & \\ \text { LIFE HISTORY } & \end{array}$

\section{Range \\ Ecology \\ ELH pattern}

Spawning

Fecundity

Age at first maturity

Longevity
Bering Sea, $54-66^{\circ} \mathrm{N}$

Epi- and mesobenthal

Oviparous, pelagic eggs, pelagic larvae

\section{Season:}

Area:

Mode:

Migration:

Range/function:

\section{EARLY LIFE HISTORY DESCRIPTION}

\section{EGGS}

Diameter

No. of oil globules

Oil globule diameter

Yolk

Envelope

Hatch size

Incubation time/temp.

Pigment

- Yolksac

- Late-stage embryo

Diagnostic characters
$1.20-1.61 \mathrm{~mm}$

None

Homogeneous

Thick, sometimes striated 4.1-5.2 mm SL

\section{LARVAE}

Preanal length

Length at flexion

Length at transformation

Sequence of fin development

Pigment

- Finfold (dorsal and anal)

- Three postanal bands

Diagnostic characters

Distinguished from other pleuronectids by

- Presence of three postanal pigment bands

- High myomere count (52-60)

- Presence of preopercular spines

Distinguished from G. zachirus by

- Fewer myomeres (52-60)

- Pigment pattern - Presence of finfold pigment

-Difference in band pattern (fewer bands)

${ }^{a}$ Ahlstrom et al. 1984a: 19-48 mm SL. 
B

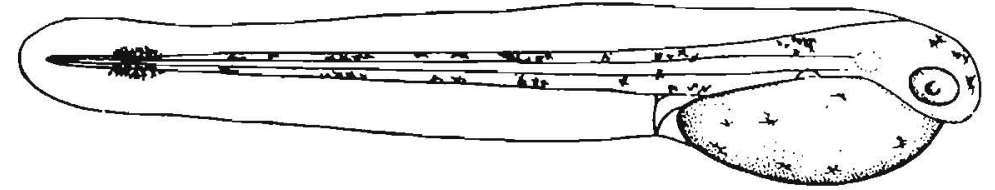

$4.6 \mathrm{~mm}$

C

Three postanal bands, finfold pigment

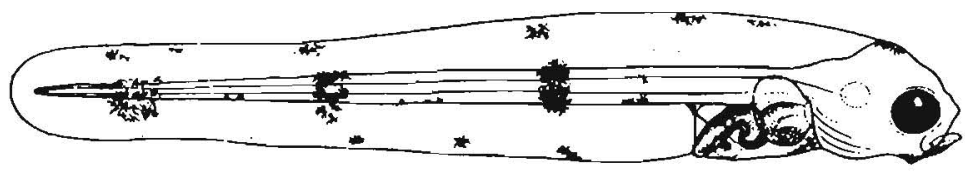

$5.4 \mathrm{~mm}$

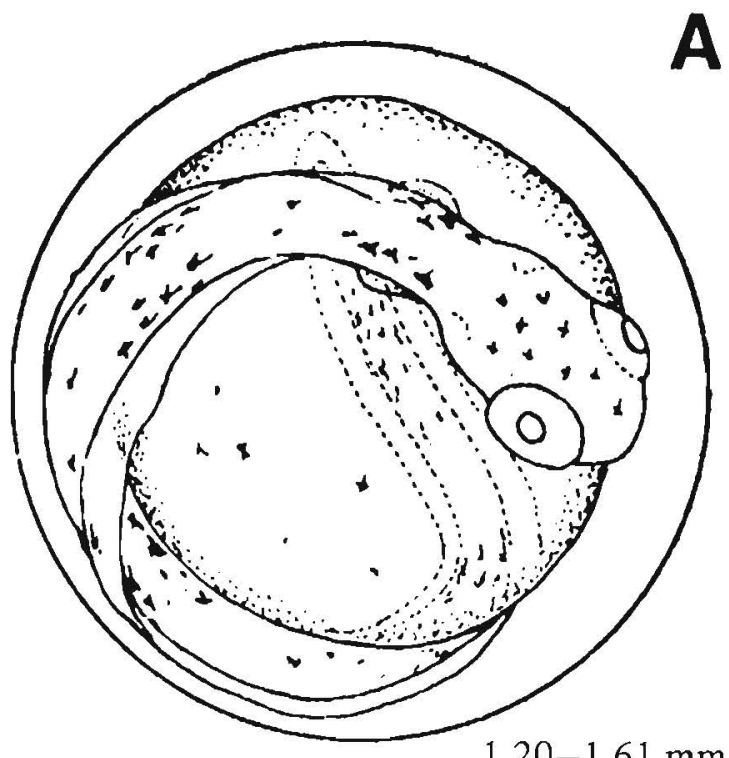

$1.20-1.61 \mathrm{~mm}$

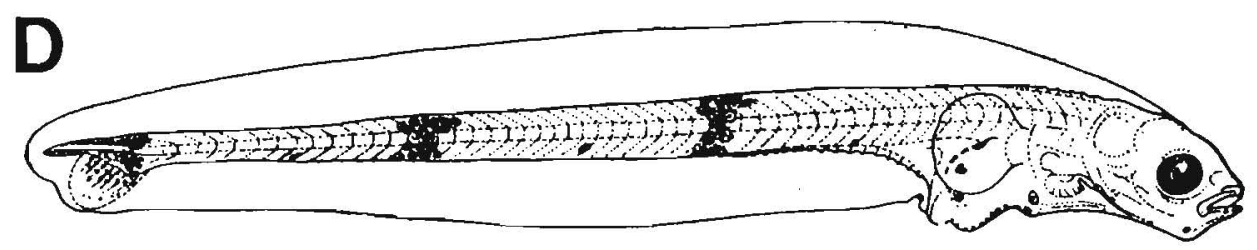

$15.2 \mathrm{~mm}$

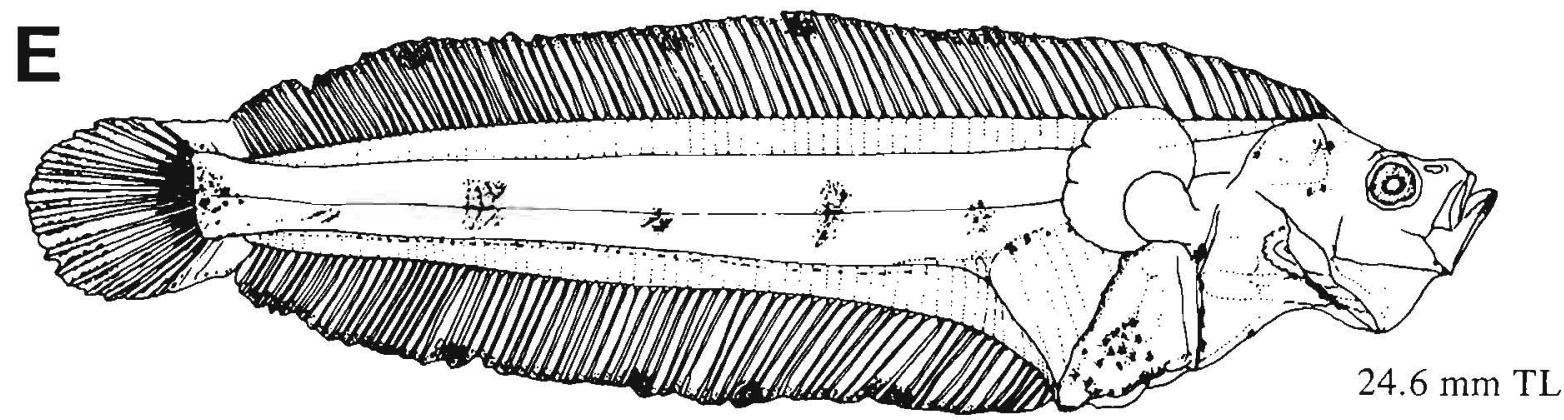

Figures A-D, Pertseva-Ostroumova 1961 (B-C, reversed); E, Okiyama and Takahashi 1976. 


\section{MERISTICS}

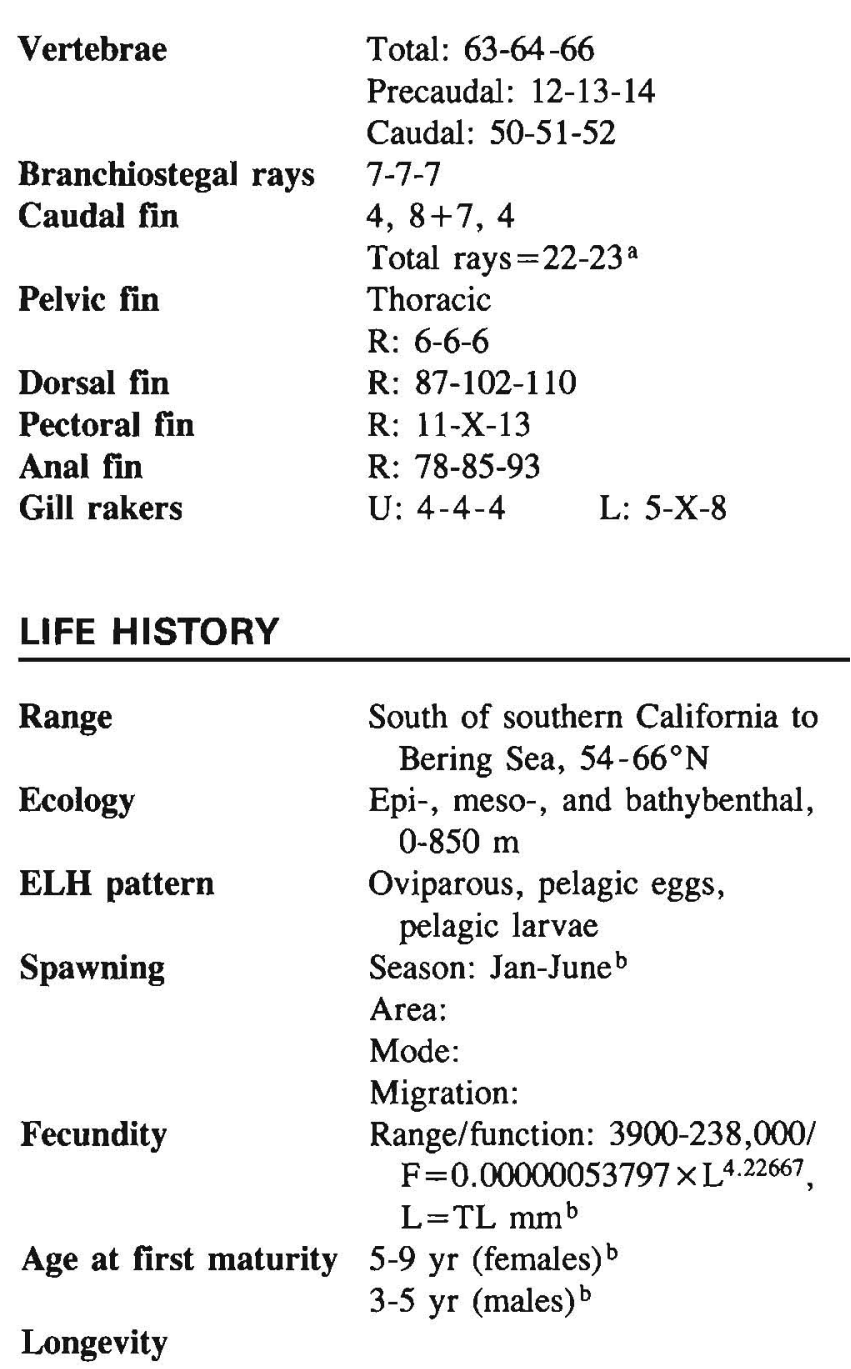

\footnotetext{
a 13 branched rays.

${ }^{b}$ Hosie and Horton 1977

${ }^{c}$ According to Ahlstrom et al. (1984a), transformation may occur as late as $72 \mathrm{~mm}$ SL.

Ref: Ahlstrom and Moser 1975, Ahlstrom et al. 1984a.
}

EARLY LIFE HISTORY DESCRIPTION

\section{EGGS}

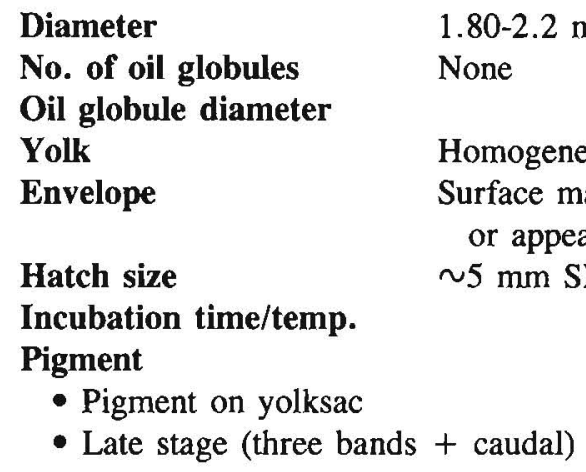

\section{Diagnostic characters}

Distinguished from Lyopsetta exilis (p. 606) and

Microstomus pacificus (p. 608) by

- Size

- Pigment on yolksac, late-stage embryo

- Wide perivitelline space

- Size of embryo (coiling in G. zachirus)

\section{LARVAE}

Preanal length $\quad<50 \%$ SL

Length at flexion $\quad 15.3-24.0 \mathrm{~mm} \mathrm{SL}$

Length at transformation $49-59 \mathrm{~mm} \mathrm{SL}^{\mathrm{c}}$

Sequence of fin Caudal, dorsal and anal, development pelvics, pectorals

Pigment

- Postanal body

- Three bands

- Caudal band (above, below, and in finfold)

-Melanophores between bands

- Head, lower jaw, gut

- With development, caudal pigment becomes restricted to hypural area

Diagnostic characters

- See G. stelleri (p. 584)

Distinguished from other pleuronectids by

- Presence of four postanal pigment bands

- High myomere count (63-66)

- Presence of preopercular spines

See Embassichthys bathybius (p. 580) and Microstomus pacificus (p. 608) for similar larvae 

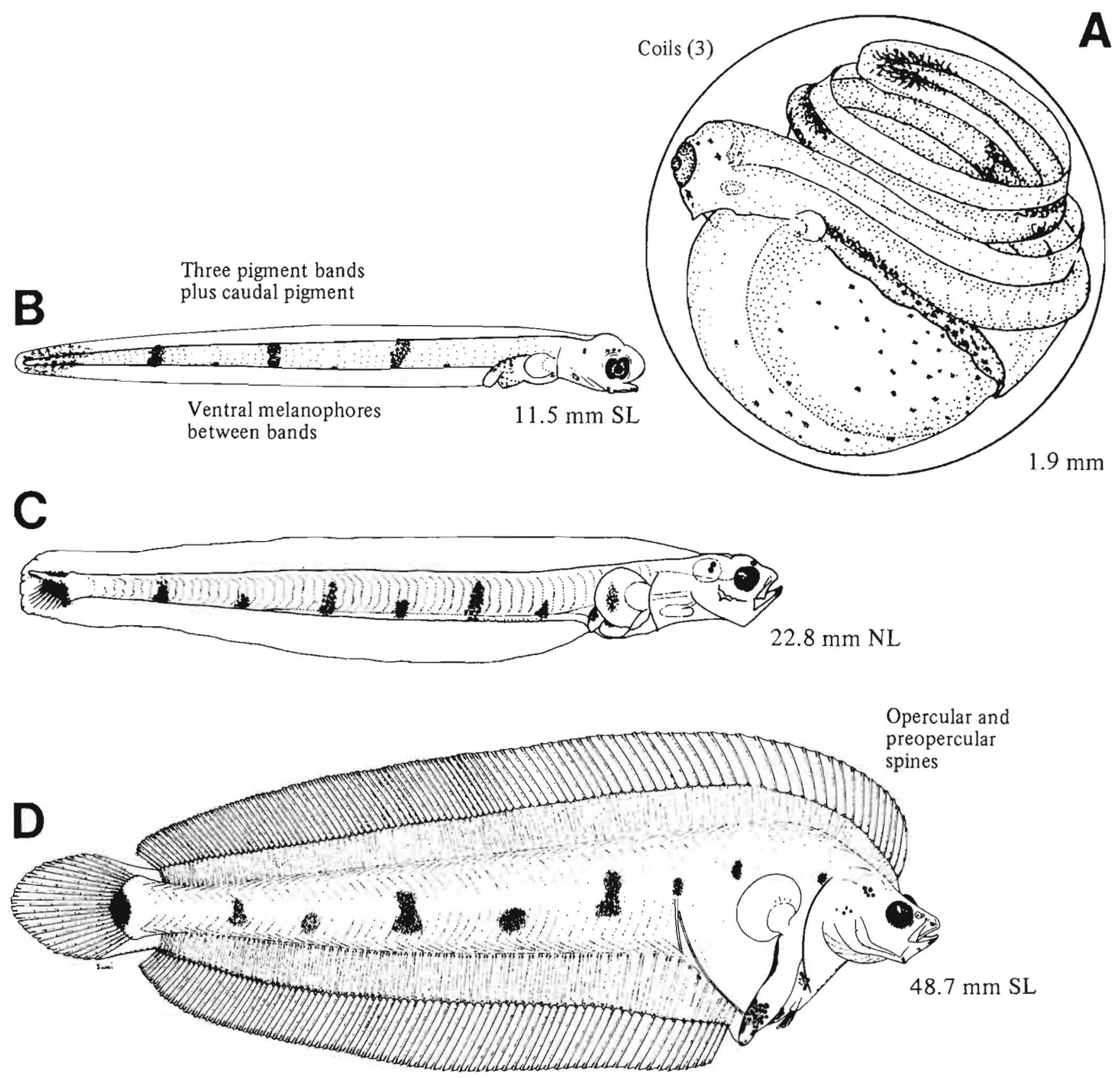

Figure A, Ahlstrom and Moser 1980; B, NWAFC original (B. Vinter); C - D, Ahlstrom et al. 1984a (after Ahlstrom and Moser 1975). 


\section{MERISTICS}

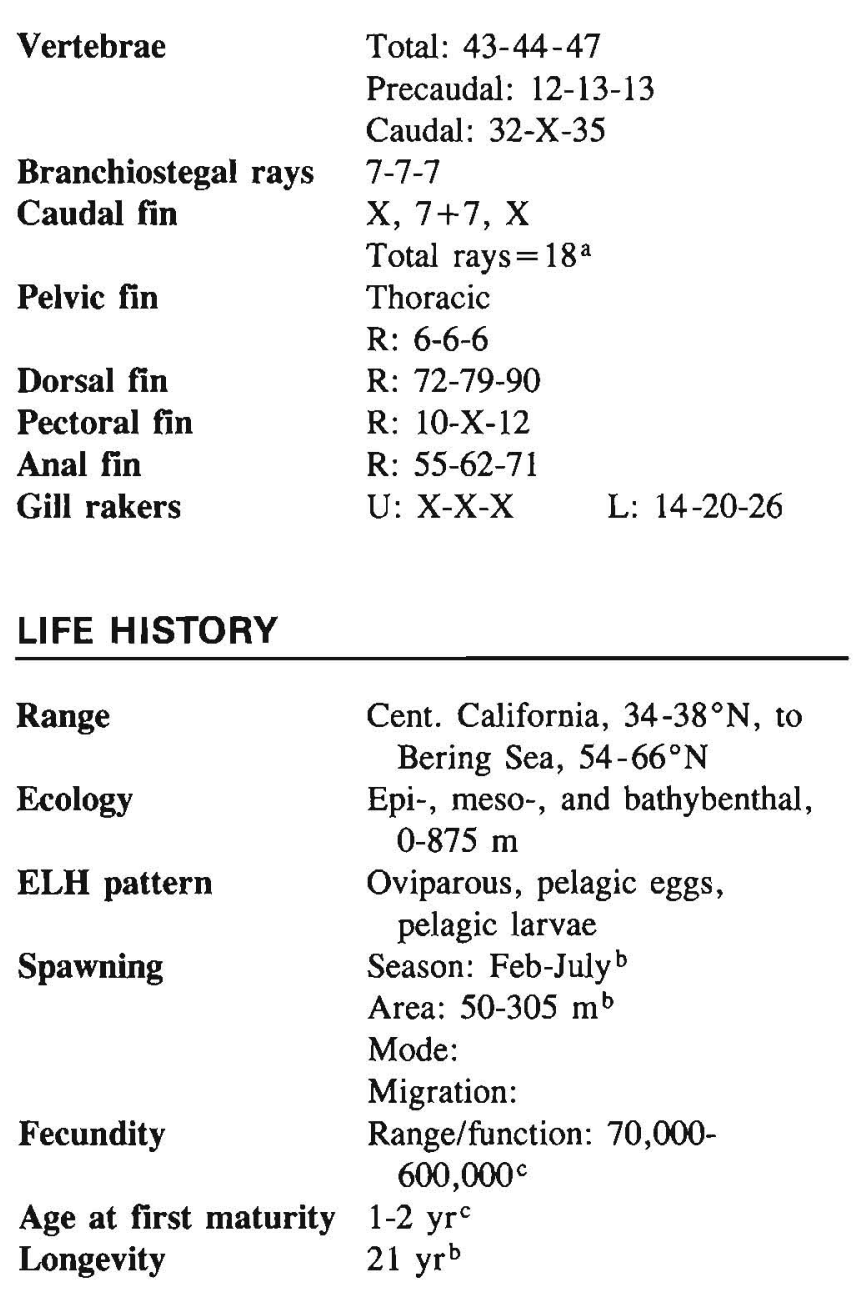

\section{EARLY LIFE HISTORY DESCRIPTION}

\section{EGGS}

Diameter

No. of oil globules

Oil globule diameter

Yolk

Envelope

Hatch size

2.75-3.75 mm (2.9-3.5 mm)

None

Homogeneous, colorless

Smooth

Incubation time/temp.

5.3-6.9 $\mathrm{mm} \mathrm{SL}^{\mathrm{d}}$

Pigment

- Yolksac

- Late-stage embryo: On body and in dorsal and ventral finfold

Diagnostic characters

- Wide perivitelline space

- Body and finfold pigment

\section{LARVAE}

Preanal length

$<50 \%$ SL

Length at flexion

9-10 mm SL

Length at transformation

Sequence of fin development

\section{Pigment}

- Three postanal bands and along ventral midline

- Around urostyle

- Finfold

- With development becoming less pigmented

Diagnostic characters

Distinguished from other pleuronectids with three postanal pigment bands by

- Presence of hypaxial, fin, and urostyle pigment

- Low myomere count (43-47)

\footnotetext{
a 12 branched rays.

${ }^{b}$ Salveson 1976

${ }^{c}$ Miller 1969

${ }^{d}$ Pertseva-Ostroumova 1961

Ref: Ahlstrom et al. 1984a, Pertseva-Ostroumova 1961.
} 

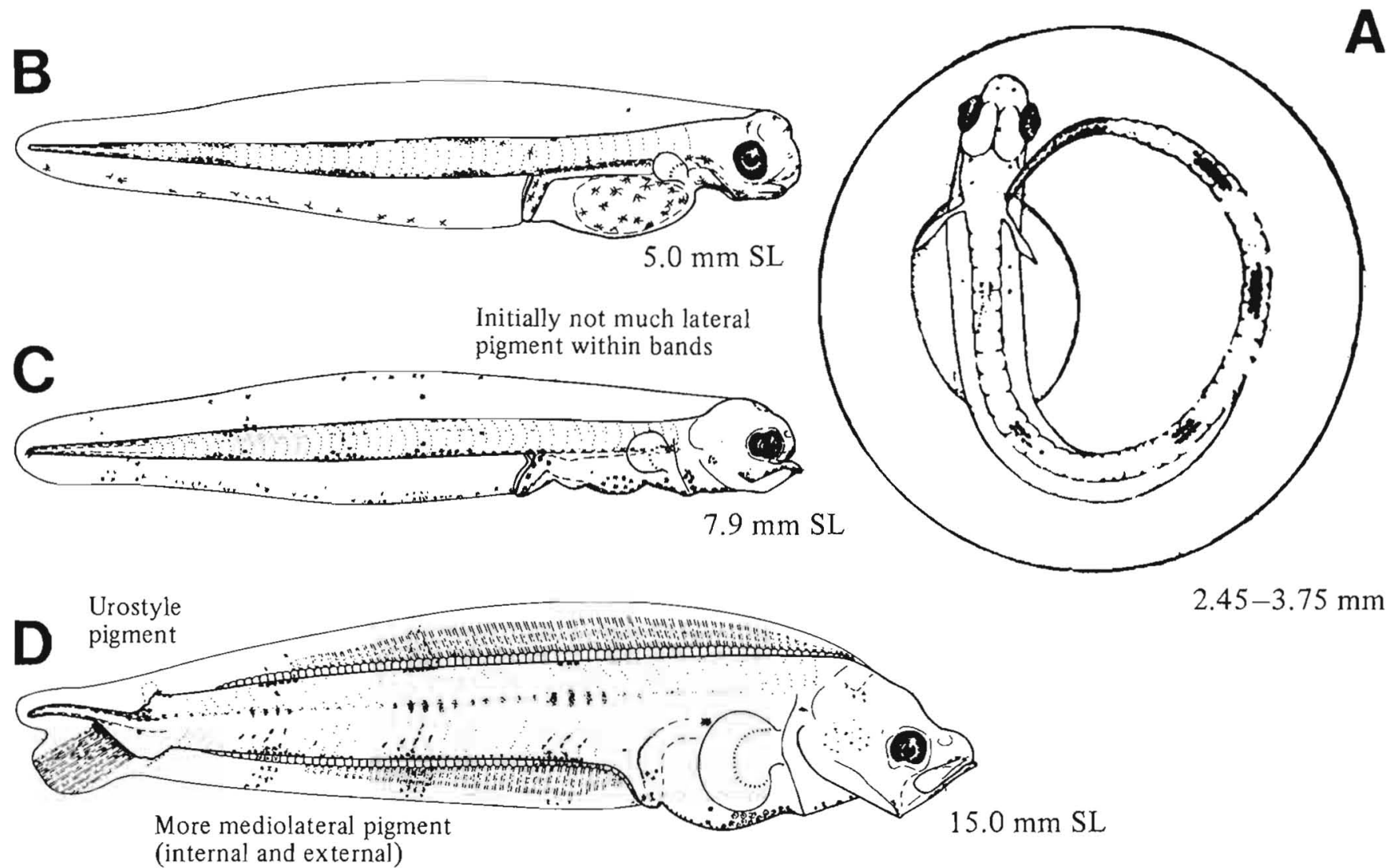

$2.45-3.75 \mathrm{~mm}$

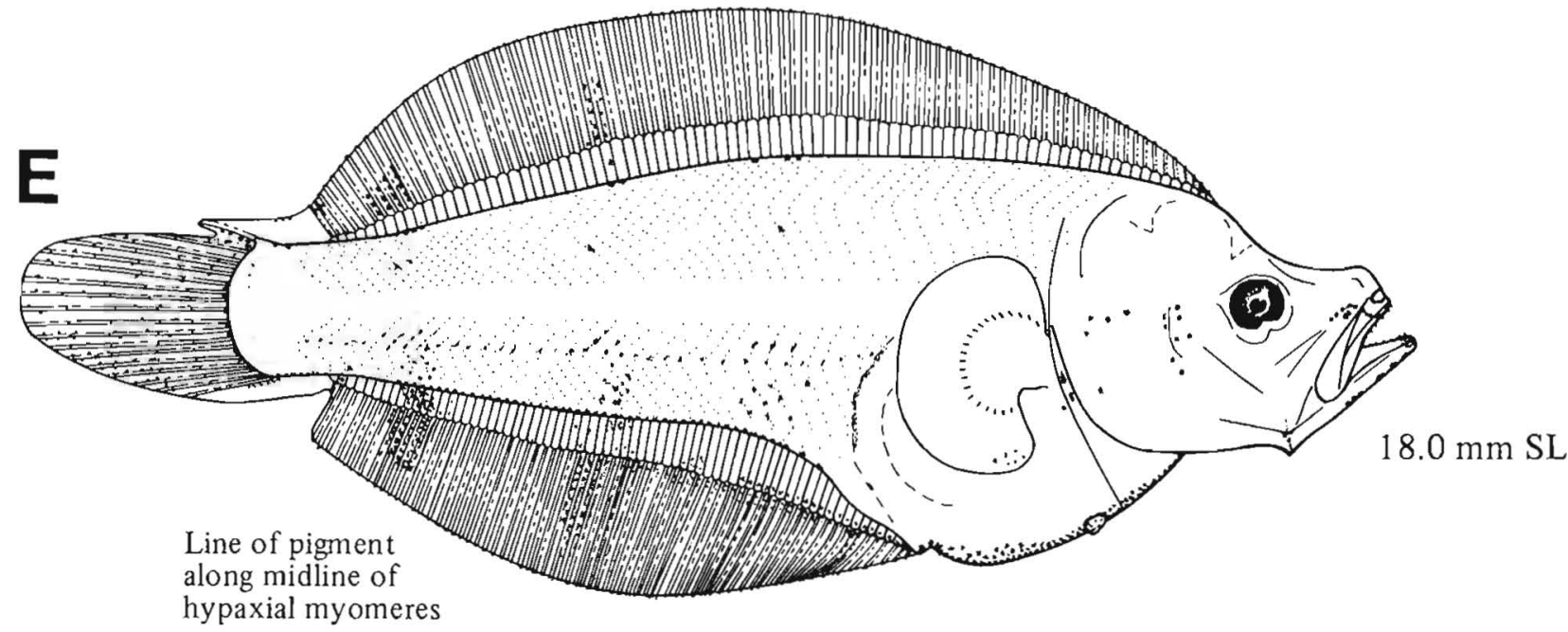

Figure A, Pertseva-Ostroumova 1961; B-E, NWAFC originals (B. Vinter). 


\section{MERISTICS}

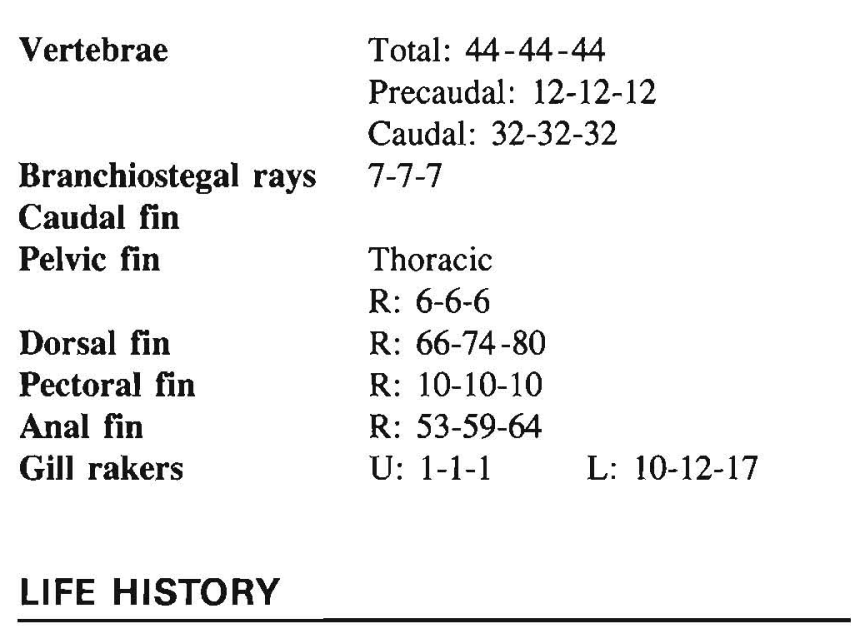

$\begin{array}{ll}\text { Range } & \begin{array}{c}\text { Aleutian Is., } 51-55^{\circ} \mathrm{N}, \text { to } \\ \text { Chukchi Sea, north of } 66^{\circ} \mathrm{N} \\ \text { Epi- and mesobenthal, 18-425 } \mathrm{m}\end{array} \\ \begin{array}{l}\text { Ecology } \\ \text { ELH pattern } \\ \text { Oviparous, pelagic eggs, } \\ \text { pelagic larvae } \\ \text { Spawning }\end{array} & \begin{array}{l}\text { Season: Apr-June } \\ \text { Area: Shallow gulfs and bays } \\ (50-150 \mathrm{~m})^{\mathrm{a}}\end{array} \\ & \text { Mode: } \\ & \begin{array}{l}\text { Migration: } \\ \text { Range/function: }\end{array} \\ \begin{array}{l}\text { Fecundity } \\ \text { Age at first maturity } \\ \text { Longevity }\end{array} & \end{array}$

\section{EARLY LIFE HISTORY DESCRIPTION}

\section{EGGS}

Diameter

No. of oil globules

Oil globule diameter

Yolk

Envelope Smooth, thin

Hatch size

$4 \mathrm{~mm} \mathrm{SL}$

Incubation time/temp.

Pigment

- Yolksac

- Late-stage embryo: On body

Diagnostic characters

- Wide perivitelline space

- Smaller in diameter than $H$. elassodon

\section{LARVAE}

Preanal length $\quad<50 \%$ SL

Length at flexion $\quad \sim 11 \mathrm{~mm} \mathrm{SL}$

Length at transformation $>28.6 \mathrm{~mm} \mathrm{SL}^{b}$

Sequence of fin development

Pigment

- Three postanal bands extending into finfold

Diagnostic characters

- See H. elassodon (p. 588) 


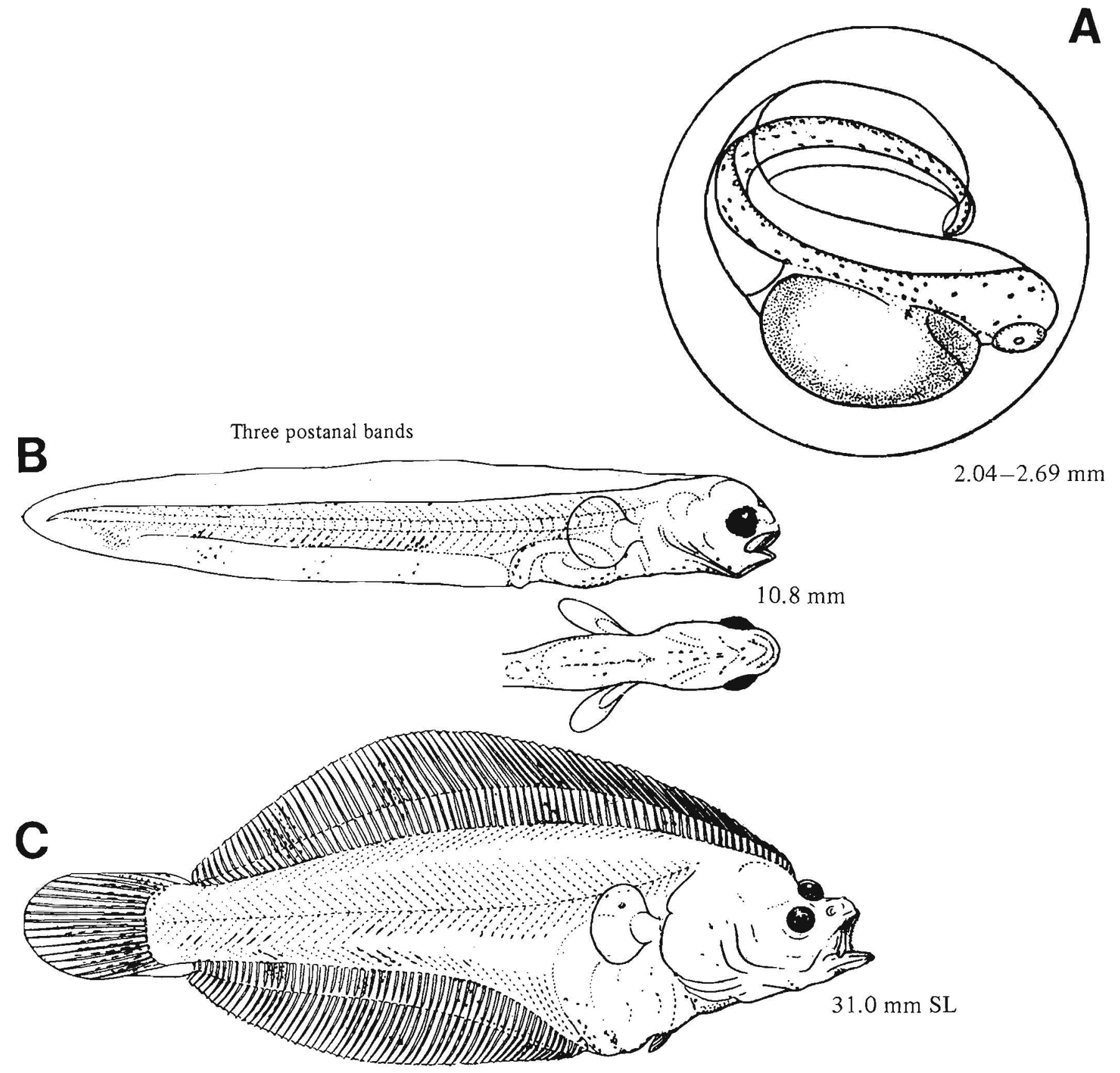

Figures $A-C$ (B insert, ventral view of head), Pertseva-Ostroumova 1961. 


\section{MERISTICS}

$\begin{array}{ll}\text { Vertebrae } & \text { Total: } 49-50-51 \\ & \text { Precaudal: } 16-16-16 \\ & \text { Caudal: } 35-35-35 \\ \text { Branchiostegal rays } & 7-7-7 \\ \text { Caudal fin } & \text { Total rays }=19 \\ \text { Pelvic fin } & \text { Thoracic } \\ & \text { R: } 6-6-6 \\ \text { Dorsal fin } & \text { R: } 89-99-109 \\ \text { Pectoral fin } & \text { R: } 19-19-19 \\ \text { Anal fin } & \text { R: } 64-75-81 \quad \\ \text { Gill rakers } & \text { U: X-X-X } \quad \text { L: X-X-X }\end{array}$

\section{LIFE HISTORY}

\begin{tabular}{|c|c|}
\hline Range & $\begin{array}{l}\text { South of southern California to } \\
\text { Chukchi Sea, north of } 66^{\circ} \mathrm{N}\end{array}$ \\
\hline Ecology & $\begin{array}{l}\text { Epi-, meso-, and bathybenthal, } \\
6-1110 \mathrm{~m}\end{array}$ \\
\hline ELH pattern & $\begin{array}{l}\text { Oviparous, pelagic eggs, } \\
\text { pelagic larvae }\end{array}$ \\
\hline \multirow[t]{4}{*}{ Spawning } & Season: Nov-Mar ${ }^{a}$ \\
\hline & Area: $180-550 \mathrm{~m}^{\mathrm{a}}$ \\
\hline & Mode: \\
\hline & $\begin{array}{l}\text { Migration: To deepwater spawn- } \\
\text { ing banks in Gulf of Alaska }{ }^{b}\end{array}$ \\
\hline Fecundity & $\begin{array}{l}\text { Range/function: } 200,000- \\
4 \text { million }^{c}\end{array}$ \\
\hline \multirow[t]{2}{*}{ Age at first maturity } & $7-20$ yr (females) ${ }^{a}$ \\
\hline & $5-20 \mathrm{yr}$ (males) $^{\mathrm{a}}$ \\
\hline \multirow[t]{2}{*}{ Longevity } & $42 \mathrm{yr}(\text { females })^{d}$ \\
\hline & $27 \mathrm{yr}$ (males) \\
\hline
\end{tabular}

\footnotetext{
${ }^{a}$ St. Pierre 1984

b Thompson and Van Cleve 1936

${ }^{\mathrm{c}}$ Schmit and Skud 1978

Webber and Alton 1976

${ }^{\mathrm{c}}$ Become juveniles at $28 \mathrm{~mm} \mathrm{SL}$.

Ref: Ahlstrom et al. 1984a, Pertseva-Ostroumova 1961, Thompson and Van Cleve 1936.
}

\section{EARLY LIFE HISTORY DESCRIPTION}

\section{EGGS}

Diameter

No. of oil globules

Oil globule diameter

Yolk

Envelope

Hatch size
$2.9-3.8 \mathrm{~mm}$

Homogeneous, dense, yellow (after preservation) Shallow honeycomb pattern (not always visible on preserved specimens but slight surface irregularities are easily discernible)

7.8-8.5 mm SL
Incubation time/temp.

Pigment

- Embryo unpigmented at hatching

Diagnostic characters

- Large size (>3.0 mm)

- Lack of pigment

\section{LARVAE}

Preanal length

Length at flexion

$<50 \%$ SL

Length at transformation

$13.6-17.8 \mathrm{~mm} \mathrm{SL}$

Sequence of fin development

$14.7-24.1 \mathrm{~mm} \mathrm{SL}^{\mathrm{e}}$

Caudal, dorsal and anal, pelvics, pectorals

\section{Pigment}

- Lack of pigment in yolksac larvae

- Series of melanophores along dorsal surface of notochord and along ventral midline

- Median finfolds pigmented along edge

\section{Diagnostic characters}

Distinguished from other pleuronectids by

- Myomeres (49-51)

- Pigment: Lack of bands, and presence of pigment on ventral midline and along edges of median finfolds

Distinguished from Reinhardtius hippoglossoides (both have large yolksac larvae) by

- See $R$. hippoglossoides (p. 622) 


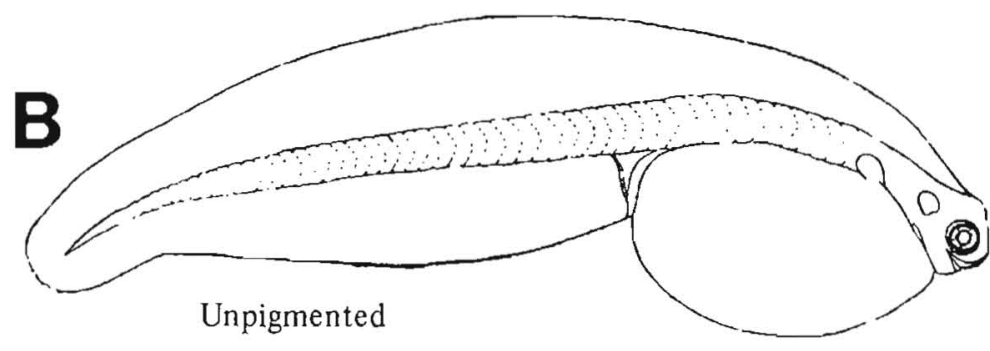

$9.5 \mathrm{~mm}$

c

Distal edges of median finfolds pigmented
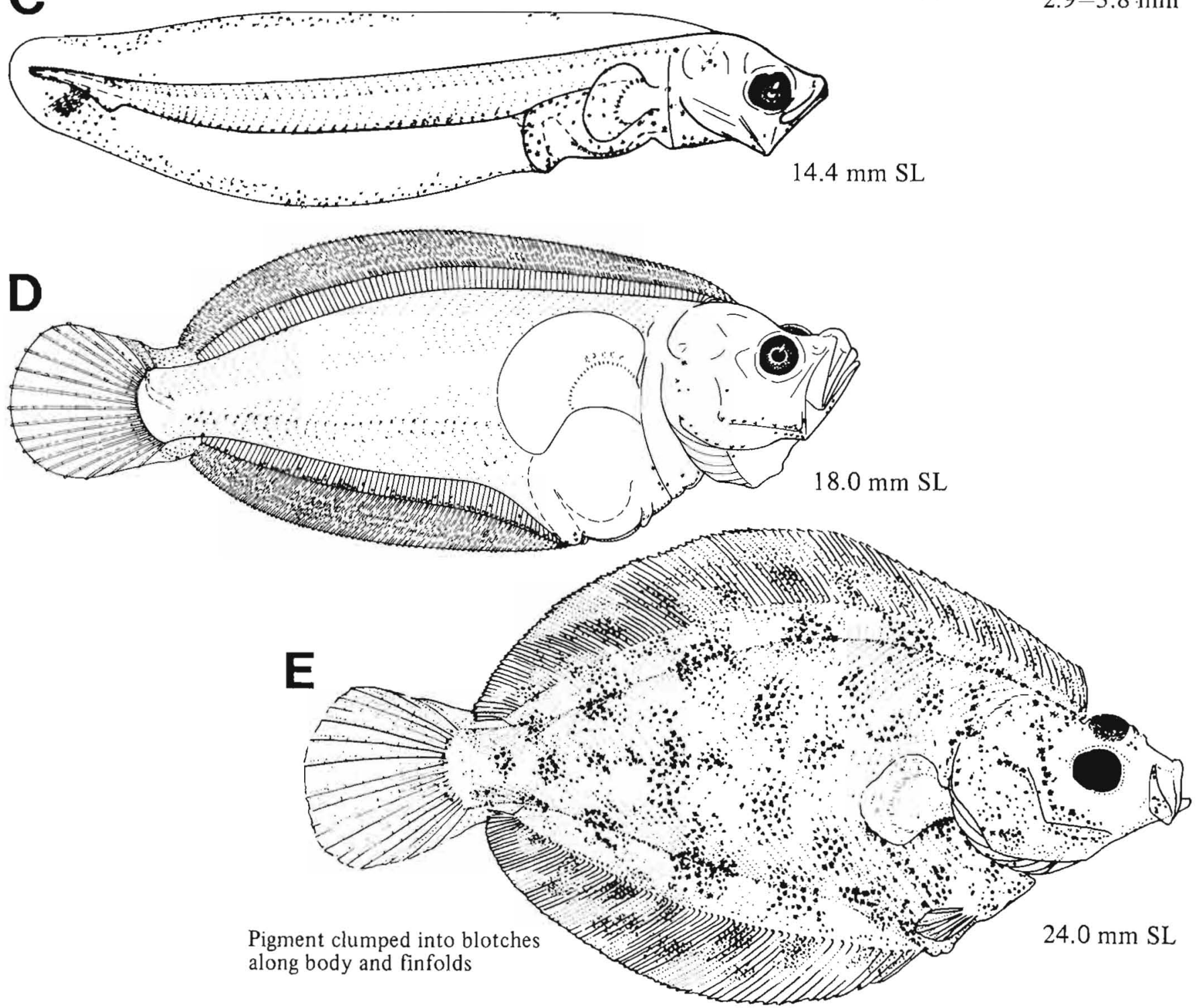

Figures A-B, Pertseva-Ostroumova 1961 (after Thompson and Van Cleve 1939); C-D, NWAFC originals (B. Vinter); E, Ahlstrom et al. 1984a. 


\section{MERISTICS}

Vertebrae

Total: $41-42-42$

Precaudal: 10-10-11

Caudal: $\mathrm{X}-\mathrm{X}-\mathrm{X}$

Branchiostegal rays $7-\mathrm{X}-8$

Caudal fin

$\mathrm{X}, 7+6, \mathrm{X}$

Total rays $=18^{\mathrm{a}}$

Pelvic fin

Thoracic

R: 6-6-6

Dorsal fin

R: 78-86-90

Pectoral fin

$\mathrm{R}: 11-\mathrm{X}-13$

Anal fin

R: $58-66-69$

Gill rakers

$\mathrm{U}: \mathrm{X}-\mathrm{X}-\mathrm{X}$

$\mathrm{L}: 7-\mathrm{X}-8$

\section{EARLY LIFE HISTORY DESCRIPTION}

\section{EGGS}

Diameter

No. of oil globules

Oil globule diameter

Yolk

Envelope

Hatch size

0.84-1.00 mm; occasionally up to $1.10 \mathrm{~mm}$

None

Homogeneous

Striated

2.7-2.9 mm SL

Incubation time/temp.

Pigment

\section{Diagnostic characters}

Very difficult to distinguish from the three other

1.0-mm pleuronectid eggs in the area:

Parophrys vetulus (p. 610)

Platichthys stellatus (p. 612)

Psettichthys melanostictus (p. 620)

Ecology

Bering Sea, $54-66^{\circ} \mathrm{N}$

ELH pattern

Epi- and mesobenthal, 20-425 m

Oviparous, pelagic eggs, pelagic larvae

Spawning

Season: Feb-Apr ${ }^{\text {b }}$

Area: Coastal waters ${ }^{b}$

Mode: Schools ${ }^{b}$

Migration:

Fecundity Range/function: 350,000$650,000^{\mathrm{c}}$

Age at first maturity $3 \mathrm{yr}$ (females) $^{\mathrm{b}}$

2 yr (males) ${ }^{b}$

Longevity $\quad 11 \mathrm{yr}$ (females) $^{\mathrm{d}}$

10 yr (males) ${ }^{d}$

\section{LARVAE}

Preanal length

Length at flexion

Length at transformation

Sequence of fin development

Pigment

- Three postanal bands at 50,67, 90\% SL

- Melanophores extend ventrally on gut and along posterior portion of abdominal cavity

Diagnostic characters

- Three pigment bands

- Low myomere count (41-42)

11 branched rays.

${ }^{b}$ Smith 1936

${ }^{\mathrm{C}}$ Forrester 1969

${ }^{\mathrm{d}} \mathrm{Har} 1973$

Ref: Richardson et al. 1980 
B

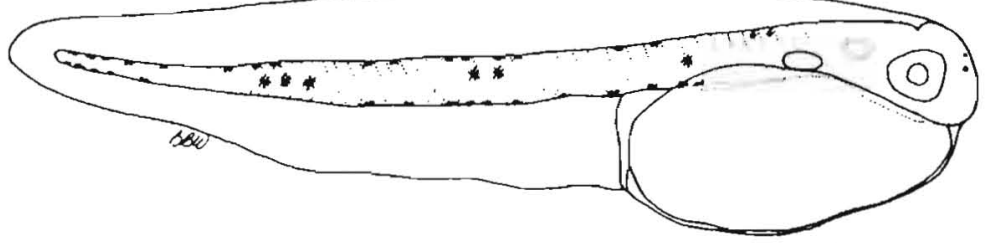

$2.9 \mathrm{~mm} \mathrm{SL}$

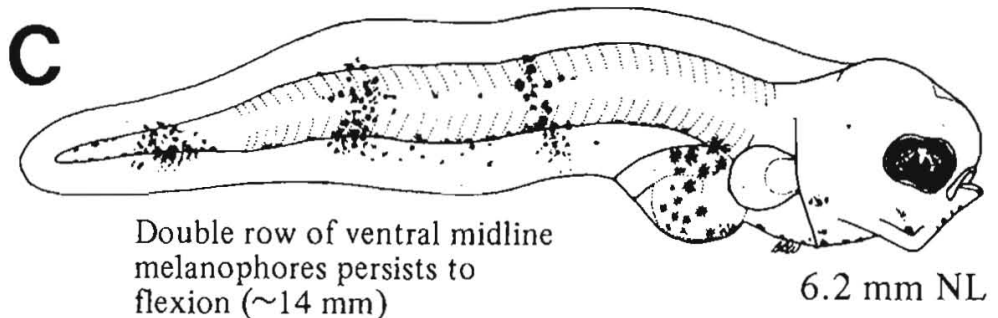

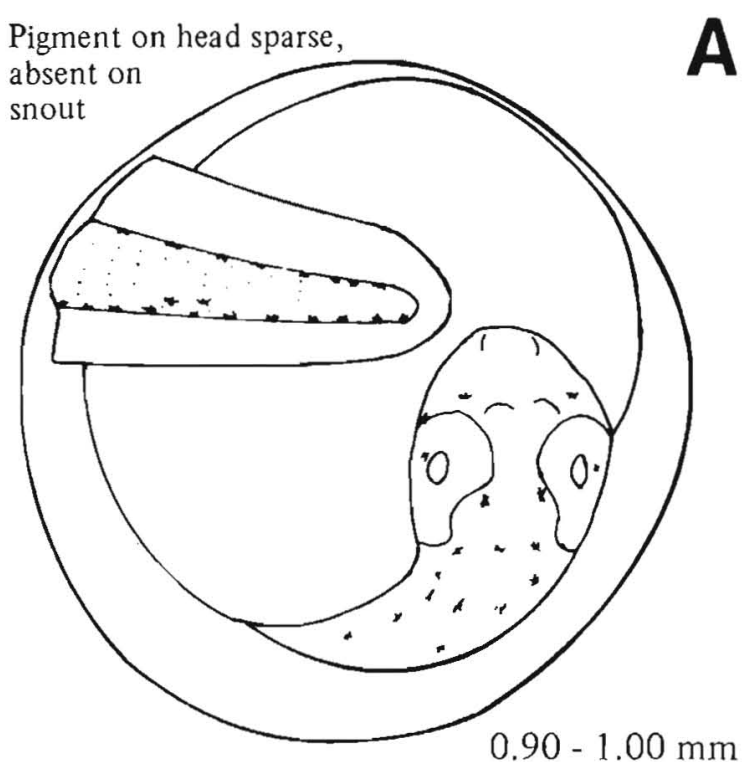
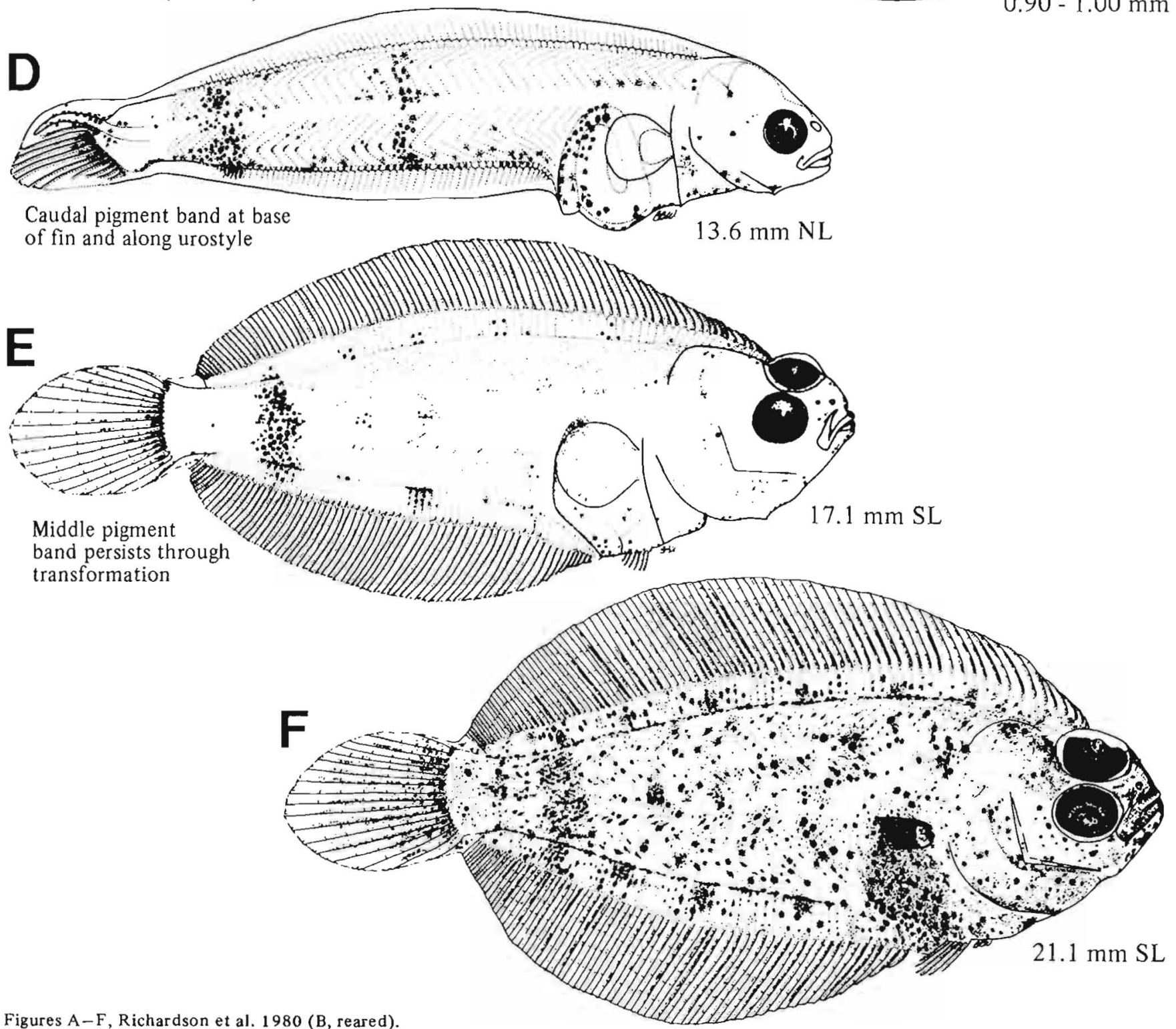

Figures A-F, Richardson et al. 1980 (B, reared). 


\section{MERISTICS}

\begin{tabular}{ll}
\hline Vertebrae & Total: $39-40-42$ \\
& Precaudal: $10-11-12$ \\
& Caudal: $28-30-31$ \\
Branchiostegal rays & $7-7-7$ \\
Caudal fin & Total rays $=18-19^{\text {a }}$ \\
Pelvic fin & Thoracic \\
& R: $6-6-6$ \\
Dorsal fin & R: $65-76-84$ \\
Pectoral fin & R: $8-11-13$ \\
Anal fin & R: $50-58-65 \quad$ \\
Gill rakers & U: $3-3-3 \quad$ L: $5-X-8$
\end{tabular}

\section{LIFE HISTORY}

\begin{tabular}{|c|c|}
\hline Range & $\begin{array}{l}\text { S. California, } 32-34^{\circ} \mathrm{N} \text {, to } \\
\text { Bering Sea, } 54-66^{\circ} \mathrm{N}\end{array}$ \\
\hline Ecology & $\begin{array}{l}\text { Epi-, meso-, and bathybenthal, } \\
0-579 \mathrm{~m}\end{array}$ \\
\hline ELH pattern & $\begin{array}{l}\text { Oviparous; demersal, adhesive } \\
\text { eggs; pelagic larvae }\end{array}$ \\
\hline Spawning & Season: Feb-Apr ${ }^{b}$ \\
\hline & Area: \\
\hline & Mode: \\
\hline & Migration: \\
\hline Fecundity & Range/function: $400,000-$ \\
\hline & $1,300,000$ (Brit. Col.); ${ }^{\mathrm{c}}$ \\
\hline & $150,000-400,000$ (Bering \\
\hline & Sea); ${ }^{\text {d } 80,000-920,000 ~}$ \\
\hline & (western Pacific)/ \\
\hline & $\begin{array}{l}\mathrm{F}=0.0004891 \times \mathrm{L}^{3.120} \\
\mathrm{~L}=\mathrm{BL} \mathrm{cm}^{\mathrm{e}}\end{array}$ \\
\hline Age at first maturity & $3-4$ yr (females) ${ }^{b}$ \\
\hline & 2 yr (males) $^{b}$ \\
\hline Longevity & 15 yr (females) ${ }^{f}$ \\
\hline & 22 yr (males) ${ }^{f}$ \\
\hline
\end{tabular}

\section{EARLY LIFE HISTORY DESCRIPTION}

\section{EGGS}

Diameter

No. of oil globules

Oil globule diameter

Yolk

Envelope

Hatch size

Incubation time/temp.

Pigment

Diagnostic characters

- Demersal egg

\section{LARVAE}

Preanal length

$<50 \%$ SL

Length at flexion

8.4-9.9 mm SL

Length at transformation

$\sim 20 \mathrm{~mm} \mathrm{SL}$;

$>17.7 \mathrm{~mm} \mathrm{SL}^{\mathrm{h}}$
Sequence of fin Caudal, dorsal, and anal; development pelvics; pectorals

Pigment

- Dorsally on peritoneum, ventrolateral gut pigment becoming posterolateral with development

- Postanal ventral melanophores 3/4 length of body

- Two dorsolateral patches, the posterior one becoming a band meeting the ventral surface

- Anal finfold lightly pigmented on posterior half

\section{Diagnostic characters}

Distinguished from other pleuronectids by

- Presence of one band posteriorly on body, not as conspicuous as most

- Size at transformation (20 mm SL)

- Advanced stage of development at hatching

See Lepidopsetta 2 (p. 599) and Psettichthys melanostictus (p. 620)

\footnotetext{
a 12 branched rays.

${ }^{b}$ Smith 1936

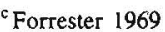

${ }^{d}$ Fadeey 1965

'Shvetsov 1979

${ }^{i}$ Hart 1973

${ }^{8}$ Pertseva-Ostroumova 1961

${ }^{h}$ Ahlstrom et al. 1984 a
}

Ref: Ahlstrom et al. 1984a, Forrester 1964, Pertseva-Ostroumova 196I. 
B

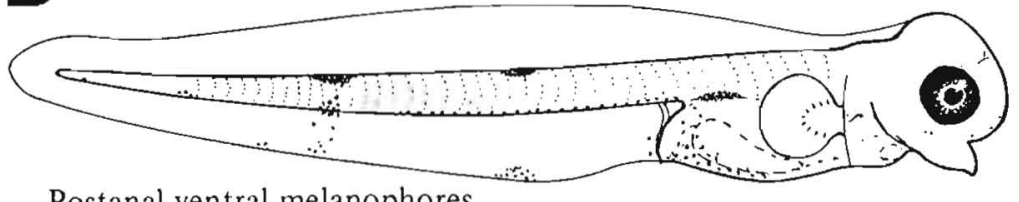

Postanal ventral melanophores extend $3 / 4 \mathrm{BL}$

$4.3 \mathrm{~mm} \mathrm{SL}$

C

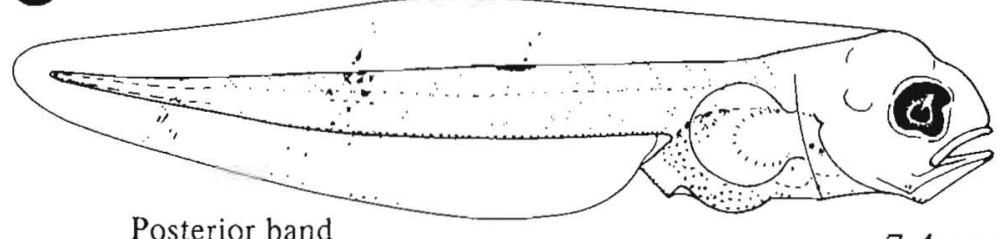

$7.4 \mathrm{~mm} \mathrm{SL}$

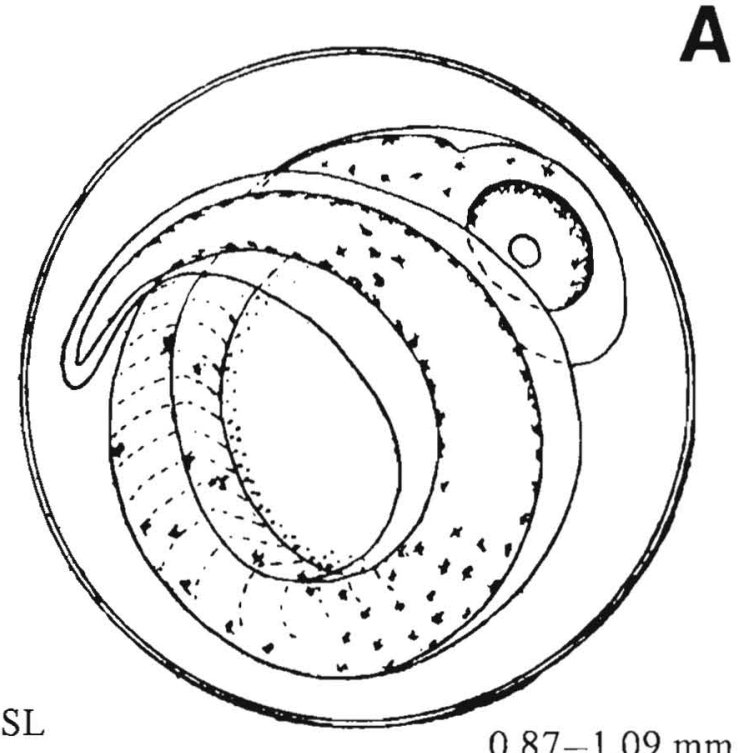

$0.87-1.09 \mathrm{~mm}$

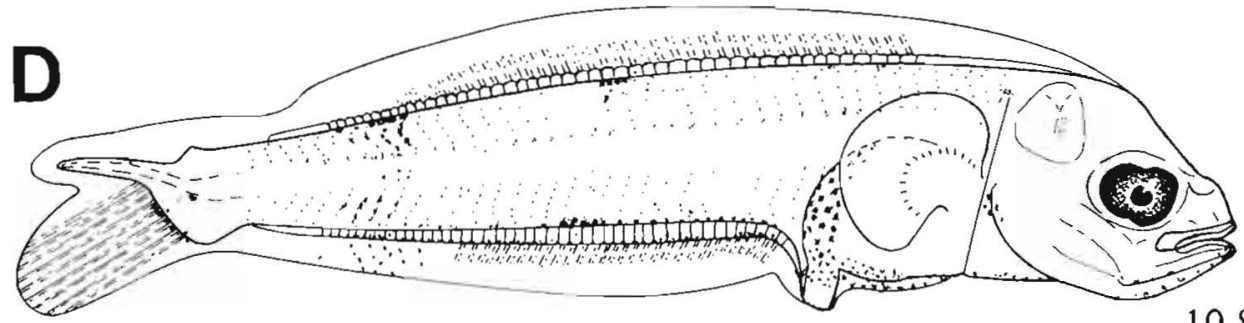

Pigment on ventral and

$10.8 \mathrm{~mm} \mathrm{SL}$

posterior portions of gut

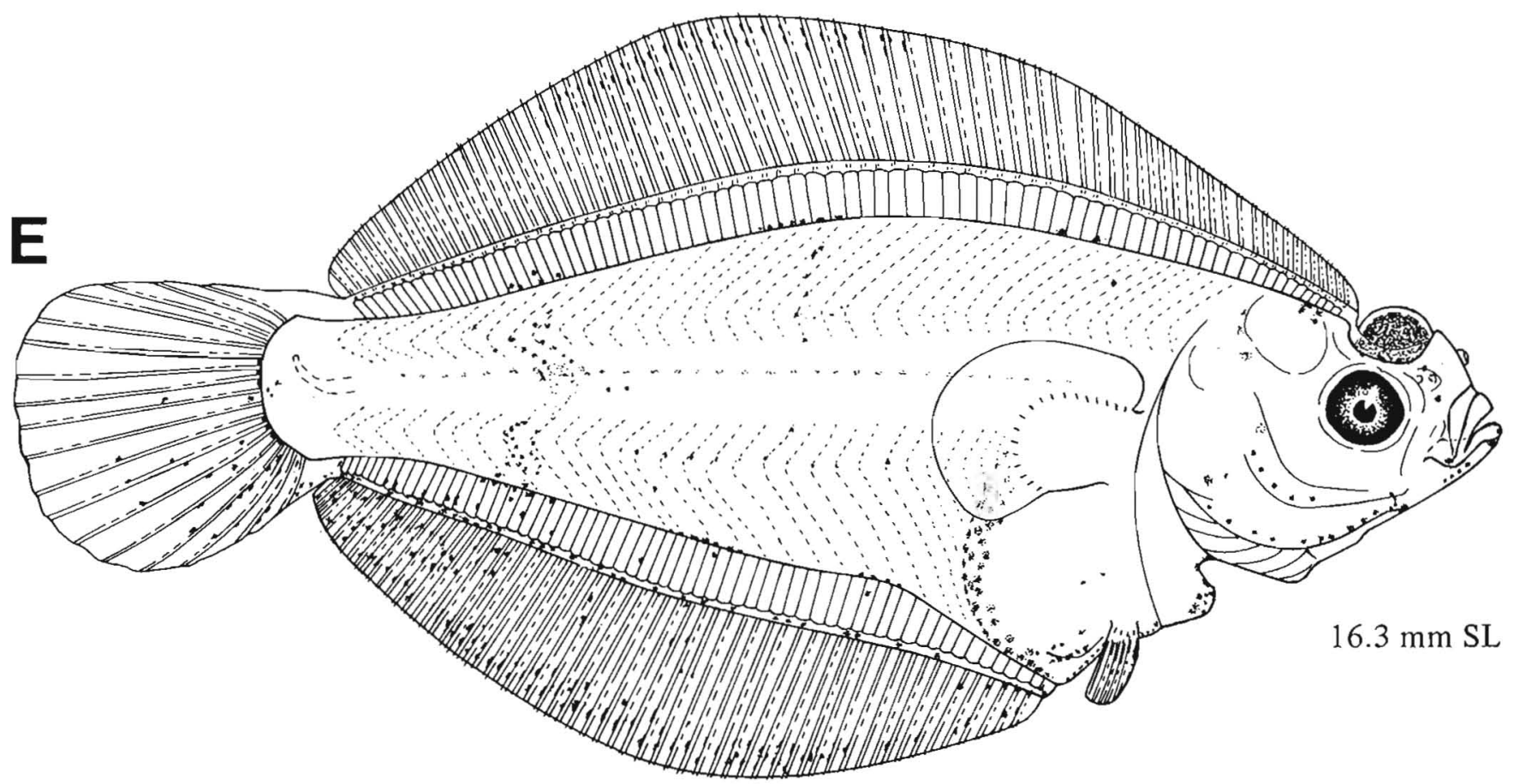

Figure A, Pertseva-Ostroumova 1961; B-E, NWAFC originals (B. Vinter). 



\section{Lepidopsetta bilineata/Lepidopsetta 2/Psettichthys melanostictus}

Two readily distinguishable types of Lepidopsetta larvae are collected in our study area which we designate Lepidopsetta bilineata and Lepidopsetta 2 . Wilimovsky et al. (1967) had previously indicated that there are two subspecies of Lepidopsetta in the Northeast Pacific based on adult specimens $(L . b$. bilineata and $L$. b. peracuata). Lepidopsetta 2 larvae are very similar to larvae of Psettichthys melanostictus at certain stages of development. We present Lepidopsetta 2 as a separate series, since these larvae are distinct from those of $L$. bilineata and $P$. melanostictus. The following pigment patterns and morphological characters separate the three kinds of larvae.

Psettichthys melanostictus (see also p. 620)

Range from California to Alaska, but the center of distribution appears to be off California, Oregon, and Washington. Pigment

- Postanal ventral midline melanophores: Usually restricted to 3-4 large spots

- Tail pigment less prominent than on Lepidopsetta 2

- Pigment on upper and lower jaw

- Isthmus pigment heavier than on others

- First spot along dorsal midline more anterior (over anus)

Morphology

- Gut coiled, shape of posterior gut

- Deeper body (wider finfold)

Lepidopsetta 2 (see p. 600, 601)

Range from Puget Sound to Alaska, and center of distribution appears to be off Southeast Alaska and in the Gulf of Alaska. Pigment

- Postanal ventral midline melanophores: A series of small melanophores extending from the gut to the last myomere; sometimes a larger spot occurs about midbody (with larvae 6.3-9.7 mm SL, there may be more spots than at other sizes)

- First spot along dorsal midline behind anus

- Other: Tail pigment more intense, but mouth and isthmus pigment less intense than on Psettichthys Morphology

- Eye relatively larger than on Psettichthys at similar ontogenetic stages

- Gut shape simple

Lepidopsetta bilineata (see also p. 596)

Range from southern California to the Bering Sea.

Pigment

- Postanal ventral midline melanophores: A series of small melanophores extending from the gut to about $2 / 3$ body

- Only two spots along dorsal midline; anterior spot at midbody, posterior spot forming a band at about myomere 30

- No heavy pigment along edges of median finfold

- Tail pigment less prominent than on others

- Pigment along hypural edge

Morphology

- Gut shape simple

Finfold pigment may or may not be an additional criterion for separating Lepidopsetta 2 and Psettichthys. Many Psettichthys specimens in our collection appear to have more prominent finfold pigment that is retained longer during development; however, many of these are laboratory-reared specimens. More wild-caught Psettichthys specimens are needed to verify whether they all have intense finfold pigment.

Yolksac larvae are more difficult to separate. Psettichthys larvae appear to possess upper and lower jaw pigment, smaller, more concentrated melanophores in the finfold and on the urostyle, and the last postanal band has a ventral stripe only. Lepidopsetta 2 yolksac larvae appear to have only lower jaw pigment, larger, fewer, and evenly spaced melanophores in the finfold and urostyle, and the last postanal band has a dorsal and ventral stripe.

Literature: Specimens illustrated by Ahlstrom et al. (1984a) are both Psettichthys based primarily on the presence of the coiled gut and the 2-3 large ventral melanophores (Fig. 351C, p. 662, and Fig. 352E, p. 663). Hickman (1959) illustrated six Psettichthys larvae from specimens he reared from eggs (his Figs. 1 and 2) and from specimens he collected in Puget Sound (his Figs. 3-6). Hickman's Figures 3 and 4 appear to resemble Lepidopsetta 2 larvae, while his other figures appear to resemble Psettichthys. 


\section{Psettichthys melanostictus}

A
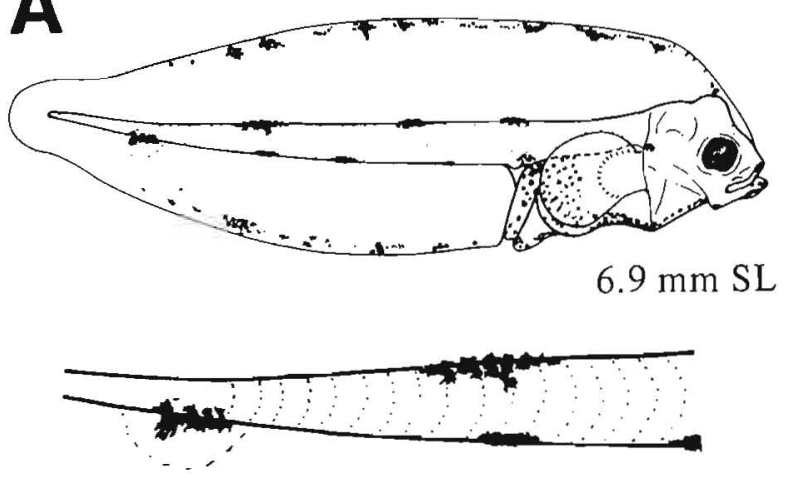

B

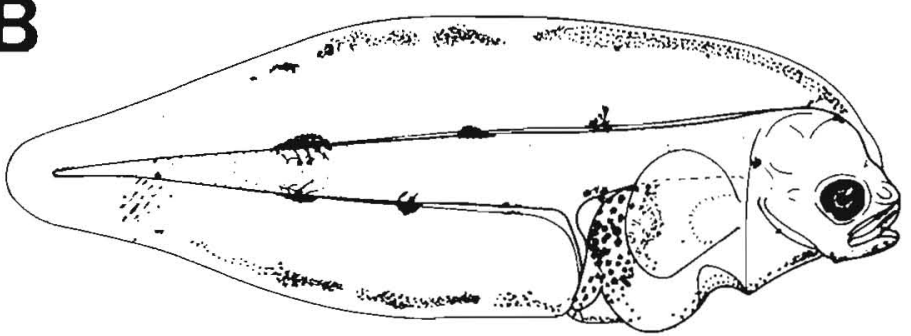

$8.1 \mathrm{~mm} \mathrm{SL}$

\section{Lepidopsetta 2}

D

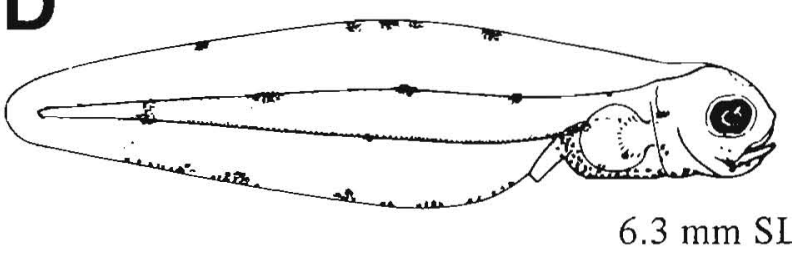

$6.3 \mathrm{~mm} \mathrm{SL}$

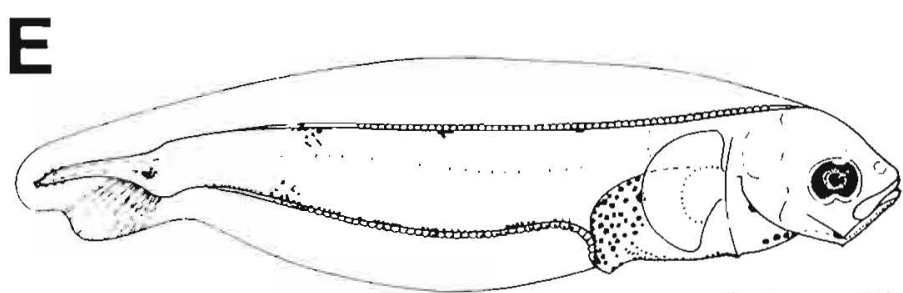

$9.7 \mathrm{~mm} \mathrm{SL}$

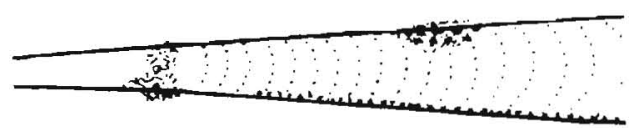

\section{Lepidopsetta bilineata}

G

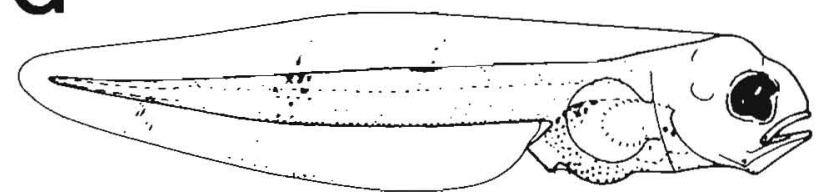

$7.4 \mathrm{~mm} \mathrm{SL}$

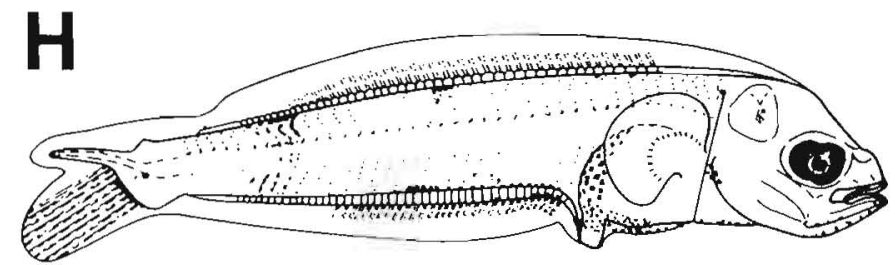

$10.8 \mathrm{~mm} \mathrm{SL}$

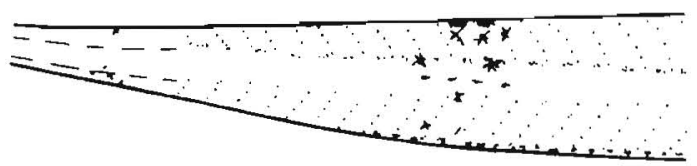

Figures A-I, NWAFC originals (B. Vinter; A, D, G, include a detailed enlargement of tail). 


\section{PSETTICHTHYS / LEPIDOPSETTA}
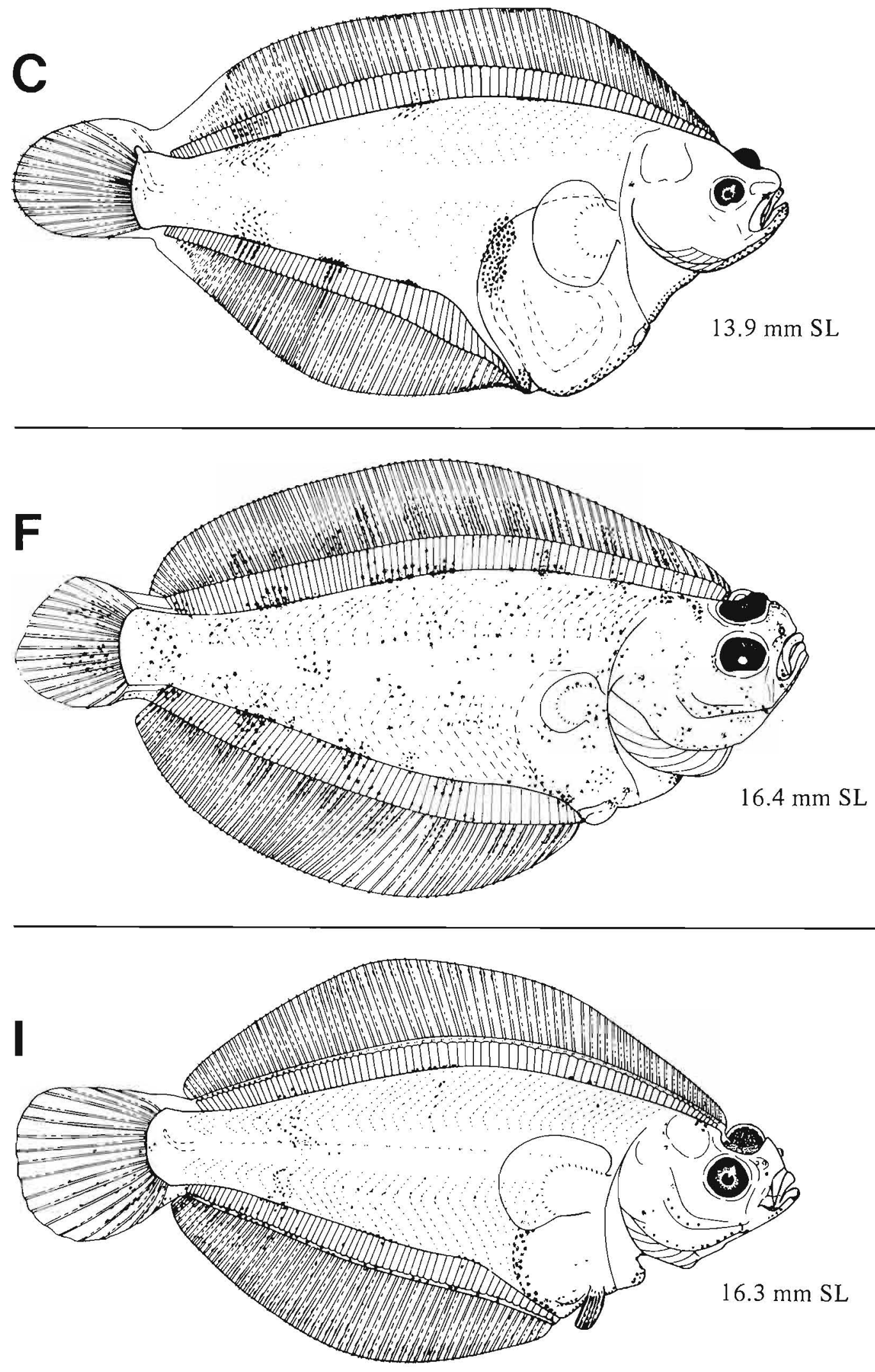


\section{MERISTICS}

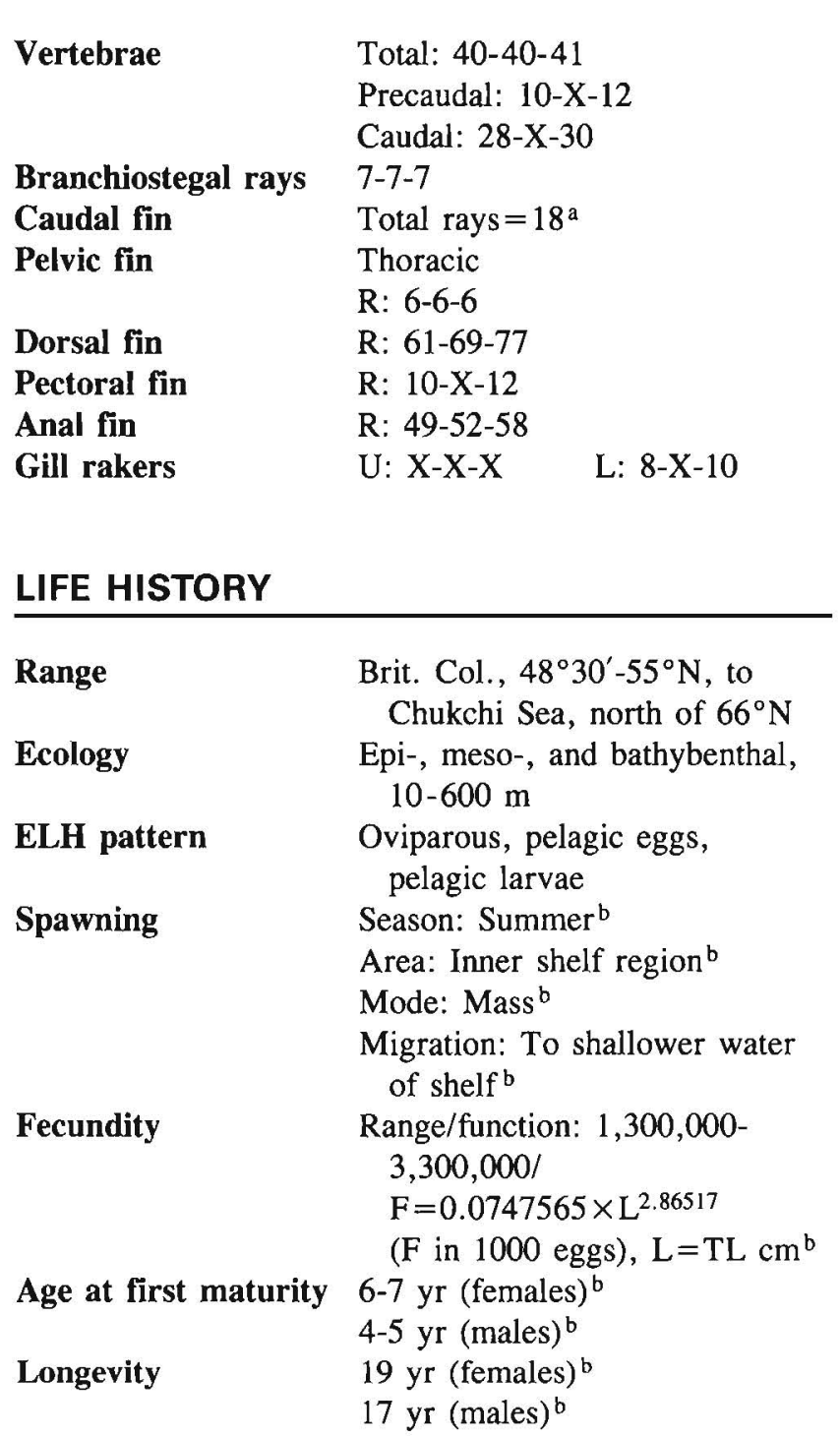

a 12 branched rays.

${ }^{b}$ Salveson and Alton $1976 \mathrm{~b}$

${ }^{c}$ Ahlstrom et al. 1984a

${ }^{d}$ According to Allen and Smith (1988), this species occurs in the Bering Sea

'Pertseva-Ostroumova 1961

Ref: Ahlstrom et al. 1984a, Pertseva-Ostroumova 1961, Schmidt 1950.

\section{EARLY LIFE HISTORY DESCRIPTION}

\section{EGGS}

Diameter

No. of oil globules

Oil globule diameter

Yolk

Envelope

Hatch size

Incubation time/temp.

Pigment

\section{Diagnostic characters}

- Size

\section{LARVAE}

Preanal lengt

Length at flexion

$<50 \% \mathrm{SL}$

$\sim 7 \mathrm{~mm} \mathrm{SL}$;

7.5-9.5 $\mathrm{mm} \mathrm{SL}^{\mathrm{c}}$

Length at transformation $15-17 \mathrm{~mm} \mathrm{SL}$; may begin at $10 \mathrm{~mm} \mathrm{SL}^{\mathrm{c}}$

Sequence of fin Caudal, dorsal and anal, development

pelvics, pectorals

Pigment

- Mediolateral, along notochord

- Ventrolateral, along hypaxial myomeres

- Anal finfold

Diagnostic characters

Distinguished from other pleuronectids without postanal pigment bands by

- Ventrolateral pigment along hypaxial myomeres

- Size at transformation (15-17 mm SL)

- Finfold pigment restricted to anal fin

- Urostyle unpigmented

$L$. proboscidea and $L$. sakhalinensis ${ }^{\mathrm{d}}$ eggs and larvae are incompletely known. The following information may aid in identification.

$\begin{array}{lcc} & \text { L. proboscidea } & \text { L. sakhalinensis } \\ \text { Egg diameter } & 0.72-0.87 \mathrm{~mm} & \\ \text { Total vertebrae } & 38-40 & \\ \quad \text { Precaudal } & 11 & \\ \quad \text { Caudal } & 27-29 & \\ \text { Dorsal fin rays } & 62-69 & 68-76 \\ \text { Pectoral fin rays } & 12 & 53-59 \\ \text { Anal fin rays } & 46-50 & \\ \text { Range } & \text { Bering Sea-Chukchi Sea } \\ \text { Spawning season } \quad \text { Spring-Summer } & \end{array}$




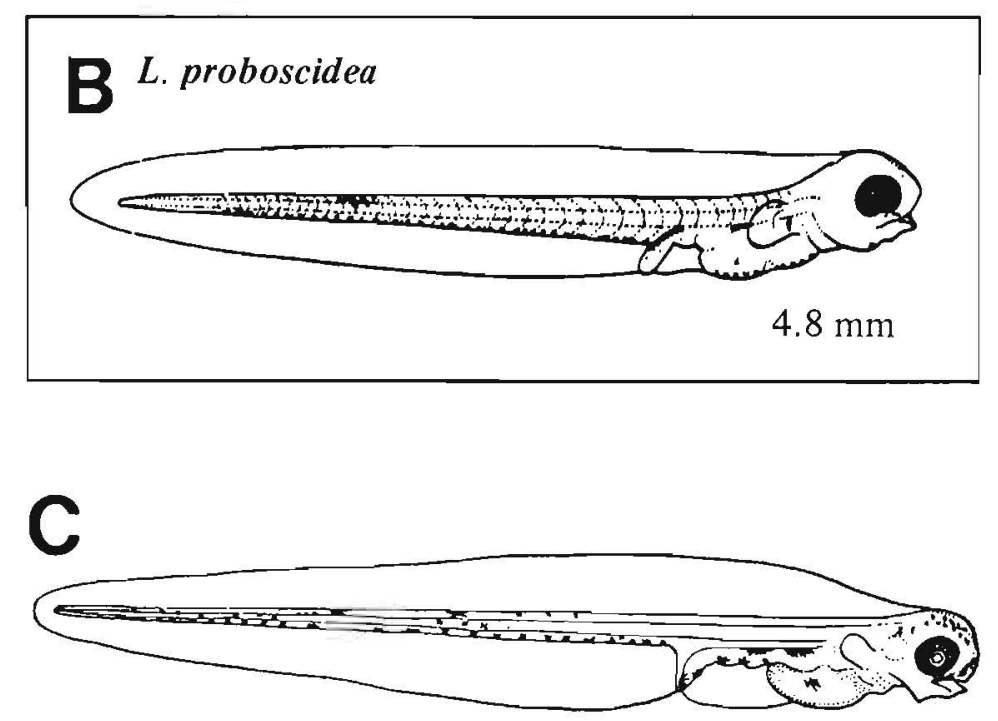

$3.2 \mathrm{~mm}$

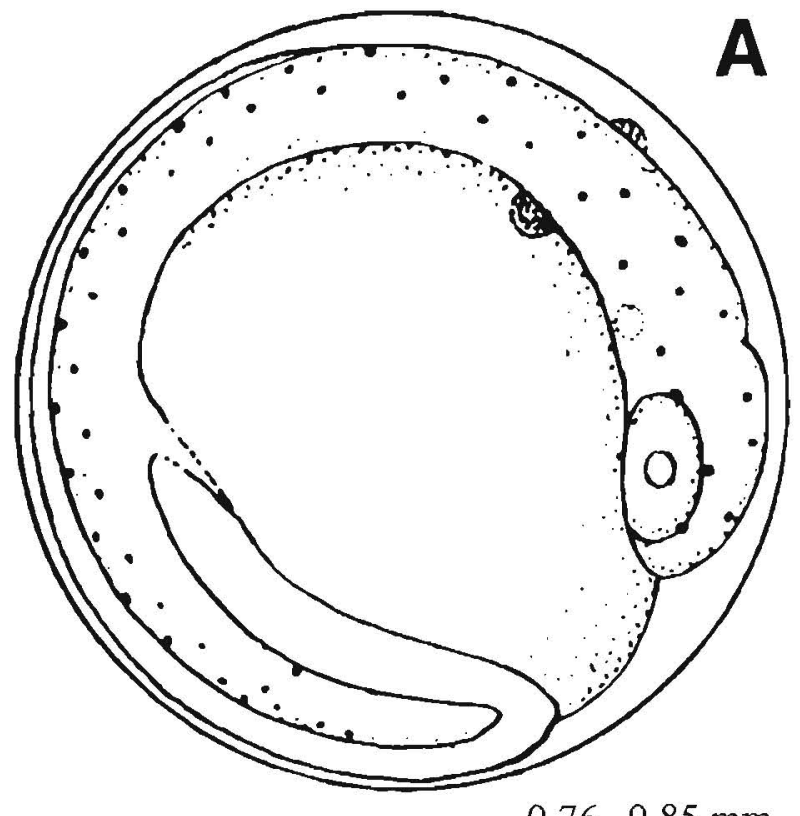

$0.76-0.85 \mathrm{~mm}$

D
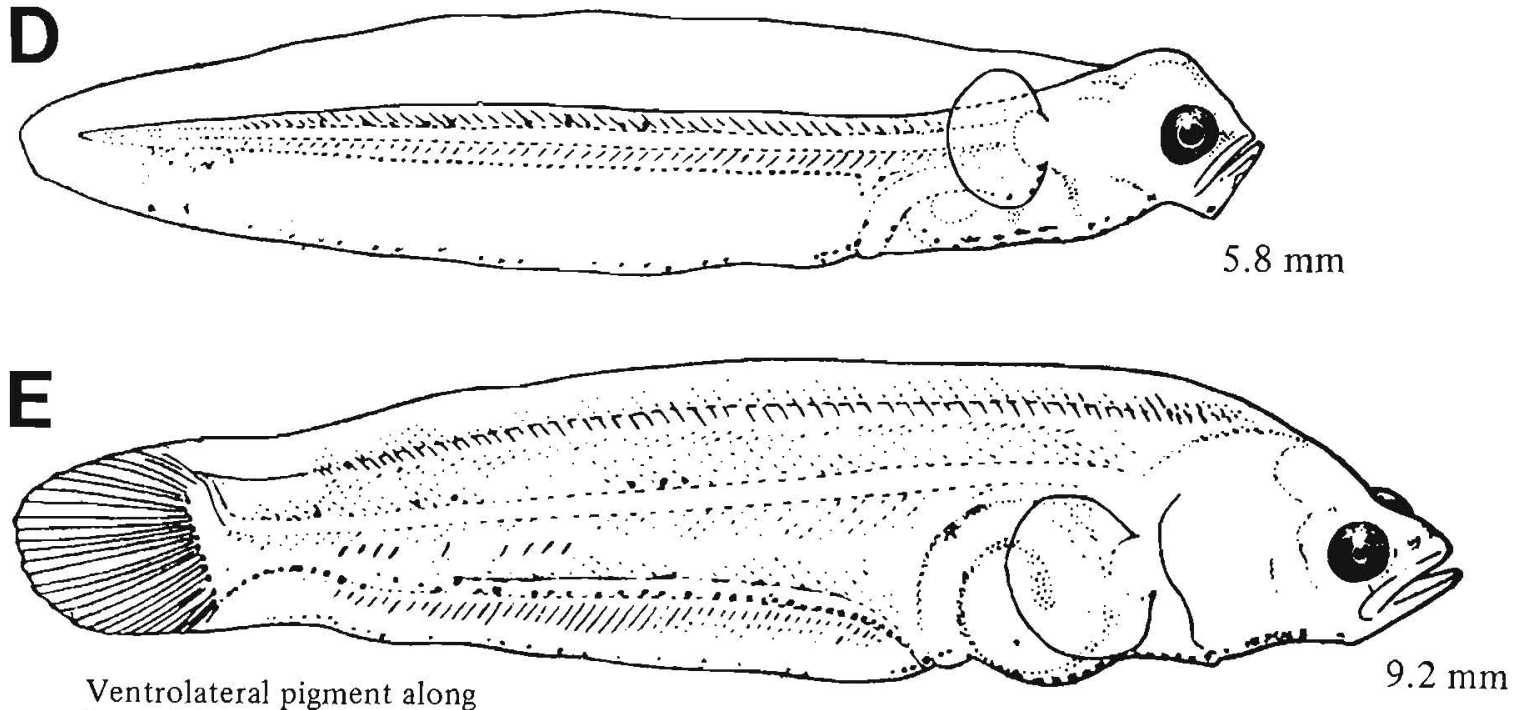
hypaxial myomeres

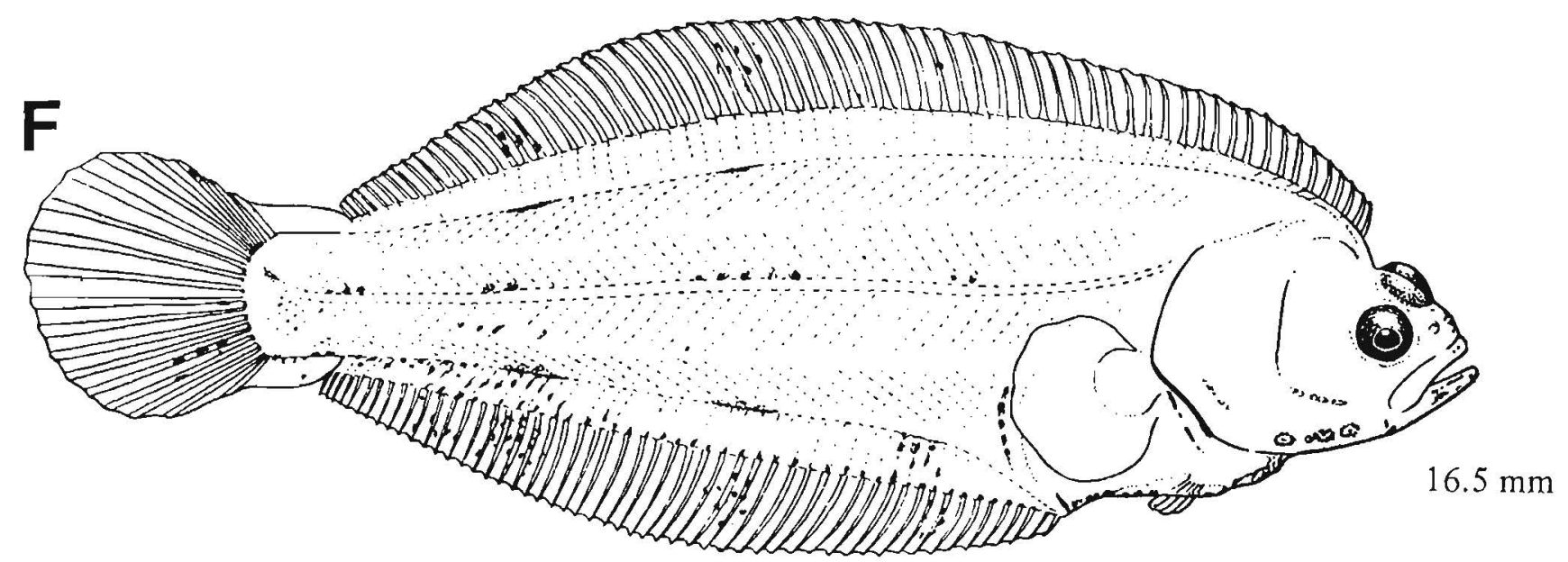

Figures A-F (C, reversed), Pertseva-Ostroumova 1961. 
MERISTICS

\begin{tabular}{|c|c|}
\hline \multirow[t]{3}{*}{ Vertebrae } & Total: $37-\mathrm{X}-41$ \\
\hline & Precaudal: $11-X-13$ \\
\hline & Caudal: $26-26-26$ \\
\hline Branchiostegal rays & $7-X-8$ \\
\hline Caudal fin & Total rays $=18$ \\
\hline \multirow[t]{2}{*}{ Pelvic fin } & Thoracic \\
\hline & $R: 6-6-6$ \\
\hline Dorsal fin & $\mathrm{R}: 48-\mathrm{X}-64$ \\
\hline Pectoral fin & $\mathrm{R}: 8-\mathrm{X}-12$ \\
\hline Anal fin & $R: 33-X-46$ \\
\hline Gill rakers & $\mathrm{U}: 2-\mathrm{X}-4$ \\
\hline \multicolumn{2}{|l|}{ LIFE HISTORY } \\
\hline Range & $\begin{array}{l}\text { Bering Sea, } 54-66^{\circ} \mathrm{N} \text {, to } \\
\text { Arctic, not specific }\end{array}$ \\
\hline Ecology & Nearshore shelf demersal \\
\hline ELH pattern & $\begin{array}{l}\text { Oviparous, demersal eggs, } \\
\text { pelagic larvae }\end{array}$ \\
\hline \multirow[t]{4}{*}{ Spawning } & Season: Jan-Mar ${ }^{\mathrm{a}}$ \\
\hline & Area: Shallow water, $5-10 \mathrm{~m}^{\mathrm{a}}$ \\
\hline & Mode: \\
\hline & Migration: \\
\hline Fecundity & Range/function: $31,000-230,000^{\mathrm{a}}$ \\
\hline Age at first maturity & $2+\mathrm{yr}$ (usually $4-5)^{b}$ \\
\hline Longevity & $>9 \mathrm{yr}^{\mathrm{b}}$ \\
\hline
\end{tabular}

\section{EARLY LIFE HISTORY DESCRIPTION}

\section{EGGS}

Diameter

No. of oil globules

Oil globule diameter

Yolk

Envelope

Hatch size

$1.2-1.7 \mathrm{~mm}(1.54-1.70 \mathrm{~mm})$

None

Clear, homogeneous

Thin, smooth

$3.7 \mathrm{~mm} \mathrm{SL}$

Incubation time/temp.

Pigment

Diagnostic characters

\section{LARVAE $^{\mathrm{C}}$}

Preanal length $\quad<50 \%$ SL

Length at flexion

Length at transformation

Sequence of fin development

Pigment

- Newly hatched larvae heavily pigmented, but with development pigment appears to be restricted to postanal body above and below notochord

\section{Diagnostic characters}

Distinguished from other pleuronectids without pigment bands by

- No pigment in finfolds or along hypaxial myomeres

- Myomere count (37-41) 
A

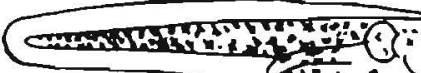

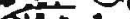

\section{$3.7 \mathrm{~mm}$}

B
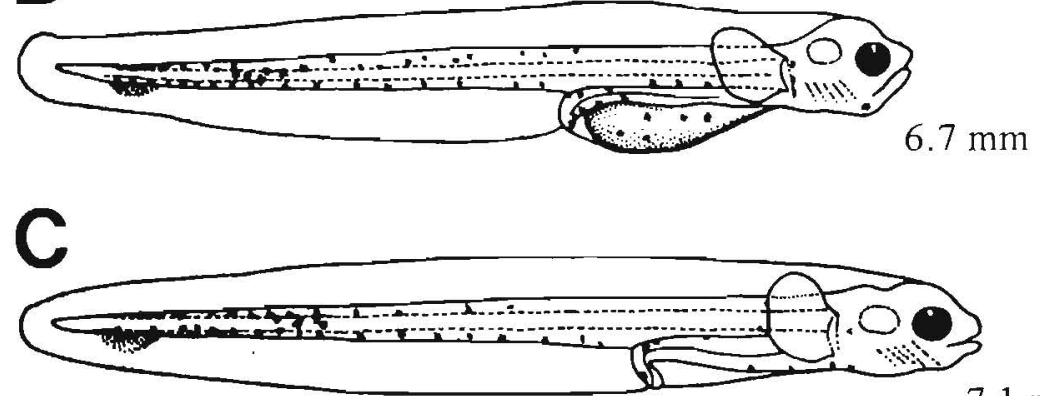

$7.1 \mathrm{~mm}$

Figures A-C, Pertseva-Ostroumova 1961 (figures reversed). 


\section{MERISTICS}

\begin{tabular}{|c|c|}
\hline Vertebrae & $\begin{array}{l}\text { Total: } 43-45-47 \\
\text { Precaudal: } 11-12-13 \\
\text { Caudal: } 32-33-35\end{array}$ \\
\hline Branchiostegal rays & $7-7-7$ \\
\hline Caudal fin & $\begin{array}{l}X, 8+7, X \\
\text { Total rays }=19^{a}\end{array}$ \\
\hline Pelvic fin & $\begin{array}{l}\text { Thoracic } \\
\text { R: } 6-6-6\end{array}$ \\
\hline Dorsal fin & R: $72-77-88$ \\
\hline Pectoral fin & R: $10-10-10$ \\
\hline Anal fin & $R: 57-60-66$ \\
\hline Gill rakers & $\mathrm{L}: 9-\mathrm{X}-11$ \\
\hline \multicolumn{2}{|l|}{ LIFE HISTORY } \\
\hline Range & $\begin{array}{l}\text { South of southern California to } \\
\text { Gulf of Alaska, } 54-60^{\circ} \mathrm{N}\end{array}$ \\
\hline Ecology & $\begin{array}{l}\text { Epi-, meso-, and bathybenthal, } \\
25-800 \mathrm{~m}\end{array}$ \\
\hline ELH pattern & $\begin{array}{l}\text { Oviparous, pelagic eggs, } \\
\text { pelagic larvae }\end{array}$ \\
\hline Spawning & $\begin{array}{l}\text { Season: Feb; }{ }^{b} \mathrm{Apr}^{\mathrm{c}} \\
\text { Area: } \\
\text { Mode: } \\
\text { Migration: }\end{array}$ \\
\hline Fecundity & Range/function: \\
\hline Age at first maturity & $\begin{array}{l}3-5 \mathrm{yr}^{(\text {females })^{\mathrm{C}}} \\
2-3 \mathrm{yr}^{(\text {males })^{\mathrm{c}}}\end{array}$ \\
\hline
\end{tabular}

\section{EARLY LIFE HISTORY DESCRIPTION}

\section{EGGS}

Diameter

No. of oil globules

Oil globule diameter

Yolk

Envelope

Hatch size

$1.47-1.71 \mathrm{~mm}$

None

Homogeneous

Surface may be irregular and appear bumpy $\sim 5.6 \mathrm{~mm} \mathrm{SL}$

\section{Incubation time/temp.}

Pigment

- Yolksac

- Late-stage embryo: Distinct caudal pigment visible

\section{Diagnostic characters}

- Caudal pigment

- Smallest size range of three similar-looking pleuronectid eggs in the $1.5-2.5 \mathrm{~mm}$ size range; see Glyptocephalus zachirus (p. 586) and Microstomus pacificus (p. 608)

\section{LARVAE}

Preanal length

Length at flexion $<50 \%$ SL

Length at transformation

Sequence of fin development 9.0-10.9 mm SL Pigment $15.7-24.7 \mathrm{~mm} \mathrm{SL}$

Caudal, dorsal and anal, pelvics, pectorals

- Early and midflexion larvae

-Dorsal and ventral midline melanophores around tail and in finfold; with development, these melanophores move laterally on body along myoseptal lines

-Finfold pigment increases to cover anal finfold and posterior half of dorsal finfold

- Gut pigment increases ventrally with development

Diagnostic characters

Distinguished from other pleuronectids without pigment bands by

- Pigment pattern: Continuous dorsal and ventral midline

- In preflexion larvae, the anterior melanophores of the dorsal midline series extend laterally

- Finfold pigment

\footnotetext{
a 12-13 branched rays.

${ }^{\text {bHart }} 1973$

${ }^{\text {c}}$ Smith 1936
}

Ref: Ahlstrom and Moser 1975, Ahlstrom et al. 1984a. 
Lateral placement of the anterior melanophores that are part of the

B same series as the posterior dorsal

\section{C} midline melanophores
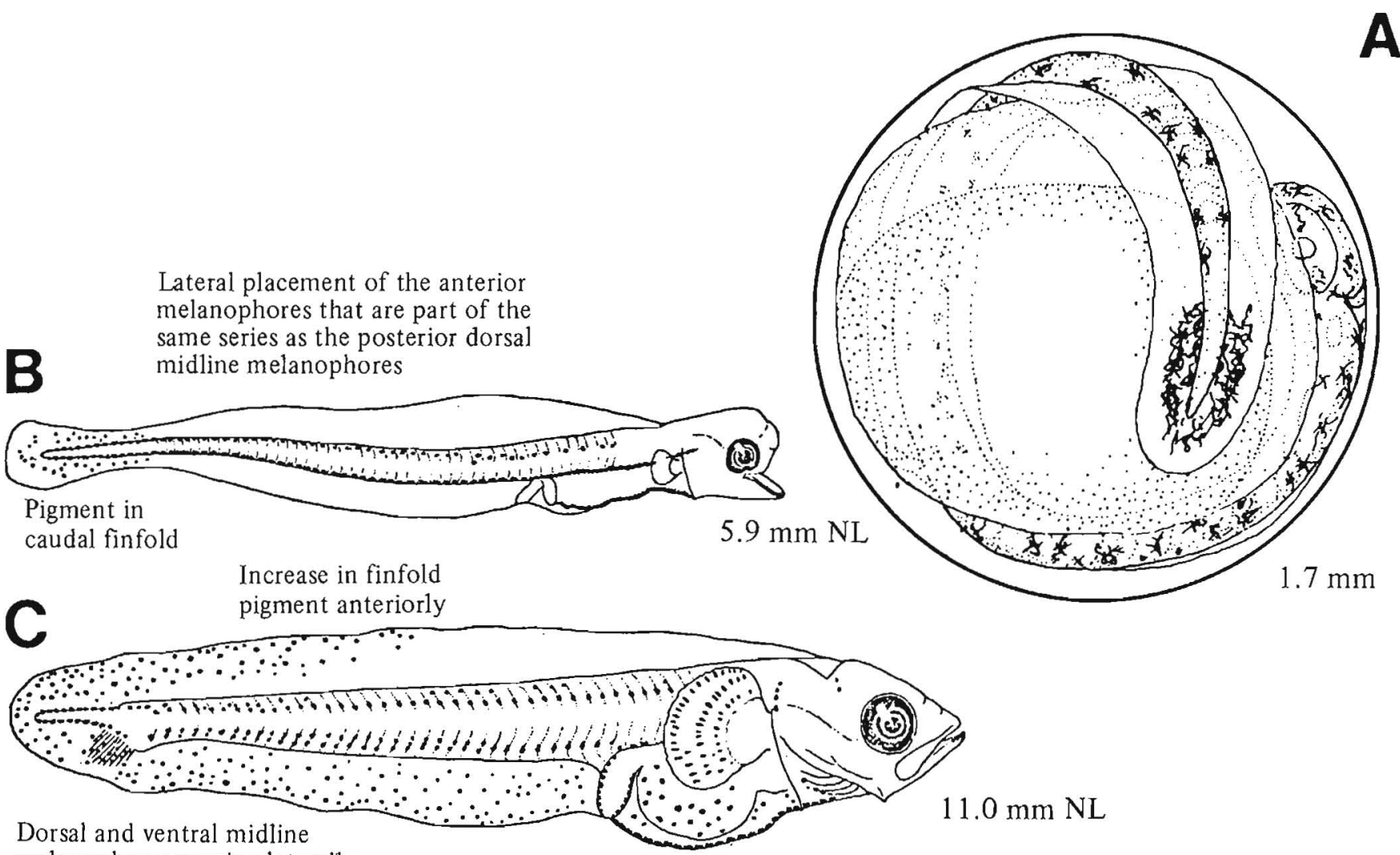

melanophores moving laterally

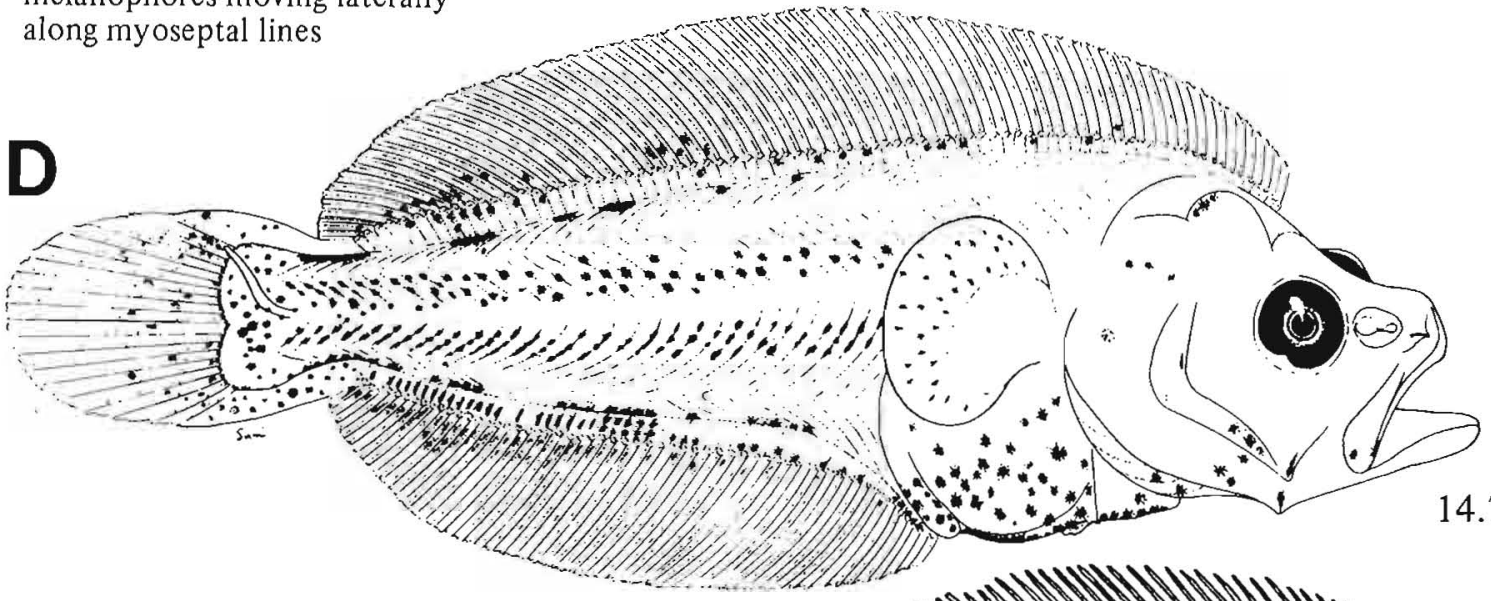

$14.7 \mathrm{~mm} \mathrm{SL}$

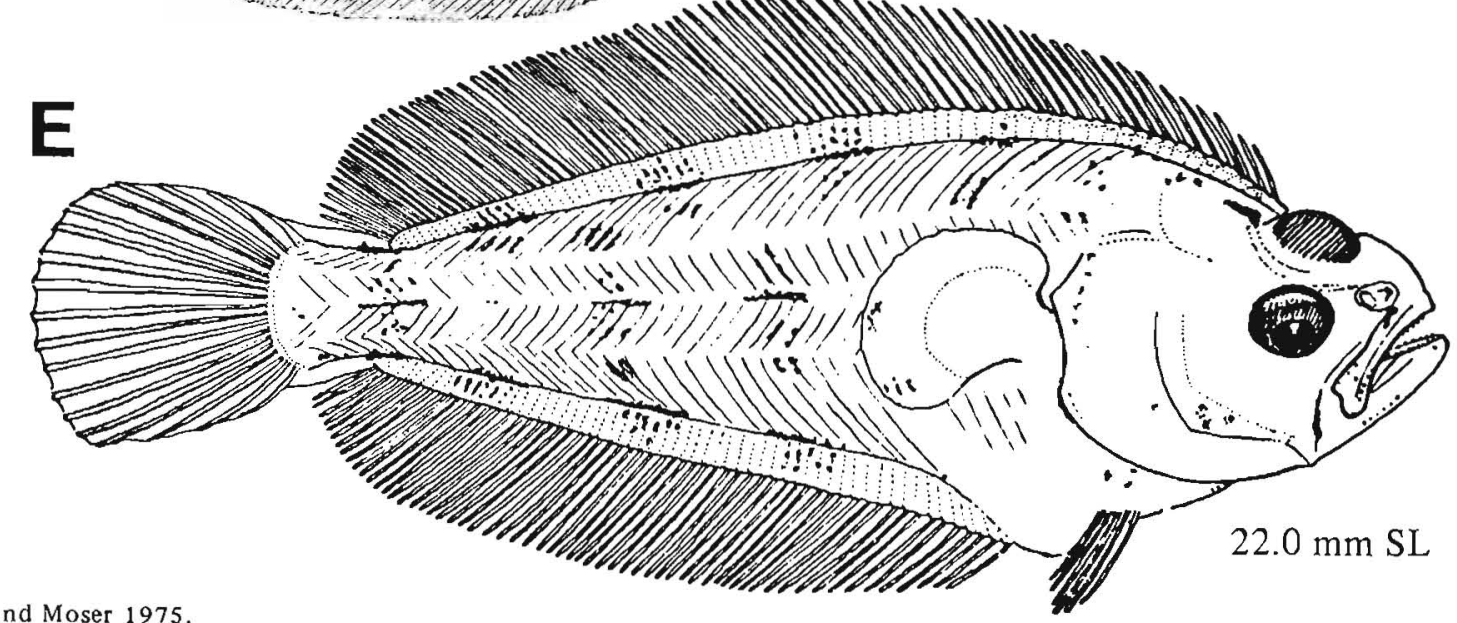

Figures A-E, Ahlstrom and Moser 1975. 


\section{MERISTICS}

\begin{tabular}{|c|c|}
\hline Vertebrae & $\begin{array}{l}\text { Total: } 50-52-55 \\
\text { Precaudal: } 11-12-13 \\
\text { Caudal: } 38-40-41\end{array}$ \\
\hline Branchiostegal rays & $7-7-7$ \\
\hline Caudal fin & $\begin{array}{l}X, 9+8, X \\
\text { Total rays }=21^{\text {a }}\end{array}$ \\
\hline Pelvic fin & $\begin{array}{l}\text { Thoracic } \\
\text { R: } 6-6-6\end{array}$ \\
\hline $\begin{array}{l}\text { Dorsal fin } \\
\text { Pectoral fin } \\
\text { Anal fin } \\
\text { Gill rakers }\end{array}$ & $\begin{array}{l}\text { R: } 94-105-116 \\
\text { R: } 8-X-12 \\
\text { R: } 80-87-96 \\
\text { U: } 5-X-8 \quad \text { L: } 8-X-11\end{array}$ \\
\hline \multicolumn{2}{|l|}{ LIFE HISTORY } \\
\hline Range & $\begin{array}{l}\text { South of southern California to } \\
\text { Bering Sea, } 54-66^{\circ} \mathrm{N}\end{array}$ \\
\hline Ecology & $\begin{array}{l}\text { Epi-, meso-, and bathybenthal, } \\
9-1189 \mathrm{~m}\end{array}$ \\
\hline ELH pattern & $\begin{array}{l}\text { Oviparous, pelagic eggs, } \\
\text { pelagic larvae }\end{array}$ \\
\hline Spawning & $\begin{array}{l}\text { Season: Nov-Aug } \\
\text { Area: } 80-550 \mathrm{~m}^{\mathrm{b}} \\
\text { Mode: } \\
\text { Migration: Offshore for } \\
\text { spawning }{ }^{\mathrm{c}}\end{array}$ \\
\hline Fecundity & $\begin{array}{l}\text { Range/function: } 37,188^{\mathrm{d}}- \\
260,000^{\mathrm{e}}\end{array}$ \\
\hline Age at first maturity & $5 \mathrm{yr}^{\mathrm{f}}$ \\
\hline Longevity & $45 \mathrm{yr}^{\mathrm{g}}$ \\
\hline
\end{tabular}

a $13-16$ branched rays.

${ }^{b}$ Hirschberger and Smith 1983

${ }^{c}$ Westrheim and Morgan 1962

${ }^{d}$ Hagerman 1952

'Harry 1959

'Frey 1971

${ }^{8}$ Chilton and Beamish 1982

Ref: Ahlstrom and Moser 1975, Ahlstrom et al. 1984a, Richardson $198 \mathrm{lb}$.

\section{EARLY LIFE HISTORY DESCRIPTION}

\section{EGGS}

Diameter

No. of oil globules

2.05-2.68 mm

Oil globule diameter

Yolk

Envelope

Hatch size

None

Incubation time/temp. $\quad 27 \mathrm{~d} / 10^{\circ} \mathrm{C}$

Pigment

- Yolksac

- Late-stage embryo: Caudal pigment not as pronounced as Lyopsetta exilis (p. 606)

Diagnostic characters

- Largest egg of three similar-looking pleuronectid eggs; see L. exilis and Glyptocephalus zachirus (p. 586)

\section{LARVAE}

Preanal length

Length at flexion

$<50 \%$ SL

Length at transformation

10-15 mm SL

Eye migration begins at $20 \mathrm{~mm}$ SL; larvae remain pelagic $>45 \mathrm{~mm} \mathrm{SL}$

Sequence of fin development Caudal, dorsal and anal, pelvics, pectorals

Pigment

- Lower jaw; dorsally and ventrally on gut, with development becoming restricted to ventral surface (isthmus to anus)

- Preflexion - Three pigment patches in addition to tail and finfold -Anterior patch restricted to ventral area; two posterior patches have dorsal and ventral components extending into finfold

- Around urostyle and in finfold

- Flexion: Pigment patches occur above notochord and along distal ends of finfold

- Postflexion: Pigment patches along dorsal and anal pterygiophores

Diagnostic characters

Distinguished from other pleuronectids with 3-4 pigment bands by

- Pigment bands extending into finfold

- Morphology: Early larvae long and slender, becoming deep-bodied during development

- Otic spines

- Myomeres (50-55)

See also G. zachirus (p. 586) and Hippoglossoides elassodon (p. 588) 

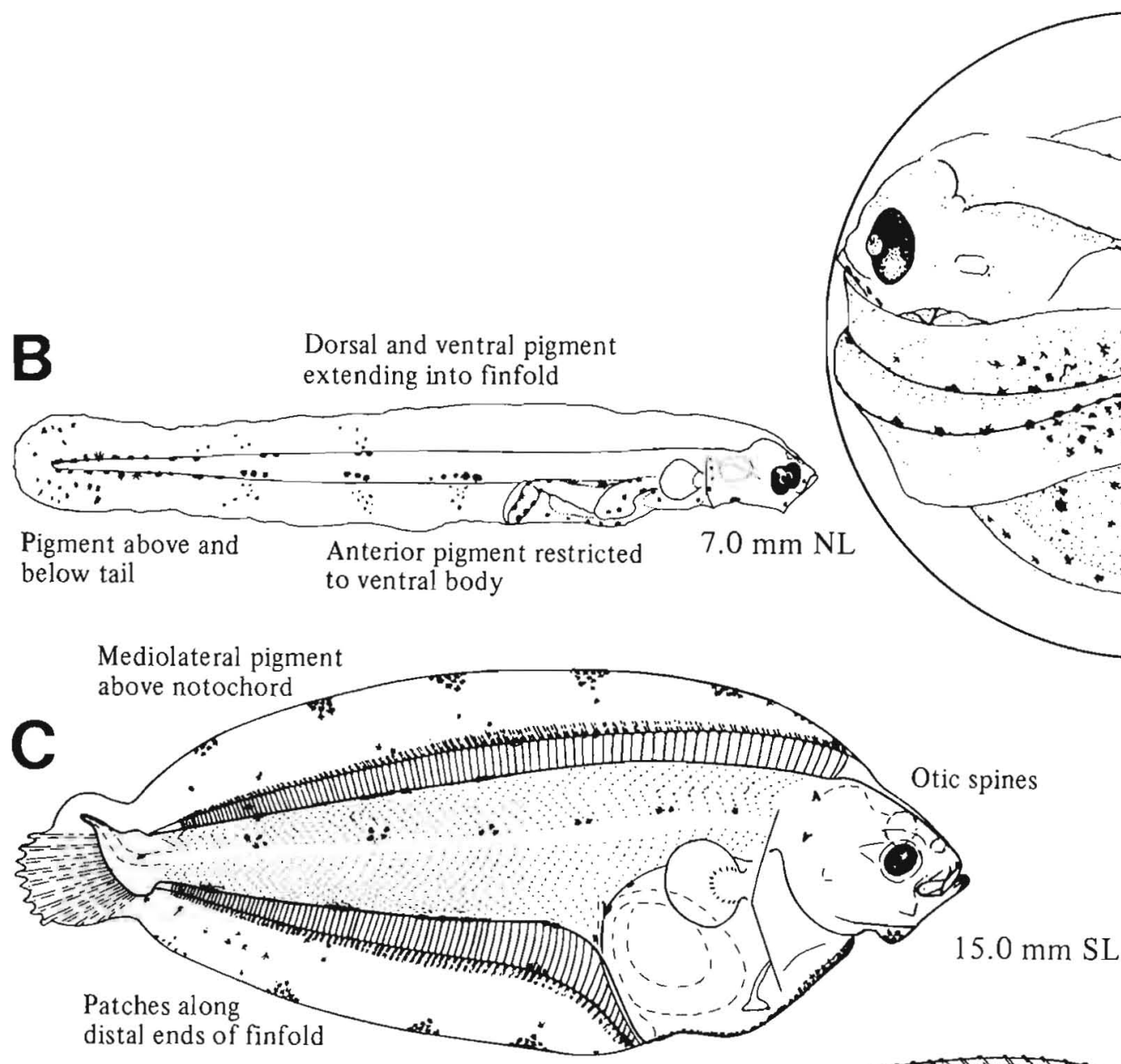

$2.05-2.68 \mathrm{~mm}$

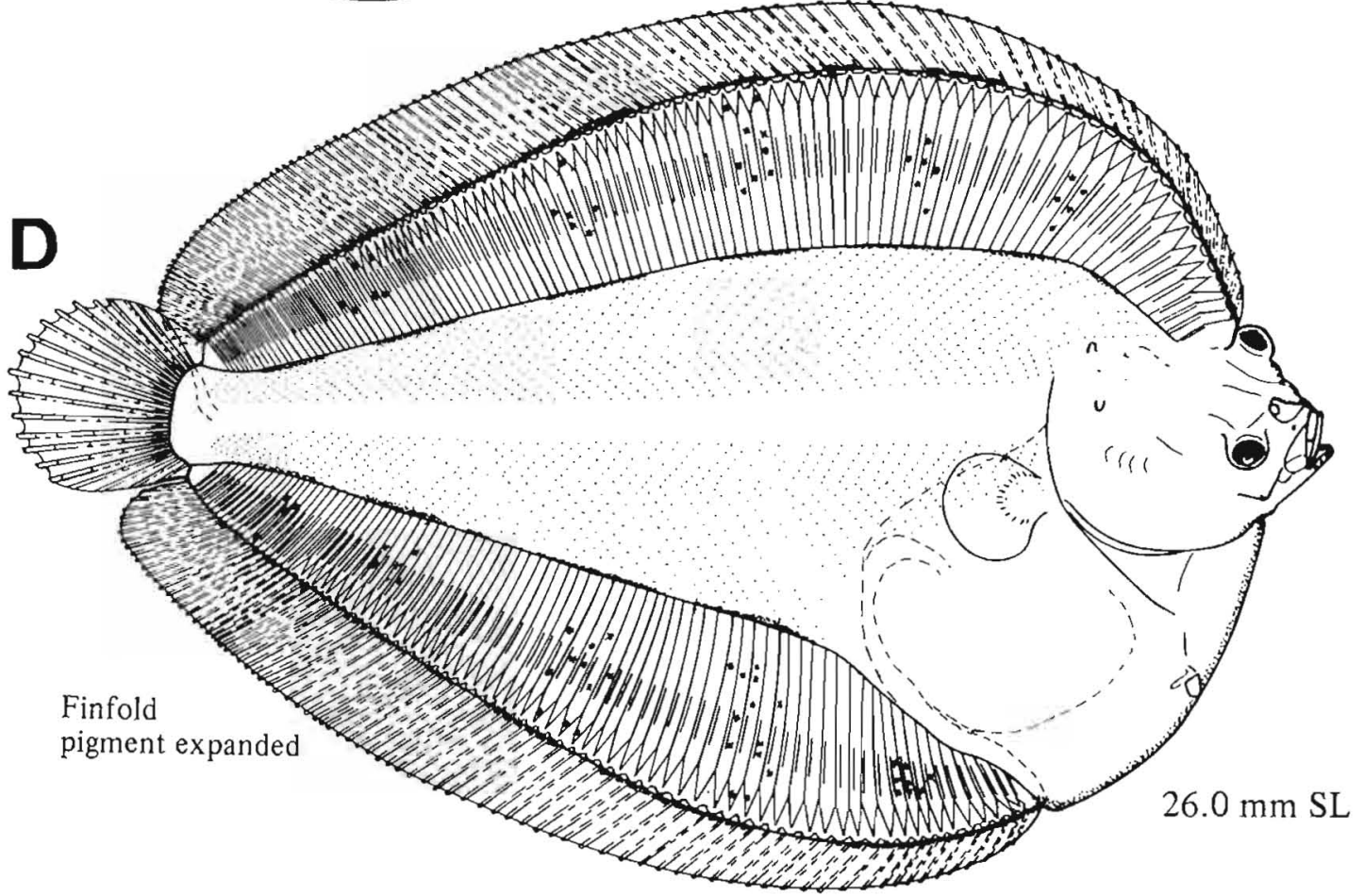

Figure A, Ahlstrom and Moser 1975; B, Ahlstrom et al. 1984a (after Ahlstrom and Moser 1975); C-D, NWAFC originals (B. Vinter). 


\section{MERISTICS}

$\begin{array}{ll}\text { Vertebrae } & \text { Total: } 42-44-47 \\ & \text { Precaudal: } 10-11-12 \\ & \text { Caudal: } 31-33-34 \\ \text { Branchiostegal rays } & 7-X-8 \\ \text { Caudal fin } & \text { X, } 7+7, \mathrm{X} \\ & \text { Total rays }=18^{\mathrm{a}} \\ \text { Pelvic fin } & \text { Thoracic } \\ & \text { R: } 6-6-6 \\ \text { Dorsal fin } & \text { R: } 72-80-82 \\ \text { Pectoral fin } & \text { R: } 10-\mathrm{X}-12 \\ \text { Anal fin } & \text { R: } 54-60-70 \\ \text { Gill rakers } & \text { U: } 4-X-6 \quad \text { L: } 10-X-13\end{array}$

LIFE HISTORY

\begin{tabular}{|c|c|}
\hline Range & $\begin{array}{l}\text { South of southern California to } \\
\text { Bering Sea, } 54-66^{\circ} \mathrm{N}\end{array}$ \\
\hline Ecology & $\begin{array}{l}\text { Epi-, meso-, and bathybenthal, } \\
\text { intertidal to } 550 \mathrm{~m}\end{array}$ \\
\hline ELH pattern & $\begin{array}{l}\text { Oviparous, pelagic eggs, } \\
\text { pelagic larvae }\end{array}$ \\
\hline Spawning & $\begin{array}{l}\text { Season: Oct-May (California); } \\
\text { Jan-Apr (Puget Sound) } \\
\text { Area: Demersal } \\
\text { Mode: } \\
\text { Migration: Southern migration } \\
\text { from feeding grounds to } \\
\text { sheltered water in channels } \\
\text { or bights }^{\mathrm{d}}\end{array}$ \\
\hline Fecundity & $\begin{array}{l}\text { Range/function: } 150,000- \\
1,950,000^{\mathrm{e}}\end{array}$ \\
\hline Age at first maturity & $\begin{array}{l}3-4 \mathrm{yr}^{(\text {females }}{ }^{\mathrm{c}} \\
\left.2 \mathrm{yr}^{-} \text {(males) }\right)^{\mathrm{c}}\end{array}$ \\
\hline Longevity & $17 \mathrm{yr}^{\mathrm{f}}$ \\
\hline
\end{tabular}

\section{EARLY LIFE HISTORY DESCRIPTION}

\section{EGGS}

Diameter

No. of oil globules

Oil globule diameter

Yolk

Envelope

Hatch size

Incubation time/temp.

Pigment
$0.80-1.05 \mathrm{~mm}$
None

Homogeneous

Thin, smooth, transparent

2.3-2.8 mm SL

\section{Diagnostic characters}

- See Isopsetta isolepis (p. 594)

\section{LARVAE}

\section{Preanal length}

Length at flexion

$$
\begin{aligned}
& 43-45 \% \mathrm{SL} \\
& \sim 10 \mathrm{~mm} \mathrm{SL} ; \\
& \quad 8.8-10.5 \mathrm{~mm} \mathrm{SL}^{\mathrm{g}}
\end{aligned}
$$

Length at transformation Eye migration prior to

$$
17.5 \mathrm{~mm} \mathrm{SL}
$$

\section{Sequence of fin development \\ Caudal, dorsal and anal, pelvics, pectorals}

Pigment

- Initially, anteroventrally on gut; with development, extending posteriorly; in postflexion, a line of pigment forms along the posterior hindgut and anus

- Dorsal and ventral midline: Ventral beginning prior to anus ( $\sim$ myomere 5 ) and dorsal at $\sim$ myomere 15 (pigment is variable); dorsal midline becoming less prominent with development as internal pigment develops above notochord through flexion stage

- Myoseptal pigment develops along hypaxial myomeres in postflexion larvae

- Finfold pigment restricted to anal finfold

\section{Diagnostic characters}

Distinguished from other pleuronectids without pigment bands by

- Dorsal and ventral midline pigment, hypaxial pigment

- Finfold pigment restricted to anal finfold

Note: The number of dorsal midline melanophores in preflexion larvae is highly variable.

\footnotetext{
${ }^{a} 12$ branched rays.

Jow 1969

${ }^{c}$ Smith 1936

${ }^{d}$ Ketchen 1956

'Ketchen 1947

'Frey 1971

${ }^{8}$ Ahlstrom et al. $1984 a$

Ref: Budo 1940, Ahlstrom and Moser 1975, Ahlstrom et al. 1984a.
} 


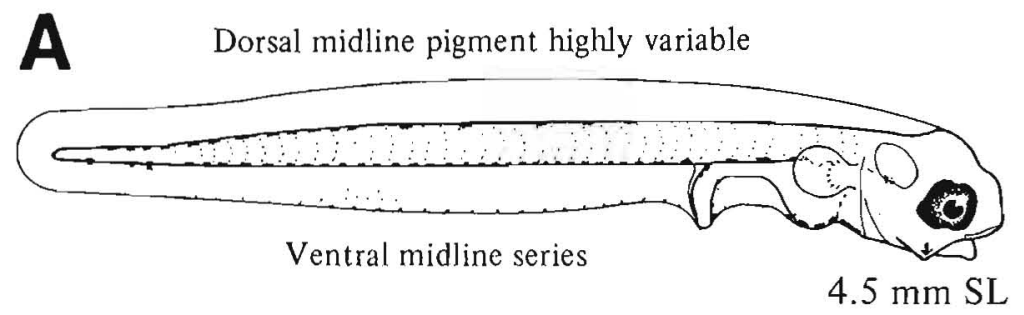

B $\begin{array}{ll}\text { Urostyle } & \text { Internal pigment } \\ \text { pigment } & \text { above notochord }\end{array}$

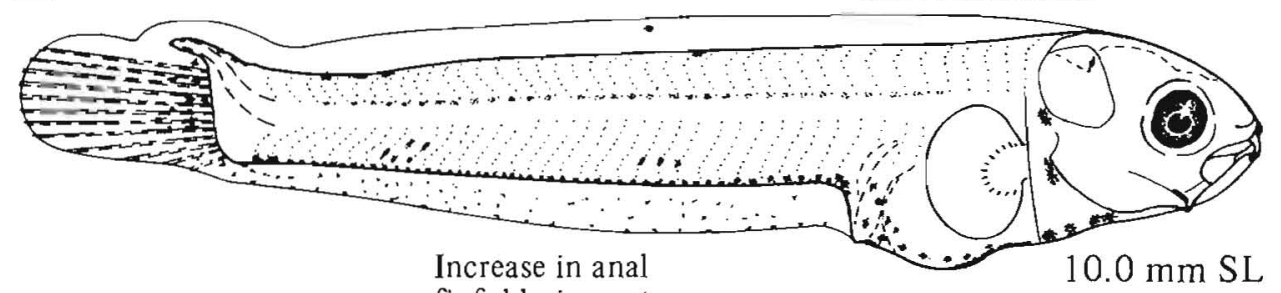

finfold pigment
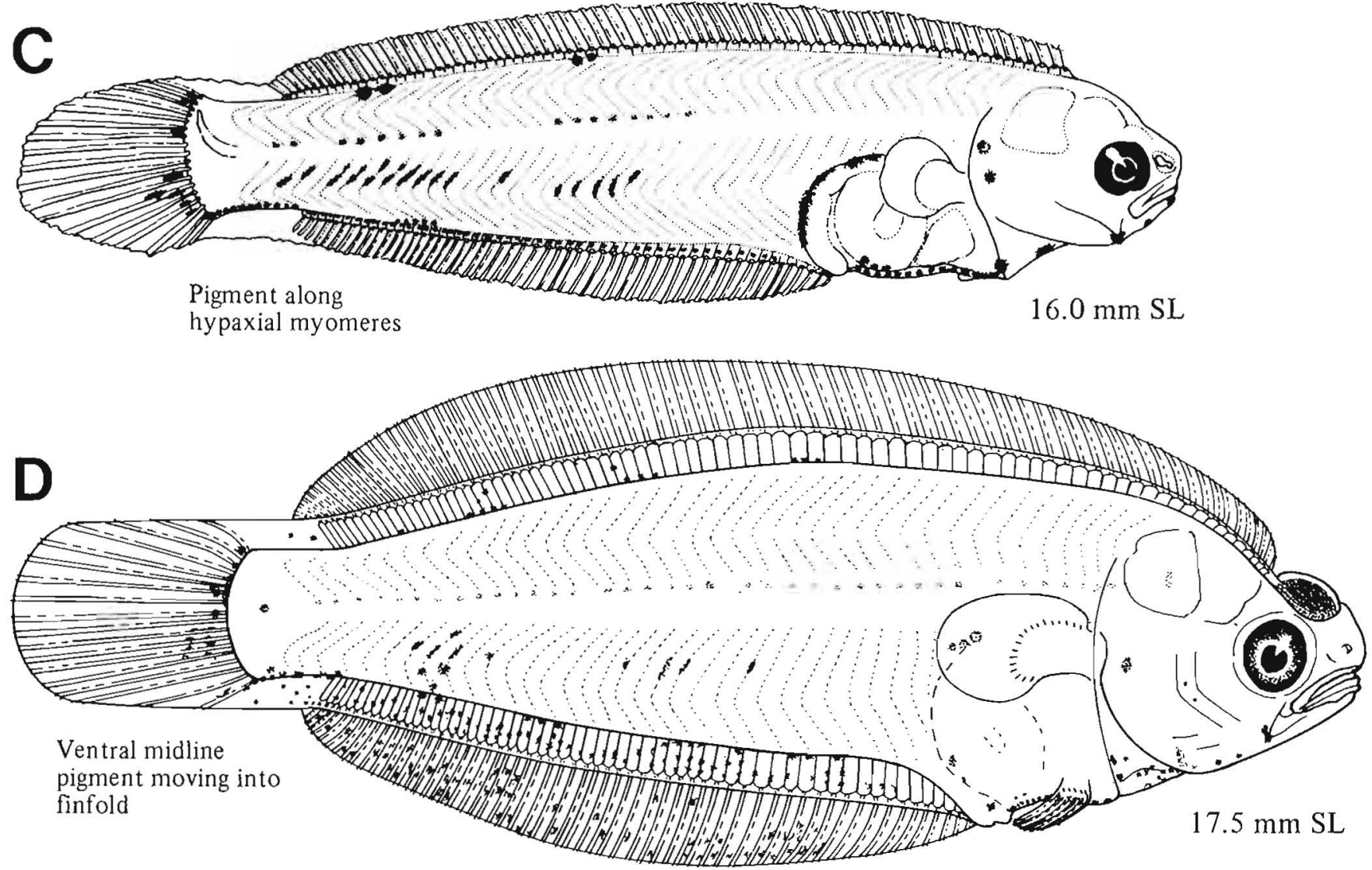

Figures A-B, D, NWAFC originals (B. Vinter); C, Ahlstrom et al. 1984a (after Ahlstrom and Moser 1975). 


\section{MERISTICS}

\begin{tabular}{|c|c|}
\hline Vertebrae & $\begin{array}{l}\text { Total: } 35-36-38 \\
\text { Precaudal: } 10-11-12 \\
\text { Caudal: } 24-25-26\end{array}$ \\
\hline Branchiostegal rays & $7-7-7$ \\
\hline Caudal fin & Total rays $=18$ \\
\hline Pelvic fin & $\begin{array}{l}\text { Thoracic } \\
\mathrm{R}: 6-6-6\end{array}$ \\
\hline Dorsal fin & $\mathrm{R}: 52-59-66$ \\
\hline Pectoral fin & R: $9-10-10$ \\
\hline Anal fin & R: $38-42-47$ \\
\hline Gill rakers & $\mathrm{U}: 3-3-3$ \\
\hline \multicolumn{2}{|l|}{ LIFE HISTORY } \\
\hline Range & $\begin{array}{l}\text { S. California, } 32-34^{\circ} \mathrm{N} \text {, to } \\
\text { Arctic, not specific }\end{array}$ \\
\hline Ecology & $\begin{array}{l}\text { Epi- and mesobenthal, freshwater } \\
\text { (upstream) to } 375 \mathrm{~m}\end{array}$ \\
\hline ELH pattern & $\begin{array}{l}\text { Oviparous, pelagic eggs, } \\
\text { pelagic larvae }\end{array}$ \\
\hline Spawning & $\begin{array}{l}\text { Season: Nov-Feb (California); }{ }^{a} \\
\text { Feb-Apr (Puget Sound); }{ }^{b} \\
\text { May-June (Bering Sea) }{ }^{c} \\
\text { Area: Shallow water }{ }^{\mathrm{a}} \\
\text { Migration: }\end{array}$ \\
\hline Fecundity & $\begin{array}{l}\text { Range/function: } 900,000^{c}- \\
11 \text { million }^{\mathrm{a}}\end{array}$ \\
\hline Age at first maturity & $3-4$ yr (females) ${ }^{a}$ \\
\hline Longevity & $21 \mathrm{yr}^{\mathrm{d}}$ \\
\hline
\end{tabular}

\section{EARLY LIFE HISTORY DESCRIPTION}

\section{EGGS}

Diameter

No. of oil globules

Oil globule diameter

Yolk

Envelope

Hatch size

\author{
$0.88-1.28 \mathrm{~mm}$ \\ None
}

Homogeneous

Striated, yellow

1.9-2.1 mm SL

Incubation time/temp.

Pigment

Diagnostic characters

- See Isopsetta isolepis (p. 594)

\section{LARVAE}

$$
\begin{array}{lc}
\text { Preanal length } & <50 \% \mathrm{SL} \\
\text { Length at flexion } & \sim 7 \mathrm{~mm} \mathrm{SL} ; \\
& 5.5-6.0 \mathrm{~mm} \mathrm{SL} \\
\text { Length at transformation } & 10.5 \mathrm{~mm} \mathrm{SL} \text { (may be } \\
& \text { slightly smaller, } \\
& 8-9 \mathrm{~mm} \mathrm{SL} \text { ) } \\
\text { Sequence of fin } & \text { Caudal, dorsal and anal, } \\
\text { development } & \text { pelvics, pectorals }
\end{array}
$$
Sequence of fin Caudal, dorsal and anal, development pelvics, pectorals

Pigment

- Lightly scattered over head and posteroventrally along gut

- Initially, in preflexion larvae, postanal pigment scattered along anal finfold and along posterior $1 / 3$ of body

- Around urostyle

- Internally above notochord, becoming less concentrated with development

- Pigment in dorsal fin disappears with development

- Postflexion larvae lightly pigmented with lateral patches

\section{Diagnostic characters}

Distinguished from other pleuronectids without pigment bands by

- Lack of dorsal midline pigment

- Small size at transformation $(<10 \mathrm{~mm} \mathrm{SL})$

\footnotetext{
Orcutt 1950

${ }^{\mathrm{b}} \mathrm{Hart} 1973$

'Fadeev 1965

${ }^{\circledR}$ Wolotira et al. 1977

${ }^{\mathrm{C}}$ Ahlstrom et al. 1984a
}

Ref: Ahlstrom et al. 1984a, Orcun 1950, Pertseva-Ostroumova 1961, Yusa 1957. 
B

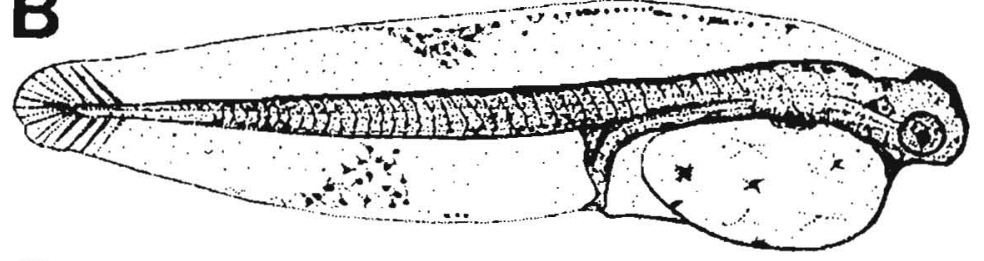

C
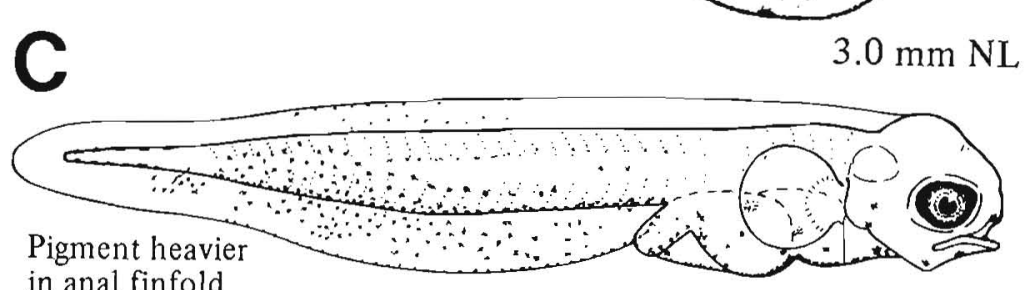
in anal finfold

\section{D} $4.8 \mathrm{~mm} \mathrm{SL}$

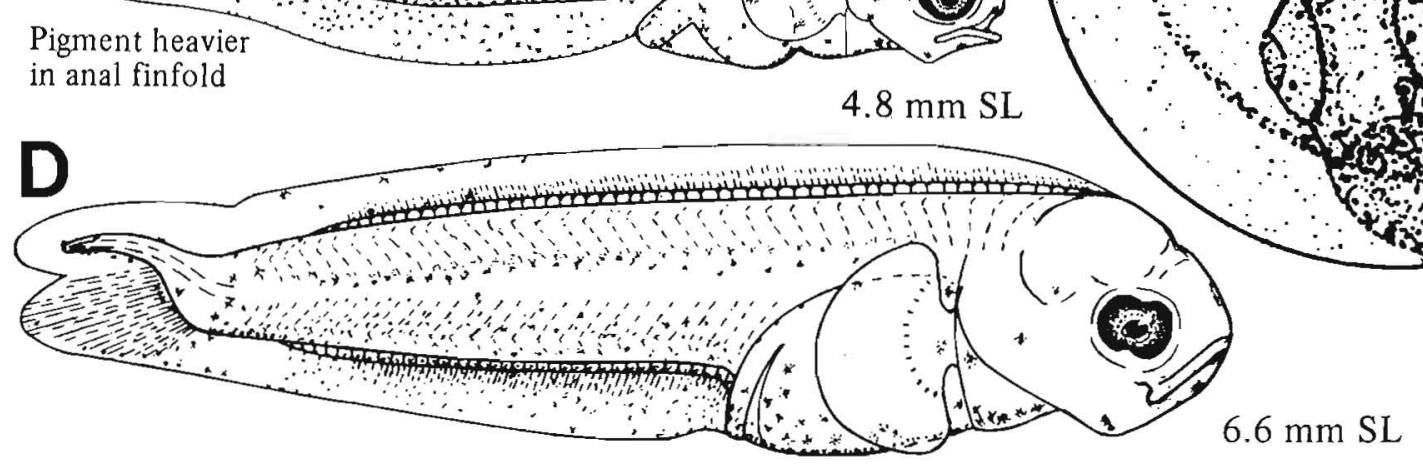

E

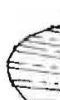

E
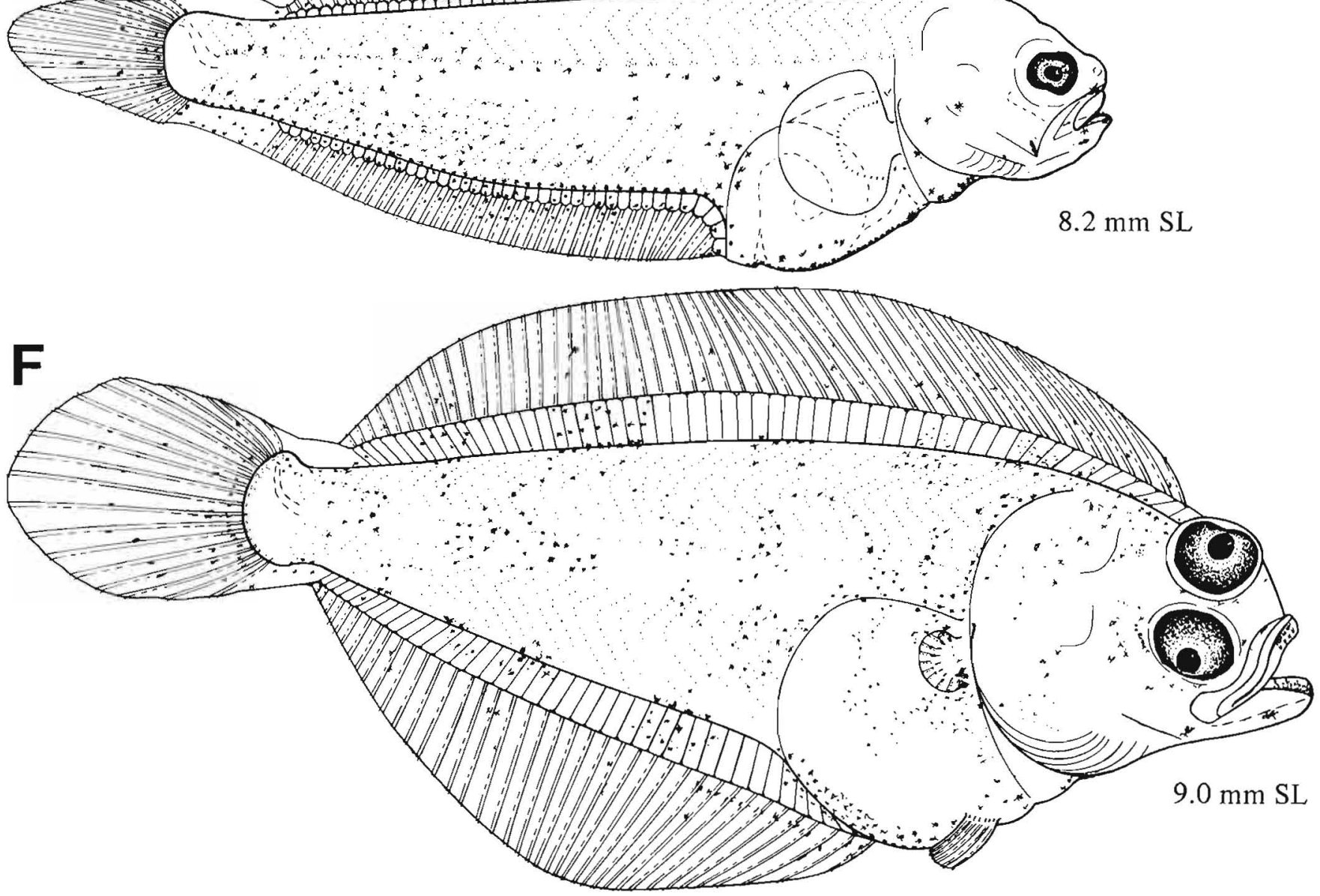

Figures A-B, Orcutt 1950 (B, reversed); C-F, NWAFC originals (B. Vinter). 


\section{MERISTICS}

\begin{tabular}{|c|c|}
\hline Vertebrae & $\begin{array}{l}\text { Total: } 41-X-42 \\
\text { Precaudal: } 18-18-19 \\
\text { Caudal: X-X-X }\end{array}$ \\
\hline $\begin{array}{l}\text { Branchiostegal rays } \\
\text { Caudal fin }\end{array}$ & $7-X-8$ \\
\hline Pelvic fin & $\begin{array}{l}\text { Thoracic } \\
\text { R: 6-6-6 }\end{array}$ \\
\hline Dorsal fin & $\mathrm{R}: 62-69-71$ \\
\hline Pectoral fin & R: $11-11-11$ \\
\hline Anal fin & R: $51-51-56$ \\
\hline Gill rakers & $\mathrm{U}: \mathrm{X}-\mathrm{X}-\mathrm{X}$ \\
\hline \multicolumn{2}{|l|}{ LIFE HISTORY } \\
\hline Range & $\begin{array}{l}\text { Gulf of Alaska, } 54-60^{\circ} \mathrm{N} \text {, to } \\
\text { Chukchi Sea, north of } 66^{\circ} \mathrm{N}\end{array}$ \\
\hline Ecology & Epi- and mesobenthal, 6-475 m \\
\hline ELH pattern & $\begin{array}{l}\text { Oviparous, pelagic eggs, } \\
\text { pelagic larvae }\end{array}$ \\
\hline \multirow[t]{4}{*}{ Spawning } & Season: Spring ${ }^{\mathrm{a}}$ \\
\hline & Area: \\
\hline & Mode: \\
\hline & Migration: Inshore ${ }^{a}$ \\
\hline Fecundity & Range/function: \\
\hline $\begin{array}{l}\text { Age at first maturity } \\
\text { Longevity }\end{array}$ & $4 y^{2}$ \\
\hline
\end{tabular}

\section{EARLY LIFE HISTORY DESCRIPTION}

\section{EGGS}

Diameter

No. of oil globules

Oil globule diameter

Yolk

Envelope

Hatch size

Incubation time/temp.

Pigment

Diagnostic characters

\section{$1.67-2.21 \mathrm{~mm}(1.7-1.9 \mathrm{~mm})$}

None

Thick wavy surface (color: bronze hue) $\sim 5.85 \mathrm{~mm} \mathrm{SL}$

\section{LARVAE}

Preanal length

Length at flexion

Length at transformation

Sequence of fin development

Pigment

- Isthmus, posteroventrally on gut

- Initially, in preflexion larvae, dorsal spots over posterior half of body that become less prominent with development

- Ventral midline melanophores extend onto finfold and on caudal region

- Internal row above notochord throughout development

Diagnostic characters

Distinguished from other pleuronectids without pigment bands by

- Pigment pattern

-Urostyle unpigmented

-No slash-like pigment along hypaxial myomeres

-Finfold pigment mainly restricted to anal finfold

- Size at transformation $\sim 10 \mathrm{~mm} \mathrm{SL}$

${ }^{\text {a }}$ Pertseva-Ostroumova 1961 Ref: Ahlstrom et al. 1984a. 
B

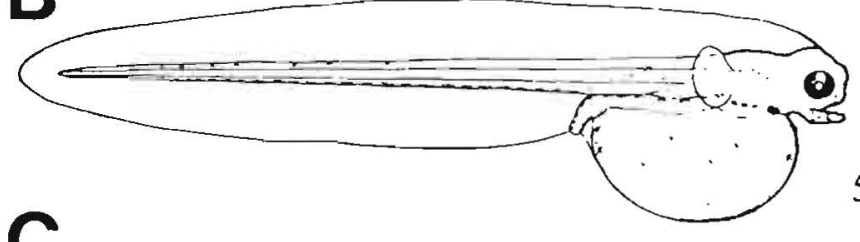

$5.9 \mathrm{~mm}$

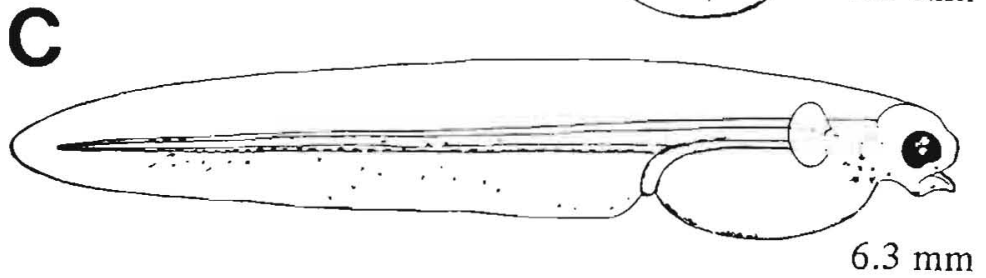

D

$6.3 \mathrm{~mm}$

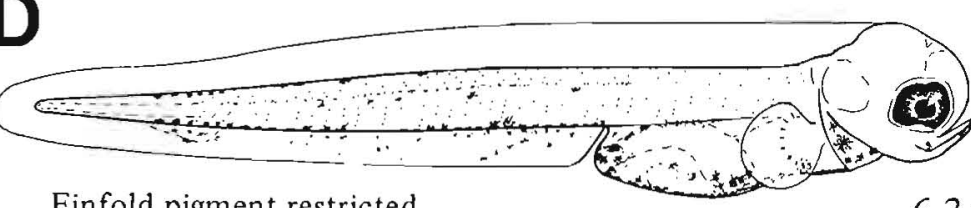

Finfold pigment restricted to anal finfold

\section{$6.3 \mathrm{~mm} \mathrm{SL}$}

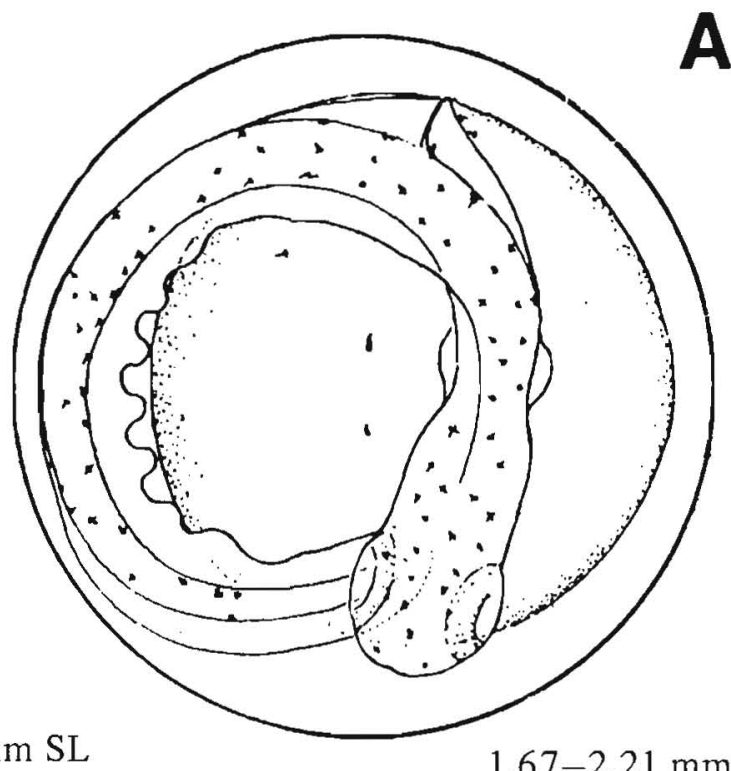

E
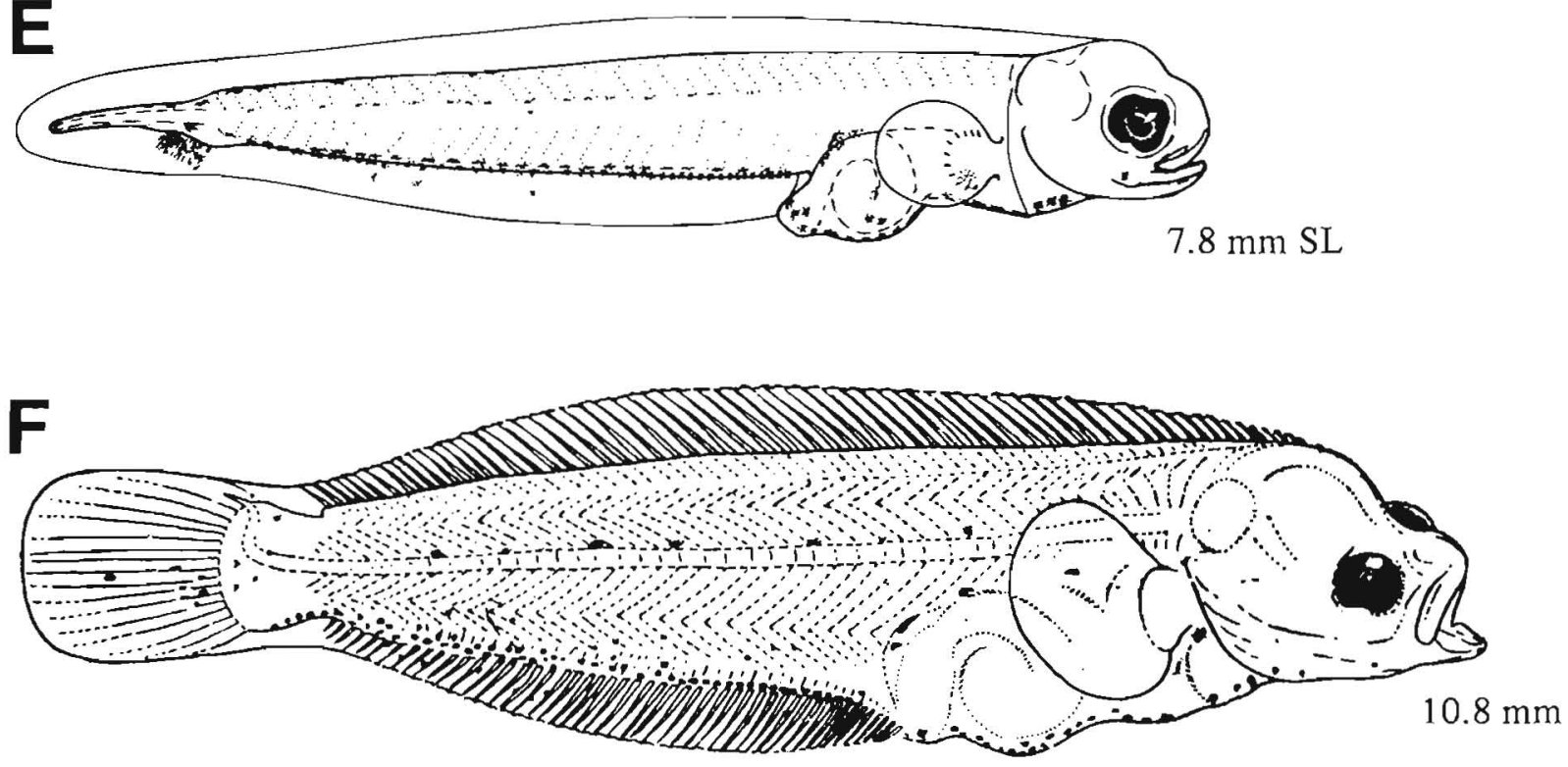

Figures A-C, F, Pertseva-Ostroumova 1961 (B-C, reversed); D-E, NWAFC originals (B. Vinter). 


\section{MERISTICS}

\begin{tabular}{ll} 
Vertebrae & Total: $37-38-39$ \\
& Precaudal: $12-13-13$ \\
& Caudal: $24-25-26$ \\
Branchiostegal rays & $7-X-8$ \\
Caudal fin & Total rays=19 \\
Pelvic fin & Thoracic \\
& R: $6-6-6$ \\
Dorsal fin & R: $65-72-78$ \\
Pectoral fin & R: $9-11-12$ \\
Anal fin & R: $46-50-56$ \\
Gill rakers & U: $3-X-4$ \\
& \\
& \\
LIFE HISTORY & \\
\hline
\end{tabular}

$\begin{array}{ll}\text { Range } & \begin{array}{c}\text { South of southern California to } \\ \text { SE Alaska, 55-59 } \mathrm{N}\end{array} \\ \text { Ecology } & \text { Epi- and mesobenthal, 0-350 m } \\ \text { ELH pattern } & \begin{array}{c}\text { Oviparous, pelagic eggs, } \\ \text { pelagic larvae }\end{array} \\ \text { Spawning } & \text { Season: Mar-Aug (California) } \\ & \text { Area: } \\ & \text { Mode: } \\ & \text { Migration: } \\ \text { Fecundity } & \text { Range/function: } \\ \text { Age at first maturity } & \\ \text { Longevity } & \end{array}$

\section{EARLY LIFE HISTORY DESCRIPTION}

\section{EGGS}

Diameter

No. of oil globules

Oil globule diameter

Yolk

Envelope

Hatch size

$1.20-1.56 \mathrm{~mm}$

None

Homogeneous

Sculptured with polygonal pattern

$3.9 \mathrm{~mm} \mathrm{SL}$

Incubation time/temp.

Pigment

Diagnostic characters

- Egg diameter (1.20-1.56 mm)

- Polygonal sculpturing on envelope surface

\section{LARVAE}

Preanal length $\quad<50 \%$ SL

Length at flexion $\quad 6.2-8.5 \mathrm{~mm} \mathrm{NL}$

Length at transformation $8.2->11.4 \mathrm{~mm} \mathrm{SL}$

Sequence of fin Caudal slightly before development dorsal and anal, pelvics, pectorals

Pigment

- Preflexion larvae: Opposing pigment clusters on dorsal and ventral finfolds, increasing with development

- Small melanophores covering all but posterior $1 / 4$ of body

Diagnostic characters (see Table 3)

Distinguished from other pleuronectids without pigment bands by

- Heavy pigment pattern

Distinguished from $P$. decurrens by

- Pigment pattern: P. coenosus has less finfold pigment than $P$. decurrens

- Precaudal vertebrae 12-13, whereas $P$. decurrens usually has 14-15

- Lack of pterotic spines

Note: Preflexion larvae occasionally have more snout and lower jaw pigment than shown on figure.

${ }^{2}$ Budd 1940

Ref: Ahlstrom et al. 1984a, Sumida et al. 1979. 
B

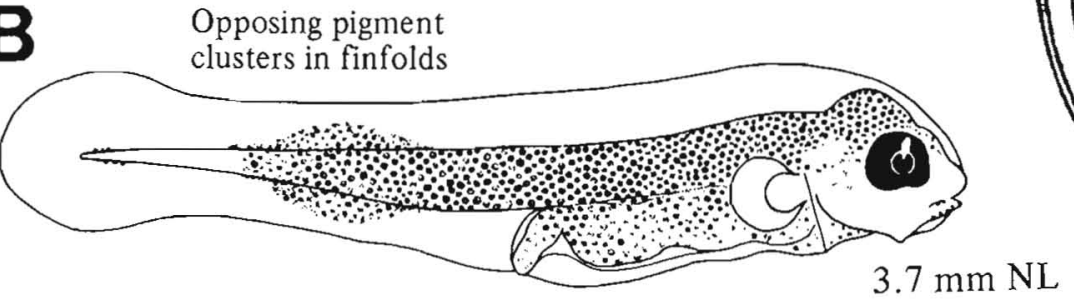

C

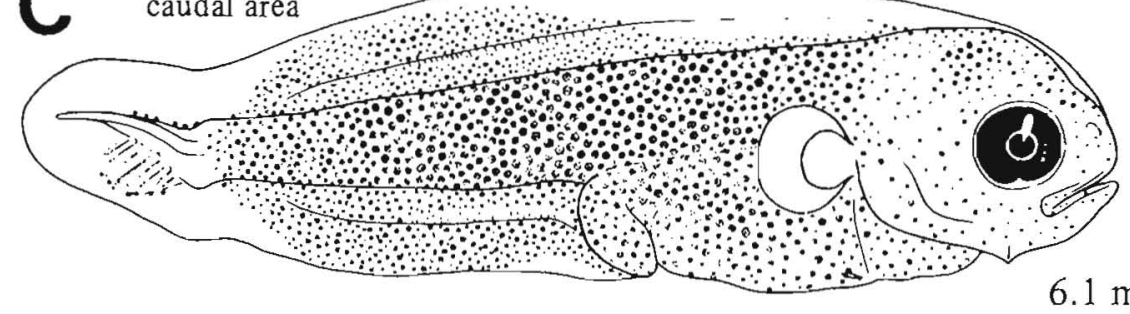

$6.1 \mathrm{~mm} \mathrm{NL}$

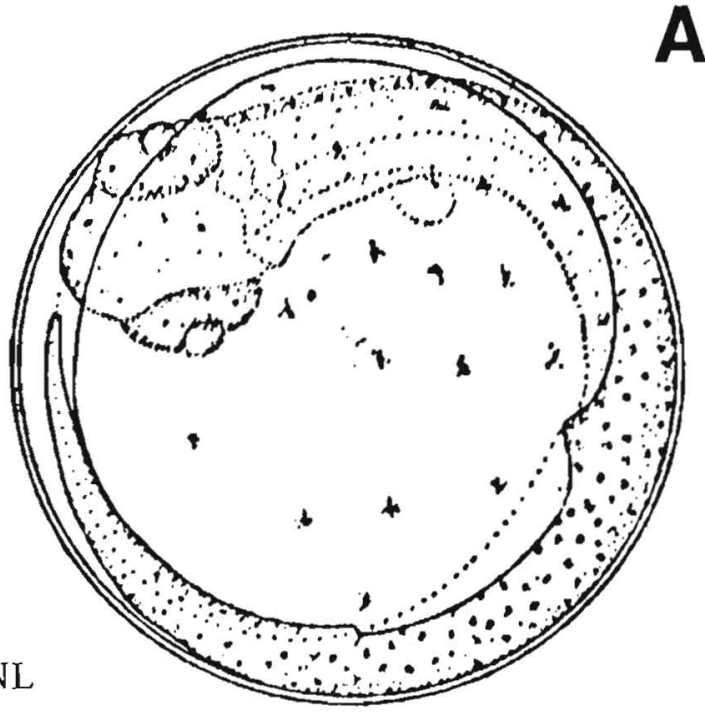

$1.20-1.56 \mathrm{~mm}$
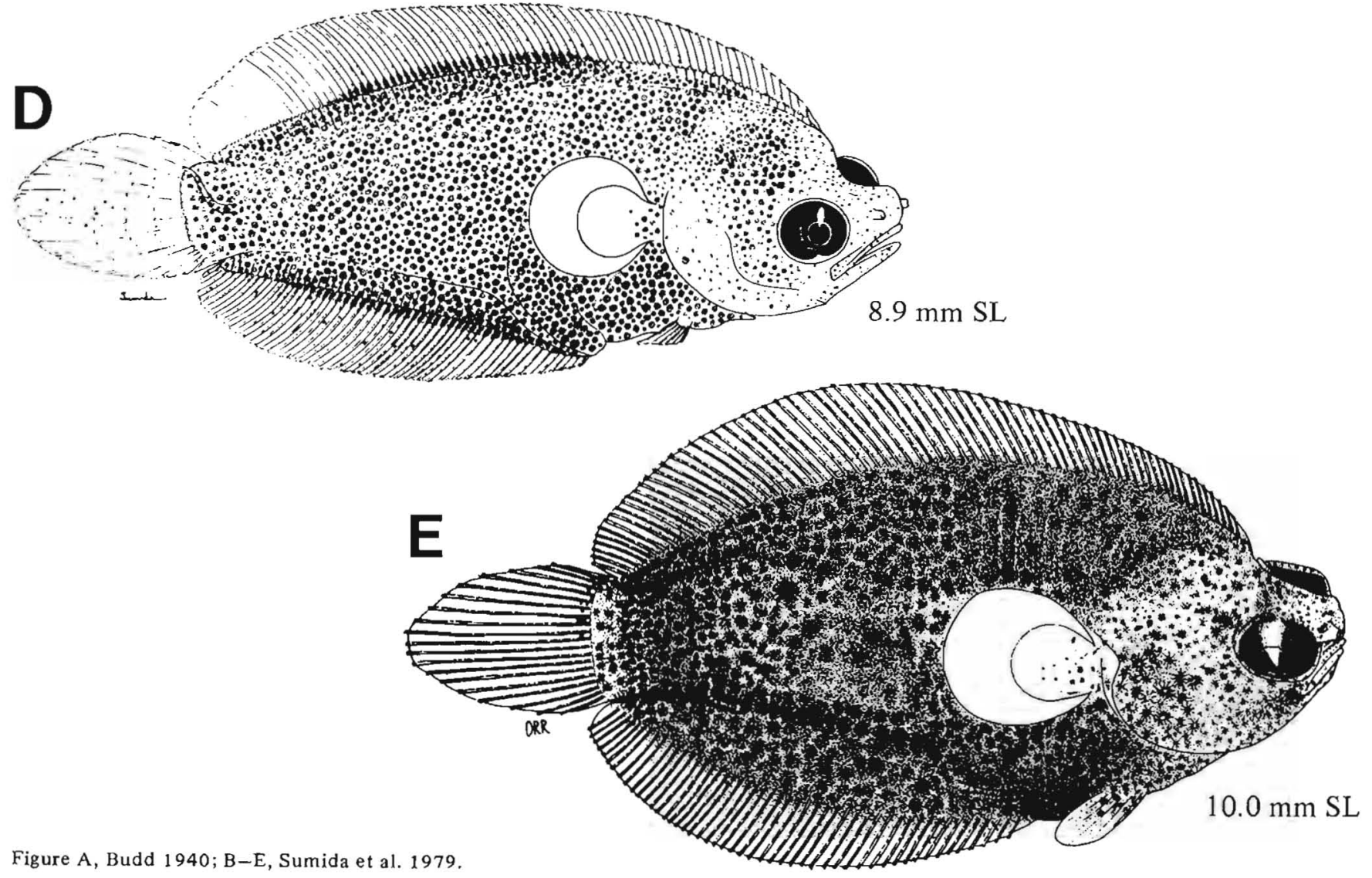

Figure A, Budd 1940; B-E, Sumida et al. 1979. 


\section{MERISTICS}

\begin{tabular}{ll} 
Vertebrae & Total: $38-39-41$ \\
& Precaudal: $13-14-15$ \\
& Caudal: $24-25-26$ \\
Branchiostegal rays & $7-\mathrm{X}-8$ \\
Caudal fin & $2,7+7,2$ \\
& Total rays $=19^{\mathrm{a}}$ \\
Pelvic fin & Thoracic \\
& R: $4-6-7$ \\
Dorsal fin & R: $67-75-81$ \\
Pectoral fin & R: $9-12-14$ \\
Anal fin & R: $45-50-55$ \\
Gill rakers & U: $3-\mathrm{X}-4$ \\
& \\
& \\
LIFE HISTORY & \\
\hline Range & South of southern California to \\
& Bering Sea, $54-66^{\circ} \mathrm{N}$ \\
Ecology & Epi-, meso-, and bathybenthal, \\
& $8-532 \mathrm{~m}$ \\
ELH pattern & Oviparous, pelagic eggs, \\
& pelagic larvae \\
Spawning & Season: \\
& Area: \\
& Mode: \\
Fecundity & Migration: \\
Age at first maturity & Range/function: \\
Longevity & \\
&
\end{tabular}

EARLY LIFE HISTORY DESCRIPTION

\section{EGGS}

Diameter

No. of oil globules

Oil globule diameter

Yolk

Envelope

Hatch size

Incubation time/temp.

Pigment
$1.84-2.08 \mathrm{~mm}$

None

Homogeneous

Sculptured with polygonal pattern

4.9-5.5 mm SL

\section{Diagnostic characters}

- Egg diameter (1.84-2.08 mm)

- Polygonal sculpturing on envelope surface

\section{LARVAE}

Preanal length

Length at flexion

Length at transformation

Sequence of fin

development $\sim 50 \% \mathrm{SL}$

7.8-11.0 mm NL

$10.5->21.0 \mathrm{~mm} \mathrm{SL}^{b}$

Caudal slightly before dorsal and anal, pelvics, pectorals

\section{Pigment}

- Nearly entire body and finfolds pigmented except posteriorly in preflexion larvae; banding pattern develops in postflexion larvae

\section{Diagnostic characters (see Table 3)}

Distinguished from other pleuronectids without pigment bands by

- Heavy pigment pattern

- Pterotic spines

Distinguished from $P$. coenosus by

- See P. coenosus (p. 616)

- In addition to having pterotic spines, more pigment, and more precaudal myomeres, $P$. decurrens is larger at various stages of development (see figures). 
B
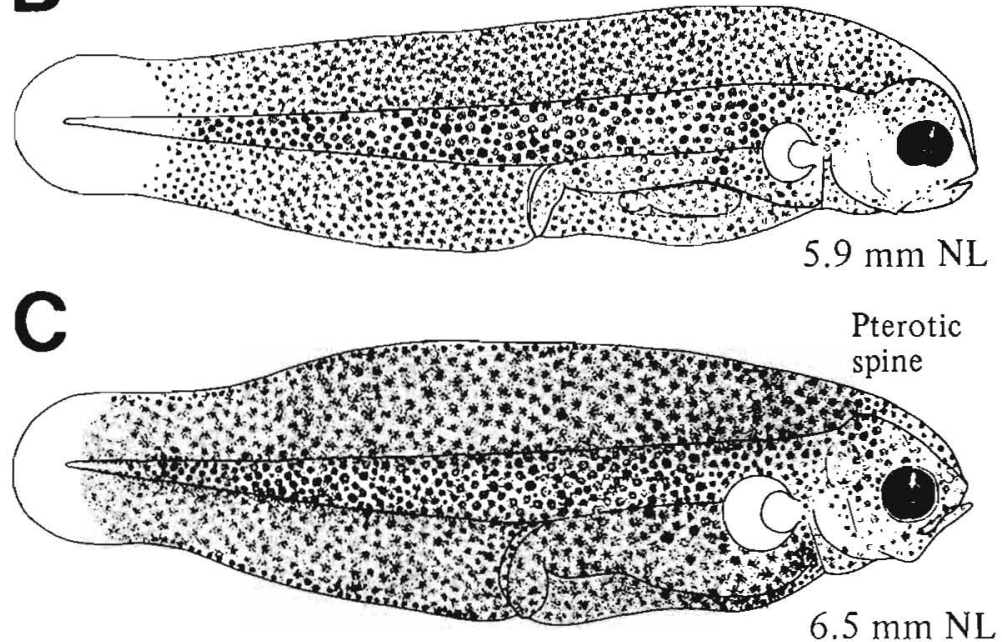

D

$6.5 \mathrm{~mm} \mathrm{NL}$
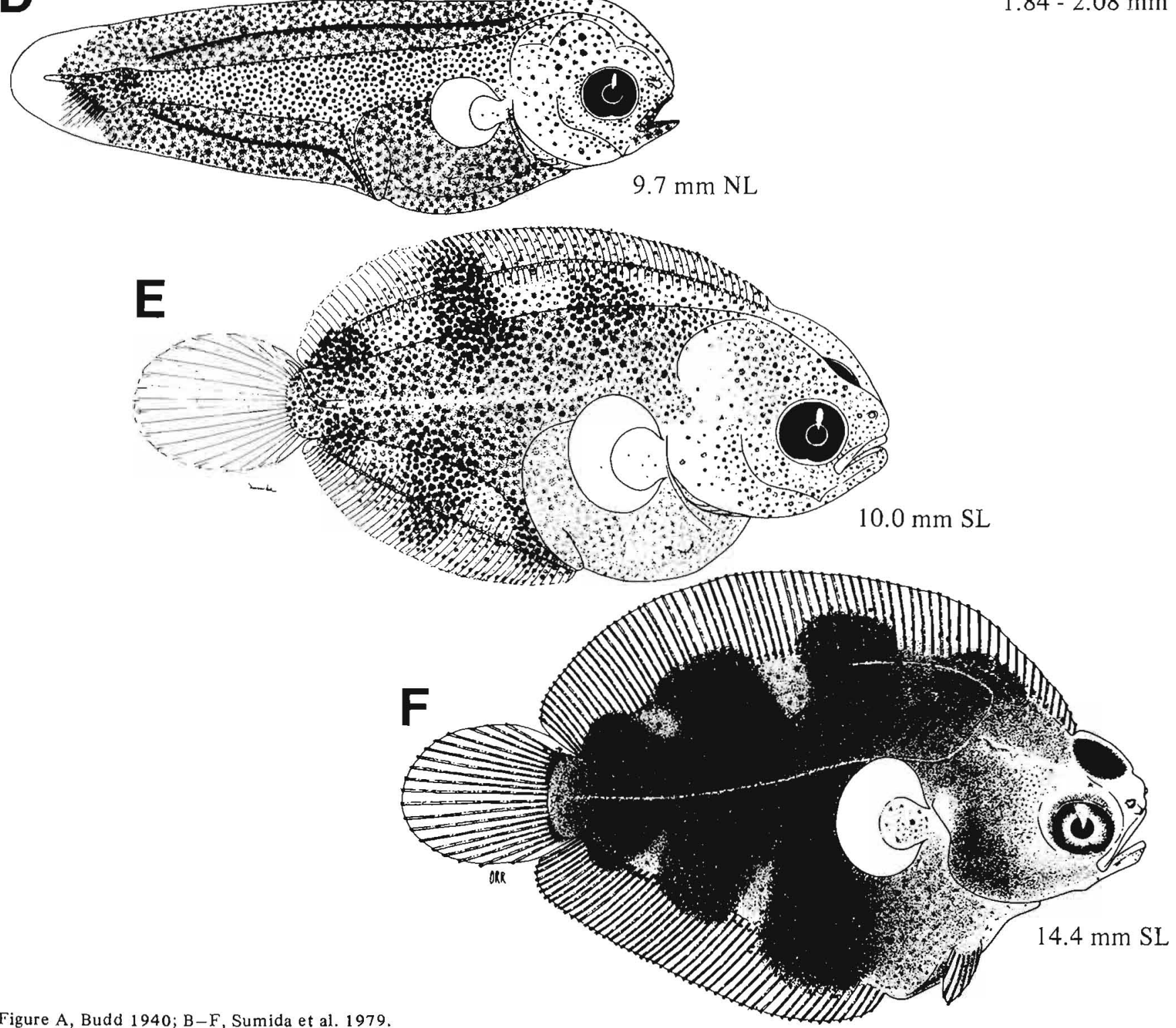

Figure A, Budd 1940; B-F, Sumida et al. 1979. 
MERISTICS

\begin{tabular}{ll} 
Vertebrae & Total: $38-39-41$ \\
& Precaudal: $11-11-12$ \\
& Caudal: $28-28-30$ \\
Branchiostegal rays & $7-7-7$ \\
Caudal fin & $2,7+7,2$ \\
& Total rays $=18^{\mathrm{a}}$ \\
Pelvic fin & Thoracic \\
& R: $6-6-6$ \\
Dorsal fin & R: $73-85-88$ \\
Pectoral fin & R: $10-\mathrm{X}-12$ \\
Anal fin & R: $53-58-62$ \\
Gill rakers & U: $5-\mathrm{X}-7$ \\
& \\
LIFE HISTORY & \\
\hline & \\
Range & S. California, $32-34^{\circ} \mathrm{N}$, to \\
& Bering Sea, $54-66^{\circ} \mathrm{N}$ \\
Ecology & Epi- and mesobenthal, $1-325 \mathrm{~m}$ \\
ELH pattern & Oviparous, pelagic eggs, \\
& pelagic larvae \\
Spawning & Season: Jan-Mar (Puget Sound); \\
& July (British Columbia) \\
& Area: \\
& Mode: \\
Fecundity & Migration: \\
Age at first maturity & $\begin{array}{l}\text { Range/function: } \\
\text { 2-3 yr (females) }\end{array}$ \\
Longevity & 2 yr (males) \\
&
\end{tabular}

EARLY LIFE HISTORY DESCRIPTION

\section{EGGS}

\begin{tabular}{|c|c|}
\hline Diameter & $0.83-1.04 \mathrm{~mm}$ \\
\hline No. of oil globules & None \\
\hline \multicolumn{2}{|l|}{ Oil globule diameter } \\
\hline Yolk & Clear \\
\hline \multicolumn{2}{|l|}{ Envelope } \\
\hline Hatch size & $<3 \mathrm{~mm} \mathrm{SL}$ \\
\hline \multicolumn{2}{|c|}{ Incubation time/temp. } \\
\hline \multicolumn{2}{|c|}{ Pigment } \\
\hline Diagnostic character & \\
\hline $\begin{array}{l}\text { See Isopsetta isolep } \\
\text { yolksac and in late }\end{array}$ & $\begin{array}{l}94) \text {; usually pigment on } \\
\text { on finfold }\end{array}$ \\
\hline
\end{tabular}
LARVAE (see discussion, p. 599)
Preanal length $\quad<50 \%$ SL
Length at flexion $\quad \sim 8-10 \mathrm{~mm} \mathrm{SL}$
Length at transformation $>22.6 \mathrm{~mm} \mathrm{SL}$
Sequence of fin Caudal, dorsal and anal, development pelvics, pectorals

Pigment

- Postanal body

-3-4 large spots along ventral midline

-Three spots along dorsal midline; the first spot more anteriorly placed than the first ventral spot

- Prominent pigment along edges of dorsal and ventral finfolds

- Mouth, isthmus

- Gut pigmented along ventral midline and posterior edge

\section{Diagnostic characters}

See Lepidopsetta bilineata (p. 596) and Lepidopsetta 2 (p. 599)

Distinguished from other pleuronectids with pigment bands by

- Distinctive pigment along edges of dorsal and ventral finfolds and dorsal and ventral body midlines

\footnotetext{
12 branched rays.

${ }^{b}$ English 1961

${ }^{\mathrm{c}}$ Manzer 1947

${ }^{d}$ Smith 1936
}

Ref: Ahlstrom et al. 1984a. 
A

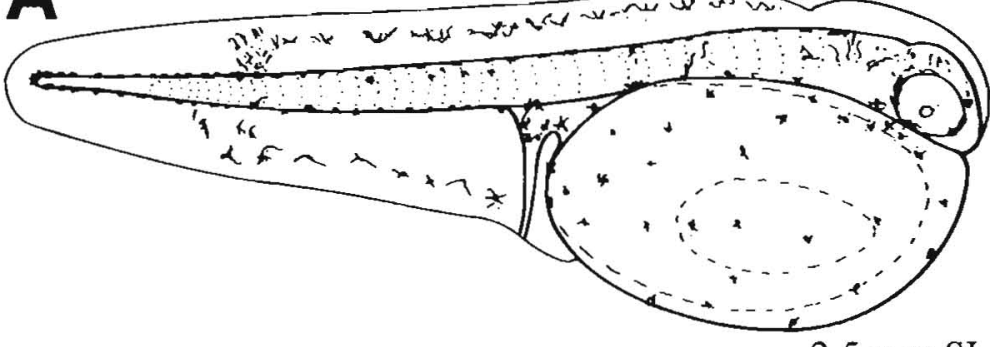

$2.5 \mathrm{~mm} \mathrm{SL}$
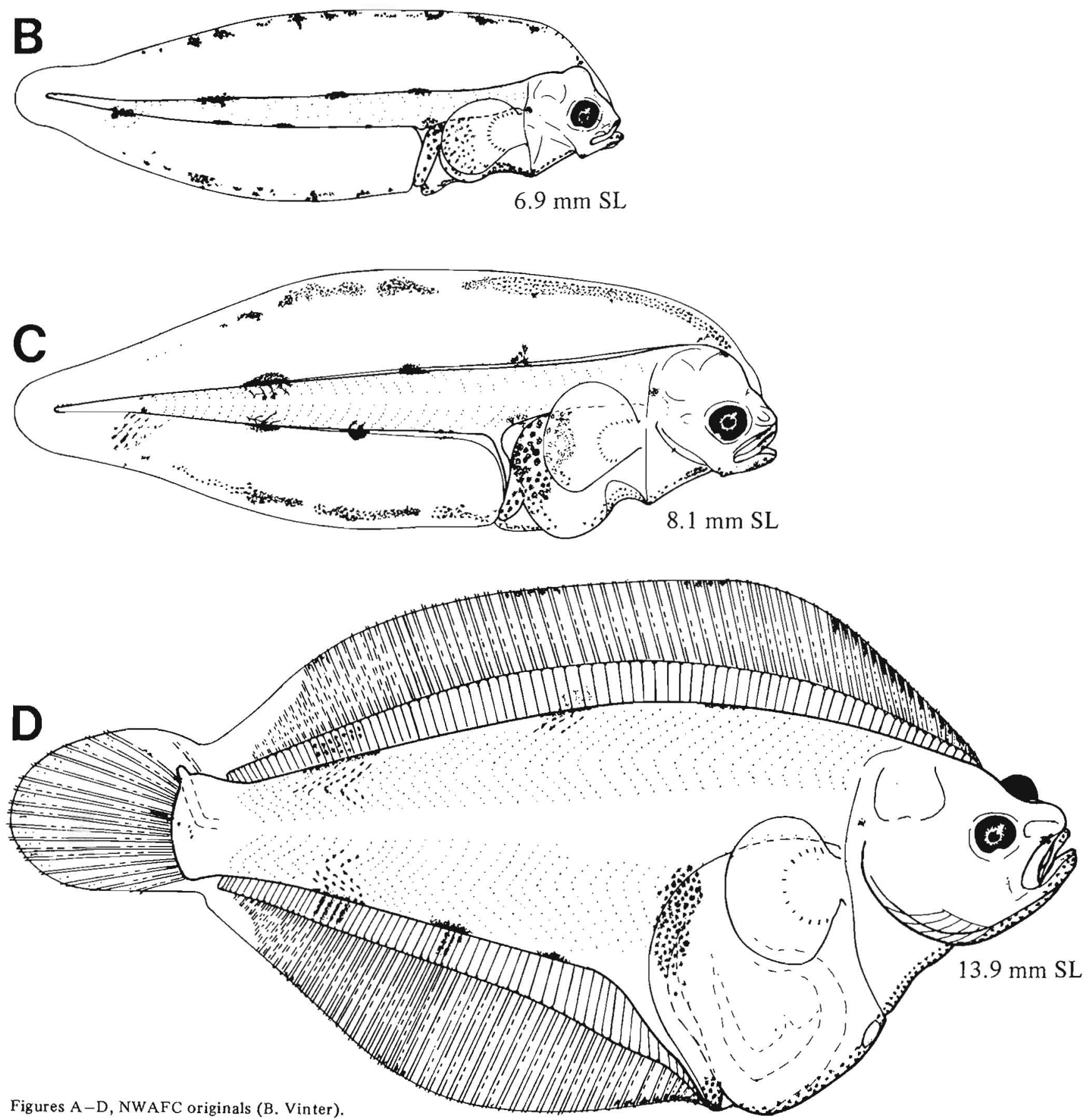


\section{MERISTICS}

Vertebrae

Total: 61-63-64

Precaudal: $17-18-19$

Caudal: $43-45-46$

Branchiostegal rays $\quad 7-7-7$

Caudal fin

Pelvic fin

Thoracic

R: 5-6-7

Dorsal fin

R: $83-97-105$

Pectoral fin

R: $11-14-15$

Anal fin

R: $63-72-79$

Gill rakers

U: $3-X-6$

L: $11-\mathrm{X}-16$

\section{EARLY LIFE HISTORY DESCRIPTION}

\section{EGGS}

Diameter

No. of oil globules

Oil globule diameter

Yolk

Envelope

Hatch size

Incubation time/temp.

Pigment

Diagnostic characters

\section{LIFE HISTORY}

\begin{tabular}{|c|c|}
\hline Range & $\begin{array}{l}\text { South of southern California to } \\
\text { Chukchi Sea, north of } 66^{\circ} \mathrm{N}\end{array}$ \\
\hline Ecology & $\begin{array}{l}\text { Epi-, meso-, and bathybenthal, } \\
14-2000 \mathrm{~m}\end{array}$ \\
\hline ELH pattern & $\begin{array}{l}\text { Oviparous, pelagic eggs, } \\
\text { pelagic larvae }\end{array}$ \\
\hline \multirow[t]{4}{*}{ Spawning } & Season: Falla \\
\hline & $\begin{array}{l}\text { Area: Continental slope } \\
\quad(>100 \mathrm{~m})^{\mathrm{a}}\end{array}$ \\
\hline & Mode: \\
\hline & Migration: \\
\hline Fecundity & $\begin{array}{l}\text { Range/function: } 15,000-215,000 \\
\text { (Atlantic)/ } \\
\mathrm{F}=0.000063 \times \mathrm{L}^{4.66}, \\
\mathrm{~L}=\mathrm{FL} \mathrm{cm} \mathrm{cm}^{\mathrm{a}}\end{array}$ \\
\hline Age at first maturity & 13 (females, Okhotsk Sea) $^{a}$ \\
\hline Longevity & $>23 \mathrm{yr}^{\mathrm{a}}$ \\
\hline
\end{tabular}

\section{LARVAE}

\begin{tabular}{ll} 
Preanal length & $<50 \% \mathrm{SL}$ \\
Length at flexion & $25-27 \mathrm{~mm} \mathrm{SL}$ \\
Length at transformation & $45-65 \mathrm{~mm} \mathrm{SL}$ \\
$\begin{array}{l}\text { Sequence of fin } \\
\text { development }\end{array}$ & Caudal, dorsal and anal, \\
\hline
\end{tabular}

Pigment

- Ventrally and posterolaterally on gut

- Light ventrolateral pigment along body from above gut, some dorsal, along peduncle; also in finfold above and below tail ( $\sim$ posterior 1/3)

- Increase in pigmentation in postflexion specimens

Diagnostic characters

- Newly hatched larvae large and unpigmented (except for eye)

Distinguished from other pleuronectids with $>60$ myomeres by

- No band pattern and overall lightly pigmented -Glyptocephalus zachirus (p. 586): Four postanal bands with more lateral intensity

-Embassichthys bathybius (p. 580): Three postanal bands

Distinguished from Hippoglossus stenolepis (p. 592) (both have large unpigmented yolksac larvae) by

- Depth of collection $(>200 \mathrm{~m})$

- High myomere count (61-64)

- Hatch size (10-16 mm SL)

\footnotetext{
${ }^{a}$ Dunn and Sample 1976
} 


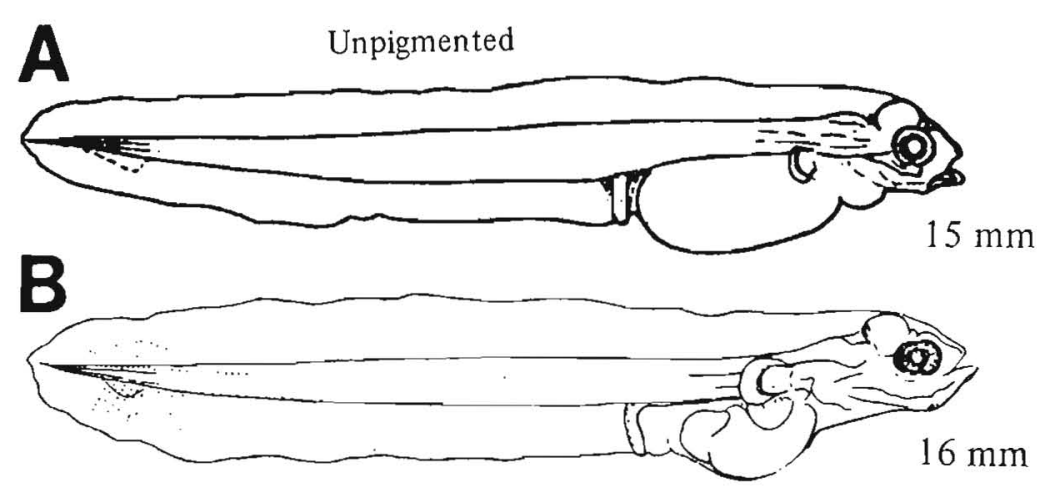

Finfold pigment restricted to posterior $1 / 3$
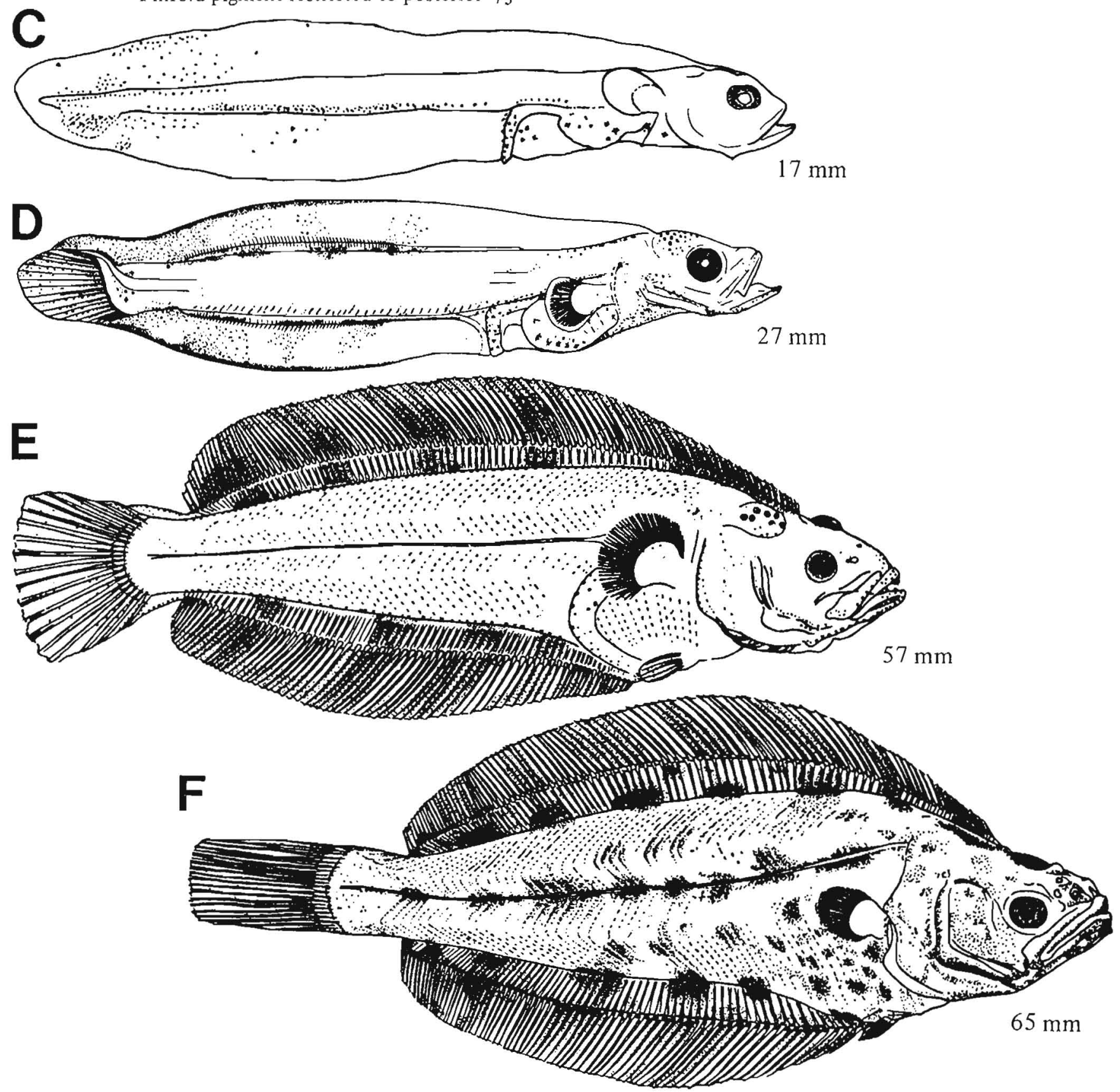

Figures A-F, Jensen 1935 (A-D, reversed; specimens collected from West Greenland waters). 


\section{MERISTICS}

$\begin{array}{ll}\text { Vertebrae } & \text { Total: } 50-51-52 \\ & \text { Precaudal: } 9-9-9 \\ & \text { Caudal: } 41-\mathrm{X}-43 \\ \text { Branchiostegal rays } & 6-6-6 \\ \text { Caudal fin } & \text { Total rays }=12^{\text {a }} \\ \text { Pelvic fin } & \text { Thoracic; } 4 \text { rays on eyed side, } \\ & \text { absent on blind side } \\ \text { Dorsal fin } & \text { R: } 95-X-106 \\ \text { Pectoral fin } & \text { Present during larval period but } \\ & \text { lacking in juvenile and adult } \\ & \text { stages } \\ \text { Anal fin } & \text { R: } 77-X-90 \\ \text { Gill rakers } & \text { U: X-X-X L: X-X-X }\end{array}$

LIFE HISTORY

\begin{tabular}{ll}
\hline Range & $\begin{array}{c}\text { South of southern California to } \\
\text { Oregon, } 42-46^{\circ} \mathrm{N}\end{array}$ \\
Ecology & Nearshore shelf demersal, \\
& $1-201 \mathrm{~m}$ \\
ELH pattern & Oviparous, pelagic eggs, \\
& pelagic larvae \\
Spawning & Season: June-Sept \\
& Area: \\
& Mode: \\
Fecundity & Migration: \\
Age at first maturity & Range/function: \\
Longevity &
\end{tabular}

\section{EARLY LIFE HISTORY DESCRIPTION}

\section{EGGS}

Diameter

No. of oil globules

Oil globule diameter

Yolk

Envelope

Hatch size

0.71-0.78 mm

Multiple, 10-23

Homogeneous

Smooth, colored

$1.9 \mathrm{~mm} \mathrm{SL}$
Incubation time/temp.

Pigment

Diagnostic characters

- Multiple oil globules

\section{LARVAE}

Preanal length

Length at flexion

Length at transformation

Sequence of fin development
$50 \%$ NL decreasing with development to $28 \%$ SL

9.4-10.8 mm SL

19.0-24.2 mm SL

Anterior dorsal; caudal, dorsal, and anal; pelvics ${ }^{c}$ (pectorals disappear at metamorphosis)

\section{Pigment}

- Small melanophores along dorsal midline, larger along ventral midline

- Single band posteriad on tail

- Large blotches at finfold margin, with development restricted to along the distal edges

- Head, gut, and swimbladder

\section{Diagnostic characters}

- Morphology: Large head and tapering body

- Gut mass trails posteriad

- Five exserted dorsal fin rays develop by $6.0 \mathrm{~mm} \mathrm{SL}$ but they are no longer than other fin rays (in other species of Symphurus, elongate anterior dorsal fin rays persist)

- Pelvic fin on blind side begins disappearing at 18.0-22.0 $\mathrm{mm} \mathrm{SLc}^{\mathrm{c}}$

\footnotetext{
${ }^{a}$ All rays on hypurals, all unbranched.

${ }^{b}$ Fitch and Lavenberg 1975

${ }^{\circ}$ E.H. Ahlstrom notes

Ref: Ahlstrom et al. 1984a.
} 

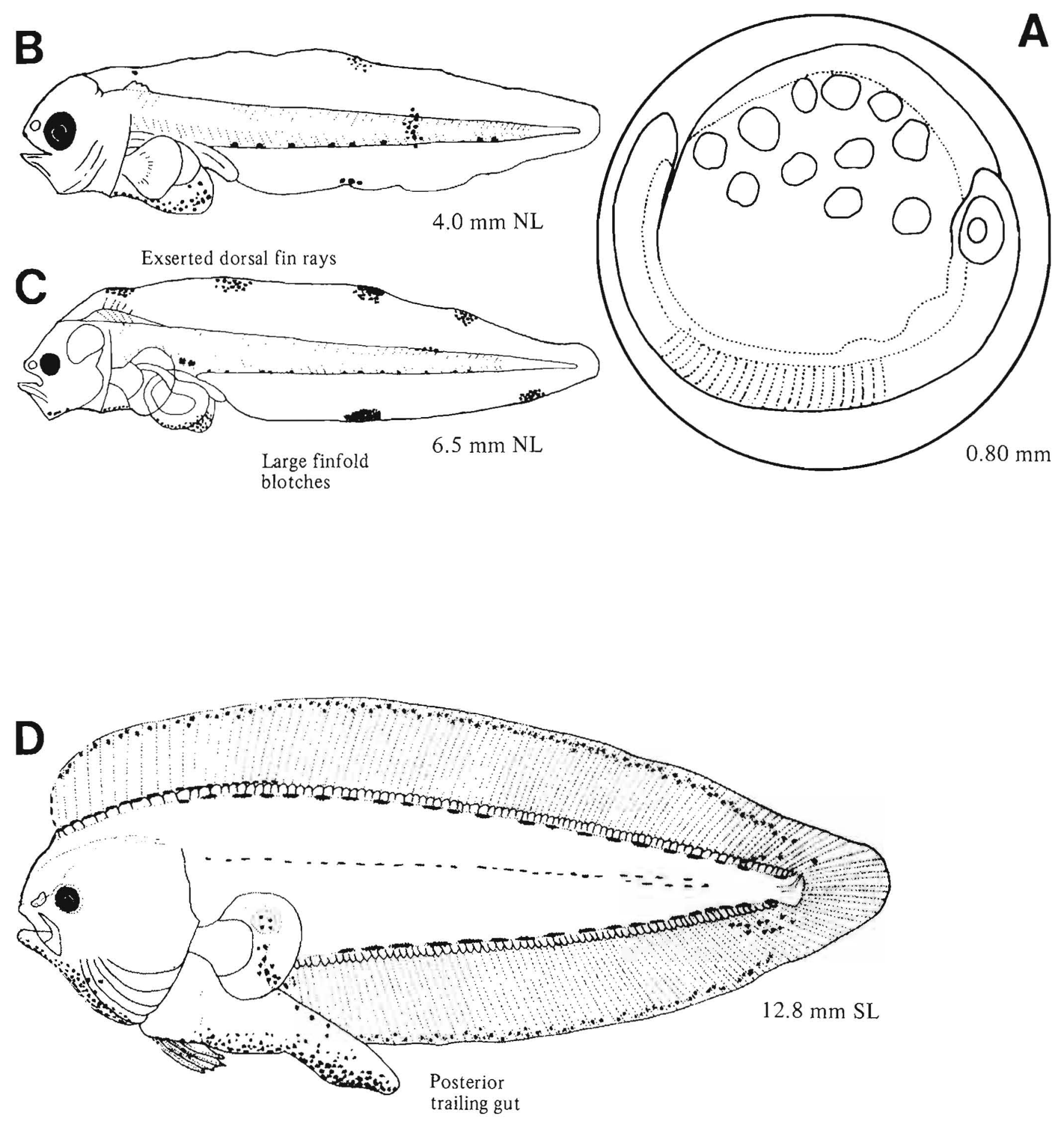

Figure A, Matarese and Sandknop 1984; B-D, Ahlstrom et al. 1984a. 



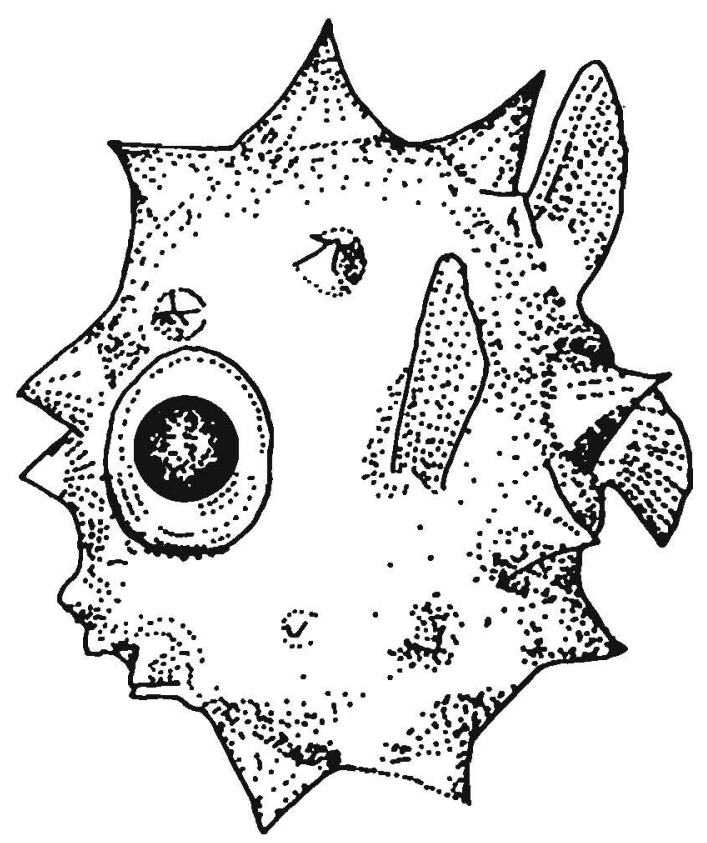

\section{Tetraodontiformes}

Although most fishes of the Tetraodontiformes are tropical and associated with the bottom, some are found in temperate zones and remain pelagic throughout their lifespans. The general body shape is rounded or boxlike and the body may be encased in a bony carapace or covered with sharp spines. Many species are able to inflate themselves with water or air. Eight families, 92 genera, and 329 species make up the order (J. Nelson 1984). Only one family, Molidae, is found in the Northeast Pacific. The most unusual of the tetraodontiforms, molids have no caudal fin and propel themselves with large dorsal and anal fins. Eggs are pelagic, 1.42-1.80 mm in diameter, and have multiple oil globules (Leis 1984). Larvae hatch with a functioning jaw, pigmented eyes, and a dermal sac enclosing the head and trunk. With development, body spines form and the tail atrophies. Molids may have a long prejuvenile stage marked by retention of spines and a shape unlike that of adults (Leis 1984).

Family in study area: Molidae 


\section{MERISTICS}

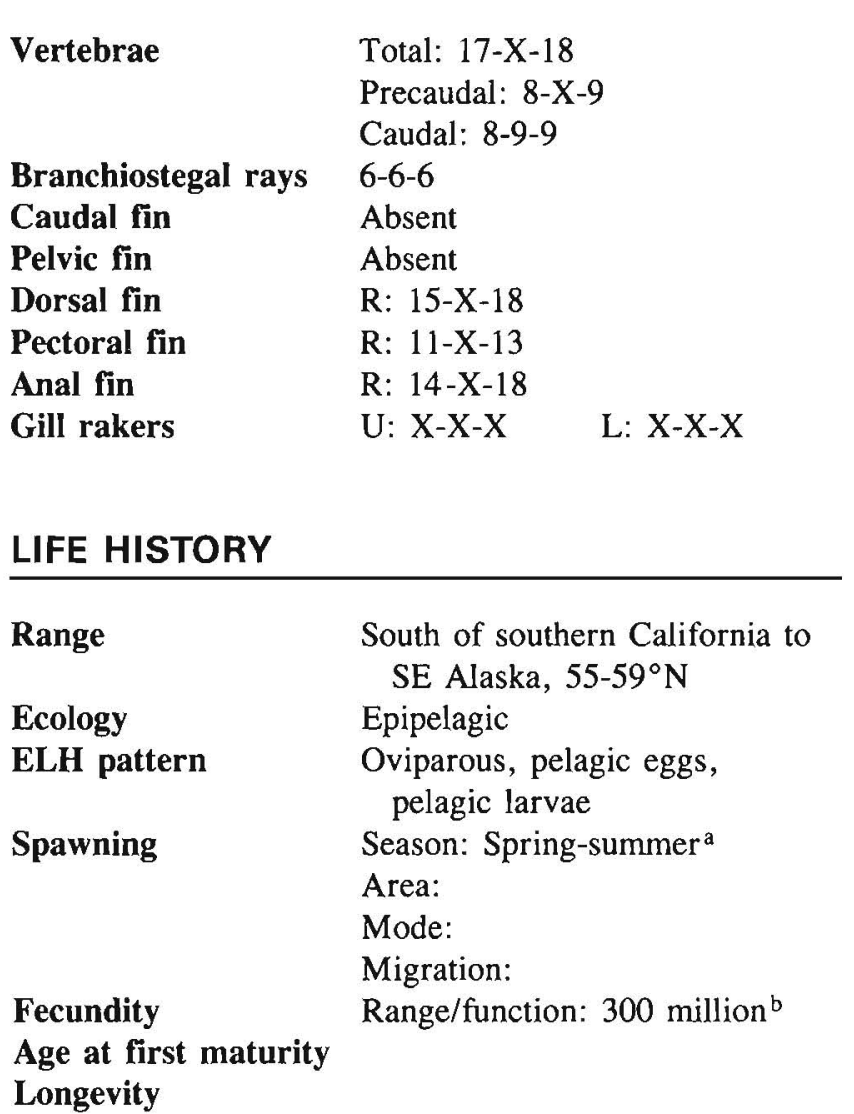

EARLY LIFE HISTORY DESCRIPTION

\section{EGGS}

Diameter

No. of oil globules

Oil globule diameter

Yolk

Envelope

Hatch size $\quad<1.84 \mathrm{~mm}$ TL

Incubation time/temp. Other members of family

Pigment
Diagnostic characters - Family
- Pelagic, large (1.4-1.8 mm), and have multiple oil globules

\footnotetext{
Larvae

Preanal length

Length at flexion

$<50 \%$ SL increasing with development to $>50 \%$ SL

Length at transformation Long ontogenetic stage between larvae and juveniles

Sequence of fin development

Pectorals and caudal, dorsal and anal

Pigment - Family

- Usually heavily pigmented over gut and dorsal surfaces

\section{Diagnostic characters}

- Morphology: Wide, deep body

- Body spines with a ventral keel (form soon after hatching)

- Tail in young is normal but soon atrophies and a true caudal never forms; notochord flexion does not take place, so the clavus (gradual thickening near the distal end) is not homologous with the caudal fin $^{\mathrm{c}}$
}

\footnotetext{
${ }^{a}$ Martin and Drewry 1978

${ }^{\mathrm{b}} \mathrm{Hart} 1973$

${ }^{c}$ Leis 1984

Ref: Leis 1984.
} 

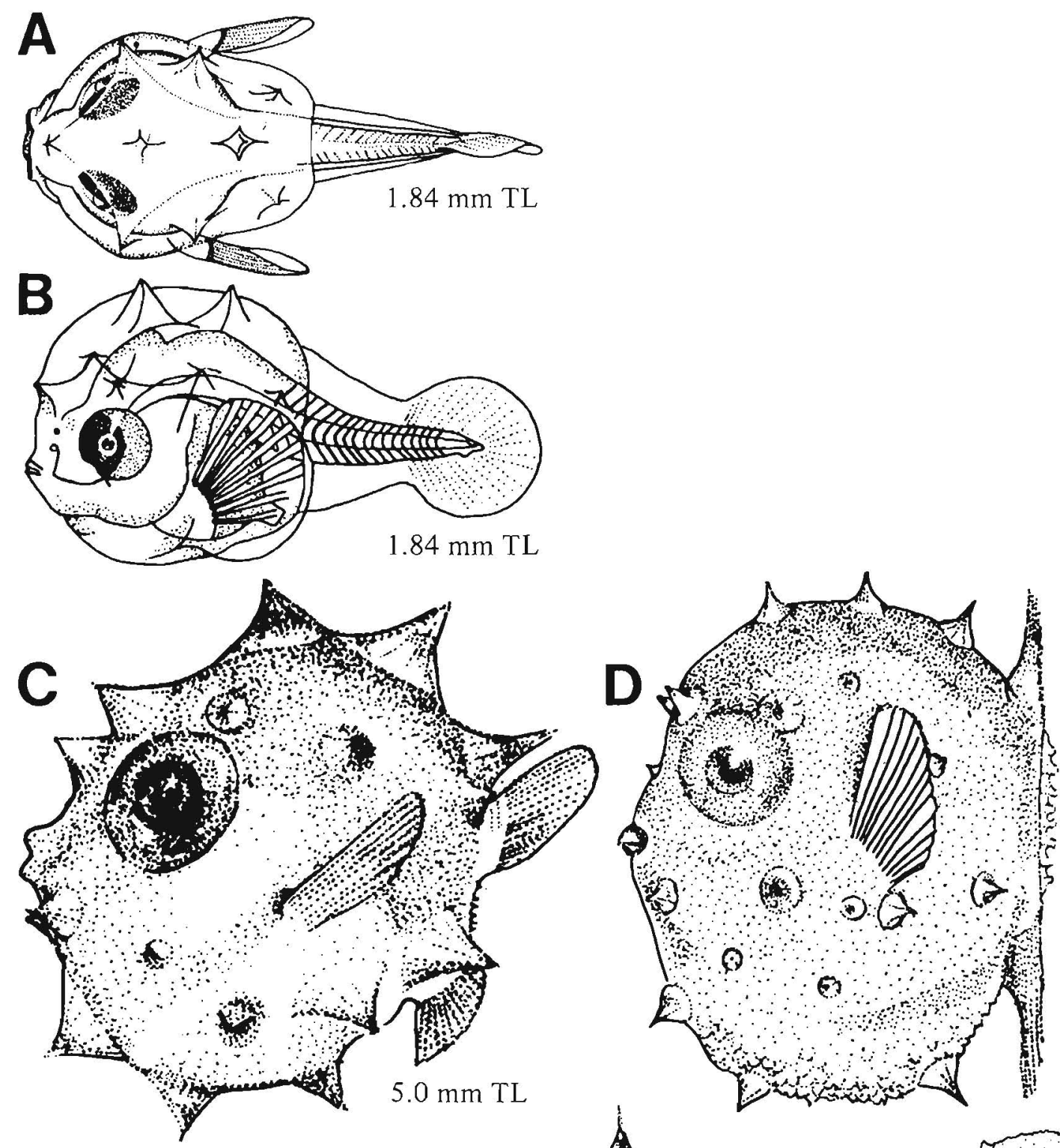

Body spines reach maximum

size at the same time the clavus forms

Figures $A-F$ (A, dorsal view; $E$, anterior view), Martin and Drewry 1978 (North Atlantic specimens).

$37.0 \mathrm{~mm} \mathrm{TL}$

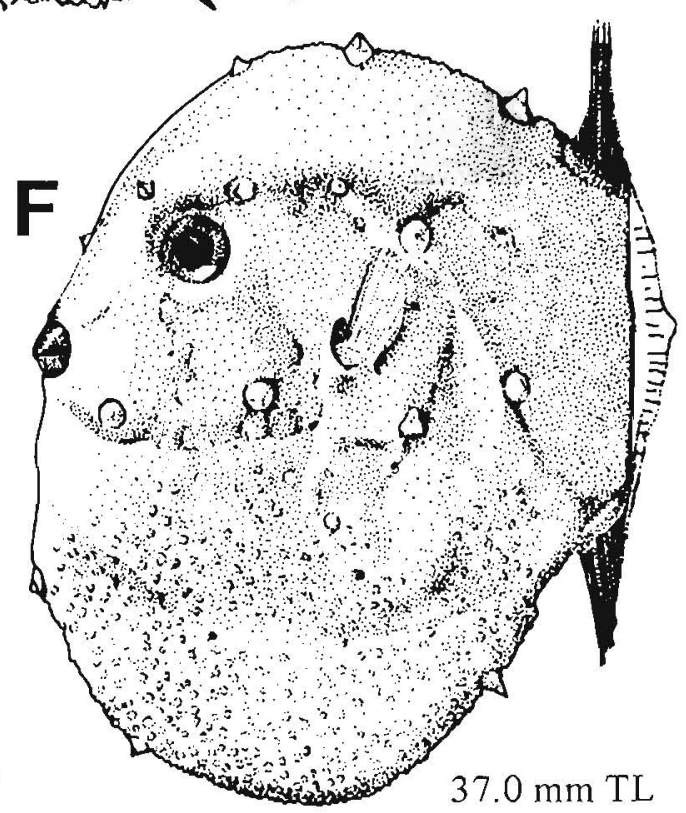


Abe, T., and S. Kaji

1972 A record of Oreosoma atlanticum (Oreosomatidae, Zeiformes, Teleostei) from Tasman Sea. UO (Sakana) No. 8:5-7, Ichthyol. Soc. Jpn., Tokyo.

Able, K.W., D.F. Markle, and M.P. Fahay

1984 Cyclopteridae: Development. In Moser, H.G., et al. (eds.), Ontogeny and systematics of fishes, p. 428-437. Spec. Publ. 1, Am. Soc. Ichthyol. Herpetol. Allen Press, Lawrence, KS.

Aboussouan, A., and R. Rasonarivo

1986 Capture d'une larve de Spectrunculus grandis (Günther, 1877) dans l'ouest de l'ocean Indien, ile de la reunion (Pisces, Ophidiiformes, Ophidiidae). Cybium 10:206-207 [in French].

Ahlstrom, E.H.

1965 Kinds and abundance of fishes in the California Current region based on egg and larval surveys. Calif. Coop. Oceanic Fish. Invest. Rep. 10:31-52.

1969 Remarkable movement of oil globules in eggs of bathylagid smelts during embryonic development. J. Mar. Biol. Assoc. India 11: 206-217.

1972 Distributional atlas of fish larvae in the California Current region: Six common mesopelagic fishes, Vinciguerria lucetia, Triphoturus mexicanus, Stenobrachius leucopsarus, Leuroglossus stilbius, Bathylagus wesethi, and Bathylagus ochotensis. Calif. Coop. Oceanic Fish. Invest. Atlas 17, $306 \mathrm{p}$.

1974 The diverse patterns of metamorphosis in gonostomatid fishesAn aid to classification, In Blaxter, J.H.S. (ed.), The early life history of fish, p. 659-674. Springer-Verlag, NY.

Ahlstrom, E.H., and O.P. Ball

1954 Description of eggs and larvae of jack mackerel (Trachurus symmetricus) and distribution and abundance of larvae in 1950 and 1951. U.S. Fish Wildl. Serv., Fish. Bull. 56:209-245.

Ahlstrom, E.H., and R.C. Counts

1955 Eggs and larvae of the Pacific hake, Merluccius productus. U.S. Fish Wildl, Serv., Fish. Bull, 56:295-329.

Ahlstrom, E.H., and H.G. Moser

1975 Distributional atlas of fish larvae in the California Current region: Flatfishes, 1955 through 1960 . Calif. Coop. Oceanic Fish. Invest. Atlas 23, $207 \mathrm{p}$.

1976 Eggs and larvae of fishes and their role in systematic investigations and in fisheries. Rev. Trav. Inst. Peches Marit. 40:379-398.

1980 Characters useful in identification of pelagic marine fish eggs. Calif. Coop. Oceanic Fish. Invest. Rep. 21:121-131.

Ahlstrom, E.H., and E. Stevens

1976 Report of neuston (surface) collections made on an extended CalCOFI cruise during May 1972. Calif. Coop. Oceanic Fish. Invest. Rep. 18:167-180

Ahlstrom, E.H., J.L. Butler, and B.Y. Sumida

1976 Pelagic stromateoid fishes (Pisces, Perciformes) of the eastern Pacific: Kinds, distributions, and early life histories and observations on five of these from the Northwest Atlantic. Bull. Mar. Sci. 26:285-402.

Ahlstrom, E.H., K. Amaoka, D.A. Hensley, H.G. Moser, and B.Y. Sumida

1984a Pleuronectiformes: Development. In Moser, H.G., et al. (eds.), Ontogeny and systematics of fishes, p. 640-669. Spec. Publ. 1, Am. Soc. Ichthyol. Herpetol. Allen Press, Lawrence, KS.

Ahlstrom, E.H., H.G. Moser, and D.M. Cohen

1984b Argentinoidei: Development and relationships. In Moser, H.G., et al. (eds.), Ontogeny and systematics of fishes, p. 155-168. Spec. Publ. 1, Am. Soc. Ichthyol. Herpetol. Allen Press, Lawrence, KS.

Ahlstrom, E.H., W.J. Richards, and S.H. Weitzman

1984c Families Gonostomatidae, Sternoptychidae, and associated stomiiform groups: Development and relationships. In Moser, H.G., et al. (eds.), Ontogeny and systematics of fishes, p. 184-198. Spec. Publ. 1, Am. Soc. Ichthyol. Herpetol. Allen Press, Lawrence, KS.
Alaska Department of Fish and Game

1985 Alaska habitat management guide. South central region, Vol. 1: Life histories and habitat requirements of fish and wildlife. Alaska Dep. Fish Game, Juneau, AK 99811, 429 p.

Alderdice, D.F., and C.R. Forrester

1971 Effects of salinity and temperature on embryonic development of the petrale sole (Eopsetta jordani). J. Fish. Res. Board Can. 28:727-744.

Allen, L.G.

1979 Larval development of Gobiesox rhessodon (Gobiesocidae) with notes on the larva of Rimicola muscarum. Fish. Bull., U.S. 77: 300-304.

1984 Gobiesociformes: Development and relationships. In Moser, H.G., et al. (eds.), Ontogeny and systematics of fishes, p. 629-636. Spec. Publ. 1, Am. Soc. Ichthyol. Herpetol. Allen Press, Lawrence, KS.

Allen, L.G., and J.M. Ilg

1983 Larval development of the northern clingfish, Gobiesox maeandricus. Copeia 1983:551-554.

Allen, M.J., and G.B. Smith

1988 Atlas and zoogeography of common fishes in the Bering Sea and northeastern Pacific. NOAA Tech. Rep. NMFS 66. Natl. Oceanic Atmos. Adm., Natl. Mar. Fish. Serv., Seattle, WA 98115-0070, $151 \mathrm{p}$.

Alton, M.S., and R.A. Webber

1976 Sablefish (family Anoplopomatidae). In Pereya, W.T., et al. (eds.), Demersal fish and shellfish resources in the eastern Bering Sea in the baseline year 1975, p. 425-438. Proc. Rep., Northwest Alaska Fish. Cent., Natl. Mar. Fish. Serv., NOAA, Seattle, WA 98115-0070.

Ambrose, D.A., J.L. Butler, H.G. Moser, B.Y. Sumida, E.M. Sandknop, and E.G. Stevens

1983 Description of the larvae of the cusk eels Ophidion scrippsae and Chilara taylori (family Ophidiidae). Calif. Coop. Oceanic Fish. Invest. Rep. 24:226-234.

Anderson, M.E.

1984a On the anatomy and phylogeny of the Zoarcidae (Teleostei: Perciformes). Ph.D. diss., Coll. William and Mary, Williamsburg, VA $23185,254 \mathrm{p}$.

1984b Zoarcidae: Development and relationships. In Moser, H.G., et al. (eds.), Ontogeny and systematics of fishes, p. 578-581. Spec. Publ. 1, Am. Soc. Ichthyol. Herpetol. Allen Press, Lawrence, KS.

Anderson, M.E., and G.M. Cailliet

1974 Crab and snailfish commensalism in Monterey Bay. Underwater Nat. 8(3):29-31.

Andriashev, A.P.

1954 Fishes of the northern seas of the U.S.S.R. Tr. Zool. Inst. Akad. Nauk SSSR 53. [In Russ., transl. by Isr. Prog. Sci. Transl., Jerusalem, 1964; avail. U.S. Dep. Commer., Natl. Tech. Inf. Serv., Springfield, VA 22161, as OTS63-11160.]

Arita, G.S.

1969 Sexual dimorphism in the cyclopterid fish Eumicrotremus orbis. J. Fish. Res. Board Can. 26:3262-3265.

Arora, H.L.

1951 An investigation of the California sanddab, Citharichthys sordidus (Girard). Calif. Fish Game 37:3-42.

Atkinson, C.E.

1939 Notes on the life-history of the tidepool johnny (Oligocottus maculosus). Copeia 1939:23-30.

Auer, N.A. (editor)

1982 Identification of larval fishes of the Great Lakes Basin with emphasis on the Lake Michigan drainage. Spec. Publ. 82-3, Great Lakes Fish Comm., Ann Arbor, MI 48105, 744 p. 
Badcock, J., and R.C. Baird

1980 Remarks on systematics, development, and distribution of the hatchetfish genus Sternoptyx (Pisces, Stomiatoidei). Fish. Bull., U.S. $77: 803-820$.

Badcock, J., and N.R. Merrett

1976 Midwater fishes in the eastern North Atlantic. I. Vertical distribution and associated biology in 30 degrees $N, 23$ degrees W, with developmental notes on certain myctophids. Prog. Oceanogr. 7:3-58.

Bailey, K.M., R.C. Francis, and P.R. Stevens

1982 The life history and fishery of the Pacific whiting, Merluccius productus. Calif. Coop. Fish. Invest. Rep. 23:81-98.

Bain, H., and A.D. Sekerak

1978 Aspects of the biology of Arctic cod, Boreogadus saida, in the central Canadian Arctic. Report prepared for Polar Gas Project by LGL Limited, Environ. Res. Assoc., Toronto, Ontario, Canada, $104 \mathrm{p}$.

Barnhart, P.S.

1932 Notes on the habits, eggs, and young of some fishes of southern California. Bull. Scripps Inst. Oceanogr. Univ. Calif. 3(4):87-99.

Barsukov, V.V.

1959 The wolffish (Anarhichadidae). Fauna SSSR Moscow, N.S. 73. [Transl. by Smithson. Inst., Wash., D.C., 1972; avail. U.S. Dep. Commer., Natl. Tech. Inf. Serv, Springfield, VA 22161, as TT67-59074.]

Bauchot, M.

1959 Etude des larvaes leptocephalus du groups Leptocephalus lanceolarus Stromman et identification a la famille des Serrivomeridae. Dana-Rep. Carlsberg Found. 48, 148 p. [in French].

Baxter, J.L.

1967 Summary of biological information on the northern anchovy Engraulis mordax Girard. Calif. Coop. Oceanic Fish. Invest. Rep. 11:110-116.

Baxter, R.

1975 Inshore marine resources of Bristol Bay, Alaska. Alaska Dep. Fish Game, Bethel, AK 99559, 97 p

Beamish, R.J.

1979 Differences in the age of Pacific hake (Merluccius productus) using whole otoliths and sections of otoliths. J. Fish. Res. Board Can. 36:141-151.

Beebe, $W$.

1933 Deep-sea fishes of the Bermuda Oceanographic Expeditions. No. 2. Family Alepocephalidae. Zoologica (NY) 16(2):15-93.

1934 Deep-sea fishes of the Bermuda Oceanographic Expeditions. Family Idiacanthidae. Zoologica (NY) 16(4):97-147.

Beebe, W., and J. Crane

1939 Deep-sea fishes of the Bermuda Oceanographic Expeditions. Family Melanostomiidae. Zoologica (NY) 24(2):65-238

Belyanina, T.N.

1983 Developmental sequences of Sternoptyx species (Sternoptychidae). J. Ichthyol. 23(4):73-86.

1984 Larvae of hatchetfishes of the genus Argyropelecus (Sternoptychidae). J. Ichthyol. 24(2):7-20.

Berrien, P.L.

1978 Eggs and larvae of Scomber scombrus and Scomber japonicus in continental shelf waters between Massachusetts and Florida. Fish. Bull. U.S. 76:95-115.

Bertelsen, E.

1951 The ceratioid fishes. Ontogeny, taxonomy, distribution, and biology. Dana-Rep. Carlsberg Found. 39, $276 \mathrm{p}$

1984 Ceratioidei: Development and relationships. In Moser, H.G., et al. (eds.), Ontogeny and systematics of fishes, p. 325-333. Spec. Publ. 1, Am. Soc. Ichthyol. Herpetol. Alten Press, Lawrence, KS.

Bertelsen, E., G. Krefft, and N.B. Marshall

1976 The fishes of the family Notosudidae. Dana-Rep. Carlsberg Found. $86,114 \mathrm{p}$.

Best, E.A.

1963 Contribution to the biology of the Pacific hake, Merluccius productus (Ayres). Calif. Coop. Oceanic Fish. Invest. Rep. 9:51-56
Bigelow, H.B., and W.C. Schroeder

1953 Fishes of the Gulf of Maine. U.S. Fish Wildl. Serv., Fish. Bull. 74(vol. 53), 577 p.

Blackburn, J.E.

1973 A survey of the abundance, distribution, and factors affecting distribution of ichthyoplankton in Skagit Bay. M.S. thesis, Univ. Wash., Seattle, WA $98195,136 \mathrm{p}$.

Boehlert, G.W., and M.M. Yoklavich

1984 Reproduction, embryonic energetics, and the maternal-fetal relationship in the viviparous genus Sebastes (Pisces: Scorpaenidae) Biol. Bull. (Woods Hole) 167:354-370.

Bolin, R.L.

1936 Embryonic and early larval stages of the California anchovy. Calif. Fish Game 22:314-321.

Bond, C.E., and D.L. Stein

1984 Opaeophacus acrogeneius, a new genus and species of Zoarcidae (Pisces: Osteichthyes) from the Bering Sea. Proc. Biol. Soc. Wash. 97(3):522-525.

Breder, C.M., and D.E. Rosen

1966 Modes of reproduction in fishes. T.F.H. Publ., Jersey City, NJ, $941 \mathrm{p}$

Brothers, E.B.

1975 The comparative ecology and behavior of three sympatric California gobies. Ph.D. diss., Univ. Calif., San Diego, CA 92037, 370 p.

Budd, P.L.

1940 Development of the eggs and early larvae of six California fishes. Calif. Dep. Fish Game, Fish Bull. 56, 50 p.

Burge, R.T., and S.A. Schultz

1973 The marine environment in the vicinity of Diablo Cove with special reference to abalones and bony fishes. Calif. Dep. Fish Game, Mar. Res. Tech. Rep. 19, 239 p.

Castle, P.H.

1965 Leptocephali of the Nemichthyidae, Serrivomeridae, Synaphobranchidae, and Nettastomatidae in Australian waters. Trans. R. Soc. N.Z. Zool. 5:131-146.

1984 Notacanthiformes and Anguilliformes: Development. In Moser, H.G., et al. (eds.), Ontogeny and systematics of fishes, p. 62-93. Spec. Publ. I, Am. Soc. Ichthyol. Herpetol. Allen Press, Lawrence, KS.

Castle, P.H., and N.S. Raju

1975 Some rare leptocephali from the Atlantic and Indo-Pacific Oceans, Dana-Rep. Carlsberg Found. 85, 25 p.

Chapman, W.M.

1939 Eleven new species and three new genera of oceanic fishes collected by the International Fisheries Commission from the northeastern Pacific. Proc. U.S. Natl. Mus. 86(3062):501-542.

Chapman, W.M., and L.D. Townsend

1938 The osteology of Zaprora silenus Jordan, with notes on its distribution and early life history. Ann. Mag. Nat. Hist. 11:89-117.

Chen, $\mathrm{L}$.

1986 Meristic variation in Sebastes (Scorpaenidae) with an analysis of character association and bilateral pattern and their significance in species separation. NOAA Tech. Rep. NMFS 45, Natl. Oceanic Atmos. Adm., Natl. Mar. Fish. Serv., Seattle, WA 98115-0070, 17 p.

Chilton, D.E., and R.J. Beamish

1982 Age determination methods for fishes studied by the groundfish program at the Pacific Biological Station. Can. Spec. Publ. Fish. Aquat. Sci. 60, 102 p.

Chung, M. 1977 The fishes of Korea. Il Ji Sa Publ. Co., Seoul, Korea

Clark, F.N., and J.B. Phillips

1952 The northern anchovy (Engraulis mordax) in the California fishery. Calif. Fish Game 38:189-207.

Clemens, W.A., and G.V. Wilby

1961 Fishes of the Pacific coast of Canada. Fish. Res. Board Can. Bull. $68,2 \mathrm{~d}$ ed., $443 \mathrm{p}$

Cohen, D.M.

1958 A revision of the fishes of the subfamily Argentininae. Bull. Fla. State Mus. Biol. Ser. 3:93-172. 
1960 New records of the opisthoproctid genus Bathylychnops, with a notice of neoteny in the related genus Dolichopteryx. Copeia 1960:147-149.

Cohen, D.M., and J.G. Nielsen

1978 Guide to the identification of genera of the fish order Ophidiformes, with a tentative classification of the order. NOAA Tech. Rep. NMFS Circ. 417, Natl. Oceanic Atmos. Adm., Natl. Mar. Fish. Serv., Seattle, WA 98115-0070, 72 p.

Collette, B.B.

1984 Atherinomorpha: Introduction. In Moser, H.G., et al. (eds.), Ontogeny and systematics of fishes, p. 334. Spec. Publ. 1, Am. Soc. Ichthyol. Herpetol. Allen Press, Lawrence, KS.

Collette, B.B., and C.E. Nauen

1983 Scombrids of the world. FAO Fish. Synop. 125, Vol. 2, $137 \mathrm{p}$.

Collette, B.B., G.E. McGowen, N.V. Parin, and S. Mito

1984a Beloniformes: Development and relationships. In Moser, H.G., et al. (eds.), Ontogeny and systematics of fishes, p. 335-354. Spec. Publ. 1, Am. Soc. Ichthyol. Herpetol. Allen Press, Lawrence, KS.

Collette, B.B., T. Pothoff, W.J. Richards, S. Ueyanagi, J.L. Russo, and $Y$. Nishikawa

1984b Scombroidei: Development and relationships. In Moser, H.G., et al. (eds.), Ontogeny and systematics of fishes, p. 591-619. Spec. Publ. 1, Am. Soc. Ichthyol. Herpetol. Allen Press, Lawrence, KS.

Craig, P.C., W.B. Griffiths, L. Haldorson, and H. McElderry

1982 Ecologial studies of Arctic cod (Boreogadus saida) in Beaufort Sea coastal waters, Alaska. Can. J. Fish. Aquat. Sci. 39:395-406.

DeLacy, A.C., C.R. Hitz, and R.L. Dryfoos

1964 Maturation, gestation, and birth of rockfish (Sebastodes) from Washington and adjacent waters. Wash. Dep. Fish., Fish. Res. Pap. 2(3):51-67.

DeMartini, E.E.

1976 The adaptive significance of territoriality and egg cannabalism in the painted greenling, Oxylebius pictus Gill, a northeastern Pacific marine fish. Ph.D. diss., Univ. Wash., Seattle, WA 98195, $286 \mathrm{p}$

1978 Spatial aspects of reproduction in buffalo sculpin, Enophrys bison. Environ. Biol. Fishes 3:331-336.

Dunn, J.R.

1983 Development and distribution of young of northern smoothtongue, Leuroglossus schmidri (Bathylagidae) in the Northeast Pacific, with comments on the systematics of the genus Leuroglossus Gilbert. Fish. Bull., U.S. 81:23-40.

1986 A catalog of Northwest and Alaska Fisheries Center ichthyoplankton cruises 1965-1985. Proc. Rep. 86-08, Northwest Alaska Fish. Cent., Natl. Mar. Fish. Serv., NOAA, Seattle, WA 981 15-0070, $164 \mathrm{p}$.

Dunn, J.R., and A.C. Matarese

1984 Gadidae: Development and relationships. In Moser, H.G., et al. (eds.), Ontogeny and systematics of fishes, p. 283-289. Spec. Publ. 1, Am. Soc. Ichthyol. Herpetol. Allen Press, Lawrence. KS. 1987 A review of the early life history of Northeast Pacific gadoid fishes. Fish. Res. (Amst.) 5:163-184

Dunn, J.R., and T.M. Sample

1976 Greenland halibut (family Pleuronectidae). In Pereya, W.T., et al. (eds.), Demersal fish and shellfish resources in the eastern Bering Sea in the baseline year 1975, p. 475-487. Proc. Rep., Northwest Alaska Fish. Cent., Natl. Mar. Fish. Serv., NOAA, Seattle, WA $98115-0070$

Dunn, J.R., and B.M. Vinter

1984 Development of larvae of saffron cod, Eleginus gracilis, with criteria for identification of gadid larvae in Pacific and Arctic waters contiguous to Canada and Alaska. Can. J. Fish. Aquat. Sci. 41: 304-318.
D'Vincent, S., H.G. Moser, and E.H. Ahlstrom

1980 Description of the larvae and early juveniles of Pacific butterfish, Peprilis simillimus (family Stromateidae). Calif. Coop. Oceanic Fish. Invest. Rep. 21:172-179.

Ebeling, A.W.

1962 Melamphaidae. I. Systematics and zoogeography of the species in the bathypelagic fish genus Melamphaes Günther. Dana-Rep. Carlsberg Found. 58, $164 \mathrm{p}$.

Ebert, E.E., and C.H. Turner

1962 The nesting behavior, eggs, and larvae of the bluespot goby. Calif. Fish Game 48:249-252.

\section{Efremenko, V.N., and L.A. Lisovenko}

1970 Morphological features of intraovarian and pelagic larvae of some Sebastodes species inhabiting the Gulf of Alaska. In Moiseev, P.A. (ed.), Soviet fisheries investigations in the northeastern Pacific, Pt.V, p. 267-286. Izv. Tikhookean. Nauchno-Issled. Inst. Rybn. Khoz. Okeanogr. (TINRO) 72; Tr. Vses. Nauchno-Issled. Inst. Morsk. Rybn. Khoz. Okeanogr. (VNIRO) 70. [Transl. from Russ.; avail. U.S. Dep. Commer., Natl. Tech. Inf. Serv, Springfield, VA 22161, as TT71-50127.]

English, T.S.

1961 An inquiry into distributions of planktonic fish eggs in a restricted area of Puget Sound. Ph.D. diss., Univ. Wash., Seattle, WA 98195, $227 \mathrm{p}$.

Eschmeyer, W.N., E.S. Herald, and H. Hammann

1983 A field guide to Pacific Coast fishes of North America from the Gulf of Alaska to Baja California. Houghton Mifflin Co., Boston, $336 \mathrm{p}$.

Evseenko, S.A.

1982 Ichthyoplankton of slope and Gulf Stream waters off Nova Scotia in late autumn 1974. J. Northwest Atl. Fish. Sci. 3:127-139.

Faber, D.J.

1976 Identification of four northern blennioid fish larvae in the Canadian Atlantic Ocean (Stichaeidae, Lumpeninae). J. Fish. Res. Board Can. 33:1798-1802.

Fadeev, N.S.

1965 Comparative outline of the biology of flatfishes in the southeastern part of the Bering Sea and condition of their resources. In Moiseev, P.A. (ed.), Soviet fisheries investigations in the Northeast Pacific, Pt. IV, p.112-119. Izv. Tikhookean. Nauchno-Issled. Inst. Rybn. Khoz. Okeanogr. (TINRO) 53; Tr. Vses. Nauchno-Issled. Inst. Morsk. Rybn. Khoz. Okeanogr. (VNIRO) 58. [Transl. from Russ. by Isr. Prog. Sci. Transl., Jerusalem, 1968; avail. U.S. Dep. Commer., Natl. Tech. Inf. Serv., Springfield, VA 22161, as TT67-51206.]

Fahay, M.P.

1983 Guide to the early stages of marine fishes occurring in the western North Atlantic Ocean, Cape Hatteras to the southern Scotian Shelf J. Northwest Atl. Fish. Sci. 4, 423 p

Fahay, M.P., and D.F. Markle

1984 Gadiformes: Development and relationships. In Moser, H.G., et al. (eds.), Ontogeny and systematics of fishes, p. 265-282. Spec. Publ. 1, Am. Soc. Ichthyol. Herpetol. Allen Press, Lawrence, KS.

Fast, T.N.

1960 Some aspects of the natural history of Stenobrachius leucopsarus Eigenmann and Eigenmann. Ph.D. diss., Stanford Univ., Stanford, CA $94305,107 \mathrm{p}$.

Feder, H.M., C.H. Turner, and C. Limbaugh

1974 Observations on fishes associated with kelp beds in southern California. Calif. Dep. Fish Game, Fish Bull. 160, 144 p.

Fedorov, V.V.

1973 Ikhtiofauna materikovogo sklona beringova morya i nekotorye aspekty ee proiskhozhdeniya i formirovaniya. Izv. Tikhookean. Nauchno-Issled. Inst. Rybn. Khoz. Okeanogr. (TINRO) 87: 3-4l. [Transl. by Transl. Bur. (DCM), Multilingual Serv. Div., Dep. of Secretary of State of Canada, Transl. Ser. 3345, 70 p.; avail. Can. Dep. Fish. Oceans, Fish. Res. Br., Pac. Biol. Stn., Nanaimo, B.C., Canada V9R 5K6.] 
Fink, W.L.

1984 Stomiiforms: Relationships. In Moser, H.G., et al. (eds.), Ontogeny and systematics of fishes, p. 181-184. Spec. Publ. 1, Am. Soc. Ichthyol. Herpetol. Allen Press, Lawrence, KS.

Fink, W.L., and S.H. Weitzman

1982 Relationships of the stomiiform fishes (Teleostei), with a description of Diplophos. Bull. Mus. Comp. Zool. Harv. Univ. 150(2): 31-93.

Fisher, J.P., and W.G. Pearcy

1983 Reproduction, growth and feeding of the mesopelagic fish Tactostoma macropus (Melanostomiidae). Mar. Biol. 74:257-267.

Fitch, J.E., and R.J. Lavenberg

1968 Deep-water fishes of California. Univ. Calif. Press, Berkeley, $155 \mathrm{p}$.

1971 Marine food and game fishes of California. Univ. Calif. Press, Berkeley, 179 p.

1975 Tidepool and nearshore fishes of California. Univ. Calif. Press, Berkeley, $156 \mathrm{p}$.

Forrester, C.R.

1964 Rate of development of eggs of rock sole (Lepidopsenta bilineata Ayres). J. Fish. Res. Board Can. 21:1533-1534.

1969 Life history information on some groundfish species. Fish. Res. Board Can. Tech. Rep. 105, 17 p.

Francis, R.C., and K.M. Bailey

1983 Factors affecting recruitment of selected gadoids in the Northeast Pacific and eastern Bering Sea. In Wooster, W.S. (ed.), From year to year: Interannual variability of the environment and fisheries of the Gulf of Alaska and the eastern Bering Sea. Rep WSG-WO 83-3, Wash. Sea Grant Prog., Univ. Wash., Seattle, WA 98195.

Frey, H.W. (editor)

1971 California's living marine resources and their utilization. Calif. Dep. Fish Game, Sacramento, CA 95814, 148 p.

Fritzsche, R.A.

1978 Development of fishes of the Mid-Atlantic Bight: An atlas of egg, larval and juvenile stages. Vol. V, Chaetodontidae through Ophidiidae. U.S. Fish. Wildl. Serv. Biol. Rep. FWS/OBS-78/12.

1984 Gasterosteiformes: Development and relationships. In Moser, H.G., et al. (eds.), Ontogeny and systematics of fishes, p. 398-404. Spec. Publ. 1, Am. Soc. Ichthyol. Herpetol. Allen Press, Lawrence, $\mathrm{KS}$.

Garrison, K.J., and B.S. Miller

1982 Review of the early life history of Puget Sound fishes. Unpubl. rep. FRI-UW-8216, Fish. Res. Inst., Univ. Wash., Seattle, WA $98195,729 \mathrm{p}$.

Giorgi, A.E.

1981 The environmental biology of the embryos, egg masses, and nesting sites of the lingcod, Ophiodon elongatus. Proc. Rep. 81-06, Northwest Alaska Fish. Cent., Natl. Mar. Fish. Serv., NOAA, Seattle, WA 98115-0070, $107 \mathrm{p}$.

Goldberg, S.R.

1980a Seasonal spawning cycle of the longspine combfish, Zaniolepis latipinnis, with notes on spawning of the shortspine combfish, Zaniolepis frenata (Zaniolepidae). Copeia 1980:882-884.

1980b Seasonal spawning cycles of two marine cottid fishes, Chitonotus pugetensis and Icelinus quadricomis, from southern California. Bull. Mar. Sci. 30:131-135.

Gorbunova, N.N.

1954 Reproduction and development of the walleye pollock, Theragra chalcogramma (Pallas). Tr. Inst. Okeanol. A kad. Nauk SSSR 11: 132-195. [In Russ., transl. by S. Pearson, 1972, Natl. Mar. Mammal Lab., NMFS, 7600 Sand Point Way N.E., Seattle, WA 981150070.]

1962 Spawning and development of greenlings (family Hexagrammidae). In Rass, T.S. (ed.), Greenlings: Taxonomy, biology, interoceanic transplantation. Tr. Inst. Okeanol. Akad. Nauk SSSR 59: 121-185. [Engl. transl. avail. U.S. Dep. Commer., Natl. Tech. Inf. Serv., Springfield, VA 22161, as TT69-55097.]
1964 Breeding and development of hemilepidotine sculpins (Cortidae, Pisces). In Rass, T.S. (ed.), Fishes of the Pacific and Indian Oceans. Biology and distribution, p. 249-266. Tr. Inst. Okeanol. Akad. Nauk SSSR 73. [Isr. Prog. Sci. Transl., Jerusalem, Transl. 1411.]

1982a Larvae of Pacific species of Cyclothone genus. Tr. Inst. Okeanol. Akad. Nauk SSSR 118:120-132 [in Russ.]

1982b Larvae of trichiuroid fishes from the collection of the International Mexican Biological Center (Gempylidae, Trichiuridae, Pisces). Proc. P.P. Shirshov. Inst. Oceanol. 118:85-104 [in Russ.].

Gordon, D.J., D.F. Markle, and J.E. Olney

1984 Ophidiiformes: Development and relationships. In Moser, H.G., et al. (eds.), Ontogeny and systematics of fishes, p. 308-319. Spec. Publ. 1, Am. Soc. Ichthyol. Herpetol. Allen Press, Lawrence, KS.

Grant, W.S.

1986 Biochemical genetic divergence between Atlantic, Clupea

Grey, M. harengus, and Pacific, C. pallasi, herring. Copeia 1986:714-719.

1964 Family Gonostomatidae. In Bigelow, H.B. (ed.), Fishes of the western North Atlantic, p. 78-240. Mem. 1 (pt. 4), Sears Found. Mar. Res., Yale Univ., New Haven, CT.

Grossman, G.D., and V. DeVlaming

1984 Reproductive ecology of female Oligocottus snyderi, a North American intertidal sculpin. J. Fish. Biol. 25:231-240.

Gunderson, D.R., P. Callahan, and B. Goiney

1980 Maturation and fecundity of four species of Sebastes. Mar. Fish. Rev. 42(3-4):74-79.

Hagerman, F.B.

1952 The biology of the dover sole, Microstomus pacificus (Lockington). Calif. Dep. Fish Game, Fish Bull. 85, 48 p.

Hamada, $T$.

1966 Studies on fluctuations in the abundance of larval sand-lance in the Harima-nada and Osaka Bay. I. Relation between the progeny abundance and the age composition of parent fish. Bull. Jpn. Soc. Sci. Fish. 32(5):393-398 [in Jpn., Engl. summ.].

Hardy, G.S.

1983 A revision of the fishes of the family Pentacerotidae (Perciformes). N.Z. J. Zool. 10:177-220.

Harling, W.R., M.S. Smith, and N.A. Webb

1971 Preliminary report on maturity, spawning season, and larval identification of rockfishes (Scorpaenidae) collected during 1970. Fish. Res. Board Can., Manuscr. Rep. 1137, 26 p.

Harry, G.Y.

1959 Time of spawning, length of maturity, and fecundity of the English, petrale, and Dover soles (Parophrys vetulus, Eopsetta jordani, and Microstomus pacificus, respectively). Fish. Comm. Oreg. Res. Briefs 7(1):5-13 (Portland, OR 97201).

Hart, J.L.

1967 Fecundity and length-weight relationship in lingcod. J. Fish. Res. Board Can. 24:2485-2489.

1973 Pacific fishes of Canada. Bull. Fish. Res. Board Can. 180, $740 \mathrm{p}$.

Hart, J.L., and J.L. McHugh

1944 The smelts (Osmeridae) of British Columbia. Bull. Fish. Res. Board Can. 64, 27 p.

Haryu, T., and T. Nishiyama

1981 Larval form of zaprorid fish Zaprora silenus from the Bering Sea and the northern North Pacific. Jpn. J. Ichthyol. 28:313-319.

Hattori, S.

1964 Studies on fish larvae in the Kuroshio and adjacent waters. Bull. Tokai Reg. Fish. Res. Lab. 40, 158 p. [in Jpn., Engl. synop.].

Hearne, M.E.

1983 Identification of larval and juvenile smelts (Osmeridae) from California and Oregon using selected morphometric characters. M.S. thesis, San Francisco State Univ., San Francisco, CA 94132, 142 p. 1984 Osmeridae: Development and relationships. In Moser, H.G., et al. (eds.), Ontogeny and systematics of fishes, p. 153-154. Spec. Publ. I, Am. Soc. Ichthyol. Herpetol. Allen Press, Lawrence, KS. 
Hedgpeth, J.W.

1957 Classification of marine environments. Geol. Soc. Am. Mem. 67(1):17-28

Hickman, C.P.

1959 The larval development of the sand sole (Psettichthys melanostictus). Wash. Dep. Fish., Fish. Res. Pap. 2(2):38-47.

Hinckley, S.

1986 Spawning dynamics and fecundity of walleye pollock (Theragra chalcogramma) in the eastem Bering Sea. M.S. thesis, Univ. Wash., Seattle, WA $98195,103 \mathrm{p}$.

Hirschberger, W.A., and G.B. Smith

1983 Spawning of twelve groundfish species in the Gulf of Alaska and Pacific Coast regions, 1975-81. Tech. Memo. NMFS F/NWC44, Northwest Alaska Fish. Cent., Natl. Mar. Fish. Serv., NOAA,

Hitz, C.R. Seattle, WA $98115-0070,50 \mathrm{p}$

1962 Seasons of birth of rockfish (Sebastodes spp.) in Oregon coastal waters. Trans. Am. Fish. Soc. 91:231-233.

Hollister, G.

1934 Clearing and dyeing fish for bone study. Zoologica 12(10): 89-101.

Horn, M.H.

1984 Stromateoidei: Development and relationships. In Moser, H.G., et al. (eds.), Ontogeny and systematics of fishes, p. 620-628. Spec. Publ. 1, Am. Soc. Ichthyol. Herpetol. Allen Press, Lawrence, KS.

Hosie, M.J., and H.F. Horton

1977 Biology of the rex sole, Glyptocephalus zachirus, in waters off Oregon. Fish. Bull., U.S. 75:51-60.

Hubbs, C.L., and T. Iwamoto

1977 A new genus (Mesobius), and three new bathypelagic species of Macrouridae (Pisces, Gadiformes) from the Pacific Ocean. Proc. Calif. Acad. Sci. 41:233-251.

Hubbs, C.L., W.I. Follett, and L.J. Dempster

1979 List of the fishes of California. Occas. Pap. Calif. Acad. Sci. 133:1-51.

Humphreys, R.L., Jr., G.A. Winans, and D.T. Tagami

1989 Synonomy and proposed life history of North Pacific pelagic armorhead, Pseudopentaceros wheeleri Hardy (Pisces: Pentacerotidae). Copeia 1989:142-153.

Ida, $\mathbf{H}$.

1976 Removal of the family Hypoptychidae from the suborder Ammodytoidei, order Perciformes, to the suborder Gasterosteoidei, order Syngnathiformes. Jpn. J. Ichthyol. 23:33-42.

Idyll, C.P.

1964 Abyss: The deep sea and the creatures that live in it. Thomas Y. Cromwell Co., NY, 395 p.

Inoue, A., S. Takamori, K. Kuniyaki, S. Kobayashi, and S. Nishina 1967 Studies on fishery biology of the sand-lance, Ammodytes personatus (Girard). Bull. Naikai Reg. Fish. Res, Lab. 25(121):1-335 [in Jpn., Engl. summ.].

Iwamoto, $\mathrm{T}$.

1975 The abyssal fish Antimora rostrata (Günther). Comp. Biochem. Physiol. 52b:7-11.

Jensen, $A$.

1935 The greenland halibut (Reinhardtius hippoglossoides), its development and migration. K. Dan. Vidensk. Selsk. Skr. 9:1-32.

Jespersen, P., and A.V. Tåning

1926 Mediterranean Sternoptychidae. Rep. Dan. Oceanogr. Exped. Mediterr. 2(A.12), 59 p.

Jewell, E.D.

1968 Scuba diving operations on lingcod spawning at a Seattle breakwater. Wash. Dep. Fish., Fish. Res. Pap. 3(1):27-36, Olympia, WA 98504 .

Johnson, C.L.

1970 Notes on the intertidal life history of the northern clingfish, Gobiesox maeandricus (Girard). Am. Midl. Nat. 83(2):625-627.
Johnson, G.D.

1984 Percoidei: Development and relationships. In Moser, H.G., et al. (eds.), Ontogeny and systematics of fishes, p. 464-498. Spec. Publ. 1, Am. Soc. Ichthyol. Herpetol. Allen Press, Lawrence, KS.

Johnson, R.K.

1974a A revision of the alepisaurid family Scopelarchidae (Pisces: Myctophiformes). Fieldiana Zool. 66:1-249.

1974b A Macristium larva from the Gulf of Mexico with additional evidence for synonomy of Macristium with Bathysaurus (Myctophiformes: Bathysauridae). Copeia 1974:973-977.

1984 Scopelarchidae: Development and relationships. In Moser, H.G., et al. (eds.), Ontogeny and systematics of fishes, p. 245-249. Spec. Publ. 1, Am. Soc. Ichthyol. Herpetol. Allen Press, Lawrence, KS.

Jones, A.C.

1962 The biology of euryhaline fish Leptocottus armatus armatus (Girard). Univ. Calif. Publ. Zool. 67(4):321-368.

Jow, T.

1969 Results of English sole tagging off California. Pac. Mar. Fish. Comm. Bull. 7:15-33.

Kabata, Z., and C.R. Forrester

1974 Atheresthes stomias (Pisces: Pleuronectiformes) and its eye parasite Phrizocephalus cincinnatus (Copepods: Lernaeoceridae) in Canadian Pacific waters. J. Fish. Res. Board Can. 31:1589-1595.

Karp, W.A.

1982 Biology and management of Pacific cod (Gadus macrocephalus) in Port Townsend, Washington. Ph.D. diss., Univ. Wash., Seattle, WA $98195,119 \mathrm{p}$.

Katz, M.

1942 The herring races of Washington, with a note on the fecundity of the Seal Rock population. M.S. thesis, Univ. Wash., Seattle, WA $98195,65 \mathrm{p}$.

Kawaguchi, K., and R. Marumo

1967 Biology of Gonostoma gracile (Gonostomatidae). 1. Morphology, life history, and sex reversal. In Information bulletin on planktology in Japan, Commemoration number of Dr. Y. Matsui's sixtieth birthday, p. 253-269. Plankton Soc. Jpn., Tokyo.

Kawaguchi, K., and H.G. Moser

1984 Stomiatoidea: Development. In Moser, H.G., et al. (eds.), Ontogeny and systematics of fishes, p. 169-180. Spec. Publ, 1, Am. Soc. Ichthyol. Herpetol. Allen Press, Lawrence, KS.

Keene, M.J., and K.A. Tighe

1984 Beryciformes: Development and relationships. In Moser, H.G., et al. (eds.), Ontogeny and systematics of fishes, p. 383-392. Spec. Publ. 1, Am. Soc. Ichthyol. Herpetol. Allen Press, Lawrence, KS.

Kendall, A.W., Jr., and R.J. Behnke

1984 Salmonidae: Development and relationships. In Moser, H.G., et al. (eds.), Ontogeny and systematics of fishes, p. 142-149. Spec. Publ. 1, Am. Soc. Ichthyol. Herpetol. Allen Press, Lawrence, KS.

Kendall, A.W., Jr., and J. Clark

1982 Ichthyoplankton off Washington, Oregon, and northern California April-May 1980. Proc. Rep. 82-11, Northwest Alaska Fish. Cent., Natl. Mar. Fish. Serv., NOAA, Seattle, WA 98115-0070, 44 p.

Kendall, A.W., Jr., and A.C. Matarese

1987 Biology of eggs, larvae, and epipelagic juveniles of sablefish, Anoplopoma fimbria, in relation to their potential use in management. Mar. Fish. Rev. 49(1):1-13.

Kendall, A.W., Jr., and B. Vinter

1984 Development of hexagrammids (Pisces, Scorpaeniformes) in the northeastern Pacific Ocean. NOAA Tech. Rep. NMFS 2, Natl. Oceanic Atmos. Adm., Natl. Mar. Fish. Serv., Seattle, WA 98115$0070,44 \mathrm{p}$.

Kendall, A.W., Jr., C.D. Jennings, T.M. Beasley, R. Carpenter, and

B.L. Somayajulu

1983 Discovery of a cluster of unhatched fish eggs of a zoarcid buried 10 to $12 \mathrm{~cm}$ deep in continental slope sediments off Washington state, USA. Mar. Biol. 75:193-199. 
Kendall, A.W., Jr., E.H. Ahlstrom, and H.G. Moser

1984 Early life history stages of fishes and their characters. In Moser, H.G., et al. (eds.), Ontogeny and systematics of fishes, p. 11-22. Spec. Publ. 1, Am. Soc. Ichthyol. Herpetol. Allen Press, Lawrence, $\mathrm{KS}$.

Ketchen, K.S.

1947 Studies on lemon sole development and egg production. Fish. Res. Board Can., Prog. Rep. Pac. Coast Stn. 73:68-70.

1956 Factors influencing the survival of lemon sole (Parophrys vetulus) in Hecate Strait, British Columbia. J. Fish. Res. Board Can. 13:647-694.

1961 Observations on the ecology of the Pacific cod (Gadus macrocephalus) in Canadian waters. J. Fish. Res. Board Can. 18:513-558.

Khan, N.Y.

1972 Comparative morphology and ecology of the pelagic larvae of nine Cottidae (Pisces) of the Northwest Atlantic and St. Lawrence Drainage. Ph.D. diss., Univ. Ottawa, Ottawa, Ontario, Canada, $234 \mathrm{p}$.

Kido, $\mathrm{K}$.

1983 New and rare liparidid species from the Okhotsk and Bering Seas and their adjacent waters. Jpn. J. Ichthyol. 29:374-384.

1984 Occurrence of the liparidid fish, Paraliparis pectoralis, in the Bering Sea. Jpn. J. Ichthyol. 31:203-204

1985 New and rare species of the genus Careproctus (Liparididae) from the Bering Sea. Jpn. J. Ichthyol. 32:6-7.

Kido, K., and D. Kitagawa

1986 Development of larvae and juveniles of Rhinoliparis barbulifer (Liparididae). In Uyeno, T., et al. (eds.), Indo-Pacific fish biology: Proceedings of the second international conference on Indo-Pacific fishes, p. 697-702. Ichthyol. Soc. Jpn., Tokyo.

Kimura, D.K., and A.R. Millikan

1977 Assessment of the population of Pacific hake (Merluccius productus) in Puget Sound, Washington. Wash. Dep. Fish. Tech. Rep. $35,46 \mathrm{p}$.

Kobayashi, K.

1961a Young of the wolf-fish, Anarhichas orientalis Pallas. Bull. Fac. Fish. Hokkaido Univ. 12:1-4 [in Jpn., Engl. abstr.].

1961b Larvae and young of the quill-fish, Ptilichthys goodei. Bull. Fac. Fish. Hokkaido Univ. 12:5-8 [in Jpn., Engl. abstr.].

1961c Larvae and young of the sand-lance, Ammodytes hexapterus. Bull. Fac. Fish. Hokkaido Univ. 12:111-120 [in Jpn., Engl. abstr.].

1962 Larvae of the smooth lumpsucker, Aptocyclus ventricosus (Pallas), with discussion on revision of the taxonomy of the species. Bull. Fac. Fish. Hokkaido Univ. 13:153-164.

Kobayashi, K., M. Mikawa, and J. Ito

1968 Descriptions of the young and one immature adult specimen of coster dory, Allocyttus verrucosus (Gilchrist) from the northern part of the Pacific. Bull. Fac. Fish. Hokkaido Univ. 19:1-6.

Kramer, D.

1960 Development of eggs and larvae of Pacific mackerel and distribution and abundance of larvae 1952-56. U.S. Fish Wildl. Serv., Fish. Bull. 60:393-438.

1970 Distributional atlas of fish eggs and larvae in the California Current region: Pacific sardine, Sardinops caerulea (Girard), 1951 through 1966. Calif. Coop. Oceanic Fish. Invest. Atlas 12, 277 p.

Kramer, D., and E.H. Ahlstrom

1968 Distributional atlas of fish larvae in the California Current region: Northern anchovy, Engraulis mordax Girard, 1951 through 1965. Calif. Coop. Oceanic Fish. Invest. Atlas 9, 282 p.

Kramer, D.E., M.J. Kalin, E.G. Stevens, J.R. Thrailkill, and J.R.

Zweifel

1972 Collecting and processing data on fish eggs and larvae in the California Current region. NOAA Tech. Rep. NMFS Circ. 370, Natl. Oceanic Atmos. Adm., Natl. Mar. Fish. Serv., Seattle, WA 98115-0070, $38 \mathrm{p}$

Kyushin, $\mathbf{K}$.

1968 The embryonic and larval stages of Hemitripterus villosus (Pallas). Bull. Fac. Fish. Hokkaido Univ. 18:277-289.
1970 Embryonic development and larvae of Gymnocanthus hertzensteini Jordan and Starks. Jpn. J. Ichthyol. 17:74-79.

LaRiviere, M.G., D.D. Jessup, and S.B. Mathews

1981 Lingcod, Ophiodon elongatus, spawning and nesting in San Juan Channel, Washington. Calif. Fish Game 67:231-239.

Laroche, W.A.

In prep. Guide to larval and juvenile rockfishes (Sebastes) of North America, 311 p. Box 216, Enosburg Falls, VT 05450.

Laroche, W.A., and S.L. Richardson

1980 Development and occurrence of larvae and juveniles of the rockfishes Sebastes flavidus and Sebastes melanops (Scorpaenidae) off Oregon. Fish. Bull., U.S. 77:901-924.

1981 Development of larvae and juveniles of the rockfishes Sebastes entomelas and $S$. zacentrus (family Scorpaenidae) and occurrence off Oregon, with notes on head spines of $S$. mystinus, S. flavidus, and S. melanops. Fish. Bull., U.S. 79:231-258.

Laroche, W.A., W.F. Smith-Vaniz, and S.L. Richardson

1984 Carangidae: Development. In Moser, H.G., et al. (eds.), Ontogeny and systematics of fishes, p. 510-521. Spec. Publ. 1, Am. Soc. Ichthyol. Herpetol. Allen Press, Lawrence, KS.

Lasker, R., and P.E. Smith

1977 Estimation of the effects of environmental variations on the eggs and larvae of northern anchovy. Calif. Coop. Oceanic Fish. Invest. Rep. 19:128-137.

Lavenberg, R.J., G.E. McGowen, and R.E. Woodsum

1984 Preservation and curation. In Moser, H.G., et al. (eds.), Ontogeny and systematics of fishes, p. 57-59. Spec. Publ. 1, Am. Soc. Ichthyol. Herpetol. Allen Press, Lawrence, KS.

Lea, R.N. and L.J. Dempster

1982 Status and nomenclatural history of Agonus vulsus Jordan and Gilbert, 1880 (Pisces-family Agonidae). Calif. Fish Game 68: 249-252.

Lea, R.N., and L.F. Quirollo

1986 First record of Hemitripterus bolini, the bigmouth sculpin, from California waters. Calif. Fish Game 72:117-126.

Lea, R.N., and R.H. Rosenblatt

1987 Occurrence of the family Notacanthidae (Pisces) from the marine waters of California. Calif. Fish Game 73:51-53.

Lee, J.U.

1985 Studies on the fishery biology of the Atka mackerel Pleurogrammus monopterygius (Pallas) in the North Pacific Ocean. Bull, 34, Fish. Res. Dev. Agency, Pusan, Korea [in Korean].

Leis, J.M.

1984 Tetraodontoidei: Development. In Moser, H.G., et al. (eds.), Ontogeny and systematics of fishes, p. 447-449. Spec. Publ. 1, Am. Soc. Ichthyol. Herpetol. Allen Press, Lawrence, KS.

Leis, J.M., and D.S. Rennis

1983 The larvae of Indo-Pacific coral reef fishes. New South Wales Univ. Press, Sydney, 269 p.

Leis, J.M., and W.J. Richards

1984 Acanthuroidei: Development and relationships. In Moser, H.G., et al. (eds.), Ontogeny and systematics of fishes, p. 547-551 Spec. Publ. 1, Am. Soc. Ichthyol. Herpetol. Allen Press, Lawrence, KS.

Limbaugh, C.

1962 Life history and ecological notes on the tubenose, Aulorhynchus flavidus, a hemibranch fish of western North America. Copeia 1962:549-555

Loeb, V.J.

1979 Larval fishes in the zooplankton community of the North Pacific central gyre. Mar. Biol. 53:73-191.

MacCall, A.D., and G.D. Stauffer

1983 Biology and fishery potential of jack mackerel (Trachurus symmetricus). Calif. Coop. Oceanic Fish. Invest. Rep. 24:46-56.

MacGregor, J.S.

1966 Fecundity of the Pacific hake, Merluccius productus (Ayres) Calif. Fish Game 52:111-116. 
1986d Early life history of the family Myctophidae in the ocean off southern Japan. In Ozawa, T. (ed.), Studies on the oceanic ichthyoplankton in the western North Pacific, p. 114-187. Kyushc. Univ. Press, Fukuoka, Japan.

1986e The larvae of the family Trichiuridae in the ocean off southern Japan. In Ozawa, T. (ed.), Studies on the oceanic ichthyoplankton in the western North Pacific, p. 290-300. Kyushu Univ. Press, Fukuoka, Japan.

Ozawa, T., and S. Aono

1986 Early ontogeny of melanostomiid fishes in the western North Pacific. In Ozawa, T. (ed.), Studies on the oceanic ichthyoplankton in the western North Pacific, p. 30-50. Kyushu Univ. Press, Fukuoka, Japan.

Ozawa, T., and K. Oda

1986 The larvae of the gonostomatid genus Cyclothone in the westem North Pacific. In Ozawa, T. (ed.), Studies on the ocearic ichthyoplankton in the western North Pacific, p. 52-67. Kyushu Univ. Press, Fukuoka, Japan.

Pacific Fisheries Management Council

1978 Northern anchovy fishery: Final environmental impact statement and fishery management plan. Pac. Fish. Manage. Counc., $526 \mathrm{~S}$. Mill St., Portland, OR 97201.

Patten, B.G.

1973 Biological information on copper rockfish in Puget Sound, Washington. Trans. Am. Fish. Soc. 102:412-416.

1980 Short term thermal resistance of hexagrammid eggs and planktonic larvae from Puget Sound. Trans. Am. Fish. Soc, 109 427-432.

Paxton, J.R.

1972 Osteology and relationships of the lanternfishes (Family, Myctophidae). Bull. Los Ang. Cty. Mus. Nat. Hist. Sci. 13,81 p.

Pearcy, W.G.

1962 Egg masses and early developmental stages of the scorpaenid fish, Sebastolobus. J. Fish. Res. Board Can. 19:1169-1173.

Peden, A.E.

1978 A systematic revision of the hemilepidotine fishes (Cottidae). Syesis 11:11-49.

Peden, A.E., and G.W. Hughes

1986 First records, confirmatory records, and range extensions of marine fishes of Canada's west coast. Can. Field-Nat. 100:1-9.

Peden, A.E., W. Ostermann, and L.J. Pozar

1985 Fishes observed at Canadian weathership ocean station Papa [50 $\mathrm{N}, 145 \mathrm{~W}]$ with notes on the transpacific cruise of the CSS Endeavor. Brit. Col. Prov. Mus. Herit. Rec. 18, Min. Prov. Sec. and Gov. Serv., Victoria, B.C., Canada.

Peppar, J.L.

1965 Some features of the life history of the cockscomb prickleback, Anoplarchus purpurescens Gill. M.S. thesis, Univ. Brit. Columbia, Vancouver, B.C., Canada, 159 p.

Pertseva-Ostroumova, T.A.

1961 The reproduction and development of far-eastern flounders. $\mathrm{Tr}$. Inst. Okeanol. Akad. Nauk SSSR, 484 p. [Transl. avail. Fish. Res. Board Can., Pac. Biol. Stn., Nanaimo, B.C., Canada V9R 5K6, Transl. Ser. 856, 1967.]

1964 Some morphological characteristics of myctophid larvae (Myctophidae, Pisces). In Rass, T.S. (ed.), Fishes of the Pacific and Indian Oceans, Biology and distribution, p. 79-97. Tr. Inst. Okeanol. Akad. Nauk SSSR 73. [Transl. avail. U.S. Dep. Commer., Natl. Tech. Inf. Serv., Springfield, VA 22161, as TT65-5120.]

Phillips, J.B.

1959 A review of the lingcod, Ophiodon elongatus. Calif. Fish Game 45: $19-27$.

1964 Life history studies on ten species of rockfish (genus Sebastodes) Calif. Dep. Fish Game, Fish Bull. 126, 70 p.

Phillips, J.B., and S. Imamura 1954 The sablefish fishery of California. Pac. Mar. Fish. Comm.
Bull. 3:6-37.
Pietsch, T.W.

1976 Dimorphism, parasitism and sex: Reproductive strategies among deepsea ceratioid anglerfishes. Copeia 1976:781-793.

1978 Evolutionary relationships of the sea moths (Teleostei: Pegasidae) with a classification of gasterosteiform families. Copeia 1978:517529.

1984 Lophiiformes: Development and relationships. In Moser, H.G., et al. (eds.), Ontogeny and systematics of fishes, p. 320-324. Spec. Publ. 1, Am. Soc. Ichthyol. Herpetol. Allen Press, Lawrence, KS. 1986 Systematics and distribution of bathypelagic anglerfishes of the family Ceratiidae (order: Lophiiformes). Copeia 1986:479-493.

Pietsch, T.W., and D. Grobecker

1987 Frogfishes of the world. Systematics, zoogeography, and behavioral ecology. Stanford Univ. Press, Stanford, CA 94305, 420 p.

Pillsbury, R.W.

1957 Avoidance of poisonous eggs of the marine fish Scorpaenichthys marmoratus by predators. Copeia 1957:251-252.

Powles, H., and D.F. Markle

1984 Identification of larvae. In Moser, H.G., et al. (eds.), Ontogeny and systematics of fishes, p. 31-33. Spec. Publ. 1, Am. Soc. Ichthyol. Herpetol. Allen Press, Lawrence, KS.

Prasad, R.R.

1958 Reproduction in Clevelandia ios with an account of the embryonic and larval development. Proc. Natl. Inst. Sci. India 25B:12-30.

Rass, T.S.

1949 Composition of the ichthyoplankton of the Barents Sea. In Rass T.S., et al. (eds.), Material on the reproduction and development of fishes of the northern seas, p. 1-68. Tr. Vses. Nauchno-Issled. Inst. Morsk. Rybn. Khoz. Okeanogr. (VNIRO) 17 [in Russ.].

Regan, C.T.

1916 Larval and post-larval fishes. Nat. Hist. Rep. Br. Antarct. Terra Nova Exped. Zool. 1:125-156

Richardson, S.L.

1977 Larval fishes in ocean waters off Yaquina Bay, Oregon: Abundance, distribution, and seasonality, January 1971 to August 1972. Publ. ORES-T-77-003, Oregon State Univ. Sea Grant Coll. Prog., Corvallis, OR 97331, $73 \mathrm{p}$.

1981a Current knowledge of larvae of sculpins (Pisces: Cottidae and allies) in Northeast Pacific genera with notes on intergeneric relationships. Fish. Bull., U.S. 79:103-121.

1981b Pelagic eggs and larvae of the deep sea sole, Embassichthys bathybius (Pisces: Pleuronectidae), with comments on generic affinities. Fish. Bull., U.S. 79:163-170.

Richardson, S.L., and C. Bond

1978 Two unusual cottoid fishes from the Northeast Pacific. Paper presented at Annu. Meet., Am. Soc. Ichthyol. Herpetol., Tempe, Arizona, 33 p. [Avail. A.C. Matarese, Northwest Alaska Fish. Cent., Natl. Mar. Fish. Serv., NOAA, 7600 Sand Point Way N.E., Seattle, WA 98115-0070.]

Richardson, S.L., and D.A. Dehart

1975 Records of larval, transforming, and adult specimens of quillfish, Ptilichthys goodei, from waters off Oregon. Fish. Bull., U.S. 73: 681-684.

Richardson, S.L., and W.A. Laroche

1979 Development and occurrence of larvae and juveniles of the rockfishes Sebastes crameri, Sebastes pinniger, and Sebastes helvomaculatus (family Scorpaenidae) off Oregon. Fish. Bull., U.S. $77: 1-46$

Richardson, S.L., and W.G. Pearcy 1977 Coastal and oceanic fish larvae in an area of upwelling off Yaquina Bay, Oregon. Fish. Bull., U.S. 75:125-145.

Richardson, S.L., and B.B. Washington

1980 Guide to the identification of some sculpin larvae from marine and brackish waters off Oregon and adjacent areas of the Northeast Pacific. NOAA Tech. Rep. NMFS Circ. 430, Natl. Oceanic Atmos. Adm., Natl. Mar. Fish. Serv., Seattle, WA 98115-0070, 56 p.

Richardson, S.L., J.R. Dunn, and N.A. Naplin 1980 Eggs and larvae of butter sole, Isopseth tidae), off Oregon and Watinger sole, Isopsetta isolepis (Pleuronec 
Robertson, D.A.

1977 Planktonic eggs of the lanternfish Lampanyctodes hectoris (family Myctophidae). Deep-Sea Res. 24:1-4

Robins, C.R., R.M. Bailey, C.E. Bond, J.R. Brooker, E.A. Lachner, R.N. Lea, and W.B. Scott

1980 A list of common and scientific names of fishes from the United States and Canada. Spec. Publ. 12, Am. Fish. Soc., Bethesda, MD 20014,174 p.

Rofen, R.R.

1966a Family Paralepididae. In Mead, G.W. (ed.), Fishes of the western North Atlantic, p. 205-461. Mem. 1 (pt. 5), Sears Found. Mar. Res., Yale Univ., New Haven, CT.

1966b Family Anotopteridae. In Mead, G.W. (ed.), Fishes of the western North Atlantic, p. 498-510. Mem. 1 (pt 5), Sears Found Mar. Res., Yale Univ., New Haven, CT.

1966c Family Omosudidae. In Mead, G.W. (ed.), Fishes of the western North Atlantic, p. 462-481. Mem. 1 (pt. 5), Sears Found. Mar. Res., Yale Univ., New Haven, CT.

Rosenblatt, R.H., and R.R. Wilson

1987 Cutlassfishes of the genus Lepidopus (Trichiuridae) with two new eastern Pacific species. Jpn. J. Ichthyol. 33:342-351.

Roule, L., and F. Angel

1930 Larves et alevins de poissons provenant det croisieres du Prince Albert I de Monaco. Result. Camp. Sci. Prince Albert I 79:1-148 [in French].

Rudomilov, O.I.

1972 Fecundity of herring of the eastern part of the Bering Sea. Izv. Tikhookean. Nauchno-Issled. Inst. Rybn. Khoz. Okeanogr. (TINRO) 82:321-332 [in Russ., Engl. abstr.].

Rugen, W.C., and A. C. Matarese

1988 Spatial and temporal distribution and relative abundance of Pacific cod (Gadus macrocephalus) larvae in the western Gulf of Alaska. Proc. Rep. 88-18, Northwest Alaska Fish. Cent., Natl. Mar. Fish. Serv., NOAA, Seattle, WA 98115-0070, 53 p.

Ruple, D.

1984 Gobioidei: Development. In Moser, H.G., et al. (eds.), Ontogeny and systematics of fishes, p. 582-587. Spec. Publ. 1, Am. Soc. Ichthyol. Herpetol. Allen Press, Lawrence, KS

Ryder, J.A.

1887 On the development of osseous fishes, including marine and freshwater forms. Rep. U.S. Comm. Fish. 13(1885):489-603.

Sakamoto, K.

1984 Interrelationships of the family Pleuronectidae (Pisces: Pleuronectiformes). Mem. Fac. Fish., Hokkaido Univ. 31(1,2):95-215.

Salveson, S.J.

1976 Flathead sole (family Pleuronectidae). In Pereya, W.T., et al. (eds.), Demersal fish and shellfish resources in the eastern Bering Sea in the baseline year 1975, p. 497-510. Proc. Rep., Northwest Alaska Fish. Cent., Natl. Mar. Fish. Serv., NOAA, Seattle, WA 98115-0070.

Salveson, S.J., and M.S. Alton

1976a Pollock (family Gadidae). In Pereya, W.T., et al. (eds.), Demersal fish and shellfish resources in the eastern Bering Sea in the baseline year 1975, p. 369-392. Proc. Rep., Northwest Alaska Fish. Cent., Natl. Mar. Fish. Serv., NOAA, Seattle, WA 98115-0070.

1976b Yellowfin sole (family Pleuronectidae). In Pereya, W.T., et al. (eds.), Demersal fish and shellfish resources of the eastern Bering Sea in the baseline year 1975, p. 439-459. Proc. Rep., Northwest Alaska Fish. Cent., Natl. Mar. Fish. Serv., NOAA, Seattle, WA 98115-0070.

Sanzo, L.

1931a Sottordinae: Stomiatoidei. In Uova, larvae e stadi giovanili di Teleostei, p. 42-49. Fauna e Flora del Golfo di Napoli. Monogr. 38,1064 p. + plates [in Ital.].

1931b Uova stadi embrionali e post-embrionali di Naucrates ductor L. Mem. Res. Com. Talass. Ital. 185 [in Ital.]
1935 Uova, sviluppo, embrionale, stadi larvali, postlarvali e giovanil di Sternoptychidae e Stomiatidae. Monogr. Res. Com. Talass. Ital. 2:123-180 [in Ital.].

1939 Nuovo contributo alla conoscenza dello sviluppo di Myctophum rissoi (Cocco). Atti. Accad. Gioenia Sci. Nat. Catania (6)3(25):1-8 [in Ital.].

Saruwatari, T., K. Betsui, and M. Okiyama

1987 Occurrence of the grunt sculpin (Rhamphocottus richardsoni) larvae from northern central Japan. Jpn. J. Ichthyol. 34:387-392.

Sazonov, Yu. I.

1981 Talismania brachycephala sp. N. (Alepocephalidae, Salmoniformes)-A new species from the northwestern Pacific Ocean. J. Ichthyol. 21(6): 151-153.

Scattergood, L.W., C.J. Sindermann, and B.E. Skud

1959 Spawning of North American herring. Trans. Am. Fish. Soc. $88: 164-168$.

Schaefer, M.B.

1937 Notes on the spawning of the Pacific herring, Clupea pallasi. Copeia 1937:57.

Schaefer, K.M.

1980 Synopsis of biological data on the chub mackerel, Scomber japonicus Houttuyn, 1782, in the Pacific Ocean. In Bayliff, W.H. (ed.), Synopses of biological data on eight species of scombrids, $p$. 394-530. Spec. Rep. 2, Inter-Am. Trop. Tuna Comm., Scripps Inst. Oceanogr., La Jolla, CA 92093.

Schmidt, J., and A. Strubberg

1918 Mediterranean Bramidae and Trichuridae. Rep. Dan Oceanogr. Exped. Mediterr. 2(A.6), 15 p.

Schmitt, C.C., and B.E. Skud

1978 Relation of fecundity to longterm changes in growth, abundance, and recruitment. Int. Pac. Halibut Comm. Sci. Rep. 66, 31 p.

Schultz, L.P.

1961 Revision of the marine silver hatchetfish (Family Sternoptychidae). Proc. U.S. Natl. Mus. 112(3449):587-649.

Schultz, L.P., and A.C. DeLacy

1932 The eggs and nesting habits of the crested blenny, Anoplar chus. Copeia 1932:143-147.

Serobaba, I.I.

1968 Spawning of the Alaskan pollock, Theragra chalcogramma (Pallas) in the northeastern Bering Sea. Probl. Ichthyol. 8(6): 789-798

Shiino, S.M.

1976 List of common names of fishes of the world, those prevailing among English-speaking nations. Sci. Rep. Shima Marineland 4, $206 \mathrm{p}$.

Shiogaki, M.

1982 Life history of the stichaeid fish Opisthocentrus ocellatus. Jpn. J. Ichthyol. 29:77-85.

Shmidt, P.Y.

1950 Fishes of the Sea of Okhotsk. Tr. Zool. Inst. Akad. Nauk SSSR 6:1-392. [Transl. by Isr. Prog. Sci. Transl., Jerusalem, Transl, 1263.]

Shvetsov, F.C

1979 Reproduction of the flounder, Lepidopsetta bilineata bilineata, off the Okhotsk Sea coast near Paramoshiro and Shumushu Islands. J. Ichthyol. 19(5):61-62.

Simenstad, C.A.

1971 The feeding ecology of the rock greenling, Hexagrammos lagocephalus in the inshore waters of Amchitka Island, Alaska. M.S thesis, Univ. Wash., Seattle, WA 98195, 131 p.

Smith, D. G.

1979 Guide to the leptocephali (Elopiformes, Anguilliformes, and Notacanthiformes). NOAA Tech. Rep. NMFS Circ. 424, Natl Oceanic Atmos. Adm., Natl. Mar. Fish. Serv., Seattle, WA 98115 0070, $39 \mathrm{p}$.

1984 Elopiformes, Notacanthiformes and Anguilliformes: Relationships. In Moser, H.G., et al. (eds.), Ontogeny and systematics of fishes, p. 94-101. Spec. Publ. 1, Am. Soc. Ichthyol. Herpetol. Allen Press, Lawrence, KS 
Smith, J.L.B.

1964 Fishes of the family Pentacerotidae. Ichthyol. Bull. Rhodes Univ. 29:567-579.

Smith, R.T.

1936 Report on the Puget Sound otter trawl investigations. Wash. Dep. Fish. Biol. Rep. 36B, 61 p.

Smoker, W., and W.G. Pearcy

1970 Growth and reproduction of the lanternfish Stenobrachius leucopsarus, J. Fish. Res. Board Can. 27:1265-1275.

Soldatov, V.K., and G.J. Lindberg

1930 A review of the fishes of the seas of the far east. Izv. Tikhookean. Nauchno-Issled. Inst. Rybn. Khoz. Okeanogr. (TINRO) 5, 576 p. [in Russ.].

St. Pierre, G.

1984 Spawning locations and season for Pacific halibut. Int. Pac. Halibut Comm. Sci. Rep. 70, 46 p.

Stahl-Johnson, K.L.

1985 Descriptive characteristics of reared Sebastes caurinus and $S$. auriculatus larvae. In Kendall, A.W., Jr., and J.B. Marliave (eds.), Description of early life history stages of selected fishes: From the 3rd international symposium on the early life history of fishes and 8th annual larval fish conference, p. 65-76. Can. Tech. Rep. Fish. Aquat. Sci. 1359.

Stein, D.L.

1980a Description and occurrence of macrourid larvae and juveniles in the Northeast Pacific Ocean off Oregon, U.S.A. Deep-Sea Res. 27a:889-900.

1980b Aspects of reproduction of liparid fishes from the continental slope and abyssal plain off Oregon, with notes on growth. Copeia 1980:687-699.

Stein, D.L., and C.E. Bond

1985 Observations on the morphology, ecology, and behavior of Bathylychnops exilis Cohen. J. Fish Biol. 27:215-228.

Stein, R.

1972 Identification of some larval Pacific cottids. M.S. thesis, Humboldt State Univ., Arcata, CA 95521, 41 p.

1973 Description of laboratory-reared larvae of Oligocottus maculosus Girard (Pisces: Cottidae). Copeia 1973:373-377.

Stepien, C.A.

1986 Life history and larval development of the giant kelpfish, Heterostichus rostratus Girard, 1854. Fish. Bull., U.S. 84:809-826.

Stevens, E.G., A.C. Matarese, and W.W. Watson

1984 Ammodytoidei: Development and relationships. In Moser, H.G., et al. (eds.), Ontogeny and systematics of fishes, p. 574-575. Spec. Publ. 1, Am. Soc. Ichthyol. Herpetol. Allen Press, Lawrence, KS.

Steyskal, G.C.

1980 The grammar of family-group names as exemplified by those of fishes. Proc. Biol. Soc. Wash, 93(1):168-177.

Sulak, K.J., C.A. Wenner, G.R. Sedberry, and L.V. Guelpen 1985 The life history and systematics of deep-sea lizard fishes, genus Bathysaurus (Synodontidae). Can, J, Zool. 63:623-642.

Sumida, B.Y., E.H. Ahlstrom, and H.G. Moser

1979 Early development of seven flatfish of the eastern North Pacific with heavily pigmented larvae (Pisces, Pleuronectiformes). Fish. Bult., U.S. 77:105-145.

Takahura, $\mathbf{T}$.

1954 The bchavior of the spawning pollock schools recorded by fish detector. Bull. Jpn. Soc. Sci. Fish. 20(1):10-12 [in Jpn., Engl. summ.].

Tåning, A.V.

1918 Mediterranean Scopelidae (Saurus aulopus, Chlorophthalmus, and Myctophum). Rep. Dan. Oceanogr. Exped. Mediterr. 2(A.7), $153 \mathrm{p}$.

Templeman, $\mathbf{W}$.

1948 The life history of the caplin (Mallotus villosus O. F. Müller) in Newfoundland waters. Res. Bull. Div. Fish. Res. Newfoundland $17,151 \mathrm{p}$.
Thompson, J.A.

1962 On the fecundity of Pacific cod (Gadus macrocephalus) from Hecate Strait, British Columbia. J. Fish. Res. Board Can. 19: 497-500.

Thompson, W.F., and R. Van Cleve

1936 Life history of the Pacific halibut. 2. Distribution and early life history. Rep. Int. Fish. Comm. 9, 184 p.

Tighe, K.A., and M.J. Keene

1984 Zeiformes: Development and relationships. In Moser, H.G., et al. (eds.), Ontogeny and systematics of fishes, p. 393-397. Spec. Publ. 1, Am. Soc. Ichthyol. Herpetol. Allen Press, Lawrence, KS.

Toole, C.L.

1982 Widow rockfish. Marine Advisory Programs Newsletter, Calif. Sea Grant, Univ. Calif., Davis, CA 95616, p. 1-2.

Trumble, R.J.

1973 Distribution, relative abundance, and general biology of selected underutilized fishery resources of the eastern North Pacific Ocean. M.S. thesis, Univ. Wash., Seattle, WA 98195, 178 p.

Tyler, J.C.

1980 Osteology, phylogeny, and higher classification of the fishes of the order Plectognathi (Tetraodontiformes). NOAA Tech. Rep. NMFS Circ. 434. Natl. Oceanic Atmos. Adm., Natl. Mar. Fish. Serv., Seattle, WA $98115-0070,422$ p.

Uchida, K., S. Imai, S. Mito, S. Fujita, M. Ueno, Y. Shojima, T. Senta, M. Tahuka, and Y. Dotsu

1958 Studies on the eggs, larvae, and juveniles of Japanese fishes. Series 1. Second Laboratory of Fisheries Biology, Fish. Dep., Fac. Agric., Kyushu Univ., Fukuoka, Japan [in Jpn.].

Waldron, K.D.

1968 Early larvae of the canary rockfish, Sebastodes pinniger. J. Fish. Res. Board Can. 25:801-803.

Wales, L.H.

1952 Life history of the blue rockfish, Sebastodes mystinus. Calif. Fish Game 38:485-498.

Walters, G.E.

1984 Ecological aspects of larval and juvenile Pacific cod (Gadus macrocephalus), walleye pollock (Theragra chalcogramma), and Pacific tomcod (Microgadus proximus) in Port Townsend, Washington. M.S. thesis, Univ. Wash., Seattle, WA 98195, 129 p.

Wang, J.C.

1981 Taxonomy of the early life stages of fishes: Fishes of the Sacramento-San Joaquin estuary and Moss Landing harbor-Elkhorn slough, California. Ecological Analysts, Inc, Concord, CA 94520, 168 p. 1986 Fishes of the Sacramento-San Joaquin estuary and adjacent waters, California: A guide to the early life histories. Interagency Ecological Study Program for the Sacramento-San Joaquin Estuary. Tech. Rep. 9, 602 p. [Avail. Ecological Analysts, Inc., 2150 John Glenn Drive, Concord, CA 94520.]

Washington, B.B.

1981 Identification and systematics of larvae of Artedius, Clinocottus, and Oligocottus (Scorpaeniformes: Cottidae). M.S. thesis, Oregon State Univ., Corvallis, OR 97331, 205 p.

1986 Systematic relationships and ontogeny of the sculpins Artedius, Clinocottus, and Oligocottus (Cottidae: Scorpaeniformes). Proc. Calif. Acad. Sci. 44(9):157-224.

Washington, B.B., W.N. Eschmeyer, and K.M. Howe

1984a Scorpaeniformes: Relationships. In Moser, H.G., et al. (eds.), Ontogeny and systematics of fishes, p. 438-447. Spec. Publ. 1, Am. Soc. Ichthyol. Herpetol. Allen Press, Lawrence, KS.

Washington, B.B., H.G. Moser, W.A. Laroche, and W.J. Richards 1984b Scorpaeniformes: Development. In Moser, H.G., et al. (eds.), Ontogeny and systematics of fishes, p. 405-427. Spec. Publ. 1, Am. Soc. Ichthyol. Herpetol. Allen Press, Lawrence, KS.

Washington, P.M., R. Gowan, and D.H. Ito

1978 A biological report on eight species of rockfish (Sebastes spp.) from Puget Sound, Washington. Proc. Rep., Northwest Alaska Fish. Cent., Natl. Mar. Fish. Serv., NOAA, Seattle, WA 98115-0070, $50 \mathrm{p}$. 
Watson, W.W.

1982 Development of eggs and larvae of the white croaker, Genyonemus lineatus Ayres (Pisces: Sciaenidae) off the southern California coast. Fish. Bull., U.S. 80:403-417.

Webber, R.A., and M.S. Alton

1976 Pacific halibut (family Pleuronectidae). In Pereya, W.T., et al. (eds.), Demersal fish and shellfish resources of the eastern Bering Sea in the baseline year 1975, p. 511-522. Proc. Rep., Northwest Alaska Fish. Cent., Natl. Mar. Fish. Serv., NOAA, Seattle, WA 98115-0070.

Weitzman, S.H.

1974 Osteology and evolutionary relationships of the Sternoptychidae with a new classification of stomiatoid families. Bull. Am. Mus. Nat. Hist. 153:327-478.

Wenner, C.A.

1978 Making a living on the continental slope and in the deep sea: Life history of some dominant fishes of the Norfolk Canyon area. Ph.D. diss., Coll. William and Mary, Williamsburg, VA 23186, 294 leaves, 105 leaves of plates.

Westrheim, S.J.

1975 Reproduction, maturation, and identification of larvae of some Sebastes (Scorpaenidae) species in the Northeast Pacific Ocean. J. Fish. Res. Board Can. 32:2399-2411.

Westrheim, S.J., and A.R. Morgan

1963 Results from tagging a spawning stock of Dover sole, Microstomus pacificus. Bull. Pac. Mar. Fish. Comm. 6:13-21.

Westrheim, S.J., W.R. Harling, and D. Davenport

1968a Preliminary report on maturity, spawning season, and larval identification of rockfishes (Sebastodes) collected off British Columbia in 1967. Fish. Res. Board Can., Manuscr. Rep. Ser. 951, 23 p.

Westrheim, S.J., W.R. Harling, D. Davenport, and M.S. Smith

1968b Preliminary report on maturity, spawning season, and larval identification of rockfishes (Sebastodes) collected off British Columbia in 1968. Fish. Res. Board Can., Manuscr. Rep. Ser. 1005, 28 p.

White, B.N., R.J. Lavenberg, and G.E. McGowen

1984 Atheriniformes: Development and relationships. In Moser, H.G., et al. (eds.), Ontogeny and systematics of fishes, p. 355-361. Spec. Publ. 1, Am. Soc. Ichthyol. Herpetol. Allen Press, Lawrence, KS.

Wiley, J.W.

1973 Life history of the western North American goby Coryphopterus nicholsi (Bean). Trans. San Diego Soc. Nat. Hist. 17(14):187-208.

Wilimovsky, N.J., A. Peden, and J. Peppar

1967 Systematics of six demersal fishes of the North Pacific Ocean. Fish. Res. Board Can. Tech. Rep. 34, 95 p.

Wingert, R.C.

1974 Comparative reproductive cycles and growth in two species of Xiphister (Pisces, Stichaeidae), from San Simeon, California. M.A. thesis, Calif. State Univ., Fullerton, CA 92631, 91 p.

Wisner, R.L.

1974 The taxonomy and distribution of lanternfishes (family Myctophidae) of the eastern Pacific Ocean. NORDA Rep.-3, Navy Ocean Research and Development Activity, Bay St. Louis, MS, 229 p.

Wolotira, R.J.

1985 Saffron cod (Eleginus gracilis) in western Alaska: The resource and its potential. Tech. Memo. NMFS F/NWC-79, Northwest Alaska Fish. Cent., Natl. Mar. Fish. Serv., NOAA, Seattle, WA 98115-0070, 119 p.

Wolotira, R.J., T.M. Sample, and M. Morin, Jr.

1977 Demersal fish and shellfish resources of Norton Sound, the southeastern Chukchi Sea, and adjacent waters in the baseline year 1976. Proc. Rep., Northwest Alaska Fish. Cent., Natl. Mar. Fish. Serv., NOAA, Seattle, WA 98115-0070, 292 p.

Wooster, W.S.

1983 From year to year: Interannual variability of the environment and fisheries of the Gulf of Alaska and the eastern Bering Sea. Rep. WSG-WO 83-3, Wash. Sea Grant Prog., Univ. Wash., Seattle, WA 98195, 208 p.
Wyllie Echeverria, $T$.

1987 Thirty-four species of California rockfishes: Maturity and seasonality of reproduction. Fish. Bull., U.S. 85:229-250.

Yatsu, A.

1985 Phylogeny of the family Pholididae (Blennioidei) with a redescription of Pholis scopoli. Jpn. J. Ichthyol. 32:273-282.

1986 Phylogeny and zoogeography of the subfamilies Xiphisterinae and Cebidichthyinae (Blennioidei, Stichaeidae). In Uyeno, T., et al. (eds.), Indo-Pacific fish biology: Proceedings of the second international conference on Indo-Pacific fishes, p. 663-678. Ichthyol. Soc. Jpn., Tokyo

Yusa, T.

1957 Eggs and larvae of flatfishes in the coastal waters of Hokkaido. I. Embryonic development of the starry flounder Platichthys stellatus (Pallas). Bull. Hokkaido Reg. Fish. Res. Lab. 15:1-14.

Zama, A., M. Asai, and F. Yasuda

1977a Records of the pelagic armorhead, Pentaceros richardsoni, from Hachijo Island and the Ogasawara Islands. Jpn. J. Ichthyol. 24: 57-60.

1977b Changes with growth in bony cranial projections and colour patterns in the Japanese boarfish, Pentaceros japonicus. Jpn. J. Ichthyol. 24:26-34. 
Acantholiparis 474

Acantholiparis caecus 474

Acantholiparis opercularis 474

Acantholumpenus mackayi 507

Acanthopsetta 573-575

Acanthopsetta nadeshnyi 567, 574, 575

Acanthuroidei $18,556-557$

Agonidae 19, 267, 456, 457, 458-463

Agonidae A 4, 456, 457

Agonomalus mozinoi 4, 456, 458, 459

Agonopsis vulsa 456

Agonus acipenserinus 456

Agonus decagonus 456

Albatrossia pectoralis 200-201

Alectridium aurantiacum 507

Alectrini 504, 505, 507

Alepisauridae $19,125,142-143$

Alepisaurus ferox 142,143

Alepocephalidae $19,51,76$

Alepocephalus tenebrosus 76

Allocyttus sp. 257-258

Allolumpenus hypochromus 507

Allosmerus elongatus 79

Ammodytes hexapterus $4,27,540,541$

Ammodytidae $19,26,27,483,540-541$

Ammodytoidei $18,540-541$

Anarhichantidae 19, 483, 524-527

Anarhichas orientalis 4, 524, 525

Anarrhichthys ocellatus 524, 526, 527

Anguilliformes 18, 31-41

Anisarchus medius 507

Anoplagonus inermis 456

Anoplarchus insignis 507

Anoplarchus purpurescens 4, 27, 505, 507, 512, 513, 514

Anoplogaster cornuta 250, 251

Anoplogastridae 19, 249-251

Anoplopoma fimbria 4, 20, 24, 338, 339

Anoplopomatidae 19, 267, 338-339

Anoplopomatoidei $18,267,338-339$

Anotopteridae 19, 125, 140-141

Anotopterus pharao 140,141

Antimora 182

Antimora microlepis 182

Aphanopus carbo 551

Aphyonidae 19, 211, 217

Apodichthys flavidus 4, 522, 523

Aptocyclus ventricosus 465-466, 467

Archaulus 361

Arctoscopus japonicus 532

Argentina sialis 52, 53

Argentinidae 19, 51-55

Argentinoidei $18,51-77$

Argyropelecus 84, 85, 92-101

Argyropelecus affinis 93-94, 95, 100
Argyropelecus hemigymnus 92-93, 96, 97, 100

Argyropelecus lychnus 93, 98, 99

Argyropelecus sladeni 93, 98, 100, 101

Aristostomias 106, 118-119

Aristostomias scintillans 106, 118, 119

Artediellichthys 361

Artedielliscus 361

Artediellus 361

Artedius 361-362, 384-385, 414-421

Artedius corallinus 420

Artedius fenestralis $414,415,416,418,420$

Artedius harringtoni $363,416,417$

Artedius lateralis 414, 416, 418, 419, 420

Artedius meanyi 361-362, 384, 385

Artedius notospilotus 420

Artedius spp. 414, 416, 418, 420

Artedius 3 414, 416, 418, 420, 421

Ascelichthys 361-363, 386-387

Ascelichthys rhodorus $363,386,387$

Asemichthys 361

Aspidophoroides bartoni 456

Aspidophoroides olriki 456

Atheresthes 573, 576-579

Atheresthes evermanni 567, 576, 577

Atheresthes stomias 5, 567, 576, 578, 579

Atherinidae 19, 235, 238-241

Atheriniformes $18,235,238-241$

Atherinomorpha 235-241

Atherinops affinis $238,239,240$

Atherinopsis californiensis 238, 240, 241

Aulopiformes 18, 125-143

Aulorhynchidae 19, 261-263

Aulorhynchus flavidus 262, 263

Avocettina infans $33,38,39,40$

Barathronus pacificus 217

Barbourisia rufa 256

Barbourisiidae 19, 249, 256

Bassozetus sp. 212

Bathophilus 106, 110-111

Bathophilus flemingi 106, 110,111

Bathyagonus alascanus 456

Bathyagonus infraspinatus 456

Bathyagonus nigripinnis 456

Bathyagonus pentacanthus 456

Bathylaco nigricans 76

Bathylagidae 19, 51, 56-69

Bathylagus bericoides 56,57, 60

Bathylagus milleri 3, 58, 59, 66

Bathylagus ochotensis 3, 56, 60, 61

Bathylagus pacificus $3,56,60,62,63$

Bathylagus wesethi $58,64,65$

Bathylychnops exilis 70, 71, 72, 74

Bathymaster A 497

Bathymaster caeruleofasciatus 496 
Bathymaster leurolepis 496

Bathymaster signatus 496

Bathymasteridae $19,26,27,483,496-497$

Bathysauridae 19, 125, 130-131

Bathysaurus mollis 130,131

Beloniformes 18, 235-237

Benthalbella dentata 126, 127

Benthalbella linguidens 126, 127

Benthodesmus elongatus 551-552, 553

Benthodesmus spp. 552, 553

Benthodesmus tenuis 551-552

Bertella idiomorpha 223

Beryciformes 18, 249-256

Blennioidei 18, 535-537

Blepsias 20, 361, 364, 448-451

Blepsias bilobus 4, 448, 449, 450

Blepsias cirrhosus 4, 22, 448, 450, 451

Boreogadus saida 3, 189-190, 191

Bothragonus swani 456, 460, 461, 462

Bothrocara brunneum 498

Bothrocara hollandi 498, 499

Bothrocara molle 498

Bothrocara pusillum 498

Bothrocara remigerum 498

Brama japonicus 488, 489

Bramidae 19, 483, 488-489

Brosmophycis marginata 3, 217-218, 219

Bryozoichthys lysimus 507

Bryozoichthys marjorius 507

Bythitidae 19, 211, 217-219

Bythitoidei 211, 217-219

Carangidae 19, 483-487

Careproctus 470, 472, 474

Careproctus abbreviatus 474

Careproctus attenuatus 474

Careproctus bowersianus 474

Careproctus cameliae 474

Careproctus canus 474

Careproctus colletti 474

Careproctus cypselurus 474

Careproctus ectenes 474

Careproctus filamentosus 474

Careproctus furcellus 474

Careproctus gilberti 474

Careproctus longifilis 474

Careproctus melanurus 474

Careproctus microstomus 474

Careproctus mollis 474

Careproctus opisthotremus 474

Careproctus oregonensis 474

Careproctus ostentum 474

Careproctus ovigerum 474

Careproctus pellucidus 474

Careproctus phasma 474

Careproctus pycnosoma 474

Careproctus rastrinus 474

Careproctus scottae 474
Careproctus simus 474

Careproctus spectrum 474

Careproctus zachirus 474

Caristiidae 19, 490, 491

Caristius macropus 490

Cataetyx rubrirostris 217

Cebidichthys violaceus 507

Centrolophidae 19, 483, 558-559

Ceratias holboelli 222-223

Ceratias sp. 222-223

Ceratiidae 221-223

Ceratioidei 221-227

Ceratoscopelus 146-147, 160-161

Ceratoscopelus townsendi 146, 160, 161

Cetomimidae 249, 256

Cetomimoidei 249,256

Cetomimus 256

Cetostoma regani 256

Chaenophryne longiceps 223-224, 225

Chaenophryne melanorhabus 223

Chauliodontidae 19, 83, 106-109

Chauliodus 106-109

Chauliodus macouni 106, 108, 109

Chilara taylori 212, 214, 215

Chirolophini 500, 501, 507

Chirolophis decoratus 507

Chirolophis nugator 507

Chirolophis snyderi 507

Chirolophis tarsodes 507

Chitonotus 361-362, 388-389

Chitonotus pugetensis 4, 388, 389

Citharichthys sordidus 5, 567-568, 569

Citharichthys stigmaeus 567, 570, 571

Clevelandia ios 4, 543-544, 545, 548

Clidoderma asperrimum 567

Clinidae 19, 483, 535-537

Clinocottus 361-362, 422-428

Clinocottus acuticeps $360,422,423$

Clinocottus embryum 424, 425

Clinocottus globiceps 426, 427

Clinocottus recalvus 426, 428, 429

Clupea pallasi $3,18,27,44,45,46,48$

Clupeidae 19, 26, 27, 43-47

Clupeiformes 18, 43-49

Cololabis saira $3,20,25,236,237$

Congridae $19,31-33,36,37$

Coryphaenoides acrolepis 200-202, 203

Coryphaenoides armatus 200-201

Coryphaenoides cinereus 200-201

Coryphaenoides filifer 200-201, 204, 205

Coryphaenoides leptolepis 200-201, 206, 207

Coryphaenoides liocephalus 200

Coryphaenoides longifilis 200

Coryphaenoides yaquinae 200

Coryphopterus nicholsi 4, 543-544, 546, 547, 548

Cottidae $18-19,267,360-362,363,364,365,366-455$

Cottoid A 436, 438, 439 
Cottoidei $18,267,360-455$

Cottus 361, 364, 444-445

Cottus aleuticus 444

Cottus asper 444, 445

Cryptacanthodidae $19,483,520,521$

Crystallichthys cyclospilus 474

Crystallichthys mirabilis 474

Cyclopteridae 4, 18-19, 267, 465-481

Cyclopterinae 465-469

Cyclopteropsis phrynoides 465

Cyclothone $84,86-88,89,90$

Cyclothone acclinidens 87-88, 89

Cyclothone atraria $87-88,89$

Cyclothone pallida 87-88, 89

Cyclothone pseudopallida 87-88, 89

Cyclothone signata 87-88, 89

Cyema atrum 33-34, 35

Cyematidae 19, 31-34, 35

Cynoglossidae $19,565,567,624-625$

Danaphos 84, 86, 92, 104-105

Danaphos oculatus 3, 93, 104, 105

Dasycottus 361, 364, 440-441

Dasycottus setiger 440, 441

Delolepis gigantea 4, 20, 25, 520, 521

Derepodichthys alepidotus 498

Diaphini 146, 176-177

Diaphus 146-147, 176-177

Diaphus theta 3, 146, 172, 176, 177

Dicrolene filamentosa 212

Dolichopteryx sp. 72, 73, 74

Dorsadena 146-147

Dorsadena yaquinae 146

Elassodiscus caudatus 474

Elassodiscus tremebundus 474

Electrona 146-149

Electrona rissoi $146,148,149$

Electronini $146-153$

Eleginus gracilis 189, 192, 193

Elopomorpha 31-41

Embassichthys 573, 580-581

Embassichthys bathybius 212, 567, 580, 581, 622

Engraulididae 19, 26, 27, 43, 48-49

Engraulis mordax 27, 44, 46, 48, 49

Enophrys 361-362, 390-391

Enophrys bison 390, 391

Enophrys diceraus 390

Enophrys lucasi 390

Eopsetta 567, 573, 582-583

Eopsetta jordani 567, 582, 583

Ericara salmoneum 76

Erilepis zonifer 338

Eumesogrammus praecisus 507

Eumicrotremus andriashevi 465

Eumicrotremus barbatus 465

Eumicrotremus birulai 465

Eumicrotremus gyrinops 465

Eumicrotremus orbis 465, 468, 469
Eumicrotremus soldatovi 465

Eumicrotremus taranetzi 465

Eurymen 361

Eurypharyngidae $19,32-33,36,37$

Eurypharynx pelecanoides 33, 37

Eustomias 106, 112, 113

Eustomias sp. 106, 112, 113

Gadidae 18-19, 181, 189-199

Gadiformes 18, 181-209

Gadus macrocephalus 3, 189, 194, 195, 196, 198

Gasterosteidae 261

Gasterosteiformes 18, 261-265

Genyonemus lineatus 492, 493

Gibbonsia metzi 535

Gibbonsia montereyensis 535

Gilbertidia 361, 364, 435-436

Gilbertidia sigalutes 434, 435, 436

Glyptocephalus 567, 573, 584-587

Glyptocephalus stelleri 567, 580, 584, 585

Glyptocephalus zachirus 5, 567, 580, 584, 586, 587, 622

Gobiesocidae 19, 229-233

Gobiesociformes 18, 229-233

Gobiesox maeandricus 3,230, 231, 232

Gobiidae 19, 483, 543-549

Gobioidei 18, 543-549

Gonichthyini $146,156-159$

Gonostoma 84, 86, 88, 90

Gonostoma atlanticum 87-88, 90, 91

Gonostoma gracile $84,87-88,90,91$

Gonostomatidae 19, 83, 87-91

Gonostomatoidei 86-105

Gymnelis hemifasciatus 498

Gymnelis popovi 498

Gymnelis viridis 498, 499

Gymnocanthus 361-362, 392, 393, 394-395

Gymnocanthus A 392, 393

Gymnocanthus detriscus 392

Gymnocanthus galeatus 392

Gymnocanthus pistilliger 392

Gymnocanthus tricuspis 392, 394, 395

Gymnoclinus cristulatus 507

Gymnoscopelini 146, 178-179

Gyrinichthys minytremus 474

Gyrinomimus sp. 256

Hemilepidotus 361-362, 368-381

Hemilepidotus gilberti 368, 370, 372, 373

Hemilepidotus hemilepidotus $368,369,370,371,374$, $375,376,380$

Hemilepidotus jordani 4, 368, 369, 370, 371, 376, 377, 380

Hemilepidotus papilio 368,370

Hemilepidotus spinosus $21,363,368,370,371,378,379$

Hemilepidotus spp. 20,369,370,382

Hemilepidotus zapus $368,369,370,371,380,381$

Hemitripterus 361, 364, 452-453

Hemitripterus bolini 452

Hemitripterus villosus 20, 23, 365, 452, 453 
Heterostichus rostratus 535-536, 537

Hexagrammidae $19,267,341-359$

Hexagrammoidei $18,267,341-359$

Hexagrammos 20, 341, 350, 351, 352-359

Hexagrammos decagrammus $341,350,351,352,353$

Hexagrammos lagocephalus $341,350,351,354,355$

Hexagrammos octogrammus 24, 341, 350, 351, 356, 357

Hexagrammos stelleri $341,350,351,358,359$

Hippoglossoides 573, 588-591

Hippoglossoides elassodon $15,18,567,588,589,590$

Hippoglossoides robustus 567, 590, 591

Hippoglossus 573, 592-593

Hippoglossus stenolepis 5, 567, 592, 593, 622

Histiobranchus bathybius 33-34

Holcomycteronus profundissimus 212

Holtbyrnia innesi 77

Holtbyrnia latifrons 77

Hypomesus pretiosus 79

Hypsagonus quadricornus 456, 457

Icelinus 361-362, 384, 388, 396, 397

Icelinus A 396, 397

Icelinus B 396, 397

Icelinus borealis 396

Icelinus burchami 396

Icelinus filamentosus 396

Icelinus fimbriatus 396

Icelinus tenuis 396

Icelus 361-362, 399

Icelus canaliculatus 399

Icelus euryops 399

Icelus scutiger 399

Icelus spatula 399

Icelus spiniger 399

Icelus uncinalis 399

Icichthys lockingtoni 558, 559, 560

Icosteidae 19, 483, 538-539

Icosteoidei $18,538-539$

Icosteus aenigmaticus 538, 539

Idiacanthidae $19,83,106,120-123$

Idiacanthus 106, 120-123

Idiacanthus antrostomus 106, 120, 121, 122

Idiacanthus fasciola 120, 122, 123

Inopsetta ischyra 567

Isopsetta 567, 573, 594-595

Isopsetta isolepis 567, 594, 595

Jordania 361

Krusensterniella pavlovskii 498

Laemonema 182

Laemonema longipes 182

Lampadena 146-147, 162-163

Lampadena urophaos 146, 162, 163

Lampanyctinae 146-147, 160-179

Lampanyctini $146,160-175$

Lampanyctus 147, 165-169

Lampanyctus fernae 146, 165

Lampanyctus jordani 146, 165

Lampanyctus regalis 3, 146, 165-166, 167, 168
Lampanyctus ritteri 146, 165, 168, 169

Lampridae 19, 243-245

Lampriformes 18, 243-247

Lampris guttatus 244, 245

Lepidogobius lepidus 4, 543-544, 548, 549

Lepidopsetta 567, 573, 596-599, 600-601

Lepidopsetta bilineata $5,18,567,596,597,599,600-601$

Lepidopsetta 2 5, 599, 600-601

Lepidopus fitchi 551

Leptochilichthys agassizi 76

Leptocottus 361, 364, 446-447

Leptocottus armatus 365, 446, 447

Lestidiops ringens $134,135,136,138$

Lethotremus muticus 465

Leuroglossus schmidti 66, 67, 68

Leuroglossus stilbius 66-68, 69

Limanda 567, 573, 602-603

Limanda aspera 567, 602, 603

Limanda proboscidea 567, 602, 603

Limanda sakhalinensis 602

Liopsetta 567, 573, 604-605

Liopsetta glacialis 567, 604, 605

Liparidinae 465, 470, 471-473, 474-481

Liparis $470,471,474-475$

Liparis bristolensis 474

Liparis callyodon 474

Liparis catharus 475

Liparis cyclopus 475

Liparis dennyi 475

Liparis florae 475

Liparis fucensis 475

Liparis gibbus 475

Liparis grebnitzki 475

Liparis mednius 475

Liparis megacephalus 475

Liparis micraspidophorus 475

Liparis mucosus 475

Liparis ochotensis 475

Liparis pulchellus 475

Liparis rutteri 475

Liparis tunicatus 475

Lipariscus nanus 475

Lophiiformes 18, 221-227

Loweina 147, 156-157

Loweina rara $146,156,157,158$

Lumpenella longirostris 503, 507

Lumpeninae 502-505, 507

Lumpenini 502, 503, 507

Lumpenus fabricii 507

Lumpenus maculatus 503, 507

Lumpenus medius 507

Lumpenus sagitta 4, 503, 507

Luvaridae 19, 483, 556-557

Luvarus imperialis 556, 557

Lycenchelys altus 498

Lycenchelys camchaticus 498

Lycenchelys crotalinus 498 
Lycenchelys hippopotamus 498

Lycenchelys jordani 498

Lycenchelys longirostris 498

Lycenchelys microporus 498

Lycenchelys pliciferus 498

Lycenchelys rassi 498

Lycenchelys ratmanovi 498

Lycenchelys roseus 498

Lycenchelys volki 498

Lycodapus derjugini 498

Lycodapus dermatinus 498

Lycodapus endemoscotus 498

Lycodapus fierasfer 498

Lycodapus leptus 498

Lycodapus mandibularis 498

Lycodapus pachysoma 498

Lycodapus parviceps 498

Lycodapus poecilus 498

Lycodapus psarosomatus 498

Lycodes brevipes 498

Lycodes concolor 498

Lycodes cortezianus 498

Lycodes diapterus 498

Lycodes mucosus 498

Lycodes pacifica 498

Lycodes palearis 498

Lycodes raridens 498

Lycodes turneri 498

Lyconectes aleutensis 4, 20, 25, 520, 521

Lyconema barbatum 498

Lyopsetta 573, 606-607

Lyopsetta exilis $567,586,606,607,608$

Macropinna microstoma 3, 72, 74, 75

Macrouridae 18-19, 181, 200-209

Malacocottus $361,364,442-443$

Malacocottus kinkaidi 442

Malacocottus zonurus 365, 442, 443

Malacosteidae $19,83,106,118-119$

Mallotus villosus 26, 27, 79-80, 81

Maulisia argipalla 77

Megalocottus 361

Melamphaes 252, 253, 254-255

Melamphaes lugubris 252, 253, 254, 255, 336

Melamphaes parvus 254

Melamphaidae $19,249,252,253,254-255$

Melanonidae $19,181,184-185$

Melanonus zugmayeri 3, 184, 185

Melanostigma pammelas 498

Melanostomiidae $19,83,106,110-117$

Merlucciidae 19, 181, 186-187

Merluccius productus 1, 186, 187, 486

Microcottus 361

Microgadus proximus $189,196,197$

Microstomus 573, 608-609

Microstomus pacificus 5, 567, 586, 608, 609

Mola mola 18, 628, 629

Molidae 19, 627-629
Moridae 19, 181-182, 183

Myctophidae 19, 125, 146-179

Myctophiformes 18, 125, 144-179

Myctophinae 146-159

Myctophini 146, 154-155

Myoxocephalus $361-362,400,401,402-403$

Myoxocephalus axillaris 400

Myoxocephalus B 400, 401

Myoxocephalus brandti 400

Myoxocephalus G 20,21,363, 400, 401

Myoxocephalus jaok 400

Myoxocephalus niger 400

Myoxocephalus polyacanthocephalus 400, 401

Myoxocephalus quadricornis 400

Myoxocephalus scorpioides 400

Myoxocephalus scorpius 400, 402, 403

Myoxocephalus stelleri 400

Myoxocephalus verrucosus 400, 402

Nalbantichthys elongatus 498

Nansenia candida $3,52,54,55$

Narcetes stomias 76

Naucrates ductor 484, 485

Nautichthys $361,364,454-455$

Nautichthys oculofasciatus 4, 20, 23, 454, 455

Nautichthys pribilovius 454

Nautichthys robustus 454

Nectoliparis pelagicus 4, 470, 475-476, 477

Nemichthyidae $19,31-33,38-41$

Nemichthys larseni 33, 40

Nemichthys scolopaceus $33,40,41$

Neoscopelidae 19, 125, 144, 145

Neoscopelus macrolepidotus 144-145

Nettastomatidae 19, 31-33, 36, 37

Nezumia stelgidolepis 200, 208-209

Notacanthidae $31-34,35$

Notacanthiformes 18, 31-35

Notacanthus chemnitzi 33-34

Notolepis rissoi 134, 136, 137

Notoscopelus 146-147, 178-179

Notoscopelus japonicus 178

Notoscopelus resplendens 146, 178, 179

Notosudidae 19, 125, 128-129

Ocella dodecaedron 456

Ocella impi 456

Ocella verrucosa 456,457

Odontoliparis ferox 475

Odontopyxis trispinosa 456

Oligocottus 361-362, 430-433

Oligocottus maculosus 430, 431, 432

Oligocottus rimensis 430

Oligocottus snyderi $430,432,433$

Oneirodes bulbosus 223, 226-227

Oneirodes thompsoni 223

Oneirodidae 19, 221-227

Opaeophacus acrogeneius 498

Ophidiidae 3, 19, 211-212, 213, 214-215

Ophidiiformes 18, 211-219 
Ophidioidei 211-215

Ophiodon elongatus $20,24,341,346,347$

Opisthocentrini 504, 505, 507

Opisthocentrus ocellatus 505, 507-508, 509

Opisthoproctidae $19,51,70-75$

Opostomias 106, 114-115

Opostomias mitsuii 106, 114, 115

Oreosomatidae 19, 257-259

Osmeridae $19,26,27,51,79-81$

Osmerus mordax 79

Osteodiscus cascadiae 475

Oxylebius pictus $341-342,343,344$

Pachycara bulbiceps 498

Pallasina barbata 456

Paralepididae 19, 125, 134-139

Paralepis atlantica 134, 138, 139

Paralichthyidae $19,565,567-571$

Paraliparis 470, 472, 475, 478-479

Paraliparis cephalus 475

Paraliparis dactylosus 475

Paraliparis deani 470, 475, 478

Paraliparis holomelas 475

Paraliparis latifrons 475

Paraliparis megalopis 475

Paraliparis melanobranchus 475

Paraliparis mento 475

Paraliparis paucidens 475

Paraliparis pectoralis 475

Paraliparis rosaceus 475

Paraliparis sp. 4, 470, 478, 479

Paraliparis ulochir 475

Paricelinus 361-362, 404-405

Paricelinus hopliticus 404, 405

Parophrys 567, 573, 610-611

Parophrys vetulus 5, 567, 594, 610, 611

Parvilux 147, 170-171

Parvilux ingens 146, 170, 171

Pelagocyclus vitiazi 465

Pellisolus eubranchus 77

Pentacerotidae 19, 483, 494, 495

Peprilus simillimus 562, 563

Perciformes 18, 483-563

Percis japonicus 456

Percoidei 18, 484-495

Phallocottus 361

Pholididae 19, 26, 27, 483, 522, 523

Pholis sp. 4, 27, 563

Pholis clemensi 522

Pholis dolichogaster 522

Pholis fasciata 522

Pholis gilli 522

Pholis laeta 522

Pholis ornata 522

Pholis schultzi 522

Phytichthys chirus 507, 512, 514, 515

Platichthys 573, 612-613

Platichthys stellatus 5, 567, 594, 612, 613
Platytroctidae $19,51,77$

Plectobranchus evides 4, 505, 507, 510, 511

Pleurogrammus monopterygius 20, 341, 348, 349

Pleuronectes 567, 573, 614-615

Pleuronectes quadrituberculatus 5, 567, 614, 615

Pleuronectidae $18-19,565,567,573-623$

Pleuronectiformes $18,565-625$

Pleuronichthys 18, 20, 573, 616-619

Pleuronichthys coenosus 567, 616, 617, 618

Pleuronichthys decurrens 22, 567, 616, 618, 619

Polyacanthonotus challengeri 33-34

Polypera beringiana 475

Polypera greeni 475

Poroclinus rothrocki 503, 507

Porocottus 361

Poromitra 252, 253

Poromitra crassiceps 253

Protomyctophum 147-148, 150-153

Protomyctophum crockeri 146, 150, 151, 152

Protomyctophum thompsoni 146, 150, 152, 153

Psettichthys 573, 599, 600-601, 620-621

Psettichthys melanostictus 5, 567, 594, 599, 600-601, 620,621

Pseudopentaceros spp. 494, 495

Psychrolutes 361, 364, 436-437

Psychrolutes paradoxus 4, 365, 434, 436, 437

Psychrolutes phrictus 436, 438

Ptilichthyidae 19, 483, 528-529

Ptilichthys goodei 528, 529

Puzanovia rubra 498

Radulinus 20, 361-362, 406-409

Radulinus asprellus 23, 406, 407, 408

Radulinus boleoides 408, 409

Rastrinus scutiger 399

Reinhardtius 573, 622-623

Reinhardtius hippoglossoides 567, 580, 622, 623

Rhamphocottus 361-362, 366-367

Rhamphocottus richardsoni 20,21, 363, 366, 367

Rhinoliparis attenuatus 475

Rhinoliparis barbulifer $470,475,480,481$

Rimicola muscarum 232, 233

Rondeletia loricata 256

Rondeletiidae 19, 249, 256

Ronquilus jordani 4, 27, 496, 497

Sagamichthys abei 77

Salmonidae 51

Salmoniformes 18, 51-81

Salmonoidei $18,79-81$

Sardinops sagax 44, 46, 47

Sarritor frenatus 456

Sarritor leptorhynchus 456

Sciadonus pedicellaris 217

Sciaenidae 19, 483, 492-493

Scomberesocidae 19, 235-237

Scomber japonicus 486, 492, 554, 555

Scombridae 19, 483, 554-555

Scombroidei 18, 551-555 
Scopelarchidae $19,125-127$

Scopeloberyx 252, 253

Scopeloberyx robustus 253

Scopelogadus 252, 253

Scopelogadus bispinosus 253

Scopelomorpha 125-179

Scopelosarus harryi 128,129

Scorpaenichthys 362-362, 382-383

Scorpaenichthys marmoratus 20, 22, 382, 383, 406

Scorpaenidae $19,267-337$

Scorpaeniformes $18,267-481$

Scorpaenoidei $18,267-337$

Sebastes $267-272,273-280,281,282-287,288-335$

Sebastes aleutianus 269,271, 273, 283, 288, 289

Sebastes alutus 269, 271, 273, 283

Sebastes auriculatus 269, 271, 273, 285, 290, 291, 296

Sebastes aurora 269, 271, 274, 287, 292, 293

Sebastes babcocki 269, 271, 274, 287, 294, 295

Sebastes borealis 269, 271

Sebastes brevispinis 3, 269,271, 274, 285

Sebastes caurinus 3, 269, 271, 275, 284, 290, 296, 297

Sebastes chlorostictus 269,271, 275, 287

Sebastes ciliatus 269, 271, 275

Sebastes crameri 269, 271, 275, 284, 298, 299

Sebastes diploproa $269,271,276,283,300,301$

Sebastes elongatus $269,271,276,287,302,303$

Sebastes emphaeus $269,271,286,304,305$

Sebastes entomelas 269, 271, 276, 282, 306, 307

Sebastes flavidus $269,271,276,282,308,309,314$

Sebastes glaucus 269, 271

Sebastes goodei 269, 271, 277, 284

Sebastes helvomaculatus $269,271,277,287,310,311$

Sebastes jordani $3,269,271,277,286,312,313$

Sebastes maliger 269, 271, 277

Sebastes melanops $3,269,271,277,282,308,314,315$

Sebastes melanostictus 269, 271

Sebastes melanostomus $269-271,277,284,316,317$

Sebastes miniatus $269,271,278,282,318,319$

Sebastes mystinus $269,271,278,282,320,321$

Sebastes nebulosus 269, 271

Sebastes nigrocinctus 269, 271, 284

Sebastes paucispinis $269,271,278,283,322,323$

Sebastes pinniger 269, 271, 278, 282, 324, 325

Sebastes polyspinis 3,269,271, 278, 286

Sebastes proriger $269,271,278,285,326,327$

Sebastes rastrelliger 269, 271, 285

Sebastes reedi $269,271,279,284,328,329$

Sebastes ruberrimus $269,271,279,287$

Sebastes rufus $3,269,271,279,286,330,331$

Sebastes saxicola $269,271,279,284,332,333$

Sebastes variegatus $3,269,271,280$

Sebastes wilsoni 269, 271, 286

Sebastes zacentrus 3, 269, 271-272, 280, 285, 334, 335

Sebastinae 268-335

Sebastolobinae 268, 336-337

Sebastolobus 268, 336-337

Sebastolobus alascanus 336, 337
Sebastolobus altivelis 336,337

Sebastolobus macrochir 336

Sebastolobus spp. 254, 336, 337

Serrivomer jesperseni $33,36,37$

Serrivomeridae $19,31-33,36,37$

Sigmistes 361

Spectrunculus grandis 211-212, 213

Spectrunculus radcliffei 212

Spirinchus starksi 79

Spirinchus thaleichthys 79

Stelgistrum 361

Stellerina xyosterna 456,457

Stenobrachius 146-147, 172-173

Stenobrachius leucopsarus 3, 146, 172, 173, 176

Stenobrachius nannochir 146, 172

Sternias 361

Sternoptychidae $19,83,92-105$

Sternoptyx 84, 86, 92, 102, 103

Sternoptyx diaphana 93, 102, 103

Sternoptyx pseudobscura 93, 102, 103

Sternoptyx spp. 102, 104

Stichaeidae $19,26,27,483,500,501,502,503,504$, 505, 507-519

Stichaeinae $500-501,507$

Stichaeini 496, 500, 501, 507

Stichaeus punctatus 496, 501, 507

Stlegicottus 361

Stomiiformes $18,83-84,85,86-123$

Stomioidei $107-123$

Stromateidae $19,483,562-563$

Stromateoidei 18, 558-563

Symbolophorus 147, 154-155

Symbolophorus californiensis 146, 154, 155

Symphurus atricauda 567, 624, 625

Synaphobranchidae $19,31-34,35$

Synchirus 361-362, 410-411

Synchirus gilli 4, 410, 411

Syngnathidae $19,261,264,265$

Syngnathus leptorhynchus 264

Synodontidae 19, 125, 132-133

Synodus lucioceps 132, 133

Taaningichthys 146-147, 174, 175

Taaningichthys bathyphilus 146, 174-175

Tactostoma 106, 116-117

Tactostoma macropus 3, 106, 116, 117

Talismania bifurcata 76

Taractes asper 488

Taranetzella lycoderma 498

Tarletonbeania 147, 158-159

Tarletonbeania crenularis $146,156,158,159$

Tarletonbeania taylori 146, 158

Taurocottus 361

Temnocora candida 475

Tetragonuridae $19,483,560-561$

Tetragonurus cuvieri 558, 560, 561

Tetraodontiformes 18, 627-629

Thalassenchelys coheni 3, 32-34, 35 
Thaleichthys pacificus 79

Thecopterus 361

Theragra chalcogramma $1,3,189-190,194,196,198,199$

Thyriscus 361

Trachinoidei $18,532-533$

Trachipteridae $19,243,246-247$

Trachipterus altivelis 3, 244, 246, 247

Trachurus symmetricus 486, 487

Trichiuridae 19, 483, 551-552, 553

Trichodon trichodon 532, 533

Trichodontidae $19,483,532-533$

Triglops $361-362,412,413$

Triglops A 412,413

Triglops B 412, 413

Triglops forficata 412

Triglops jordani 412

Triglops macellus 412

Triglops metopias 412

Triglops pingeli 412

Triglops scepticus 412, 413

Venefica sp. 33, 36

Xeneretmus latifrons 456, 460, 462, 463

Xeneretmus leiops 456

Xeneretmus triacanthus 456

Xenocongridae $19,31-34,35$

Xenomystax atrarius 33,36

Xererpes fucorum 522

Xiphister atropurpureus 4, 505, 507, 516, 517, 518

Xiphister mucosus 507, 516, 518, 519

Xiphisterinae 504-505, 507

Xiphisterini 504, 505, 507

Zaniolepis frenata $341,344-345$

Zaniolepis latipinnis 341, 344-345

Zaniolepis spp. $341-342,344,345$

Zaprora silenus 20, 24, 530, 531

Zaproridae $19,483,530-531$

Zeiformes 18, 257-259

Zesticelus 361

Zoarcidae 19, 483, 498, 499

Zoarcoidei 18, 496-531

Italic page numbers indicate illustrations. 
Anchovies 43, 48-49

Anchovy, northern 48, 49

Argentine

bluethroat 54,55

Pacific 52, 53

Argentines 52-55

Armorheads 494, 495

Arrowtail 184,185

Arrowtails 184-185

Barracudina

duckbill 138,139

ribbon 136,137

slender 134,135

Barracudinas 134-139

Barreleye 74, 75

Bigscale, highsnout 254,255

Bigscales 252, 253, 254-255

Blackdragon, Pacific 120,121

Blackdragons 120-123

Blacksmelt

Pacific 62, 63

popeye 60,61

robust 58,59

snubnose 64,65

Blenny, ocellated 508, 509

Bocaccio 322, 323

Bottlelights 104,105

Bristlemouths $87-88,89,90,91$

Brotula, red 218, 219

Brotulas 218-219

Butterfishes 562-563

Cabezon 382, 383

Capelin 80,81

Clingfish

kelp 232, 233

northern 230,231

Clingfishes 229-233

Cockscomb, high 512,513

Cod

Arctic 190,191

Pacific 194, 195

saffron 192,193

Codfishes 189-199

Codlings 182,183

Combfishes 344,345

Croaker, white 492, 493

Croakers 492-493

Cusk-eel, spotted 214, 215

Cusk-eels 212, 213, 214-215

Cutlassfishes $551-552,553$

Daggertooth 140,141

Daggertooths 140-141

Deepsea smelts 51, 56-69
Dragonfish

highfin 110,111

longfin 116,117

pitgum 114,115

Dragonfishes, scaleless 110-112, I13, 114-117

Dreamer, smoothhead 224, 225

Dreamers 222-227

Eelpouts 498,499

Eels $31-34,35,36,37,38-41$

Fangtooth 250, 251

Fangtooths 250-251

Flashlightfish

California 150,151

chubby 148,149

northern 152,153

Flounder

Arctic 604, 605

arrowtooth 578,579

Bering 590, 591

Kamchatka 576, 577

long 584,585

spiny 574,575

starry 612,613

Flounders

lefteye 568-571

righteye 573-623

Glowingfish 144-145

Gobies 543-549

Goby

arrow 544,545

bay 548,549

blackeye 546,547

Greenling

kelp 352,353

masked 356,357

painted 342,343

rock 354,355

whitespotted 358,359

Greenlings $341-350,351,352-359$

Grenadier

ghostly 206, 207

Pacific 202, 203

threadfin 204,205

Grenadiers 200-209

Gunnels 522, 523

Hake, Pacific 186,187

Hakes 186-187

Halibut

Greenland 622, 623

Pacific 592, 593

Hatchetfish

lowcrest 100,101

slender 94, 95

spurred 96, 97

tropical 98,99 
Hatchetfishes 92-105

Headlightfish, California 176, 177

Herring, Pacific 44, 45

Herrings 43-47

Irish lord

banded 372,373

brown 378,379

longfin 380,381

red 374,375

yellow 376,377

Irish lords $368,369,370,371,372-381$

Jacks 484-487

Jacksmelt 240, 241

Kelpfish, giant 536, 537

Kelpfishes 535-537

King-of-the-salmon 246, 247

Lampfish

broadfin 168,169

dogtooth 160,161

giant 170,171

patchwork 178,179

pinpoint 166,167

sunbeam 162,163

Lancetfish, longnose 142,143

Lancetfishes 142-143

Lanternfish

blue 158,159

California 154,155

northern 172,173

Lanternfishes $146-179$

Lingcod 346, 347

Lizardfish

California 132,133

highfin 130,131

Lizardfishes 132-133

Lizardfishes, deepsea 130-131

Loosejaw, shiny 118,119

Loosejaws 118-119

Louvar 556,557

Louvars 556-557

Lumpsucker

Pacific spiny 468,469

smooth 466,467

Lumpsuckers 465-469

Mackerel

Atka 348,349

chub 554,555

jack 486,487

Mackerels 554-555

Medusafish 558, 559

Medusafishes 558-559

Molas 628-629

Opah 244, 245

Opahs 244-245

Oreos 258, 259

Paperbone, scaly 128,129

Paperbones 128-129
Pearleyes 126,127

Pilotfish 484, 485

Pipefishes 264, 265

Plaice, Alaska 614, 615

Poacher

blacktip 462,463

kelp 458,459

Poachers 456, 457, 458-463

Pollock, walleye 198, 199

Pomfret, Pacific 488, 489

Pomfrets 488-489

Pompano, Pacific 562, 563

Prickleback

black 516,517

bluebarred 510,511

ribbon 514,515

rock 518,519

Pricklebacks 500, 501, 502, 503, 504, 505, 507-519

Prowfish 530, 531

Prowfishes 530-531

Quillfish 528, 529

Quillfishes 528-529

Ragfish 538, 539

Ragfishes 538-539

Ribbonfishes 246-247

Rockfish

aurora 292,293

bank 330,331

black 314,315

blackgill 316,317

blue 320,321

brown 290,291

canary 324,325

copper 296, 297

darkblotched 298, 299

greenstriped 302,303

Puget Sound 304, 305

redbanded 294, 295

redstripe 326,327

rosethorn 310,311

rougheye 288,289

sharpchin 334,335

shortbelly 312,313

splitnose 300,301

stripetail 332,333

vermilion 318,319

widow 306,307

yellowmouth 328,329

yellowtail 308,309

Rockfishes $268-272,273-280,281,282-287,288-335$

Rockhead 460,461

Ronquils 496, 497

Sablefish 338,339

Sablefishes 338-339

Sanddab

Pacific 568, 569

speckled 570,571 
Sanddabs 568-571

Sandfish, Pacific 532, 533

Sandfishes 532-533

Sandlance, Pacific 540, 541

Sandlances 540-541

Sardine, Pacific 46, 47

Sauries 236-237

Saury, Pacific 236, 237

Sculpin

Arctic staghorn 394,395

bald 428,429

buffalo 390,391

calico 424,425

crested 448,449

darkfin 442,443

darter 408, 409

fluffy 432,433

grunt 366,367

manacled 410,411

mosshead 426, 427

Pacific staghorn 446, 447

padded 414,415

prickly 444,445

Puget Sound 384, 385

rosylip 386,387

roughback 388,389

sailfin 454,455

scalyhead 416,417

sharpnose 422,423

shorthorn 402, 403

silverspotted 450,451

$\operatorname{slim} 406,407$

smoothhead 418,419

soft 434,435

spinyhead 440,441

tadpole 436, 437

thornback 404, 405

tidepool 430, 431

Sculpins $360-362,363,364,365,366-455$

Seadevils 222-223

Sea raven, shaggy 452,453

Silversides 238-241

Skilfish 338

Slickheads 76

Smelts 51, 79-81

Smoothtongue

California 68,69

northern 66,67

Snailfish

longnose 480,481

prickly 478,479

tadpole 476,477

Snailfishes $465,460,471-473,474-481$

Snipe eel

blackline 38,39

slender 40,41

Spiny eels $31-35$
Sole

butter 594,595

C-O 616,617

curlfin 618,619

deepsea 580,581

Dover 608, 609

English 610,611

flathead 588,589

petrale 582,583

rex 586,587

rock 596,597

sand 620,621

slender 606,607

yellowfin 602,603

Spookfish

javelin 70,71

winged $72-73$

Spookfishes 70-72, 73, 74-75

Squaretail, smalleye 560,561

Squaretails $560-561$

Sunfish, ocean 628,629

Thornyheads 336, 337

Tomcod, Pacific 196, 197

Tonguefish, California 624,625

Tonguefishes $624-625$

Topsmelt 238, 239

Tubeshoulders 77

Tube-snout 262, 263

Tubesnouts 262-263

Veilfin, bigmouth 490

Veilfins 490, 491

Viperfish, Pacific 108, 109

Viperfishes 108-109

Whalefishes 256

Wolf-eel 526, 527

Wolffish, Bering 524, 525

Wolffishes 524-527

Wrymouths 521,522

Italic page numbers indicate illustrations. 